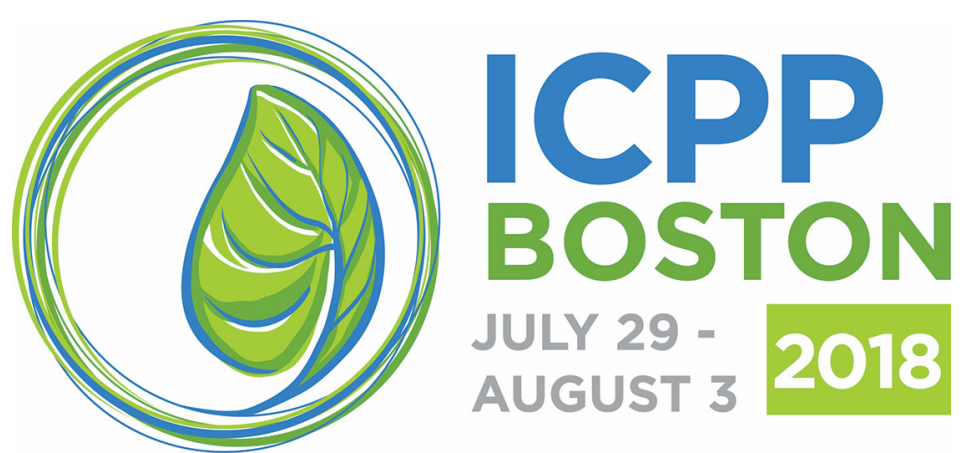

\title{
ICPP2018 Abstracts of Poster Presentations
}

\author{
Abstracts submitted for presentation at ICPP2018 in Boston, Massachusetts, U.S.A., July 29-August 3, 2018. The recommended format for citing \\ congress abstracts, using the first abstract below as an example, is as follows:
}

Kim, D. H. 2018. Comparative transcriptomic analysis of MAPK-mediated regulation of sectorization in Cryphonectria parasitica. (Abstr.) Phytopathology 108:S1.1. https://doi.org/10.1094/PHYTO-108-10-S1.1 The abstracts are published as a supplement to Phytopathology for citation purposes. They were not reviewed by the Phytopathology Editorial Board and
were not edited by the APS editorial staff. Please send questions or comments to aps@scisoc.org.

https://doi.org/10.1094/PHYTO-108-10-S1.1

(C) 2018 The American Phytopathological Society

Comparative transcriptomic analysis of MAPK-mediated regulation of sectorization in Cryphonectria parasitica D. H. KIM, Chonbuk National University, Jeonju, SOUTH KOREA

Fungal phenotypic sectorization is a complex trait and still not fully understood. The unique phenotypic change of sporadic sectorization in the mutants of $C p B c k 1$, a MAPKKK gene, and CpSlt2, a MAPK gene, in cell wall integrity pathway of the chestnut blight fungus Cryphonectria parasitica have been previously studied. Although there are several environmental and physiological factors causing this sectoring phenotype, genetic variants can also impact this complex morphogenesis. Therefore, transcriptome analysis was employed to discover candidate genes that are associated with the sectorization traits and to understand the genetic mechanism of this phenotype. Transcriptome analysis of the mutants of $C p B c k 1$ and $C p S l t 2$, and their sectored progenies revealed a number of differentially expressed genes (DEG) related to various cellular processes. Functional description of DEG's between the parental mutants and their corresponding sectored progenies revealed several key pathways including the biosynthesis of secondary metabolites, amino acid metabolism, and carbohydrate metabolism, among which the pathway for secondary metabolism appeared the most represented pathway. Our results of this comparative study provide better understanding the basis of genetic regulation of fungal sector formation and suggest a complex interplay between the secondary metabolites and morphogenesis.

Regulation of the Cryparin, Hydrophobin, by MAPK Signaling Pathways from the Chestnut Blight Fungus Cryphonectria parasitica D. H. KIM, Chonbuk National University, Jeonju, SOUTH KOREA

We assessed the regulation of cryparin, a typical type II hydrophobin, by three representative mitogen-activated protein kinase (MAPK) pathways in Cryphonectria parasitica. The mutation in the CpSlt2 gene, an ortholog of the cell wall integrity (CWI) pathway of Saccharomyces cerevisiae, showed dramatic decrease in the cryparin production. The mutant of $C p B c k l$ gene, a MAPKKK gene in CWI pathway, showed similar decreased cryparin production. In addition, the mutant of cpmk 1 gene, an ortholog of yeast HOG1, showed the decreased cryparin production. However, the mutant of cpmk2 gene, an ortholog of yeast Kss1/Fus3, showed the increased cryparin production. Easy-wet phenotype and accumulation of cryparin transcript of corresponding mutants were in good agreement with the cryparin production. In silico analysis of the promoter region of cryparin gene revealed the presence of binding motifs related to the downstream transcription factors of CWI, HOG1, and pheromone responsive pathways including MADS boxand Ste12-binding domains. Real time RT-PCR analyses indicated that both CpRlm1, an ortholog of yeast RLM1 in CWI pathway, and cpst12, an ortholog of yeast STE12 in mating pathway, showed significantly reduced transcription level in the mutant strains showing the less production of cryparin. However, the transcription of $C p M c m 1$, an ortholog of yeast $M C M 1$, did not correlate with the mutant strains showing the down-regulation of cryparin. These results indicated all three representative MAPK pathways in filamentous fungi played a role in regulation of the cryparin production. In addition, protein expressions of CpRlm1 and cpst 12 are underway to check the binding affinity of CpRLM1 and CpST12 into promoter region of cryparin.

Metagenomic signatures of bacteria harbored in sorghum leaf tissue

K. MASENYA (1), M. Tekere (2), G. Thompson (1), J. Rees (2,3), (1) Agricultural Research Council-Biotechnology Platform, Pretoria, SOUTH AFRICA; (2) University of South Africa, Johannesburg, SOUTH AFRICA; (3) University of South Africa, Pretoria, SOUTH AFRICA

Sorghum is a promising biofuel resource and one of the most important cereal crops globally. Large-scale planting would however require improved varieties that are tolerant to a range of stressors, including plant pathogens. This study aimed to extensively assess the bacterial diversity present in sorghum lines developed for cultivar breeding programs using $16 \mathrm{~S}$ metagenomic sequencing. The observed patterns revealed a highly heterogenous bacterial community and a link between the presence of plant pathogens and the composition of the endophytic bacterial community. Diseased plants 
showed high abundances of pathogens causing bacterial wilt and leaf blight, as well as food-borne pathogens. A higher abundance of bacterial species that have been reported to have bio-control capabilities were retrieved in healthy sorghum plants i.e. Bacillus subtilis, Enterobacter cloacea, Pantoea sp., and Bacillus casamancenscis. Understanding these multitrophic interactions is key to developing new strategies for plant protection.

\author{
Strawberry $B$. cinerea IPM optimisation by iMETOS $^{\circledR}$ sm forecasting model \\ N. RASIUKEVICIUTE, A. Valiuskaite, Lithuanian Research Centre for Agriculture and Forestry Institute of Horticulture, Babtai, Kaunas dist., \\ LITHUANIA
}

Botrytis cinerea has capability to remain quiescent in unripe strawberry. Grey mould is responsible for a $50 \%$ reduction in annual yields. Meteorological conditions play an important role for the grey mould and strawberry post-harvest shelf-life. In IPM forecasting models can help to evaluate the conditions for the disease development and assist with management decisions. The forecasting models provide producers, consultants and researchers with a tool to evaluate disease management and obtain control recommendations. The aim of this study was to evaluate the iMETOS ${ }^{\circledR}$ sm forecasting model for strawberry grey mould management and postharvest control. The research was carried out at the LAMMC Institute of Horticulture, Lithuania. There were open field experiments in 2008-2009 and 2013-2014 on a cultivar 'Elkat', planted at a spacing of $0.8 \times 0.3 \mathrm{~m}$. on two rows white film-mulch The treatments were replicated four times at randomized complete block, each replicate consisted $\sim 3.2 \mathrm{~m}^{2}$. There were two plant protection systems: conventional and iMETOS ${ }^{\circledR}$ sm model's. The data revealed that the most favourable conditions for strawberry grey mould development were in June. Comparison of different systems showed that both disease management systems reduced the spread of grey mould, but most efficient were iMETOS ${ }^{\circledR}$ sm. The applications by model gave yield increases in 2008-2009 and 2013-2014 of $0.95 \mathrm{t} \mathrm{ha}^{-1}, 9.5 \mathrm{t} \mathrm{ha}^{-1}, 4.28 \mathrm{tha}^{-1}$ and $3.21 \mathrm{t}^{-1}$, respectively compared to the control. There was less rotten fruits in iMETOS ${ }^{\circledR} \mathrm{sm}\left(0.67 \mathrm{tha}^{-1}, 2.28 \mathrm{tha}^{-1}, 0.25 \mathrm{t} \mathrm{ha}^{-1}\right.$ and $\left.0.60 \mathrm{t} \mathrm{ha}^{-1}\right)$. iMETOS ${ }^{\circledR}$ sm model helped to optimize fungicide application and allows more efficient, ecologically and economically accepted control of strawberries grey mould.

Analysis of seed potato certification data for limiting potato disease incidence in Colorado

Y. ZENG (1), A. C. Fulladolsa (2), A. Houser (1), A. O. Charkowski (2), (1) Colorado State University, Center, CO, USA; (2) Colorado State University, Fort Collins, CO, USA

Seed potato certification programs aim to limit disease incidence in planting material to levels below a threshold that causes significant losses to seed potato buyers. Records maintained for seed potato certification also can be analyzed for trends in seed potato varietal mixture and disease incidences over time. In the present study, generalized linear models (GLM) were conducted to analyze effects of year, potato variety, and their interaction on the incidence of potato diseases and disorders, and to determine the effect of seed generation of important varieties on the incidence of common potato diseases based on seed potato certification data collected in San Luis Valley, Colorado from 2012 to 2016. Among documented diseases, mosaic and blackleg have been persistent problems leading to high percentage of seed lot rejection. The GLM demonstrated year, potato variety, and their interaction were key factors contributing to incidence of varietal mixture, or one or more potato diseases and disorders. Our models also predicted 49, 54, and 4 varieties were susceptible to mosaic, blackleg, and leafroll, respectively, whereas there were 12,6 , and 5 varieties that were disease tolerant or resistant regardless of pathogen pressures in the environment. Seed generation effects on mosaic and blackleg was not consistent. In addition, we observed a significantly positive correlation between disease incidence of leafroll and the relative abundance of the green peach aphid, Myzus persicae. Mosaic incidence was not influenced by common aphid species captured in the valley and leafroll was not influenced by other common aphid species captured in the valley. These results identify the major causes of seed potato losses, identification of susceptible and resistant varieties, and provide suggestions for improving integrated crop management practices in Colorado.

Relationship between weather, colonization and mycotoxins produced by Fusarium graminearum species complex on sorghum grain L. ROTHMANN, N. W. McLaren, University of the Free State, Bloemfontein, SOUTH AFRICA

Sorghum grain mold (SGM) is one of the most important biotic constraints in sorghum production. The Fusarium graminearum species complex (FgSC) is responsible for the majority of important mycotoxins associated with SGM, including deoxynivalenol (DON), nivalenol (NIV) and zearalenone (ZEA), Weather variables that favour SGM development with specific reference to FgSC colonisation and concomitant mycotoxin production were studied with the aim of developing a risk forecast model. Sorghum grain was collected from field trials from 2007-2014 at 11 South African sorghum production localities. Site-specific weather data were also collected for each locality. Grain samples were analysed for $F g S C$ colonisation using qPCR and mycotoxin (DON, NIV and ZEA) contamination with LC-MS-MS. Multivariate data analyses were completed using GenStat $\left(18^{\text {th }} \mathrm{Ed}\right.$.). FgSC colonisation and concomitant mycotoxin accumulation coincided with weather conditions during late flowering and soft-dough stage of grain development, which are the critical periods for disease development. Grain characteristics also influenced mycotoxin production. An epidemiological model which identifies specific sorghum production risk regions, will enable producers to ensure that timely management decisions are made to reduce FgSC infection and mycotoxin contamination.

Sclerotinia stem rot of soybean: The South African approach

N. W. MCLAREN, L. Rothmann, University of the Free State, Bloemfontein, SOUTH AFRICA

Average incidences of Sclerotinia stem rot for the period 2006-2015 exceeded 20\% in key South African production areas. AFLP analysis indicated a relatively uniform geographic distribution of isolates, with evidence of genetic diversity. The significance of the genetic diversity in host response and locality interactions still requires elucidation. Disease responses in multi-location germplasm trials indicated highly significant genotype $\times$ environment interactions. Nonlinear regression analysis was used to quantify genotype responses and their stability to changing disease potentials. Genotypes with varying levels of tolerance to disease pressure were identified which could reduce the risk of crop losses. Regression parameters indicated disease potential required to initiate disease and subsequent rates of disease development. Ten year weather data were bulked into periodic running means and multivariate analyses were used to estimate critical growth stages and the length of infection and colonization periods. Analyses identified 21 day periods associated with R3-R5 as critical growth stages and stepwise variable selection identified rainfall, minimum humidity and minimum temperature as key driving variables. An intervention threshold model which integrates genetic resistance and weather variables is being developed with the aim of optimizing fungicide applications.

A rule-based prediction system improves spray precision for the control of strawberry powdery mildew H. WILEMAN (1), B. Liu (1), J. Dodgson (2), A. Hall (1), (1) University of Hertfordshire, Hatfield, Herts, UNITED KINGDOM; (2) University of Mahasarakham, Maha Sarakham, THAILAND 
Work at the University of Hertfordshire has shown that environmental conditions such as temperature and relative humidity affected strawberry powdery mildew (Podosphaera aphanis) conidial conidiation, germination and disease severity. A rule-based prediction system was developed into a CD software to record the accumulated number of hours (up to 144) of disease-conducive conditions (temperature $15-25^{\circ} \mathrm{C}$ and $\mathrm{RH}>60 \%$ ) needed for the development of the pathogen. It identifies high risk days when sporulation may occur thus allowing growers to spray at the optimal time (primary infection). Two-weekly monitoring of disease severity in field trials using this software in 2014 and 2015 showed that strawberry crops from the prediction spray programme had fewer fungicide applications ( 7 sprays in 2014, 3 in 2015) compared with the commercial programme (9 sprays in 2014 7 in 2015), as well as less severe disease. A more user-friendly web-based system was introduced in 2017 and has shown additional results in improving the efficient use of the fungicide mode of actions and reducing fungicide applications by 5, saving $£ 300$ per hectare. The 2014-17 results showed that the use of the prediction system achieved satisfactory control of $P$. aphanis with fewer fungicide applications, enabling growers to spray with precision thus maximising fungicide effectivity for disease control.

\section{Analysis of the Influence of Climate on Arceuthobium sichuanense \\ C. TIAN, D. Xiong, C. Zhang, Beijing Forestry University, Beijing, CHINA}

In recent years, with the global warming and extreme weather are more frequently, structure and types of forest are undergoing tremendous changes. Arceuthobium sichuanense is a parasitic seed plant disease that mainly harm to spruce trees, and causes a seriously economic loss in nature spruce forests of "san jiang yuan" areas recently. In this study, we tried to find out the suitable areas for $A$. sichuanense growth and the impact of climate change for $A$. sichuanense by using the niche models (GARP and MaxEnt) and dendrochronology. The result showed that suitable areas of A. sichuanense were mainly concentrated in Qinghai, Gansu, Sichuan, Xizang provinces, and the central areas of $A$. sichuanense cross the land of Qinghai, Gansu, Sichuan according to combine the prediction results of GARP and MaxEnt model with 4:1 weight. Interestingly, we also found that the suitable areas of $A$. sichuanense trended to expand to the northwest and southwest from 1961 to 2000. Additionally, we measured the width of tree rings of the infected spruce with disease index $>3$, and the results showed that the width of tree rings in 1992 was significantly reduced compared to other years, which may cause by $A$. sichuanense. We also revealed that temperature showed more correlative with volume increasing of host trees compared to precipitation, and the temperature had inverse impact on healthy and sick spruce which had a positive influence on healthy spruce and a negative influence on sick spruce. Overall, our data would increase our knowledge on A. sichuanense, which may be helpful for the disease control.

A Meta-Analytical Approach Towards Optimizing Peanut Digging Decisions in the Presence of Late or Early Leaf Spot Defoliation D. J. ANCO (1), D. Jordan (2), B. Shew (2), A. K. Culbreath (3), W. S. Monfort (3), H. L. Mehl (4), N. S. Dufault (5), B. Tillman (6), I. M. Small (7), J. Sarver (8), (1) Clemson University, Blackville, SC, USA; (2) North Carolina State University, Raleigh, NC, USA; (3) University of Georgia, Tifton, GA, USA; (4) Virginia Tech Tidewater AREC, Suffolk, VA, USA; (5) University of Florida, Gainesville, FL, USA; (6) University of Florida, Marianna, FL, USA; (7) University of Florida, Quincy, FL, USA; (8) Mississippi State University, Mississippi State, MS, USA

Late and early leaf spot, respectively caused by Cercosporidium personatum and Cercospora arachidicola, are damaging diseases of peanut (Arachis hypogaea) capable of defoliating canopies and reducing yield. While these diseases each may be more predominant in a given area, both are important on a global scale. To better guide management decisions and quantify relationships of end-of-season defoliation and yield loss, a series of meta-analyses was conducted over more than 80 data sets meeting established criteria. Linear slopes of proportion yield loss with increasing defoliation were estimated separately for runner- and Virginia-type varieties. Results for runner-types indicated yield loss to increase 1.3 to $2.8 \%$ per $10 \%$ increase in defoliation. Yield loss for Virginia-types was at the rate of 1.6 to $3.2 \%$ per corresponding $10 \%$ increase, with meta-regression indicating this slope to increase as a function of mean defoliation by approximately $4.0 \%$ per $10 \%$ increase in defoliation. A second meta-analysis estimated the rate of relative yield increase towards a relative optimum from digging dates within 21 days of optimal relative yield to be 0.7 to $1.4 \%$ per day. A third meta-analysis estimated the rate of defoliation progress from related data sets according to the logistic function at 0.094 /day. The integration of these findings should help inform recommendations regarding digging under varying defoliation pressures and peanut maturities in order to minimize losses.

Prediction and warning system in Chile: A way to face the risk of late blight

I. ACUNA (1), R. Bravo (1), J. Gatica (1), J. Quintana (2), (1) Agricultural Research Institute INIA Chile, Osorno, CHILE; (2) National Weather Service DMC Chile, Santiago, CHILE

There is a large amount of weather information available that can be accessed to enable forecasting using a decision support system (DSS) for development of integrated pest management for late blight. In Chile, late blight DSS forecasting system based on Blitecast has been available since 2007. This system utilizes weather station data to predict late blight risk which is delivered to the farmers through a web page, SMS and e-mail (http://tizon.inia.cl). A survey shows that $42 \%$ of the registered farmers use the system to make fungicide application decisions. To improve the opportunity of application and efficacy of chemical control, an early warning system using weather prediction data has been evaluated. Field experiments have been performed during 3 crop seasons to evaluate the system using 24,48 and 72 hour forecast data to monitor incidence and severity of late blight. Disease severity was different for each crop season. No significant differences in disease damage were found using weather station data, weather prediction data (24, 48 y $72 \mathrm{hrs)}$ or scheduled application (RAUDPC: $0.06,0.05,0.02,0.01,0.05$, respectively) but all treatments were better than the untreated control (RAUDPC: 0.41 ). The weather prediction system resulted in $50 \%$ reduction in spray number, compared to scheduled applications in all seasons, however, using the weather station data, the number of timely sprays was reduced by $75 \%$ under a low disease pressure, reducing the risk of late blight.

Climatic and spatial factors associated with Xylella fastidiosa outbreaks in Italy and mainland Spain

J. Martínez-Minaya (1), D. Conesa (1), A. López-Quílez (1), M. Saponari (2), A. VICENT CIVERA (3), (1) Departament d'Estadística i Investigació Operativa, Universitat de València, Burjassot, SPAIN; (2) CNR - Institute for Sustainable Plant Protection, Bari, ITALY; (3) Instituto Valenciano de Investigaciones Agrarias (IVIA), Moncada (Valencia), SPAIN

Diseases caused by the bacterium Xylella fastidios $a$ were recently detected in the Mediterranean Basin. The olive quick decline, caused by $X$. fastidiosa subsp. pauca, has devastated extensive areas in the Salento peninsula, south east Italy. An outbreak of almond leaf scorch, caused by X. fastidiosa subsp. multiplex, was detected in 2017 in Alicante province, mainland Spain. Climate is known to play an important role limiting the geographic range of Xylella diseases, but different relationships were described between $X$. fastidiosa and climatic covariates depending on the subsp. and the host. In this study, species distribution models were used to infer about the potential geographic distribution of olive quick decline and almond leaf scorch in the Mediterranean Basin. Data on the prevalence of X. fastidiosa in Italy and mainland Spain were gathered from official surveys. In Italy, different sampling strategies were followed each year, so the analysis was focused only in the Lecce province for the 2013-14 surveys, where a more regular sampling was observed. Monthly average climate data from 1950 to 2000 were obtained from the WorldClim database. Bayesian inference by INLA was used and the geostatistical effect was implemented by the SPDE approach. Results indicated a strong effect of the geostatistical component in the models, indicating 
that spatial structure has a profound impact on disease spread dynamics. Due to relatively small extent of the study areas, little variability was observed in the climate covariates and so they were not much informative in the models. Nevertheless, increasing minimum winter temperature was associated with higher probability of olive quick decline presence in Italy.

A forecasting system for bacterial spot disease of stone fruits caused by Xanthomonas arboricola pv. pruni I. LLORENTE, G. Morales, E. Montesinos, C. Moragrega, University of Girona, Girona, SPAIN

Bacterial spot disease of stone fruits, caused by Xanthomonas arboricola pv. pruni, is of high economic importance in the major stone-fruit-producing areas worldwide. As no effective chemical control is available, quarantine measures are needed to avoid pathogen introduction and dissemination. In affected areas, control is mainly based on preventative copper spray applications and antibiotics (where they are registered). Appropriate and rational timing of copper would increase the efficacy of disease control. The study was aimed at developing a mechanistic forecasting system for this disease based on three components: i) epiphytic inoculum potential, ii) weather conditions conducive to infections, and iii) disease symptom appearance. The effects of environmental parameters and inoculum populations on different steps of the disease cycle were quantified and modeled. Models for predicting bacterial growth as a function of temperature, infection risk based on wetness period duration and temperature, and symptom development based on cumulative degree day were developed. Inoculum dose threshold and infection risk threshold were determined. The results obtained, performed under controlled environment conditions, contribute with new knowledge on the epidemiology of the bacterial spot disease and offer new possibilities in the disease management. This research was supported by grants AGL2013-41405-R (Spain) and EU FP7 number 613678 (DROPSA).

Identification of weather variables associated with epidemics of sugarcane orange rust in Florida

B. CHAULAGAIN (1), I. M. Small (2), R. N. Raid (1), P. C. Rott PhD (1), (1) University of Florida, Belle Glade, FL, USA; (2) University of Florida, Quincy, FL, USA

Most sugarcane cultivars grown in Florida are susceptible to orange rust caused by Puccinia kuehnii, thus threatening sugarcane production. Currently, Florida's growers rely on fungicides for orange rust management and application schedules are based solely on disease scouting. The objective of this study was to identify weather variables associated with disease progress in order to develop a weather-based model for prediction of orange rust epidemics. Disease severity (percent rust-affected area of the top visible dewlap leaf) was recorded every two weeks on two to four sugarcane cultivars (CL85-1040, CP80-1743, CP88-1762, CP89-2143) susceptible to orange rust and grown at Belle Glade, FL, from 2014 through 2016. Hourly weather data for 30-day periods prior to each orange rust assessment were retrieved from the South Florida water management district's weather station, located within one mile from the experimental fields. Two hundred fifty variables were derived from weather data and evaluated as potential predictors of rust severity under field conditions. For all three years of disease observation, the number of hours with an average air temperature of $20.0-22.2^{\circ} \mathrm{C}$ and $\mathrm{RH} \geq$ $90 \%$ from $8 \mathrm{pm}$ to 8 am over the 30-day period prior to disease assessment correlated $(\mathrm{r}>0.80)$ with rust severity. These variables will be used to develop a sugarcane orange rust prediction model and improve Florida's orange rust management by optimizing the timing of fungicide applications.

Predicting emergence of hop shoots systemically infected by Pseudoperonospora humuli in Wisconsin using a simple degree-day model M. E. MARKS, A. J. Gevens, University of Wisconsin-Madison, Madison, WI, USA

Hop (Humulus lupulus) downy mildew, caused by the oomycete Pseudoperonospora humuli, is the most important disease infecting hops in Wisconsin (WI) and many other temperate growing regions. The pathogen overwinters in the perennial hop rootstock and emerges in spring from systemically infected shoots, or 'basal spikes'. A predictive model based on growing degree-days (GDDs) calculated from air temperatures was developed in the Pacific Northwest (PNW). A threshold of 111.3 GDDs was determined to accurately predict basal spike emergence within 5 days. This threshold was calculated with a base temperature of $6.5^{\circ} \mathrm{C}$ and initiated on a date after which significant cold periods no longer persisted. Our goal was to validate this threshold for use in WI and, if necessary, develop a new threshold. Air temperatures and observations of basal spikes (weekly from late March-early May) were collected at 4 commercial hop yards during 2015-17. GDD accumulations were calculated for each location, and the day of year (DOY) at which the threshold was met for each location/year combination was calculated and compared to the date of actual observed spike emergence. For all years and locations, the DOY at which 111.3 GDDs was met occurred within 6 days of spike emergence (dates ranged from 18 April to 7 May). We determined that the PNW model using a 111.3 GDD threshold for basal spike emergence is valid for use in WI and can help growers enhance early season disease management.

Geostatistical analysis of rice blast in China at three different scales

F. GUO, X. Chen, S. W. Wang, L. Yang, N. Wang, B. M. Wu, China Agricultural University, Beijing, CHINA

Blast caused by Magnaporthe oryzae is one of the most destructive diseases in rice worldwide. Knowledge on the spatial pattern of disease incidence is essential to understand its epidemic. In this study, spatial patterns of rice blast were analyzed using geostatistical tools at three different scales. At largescale, rice blast incidences at 47 to 107 stations in major rice production regions in China were surveyed from 2009 to 2014. At middle-scale, 53, 151, 142 fields in an area of 190000, 60000, 1900000 hectares were surveyed at three locations in North of Jiangxi, South of Jiangxi, and the junction area of Jiangxi, Hubei, and Anhui Provinces, respectively. At small-scale, rice blast incidence was determined on grids within 3 fields each in Jiangxi and Liaoning Provinces. Results demonstrated that rice blast incidence exhibited significant spatial dependence with influence ranges from 398 to $1080 \mathrm{~km}$ on 10 dates out of the 101 survey dates. At middle-scale, a significant linear trend was detected in 2 out of the 3 locations, but no significant spatial dependence was observed. At small scale, strong spatial dependence with an influence range of $5 \mathrm{~m}$ was detected in one out of 3 fields in Jiangxi, while strong spatial dependence with an influence range of 7.2 to $10 \mathrm{~m}$ was detected in all the three fields in Liaoning. The findings from this study provide helpful information to sampling and management of rice blast.

Integrating real-time edaphics into epidemic models for predicting risk in soilborne pathogen systems J. HAYTER (1), T. Chappell (2), (1) Texas A\&M University Department of Plant Pathology and Microbiology, College Station, TX, USA; (2) Texas A\&M University, College Station, TX, USA

Soil conditions are relevant to pathosystems involving soilborne pathogens, and pathogenesis occurring in the rhizosphere. Epidemic variation in many such pathosystems is economically important, and has been the focus of modeling efforts aimed at predicting risk of disease in these contexts. In many such models, typological information about soils is utilized; however, existing and novel models stand to benefit from increasing descriptions of soils beyond typology by incorporating real-time estimates of soil physical (structural, chemical, etc.) and edaphic (microbial, nutritional) conditions. Means of providing these estimates through the use of weather and other data are introduced: edaphological models are developed through experimentation and statistical methods, and thereafter used to project relevant soil conditions in real time using available weather data. These conditions are then used in the 
development of epidemic models used to predict disease risk. Examples of how these benefits are realized in predictive performance are provided through the use of historical (pathosystems involving Fusarium oxysporum) and simulated datasets. Significant improvement in the performance of risk prediction following this integration results, and applications to similar systems is discussed.

\section{Spatial Distribution of Foliar Diseases in Soybeans}

M. PATTERSON (1), A. C. Tolbert (2), T. N. Spurlock (3), (1) University of Arkansas-Fayetteville, MONTICELLO, AR, USA; (2) University of Arkansas Division of Agriculture, Monticello, AR, USA; (3) University of Arkansas, Monticello, AR, USA

Asian soybean rust, caused by Phakopsora pachyrhizi, Cercospora leaf blight, caused by the fungus Cercospora kikuchii, and Frogeye leaf spot, caused by the fungus Cercospora sojina were identified in a field near Hamburg, AR. Fifty GPS points were marked at approximately equal distances and foliar disease levels were rated in the top $1 / 3$ of the soybean canopy. At each point, 10 leaflets were collected and imaged using a flatbed scanner. Leaflet images were analyzed for percent defoliation of each of the three diseases present by point. Spatial analysis of disease levels were conducted to determine distributions using Moran's I in Geoda. Asian soybean rust and Cercospora leaf blight were significantly clustered (P=0.05) while Frogeye leaf spot occurred at very low levels and was randomly distributed throughout the field $(N S)$. When distributions of Asian soybean rust and Cercospora leaf blight were compared, their distributions were significantly dispersed $(\mathrm{P}=0.05)$ indicating some level of biological competition. These results are significant as foliar diseases are typically considered to be randomly distributed in fields. However, the clustered natures of the diseases found in this location are in disagreement with this idea. These results point to opportunities for predictive scouting or preferential fungicide trial placement in the future.

Development and validation of standard area diagrams for assessment of coffee leaf rust (Hemileia vastatrix Berk. \& Br.) severity in Colombia J. M. López-Vasquez, A. L. Gaitan, C. A. ANGEL, National Coffee Research Center -Cenicafe, Manizales, COLOMBIA

Coffee Leaf Rust (CLR, Hemileia vastatrix) is one of the most devastating diseases for coffee worldwide, causing yield and quality losses between 23 and $50 \%$ in susceptible varieties without CLR management in Colombia. To support epidemiological, host resistance and management studies, two Standard Area Diagrams (SADs) to assess CLR severity on infected leaves from the field and from artificially inoculated plants were developed and validated. The SADs were composed of two sets of images with seven different percentages of affected leaf area $(1,2,4,8,16,32$ and $64 \%)$, and validated by 10 non-expert and 10 expert raters based on their experience evaluating coffee diseases. Both groups estimated CLR first without any help; followed by two other CLR severity diagrams, and finally they used the new SADs. Lin's concordance correlation analysis of actual field CLR vs. estimated CLR using SADs showed 2.7 and 1.7\% overestimation for non-experts and experts respectively, versus 24 and $7.6 \%$ when they did not use any diagram respectively. Interestingly, overestimation for artificially inoculated leaves were 1.59 and $5.24 \%$ respectively, and obtained similar values to the field CLR test when did not use any diagram. When the new SADs were used, $92.1 \%$ for field CLR and $50.5 \%$ for inoculated CLR of any possible rating combinations for raters in pairs were obtained within the highest frequency range of determinations $\left(\mathrm{R}^{2} 0.9\right.$ to 1.0). The developed SADs for CLR severity visual assessment significantly improved reliability, accuracy and precision, for both experts and non-experts evaluators.

Infectivity titration dose response curves within Aspergillus flavus: A Case for Infection Specificity R. R. SWEANY, C. DeRobertis, K. E. Damann, Louisiana State University AgCenter, Baton Rouge, LA, USA

Aspergillus flavus contaminates corn and other oil-seed crops with carcinogenic aflatoxin. Soil A. flavus populations differ from crop populations. Among Louisiana soil and corn A. flavus populations, only six of 16 vegetative compatibility groups (VCG) infected corn. Infection ability of two soil VCGs and two frequent corn VCGs was assessed with a 2-year field infectivity titration experiment. Silks were dipped into doses of $1 \times 10^{5}, 10^{7}$ and $10^{9}$ conidia/ml for each VCG. To determine infection differences between VCGs, generalized linear models with logit link for binomial distribution predicted infectivity dose response curves (infected kernels/ear vs. dose). Dose response curves were not sigmoidal as expected, but u-shaped (parabolic). Dose response curves differed between soil and corn VCGs. Soil VCGs resembled inverted u-curves but the curve estimates were not statistically significant, indicating relatively low infection. Corn VCGs had statistically significant $\mathrm{u}$-shaped curves, indicating greater infection than soil VCGs especially at $10^{9}$. When silks were inoculated with $10^{9}$ conidia, soil VCGs infected $6 \pm$ s.e. $3 \%$ and $8.2 \pm 2 \%$ of kernels and corn VCGs infected $17 \pm 3 \%$ and $12 \pm 3 \%$. If dose was treated as a class variable, the most frequently isolated corn VCG infected more frequently than the other corn VCG in only one year. Though considered an opportunistic pathogen, certain strains are more frequently isolated from corn due to increased virulence on corn.

30 years of polyetic development of the polycyclic onion disease Botrytis Leaf Blight H. VAN DER HEYDEN (1), J. B. Charron (1), O. Carisse (2), (1) McGill University, Ste. Anne de Bellevue, QC, CANADA; (2) Agric \& Agri-Food Canada, Saint-Jean-sur-Richelieu, QC, CANADA

Despite our deep understanding of cultural, genetic and chemical control, epidemics of plant diseases are still responsible for significant losses amongst several agrosystems. For diseases of annual crops the natural time scale for the study of an epidemic is often considered to be a single growing season. However, the temporal framework for their study should encompass several scales from within-field to farms and regions and between seasons. In Québec, onions are grown on more than 2000 hectares of land. This production is threatened by several diseases including Botrytis Leaf Blight caused by Botrytis squamosa, a well-described endemic polycyclic disease. This disease was used to investigate the general hypothesis that final disease severity in a given field and a given season influences disease onset and disease progress rate in neighboring fields the following seasons. Disease severity, collected once a week over the past 30 years in about 120 georeferenced fields was used to characterize spatial and temporal heterogeneity at different hierarchical scales: from within field to regional level. In a first step, Gompertz, logistic and sigmoid models were fitted to the data to characterize individual disease progress curves. Secondly, seasonal spatio-temporal variations were characterized by adjusting Taylor's power law on raw data as well as on the parameter estimates of the disease progress curves. Finally, the presence of autocorrelation between growing seasons was estimated using a Box-Jenkins approach. Data analysis suggests that at the regional scale, a reduction of disease intensity toward the end of a season influences disease onset the following year.

Transcriptome alteration in Phytophthora infestans in response to phenazine-1-carboxylic acid production by Pseudomonas fluorescens LBUM223

M. FILION, R. Roquigny, A. Novinscak, D. L. Joly, Université de Moncton, Moncton, NB, CANADA

Phytophthora infestans is responsible for late blight, one of the most devastating potato diseases. Phenazine-1-carboxylic acid (PCA)-producing Pseudomonas fluorescens LBUM223 shows biocontrol potential against various plant pathogens, including $P$. infestans. We wished to further characterize the effect of PCA-producing LBUM223 on the transcriptome of $P$. infestans using an in vitro time-course confrontational study with $P$. 
infestans inoculated alone (control) or with wild-type LBUM223, its isogenic mutant (no PCA production), or synthetic PCA. Destructive sampling was performed at 6,9 and 12 days, the transcriptome was analysed using RNA-Seq and the expression of a subset of differentially expressed genes was validated by RT-qPCR. Both LBUM223 and synthetic PCA significantly repressed $P$. infestans' growth at all times. Compared to the control treatment, transcriptomic analyses showed that the percentage of genes significantly altered by LBUM223 and synthetic PCA increased as time progressed, up to $61 \%$ and $46 \%$, respectively. When all time points were combined, PCA, either synthetic or produced by LBUM223, led to 214 differentially expressed genes. These key genes are involved in major cell functions, like oxidoreduction activity and phosphorylation mechanisms. Interestingly, even though no host plant tissue was present in the in vitro system, PCA also led to the overexpression of several genes encoding effectors, suggesting an implication in biocontrol.

Genetic analysis of the contribution of bacterial phenyl acetic acid production to virulence of Rhizoctonia solani AG2-2IIIB K. OBASA (1), F. F. White (2), Z. Peng (3), A. Block (4), (1) UNIVERSITY OF FLORIDA, GAINESVILLE, FL, USA; (2) University of Florida, Gainesville, FL, USA; (3) University of Florida, GAINESVILLE, FL, USA; (4) USDA, ARS, SAA, CMAVE, Gainesville, FL, USA

Rhizoctonia solani is species complex of genetically distinct subgroups described as anastomosis groups (AGs), and strains of fungus infect many economically important crops, resulting in significant yield losses. We have demonstrated that an endosymbiont bacterium in a strain of AG 2-2IIIB contributes to pathogenicity on creeping bentgrass. Loss of the bacterium, Enterobacter sp. EnCren, was shown to affect the production of phenylacetic acid (PAA), which is considered a virulence factor of the fungus. PAA production was significantly reduced in the bacteria-free fungus. The correlation between PAA biosynthesis by the bacterium and virulence of the host fungus in sugarbeet was examined. An indole-3-pyruvate decarboxylase (IpdC) bacteria mutant $\left(\right.$ EnCren $\left.^{-I p d C}\right)$, which in the free-living state showed reduced PAA levels, was reintroduced into a bacteria-free strain of the fungus to generate Restored ${ }^{-I P d C}$-RsCren. An IpdC-complemented strain of $\mathrm{EnCren}^{-I p d C}$, designated as EnCren ${ }^{+I p d C}$, was also reintroduced into the bacteria-free fungus to generate Restored ${ }^{+I P d C}$-RsCren. Disease assays of Restored ${ }^{-I P d C}$-RsCren and Restored ${ }^{+I P d C}$-RsCren on sugarbeet seedlings under growth-chamber conditions indicate reduced virulence in the Restored ${ }^{-I P d C}$-RsCren strain. Findings from this study could lead to better understanding of the underlying mechanism(s) of bacterial-endosymbiont-mediated pathogenicity in $R$. solani AG 2-2IIIB and the development of novel disease management strategies.

Dissecting the intercellular trafficking of the movement protein of Ourmia melon virus

N. OZBER (1), C. Anderson (1), M. Turina (2), C. Rosa (1), (1) The Pennsylvania State University, University Park, PA, USA; (2) Institute for Sustainable Plant Protection, National Research Council, Italy, Turin, ITALY

The movement protein (MP) of Ourmia melon virus (OuMV) is targeted to plasmodesmata and forms tubules to facilitate cell-to-cell movement of virions. It is, however, still unknown how OuMV MP reaches the cell periphery. To understand the transport mechanism of OuMV MP, we introduced glycine substitutions into tyrosine (Y)- and dileucine (LL)-based sorting motifs in OuMV MP and examined the virus movement of mutated agroclones in Nicotiana benthamiana and Arabidopsis thaliana. Four out of five mutants failed to move systemically. Then, we investigated the subcellular localization of GFP tagged MP mutants in $N$. benthamiana. Y-based motif mutants were distributed in the cytoplasm but failed to form puncta at the cell periphery, the typical localization of wild-type MP, whereas LL-based motif mutants localized to puncta in the cytoplasm and along the cell periphery. Staining of epidermal cells with propidium iodide and aniline blue revealed that movement deficient LL-based motif mutants failed to localize to the cell wall and puncta were not associated with callose deposits at plasmodesmata. Moreover, movement deficient GFP tagged Y-and LL-based motif mutants failed to form tubules in protoplasts. Taken together, Y- and LL-based motifs are essential for targeting of OuMV MP to plasmodesmata and cell-to-cell movement of OuMV, but show differences in functionality. Elucidating the transport mechanism of OuMV will provide tools to better control virus diseases.

Digital imaging to investigate root architectural changes associated with a root rot disease C. MATTUPALLI, A. Seethepalli, L. M. York, C. A. Young, Noble Research Institute, LLC, Ardmore, OK, USA

Phymatotrichopsis omnivora is the causal agent of Phymatotrichopsis Root Rot (PRR) disease that severely limits alfalfa production in southern Oklahoma. The disease manifests as circular to irregular shaped areas in the field categorized into three zones based upon plant status: asymptomatic, disease front and survivor. To study root architectural changes associated with PRR, a four-year old 23.8 hectare alfalfa stand infested with PRR was selected at the Red River Farm, Burneyville, OK during October 2017. Line transect sampling was conducted from four actively growing PRR disease rings. At each disease ring, six line transects were positioned spanning $\sim 15 \mathrm{~m}$ on either side of the disease front with one alfalfa root sampled at every $\sim 3$ $\mathrm{m}$ interval. Each root was imaged using the RhizoVision-Crown platform using a backlight and a high-resolution monochrome CMOS camera. The platform's image analysis software automatically segmented images, skeletonized, and extracted a suite of features. Total root length ranged from $211.7 \pm 133.2 \mathrm{~cm}$ for survivors to $170.6 \pm 104.2 \mathrm{~cm}$ for roots sampled from the asymptomatic zone. Survivor plants compensated for damage or loss to the taproot through the development of more lateral and crown roots. The mean time to extract 24 root morphological features from an alfalfa root image was $8.4 \pm 6.0 \mathrm{~s}$. This study demonstrates the benefits of a low-cost, efficient and high-throughput digital imaging approach to study root architectural changes.

Nucleobase transport in Erwinia amylovora

N. P. SCHULTES (1), A. Stoffer (2), C. Alexander (2), I. Gatchell (2), A. Funk (2), G. Mourad (2), (1) The Connecticut Agricultural Experiment Station, New Haven, CT, USA; (2) Department of Biology, Indiana University-Purdue University Fort Wayne, Ft. Wayne, IN, USA

Erwinia amylovora, a phytopathogenic bacterium, causes fire blight of apples and pears. It derives nutrients from the host during disease progression. Our research 1) identifies E. amylovora nucleobase transporter loci, 2) biochemically characterizes the encoded transporters and 3) determines if the loci are necessary for disease. Previous work revealed that E. amylovora nucleobase biosynthetic mutants differ in their ability to cause disease, suggesting either varied uptake or importance of nucleobases. Here the transport function and biochemical properties for four nucleobase cation symporter 2 transporters in E. amylovora are determined through heterologous expression in nucleobase transport deficient Escherichia coli Keio strains coupled with radiolabeled nucleobase uptake studies and toxic analog growth studies. Under in vitro culture conditions, the uracil transporter, EaUraA, is able to transport uracil and 5-fluorouracil into E. coli cells. Similarly, the guanine/hypoxanthine transporter, EaG/HXP, moves guanine, hypoxanthine, 8azaguanine and 6-thioguanine; the adenine transporter, EaAdeP, transports adenine, guanine and 8-azaadenine; and the xanthine transporter, EaXanP, moves xanthine. Finally, mutations of the $u r a A$ or $g h x P$ or adeP loci in E. amylovora do not impair pathogenicity. Our results confirm that nucleobase transporter genes are present and functional in E. amylovora yet they are not required for disease progression.

Chitin synthases $P$ CCHS and PSCHS1 are involved in sporangial development, zoospore production, and plant infection in Phytophthora C. Zhang, W. Wang, Z. Xue, Y. Fang, B. Zhang, X. LIU, China Agricultural University, Beijing, CHINA 
Oomycetes exhibit a filamentous growth morphology that is very similar to that of many fungi, but they are not fungi or even not related to fungi. A low content or lack of chitin in oomycetes cell walls has been taken as a criterion for that differentiates them from true fungi. Phytophthora capsici and $P$. sojae are both representative oomycetes species and have a worldwide distribution. Interestingly, no chitin was detected in Phytophthora cell walls, but putative chitin synthase genes are presented in Phytophthora species. In this study, we identified a CHS gene named PcCHS from $P$. capsici, and a CHS genes named PsCHS1 from P. sojae. The transcript patterns of PcCHS and PsCHS1 were similar in different stages; both of them had higher expression level in zoospores, cysts and infection stages. By systematic characterization of the impact of individual gene knockout, silencing, and overexpression on asexual and sexual growth, cell wall composition, chemical sensitivity, and virulence on plants, it showed that PcCHS and PsCHS1 were mainly involved in sporangial development, zoospore release and pathogenicity on plants. In addition, PsCHS1-GFP fluorescence was concentrated in brightly fluorescent cell membrane in the sporangia and hyphae with some diffuse fluorescence in the cytoplasm. Thus, our results suggest that $P c C H S$ and PsCHS1 are not involved in chitin biosynthesis, but required for the asexual growth and plant infection in Phytophthora.

Elucidating the functions of methyl-accepting chemotaxis (mcp) proteins of Dickeya dianthicola A. S. B. NASARUDDIN (1), N. T. Perna (2), J. D. Glasner PhD (3), A. O. Charkowski (1), (1) Colorado State University, Fort Collins, CO, USA; (2) UW-Madison, Madison, WI, USA; (3) University of Wisconsin Madison, Madison, WI, USA

Potato is the fourth most consumed food globally, and the most important non-grain crop worldwide. In the U.S., potato has an annual approximate of $\$ 4$ billion farm gate value. Every year, soft rot disease of potato caused by Dickeya spp. and Pectobacterium spp. contributes to at least $1 \%$ crop losses, which is about $\$ 400$ million. In 2015, an aggressive species of Dickeya, identified as Dickeya dianthicola, created a new threat to the U.S. potato industry. D. dianthicola was reported in 10 states and its spread had been confirmed in 23 states by 2016. A genomic comparison of 307 Enterobacteriaceae showed that Pectobacterium and Dickeya genomes are enriched in methyl-accepting chemotaxis proteins (MCP) and ATP-binding cassette $(\mathrm{ABC})$ transporters compared to other closely related animal pathogens in this family. Some of these MCP and ABC transporters may detect and transport plant metabolites that affect bacterial growth. A phenotype array identified compounds metabolized by soft rot Enterobacteriaceae and some of the same molecules were identified in the potato stem and stolon metabolomes. D. dianthicola ME23 showed chemotaxis towards potato tuber extract. To determine which compounds MCPs may sense, we transformed $42 \mathrm{mcp}$ genes from D. dianthicola ME23 into Escherichia coli UU1250 and assayed transformants via qualitative plate-based chemotaxis using various carbon sources, amino acids, and other metabolites found in potato.

Sclerotinia sclerotiorum oxalate-minus mutants accumulate fumaric acid in a pH-responsive manner and remain pathogenic on most host plants L. Xu (1), W. CHEN (2), G. Li (3), D. Jiang (3), (1) Northwest A\&F University, Yangling, CHINA; (2) USDA ARS, Pullman, WA, USA; (3) State Key Laboratory of Agricultural Microbiology, Huazhong Agricultural University, Wuhan, CHINA

The necrotrophic pathogen Sclerotinia sclerotiorum has a wide host range of $>400$ plant species. It produces and accumulates high levels of oxalic acid in culture and in diseased tissue, which is considered to be required for S. sclerotiorum to cause disease. Using genetically defined mutants defective at the oxaloacetate acetylhydrolase (oah) gene, we recently showed that the oah mutants lost oxalate production, but instead accumulated fumaric acid in culture, and remained pathogenic on some legume plants. This study was designed to determine the kinetics of fumaric acid accumulation in the oah mutants and test the pathogenicity of the oah mutants on additional hosts of several plant families (Asteraceae, Brassicaceae, Cucurbitaceae and Solanaceae). Production of fumaric acid and other acids under $\mathrm{pH} 3,5$, and 7 was monitored using HPLC. The oah mutants produced increasingly higher levels of fumaric acid as ambient $\mathrm{pH}$ increased, showing the same $\mathrm{pH}$ response of oxalic acid production found in the wild type. Interestingly, both wild type and the oah mutants produced more kinds of organic acids as the ambient $\mathrm{pH}$ increased. Pathogenicity assays showed that the oah mutants were not only able to initiate infection, but also caused disease levels similar to that of the wild type on most host plants. The results showed that the oah mutants remained $\mathrm{pH}$-responsive in acid production and can cause significant disease in the absence of oxalic acid at least on some plants.

Disruption of the Rice Blast genome to identify genes involved in production of Reactive Oxygen Species J. PANCAKE, D. Mikolajewski, K. Huang, N. M. Donofrio, University of Delaware, Newark, DE, USA

Rice Blast disease, caused by the fungus Magnaporthe oryzae, results in devastating losses to crops worldwide, especially South Asia. To better understand how to take advantage of basal immunity in rice against $M$. oryzae, we are performing forward genetic screens. We use a barley-infecting strain of M. oryzae, 4091, that had previously been transformed with a live sensor of reactive oxygen species (ROS) called Hyper-sensor (HS) gene into the fungus, which gives 4091 the ability to fluoresce when in the presence of ROS. We are in the process of randomly disrupting the genome of 4091-HS via random integration of a DNA construct carrying resistance to Hygromycin (Hyg), and double, outward-facing promoters. Our goal is to identify genes that disrupt the process of ROS detection and production by the fungus, by altering the quantifiable level of the HyPer sensor. Such genes could potentially play a role in ROS production, directly linked to basal immunity. Changes in ROS levels will be detected using confocal microscopy, and currently, we have over 500 transformed candidates that have gone through a primary screen. Results will be presented on our screening strategy, the initial screen and some preliminary data of our secondary and confocal screens.

Loss-of-function mutations in the Dpp and Opp permeases render Erwinia amylovora resistant to kasugamycin and blasticidin $\mathbf{S}$ Y. GE (1), J. H. Lee (2), B. Hu (3), Y. Zhao (2), (1) University of Illinois, Urbana, IL, USA; (2) University of Illinois at Urbana-Champaign, Urbana, IL, USA; (3) Nanjing Agricultural University, Nanjing, CHINA

Extensive use of antibiotic streptomycin to control fire blight disease of apples and pears, caused by Erwinia amylovora, leads to the development of streptomycin-resistant strains in the United States and elsewhere. Kasugamycin (Ksg) has been considered as an alternative to control this disease. In this study, we investigated the role of two major peptide $\mathrm{ABC}$-transporter systems in E. amylovora, the dipeptide permease (Dpp) and oligopeptide permease $(\mathrm{Opp})$, in conferring sensitivity to Ksg and blasticidin S (BcS). Minimum inhibitory concentration and spot dilution assays showed that the $d p p$ and $o p p$ deletion mutants exhibited enhanced resistance to Ksg and BcS. Deletion of both $d p p$ and $o p p$ conferred higher level of resistance to Ksg as compared to the single mutant. In addition, bioinformatic analysis combined with qRT-PCR showed that the Rcs system negatively regulates opp expression and the $r c s B$ mutant was more sensitive to both $\mathrm{Ksg}$ and $\mathrm{BcS}$ as compared to the wild-type. Electrophoresis motility shift assay further confirmed the direct binding of the RcsA/RcsB proteins to the promoter region of the opp operon. However, neither the Dpp nor the Opp permeases contributed to disease progress on immature pears, hypersensitive response on tobacco leaves, motility, and amylovoran production. These results suggested that Ksg and BcS hijack the Dpp and Opp permeases to enter E. amylovora cells, and the Dpp and Opp permeases act synergistically for illicit transport of antibiotics. 
Stemphylium lycopersici isolates virulence depends on the synthesis of phytotoxic metabolites, which is modified by the environment R. Medina (1), M. E. E. Franco (1), G. Lucentini (1), S. M. Y. Lopez (1), M. C. N. Saparrat (2), P. A. BALATTI (1), J. Rosso (3), (1) Centro de Investigaciones de Fitopatologia (CIDEFI), La Plata, ARGENTINA; (2) Instituto de Fisiologia Vegetal, La Plata, ARGENTINA; (3) Instituto de Investigaciones Fisicoquímicas Teóricas y Aplicadas (INIFTA), La Plata, ARGENTINA

Stemphylium lycopersici is one of the causative agents of Tomato grey leaf spot. We found that the genome of $S$. lycopersici CIDEFI-216 contains several putative secondary metabolite (SM) gene clusters, which have been associated to different biological roles. We hypothesized that isolates of $S$. lycopersici that differ in their virulence and ability to sporulate synthesize a different array of SMs. Three isolates of the fungus that differ in virulence and sporulation capacity were grown on V8 and PDA plates at $25^{\circ} \mathrm{C}$ for 14 days. SMs were obtained from lyophilized cultures through sonication and ultrafiltration. Spectroscopic UV-Vis absorption spectra and fluorescence-excitation-emission matrices as well as phytotoxicity of the fungal extracts were evaluated. The absorbance spectrum showed that all the extracts had a 450-nm peak and that isolate CIDEFI-216 grown on V8 medium contained the most complete array of peaks, metabolites. Fluorescence intensity and emission was a function of the fungal isolate and the culture medium. Extracts from CIDEFI-216 presented the highest fluorescence emission spectra, and all of them provoked lesions on tomato leaflets $(p<0.05)$, which were greater provided the fungus was grown in V8 medium. Here we demonstrate that SMs are key variable factors in the necrotrophic ability of S. lycopersici isolates, which can be drastically modified by the environment where the fungus grows.

\section{The infection process of Exserohilum turcicum: A microscopy investigation}

R. Kotze (1), Q. KRITZINGER (1), B. Crampton (1,2), (1) Department of Plant and Soil Sciences, University of Pretoria, Pretoria, Gauteng, SOUTH AFRICA; (2) Forestry and Agricultural Biotechnology Institute (FABI), University of Pretoria, Pretoria, Gauteng, SOUTH AFRICA

Northern corn leaf blight (NCLB) is a foliar disease of maize caused by the fungal pathogen, Exserohilum turcicum. The aim of this study was to determine the infection strategy of $E$. turcicum race $23 \mathrm{~N}$ in maize leaves by using various microscopy techniques to better understand the role of the fungus in NCLB development. Leaf samples were collected from inoculated B73 maize plants at 1, 4,11,14 and 18 days post inoculation (dpi). Samples were prepared according to standard microscopy procedures and analysed using light and electron microscopy. At 1 dpi the fungus penetrated the leaf epidermis, and at 4 dpi hyphal growth was seen in the epidermis. At $11 \mathrm{dpi}$, lesions formed on the leaves. Scanning electron microscopy revealed hyphal growth in the xylem at $11 \mathrm{dpi}$ and almost completely blocked by 14 dpi. Hyphae spread into the adjacent bundle sheath cells causing cellular damage, characterised by plasmolysis at $18 \mathrm{dpi}$. Conidiophores formed through the stomata at $18 \mathrm{dpi}$. Additionally, the lesions enlarged and started to coalesce and wilting of the leaves was observed. Extensive plugging of the xylem could inhibit water flow through the parasitized tissue, which could account for the formation of lesions. Increased colonization of the fungus in the xylem corresponded to an increase in size of the lesions, which leads to reduction in photosynthetic area. Understanding the infection strategy of the fungus could aid in development of better control and prevention strategies.

Hypoxia, denitrification, and the fitness of Fusarium verticillioides as a fungal pathogen of maize

B. OAKLEY (1), L. Pierce (2), J. Vaughn (3), G. Hibbs (1), S. E. Gold (2), A. E. Glenn (2), (1) Department of Plant Pathology, The University of Georgia, Athens, GA, USA; (2) USDA-ARS Toxicology and Mycotoxin Research Unit, Athens, GA, USA; (3) USDA-ARS Genomics and Bioinformatics Research Unit, Athens, GA, USA

Fusarium verticillioides is a globally distributed fungal pathogen of maize and is of concern both for its impact on grain quality and its contamination of grain with fumonisin mycotoxins. The fungus is noted for its endophytic infection of maize, but it also colonizes plant debris in the soil. Since the plant's internal tissues and the soil environment are both prone to periods of hypoxia (low oxygen), we hypothesized that $F$. verticillioides utilizes physiological processes conferring hypoxia tolerance and that the genes encoding these processes are differentially regulated. RNA-seq analysis identified 1444 genes up-regulated at least 8 -fold in response to $24 \mathrm{~h}$ hypoxic growth. Three of these genes encode a dissimilatory nitrate reductase (10-fold), a dissimilatory nitrite reductase (832-fold), and a P450 nitric oxide reductase (17560-fold), which are all components of denitrification. Deletion mutants were created for these genes and others likely associated with denitrification and other responses to hypoxia. We are evaluating the impact of these genes on $F$. verticillioides virulence, endophytic infection, and general tolerance to hypoxic growth. Since nitrous oxide $\left(\mathrm{N}_{2} \mathrm{O}\right)$ is the final product of fungal denitrification, we are also assessing the impact of these genes on production of this greenhouse gas. This is important since agriculture is the primary source of global emissions of $\mathrm{N}_{2} \mathrm{O}$, which has 300-times the warming potential of carbon dioxide.

Regulation of toxin production by Rathayibacter toxicus, causative agent of bacterial head blight of grasses and annual ryegrass toxicity E. E. ROGERS (1), A. Sechler (2), M. Tancos (2), M. B. McMahon (2), W. Schneider (1), D. Luster (2), (1) USDA ARS FDWSRU, Fort Detrick, MD, USA; (2) USDA ARS FDWSRU, Frederick, MD, USA

The USDA-APHIS select agent Rathayibacter toxicus is a gram-positive bacterium that produces a lethal tunicamycin-like toxin in many forage grasses in association with seed gall nematodes from Anguina and related genera. To date, $R$. toxicus has only been found in Australia; however, anguinid seed gall nematodes are widespread, including in the grass seed production areas of the U.S. Pacific Northwest. At this point, little is known about when or why $R$. toxicus produces tunicamycin. Whole genome sequencing has identified a putative tunicamycin biosynthetic gene cluster (TGC) which contains genes similar to all 12 essential genes necessary for tunicamycin production in the characterized TGC from Streptomyces chartreusis. Triggers of toxin production under field conditions are unknown, although tunicamycin concentration increases in the final stages of seed maturation and drying. In the laboratory, toxin production can be turned on using a bacteriophage (NCPPB 3778). However, $R$. toxicus rapidly stops producing tunicamycin under standard laboratory growth conditions. Preliminary qRT-PCR experiments have shown that the TGC is expressed constitutively under both toxinproducing and non-producing conditions, suggesting post-transcriptional regulation of tunicamycin biosynthesis. Results from ongoing experiments examining the transcription, translation, and protein function of the TGC will be presented.

The mitogen-activated protein kinase kinase kinase SsOS4 regulates vegetative growth and fungicide sensitivity in Sclerotinia sclerotiorum T. LI, Y. Duan, Y. Yang, M. Zhou, Nanjing Agricultural University, Nanjing, CHINA

The osmotic signaling system, which consists of the upstream two-component regulatory system and the downstream HOG MAP kinase signal system, plays an essential role in regulating morphology, virulence, osmotic stress, and fungicide sensitivity in filamentous fungi. HOG system can be activated by phosphorylation through the upstream two-component regulatory system and regulates intracellular glycerol synthesis and cell adapts to the environmental stresses. Group-III histidine kinase is believed to regulate the two-component signaling system. Dicarboximide and phenylpyrrole fungicides have a strong antifungal activity against many phytopathogenic fungi. Action target of the two classes of fungicides has been documented to be involved in the osmotic signaling system. But fungicidal mechanism and resistant mechanism have not been fully elucidated. In our previous study, we characterized group-III histidine kinase Shk1 in Sclerotinia sclerotiorum and revealed that Shk1 was involved in the sensitivity to fludioxonil and osmotic stresses. In this study, we characterized the MAP kinase kinase kinase SsOS4 (SS1G_06598), a key kinase in HOG system in $S$. 
sclerotiorum. Compared with the wild type isolate, disruption of SsOS4 showed a significant reduction in vegetative growth, a decreased sensitivity to phenylpyrrole and an increased sensitivity to osmotic stress. The virulence of the mutant was also assessed on leaves of rapeseed, but no significant difference was detected in the size of lesions. Meanwhile, Western blotting test showed that Hog1 phosphorylation was present in the wild type strain but not in the SsOS4-deleted mutant. This indicated that SsOS4 regulated the phosphorylation transmitting to the downstream Hog1 in HOG system.

\author{
Dickeya dadantii forms elongated cells during the infection of potato tubers: Causal conditions, molecular basis, and implications to \\ pathogenicity \\ Z. CUI (1), Q. Zeng (2), (1) Department of Plant Pathology \& Ecology, The Connecticut Agricultural Experiment Station, New Haven, CT, USA; (2) \\ Department of Plant Pathology and Ecology, The Connecticut Agricultural Experiment Station, New Haven, CT, USA
}

Dickeya dadantii is a phytopathogenic bacterium that causes soft rot disease on potatoes. Tubers are nutrient storage organs of potato and are highly susceptible to $D$. dadantii infection. We observed that during the infection of potato tubers, some $D$. dadantii cells formed elongated cell morphology. To better understand this phenotype and its biological significance, we first investigated the conditions under which the long cells are formed. Our results demonstrated that $D$. dadantii formed more long cells under dry conditions with no free water, at the surface of the infected tubers, as oppose to under the wet conditions with free water, and at the junction of healthy and infected tuber tissues. We further characterized the molecular basis for the cell elongation. We observed a negative correlation between the cell length and the level of nucleotides guanosine tetraphosphate (ppGpp) in D. dadantii. Average cell length of $D$. dadantii is longer in mutant of ppGpp synthesis gene relA, and shorter in mutant of ppGpp degradation gene spoT. We also report a positive correlation between the ppGpp level and the bacterial virulence, type III secretion system, production of pectate lyase, and motility. Our results suggest that by adjusting cellular levels of ppGpp, $D$. dadantii places the elongated, non-mobile and low virulent cells at the surface of infection whereas places the shorter, motile and more virulent cells at the interface of infection.

Identification and characterization of hemagglutinins at different stages of bacterial wilt disease

D. KHOKHANI (1), M. Hayes (2), C. Allen (3), (1) University of Wisconsin Madison, Madison, WI, USA; (2) UW-Madison, Madison, WI, USA; (3) University of Wisconsin, Madison, WI, USA

Attachment to host surfaces is a critical early step in plant pathogenesis. The wide host range bacterial wilt pathogen Ralstonia solanacearum (Rs) must attach to roots before it invades and colonizes host plant xylem vessels. The $R s$ genome encodes 18 predicted non-fimbrial adhesins (hemagglutinins). Interestingly, many of these hemagglutinin genes are upregulated in a quorum sensing mutant which is locked in the pathogen's low cell density mode, presumably corresponding to early disease. However, a distinct set of hemagglutinin genes are upregulated only at high cell density. Using scanning electron microscopy, tomato root attachment assays, and plant colonization experiments, we found that quorum sensing mutants attach to roots, xylem walls, and other $R s$ cells much better than wild-type, but are defective in spreading in the plant. These results suggest that $R s$ attaches to host surfaces avidly early in the infection process, but eventually becomes less sticky to facilitate dispersal within the plant. Phylogenetic analysis revealed a cluster of hemagglutinins with novel bacterial collagen-like domains. $R s$ mutants lacking one or more of these collagen-domain adhesins did not colonize xylem effectively. Together the study suggests that different kinds of attachment are adaptive at various points in bacterial wilt disease development and also provides novel targets for disease control.

Role of the ubiquitin-conjugating enzymes in plant innate immunity

L. ZENG (1), B. Zhou (1), R. Mural (1), X. Chen (2), M. Oates (3), R. Connor (4), G. B. Martin (5), J. Gough (3), (1) University of Nebraska, Lincoln, NE, USA; (2) Fujian Agriculture and Forestry University, Fuzhou, CHINA; (3) University of Bristol, Bristol, UNITED KINGDOM; (4) University of Arkansas, Little Rock, Little Rock, AR, USA; (5) School of Integrated Plant Science, Cornell University, Ithaca, NY, USA

Ubiquitination has emerged in recent years as a key regulatory mechanism underlying plant immunity against various pathogens. Of the three classes of enzymes involved in ubiquitination, few ubiquitin-conjugating enzymes (E2) have been investigated in plants. Our recent study revealed the tomato UBC13 type E2s, Fni3 and SIUBC13-2 and their cofactor, Suv positively regulate plant immunity. To further characterize the role of E2 in plant innate immunity, we identified and cloned forty tomato genes encoding E2 proteins. Thioester assays indicated the majority of the genes encode enzymatically active E2. Phylogenetic analysis classified the forty tomato E2 into thirteen groups, of which only members of group III were found to act with AvrPtoB, a Pseudomonas syringae pv. tomato effector that uses its ubiquitin ligase (E3) activity to suppress host immunity. Knocking-down the expression of group III E2 genes in Nicotiana benthamiana diminished AvrPtoB-promoted degradation of the Fen kinase and AvrPtoB suppression of host immunityassociated PCD. Importantly, silencing group III E2 genes also resulted in reduced pattern-triggered immunity (PTI). By contrast, PCD induced by several effector-triggered immunity (ETI) elicitors was not affected on group-III-silenced plants. Functional characterization suggested redundancy among group III members for their role in the suppression of plant immunity by AvrPtoB and in PTI. These results suggest AvrPtoB has evolved a strategy for suppressing host immunity that is difficult for the plant to thwart. We also uncovered the involvement of non-group III E2 members in plant immunity through functioning at the endoplasmic reticulum (ER) site. Our work builds a foundation and provides critical inroads into understanding the regulation of plant immunity by an often-overlooked group enzymes in ubiquitination.

An optimized Agrobacterium tumefaciens-mediated transformation protocol for Ceratocystis albifundus

M. Sayari (1), E. Steenkamp (2), M. van der Nest (2), B. D. WINGFIELD (2), (1) Dept Genetics, University of Pretoria, Pretoria, SOUTH AFRICA; (2) Forestry and Agricultural Biotechnology Institute (FABI), University of Pretoria, Pretoria, SOUTH AFRICA

Despite the availability of genome sequences for the wattle pathogen, Ceratocystis albifundus, functional association of loci with specific biological traits remains lacking. This is mainly because an efficient transformation system is not available for the fungus. Here, we present an optimized Agrobacterium tumefaciens-mediated transformation protocol for genetic manipulation of this fungus. For the development of this protocol, $A$. tumefaciens strain AGL-1 and four binary T-DNA vectors (conferring hygromycin and geneticin resistance and expressing green fluorescent protein [GFP]) were used for transforming germinated conidia of three C. albifundus strains. Stable expression of GFP or the antibiotic resistance genes was confirmed through sequential sub-culturing of transformants on selective and non-selective media. Incorporation of these genes into the genome of the fungus was also confirmed using PCR, Sanger sequencing and Southern hybridization analysis. The range of experimental parameters optimised included: (i) the concentrations of hygromycin and geneticin required for inhibiting growth of the fungi, (ii) concentration of the fungal conidial suspension, (iii) bacterial cell concentration, and (iv) fungus-bacterium co-cultivation durations. To the best of our knowledge, this is the first report of a transformation protocol for C. albifundus and its availability will be invaluable for functional genetics studies in this important fungus. 
Differential Roles of Glucosinolates and Camalexin at Different Stages of Agrobacterium-Mediated Transformation

P. Y. Shih (1), S. J. Chou (1), C. Muller (2), B. A. Halkier (3), R. Deeken (4), E. M. LAI (1), (1) Institute of Plant and Microbial Biology, Academia Sinica, Taipei, TAIWAN; (2) Bielefeld Univ, Bielefeld, GERMANY; (3) DynaMo Center, Copenhagen, DENMARK; (4) Univ of Wuerzburg, Wuerzburg, GERMANY

Agrobacterium tumefaciens causes crown gall disease on plants via transforming T-DNA into the plant genome and is a useful tool for genetic modification. To clarify novel plant components which regulate the transformation process, we used Arabidopsis thaliana seedlings as a model system to reveal the gene expression profiles on Agrobacterium infection. Our transcriptome analysis of Agrobacterium-infected seedlings identified the differential regulation of genes involved in metabolism of secondary metabolites glucosinolates (GSs) and camalexin, suggesting their potential roles in regulating Agrobacterium transformation processes. Thus, we evaluated the impacts of GSs and camalexin during different stages of Agrobacteriummediated transformation combining Arabidopsis mutant studies, metabolite profiling, and exogenous applications of various GS hydrolysis products or camalexin. The results showed that indole GS hydrolysis played an inhibitory role in Agrobacterium-mediated transient transformation efficiency on Arabidopsis seedlings. Later in the Agrobacterium infection process, camalexin accumulation was a key factor inhibiting tumor development on Arabidopsis inflorescence stalks. No correlation could be found between transformation efficiency and bacterial growth and/or colonization upon GSs and camalexin applications, suggesting their impacts on plant transformation likely function as signals in regulating plant defense. Based on our findings, we suggest the hydrolysis products of indole GSs are used by Arabidopsis to defend Agrobacterium at early stage, and camalexin inhibits tumor development at the later stages. In addition, the differential effects of certain GS hydrolysis metabolites on Agrobacterium transformation may be used to control crown gall diseases or to enhance the transformation efficiency.

Streptomyces scabies, a causal agent of potato common scab, has the ability to degrade aromatic constituents of tuber periderm C. BEAULIEU, M. Khalil, Université de Sherbrooke, Sherbrooke, QC, CANADA

The potato periderm contains layers of suberized cells. Suberin is composed of an aliphatic domain covalently linked to an aromatic domain. S. scabies has the ability to degrade the aliphatic domain but degradation of the aromatic domain has not been documented. The last domain is composed of cinnamic acids such as ferulic and coumaric acids. These compounds were added to $S$. scabies culture medium and it was shown that the pathogen efficiently utilized these aromatics. In contrast, other common scab-inducing species exhibited a poor utilization capacity. S. scabies thus appears to be more adapted to its host than the new emerging pathogens. A bioinformatics study suggests that ferulic and coumaric acids catabolism occurred via the $\beta$-ketoadipate pathway. The gene SCAB_15301 encoding a vanillate monooxygenase was deleted from $S$. scabies genome. The mutant retained its ability to catabolize ferulic acid into vanillate but could not further degrade the latter compound. While ferulic acid induced in the wild strain the expression of genes associated with the $\beta$-ketoadipate pathway, no gene expression was recorded in the mutant. When grown in the presence of suberin-enriched potato periderm, accumulation of vanillic acid was observed only for the mutant. This work thus brought evidence that $S$. scabies could degrade not only the aliphatic part of suberin but also constituents of the aromatic domain. Suberin degradation may facilitate colonization as well as tuber infection.

Ralfuranones feedback-regulate the quorum sensing, contributing to virulence of Ralstonia solanacearum strain OE1-1 Y. HIKICHI (1), K. Hayashi (2), K. Ohnishi (3), A. Kiba (3), K. Kai (4), (1) Kochi University, Nankoku, JAPAN; (2) Kochi University, Nankoku, Kochi, JAPAN; (3) Kochi Univ, Nankoku, Kochi, JAPAN; (4) Osaka Prefecture University, Sakai, Osaka, JAPAN

Ralstonia solanacearum strain OE1-1 produces and secretes methyl 3-hydroxymyristate (3-OH MAME) as a quorum sensing (QS) signal, which contributes to its virulence. A global virulence regulator, PhcA, functioning through the QS positively regulates the expression of ralA, which encodes furanone synthase, to produce aryl-furanone secondary metabolites, ralfuranones. A ralfuranone-deficient mutant $(\triangle \mathrm{ral} A)$ is weakly virulent on tomato plants when directly inoculated into tomato xylem vessels. However, when inoculated using the root dipping, $\Delta$ ralA did not systemically infect tomato plants and lost its virulence. To investigate the functions of ralfuranones, we analysed $R$. solanacearum transcriptome data generated by RNA sequencing technology. $\triangle$ ralA exhibited downregulated expression of more than $90 \%$ of the QS-positively regulated genes, and upregulated expression of more than $75 \%$ of the QS-negatively regulated genes. Quantitative real-time PCR assays revealed that $p h c B$, which is associated with 3-OH MAME production, and $p h c A$ in $\triangle \mathrm{ral} A$ were expressed at levels similar to those in strain OE1-1. Additionally, Ralfuranone supplementation restored the ability of $\triangle$ ralA cells to aggregate dependent on the QS. Ralfuranones A and B restored the swimming motility of $\triangle$ ralA to wild-type levels. Additionally, the deletion of ralA resulted in downregulated expression of eps $B$, which is induced by the QS and involved in major exopolysaccharide, EPS I production, and upregulated expression of $f l i C$, which is suppressed by the QS and involved in swimming motility. Overall, our findings indicate that integrated signalling via ralfuranones influences the QS and virulence of $R$. solanacearum.

Down-regulation of the mycotoxin beauvericin in a phytopathogen-endophyte interaction M. BÄRENSTRAUCH (1), S. Prado (1), S. Mann (1), C. Kunz (2), (1) Museum of Natural History, Paris, FRANCE; (2) Univ Paris VI, Paris Cedex 05, FRANCE

The endophytic fungus Paraconiothyrium variabile, inhabiting the needles of the conifer Cephalotaxus harringtonia, is responsible for a strong decrease of beauvericin in Fusarium oxysporum, an ubiquitous soil-borne phytopathogen. The mycotoxin beauvericin provokes cell death in mammals and is supposed to be a virulence factor for phytopathogens. The reduction of beauvericin production is correlated to the formation of two oxylipins, 13hydroperoxy-9,11-octadecadienoic acid (13-HpODE) and 13-keto-9,11-octadecadienoic acid (13-KODE) in the interaction zone between the two antagonists. Such oxidized fatty acids are known for their role in mycotoxin regulation and are synthetized by lipoxygenase (LOX) enzymes. Two lox genes are present in P. variabile (pvlox 1 and 2) and F. oxysporum (foxlox and foxlox I) respectively. We emitted the hypothesis that endophytic oxylipins were inhibiting the transcription of the beauvericin synthetase gene (beas). qPCR results showed that the foxlox gene from $F$. oxysporum and the gene pvlox 2 from $P$. variabile were upregulated during the interaction, as well as the beas gene. These unexpected results indicate that the presence of the endophyte induces the beas gene of the phytopathogen, probably through an oxylipin signal. How can we then explain reduced beauvericin production during the interaction? Our hypothesis is that $P$. variabile hydrolyses beauvericin, as we have shown before for bacterial surfactins.

Effects of codon bias on heterologous expression of fluorescent proteins in Xylella fastidiosa

T. LOWE-POWER, S. Lindow, University of California Berkeley, Berkeley, CA, USA

The xylem pathogenic bacterium Xylella fastidiosa is characterized by slow growth, a small genome, and low codon usage bias in protein-coding genes. In most organisms, genes have bias in codon usage that reflects availability of cognate tRNAs charged with amino acids. Codon usage mismatch between a genome and a heterologous gene can reduce protein expression. Although a constitutive GFP-expressing $X$. fastidiosa strain was created by expressing the $g f p$ gene from a strong ribosomal promoter, previous attempts to create moderately-expressed fluorescent biosensors in X. fastidiosa have been unsuccessful. Therefore, we hypothesize that a codon-harmonized fluorescent protein expresses better in X. fastidiosa. To test this hypothesis, we 
adapted the fluorescent protein $\mathrm{mNeonGreen.} \mathrm{After} \mathrm{normalization} \mathrm{to} X$. fastidiosa codon usage the gene was synthesized and the codon-harmonized and wildtype $m$ NeonGreen alleles were fused to the $h x f A$ promoter and transformed into $X$. fastidiosa Temecula1 to generate biosensors that are specific for the $X$. fastidiosa quorum sensing signal diffusible signal factor (DSF). The effect of codon normalization on protein expression and fluorescence output in bacteria will be discussed.

Battles in the outer space: Extracellular DNases secreted by Pectobacterium carotovorum and its host plants N. Yu (1,2), A. Cochran (2), S. Trinh (2), F. Wen (2), H. VanEtten (2), M. C. Hawes (2), Z. XIONG (2), (1) Chinese Academy of Tropical Agricultural Sciences, Haikou, CHINA; (2) University of Arizona, Tucson, AZ, USA

Extracellular DNA (exDNA) plays a plethora of roles in the microbe and host interactions. exDNA is an important component of bacterial biofilms, aiding bacterial colonization and survival while exDNA secreted by human, animal, and plant hosts plays significant roles in defense against microbial infections. To investigate the roles of extracellular DNases (exDNases) in this warfare, we characterized the exDNases secreted by Pectobacterium carotovorum and pea (Pisum sativum) root tips. In DNase agar plate assays, exDNase activities were detected from both P. carotovorum and pea root tips, but the activities increased significantly when the two were co-cultured together. Biochemical characterizations indicated the presence of DNase IIlike endonuclease and exonuclease activities secreted from pea root tips and DNase I-like endonuclease and exonuclease activities secreted from $P$. carotovorum. In-gel PAGE DNase assays determined a single protein of $\sim 24 \mathrm{kd}$ with strong DNase activities from the culture filtrate of $P$. carotovorum and a single protein of $\sim 40 \mathrm{kd}$ with a moderate level of DNase activities from culture filtrate of pea root tips. Genes coding for both the $P$. carotovorum DNase and the pea DNase have been identified by Orbitrap LC-MS/MS. As mammalian DNase II is known to play a role in the degradation of exogenous DNA encountered by phagocytosis, the defensive role of pea exDNase is anticipated.

Phyllosphere colonization strategies related to successful infection in Xanthomonas vesicatoria A. M. ROMERO (1), V. Felipe (2), M. Montecchia (1,3), M. I. Bianco (4), P. Yaryura (2,5), (1) Facultad de Agronomia, University of Buenos Aires, Buenos Aires, ARGENTINA; (2) IAPCByA, Universidad Nacional de Villa María, Villa María, Cordoba, ARGENTINA; (3) INBA, CONICET Universidad de Buenos Aires, Buenos Aires, ARGENTINA; (4) Instituto de Ciencia y Tecnología Dr. César Milstein, Fundación Pablo Cassará, CONICET, Buenos Aires, ARGENTINA; (5) CIT Villa María, CONICET, Universidad Nacional de Villa María, Villa María, Cordoba, ARGENTINA

Bacterial spot, induced by Xanthomonas vesicatoria, is a serious constrain of field grown tomatoes in Argentina. To better understand strains aggressiveness, several characteristics of three local strains that induce different levels of disease were evaluated. For each strain, we determined in vitro swarming and twitching motilities, biofilm formation, xanthan production and xanthan viscosity. All assays were performed in triplicate. We also evaluated the number and size of the lesions induced by each strain and bacterial multiplication in planta; in both cases we used four plants per strain. Strain BNM208 was the most aggressive: it induced more and bigger lesions than BNM216; BNM214 showed intermediate values. When inoculated by infiltration, avoiding the need for phyllosphere colonization, all strains reached similar population sizes. Among the strains evaluated, BNM208 presented the greatest swarming and twitching motilities. BNM 208 also developed a mature biofilm with presence of defined cell clusters, a homogeneous and compact structure, and higher biomass and substratum coverage than BNM214 and BNM216. The three strains produced similar amounts of xanthan, however, BNM208 produced the most viscous polysaccharide, which possibly relates to the better characteristics of its biofilm. The ability of BNM208 to move over surfaces and form mature biofilms may allow a better colonization of the phyllosphere, resulting in a high infection success of this strain.

\section{Unraveling the structure and function of an uncharacterized nuclease with putative DNA activities in Xanthomonas campestris pathovar campestris}

F. C. PERITORE-GALVE (1), C. Schiltz (2), A. Lee (2), A. Han (1,3), J. S. Chappie (2), C. D. Smart (1), (1) Plant Pathology and Plant-Microbe Biology Section, Cornell University, Geneva, NY, USA; (2) Department of Molecular Medicine, Cornell University, Ithaca, NY, USA; (3) Department of Biology, Duke University, Durham, NC, USA

Black rot of crucifers caused by the gamma-proteobacterium Xanthomonas campestris pv. campestris $(X c c)$ is an economically devastating bacterial disease of brassica crops with a worldwide distribution. Infections often begin through contaminated seed, and secondary inoculum enters through the hydathodes or plant wounds, colonizing the vascular system. Despite the importance of seed contamination and bacterial survival, there is limited information on the mechanisms used during periods of low bacterial replication. The OLD nuclease has been hypothesized to play a virulence-lifestyle associated role in $X c c$ by mediating DNA repair under stressful environmental conditions. Preliminary structural analysis indicates that the ATPase domains of OLD family enzymes share structural homology with Rad50, a protein involved in eukaryotic double strand break repair. Additional structural and functional characterization of the C-terminal catalytic regions from Xcc OLD and Burkholderia pseudomallei OLD reveals surprising homology to DnaG primases and bacterial RNAse M5 maturases and supports a two-metal catalysis mechanism for nuclease cleavage. We have generated knockout and complement old gene mutants in $X c c$, and these isolates are being functionally characterized for biofilm formation, in vitro growth, epiphytic survival, and virulence to better understand the influence of OLD nuclease. Studying the molecular machinery underlying survival and colonization may enable the identification of new targets for resistance breeding or chemical control.

Fusel alcohol biosynthesis in the Ceratocystidaceae

D. Mailula, M. VAN DER NEST, B. D. Wingfield, A. Hammerbacher, Forestry and Agricultural Biotechnology Institute (FABI), University of Pretoria, Pretoria, SOUTH AFRICA

Fungi in the family Ceratocystidaceae produce fusel alcohols and acetates which have fruity and floral odours. These compounds are valuable additives of food products, perfumes and soaps. The of the study aim was to analyse the volatiles produced by the Ceratocystidaceae and to study their biosynthesis. All the stains included in our study produced isoamyl acetate from leucine, while members of the Endoconidiophora and Huntiella genera also produced phenylethyl acetate from phenylalanine. Species of Ceratocystis and Huntiella produced isobutyl acetate using valine as a substrate. We discovered four genes encoding aromatic amino transferases and three genes encoding branched-chain amino transferases in the available genomes of the Ceratocystidaceae that might be involved in catalysing the first steps in the production of the fusel alcohols produced by these fungi. This, however, represents a lower number of genes encoding these enzymes than in other fungi of the Sordariomycetes that do not produce fruity or floral smells. Further functional analysis of these genes will therefore be necessary to confirm their involvement in the biosynthesis of these volatiles.

Regulatory proteins involved in cyclic-di-GMP-mediated transcriptional regulation of amylovoran production in Erwinia amylovora R. R. KHARADI, G. W. Sundin, Michigan State University, East Lansing, MI, USA 
Cyclic-di-GMP (c-di-GMP) is a ubiquitous bacterial second messenger critical in signaling the transition from a mobile lifestyle to an attached lifestyle in the form of biofilms. In Erwinia amylovora, c-di-GMP is known to positively regulate the production of amylovoran, which is a pathogenicity factor, the most abundant exopolysaccharide in the cell, and is also required for biofilm formation. In order to evaluate whether this regulation occurs at a transcriptional level, we mapped the promoter region of $a m s G$, the first gene of the amylovoran biosynthetic operon and determined the location of the transcriptional start site (TSS). Through the systematic cloning of various sequence regions of the ams $G$ promoter into a GFP reporter transcriptional fusion vector, we were able to determine that a ca. 170-bp region located upstream of the TSS is critical in the c-di-GMP mediated transcriptional regulation of ams $G$. In order to identify potential c-di-GMP dependent effectors that transcriptionally regulate the ams $G$ promoter, we screened a library of transposon mutants generated using the transcriptional fusion reporter strains via fluorescence based cell sorting. We identified six potential positive regulators of the $a m s G$ promoter. Based on evaluating these candidates for their effect on amylovoran production, biofilm formation as well as $a m s G$ expression, we illustrate the role of two novel positive transcriptional regulators of amylovoran production.

Engineering citrus canker and Huanglongbing resistance by overexpressing of glutamate decarboxylase in citrus S. ZHANG (1), Q. Shi (2), M. Pitino (2), D. Hall (2), G. Gupta (1), E. Stover (2), (1) New Mexico Consortium, Los Alamos, NM, USA; (2) U.S. Horticultural Research Laboratory, USDA-ARS, Fort Pierce, FL, USA

Gamma-Aminobutyric acid (GABA) is a ubiquitous four-carbon, nonprotein amino acid synthesized by glutamate decarboxylase (GAD) in bacteria, animals and plants. Plant-derived GABA has been shown to modulate quorum sensing of Agrobacterium tumefaciens C58 in tobacco (Chevrot et al., 2006), and to repress the expression of the type III secretion system of Pseudomonas syringae pv. tomato DC3000 in Arabidopsis (Ward et al., 2010). Overexpression of GAD in tobacco plants with elevated GABA levels was sufficient to impart enhanced resistance to an insect, a nematode and a bacterial pathogen (McLean et al., 2003; MacGregor et al., 2003; Chevrot et al., 2006). Citrus canker and Huanglongbing are two destructive bacterial diseases in citrus production worldwide. They are caused by Xanthomonas citri subsp. citri (Xcc) and Candidatus Liberibacter spp., respectively. Here, a gene expression survey of citrus GAD orthologs was conducted in 'Valencia' sweet orange, 'Cleopatra' mandarin, 'Carrizo' citrange, rough lemon and citron. The expression levels of two citrus GAD genes were significantly higher in all Liberibacter-infected trees compared with uninfected trees regardless genotype, indicating the involvement of GABA in citrus-Liberibacter interactions. GABA at various concentrations didn't directly inhibit Xcc growth in medium. However, GABA eliminated Xcc-mediated hypersensitive response (HR) in the non-host Nicotiana benthamiana and promoted bacterial growth, suggesting a suppression effect of GABA on Xcc type III secretion. Full-length cDNA of two GAD homologs from 'Valencia' sweet orange was cloned and genetically transformed into Carrizo citrange. GAD-overexpressing plants have been identified and are being characterized for increased canker and HLB resistance.

Design of a flagella-propelled bio-inspired submersible robot for hydroponic production and irrigation system inspection F. BAYSAL-GUREL (1), E. Erdemir (2), T. Darrah (2), E. Kaplanoglu (2), R. Turner (2), S. K. Hargrove (2), (1) Tennessee State University, McMinnville, TN, USA; (2) Tennessee State University, Nashville, TN, USA

The goal of this research is to design and optimize a flagella-propelled bio-inspired submersible robot that can operate in hydroponic systems to assess the root zone health of individual plants and operate in irrigation pipes to detect leaks by data fusion and image processing techniques. In recent years, hydroponic farming has experienced exponential growth due to several factors including rural land scarcity and increasing demand for food. Hydroponic production requires a high degree of monitoring, maintenance and precise control of several factors to be successful. Root diseases such as Pythium occur in all types of hydroponic systems. Root diseases are easily detected in solution culture systems as the roots can be seen and disease development on the roots is often noticed before symptoms are visible in plant shoots. Nevertheless, root assessment by the growers is time consuming and may stress or damage the plants. Also, water leak detection for underground irrigation systems is time consuming. The research team designed a flagella-propelled submersible inspection robot and verified its maneuverability and operability under/around/through plant roots autonomously and through the irrigation pipes. Future studies will be on evaluation of robot performance for early determination of stress conditions (root rot etc.) in hydroponic systems, as well as determination of leaks in irrigation pipes.

CRISPR-Cas mediated RNA modulation for improved plant defense

D. Cook, J. Huang, V. SHARMA, Kansas State University, Manhattan, KS, USA

Application of the prokaryotic immune system, defined by clustered regularly interspaced short $p$ alindromic $r$ epeats (CRISPR) and CRISPR associated (Cas) proteins, for eukaryotic genome editing has significantly impacted diverse biologic fields. The CRISPR-Cas system targeting DNA has been used to delete DNA, direct gene insertion, gene-drive, DNA viral resistance, transcriptional regulation and epigenome modification. One area of interest, is the use of CRISPR-Cas for targeted gene deletion in crops to improve agronomics. However, removing or inactivating a gene(s) can result in unanticipated negative pleiotropic effects. Additionally, the use of the CRISPR-Cas system for anti-viral immunity is limited to targeting DNA viruses. To overcome these challenges, we have developed a newly described RNA-guided RNA-targeting CRISPR-Cas system for use in plants. We are developing the system for two purposes- 1) to direct viral resistance to both DNA and RNA viruses; 2) regulate plant defense through mRNA degradation and translational manipulation. The ability to target and manipulate mRNA will open the door to a range of new applications, continuing the innovative use of the prokaryotic CRISPR-Cas system to study and improve crops.

Engineering $x o p A G$ induced expression by Xanthomonas citri TALE confer resistance to citrus canker

D. SHANTHARAJ (1,2), P. Römer (3), V. Orbovic (4), G. V. Minsavage Jr. (5), D. M. Horvath (6), T. Lahaye (7), G. A. Moore (8), J. B. Jones (5), (1) Auburn University, Department of Biological Sciences, Auburn, AL, USA; (2) University of Florida, Plant Pathology Department, Gainesville, FL, USA; (3) Genetics, Department of Biology, Ludwig-Maximilians-University Munich Germany., Munich, GERMANY; (4) CREC, University of Florida, Lake Alfred, FL, USA; (5) Department of Plant Pathology, University of Florida, Gainesville, FL, USA; (6) 2Blades Foundation, Evanston, IL, USA; (7) Zentrum für Molekularbiologie der Pflanzen (ZMBP), Eberhard-Karls-Universität Tübingen, Auf der Morgenstelle, GERMANY; (8) University of Florida, Horticultural Sciences Department, Gainesville, FL, USA

Xanthomonas citri ssp. citri (Xcc), causal agent of citrus canker, delivers transcription activator-like effectors (TALEs) into plant cells via the type III secretion system. One group of TALEs targets effector binding elements (EBEs) in the host genome to activate expression of a susceptibility gene, essential for typical disease. Predictably, TALEs bind EBEs in host promoters. We introduced 14 EBEs, matching distinct Xcc TALEs, into the promoter of the pepper $B s 3$ gene (ProBs $3_{1 \mathrm{EBE}}$ ), and fused this engineered promoter (ProBs $3_{14 \mathrm{EBE}}$ ) to the $X$. fuscans subsp. aurantifolii gene, avrGf2, which encodes a hypersensitive response (HR)-eliciting effector in grapefruit, to create ProBs $3_{14 \mathrm{EBE}}$ :avrGf2. This construct was placed in the binary vector, pTLAB21. Agrobacterium strain EHA101 carrying the binary vector pTLAB21 with ProBs $3_{14 \mathrm{EBE}}$ :avrGf2 was used to produce genetically engineered grapefruit plants. The plant transformed with ProBs $3_{14 \mathrm{EBE}}$ :avrGf2 was evaluated for disease resistance. Infiltration of a bacterial suspension $\left(10^{8} \mathrm{CFU} / \mathrm{ml}\right)$ of Xcc into leaves elicited an HR in leaves of the transgenic grapefruit, but not wild-type grapefruit. Similarly, when a bacterial suspension ( $\left.10^{5} \mathrm{CFU} / \mathrm{ml}\right)$ of Xcc 
was infiltrated into leaves, bacterial populations were ten-fold lower in leaves of the transgenic plant than in the wild-type grapefruit, 5 days after infiltration. Grapefruit transformed with ProBs $3_{14 \mathrm{EBE}}: a v r G f 2$ offers resistance to a broad spectrum of citrus canker strains.

Development of a protein-luciferase-based high-throughput screening system to monitor degradation of Jasmonate ZIM-domain family proteins H. ISHIDA, R. Ogura, K. Hiratsuka, Graduate School of Environment and Information Sciences, Yokohama National University, Yokohama, JAPAN

We previously demonstrated that the system using Arabidopsis seedlings harboring a promoter-reporter fusion gene, such as luciferase (LUC) reporter fusion, is suitable for monitoring of regulated gene expression of pathogen responsive genes such as PR1, PDF1.2, and VSP1. Using the system we could successfully carry out the screening and evaluation of bioactive compounds capable of inducing the expression of defense-related genes involved in systemic acquired resistance or induced systemic resistance. However, the system provides us with indirect evidence for the resulting gene expression and the processes involved in the signaling pathway are not necessarily clear. On the other hand, protein degradation involved in a particular signaling pathway can be a suitable marker for specific regulation of the signaling process. Using transgenic plants expressing LUC-protein fusion, we are able to monitor the protein degradation process as the decay of luciferase activity in vivo. To monitor defense response against necrotrophic pathogens mediated by jasmonates (JA), we attempted to observe the dynamics of Jasmonate ZIM-domain family proteins (JAZ) which are rapidly degradated in the presence of jasmonyl-L-isoleucine. Using transgenic Arabidopsis seedlings expressing JAZ-LUC fusion proteins, we could observe a rapid decay of the bioluminesence in planta in response to JA treatment. The system allows us to monitor JAZ-mediated signaling response within less than one hour and to perform high-throughput screening for JA agonists using 384-well plates.

\section{Development of the VIGS system towards enhancing the production level of hatching factors for potato cyst nematode using Nicotiana} benthamiana

G. ATSUMI (1), N. Itchouda (2), U. Kagaya (2), A. Haseda (2), K. Hayashi (2), K. Furuta (2), N. Tabayashi (2), T. Matsumura (1), (1) National Institute of Advanced Industrial Science and Technology, Sapporo, Hokkaido, JAPAN; (2) Hokusan Co., Ltd., Kitahiroshima, Hokkaido, JAPAN

Cyst nematodes threaten stable productions of economically important crops such as potato. Host potato plants release chemicals such as triterpenoids including solanoeclepin A from the roots to stimulate hatching of the nematode. However, the biosynthetic pathways are unknown. In this study, we developed a system to evaluate a hatching activity towards potato cyst nematode using root exudates from Nicotiana benthamiana which is a suitable host for a rapid analysis of gene functions using technologies such as virus-induced gene silencing (VIGS). We found that root exudates from $N$. benthamiana have a hatching activity for potato cyst nematode in in vitro hatching assay. To enhance overall triterpenoid synthesis, we down-regulated the competitive pathways leading to cholesterol and/or phytosterols using tobacco rattle virus (TRV) vector-based VIGS in hydroponically grown $N$. benthamiana. We isolated the genes encoding enzymes, cycloartenol synthase 1 [CAS1], sterol side chain reductase 2 [SSR2], and S-adenosyl-L-Metdependent C-24 sterol methyltransferase 1 [SMTI] from N. benthamiana, and constructed TRV vectors to down-regulate respective genes. VIGS using the constructed TRVs successfully reduced CAS1, SSR 2 and SMT1 expressions to approximately 14, 11, and 6\% in root tissues 16 days after virus inoculation, respectively. Their expressions were similarly down-regulated in leaf and stem tissues. We believe that the TRV-based VIGS system in $N$. benthamiana will be a powerful tool to elucidate the biosynthetic pathways of hatching factors, eclepins. This work was supported by a grant from the New Energy and Industrial Technology Development Organization (NEDO) of Japan.

Regulation of citrus DMR6 via RNA interference and CRISPR/Cas9-mediated gene editing to improve Huanglongbing tolerance S. ZHANG (1), Q. Shi (1), Y. Duan (1), D. Hall (1), G. Gupta (2), E. Stover (1), (1) U.S. Horticultural Research Laboratory, USDA-ARS, Fort Pierce, FL, USA; (2) New Mexico Consortium, Los Alamos, NM, USA

Plant innate immunity involves the activation of inducible defenses that are tightly controlled by gene regulators. Arabidopsis Down Mildew Resistance 6 (DMR6) is a $2 \mathrm{OG} \mathrm{Fe}$ (II)-dependent oxygenase that acts as a suppressor of plant immunity, and its expression is upregulated during pathogen infection (Zeilmaker et al., 2015). Mutation of Arabidopsis DMR6 and its orthologs in potato and tomato resulted in elevated salicylic acid level and conferred broad-spectrum disease resistance (de Toledo Thomazella et al., 2016; Sun et al., 2016). Huanglongbing (HLB) is a devastating disease in citrus worldwide. Genetic resistance from commercial varieties is lacking but tolerance has been observed. Previously, we found DMR6-like genes were downregulated in the more tolerant 'Jackson' grapefruit compared with those in the susceptible 'Marsh' grapefruit. Here, we conducted an expression survey of citrus DMR6 orthologs in 'Hamlin' sweet orange, 'Clementine' mandarin, 'Carrizo' citrange, rough lemon, sour orange and citron. The expression levels were significantly higher in infected trees than in uninfected ones regardless genotype, suggesting a universal role of DMR6 in the defense against the HLB bacterium, Candidatus Liberibacter asiaticus. RNA interference was used for gene knockdown in Carrizo, and resulted in an average of $41.4 \%$ silencing efficiency. DMR6 silenced plants exhibited higher expression of defense-related genes than non-transgenic controls. A CRISPR/Cas9 system was also used for gene knockout. Three sgRNAs were designed and shown to be effective in DNA cleavage in vitro. Agrobacterium-mediated transformation of these CRISPR constructs in citrus is underway to generate DMR6 mutants for increased HLB tolerance/resistance.

Random T-DNA mutagenesis reveals gene candidates modulating pathogen virulence in postharvest Penicillium-apple fruit interactions H. Peng (1), W. M. JURICK II PHD (1), V. L. Gaskins (1), K. D. Cox (2), W. J. Janisiewicz (3), K. A. Peter (4), (1) USDA-ARS Food Quality Laboratory, Beltsville, MD, USA; (2) Cornell University, Geneva, NY, USA; (3) USDA-ARS AFRS, Kearneysville, WV, USA; (4) Penn State University, Biglerville, PA, USA

Postharvest blue mold decay of apple fruit is mainly caused by the fungus, Penicillium expansum. Problems with controlling this disease are exacerbated by a limited number of fungicides, no known resistance in apple, and emergence of fungicide resistant strains. Sustainable strategies are needed to control blue mold to maintain fruit quality, reduce economic losses for producers, and eliminate patulin contamination. A forward genetics approach was implemented, using Agrobacterium tumefaciens-mediated transformation, to generate a $P$. expansum mutant library. A total of $\sim 450$ transformants were screened in vivo for defects in virulence on apple fruit. Phenotypes ranged from wild type to completely non-pathogenic and were conducted in two different apple varieties. Strains that were reduced in virulence during apple decay and did not differ greatly from wild type growth in culture on were further analyzed. Specific mutants were characterized to determine T-DNA copy number, location of integration, and the nature of the insertion using molecular tools. Our analysis revealed the identities of several new genes, some with uncharacterized domains/function, that are associated with virulence defects during apple fruit decay. Future studies will utilize well characterized T-DNA mutant strains for omics-based approaches to ascertain the molecular mechanisms of Penicillium virulence with the end goal of translating our findings into practical blue mold controls. 
Rice blast, caused by the hemibiotrophic fungus Magnaporthe oryzae, is the most destructive disease of rice world-wide. As an alternative to readily broken-down disease resistance, HIGS (host induced gene silencing) is being developed as a strategy to create a new type of disease resistant plants. Eight genes (CRZ1, Pmc1, MagB, Lhs 1, CYP51A, CYP51B, Exo70, Sec5) that play important roles in pathogenicity and development of M. oryzae were chosen for HIGS. HIGS vectors were transformed into rice calli through the Agrobacterium-mediated method. T0, T1 and T2 generations of transgenic rice plants were generated and the insertion of HIGS fragments confirmed. HIGS transgenic rice plants challenged with $M$. oryzae showed significantly reduced disease compared with non-silenced control plants. Following infection with M. oryzae of HIGS transgenic plants, expression levels of target genes were reduced as demonstrated by Quantitive RT-PCR. In addition, treating M. oryzae with naked diced siRNA derived from the target genes silenced their expression in the fungus and caused functional collapse. These findings suggest RNA silencing signals can be transferred from host to invasive fungus and HIGS has potential to generate resistant rice against M. oryzae.

Non-transgenic gene editing of Citrus sinensis using CRISPR/Cas9 ribonucleoprotein complexes Y. WANG, CREC, University of Florida, Lake Alfred, FL, USA

Citrus is one of the most important tree crops in the world. However, citrus production is facing many challenges including many biotic and abiotic stresses. Among them, both citrus canker caused by Xanthomonas citri and Huanglongbing caused by Candidatus Liberibacter asiaticus cause severe economic losses to citrus production. Breeding disease-resistant varieties is the most efficient and sustainable approach to controlling most plant diseases. Traditional breeding to generate disease-resistant citrus varieties has been hindered by polyembryony, pollen-ovule sterility, sexual and graft incompatibilities, and extended juvenility. CRISPR technology has shown promise to speed up the breeding process for citrus. Here, we describe our current progress in generating transgene-free citrus using the CRISPR/Cas9 ribonucleoproteins (RNPs) method. The purified Cas9 shows nuclease activity at the presence of sgRNA. Mutation of $C S P D S$ was observed. No off-targets were detected.

Engineering Resistance to Wheat Stripe Rust (Puccinia striiformis f. sp. tritici) Using a Protease Recognition System M. HELM, R. W. Innes, Indiana University, Bloomington, IN, USA

This project focuses on a novel 'decoy' system for engineering resistance to wheat stripe rust (Puccinia striiformis f. sp. tritici; Pst) using endogenous disease resistance (R) proteins to detect Pst proteases. This approach represents an extension of our work on the Arabidopsis R protein RPS5, which mediates recognition of the protease AvrPphB by detecting cleavage of one of AvrPphB's targets, PBS1. We have recently shown that we can expand the recognition specificity of RPS5 by adding PBS1 'decoys' that function as substrates for other pathogen proteases. Cleavage of these modified PBS1 proteins by pathogen proteases activates RPS5, thereby conferring resistance to multiple pathogens. Significantly, AvrPphB is recognized by most wheat cultivars; thus, wheat likely contains an $R$ protein functionally analogous to RPS5 that recognizes cleavage of a wheat PBS1. We predict we can change the specificity of the wheat R protein simply by modifying the AvrPphB cleavage site in a wheat PBS1 ortholog. We have identified conserved Pst proteases that are expressed during infection and which may be translocated into host cells. Creation of wheat PBS1 decoys that are cleaved by one or more of these proteases should activate the endogenous $R$ protein and thus resistance to $P$ st, one of the most economically important pathogens of wheat.

\section{Development of CRISPR/Cas9 mediated virus resistance}

A. CHAKRABORTY (1,2), H. Li (1), S. Iqbal (1), M. G. K. Jones (1), S. J. Wylie (1), (1) Murdoch University, Perth, WA, AUSTRALIA; (2) Shahjalal University of Science and Technology, Sylhet, BANGLADESH

Plant RNA viruses are a very diverse group of viruses, capable of causing significant damage in crops, and potentially posing a threat to food security on local and global scales. Gene editing has enormous potential in the control of plant pathogens in agricultural crops. However, RNA viruses are not an obvious target for gene editing technologies because CRISPR/Cas9 targets DNA, not RNA. In this study we investigated novel strategies to control RNA viruses in plants using a highly flexible CRISPR/Cas9-based approach. The strategy depends on both specific and degenerate sgRNAs and their targeted delivery. Studies focused on design of the sgRNAs, their specific (species) and generalised (genera, family) targets, delivery to sites of action, and stability over time. Current studies will test the concept on one family of RNA viruses, the Potyviridae, but future studies will test the system against other groups of RNA viruses. The current study was done using Nicotiana tabacum L plants as a model system because of ease of transformation and large leaf surface area and biomass. Research in this model plant will provide proof of concept for applications to economically important crop species. Implications of a successful outcome of this research will be novel control strategies against, potentially, all plant RNA viruses.

Improve tobacco rattle virus-based microRNA silencing by special viral RNA interference suppressor J. ZHAO, Texas A\&M University, AgriLife Research Center at Dallas, Dallas, TX, USA

Virus-based microRNA silencing (VbMS) is a powerful tool to dissect the function of plant endogenous microRNAs (miRNA). Target mimic (TM) molecules expressed from virus backbones block the function of convergent miRNAs by knocking down miRNA targets and several members of the miRNA family. To date, various VbMS systems have been developed in tomato, Nicotiana benthamiana, and cotton. The tobacco rattle virus (TRV) based vectors has been the most applied approach for virus induced gene silencing (VIGS), while the TRV-based VbMS system so far has not been widely used and needs improvement for higher efficiency. Here, we report an improved TRV-based VbMS system by integrating special viral RNA interference suppressors (VSRs) into the virus backbone. In this system, two geminivirus VSRs under the control of TRV expression promoter could increase the level of TM molecules. Expression of the artificial designed short tandem target mimics (STTM) of miR165/166 from the improved TRV vectors blocked the function of endogenous miR165/166, causing distinct developmental defects in $N$. benthamiana, such as ectopic growth of leaf lamina on the abaxial leaf surface along vein and midrib. Molecular analysis confirmed the suppression of endogenous miR $165 / 166$ and the increase of mRNA transcripts of the miR165/166 target HDZIP like gene. The improved TRV VbMS system presented approximately 100\% efficiency and very mild viral symptom in inoculated plants. Since the TRV VIGS system possesses wider host range than other systems, this improved TRV VbMS system could be applied to a wider range of plant species for functional characterization of miRNAs.

Increase of sweet orange resistance against Xanthomonas citri pv citri through translocation of DSF molecules from transgenic rootstocks R. CASERTA (1), R. de Souza (2), S. Lindow (3), A. A. de Souza (4), (1) Centro de Citricultura Sylvio Moreira, Cordeirópolis, BRAZIL; (2) Centro de Citricultura Sylvio Moreira, Cordeiropolis, BRAZIL; (3) University of California Berkeley, Berkeley, CA, USA; (4) Centro de Citricultura Sylvio Moreira - Agronomic Institute (IAC), Cordeiropolis, BRAZIL

Rootstocks are widely used to improve citrus scions characteristics. We have shown that transgenic sweet orange scions producing XfDSF, a diffusible signal factor molecule used by Xylella fastidiosa $(X f)$ for Quorum Sensing (QS), were more resistant for both citrus variegated chlorosis and citrus 
canker diseases. Citrus canker is caused by Xanthomonas citri subsp. citri $(X c c)$, and the resistance of these transgenic plants was attributed to the antagonist effect of the XfDSF-producing plants. As the production of XfDSF increased the resistance in sweet orange scions, we hypothesized that transgenic rootstocks could increase the resistance of non-transgenic grafted scions by the translocation of DSF molecules. Our goal was to obtain transgenic plants of the rootstock Carrizo producing DSF and to evaluate the resistance of grafted Valencia and Hamlin sweet orange varieties against $X c c$. The challenge of plants grafted onto transgenic rootstocks demonstrated reduced symptoms of canker lesions. Expression of key virulence-related genes of $X c c$, known to be positively regulated by the $X c c$ DSF, such as bacterial movement, cellulose degradation and type III secretion system were downregulated in $X c c$ isolated from transgenic plants. This strategy is innovative for citrus once a molecule produced by a transgenic rootstock increased the resistance of non-transgenic scions through the alteration of bacterial behavior.

A TMV-based viral vector for delivering gene editing tools

K. T. CHIONG (1), W. B. Cody (1), T. E. Mirkov (2), H. B. Scholthof (1), (1) Texas A\&M University, College Station, TX, USA; (2) Texas A\&M AgriLife Research, Weslaco, TX, USA

Plant viruses can be engineered and utilized as overexpression tools for transient gene modification studies and large-scale production of high value proteins. An efficient example is the agroinfiltratable TMV-based overexpression vector (TRBO), a coat protein gene deletion mutant of TMV. The CRISPR/Cas9 gene editing system involves two main components, a single guide RNA (sgRNA) and a Cas9 endonuclease which, as a complex, creates double-stranded breaks in complementary DNA sequences. Previously, our group used the TRBO vector to express both a green fluorescent protein (GFP) and a biologically active sgRNA (TRBO-G-3'gGFP) in Nicotiana benthamiana. Here, we utilized a TRBO vector to transiently express high levels of Cas9, and another for simultaneous delivery of a sgRNA which targets the $m g f p 5$ gene (gGFP) in the $N$. benthamiana GFP-expressing line $16 \mathrm{c}$. The engineered Cas9-expressing TRBO vector (TRBO-Cas9) was able to express a biologically functional protein, despite the large insert size ( 4.2 kb). Co-delivery of TRBO-Cas9 and TRBO-G-3'gGFP along with P19, an RNA silencing suppressor protein of Tomato bushy stunt virus (TBSV), resulted in high levels of Cas 9 protein and efficient gene editing in planta. Additionally, we combined both Cas9 and the gGFP in a single delivery vector that resulted in gene editing events. Our system demonstrates the potential of virus vectors to rapidly create non-transgenic knockout plants for plant functional genomics and proteomics.

\section{Development of high expression system of a foreign gene replacing a coat protein region in the cucumber mosaic virus vector through agroinfection \\ N. FUKUZAWA (1), C. Masuta (2), T. Matsumura (1), (1) National Institute of Advanced Industrial Science and Technology, Sapporo, Hokkaido, JAPAN; (2) Research Faculty of Agriculture, Hokkaido University, Sapporo, Hokkaido, JAPAN}

To enable further improvement in the expression levels of the foreign gene using viral vector, we have developed the cucumber mosaic virus (CMV)agroinfection methods using a plant-based platform. CMV is known to have a very broad host range and contains three segmented, genomic RNAs (RNAs 1-3). In this study, we engineered CMV RNA3 to replace the coat protein (CP) gene with a green fluorescence protein (GFP) as a marker protein in a Ti plasmid (pBI-CR3 $\triangle \mathrm{CP}-\mathrm{GFP}$ ). RNA1 and RNA2 were also designated pBI-CR1 and pBI-CR2. As the platform plant to produce the foreign protein, we prepared transgenic Nicotiana benthamiana expressing either RNA1 or RNA2 (CR1 Tg or CR2 Tg plant). When wild-type plants were infiltrated with Agrobacterium suspension harboring the pBI-CR1, pBI-CR2 and pBI-CR3 $\triangle$ CP-GFP construct, we observed sporadic GFP fluorescence in the infiltrated leaves. When the pBI-CR1 and pBI-CR3 $\triangle$ CP-GFP construct were infiltrated in CR2 Tg plants, we found that the GFP fluorescence was dispersed in the infected leaves. However, when CR1 Tg plants were infiltrated with bacteria containing the pBI-CR2 and pBI-CR3 $\triangle$ CP-GFP construct, the yield of GFP in CR1 Tg plants was calculated to be $750 \mathrm{mg} / \mathrm{kg}$ fresh weight within 3 dpi. This study demonstrates that a transgenic plant expressing CMV RNA1 can be used as an excellent plant-based platform for high protein production.

\section{Editing citrus genome via SaCas9/sgRNA system}

H. JIA (1), N. Wang (2), (1) CREC, University of Florida, Lake Alfred, FL, USA; (2) University of Florida, Lake Alfred, FL, USA

SaCas9/sgRNA, derived from Staphylococcus aureus, is an alternative system for genome editing to Streptococcus pyogenes SpCas9/sgRNA. The smaller SaCas9 recognizes a different protospacer adjacent motif (PAM) sequence from SpCas9. SaCas9/sgRNA has been employed to edit the genomes of Arabidopsis, tobacco and rice. In this study, we aimed to test its potential in genome editing of citrus. Transient expression of SaCas9/sgRNA in Duncan grapefruit via Xcc-facilitated agroinfiltration showed it can successfully modify $C s P D S$ and $C s 2 g 12470$. Subsequently, binary vector GFPp1380N-SaCas9/35S-sgRNA1:AtU6-sgRNA2 was developed to edit two target sites of Cs $7 g 03360$ in transgenic Carrizo citrange. Twelve GFP-positive Carrizo transformants were successfully established, designated as $\# \mathrm{Cz} 1$ to \#Cz12. Based on targeted next generation sequencing results, the mutation rates for the two targets ranged from $15.55 \%$ to 39.13 for sgRNA1 and $49.01 \%$ to $79.67 \%$ for sgRNA2. Therefore, SaCas $9 / \operatorname{sgRNA}$ can be used as an alternative tool to SpCas9/sgRNA for citrus genome editing.

Use of biotechnological tools to incorporate broad virus resistance into wheat

M. NAVIA-URRUTIA (1), J. Rupp (2), J. P. Fellers (3), H. N. Trick (1), (1) Kansas State University, Manhattan, KS, USA; (2) Department of Plant Sciences and Plant Pathology, Montana State University, Bozeman, MT, USA; (3) USDA ARS HWWGRU, Manhattan, KS, USA

Viruses are a serious threat to global wheat production. In Kansas, the wheat streak mosaic complex, caused by Wheat streak mosaic virus (WSMV), Triticum mosaic virus (TriMV), and Wheat mosaic virus (WMoV), ranked as the second most important disease of 2017, accounting for 5.6\% yield loss. Control of the vector is not fully effective and few sources of resistance to these viruses have been identified. Viruses of the family Potyviridae (WSMV and TriMV) recruit the host eukaryotic translation initiation factors to complete their infection cycle. Our aim was to silence and edit the wheat initiation factors $e I F$ (iso) $4 E-2$ and $e I F 4 G$, for conferring resistance to WSMV and TriMV. Calli of the susceptible cultivar 'Bobwhite' were independently bombarded with $e I F$ (iso) $4 E-2$ and $e I F 4 G$ RNAi expression vectors. Silenced lines were resistant to WSMV and TriMV in single and mixed infections, through multiple generations and in crosses with the variety 'Karl 92'. Relative expression assays confirmed reduction in eIF(iso)4E-2 and eIF4G expression, and in viral titer. For the gene-editing approach, four regions were selected in each gene to be targeted by Cas9. 'Bobwhite' calli were bombarded with target-specific sgRNA scaffold vectors and vectors carrying Cas 9 . Sixty-four independent biolistic experiments have been completed. Regeneration of mutants, performance against viruses, and the effectiveness of these tools to incorporate virus resistance into wheat, will be discussed.

A technique to reduce DNA methylation in a sequence-specific manner by using a ribozyme-expressing cucumber mosaic virus vector W. Matsunaga (1), R. ISODA (1), T. Inukai (1), T. Matsumura (2), C. Masuta (1), (1) Research Faculty of Agriculture, Hokkaido University, Sapporo, Hokkaido, JAPAN; (2) National Institute of Advanced Industrial Science and Technology, Sapporo, Hokkaido, JAPAN 
Host epigenetic status has been found to be modified by some plant virus infections. For example, cucumber mosaic virus (CMV) has been shown to affect genome-wide DNA methylation in infected plants. We have been studying the interactions between CMV and host in epigenetic modifications. Taking advantage of our findings on CMV, we here tried to use the CMV vector to develop a technique to induce demethylation in a sequence-specific manner. We first induced transcriptional gene silencing (TGS) to the 35S promoter upstream of the GFP gene in the transgenic 16C plants; TGS has been stably maintained through at least several generations (GFP-TGS). Short RNAs and scaffold RNAs (scRNAs) are known to play a role in guiding the DNA methylation complex to the target sequence. We thus tried to destroy short RNAs and scRNAs by sequence-specific hammer-head ribozyme (Rz) cleavage. An Rz expressed through the CMV vector was designed to cut scRNAs before the transcription start site (TSS). When we inoculated the CMV vector with an Rz onto the GFP-TGS plants, we found that DNA methylation levels were decreased over the 35S promoter and especially around the TSS, resulting in recovery of GFP fluorescence. To confirm whether the designed Rzs were really functional, we conducted in vitro cleavage and in vivo sensor experiments. These results together indicate that we could successfully develop a technique for DNA demethylation by using an Rz-expressing CMV vector.

Soil-borne diseases identified as key yield-limiting factors in potato crops R. FALLOON, S. Sinton, F. Shah, S. Dellow, A. Michel, The New Zealand Institute for Plant \& Food Research Limited, Christchurch, NEW ZEALAND

The climate in Canterbury, New Zealand, supports potato yields of $90 \mathrm{t} / \mathrm{ha}$ in most growing seasons, as indicated by a verified simulation model. However, current yields from processing (French fry) potato crops are 50-60 t/ha. Soil-borne diseases, particularly Rhizoctonia stem canker and Spongospora root galling, were identified in a previous survey as commonly occurring yield-limiting factors in potato crops. Three irrigated crops (each $\cong 25 \mathrm{ha}$, but with different soils and cropping histories) were intensively monitored (2014/15 growing season) to determine causes of this "yield gap". At eight sites in each crop, selected to represent local soil variability, observation plots (eight rows by $10 \mathrm{~m}$ ) were marked, and plants were assessed at 10 to $14 \mathrm{~d}$ intervals for potential yield-limiting factors and tuber yields. In one crop, where little soil- or seed tuber-borne disease occurred, measured yields approached potential (mean $=78 \mathrm{t} / \mathrm{ha}$ ). In the second crop, yields were slightly reduced (mean $=72 \mathrm{t} / \mathrm{ha}$ ), where Rhizoctonia stem canker developed late in the growing season, and Spongospora root galling was slight (mean $=6-8$ galls/plant) and also late-developing. Yields from the third crop averaged 55 t/ha, where Rhizoctonia stem canker was severe, killing many plant stems, and Spongospora galling developed early and was severe on roots and underground stems. This study, of three contrasting potato crops, highlights the need for effective strategies to reduce soil-borne diseases, to obtain yields from intensively managed potato crops that approach their modelled potential.

Impact of berry blotch disease (Cercospora coffeicola Berk. \& Cooke.) on coffee quality and value in Colombia C. A. ANGEL (1), G. Puerta (1), H. Duque (1,2), (1) National Coffee Research Center -Cenicafe, Manizales, COLOMBIA; (2) Colombian Coffee Growers Federation -FNC, B, COLOMBIA

Coffee Berry Blotch Disease (BBD, Cercospora coffeicola) is relevant worldwide, causing defoliation and yield losses to berries, but limited data exist on assessing these losses. To determine economic loss functions and BBD impact on physical and beverage quality, samples of Coffea arabica cv. Colombia ripe-berries from a commercial field were collected according to a BBD severity grading scale, from grade 1 (superficial spots) to grade 5 ( $\geq$ $75 \%$ of berry's pericarp and pulp necrosed and sunken). Berries were processed to obtain dried green beans, and weight conversion factors, yield, defective beans and commercial price were determined based on Colombian coffee standards. A coffee tasting panel analyzed 15 cups per each healthy and BBD grade samples, and spiked healthy samples with 5 and $10 \%$ per grade in berries weight. Grades 1 and 2 (superficially diseased) showed neither economic losses nor physical and beverage quality defects. However, significant differences occurred for grades 3,4 and 5 , which weight losses ranged from 19.8 to $75.2 \%$ for berries, and from 10.2 to $30.6 \%$ for green beans, whereas healthy beans ranged from 87.5 to $56.4 \%$ respectively. Grade 3 beans lose $61.6 \%$ of market price and $100 \%$ for grades 4 and 5 . Significant exponential loss functions were obtained with determination coefficients $\mathrm{R}^{2}$ higher than 0.74 . Beverage from grade 3 rejected $83 \%$ of the cups and $100 \%$ from grades 4 and 5 . Healthy spiked samples with grades 3 to 5 rejected 42 to $100 \%$ of the cups because tasted to phenolic, astringent, fermented, immature and dirty defects. Consequently, BBD severely affects coffee yield, price, and physical and beverage quality.

Effect of Puccinia kuehnii on two sugarcane cultivars with intermediate resistance to orange rust F. ALINE CAVALCANTE LEITE, R. Gazaffi, A. Seiiti Urashima, Federal University of São Carlos, Araras, BRAZIL

The first report of the first outbreak of orange rust (Puccinia kuehnii) in Brazil was in 2010. The main strategy of control was the elimination of susceptible cultivars, as done in Australia. Nevertheless, RB92579, and RB865156 show intermediate resistance and are ranked third and fourth to establish new sugarcane fields in 2015, demonstrating that intermediate canes will persist in the Brazilian sugarcane industry. Therefore, the present study aimed to examine the effect of $P$. kuehnii on two intermediate cultivars towards orange rust. Plants derived from single eye bud were transplanted to plastic pots three months after planting and divided in inoculated and uninoculated treatments. Two-way design was considered, with six repetitions and single potted plant as experimental unit. Inoculation of $P$. kuehnii was carried out biweekly with concentration of $10^{5}$ urediniospore.ml ${ }^{-1}$ and volume of 13-30 ml, according to plant growth. Uninoculated plants were protected with fungicide to ensure disease-free control plants. After six months, wet and dry weight were measured. Results indicated that the effect of inoculation was significant, i.e., wet and dry weight were significantly reduced when plants were submitted to the disease, and no interaction between disease and cultivars was verified. This indicated that chemical protection is necessary for these two intermediate cultivars under high disease pressure and when favorable conditions for orange rust are prevalent.

Tomato chlorosis virus: Purification, antiserum production and yield loss on potato plants L. R. Pinto, A. BERGAMIN FILHO, J. A. M. Rezende, University of São Paulo - ESALQ, Piracicaba, BRAZIL

Tomato chlorosis virus (ToCV) is a species of the genus Crinivirus, which is transmitted by Bemisia tabaci MEAM1 in a semi persistent manner. Considering the patosystem potato/ToCV, there is a lack of information on occurrence, symptomatology in different varieties, and damages caused by this crinivirus. In addition, there is no polyclonal antiserum for the Brazilian isolate of ToCV for use in diagnosis. The objectives of the present work were: to purify the virus and produce a polyclonal antiserum; and to evaluate the yield loss caused by this crinivirus on two potato cultivars. The virus purification was conducted using ToCV-infected tomato leaves. Purified virus was injected in a rabbit for antiserum production. Plants of cultivars Ágata and Asterix were inoculated with ToCV, by viruliferous vectors. Ninety days after inoculation plant development biomass was evaluated based on the fresh mass weight of the aerial part and weight of harvested tubers. The production of polyclonal antiserum in rabbit was satisfactorily accomplished. However, it was only efficient for ToCV detection in dot-blot assays, when diluted 1:20. For two independent experiments, average reductions in aerial fresh weight of the plants were $60.1 \%$ for Ágata and $46 \%$ for Asterix. Reductions in the yield of tubers from infected plants of the Ágata and Asterix, for the first experiment, were $99.5 \%$ and $98.1 \%$, respectively. For the second experiment, the values were $82.3 \%$ and $56.2 \%$, respectively. 
Yield losses from foliar and soilborne peanut diseases

J. P. DAMICONE, K. Jackson, Oklahoma State University, Stillwater, OK, USA

The foliar disease early leaf spot and the soilborne diseases stem rot and Sclerotinia blight are important disease of peanut in Oklahoma and worldwide. Quantifying yield loss is important for yield loss assessment on a regional scale and for evaluating the effectiveness of disease management programs in individual fields. Trials evaluating various fungicides and application timings were used to produce differential levels of disease and resulting yields. In evaluating associations between disease incidence and yield, critical point ratings near the end of the growing season generally were as effective as area under the disease progress curve. Relative yield was calculated as a percentage of the y intercept $($ disease $=0)$ in regression analysis of disease incidence vs. yield. For early leaf spot on spanish-type cultivars, relationships of defoliation with relative yield were curvilinear with little decline in yield until defoliation reached ca. $60 \%$. Thereafter, yield declined to reach ca. $50 \%$ yield loss at $100 \%$ defoliation. For Sclerotinia blight and stem rot, relationships between relative yield and incidence of blighted plants were linear. Yield loss declined about $1.5 \%$ for every percent increase in disease suggesting that disease was more extensive underground than was apparent aboveground. Cultivars with resistance to Sclerotinia blight had similar yield loss slopes as susceptible cultivars, but lower maximum levels of disease than susceptible cultivars.

Impact of Sugarcane yellow leaf virus on sugarcane yield traits in the progenies from four diverse crosses S. G. SOOD, J. C. Comstock, D. Zhao, USDA ARS, Canal Point, FL, USA

Yellow leaf caused by Sugarcane yellow leaf virus (SCYLV) is an important disease for sugarcane industries worldwide. Yield losses up to $50 \%$ were reported in susceptible varieties. Most commercial cultivars in Florida are infected with SCYLV. Therefore, a study was conducted to determine the impact of SCYLV in sugarcane progenies originated from four crosses [CP 80-1827 (susceptible) selfed, Green German (susceptible) $\times$ Ind 81-146 (resistant), and reciprocal crosses between two susceptible varieties CP95-1039 and CP88-1762]. These progenies were exposed to the natural infestation of SCYLV vector aphids (Melanaphis sacchari; Rhopalosiphum species) for more than eight years. However, most of the individuals in the four progenies were asymptomatic. Individuals of these progenies were tested for SCYLV infection by tissue blot immunoassay. The impact of SCYLV on sugarcane stalk weight and sucrose content was estimated by evaluating ten random stalks from a seven-foot plot for each individual. Juice was collected and measured only in the individuals of the progeny obtained from the cross between Green German x Ind 81-146. Results showed that 52\% of individuals of CP80-1827 selfed progeny were infected with SCYLV after 32 years of the exposure to SCYLV vectors, while only 5-10\% individuals in the other crosses were infected after more than eight years of the exposure to the SCYLV vectors. No significant difference was observed in average stalk weight, juice brix, pol and volume between the SCYLV infected and uninfected individuals in all the four progenies. Although previous studies showed an impact of SCYLV on sugarcane yield in Florida, SCYLV had no negative effect on the important parameters of sugarcane production in the progenies of the crosses studied.

Effect of inoculation timing and hybrid resistance on yield loss attributed to Goss' wilt and leaf blight in North Dakota E. BAUSKE, P. Beauzay, J. Knodel, C. Schuh, A. J. Friskop, North Dakota State University, Fargo, ND, USA

Goss' wilt and leaf blight (Goss') of corn (Zea mays), caused by Clavibacter michiganensis subsp. nebraskensis, was first documented in North Dakota (ND), USA in 2011. Grain corn acreage in ND has increased by $68 \%$ since 2011 . To assess yield loss attributed to Goss' in ND, inoculation timing by hybrid susceptibility field trials were established over six location-years. Trials were designed in a randomized complete block with a split-plot arrangement and four replications. Three corn hybrids (susceptible, moderately susceptible and resistant) with sub- 88 day relative maturities were sown at each location. Inoculation timings included a non-inoculated check, V6-V10 (early-season), R1 (late season), and a sequential inoculation of V6-V10 and R1 (combination). Beginning 14 days after inoculation and continuing until corn reached maturity, disease severity was assessed in each plot. Test weight and yield were recorded at harvest. Under high disease pressure in 2016 and 2017, year-end disease severity values ranged from 21-52\% and $12-$ $61 \%$ on susceptible hybrids, respectively. Yield loss values in excess of $35 \%$ in 2016 and $30 \%$ in 2017 were associated with early season inoculations. Minor yield losses were observed on the resistant hybrid and the R1 inoculation treatment. Results from this research demonstrate the importance of hybrid selection when reducing economic losses attributed to Goss'.

Effect of Sugarcane Mosaic caused by Sorghum mosaic virus on Sugarcane in Louisiana

M. P. GRISHAM (1), K. Warnke (2), J. Maggio (2), (1) USDA-ARS, SRU, Houma, LA, USA; (2) USDA, ARS, Sugarcane Research Unit, Houma, LA, USA

To determine the effect of mosaic caused by the Sorghum mosaic virus on sugarcane, experimental field plots were planted with seed cane with mosaic symptoms (mosaic plots) or without mosaic symptoms (control plots). Commercial (Ho 05-961 and HoCP 09-804) and advanced candidate (Ho 09-832, L 08-88, and L 09-117) sugarcane cultivars were included. The plant-cane (first-year crop) and three subsequent ratoon crops were harvested annually from 2013 through 2016. Cane and sugar yields were determined using total plot weights and laboratory determined theoretically recoverable sucrose (TRS). The average cane yield $\left(\mathrm{Mg} \mathrm{ha}^{-1}\right)$ was numerically lower among all mosaic plots in plant-cane compared to control plots. Average cane yields were reduced in mosaic plots across all crops for Ho 05-961, HoCP 09-804, and L 08-88, except in the first ratoon of HoCP 05-961. Because average TRS ( $\mathrm{kg} \mathrm{Mg}^{-1}$ ) was higher in many mosaic plots compared to control plots, sugar yields $\left(\mathrm{kg} \mathrm{ha}^{-1}\right)$ were not reduced in some crops where cane yields were reduced. Across all crops, mosaic reduced sugar yields in L08-88 and HoCP $09-804$ by $21 \%$ and $7 \%$ as compared to the controls, respectively. Mosaic incidence (\% shoots with symptoms) was determined each spring. Natural spread of mosaic was noted among control plots of all cultivars resulting in mosaic incidence increasing from crop to crop. In the third ratoon, mosaic incidence among control plots ranged from 16 to $47 \%$.

Brome mosaic virus reduces wheat yield in both early and late growth stage infections B. A. HODGE (1), P. A. Paul (1), L. R. Stewart (2), (1) The Ohio State University, Wooster, OH, USA; (2) USDA ARS, Wooster, OH, USA

Brome mosaic virus (BMV) is a cereal virus of global distribution that is reported to have little economic impact on wheat production. BMV was detected in 25 of 107 Ohio wheat fields surveyed between 2012 and 2017, highlighting the need to assess its potential impact. Field trials were conducted in the 2016 and 2017 growing seasons, in which wheat cultivars were tested for responses to BMV when inoculated at the 2-leaf, 5-leaf, flag leaf emergence, or boot growth stages. Three out of four cultivars were susceptible to infection at all inoculated growth stages, with BMV inducing yield loss of up to $61 \%$ compared to the non-inoculated controls. The main and interaction effects of cultivar and inoculation timing on grain yield were statistically significant $(\mathrm{p}<0.05)$. The magnitude of yield loss varied among cultivars and inoculation timings. In contrast to reports with other cereal viruses, BMV induced up to 26.5 and $36.4 \%$ yield reduction when inoculated at flag leaf emergence and boot, respectively. Most inoculated treatments reduced test weight and kernel weight, but only inoculation at the 2-leaf growth stage reduced plant population and height. This research demonstrates that BMV infection impacts wheat development, grain yield and quality, but the magnitude of these effects depends on inoculation timing and cultivar. This suggests that BMV can have a substantial economic impact on wheat production, and more research is needed to develop management practices. 
Soybean losses due to diseases and nematodes in the USA since 1996: General trends and observations

P. ESKER (1), T. W. Allen Jr. (2), C. A. Bradley PhD (3), (1) Penn State University, UNIVERSITY PARK, PA, USA; (2) Mississippi State University, Stoneville, MS, USA; (3) University of Kentucky, Princeton, KY, USA

Since 1996, losses due to soybean diseases including nematodes have been collected by experts in the USA from 28 soybean producing states. During this period, numerous technological advances have occurred in soybean production, ranging from the advent of GMO soybean to the discovery of soybean rust, as well as increased use of different seed treatment and foliar fungicide products. The objective of the current work was to estimate the overall impact of soybean diseases, as well as examine how losses due to specific variables have changed over time. First, the overall loss for each state and year was estimated using Padwick's calculation. Second, annual soybean price data was obtained from USDA-NASS to estimate the economic impact of individual diseases and nematodes for each state and year combination. Temporal trends were examined based on heat maps, and physical losses (i.e., yield) were calculated to re-estimate yield, which were compared empirically to current yield in consult with soybean agronomists. In the northern region, losses ranged from 10 to $17 \%$, while in the southern region, losses ranged from 7 to $14 \%$. Economically, losses on a per hectare basis were judged to be between $\$ 48$ and $\$ 351$ in the northern region, and $\$ 32$ and $\$ 116$ in the southern region. Overall, losses have been variable across states, depending on the year, although results illustrate the extreme importance and impact of soybean pests and diseases on production in the USA.

Chemosensitization of Zymoseptoria tritici isolates resistant to DMI and strobilurin fungicides

J. A. DELGADO (1), G. Gustafson (2), (1) Dow AgroSciences, Indianapolis, IN, USA; (2) Dow AgroSciences LLC, Indianapolis, IN, USA

Chemosensitization was first explored as a tool for improving control of cancer cells resistant to chemotherapy. Subsequently, chemosensitizers have been shown to enhance the activity of antimicrobials against resistant bacteria and fungi, while having little or no activity against the target organism and no apparent mammalian toxicity. In this study, we evaluated the utility of farnesol, a natural product chemosensitizing agent, for improving control of isolates of the wheat fungal pathogen Zymoseptoria tritici that are resistant to strobilurin and 14a-demethylase-inhibiting (DMI) fungicides. In mixture with strobilurin or DMI fungicides, farnesol enhanced growth inhibition of resistant $Z$. tritici isolates in vitro. Farnesol also enhanced fungicidal activity of strobilurin and DMIs against resistant Z. tritici on wheat seedlings. However, the ability of farnesol to improve disease control on wheat seedlings was dependent on application coverage. In a separate study, the activity spectrum of farnesol and two other analogs was investigated using BIOLOG plates to identify additional chemosensitizer-fungicide combinations for characterization on infected wheat seedlings. Farnesol enhanced the fungicidal activity of 51 antifungals out of the 120 BIOLOG compounds. These results demonstrated that BIOLOG plates are a suitable tool for high-throughput screening of chemosensitizers against $Z$. tritici.

Resistance to SDHI fungicides in Botrytis cinerea from commercial strawberry fields in Spain D. FERNANDEZ-ORTUNO (1), A. Perez Garcia (2), J. A. Tores (1), A. De Vicente (2), (1) IHSM-UMA-CSIC La Mayora, Algarrobo-Costa, Malaga, SPAIN; (2) IHSM-UMA-CSIC La Mayora, University of Malaga, Malaga, SPAIN

Gray mold, caused by Botrytis cinerea Pers., is one of the most economically important diseases of strawberries. Gray mold control involves the application of fungicides throughout the strawberry growing season; however, $B$. cinerea isolates resistant to multiple classes of site-specific fungicides have been recently reported in the Spanish B. cinerea population. Succinate dehydrogenase inhibitors (SDHIs) constitute a relatively novel class of fungicides registered for gray mold control representing new alternatives for strawberry growers. In the present study, 37 B. cinerea isolates previously characterized for their sensitivity to boscalid and amino acid changes in the SdhB protein, were used to determine the effective concentration that reduces mycelial growth by $50 \%\left(\mathrm{EC}_{50}\right)$ to fluopyram, fluxapyroxad and penthiopyrad. The $\mathrm{EC}_{50}$ values ranged from 0.01 to $>100 \mu \mathrm{g} / \mathrm{ml}$ for fluopyram, from $<0.01$ to $4.19 \mu \mathrm{g} / \mathrm{ml}$ for fluxapyroxad, and finally, from $<0.01$ to $59.65 \mu \mathrm{g} / \mathrm{ml}$ for penthiopyrad. The present study was also conducted to obtain discriminatory doses to monitor SDHI fungicide resistance in 580 B. cinerea isolates collected from 27 commercial fields in Spain during $2014-2016$. Over the course of the three-year monitoring period, the overall frequencies of resistance to boscalid, fluopyram, fluxapyroxad and penthiopyrad were $56.9,6.9,12.9$, and $24.6 \%$, respectively. Four SDHI resistance patterns were observed in our population, which were associated with different aminoacid substitutions (H272R/Y, N230I) in the SdhB subunit. For gray mold management, it is suggested that the simultaneous use of boscalid and penthiopyrad should be limited to one application per season; however, the use of fluxapyroxad and, especially, fluopyram could be used as valid SDHI alternatives for gray mold control, but they should be applied with caution.

The role of heteroplasmy for the cytochrome b gene in resistance to QoI fungicides in Podosphaera xanthii A. VIELBA-FERNANDEZ (1), J. A. Tores (1), A. De Vicente (2), D. Fernandez-Ortuno (1), A. Perez Garcia (2), (1) IHSM-UMA-CSIC La Mayora, Algarrobo-Costa, Malaga, SPAIN; (2) IHSM-UMA-CSIC La Mayora, University of Malaga, Malaga, SPAIN

Powdery mildew elicited by Podosphaera xanthii is a devastating disease of cucurbits worldwide and one of the most important diseases affecting these crops in Spain. Application of fungicides is the main control practice in most cucurbit crops for managing powdery mildew. The quinone outside inhibitors (QoI) represent an important class of agricultural fungicides, which are widely used in Spain against the powdery mildew disease. QoI have a single-site mode of action inhibiting mitochondrial respiration by binding to the Qo site of the cytochrome bc $_{1}$ enzyme complex, thus blocking electron transfer in the respiration pathway and leading to and energy deficiency due a lack of ATP. In a previous report, widespread resistance to QoI fungicides in populations of $P$. xanthii in South central Spain was documented, but the molecular mechanisms of resistance remained unclear. In this work, the role of cytochrome $b(c y t b)$ gene mutations in QoI resistance of $P$. xanthii was examined. Sequence analysis of a cloned fragment of $c y t b$ revealed the presence of a G143A substitution that occurs in many QoI-resistant fungi. This mutation was always detected in QoI-resistant isolates of $P$. xanthii; however, it was also detected in sensitive isolates. To better understand the role of heteroplasmy for $c y t b$ in QoI resistance of $P$. xanthii, an allele-specific quantitative polymerase chain reaction was developed to quantify the relative abundance of the A143 (resistant) allele. Our data suggest that G143A mutation in cytb is the primary factor involved in QoI resistance of $P$. xanthii; however, the proportion of G143 and A143 alleles in an isolate may determine its QoI resistance level. In addition, the role of the Rieske-FeS protein (ISP), the other protein component of the Qo site, in QoI resistance was also examined.

Managing QoI resistant Cercospora beticola on sugar beet (Beta vulgaris L.) in the USA

M. KHAN, North Dakota State University \& University of Minnesota, Fargo, ND, USA

Cercospora leaf spot (CLS), caused by the fungus Cercospora beticola, is one of the most damaging foliar diseases of sugar beet in warm and humid regions. Fungicide application is an important component of an integrated pest management program for controlling CLS. In the last two decades, growers in Europe and USA have been using mainly single site mode of action fungicides in mixtures or individually with rotation to control $C$. beticola Recently, resistance of $C$. beticola to QoI fungicides were reported in Austria, Serbia, and the USA. In 2016, USA growers suffered over \$200 million economic losses due to QoI fungicide resistance. Therefore, we conducted field studies in 2016 and 2017 to evaluate the efficacy of fungicides 
individually, in mixtures, and in a rotation program for controlling a $C$. beticola population that was resistant to QoI fungicides and had reduced sensitivity to demethylation inhibitors (DMI). In both years, QoI fungicide treatments showed no significant difference in disease control from the nontreated check. However, the DMI fungicides had an improved efficacy when used in mixtures with broad-spectrum fungicides, such as triphenyltin hydroxide and mancozeb. The use of multiple applications of fungicide mixtures at 10 day intervals were the most effective at controlling CLS resulting in the highest recoverable sucrose content. This work provides a practical way for better controlling CLS in areas with known QoI resistant $C$. beticola population.

Identification and Characterization of Inherent Resistance to 14a-demethylation Inhibitors in Colletotrichun truncatum

S. CHEN (1), G. Schnabel (2), H. Yuan (3), (1) Institute of Plant Protection, Chinese Academy of Agricultureal Sciences, Beijing, CHINA; (2) Clemson University, Clemson, SC, USA; (3) Institute of Plant Protection, Chinese Academy of Agricultural Science, Beijing, CHINA

Anthracnose disease caused by Colletotrichum truncatum is a major economic constraint to fruits and vegetables in the tropical and subtropical regions of the world. Isolates of C. truncatum were collected from the US and China from peach, soybean, and citrus and their sensitivity to demethylation inhibitor fungicides (DMIs) including difenconazole, propiconazole, tebuconazole, metconazole, flutriafol, and fenbuconazole was determined. In contrast to Colletotrichum fructicola, Colletotrichum siamense, and Colletotrichum fioriniae isolates, all C. truncatum isolates were resistant to tebuconazole, metconazole, flutriafol, and fenbuconazole, and less sensitive to difenconazole and propiconazole. The Colletotrichum spp. genome contained two homologous CYP51 genes, CYP51A and CYP51B that putatively encode sterol $14 \alpha$-demethylase enzymes. The CYP51A and CYP51B from $C$. truncatum and other species were cloned to investigate the molecular basis of inherent resistance to DMI fungicides in $C$. truncatum. Quantitative expression analysis did not show correlations between the CYP51 expression level and fungicides senstitivity. Amino acid sequences were compared among different Colletotrichum species within substrate recognition sites. Four amino acid variations L208Y, H238R, S302A, I366L from CYP51A, and three variations H373N, M376L, S511T from CYP51B correlated with the DMI resistance phenotype. Molecular docking with CYP51A structure models suggested the four variations in CYP51A reduce azoles interactions with critical residues in the binding cavity, thereby causing inherent reistance to DMI fungicides in C. truncatum.

Colletotrichum Species Composition and Fungicide Tolerance in Isolates Causing Bitter Rot of Apples in Pennsylvania P. MARTIN, K. A. Peter, Penn State University, Biglerville, PA, USA

Bitter rot of apples caused by a Colletotrichum spp. complex is an increasing problem in the mid-Atlantic and northeastern US. Several causes for this increase have been hypothesized, including increased tolerance of widely used fungicides and selection of species with increased fitness. Colletotrichum spp. were isolated from $>100$ apples with bitter rot symptoms grown under both conventional and organic management practices from across Pennsylvania. Partial sequences of the beta-tubulin and GAPDH genes, and the ITS region of ribosomal DNA were amplified and sequenced to identify the fungi to species. Isolates were tested for tolerance to commonly used fungicides with differing modes of action using mycelial growth and conidial germination assays. Colletotrichum fioriniae was the dominant species identified and increased tolerance to FRAC (Fungicide Resistance Action Committee) group 11 fungicides was observed. Evaluations of FRAC Groups 1 and 7 are in progress. C. fioriniae is a widely distributed fungus that has been isolated from apple, forest trees, shrubs, and scale insects, and infects strawberries, lettuce, blueberries, peaches, apricots and peppers, among others. Findings from the current study will be discussed and are predicted to uncover aspects of understanding cross-infectivity between different crop hosts, elucidating inoculum sources, and for evaluating selection pressures from different mode of action fungicides.

Phenotypic and molecular characterization of Botrytis cinerea isolates from strawberry to isofetamid and cross-resistance with other SDHI fungicides

A. ZUNIGA (1), N. Peres (2), (1) Gulf Coast Research and Education Center; University of Florida, Wimauma, FL, USA; (2) University of Florida, Wimauma, FL, USA

Botrytis cinerea, the causal agent of Botrytis fruit rot on strawberry, has rapidly selected resistance to the SDHI fungicides. In this study, we identified mutations conferring resistance to isofetamid, a SDHI newly registered for strawberry, and determined resistance frequencies of 565 Botrytis isolates to five SDHI fungicides, i.e. boscalid, penthiopyrad, fluopyram, benzovindiflupyr, and isofetamid. Isolates were collected during two consecutive seasons (2015-16 and 2016-17), and used for fungicide sensitivity assays. Molecular characterization to isofetamid was based on mycelial growth inhibition assays of previously characterized isolates with $\mathrm{B}^{\mathrm{H} 272 \mathrm{R}}, \mathrm{B}^{\mathrm{H} 272 \mathrm{Y}}, \mathrm{B}^{\mathrm{N} 230 \mathrm{I}}$, and $\mathrm{B}^{\mathrm{P} 225 \mathrm{~F}}$ mutations known to confer resistance to SDHI fungicides, but only $\mathrm{B}^{\mathrm{N} 2301}$ and $\mathrm{B}^{\mathrm{P} 225 \mathrm{~F}}$ were found in isolates moderately and highly resistant to isofetamid, respectively. Among the $B$. cinerea isolates collected during the first season, $95,33,21,25$, and $0 \%$ were resistant to boscalid, penthiopyrad, fluopyram, benzovindiflupyr, and isofetamid, respectively. The respective resistance frequencies for the second season were $91,95,44,27$, and 1.3\%. Three moderately resistant isolates to isofetamid were found during $2016-17$ and the $\mathrm{B}^{\mathrm{N} 230 \mathrm{I}}$ mutation was confirmed. Isolates moderately resistant to isofetamid were also highly resistant to the other SDHI's tested, suggesting alternative disease control strategies are needed to avoid cross-resistance and preserve efficacy of newer SDHI fungicides.

Virulence of multi-fungicide resistant $Z$ ymoseptoria tritici isolates under greenhouse conditions

C. AVILA-ADAME (1), T. Slanec (2), (1) Dow AgroSciences LLC, Indianapolis, IN, USA; (2) Dow AgroSciences, Indianapolis, IN, USA

Zymoseptoria tritici (causing Septoria leaf blotch of wheat) is managed with fungicides differing in mode of action (MoA), including beta-tubulin, cytochrome b-Qo, and 14-alpha demethylase inhibitors. Concurrently, the European fungal population developed resistance to these chemistries. To determine whether accumulation of mutations conferring resistance to multiple MoAs impairs virulence/aggressiveness, 10 isolates from 5 countries were compared through 5 cycles of infection on wheat seedlings. Five isolates carried the E198A and G143A mutations conferring resistance to betatubulin and Qo inhibitors, respectively, as well as multiple mutations in CYP51 decreasing sensitivity to 14-alpha demethylase inhibitors. Three other isolates had mutations associated with resistance to 1 or 2 fungicide classes but not to all 3 . Two isolates were fully sensitive to all 3 fungicide classes. The resistant and sensitive isolates were all virulent, infecting wheat with typical disease symptoms; however, only 8 isolates caused more than $95 \%$ disease severity 20 days after inoculation at the end of the 5 cycle. Two isolates resistant to 3 fungicide classes were less aggressive than fully sensitive isolates but overall there was no consistent effect of resistance phenotype on disease development.

Development of resistance in field populations of Botrytis cinerea following exposure to various fungicide programs S. D. COSSEBOOM (1), G. Schnabel (2), K. L. Ivors (1), G. J. Holmes (1), (1) Strawberry Center, California Polytechnic State University, San Luis Obispo, CA, USA; (2) Clemson University, Clemson, SC, USA 
Gray mold of strawberry, caused by Botrytis cinerea, is a very destructive fruit rot and is routinely managed by aerial fungicide applications. However, multiple chemical-class-resistant (CCR) populations reside in strawberry fields. We conducted two replicated field trials to examine the rate of resistance development to the active ingredients boscalid, fenhexamid, and fludioxonil. Treatments were applied weekly to plots for six weeks followed by a sixweek period of no applications. Throughout this 12-week trial, 112 isolates of $B$. cinerea were collected from sporulating strawberry fruit five times. A mycelial growth assay using discriminatory dosages of fungicides differentiated sensitive from resistant isolates. Prior to the first fungicide application, low frequencies of resistance were observed to boscalid and fludioxonil, but $30 \%$ of all isolates were resistant to fenhexamid. Resistance to fenhexamid in control plots remained steady throughout the trial, yet treatments involving fenhexamid revealed a sharp increase in resistance to fenhexamid after the sixth application and an equally sharp decrease in resistance during the period of no applications. Our "resistance management" treatments (tank mixtures and chemical class rotation) offer insight into the effectiveness of these tactics. These trials indicate that the frequencies of resistance in a field population can drastically change within one season depending on the fungicides applied.

Evaluating Helminthosporium solani, causal agent of potato silver scurf blemish disease, for sensitivity to the fungicide azoxystrobin S. MACCHIAVELLI GIRÓN, Y. Chen, J. Spychalla, A. J. Gevens, University of Wisconsin-Madison, Madison, WI, USA

Silver scurf of potato, caused by the ascomycetous fungus Helminthosporium solani, is a tuber blemish disease causing negative impacts of increasing concern in potato production. Growers have utilized variably-effective cultural controls and fungicides for disease management. After $H$. solani developed resistance to the fungicide thiabendazole, QoI fungicides (such as azoxystrobin) were adopted with some control success. However, recently there has been an increase in disease severely affecting quality and storability. Genetic mutations associated with QoI fungicide resistance have been identified in other ascomycetes, such as Alternaria solani. For this reason, we hypothesized that $H$. solani might currently be poorly controlled due to QoI resistance after over 2 decades of use. In our preliminary screening we selected five $H$. solani isolates representing different production regions and management regimes. Five of these isolates were exposed to azoxystrobin at 8 concentrations to determine fungicide sensitivity. Isolates were grown on clarified V8 agar amended with azoxystrobin $(0-100 \mu \mathrm{g} / \mathrm{mL})$. Relative growth was measured after 20 and 40 days of incubation at $23^{\circ} \mathrm{C}$ under darkness. There were significant differences in growth between isolates, as well as between treatments. One of the isolates grew on all concentrations, including the highest rate, suggesting some level of insensitivity. Further studies will focus on evaluating QoI sensitivity in a larger population and we will investigate the presence of mutations associated with QoI sensitivity in other ascomycetes pathogens in our $H$. solani isolates. Our results could greatly influence continued use of azoxystrobin in potato production for the control silver scurf.

Heterogenous expression of Sclerotinia sclerotiorum $\beta$-tubulin conferring benzimidazole-resistance in Fusarium asiaticum Y. YANG, M. Li, Y. Duan, M. Zhou, Nanjing Agricultural University, Nanjing, CHINA

In many filamentous fungi, only one $\beta$-tubulin exists in the genome and its specific point mutations confer resistance to benzimidazole fungicides. Fusarium asiaticum has two $\beta$-tubulins, $\beta_{1}$ - and $\beta_{2}$-tubulin. Homology of $\beta_{1}$-tubulin is higher than $\beta_{2}$-tubulin with $\beta$-tubulin of other filamentous fungi. Interestingly, the resistance of $F$. aisaticum to benzimidazoles is primarily due to the point mutations of $\beta_{2}$-tubulin. Recently, many studies that the point mutation of $\beta$-tubulin regulates resistance to benzimidazoles were widely reported in filamentous fungi. Molecular docking between drugs and target $\beta$ tubulin was analyzed by computer software. However, molecular mechanism of resistance has not been elucidated. In our previous study, the E198A genotype of Botrytis cinerea conferring benzimidazole-resistance replaced the $\beta_{2}$-tubulin locus in $F$. asiaticum. This heterogenous expression can't exhibit benzimidazole resistance. One possible cause is that the E198A genotype was not reported in F. asiaticum. To further confirm whether heterogenous expression of the same mutated genotype existing in both $F$. asiaticum and Sclerotinia sclerotiorum can regulate drug-resistance, the $\beta_{1}$ tubulin and $\beta_{2}$-tubulin locus of $F$. asiaticum was replaced with the F200Y genotype of $S$. sclerotiorum, respectively. Both the mutants were still sensitive to benzimidazoles. Furtherly, both $\beta_{1}$-tubulin and $\beta_{2}$-tubulin locus of $F$. asiaticum were replaced with the F200Y genotype of $S$. sclerotiorum. Surprisingly, the mutants were resistant to benzimidazole. These results indicate that genetic and resistance evolution of $\beta$-tubulin in filamentous fungi is more complex than we thought and provide critical insights not only into possible drug-resistance mechanism but also on the design of novel tubulin inhibitors with improved properties.

Identification of QoI mutation in soybean pathogens in Brazil

F. E. DE MELLO (1), V. S. Lopes-Caitar (2), S. A. Xavier (1), A. Mehl (3), F. C. Marcelino-Guimaraes (2), J. A. Verreet (4), M. I. Balbi-Peña (5), C. V. Godoy (6), (1) Londrina State University, Londrina, BRAZIL; (2) Embrapa Soybean, Londrina, BRAZIL; (3) Bayer CropScience, Monheim, GERMANY; (4) Christian-Albrechts-Universität zu Kiel, kiel, GERMANY; (5) Universidade Estadual de Londrina, Londrina, Paraná, BRAZIL; (6) EMBRAPA, Londrina, Parana, BRAZIL

Excessive sprays of quinone outside inhibitors fungicides (QoI) in Brazilian soybean crops have contributed to the development of resistant populations of Cercospora kikuchii, Colletotrichum truncatum and Corynespora cassiicola. We investigated the molecular mechanisms of resistance to QoIs in these populations for the target gene cytochrome $\mathrm{b}$ and the occurrence of cross resistance. Fungi were isolated from several soybean fields sampled in different seasons. Cross resistance was tested for C. kikuchii and C. cassiicola by radial growth assay in amended media using discriminatory dose $\left(10 \mu \mathrm{g} \mathrm{mL}^{-1}\right)$ of Azoxystrobin (AZX), Picoxystrobin (PCX) and Pyraclostrobin (PRC) fungicides. The change from glycine to alanine at position 143 (G143A) was detected for C. kikuchii, C. cassiicola and C. truncatum isolates. The nucleotide sequence at amino acid position 143 was converted from GGT in sensitive to GCT in resistant isolates. Positive cross-resistance was observed in more than $86 \%$ of $C$. cassiicola and C. kikuchii isolates. Negative crossresistance between PRC x PCX and PRC x AZX was observed in C. cassiicola and C. kikuchii, respectively and for AZX x PCX for C. kikuchii. This is the first report of the G143A mutation of these pathogens in Brazil.

QoI sensitivity in Alternaria solani, causal agent of potato early blight, is dependent upon the quantity of wildtype cytochrome $b$ S. DING, K. Meinholz, A. J. Gevens, University of Wisconsin-Madison, Madison, WI, USA

Potato early blight caused by Alternaria solani is a perennial and destructive fungal disease. Management currently relies on fungicides due to lack of cultivar resistance. A commonly used class of fungicides is quinone outside inhibitors (QoIs). Mutations at amino acid position 129 of cytochrome $b$ in A. solani isolates lead to partial resistance to QoIs. In this study, three $A$. solani isolates with different quantities of the mutated single nucleotide polymorphisms (SNPs) were subcultured onto clarified V8 juice agar (CV8) and CV8 amended with azoxystrobin (AZ) at 1mg/L every ten days for a total of 5 subcultures. Mycelial growth rate, EC50 of conidial germination rate, and the quantity of the mutated SNPs were monitored. Results showed no significant difference in mycelial growth rate, EC50, or quantity of mutated SNPs across subcultures when grown on CV8. However, when grown on CV8 amended with AZ, the mycelial growth rate increased by $60 \%$, the quantity of mutated SNPs increased by $28 \%$, and the EC 50 increased by $25 \%$. EC50 was positively related to the increasing quantity of mutated SNPs $\left(\mathrm{r}^{2}=0.68\right)$; isolates became resistant when mutated SNPs accumulated. While the increase in mutated SNPs occurs relatively quickly, AZ may still be effective if a population has a low level of mutations prior to selection. Fungicide resistance management and resistance monitoring are critical in maintaining effectiveness of AZ and other single site mode of action fungicides. 
Resistance of Phytophthora cactorum isolates causing crown and leather rot in Florida strawberries to Mefenoxam M. MARIN (1), T. E. Seijo (2), M. Oliveira (1), E. Zuchelli (3), J. C. Mertely (4), N. Peres (1), (1) University of Florida, Wimauma, FL, USA; (2) Univ of Florida - GCREC IFAS, Wimauma, FL, USA; (3) Universidade de Passo Fundo, Rio Grande Do Sul, BRAZIL; (4) University of Florida-GCREC, Wimauma, FL, USA

Phytophthora cactorum causes leather rot on fruit and crown rot (PhCR) of strawberry plants. Leather rot is not a common disease in Florida; however, up to $50 \%$ yield loss has been reported after high rainfall events. PhCR is an important disease during plant establishment when infected transplants from nurseries are overhead irrigated for 10 days during plant establishment. Mefenoxam is the most effective and widely used fungicide to control both diseases. P. cactorum isolates from both leather rot and PhCR have been collected from multiple strawberry fields in Florida since 1997 and sensitivity to mefenoxam has been monitored. Isolates $(\mathrm{n}=230)$ were tested for mefenoxam sensitivity at $0,0.05,0.5,5 \mathrm{and} 100 \mathrm{mg} / \mathrm{L}$. EC $\mathrm{E}_{50} \mathrm{values}$ of sensitive isolates ranged from 0.05 to $1 \mathrm{mg} / \mathrm{L}$. Resistance to mefenoxam $\left(\mathrm{EC}_{50}\right.$ values $>100 \mathrm{mg} / \mathrm{L}$ ) was found among leather rot and PhCR isolates collected after 2015. During 2015-16 and 2016-17 growing seasons, $10 \%(\mathrm{n}=10)$ of all isolates collected were resistant. These isolates were from six different commercial farms and three transplant sources. This is the first report of occurrence of $P$. cactorum resistance to mefenoxam in Florida, which suggests that alternative control strategies are needed to avoid the increase of $P$. cactorum-resistant populations in Florida fields.

First report of mandipropamid resistance of grapevine downy mildew in North America X. Feng, A. BAUDOIN, Virginia Tech, Blacksburg, VA, USA

Carboxylic acid amide (CAA) fungicides constitute a group of fungicides highly effective against downy mildews. Two members are registered for use against grape downy mildew (Plasmopara viticola) in the United States, mandipropamid since 2008 and dimethomorph since 2009. Resistance of $P$. viticola against CAA fungicides, first detected in Europe in 1994, has gradually spread and is now considered widespread in most of Europe's viticultural regions. CAA resistance has been attributed to a mutation in the cellulose synthase gene (PvCesA3). Since 2011, control failures and the presence of this mutation have also been reported from India, Japan and China, but not from North America. In 2016, we received a report of suspected control failure of mandipropamid in a west-central Virginia vineyard. All eight isolates collected from this location grew well on leaves and potted plants treated with a field rate of mandipropamid (156 mg/liter) whereas an older isolate produced no growth under these conditions. The $144 \mathrm{bp} P v C e s A 3$ gene of two resistant $P$. viticola isolates was amplified and sequenced. The sequenced PCR products revealed a GGC-to-AGC substitution at codon 1105 of the $P v C e s A 3$ gene, the same G1105S mutation previously associated with CAA resistance of $P$. viticola in other regions.

Diversity of RPA190 in Phytophthora infestans resistant to metalaxyl

Q. Zhou, Y. Li, F. CHEN, J. Zhan, Fujian Agriculture and Forestry University, Fuzhou, CHINA

Metalaxyl is a phenylamide fungicide, introduced for oomycete management since 1970s. The RPA190 gene, the largest subunit of RNA polymerase I, was indicated recently involved in metalaxyl resistance. The present study described the metalaxyl resistance and diversity of RPA190 in Phytophthora infestans collected consecutively in three years from Yunnan province in China. All isolates were resistant to metalaxyl, whose $\mathrm{EC}_{50}$ values greater than $5 \mu \mathrm{g} / \mathrm{ml}$. The difference in sensitivity between the isolates at each end of the spectrum was more than seven folds. Metalaxyl sensitivity tended to decrease over sampling time for isolates collected in 2012,2103, and 2014, respectively. The nucleotide diversity in the three temporal populations ranged from 0.00273 to 0.00282 with an overall diversity of 0.00273 when sequences from the three years were combined. The haplotype diversity in the three years ranged from 0.787 to 0.828 with an overall diversity of 0.776 when sequences from the three years were pooled. Haplotype diversity directionally increased over temporal scale but the correlation between sampling year and haplotype diversity was not significant. The nucleotide sequences of haplotypes clustered into six clades with five mutations were shown to be under positive selection. The study indicated high diversity of RPA190 gene in metalaxyl resistant $P$. infestans.

Fungicide resistance in Botrytis spp. from strawberry fields in Norway

G. M. Strømeng (1), M. Nordang Skårn (1), K. Ørstad (1), A. Stensvand (1,2), K. A. GREDVIG NIELSEN $(1,2)$, M. B. Brurberg (1,2), (1) NIBIO / Norwegian Institute of Bioeconomy Research, Ås, NORWAY; (2) Norwegian University of Life Sciences, Ås, NORWAY

In 2016, Botrytis fruit rot caused unexpectedly high strawberry yield losses in southern Norway. Farmers suspected fungicide resistance in the pathogen to be a major cause, and an investigation into the presence of such resistance was thus initiated. Isolates of Botrytis sp. were collected from plant materia in 19 strawberry fields. Resistance to boscalid, fenhexamid, fludioxonil, iprodione, pyrimethanil and pyraclostrobin were analyzed with a germination assay in 157 Botrytis isolates (148 for boscalid). Spores were allowed to germinate on nutrient agar amended with fungicides, and germ tube growth was recorded. We found that $89.7,86.0$, and $65.0 \%$ of the isolates were resistant to boscalid, pyraclostrobin, and fenhexamid, respectively. Moreover, $25.0 \%$ were resistant to pyrimethanil. Few isolates $(2.6 \%)$ were resistant to iprodione, and none were resistant to fludioxonil. Only $8.9 \%$ of the isolates were sensitive to all the fungicides, while $5.1 \%$ were resistant to one fungicide. The remaining isolates were resistant to two $(21.0 \%)$, three $(43.9 \%)$, four (19.1\%) and five (1.9\%) fungicides. Approximately $80 \%$ of the isolates were tested using PCR assays for mutations known to confer fenhexamid and pyraclostrobin resistance, and good correlation was observed between detected mutations and resistance phenotypes. We conclude that fungicide resistance requires consideration in practical disease management of Botrytis fruit rot in Norwegian strawberry production.

Management of Monilinia fructicola resistance to tebuconazole in the field

W. Pereira (1), R. Morales (2), K. Kudlawiec (3), L. L. MAY DE MIO (4), (1) Universidade Federal do Paraná, Curitiba, BRAZIL; (2) Empresa de Pesquisa Agropecuária e Extensão rural de Santa Catarina, itajaí, BRAZIL; (3) Departamento Entomologia e Acarologia, Universidade de São Paulo, Piracicaba, SP, BRAZIL; (4) Federal University of Parana, Curitiba, BRAZIL

Brown rot is the main peach disease in the world and the main control is based in fungicides. The tebuconazole has been the most used one in the last decade in Brazil. Several resistant individuals are present in the population and still exist a lack in information how to manage the resistance in the field. The frequency of resistance in $M$. fructicola population from orchards with different managements of tebuconazole was monitored during 3 seasons (2012/13, 2013/14 and 2014/15) in the states of Paraná (PR) and São Paulo (SP). The number of sprayings with demethylation inhibitors (DMI) fungicides varied from 0 to 2 (PR) and 6 to 16 (SP) until the 2011/12 season. The application of DMI was reduced to 0 to 1 in subsequent seasons and isolates growth at discriminatory concentration of $0.3 \mu \mathrm{g} / \mathrm{ml}$ of tebuconazole. Changes in density curves and sensitivity distributions revealed that SP and PR populations differed $(p$ value $<0.05)$. PR population has remained sensitive during the seasons. The population from SP had high, intermediate and low frequency of the resistance isolates in the season 2012/13,2013/14 and 2014/15, respectively. The absence of tebuconazole fungicide applications associated with fitness penalty of resistant isolates could restore sensitivity of the population in the field. To confirm resistant phenotypes a sample of isolates was analyzed and the G461S mutation in the MfCYP51 gene was detected in $88 \%$ of the isolates. 
Evolution of fungicide resistance in UK field populations of Zymoseptoria tritici

B. B. FRAAIJE (1), P. Diez (2), N. Hawkins (3), (1) Rothamsted Research, Hertfordshire, UNITED KINGDOM; (2) Rothamsted Research, Biointeractions and Crop Protection, Harpenden, UNITED KINGDOM; (3) Rothamsted Research, Harpenden, UNITED KINGDOM

The fungal wheat pathogen Zymoseptoria tritici is becoming more difficult to control in NW-Europe. Using archived infected wheat leaf samples from Broadbalk, the long-term winter wheat experiment at Rothamsted, resistance to methyl benzimidazole carbamates and quinone outside inhibitors due to alterations in $\beta$-tubulin (E198A) and cytochrome $b$ (G143A) was detected in 1985 and 2002, respectively. UK field populations have also become less sensitive to azoles due to three different mechanisms, alterations in the target protein sterol 14 $\alpha$-demethylase (CYP51) with the first mutation, Y137F, detected in 1991, CYP51 over-expression (2009) and altered efflux pump activity (2013). All three mechanisms have been found simultaneously in some isolates. Resistance conferring mutations to succinate dehydrogenase inhibitors (SDHIs) have also been detected since 2015, with up to 15 different mutations found in $S d h B, S d h C$ and $S d h D$ in 2017. Two isolates carried two mutations simultaneously, but these strains were not found in subsequent years. Here we present the latest monitoring data from the four most frequently occurring Sdh mutations (SdhC-T79N, C-W80S, C-N86S and C-H152R) in the UK at the start of the season in different geographical regions and late season after selection by different fungicide programmes. Previous studies and results from our current monitoring studies indicate that isolates carrying C-H152R might harbour a fitness cost. Quantitative detection of the four targeted $S d h$ mutations show that selection for these mutations was influenced by the dose rate, spray number and mixing partner of SDHI fungicides.

Fungicide sensitivity of Rhizoctonia spp. isolated from soybean fields in Nebraska

N. GAMBHIR (1), S. Kodati (2), A. O. Adesemoye (2), S. E. Everhart (1), (1) University of Nebraska, Lincoln, NE, USA; (2) University of Nebraska Lincoln, North Platte, NE, USA

Soybean seedling diseases reduce stand establishment and cause economic losses. Rhizoctonia species are important causal agents in Nebraska. For effective disease control, the prevalent Rhizoctonia spp. need to be characterized. Soil and symptomatic plants were collected from a total of 54 soybean fields in Nebraska in 2015, 2016, and 2017. Soil samples were collected in a 'W' or 'Z' pattern from a depth of 0-6'. Sugarbeet and toothpick bait methods were used to isolate Rhizoctonia spp. from soil. A total of 48 Rhizoctonia spp. were isolated. ITS sequencing was used to determine species and AG. The most abundant were $R$. zeae (26) and R. solani AG 4 HG-II (14). Fungicide seed treatments are commonly used to manage soybean seedling disease and include several modes of action, such as SDHI, QoI, and Phenylpyrroles. Few studies have examined fungicide sensitivity of Rhizoctonia from soybean fields and none have characterized sensitivity of populations in Nebraska. Current work is underway to determine fungicide sensitivity of isolates obtained in the present study. Fungicide sensitivity of isolates will be determined using a plate dilution method for three fungicides: Fluxapyroxad (SDHI), Azoxystrobin (QoI), and Fludioxonil (Phenylpyrroles). Effective concentration for 50\% inhibition (EC 50 ) will be estimated from a dose-response curve. Results will establish sensitivity of Rhizoctonia spp in Nebraska and can be used for monitoring sensitivity shifts in future.

Fungicide resistance profiles of Botrytis cinerea isolated from berry crops in Oregon V. O. STOCKWELL (1), B. Shaffer (2), L. Jones (3), J. W. Pscheidt (4), (1) USDA ARS, Horticultural Crops Research Unit, Corvallis, OR, USA; (2) USDA-ARS, Corvallis, OR, USA; (3) New Zealand Institute for Plant \& Food Research Ltd, Motueka 7198, NEW ZEALAND; (4) Oregon State University, Corvallis, OR, USA

The gray mold pathogen, Botrytis cinerea, causes pre- and post-harvest fruit rot and stem disease of berry crops. Disease control measures involve applications of fungicides in rotation or in combination from bloom to near harvest. $B$. cinerea can rapidly develop resistance to fungicides, which reduces control efficacy. Little was known about the fungicide resistance profiles of $B$. cinerea from berry crops grown in Oregon. We isolated $B$. cinerea from blackberry, blueberry, raspberry, and strawberry fruits in western Oregon fields from 2014 to 2017. Resistance to fungicides was determined by growth inhibition assays on fungicide-amended media. The media and discriminatory doses of formulated fungicides tested were: 1) potato dextrose agar amended with $0.5 \mathrm{ppm}$ fenhexamid, $0.3 \mathrm{ppm}$ fludioxonil, or $3 \mathrm{ppm}$ iprodione, 2) yeast extract agar with $5 \mathrm{ppm}$ boscalid, and 3 ) the defined medium, cyprodinil test agar (CTA), amended with 1 or $10 \mathrm{ppm}$ cyprodinil. Among the 511 isolates evaluated, $61 \%$ were resistant to at least one fungicide. Boscalid resistance was common and resistance to fenhexamid or cyprodinil was frequently detected. Among the isolates with fungicide resistance, $64 \%$ were resistant to at least two fungicides and 36\% were resistant to three or four of the fungicides. Although $39 \%$ of the isolates were sensitive to the fungicides tested, the emergence of multi-fungicide resistance may compromise the efficacy of chemical control of gray mold.

In vitro and in planta assessment of the effect of mefenoxam-acquired resistance on sporulation in isolates of Phytophthora infestans M. REGNIER, J. Gonzalez Tobon, G. Danies, S. Restrepo, Universidad de los Andes, Bogota, COLOMBIA

Phytophthora infestans is an oomycete pathogen that causes potato and tomato late blight disease, a worldwide concern for farmers given its explosive epidemic potential. In order to control the disease, an integral management approach, which includes the use of fungicides, has been implemented. Unfortunately, populations of $P$. infestans resistant to fungicides have been documented. Furthermore, studies have shown that isolates of $P$. infestans are able to acquire resistance to mefenoxam in vitro, after a single exposure through sub-lethal concentrations of this widely used fungicide. The aim of this study was to evaluate if there is an effect on sporangial production in isolates of $P$. infestans that acquire resistance to mefenoxam in vitro. An originally susceptible isolate of $P$. infestans was exposed to different concentrations of mefenoxam-amended media and compared to a resistant control. Sporangia production was significantly diminished in isolates that acquired resistance. Interestingly, naturally resistant isolates sporulated significantly more in the presence of mefenoxam. Possible implications of the acquisition of resistance on sporangia production are discussed. These results provide further insights into the phenomenon of mefenoxam-acquired resistance.

Detection of QoI fungicide resistant Cercospora beticola airborne inoculum using quantitative PCR

K. CHITTEM (1), A. Milosavljevic (2), L. E. del Rio Mendoza (3), M. Khan (4), (1) North Dakota State University, Fargo, ND, USA; (2) Institute for plant protection and environment, Belgrade, REPUBLIC OF SERBIA; (3) North Dakota State Univ, Fargo, ND, USA; (4) North Dakota State Univ \& Univ of MN, Fargo, ND, USA

Cercospora leaf spot caused by Cercospora beticola is a devastating foliar disease of sugar beet (Beta vulgaris) worldwide. This disease is considered the most important constraint for sugar beet production in North Dakota and Minnesota, and warrants multiple fungicide applications per growing season. Development of QoI fungicide resistance in pathogen populations results in unsatisfactory control of this disease. Thus monitoring of the pathogen population for prevalence of QoI fungicide resistance is essential for effective management of the disease using fungicides. The objective of this study was to assess the possibility of using qPCR methods to monitor QoI-resistant C. beticola in airborne inoculum. Spore samplers were set up in 2016 and 2017 growing seasons in research plots at Foxhome, MN and airborne spore samples were collected using either a Burkard volumetric samplers or cyclonic samplers. DNA was extracted from the samples, and quantitative PCR assay designed to detect G143A mutation was used to determine the proportion of QoI resistant and sensitive populations. QoI-resistant C. beticola isolates were detected in varying proportions in both 2016 and 2017 
growing seasons. The preliminary results suggest that by using cyclonic samplers and qPCR assay, C. beticola can be monitered for QoI-resistance in real time.

Assessment of boscalid, fluopyram, and fluxapyroxad sensitivity in Michigan populations of Blumeriella jaapii

J. C. GLEASON (1), T. J. Proffer (2), C. A. Outwater (1), N. Rothwell (1), G. W. Sundin (1), (1) Michigan State University, East Lansing, MI, USA; (2) Kent State Univ, Salem, OH, USA

Cherry leaf spot (CLS), caused by Blumeriella jaapii is the most important disease affecting tart cherry trees in the Great Lakes region of the United States. Early defoliation affects fruit quality and yield, and if severe, can impact the ability of trees to survive over winter. Succinate dehydrogenase inhibitor fungicides (SDHIs) have become the mainstay for control of CLS recently. Tart cherry orchards were sampled in 2016 and 2017 , and more than 800 single conidium isolates were established in each year. The in vitro sensitivity of these isolates to boscalid, fluopyram and fluxapyroxad was examined using a minimum inhibitory concentration test (MIC). At least $50 \%$ of isolates with MICs at concentrations of $25 \mu \mathrm{g} / \mathrm{ml}$ or greater to boscalid, fluopyram and fluxapyroxad were present in 17, 30, and 17 of 36 orchards, respectively, in 2016 and $50 \%$ of isolates with MICs of $40 \mu \mathrm{g} / \mathrm{ml}$ or greater were present in 12, 14 and 1 orchard, respectively. In $2017,50 \%$ of isolates from at least 24 and 11 of 43 screened orchards had MICs of $25 \mu \mathrm{g} / \mathrm{ml}$ or 40 $\mu \mathrm{g} / \mathrm{ml}$, respectively. We have also shown that isolates with the higher levels of reduced sensitivity observed in vitro are not controlled by field rates of fungicides in vivo. Further testing of fluopyram and boscalid is ongoing.

Selection of boscalid resistance in Blumeriella jaapii populations treated with boscalid, fluopyram, or fluxapyroxad C. A. OUTWATER (1), G. W. Sundin (1), T. J. Proffer (2), (1) Michigan State University, East Lansing, MI, USA; (2) Kent State Univ, Salem, OH, USA

Cherry leaf spot (CLS), caused by the fungus Blumeriella jaapii, is a major disease of tart cherry (Prunus cerasus) trees, leading to early defoliation which results in poor fruit quality, reduced fruit set, and tree death. Pristine, a commonly-utilized fungicide for CLS management in Michigan from 2004-2011, is a premix of boscalid, a succinate dehydrogenase inhibitor (SDHI), and pyraclostrobin, a quinone outside inhibitor (QoI). We conducted a two year statewide survey collecting 1346 isolates from 49 orchards and found a shift in boscalid sensitivity with $22 \%$ and $35 \%$ of the population exhibiting high levels of boscalid resistance in 2010 and 2011, respectively. The presence of the H272R mutation in the $s d h B$ gene known to confer boscalid resistance was identified in all ten of the highly resistant isolates sequenced. Beginning in 2013, Luna Sensation, a premix of fluopyram (SDHI) and trifloxystrobin (QoI), and Merivon, a premix of fluxapyroxad (SDHI) and pyraclostrobin, were widely adopted to replace Pristine for CLS management. To assess the potential of these new SDHIs for the selection of boscalid resistance, 24 isolates from four-tree replicates that were either non-treated or treated with Pristine, Merivon or Luna Sensation were collected at three time points during 2012. At the end of the sampling period Pristine, Merivon and Luna Sensation appeared to be selecting for boscalid resistance with $54 \%, 79 \%$ and $33 \%$ of isolates recovered with MIC values $>25 \mathrm{mg} / \mathrm{ml}$.

Rhizoctonia cerealis sensitivity to fludioxonil in China and analysis of laboratory fludioxonil-resistant mutants H. SUN, W. Li, Y. Deng, H. Chen, Jiangsu Academy of Agricultural Sciences, Nanjing, CHINA

Rhizoctonia cerealis is a major soilborne fungal pathogen causing wheat sharp eyespot (WSE) in winter wheat in China. The phenylpyrrole fungicide fludioxonil effectively controls WSE, but the sensitivity and the potential for $R$. cerealis to develop resistance were little reported. We studied fludioxonil sensitivity in a total of ninety-two $R$. cerealis field isolates and evaluated the resistance risk in laboratory fludioxonil-resistant mutants. Our results showed that fludioxonil was effective against all field isolates. The $\mathrm{EC}_{50}$ (effective concentration inhibiting $50 \%$ mycelial growth) values ranged from 0.009 to $0.064 \mathrm{mg} \mathrm{l}^{-1}$ and were distributed unimodal around an average of $0.027 \mathrm{mg} \mathrm{l}^{-1}$. We further isolated fifteen $R$. cerealis laboratory mutants with low or high resistance levels to fludioxonil by exposing mycelia plugs on PDA plates containing fludioxonil. Fludioxonil-resistant mutants showed parental sensitivity to thifluzamide and tebuconazole, but resistance to iprodione, and slight resistance to jinggangmycin. Studies on fitness parameters of fludioxonil-resistant mutants revealed a fitness cost on mycelial growth and pathogenicity. Moreover, the mutants were more sensitive to osmotic stress (high sugar and high salt) than their wild-type isolates. Therefore, $R$. cerealis has a potentially low risk of developing resistance to fludioxonil.

Mutation in the rpsL gene are responsible for streptomycin resistance of Clavibacter michiganensis subsp. michiganensis Q. LYU, China Agricultural University, Beijing, CHINA

Streptomycin is an aminoglycoside antibiotic effective against a wide variety of bacteria and has been used to control many plant bacterial diseases, including bacterial canker of tomato caused by Clavibacter michiganensis subsp. michiganensis (Cmm), but no resistant Cmm strain has been reported. The induced streptomycin-resistant $\left(\mathrm{Sm}^{\mathrm{r}}\right)$ mutant of $\mathrm{Cmm}$ was isolated by culturing the streptomycin-sensitive strain BT-0505 on streptomycin containing LB agar, the isolate can grow on LB agar in the presence of $512 \mu \mathrm{g} / \mathrm{mL}$ streptomycin while the minimum inhibitory concentration was more than $5000 \mu \mathrm{g} / \mathrm{mL}$. The gene related with the streptomycin resistance was amplified from the $\mathrm{Sm}^{\mathrm{r}}$ mutant by PCR and confirmed by sequencing. There was an A to $\mathrm{G}$ point mutation in rpsL gene at nucleotide 128 in the $\mathrm{Sm}^{\mathrm{r}}$ mutant, resulting a lysine to arginine change in the RpsL protein. The complementation assay further confirmed that the point mutation in the $r p s L$ gene was responsible for the streptomycin resistance in BT-0505. These results are consistent with the current understanding of the mechanism on streptomycin resistance in Escherichia coli and other bacteria.

Evaluating SDHI Fungicide Insensitivities in Sclerotinia homoeocarpa

A. ANTHONY, J. P. Kerns, Department of Entomology and Plant Pathology, North Carolina State University, Raleigh, NC, USA

Dollar spot is a disease of turfgrass caused by the fungus Sclerotinia homoeocarpa and is considered the most economically important disease of amenity turfgrass. Succinate dehydrogenase inhibitors (SDHIs) make up a group of fungicides with a growing list of products registered for turf and ornamentals, but resistance and cross-resistance within the class have been reported in some fungal pathogens. In a 2016 fungicide trial in Raleigh, NC, three SDHIs (boscalid, penthiopyrad, and fluxapyroxad) failed to control dollar spot. Twenty-four isolates were collected from dollar spot foci in the SDHI-treated and non-treated plots. A fungicide amended media assay was attempted with full-strength and $1 / 4$-strength potato dextrose agar, both including two runs of each SDHI at six concentrations $(0,0.01,0.1,1,10,100 \mathrm{mg}$ fungicide/ $\mathrm{L})$. A plug of each isolate was placed in the center of amended media plates and radial growth was measured after 40 hours. An in vivo experiment was performed in which plugs of creeping bentgrass were greenhouse grown to maturity and sprayed with SDHIs at field application rates. Plants were inoculated via rye grain infested with isolates used in plate assays and kept in a growth chamber with enhanced disease conditions. Disease severity and turf quality ratings were taken every 3 days for 21 days. Results of these experiments suggest alternative plate assay methods will be needed to capture reduced sensitivity displayed by isolates in vivo. 
Rapid sampling techniques to determine QoI fungicide resistance in Erysiphe necator

S. LOWDER (1), T. Neill (2), M. M. Moyer (3), T. Miles (4), W. Mahaffee (2), (1) Oregon State University, Corvallis, OR, USA; (2) USDA, Corvallis, OR, USA; (3) Washington State University, Prosser, WA, USA; (4) California State University Monterey Bay, Seaside, CA, USA

Control failures of grape powdery mildew (GPM) after the use of quinone outside inhibitor (QoI) fungicides (FRAC Group 11) have been reported by vineyard managers throughout OR, WA, and CA. Quick, cost-effective methods to detect fungicide resistance in Erysiphe necator are necessary to reduce the selection for resistant alleles and mitigate ineffective fungicide applications. E. necator samples were collected via linear stratified sampling techniques by placing a ToughSpot adhesive dot directly onto a mildew colony $(\mathrm{n}=119)$ or by wiping gloves with sterile cotton swabs $(\mathrm{n}=65)$ after simulated vine training. A competitive TaqMan qPCR assay, that was previously demonstrated to be in $100 \%$ agreement with germination assays, was used to detect the G143A mutation in the cytb gene conferring QoI resistance. Both methods detect similar distributions (of samples with resistant, sensitive and mixed genotypes: ToughSpots: $48.7 \%$ resistant, $43.7 \%$ sensitive, $7.6 \%$ mixed; Swabs: $59.2 .0 \%$ resistant, $24.5 \%$ sensitive, $16.3 \%$ mixed), indicating that either could be a viable, rapid sampling technique with minimal interruption to grower production schedules. A rapid sampling technique for chasmothecia (syn. cleistothecia) overwintering in grape bark is under development to provide managers with an assessment of the risk of fungicide resistance in the next season. The resistant genotype can be determined from a single chasmothecium in $2 \mathrm{~g}$ of dry bark.

Evidence for CYP51-mediated reduced sensitivity to triazole fungicides in Calonectria henricotiae

J. HULVEY (1), R. E. Marra (2), (1) Eastern Connecticut State University, Willimantic, CT, USA; (2) Connecticut Agric Exp Sta, New Haven, CT, USA

Boxwood blight, caused by Calonectria pseudonaviculata and C. henricotiae, is a serious threat to Buxus spp. worldwide. Evidence for reduced sensitivity to triazole fungicides in C. henricotiae warranted comparative analyses of the CYP51 gene in C. henricotiae and C. pseudonaviculata. BLAST searches of genomic contigs uncovered three CYP51 paralogs, two of which, CYP51A and CYP51B, are known to mediate resistance to azoles. Phylogenetic analyses support the identity of the CYP51 paralogs, which cluster with those from previously characterized fungal species. In the $C Y P 51 B$ coding sequence we identified only synonymous SNPs between $C$. henricotiae and $C$. pseudonaviculata, while comparison of the coding sequences of CYP51A uncovered non-synonymous SNPs, two of which are located in regions of the CYP51A active site that have been associated with azole binding. In addition, we report a premature stop codon in CYP51A of C. pseudonaviculata. These findings support a role for CYP51A in the reduced sensitivity exhibited by $C$. henricotiae and have implications for future management of boxwood blight.

In-season dynamics in sensitivity to azoxystrobin in the tobacco frogeye leaf spot pathogen, Cercospora nicotianae G. Walles, W. Barlow, E. W. Dixon, R. Pearce, E. E. PFEUFER, University of Kentucky, Lexington, KY, USA

Reduced sensitivity to azoxystrobin fungicide in the tobacco frogeye leaf spot pathogen, Cercospora nicotianae, was documented in 2016. To determine the influence of fungicide programs on azoxystrobin sensitivity patterns in C. nicotianae populations, a randomized complete block trial was conducted in 2017. Fungicide programs were sequences of azoxystrobin and mancozeb applied on three dates. Symptomatic samples were taken at season outset, after each spray, and at season conclusion. Single-spore isolates were generated and spore germination was determined on $0,0.001,0.01,0.1,1$, and 10 ppm azoxystrobin-amended $1 / 4$-strength potato dextrose agar with SHAM. For each isolate, the effective concentration to inhibit $50 \%$ of spore germination $\left(\mathrm{EC}_{50}\right)$ was calculated based on the dose response curve. $\mathrm{EC}_{50} \mathrm{~S}$ ranged from 0.04 to $0.91 \mathrm{ppm}$ azoxystrobin. C. nicotianae populations from the untreated control and azoxystrobin-intensively treated plots had mean and median $\mathrm{EC}_{50} \mathrm{~s}$ of 0.20 and 0.06 , and 0.62 and 0.58 ppm azoxystrobin, respectively, after two applications. The $\mathrm{EC}_{50}$ mean and $\mathrm{EC}_{50}$ distribution of the azoxystrobin-intensive treated population were significantly greater than the nonexposed $C$. nicotianae population as determined by a two-sample t-test $(P=0.002)$ and a Kolmogorov-Smirnov test $(P=0.003)$, respectively. This suggests that measurable changes in $C$. nicotianae populations' sensitivity to azoxystrobin may occur over the course of a single cropping season.

N837 deletion in oxysterol binding protein-related protein confers oxathiapiprolin resistance in Phytophthora capsici and $P$. sojae J. MIAO, D. Lin, Q. Peng, Z. Wang, X. Liu, China Agricultural University, Beijing, CHINA

Oxathiapiprolin is a novel fungicide which was registered in China in 2015 to control pant-pathogenic oomycetes, such as Phytophthora spp. and downy mildews. In our previous study, point mutation of G770V and G839W in PcORP1 were detected in oxathiapiprolin resistant mutants of $P$. capsici obtained by fungicide adaption. In the current study, CRISPR/Cas9 system was used to verify the effect of the two-point mutation on characteristics of $P$. capsici BYA5. Transformants contained heterozygous G770V in PcORP1 showed similar phenotype with wild-type isolate BYA5, including sporangia, zoospores production, cyst germination and pathogenicity. However, two transformants with heterozygous G839W in PcORP1 could not produce sporangia. Three transformants with a new point mutation, $\triangle \mathrm{N} 837$ in PcORP1, showed high oxathathiapirplin resistance and survival ability. Point mutation, $\triangle \mathrm{N} 837$ in PsORP1, was also confirmed that it could confer oxathiapiprolin resistance in $P$. sojae P6497. These $P$. sojae mutants also showed certain fitness with wild type P6497. PsORP1- $4 \mathrm{~N} 837$ with the original promoter was also transferred into P6497, and positive transformants also showed oxathiapiprolin resistance and similar phenotype with P6497. These results showed that G770V, G839W and $\triangle \mathrm{N} 837$ in ORP1 could confer oxathiapiprolin resistant with no fitness cost. The new information will be useful for the development of series novel OSBPI fungicides.

Studying Xanthomonas arboricola pv. corylina strains from Serbia for streptomycin and kasugamycin resistance and copper sulfate sensitivity in vitro

A. PROKIĆ (1), M. Ivanović (1), K. Gasic (2), N. Kuzmanovic (3), N. Zlatković (1), A. Obradovic (1), (1) University of Belgrade, Faculty of Agriculture, Belgrade, SERBIA AND MONTENEGRO; (2) Institute for Plant Protection and Environment, Belgrade, SERBIA AND MONTENEGRO; (3) Julius Kühn-Institut, Federal Research Centre for Cultivated Plants (JKI), Braunschweig, GERMANY

Hazelnut bacterial blight, caused by Xanthomonas arboricola pv. corylina (Xac), has been observed in Serbia since 1950s. While the application of antibiotics is prohibited, different copper compounds are routinely used in disease control which can lead to the pathogen resistance. Therefore, we studied the in vitro effect of different concentrations of copper sulfate, streptomycin and kasugamycin on development and growth of 46 Xac strains originating from Serbia. Droplets of each strain suspension were spotted on the surface of sucrose peptone agar (SPA) plates amended with either $\mathrm{CuSO}_{4}$ x $5 \mathrm{H}_{2} \mathrm{O}(100,200 \mathrm{ppm})$, streptomycin sulfate $(25,50 \mathrm{ppm})$ or kasugamycin $(50,100 \mathrm{ppm})$ or on SPA without any of the bactericides. Xanthomonas euvesicatoria, strain E-3, resistant to these compounds was used as a positive control. Plates were incubated for $48 \mathrm{~h}$ at $28^{\circ} \mathrm{C}$ and observed for bacterial growth. Results of these experiments showed that all tested Xac strains were sensitive to the lowest concentration of streptomycin sulfate (25 ppm). Kasugamycin inhibited growth of two strains at $50 \mathrm{ppm}$, while 44 were able to grow. Two times higher concentration of kasugamycin inhibited growth of 35 strains while 11 strains showed reduced growth. None of the strains developed on SPA amended with 200 ppm of copper sulfate, however 39 strains showed tolerance to $100 \mathrm{ppm}$, indicating certain level of copper tolerance. This study suggests the possibility of resistance development to copper ions in Serbian Xac population that may be due to intensive use of this compound in disease control. 
Resistance to host damage is distinct from resistance to pathogen reproduction in the major wheat pathogen Zymoseptoria tritici P. Karisto (1), S. Yates (2), B. A. McDonald (1), A. MIKABERIDZE (3), (1) Plant Pathology, ETH Zurich, Zurich, SWITZERLAND; (2) Molecular Plant Breeding, ETH Zurich, Zurich, SWITZERLAND; (3) Epidemiology of Plant Diseases, ETH Zurich, Zurich, SWITZERLAND

Septoria tritici blotch (STB) caused by the fungus Zymoseptoria tritici is a major disease of wheat worldwide. The pathogen adapts rapidly to deployment of fungicides and qualitative host resistances. Quantitative resistance is likely to be more durable, but phenotyping based on conventional visual scoring does not readily detect quantitative differences between cultivars, which hinders breeding for quantitative resistance. We used automated analysis of digital images of 21420 leaves from 335 elite winter wheat cultivars naturally infected by a diverse population of $Z$. tritici to evaluate quantitative STB resistance. This novel, high-throughput phenotyping method not only quantifies leaf necrosis, but also counts asexual fruiting bodies of the pathogen. This allowed us to obtain precise and reproducible quantitative measures of STB intensity and separate resistance affecting host damage from resistance affecting pathogen reproduction. Cultivar rankings with respect to the two measures exhibited only a weak correlation $\left(r_{\mathrm{s}}=0.17\right)$, indicating that the two measures are largely independent. Moreover, a genome-wide association study revealed that the two forms of resistance are under separate genetic control. Our findings suggest that resistance to host damage and resistance to pathogen reproduction can be considered separately in breeding programs, but can also be combined to form new cultivars highly resistant to STB.

Rapid screening for resistance against Pseudocercospora banana pathogens using relatively long detached banana leaves under controlled conditions

A. E. Alakonya (1), A. ORTEGA-BELTRAN (1), G. S. Mahuku (2), R. Bandyopadhyay (1), R. Swennen (1), (1) International Institute of Tropical Agriculture, Ibadan, NIGERIA; (2) International Institute of Tropical Agriculture (IITA), Dar es Salaam, TANZANIA

Banana and plantain (Musa spp.) are important food and cash crops throughout the tropics and subtropics. However, they are threatened by the 'Sigatoka Disease Complex' (SDC) caused by Pseudocercospora fijiensis, P. musae, and P. eumusae. Invasion by these pathogens results in poorly filled fruits and smaller, lower yielding bunches. In addition, severe infections induce physiological changes that result in a heightened climacteric rise. This leads to reduced green life and is associated with premature and uneven fruit ripening, reduced pulp color clarity, and altered fruit taste. Although significant progress has been made in breeding for resistance to SDC, these efforts are constrained by lack of rapid, robust screening methods aimed to identify sources of resistance. We developed a robust, rapid, and reliable screening method to evaluate susceptibility to SDC using detached banana leaves $(25$ $\mathrm{cm} \times 10 \mathrm{~cm}$ ) placed in plastic boxes containing technical agar amended with 5 ppm gibberellic acid and separately inoculated with $P$. fijiensis and $P$. musae. Leaves remained viable $>100$ days and showed similar reaction as when infected in the field. For instance, the resistant accessions Calcutta 4 and Yangambi KM5 showed significantly lower $(\mathrm{P} \leq 0.05)$ disease severity compared to the highly susceptible cultivars Gros Michel and Agbagba. The method allows both identifying resistant germplasm and studying host-pathogen interactions. This method will be useful to banana/plantain breeding programs across the globe to rapidly screen hundreds of banana and plantain accessions for resistance to SDC and other diseases under controlled conditions.

Evaluating inoculum source, application and timing in screening for resistance to Sclerotinia sclerotiorum on sunflower cultivars M. BESTER, N. W. McLaren, University of the Free State, Bloemfontein, SOUTH AFRICA

Sunflower head rot, caused by Sclerotinia sclerotiorum, is responsible for economic losses of up to $75 \%$. Resistance screening requires consistent inoculation techniques (IT) that replicate natural field reactions of the host to the pathogen. The aim was to optimize laboratory inoculum production and to evaluate the viability and efficacy of inoculum sources, application methods and timing on disease incidence in field and greenhouse trials. Techniques used were sclerotia planted adjacent to seedlings, spray and milled grain mycelium, ascospore suspension and head punch using $S$. sclerotiorum colonized grains. Treatments were applied at bud, flower and head ripening growth stages, respectively. Inoculation technique and cultivar main effects were observed but no IT $\mathrm{x}$ cultivar interactions were recorded in the field. IT $\mathrm{x}$ growth stage interactions were observed in both greenhouse and field trials. An ascospore suspension sprayed at flowering resulted in the highest disease level in the greenhouse. In contrast, spray mycelium at bud stage yielded the highest disease level in the field. Cultivar responses to IT were quantified as percentage head rot incidence (HRI) and regression analysis was used to determine response type and relationship between observed HRI within a cultivar and disease potential. Results highlight the need for multiple considerations when screening for S. sclerotiorum resistance.

Fungal Diseases, Susceptibility of Nematodes, Efficacy of Herbicides, and Drought Tolerance/Heat of Birdsfoot Trefoil (Lotus corniculatus) Varieties

H. MOYE (1), N. Xiang (1), K. S. Lawrence (1), E. van Santen (2), J. Tredaway (1), (1) Auburn University, Auburn, AL, USA; (2) University of Florida, Gainesville, FL, USA

Lotus corniculatus (birdsfoot trefoil) is a common flowering plant in the pea family Fabaceae and native to Eurasia and North Africa. It is used in agriculture as a forage plant and also grown for pasture, hay, and silage due to its non-bloating properties; along with being used as a cover crop. Auburn University's breeding program for birdsfoot trefoil is attempting to extend the forage's geographic adaptation across the southern United States. Stand decline of the birdsfoot trefoil breeding lines due to fungal diseases and nematode pressure was observed at the Plant Breeding Unit of the E.V. Smith Research Center in Tallassee, Alabama in the 2015 season. We were able to isolate Macrophomina phaseolina from symptomatic plants. This pathogen causes stand decline, root rot, and charcoal rot in more than 500 crop and non-crop host plants (Smith and Carvil, 1977). The successful completion of Koch's Postulates indicates Fusarium oxysporum is a causal agent of seedling disease resulting in stand decline of birdsfoot trefoil in Alabama. Meloidogyne incognita (root-knot nematode) had a higher population and reproductive factor and can increase M. incognita populations on three varieties of birdsfoot trefoil tested. Two herbicides (Butyrac 200 and Panoramic) reduced the fresh shoot mass of cultivars tested in our greenhouse trial; along with the Pardee cultivar being the most susceptible to herbicide damage. Selection for drought/heat tolerance of a birdsfoot trefoil cultivar using three commercially available cultivars (Empire, Norcen, and Pardee) to improve stand endurance is the goal in developing a trans-regional birdsfoot trefoil cultivar with a wide geographic adaptation that has a longer stand life than existing cultivars.

Rapid, reliable and efficient phenotyping for crown rot resistance and tolerance in wheat C. PERCY (1), T. Nguyen-Ky (1), B. MacDonald (2), (1) University of Southern Queensland, Toowoomba, AUSTRALIA; (2) Department of Agriculture and Fisheries, Queensland, Toowoomba, AUSTRALIA

Crown rot caused by Fusarium pseudograminearum $(F p)$ is a significant soil borne disease of winter cereals. Phenotyping for this disease is laborious and often challenged by high variation between experiments. Current and alternative methods of measuring the host response to $F p$ infection are being tested in field experiments in the northern grain-growing region of Australia. Sampling strategies, disease severity, reflectance patterns, yield and yield 
components are being measured in inoculated and non-inoculated plants of a standard set of 12 wheat genotypes ranging in susceptibility to crown rot. Results have shown assessing 30 to 60 tillers per plot is the most efficient sampling strategy for disease severity ratings. It is more efficient to allocate resources to replicates, then plants and then tillers, however this will vary considering which tillers are sampled. Hyperspectral images analysed by the wavelet and filter band methods ranked the 12 wheat genotypes into three classes as most resistant, middle, and susceptible, with an accuracy of $80 \%$ to $90 \%$ compared with the expected crown rot ranking. This research broadens our understanding of this host-pathogen relationship, improves the way we assess crown rot disease in field environments, and provides a rapid, reliable and efficient screening tool to researchers and breeding companies which will enhance capacity to deliver crown rot resistant and tolerant varieties to growers in Australia and internationally.

\author{
The use of ascospores of the dieback fungus Hymenoscyphus fraxineus for infection reveals a period of biotrophic interaction in penetrated ash \\ cells \\ J. W. MANSFIELD (1), N. Galambos (2), R. Saville (3), (1) Imperial College London, London, UNITED KINGDOM; (2) East Malling Research, East \\ Malling, UNITED KINGDOM; (3) East Malling Research, Maidstone, UNITED KINGDOM
}

Ascospores, discharged naturally from apothecia growing on rachis debris, were used as inoculum to examine the invasion of ash tissues by the dieback fungus in order to understand the critical, but poorly understood, early interactions between host and pathogen. Methods were developed to collect ascospores for controlled infection assays on detached leaves, petiole and stem internode tissues. Light microscopy, including plasmolytic techniques, allowed the invasion of living plant cells to be observed. Ascospores were readily available from late May to September. On the plant surface, most spores differentiated directly to form appressoria without germ-tube growth. Direct penetration was followed by a significant period of biotrophic fungal growth before lesions developed. Following the formation of a vesicle-like structure after penetration, bulbous and elongated intracellular hyphae were produced in living plant cells. The use of ascospore inoculum, rather than mycelia, will allow natural and rapid screening of ash genotypes for resistance to the devastating dieback disease. The identification of the biotrophic phase of infection suggests that virulence is controlled by effector triggered immunity. Consequently, the emergence of the ongoing epidemic may be through loss of an avirulence determinant that has allowed a "host jump", comparable to the wheat blast outbreak across Asia.

Host resistance: The key to effectively manage Sclerotinia stem rot (Sclerotinia sclerotiorum) in canola (Brassica napus) M. A. KHAN (1,2,3), W. A. Cowling (2), M. You (2,4), J. Batley (5), S. S. Banga (6), M. Barbetti (1,2), (1) School of Agriculture and Environment Faculty of Science The University of Western Australia, Perth, AUSTRALIA; (2) The University of Western Australia Institute of Agriculture, Faculty of Science, Perth, AUSTRALIA; (3) Punjab Bio-Energy Institute, University of Agriculture, Faisalabad, PAKISTAN; (4) The University of Western Australia, School of Agriculture and Environment, Perth, AUSTRALIA; (5) School of Biological Sciences Faculty of Science The University of Western Australia, Perth, AUSTRALIA; (6) Department of Plant Breeding and Genetics, Punjab Agricultural University, Ludhiana, INDIA

Recent decades have seen increased areas sown to canola (Brassica napus) and associated changes in fungal disease incidence and/or severity of canola disease epidemics across Australia. One of the most devastating diseases of canola is caused by the fungal pathogen, Sclerotinia sclerotiorum. To manage this pathogen cost-effectively for the long term, it is essential to locate, develop and deploy new and effective host resistances in order to protect and ensure economic canola yields. However, while some high level resistance has been located in historic cultivars no longer available and also across a range of diverse Brassicaceae species, currently there are no commercial canola cultivars in Australia targeting resistance to Sclerotinia stem rot. To address this, cohorts of canola genotypes consisting of parents with known resistance to Sclerotinia stem rot and their succeeding generations are being evaluated for resistance against S. sclerotiorum. Phenotypic variation occurred among segregating populations at seedling and adult stages, ranging from highly resistant to susceptible types. Close relationships between phenotypic expressions of host resistance within and between segregating populations were identified under both controlled and field environments. Phenotypic selection in $F_{2}$ and $F_{3}$ coupled with QTL mapping in $F_{2}$ genotypes is being utilized to define the basis of genetic control of resistance against this pathogen in canola. These outcomes will be of critical value to plant breeders in developing new cultivars with improved resistance against this major disease of canola both within Australia and worldwide.

Differential responses of potato cultivars to Meloidogyne hapla

A. M. GORNY (1), F. S. Hay (1), S. J. Pethybridge (2), (1) Plant Pathology \& Plant-Microbe Biology Section, Cornell University, Geneva, NY, USA; (2) Cornell University, Plant Pathology \& Plant-Microbe Biology Section, Geneva, NY, USA

The Northern root-knot nematode (Meloidogyne hapla) is an important soilborne pathogen of potato in the northeastern United States, which may result in reductions in yield. Damage from M. hapla to potato tubers also reduces quality and may render them unmarketable. Knowledge of cultivar differences in susceptibility to $M$. hapla may enable the design of targeted management strategies and mitigate the need for control tactics such as nematicides. Eleven potato cultivars commonly grown in New York State, USA, were evaluated for their response to M. hapla in a replicated pot trial conducted in the greenhouse. Plants were inoculated with one of three population densities (500 or 1,500 second-stage juveniles per pot, or a noninoculated control). Plants were evaluated for tuber yield, shoot fresh weight, root fresh weight, number of tubers formed, tuber diameters, and root galling severity. Meloidogyne hapla reproduction factor for each cultivar was also calculated. Data were analyzed and significant effects investigated for individual factors and interactions. Inoculation with $500 \mathrm{M}$. hapla second-stage juveniles per pot significantly reduced yield. Interactions between cultivar and inoculation levels also had significant effect on M. hapla reproduction factor. Results of this study will be used to inform multi-variate disease risk prediction models for $M$. hapla in potato.

Developing of new multi rust resistant bread wheat cultivar "Maaroof" for irrigated and rain-fed zones of Iraq E. AL-MAAROOF, College of Agricultural Sciences, University of Sulaimani, Sulaimani, IRAQ

Rust diseases are the most critical grain yield-limiting factor of wheat in Iraq. Breeding for rust resistance is the most sustainable strategy to reduce yield losses. The present study represents a long term breeding program for developing a new multi resistant bread wheat cultivar to brown, yellow, and stem rust diseases. Following designation of R-30 as a source of resistance to the three rusts; it was successfully introduced in a breeding program with Tamuz 2 using the pedigree method in 1997. The progenies of three successive generations (F3-F5) were screened to the three rusts under artificial inoculation conditions at adult plant stage in Twaitha. Out of 135 hybrid lines, 12 promising lines were selected. The selected lines were introduced in comprehensive evaluation test for yield potential and response to rusts and bunt diseases in different locations. As a result, the promising line "63" was selected due to its high grain yield in the presence and absence of rusts and bunt diseases. Yield potential of the genotype is $10-19 \%$ higher than the local commercial cultivars Araz and Tamuz 2. Maaroof was registered and released as a new cultivar with high yield potential and multi resistance to rusts and bunt diseases by the National Committee of Registration and Release of Agricultural Cultivars/Iraqi ministry of agriculture according to order no. 40 in 30/10/2014. Great emphasis was conducted on multiplication and delivering the seeds to farmers. Yield potential of Maaroof on farm scale was 3750 and $4750 \mathrm{Kg} / \mathrm{H}$ under rain-fed and irrigated conditions respectively. 
Evaluating the stability of hybrid field maize reactions to gibberella ear rot and deoxynivalenol across environments

F. DALlA LANA (1), R. Minyo (2), P. Thomison (3), L. V. Madden (4), P. A. Paul (4), (1) Ohio State University, Wooster, OH, USA; (2) Department of Horticulture and Crop Science 119 Williams Hall 1680 Madison Avenue, Wooster, OH, USA; (3) Department of Horticulture and Crop Science, Ohio State University, Columbus, OH, USA; (4) The Ohio State University, Wooster, OH, USA

Gibberella ear rot (GER), caused by the necrotrophic fungus Fusarium graminearum, and its associated mycotoxins, particularly deoxynivalenol (DON), have increased in prevalence in the Corn Belt. However, disease severity and DON contamination vary among hybrids, and for a given hybrid, among environments. In this study, we evaluated the stability of hybrid reactions to GER and DON across 10 location-years (environments). F. graminearum inoculated ears were harvested from plots of the same nine hybrids planted in each environment and rated for GER severity and DON. Averaged across environments, mean severity ranged from 0.2 to $19 \%$ and DON from 1.5 to $66 \mathrm{ppm}$. Hybrids were ranked based on severity and DON, and nonparametric analyses performed to determine their stability based on Kendall coefficient of concordance $(W)$. Mean ranking of the hybrids across environments ranged from 3.3 to 7.8 for severity and from 3.5 to 7.5 for DON, and the variance of rankings, calculated as a measure of rank stability, ranged from 1.7 to 8.9 and 1.6 to 7.5 for severity and DON, respectively. There was a significant concordance $(P<0.002)$ of rankings across environments for both severity and DON. However, $W$ was considerably lower than 1 ( 0.35 for severity and 0.31 for DON), indicating that the rankings were not identical across environments. Results from this study will be useful for selecting hybrids for GER management and for future field experiments.

Development of laboratory bioassays to study powdery mildew pathogens of $\mathrm{Phlox}$ in vitro C. FARINAS (1), F. Peduto Hand PhD (2), (1) The Ohio State University, Columbus, OH, USA; (2) Ohio State Univ, Dept of Plant Pathology, Columbus, OH, USA

The genus Phlox consists of about 65 species that include some of the most prevalent ornamental plants in the temperate zone. These popular ornamentals are extremely susceptible to powdery mildew caused by the biotrophic pathogens Golovinomyces magnicellulatus and Podosphaera sp. Our long-term goal is to identify Phlox species that may be resistant to the disease by screening a germplasm collection stored at the Ornamental Plant Germplasm Center in Columbus, OH. To do so, we developed a set of laboratory tools to study the pathogens in vitro, including a detached leaf and a plantlet bioassay. In each experiment, we used colony diameter and spore production to assess pathogen growth under different experimental conditions, which included the use of different inoculation techniques, media, age of pathogen inoculum, and phenology of the host. As a result, the following protocol was established for germplasm screening. Single pathogen spores are transferred using an eyelash glued to a stick on a detached disinfected Phlox leaf maintained on $1 / 2$ MS medium supplemented with benzimidazole. The pathogen is allowed to grow for 18 days after which colonies are transferred using a paintbrush on the entire surface of micropropagated plantlets. Disease severity and spore production are then used to assess plant susceptibility to the disease. These tools will facilitate studies on powdery mildew of Phlox and possibly other ornamental species attacked by the same fungi.

Assessment of Xanthomonas campestris pv. musacearum host range and banana cultivars susceptibility in Rwanda

F. UWAMAHORO (1,2), A. Berlin (3), C. Bucagu (2), H. Bylund (1), J. Yuen (1), (1) Swedish University of Agricultural Sciences, Uppsala, SWEDEN; (2) University of Rwanda, Musanze, RWANDA; (3) Swedish Univ of Agricultural Sciences, Uppsala, SWEDEN

The bacteria, Xanthomonas campestris pv. musacearum causing banana Xanthomonas wilt is widely spread in Rwanda and infects all cultivated bananas Effective management necessitates the knowledge of host range and cultivars' susceptibility. Twelve plants of each of 17 suspected hosts (banana, blood banana, beans, Canna edulis, C. indica, C. pretolia, capsicum, cassava, colcasia, enset, groundnuts, maize, potato, pumpkins, sorghum, potato and wheat) and ten plants of each of 5 banana cultivars (Fhia-17, Fhia-25, Injagi, Mporogoma and Nkazikamwa) were inoculated with $X$. campestris pv. musacearum isolate in controlled environment. Control plants were inoculated with sterile water. Only banana and its relatives (enset, blood banana and Canna spp.) developed Xanthomonas wilt symptoms. Latency phase varied from 16 to 29 days after inoculation (dai), rapid complete wilting was observed in banana (44 dai) and late complete wilting in $C$. indica (72 dai). None of the 5 cultivars tested was resistant to $X$. campestris pv musacearum . Latency phase (13-19 dai) and days to complete wilting (39-65 dai) varied among cultivars. Distributed cultivars are highly susceptible, thus, access to resistant transgenic bananas and information on how to limit the disease spread is recommended. Since $X$. campestris pv. musacearum can infect banana relatives, these plants should be avoided in neighbouring farms or gardens to banana and flower industry should be notified about the potential threat.

Rootstocks in Washington State winegrape vineyards: Effects on plant-parasitic nematodes and vineyard establishment M. M. MOYER (1), K. East (1), I. A. Zasada (2), (1) Washington State University, Prosser, WA, USA; (2) USDA ARS, Corvallis, OR, USA

Management of plant parasitic nematodes in Washington State vineyards has been dominated by preplant soil fumigation. One practice that may mitigate economic loss due to nematodes is the adoption of nematode-"resistant" rootstocks; unfortunately, own-rooted vines are preferred in Washington given their ease of retraining after damaging winter cold events. There is little information on the performance of most rootstocks against northern root-knot nematode (Meloidogyne hapla), the main plant-parasitic nematode species in the state, and even less information on dual performance against dagger nematode (Xiphinema sp.). In 2015, we established a trial evaluating currently-available rootstocks in a commercial vineyard undergoing replanting after $20+$ years of production. The rootstocks were planted in replicated plots of fumigated (metam sodium), nonfumigated, and nonfumigated inoculated with M. hapla. Fumigation was only effective against M. hapla for the first 6 months after application yet it remained effective against Xiphinema for 2 growing seasons. Rootstocks were poor hosts for M. hapla relative to own-rooted Vitis vinifera, but all were acceptable hosts for Xiphinema sp. Several rootstocks (e.g., Harmony, 1103P) had greater shoot biomass at the end of year 2 compared to own-rooted $V$. vinifera. The goal of this project is to understand the long-term performance of rootstocks and the impacts of nematodes on vineyard lifespan in Washington State.

Improving resistance to Fusarium head blight in winter wheat by genomic selection T. MIEDANER (1), C. Herter (1), S. Kollers (2), V. Korzun (2), E. Ebmeyer (2), (1) University of Hohenheim (720), Stuttgart, GERMANY; (2) KWS CEREALS, Bergen, GERMANY

Fusarium head blight (FHB), caused by Fusarium graminearum, F. culmorum and other species, is a devastating disease in many wheat growing areas resulting in yield and quality losses and contamination of mycotoxins harmful for man and livestock. Although fungicides exist, breeding resistant cultivars is the most effective and environmentally friendly measure to control FHB. Resistance is inherited quantitatively by many genes with small effects making marker-assisted selection inefficient. The availability of phenotypes and a high-density marker array in wheat allows to use the information from the whole genome to predict FHB resistance (=genomic selection, GS). We analyzed two training populations with a total of 1,180 winter wheat lines for FHB resistance, heading date, and plant height at four locations and genotyped them by an 15k Illumina SNP assay. Mean FHB 
symptoms varied from 5 to $60 \%$ among the lines. Prediction accuracies for FHB resistance ranged from 0.36 to 0.74 depending on the genetic relatedness of the progenies. From a test population of 2,500 progenies the best $10 \%$ for FHB resistance have been selected by their genomic composition. We explore now the realized selection gain for this GS approach by field phenotyping the genomically selected progenies in 2018. In conclusion, GS would make FHB resistance more efficient, because larger populations can be screened in a shorter time period with less efforts for field plots and locations.

Identification of resistances in pumpkin (Cucurbita moschata) accessions against Squash leaf curl Philippines virus in Taiwan W. S. TSAI, National Chiayi University, Chiayi, TAIWAN

The squash crops (Cucurbita spp.) are important vegetables of the world. They have nutrient-dense fruits, while their young shoots and leaves are also often consumed as vegetable. However, leaf curl diseases caused by whitefly-transmitted begomoviruses are a major constraint to squash crop production. The disease symptoms on squash include leaf curling, yellowing, mosaic and plant stunting. In Taiwan, the disease was first observed in 2001 and currently prevalent in squash fields. The causal agent was identified as Squash leaf curl Philippines virus (SqLCPV). The SqLCPV was identified as major virus, distributed widely and revealed high nucleotide sequence identity among isolates (>94\%). The pumpkin accession 'Nigerian Local' (Cucurbita moschata) with good resistance to two potyviruses, Zucchini yellow mosaic virus and Papaya ringspot virus, was found to be susceptible to SqLCPV. Fortunately, the SqLCPV resistances were screened in two pumpkin accessions by whitefly transmission in an insect-proof cage following the virus detection by PCR. The results of resistance assay in patents, F1 and F2 plants indicated one SqLCPV resistance is a recessive inheritance and the other one is a dominant inheritance. In the future, the candidates related to both of SqLCPV resistances should be identified following development of molecular markers for marker-assisted selection to accelerate the pumpkin breeding with begomovirus-resistance.

Cultivar screening for tolerance to Sclerotinia sclerotiorum using oxalate oxidase gene activity and detached leaf assays L. VAN DER HOVEN, J. van der Waals, University of Pretoria, Pretoria, SOUTH AFRICA

In South Africa, there is limited information on the susceptibility and tolerance of soybean cultivars currently being planted to the fungal pathogen Sclerotinia sclerotiorum. Detached leaf assays and histological assays were conducted using twenty-nine soybean cultivars commonly planted in South African to screen for tolerance to $S$. sclerotiorum. No oxalate oxidase gene activity was shown by histological assays, possibly indicating low or no gene expression in cultivars tested. Cultivars differed significantly $(\mathrm{P}<0.001)$ regarding percentage diseased leaf area during detached leaf assays, however, temperature and relative humidity means were not significantly different from each other. Cultivar LS $6444 \mathrm{R}$ was found to be most susceptible to $S$. sclerotiorum at both high and low relative humidity, and cultivars DM 6.8i RR and PAN 1583 R showed higher tolerance under high and low relative humidity, respectively. Cultivars NS $6448 \mathrm{R}$ and LS $6444 \mathrm{R}$ showed more susceptibility at $20^{\circ} \mathrm{C}$ and $25^{\circ} \mathrm{C}$, respectively, while PAN $1614 \mathrm{R}$ and LS 6466 $\mathrm{R}$ were the most tolerant cultivars at $20^{\circ} \mathrm{C}$ and $25^{\circ} \mathrm{C}$, respectively. Short to medium growing cultivars showed higher susceptibility to $S$. sclerotiorum, reaffirming the importance of planting dates in South Africa, to ensure that shorter growing cultivars escape pathogen infection in the field.

Evaluation of development and production of common beans cultivars under infection of Curtobacterium flaccumfaciens pv. flaccumfaciens A. C. MARINGONI (1), J. M. Soman (1), T. Domingues Tomasini (1), J. C. Silva (1), T. A. Fernandes da Silva Junior (2), (1) São Paulo State University, Botucatu, BRAZIL; (2) Universidade do Sagrado Coração, Bauru, BRAZIL

The bacterial wilt, induced by Curtobacterium flaccumfaciens pv. flaccumfaciens (Cff), is one of the most important disease of common beans in Brazil. The aims of this work were to evaluate the development and agronomic parameters of common beans cultivars resistant (IAC Alvorada, IAC Diplomata and IPR Corujinha) and susceptible (IAC Carioca and Pérola). To this, assays were performed in greenhouse condition. At the first assay, repeated in three times, were evaluated number of pods, grain per pod, mass of one hundred grains, and disease severity on plants inoculated or not with Cff. Disease was evaluated every seven days and the plants were conducted until pods harvest. About the second assay, repeated in two periods, percentage of dry weight reduction of inoculated plants was evaluated in relation to uninoculated at the same cultivars, after 30 days. It was observed that agronomic parameters were directly affected by disease in susceptive cultivars, highlighting the Pérola cultivar, which presented $79.8 \%$ losses in mass of one hundred grains. Cultivars IAC Alvorada, IAC Diplomata and IPR Corujinha showed a dry weight reduction of $21.6 \%, 15.7 \%$ and $17.3 \%$ respectively. Cultivars IAC Carioca and Perola showed a reduction of $30.3 \%$ and $26.3 \%$, respectively. These study showed that resistant cultivars presented less significant losses in the presence of the disease.

Efficient field phenotyping for multiple disease resistance in a winter wheat panel

K. FLATH (1), A. Jacobi (2), M. Taylor (3), T. Miedaner (4), (1) Julius Kuehn-Institut, Kleinmachnow, GERMANY; (2) Strube Research GmbH \& Co. KG, Söllingen, GERMANY; (3) Limagrain Field Seeds, Peine, GERMANY; (4) University of Hohenheim (720), Stuttgart, GERMANY

Wheat is challenged by many diseases, among them yellow/stripe rust (YR) caused by Puccinia striiformis, stem rust (SR) caused by P. graminis, and Fusarium head blight (FHB) caused by Fusarium spp. are the most important. For reducing the use of pesticides it is important to breed cultivars for the market with multiple disease resistance (MDR). Our objective was to analyze the resistance of 36 commercially grown European winter wheat cultivars to YR, SR, and FHB in three individual and one combined field inoculations across seven (YR, FHB) and three (SR) Location $\times$ year combinations to evaluate MDR in an efficient way. Large variation among wheat cultivars was detected for YR, SR, and FHB resistance ranging from $0 \%$ to $48 \%, 0.8 \%$ to $80 \%$ and $10 \%$ to $51 \%$, respectively, in the combined inoculation variant. Interaction between inoculation treatments (individual vs. combined) and wheat genotype was not significant $(\mathrm{P}>0.1)$ for each of the three diseases. Accordingly, correlations between both inoculation treatments were very high $\left(\mathrm{R}^{2}=0.95-0.99\right)$. Several cultivars showed MDR to two or even three of the pathogens. In conclusion, resistance ranking among genotypes was not changed when plants were challenged with all three pathogens together. Substituting factorial inoculation trials by multi-pathogen inoculation makes it more efficient to select for MDR in practical breeding programs.

A reliable glasshouse screening technique to detect BYDV-PAV disease resistance in cereal crops at early and late growth stage S. CHOUDHURY, TIA, Launceston, AUSTRALIA

Barley yellow dwarf virus-PAV (BYDV-PAV) is one of the major virus causing a widespread and serious viral disease affecting cereal crops. A reliable method was developed to screen cereal crops (wheat, barley and oats) for BYDV-PAV resistance in glasshouse experiments. We evaluated traits associated with BYDV-PAV infection such as leaf discoloration, reduction in growth and yield traits, and percentage of virus-infected plants using ELISA and TBIA tests. Inoculation of plants using viruliferous aphids as vectors proved effective establishing infections and reducing plant growth in susceptible varieties, and in inducing leaf discoloration. Increasing the numbers of viruliferous aphids per plant led to very high percentages of infection in susceptible cultivars. Visual evaluation of symptoms on barley and oats is considered adequate for assessing BYDV-PAV resistance, but in wheat, it is necessary to evaluate BYDV-PAV resistance by an ELISA test (at early stage) and grain number and yield measurements (at late stage). Wheat varieties 
containing the $B d v 2$ gene demonstrated a high level of resistance for BYDV-PAV. The $Y d 2$ gene-containing barley variety showed leaf discoloration and plant height reduction but no significant reductions in yield, which suggests the need for yield analysis for assessing BYDV-PAV tolerance.

\author{
Evaluation of soybean breeding lines for resistance to Phomopsis seed decay: Results of 2014, 2015, and 2016 field trials in Stoneville, \\ Mississippi \\ S. LI (1), J. R. Smith (2), (1) USDA ARS CGRU, Stoneville, MS, USA; (2) USDA-ARS, Stoneville, MS, USA
}

Soybean [Glycine $\max$ (L.) Merr.] is one of the most important crops in the world. Phomopsis seed decay (PSD) is a soybean seed disease that causes poor seed quality. This disease is caused primarily by a fungal pathogen, Phomopsis longicolla (syn. Diaporthe longicolla). Planting PSD-resistant soybean cultivars is the most effective means to control PSD. In this study, breeding lines were developed from three crosses between PSD-resistant and high germination parents (DS25-1 x PI 424324B; DS25-1 x PI 417274; DS34-1 x PI 424324B) and from one cross between two PSD-resistant parents (PI 80837 x SS93-6181). Results from seed plating assays of 122 breeding lines from field trails in 2014, 2015, and 2016 showed that the range of percent seed infection by $P$. longicolla among all lines tested was from 0 to $52 \%$. Fourteen breeding lines were completely free of PSD based on seed plating assays in 2014, 2015, and 2016 using F5, F6, and F7 seed, respectively. The mean germinations of the 14 lines were $95.5 \%$ and $92.9 \%$ in the tests in 2015 and 2016, respectively. Breeding lines with resistance to PSD and high-germination were entered into multi-year yield tests in 2017 and the most resistant lines will be selected for future use in developing PSD-resistant soybean cultivars.

Symptoms of infected plants and selection of resistance to bacterial canker in Kiwifruit accessions (Actinidia deliciosa \& Actinidia chinensis) M. LEE, Y. B. Kwack, H. L. Kim, H. W. Jeong, H. C. Rhee, Namhae branch, NIHHS, RDA, Namhae-gun, KOREA

Bacterial canker that causes serious damage to the Kiwifruit (Actinidia deliciosa \& Actinidia chinensis) industry is caused by Pseudomonas syringae pv. actinidiae (Psa). This study was carried out to identify symptoms of bacterial canker and to select resistance resources. Bacteria, Psa2 (KBE9) and Psa3 (SYS2), were cultured. Before inoculation, the grafted kiwifruits, one or two-year-old, were disinfected by $80 \%$ ethanol and adapted at a temperature of 18 to 20 degrees Celsius and relative humidity of $80 \%$ or higher. After that, the plantlets were inoculated by spraying without no-wounds. Main symptoms were composed of angular necrotic dark leaf spot with a halo, angular necrotic dark leaf spot without a halo, yellowish chlorotic halos and necrosis like wilting. In A. deliciosa, Psa2 inoculation gave an average disease index 2.0 and Psa3 did 2.29. Jecy Green (A. hybrid), Chin Mei and M51-2 showed no or fewer symptoms. In A. chienesis, Psa2 and Psa3 showed 2.56 and 1.96 disease index respectively. Golden King and Songoku also showed no or fewer symptoms. We selected germplasms that has low disease index and the second test is underway.

Aggressiveness evaluation of Diaporthe species causing soybean stem canker in the United States

K. PETROVIC (1,2), K. Ghimire (2), P. N. Okello (2), C. A. Bradley PhD (3), M. Ćeran (1), M. Chilvers (4), V. Djordjevic (1), V. Djukic (1), J. Miladinović (1), Z. Miladinov (1), D. S. Mueller (5), D. Smith (6), S. Balešević Tubić (1), M. Vidic (1), K. Wise (3), F. M. Mathew (2), (1) Institute of Field and Vegetable Crops, Novi Sad, SERBIA; (2) South Dakota State University, Brookings, SD, USA; (3) University of Kentucky, Princeton, KY, USA; (4) Michigan State University, East Lansing, MI, USA; (5) Iowa State University, Ames, IA, USA; (6) University of Wisconsin-Madison, Madison, WI, USA

In 2014, the estimated yield loss of soybean (Glycine max L.) caused by stem canker was 350,000 tons (175 million dollars). A total of 135 isolates were recovered from symptomatic soybean stems collected from six U.S. states (IA, IN, IL, KY, MI, and SD) between 2002 and 2016. Using morphology and DNA sequence analysis, isolates were identified as D. longicolla $(66 \%), D$. aspalathi $(25 \%)$ and $D$. caulivora $(9 \%)$. In a greenhouse experiment, aggressiveness of five isolates each of D. caulivora and D. aspalathi was compared in two independent experiments using four inoculation methods (toothpick, stem-wound, mycelium-contact, and spore-injection). Isolates of D. caulivora were compared on cv. Hawkeye, whereas D. aspalathi isolates were compared on cv. Bragg. The experiment was established in a completely randomized design in a factorial arrangement with inoculation methods and isolates as factors. At 21 days after inoculation, lesion length and stem length were measured; both were affected by a two-way interaction $(P<$ $0.001)$ between isolates and inoculation methods. For $D$. caulivora, isolate SD-29 produced significantly $(P \leq 0.05)$ longer lesions and shorter stems by the stem-wound method compared to other inoculation methods. For $D$. aspalathi, isolate KY-41 produced significantly $(P \leq 0.05)$ longer lesions compared to the other isolates. The toothpick method resulted in significantly $(P \leq 0.05)$ shorter stems across all $D$. aspalathi isolates compared to the other methods. Our study determined that the toothpick and stem-wound methods can be used effectively for screening soybean germplasm for resistance to $D$. aspalathi and D. caulivora.

Utilization of a diversity panel to address genetic bottlenecks in cultivars of lima bean while improving their resistance to Phytophthora phaseoli T. MHORA (1), N. F. Gregory (1), N. M. Donofrio (1), T. A. Evans (1), E. Ernest (2), R. J. Wisser (1), C. Scanlan (3), S. Davis (3), D. R. Lester (3), (1) University of Delaware, Newark, DE, USA; (2) University of Delaware, Georgetown, DE, USA; (3) Dept. of Plant and Soil Science, University of Delaware, Newark, DE, USA

Delaware and the Mid-Atlantic region are the largest producers of lima bean (Phaseolus lunatus) in the US. Downy mildew of lima bean, caused by the oomycete pathogen Phytophthora phaseoli, is the most important yield-limiting disease resulting in continued loss of profitable production. In 2004 the University of Delaware initiated a lima bean breeding program. Existing cultivars with quality characteristics required by the market have a narrow genetic diversity and more diverse germplasm must be used to improve disease resistance and agronomic traits. A diversity panel (DP) comprised of 261 accessions sampled from the major genepools of lima bean was obtained from the species' centers of domestication. Accessions of the DP were evaluated for reactions to two major races (E and F) of $P$. phaseoli and to $P$. capsici, an emerging pathogen of importance. A population genetic analysis using genotyping-by-sequencing of the DP and crosses derived from it is being conducted to understand the extent of the genetic bottleneck in local cultivars and its effect on disease resistance. Concurrent resistance to races $\mathrm{E}$ and $\mathrm{F}$ has not previously been identified nor bred into existing cultivars. Phenotyping data revealed accessions that have resistance to one or both races and their geographic distribution suggests alternative resistance alleles. These data coupled with the lima bean genome recently completed by our group are being used to investigate these hypotheses.

Demystifying the endophytic and saprophytic ecology of Fusarium oxysporum f. sp. lycopersci race 3 for improved Fusarium wilt management in tomato

C. L. SWETT (1), E. Hellman (2), R. Oguchi (3), (1) Department of Plant Pathology, University of California - Davis, Davis, CA, USA; (2) University of California, Davis, Davis, CA, USA; (3) Department of Plant Pathology, Univeristy of California, Davis, Davis, CA, USA

The life history strategies of pathogens in the Fusarium oxysporum species complex include abilities as non-pathogenic endophytes and saprophytes; these stages perpetuate persistence of soil-borne inoculum and enhance the risk of resistance-breaking race emergence. With recent geographic 
expansion of the Fusarium wilt pathogen of tomato, Fusarium oxysporum f. sp. lycopersci race 3 (Fol R3) within California, population management has become critical. We evaluated cryptic pathogen sources as contributors to Fol R3 population persistence and growth. Five Fol R3 isolates all systemically colonized a resistant tomato cultivar (20-80\% incidence). Compared with susceptible cultivars, infection incidence in resistant cultivars was the same $(100 \%)$, but extent of root and shoot colonization was $65 \%$ and $97 \%$ lower respectively. Fol R3 colonized diverse rotation crops; plant infection incidence in cantaloupe (Cucurbitaceae) and sunflower (Asteraceae) were the same as tomato but 20\% lower in rice and corn (Poaceae). All rotation crops could be systemically colonized, but the extent of shoot colonization was $40-50 \%$ lower in Poaceae crops than tomato. Studies are underway to connect colonization extent with soil inoculum loads and wilt development. These studies reveal that resistant tomato cultivars, non-tomato rotation crops, and Fol R3 hosts may be high risk rotations, whereas certain grass crops may help manage soil inoculum loads.

Resistance of Brazilian sugarcane cultivars to Ceratocystis paradoxa, the causal agent of pineapple sett rot J. UZAN, A. Seiiti Urashima, Federal University of São Carlos, Araras, BRAZIL

Sugarcane is a versatile and renewable crop used in Brazil to produce sugar, ethanol, and bioelectricity. The high demand for its raw materials has forced all year-round planting. Pineapple sett rot has become a major problem of sugarcane seed pieces during winter when low soil temperature and moisture delay germination and setts decay before bud germinates. Resistant cultivars is the ideal method to control this disease, and reports from other countries indicated a wide range of susceptibility among cultivars. Therefore, this study aimed to examine the reaction of four of the most important Brazilian sugarcane cultivars to pineapple sett rot. Ten buds of each cultivar (RB855156, RB867515, RB92579, RB966928) were planted in a plastic tray of 2,700 $\mathrm{cm}^{3}$ of substrate and contaminated with $0.04,0.4,4,40$ spores. $\mathrm{cm}^{-3}$ and compared to uninoculated control. The experiment was kept at $25^{\circ} \mathrm{C}$, photoperiod of $12 \mathrm{~h}$, and $25 \%$ of soil humidity in a completely randomized design with four replications for 28 days, when germination failure due to the disease was evaluated. Percentage of germinated buds was submitted to analysis of variance and to the Tukey test using software ASSISTAT. RB92579 was the most susceptible cultivar, being the first to have bud germination significantly affected as pathogen level increased $\left(4 \mathrm{spores} . \mathrm{cm}^{-3}\right)$. On the other hand, RB867515 showed the highest resistance, with no significant difference to uninoculated control in all pathogen levels.

Disease resistance and yield performance of rice cultivars under organic production

X. ZHOU (1), A. M. McClung (2), F. Dou (1), (1) Texas A\&M AgriLife Research, Beaumont, TX, USA; (2) USDA ARS Dale Bumpers National Rice Research Center, Stuttgart, AR, USA

Disease resistance and yield performance of rice cultivars under organic production systems in the US remain largely unknown. Narrow brown leaf spot (NBLS) caused by Cercospora janseana and brown spot caused by Cochliobolus miyabeanus are two common diseases affecting organic rice production. A 2-year study was conducted in a field under organic management at Beaumont, Texas to evaluate 20 (2016) or 10 (2017) rice cultivars and germplasm lines for their resistance to these diseases and yield performance. Jasmine 85, PI312777, Rondo, Tesanai 2, XL723, and XL753 consistently showed high levels of resistance to NBLS while Cocodrie, Jazzman 2, Presidio, and Sierra were either susceptible or very susceptible to NBLS. None of the cultivars evaluated showed complete resistance to brown spot. Most of the cultivars, including Cocodrie, Jasmine 85, XL723 and XL753, were either susceptible or very susceptible to brown spot. Jasmine 85, PI312777, Rondo, Tesanai 2, and XL753 were among the best cultivars showing good stand establishment, vigorous growth, and high yield potential while Cocodrie, Jazzman 2, Presidio, and Sierra showed lowest yields. Selection of a resistant cultivar with high yield potential is the best approach for effective management of NBLS and brown spot and maximizing the returns of organic rice production.

Varietal susceptibility to multiple Phytophthora species in macadamia

O. JEFF-EGO (1), O. Akinsanmi (1), B. Topp (1), J. Henderson (2), A. Drenth (1), (1) The University of Queensland, Brisbane, AUSTRALIA; (2) Department of Agriculture and Fisheries, Brisbane, AUSTRALIA

Phytophthora root rot caused by Phytophthora cinnamomi is an important disease of macadamia worldwide, while several other Phytophthora species are known to cause disease on macadamia. Limited information exists on varietal resistance to these pathogens in macadamia. Since traditional screening methods for host resistance to Phytophthora root rot in tree crops are time-consuming, we sought to identify tolerant germplasm to multiple Phytophthora spp. associated with macadamia using a rapid high-throughput bioassay. Using this new assay, we determined the response to infection by seven Phytophthora spp. in 470 macadamia genotypes, including 20 accessions of the four wild Macadamia spp., the main commercial cultivars, and segregating populations from the macadamia breeding program. Results revealed a wide spectrum of varietal susceptibility. 'HAES 816 ' was the most susceptible commercial cultivar and M. ternifolia was the most susceptible wild macadamia species. The results correlate with the observed disease severity to P. cinnamomi in the field and our early screening methods may be a useful tool in the breeding program, to increase the level of resistance in new cultivars.

Differential regulatory systems of virulence-related functions between two strains of Burkholderia glumae require a common master regulator qsmR

T. DE PAULA LELIS (1), I. Barphagha (2), J. H. Ham (3), (1) Louisiana State University, Baton Rouge, LA, USA; (2) Louisiana State Univeersity, BATON ROUGE, LA, USA; (3) Louisiana State University, BATON ROUGE, LA, USA

Burkholderia glumae is a major bacterial pathogen of rice in the United States. Significant phenotypic and genotypic variations have been reported within this specie. B. glumae strains $336 \mathrm{gr}-1$ and $411 \mathrm{gr}-6$ show phenotypic variations in virulence and pigmentation. Virulence-related genes are mostly regulated by the TofI/TofR-mediated quorum sensing (QS) system. To study the function of QS in B. glumae 336gr-1 and 411gr-6, a series of deletion mutants were generated for $\Delta t o f I / t o f R$ derivatives using the pkkSacB suicide vector. The $\Delta$ tofI/tof $R$ derivative of 336gr- 1 completely lost its ability to produce toxoflavin in LB media, and its extracellular protease activity in NA agar supplemented with $1 \%$ skim milk. However, the $\Delta$ tofI/tofR derivative of $411 \mathrm{gr}-6$ retained the ability to produce toxoflavin, and extracellular protease in a lower level compared to its parent strain $411 \mathrm{gr}-6$. Additionally, virulence of each quorum-sensing-defective derivatives was tested with the susceptible rice cultivar Trenasse. 336gr- $1 \Delta$ tofI/R and $411 \mathrm{gr}-6 \Delta \mathrm{tofI} / \mathrm{R}$ exhibited retained virulence to cause disease in the rice plants with disease severity scoring as much as $20 \%$ and $60 \%$, respectively, at 14 days after inoculation. Remarkably, it was found that deletion of $q \operatorname{sm} R$, a regulatory gene for quorum-sensing, abolished toxoflavin production, and extracellular protease activity in both $336 \mathrm{gr}-1$, and $411 \mathrm{gr}-6$ strains, indicating the master regulatory role of this gene for the bacterial pathogenesis.

Mixed messages: The role of nitric oxide in Ralstonia solanacearum Type III Secretion and virulence C. HENDRICH (1), B. L. Dalsing (2), A. Truchon (1), C. Allen (1), (1) University of Wisconsin, Madison, WI, USA; (2) Syngenta, Greensboro, NC, USA 
Ralstonia solanacearum (Rs) is a xylem-dwelling bacterial plant pathogen that can infect a wide range of hosts around the world. The xylem fluid of tomato, a model Rs host, is low in oxygen but high in nitrate, and nitrogen metabolism is an important factor in Rs biology. Nitric oxide (NO), a potent signal and metabolic byproduct that is produced by both $R s$ and its plant hosts is positively correlated with the Rs Type III Secretion System (T3SS), a key $R s$ virulence factor. Both exogenously applied and endogenously produced NO induced T3SS expression in a range of conditions. NO induces T3SS gene expression in a dose-dependent manner. Induction of T3SS is specific to NO, and is not shared by other toxins or stressors. We used an NO-reactive fluorescent dye to measure NO levels in tomato xylem and found that the levels of NO in the xylem are around $20 \mathrm{uM}$. In vitro, T3SS can be induced by NO concentrations above $20 \mathrm{uM}$, implying that the physiological levels of NO present in tomato xylem may be sufficient to induce $R s$ T3SS gene expression in planta. These findings suggest that $R s$, and potentially other xylem dwelling microbes, experience NO stress in the xylem, and may use the presence of $\mathrm{NO}$ as a signal to recognize and adapt to their in-host niche.

Critical role of cytochrome bc1 in tolerance of Xanthomonas campestris pv. campestris to Phenazine-1-carboxylic acid

J. WU (1), X. Pan (1), S. Xu (1), Y. Duan (2), M. Zhou (2), (1) Nanjing agricultural university, Nanjing, CHINA; (2) Nanjing Agricultural University, Nanjing, CHINA

Xanthomonas spp. is a class of plant Pathogen that infected a wide range plants, causing serious damage in global agriculture production. Phenazine-1 carboxylic acid (PCA) is a microbial antibiotic, primarily produced by Pseudomonas spp. PCA had been used in control of rice bacterial leaf streak in rice planting region of South China. In previous study, we found twin-arginine translocation (Tat) system and cytochrome $c$ synthesis (CCM) system were important in regulating the tolerance of PCA-insensitive bacterium Xanthomonas campestris pv. campestris (Xcc, the the causal agent of black rot of vegetable brassica crops). In our current study, we found that this phenomenon was caused by influencing the cytochrome bc1, which is the functional downstream of Tat system and CCM system. The $\mathrm{EC}_{50}$ value of the cytochrome bc1 deletion mutant of Xcc to PCA decreased about 100 times than that of the WT. This results may provide some suggestions of the plant disease caused by Xanthomonas campestris pv. campestris in field control. In conclusion, the cytochrome bc1 was critical in tolerance of Xanthomonas campestris pv. campestris to PCA.

A cellulase as an essential virulence factor of Clavibacter michiganensis subsp. michiganensis causing bacterial canker in tomato I. S. HWANG, E. J. Oh, C. S. Oh, Department of Horticultural Biotechnology, Kyung Hee University, Yongin, KOREA

Clavibacter michiganensis $(\mathrm{Cm})$ subsp. michiganensis causes bacterial canker in tomato plants. This bacterial pathogen carries the plasmid (pCM1)borne cellulase gene, celA that is required for its pathogenicity in plants. CelA consists of the cellulase domain, the cellulose binding domain, and an expansin domain in its C-terminus. Based on the previous reports, the removal of pCM1 from Cm subsp. michiganensis strain NCPPB382 caused very mild and delayed wilting symptoms in tomato. In addition, this strain carries the second cellulase gene, $c e l B$, which has not been functionally characterized. To better understand how cellulase genes contribute to the pathogenicity of $\mathrm{Cm}$ subsp. michiganensis, celA mutants of $\mathrm{Cm}$ subsp. michiganensis type strain LMG7333 were generated by transposon mutagenesis, and these mutants almost lost the ability to cause severe wilting symptoms in tomato. This strain was complemented with full or truncated forms of celA or celB genes. The truncated celA gene only carrying two domains, cellulase and cellulose binding domains, was enough to recover the ability to cause severe wilting symptoms in tomato. Moreover, the functional $c e l B$ genes could complement $c e l A$ mutants only partially. The $c e l A$ overexpression in $C m$ subsp. capsici strain that originally lacks $c e l A$ and almost non-pathogenic in tomato was able to cause disease symptom in tomato. These results indicate that the celA gene acts as a major virulence factor in a host-specific manner.

Functional analysis of Xylella fastidiosa PD0576 gene encoding a histidine kinase and response regulator hybrid protein H. CHEN (1), L. De La Fuente (2), (1) Auburn University, Auburn, AL, USA; (2) Department of Entomology and Plant Pathology, Auburn University, Auburn, AL, USA

Xylella fastidiosa (Xf), a xylem-limited insect-vectored pathogenic bacterium, is the causative agent of devastating plant diseases in many economically important crops in the Americas, Europe and Asia. Diseases caused by this pathogen are associated with the blockage of the host xylem system by biofilm formation, as well as twitching motility. Previous studies of our group found that the mineral nutrient calcium can affect these traits in Xf, but mechanisms involved in these processes are poorly understood, especially molecular mechanisms responsible for sensing environmental Ca. We focused on two component regulatory systems, which are important for bacteria to sense and respond to environmental signals. In silico, we found that PD0576, a histidine kinase and response regulator hybrid protein, had two putative Ca-dependent phosphotriesterase domains which are located in the $\mathrm{N}$ terminal sensor part. Taking the advantage of Xf natural competence, a novel gene knockout protocol was used to construct a gene deletion mutant. The gene deletion did not affect the growth of Xf, but the mutation increased twitching motility of the WT strain WM1-1 on PW plates, and decreased cell to cell aggregation and biofilm formation by WM1-1 under flow conditions in microfluidic chambers. We proposed that PD0576 in Xf may sense the environmental $\mathrm{Ca}$ to regulate switching from planktonic to biofilm growth. Further characterization of this mutation is ongoing.

Effect of Hop stunt viroid on host (Humulus lupulus) transcriptome and its interactions with hop powdery mildew (Podospheara macularis) M. KAPPAGANTU (1), J. Bullock (2), M. E. Nelson (2), K. C. Eastwell (2), (1) Washington State University, Pullman, WA, USA; (2) Washington State University, Prosser, WA, USA

Hop stunt viroid (HSVd) and hop powdery mildew fungus (Podospheara macularis) (HPM) are the most economically important pathogens that infect hops. Interactions of these two pathogens during mixed infections were investigated, and changes in the hop transcriptome during HSVd infection were quantified. In a detached leaf assay, HSVd-free and HSVd-positive hop leaves of cultivar Willamette were inoculated with HPM, which revealed reduced growth of HPM on HSVd infected leaves. To identify the changes in hop transcriptome during HSVd infection that may cause HSVd antagonism towards HPM, RNA from hop leaves representative of each treatment (i.e. HSVd/ HPM-free control, HSVd infected, HPM infected; and leaves infected with HSVd and HPM) were subjected to high throughput sequencing. Transcriptome analysis of HSVd-infected leaves revealed dynamic changes in host gene expression. Expression of genes related to defense, and to lipid and terpenoid metabolism were most strongly altered as a result of HSVd infection. Further analysis by quantitative reverse transcription-polymerase chain reaction analysis showed that transcript levels of pathogenesisrelated (PR) genes such as PR 1, chitinase, and thaumatin-like protein genes were induced in leaves infected with HPM alone, but were suppressed in leaves previously infected with HSVd, thus indicating that HSVd acts as a negative regulator of certain PR genes which are typically elicited by other pathogen infections.

An effector from the Huanglongbing-associated pathogen repressing host hypersensitive reaction to facilitate pathogenesis Z. PANG (1), J. Li (1), S. Duan (2), S. Duan (2), N. Wang (1), (1) University of Florida, Lake Alfred, FL, USA; (2) Univerisity of Florida, Lake Alfred, FL, USA 
Citrus Huanglongbing (HLB) also known as citrus greening disease is caused by the psyllid-transmitted, phloem-limited bacteria 'Candidatus Liberibacter. asiaticus' (CLas), 'Ca. Liberibacter. africanus' (CLaf), and ' $\mathrm{Ca}$. Liberibacter. americanus' (CLam). HLB is the most destructive disease which eliminates the productivity of citrus trees, with reported losses of 30 to $100 \%$. However, the pathogenicity mechanisms of Liberibacters remain unknown partially because ' $\mathrm{Ca}$. Liberibacter' species have not been cultured. Sec-dependent effectors (SDEs) have been known to play critical roles in the pathogenicity of phloem-limited bacteria. It has been suggested that SDEs are secreted into phloem or companion cells and interact with host proteins that facilitate compatibility or disease development. Here, we characterized a Sec-dependent effector. This effector is highly expressed in citrus host at early stage of CLas infection. Constitutively expressing this effector in plants not only affects the growth and development, but also regulates plant innate immunity by prohibiting defense responses of plants to make plants more susceptible to pathogen infections. Its targets were identified by yeasttwo-hybrid analysis, pull-down assay, or bimolecular fluorescence complementation. We also analyzed how those targets are involved in HLB disease development.

Bacterial enhancer binding protein $\mathrm{HrpS}$ is regulated by three two-component systems and Lon protease in Erwinia amylovora J. H. Lee (1), Y. ZHAO (2), (1) University of Illinois, Urbana, IL, USA; (2) University of Illinois at Urbana-Champaign, Urbana, IL, USA

The bacterial enhancer binding protein $\mathrm{HrpS}$ is essential in activating hrpL and the type III secretion system (T3SS) in Erwinia amylovora. However, how the hrpS gene is regulated remains unknown in E. amylovora. In this study, we investigated the role of various two-component systems (TCSs) and Lon in regulating HrpS. Promoter deletion analyses and 5' RACE showed that the hrpS gene contains two promoters driven by HrpX/HrpY and the Rcs system, respectively. EMSA and qRT-PCR assays demonstrated that integration host factor IHF positively regulates $h r p S$ expression through directly binding the $h r p X$ promoter and positively regulating $h r p X / h r p Y$ expression. Western blot analyses showed that mutation in lon directly affected the accumulation and stability of HrpS. Mutation in lon indirectly influenced the expression of $h r p S$ through accumulation of the RcsA/RcsB proteins. Furthermore, the $\operatorname{csr} A$ mutant showed significantly reduced transcripts of the $h r p X / h r p Y, \operatorname{rss} A$ and $r \operatorname{cs} B$ genes, indicating that CsrA is required for full $h r p S$ expression. On the other hand, the $\operatorname{csr} B$ mutant exhibited up-regulation of the $r c s A$ and $r c s B$ genes, and $h r p S$ expression was largely diminished in the $\operatorname{csr} B / r c s B$ mutant, indicating that the Rcs system is mainly responsible for the increased $h r p S$ expression in the $\operatorname{csr} B$ mutant. These findings suggest that E. amylovora recruits multiple TCSs to regulate $h r p S$ at the transcriptional level and HrpS is also regulated at posttranslational level by Lon.

A glycine-rich poly(U)-binding nuclear protein regulates asexual development and virulence of Magnaporthe oryzae J. YANG PHD, X. Gao, C. Yin, X. Liu, W. Zhao, Y. L. Peng, China Agricultural University, Beijing, CHINA

Glycine-rich RNA-binding proteins (GRPs) are involved in diverse biological processes and play important roles through binding RNAs and proteins. We here identified a novel gene MoGRP1 encoding a GRP from Magnaporthe oryzae, which was important to fungal virulence and development. The $\Delta$ Mogrp1 mutants exhibited severe defects in growth, conidiation, appressorium formation, invasive hyphal development, and virulence. The $\Delta$ Mogrp 1 mutants were also deficient in cell wall integrity and responses to different stresses. MoGrp1 was localized in nuclei and co-immunoprecipitated with several components of the spliceosome, including subunits of U1 snRNP and U2 snRNP. Moreover, MoGrp1 possessed a high binding capacity to poly(U). MoGrp1 has no homologs in yeasts, plants, and animals, suggesting it might function as a unique auxiliary splicing factor. MoGrp1 has an Nterminal RNA recognition motif (RRM) domain and a C-terminal glycine-rich domain with four Arg-Gly-Gly (RGG) repeats. Domain deletion assays showed that both the RRM domain and RGG repeats were essential to fully function of MoGrp1. The full sequence of nine amino acids between the first and the second RGG repeats was essential to nuclear localization and biological functions of MoGrp1. In summary, our results indicate that MoGrp1 is a novel component of the spliceosome unique to filamentous fungi and regulates fungal virulence, development, and responses to stresses in the rice blast fungus.

\author{
The Colletotrichum orbiculare MTF4 is a transcription factor downstream of MOR required for plant-derived signal dependent appressorium \\ development \\ S. Kodama (1), T. Nishiuchi (2), Y. KUBO (1), (1) Kyoto Prefectural University, Kyoto, JAPAN; (2) Kanazawa University, Kanazawa, Ishikawa, \\ JAPAN
}

Many plant pathogenic fungi including Colletotrichum species form a specialized infection structure, called an appressorium. Appressorium formation relies on perception of physical and biochemical signals at the plant surface. In cucumber anthracnose fungus $C$. orbiculare, the morphogenesis-related NDR (nuclear Dbf2-related) kinase pathway (MOR) is crucial for translating plant-derived signals for appressorium development (Kodama et al., 2017), Based on whole genome transcript profiling, we identified the transcription factor MTF4 (MOR-related transcription factor 4) as a putative downstream target of MOR. The $m t f 4$ mutant phenotype was closely similar to the MOR component pagl mutant. Introduction of the constitutively active variant of the NDR kinase Cbk1 had no effect on mtf4 mutant phenotypes, while introduction of overexpression construct of MTF4 to pagl mutant complemented pag1 defects. These results suggest that MTF4 acts downstream of MOR for appressorium morphogenesis and pathogenesis. Furthermore, Mtf4 was recognized at nuclei during appressorium formation in planta but not in vitro. Mtf4 mis-localized by the conditional inactivation of Cbk1 and pag1 deletion, suggesting that MOR-dependent localization of Mtf4 responds to plant-derived signals during appressorium development. Therefore, MTF4 is a key downstream transcription factor of MOR in C. orbiculare for appressorium development in response to plant-derived signals.

\title{
Microbial small molecules - weapons of plant subversion
}

R. DE JONGE (1,2,3), I. A. Stringlis (1), H. Zhang (1), C. M. J. Pieterse (1), M. D. Bolton (4), (1) Plant-Microbe Interactions, Department of Biology, Faculty of Science, Utrecht University, Utrecht, NETHERLANDS; (2) VIB Center for Plant Systems Biology, Ghent, BELGIUM; (3) Plant-Microbe Interactions, Department of Biology, Faculty of Science, Utrecht, NETHERLANDS; (4) USDA-ARS, Red River Valley Agricultural Research Center, Fargo, ND, USA

Plants live in close association with myriads of microbes in nature. Most of these interactions are harmless, but a multitude of pathogenic microbes can cause severe disease and therefore impact plant health. In the case of crop plants, plant diseases often reduce yield and quality while endangering food security. Beneficial microbes, unlike their pathogenic counterparts, provide plants with important services such as enhanced nutrient uptake and protection against pathogenic ones. Like plant pathogens, beneficial microbes may modulate host immunity to efficiently colonize the nutrient-rich niches found within and around the roots and aerial tissues of a plant, a situation roughly analogous to the establishment of commensal gut microbes in human. Various mechanisms have been described by which pathogenic and symbiotic microbes communicate with their host, including the delivery of effector proteins through dedicated secretion machineries and the production of phytohormones, toxins and other small molecules. Plants on the other hand attract and signal to their associated microbes via exudation of photosynthetically fixed carbon sources, quorum-sensing mimicry molecules and selective compounds such as the strigolactones and flavonoids. Plant - microbe communication by means of exchanging signalling compounds thus forms an integral part of the establishment of both beneficial and pathogenic interactions. In parallel with microbe - microbe communication, plant - 
microbe communication shapes plant-associated microbial communities both within and around the plant. Here we discuss large-scale genome comparisons of small molecule biosynthesis capabilities within the Pseudomonas genus, and highlight the vast and diverse biosynthetic potential of individual microbial genomes and the population as a whole.

Xanthomonas euvesicatoria Effector AvrRxo1 Interacts with AIN1 to EnhanceBacterial Growth on Pepper and Tobacco Plants Z. WANG, J. Miao, B. Zhao, Virginia Tech, Blacksburg, VA, USA

Bacterial leaf spot disease caused by Xanthomonas euvesicatoria (Xe) is one of most important pepper diseases. We previously cloned a type III effector (T3E) gene AvrRxo1 from Xanthomonas oryzae pv. oryzicola, which encodes a NAD kinase. The kinase activity is required for the virulence function of AvrRxo1. However, the virulence mechanism of AvrRxo1 is still elusive. AvrRxo1 homologue, Xe-AvrRxo1, was identified from Xe. However, deletion of Xe-AvrRxo1 in Xe strain did not compromise the bacterial virulence on pepper. The virulence function of Xe-AvrRxo1 was masked by other Xe effectors. We developed Xe mutants with deletion of multiple virulence effectors in addition of Xe-AvrRxo1, which were used to inoculate pepper and tobacco plants. The Xe mutants carrying Xe-AvrRxo1 but with the deletion of T3E XopQ or a putative glycerol-transporter (GT) would proliferate better on pepper and tobacco than the isogenic strains without Xe-AvrRxo1. Therefore, Xe-AvrRxo1 indeed possesses virulence function that is redundant with XopQ and GT. To identify host proteins required for the virulence function of Xe-AvrRxo1, we performed a yeast two-hybrid (Y2H) screening by using Xe-AvrRxo1 as bait. Plant protein AIN1 was identified as a putative Xe-AvrRox1 interactor. Silencing or knockout Ain1 by Virus Induced Gene Silence (VIGS) or CRISPR/Cas9 approach compromised the virulence function of Xe-AvrRxo1. Therefore, we confirmed that XeAvrRxo1 could interact with AIN1 to enhance bacterial growth on pepper and tobacco plants. The interplays between Xe-AvrRxo1, XopQ, GT and plant protein AIN1 will be discussed in the poster.

Xylella fastidiosa utilizes a $\beta$ 1,4 endoglucanase to modulate exopolysaccharide production and the dynamics of biofilm development C. CASTRO, B. M. Ingel, M. C. Roper, University of California, Riverside, Riverside, CA, USA

Xylella fastidiosa $(X f)$, a gram-negative bacterium that causes numerous severe diseases in economically important crops, resides in the host xylem and in the mouthparts of its insect vectors where it produces exopolysaccharides (EPS) and forms robust biofilms. We investigated the role of an endoglucanase (EngXCA2) in $X f$ subsp. fastidiosa, the causal agent of Pierce's disease of grapevine, in phenotypes associated with specific stages of biofilm development, surface attachment, and cell-cell aggregation. The $\triangle e n g X C A 2$ mutant strain was significantly compromised in attachment to glass and was unable to develop a complete biofilm relative to the wild-type strain. In addition, $\triangle e n g X C A 2$ mutant was impaired in cell-cell aggregation. Furthermore, $\triangle e n g X C A 2$ mutant produced significantly more EPS than the wild-type strain in vitro. We hypothesize that the endoglucanase, EngXCA2, plays a role in modulating $X f$ biofilm dynamics by facilitating the turnover of EPS during the biofilm developmental cycle through enzymatic degradation of the predicted $\beta 1,4$ glucan backbone of EPS

The Fusarium graminearum Histone Acetyltransferases are Important for Morphogenesis, DON Biosynthesis, and Pathogenicity H. ZHANG, Institute of Plant Protection, Chinese Academy of Agricultural Sciences, Beijing, CHINA

Post-translational modifications of chromatin structure by histone acetyltransferase (HATs) play a central role in the regulation of gene expression and various biological processes in eukaryotes. Although HAT genes have been studied in many fungi, seldom of them have been functionally characterized in plant pathogenic ascomycete Fusarium graminearum. In this study, we identified and characterized four putative HATs (FgRTT109, FgSAS2, FgSAS3 $F g G C N 5)$ in $F$. graminearum, the causal agent of Fusarium head blight of wheat and barley. We observed that all mutants inhibited the growth of $F$. graminearum. The $\triangle F g S A S 3$ and $\triangle F g G C N 5$ mutant increased sensitivities to oxidative and osmotic stresses. Additionally, $\triangle F g S A S 3$ showed reduced conidia sporulation and perithecium formation than wide type PH-1. $\triangle F g G C N 5$, however, was unable to generate any conidia and lost the ability of perithecium formation. Our data showed that $F g S A S 3$ and $F g G C N 5$ are pathogenicity factors required for infecting wheat heads. Importantly, almost no DON was produced in $\triangle F g S A S 3$ and $\triangle F g G C N 5$ mutants, which was consistent with a significant downregulation of TRI genes expression levels. Furthermore, we showed that $F g S A S 3$ is indispensable for the acetylation of H3K4 and H3K14. FgGCN5 is essential for the acetylation H3K9, H3K14, H3K18 and H3K27. RNA-seq analysis expression patterns of the two mutant strains provide insight into their function in development and metabolism. Results from this study clarify the functional divergence of HATs in F. graminearum, and may provide novel targeted strategies to control secondary metabolite expression and infections of $F$. graminearum.

Comparative analysis of the $\operatorname{cyp} A-$ norB gene region of Aspergillus pseudotamarii

C. CHING'ANDA (1), J. Atehnkeng (2), R. Bandyopadhyay (3), P. J. Cotty (4), (1) University of Arizona, Tucson, AZ, USA; (2) International Institute of Tropical Agriculture, Lilongwe, MALAWI; (3) International Institute of Tropical Agriculture, Ibadan, NIGERIA; (4) USDA-ARS, University of Arizona, Tucson, AZ, USA

Aflatoxins are fungal metabolites that often contaminate food and feed and cause immune suppression, stunting, and cancer. Members of Aspergillus section Flavi vary in aflatoxin producing ability. Some members, such as Aspergillus parasiticus and Aspergillus nomius, produce both B and G aflatoxins while Aspergillus flavus and Aspergillus pseudotamarii produce only B aflatoxins. Aflatoxin biosynthesis involves at least 25 genes clustered within a $70 \mathrm{~kb}$ region. Lack of ability to produce $\mathrm{G}$ aflatoxins is attributed to loss of function of the cytochrome $\mathrm{P} 450 \mathrm{monooxygenase}$ encoded by $c y p A$. Several Aspergillus taxa which produce B aflatoxins but not $\mathrm{G}$ aflatoxins have deletions ( 0.8 to $2.2 \mathrm{~kb})$ bridging from $c y p A$ to norB. However, the basis for lack of $\mathrm{G}$ toxin production in $A$. pseudotamarii is not known. Primers designed to characterize partial deletions of $c y p A$ in the $\mathrm{L}$ and $\mathrm{S}$ morphotypes of $A$. flavus and in the Lethal Aflatoxicosis Fungus from Kenya failed to produce PCR products from A. psuedotamarii. Successful amplification of a 1 $\mathrm{kb}$ portion of cypA distal from norB resulted in sequence $90 \%$ similar to $A$. flavus (GeneBank accession number AY 510452.1 ). Efforts to amplify and sequence regions extending from nor $B$ towards the cyclopiazonic acid cluster are underway. Determination of the mechanism of loss of function of $c y p A$ will enhance our understanding of the evolutionary events leading to loss of G aflatoxin production in Aspergillus section Flavi.

Domain-based interactions between a kinase of Gossypium hirsutum and a protein encoded by a betasatellite associated with Cotton leaf curl virus

H. Kamal (1), F. Minhas (2), H. R. PAPPU (3), S. Mansoor (4,5), I. Amin (5), (1) Department of Plant Pathology, Washington State University, PULLMAN, WA, USA; (2) Pakistan institute of engineering and applied sciences, ISLAMABAD, PAKISTAN; (3) Department of Plant Pathology, Washington State University, Pullman, WA, USA; (4) National Institute for Biotechnology and Genetic Engineering (NIBGE), Faisalabad, PAKISTAN; (5) National Institute for Biotechnology and Genetic Engineering (NIBGE), FAISALABAD, PAKISTAN 
Geminiviruses infect several economically important crops that include cassava, corn, cotton, and tomato. Cotton leaf curl disease (CLCuD) is one of the major limiting factors for cotton production in Pakistan, caused by begomoviruses belonging to family Geminiviridae. CLCuD is predominantly caused by a complex that includes Cotton leaf curl Kokhran virus-Burewala (CLCuKoV-Bu) and a beta satellite molecule $\beta \mathrm{C} 1$ known as pathogenicity determinant. Yeast two hybrid assay (Y2H) was used to find cotton plant (Gossypium hirsutum) proteins interacting with $\beta \mathrm{C} 1 \mathrm{protein}$. In $\mathrm{Y} 2 \mathrm{H}, \mathrm{G}$. hirsutum SNF1-related kinase (GhSNF1), a key enzyme in energy metabolism and protection of plants against abiotic stresses, was found to interact with $\beta C 1$. In-silico study predicted four domains in GhSNF1, namely Conserved kinase domain (KD), Ubiquitin a associated domain (UBA), Autoinhibitory sequence domain and $\mathrm{C}$-terminal domain. Among these, the UBA domain preferentially interacted with the $\beta \mathrm{C} 1 \mathrm{protein}$. Y2H confirmed the computational predictions that the UBA domain among all of the domains helps GhSNF to interact with $\beta \mathrm{C} 1$ and further supports CLCuD to spread and cause infection. Furthermore, to study the in vivo protein-protein interaction of $\beta C 1$ and GhSNF1, full length genes and domain based mutants were cloned for studying bimolecular fluorescence complementation system and pull-down assay. These studies contributed to better understand the virus-host interactions at the molecular level.

Exploring the grapevine fanleaf virus RNA-dependent RNA polymerase-host protein interactome for insights into symptom development L. J. OSTERBAAN, M. Fuchs, Cornell University, Geneva, NY, USA

Grapevine fanleaf virus (GFLV) is one of the greatest viral threats to grapevine cultivation worldwide. GFLV-infected hosts exhibit diverse symptoms, yet the molecular mechanisms underpinning symptom development remain largely unknown. This bipartite positive-sense RNA virus requires both genomic RNAs for systemic infection, which can be established in the model species Nicotiana benthamiana by agroinoculation using infectious cDNA clones. Most GFLV strains are asymptomatic in $N$. benthamiana. An exception is GFLV strain GHu, which causes a systemic vein clearing. Symptoms of GFLV-GHu are associated with a region near the C-terminus of the RNA-dependent RNA polymerase (1E $\left.\mathrm{E}^{\mathrm{Pol}}\right)$. Using targeted mutagenesis, we recently showed that vein clearing symptom can be abolished, without affecting virus infectivity, by modifying a single codon in the $1 \mathrm{E}^{\mathrm{Pol}}$ coding region. Furthermore, we demonstrated that symptom development is dependent on amino acid identity, not nucleotide sequence, at this site. To further explain the mechanisms of symptom development, we hypothesized the existence of a specific GFLV-GHu- $N$. benthamiana protein interaction network. Mapping the $1 \mathrm{E}^{\mathrm{POI}}$-host protein interactome will provide insights into the molecular context of this protein central to GFLV replication and allow us to determine host interactants unique to GFLV-GHu, which may be associated with vein clearing symptom development in $N$. benthamiana .

Using Virulence Mutants to Identify Avr Genes in the wheat stem rust fungus, Puccinia graminis f. sp. tritici N. M. Upadhyaya (1), D. Ortiz (1), J. Chen (2), J. Ellis (1), F. Li (3), R. Mago (1), K. Kanyuka (4), M. Figueroa (5), R. F. Park (2), P. DODDS (1), (1) CSIRO Agriculture and Food, Canberra, AUSTRALIA; (2) Univ of Sydney Plant Breeding Institute, Cobbitty, AUSTRALIA; (3) University of Minnesota, St. Paul, MN, USA; (4) Rothamsted Research, Harpenden, UNITED KINGDOM; (5) Department of Plant Pathology, University of Minnesota, St. Paul, MN, USA

The wheat stem rust fungus Puccinia graminis f. sp. tritici (Pgt) is one of the most destructive pathogens of wheat. Resistance of host lines is often governed by recognition of fungal effector proteins (avirulence/virulence proteins) by plant resistance proteins (R proteins). We have taken a mutational genomics approach to identify Avr genes in Pgt. We isolated spontaneous mutants with virulence for $S r 50, S r 5, S r 27, S r 21$ or $S r 45$ by selection on resistant host lines. Sequence analysis of the $S r 50$ virulent mutant revealed that virulence resulted from the exchange of a whole chromosome between the two haploid nuclei of this dikaryotic organism, resulting in loss of the avirulence allele. This confirms the important role of somatic exchange events in virulence evolution in Pgt. The AvrSr50 gene was identified from the 25 candidate effector genes from this chromosome by transient co-expression with the cloned $\mathrm{Sr} 50$ gene in $\mathrm{N}$. benthamiana. AvrSr50 recognition was confirmed in wheat by viral expression. AvrSr 50 is expressed early during infection and is highly expressed in haustoria. Three mutants with virulence for $S r 27$ contain overlapping deletions and a single candidate gene for AvrSr 27 has been identified. Likewise, AvrSr 5 mutants contain large deletions spanning several candidate effector genes. New expression assays are being developed for confirmation of avirulence gene function in wheat.

\section{Development of an Arabidopsis - Pseudomonas syringae co-culture system to investigate mechanisms of plant immunity against bacterial pathogens \\ Q. YAN (1), J. Anderson (2), (1) Oregon state University, Corvallis, OR, USA; (2) Department of, Corvallis, OR, USA}

Plants recognize microbial features termed pathogen-associated molecular patterns (PAMPs) and activate defense responses termed pattern-triggered immunity (PTI). To overcome PTI, bacterial pathogens such as Pseudomonas syringae use a type III secretion system (T3SS) to deliver immunesuppressing effector proteins into host cells. Although PTI elicits massive changes in plant cell physiology, the exact mechanism(s) of how PTI limits bacterial growth is(are) largely unknown. Here we report the development of an infection system using Arabidopsis suspension cells and $P$. syringae that allows for well-defined analyses of signaling events between host and pathogen during infection. Using this co-culture system, we found that $P$. syringae grows to high levels in the presence of plant cells in a T3SS-dependent manner, and that this growth can be effectively suppressed by pre-treating the plant cells with PAMPs. To investigate the underlying mechanism of this growth inhibition, we performed a metabolomics analysis and found several compounds that are known inducers of T3SS genes were exuded in lower amounts by PAMP-treated plant cells. Furthermore, exogenous application of these metabolites effectively suppressed PAMP-mediated inhibition of $P$. syringae growth in this co-culture system. These data support the hypothesis that decreased metabolite release from defense-elicited plant cells is a contributing to limiting pathogen growth during PTI.

The $h r p X$ and $h r p G$ play important roles in virulence of Acidovorax citrulli, the causal agent of bacterial fruit blotch of cucurbits X. Zhang (1), M. Zhao (2), J. Yan (1), L. Yang (1), Y. Yang (1), W. Guan (1), R. R. Walcott PhD (2), T. ZHAO (1), (1) Institute of Plant Protection, CAAS, Beijing, CHINA; (2) The University of Georgia, Athens, GA, USA

Acidovorax citrulli causes bacterial fruit blotch, a global threat to watermelon and melon production. Despite its economic importance, relatively little is known about the molecular mechanisms of $A$. citrulli pathogenicity and virulence. Like other foliar plant pathogenic bacteria, $A$. citrulli relies on a type III secretion system (T3SS) for pathogenicity. Based on sequence analysis and operon arrangement, $A$. citrulli has a class II $h r p$ gene cluster, similar to those of Xanthomonas and Ralstonia spp. For class II $h r p$ clusters, hrpG and $h r p X$ play key roles in the regulation of T3S effectors (T3Es). However, little is known about the regulation of T3SS in A. citrulli. The objective of the study was to investigate the roles of $h r p G$ and $h r p X$ in $A$. citrulli. We found that when $h r p G$ or $h r p X$ was deleted in the A. citrulli group II strain Aac5, both mutants completely lost pathogenicity on watermelon seedlings, failed to induce a hypersensitive response on tobacco, and elicited higher levels of reactive oxygen species in Nicotiana benthamiana compared to the wildtype strain. We also demonstrated that HrpG activates HrpX in A. citrulli. Moreover, the expression of the T3E, Aave_2166 was suppressed in transcription and translation in $h r p G$ and $h r p X$ mutants. Notably, $h r p G$ and $h r p X$ appeared to modulate biofilm formation. These results suggest that $h r p G$ and $h r p X$ are essential for pathogenicity, regulation of T3Es, and biofilm formation in A. citrulli. 
The Xanthomonas genus contains a large group of plant pathogens, which cause severe diseases on many crops worldwide. The diffusible signal factor (DSF) - mediated quorum sensing (QS) regulatory system coordinates expression of virulence factors in these pathogens. However, the regulatory effects of this system during the Xanthomonas- plant interactions remain unclear from both the pathogen and host aspects. Here, we investigated the in planta DSF- mediated QS regulon of $X$. citri subsp. citri (Xac), the causal agent of citrus canker, and the transcriptional responses of citrus to DSF-mediated Xac infection using the dual RNA-seq approach. Comparative transcript profiles of Xac strain 306 and its DSF-deficient $(\Delta \mathrm{rpfF})$ mutant revealed that DSF- mediated QS specifically modulates bacterial adaptation, nutrition uptake and metabolisms, stress tolerance, virulence, and signal transduction to favor host infection. The transcriptional responses of citrus to DSF-mediated Xac infection are characterized by downregulation of photosynthesis genes and plant defense related genes. Alterations of plant hormone signaling pathways were also triggered by DSF-mediated Xac infection to benefit the pathogen. These findings suggest that the DSF/Rpf QS system modulates the citrus phenotypes to favor Xac infection. Collectively, our results provide new insight into the DSF- mediated QS regulation during plant-pathogen interactions and advance the understanding of traits used by Xanthomonas to adapt to the in planta environments.

TAL effector targets the abscisic acid biosynthesis pathway for disease susceptibility in bacterial leaf streak of wheat Z. PENG (1,2), Y. Hu (2), J. Zhang (1), S. Park (2), Z. Liu (3), S. Liu (2), F. F. White (1), (1) University of Florida, Gainesville, FL, USA; (2) Kansas State University, Manhattan, KS, USA; (3) North Dakota State University, Fargo, ND, USA

Plant susceptibility (S) genes are targeted by transcription activator-like (TAL) effectors in a variety of disease complexes caused by Xanthomonas species. Here, we show that the TAL effector Tal8 from X. translucens pv. undulosa (Xtu) XT4699 targets a novel S gene, encoding nine-cisepoxycarotenoid dioxygenase (NCED), to enhance susceptibility in bacterial leaf streak of wheat. The mutant M6 sustained the loss of tal8, resulting in reduced lesion expansion compared to the wild type (WT) strain. Transcriptional profiling of samples inoculated with M6 or WT revealed two candidate upregulated S genes, TaNCED and TaERF. Homeologs of TaNCED encode enzymes that catalyze the rate-limiting step in the biosynthesis of phytohormone abscisic acid (ABA), while homeologs of TaERF encode ethylene-responsive transcription factors. Expression of TaNCED and TaERF was associated with higher virulence of some Xtu strains but not with low virulence strains. The transformation of tal8 into the low virulence strain LW16 resulted in elevated expression of TaNCED and TaERF and enhanced virulence. Synthetic designer TAL effectors (dTALes) targeting TaNCED resulted in elevated ABA levels and enhanced virulence, while dTALes targeting TaERF were ineffective. Exogenous application of ABA onto plant leaves greatly promoted bacterial virulence. The results suggest that Xtu strains gain virulence by targeting ABA biosynthesis genes and elevating the ABA content of the infected tissue.

The calcium-dependent protein kinase OsCPK4 regulates a buffering mechanism that fine-tunes innate immunity in rice J. Wang (1), S. Wang (1), K. Hu (2), J. Yang (3), X. Xin (2), W. Zhou (1), J. Fan (4), F. Cui (1), B. Mou (1), S. Zhang (3), G. L. Wang (4), W. SUN (1), (1) Department of Plant Pathology, China Agricultural University, Beijing, CHINA; (2) College of Biological Sciences, China Agricultural University, Beijing, CHINA; (3) Rice Research Institute, Shandong Academy of Agricultural Science, Jinan, CHINA; (4) Department of Plant Pathology, Ohio State University, Columbus, OH, USA

The calcium-dependent protein kinase OsCPK4 has been demonstrated to play important roles in salt and drought tolerance, plant growth and development in rice. However, little is known on molecular mechanisms underlying OsCPK4 function in rice immunity. In this study, we demonstrated that the generation of oxidative burst and pathogenesis-related gene expression triggered by microbe-associated molecular patterns (MAMPs) were significantly enhanced in the oscpk4 mutants. These mutant lines are more resistant to bacterial blight and fungal blast diseases than the wild-type plants, indicating that OsCPK4 negatively regulates innate immunity in rice. OsCPK4 was further identified to interact with a receptor-like cytoplasmic kinase OsRLCK176. OsRLCK176 accumulation is negatively regulated by OsCPK4. Interestingly, the kinase-dead OsCPK4 promotes OsRLCK176 degradation more strongly than the wild-type protein. OsCPK4 and OsRLCK176 mutually phosphorylate each other and form a feedback loop. Moreover, the kinase activity and phosphorylation of OsCPK4 and OsRLCK176 contribute to the stability of OsRLCK176. These findings indicate that the kinase-inactive OsCPK4 promotes OsRLCK176 degradation and restricts plant defenses; whereas the activation of OsCPK4-OsRLCK176 phosphorylation circuit invalidates the OsRLCK176 degradation machinery and thus enhancing plant immunity. Collectively, the study proposes a novel defense buffering mechanism mediated by OsCPK4, which fine-tunes MAMP-triggered immunity in rice.

\title{
Identification and characterisation of in-planta expressed Zymoseptoria tritici effectors
}

\author{
S. J. KARKI, A. Feechan, University College Dublin, Dublin, IRELAND
}

Zymoseptoria tritici is an ascomycete fungus that causes Septoria Tritici Blotch, an important foliar disease of wheat which can cause up to $50 \%$ crop losses. Infection occurs through the stomata, followed by a latent period of 2-3 weeks where $Z$. tritici remains within the plant apoplast. The fungus then shifts to a necrotrophic phase, resulting in necrosis of the plant tissue corresponding with an increase in fungal biomass. $Z$. tritici is predicted to produce a series of small secreted proteins (SSPs) throughout its interaction with the host (Rudd et al., 2015). To understand the role of $Z$. tritici SSPs during infection and host colonisation, we mined publically available data and a second dataset was generated in the lab from $Z$. tritici infected wheat leaves. Using this information and the NCBI conserved domain search, we identified 50 SSPs with no homology to known proteins. 30 out of 50 SSPs were then cloned into overexpression vectors for transient expression in planta. In order to test these SSPs as potential effectors, the induction of cell death in the non-host model plant Nicotiana benthamiana was tested. We identified 6 prominent effector candidates that resulted in a cell death phenotype. These candidates will then be further characterised for their role in pathogenicity.

Transcriptome analysis of virulence-differentiated Fusarium oxysporum f. sp. cucumerinum during their colonization of cucumber X. LU, X. Huang, M. H. Sun, S. Li, Institute of Plant Protection, Chinese Academy of Agricultural Sciences, Beijing, CHINA

Fusarium oxysporum f. sp. cucumerinum (Foc) causes cucumber wilt worldwide. Previously, we found that the virulence of $F o c$ was significantly enhanced after 5 successive cycles of inoculation and re-isolation on a Foc-resistant cucumber cultivar. To understand its mechanism, a high coverage of RNA-Seq was obtained from cucumber roots at early (24 hours post inoculation, or hpi) and later (120 hpi) stages of infection by the original strain foc$3 \mathrm{~b}$ and its virulence-increased variant Ra-4, and from vegetative cultures grown on agar plates. Analysis of differentially expressed genes (DEG) induced in planta showed that at both 24 and $120 \mathrm{hpi}$, the number of DEGs of Ra-4 was greater than that of foc-3b, but the numbers of repressed DEGs were similar between the two types of strain, indicating that more genes were highly and rapidly expressed in the highly virulent strain when colonizing the 
host. Among these DEGs, 190 up-regulated and 360 down-regulated genes were overlapped between foc-3b and Ra-4, suggesting their apparent involvement in infection. Meanwhile, 286 and 366 DEGs, up-regulated at early and later stages respectively, were unique to Ra-4. These unique DEGs with a high potential for contribution to virulence enhancement, were mainly associated with secondary metabolism and signaling pathways by analysis of their protein characteristics. This result provides a critical resource for studying rapid adaption and evolution of Foc under host selection pressure.

\section{Identification of novel elicitors from Phytophthora parasitica}

T. Y. KE, C. C. Chen, C. C. Cho, R. F. Liou, Natl Taiwan Univ, Taipei, TAIWAN

In response to infection by the pathogens, plants display the first layer of plant immunity, pattern-triggered immunity (PTI), which is elicited upon recognition by plants of microbe-associated molecular patterns originated from elicitors (or apoplastic effectors). To elucidate the mechanisms underlying PTI, it is essential to identify novel elicitors and the corresponding patterns, and furthermore, to find out how these molecules trigger defense response. The aim of this study is to identify novel elicitors from Phytophthora parasitica, a notorious oomyceteous plant pathogen of a wide host range, with special interest in glycoside hydrolases (GHs). Search of the genome database of $P$. parasitica revealed the presence of a wide array of genes encoding different families of glycoside hydrolases. As a strategy for preliminary screening, we performed quantitative reverse transcriptase-PCR to analyze the expression profiles of candidate genes in the infection process of $P$. parasitica. Meanwhile, we assessed their elicitor activity by agroinfiltration for transient expression of proteins on Nicotiana benthamiana and Nicotiana tobacum (cv. Samsun-NN). Of the genes analyzed, G_05 and G_07, both contain a GH17 domain, cause severe yellowing symptom, and G_16 containing a GH16 domain causes necrosis on N. tobacum. Genes of $\mathrm{GH}_{1} 1$ encodes putative beta-1,3-glucanase, whereas the enzymes encoded by GH16 can be diverse. Further analysis by infiltration of recombinant proteins obtained from $E$. coli indicate that G_16 causes necrosis and reactive oxygen species (ROS) production on the leaves of $N$. tabacum. These results suggest that $\mathrm{G}_{-} 16$ of $P$. parasitica may encode an elicitor protein.

\section{Quorum sensing systems in Dickeya solani with different virulence levels}

M. POTRYKUS (1), F. Decorosi (2), C. Viti (2), A. Mengoni (3), N. Hugouvieux-Cotte-Pattat (4), E. Lojkowska (1), (1) Intercollegiate Faculty of Biotechnology University of Gdansk Medical University of Gdansk, Gdansk, POLAND; (2) Department of Agrifood Production and Environmental Sciences, University of Florence, Florence, ITALY; (3) Department of Biology, University of Florence, Sesto Fiorentino, ITALY; (4) UMR5240 Microbiologie Adaptation et Pathogénie, CNRS, Univ Claude Bernard Lyon 1, INSA Lyon, Villeurbanne, FRANCE

Dickeya solani is a highly homogenous species at the genomic level but single strains differ in their virulence level. Two quorum sensing (QS) mechanisms were described so far in Dickeya spp.: classic QS based on N-acyl-homoserine lactone (AHL) signals utilized by different microorganisms, and a specific Virulence Factor Modulating QS (VFM-QS) used exclusively by Dickeya genus. In this study, the influence of both QS on the D. solani virulence has been verified by mutagenesis. Single mutants inactivated in $v f m A, v f m E, v f m H, v f m I$ and $v f m K$ genes as well as $\operatorname{expI}$ and $\operatorname{expR}$ genes were constructed via transduction. Moreover, double mutants of both QS systems ( $\mathrm{vfmI} / E$-expI/R) were obtained. All mutants were evaluated for their ability to macerate plant tissue, pectate lyase, protease and cellulase activities, as well as swimming and swarming capacity. For some of the mutants, the difference in the phenotypes was verified with $\mathrm{Omnilog}^{\mathrm{R}}$ Phenotype Microarrays PM1-10 plates. In most $D$. solani strains, the expI/R mutations caused a decrease in chicory maceration, with a stronger effect for $\exp R$ than for $\exp I$. Similarly, all $D$. solani $v f m$ mutants showed an attenuation of chicory maceration as well as decreased protease and cellulase activity. No additive effect on tested features was observed in double mutants background. Thus, no synergy between VFM- and AHL-QS is observed in fine-tuning the production of different virulence factors and the ability to macerate plant tissue.

\section{Identification of putative PAMPs in Ralstonia solanacearum proteins using Tajima's D test} N. ECKSHTAIN-LEVI, A. J. Weisberg, B. A. Vinatzer, PPWS Department, Virginia Tech, Blacksburg, VA, USA

Host perception of pathogen-associated molecular patterns (PAMPs) constitutes the first layer of plant innate immunity with the best known PAMP being the flg22 epitope of flagellin. Plants unable to recognize PAMPs exhibit enhanced disease susceptibility. Therefore, bacterial pathogens have evolved several strategies to evade recognition. For example, Ralstonia solanacearum, the causal agent of bacterial wilt disease, has multiple non-synonymous mutations in the flg22 region that enable it to escape recognition by the pattern recognition receptor FLS2. To date, elf18, an epitope of elongation factor $\mathrm{Tu}(\mathrm{EF}-\mathrm{Tu})$, is the only known PAMP of $R$. solanacearum. In order to discover new PAMPs in this pathogen, we applied a population genetics test, Tajima's D, to the genome sequences of 37 R. solanacearum strains to search for genes that are under strong selective pressure. The screen identified 49 genes, including the one encoding EF-Tu, of the 3003 genes annotated in $R$. solanacearum. A gene encoding a cold shock protein, which contains a sequence corresponding to the Pseudomonas syringae PAMP csp22, was identified by the Tajima's D test and experimentally confirmed. $R$. solanacearum genes encoding the $P$. syringae PAMPs flg22 and xup25 were not identified in our screen and were confirmed not to be PAMPs. We conclude that identification of non-neutrally evolving genes using Tajima's D is a promising approach to identify novel PAMPs in bacterial plant pathogens.

\section{Role of type IV pili in biofilm formation and virulence of Xylophilus ampelinus}

Y. PETERSEN (1), N. Nyembe (1,2), (1) Agricultural Research Council, Stellenbosch, SOUTH AFRICA; (2) University of the Western Cape, Bellville, SOUTH AFRICA

Grapevine bacterial blight and necrosis caused by the bacterium Xylophilus ampelinus, has been a problem in South African vineyards for many decades. The disease reduces productivity and shortens the lifespan of infected vines. Although type IV pili (T4P) have been shown to be important in attachment, motility and biofilm formation for several plant and animal pathogens, this has not yet been explored for $X$. ampelinus. Therefore, we undertook a preliminary study to evaluate the importance of $X$. ampelinus T4P in virulence on grapevine. The study was conducted utilizing two mutants from a transposon mutant library with insertions in the genes, pilYl and pilQ. The presence of a single pilY1 homolog in the $X$. ampelinus genome was confirmed. In vitro biofilm formation on glass surfaces as well as twitching motility on $1 \%$ agar plates was impaired in both mutants. Assessment of virulence susceptible grapevine cultivar, Redglobe, showed that although the rate of symptom development and disease severity for the pilQ mutant was comparable to the wild type, plants inoculated with the pilY1 mutant developed symptoms faster than the wild type. However, symptom expression three months post-inoculation was comparable to the wild type. Both mutants were able to migrate up- and downstream from the point of inoculation on the shoots. Results from the in vitro assays confirmed the role of T4P in biofilm formation on abiotic surfaces and twitching motility. The in planta results, however, are contrary to those for vascular plant pathogens Xylella fastidiosa and Acidovorax citrulli. Further examination of the role of T4P in the in planta biofilm formation is currently under way. 
Erwinia amylovora causes the devastating fire blight disease of apple and pear trees by successfully colonizing and infecting through coordinated regulation of attack and defense strategies. E. amylovora uses flagellar motility to migrate to susceptible host tissues, produces exopolysaccharides to build large protected populations, and translocates effectors via a type III secretion system to manipulate host cells. Each of these virulence factors is regulated by the Hfq-dependent small RNA ArcZ, and we have shown that ArcZ positively regulates flagellar motility. However, ArcZ negatively regulates translation of the flagellar master regulator $f l h D$ through a direct interaction. In order to clarify this contradiction and to identify additional regulators in this pathway, we conducted a transposon mutagenesis in an $\operatorname{arc} Z$ mutant background and screened 18,000 mutants for reversion to wildtype motility. This screen identified the leucine-responsive regulatory protein (Lrp) as a major regulator of flagellar motility epistatic to ArcZ. RNAseq transcriptomic analysis of the $\operatorname{arc} Z$ mutant confirmed that Lrp is a part of the ArcZ regulon. Virulence testing indicated that Lrp not only regulates flagellar motility but also production of the exopolysaccharides amylovoran and levan. These findings identify Lrp as a novel virulence regulator in $E$. amylovora, improve current understanding of flagellar regulation, and further elucidate the role of small RNAs in E. amylovora virulence.

Type II toxin-antitoxin systems are essential for the survival of Erwinia amylovora under lethal stress conditions T. SHIDORE, Q. Zeng, L. R. Triplett, Department of Plant Pathology and Ecology, The Connecticut Agricultural Experiment Station, New Haven, CT, USA

Toxin-Antitoxin systems (TAs) are ubiquitous bacterial self-killing systems comprised of an antibacterial toxin and a neutralizing antitoxin. These multifunctional systems are involved in plasmid maintenance, virulence, biofilm formation, persistence to antibiotics and other stresses. The majority of known systems are "type II" TAs, comprising of protein toxins that are suppressed by protein antitoxins. While many studies have highlighted the significance of TAs in human pathogens, not much is known about their functions in plant-associated bacteria. Erwinia amylovora is the causal agent of fire blight, a devastating disease of pears and apples. Genomic analysis identifies 9 putative type II TAs in E. amylovora strain CTBT3-1. We generated deletion mutants for six of these TAs and assessed their rate of survival upon prolonged log-phase exposure to streptomycin, a key antibiotic used in management of fire blight. Survival rates of individual knockout mutants were compared to the wild type using antibiotic survival assay. Deletion of the cytoskeleton-binding TAs yeeUV significantly reduced survival to streptomycin despite having no growth defects, suggesting a contribution to persistence to the antibiotic. Effects of either deletion or overexpression of the six TAs on virulence, biofilm formation, and survival to other stresses are also being evaluated. This study demonstrates that TAs may impact the efficacy of bacterial disease management strategies.

XopJ6, a new member of the XopJ family of type III effectors, in Xanthomonas perforans

F. IRUEGAS-BOCARDO (1), P. Abrahamian (2), G. V. Minsavage Jr. (1), N. Potnis (3), G. E. Vallad (2), J. B. Jones (1), E. M. Goss (1), (1) Department of Plant Pathology, University of Florida, Gainesville, FL, USA; (2) Gulf Coast Research and Education Center, University of Florida, Wimauma, FL, USA; (3) Department of Entomology and Plant Pathology, Auburn University, Auburn, AL, USA

Bacterial leaf spot on pepper and tomato is caused by four Xanthomonas species. Of these, X. perforans $(X p)$ is typically a specific pathogen of tomato. The type III secretion effector AvrBsT (XopJ2) belongs to the XopJ effector family. It induces a hypersensitive response (HR) in pepper, but behaves as a virulence gene in tomato, compromising its defense responses. We describe a new member of the XopJ effector family, XopJ6. An homology analysis using HMMER showed that this gene belongs to the serine/threonine acetyltransferase XopJ effector family, and contains its signature catalytic core (His, Glu and Cys), required to induce HR in resistant plants. A tBLASTn analysis revealed its presence in 14 out of 137 sequenced strains collected from Florida, China, Thailand and Nigeria, all belonging to a unique phylogenetic lineage. XopJ6 is closely related to AvrBsT, sharing $71.5 \%$ sequence identity, but a genomic context analysis revealed that it is located at different locus. We demonstrated that XopJ6 induces an hypersensitive response in pepper after cloning the gene into pepper pathogenic X. euvesicatoria strain 85-10. Our results provide further evidence that the XopJ family of effectors are important virulence and host-range delimiting factors in $X p$.

Molecular mechanisms of mutation to virulence in Leptosphaeria maculans populations in the UK L. H. GAJULA, G. Mitrousia, B. Fitt, Y. Huang, University of Hertfordshire, Hatfield, UNITED KINGDOM

Leptosphaeria maculans, the cause of phoma stem canker disease of oilseed rape, develops gene-for-gene interactions with its host plant resistance genes. Pathogens develop new effector proteins to overcome recognition by the host and plants evolve new recognition proteins to target novel $A v r$ proteins. In this study, the regional distribution of the L. maculans races in the UK was monitored and the molecular mechanisms of mutation to virulence were investigated. Field experiment sites were set up at different sites in the UK: from leaf spot lesions on Drakkar (susceptible cultivar, trap crop) and other cultivars (with $\operatorname{Rlm} 7$ resistance gene), 64 and 88 L. maculans isolates, were obtained in the 2015/2016 and 2016/2017 cropping seasons, respectively. Changes in frequencies of avirulent $A v r L m 1, A v r L m 4$ or $A v r L m 7$ alleles were investigated by testing isolates on cotyledons of a differential set of cultivars. Isolates virulent towards $R \operatorname{lm} 1, R \operatorname{lm} 4$ or $\operatorname{Rlm} 7$ were investigated for molecular events. There were variations in the frequencies of avirulent $A v r L m 1$ and $A v r L m 4$ alleles between cropping seasons. All the isolates from different sites were avirulent against $R \operatorname{lm} 7$ in the $2015 / 2016$ season. In the 2016/2017 season, $6.8 \%$ of isolates were virulent towards $R \operatorname{lm} 7$. The molecular mechanism of mutation to virulence in $A v r L m 1$ was observed to be whole gene deletion in $86 \%$ of isolates. The other $13 \%$ were sequenced to investigate events leading to virulence. Whole gene deletion was observed in $6 \%$ or $50 \%$ of isolates carrying the virulent alleles of $A v r L m 4$ or $A v r L m 7$, respectively. The others need to be sequenced for further investigation.

Hypoxia tolerance is a virulence component in the colonization of maize seeds by Aspergillus flavus

S. CHALIVENDRA (1,2), P. K. Chang (3), K. E. Damann (4), (1) Louisiana State University, Baton Rouge, LA, USA; (2) Louisiana State UniversityAgCenter, Baton Rouge, LA, USA; (3) Southern Regional Research Center, USDA-ARS, NEW ORLEANS, LA, USA; (4) Louisiana State University AgCenter, Baton Rouge, LA, USA

Aspergillus flavus is an opportunistic pathogen that contaminates corn and key oilseed crops with carcinogenic aflatoxins (AFs). As an obligate aerobe, A. flavus is severely limited in its growth, sporulation and $\mathrm{AF}$ synthesis under hypoxia and becomes dormant under anoxia when grown in vitro. Yet, the fungus successfully invades the severely hypoxic corn seeds $\left(3 \% \mathrm{O}_{2}\right.$ in the embryo and $\sim 0 \%$ in the endosperm) and produces abundant $\mathrm{AF}$, implicating hypoxia tolerance as a key virulence factor. Understanding the basis of this adaptability is important to identify new targets for limiting AFcontamination of the food chain. My presentation will focus on the role of MedA, a transcription factor, in hypoxic tolerance. A medA deletion mutant in A. flavus showed fewer conidia as expected from MedA's role in conidiogenesis. The mutant also made less AF than the wild type but showed superior growth in vitro and on intact corn seeds. The greater vegetative growth of the mutant was correlated with impaired biofilm formation and in turn, enhanced fungicide sensitivity under hypoxia. This suggests a role for MedA in biofilm formation, a hypoxic structure that protects the fungus from adverse conditions including antifungals and anoxic injury, and is consistent with a report that $A$. flavus forms biofilm at the surface of anoxic seed tissues only in a resistant hybrid. Based on these new insights, we are exploring potent host-induced gene silencing strategies for AF-mitigation in corn. 
hok-sok toxin-antitoxin system plays important roles in morphological plasticity, bacterial persistence, and catalase activity in Erwinia amylovora

J. PENG (1), L. R. Triplett (2), G. W. Sundin (1), (1) Michigan State University, East Lansing, MI, USA; (2) Department of Plant Pathology and Ecology, The Connecticut Agricultural Experiment Station, New Haven, CT, USA

Erwinia amylovora is a plant pathogenic bacterium that causes devastating fire blight disease on apple, pear and cherry. Recently, we identified a chromosomally-encoded hok-sok toxin-antitoxin system in E. amylovora Ea1189. The ectopic expression of hok in E. amylovora is highly toxic and turns a subpopulation of cells into the viable but non-culturable (VBNC) state. Overexpression of small RNA sok partially reversed the toxicity of hok by indirectly inhibiting the translation of mok, a modulator gene of hok. A hok-sok knockout mutant, $\Delta$ hok-sok, exhibited slower exponential growth than the WT. Using scanning electron microscopy, we observed that subpopulations of the $\Delta$ hok-sok mutant had switched to filamentous growth with the longest cell observed measuring $42 \mathrm{~nm}$ in length compared to $2 \mathrm{~nm}$ in the WT. The hok-sok locus appears to be important for bacterial persistence. In both the WT and an obg (a GTPase gene that is important for bacterial persistence in E. coli) overexpression background, the $\Delta h o k$-sok strain exhibited a greatly reduced number of persister cells under several antibiotics tested. $\Delta$ hok-sok also had significantly-impaired catalase activity, and we are currently investigating the hok-sok locus-linked crosstalk between catalase activity and persister cell formation. Taken together, E. amylovora retains the chromosomal hok-sok locus, a potentially self-toxic system, in exchange for advantages that could affect environmental fitness.

The role of TAL effectors in virulence of Xanthomonas campestris pv. campestris

Z. DUBROW (1), H. W. Lange (2), C. D. Smart (3), A. J. Bogdanove (1), (1) Cornell University, Ithaca, NY, USA; (2) SIPS, Cornell University, Geneva, NY, USA; (3) Plant Pathology and Plant-Microbe Biology Section, Cornell University, Geneva, NY, USA

Xanthomonas campestris pv. campestris $(X c c)$, the causal agent of black rot of crucifers, is one of the most important Brassica pathogens worldwide. Transcription activator-like effectors (TALEs) are a large family of type III secreted effectors present in some Xanthomonas species that play a role in bacterial colonization of host plants. The first four Xcc genomes published showed no evidence of TALEs, suggesting Xcc lacked them. However, more recent work and reclassification of Xanthomonas campestris pathovars have shown that some Xcc strains do contain TALEs. We used PCR and western and Southern blotting to survey $124 \mathrm{Xcc}$ isolates from a 10-year New York State collection and found 30\% of the isolates have TALE genes and that the TALEs are expressed. The New York Xcc isolates have different TALE repertoires even among isolates found by MLSA to be closely related. To understand the role of TALEs in black rot of crucifers, we are sequencing the Xcc TALEs and determining by targeted mutagenesis whether they contribute to $X c c$ virulence. We will identify susceptibility (S) genes upregulated by important TALEs. These data could inform targeted resistance breeding approaches for cruciferous vegetables.

Validation of predicted miRNAs in Phytophthora sojae and Phytophthora infestans C. Madison, M. D. OSPINA-GIRALDO, Lafayette College, Easton, PA, USA

The post-transcriptional regulatory environment in the oomycetes Phytophthora infestans and $P$. sojae is poorly understood and the expression of microRNAs (miRNAs), and their potential roles in infection, is a topic of increasing interest. We have attempted to experimentally verify the existence of several miRNAs from both $P$. infestans and $P$. sojae, which had been predicted using in silico approaches. In addition, we investigated the possibility of these miRNAs playing a role in Phytophthora pathogenicity. The presence of two of the five miRNAs, namely psj-miR8788 (predicted based on its stem-loop structure and sequence conservation between $P$. sojae, $P$. ramorum, and $P$. infestans) and PimiRNA3, predicted in $P$. infestans, was experimentally confirmed in vivo by amplification using specific stem-loop primers and subsequent cloning and sequencing. The predicted targets of psjmiR8788 include members of the amino acid/auxin permease family, which serve amino acid transport functions, while the targets for PimiRNA3 include a diverse group of proteins, some of them with enzymatic activity. We also measured the transcriptional activity of psj-miR8788 during infection of soybean plants and determined that psj-miR8788 is upregulated mostly at the earlier stages of infection. This suggests that psj-miR8788 is important during the biotrophic stage of infection, and perhaps plays a role in suppressing plant defense systems by modulating amino acid transport systems in the plant.

The Ustilago maydis transcription factor, Zfp1 influences pathogenic development through the control of effector gene expression H. Y. K. Cheung, M. Donaldson, K. Spence, J. Fetsch, M. Harrison, B. SAVILLE, Trent University, Peterborough, ON, CANADA

Control of the pathogenic/sexual cycle in the corn smut pathogen Ustilago maydis was investigated through functional characterization of meiosis gene orthologs. $z f p 1$, encoding a $\mathrm{Zn}(\mathrm{II})_{2} \mathrm{Cys}_{6}$ transcription factor, was identified as an ortholog to Saccharomyces cerevisiae Ume6. Deleting zfp 1 in compatible haploid U. maydis strains did not affect meiosis, but altered pathogenesis. Deletion in a solopathogenic strain, SG200, reduced anthocyanin production, inhibited in planta growth, and blocked the progression of pathogenesis. Zfp1 localizes to the nucleus and comparative transcriptome

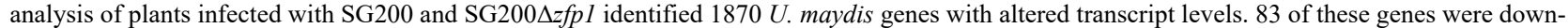
regulated effectors, including tin 2 which induces anthocyanin production during infection. The sub-cellular location, pattern of transcript level changes and results of complementation experiments support a hypothesis that Zfp1 differentially controls effector gene expression by interacting with distinct binding partners. Transcription factor gene transcript levels were also altered in SG200 $\Delta z f p 1$ suggesting Zfp1 is part of a regulatory cascade. GO analysis of Zea mays genes that were differentially represented in SG200 $\Delta z f p 1$ infections, identified plant processes potentially influenced by Zfp1 controlled $U$. maydis effectors. This study advances the molecular knowledge of smut pathogenesis to aid control measure development.

\author{
A fliC flagellin mutant of Pseudomonas syringae effectorless polymutant DC3000D36E reveals novel death elicitation activity in Nicotiana \\ benthamiana \\ W. ZHANG (1), A. Collmer (1), H. L. Wei (2), (1) Cornell University, Ithaca, NY, USA; (2) Chinese Academy of Agricultural Sciences, Beijing, \\ CHINA
}

Pseudomonas syringae pv. tomato DC3000 injects multiple effector proteins into plant cells via the type III secretion system (T3SS) to cause bacterial speck disease in Arabidopsis, tomato, and Nicotiana benthamiana (if $\Delta$ hopQ1-1). In N. benthamiana, effectorless polymutant DC3000D36E grows 4 logs less than DC3000 4 hopQ1-1, fails to trigger cell death, and displays fliC (flagellin)-dependent elicitation of a 15-h post-inoculation reactive oxygen species (ROS) burst that is associated with pattern-triggered immunity (PTI). In contrast, DC3000D36E $\Delta$ fliC loses ROS elicitation activity and triggers rapid cell death in $N$. benthamiana. A T3SS-deficient derivative of DC3000D36E $\Delta$ fliC does not elicit cell death under the same conditions. These observations suggest that plant cell death may be triggered by one or more T3SS machinery components but is suppressed by flagellin-triggered PTI. AvrPtoB $_{\mathrm{M} 3}$, a derivative of a key DC3000 effector that has site-specific mutations disrupting its E3 ubiquitin ligase domain and the two known domains 
for interacting with immunity-associated kinases, is a suppressor of effector-triggered immunity elicited by certain effectors. AvrPtoB $\mathrm{B}_{\mathrm{M} 3}$ also suppresses death elicitation by DC3000D36E $\Delta f l i C$ in $N$. benthamiana. Ongoing experiments are exploring the basis for death elicitation by DC3000D36E $\Delta f l i C$ and its suppression by flagellin and $\mathrm{AvrPtoB}_{\mathrm{M} 3}$ in this minimalized pathosystem that features native deployment and interplay of core interaction factors.

\section{Dual dissection of fungi effectors and plant susceptibility factors reveals new candidate genes involved in the wheat/Fusarium graminearum} interaction

L. BONHOMME (1,2), F. Fabre (1,2), M. Vignassa (1,2), T. Langin (1,2), (1) University of Clermont Auvergne, clermont ferrand, FRANCE; (2) INRA, clermont ferrand, FRANCE

Coordinated actions of microbial effectors and host-susceptibility factors are key determinants of disease development, promoting pathogen growth and spreading in plant tissues. In the wheat/Fusarium graminearum (Fg) interaction determining the Fusarium head blight (FHB) disease, these primary players of plant susceptibility are still widely unknown despite their great potential to guide original breeding programs. $F g$ effectors and host susceptibility factors were investigated using high resolution dual proteomics in the grains of a susceptible wheat cultivar and over four early FHB infection stages (24 to $96 \mathrm{hpi}$ ). The abundance of about 5,800 wheat proteins were profiled along with that of nearly $1,100 \mathrm{Fg}$ proteins including more than 100 predicted effectors according to EffectorP. The amounts of about 1,000 wheat proteins were significantly regulated during early stages of FHB and the abundance of nearly $200 \mathrm{Fg}$ proteins, including 9 effectors, was changed independently of the fungal biomass progress. Interestingly, mRNA quantity did not correlate with the abundance of part of the effectors suggesting their earlier accumulation during spore development. A co-occurring molecular remodeling was evidenced in both wheat and $F g$ between $48 \mathrm{hpi}$ and $72 \mathrm{hpi}$ depicting two distinct steps. These were characterized by specific molecular events shaping both initial infection and $F g$ spreading in plant tissues evidencing putative susceptibility factors.

A genomic island carrying a type III effector enters stealth mode in a pathogen population infecting a resistant plant H. Neale (1), R. Laister (1), J. Payne (1), G. M. Preston (2), R. W. JACKSON (3), D. L. Arnold (1), (1) University of the West of England Bristol, Bristol, UNITED KINGDOM; (2) Oxford Univ, Oxford, UNITED KINGDOM; (3) University of Reading, Reading, UNITED KINGDOM

Bacterial pathogens evolve to overcome host resistance by different means. We have discovered that a Pseudomonas syringae pv. phaseolicola (Pph) bean pathogen carrying a genomic island encoding the type III effector $a v r P p h B$ which triggers hypersensitive response (HR) immunity in a resistant bean plant, can evade the resistance response. This happens initially by the island excising from the chromosome to become a supercoiled episome that results in the downregulation of $a v r P p h B$. The pathogen then evolves over time by losing the genomic island from the cells - but this does not lead to island extinction because a sub-population ( $0.5 \%$ of the population) of cells with the island co-exist in a stealth mode with the dominant islandless population. We have developed a mathematical model to predict if the genomic island PPHGI-1 would be maintained in the population. We empirically tested the predictions made by the model and determined that PPHGI-1 frequency in the bacterial population drops during the HR. The island is then stably maintained in approximately $0.5 \%$ of the population over the long term. However, when a population of $P p h$ that contains $0.5 \%$ cells carrying PPHGI-1 is inoculated into a bean cultivar that does not produce the HR, the proportion carrying PPGHI-1 increases rapidly suggesting that PPHGI-1 confers a fitness benefit. These results provide insights into the long term population dynamics of mobile genetic elements influencing pathogen evolution.

Validation of a conserved effector associated with avirulence on Harbin and Tifang barley N. WYATT (1), J. Richards (2), R. S. Brueggeman (3), T. L. Friesen (4), (1) North Dakota State University, Fargo, ND, USA; (2) Plant Pathology Department, North Dakota State University, Fargo, ND, USA; (3) Department of Plant Pathology, North Dakota State University, Fargo, ND, USA; (4) USDA-ARS, Cereal Crops Research Unit, Northern Crop Science Lab, Fargo, ND, USA

Net form net blotch (NFNB) is a foliar disease of barley caused by the fungal pathogen Pyrenophora teres f. teres. Although economically important, little is known of the molecular mechanisms involved in this host-pathogen interaction. $P$. teres f. teres bi-parental mapping populations have been used to investigate the avirulence/virulence loci contributing to NFNB disease. These loci commonly harbor small secreted proteins (SSPs) that have been shown in many systems to be effectors used to manipulate the host defense. The pathogen population $15 \mathrm{~A} \times 0-1$ was used to map a genetic locus (AvrHar) associated with avirulence on Harbin and Tifang barley with avirulence being contributed by isolate 15A. Here, using a genome wide association study approach (GWAS) with a $P$. teres f. teres global population, we identified a strong marker trait association with avirulence on Tifang barley that maps to the same AvrHar region. The strongest associated SNP marker is located within the 5'UTR of a small secreted protein (SSP). Comparative analysis of this SSP between 15A and 0-1 revealed a six bp in-frame indel that results in the insertion of the amino acids alanine and aspartic acid in the virulent $0-1$ isolate. Functional validation of this effector gene via split marker gene knock out, gain-of-function transformation, and heterologous expression will be presented.

Candidate effector gene of spot form net blotch identification using genetic mapping and whole genome sequencing S. CLARE, North Dokata State University, Fargo, ND, USA

Net blotch is a destructive necrotrophic foliar pathogen of barley and occurs as net and spot forms caused by Pyrenophora teres f. teres and $P$. teres $\mathrm{f}$. maculata (Ptm), respectively. Recently, Ptm has become prevalent in the Northern Great Plains, USA. A 118-progeny bi-parental mapping population was developed from the virulent Montana isolate 'PA14' and the avirulent California isolate 'CAWB' (collected from wild barley) to investigate the Ptm-barley interaction. Progeny isolates were phenotyped on a differential set of barley lines and genotyped using RAD-GBS. Genetic maps were developed for QTL analysis identifying a total of four QTL across three of the 12 linkage groups. One locus mapped to an $11.9 \mathrm{cM}$ genetic interval and accounted for 21-39\% (LOD 7.0-18.9) of the phenotypic variation. A reference quality sequence was assembled for North Dakota isolate 'FGO' using PacBio sequencing. Analysis of the $11.9 \mathrm{cM}$ interval in the reference isolate revealed a $113.5 \mathrm{~kb}$ region containing 34 genes. Five of the 34 genes were predicted to contain a secretion signal, of which only two were under 250 amino acids. Only one of the genes was expressed and is therefore the top candidate effector gene. This locus has been identified in two additional mapping populations, with parents contributing virulence all harboring an identical candidate gene sequence to 'PA14'. Validation of this gene utilizing gain- and loss-of-function gene transformation is in progress.

Xylella fastidiosa requires Type II-secreted endoglucanases for virulence in grapevine B. M. INGEL, M. C. Roper, University of California, Riverside, Riverside, CA, USA

The ability of Xylella fastidiosa $(X f)$, the causal agent of Pierce's Disease in grapevine, to systemically colonize the xylem directly correlates with symptom development. $X f$ facilitates its colonization of the xylem by dismantling vessel pit membranes, the components of which are substrates for $X f$ 's cell wall-degrading enzymes (CWDEs). $X f$ utilizes at least one polygalacturonase (PG) and one endoglucanase (EGase) to breach pit membranes that 
separate one xylem vessel element from another. We have assessed the roles of two EGases in $X f$ in both their individual and collective roles in virulence and determined that these EGases act as additive virulence factors during the grapevine infection process. Furthermore, a proteomic analysis of the $X f$ secretome indicates that these two EGases are secreted via the Type II secretion system. Taken together, these data indicate that EGases are critical to the $X f$ infection dynamics in planta.

Genomic Analyses Reveal Localized Effector Diversification and Candidate SnTox 5 in Parastagonospora nodorum J. RICHARDS (1), J. Carpenter (2), Z. Liu (2), J. D. Faris (3), T. L. Friesen (4), (1) Plant Pathology Department, North Dakota State University, Fargo, ND, USA; (2) North Dakota State University, Fargo, ND, USA; (3) USDA-ARS, Fargo, ND, USA; (4) USDA-ARS, Cereal Crops Research Unit, Northern Crop Science Lab, Fargo, ND, USA

Parastagonospora nodorum, a necrotrophic fungal pathogen of wheat, utilizes an array of effectors to intentionally elicit programmed cell death. These molecules are typically small secreted proteins (SSPs), may be cysteine-rich, and lack conserved domains or homology with other organisms. Eight $P$. nodorum-wheat interactions have been identified and three effectors have been cloned, including SnToxA, SnTox1, and SnTox3. However, little is known of the genic diversity of potential effectors within populations in the USA and the effectors underlying previously identified interactions remain to be cloned. Genome sequencing of 175 P. nodorum isolates led to the identification of 322,613 SNPs/INDELs, including 132,650 nonsynonymous SNPs. Results show the population-specific accumulation of nonsynonymous SNPs in effector candidates, with 24 and 15 genes from the Midwest and South/East populations, respectively, containing more than two nonsynonymous SNPs/kb. Additionally, $48 \mathrm{SSPs}$ exhibited presence/absence variation (PAV) throughout the entire population. GWAS using SNP/INDEL/PAV markers identified a SSP with PAV strongly associated with virulence on the SnTox 5 differential line LP29 and durum wheat line Scoop1. This gene is currently being validated via heterologous expression and gene-disruption transformants. Results of this research illustrate the population-specific diversity present in effector candidates and the utility of pathogen GWAS for effector identification.

Screening candidate effectors from Botryosphaeria dothidea

C. J. Zhang, S. X. Wang, B. Z. Dong, S. H. Wen, X. ZHU, L. Guo, China Agricultural University, Beijing, CHINA

Botryosphaeria dothidea is an ascomycetous fungus causing apple white rot, which is a serious disease on apple in China. The genome of $B$. dothidea was sequenced and predicted to contain about 320 effector genes with less than 300 amino-acid in size and more than four cysteine residues. Fungal effectors often play essential roles in host-pathogen interactions. However, little is known about the functions of $B$. dothidea effectors. Among the selected 100 candidate effectors in this study, 30 candidate effectors (without signal peptide regions) were successfully cloned into PVX vector pGR107 and introduced into Agrobacteria tumefaciens strain GV3101 by electroporation. Potential effects of the candidate effector genes on cell death were examined using the BAX system. Candidate effector was expressed in agro-infiltrated leaves of Nicotiana benthamiana. At $24 \mathrm{~h}$ after the initial inoculation, the infiltrated leaves were challenged with A. tumefaciens cells carrying the gene of the BAX. In total, 22 candidate effectors completely inhibited programmed cell death (PCD) of $N$. benthamiana induced by the BAX gene. Expression patterns of four effectors analyzed by Reverse Transcript-quantitative PCR showed all were highly expressed from 36 to $72 \mathrm{~h}$ post inoculation in apple fruit. The secretional signal peptides were tested in yeast system (pSUC2 vector) and found that 10 of 12 effectors showed secretory activity. These results suggested that candidate effectors of $B$. dothidea might play important roles in its interaction with apple.

GWAS-based analysis of quantitative traits in Ceratocystis albifundus

M. VAN DER NEST, N. Soal, E. Steenkamp, A. Chan, A. Fourie, A. McTaggart, L. de Vos, B. Swalarsk-Parry, M. J. Wingfield, B. D. Wingfield, Forestry and Agricultural Biotechnology Institute (FABI), University of Pretoria, Pretoria, SOUTH AFRICA

Ceratocystis albifundus is an important pathogen of commercially propagated Acacia mearnsii and Protea cynaroides in South Africa. It also infects wounds on a wide range of native African tree species without causing disease. Previous work has shown that C. albifundus exhibits quantitative traits (i.e. growth rate, laccase activity and virulence) likely involved in its ability to infect and cause disease on its plant hosts. In this study, we identified polymorphisms and genes associated with these quantitative traits by employing a genome-wide association study (GWAS). For this purpose, 36 genetically diverse C. albifundus isolates, originating from a wide range of host species and geographic regions, were used. The isolates were phenotyped using growth rate measurements on potato dextrose agar medium, alpha-napthol-based laccase assays, and pathogenicity tests on $A$. mearnsii seedlings. To determine their genotypes, isolates were subjected to low-coverage genome sequencing using Ion Torrent technology. These sequences were mapped to a reference genome, which was generated with the PacBio and Illumina sequencing platforms. Correlation analysis between genotypes and the respective phenotypes allowed identification of genomic regions that are significantly associated with the three quantitative traits examined. Future characterization of these genomic regions will reveal their role in growth rate, laccase activity, virulence and the overall biology of $C$. albifundus.

F-box like motif of the brassica yellows virus silencing suppressor P0 protein facilitates its stability in vivo Y. LI (1), Q. Sun (1), T. Zhao (1), H. Xiang (1,2), X. Y. Zhang (1), Z. Wu (1), D. Li (1), J. Yu (1), X. Wang (1), Y. Zhang (1), Y. Wang (1), S. P. Dinesh-Kumar (3), C. Han (1), (1) China Agricultural University, Beijing, CHINA; (2) Yunnan Academy of Tobacco Science, Kunming, CHINA; (3) University of California, Davis, Davis, CA, USA

Several studies have shown that P0 of some poleroviruses can target Argonaute 1 (AGO1) to suppress RNA silencing. Although P0 harbor F-box like motif reported to be essential for RNA silencing suppression activity, it is the autophagy pathway that was shown to contribute to the AGO1 degradation. Therefore, the role of P0 F-box like motif in silencing suppression remains unclear. Here, global mutagenesis and comparative functional analysis of the $\mathrm{P} 0$ encoded by Brassica yellows virus genotype $\mathrm{A}\left(\mathrm{P} 0^{\mathrm{BrA}}\right.$ ) revealed different residues within $\mathrm{P}^{\mathrm{BrA}}$ are required for local and systemic silencing suppression activities. Furthermore, $\mathrm{P} 0^{\mathrm{BrA}}$-mediated destabilization of $\mathrm{AGO} 1$ is only required for the suppression of local but not systemic silencing. Remarkably, the F-box like motif mutant of $\mathrm{P}^{\mathrm{BrA}}$ is destabilized in vivo. Inhibitor treatment assay indicated involvement of both $26 \mathrm{~S}$ proteasome system and autophagy pathway in this process. Genetic analyses indicated that, although $\mathrm{P} 0^{\mathrm{BrA}} \mathrm{F}$-box like motif is required for its interaction with SKP1, it is not required for its silencing suppression activity. Therefore, the $\mathrm{F}$-box like motif is required for the stability of $\mathrm{P}^{\mathrm{BrA}}$ protein to assure efficient $\mathrm{RNA}$ silencing suppression. Consistent with these findings, efficient systemic infection of $\mathrm{BrYV}$ requires $\mathrm{P} 0^{\mathrm{BrA}}$. Results presented here provide a novel insight into the polerovirus P0 protein stability and RNA silencing suppression activities and the function of P0 F-box like motif.

Virulence of Fusarium oxysporum f. sp. cucumerinum is affected by its successive generations on resistant and susceptible cucumber cultivars X. Huang, M. H. Sun, X. Lu, S. LI, Institute of Plant Protection, Chinese Academy of Agricultural Sciences, Beijing, CHINA 
Fusarium oxysporum f. sp. cucumerinum $(F o c)$ is the causal pathogen of cucumber Fusarium wilt resulting in losses to cucumber production. To investigate the effects of the selective pressures of host plants on the virulence of $F o c$, we successively inoculated foc- $3 \mathrm{~b}$, a low virulence isolate, on resistant and susceptible cucumber cultivars for five generations. Pathogenecity tests showed that virulence of the original isolate diverged after serial passage. The virulence was significantly strengthened on the resistant cultivar and weakened on the susceptible plants $(P<0.05)$. The expression of four virulence-related genes of $F$. oxysporum, G-protein a subunit gene $f g a l$, sucrose non-fermenting 1 gene snfl, F-box protein gene $f r p l$, and Class $\mathrm{V}$ chitin synthase gene $c h s V$, was detected using real-time PCR. All 4 genes were significantly up-regulated after serial passage on the resistant cultivar, compared to the original strain, and the expression of snf1 was down-regulated in strains re-isolated from the susceptible plants $(P<0.05)$. A significant positive correlation was found between the expression levels of gene snfl, frpl and chs $V$ and disease severity of cucumber Fusarium wilt, suggesting these genes may impact virulence differentiation. This study will improve the management of cucumber Fusarium wilt and provide insight into the mechanisms underlying virulence of $F$. oxysporum.

Hfq is important for biofilm formation, motility and pathogenicity of the plant pathogen Pantoea ananatis G. Y. SHIN (1), D. Shyntum (2), L. Moleleki (2), T. A. Coutinho (1), (1) Centre for Microbial Ecology and Genomics, University of Pretoria, Pretoria, SOUTH AFRICA; (2) Department of Microbiology and Plant Pathology, University of Pretoria, Pretoria, SOUTH AFRICA

Bacterial phytopathogens constantly encounter changing environmental conditions. To ensure their survival and persistence, they rapidly respond to a change by modulating gene expression, resulting in an altered phenotype. The RNA chaperone Hfq is the key protein that controls the gene expression at the post-transcriptional level through mediating a regulatory interaction between the small RNA with its target messenger RNA. The outcome of this regulation allows the bacterium to adapt to change accordingly. Pantoea ananatis is a versatile plant pathogen of many agro-economically important plant species including corn, onion, rice, pineapple, melon and Eucalyptus and its motility and ability to form biofilm helps the pathogen to infect and proliferate in its plant host. The bacterium is also found in a diverse range of ecological niche as a saprophyte and epi- or endophyte. We hypothesize that the versatility of $P$. ananatis in infecting and colonizing different hosts and environments may be attributed to rapid Hfq-mediated response to various environmental conditions. Thus the functional role of $\mathrm{Hfq}$ was investigated by constructing an $h f q$ deletion mutant of $P$. ananatis $\mathrm{LMG} 2665^{\mathrm{T}}$ and the effect of the mutation in biofilm formation, motility and pathogenicity was characterized. An $h f q$ null mutant displayed significantly decreased biofilm production and motility and there was an absence of pathogenicity on onion seedlings. The findings suggest that Hfa is an important regulator of biofilm formation, motility and pathogenicity in $P$. ananatis, providing an insight into functional roles of Hfq in this versatile plant pathogen.

The Proline18 in P3a is important for Brassica yellows virus systemic infection which can be rescued by ectopically expressed P3a X. Y. Zhang, T. Zhao, Y. Li, H. Xiang, S. Dong, Z. Zhang, Y. Wang, D. Li, J. Yu, C. HAN, China Agricultural University, Beijing, CHINA

ORF3a, a newly identified non-AUG-initiated ORF encoded by members of genera Polerovirus and Luteovirus, is required for long-distance movement in plants. However, the mechanism of how P3a functions in viral systemic movement is still not clear. In this study, sequencing of a brassica yellows virus (BrYV) mutant defective in systemic infection revealed two-nucleotide variation at positions 3406 and 3467 in the genome. Subsequent nucleotide substitution analysis proved that only the nonsynonymous substitution $(\mathrm{C} \rightarrow \mathrm{T})$ at position 3406 , resulting in $\mathrm{P} 3 \mathrm{a}^{\mathrm{P} 18 \mathrm{~L}}$, abolished the systemic infection of BrYV. Preliminary investigation showed that wild type BrYV was able to load into the petiole of the agroinfiltrated Nicotiana benthamiana leaves, whereas the mutant displayed very low efficiency. Further experiments revealed that the P3a and its mutant P3a ${ }^{\mathrm{P} 18 \mathrm{~L}}$ localized to the Golgi apparatus and near plasmodesmata, as well as the endoplasmic reticulum. Both of $\mathrm{P} 3 \mathrm{a}$ and $\mathrm{P} 3 \mathrm{a}^{\mathrm{P} 18 \mathrm{~L}}$ were able to self-interact in vivo, however, the mutant seemed to form irreversible dimer and lose its function. More interestingly, we confirmed for the first time that the ectopic expression of P3a of other poleroviruses and luteoviruses, as well as co-infection with Pea enation mosaic virus 2 (PEMV 2), restored the ability of systemic movement of BrYV P3a defective mutant, indicating that the P3a is functionally conserved in poleroviruses and luteoviruses and is redundant when BrYV co-infects with PEMV 2. These observations provide a novel insight into the conserved function of $\mathrm{P} 3 \mathrm{a}$ and its important residue in the systemic infection.

Examining the role of an EF-Hand Protein in regulating virulence in Xanthomonas

S. BIBI (1), J. Pereira (1), S. Timilsina (1), G. V. Minsavage Jr. (1), J. C. Hulbert (2), J. B. Jones (1), (1) Department of Plant Pathology, University of Florida, Gainesville, FL, USA; (2) Department of Chemistry, Physics and Geology, Winthrop University, Rock Hill, SC, USA

Bacterial spot is a major disease of tomato and pepper. Four species of Xanthomonas are causal agents of this disease: Xanthomonas euvesicatoria, X. vesicatoria, X. perforans and X. gardneri. A gene was identified in X. fuscans subsp. aurantifolii that when expressed in X. perforans, elicited an HR in tomato. Based on analysis of the gene using bioinformatics tools, the protein is predicted to contain a region of unknown function at the amino-terminus, a single putative transmembrane helix and two EF-Hand calcium-binding domains at the carboxy-terminus. Genomic searches have revealed that the gene is also present in X. perforans and X. euvesicatoria. Interestingly, in X. euvesicatoria strains isolated from pepper, this gene is intact, whereas in strains isolated from tomato, there is a stop codon immediately upstream of the EF-hand domains. $X$. perforans strains that are associated with tomato contain a stop codon preceding the EF-Hand domain sequences. Expression of the full-length form of the gene in strains of X. perforans isolated from peppers resulted in reduced ability to grow in tomato leaflets compared to the wild-type strain. The preliminary data of population and electrolyte leakage of these mutants indicate possible role in virulence. Our aim is to look into the possible role of the transmembrane region and EF-hands in the regulation of pathogenicity of these strains. The next step is to use this knowledge to identify the function of this protein in Xanthomonas and try to understand its role in the calcium environment by generating various mutants.

Characterization of winter squash age-related resistance to Phytophthora capsici through fruit peel transcriptome profiling S. A. ALZOHAIRY (1), B. Moore (2), R. Hammerschmidt PhD (1), S. H. Shiu (2), M. K. Hausbeck (1), (1) Department of Plant Soil and Microbial Sciences, Michigan State University, East Lansing, MI, USA; (2) Department of Plant Biology, Michigan State University, East Lansing, MI, USA

Fruit of winter squash (Cucurbita moschata) cultivars develop resistance to the oomycete plant pathogen Phytophthora capsici 21 days post pollination (dpp); young fruit (7 dpp) are susceptible. This phenomenon is known as age-related resistance (ARR). Previous studies demonstrated that exocarp cuticle and epidermal walls are thicker at $21 \mathrm{dpp}$ than at $7 \mathrm{dpp}$ and that wounding of mature fruit results in susceptibility. To determine the molecular mechanisms of ARR, two C. moschata cultivars ('Chieftain' and 'Dickenson') that exhibit different ARR onsets were selected for transcriptome profiling with RNA-sequencing. The sequencing was conducted using RNA samples from fruit at the following dpp: 7, 10, 14, and 21 from 'Chieftain' that develops ARR at $14 \mathrm{dpp}$ and 'Dickenson' that develops ARR at $21 \mathrm{dpp}$. Functional annotation of the differentially up-regulated genes in the resistant fruit peel relative to the susceptible one revealed enrichment for genes associated with cell wall structures biosynthesis. Pathway enrichment analysis revealed enrichment of the upregulated genes in cutin, suberin monomers and phenylpropanoids biosynthesis. Our findings suggested that ARR is highly associated with monolignols biosynthesis and lignin deposition at the cell wall of the resistant fruit peel. This is the first time that transcriptome profiling has been used to uncover the mechanisms of ARR in winter squash to P. capsici. Identifying the genetic mechanism of ARR in winter squash will 
facilitate the development of Cucurbita varieties with resistance to $P$. capsici and assist the development of $P$. capsici disease management schemes for producers' benefit.

$\mathrm{Ca}$. Liberibacter asiaticus peroxiredoxin and peroxidase are virulence factors critical for survival and colonization of citrus

D. W. GABRIEL (1), M. Jain (1), A. Munoz Bodnar (2), S. Zhang (3), (1) Institute of Food and Agricultural Sciences, University of Florida, Gainesville, FL, USA; (2) University of Florida, Gainesville, FL, USA; (3) U.S. Horticultural Research Laboratory, USDA-ARS, Fort Pierce, FL, USA

A seminal feature of plant defense reactions in response to an attempted pathogen invasion is engagement of an oxidative $\left(\mathrm{H}_{2} \mathrm{O}_{2}\right)$ burst. In $C a$. Liberibacter asiaticus (Las) strain UF506, the expression of an SC2 peroxidase increases bacterial fitness and delays symptom development in the host plant. Two peroxiredoxin genes, CLIBASIA_00980 (C980) and CLIBASIA_00485 (C485) are conserved among all sequenced Las strains (including those lacking prophages). Both peroxiredoxins were functionally characterized in L. crescens, a culturable proxy for Las. C980 appears to be a housekeeping gene with similar moderate transcript abundance in both Las-infected psyllids and citrus, whereas C485 expression completely repressed in psyllids, reminiscent of SC2 peroxidase expression. Both SC2 peroxidase and C485 were demonstrated to be non-classically secreted. C485 conferred significant resistance against both $\mathrm{H}_{2} \mathrm{O}_{2}$ and an organic oxidizing agent $t \mathrm{BOOH}$. Transient overexpression of $\mathrm{C} 485$ in tobacco resulted in (a) transcriptional inactivation of $\mathrm{RbohB}$, and (b) suppression of oxidative damage to host cell lipid membranes. In addition to mitigating the RBOHmediated long-distance systemic defense signaling cascades, C485 may also play a critical role in alleviating the chain reaction like spread of lipid peroxidation, and subsequent accumulation of oxylipins that are both antimicrobial as well as positive transcriptional regulators of hypersensitive cell death response.

Fungal $\beta$-lactamases, what's up with that?

S. E. GOLD (1), M. Gao (2), N. Crenshaw (1), G. Hibbs (2), M. Nadal (1), A. E. Glenn (1), (1) USDA-ARS Toxicology and Mycotoxin Research Unit, Athens, GA, USA; (2) Department of Plant Pathology, The University of Georgia, Athens, GA, USA

We are characterizing the role of fungal lactamase encoding genes in the environmental competitiveness of Fusarium verticillioides (Fv), a fungus commonly infecting maize and producing the fumonisin mycotoxins. The role of bacterial $\beta$-lactamases as a mechanism of resistance to $\beta$-lactam antibiotics (such as penicillin) is well documented. Large families of genes encoding metallo- (PFAM PF00753) and serine-based (PF00144) $\beta$ lactamases exist in some fungi. Interestingly, soil borne fungal genomes are highly enriched in lactamase encoding genes. For example, $F v$ has 46 genes. In contrast, the environmentally isolated foliar obligate plant pathogens, such as Puccinia graminis, Blumeria graminis, and Taphrina deformans genomes encode only 5,2, and 5 lactamases. Our central hypothesis is that in the microbially diverse soil environment, fungi are exposed to numerous xenobiotics that they must detoxify. We currently know the function of only one of the $F v$ lactamase genes. $M B L 1$ (FVEG 08291) is required for degradation of the plant-derived benzoxazinone phytoanticipins like BOA. MBL1 expression is highly and specifically induced upon exposure to BOA. This then serves as our paradigm: lactamase genes induced by specific xenobiotics are predicted to confer resistance to the inducing xenobiotic. We are taking a broad approach to identify interactions that induce specific $F v$ lactamases. Assays for expression of specific lactamases are underway and include interactions with soil isolated Streptomyces species and with Sarocladium zeae, a fungal co-inhabitant with $F v$ in maize seed. We have generated a set of single lactamase gene deletion mutants to test the role of individual genes in conferring resistance to specific stimuli.

Characterization of a novel transcription factor from Sclerotinia sclerotiorum induced during infection of pea

H. SANG, H. X. Chang, M. Chilvers, Michigan State University, East Lansing, MI, USA

The plant pathogenic fungus Sclerotinia sclerotiorum is the primary causal agent of white mold and is capable of infecting over 400 host species including pea (Pisum sativum L.). Pea is an important legume crop in the United States but white mold can cause substantial yield loss. Understanding mechanisms of Sclerotinia pathogenicity is essential to improving white mold management in pea but the pathogenicity factors to pea have been poorly studied. A white mold partially resistant pea line (PI 240515) and susceptible cultivar 'Lifter' were inoculated with S. sclerotiorum colonized potato dextrose agar (PDA) plugs of strain Sc102-05 and uncultured PDA media inoculated with the plugs were used as a control. Samples were harvested at 12, 24, and 48 hours post inoculation (hpi) for RNA-seq analysis. A total of 317 transcripts of S. sclerotiorum were significantly induced in both pea lines at 24 and 48 hpi compared to the expression on the PDA plugs alone. Among these transcripts, one putative S. sclerotiorum transcription factor (TF) showed higher expression in the susceptible cultivar than the partially resistant line at $24 \mathrm{hpi}$. This TF was silenced by constitutively overexpressing a hairpin double stranded RNA of target sequences to investigate its function in pathogenicity and development of $S$. sclerotiorum. This study will provide molecular insights of $S$. sclerotiorum pathogenicity into pea and other economically important crops.

Oxidative stress tolerance is critical for xylem colonization and virulence of xylem-limited pathogens Xanthomonas albilineans and Xylella fastidiosa

M. JAIN (1), S. Zhang (2), D. W. Gabriel (1), (1) Institute of Food and Agricultural Sciences, University of Florida, Gainesville, FL, USA; (2) U.S Horticultural Research Laboratory, USDA-ARS, Fort Pierce, FL, USA

Xanthomonas albilineans (Xal) and Xylella fastidiosa (Xf) are xylem-limited bacteria causing leaf scald of sugarcane and Pierce's disease in grapevines, respectively. Hydrogen peroxide $\left(\mathrm{H}_{2} \mathrm{O}_{2}\right)$ is essential for protoxylem differentiation and cell wall lignification during xylem maturation, resulting in an oxidative environment in differentiated (dead) xylem vessels. We hypothesize that xylem-limited pathogens such as Xal and Xf must therefore breach an intrinsic oxidative burden for successful colonization. Genes encoding a bifunctional heme-dependent catalase and broad spectrum peroxidase (E. coli $k a t G$ homologs) are annotated in both Xal and Xf genomes. The Xal kat $G$ (VB87_RS05200) was replaced by an enhanced Green florescent protein gene $(g f p)$ via marker exchange. Xal $\Delta k a t G / g f p$ mutant strains exhibited significantly reduced pathogenicity on newly emerged sugarcane leaves and compromised viability in planta as compared to wild type Xal. Similarly, Xf katG (PD_RS06770) was replaced by nptII via marker exchange. Challenge inoculations of Vitis vinifera grapevines with $\mathrm{Xf} \Delta \mathrm{katG} / \mathrm{nptII}$ mutant strains exhibited only limited disease symptoms near the inoculation sites. The pathogenicity of the Xal $\Delta$ katG/gfp and $\mathrm{Xf} \Delta \mathrm{kat} / \mathrm{nptII}$ mutant strains were completely restored by complementation with full-length Xal kat $G$ (cloned in pUFR047) and Xf katG (cloned in pBBRIMCS-5). Since Xf has only a single peroxidase gene, it is being evaluated as a target for chemical control.

Comparative transcriptome profiling of compatible and incompatible Magnaporthe grisea-pearl millet interaction R. SHARMA (1), S. Singh (2), S. Nayak (3), P. Boyapati (4), A. Khan (1), R. Varshney (5), (1) ICRISAT, Hyderabad, INDIA; (2) ICAR National Organic Farming Research Institute, Gangtok, INDIA; (3) University of Agricultural Sciences, Dharwad, Dharwad, INDIA; (4) Professor Jayashankar Telangana State Agricultural University, Hyderabad, INDIA; (5) International Crop Research Institute for the Semi-Arid Tropics, Hyderabad, INDIA 
Blast of pearl millet caused by Magnaporthe grisea has recently become a disease of economic concern in India. For the better understanding of plantpathogen interactions, the transcriptomes were captured by RNA-seq analysis of a pearl millet genotype (ICMB 93333) inoculated with $M$. grisea isolates Pg 45 and Pg 174 having avirulent and virulent reaction, respectively. The experiment was set up with two un-inoculated controls at 0 and 72 hours post-inoculation (hpi) and five infection time points 6, 12, 24, 48 and $72 \mathrm{hpi}$. The RNA-seq generated a total of 1103 million reads with an average of 92 million reads at pre-processing. A significant differential expression levels between the resistant and susceptible samples were found in 1985 genes; 1304 genes were up-regulated and 1093 genes (non-redundant) were down-regulated in the incompatible interaction. The transcriptional changes in the incompatible interaction were observed predominantly for PR protein families encoding defense response genes; genes functional in reactive oxygen species; signal transduction (cellular communication and signaling); carbohydrate, protein and lipid metabolism processes; secondary metabolic processes and cell wall alterations. The study was focused on functional classification and identification of novel genes or gene family members involved in plant-pathogen interactions, the expression pattern of which could help in the development of new disease control strategies.

Identifying oxalic acid independent compatibility factors from Sclerotinia sclerotiorum P. L. YU (1), Y. Zhang (2), J. A. Rollins (2), (1) University of Florida, Gainesville, FL, USA; (2) Department of Plant Pathology, University of Florida, Gainesville, FL, USA

Previous studies have shown that oxalic acid (OA) accumulation is required for Sclerotinia sclerotiorum to fully colonize and produce symptoms in a broad range of hosts. We hypothesize that limited lesion-causing OA-minus mutants (oahl) are able to establish a basic compatibility with their hosts but are unable to transition to the OA-dependent colonization phase of compatibility. To test this hypothesis we are seeking to identify and characterize genes important for the establishment of primary lesions independent of OA accumulation. We have approached this experimentally by comparing transcriptome expression profiles from the wild type and OA-minus mutants during pre-penetration, penetration, and colonization phases of disease development. Of specific interest, cyanide hydratase genes ( $\operatorname{chn} 1$ and $\operatorname{chn} 2)$ are highly expressed by both wild type and the OA-minus mutant during the infection of Brassicaceae and strongly up-regulated in the OA-minus mutant relative to the wild type. As glucosinolates are major phytoanticipins in Brassicaceae, these enzymes, through breakdown of toxic glucosinulate hydrolysis products, are candidates for the establishment of basic compatibility in Brassicaceae. From functional analyses, we have observed that $\operatorname{chn} 1$ mutants retain wild type virulence and chn2, chn1-chn2, and chn-oah1 mutants are being developed to further test the role of cyanide hydratases in establishment of basic compatibility in this broad host range necrotrophic pathogen.

Transcriptomic and Phenotypic Responses of Arabidopsis thaliana to Infection with Pathogenic or Non-Pathogenic Fusarium oxysporum K. VESCIO (1), L. Guo (1), L. J. Ma (2), (1) University of Massachusetts, Amherst, MA, USA; (2) Plant Biology Graduate Program, University of Massachusetts Amherst, Amherst, MA, USA

Fusarium oxysporum $(F o)$ is a soil-borne fungal pathogen that causes vascular wilt disease on a broad range of plants, including agricultural crops and the model plant Arabidopsis thaliana. There are non-pathogenic members of the $F o$ species complex that confer various defense benefits against other pathogens to the host plant. Transcriptomic analysis of Arabidopsis thaliana infected by the pathogenic strain Fo5176 and non-pathogenic strain Fo47 has identified shifts in plant gene expression specific to treatment. Annotation of protein-protein interaction networks highlighted an enrichment of genes and pathways related to nutrient acquisition and assimilation, and transcriptional reprogramming in Fo47 infected A. thaliana roots. In contrast, when responding to pathogen infection, genes and pathways related to protein homeostasis, jasmonic acid signaling and systemic defense responses are induced. Phenotypic characterization of $A$. thaliana plants inoculated with Fo47 or Fo5176 has revealed significant increases in shoot and root biomass, and alterations in lateral root architecture in Fo47 inoculated plants. With insights from RNA-seq and phenotypic data, we propose that infection with Fo47 improves plant tolerance to stress, and mechanisms taking place on the molecular and cellular levels will be discussed.

Clathrin is essential for virulence factors delivery in the necrotrophic fungus Botrytis cinerea

E. Souibgui (1), C. Dieryckx (2), C. Bruel (1), M. P. Latorse (3), N. POUSSEREAU (1), (1) University Lyon 1, Lyon, FRANCE; (2) CNRS, Lyon, FRANCE; (3) BAYER SAS, Lyon, FRANCE

Fungi are important plant pathogens on agricultural and horticultural crops. Study of these microorganisms remains essential to understand pathogenic process and control plant diseases. In this context, a random insertional mutagenesis strategy, based on the Agrobacterium tumefaciens-mediated transformation (ATMT), was used to create a mutant library of the grey mould fungus Botrytis cinerea. A nonpathogenic mutant altered in the expression of a gene encoding clathrin heavy chain was selected. Clathrin heavy chain is well conserved from yeast to human and is described to be mainly involved in intracellular coat vesicles formation for endocytosis and post-Golgi trafficking of proteins. The characterization of the mutant was performed to precise the role of this protein in the infectious process of this typical necrotrophic fungus. A secretion defect of numerous proteins including known virulence factors, as Plant Cell Wall Degrading Enzymes and elicitors was observed. A clathrin-coated vesicle enriched fraction was isolated from the wild type and mutant strains. Using a proteomic approach, we analyzed and compared the proteomic composition of these clathrin-coated vesicles and present here the results. This study demonstrated for the first time the essential role of clathrin in the infectious process of a fungal pathogen and its importance in virulence factors secretion.

\section{Bacillus pumilus enhances the safflower (Carthamus tinctorius L.) growth under chromium stress by an antioxidative potential and nutrient acquisition}

M. T. JAVED (1), M. S. Akram (1), A. Abid (1), M. Shahid (2), Q. Ali (1), N. Iqbal (1), (1) Department of Botany, Government College University, Faisalabad, PAKISTAN; (2) Department of Biotechnology and Bioinformatics, Government College University, Faisalabad, PAKISTAN

Chromium (Cr) is a significantly lethal metal and exerted negative effect on plant physiology and healthy developments. Free living soil bacteria like Bacillus pumilus can improve the plant growth and health in stressed environment including metal toxicity and are considered as defensive approach to mitigate the toxic effects. The current study is, therefore, aimed to check physiology, morphology, growth parameters as well as the capacity to tolerate the $\mathrm{Cr}$ stress in safflower (Carthamus tinctorius $\mathrm{L}$.) based upon better nutrient homeostasis. Plants were grown in soil spiked with different concentration of $\mathrm{K}_{2} \mathrm{Cr}_{2} \mathrm{O}_{7}$ i.e. 0, 2, 4, 6 and $10 \mathrm{mg} / \mathrm{kg}$ soil with and without Bacillus pumilus inoculation. Higher Cr treatment levels decreased the plant fresh and dry weight as well as the photosynthetic pigments of $C$. tinctorius. The plant root and shoot $\mathrm{Cr}$ contents increased while nutrient $(\mathrm{P}, \mathrm{K}, \mathrm{Ca}$, and $\mathrm{Na})$ contents decreased with increasing Cr levels particularly at $10 \mathrm{mg} / \mathrm{kg} \mathrm{Cr}$ treatment. However, the C. tinctorius plants maintained with B. pumilus inoculation prompted a significant increase in plant biomasses together with decreased reactive oxygen species and enhanced activity of cellular antioxidant enzymes under $\mathrm{Cr}$ stress as compared with their respective un-inoculated controls. In parallel, the inoculation with B. pumilus under $\mathrm{Cr}$ stress enabled the $C$. tinctorius plants to accumulate the nutrients ( $\mathrm{P}, \mathrm{K}, \mathrm{Ca}$, and $\mathrm{Na}$ ). It can be concluded that $B$. pumilus inhibited the uptake as well as root to shoot translocation of $\mathrm{Cr}$ in C. tinctorius which in turn resulted in healthy plants under $\mathrm{Cr}$ stress. Hence, $\mathrm{Cr}$ stress can be significantly mitigated by $B$. pumilus inoculation to get healthy plants of $C$. tinctorius in $\mathrm{Cr}$ contaminated areas. 
Fusarium graminearum chemotype differences and virulence

G. WIESENBERGER, K. Twaruschek, H. Michlmayr, E. Varga, M. Vaclavikova, M. Piatkowska, F. Berthiller, G. Adam, University of Natural Resources and Life Sciences, Vienna, Tulln, AUSTRIA

In $F$. graminearum several "chemotypes" producing different trichothecene virulence factors exist. Nivalenol production seems to be the ancestral state, loss of TRI13 leads to deoxynivalenol production. Allelic differences at TRI8 determine whether preferentially 3-acetyl- or 15-acetyl-deoxynivalenol is produced. Recently a $F$. graminearum population was identified in North America (3-ADON genotype), which due to a different TRII allele produces a novel type A-trichothecene, NX-2, lacking a C-8 keto-group. Comparing the virulence of natural isolates with different chemotypes is usually inconclusive due to abounding differences in the genomic background. We have developed positive-negative selectable transformation markers, which allow construction of near isogenic strains containing swapped alleles. Despite the fact that TRII is unlinked to the core TRI cluster, no NX-4 producers have so far been found in nature. Introduction of the NX-TRII allele into the 15-ADON background caused NX-4 production in vitro and drastically reduced virulence on wheat. Recently up to $20 \%$ NX-producers were detected in an area of New York State, a much higher frequency than previously reported. A selective advantage could be that NX-type toxins can escape inactivation by Michael-adduct formation with glutathione. Yet, NX toxins can undergo a non-enzymatic rearrangement to non-toxic M1-derivatives lacking the epoxide, which occurs during extended grain storage and food processing.

Identification and characterization of a carbonic anhydrase involved in virulence and bacterial competition of Pseudomonas syringae pv. tomato DC3000

M. Fishman (1), A. Cohn (1), M. FILIATRAULT (2), (1) Cornell University, Ithaca, NY, USA; (2) USDA ARS, Ithaca, NY, USA

Carbonic anhydrases play important roles in balancing $\mathrm{pH}$, lipid biogenesis, calcification, and pathogenesis of bacteria. We recently discovered a gene (cynT) in the plant pathogenic bacterium Pseudomonas syringae pv. tomato DC3000 (Pto) that encodes a putative carbonic anhydrase. To determine the role of cynT in Pto, we investigated the transcriptional regulation and the impact of deleting this gene in Pto. Bioinformatics analysis identified alternative transcripts in the 5' UTR of cynT as well as a predicted binding site for the sigma factor RpoF within the promoter region. We demonstrate that RpoF regulates transcription of $c y n T$ and a putative small RNA is produced within this genomic location. A $\Delta c y n T$ strain displayed reduced symptoms and growth in tomato plants and showed a delay in the hypersensitive response in Nicotiana benthamiana. Furthermore, we show that cynT controls cellulose production in Pto and affects bacterial competition of Pto with other bacteria. Our data support a role of cynT in deployment of effectors into host cells and other bacterial cells, linking cynT to regulation of the Type III and VI secretion systems. The involvement of $c y n T$ in critical steps of the Pto life cycle suggests an important role for carbonic anhydrases during bacterial plant-pathogen interactions. Since carbonic anhydrases are conserved among bacterial plant pathogens, these enzymes represent promising new targets for the development of anti-bacterial agents.

Cross-kingdom communication between Ralstonia and Fusarium mediate tomato wilt disease and microbial survival N. VENKATESH, J. Spraker, P. Wiemann, N. P. Keller, University of Wisconsin, Madison, WI, USA

Ralstonia solanacearum (Rs) and Fusarium oxysporum f. sp. lycopersici $(\mathrm{Fol})$ are vascular wilt pathogens that result in heavy yield losses in susceptible hosts such as tomato. Although both pathogens occupy the xylem, the consequences of mixed infections on wilt disease are unknown. Our previous in vitro studies had identified a $R s$ lipopeptide, ralsolamycin, that induces chlamydospore formation in fungi including Fol. Bacteria have been observed to invade the chlamydospores and is proposed to impact the survival of both microbes. Here we show that $F o l$ responds to ralsolamycin exposure with the production of antibacterial metabolites (e.g. bikaverin and beauvericin) and explore interactions between the pathogens in tomato plants with corresponding secondary metabolite mutants of both the bacterium and the fungus. In planta co-infection experiments with $R s$ and $F o l$ provide evidence for an antagonistic behavior of the fungus towards $R s$ with a significant reduction in bacterial wilt severity. Co-infections with metabolite mutants of both $R s$ and $F o l$ will be assessed for disease progress and survival of both pathogens, including the ability of the bacterium to overwinter in fungal chlamydospores. These experiments coupled with RNAseq and metabolomics analyses are expected to reveal key communication molecules which may form the basis for novel strategies to control vascular wilt diseases.

A polyketide biosynthesis gene cluster is required for production of bactericidal activity by Burkholderia contaminans strain MS14 P. Deng, J. Jia, S. Baird, S. LU, Mississippi State University, Mississippi State, MS, USA

Burkholderia contaminans MS14 produces the antifungal glycopeptide occidiofungin via a nonribosomal peptide synthase mechanism. MS14 also possesses a broad range of antibacterial activities against Gram negative bacteria, such as Erwinia amylovora and Xanthomonas citri. Random mutagenesis and gene complementation revealed that four genes are required for production of MS14 antibacterial activity. However, sequence-based function prediction indicated that none of them are directly involved in biosynthesis of the bactericidal activity. In order to identify the biosynthetic gene cluster for the unknown antibacterial compound production, RNA-Seq was conducted to analyze the transcriptomes of the MS14 wild type and its mutants that lack antibacterial activity. A polyketide synthases (PKS) gene cluster was predicted to be directly involved in antibacterial activity. Mutants of the two PKS genes were generated using kanamycin cassette insertion and CRISPR-Cas9 system. Plate bioassays showed that the mutations in the two PKS genes resulted in undetectable antibacterial activity against $E$. amylovora. As expected the mutants defective in antibacterial activity retained the wild-type production level of occidiofungin. The results suggest the PKS gene cluster is directly responsible for the production of MS14 bactericidal activity.

Novel biosynthetic gene cluster in Pantoea ananatis is critical to foliar lesion development in center rot of onion J. A. ASSELIN, J. M. Bonasera, S. V. Beer, Cornell University, Ithaca, NY, USA

Center rot of onion, caused by the bacteria Pantoea ananatis, P. allii, and P. agglomerans, results in economic losses in many onion-growing areas worldwide. The disease is characterized by lesions on leaves and water soaking, discoloration, and scale shrinkage within onion bulbs. $P$. ananatis is arguably the best studied center rot pathogen. However, few pathogenicity or virulence factors have been identified and verified empirically. Also, pathogenicity to onion by $P$. ananatis is variable by strain. The goal of this work was to identify additional pathogenicity/virulence factors in $P$. ananatis towards onion. In this study, genome comparisons from two onion-pathogenic and two onion-non-pathogenic $P$. ananatis strains yielded a list of candidate genes that appeared well-conserved in onion-pathogenic strains, but absent or poorly-conserved in onion-non-pathogenic strains. Screening of additional strains with PCR primers designed to amplify some of these genes suggested the presence of a previously uncharacterized biosynthetic gene cluster present in only the onion-pathogenic strains. Preliminary results demonstrate that the gene cluster is necessary for $P$. ananatis to cause leaf lesions and water soaking in onion bulbs. Furthermore, genes from this cluster were detected in onion-pathogenic strains of $P$. allii and $P$. agglomerans. These results suggest that activity of this cluster is involved in center rot of onion. 
Modulation of growth, twitching movement and biofilm formation in Xylella fastidiosa mediated by gene PD0913 under different calcium concentrations

L. M. GÓMEZ (1), L. De La Fuente (2), (1) Auburn University, Auburn, AL, USA; (2) Department of Entomology and Plant Pathology, Auburn University, Auburn, AL, USA

Xylella fastidiosa (Xf) is an insect transmitted, xylem-limited bacterium that causes disease in many economic and ecologically important host plants. A key feature involved in the virulence mechanism of $\mathrm{Xf}$ is the formation of biofilm that blocks the flow of water and nutrients from the roots to the leaves. Mineral nutrients such as calcium $(\mathrm{Ca})$ are transported through the xylem vessels. In Xf, calcium was reported to have an important role modulating biofilm formation and twitching motility. Through the study of whole transcriptome analysis and comparative genomics in Xf, a set of genes regulated by calcium was identified. PD0913 was selected for functional analysis since it is also part of a putative genomic island absent in non-virulent strains. Two PD0913-Xf mutants were generated by site-directed mutagenesis and the total, planktonic and biofilm growth was quantified. Compared to the WM1-1 wild type strain, growth of the mutants was reduced under different concentrations of $\mathrm{CaCl}_{2}(2 \mathrm{mM}, 4 \mathrm{mM}$ and $8 \mathrm{mM})$. Biofilm growth was significantly higher under $8 \mathrm{mM}$ of $\mathrm{Ca}$, in which WT values were greater than PD0913-Xf mutants. When twitching movement was analyzed, differences between WT and mutant were evident at $4 \mathrm{mM} \mathrm{Ca}$, where mutants were impaired in movement. These results suggest that PD0913 gene, so far annotated as a hypothetical protein, is involved in growth and regulation of virulence traits in Xf.

Gene conservation reveals perylenequinone toxin biosynthesis clusters in multiple plant pathogenic fungal species R. SPANNER (1), M. K. Ebert (2), D. Smith (3), G. A. Secor (4), B. P. H. J. Thomma (2), R. de Jonge (5), M. D. Bolton (6), (1) North Dakota State University, Fargo, ND, USA; (2) Wageningen University, Wageningen, NETHERLANDS; (3) USDA - ARS, Fargo, ND, USA; (4) Department of Plant Pathology, North Dakota State University, Fargo, ND, USA; (5) Plant-Microbe Interactions, Department of Biology, Faculty of Science, Utrecht, NETHERLANDS; (6) USDA-ARS, Red River Valley Agricultural Research Center, Fargo, ND, USA

Perylenequinones are a family of structurally related secondary metabolite fungal toxins that absorb light energy to produce reactive oxygen species. This potent mechanism serves as an effective weapon for plant pathogens in disease establishment. The foliar sugar beet pathogen Cercospora beticola secretes its namesake perylenequinone cercosporin during infection. Recent studies have shown that the cercosporin toxin biosynthesis cluster is present in other plant pathogenic fungal species outside of the genus Cercospora. For the apple fruit pathogen Colletotrichum fioriniae, the production of cercosporin in vitro was demonstrated and we are investigating the role of the toxin in post-harvest decay of apple via gene expression studies. These results have prompted the search for biosynthetic gene clusters of structurally-similar perylenequinones in other fungi. We report the identification of the elsinochrome biosynthetic cluster of Elsinoë fawcettii based on gene cluster conservation and validation by targeted gene replacement. Phylogenetic analysis of the core polyketide synthase encoding genes as well as alignment of putative melanin clusters revealed high conservation between established Magnaporthe grisea and newly identified C. beticola, E. fawcettii, and Cladosporium phlei melanin pathways. Our results emphasize the high conservation level between biosynthetic clusters of structurally related secondary metabolites and how it can be exploited as a reliable method to identify new biosynthetic pathways that are of agronomic importance.

Auto-activated maize $\mathbf{R}$ protein recognizes a bacterial effector to trigger incomplete disease resistance in Arabidopsis thaliana J. Miao, Y. Liu, C. Zhou, D. Kong, Z. Yang, S. Wu, Z. Wang, Q. LI, B. Zhao, Virginia Tech, Blacksburg, VA, USA

Rice bacterial leaf streak (BLS) disease caused by the gram-negative bacterium Xanthomonas oryzae pv. oryzicola (Xoc) is one of the most important rice diseases in Asia and Africa. We previously identified a maize disease resistance gene, Rxol, which conditions resistance to Xoc strains carrying the type 3 effector AvrRxo1 in transgenic maize and rice plants. However, the mechanism of Rox1/AvrRxo1-mediated disease resistance is still elusive. It is also unclear if Rxo1 can be functional transferred to other plant species. To this end, we generated transgenic Arabidopsis plants expressing Rxo1. However, the Arabidopsis-Rxo1 plants failed to confer disease resistance to Pseudomonas syringae pv. tomato (Pst) DC3000 expressing the XocavrRxol gene. To understand why Rxol is not functional in Arabidopsis, and test the possibility of modifying Rxol to reconstitute its functionality in Arabidopsis, we generated an Rxol autoactivation mutant D508V. We demonstrated that Rxo1-D508V triggers cell death in N. benthamiana and Arabidopsis plants. Interestingly, transgenic Arabidopsis plants expressing Rxo1-D508V can specifically recognize Xoc-avrRxo1, and trigger incomplete disease resistance to Pst DC3000 carrying Xoc-avrRxo1. We further demonstrated that Xoc-avrRxo1 directly interacts with the NBS domain alone but not the full length of Rxo1 in vitro and in vivo. We hypothesize that Rxo1-D508V may have altered protein structure allowing it to expose the NBS domain and interact with AvrRxo1 in Arabidopsis plant cells. The direct interaction may partially explain the functionality of Rox1-D508V/AvrRxo1 in Arabidopsis.

Sethoxydim herbicide at sublethal dose synergizes biocontrol of green foxtail by Pyricularia setariae via triggering ABA-activated pathways and bZIP60

T. Song (1), M. Chu (1), J. Zhang (2), R. Wen (1), F. Yu (1), B. D. Gossen (1), G. PENG (1), (1) Agric \& Agri-Food Canada, Saskatoon, SK, CANADA; (2) China National Rice Research Institute, Hangzhou, CHINA

Applying sethoxydim at $0.1 \times$ label rate (sublethal) dramatically enhances the biocontrol of herbicide-sensitive (HS) Setaria viridis [green foxtail (GFT)] by the mycoherbicide fungus Pyricularia setariae. In this study, GFT transcriptomes were analyzed to elucidate the mechanism for the herbicide effect. Reference transcriptomes of HS and herbicide-resistant (HR) GFT were constructed via de novo assembly of RNA-seq data using a mixture of samples from several growth stages of HS and HR GFT biotypes, separately. Analysis of differentially regulated biological processes showed that the sethoxydim treatment suppressed photosynthesis in HS plants, especially the C4-specific phosphoenolpyruvate carboxylase kinase. It also induced ABA-activated signaling pathways as well as a bZIP transcriptional factor 60 (bZIP60), coinciding with the enhanced biocontrol of GFT-HS by P. setariae. On HR plants, the herbicide treatment failed to induce the ABA pathway or bZIP60, and did not improve the biocontrol. An exogenous application of ABA to HS plants produced effects similar to that by sethoxydim; it enhanced the expression of bZIP60 and synergized the biocontrol by $P$. setariae. This is the first use of RNA-seq to decipher the mechanism of a sublethal rate of herbicide to synergize weed biocontrol. This method provides a better understanding of the modes of action in the synergized GFT biocontrol, and a new approach to improve the efficacy and practicality of weed biocontrol.

Understanding the role of Type VI Secretion Systems for intra-specific competition and pathogenicity in Erwinia tracheiphila C. M. VRISMAN (1), J. Rocha (2), L. R. Shapiro (3,4), C. G. Taylor (1), G. Rajashekara (5), S. A. Miller (1), (1) Department of Plant Pathology, The Ohio State University, Wooster, OH, USA; (2) Centro de Investigacion en Alimentacion y Desarrollo, Hermosillo, MEXICO; (3) North Carolina State University, Raleigh, NC, USA; (4) Department of Microbiology \& Immunology, Harvard Medical School, Boston, MA, USA; (5) Food and Animal Health Research Program and Sciences, The Ohio State University, Wooster, OH, USA 
In many pathogenic bacteria, Type VI Secretion Systems (T6SS) are important for intra-specific bacterial competition. Genetically distinct populations have recently been observed within the phytopathogen Erwinia tracheiphila (Et). To assess whether genetically distinct Et strains interact in planta, coinoculation assays using TedCu10 (TC) and BHKY (BH), strains isolated from cucumber and squash, respectively, were performed in vitro and in planta using squash. In single inoculations BH, but not TC, is pathogenic to squash. All BH inoculated plants died within 26 days post inoculations. For coinoculations, the second true leaves of squash plants were wound inoculated with TC at concentrations of $10^{8}, 10^{7}, 10^{6}, 10^{5}$ or 0 colony forming units $(\mathrm{CFU}) / \mathrm{ml}$ and then immediately inoculated with $\mathrm{BH}$ at $10^{8} \mathrm{CFU} / \mathrm{ml}$ in the same sites. Plants inoculated with TC at $10^{8} \mathrm{CFU} / \mathrm{ml}$ followed by BH did not die. Approximately $70 \%$ of plants inoculated with TC at $10^{5} \mathrm{CFU} / \mathrm{ml}$ followed by $\mathrm{BH}$ died. A decrease in $\mathrm{BH}$ population was observed in vitro when $\mathrm{BH}$ was mixed at a ratio of 1:1 with TC on solid but not in liquid medium, suggesting that the interaction effect observed in planta could be contact dependent T6SS mediated. Whole genome sequencing showed that TC has three T6SS loci while BH contains two. Deletion mutants of the different T6SS loci in TC and BH are being generated to evaluate the effect of these loci on pathogenicity, disease suppression and within host bacterial competition.

Utilizing genomic tools to identify and characterize effectors in the novel sugar beet pathogen Fusarium secorum S. SHRESTHA (1), K. M. Webb (2), Z. Bian (3,4), M. K. Ebert (5), B. P. H. J. Thomma (5), G. A. Secor (6), R. de Jonge (7), M. D. Bolton (8), (1) North Dakota State University, Fargo, ND, USA; (2) USDA-ARS, Soil Management and Sugar Beet Research Unit, Fort Collins, CO, USA; (3) NDSU, Fargo, ND, USA; (4) Purdue, West Lafayette, IN, USA; (5) Wageningen University, Wageningen, NETHERLANDS; (6) Department of Plant Pathology, North Dakota State University, Fargo, ND, USA; (7) Plant-Microbe Interactions, Department of Biology, Faculty of Science, Utrecht, NETHERLANDS; (8) USDA-ARS, Red River Valley Agricultural Research Center, Fargo, ND, USA

Sugar beet is an important source of sucrose for human consumption throughout the world. Fusarium yellowing decline, caused by the novel fungal pathogen Fusarium secorum, is a new disease of sugar beet which was recently found in the Red River Valley of Minnesota and North Dakota. This disease is characterized by yellowing of leaves, vascular discoloration in the petiole, damping off of seedlings and the rapid death of plant at early stage. $F$. secorum produces effectors that are secreted during infection of the host. To gain a better understanding of the molecular basis of pathogenicity of this new disease, we utilized genome sequencing, transcriptome analysis, and xylem sap mass spectrometry of $F$. secorum infected-sugar beet plants to report 15,900 predicted genes, among which 527 exhibited effector characteristics. Among these candidate effector genes, 30 were highly expressed during $F$. secorum infection in planta. We developed a transformation system for this fungus and performed targeted gene replacement to characterize 11 candidate effector genes of which FSECE8 was identified as a virulence factor. Further studies will be directed to characterize the role of FSECE8 in pathogenicity. This study provides a valuable genomic resource for F. secorum and other closely related Fusarium spp. and sheds light on the virulence strategies of this novel pathogen.

Identification of Pseudomonas syringae Genes Required for Initiating Type III Secretion in Response to Host Plant-derived Metabolite Signals S. Turner, M. O’Malley, Y. Y. Pang, A. J. Weisberg, C. Rogan, Q. Yan, J. Chang, J. ANDERSON, Department of Botany and Plant Pathology, Oregon State University, Corvallis, OR, USA

Pseudomonas syringae is a bacterial pathogen that relies on a type III secretion system (T3SS) to inject immune-suppressing effector proteins into host cells during infection of plants. Although critical for virulence, the genes encoding the T3SS are not constitutively expressed and must be induced during infection. Recently, several plant-derived metabolites were identified as signals that increase expression of T3SS-associated genes in the model strain $P$. syringae pv tomato DC3000. To investigate how these chemical signals regulate the T3SS, we performed a large-scale Tn5 transposon mutagenesis of DC3000 to identify mutants with decreased response to the bioactive metabolites. We identified $200 \mathrm{Tn} 5^{+}$strains with confirmed loss-of-response phenotypes. Using a whole genome Illumina sequencing strategy, we identified essentially all $\operatorname{Tn} 5$ insertion sites within our collection of 200 mutants in less than one month's time. Within this set of mutants we found $\operatorname{Tn} 5$ insertions in all genes that encode known positive regulators of T3SS signaling in $P$. syringae, thus validating our screening method. Furthermore, we identified $\operatorname{Tn} 5$ insertions in several previously uncharacterized genes predicted to encode two-component systems, membrane transporters and transcriptional regulators, suggesting a role for these genes as positive regulators of T3SS expression. These results, as well as our efforts to functionally characterize how these genes regulate the T3SS, will be presented.

\section{Interactions among severity of spot blotch disease of wheat caused by Bipolaris sorokiniana, nitrogen supply and WRKY transcription factor functions}

S. BABA, Newcastle University, Newcastle upon Tyne, UNITED KINGDOM

Spot blotch caused by Bipolaris sorokiniana is a serious disease of wheat grown in warm climates. WRKY transcription factors (WRKYTFs) have been reported to be involved in plant responses to pathogens and to nutrient stress. We have found that in wheat, nutrient stress affects spot blotch severity, and nutrient stress and infection cause interacting changes in WRKYTF gene expression. Here we report on time dependence of pathogen development and WRKYTF gene expression in leaves of two Iraqi wheat cultivars, Rasheed (more susceptible to spot blotch) and Latifia (more resistant), and on pathogen development in wheat TILLING lines in which the WRKYGQK sequence characteristic of WKRYTFs is mutated. Seedlings of the two Iraqi wheats were supplied with $7.5 \mathrm{mM}$ nitrogen and TILLING lines with $0.75 \mathrm{mM}, 3.75 \mathrm{mM}$ or $7.5 \mathrm{mM}$ nitrogen. Fourteen-day-old seedlings were inoculated with B. sorokiniana. Pathogen development was assessed by binding of FITC-conjugated wheat germ agglutinin and RT-qPCR. The two methods gave consistent results. Fungal growth decreased as nitrogen input was reduced. Expression of different WRKYTF genes increased over time in Latifia, but decreased in Rasheed at $96 \mathrm{~h}$ after infection. Fungal growth was altered in TILLING lines. In one line there was higher fungal growth when $\mathrm{N}$ supply was reduced, the opposite pattern to wild-type plants. The results demonstrate that wheat WKRYTFs influence pathogen development and its interaction with nitrogen supply.

The PacC transcription factor regulates pH-dependent fungal development and virulence in the barley pathogenic fungus Cochliobolus sativus Y. LENG, A. Ahmadpour PhD, Y. Liu, S. Shrestha, B. Poudel, S. Zhong PhD, North Dakota State University, Fargo, ND, USA

Fungi can grow in a wide range of $\mathrm{pH}$ in nature. The ambient $\mathrm{pH}$ regulatory system that controls the expression of both acid and alkaline expressed genes have been identified in Aspergillus and other fungi. Among the genes involved in this system, $P a c C$, a gene encoding the zinc-finger transcription factor, plays a central role. In this study, we identified and characterized a homologous gene (CsPacC) of PacC from Cochliobolus sativus, the causal agent of spot blotch on barley. The deletion of $C s P a c C$ resulted in poor vegetative growth at high pH (9.0), suggesting that $C s P a c C$ is involved in activation of the genes when grown under alkaline conditions. Conidial production increased in the wild type isolate when the $\mathrm{pH}$ values increased from 5 to 9 ; however, the $\triangle C s P a c C$ mutant produced less conidia compared to the wild type under the same conditions. Real time quantitative PCR revealed that the expression of $C s P a c C$ was $\mathrm{pH}$ dependent. The relative expression of $\mathrm{CsPacC}$ was about four times more at $\mathrm{pH} 9.0$ than at $\mathrm{pH} 5.0$. The virulence of the $\triangle C s P a c C$ mutant was significantly reduced compared to the wild type. Complementation of the mutant with the wild type CsPacC restored the 
phenotypes to the wild type. RNA-seq revealed differential expression patterns of some genes between wild type and the $\triangle C s P a c C$ mutant under different $\mathrm{pH}$ conditions. These results indicated that $C s P a c C$ regulates transcription of alkaline expressed genes and also genes involved in virulence on barley.

HGT or Something More Interesting? Phylogeny of a Family of Enzymes Including One for a Bioprotective Alkaloid Produced by Epichlö̈ spp. C. L. SCHARDL, University of Kentucky, Lexington, KY, USA

Fungal genes encoding biosynthetic enzymes for specialized metabolites (SM) are often reported as examples of horizontal gene transfer (HGT) between highly divergent fungal taxa, but multiple paralogy (gene duplication) and loss events are the likeliest alternative. Among the suggested HGT examples are genes for lolines, anti-insect alkaloids produced by grass-symbiotic Epichloë species. One loline biosynthesis enzyme, LolC, is a member of a wellcharacterized family including several housekeeping enzymes. I conducted phylogenetic analysis of this family to investigate whether SM genes tend to exhibit more paralogy and loss than do related housekeeping genes. Included were all LolC-related genes in genomes of 24 species from five classes of Ascomycota. The clade for each of three housekeeping genes contained a single representative from each genome, and phylogenies within these clades were mostly consistent with each other and currently accepted species relationships. In contrast, related SM genes and genes with unknown functions exhibited extensive paralogy. These included a well-supported clade of 23 genes that had five sets of paralogs, were absent from several genomes, and had a substantially different phylogeny from those of housekeeping genes. I conclude that duplications and losses of non-housekeeping genes, together with selection for their abundance or paucity in different fungal groups as observed for loline genes, may be much more important than HGT in evolution and niche specialization of plant-pathogenic and plant-symbiotic fungi.

The expanded lineage-specific $\mathrm{C}_{2} \mathrm{H}_{2}$-homeobox transcription factors regulate microsclerotia formation and virulence in Verticillium dahliae Y. Fang, C. Deng, C. Tian, Y. WANG, Beijing Forestry University, Beijing, CHINA

The fungus Verticillium dahliae causes vascular wilt diseases on a broad range of plant species. $V$. dahliae is soilborne and widely distributed. It forms melanized microsclerotia that are capable of survival in the soil for more than ten years and can act as the primary source of infection. Genome-wide analyses of the $\mathrm{C}_{2} \mathrm{H}_{2}$-homeobox-encoding genes revealed significant expansion of this gene family in Verticillium. Out of seven genes identified, two of $\mathrm{C}_{2} \mathrm{H}_{2}$-homeobox genes were located in the lineage-specific region 2 of VdLs.17 isolate. In this study, we investigated the expansion of this gene family and the specific roles of these genes in microsclerotia formation and virulence in $V$. dahliae. Interestingly, though not encoded in the core genome, the LS-associated gene deletion mutant VDAG_04891 showed deficiencies not only in virulence, but also in microsclerotia formation. Other gene deletion mutants exhibited undistinguishable phenotypes compared with the wild-type. Using RNA-Seq methods, we identified genes regulated by $\mathrm{C}_{2} \mathrm{H}_{2}-$ homeobox during microsclerotia formation and plant infection. Furthermore, we explored the specific roles of each $\mathrm{C}_{2} \mathrm{H}_{2}$-homeobox transcription factors in growth, development and pathogenesis. The results reveal the biological significance of expanded $\mathrm{C}_{2} \mathrm{H}_{2}$-homeobox transcription factors and provide evidence to assign functions of the LS-associated transcription factor to roles in both microsclerotia formation and plant infection in $V$. dahliae.

Use of a Tobacco mosaic virus-based vector for the identification of $16 \mathrm{SrIII-J}$ phytoplasma effector proteins C. Gamboa, N. Quiroga, N. Fiore, A. ZAMORANO, University of Chile, Santiago, CHILE

16SrIII-J phytoplasma is a pathogenic bacteria with an extensive distribution in Chile and South America, and it is associated to several diseases in woody and herbaceous hosts. Regarding the importance of these phytoplasma, it is necessary to understand the mechanisms of pathogenicity involved in the symptom induction. Many phytoplasma belonging to different ribosomal groups, are able to induce symptoms in plants by a single protein that interferes in gene expression. Thus, in this work we propose to determine the impact of the transient expression of two potential $16 \mathrm{SrIII-J}$ phytoplasma effectors, orthologs to SAP54 and SAP05, using a viral vector based in a hypovirulent strain of TMV (pBSG-1057). After vector construction by stickyend ligation and T7 polymerase in vitro transcription, 10 plants of Nicotiana benthamiana and Arabidopsis thaliana ecotype Shahdara were inoculated with viral RNA using bentonite protocol. Phytoplasma genes were detected in non-inoculated leaves 7 days post-inoculation. 4 weeks post-inoculation, plants exhibited symptoms of leafy-flower and leaf alterations in SAP54-like and SAP05-like inoculations, respectively. No symptoms were observed in TMV-GFP inoculated plants. This is the first identification of effector proteins in a phytoplasma belonging to 16SrIII ribosomal group. This work also represents the initial step for the understanding of the mechanisms underlying the pathogenicity and polyphagia associated 16SrIII-J phytoplasma.

\section{Evaluation of potential trap crops for management of root-knot nematode on carrots} B. B. WESTERDAHL, University of California, Davis, Davis, CA, USA

In trap cropping, a host is planted and larvae of a sedentary parasitic nematode such as root-knot are induced to enter and establish a feeding site. Once this has occurred, and the female begins to mature, she is unable to leave the root. The plants are then destroyed before egg laying by nematodes is initiated, trapping nematodes within the root. A field trial was conducted in a field with an established population of root-knot nematode (Meloidogyne javanica). There were 20-treatments, consisting of five replicates in a randomized complete block design. Five trap crops (carrots, beans, sugarbeets, tomatoes, sesame), plus 1,3-D (Telone II) as a chemical standard, and a wet fallow treatment to germinate weeds were tested. Trap crops were destroyed at three weeks after planting either by tillage, application of glyphosate (Roundup), or both; followed by planting of carrots. Dry fallow $+1,3-\mathrm{D}+$ tillage, Sesame+tillage, and Carrot+tillage provided an increase in the percent marketable carrots based on either number of carrots or weight of carrots compared to the untreated $(P=0.05)$. At $P=0.05$, the following 11 treatments had fewer root-knot nematode juveniles in soil at harvest than the untreated control: Dry fallow+1,3-D+tillage, Wet fallow+Glyphosate, Sesame+Glyphosate+tillage, Carrot+tillage, Carrot+Glyphosate, Carrot+Glyphosate+tillage, Beans+Glyphosate, Sugarbeet+Glyphosate, Sugarbeet +Glyphosate+tillage, Tomatoes+Glyphosate, and Tomatoes+Glyphosate+tillage.

Reproduction potential of soybean cyst nematode, Heterodera glycines, and synergetic interaction with Fusarium virguliforme on dry bean cultivars

M. L. FALL (1,2), J. L. Jacobs (2), A. Stouffer-Hopkins (2), F. W. Warner (2), M. Chilvers (2), (1) Agriculture and AgriFood Canada, Saint-Jean-surRichelieu, QC, CANADA; (2) Michigan State University, East Lansing, MI, USA

Dry bean has been known as a host of soybean cyst nematode (SCN), by Heterodera glycines, since it was first reported in Japan in the 1930s. However, there are few studies on SCN reproduction potential on dry bean and its interaction with Fusarium virguliforme, the causal agent of sudden death syndrome (SDS). Three growth chamber studies and two years of field experiments were conducted to determine the reproduction potential of $H$. glycines and its interaction with $F$. virguliforme on 10 dry bean classes grown in the Midwestern United States in comparison with 3 soybean varieties. Soybean cyst nematode reproduced on all 10 dry bean classes, on the susceptible soybean variety (Archer) and on the resistant soybean variety (PI 88788). Based on the female indices (FIs), only Peking (soybean) was consistently classified as resistant $(\mathrm{FI}<10)$ across all the experiments. According 
to the least-squares means analysis, only the Zorro, Matterhorn and Merlot dry bean classes were less susceptible to SCN than the susceptible soybean variety. Foliar SDS like symptoms were observed on the soybean check and on most of the dry bean classes. Among all soybean varieties and dry bean classes, only Vista did not express foliar symptoms despite the presence of $F$. virguliforme in the roots. A polynomial model described a synergetic relationship between the number of cysts, the female index and the relative DNA concentration of $F$. virguliforme. The results suggested that SCN and SDS represent a serious threat not only to soybeans, but also to dry beans.

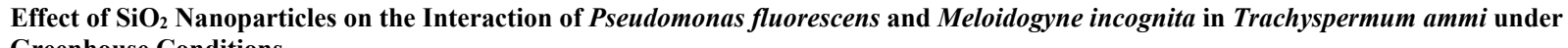
Greenhouse Conditions

M. DANISH (1), H. Sheikh (2), (1) Section of Plant Pathology and Nematology, Dept of Botany, Aligarh Muslim University, Aligarh, INDIA; (2) Section of Plant Pathology and Nematology, Dept of Botany, Aligarh Muslim University, Aligarh, INDIA

Root-knot nematodes, Meloidogyne spp. are a major threat to all the agriculture crops. Chemical control is very effective for nematode management, but it leaves several non-specific effects on beneficial organisms along with the pathogen. For alleviation of such problems an eco-friendly method is to be explored that could control nematode population effectively and efficiently. A greenhouse experiment was carried out with the aim to provide an insight into the effects of $\mathrm{SiO}_{2}$ nanopaticle induced changes in Trachyspermum ammi, an important medicinal plant, inoculated with Meloidogyne incognita and Pseudomonas fluorescens. The results revealed that application of $200 \mathrm{ppm}$ concentration of $\mathrm{SiO}_{2}$ nanaoparticles in the plants infected with M. incognita and $P$. fluorescens caused significant increase in both the plant growth as well as physiological parameters, in comparison to nematode inoculated plant and the values were found to be at par with the control plants. Higher concentrations, however, produced inhibitory effects in a concentration dependent manner. The plant inoculated with $M$. incognita and $P$. fluorescens, and treated with low concentration of $\mathrm{SiO}_{2}$ nanoparticles exhibited increase in colonization of $P$. fluorescens in the rhizospheric environment and reduction in nematode infestation and gall number. Hence, it was concluded that low concentration of $\mathrm{SiO}_{2}$ nanoparticles along with $P$. fluorescens could be used as an effective measure for managing the disease caused by $M$. incognita.

Occurrence and distribution of plant- parasitic nematodes of blueberry in Georgia

G. B. JAGDALE, L. Arnold-Smith, J. P. Noe, P. M. Brannen, University of Georgia, Athens, GA, USA

In Georgia (U.S.), blueberry (Vaccinium spp.) currently grow on 12,141 hectares with a $\$ 255$ million farm gate value, but the widespread occurrence of ring nematodes, Mesocriconema ornatum indicates blueberry replant disease (BRD), characterized by stunted growth and reduced yields, is a major limitation to continued production on existing farms. Since other plant-parasitic nematodes (PPNs) can also limit yield, we examined their occurrence on rabbiteye (RBE) and southern highbush (SHB) blueberries from 2012-2017. The UGA Nematode Diagnostic Laboratory received 241 soil samples (161 samples from SHB +80 samples from RBE) from 50 blueberry farms across 17 counties. PPNs extracted from soil were identified to genus and densities were recorded. Ten PPNs including Xiphinema, Hoplolaimus, Pratylenchus, Mesocriconema, Meloidogyne, Hemicycliophora, Helicotylenchus, Paratrichodorus, Belonolaimus and Tylenchorhynchus were common to both RBE and SHB spp., but Dolichodorus and Scutellonema were associated only with SHB. The frequency of PPN occurrence varied by blueberry species; Mesocriconema, Paratrichodorus, Pratylenchus, Xiphinema and Belonolaimus occurrence was higher in RBE $(87.5,35.0,12.5,8.8$ and 5.0\%, respectively) than in SHB $(74.5,23.6,6.2,4.3$ and $1.9 \%$, respectively); in contrast, the frequency of occurrence of Hemicycliophora and Meloidogyne (6.8, and 5.0\%, respectively) was higher in SHB than in RBE (2.5 and $1.3 \%$, respectively). Frequencies of occurrence of Helicotylenchus, Tylenchorhynchus and Hoplolaimus were comparable in both SHB (35.4, 20.5 and 6.8\%, respectively) and $\operatorname{RBE}(32.5,17.5$ and $6.3 \%$, respectively). Knowledge of PPN species distribution can help growers develop control strategies that promote long-term control, reduce the impact of BRD and improve yields.

Adaptation of the causal agent of late blight, Phytophthora infestans, to climate change A. CORDOBA, G. Danies, S. Restrepo, Universidad de los Andes, Bogota, COLOMBIA

Phytophthora infestans, is the causal agent of late blight disease of potatoes and tomatoes. This pathogen is highly dependent on environmental factors such as temperature and relative humidity to fully develop its life cycle. Due to climate change, patterns in the development of plant diseases are expected to occur. Previous studies have investigated the effect of temperature on the rate of sporangial germination, the development of the infection, and mycelial growth. However, the effect relative humidity variation on the pathogen's life cycle has been scarcely studied. Here, we investigated the effect of temperature and relative humidity on growth and disease progression of isolates of $P$. infestans to assess the adaptability of this pathogen under different climate change scenarios. The in vitro biomass production was evaluated at five temperatures $\left(5,10,15,20\right.$, and $\left.25^{\circ} \mathrm{C}\right)$ and a relative humidity of $100 \%$. The in vivo lesion area and production of sporangia were assessed by means of detached-leaf assays under the same five temperatures and a relative humidity of $100 \%$. Lastly, both in vitro and in vivo assays were conducted at three different relative humidity ranges $(40-50 \%, 60-70 \%$, and $80-$ $90 \%)$ at a temperature of $18^{\circ} \mathrm{C}$. This study provides further insights about $P$. infestans' adaptability to changes in temperature and relative humidity.

Pythium and Phytopythium associated with Soybean in Buenos Aires (Argentina)

P. E. GRIJALBA (1), A. D. C. Ridao (2), M. Steciow (3), (1) Univ. de Buenos Aires, Ciudad Autónoma de Buenos Aires, ARGENTINA; (2) Facultad de Ciencias Agrarias. Universidad de Mar del Plata, Balcarce, ARGENTINA; (3) Instituto Spegazzini. FCNyM. Universidad Nacional de La Plata, La Plata, ARGENTINA

Pythium and Phytopythium cause seed and root rot, and damping off in diverse plant species. The aim of this research was to identify these pathogens associated with soybean in Argentina. From 2013 to 2016, 85 soybean production fields were surveyed in Northern (NBA) and South-Eastern (SEBA) Buenos Aires Province. Isolates were obtained from seedlings and soil, and also from harvested seeds, using selective media. The isolates were characterized by classical and molecular methods. From seedlings and soil, from NBA 181 isolates were recovered which corresponded to 8 species of Pythium: ultimum, irregulare, sylvaticum, inflatum, aphanidermatum, dissotocum, catenulatum, longandrum and $P$. sp., and to 4 species of Phytopythium: helicoides, frezium sp. nov., chamaehyphon and Phy. sp. P. ultimum and P. irregulare were the most abundant $(41,7 \%$ and $22,5 \%$ respectively). From SEBA, 102 isolates were recovered, which belonged to Pythium: ultimum, irregulare, sylvaticum and paroecandrum, being the first two the most abundant (66,4\% y $23,2 \%$ respectively). From seeds, 2 isolates, $P$. sylvaticum and $P$. nunn, were obtained from SEBA, and 30 from NBA, identified as $P$. irregulare, $P$. paroecandrum, $P$. heterothallicum, $P$. acanthicum, $P$. sp. and Phy. vexans. The isolates presented different degrees of pathogenicity, ranging from highly pathogenic to non-pathogenic. This is the first report of P. nunn, P. longandrum, P. paroecandrum and $P$. heterothallicum, in Argentina.

Isolation and identification of Oomycete species from cocoa farm soils in Nigeria based on PCR analysis S. O. AIGBE (1), S. Woodward (2), (1) Ambrose Alli University, Ekpoma, NIGERIA; (2) Univ of Aberdeen, Aberdeen, SCOTLAND 
In Nigeria, Phytophthora megakarya and P. palmivora are the predominant pathogens causing black pod of cocoa (Theobroma cacao L.) resulting in yield losses of $10-80 \%$. Cocoa is the most important tree crop to the Nigerian economy. Since soil is the primary source of Oomycetes infecting cocoa pods, their diversity was studied in soil around black pod-infected cocoa trees on 50 farms in the major cocoa-producing states of Southern Nigeria. Soil samples (500 total) were collected from April to June 2015. Isolations were done by baiting with young oak and Rhododendron leaves and plating on PARPH-V8 selective medium. DNA was extracted using a QiagenDNeasy Plant Mini Kit and PCR products were purified with a QIAquick PCR Purification Kit after electrophoresis. PCR amplifications were run using the Oomycete-specific primer pair ITS4/ITS6. DNA sequences were compared to published sequences in GenBank using BLASTn. An abundance of Phytopythium and Pythium species were found, but $P$. megakarya or $P$. palmivora were not detected. The most common species obtained were Phytopythium vexans, Pythium cucurbitacearum, $P$. deliense, $P$. acanthicum, $P$. oligandrum and $P$. acanthophoron. Pythium cucurbitacearum, $P$. deliense and Phytopythium vexans have been reported previously as damping-off and rot pathogens of many economically important crops but their impact on cocoa needs to be investigated. Pythium oligandrum, P. acanthicum and P. acanthophoron are mycoparasites of important crop pathogens, including Phytophthora spp. Further isolations of Oomycete pathogens directly from infected cocoa pods and stems are currently being planned.

Phytophthora nicotianae and P. palmivora: Emerging pathogens of hybrid lavender (Lavandula $\times$ intermedia) D. DLUGOS, S. N. Jeffers, Clemson University, Clemson, SC, USA

Hybrid lavender (Lavandula $\times$ intermedia) is one of the most popular lavender species grown commercially in the United States due to its hardiness and oil yields. Phytophthora root and crown rot (PRCR), caused by several different species of Phytophthora, is known to occur on English lavender ( $L$. angustifolia) but has not been documented on hybrid lavender. Since 2015, hybrid lavender plants from farms and nurseries across the US have been assayed for PRCR, and P. nicotianae and P. palmivora have been isolated most frequently. To complete Koch's Postulates, isolates of both species were obtained from infected roots or infested soil around diseased plants. Inocula were prepared from two isolates of each species by growing isolates on vermiculite moistened with V8 broth. Four-month-old plants of $L$. ×intermedia 'Grosso' and 'Phenomenal' were not inoculated or were inoculated with individual isolates by mixing inoculum into the top $2 \mathrm{~cm}$ of container mix in each pot. Plants were grown for 11 weeks on a greenhouse bench and rated weekly for foliage symptoms. Plants then were harvested and evaluated for root rot, fresh weights were measured, and pathogens were isolated from washed roots. PRCR symptoms developed on all inoculated plants; P. nicotianae and P. palmivora were isolated from these plants and killed some plants in each treatment. Consequently, both $P$. nicotianae and $P$. palmivora are pathogenic on hybrid lavender and pose a threat to the lavender industry.

Identification and characterization of Pythium Phytopythium and Phytophthora species in Argentina

H. E. PALMUCCI (1), P. E. Grijalba (2), R. Zapata (3), P. Berón (3), M. Steciow (4), S. M. Wolcan (5), (1) Universidad de Buenos Aires, Buenos Aires, ARGENTINA; (2) Univ. de Buenos Aires, Ciudad Autónoma de Buenos Aires, ARGENTINA; (3) University Buenos Aires, Buenos Aires,

ARGENTINA; (4) Instituto Spegazzini. FCNyM. Universidad Nacional de La Plata, La Plata, ARGENTINA; (5) CICBA, Univ Nacional de La Plata, La Plata, ARGENTINA

Many Oomycetes including species of Phytophthora, Phytopythium and Pythium cause important plant diseases around the world. In Argentina very little was known about the importance of Oomycetes; for this reason a survey was conducted in areas surrounding Buenos Aires during $2009-2017$ to learn about diseases on intensive crops. Isolates obtained between the years 2000-2008 were also received from La Plata University. Colony morphology, cardinal temperatures, morphological and molecular characters (ITS rDNA and beta tubulin gene) were evaluated. Phytophthora capsici, $P$. cinnamomi, P. cryptogea, P. nicotianae, P. taxon kelmania, P. aff. cryptogea, P. multivora and Pythium aphanidermatum, P. cylindrosporum, $P$. graminicola, $P$. intermedium, $P$. irregulare, $P$. spinosum, $P$. sylvaticum, $P$. ultimum var. ultimum, $P$. ultimum var. sporangiferum and one putative species of Phytopythium were identified. Pathogenicity test of all isolates was confirmed, fulfilling Koch's postulates. Forty four host-pathogen relationships in 29 different hosts were studied, 30 of which were new diseases in the country. Phytophthora multivora, Pythium cylindrosporum, $P$. sylvaticum and $P$. splendens were recorded for the first time in Argentina.

A proposed model for mating type determination in Phytophthora infestans

L. GUO, X. Wang, China Agricultural University, Beijing, CHINA

Phytophthora infestans is the causal agent of late blight, the most devastating disease on potato. P. infestans is a heterothallic oomycete, which commonly requires two opposite mating types, A1 and A2 to complete sexual reproduction. Sexual reproduction is important for disease epidemics and the evolution of the pathogen, which will input difficulty for disease management. In a recent survey of $P$. infestans population in China, many selffertile isolates were obtained and their ability of $\alpha$ hormones production and reception were assayed using polycarbonate membrane method described by Ko. Results showed that these self-fertile isolates could receive $\alpha$ hormone produced by themselves, just like homothallic Phytophthora spp. Based on these findings and the facts that the inheritance of the mating type doesn't follow the Mandelian inheritance and the sexual reproduction of Phytophthora is hormonal regulated, we proposed a hypothesis that the genes encoding the production and the reception of $\alpha 1$ and $\alpha 2$ hormones co-exist in each genome of heterothallic $P$. infestans isolate, the expression of these genes are regulated by a epigenetic mechanism and determine the appearance of mating type of the isolate. This proposed model is helpful to explore the mechanisms of sexual reproduction and the regulation of hormone production and reception in oomycete.

Root and Crown Rot of Walnut in Chile primarily affected by Phytophthora species

N. RIQUELME (1), J. Guajardo (1), S. Sáa (1), G. T. Browne (2), C. Youlton (1), X. Besoain (1), (1) Pontificia Universidad Católica de Valparaíso, Quillota, CHILE; (2) USDA ARS, Davis, CA, USA

Between 2015 and 2017, a survey was carried out in orchards of walnut trees affected by root rot and crown rot, in five regions of central Chile. In each region, 9 orchards between 1 and 21 years of age were selected, the incidence and severity of damage associated with root and crown rot was analyzed. Roots samples from symptomatic trees were collected and cultured in semi-selective P5ARP medium to isolate pathogens from the oomycete class. From collected soil samples Phytophthora was recovered employing Rhododendron leaves bates. Pathogen identification was carried out by morphological analysis and ITS and beta-tubulin sequences in the rDNA. The most frequently isolated species was Phytophthora cinnamomi. Pathogenicity tests were performed with representative isolates of each Oomycete obtained. Phytophthora cinnamomi, Phytophthora citrophthora and Pythium ultimum proved to be pathogenic in Juglans regia; the test also showed that the $P$. cinnamomi and $P$. citrophthora isolates were more aggressive than $P$. ultimum. Multigene phylogeny based on the sequences of the nuclear ITS and beta-tubulin, and the mitochondrial cox 1 genes, showed that all isolates of $P$. cinnamomi recovered from J. regia in Chile belong to the same clade as the isolates of the pathogen from other places and hosts. In conclusion, Phytophthora cinnamomi is the main Phytophthora species affecting walnut orchards in Chile. 
Spatiotemporal dynamics of Phytophthora and Pythium communities in recycled irrigation water in a container nursery N. REDEKAR, J. Eberhart, J. L. Parke, Oregon State University, Corvallis, OR, USA

Recycling of irrigation water reduces crop production costs, but increases the risk of disease from waterborne plant pathogenic oomycetes. In a year-long study, we examined oomycetes in irrigation water sources within a commercial container nursery in Oregon. We compared filtration and leaf baiting methods using a metabarcoding approach. ITS1-amplicons were amplified from DNA on filters and rhododendron leaf baits, followed by Illumina MiSeq sequencing and analysis using manually curated sequences of oomycetes. We detected high OTU abundance of Phytophthora citricola-complex, $P$. syringae, $P$. parsiana-complex, $P$. chlamydospora, $P$. gonapodyides, $P$. irrigata, $P$. taxon oaksoil-complex, $P$. citrophthora-complex, $P$. megaspermacomplex, Pythium condricola-complex, Pythium dissotocum-complex, and Phytopythium litorale-complex. The leaf baiting method was more selective for biologically active, plant-associated Phytophthora and a few Pythium and Phytopythium species, but did not capture the entire spectrum of oomycetes detected by filtration. Species diversity and biological activity were highly influenced by season, with the greatest number of biologically active species detected in the winter and the fewest in the summer. The metabarcoding approach allowed spatiotemporal analysis of oomycete communities, contributing insights on pathogen load and dissemination, facilitating evaluation of disease risks associated with the use of recycled irrigation water.

Effect of temperature on aggressiveness of newly discovered $f s p$ of the grape downy mildew pathogen Plasmopara viticola $f$ sp riparia and $f s p$ aestivalis

R. A. MOUAFO TCHINDA (1), C. Beaulieu (2), M. L. Fall (3), O. Carisse (4), (1) Sherbrooke University, Sherbrooke, QC, CANADA; (2) Université de Sherbrooke, Sherbrooke, QC, CANADA; (3) Agriculture and Agri-Food canada, St-Jean-sur-Richelieu, QC, CANADA; (4) Agric \& Agri-Food Canada, Saint-Jean-sur-Richelieu, QC, CANADA

Grape downy mildew, caused by the oomycete Plasmopara viticola, is among the most important grapevine diseases worldwide. In recent studies, two different cryptic species of $P$. viticola were found in eastern Canada: $P$. viticola f. sp. riparia and f. sp. aestivalis. But, their epidemiology, ecology and significance for management purposes are unknown. This research aims at studying the influence of temperature on zoospores release and aggressiveness of the two newly discovered formae spacialis. The temporal dynamic of zoospore release, infection and sporulation was evaluated on grape leaf disks at six temperatures $\left(5\right.$ to $30^{\circ} \mathrm{C}, 5^{\circ} \mathrm{C}$ increments). For both genotypes, the highest zoospore release was observed after six hours at temperature ranging from 15 to $20^{\circ} \mathrm{C}$, but there was no significant difference between genotypes, except at $25^{\circ} \mathrm{C}$ where $P$. v. aestivalis showed more empty sporangia than $P$. $v$. riparia. The two genotypes germinated at all tested temperatures except at $30^{\circ} \mathrm{C}$. Both genotypes were able to infect at temperature ranging from 15 to $25^{\circ} \mathrm{C}$ and sporulated at temperature ranging from 10 to $25^{\circ} \mathrm{C}$. However, according to the least square mean analysis, combining infection efficiency, disease incidence, sporulation and latency, $P$. v. aestivalis was significantly more aggressive than $P$. v. riparia (temperature range between 15 to $25^{\circ} \mathrm{C}$ ). The knowledge gained during this project will be used to adapt downy mildew management tools and to improve disease management strategies.

Enabling recycled water use: The diversity and management of cryptic oomycete pathogens in recycled irrigation water in Mid-Atlantic nurseries

J. BEAULIEU (1), J. M. Del Castillo Munera (2), C. Delgado (1), C. L. Swett (3), (1) University of Maryland, College Park, MD, USA; (2) University of California, Davis, Davis, CA, USA; (3) University of California, Davis, CA, USA

While recycling irrigation water can reduce constraints and costs in nurseries, there is an increased risk of recirculating and spreading waterborne pathogens. Culture-based analysis revealed diverse Oomycetes (16 species) present in recycled pond water at two Mid-Atlantic nurseries in 2015, 2016, and 2017. Of these, Pythium oopapillum, Pythium aff. diclinum, Phytophthora cryptogea and Phytopythium helicoides were all pathogenic in seedling trials (90\% damping off). Pythium oopapillum, Ph. cryptogea and Ph. helicoides increased root rot severity in chrysanthemum trials (although differences were not significant $P=0.06)$, and reduced stem growth $(P<0.05)$; the latter two species also increased wilt severity $(P=0.03)$. Similar results were obtained in geranium trials (analyses underway). Slow sand filtration of pond water (site 1) reduced oomycete recovery from $100 \%$ of baits in the holding pond to $0 \%$, based on monthly analyses (April-November 2017), although recovery increased to $50 \%$ following transport to the greenhouse (species were primarily non-pathogens). Chlorine treatment (site 2) reduced oomycete recovery from $100 \%$ of baits in the pond to $33 \%$ in the spring and summer, and to $0 \%$ in the fall (2017). These results indicate that recycled water is a source of many pathogens which have not been previously described in Mid-Atlantic nurseries, and that slow sand filtration appears to be more effective than chlorine treatment in reducing pathogen load.

Detection, diversity and distribution of Phytophthora species associated with citrus decline in India A. K. DAS, ICAR-Central Citrus Research Institute, Nagpur, INDIA

Phytophthora spp. induce an array of diseases viz. root rot, crown rot, foot rot, gummosis and brown rot of fruits in citrus causing severe decline and yield losses in India. Between 2008 and 2016, over 300 citrus orchards in fifteen states (Maharashtra, Punjab, Rajasthan, Madhya Pradesh, Karnataka, Andhra Pradesh, Tamil Nadu, Odisha, West Bengal, Sikkim, Nagaland, Assam, Tripura, Arunachal Pradesh and Mizoram) were surveyed for the occurrence of Phytophthora. P. nicotianae was found predominant species followed by P. palmivora. A total of 257 isolates belonging to 11 different Phytophthora species (180 isolates of $P$. nicotianae, 47 isolates of $P$. palmivora, 7 isolates $P$. citrophthora, 9 isolates of $P$. insolita, 4 isolates of $P$. boehmeriae, 3 isolates of $P$. heveae, 2 isolates of $P$. tropicalis, 2 isolates of $P$. macrochlamydospora, 1 isolate of $P$. inundata 1 isolate of $P$. virginianalike and 1 isolate of $P$. lacustris) were recovered from rhizosphere soil and water, root, leaf, bark and fruit samples. Isolates were identified to the species level through morphological traits, PCR-RFLP analyses of the internal transcribed spacer (ITS) region and sequence analyses of the ITS region, $\beta$ tubulin gene, translation elongation factor $1 \alpha$ and cox 1 and 2 gene fragments. Detection of new spp. adds to the biodiversity of citrus Phytophthora in India. Pathogenicity tests supported their possible involvement in the decline of citrus plantations in different parts of India.

Cultural and morpho-molecular characterization of Fusarium solani and Alternaria spp. associated with fruit rot of strawberry N. MEHMOOD (1), A. Riaz (2), F. Naz (3), (1) PMAS-Arid Agriculture University, Rawalpindi, Rawalpindi, PAKISTAN; (2) PMAS- Arid Agriculture University, Rawalpindi, PAKISTAN; (3) Department of Plant Pathology, PMAS Arid Agriculture University Rawalpindi, Rawalpindi, PAKISTAN

In Pakistan, Strawberry is emerging as commercial fruit crop with good economic returns. During 2014-15 and 2015-16 survey was conducted in important strawberry growing areas of Punjab, Khyber Pukhtunkhwa and Islamabad, Pakistan. Maximum mean disease incidence of $56 \%$ fusarium fruit rot (Fusarium solani) and 8\% of Alternaria fruit rot (Alternaria alternata \& Alternaria tenuissima) was recorded respectively. Fusarium fruit rot was observed as thick, white fluffy masses of mycelium while Alternaria fruit rot was characterized as small, brown to black, irregular lesions. Pure cultures of fungi were obtained from symptomatic fruit sections and subsequently identified on morphological and molecular bases. Seventy nine isolates of $F$. solani and 19 isolates of Alternaria spp. (13 of A. tenuissima \& 6 of A. alternata) were undergone for identification and variation in cultural, pathogenic and morphological features. Significant genetic variability was seen among isolates belonging to different geographical regions. Nucleotide sequences of 
11 highly pathogenic isolates of $F$. solani and 7 of Alternaria spp. were amplified with transcribed spacer region (ITS), translation elongation factor 1alpha (TEF1- $\alpha$ ) and Endo-polygalacturonase gene regions $($ Endo- $P G)$ and submitted in the GenBank. Evolutionary history was analyzed with available sequences of $F$. solani and Alternaria spp. Evolutionary trees were constructed by MEGA 7.0 using maximum likelihood method having isolates falling under different sub-trees due to genetic variation in nucleotides. Cultural, morphological, pathogenic and molecular characterization of pathogens associated with fruit rot of strawberry was first time recorded in Pakistan.

Colletotrichum spp. causing anthracnose of Capsicum annuum and Cap. frutescens in Peninsular Malaysia L. ZAKARIA, N. Mohd Nor, Universiti Sains Malaysia, Minden, MALAYSIA

Colletotrichum species are pathogens of chili anthracnose worldwide. Most reports on chilli anthracnose in Peninsular Malaysia were based on the work done in the 1980s and relied on morphological characteristics as well as ITS sequences for species identification. Recent studies based on molecular identification and phylogenetic analysis of ITS regions, as well as $\beta$-tubulin, actin, and glyceraldehyde-3-phosphate dehydrogenase genes, identified five species - C. siamense, C. fructicola, C. scovillei, C. fioriniae, and C. truncatum - that were associated with anthracnose of green and red Cap. annuum and Cap. frutescens in Peninsular Malaysia. Phylogenetic analysis using combined sequences showed that the isolates of the same species were grouped with the epitype strains. Pathogenicity testing showed that the tested isolates from each species were pathogenic to green and red Cap. annuum and Cap. frutescens upon treatment of wounded fruit, using both mycelial plugs and conidial suspensions as inoculum. Only five isolates of $C$. truncatum and seven isolates of $C$. scovillei were found to be pathogenic upon treatment of non-wounded fruit. The occurrence of five Colletotrichum spp. associated with chili anthracnose indicates that correct species identification is important to formulate not only effective disease management, but also effective quarantine policy.

Characterization of disease causing agent of apical necrosis of mango

S. IRAM, Fatima Jinnah Women University, Rawalpindi, Rawalpindi, PAKISTAN

Bacterial apical necrosis is observed as a major threat for healthy mango production in different mango orchards of Punjab, Pakistan during the field survey conducted in three selected districts of Punjab viz. Multan, Khanewal and Muzaffargarh in 2015. Symptoms of bacterial apical necrosis were noticed on newly buds, stems and leaves during the survey. To validate the status of mango apical necrosis thirty eight orchards were visited in these three selected districts with the objective to assess the prevalence, incidence and severity of disease and to characterize the pathogen causing the disease at molecular level. According to the results, disorder was found widely distributed with $100 \%$ prevalence in Multan and Khanewal and $70 \%$ in Muzaffargarh. The highest $(0.9 \%)$ bacterial apical necrosis incidence was recorded in Multan and Khanewal followed by Muzaffargarh where the lowest $(0.5 \%)$ incidence was recorded. The maximum (4-5\%) severity of bacterial apical necrosis was observed in Multan and Khanewal while minimum (3$4 \%$ ) disease severity was observed in Muzaffargarh. Overall disease index in Multan (31\%), Khanewal (12\%) and Muzaffargarh (8\%) were recorded. Samples of diseased mango buds were collected from each location surveyed, isolated on Nutrient Agar and Kings' B media. Using "toothpick method" DNA with high molecular weight was extracted and molecular based analysis was done using the primers 27F and 1492R. The strains was identified based on 16S rRNA gene analysis as Pseudomonas syringae strain ICMP 3023, Pseudomonas syringae strain NCPPB 281, Pseudomonas syringae strain ATCC 19310, Pseudomonas syringae pv. tomato strain DC3000, Pseudomonas putida strain ATCC 12633, Pseudomonas savastanoi strain ATCC 13522, Pseudomonas syringae pv. phaseolicola 1448A strain.

\author{
Identification of species of Ganoderma and Assessment of Basal Stem Rot Disease in Oil palm Plantations of the Cameroon Development \\ Cooperation \\ T. ROSEMARY KINGE (1,2), A. Mathias Mih (3), (1) University of Florida, Gainsville, FL, USA; (2) The University of Bamenda, Bamenda, \\ CAMEROON; (3) University of Buea, Buea, CAMEROON
}

Oil palm is an important estate crop in Cameroon because it produces crude and kennel oil which has diverse uses in cooking and industrial applications. However, basal stem rot disease caused by different Ganoderma species seriously reduced yield in plantations. The objective was to identify the species, carryout a disease assessment and elucidate the effect of soil physiochemical properties on disease incidence and severity. The Incidence and severity of basal stem rot disease was studied in five plantations. Seasonal monitoring on 2 ha plots of different ages at these locations was done. Soil

physiochemical analysis was carried out. Molecular identification was inferred using the ITS and mtSSU rDNA. The results showed that during the first year of observation disease incidence ranged from $3.9 \%$ in Bota to $23 \%$ in Mungo of 16 year old palms. By the second year of observation, the incidence had more than doubled in all the estates surveyed, ranging from 6.8 in Bota to as high as 55\% in Mungo. Severity was also highest at Mungo and least at Bota. Although the first four principal components were strongly associated with soil properties and accounted for $100 \%$ variation in incidence and severity, disease incidence and severity only had a strong positive correlation with fine sand content and a strong negative correlation with $\mathrm{C} / \mathrm{N}$ ratio. Seven species; G. ryvardense, G. lobenense, G. tornatum, G. chalceum, G. steyaertanum, G. zonatum and Ganoderma sp. 3. were associated with basal stem rot disease of oil palm. This study has established the serious epiphytotic potential of the basal stem rot disease of oil palm in Cameroon which is important in establishing appropriate control measures.

New methods for testing rice seed: LAMP assays for the detection of Fusarium fujikuroi and Magnaporthe oryzae S. FRANCO ORTEGA (1), J. A. Tomlinson (2), J. Hodgetts (3), D. Spadaro (4), N. Boonham (3), M. L. Gullino (1), A. Garibaldi (1), (1) Agroinnova University of Torino, Grugliasco, Torino, ITALY; (2) Fera, York, UNITED KINGDOM; (3) Fera Science Ltd, York, UNITED KINGDOM; (4) DISAFA and AGROINNOVA, University of Torino, Torino, ITALY

Fusarium fujikuroi and Magnaporthe oryzae are the causal agents of bakanae and rice blast, respectively. The estimated worldwide losses caused by both pathogens can reach up to $30 \%$ of the total production. The identification of both fungi on rice seed is a prerequisite for the pathogen-free certification and essential for the control of the pathogens. ISTA currently recomends methods for the detection of rice pathogens, based on the plating of 400 seeds and morphological identification of cultures growing on the seeds 10-days post-plating. This method may result in misidentifications due to the high number of shared characteristics among closely related species and the co-growth of multiple organisms. The LAMP assays developed in this study can overcome the drawback caused by culturing-methods. The LAMP assays were designed from the elongation factor 1-alpha and calmodulin genes for $F$. fujikuroi and M. oryzae, respectively. Both assays were validated according to the international EPPO standard (PM7/98) in terms of specificity, sensitivity, reproducibility and repeatibility. The results showed a limit of detection of 100-999 fg DNA for $F$. fujikuroi and 10-99 pg for M. oryzae. Five infected rice seed lots were used to compare the traditional culturing method with the LAMP method using a commercial DNA extraction kit. The results demonstrated the reliability of the LAMP methods for the surveillance of $F$. fujikuroi and M. oryzae in seed testing laboratories. 
Development of genome-informed diagnostics for detection of Pectobacterium species using recombinase polymerase amplification coupled with LFD

F. A. AHMED (1), A. Larrea (2), M. Arif (2), A. M. Alvarez (2), (1) University of Hawaii At Manoa, Honolulu, HI, USA; (2) University of Hawaii at Manoa, Honolulu, HI, USA

Pectobacterium species can be devastating pathogens that cause bacterial soft rot diseases of a number of agroeconomically important fruit and vegetable crops worldwide, including tomato and potato. A rapid, accurate, and simple method is required to detect bacterial infections for timely management of disease. Recombinase Polymerase Amplification (RPA) coupled with a Lateral Flow Device (LFD) was developed for specific and sensitive detection of Pectobacterium directly from infected plant tissue without the need for DNA purification. The specificity of RPA was tested against 27 strains of different Pectobacterium species and 12 non-Pectobacterium sp. including Dickeya sp. No false positives or negatives were observed. RPA primers and probes were designed to target tomato and potato host genome regions to enhance the reliability of the assay. Assays specifically detected the target pathogen in infected host tissues. The detection limit for both sensitivity and spiked sensitivity assays was down to 10 fg. RPA showed no inhibitory effects when pathogen or host targets were directly detected in infected potato and tomato sap. This RPA method has applications in diagnostics at pointof-care, surveillance, biosecurity, disease management and epidemiological studies.

Biosurveillance for precision disease management of Pseudoperonospora cubensis, the cucurbit downy mildew pathogen A. RAHMAN (1), L. M. Quesada (2), (1) NCSU, Raleigh, NC, USA; (2) North Carolina State University, Raleigh, NC, USA

Pseudoperonospora cubensis, an obligate oomycete pathogen, causes cucurbit downy mildew (CDM) on a broad range of host plants including cucumber, cantaloupe, watermelon, pumpkin, and squash. In 2004, CDM re-emerged in the United States (US) by overcoming host resistance in cucumber and fungicides used for disease control in other cucurbits. Airborne sporangia of $P$. cubensis, the most important source of inoculum, can survive in no frost areas and travel long distances infecting cucurbits along the eastern US throughout the growing season. Using Next Generation Sequencing (NGS) and bioinformatics, species-specific and host-preference diagnostic markers were identified in the nuclear genome and developed into qPCR assays. Assays were tested for specificity and sensitivity using spore trap sampling rods, and a threshold detection level of $P$. cubensis sporangia and sporangia-DNA was determined to be 10 spores and $10 \mathrm{pg} / \mu 1$, respectively. Assays for detection of fungicide resistance are also being developed. Pathogen trap plots and roto-rod spore traps were deployed at two Research Stations in North Carolina for field validation. Our findings will significantly improve $P$. cubensis biosurveillance efforts and decision tools for growers by monitoring inoculum levels, cucurbit crops at risk of infection, and fungicide resistance occurrence throughout the growing season.

Specific detection of the wheat blast pathogen (Magnaporthe oryzae Triticum) by loop-mediated isothermal amplification J. YASUHARA-BELL (1), K. F. Pedley (2), J. P. Stack (1), (1) Kansas State University, Manhattan, KS, USA; (2) USDA ARS FDWSRU, Ft. Detrick, MD, USA

Wheat blast, caused by Magnaporthe oryzae Triticum (MoT) pathotype, is an economically important fungal disease of wheat. Fusarium head scab of wheat produces symptoms similar to blast and can cause confusion in the field. Currently, no in-field diagnostic exists for MoT. To address this, loopmediated isothermal amplification (LAMP) primers were designed to target the PoT2 and MoT3 loci, previously shown specific for M. oryzae and MoT, respectively. Assay specificity was determined using gDNA from $104 \mathrm{M}$. oryzae strains collected from infected wheat and other grasses and representing geographic and temporal variation. Negative controls included Fusarium graminearum DNA. Sensitivity was assessed using 10-fold serial dilutions of MoT gDNA. PoT2- and MoT3-based assays showed high specificity for M. oryzae and MoT, respectively. Both assays were sensitive to $\sim 5 \mathrm{pg}$ DNA/reaction. For field application, PoT2 and MoT3 assays were tested on MoT-infected wheat seed and spikes and identified M. oryzae and MoT, respectively, using a field DNA extraction kit and the portable Genie II system. The mitochondrial nad5 gene (NADH-dehydrogenase) was chosen as an internal control to target plant DNA. Nad5 was multiplexed with PoT2 and MoT3 and showed comparable results to individual assays. These results show applicability of these assays for MoT field surveillance, as well as identifying non-wheat species that may serve as a reservoir and/or source of inoculum for nearby wheat fields.

Development of a non-destructive high-throughput DNA extraction method for pulse seed-borne disease diagnostics and breeding applications F. CRUTCHER (1), S. Hoesel (1), B. Agindotan PhD (2), A. Owati (2), K. Mcphee (2), (1) Montana State University EARC, Sidney, MT, USA; (2) Montana State University, Bozeman, MT, USA

Pulses are an important rotational crop for Montana crop production. Chickpea, lentil, and pea have replaced fallow in many growing systems, improving sustainability in the region. Pulses use less water, reduce the need for fertilization due to symbiosis with nitrogen fixing rhizobia, and result in improved soil structure and decreased erosion. Montana pulse production increased to more than $1.2 \mathrm{M}$ acres in 2016 . However, this increase has resulted in a proliferation of pulse diseases, some of which are spread through seed infestation, threatening regional pulse production. Diagnostic laboratories identify seed-borne diseases, in particular those caused by fungi and bacteria, using traditional seed certification methods. These methods require extensive time and manpower, which can increase costs. A rapid seed DNA extraction protocol has been developed to meet the challenges of disease diagnostics in pea, specifically for Ascochyta pisi. This protocol has also been modified for marker screening of germplasm developed by the MSU Pulse Breeding Program for PSbMV resistance. The methods developed from this work will increase marker screening efficiency for the MSU Breeding Program and be used for rapid pathogen identification to decrease certification times, providing growers more planting options.

Prevalence of Fungal Diseases in Amurum Forest Reserve, Plateau State, Nigeria C. A. AMIENYO, University of Jos, Nigeria, Jos, NIGERIA

A study on the prevalence of fungal diseases in Amurum Forest Reserve, Jos Plateau State, recognized internationally as an Important Bird Area (IBA) was carried out between August and September 2016. The reserve comprise of three major habitats-The Gallery Forest, Dry Savanna, and Rocky Outcrops. The tree surveys were done on Croton L. species, Dichrostachys cinerea (L.) Wight \& Arn, Ficus L. species, Maranthes L. species, Parkia biglobosa (Jacq.) R. Br. ex G. Don f. and Syzygium guineense (Willd.) DC. being the most abundant tree species. Stratified random sampling was used in the laying of the temporary quadrat point and the location of the quadrat point was established using a Garmin Etrex ${ }^{\circledR}$ Global Positioning System device. Visual observations were made on 383 trees in 30 sample plots. Out of 383 trees recorded, $69.19 \%$ of the trees showed external disease symptoms. The highest disease incidence and severity were found to be $89.91 \%$ and $43.89 \%$ respectively in the gallery forest. Symptoms such as branch split, hollow trunk, canker, leaf blotch, dieback, leaf spots, leaf curl, and chlorosis were observed. The major genera of fungal pathogens isolated from diseased leaves were: Choanephora, Collectotrichum, Fusarium, Pestalotiopsis, Thermomyces and Trichoderma. Therefore, constant surveillance of forest tree species is necessary to help detect any threat to extinction of tree species in the reserve. 
Molecular detection and quantification of leaf rust spores in wild blueberry

N. NGUYEN, S. L. Annis, University of Maine, Orono, ME, USA

Microscopy has traditionally been incorporated with spore trap sampling to detect and quantify spores of fungal pathogens that cause wild blueberry leaf spots in Maine. However, this method is laborious and requires a great deal of technical training. To improve spore detection and quantification, quantitative polymerase chain reaction (qPCR) and quantitative loop mediated isothermal amplification (qLAMP) are being developed for Thekopsora minima, which causes leaf rust in wild blueberry. Rust urediniospores were collected from four different fields in Maine, from which DNA was extracted and then PCR was used to amplify the internal transcribed spacer (ITS) regions of ribosomal DNA. The amplicons were sequenced and analyzed to develop primers specific to T. minima. Six potential primer sequences unique to rust in the ITS regions were selected. One primer pair showed specificity for rust compared to other fungal species and was optimized for its detection limit and for qPCR. These specific primers will be modified or developed anew for qLAMP. The molecular methods will be compared to microscopy for spore quantification using spore traps and then tested for their efficacy in detecting early infection in plants. Early detection in plants and better spore counts will be coupled with weather data to determine factors affecting spore dispersal and infection to develop a more efficient forecasting system for leaf rust in blueberry.

Development of a TaqMan probe-based insulated isothermal PCR (TiiPCR) in seed detection of watermelon fruit blotch W. PEI-YI (1), Y. H. Lin (2), (1) National Pingtung University of Science and Technology, pingtung, TAIWAN; (2) National Pingtung Univ of Science $\&$ Tech, Pingtung, TAIWAN

Watermelon is an important crop of the Cucurbitaceae family in fruit production worldwide. During its production, Acidovorax citrulli is the causal agent of bacterial fruit blotch (BFB). BFB is an important international quarantine disease with zero tolerance and the infested seeds can be a primary source of inoculum in the field. Hence, a rapid and sensitive method for detecting $A$. citrulli contaminated seeds would play an important role in management of BFB. In this study, we sought to develop a method to detect contaminated seeds with $A$. citrulli based on a TaqMan probe-based insulated isothermal PCR (TiiPCR). First, specific primers and probes were designed based on the DNA fragment of $A$. citrulli genome. In our reacting conditions, results revealed the detection rate could reach $100 \%$ under 10 cells/tube. In addition, the minimal fluorescent signal intensification in this condition was over 1.8. Furthermore, detection carried out with $10 \%$ or $2 \%$ contaminated seeds still revealed $100 \%$ detection rate by TiiPCR. Therefore, we demonstrated that a rapid and sensitive detection method of BFB on seeds were established based on TiiPCR in this study.

Morphological and molecular identification of seedborne fungi in squash (Cucurbita maxima)

M. Moumni (1), M. B. Allagui (2), V. Mancini (3), S. Murolo (3), G. ROMANAZZI (4), (1) National Agricultural Institute of Tunisia, Tunis, TUNISIA; (2) National Institute for Agronomic Research of Tunisia, Ariana, TUNISIA; (3) Marche Polytechnic Univ, Ancona, ITALY; (4) Marche Polytechnic University, Ancona, ITALY

Squash (cucurbita maxima) is one of the important vegetable in tropical and temperate regions. This crop can be affected by several diseases transmitted by seeds, which become a responsible for the dispersion of many pathogens. Low percentages of seed infection can still result in severe economic losses. Early detection of seedborne fungi is the first step to control diseases. Squash fruits were collected from two regions in Tunisia and were evaluated according to three levels of lesion extension: asymptomatic fruit, infected fruit showing lesions on the squash skin without colonization of the fruit cavity, and infected fruit showing lesions that had colonized the fruit cavity. Using blotter method, morphological identification of seedborne fungi was showed that Didymella bryoniae, Alternaria alternata, Fusarium moniliforme, Fusarium solani, Myrothecium verrucaria, Myrothecium roridum and Pleospora herbarum were the most frequent fungi. Morphological identification was confirmed by molecular diagnosis using the available genusspecific and species-specific primers. Furthermore, specific primers were designed from DNA sequence in the internal transcribed spacer (ITS) region of nuclear rDNA, to identify Myrothecium spp., M. verrucaria, M. roridum and Pleospora spp. Those results can be useful to prevent squash seedborne pathogen dispersal and related losses of production later occurring in the field.

\section{Development of a novel and rapid loop-mediated isothermal amplification assay for specific detection of Alternaria alternata and Alternaria solani \\ R. CAIAZZO, T. Wood, J. Thomas, National Institute of Agricultural Botany, Cambridge, UNITED KINGDOM}

Early blight is the most significant foliar disease of potato in the USA, Asia and Africa, and incidence has been increasing in Europe and the UK too. The main species involved in the early blight complex are Alternaria solani and Alternaria alternata. Symptoms can initially appear very similar and diagnosis at the species level is only possible with microscopic assessment of spores, which is both time-consuming and requires appropriate training. Accurate identification is necessary to select appropriate control programmes. Thus a rapid, specific diagnostic assay would provide a more efficient strategy for distinguishing between Alternaria species and help to optimise control strategies. In this study, we developed and evaluated two loopmediated isothermal amplification (LAMP) assays for differential diagnosis of the two pathogens, targeting the cytochrome $\mathrm{b}$ (cytb) to detect $A$. solani, and the major allergen Alt a gene to detect $A$. alternata. The specificity of the assays was tested against a panel of closely-related species and other pathogens affecting potato. The detection limit of the LAMP assays was $3 \mathrm{pg}$ of genomic DNA per reaction for $A$. solani, and $0.3 \mathrm{pg}$ for $A$. alternata. The assays were optimized at $64^{\circ} \mathrm{C}$ and $60^{\circ} \mathrm{C}$, respectively using a $30 \mathrm{~min}$ amplification cycle. These LAMP assays have the potential to be used for monitoring the disease in the field, providing a specific, sensitive and rapid diagnostic tool for distinguishing between A. solani and A. alternata.

A multiplex PCR assay for Xanthomonas citri subsp. citri identification and pathotype determination G. Santillana (1), V. A. MAVRODIEVA (2), M. K. Nakhla (1), (1) USDA-APHIS-PPQ-S\&T-CPHST, Beltsville, MD, USA; (2) USDA APHIS PPQ S\&T CPHST, Beltsville, MD, USA

Asiatic citrus canker is a disease caused by the bacterial plant pathogen Xanthomonas citri subsp. citri (Xanthomonas citri pv. citri; Xanthomonas axonopodis pv. citri $(\mathrm{Xac})$ ). It causes distinctive necrotic raised lesions and severe infection may cause defoliation, twig dieback and general tree decline. This disease has both national and international trade implications. Three main citrus canker pathotypes have been identified namely $\mathrm{A}, \mathrm{A}^{*}$ and $\mathrm{A}^{\mathrm{w}}$, which vary in their host range. Currently, there is no simple molecular diagnostic assay to differentiate them. To address this deficiency, a multiplex PCR assay was developed that confirms citrus canker and determines the pathotype of a sample. PCR primers were designed to target pathotype-specific genomic regions identified after aligning whole genome sequences of representative subspecies. Primers were designed in Geneious ${ }^{\circledR}$ and evaluated in silico using Primer-BLAST to check cross-reactivity with non-targets. In silico analysis to evaluate the likelihood of cross-reactions among the pathotype-specific primer sets as well as with the general Xanthomonas citri subsp. citri primers was also performed. The multiplex assay correctly identified the pathotype of 59 out of 62 isolated citrus canker strains from various hosts and geographic locations. This method allows for the confirmation of citrus canker and the identification of pathotype in a single reaction, which saves time and money in the event of an outbreak. 
Detection of latent infections caused by Botrytis cinerea in flowers and fruits on apple using conventional and molecular methods in MauleRegion, Chile

E. E. FERRADA (1), B. A. Latorre (2), M. A. Lolas (1), G. A. Diaz (1), (1) Universidad de Talca, Talca, CHILE; (2) Pontificia Universidad Católica de Chile, SANTIAGO, CHILE

The major fungal problem in apple fruits in Chile is the calyx-end rot caused by Botrytis cinerea, during pre and post-harvest. The objective of this study was to determine the most effective method for early detection of latent infections caused by $B$. cinerea, using conventional and molecular methods on apples. Plant material of cvs. Cripps Pink and Fuji were collected from flowers (full bloom) and fruits (during fruit set and harvest) in three commercial orchards located in the Maule Region, Chile. Three conventional methods were assessed: i) ONFIT (overnight freezing incubation at $-20^{\circ} \mathrm{C}$ ), ii) immersion in paraquat, and iii) immersion in distillate sterile water (control), varying the times and concentrations, according to the sample (flower, fruit set and mature fruits). Molecular detection assessed conventional PCR, with specific primer of $B$. cinerea, and qPCR by using a TaqMan probe, describe previously. Results indicate that in flowers and fruit set, the most effective treatment for detection of $B$. cinerea was ONFIT, while immersion in paraquat was the most effective method with mature fruits. Conventional PCR and qPCR allow fungal detection in all flowers and fruits analyzed, with qPCR indicated variable inoculum charges according to the cultivar and locality. The use of these methods could allow to determine the inoculum pressure present in the apple orchards and estimating the prevalence of the disease in situ.

High-Resolution melting assay for identification of Colletotrichum species from strawberry and endpoint genotyping for detection of the G143A mutation

B. B. FORCELINI (1), S. Lee (2), M. Oliveira (3), N. Peres (3), (1) Gulf Coast Research and Education Center, University of Florida, Wimauma, FL, USA; (2) University of Florida, WIMAUMA, FL, USA; (3) University of Florida, Wimauma, FL, USA

Colletotrichum species cause major diseases of strawberry and disease management depends on the species present. However, species identification based on symptoms and spore morphology is difficult. Therefore, development of molecular techniques for trustworthy and high-throughput identification of Colletotrichum species are desirable. A High-Resolution Melting (HRM) assay was developed for simultaneous identification and differentiation of Colletotrichum species from fungal colonies or from symptomatic strawberry tissues. HRM markers were designed based on the ITS region of C. acutatum and C. gloeosporioides from strawberry and accurately identified and differentiated C. acutatum from C. gloeosporioides. In addition, an endpoint genotyping analysis was developed for the rapid detection of a single nucleotide polymorphism at codon 143 of the cytochrome $b$ (cytb) gene of C. acutatum and C. gloeosporioides associated with resistance to quinone-outside inhibitor fungicides. The results of this study suggest that the HRM and endpoint genotyping analyses are useful tools that can be implemented in plant diagnostic clinics for the rapid and accurate identification of Colletotrichum spp. and detection of the G143A mutation in the cytb gene of C. acutatum and C. gloeosporioides.

Identity and disease cycle of a smut fungus on wiregrass in a longleaf pine-grassland ecosystem in the southeastern USA A. ALQURASHI (1), J. L. Kerrigan (1), J. Walker (1), K. Savchenko (2), (1) Clemson University, Clemson, SC, USA; (2) Washington State University, Pullman, WA, USA

A smut fungus that hinders wiregrass restoration efforts in longleaf pine-grassland ecosystems is being investigated in North and South Carolina and Georgia. These ecosystems are unique to the southeastern USA; they are characterized by an open canopy of primarily longleaf pine (Pinus palustris) and a dense ground layer of herbaceous species. Wiregrasses, Aristida stricta and A. beyrichiana, are perennial bunchgrasses and the dominant grass found in longleaf pine forests. Once the predominant forest type in the southeast, longleaf pine forests have been reduced to a fraction of area they once covered due to land use changes and fire suppression. Seeds of Aristida species are required for regeneration efforts, but seed production has been affected adversely by a smut fungus. Smut fungi can be damaging pathogens of grasses and typically infect inflorescences of host plants, replacing the seeds with teliospores. Our objectives are to identify the smut species from A. beyrichiana and A. stricta, and to investigate the disease cycles. Based on microscopic examinations and comparisons of DNA sequences of the ITS, LSU, and GADPH regions, it is a previously undescribed species of Langdonia, which is a monophyletic genus found on Aristida species. Investigations are underway to study infection and colonization of the host by this smut pathogen. Understanding this host-pathogen system will help management efforts to increase the availability of Aristida spp. seeds.

Development of dual-labeled PNA probe-based fluorescence melting curve analysis as molecular diagnostic tool for Erwinia amylovora and $E$. pyrifoliae

B. R. Lim (1), J. Y. Song (2), S. J. Lee (3), K. H. Lee (3), J. F. Kim (2), S. H. Jo (1), M. NAM (1), (1) Xenotype Company Limited, Daejeon, KOREA; (2) Yonsei University, Seoul, KOREA; (3) Animal and Plant Quarantine Agency, Gimcheon, KOREA

Due to the recent report of fire blight outbreaks of apples and pears in Anseong, South Korea in 2015, the development of effective diagnostic methods for bacterial pathogens of fire blight is becoming more important. The diagnosis of E. amylovora has been developed in various ways, such as normal PCR assay, Taqman assay, SYBR Green assay, and lateral-flow immunographic strip methods. In this study, we identified genome-wide single nucleotide polymorphism (SNP) markers using smart sequencing by PACBIO to discriminate between E. amylovora and E. pyrifoliae and used the markers in epidemiological survey. Nine candidate SNP markers were selected and the three of them were evaluated for the performance of dual-labeled peptide nucleic acid (PNA) probe-based fluorescence melting curve analysis (FMCA) as a detection tool of E. amylovora and E. pyrifoliae. A total of $E$. amylovora isolates from various regions and related Erwinia sp. isolates (E. pyrifoliae and E. rhapontici) were investigated. Compared with the conventional methods, PNA probe-based FMCA showed improved sensitivity and more reliable diagnostic performance without false-positive results. The newly developed diagnostics method, PNA probe-based FMCA will provide more reliability and rapidity in confirmation of the E. amylovora in apple and pear trees.

The Characterization and pathology of Colletotrichum on papaya in South Africa

N. P. MTSWENI (1), E. J. van der Linde (2), M. Thaoge (3), T. Regnier (3), (1) Agricultural research council, South Africa, pretoria, SOUTH AFRICA; (2) Agricultural Research Council, Pretoria, SOUTH AFRICA; (3) Tshwane University of Technology, pretoria, SOUTH AFRICA

Colletotrichum species could cause anthracnose of papaya pre- and post-harvest, leading to crop losses worldwide. Colletotrichum isolates collected from infected papaya from various localities in the subtropical fruit producing areas of South Africa are preserved in the National Collection of Fungi. Molecular identification of the isolates was carried out through amplification of the rDNA ITS region. Seven different Colletotrichum species were identified, some of which were not previously recorded from South Africa, and/or not previously recorded from papaya. Pathogenicity tests using selected representative isolates were done by inoculation onto seemingly healthy fruit. The results revealed that various species of Colletotrichum are 
responsible for papaya anthracnose in South Africa. Sequencing results of the isolates, Koch's postulates and experiments on the potential to crossinfection of other fruits are presented. Research to develop an early detection method of these pathogens are is underway to prevent crop losses.

Specific TaqMan assay for the detection of Acidovorax valerianellae on the cotyledons of corn salad (Valerianella locusta) S. BERENDSEN, M. Westerdijk, J. Oosterhof, Rijk Zwaan Breeding B.V., De Lier, NETHERLANDS

The causal agent of the bacterial black spot disease on corn salad (Valerianella locusta) are the Gram negative bacteria Acidovorax valerianellae (Av). This bacterium has been proven to be soil and seed transmittable. The primary way to prevent corn salad infection is to start with Av free soil and seeds. ISHI-Veg has described a method for the detection of Av on corn salad seeds in which bacteria on symptomatic corn salad cotyledons from a grow-out test are confirmed by PCR. The described PCR is time consuming and lacks an internal amplification control (IAC). Therefore, a new TaqMan PCR assay was designed based on the amplification region of the ISHI-PCR. The TaqMan was validated and compared with the described ISHI-PCR utilizing a collection of Av isolates. Data reveals that the TaqMan PCR functions as good as the described PCR. To turn the reaction into a Real Time PCR duplex, we added the IAC specific TaqMan for corn salad non-pathogen bacterium Clavibacter michiganensis subsp. tesselarius (Cmt) to this assay. Av suspected and negative cotyledons were grinded in $0.85 \%$ sterile saline that was spiked with a known concentration of Cmt. Samples were processed as described by ISHI-Veg method and the crude DNA samples were tested by ISHI-PCR and the new Real-Time PCR. Based on our findings the new RealTime PCR is an improved alternative for the confirmation of Av suspected corn salad cotyledons, and will be evaluated within ISHI-Veg.

Towards improved methods for detection of Xylella fastidiosa in plant material using triplex TaqMan PCR and NGS analysis P. J. M. BONANTS, Y. Griekspoor, I. Houwers, M. Krijger, P. Zouwen van der, T. van der Lee, J. van der Wolf, Wageningen Plant Research, Wageningen, NETHERLANDS

Xylella fastidiosa is a gram-negative bacterial plant pathogen with a wide host range covering over 300 plant species. In Europe, the pathogen is causing severe disease problems since 2013, when the olive decline syndrome, caused by a specific strain of $X$. $f$. subsp. pauca, was found for the first time in the Southern part of Italy. More recently introductions in France and Spain were detected on different host plants, caused by various $X$. fastidiosa subspecies. Also interceptions of various subspecies in imported ornamental plants were diagnosed in Europe, among them Olea europaea, Coffea arabica and Nerium oleander. The host range of the pathogen can vary dependent on the subspecies and even the strain. Therefore the availability of subspecies or strain specific detection methods are of high importance in management strategies. In this study we combined two specific and sensitive TaqMan assays previously designed for $X$. fastidios $a$ with an internal control into a triplex TaqMan assay. The triplex TaqMan was compared with a single TaqMan assay often used for $X$. fastidiosa. Recently the usefulness of Next Generation Sequence (NGS) analysis was demonstrated in the field of diagnostics. Several DNA extracts from naturally infected or artificially inoculated hosts plants or plants from interceptions from France, Austria, The Netherlands, Italy and Spain, were evaluated for the presence of Xf using NGS and compared with the results of the triplex TaqMan PCR.

Potential role of soil and plant microbial communities in rapid and sudden decline of established apple trees K. J. SILVA, D. Strickland, A. Khan, Cornell University, Geneva, NY, USA

Rapid decline of established apple trees across Central and Northeastern regions of the United States, and in Ontario, Canada has been more frequently reported in recent years. Typical characteristics of sudden or rapid apple decline (RAD) syndrome are chlorotic leaves throughout the entire canopy followed by tree collapse within weeks. Possible causes include abiotic and biotic stresses, viruses, rootstock and scion compatibility, and their interactions. However, the potential role of soil and endophytes in RAD has not been studied to date. Next-generation sequencing has shown tremendous opportunity for studying phytopathogen composition and their association with plant diseases. We are analyzing the diversity of bacterial and fungal communities in the soil and rhizosphere, as well as endophytes within the rootstock and scion of both declining and healthy looking apple trees from affected orchards at sequence level to narrow down potential causes of RAD. We are using high-throughput 16S rRNA and ITS1/ITS4 rRNA amplicon sequencing, targeting V3-V4 and ITS1-ITS4 regions, respectively, of Honeycrisp cultivar grafted on M9 rootstock. A comprehensive list of endophytic microorganisms that might play a crucial role (directly or indirectly) in RAD has been developed. A more narrowly focused selection will be used for indepth analysis. The future direction of research to further understand plant-microbe interactions and their role in RAD will also be presented.

Loop Mediated Isothermal Amplification Assay for The Detection of Sugarcane White Leaf Disease

Q. NGUYEN (1), X. Nguyen (1), T. Ho (2), P. Nguyen (1), C. Nguyen (3), (1) Nong Lam University, Ho Chi Minh, VIETNAM; (2) InterInternational University, Ho Chi Minh, VIETNAM; (3) Ho Chi Minh City Open University, Ho Chi Minh, VIETNAM

Phytoplasma group 16SrXI has been known to be a causal agent of Sugarcane White Leaf Disease (SWLD) resulting in severe yield losses to the sugar industry. Although microscopy, serology based method, dot blot DNA hybridization and PCR have been developed, these techniques require intensive labor, costs, are time-consuming and can only be undertaken in well-equipped labs. Therefore, the objective of this study was to develop a closed tube loop-mediated isothermal amplification (LAMP) assay as an alternative approach for the detection of specific phytoplasmas quickly and efficiently within 30 minutes. Three LAMP primer sets including universal 16S rDNA primers, group XI 16S rDNA primers and plant cytochrome oxidase (COX) gene primers were used to detect phytoplasma DNA sequences and the house keeping gene in plant. LAMP assays using a commercially available master mix and a realtime fluorometer allowed us to detect phytoplasma within 30 minutes at $63^{\circ} \mathrm{C}$ via the visualization of amplification plot and melting curve on screen. The results obtained in this study revealed potential applicability of the closed tube LAMP assay for in- field detection of the phytoplasma causing SWLD in comparison with traditional methods and opened tube LAMP assays which are prone to cross- contamination during the implementation.

LAMP based identification of phytoplasmas associated with cassava witches' broom and sesame phyllody diseases in Vietnam X. Nguyen (1), C. Nguyen (2), T. Ho (3), P. Nguyen (1), Q. NGUYEN (1), (1) Nong Lam University, Ho Chi Minh, VIETNAM; (2) Ho Chi Minh City Open University, Ho Chi Minh, VIETNAM; (3) International University, Ho Chi Minh, VIETNAM

Witches' broom and phyllody symptoms have been caused by various groups of phytoplasmas resulting in severe yield losses of cassava and sesame respectively in Vietnam. Until now, many worldwide scientists have developed numerous techniques for phytoplasmas diagnostics, in particularly the use of nested PCR, RFLP, and sequencing. However, such methods are normally labor and time intensive and can only be taken in well-equipped labs. Therefore, an alternative method known as Loop Mediated Isothermal Amplification (LAMP) has been applied as a rapid, simple and low-cost diagnostic tool for phytoplasmas within one hour of sampling in the field that is appropriate to the poor and developing countries. In this study, the diagnostic procedures for cassava witches' broom and sesame phyllody diseases were set up and validated with universal and specific LAMP primers of 16S rRNA and plant cytochrome oxidase (COX) primers used as amplification control. Furthermore, the reliability of LAMP assay was evaluated by 
using the nested PCR and phylogenetic analysis. We found the presence of phytoplasmas groups' $16 \mathrm{SrI}$, $16 \mathrm{SrII}$ in sesame and $16 \mathrm{SrI}$, $16 \mathrm{SrII}, 16 \mathrm{SrIV}$ in cassava. The results from this study will be helpful for practical applications of LAMP-based diagnostics for the detection and control of different phytoplasmas associated diseases in plants.

Application of molecular quantification of Plasmodiophora brassicae in soil B. D. GOSSEN (1), F. Al-Daoud (2), A. Sedaghatkish (2), M. R. McDonald (2), (1) Agric \& Agri-Food Canada, Saskatoon, SK, CANADA; (2) University of Guelph, Guelph, ON, CANADA

Plasmodiophora brassicae Wor. causes clubroot, a disease with serious impact on canola (Brassica napus L.) and other brassica crops. Its long-lived resting spores and ability to erode genetic resistance make it difficult to manage, especially in an extensive field crop like canola. The resting spores are tiny and nondescript, so counts of spores extracted from soil are highly variable and inaccurate. Spore numbers are often so high in heavily contaminated soil $\left(10^{6}\right.$ to $10^{9}$ spores $\left.\mathrm{g}^{-1}\right)$ that assessment of the efficacy of IPM strategies using bioassays is impossible. Estimates of spore concentration based on molecular assessments have recently been enhanced using a competitive internal positive control (qPCR CIPC) and assessment of spore viability using pretreatment with propidium monoazide (qPCR PMA). These techniques have demonstrated that spore numbers decline rapidly in the first 2 years after a susceptible crop, and that high levels of viable spores can be present deep (e.g., 0.5-1 m) in soil. Field trials in southern Ontario Canada demonstrated that solarisation for 2 weeks can result in a substantial reduction in spore concentration. Also, forage grasses (non-hosts) that form a dense sod can stimulate germination of resting spores and reduce populations in soil. Despite these improvements, issues around collecting and subsampling representative samples are a huge constraint to the adoption of these technologies in risk assessment and disease forecasting.

Development and validation of a multiplex real-time RT-PCR for detection of citrus and hibiscus-infecting Citrus leprosis virus C2 B. ADDUCCI (1), G. Wei (1), A. Roy (1), V. A. Mavrodieva (2), M. K. Nakhla (1), (1) USDA-APHIS-PPQ-S\&T-CPHST, Beltsville, MD, USA; (2) USDA APHIS PPQ S\&T CPHST, Beltsville, MD, USA

Citrus leprosis is one of the destructive viral diseases of citrus in the Americas. Citrus leprosis virus $\mathrm{C} 2$ (CiLV-C2), the second member of the genus Cilevirus was discovered on sweet orange in Colombia in 2013. A Cilevirus infecting ornamental hibiscus was reported from Hawaii in 2013 and from Tampa, Florida in 2017. Based on amino acid sequence identity, Hibiscus-infecting Cilevirus is taxonomically reclassified as a hibiscus strain of Citrus leprosis virus $\mathrm{C} 2$ (CiLV-C2H). In order to detect both citrus and hibiscus-infecting CiLV-C2, a multiplex real-time RT-PCR (qRT-PCR) assay was developed for simultaneous detection and discrimination of CiLV-C2 and CiLV-C2H with a plant internal control. Reported here are the results of developing and validating the multiplex qRT-PCR assay on the ABI QuantStudio 7 real-time PCR platform and improvement of the internal control for the assay. The described multiplex assay can be used for a quick and reliable detection of CiLV-C2 and CiLV-C2H in single or dual infection.

Molecular detection and quantification of Xanthomonas arboricola pv. juglandis, the causal agent of walnut blight K. A. Nguyen (1), H. C. Förster (1), J. E. ADASKAVEG (2), (1) Department of Plant Pathology and Microbiology, University of California, Riverside, CA, USA; (2) Department of Microbiology and Plant Pathology, University of California, Riverside, CA, USA

Walnut blight, caused by Xanthomonas arboricola pv.juglandis (Xaj), is a serious disease worldwide. Identification of the bacterium is limited to using morphological and biochemical methods that are time- and labor-intensive. Identification of Xaj on isolation plates is often difficult because multiple bacteria of similar appearance to Xaj are often recovered from walnut tissues (Arthrobacter, Curtobacterium, Frigoribacterium, Microbacterium spp.). Using a whole genome sequence of an Xaj strain in GenBank, we identified a gene conserved in Xaj that was not shared with closely related $X$. arboricola pvs. pruni and corylina. Sequence alignment using BLASTN resulted in exclusive matches with Xaj strains. A TaqMan-based probe was designed for the gene sequence. In qPCR, 9 Xaj strains that were genetically diverse based on Rep-PCR resulted in a positive signal, whereas 15 strains of other genera listed above that were morphologically similar to Xaj did not amplify. A standard curve that was constructed using walnut bud tissue spiked with 10-fold dilutions of Xaj resulted in a detection threshold of 10 cells/ $\mu 1$. The standard curve had an $\mathrm{r}^{2}$ value of 0.999 . Quantification of Xaj in walnut buds as determined by qPCR Ct values corresponded with the number of Xaj cells recovered after isolation. These results indicate that the TaqMan probe specifically and sensitively detected Xaj in walnut tissue and can be used to identify and quantify the pathogen rapidly and efficiently.

Development of a molecular tool for the diagnosis of the different avirulence genes of Phytophthora sojae isolates found in Canada C. DUSSAULT-BENOIT (1), G. Arsenault-Labrecque (2), H. Sonah (1), R. R. Bélanger (1), (1) Université Laval, Quebec, QC, CANADA; (2) Université Laval, Québec, QC, CANADA

Phytophthora sojae is an oomycete pathogen of soybean, responsible for yearly economic losses of \$1-2 billion worldwide. The most effective method to fight this pathogen is the use of resistant genes (Rps), counterparts of avirulence genes ( $A v r)$ in $P$. sojae. In Canada, a recent survey of nearly $300 P$. sojae isolates identified many pathotypes deficient in one or several of seven Avr genes: Avrla, Avr1b, Avrlc, Avrld, Avrlk, Avr3a, Avr6. Currently, the identification of $P$. sojae pathotypes is often based on a phenotyping approach (hypocotyl assay) using soybean differential carrying $R p s$ genes, but this method is cumbersome and imprecise. In this study, our objective was to develop a molecular tool that would lead to a rapid and precise diagnosis of the presence of $A v r$ genes in $P$. sojae isolates. For this purpose, we relied on genome sequencing data of 31 isolates. Discriminant markers were identified for each $A v r$ gene. Specific primers for these markers were designed to characterize the virulence profile of each isolate. Each primer amplified DNA fragments of different lengths on an agarose gel. Subsequently, the primers were pooled together in a multiplex PCR and tested against all isolates to confirm their specificity and ability to identify the exact pathotypes of each isolate. This new molecular tool will find applications in the characterization of the precise virulence profiles of $P$. sojae isolates found in expanding soybean productions in Canada and elsewhere.

Rapid methods for detection of Phytophthora ramorum in nursery water

D. LUSTER (1), M. B. McMahon (1), S. Sharma (2), W. Schweigkofler (2), K. G. Suslow (2), T. L. Widmer (3), (1) USDA ARS FDWSRU, Frederick, MD, USA; (2) Dominican University of California, San Rafael, CA, USA; (3) USDA ARS FDWSRU, Ft. Detrick, MD, USA

Phytophthora ramorum, causal agent of sudden oak death (SOD), continues to threaten U.S. forest ecosystems and the U.S. nursery industry. Presently, USDA APHIS relies upon water baiting for P. ramorum diagnosis and confirmation in nurseries inside the boundaries of regulated zones. USDA's national water baiting program utilizes the Bottle of Bait (BOB) recovery method for $P$. ramorum, which requires collecting water from a source stream, baiting with healthy rhododendron leaves for a 3-day incubation/infection period, followed by plating on semi-selective media. More rapid methods are needed for recovery and detection of $P$. ramorum propagules from nursery water sources. We have developed and refined rapid water filtration methods for recovery of $P$. ramorum propagules using tandem filters with defined pore sizes. Novel antibodies, raised against $P$. ramorum -specific peptide epitopes in cell wall, flagellar and secreted proteins, were applied for detection of propagules by immunofluorescence directly on filters and in 
immunoassays with filter extracts. Results indicate that $P$. ramorum propagules recovered by filtration from spiked nursery water samples can be detected in immunoassays in $24 \mathrm{~h}$ or less.

The use of third generation sequencing tool for detection and identification of plant pathogens

L. CHALUPOWICZ (1), A. Beerman (1), A. Dombrovsky (2), V. Gaba (3), M. Reuven (3), G. Nissan (4), S. Manulis-Sasson (3), (1) ARO, The Volcani Center, Rishon LeZion, ISRAEL; (2) ARO Volcani Center, Rishon LeZion, ISRAEL; (3) ARO The Volcani Center, Rishon LeZion, ISRAEL; (4) Tel Aviv University, Tel Aviv, ISRAEL

Crop infections by plant pathogens are a persistent economic threat worldwide. Reliable identification of the disease causal agent is essential for disease management strategies. The diagnostic methods commonly used to detect plant pathogens have some limitations such as low sensitivity, requirement of prior knowledge of the genome sequence, and are limited in detection of several pathogens simultaneously. In recent years, the development of advanced DNA sequencing technologies has enabled determination of total nucleic acid content in biological samples and thus provides a powerful diagnostic tool In this study, we used the third generation sequencing platform of Oxford Nanopore, which enables sequencing of long reads when a single-stranded DNA molecule passes through a biological pore of the MinION sequencer. Extraction methods to obtain high molecular weight total DNA and sequence runs for 6-16 $\mathrm{h}$ were sufficient to yield enough data for real time species identification. The applicability of this technology for detection of bacterial and fungal pathogens in tomato seeds has been tested. In addition, DNA extracted from samples of symptomatic plants of unknown source were sequenced and the results provide indications for possible disease causal agents. This technology has the potential use for laboratory and field detection of plant pathogens including those that are unculturable or unknown.

Morphological and molecular characterization of Colletotrichum species causing anthracnose in soursop (Annona muricata) N. ADIKARAM (1), B. Wijeratne (2), D. Yakandawala (3), K. Yakandawala (2), (1) National Institute of Fundamental Studies, Kandy, SRI LANKA; (2) Wayamba University of Sri Lanka, Gonawila, SRI LANKA; (3) University of Peradeniya, Peradeniya, SRI LANKA

Soursop (Annona muricata), belonging to the family Annonaceae, is a popular source of fruit juice and has numerous medicinal properties. Anthracnose is a major postharvest disease of ripe soursop responsible for substantial postharvest losses. The disease was earlier believed to be caused by Colletotrichum gloeosporioides and Colletotrichum acutatum which are presently considered as species complexes. The objective of the study was to identify Colletotrichum species associated with anthracnose in ripe soursop fruit using morphological characters and multi-gene sequence analysis. Colletotrichum was isolated from anthracnose lesions on ripe soursop, collected from the Central and North Western Province of Sri Lanka. Morphometric analysis, using eleven characters ranging from colony, conidial to appressorial morphology, divided the twenty isolates into two main clusters, conidial size being the most contributing factor for cluster separation. Ten isolates representing the two clusters were subjected to DNA sequence analysis, using ITS, $\beta$-tubulin 2 (TUB2) and GAPDH as gene regions. For resulting sequences, the species affiliations and identities were determined through similarity-based searches of the NCBI GenBank Database. Considering $95 \%$ similarity, Colletotrichum siamense and Colletotrichum fructicola were identified as pathogens associated with soursop anthracnose. Both species are belonging to the C. gloeosporioides complex. ITS region contributed in placing the taxa within $C$. gloeosporioides while TUB2 and GAPDH determined their identity to species level. This is the first report of $C$. fructicola and C. siamense from soursop anthracnose.

Fatty Acid Methyl Ester (FAME) Analyses for Characterization and Detection of Grapevine Pathogens C. M. WALLIS, USDA ARS, Parlier, CA, USA

Grapevines can become infected by a variety of devastating pathogens, including the bacterium Xylella fastidiosa and canker fungi. Multiple strains of Xylella fastidiosa exist, each causing different diseases on various hosts. Although sequence-based genotyping can assist in distinguishing these strains, there is a need for phenotyping to confirm taxonomy. Likewise, there are a wide variety of fungi associated with grapevine canker diseases, but most species are difficult to distinguish from one another using existing methodologies. To address both of these issues, fingerprinting of fatty acids that comprise cell membranes of each was performed using gas chromatography-based fatty acid methyl ester analyses (FAME). Findings allow for an alternative to DNA-based identification, which could be needed in cases where nucleic acid degradation is a concern or an alternative methodology is required. Furthermore, because cell membranes mediate interactions between microorganisms and hosts, certain fatty acid profiles may be linked to increased virulence.

Soaking petiole cross-sections provides an alternative method to prepare samples for Xylella fastidiosa detection using the AmplifyRP kit R. LI, P. Russell, S. Zhang, B. Davenport, A. Eads, D. Groth-Helms, K. Schuetz, Agdia, Inc., Elkhart, IN, USA

Xylella fastidiosa (Xf), a xylem-limited bacterium, infects more than 350 plant species (including hybrids) from 204 genera of 75 different botanical families. Establishing optimized DNA extraction protocols is important for achieving reliable detection of $X f$ from different plant species. Traditional DNA extraction by grinding plant tissues and subsequent purification steps is time-consuming and laborious. Agdia has developed a sensitive isothermal AmplifyRP kit for detecting $X f$ using crude extract. However, the positive response can be inhibited when fully macerated crude extracts $(1: 10 \mathrm{wt} / \mathrm{vol})$ from select hosts and/or tissue types are used directly in the reaction. Here we have developed a simple method, called Soaking Petiole Cross-Sections (SPCS), to release $X f$ DNA from petiole tissue in AMP1 buffer (Agdia). Petiole cross-sections (2-3 $\mathrm{mm}$ in thickness) were prepared from $X f$ infectedalmond, blueberry, grapevine, and maple and soaked in AMP1 buffer $(1: 10 \mathrm{wt} / \mathrm{vol})$ for $10 \mathrm{~min}, 30 \mathrm{~min}$, and $5 \mathrm{hr}$ at room temperature. No inhibition was observed in serial dilutions of the soaking extract for the above tested plant species. Soaking for 10 min was enough to generate a similar amplification signal as for $5 \mathrm{hr}$. For leaf tissues that contain lower bacterial concentrations and/or more inhibitors SPCS can be used as an alternative method for $X f$ detection using the AmplifyRP kit. Extensive comparative evaluation of other host species is ongoing.

Detecting and quantifying latent infection of canker- and blight-causing pathogens in stone fruit and nut crops in California Y. Luo (1), F. Niederholzer (2), D. Lightle (3), T. J. Michailides (1), P. D. S. F. LICHTEMBERG (1), (1) University of California - Davis, Parlier, CA, USA; (2) Univ of California Coop Ext, Yuba City, CA, USA; (3) UC Cooperative Extension, Glenn, Butte \& Tehama Cos., Orland, CA, USA

Botryosphaeriaceae is considered as a fungal family consisting of plant pathogens, including a number of species causing canker and shoot blight diseases of woody plants. These pathogens cause latent infections of plant tissues. In order to quantify the latent infections of major canker-causing pathogens in different tree crops important in California, shoot and bud samples were randomly collected from almond, dried plum, pistachio, and walnut trees. The previously developed and used DNA primers and a $q$ PCR assay were applied to process these samples to detect and quantify six selected canker-causing pathogen groups, such as Phomopsis spp., Botryosphaeria dothidea, Lasiodiplodia spp., Cytospora spp., Neofusicoccum spp., and Diplodia spp. The concepts of molecular severity (MS) and index of latent infection (ILI) were introduced and applied to quantify the levels of latent 
infection in these samples. Variation of incidence of latent infection among pathogen groups was observed, while, Phomopsis spp. and Diplodia spp. showed low levels as compared to those of other four pathogen groups. High incidences caused by Cytospora spp. were observed for two dried plum orchards, while most orchards showed high incidence of $B$. dothidea and Lasiodiplodia spp. and moderate levels caused by Neofusicoccum spp. The MS were maintained at a certain range among pathogen groups and tree crops. The ILI values were also variable among pathogen groups and tree crops.

Detection and distribution of Aphanomyces euteiches in the United Kingdom

B. Ó LOINSIGH (1), M. J. Dickinson (2), L. Herold (3), (1) The University of Nottingham, Loughborough, UNITED KINGDOM; (2) University of Nottingham, Loughborough, UNITED KINGDOM; (3) Processors and Growers Research Organisation, Peterborough, UNITED KINGDOM

Aphanomyces root rot, caused by the oomycete Aphanomyces euteiches is one of the major constraints to pea production globally, causing up to $80 \%$ crop/yield losses per annum. A. euteiches is a strict soil borne pathogen completing its entire lifecycle in the host roots and surrounding soil. Even though the pathogen can cause such great yield losses, the spread and severity of A. euteiches across the UK growing regions is currently unknown. Therefore, due to the devastating effect Aphanomyces root rot can have on yield and a lack of effective control methods, the need for a rapid, simple, and inexpensive diagnostic detection tool is vital. In this study, we developed a loop mediated isothermal amplification (LAMP) assay for the specific detection and evaluation of $A$. euteiches from pea plate baiting experiments. The results obtained has shown high correlation between visual disease grading and LAMP amplification times, proving that this assay can be used to detect $A$. euteiches DNA on the roots of infected seedlings within days of receiving the samples. In order to estimate the degree of infestation within pea fields, we surveyed 67 sites, where soils was collected spanning the vining pea areas and tested for the presence of Aphanomyces root rot. A. euteiches was found to be present in over $60 \%$ of fields tested. Information from this study will allow a farmer to predict the inoculum potential within their fields, which, when combined with current cultural disease control methods may prevent unwarranted crop losses.

Identification of Rathayibacter and other bacteria associated with gumming disease of grasses in Oregon, U.S.A. W. Thomas, M. Wiseman, M. Wiseman, E. W. Davis, J. Chang, M. PUTNAM, Oregon State University, Botany and Plant Pathology, Corvallis, OR, USA

Rathayibacter toxicus is toxin-producing bacterium that is designated a U.S. Select Agent. It is closely related to other, non-toxic bacteria associated with gumming disease of grass seed produced in the U.S. Pacific Northwest. Accurate, rapid identification of Rathayibacter-like bacteria is an essential need. Rathayibacter can be detected with species-level (SL) PCR primers, but there is no genus-level (GL) detection to distinguish Rathayibacter from other bacterial genera, nor is there a rapid means of discriminating infected seed. We used seed washes from 699 seed lots collected between 2012 and 2015 from 13 counties in Oregon to recover bacteria associated with gumming disease. We tested whether seed rinsate could be used for culture-free detection of Rathayibacter using SL PCR primers. To identify GL primers, we analyzed whole genome sequences from approximately 120 Rathayibacter isolates and identified regions predicted to distinguish it from other genera. We tested 93 Rathayibacter spp. and genetically distant bacteria with the primers. We found the intra-genus diversity of Rathayibacter at the nucleotide level too great for sufficiently specific GL primers. Preliminary results showed seed washes to be an effective way to directly detect Rathayibacter species using SL PCR without culturing, reducing detection time from 10-14 days to two days. Additionally, we identified a new species of Rathayibacter from four different grass species.

Phylogeny and molecular diagnosis of nectriaceous fungi associated with black root rot in avocado L. PARKINSON (1), R. Shivas (2), E. K. Dann (1), (1) Queensland Alliance for Agriculture and Food Innovation, The University of Queensland, Brisbane, AUSTRALIA; (2) Centre for Crop Health, University of Southern Queensland, Toowoomba, AUSTRALIA

Root infection of avocado by some nectriaceous fungi causes black root rot, and may severely impact establishment of nursery plants in orchards. Symptoms are frequently masked in the nursery, but after transplanting include black necrotic roots, tree stunting, wilt, leaf drop, rapid decline and death within months. Nectriaceous fungi (153 isolates) from avocado and other hosts were collected from all avocado growing regions in Australia. Phylogenetic analyses of concatenated ITS, $\beta$-tubulin and histone H3 loci identified six genera, Gliocladiopsis, Cylindrocladiella, Mariannaea, Calonectria, Ilyonectria and Dactylonectria, associated with black root rot in Australia. Three new Gliocladiopsis species were formally classified and over 20 putative new species (and species complexes) across the genera remain to be resolved. Primers were designed from $\beta$-tubulin and histone $\mathrm{H} 3$ sequence data of C. ilicicola and D. macrodidyma, the two most pathogenic species, for development of a loop-mediated isothermal amplification (LAMP) assay. Specificity of detection was confirmed with 65 target, closely related and non-target species across several genera. The limit of sensitivity was $0.001 \mathrm{ng} / \mu 1$ DNA for $C$. ilicicola and $0.01 \mathrm{ng} / \mu 1$ DNA for $D$. macrodidyma. The diagnostic assay was validated using pure fungal mycelia and necrotic avocado roots from inoculated seedlings, and positive detections obtained within 12-23 min for both pathogens.

\section{Development and evaluation of a novel and rapid detection assay for Blumeria graminis f. sp. tritici based on Loop-Mediated Isothermal} Amplification

S. GONG (1,2,3), B. Yuan (1,2,3), L. Yang (1,2,3), M. Xue (1,2,3), Y. Wang (1,2,3), (1) Institute of Plant Protection and Soil Science, Hubei Academy of Agricultural Sciences, WUHAN, CHINA; (2) Key Laboratory of Integrated Pest Management on Crop in Central PRC, Ministry of Agriculture, WUHAN, CHINA; (3) Hubei Key Laboratory of Crop Diseases, Insect Pests and Weeds Control, WUHAN, CHINA

Powdery mildew, caused by Blumeria graminis f. sp. tritici, is the most common foliar disease of wheat in China. In this study, we developed a rapid method, loop-mediated isothermal amplification (LAMP), to detect B. graminis f. sp. Tritici(Bgt) in field. Eight pairs of primers were designed to amplify Bgt specific sequences, two pairs of primers showed both high efficiency and high specificity with 35 Bgt strains from wheat and 7 powdery mildew strains from other plant host. Less than $300 \mathrm{fg}$ genomic DNA is needed for detection by this method, showed comparable sensitivity with normal PCR method. The LAMP reaction could be quickly done within 60 minutes at $65^{\circ} \mathrm{C}$, and color change in magnesium pyrophosphate precipitation after adding calcein could be observed with naked eye. Since its technique simplicity, time efficiency and does not require expensive equipment, this LAMPbased diagnostic method has potential to be used under field conditions to make disease forecasting more accurate and efficient.

Root Rot Disease Caused by Fusarium solani on Gromwell in Korea

G. H. KIM, S. Y. Park, Y. J. Koh, Sunchon National University, Suncheon, KOREA

Wilted plants caused by root rot were frequently observed in the open-fields of gromwell in Jindo, Jeonnam province in Korea. Early in the course of the disease, affected plants showed wilted leaves and dark brown necrosis symptoms on stems near soil line. As the disease progressed, rotted roots were disorganized and whole plants resulted in death. A fungal isolate of Fusarium sp. was consistently obtained from symptomatic stems or roots and produced microconidia, macroconidia and chlamydospores on a carnation leaf agar plate. The fungal isolate of Fusarium sp. fulfilled Koch's postulates 
for establishing the causal agent of root rot on gromwell. On the basis of mycological, pathological characteristics and ITS rDNA sequences, the pathogen of root rot on gromwell was identified as Fusarium solani. Optimum temperature ranges for the mycelial growth of the pathogen were $24 \sim 27^{\circ} \mathrm{C}$. Wounds were necessary for the pathogen to infect gromwell plants in the fields. Overall incidence rates of root rot on gromwell were $53.7 \sim 100 \%$ in open fields during the survey periods from 2009 to 2011 , but they were $0 \sim 35.0 \%$ in rain-proof polyvinyl houses. Installation of rain-proof shelter could alleviate the occurrence of root rot epidemics known to be the most problematic limiting factor for the stable cultivation of gromwell in Jindo Island which heavy rainfalls and strong winds occurred frequently during the growing seasons of gromwell.

Canker and wood rot pathogens associated with young apple trees and propagation material in South Africa

M. Havenga (1), G. Gatsi (1), F. Halleen (2,3), C. Spies (4), R. van der Merwe (1), L. MOSTERT (3), (1) Stellenbosch University, Matieland, SOUTH AFRICA; (2) Agricultural Research Council, Stellenbosch, SOUTH AFRICA; (3) Stellenbosch University, Stellenbosch, SOUTH AFRICA; (4) Agricultural Research Council - Plant Health and Protection, Stellenbosch, SOUTH AFRICA

Canker and wood rot pathogens can cause dieback and decline of young apple trees. This study assessed the occurrence of canker and wood rot pathogens in nursery apple trees and propagation material. Isolations were made from discoloured vascular tissue of certified nursery apple trees collected from four nurseries as well as scion and rootstock mother plant material. Possible fungal pathogens were identified by sequencing relevant genes and phylogenetic analyses. Similar canker and wood rot fungi were isolated from nursery apple trees and from the propagation material. Fourtyfive fungal species associated with canker or wood rot were identified with Didymosphaeria rubi-ulmifolii s.l. the dominant species in certified nursery apple trees. Other canker and wood rot pathogens isolated belonged to the Basidiomycetes, Botryosphaeriaceae and Diatrypaceae. It also included species of Cadophora, Coniochaeta, Cytospora, Diaporthe, Didymella, Didymosphaeria and Phaeoacremonium. Sixty-five percent of certified nursery apple trees were infected with canker and wood rot pathogens. Two-year-old branches of 14-year-old Golden Delicious trees were inoculated with 39 species to evaluate pathogenicity. All the species caused lesions that were significantly longer than the control 5 months after inoculation. This study confirmed the presence of canker and wood rot pathogens in apple propagation material as well as certified nursery apple trees.

\section{Diaporthe species identified from woody plants close to vineyards in South Africa}

P. Lesuthu (1), L. Mostert (2), C. Spies (1,2), P. Moyo (2), F. HALLEEN (1,2), (1) Agricultural Research Council, Stellenbosch, SOUTH AFRICA; (2) Stellenbosch University, Stellenbosch, SOUTH AFRICA

Many Diaporthe species are known to have broad host ranges, and different species can occur on the same host. In South Africa, D. ampelina is considered a major grapevine pathogen, however, several other Diaporthe species have also been associated with grapevine diseases. These include $D$. amygdali, $D$. helianthi, $D$. cynaroidis, $D$. novem and $D$. serafiniae that are also known from other hosts. These findings have led to an investigation of Diaporthe species associated with woody plants in close proximity to grapevines to assess the risk these plants pose as potential inoculum sources of grapevine pathogens. Isolations were made from 25 different species of woody plants near grapevines in the Western Cape Province. Cultures identified as Diaporthe based on cultural and morphological features were identified to species level by sequencing the internal transcribed spacer region (ITS) and, for representative isolates, the beta-tubulin (TUB2) region. Phylogenetic analysis of the combined ITS and TUB2 data revealed 16 Diaporthe species associated with the 25 woody hosts. Seven of these Diaporthe species have been reported as possible pathogens of grapevines in South Africa. These findings suggest possible inoculum movement of several Diaporthe species between grapevines and other woody plants. Diaporthe ampelina was not recovered from non-grapevine hosts, confirming previous suggestions that this species is host-specific.

Fungal trunk disease pathogens in South-African olive nurseries

M. VERMEULEN (1,2), C. Spies (1,2), L. Mostert (1), F. Halleen (1,2), (1) Stellenbosch University, Stellenbosch, SOUTH AFRICA; (2) Agricultural Research Council, Stellenbosch, SOUTH AFRICA

The olive industry has been identified as a high growth sector in the South African National Development Plan and therefore of significant importance to the economy. Several trunk disease pathogens were recently associated with diseased olive trees in South Africa. Little is known about the biology of these pathogens and no specific management strategies are in place. The aim of this study was to investigate the status of trunk pathogens in nurseries. Isolations were made from asymptomatic cuttings from mother blocks (stage 1), rooted cuttings (stage 2) and 1-2-year-old trees (stage 3) of eight cultivars in two nurseries. Known olive trunk pathogens of Nectriaceae, Diaporthaceae, Botrysphaeriaceae, Togniniaceae, Phaeomoniellaceae and Pleurostomataceae were recovered. Stage 3 showed the highest incidence, Pleurostoma richardsiae being the most frequently isolated pathogen occurring in $82 \%$ and $37 \%$ of the trees in the two nurseries, respectively. Phaeoacremonium parasiticum was present in $29 \%$ of the trees from one nursery (stage 3). Neofusicoccum australe was detected in stage 1 . The remaining pathogens occurred in $10 \%$ or less of the material. These results indicate that propagation material from mother blocks harbour low levels of trunk disease pathogens, but that additional infections occur during the nursery process. Management strategies should focus on the prevention and elimination of infections in mother blocks, as well as during the propagation process.

A species-specific PCR assay for the newly observed root lesion nematode, Pratylenchus vulnus, in Taiwan Y. P. LIN (1), J. I. Yang (2), (1) National Taiwan university, Taipei, TAIWAN; (2) National Taiwan University, Taipei, TAIWAN

The root lesion nematode Pratylenchus sp. is the third most agriculture- impacting plant parasitic nematode genus. On March 2017, Pratylenchus vulnus was extracted from a strawberry field soil in Taiwan. The nematode was identified both morphologically and molecularly. Measurements were taken under the view of compound microscopic observation. Molecular analysis were done on the sequences of the cytochrome oxidase subunit I (COI) gene of mitochondria DNA (mtDNA) and multiple regions of the ribosome gene, 18S, ITS region, and the D2/D3 region of $28 \mathrm{~S}$. It was the first record of $P$. vulnus in Taiwan. A species-specific PCR detection assay targeting a 138-bp long region of the mtDNA COI gene of $P$. vulnus was then developed for rapid detection and identification. The detection limit of the assay is $10^{4}$ copy per $\mu 1$ when examined with cloned target DNA. The assay is highly specific and can differentiate the target from the closely related and easily misdiagnosed spscies, such as Pratylenchus speijeri, Pratylenchus coffeae, Pratylenchus kumamotoensis, and other common plant parasitic nematodes including Helicotylenchus sp., Rhadinaphelenchus sp., Radopholus sp., Tylenchorhynchus sp. Ditylenchus sp., and Bursaphelenchus xylophilus.

A loop-mediated isothermal amplification assay combined with lateral flow dipstick for rapid detection of Aphelenchoides besseyi J. I. YANG, G. Y. Yu, National Taiwan University, Taipei, TAIWAN

The rice leaf and bud nematode, Aphelenchoides besseyi, is an economically important plant parasitic nematode and a concerned quarantine pest to many countries globally. It causes the white tip disease on rice, and some susceptible rice varieties were recorded with $70 \%$ yield loss. Early detection of the $A$. 
besseyi-infected rice seedling during the nursery stage could prevent the spread of this seed-borne pathogen. A loop-mediated isothermal amplification (LAMP) assay combined with lateral flow dipstick was developed for the rapid screening and early detection purposes. A set of 4 primers, named ABID37, was designed to target the mitochondria cytochrome oxidase I (COI) gene. The ABID37 LAMP assay was optimized to complete within 60 minutes at $63^{\circ} \mathrm{C}$, with the sensitivity of $10^{4}$ copies cloned DNA plasmid per $\mu 1$. The assay is highly specific and can effectively exclude detection of the very closely related fern biotype of $A$. bessey $i$ and other common aboveground plant-parasitic nematodes. The specific lateral flow dipstick was successfully developed for immediate ABID37 LAMP result analysis without agarose gel electrophoresis, and no false positive was detected when examined with mix total DNA of rice and $A$. besseyi.

Surveillance and monitoring of the invasive forest pathogen Heterobasidion irregulare in Europe through an optimized LAMP assay F. Sillo (1), L. Giordano (1,2), P. GONTHIER (1), (1) University of Torino / DISAFA, Grugliasco, ITALY; (2) University of Torino / AGROINNOVA, Grugliasco, ITALY

The North American pine pathogen Heterobasidion irregulare was introduced and became invasive in Italy. The pathogen is recommended for regulation in Europe by the European and Mediterranean Plant Protection Organisation (EPPO). Here, we report on the development and optimization of an efficient molecular diagnostic tool based on Loop-mediated isothermal AMPlification (LAMP) coupled with two different DNA extraction methods for the detection of $H$. irregulare. The LAMP assay showed a limit of detection of approximately 20 picograms of target DNA and detection time of about 40 minutes. The assay was successfully validated both in controlled conditions and in the field on a plethora of different samples, including fungal fruiting bodies, infected plants, wood colonized by the pathogen, and woody spore traps. The process of pathogen detection through LAMP on woody spore traps was optimized both in terms of methods and in terms of length of incubation period of traps. Practical applications of this molecular diagnostic tool developed and optimized in the frame of the EU Project EMPHASIS (grant agreement 634179) include the surveillance in the ports of entry of wood imported from North America, the laboratory or in field analyses of samples from symptomatic pines, and the routinely monitoring of pine forests by the aid of woody spore traps.

\section{Reliable detection of Peach latent mosaic viroid (PLMVd) by real-time RT-PCR}

C. MARTíNEZ (1), P. Serra (2), E. Bertolini (3), M. J. Cambra (4), R. Flores (5), (1) Instituto Valenciano Investigaciones Agrarias (IVIA), Moncada, Valencia, SPAIN; (2) Instituto de Biología Molecular y Celular de Plantas (UPV-CSIC), Valencia, SPAIN; (3) Inst Valenciano de Investigaciones Agrarias, Moncada Valencia), SPAIN; (4) Inst Valenciano De Invest Agrarias (IVIA), Moncada (Valencia), SPAIN; (5) IBMCP UPV CSIC, Valencia, SPAIN

Peach latent mosaic viroid (PLMVd) is a worldwide-distributed pathogen that causes leaf mosaics, delays in foliation, flowering and ripening, irregularly-shaped and discolored fruits with cracked sutures and, in a few cases, albinism (peach calico). PLMVd variants are clustered into two different classes, I and II, with the latter presenting multiple changes with respect to the former in the region delimited by positions 161-197 (stems p6a and p6b). We have developed a real-time RT-PCR method able to detect PLMVd variants. Three different TaqMan probes were designed: P1 and P2 specific for class I and II variants, and P3 for all PLMVd variants deposited in databases. Our real-time RT-PCR approach has been used to characterize PLMVd populations present in the peach indicator GF305 after independent inoculation of representative variants of class I and II, and co-inoculation with both variants. Plants were analyzed by hybridization with a full-length PLMVd riboprobe, sequencing of conventional RT-PCR products, and realtime RT-PCR. Under greenhouse conditions the results showed a strong competition between both variants during host colonization, since only class II variants were detected in the co-inoculated plants. These results point to a higher biological fitness of class II PLMVd variants.

Investigating spatiotemporal and genotypic characters of Phytophthora infestans, causal agent of late blight, in Wisconsin during 2009-2017 T. WU, Y. Chen, A. J. Gevens, University of Wisconsin-Madison, Madison, WI, USA

Phytophthora infestans causes late blight on tomatoes and potatoes and is a major agricultural concern worldwide. In Wisconsin (WI), where vegetable and potato production is ranked $2^{\text {nd }}$ and $3^{\text {rd }}$ in the nation, respectively, monitoring for disease risk each season is a top priority. Our objectives included 1) diagnose, genotype, and track location of $P$. infestans in WI from 2009 to 2017, and 2) investigate the timing of initial late blight incidence per WI county and compare to accumulation of Blitecast disease severity values (DSV) in each year from 2009 to 2017. Blitecast is a disease forecasting tool which utilizes units of DSVs based on temperature and relative humidity promotive to late blight. When DSVs surpass the threshold level of 18 in WI, growers initiate preventative fungicide regimes. The use of Blitecast as an integrated management tool has been useful with an average of 47 days between 18 DSV thresholds and first identification of late blight in central WI. During the 8 years of this investigation, between 3 to 22 counties had confirmed late blight each year. First detections by county varied from 7 July to 26 August. While genotypes included US-8, $-22,-23$, and -24 , during 2009-2017, US-23 (mating type A1) was the most predominant genotype. Recent re-emergence of US-8 (mating type A2) suggests a continued need for monitoring of the pathogen to limit potential interaction of these two compatible mating types to avoid risk of sexual reproduction and resulting soilborne oospores and increased population variation.

Characterization of fungi species associated with Ascochyta blight of field peas in Montana A. OWATI (1), B. Agindotan PhD (1), M. E. Burrows (2), (1) Montana State University, Bozeman, MT, USA; (2) Department of Plant Sciences and Plant Pathology, Montana State University, Bozeman, MT, USA

The production of pea (Pisium sativum L) in the Northern Great Plains of the United States is rapidly increasing. Montana is the leading producer of peas in the US, where 610,000 acres were planted in 2016, accounting for $44 \%$ of the total production in the US. Chief among fungal diseases of peas is Ascochyta blight (AB). This disease is caused by a complex of fungal pathogens (Didymella pisi, Peyronellaea pinodes, and Peyronellaea pinodella). D. pisi is predominant in Montana's fields. Recently, a shift in pathogen composition has been observed in Montana from D. pisi to $P$. pinodes and $P$. pinodella. In addition, a Phoma spp. associated with diseased seeds were included in this study. To characterize these pathogens, we evaluated the effects of temperature on their mycelial growth rate and sporulation. Four levels of temperature were used; $15,20,25$, and $30^{\circ} \mathrm{C}$. Mycelial growth on PDA was measured daily and the number of spores quantified after two weeks on 1/3 PDA. The results in all the temperature levels showed that Phoma spp had the highest growth rate $(\mathrm{p}$-value $=<0.001)$ and produced more spores than the other species ( $\mathrm{p}$-value $=<0.001$ ). Pathogenicity assays on pea indicated that $P$. pinodes caused greater disease severity than the other species when inoculated on pea plants $(\mathrm{p}$-value $=<0.001)$. These results will be used to understand the changes in predominant species composition causing $\mathrm{AB}$ in pea in Montana.

Rapid diagnostic and soil inoculum quantification tools for soilborne pathogens of strawberry

A. BURKHARDT (1), M. Ramon (1), S. T. Koike (2), F. N. Martin (1), (1) USDA ARS, Salinas, CA, USA; (2) Univ of California, Salinas, CA, USA 
The ability to rapidly identify strawberry pathogens from diseased plants in the field or to quantify their soil inoculum levels allows growers to better manage diseases. Assays to diagnose pathogens that severely damage strawberry, specifically Macrophomina phaseolina, Fusarium oxysporum f. sp. fragariae (Fof) and Verticillium dahliae, have been developed. These assays provide results in a few hours using DNA extracted from soil (TaqMan) or in as little as 20 minutes using tissue macerate directly from field crops (isothermal recombinase polymerase amplification; RPA). Comparative genomics was used to identify a genotype-specific locus for M. phaseolina isolates that exhibit a host preference for strawberry as well as a locus for specific detection of Fof. An RPA assay for M. phaseolina with a detection limit of $200 \mathrm{fg}$ of pathogen DNA was validated with field samples. A single tube nested qPCR TaqMan assay for this pathogen can accurately quantify as little as $3 \mathrm{~ms} / \mathrm{g}$ soil. An RPA assay for $F$ of is in the final stages of field validation while a TaqMan assay to quantify $F$ of in soil samples is specific and has a detection limit of $50 \mathrm{fg}$ of pathogen DNA. Future work will correlate $\mathrm{Ct}$ values from the qPCR assay to plate counts of $F$ of from infested soil. An RPA assay for detection and quantification of $V$. dahliae was developed from a published qPCR assay. These assays provide new time-effective tools for pathogen detection and assessment of risk prior to planting.

Diagnosis of huanglongbing-associated Candidatus Liberibacter species in citrus roots by real-time PCR using primers targeting 16s rDNA and $n r d B$ genes

J. W. Park (1), J. Brockington (1), E. Louzada (1), B. Kostyk (2), P. Stansly (2), G. Mccollum (3), J. V. da Graça (1), W. E. Braswell (4), M. KUNTA

(1), (1) Texas A\&M University Kingsville Citrus Center, Weslaco, TX, USA; (2) University of Florida/IFAS/SWFREC, Immokalee, FL, USA; (3)

USDA, ARS, USHRL, Fort Pierce, FL, USA; (4) USDA APHIS PPQ CPHST, Edinburg, TX, USA

Huanglongbing (HLB) is a destructive disease of citrus which has been associated with three 'Candidatus Liberibacter' species, 'Ca. Liberibacter' asiaticus (CLas), $\mathrm{Ca}$. L. americanus and $\mathrm{Ca}$. L. africanus. Since HLB has no known cure or resistant cultivars, early detection of its associated bacteria is critical for disease management. Slow symptom development and uneven distribution of associated bacterium in a tree canopy can lead to misdiagnosis when leaves are sampled. Citrus roots have more uniform distribution of CLas compared to the aerial parts of a tree and CLas can be detected in fibrous roots of pre-symptomatic trees. Since the ribonucleotide reductase (RNR) detection assay targets $n r d B$, a five copy (1.7 X the number of copies of $16 \mathrm{~S}$ ) CLas gene, it is assumed that it will likely be a more sensitive method compared to other CLas detection methods. We compared the incidence of CLas detection of 85 fibrous root samples collected from citrus trees with different rootstock and scion combinations growing in the field using newly designed TXCChlb primers and probe developed based on 16s rDNA sequence with the RNR assay. Sixty-five and 67 of the 85 root samples were CLas positive with the TXCChlb and RNR assays, respectively based on a threshold cycle $(\mathrm{Ct})$ value of $\leq 37$. Evaluation of comparative sensitivities of both assays for CLas detection in citrus fibrous root tissues is under progress.

Digital (d)PCR protocol and tissue sample processing for detection and quantification of live Erwinia amylovora cells in fire blight cankers R. SANTANDER, K. Gašić, C. Meredith, Ž. Pavlović, S. G. Aćimović, Cornell University, Plant Pathology and Plant-Microbe Biology Section, Highland, NY, USA

Overwintering fire blight cankers are the main sources of inoculum for disease renewal in spring. Despite their importance in disease epidemiology, $E$. amylovora (Ea) amount, viability and survival in cankers are less understood. To quantify and distinguish live and total $E a$ cells in cankers, we optimized a sample processing and combined propidium monoazide (PMA)-dPCR protocol, using previously reported Ea specific Taqman probe and primers. The grinding of $E a$ inoculated apple branch sections in antioxidant maceration buffer improved $E a$ recovery on different media. PMA treatment of inoculated plant material allowed differentiation of live and dead cells. Using dPCR, the average $E a$ cell numbers per canker was determined at $6.7 \times 10^{4} \mathrm{cp}$ (average number of target DNA copies), out of which $2.7 \times 10^{4} \mathrm{cp}$ were live cells. We found a radial decline of $E a$ cell numbers, outwards from the canker edge. Majority of cells were in the first $4 \mathrm{~mm}$ of symptomless bark tissue around the canker edge. A total of $115 \mathrm{and} 81 \mathrm{cp} / \mathrm{mg}$ were detected in 0 $2 \mathrm{~mm}$ and 2-4 mm sections from the canker edge, respectively. PMA-dPCR of the same samples determined 58 and $22 \mathrm{cp}$ of live cells $/ \mathrm{mg}$ tissue, respectively. Our results suggest that PMA-dPCR is a valid technique to detect and quantify total and live cells of $E a$ in cankers. PMA-dPCR has a potential to elucidate unknown aspects of the $E a$ ecology, as well as the significance of different infection sources in disease epidemiology.

\section{Development of a Multiplex-PCR diagnostic tool for the main pathogenic fungi causing cranberry fruit rot}

M. CONTI (1), B. Cinget (2), J. Vivancos (3), C. Labbe (4), P. Oudemans (5), R. R. Bélanger (4), (1) Université Laval, département de phytologie, Québec, QC, CANADA; (2) Phytopathology laboratory, Département de phytologie, Université Laval, Québec, QC, CANADA; (3) Laboratoire d'expertise et de diagnostic en phytoprotection, Quebec, QC, CANADA; (4) Université Laval, Quebec, QC, CANADA; (5) Rutgers University, New Brunswick, NJ, USA

Vaccinium macrocarpon, the most cultivated species of cranberry, is known to host a large spectrum of pathogenic fungi, responsible for $25-33 \%$ of production losses through fruit rot. Currently, the diagnosis of fungal pathogens is carried out with standard plating and microscopic techniques, requiring both time and excellent mycological knowledge. Therefore, the aim of this study was to develop a Multiplex-PCR diagnostic tool able to analyse and detect rapidly and precisely the most important fungal pathogens of cranberry fruit. For this purpose, the most common barcode DNA regions were screened to identify potentially discriminant specific markers. In order to confirm the interspecific polymorphism, the selected markers were sequenced for all targeted pathogen species and compared with sequences of related species available on usual nucleotide databases. It was possible to design specific primers able to amplify DNA fragments of different length for each pathogen. For practical purposes, the twelve primer pairs were split up in three groups of four primers per PCR-reaction. This allowed a simpler visualisation on the electrophoresis gel. Having established the optimum PCR conditions, the multiplex test was validated with pure fungal specimens of the pathogens and with infected fruits. This diagnostic tool will assist cranberry growers in defining with precision 1) the major fungal pathogens present in their field and 2) the control methods to implement based on this diagnosis.

\section{Rhizoctonia solani AG-11 causes rice seedling disease in Texas}

S. GAIRE (1), Y. K. Jo (2), S. Zhou (1), (1) Texas A\&M AgriLife Research, Beaumont, TX, USA; (2) Texas A\&M University, College Station, TX, USA

Seedling diseases are important in Texas rice production and can be caused by several seed-borne and soil-borne fungi. This study was performed to identify the causal agents of rice seedling diseases in Texas. Rice seedlings showing abnormal growth were collected from Wharton County, Texas in 2017. Infected tissues were plated in water agar and Rhizoctonia solani isolates were isolated based on culture morphology. These isolates were further identified as $R$. solani AG-11 based on internal transcribed space (ITS) sequence. Pathogenicity assays were conducted by planting rice in potting mix inoculated with the $R$. solani isolates and incubating them at $25^{\circ} \mathrm{C}$ for 15 days in a growth chamber. The $R$. solani isolates reduced seedling emergence by $96 \%$, root growth by $55 \%$, and shoot growth by $76 \%$ compared to non-inoculated rice seedlings. These isolates also caused necrotic lesions on mesocotyls and coleoptiles resulting root and shoot rot. $R$. solani AG-11 was determined to be a causal agent of rice seedling disease. 
Prevalence of 'Candidatus Liberibacter asiaticus' in citrus and the Asian citrus psyllid in Texas over a 10-year period (2007-2016)

O. J. ALABI (1), M. Setamou (2), M. Kunta (3), J. Dale (4), J. V. da Graça (3), (1) Dept. of Plant Pathology \& Microbiology, Texas A\&M University, Weslaco, TX, USA; (2) Texas A\&M University-Kingsville Citrus Center, Weslaco, TX, USA; (3) Texas A\&M University Kingsville Citrus Center, Weslaco, TX, USA; (4) Texas Citrus Pest \& Disease Management Corporation, Mission, TX, USA

Candidatus Liberibacter asiaticus (CLas), vectored by the Asian citrus psyllid (ACP), is consistently associated with huanglongbing (HLB) in US citrusproducing states. Surveys were conducted in Texas over a ten-year period (2007-2016) to assess the prevalence and titer of CLas in ACP and citrus trees. ACP cohorts and leaf tissues from suspect trees were sampled in residential areas and groves and tested for CLas by qPCR using standard assays. CLas detection in ACP (2011) preceded that of plant tissue (2012) by 10 months. Annual percentages of CLas-positive ACP and leaf tissue over the detection period followed an exponential growth pattern, varying from $0.03 \%$ to $13.7 \%$ and from $1.2 \%$ to $17.4 \%$, respectively. The proportion of HLB detection sites dramatically increased with time reaching $20 \%$ of all commercial blocks and $30 \%$ of all residential trees surveyed by 2016 . Seasonal variations were observed in the percentages of CLas-positive ACP and trees within a year. Significantly more CLas-infected ACP and trees were recorded during fall and winter relative to the hottest summer months under Texas growing conditions. Although mean Ct values of CLas-positive ACP did not vary with time, there was a significant trend of higher bacterial titers in infected leaf tissues from 2012 to 2016. These findings have implications for HLB management.

\section{A three-year analysis of rust fungi intercepted at Arizona ports of entry} D. C. SANDBERG, USDA-APHIS-PPQ, Nogales, AZ, USA

Rust fungi (Basidiomycota, Pucciniales) are highly specialized obligate parasites that cause significant damage to agricultural and forestry crops. Many of the rust fungi intercepted at Arizona ports of entry that transit from Mexico are considered quarantine significant pests and account for a substantial portion of the pathogens requiring phytosanitary action when identified at the border by PPQ personnel. From January 1, 2015 to December 31, 2017, around 700 rust fungi were intercepted from 6 different Arizona ports of entry. The intercepted rust fungi belonged to 5 known rust families, 9 genera, and over 20 different species. Interceptions of rust fungi found at Arizona ports of entry increased slightly from 2015 to 2016 , but decreased in 2017. From the 3-year study, it was discovered that 2015 had the highest diversity of rust fungi. The most prevalent rust fungus intercepted was Chrysanthemum White Rust (Puccinia horiana). Chrysanthemum White Rust accounted for over half of the rust fungi found, and interceptions gradually increased from 2015 to 2017.

Isolation and Identification of Bacteria Causing Blackleg and Soft Rot of Potato in Northeastern U.S. T. GE (1), N. F. Marangoni (2), J. Hao (2), S. Johnson (3), R. P. Larkin (4), (1) University of Maine, ORONO, ME, USA; (2) University of Maine, Orono, ME, USA; (3) University of Maine Cooperative Extension, Presque Isle, ME, USA; (4) USDA-ARS, New England Plant, Soil, \& Water Lab, Orono, ME, USA

Both Dickeya and Pectobacterium spp. are important causal agents of blackleg and soft rot of potato and result in significant economic losses. Since 2015, when an outbreak occurred, blackleg has been a problem in the Northeastern U.S. Samples from symptomatic plants, dormant tubers, and surface irrigation water were collected in 2015, 2016 and 2017 to study this disease. These samples were mainly from Maine, with small portion also from other Eastern states where Maine seed tubers were planted. Dickeya and Pectobacterium spp. were isolated and purified from the samples on selective medium crystal violet pectate agar. Genomic DNA of the bacteria was extracted and amplified in polymerase chain reactions using Dickeya and Pectobacterium genus-specific primers to confirm their taxonomy. Furthermore, the gapA gene of the isolates was amplified and the amplicons were sequenced and blasted against the GenBank database to identify bacteria to species. Dickeya dianthicola, P. parmentieri, and P. carotovorum were identified from potato plant and tuber isolates. Nearly $43 \%$ of water samples were either Dickeya or Pectobacterium positive; Dickeya zeae, D. dianthicola, D. dadantii, $P$. parmentieri, and P. carotovorum were found in water samples. Isolates will be used in genetic diversity analysis of Dickeya and Pectobacterium spp. on potato. Further investigation will help to understand the epidemiology and impact of irrigation water on the disease.

Use of LAMP detection to identify potential contamination sources of Pythium irregulare in hydroponic culture system of eustoma W. FENG (1), A. Nukaya (2), M. Satou (3), H. Suga (4), K. Kageyama (5), (1) United Graduate School of Agricultural Science, Gifu University, Gifu, JAPAN; (2) Faculty of Agriculture, Shizuoka University, Shizuoka, JAPAN; (3) National Agriculture and Food Research Organization, Ibaraki, JAPAN (4) Life Science Research Center, Gifu University, Gifu, JAPAN; (5) River Basin Research Center, Gifu University, Gifu, JAPAN

Hydroponic culture systems are subject to high risks of diseases caused by zoosporic plant pathogens. Control is generally difficult because of the rapid spread of zoospores in the nutrient solutions. In Japan, the cut flower eustoma which is cultivated using the nutrient film techniques, is susceptible to the disease of root rot caused by the Pythium irregulare. We used loop-mediated isothermal amplification to identify potential contamination sources of this pathogen by monitoring its presence in the water supply wells, seedling terraces, nutrient solutions, plants, and ground soils of a eustoma greenhouse complex. The results indicated that the infestation of $P$. irregulare had become established and became more and more serious, and that the pathogen may invade the culture system from the soils around the greenhouses. The infestation most likely occurs when seedlings are moved from the seedling terraces to the greenhouses. Another possibility is that the sterilization of the hydroponic system after harvesting may not be sufficient. Pythium irregulare infested healthy plants before the disease symptoms are visible. Therefore, the periodic monitoring of pathogens in the culture system and ground soils is very important for the management and prevention of disease.

Application of a carbon-nanotube filter that traps and concentrates virions to improve the limits of detection of Tomato spotted wilt virus in tomato

J. F. ITURRALDE MARTINEZ (1), C. Rosa (2), C. Rotella (1), M. Terrones (1), E. Rajotte (1), (1) Penn State University, University Park, PA, USA; (2) The Pennsylvania State University, University Park, PA, USA

CNT-STEM (carbon nanotube size-tunable enrichment microdevice) is a 3D nanofilter that traps virus particles in a nanotube forest and enriches them according to their size. The CNT-STEM production process is standardized and can be engineered to filter any virion based on its size. Virus enrichment improves the limits of detection of serological and molecular techniques that rely on the number of viral particles or genomes present, and has been used in the past to discover and characterize new strains of Avian influenza virus. For a plant pathology application, CNT-STEM enrichment was evaluated for the possibility of improving the limits of detection of qPCR, ELISA, and deep-sequencing in Tomato spotted wilt virus-infected tomatoes. To date, the device, coupled with qPCR, enabled detection of 100 TSWV genomic segments from tomato leaf samples, which previously tested negative by uncoupled qPCR. The ultimate goal is to detect low titers of TSWV at early stages of infection in seedlings before they are transplanted to large field settings, mimicking a natural infection started by thrips (natural vectors of TSWV). The devices are portable, disposable, and once optimized, could be 
used in a greenhouse or field setting. Timely, reliable TSWV detection will give growers confidence that their transplants are clean and could reduce the risk of crop losses to this disease.

Fungal pathogens associated with red-skin root disease of Panax ginseng in Northeast China

X. Lu, X. Jiao, X. Zhang, W. W. GAO, Institute of Medicinal Plant Development, Chinese Academy of Medical Sciences, Beijing, CHINA

Red-skin root disease of Asian ginseng (Panax ginseng) is a major problem that significantly reduces the ginseng quality and yield in Northeast China. The disease has been considered as physiological disorders in most of reports but infectious disease in very few studies. In Jun. 2012 and Sep. 2013 , a total of 230 fungal isolates were obtained from 206 red-skin root samples collected from 13 commercial fields in 9 counties of 7 cities, in Jilin and Heilongjiang provinces, representing the main ginseng growing areas in China. 151 isolates with Cylindrocarpon-anamorph were identified into 7 species in 2 genera, by using a muti-gene analysis along with morphological characters. The seven species included Dactylonectria sp., D. hordeicola, Ilyonectria mors-panacis I. robusta and 3 unclassified Ilyonectria species. 74 Fusarium isolates were identified into $F$. acuminatum, F. avenaceum, F. solani and F. torulosum. The rest 5 isolates were Rhexocercosporidium panacis. Among these species, Ilyonectria sp. 1, I. robusta and F. solani were the three commonest species with isolation frequency of $36.1 \%, 20.9 \%$ and $23.9 \%$, respectively, but all of them were weakly aggressive by pathogenicity test on detached 3-year-old ginseng roots using at least 3 isolates for each species. All other species isolated showed pathogenicity on ginseng. The identification of fungal pathogen complex causing red-skin root disease in $P$. ginseng would help to devise disease control strategies.

Evaluation of High Resolution Melting Analysis to discriminate between parental and hybrid Phytophthora species M. F. Ratti, E. M. GOSS, Department of Plant Pathology, University of Florida, Gainesville, FL, USA

Phytophthora hybridization events have been reported repeatedly, resulting in new species that conserve some of their parental phenotypic traits but differ in genotypes and host range. $P$. andina and $P$. x pelgrandis are pathogens of Solanaceae and ornamentals respectively, although the extent of their host ranges and distributions are unknown. $P$. andina emerged from hybridization of $P$. infestans and an unidentified related species, $P$. $x$ pelgrandis from $P$. nicotianae and $P$. cactorum. Considering that hybrids and parental species can co-exist in the same areas and identification of hybrids usually involves sequence analysis, we aimed to develop a rapid tool to discern hybrids from parental species. We used High Resolution Melting (HRM) assays to differentiate genotypes based on their amplicon melting profiles. We designed primers for $P$. $x$ pelgrandis based on available sequences of nuclear genes of $P$. nicotianae and $P$. cactorum containing one or more polymorphisms. For $P$. andina, heterozygous sites from Illumina short reads mapped against $P$. infestans were used for the same purpose. We evaluated the discriminative potential of amplicons using uMelt prediction software; then we ran HRM experiments to obtain actual melting profiles. Significant differences in melting curves were detected for each species and hybrid. These assays could be used to identify $P$. andina and $P$. x pelgrandis hybrids, and potentially as a versatile diagnostic tool when suspected infections arise.

Botryosphaeriaceae associated with stem blight and dieback of blueberry (Vaccinium corymbosum) in Australia R. DANIEL (1), K. Scarlett (2), C. T. Rothwell (3), L. Shuttleworth (4), D. I. Guest (5), (1) NSW Department of Primary Industries, Ourimbah, AUSTRALIA; (2) Forest Research, Farnham, UNITED KINGDOM; (3) University of Sydney, Sydney, AUSTRALIA; (4) University of Pretoria, Farnham, UNITED KINGDOM; (5) University of Sydney, Eveleigh, AUSTRALIA

Stem blight and dieback due to Botryosphaeriales fungi have become more prevalent on blueberry in Australia. A small-scale survey was undertaken to determine the species associated with the disease and to gather information on possible causal agents. Fifty-two isolates were collected from two orchards in the main blueberry growing areas in New South Wales and four isolates from a single orchard in Western Australia. A multi-locus sequencing approach was used with the internal transcribed spacer region of rDNA including 5.8S (ITS), partial translation elongation factor 1-alpha (tef1- $\alpha$ ), and DNA-directed RNA polymerase II second largest subunit (rpb2). Eight species from three genera of the Botryosphaeriales were identified; the most common was Neofusicoccum parvum $(\mathrm{n}=34)$, followed by $N$. kwambonambiense $(\mathrm{n}=7), N$. occulatum $(\mathrm{n}=5)$, Lasiodiplodia theobromae $(\mathrm{n}=2)$, Botryosphaeria dothidea $(\mathrm{n}=1)$, N. australe $(\mathrm{n}=1), N$. macroclavatum $(\mathrm{n}=1)$ and L. pseudotheobromae $(\mathrm{n}=1)$. A detached stem assay used to evaluate pathogenicity showed that all isolates were able to initiate lesions on twigs of blueberry cultivar C99-42. Botryosphaeriales isolated from blueberry have also been reported in other horticultural crops and in native Australian woody plants. Blueberry orchards often occur near natural vegetation or other crops. Cross-infectivity has been demonstrated for some species. Diseases caused by wood-infecting pathogens are a major impediment to blueberry production. This study provides the first survey of Botryosphaeriales associated with blueberry stem blight and dieback in Australia, and is a valuable resource for plant pathologists and growers trying to manage the disease.

The identification of powdery mildews on Brassica chinensis var. oleifera in China S. LIU (1), P. Qiu (2), (1) Jilin Agricultural Univ, Changchun, Jilin Prov, CHINA; (2) Jilin Agricultural University, Changchun, CHINA

Brassica chinensis var. oleifera (Brassicaceae) is a widely consumed vegetable in China. Severe powdery mildews on them were observed in Jilin Agricultural University, China in May 2017. White mycelia densely covered both the upper and the lower side of leaves, stems and pods. The teleomorph stages of the fungus were not found. The morphological characteristics of the fungus were consistent with Erysiphe cruciferarum. To confirm the identification, the complete ITS region including 5.8S rDNA and partial $28 \mathrm{~S}$ rDNA sequences were amplified and sequenced. The resulting 607 bp ITS sequence (MF192845) and 629 bp partial 28S rDNA sequence (MF192846) showed 100\% identity with E. cruciferarum from Brassica juncea (KM260718) and Cardaria draba (AB102944), respectively. The pathogenicity tests were conducted by gently pressing a diseased leaf onto three asymptomatic leaves. Three uninoculated leaves served as controls. The plants were maintained in the lab at room temperature $\left(23\right.$ to $\left.25^{\circ} \mathrm{C}\right)$ and relative air humidity of 60 to $70 \%$. Symptoms developed 5 days after inoculation. No symptoms developed on the control plants. Powdery mildew on $B$. chinensis var. oleifera has been reported as E. cruciferarum only in Estonia, Germany, Switzerland on Sysmatic Mycology and Microbiology Laboratory Fungus-Host Database. To our knowledge, this is the first record of E. cruciferarum on B. chinensis var. oleifera in China and the results extended the distribution and the host range of E. cruciferarum.

Identification of the Alternaria and Fusarium Species Associated with Seeds of Brassica rapa S. Chu, Q. Liu, H. Hua, X. WU, China Agricultural University, Beijing, CHINA

Brassica rapa is an important vegetable in China and some diseases incited by plant pathogenic fungi are reported to cause yield loss on it. Among these plant pathogens, the Alternaria and Fusarium species can be seedborne. In the present study, the detection of seedborne Alternaria and Fusarium for twenty one varieties of Brassica rapa seeds from China were carried out by washing test and PDA plate methods. Besides, the effect on seed germination of the two kinds of fungi and their pathogenicity tests on detached leaves or seedlings of Brassica rapa were conducted as well. On the basis of morphological traits and molecular characteristics, the species of 113 Alternaria isolates from B. rapa seeds are A. tenuissima, A. alternata, A. infectoria, 
A. brassicicola, and A. obovoidea; and the species of 22 Fusarium isolates from B. rapa seeds are F. equiseti, F. proliferatum, $F$. verticillioides, $F$. oxysporum, and $F$. tricinctum. After dipping into spore suspension, the germination energy, percentage germination, germination index, and vigor index of Brassica rapa seeds decreased. Both Alternaria and Fusarium isolates selected could cause disease on the detached leaves or roots of seedlings of Brassica rapa. To the best of our knowledge, it's the first report in China that Brassica rapa seeds can carry pathogenic strains of Alternaria and Fusarium.

Secreted in Xylem genes used for PCR-based diagnostics of distinct Fusarium oxysporum f. sp. cubense races and vegetative compatibility groups L. COSTA CARVALHAIS (1), J. Henderson (2), C. O'Dwyer (3), E. Czislowski (4), V. Rincon-Florez (3), E. Aitken (4), A. Drenth (3), (1) Queensland Alliance for Agriculture and Food Innovation, The University of Queensland, Brisbane, AUSTRALIA; (2) Department of Agriculture and Fisheries, Brisbane, AUSTRALIA; (3) The University of Queensland, Brisbane, AUSTRALIA; (4) School of Agriculture and Food Sciences, The University of Queensland, Brisbane, AUSTRALIA

Bananas are in danger of being decimated worldwide by Panama disease tropical race 4 . Bananas are not only a major staple food in numerous developing countries but are also one of the most consumed fruits worldwide. The soil-borne fungus Fusarium oxysporum f. sp. cubense (Foc) tropical race 4 is highly virulent on varieties belonging to the AAA Cavendish subgroup. Eradication is very challenging due to the fungus capacity to survive for long periods in the soil, plant debris or even in alternative plant hosts which act as pathogen reservoirs. An effective measure to avoid further losses to the banana industry and small holders is to keep areas free of the pathogen and containment of new incursions, which relies on sensitive and specific diagnostics methods. Here we report a new PCR-based method targeting Secreted in Xylem (SIX) genes which currently comprise the only known family of effectors in F. oxysporum. Primers were designed and tested to amplify specific SIX gene homologues of the different Foc races and within the race 4 of specific vegetative compatibility groups which cannot be detected using previously reported PCR-based methods. We validated our assay on an array of $134 \mathrm{~F}$. oxysporum isolates comprising of 21 different VCG groups. Our method offers the advantages of being simple, robust and of requiring basic apparatus that are routinely used in laboratories equipped for molecular biology in any country.

Development of two LAMP assays for the diagnostic screening of the downy mildew pathogens Peronosclerospora philippinensis, P. sacchari and P. sorghi

M. Galvez, Y. RIVERA, J. Sutherland, Z. G. Abad, USDA-APHIS-PPQ-S\&T-CPHST, Beltsville, MD, USA

Exotic graminicolous downy mildews are recognized as important biosecurity threats of corn, sorghum, and sugarcane in the U.S. and other parts of the world. In particular, Peronosclerospora philippinensis and P. sacchari cause destructive diseases of corn in tropical Asia and are currently listed as two out of seven USDA Select Agents for plants in the U.S. To date, P. sorghi is the only species in the genus Peronosclerospora that has been reported in the U.S. In this study, we developed two Loop-mediated isothermal amplification (LAMP) assays targeting a variable region of the mitochondrial cytochrome oxidase 2-1 spacer for the detection of the U.S. resident species $P$. sorghi (Assay 1 ) and exotic species $P$. philippinensis and $P$. sacchari (Assay 2). The assays were optimized for reaction time and temperature, and evaluated against other downy mildew pathogens. The LAMP reactions were optimal at $67^{\circ} \mathrm{C}$ (Assay 1) and $68^{\circ} \mathrm{C}$ (Assay 2) and a 30 minute reaction time. Optimized assays are able to detect positive samples in less than 15 minutes and for target concentrations as low as $0.5 \mathrm{pg} / \mu \mathrm{l}$. No cross-reaction was observed against other Peronosclerospora species and other closely related pathogens downy mildew pathogens in the genera Bremia, Baobaposis, Sclerophthora and Sclerospora. These two LAMP assays are the first rapid screening methods for graminicolous downy mildew pathogens, thus enhancing our ability to protect the production of sorghum, sugarcane and corn.

Improvements in assessments of disease severity in conventional scouting using UAV-assisted multispectral imaging in watermelon M. KALISCHUK (1), M. Paret (1), D. Rajendranath (2), J. H. Freeman (1), S. Wright (1), S. Eubanks (3), D. Wiggins (3), M. Lollar (4), (1) North Florida Research and Education Center, University of Florida, Quincy, FL, USA; (2) Agribugs, Tallahassee, FL, USA; (3) University of Florida Cooperative Extension, Quincy, FL, USA; (4) University of Florida Cooperative Extension, Marianna, FL, USA

Agriculture-based multispectral imaging is popular but documentation of operational field-based benefits are limited. Multispectral imaging data were collected and analyzed from two commercial watermelon fields located in north Florida. The fields were rated for disease incidence and severity at random locations (conventional scouting) first followed by assessments at locations that were identified by differences in stress index and Normalized Difference Vegetation Index (NDVI) through multispectral imagery on an Unmanned Aerial Vehicle (UAV) platform (UAV-assisted scouting). Diseases identified in the watermelon fields included gummy stem blight, anthracnose, fusarium wilt, Phytophthora fruit rot, Alternaria leaf spot, and Cucurbit leaf crumple disease. A test for marginal homogeneity indicated that the severity ratings were different between conventional and UAV-assisted scouting. Higher severity ratings of 3 and 4 (on a scale of $0-5$ from no disease to complete loss of the canopy) were more common after the scouts used the stress images in determining the locations for sampling. Experienced scouts tended to rate higher severities than lesser-experienced scouts. The UAV-assisted scouting locations had significantly greater green, red and red edge NDVI and lower stress index values than the scouted areas located using multispectral imagery. While progress has been made to identify some diseases using multispectral imaging, conventional scouting involving human evaluation remains necessary to validate other types of diseases. Multispectral imagery improved the output of watermelon field scouting due to the increased ability to identify hot spots more rapidly than conventional scouting practices.

\section{Multiplex qPCR of Ribonucleotide Reductase, 16S rRNA, Heat Shock Protein and Chaperonin genes for different Huanglongbing (HLB) detection purposes Z. Yan, J. RASCOE, M. K. Nakhla, USDA-APHIS-PPQ-S\&T-CPHST, Beltsville, MD, USA}

Huanglongbing (HLB) is a serious disease affecting citrus production worldwide. Among the three bacteria species associated with HLB, "Candidatus" Liberibacter asiaticus (CLas) is the only one currently present in the United States. Detection of infected trees with methods of optimal sensitivity and/or specificity may be tailored for different purposes of HLB research, quarantine and disease management. Five assays (four CLas specific and one COX control) were multiplexed in various combinations with probes of different dyes, and used to test well-characterized HLB CLas plant extract dilutions using ABI QuantStudio7. The CLas assays target individual 5-copy-per-genome Ribonucleotide Reductase/RNR, 3-copy 16S, single-copy Heat Shock Protein/HSP and Chaperonin/CPR genes. Sensitivity was slightly increased using an RNR-FAM/16S-FAM/COX-JOE assay that detects 8-copy targets per genome compared to RNR-FAM/COX-JOE ( 5 copies), producing $0.90 \pm 0.49 \mathrm{Ct}$ lower $(\mathrm{n}=40)$ on higher titer dilutions, and showed $64 \%$ vs. $33 \%$ $(\mathrm{n}=95, \mathrm{P}<0.0001)$ positive detection of very low titer dilutions where stochastic error becomes a factor. In additional testing, preliminary data of RNRABY/16S-FAM/COX-JOE and RNR-JUN/16S-FAM/CPR-VIC/HSP-ABY assays also showed promising sensitivity, and ability to detect up to three targets simultaneously in low titer samples. This study demonstrates the value of multiplexing previously validated $C$ Las qPCR assays to increase either sensitivity or specificity. 
Information flow through diagnostic networks: Understanding pepper disease risk in Florida

J. FULTON (1), C. Lapaire Harmon (2), S. Turner (3), K. A. Garrett (1), (1) Plant Pathology Department, University of Florida, Gainesville, FL, USA; (2) Univ of Florida PDC, Gainesville, FL, USA; (3) University of Florida, Gainesville, FL, USA

The network of institutions in the United States' National Plant Diagnostic Network (NPDN) has been designed for efficient communication. The network of information flow that links growers with NPDN institutions is key to the success of US diagnostic networks, but its structure is mostly uncharacterized. We surveyed Florida pepper stakeholders to parameterize a simulation of the likely response of the Florida pepper industry to the introduction of a new pathogen during its dispersal throughout the state. This simulation models pathogen dispersal to naïve areas as latently infected young transplants are transported throughout the state's regional pepper production areas. A novel component of this research is a comparison of the effects of active farmer decision-making based on a range of theoretical farmer behavior. This integrated model incorporates information and risk uncertainty to evaluate the likely consequences of farmer management strategies. Behavioral modifications and differences between the efficacies of various management strategies then impact, to varying degrees, pathogen fitness and yield impacts. In this linked social and biological network analysis, our simulation integrates (a) emerging pathogen dispersal, (b) information dissemination among participants in the NPDN, local diagnostic networks, and industry stakeholders, and (c) host material transport. Initial analyses indicate the key role of hub communicators for linking farmers into the NPDN system.

Detection and identification of two phytoplasma subgroups associated with strawberry phyllody and red leaf disease in Chile W. CUI, A. Zamorano, N. Fiore, University of Chile, Santiago, CHILE

Strawberry (Fragaria $\mathrm{x}$ ananassa Duch.) production plays an important part in Chile's agriculture, serving both domestic consumption and export. Since the year of 2015, strawberry plants with symptoms of fruit phyllody, achenes' hypertrophy and leaf reddening have been identified in individual surveys, leading to our first report of phytoplasma infection in Chilean strawberry. In recent surveys of four individual orchards in Bio-bio and Araucanía regions of Chile, samples were collected from symptomatic plants and genomic DNA was extracted. Three genomic regions corresponding to the 16SrRNA, elongation factor $\mathrm{Tu}(t u f)$ and large ribosomal subunit protein $12 p$ genes were amplified by nested PCR. Each amplification product was cloned into the pGEM-T vector and multiple colonies were selected for sequencing to discriminate potentially multiple infections. Sequences alignments, phylogeny construction and in silico RFLP revealed two different phytoplasmas. One belongs to our previously-reported ribosomal subgroup $16 \mathrm{SrXIII-F}$ ( $100 \%$ of identity), whereas the other isolate lies outside all the eight known subgroups of $16 \mathrm{SrXIII}$ group (similarity coefficient $<0.91$ ). Our research shows widespread phytoplasma infection on Chilean strawberry crops, which calls for attention from private producers as well as seedling suppliers. We also report the detection and identification of a novel $16 \mathrm{SrXIII}$ subgroup of phytoplasma associated with strawberry phyllody and red leaf disease.

Specific detection of quarantine species, Phytophthora ramorum, P. kernoviae and P. lateralis by loop-mediated isothermal amplification (LAMP) assay

A. HIENO (1), K. Otsubo (1), H. Suga (2), K. Kageyama (1), (1) River Basin Research Center, Gifu University, Gifu, JAPAN; (2) Life Science Research Center, Gifu University, Gifu, JAPAN

Phytophthora species cause destructive diseases in agriculture and forest worldwide. P. ramorum is a causal agent of sudden oak death, most commonly observed on Camellia, Magnolia, Pieris and Quercus spp., and has now reached epidemic proportions in North America and Europe. P. kernoviae causes bleeding stem cankers on members of the Fagaceae and foliar blight and shoot dieback on other hosts in UK and New Zealand. P. lateralis is highly aggressive to Chamaecyparis lawsoniana, a native forest tree in California and Oregon. Spread of these pathogens were probably associated with import/export ornamental plants. Therefore, an effective quarantine control of these Phytophthora species is highly required. In this study, we developed a species specific detection method using LAMP and simple DNA extraction kit (whole procedure completed within 30 min). LAMP primer sets were designed in rDNA-ITS, cox 1 and Ypt1, and the specificity were checked with extracted DNA from 27 species of Phytophthora belonging to Clade 1-10, 13 species of close genus Pythium and Phytophthium, and 7 genus of other soil-borne pathogens. Selected LAMP primer sets, designed in cox 1 gene for $P$. ramorum and $P$. lateralis, and in rDNA-ITS for P. kernoviae were able to detect each target from DNA samples extracted from inoculated leaves of Rhododendron, Pieris and Camellia within $20 \mathrm{~min}$. This method can be utilized for effective detection of the three species in quarantine.

Rapid detection and characterization of Phytophthora infestans isolations in the field

S. Dangi (1), J. Woodhall (2), K. Fairchild (1), A. Oropeza (2), P. S. WHARTON (1), (1) University of Idaho, Aberdeen Research and Extension Center, Aberdeen, ID, USA; (2) University of Idaho, Parma Research and Extension Center, Parma, ID, USA

Late blight of potato, caused by Phytophthora infestans, is a major constraint to potato production. In recent years, new genotypes, some with different epidemiological characteristics, have emerged in the global populations of $P$. infestans. Early detection, coupled with knowledge of the particular genotype present, can ensure the timely implementation of the most optimum disease management strategy. Recently, loop mediated isothermal amplification (LAMP), is becoming more widely used for the rapid on-site detection of plant pathogens. Similarly, allele specific oligonucleotide markers have been developed that can determine the genetic diversity of $P$. infestans using real time PCR. In this study, we developed a new LAMP assay for $P$. infestans which can easily distinguish $P$. infestans from other oomycetes such as Phytophthora erythroseptica within 20 minutes on a Genie IIIC platform. In addition, ASO-PCR was performed with US, European and Indonesian isolates. ASO-PCR profiles of the US reference isolates were concordant with those reported previously in Gagnon et al. (2016). ASO-PCR profiles also showed that the Indonesian isolates were different to the US isolates but more similar to the European Pink-6 isolates. LAMP and ASO-PCR, coupled with a portable platform such as the Genie IIIC, can therefore enable the rapid on-site detection and characterization of $P$. infestans.

Validation of molecular diagnostic methods for specific detection of Magnaporthe oryzae Triticum pathotype, the causal agent of wheat blast J. C. BIENAPFL (1), K. Jennings (1), K. F. Pedley (2), J. P. Stack (3), Z. G. Abad (1), (1) USDA-APHIS-PPQ-S\&T-CPHST, Beltsville, MD, USA; (2) USDA ARS FDWSRU, Ft. Detrick, MD, USA; (3) Kansas State University, Manhattan, KS, USA

Wheat blast, caused by the fungal pathogen Magnaporthe oryzae Triticum pathotype (MoT), first emerged as a serious threat to wheat production areas of Brazil and subsequently spread to Argentina, Bolivia, and Paraguay. More recently, wheat blast has emerged in Bangladesh. With yield losses that can reach nearly $100 \%$ under optimal environmental conditions, there is growing concern that MoT will continue to spread to other wheat-growing regions of the world. Until recently, the diagnosis of wheat blast relied on morphological identification of M. oryzae spores or PCR-based assays, both of which are limited to confirmation at the species level and cannot distinguish pathotype. There are currently no approved regulatory methods for detection of MoT in the USA, and the International Plant Protection Convention (IPPC) has listed MoT as a pest where there is an immediate need for internationally harmonized diagnostic protocols. In a recent publication, whole-genome sequences of multiple M. oryzae strains were used to develop PCR-based assays 
to differentiate MoT strains from other M. oryzae pathotypes. The resulting PCR assays have been optimized, evaluated, and subjected to a robust validation process by the CPHST Beltsville Laboratory. Validation of the assays yielded one PCR assay for use with screening wheat samples to detect M. oryzae, and two additional PCR assays that specifically detect MoT. Data from the validation testing process will be presented.

Brn1 as a novel barcode for culture-independent identification of Bipolaris species

B. LANE, A. Adhikari, P. Harmon, E. M. Goss, Department of Plant Pathology, University of Florida, Gainesville, FL, USA

The genus Bipolaris is found worldwide and consists of many plant pathogenic species. Members of this genus cause leaf spots, leaf blights, melting out, root rots, and other diseases. Bipolaris spp. are of particular concern on gramineous crop hosts where they can result in significant economic impacts. Microstegium vimineum is a weedy annual grass that has invaded many temperate forests in the Eastern USA. In recent years, we have studied the emergence of novel Bipolaris spp. within some of these M. vimineum populations as a model system. Bipolaris spp. typically are identified by morphological features together with phylogenetically informative, multilocus sequences. However, isolation of Bipolaris spp., especially from lesions that also have been colonized by saprophytes, poses a research bottleneck. Further, current multilocus sequencing techniques are not amenable to highthroughput amplicon sequencing directly from plant tissue. We investigated Brn1, a reductase gene involved in melanin biosynthesis, as a potential single-locus barcoding region for the high throughput identification of Bipolaris spp. in diseased plant tissue. Our results suggest high interspecific variation and low intraspecific variation, which supports the potential use of Brn1 as a novel single-locus barcoding region for Bipolaris spp.

Accurate detection of three waterborne plant virus genera allows fine screening to species level by High Resolution Melting analysis F. OCHOA CORONA, S. Dobhal, A. Olmedo Velarde, Y. Carrillo Tarazona, J. Garcia-Suarez, B. Mazziero, Oklahoma State University, Stillwater, OK, USA

Plant viruses are released into the soil and water from infected and decaying plants, and living root tissues. Stable viruses i.e. Potexvirus, Tobamovirus, and Tombusvirus are reported to contaminate soil and water in forest ecosystems, agricultural fields, hydroponic cultures, and irrigation water. These three virus genera were selected as model groups for their morphological and taxonomical differences. High Resolution Melting (HRM) analysis of RTqPCR products enabled sensitive detection and discrimination of species with no need for electrophoretic resolution of products. Four assays were developed. The first consisted of three genus-specific sets of primers for single and/or multiplex endpoint RT-PCR for genus detection in water samples. Genus confirmation and screening of virus species was aided by three other sets of genus-specific primers designed for HRM discrimination. Analysis of expected PCR-product sequences using $\mathrm{uMELT}{ }^{\mathrm{SM}}$ allowed prediction of HRM scenarios. Normalized data derived from raw plots of loss of fluorescence vs. temperature and difference graphs showed an accurate discrimination of 11 Potexvirus, 11 Tobamovirus and six Tombusvirus with high confidence ( $p$-value $0.003-0.004)$. Low resolution melting temperture profiles derived from - $d F / d T$ plots in vitro were similar to predictions plotted by uMELT ${ }^{\mathrm{SM}}$ in silico. This detection-discrimination strategy was applied to glass wool filtered water samples.

The infection cushion: "A fungal weapon for plant biomass destruction"?

M. CHOQUER (1), C. Bruel (1), C. Rascle (2), C. Ribot (1), C. Dieryckx (2), A. Devallée (1), V. Girard (2), I. R. Gonçalves (1), N. Poussereau (1), (1) University Lyon 1, Lyon, FRANCE; (2) CNRS, Lyon, FRANCE

The infection cushion (IC) is a structure differentiated by several phytopathogenic fungi in order to penetrate the host plant. It is described as a melanized appressorium, made of several aggregated hyphae that induce an oxidative burst. Few molecular data have been produced to further characterize the role of this fungal structure in plant pathogenicity. Here we analyzed the transcriptome and the secretome of the IC in Botrytis cinerea. This necrotrophic fungus causes gray mold on more than a thousand plant species, such as grapevine or vegetable crops. Our data revealed a significant enrichment in membrane transporters and secreted proteins suggesting a high potential of nutrition and secretion, respectively. Indeed, we confirmed that IC is a structure dedicated to the production of secreted lytic enzymes such as proteases, xylanases, cellulases and pectinases. Several biosynthetic pathways are strongly induced in IC, including that of melanin and other secondary metabolites, among which botrydial and botcinic acid, the two major phytotoxins produced by $B$. cinerea. At last, and surprisingly, the transcriptomic data and labelling results suggest that chitin may be deacetylated in the cell wall of IC, a modification that is described in biotrophic fungi as a mechanism to escape the host plant immunity. Altogether these results suggest that IC may not be useful for penetration of the fungus only, but that it could also contribute to necrotrophy.

Multilocus sequence analysis reveals Colletotrichum nymphaeae as the dominant species causing strawberry anthracnose in the United States N. Y. WANG (1), B. B. Forcelini (2), N. Peres (1), (1) University of Florida, Wimauma, FL, USA; (2) Gulf Coast Research and Education Center, University of Florida, Wimauma, FL, USA

Strawberry anthracnose, caused by Colletotrichum acutatum, is one of the primary limiting factors in fruit production fields in the United States. Recent research focusing on the phenotypic and genetic diversity of this species has shed light on the complexity of C. acutatum species. In this study, we performed multilocus sequence analysis of four housekeeping genes to characterize 218 anthracnose-associated C. acutatum isolates collected over a 23 yr period from the symptomatic roots, crowns, or fruit of strawberry from Florida, North Carolina, Virginia, and California, including those that have been confirmed to be resistant to quinone-outside inhibitor (QoI) fungicides. The results revealed a dominant species, $C$. nymphaeae, accounting for $97.7 \%$ of the isolate collection (212/217), whereas the other identified species, C. fioriniae, comprised $2.3 \%$ of the population (5/217). All QoI-resistant isolates were found in the C. nymphaeae clade. A subset of 12 isolates representing C. nymphaeae populations sensitive or resistant to QoI fungicides from root or fruit tissue of strawberry was then selected for comparison of pathogenicity on strawberry. Of these isolates tested, all exhibited a similar degree of aggressiveness and caused indistinguishable symptoms on strawberry crowns and fruit. Overall, our results indicate genetic and pathogenic homogeneity in the C. acutatum populations associated with strawberry production in the United States.

Investigating the cause of red blotch disease in Grapevines (Vitis spp) in Oklahoma

S. WALLACE (1), J. D. Olson (1), F. Ochoa Corona (1), A. Harris (2), (1) Oklahoma State University, Stillwater, OK, USA; (2) Oklahoma State university, Stillwater, OK, USA

The value of the Oklahoma grape industry was $\$ 98$ million in 2010. Grapevines (Vitis spp) in Oklahoma were surveyed to elucidate the cause of red blotches on leaves. A 2-year Cooperative Agricultural Pest Survey was initiated and 121 grapevines were sampled in 13 counties in 2016 and in 2017 samples were collected from 96 grapevines from 14 counties. Each sample was tested for Grapevine red blotch associated virus (GRBaV), Pierce's Disease (Xylella fastidiosa), and Phytoplasma spp by Polymerase Chain Reaction (PCR). ELISA was used to test for GLRaV strains 1+3 and 4-9. A chemical test and microscopic morphological identification were conducted for Rotbrenner (Psudopezicula tracephila). GRBaV was detected in 38\%, GLRaV 1+3 16\%, GLRaV 4-9 2\%, and Pierce's 2\%, there were no detections of Phytoplasmas in 2016 and 2017. Rotbrenner was not tested in 2016. 
GRBaV was detected in 34\%, GLRaV 1+3 17\%, GLRaV 4-9 3\%, and Pierce's 3\% in 2017. No Phytoplasmas nor Rotbrenner were detected in any of the samples in 2017. Mixed infections were found in 20/121 in 2016 and 19/96 in 2017. The data generated by this survey is informative to the grape industry, growers, and extension personnel about which diseases are affecting grapevines in Oklahoma to communicate effective management strategies since each of the surveyed diseases has a different vector.

Incidence of grape diseases in Maryland vineyards

R. R. POKHAREL, Maryland Dept of Agric, Annapolis, MD, USA

In Maryland, grape growers have been observing symptoms similar to those caused by diseases, nutritional imbalances, and abiotic factors, leading to reduced yield, vine decline and/or death. From 2015-2017, 33 vineyards were surveyed for two exotic pathogens, grapevine yellows (Candidatus phytoplasma austrialense and brown root rot (Phellinus noxius), based on symptoms. Surveys also recorded incidence of downy mildew and crown gall based on symptoms, and collected information on red blotch virus. In addition, the incidence of Xylella fastidiosa and virus diseases, such as grapevine leafroll-associated virus (GLRaVs)1+3, and 2, grapevine fanleaf virus (GFLV), Arabis mosaic virus (ArMv), and tomato ringspot virus (ToRSV), was also studied by collecting 1247 leaf samples and testing them in the laboratory, using ELISA kits. No incidence of exotic pathogens was observed. Downy mildew was commonly noted each year in most vineyards and cultivars. Crown gall symptoms were recorded in several vineyards in 2015 and 2016, and one sample in 2017. Out of several red blotch symptomatic vineyards, eight growers reported verified positives for the virus. No samples were positive for GFLV. Low but similar incidences of GLRaVs 1+3 were observed in 2015-2017 in widespread areas and cultivars, whereas incidence of GLRaV 2 was found only in two cultivars. Xylella fastidiosa, not tested in 2015, was commonly found across the region in many cultivars in 2016, with only 5 positive samples found in one cultivar in 2017. In 2017, ToRV was observed in two vineyards in four cultivars.

Comparison of visual vegetation indices from aerial images to measure turfgrass health using small unmanned aircraft H. J. SOMMER (1), T. T. Lulis (1), J. E. Kaminski III (2), (1) The Pennsylvania State University, University Park, PA, USA; (2) Penn State University, University Park, PA, USA

Normalized Difference Vegetation Index (NDVI) is difficult to measure over large areas of golf courses using handheld devices, and cost-effective multispectral cameras are still not widely available. To facilitate using standard red-green-blue (RGB) cameras carried by small unmanned aircraft (UA), ten traditional visual vegetation indices (VVI) and three new VVI were computed from UA images of turfgrass. The VVI values were then correlated to handheld NDVI ground-truth measurements to assess their efficacy in predicting turfgrass health. Initial correlations were developed for A4 creeping bentgrass in which plots were stressed over a 14 day study by mowing at different heights. Correlations were validated for a second study of A4 creeping bentgrass in which the plots were subjected to water stress over 30 days. The new VVI include L*a*b* color chromaticity defined by the International Commission on Illumination (CIE) and a novel optimization method to tailor VVI for specific species. Visible Atmospherically Resistant Index (VARI) was the most effective among traditional indices. The new General Visible Vegetation Index (GVVI) using optimal RGB weighting coefficients performed better than VARI. Direct linear correlation to L*a*b* chromaticity components had almost identical efficacy as GVVI. These VVI allow expanded use of UA to scout golf courses and identify plant pathology for early identification/intervention.

Apple bitter rot fungi of New York and Virginia - which Colletotrichum species are there?

Ž. PAVLOVIĆ (1), C. Meredith (1), K. Gašić (1), R. Santander (1), K. S. Yoder (2), S. G. Aćimović (1), (1) Cornell University, Plant Pathology and Plant-Microbe Biology Section, Highland, NY, USA; (2) Virginia Tech, Agricultural Research and Extension Center, Alson H Smith Jr., Winchester, VA, USA

Bitter rot disease of pome fruits is an emerging problem in southern New York (NY) orchards and intermittently on apple fruit in storages. Factors contributing to disease outbreaks might include increasing frequency of warm wetting events during the summer, planting of susceptible and latematuring cultivars, reduced number of cover sprays, and 77 days preharvest interval limit in use of an effective fungicide, mancozeb. Fruit losses in 2016 at some NY locations reached $20 \%$ on high income cultivars like Honeycrisp. Previous research based on gene sequencing identified at least 18 Colletotrichum species that infect apples or pears worldwide. Preliminary data indicated a prevalence of C. fioriniae from C. acutatum species complex in NY. We isolated nearly 600 fungal isolates from apple and pear fruits collected at 12 locations in NY and three in Virginia (VA), and 60 of them were selected for further characterization. Based on multiplex two-step PCR assays with previously reported primers, at least two species from both $C$. acutatum and $C$. gloeosporioides species complexes were present in NY and VA. Moreover, RAPD-PCR analysis indicated species variability among both species complexes. This is the first report of PCR-validated species in C. gloeosporioides complex in NY. Different biology and variable susceptibility of Colletotrichum spp. to fungicides may impact best management practices for minimizing selection for fungicide resistance in bitter rot species.

Novel Cytohabdovirus Identified in Native Rubus Exhibiting Virus-like Symptoms M. GUZMAN (1,2), P. L. Di Bello (1), R. R. Martin (2), (1) Oregon State University, Corvallis, OR, USA; (2) USDA ARS, Corvallis, OR, USA

Raspberry leaf curl disease (RLCD) was first reported in the late 19th century in red and black raspberry and blackberry across northeastern United States and Canada. Symptoms of infection include small fruit, stunted shoots, leaf chlorosis, and downward curling of the leaves. Aphis rubicola transmits the disease in a persistent manner. Molecular tests for detection of this disease are not available. Combinations of Black raspberry necrosis, Rubus yellow net, Raspberry leaf mottle, a novel Enamovirus and two novel Cytorhabdoviruses were identified in a survey of native symptomatic Rubus from prevalent disease areas using next generation sequencing. Individual viruses were not consistently associated with symptoms observed in native Rubus. We focused on a Cytorhabdovirus detected in a Rubus from Pennsylvania because of its membership in one of two virus genera first associated with the disease. Primers were developed from the sequence of one of the detected Cytorhabdoviruses and used to test additional Rubus samples; the virus was detected in one symptomatic sample from Ontario, Canada. We determined the sequence of $14 \mathrm{~kb}$ of the genome of this Cytorhabdovirus. These molecular tools for the detection of the Cytorhabdovirus will be useful in studies to correlate its presence in planta and the development of RLCD.

Identification of Pythium spp. associated with diseased cucurbits in South Carolina

S. TOPOREK, A. P. Keinath, Coastal Research and Education Center, Clemson University, Charleston, SC, USA

Root rot and damping-off of cucurbits is commonly caused by Pythium spp., but little is known about their diversity and abundance in this crop group. A survey was conducted in South Carolina to identify Pythium species associated with diseased cucurbits. Four common cucurbit crops, bottlegourd, cucumber, Hubbard squash, and watermelon, were transplanted into four different fields, two managed organically and two managed conventionally, in May, July, and September 2017. Of 960 transplants, 236 had symptoms of root and stem rot. A total of 165 Pythium isolates was recovered from 
diseased root and stem tissues. Isolates were identified to species level by sequencing the ribosomal internal transcribed spacer region and the mitochondrial cytochrome oxidase I region. P. aphanidermatum $(40.0 \%)$ and P. myriotylum (39.4\%) were the most commonly identified species. Six additional species were detected at much lower frequencies (20.6\% total). Planting date and host significantly influenced the abundance of $P$. aphanidermatum recovered, with a significantly higher recovery rate in July compared to May and September and a significantly higher recovery rate in watermelon and cucumber than in squash and bottlegourd. Results of this survey will help manage the economically important Pythium species affecting cucurbit production. To the best of our knowledge, this is the first survey of Pythium spp. associated with root rot and damping-off in cucurbits in the United States.

Genome-informed LAMP assays for specific detection of bacterial spot-causing bacteria, Xanthomonas euvesicatoria and X. vesicatoria L. Fatdal (1), G. Boluk (1), A. Larrea (1), U. Dhakal (1), A. M. Alvarez (1), A. L. Strayer (2), M. Paret (3), J. B. Jones (4), D. Jenkins (1), M. ARIF (1), (1) University of Hawaii at Manoa, Honolulu, HI, USA; (2) University of Florida, Department of Plant Pathology, Gainesville, FL, USA; (3) North Florida Research and Education Center, University of Florida, Quincy, FL, USA; (4) Department of Plant Pathology, University of Florida, Gainesville, FL, USA

Bacterial leaf spot (BLS) is an economically important bacterial disease of tomato and pepper caused by four species, Xanthomonas euvesicatoria (Xe), $X$. vesicatoria $(X v), X$. gardneri $(X g)$ and $X$. perforans $(X p)$. Symptoms produced by all four species are nearly indistinguishable. Currently, no point-ofcare diagnostics exist for BLS. In this research, we developed two LAMP assays to rapidly and accurately identify and differentiate $X e$ and $X v$ using a field-deployable portable BioRanger instrument. Genomes of $X e, X v, X g, X p$ and other species of Xanthomonas were retrieved from NCBI GenBank genome database and were aligned and curated using Mauve and Geneious, respectively. Unique genes for $X e$ and $X v$ were selected for design of LAMP primers. Assays were tested for specificity against 74 strains of Xanthomonas species and other closely related species. No false negatives or false positives were detected. Detection limits of both assays were determined using 10-fold serially diluted purified genomic DNA of $X e$ and $X v$; both assays detected down to $100 \mathrm{fg}$ of genomic DNA. However, in spiked assays when $1 \mu \mathrm{l}$ of host DNA was added to each serial dilution of target genomic DNA, sensitivity was reduced to $1000 \mathrm{fg}$. Assays specifically detected target pathogens in infected tomato plant samples. The developed assays have applications in accurate diagnostics at point-of-care, surveillance, disease management and epidemiological studies.

TaqMan-based qPCR detection of Xylella fastidiosa subspecies pauca CoDiRO strain

T. Phannareth (1), M. J. STULBERG (1), Q. Huang (2), J. Rascoe (1), M. K. Nakhla (1), (1) USDA-APHIS-PPQ-S\&T-CPHST, Beltsville, MD, USA; (2) Floral and Nursery Plants Research Unit, U. S. National Arboretum, USDA/ARS, Beltsville, MD, USA

The CoDiRO strain of Xylella fastidiosa subsp. pauca is associated with olive quick decline syndrome in southern Italy. Spread of this pathogen to the U.S. could devastate the U.S. olive industry. A TaqMan-based qPCR method identifying this strain would aid U.S. diagnosticians as current identification is limited to more time-consuming methods of sequence analysis and conventional PCR. This subspecies has an open reading frame (ORF) that it shares only with olive-, mulberry-, and hibiscus-infecting $X$. fastidios $a$ strains. Detection of this ORF, when combined with a previously designed citrus variegated chlorosis strain-specific assay, allows for CoDiRO differentiation from other pauca strains. We designed qPCR primers and probes (XfOMH) to amplify a target within this ORF. To test our primer set we used a gBlock with the conserved ORF, and two other gene sequences for duplexed qPCR reaction positive control targets (Citrus COX and X. fastidiosa 16S rRNA gene). XfOMH efficiently amplified the gBlock target (104\%), and its performance was not significantly changed when duplexed with $16 \mathrm{~S}(100 \%)$ or COX $(100 \%)$ primers. In duplex conditions, XfOMH detected as few as 1.93 LOG gBlock molecules $100 \%$ of the time, and 0.23 LOG gBlock molecules $85 \%$ of the time. XfOMH did not amplify Xylella from environmental oak samples, but did amplify spiked gBlock targets. This assay will be further developed by testing against a collection of $X$. fastidiosa strains.

Immunoassay test based on L-cysteine functionalized gold nanoparticles for direct detection of Chrondrostereum purpureum J. MEJÍAS (1), K. Villena (1), S. Meier (1), D. Grinbergs (2), J. Chilian (3), A. France (3), (1) Instituto de Investigaciones Agropecuarias, Temuco, CHILE; (2) Instituto de Investigaciones Agropecuarias, Chillán, CHILE; (3) INIA Quilamapu, Chillan, CHILE

Chrondrostereum purpureum is the causative infectious agent of silverleaf disease in different fruit trees species, involving significant economic losses in Chile. It produces wood necrosis and leaves silvering. Necrosis is evident soon after the pathogen infects wood, but foliar silvering is visible after two or more seasons. On the other hand, a non-destructive and rapid method to detect this disease is not yet available. Thus, the objective was to develop an immunoassay based on gold nanoparticles (AuNPs) for the detection of the enzyme Endopolygalacturonase (endo-PG), produced by C. purpureum. AuNPs with a mean diameter of $20 \mathrm{~nm}$ were synthesized by citrate reduction of $\mathrm{HAuCl}_{4}$, and functionalized with L-cysteine (AuNPs-Cys). Nanoparticles were characterized using TEM, UV- VIS spectroscopy, FTIR, Particle size Analyzer and Zeta potential. The optimum concentration of L-cysteine was estimated by testing a range of $(0.1-4.0 \mu \mathrm{M})$. Antibody-coated AuNPs were prepared by mixing an AuNPs-Cys suspension and a polyclonal anti- endoPG antibody solution. BSA was added to the mixture, incubated at room temperature and washed with PBS. The assay was able to detect endopolygalacturonase in an aqueous system. Therefore. antibody-coated AuNPs-Cys were proved to be a reliable method for the detection of the pathogen and proposed as the basis for the development of a diagnostic tool for silverleaf disease, that will reduce disease dissemination and economic losses.

Whole Genome Sequencing for Development of PCR specific detection of Monilinia species for Quarantine applications S. SRIVASTAVA, K. A. Zeller, Y. Rivera, J. Sutherland, Z. G. Abad, M. K. Nakhla, USDA-APHIS-PPQ-S\&T-CPHST, Beltsville, MD, USA

Monilinia species cause important and economically devastating brown fruit rot diseases of pome and stone fruits and blossom blights. Two Monilinia species, M. fructigena and M. polystroma, are of regulatory concern to US Agriculture, but cannot be easily distinguished from native species. To help us improve diagnostics methods, we sequenced the genome of Monilinia fructigena with both Illumina Mi-Seq and PacBio platforms to compare quality and enhance marker discovery. We generated 20 million and 1.08 million reads to assemble into 1,635 and 1,324 contigs with over 150X and 50X coverage for the Illumina and PacBio platforms, respectively. We used CLC and Canu assemblers to assemble these Illumina and PacBio reads, respectively, and PacBio reads were further polished to improve the quality of bases with Illumina using PILON (hybrid correction/polisher). The Illumina assembly covers $93.67 \%$ of PacBio assembly, meaning we added $7 \%$ more genome using PacBio assembled contigs. We observed $41.2 \%$ in Accessory Genetic Material (AGM), or unique sequences, and 58.8\% Core Genetic Material (CGM) when this assembly is compared to M. fructicola. We also observed $23.8 \%$ unique (CGM 76.2\%) when compared with M. laxa, and $11.2 \%$ unique (CGM 88.8\%) when compared with M. polystroma. We plan to annotate these genomes (AGM as well as CGM) and analyze for identification of uniqueness between these closely related species to develop DNA based markers for quarantine applications. 
Reliable and inexpensive Real-time RT-PCR method for Apple stem grooving virus and Apple stem pitting virus detection E. BEAVER-KANUYA, S. J. Harper, Washington State University, Prosser, WA, USA

Apple stem grooving virus (ASGV) and Apple stem pitting virus (ASPV) negatively impact production, maintenance, and distribution of apples and other Malus species world-wide. Control of these two pathogens relies on rapid detection and diagnosis, particularly to identify newly infected plants for removal before they become a source of inoculum for further spread. Despite the increasing diversity of isolates found by high-throughput sequencing, little effort has been made to reassess or improve the diagnostic assays used for these two viruses. In this study we aimed to develop robust and reliable real-time PCR assays to meet these requirements. Primers and probes were designed against alignments of representative extant sequences from across the globe, and reaction conditions were optimized for speed, sensitivity, and specificity. Each assay was validated against a panel of ASGV and ASPV isolates and against related non-target virus species and common host species to ensure reaction specificity. These new assays showed increased detection of these two viruses in a quarantine setting. Similar results were obtained comparing the real-time RT-PCR assay and the high-throughput sequencing method in a panel of 13 samples. Adoption of the real-time RT-PCR platform for these two viruses reduced time-to-answer from 2-3 hours to 1:20 hour. The new method worked well for both domestic and foreign apple isolates and reduced the time and the cost for diagnosis.

Sesame Root Rots of South Texas: A Fresh Look

K. COCHRAN (1), T. N. Spurlock (2), J. Rose (3), G. De La Fuente (3), (1) Texas A\&M University, Uvalde, TX, USA; (2) University of Arkansas, Monticello, AR, USA; (3) Sesaco Corporation, Austin, TX, USA

Sesame is grown throughout the south United States and abroad as an oilseed, seed, and game bird habitat crop. In South Texas, sesame is often grown as a dryland or irrigated crop with both methods generating favorable conditions for soilborne pathogens, which can cause crop loss or failure. Historically, sesame root rot in south Texas was mainly attributed to Phytophthora sp. In recent years, little work has been done in south Texas and the south United States to assess root rot disease challenges and their prevalence. The objective of this work was to determine current disease challenges to sesame production in south Texas. Four sesame fields with suspected root rot in Uvalde Co., Texas were surveyed for root rot symptoms near the end of the production season in September-October 2015-2017. Plant samples were collected and roots and stem tissue from near the soil line were assayed in water agar and fungal isolates identified and transferred to pure culture. All fields had evidence of root rot with Rhizoctonia solani Kuhn and Macrophomina phaseolina (Tassi) Goid. being most commonly isolated. Incidence of $R$. solani and M. phaseolina were associated with increased lodging near harvest, resulting in dramatically decreased yields in affected areas. A better understanding of the current soilborne pathogen distribution in sesame production will assist producers with management choices and future rotation crop selections.

Design and testing of PCR-based diagnostics generated from NGS for distinguishing among Monilinia species of economic importance to US agriculture

J. SUTHERLAND, S. Srivastava, Y. Rivera, K. A. Zeller, Z. G. Abad, USDA-APHIS-PPQ-S\&T-CPHST, Beltsville, MD, USA

Fungi in the genus Monilinia cause a devastating brown rot disease of stone and pome fruits. Monilinia fructicola and M. laxa are established in the USA, while M. fructigena and M. polystroma are pathogens of regulatory significance. We have completed draft genomes of all 4 Monilinia species using Illumina Next Generation Sequencing (NGS) platform. Prior to our work, limited genomic information of M. fructigena, M. laxa and M. polystroma were available to use in diagnostics development. These draft genome assemblies are between $39-45 \mathrm{Mb}$ and consistent with the genome sizes of related fungal species. We have developed comparative genomics pipelines that are allowing us to screen this new wealth of NGS data for candidate genomic targets that can then be tested on a broader set of strains from these 4 Monilinia species. Our goal for the current phase of this project is use these comparative genomics pipelines to identify candidate targets, to design PCR primers targeting these, and to evaluate and validate speciesspecific molecular markers for the detection and differentiation of Monilinia species of concern (M. fructigena and M. polystroma) from other Monilinia species already established within the US. We will discuss these pipelines and progress toward testing, optimizing and validating molecular methods that can distinguish among these species of regulatory concern.

Rapid detection of leaf spot pathogens on spinach using PCR and real-time PCR assays B. LIU (1), C. Feng (2), J. C. Correll (2), (1) Univ of Arkansas, Fayetteville, AR, USA; (2) University of Arkansas, Fayetteville, AR, USA

Leaf spot diseases of spinach, caused by Colletotrichum spp., Stemphylium botryosum, Cladosporium variabile, and Myrothecium verrucaria, have become a major concern in spinach production in the U.S. Rapid detection and identification of these pathogens is necessary for epidemiological studies and effective disease management. Based on sequence data of the internal transcribed spacer (ITS) region of 30 representative isolates of each species (Stemphylium botryosum, Cladosporium variabile, and Myrothecium verrucaria), and the introns of glutamine synthetase (GS) and glyceraldehyde-3phosphate dehydrogenase (GPDH) genes of 30 isolates of each species (Colletotrichum dematium, C. coccodes, and C. truncatum) from GenBank, and isolates of each pathogenic species recovered from the field, five sets of primers were designed and evaluated for their species-specificity. Results showed that two sets of primer pairs from each species were highly sensitive and specific for detection of each individual species using DNA recovered from single lesion on leaves by a rapid DNA extraction procedure. Moreover, a specific primer pair was designed and used for real-time PCR assay for rapid detection and quantification of each pathogen on spinach leaves. The PCR based diagnostic analysis can be completed within $24 \mathrm{~h}$, compared with 1-2 weeks for doing isolations and identification. In addition, large-scale field testing was evaluated in order to further confirm the reliability of the newly developed molecular diagnostic techniques.

Third generation sequencing and EDNA for detection of aflatoxin production in the soil A. ESPINDOLA, K. F. Cardwell, Oklahoma State University, Stillwater, OK, USA

Aspergillus flavus is a major food safety concern for the food industry. Toxic strains produce varying amounts of the carcinogen aflatoxin B1. This study aims to improve the time-consuming soil sampling, isolation, culturing, toxin detection and exploration of the soil microbiome needed to manage aflatoxin contamination. Third Generation Sequencing (TGS) using a MinION ${ }^{\mathrm{TM}}$ portable sequencer allows sequencing rapidly metatranscriptomes. Yet, traditional microbiome analyses of TGS samples offers only a hint of the functional characteristics of the microbiome in the soil. E-probe Diagnostic for Nucleic acid Analysis (EDNA) is a bioinformatic pipeline that can infer the production of aflatoxin and detect $A$. flavus strains rapidly. In this study, the EDNA pipeline was used to measure the presence of atoxigenic and toxigenic A. flavus in soil and the production of aflatoxin. Soil inoculated with AF70 (toxigenic strain) at different titers and AF36 (atoxigenic, control) were incubated at $28^{\circ} \mathrm{C}$. RNA was extracted from the inoculated soil and mRNA sequenced using the MinION ${ }^{\mathrm{TM}}$. The sequencing output was analyzed using EDNA transcriptomics and differences were found between titers and aflatoxin gene regulation using e-probes. The obtained results suggest that EDNA can discriminate semi-quantitatively between strain taxonomic units and relative aflatoxin production in soil samples. This is the first time aflatoxin has been directly assessed in situ in the soil environment. 
Inonotus obliquus, commonly known as chaga, is a fungal pathogen found almost exclusively on birch trees. The sterile conk of chaga has an extensive history as a folk remedy for stomach ailments and cancer in Northern Europe and increasingly in North America. Due to its emerging economic value, chaga has become the target of harvest in the White Mountains National Forest (WMNF). Consequently, WMNF managers are concerned about the potential impacts of chaga harvest on forest health, and are interested in developing guidelines for sustainable harvest. These guidelines may provide a basis for special use permitting. However, these efforts to develop best management practices are currently constrained by a lack of knowledge about the abundance, preferred habitat, and volume of the chaga resource in the WMNF. The objectives of this study are to: quantify chaga abundance and preferred habitat in the WMNF; determine the incidence of chaga by tree species and other correlating variables; and from these data develop best management practices for chaga harvest in the WMNF. A survey conducted in 2017 assessed the characteristics of chaga in 109 trees carrying conks: results indicated that $48.5 \%$ of white and yellow birch, $2 \%$ of black birch, and $1 \%$ of sugar maple trees surveyed had chaga present on their stems. We will also present results about the incidence of chaga in relation to correlating variables such as DBH, Live Crown Ratio, and stand species composition.

Comparison between high throughput sequencing and current protocol for virus detection in berry crops

D. E. V. VILLAMOR (1), R. R. Martin (2), I. E. Tzanetakis (1), (1) University of Arkansas, Fayetteville, AR, USA; (2) USDA ARS, Corvallis, OR, USA

A combination of biological indexing, serological and molecular assays is the current standard for virus detection in berry fruit crops (Fragaria, Rubus and Vaccinium, spp.). This panel of assays had been reliable in ensuring the production and release of virus tested propagation materials for these specialty crops. In recent years, high throughput sequencing (HTS) has gained wide acceptance for use in virus detection. Here, we compared HTS and the standard protocols for virus detection on 32 berry accessions (12 Fragaria, 10 Rubus and 10 Vaccinium). HTS gave better results than the alternative assays because it was able to detect new viruses with a lower overall cost. These results not only confirm the efficacy of HTS for virus detection but also further suggest its use to augment or possibly replace the current virus detection protocols for berry crops.

Digital PCR reveals different effects of plant matrices on the recovery of Xylella fastidiosa DNA

T. DREO, M. Pirc, M. Ravnikar, National Institute of Biology, Ljubljana, SLOVENIA

Xylella fastidiosa infects many different host plants and some present a challenge for its reliable detection and quantification in both, diagnostics and research. To elucidate the inhibition arising from different host plants and the efficiency of molecular detection methods, altogether 129 samples of 29 genera were spiked with a low concentration of $X$. fastidios a cells. The recovery of $X$. fastidiosa was determined with digital PCR after automated magnetic beads DNA extraction, and the results compared with the results of real-time PCR. Overall, real-time PCR was less sensitive and less resistant to inhibition than dPCR, in particular in Rosmarinus, Lavandula, Origanum and Prunus samples that necessitate analysis of diluted DNA. For samples of olives (Olea europaea), critical samples extensively tested for $X$. fastidiosa in Europe, high inhibitory effect was not alleviated with analysis of dilutions and dPCR revealed low recovery of $X$. fastidiosa DNA in addition to inhibitors. In olives and other samples with high inhibition and/or low DNA recovery, dPCR provided a more reliable and accurate detection and quantification method than real-time PCR at lower number of technical repeats.

Development of a recombinase polymerase amplification assay with qualitative end-point detection for diagnosis of thousand cankers disease in walnut

J. SIMMONS (1), S. Seybold (2), R. M. Bostock (1), (1) University of California, Davis, CA, USA; (2) United States Department of Agriculture, Forest Service, Davis, CA, USA

Geosmithia morbida, the causal fungus of thousand cankers disease, forms cankers on the trunk and branches of trees of various walnut species following attack by the walnut twig beetle, which vectors the pathogen. Diagnosis typically requires isolating the fungus from bark tissue on media, which can be confounded by the presence of morphologically similar, nonpathogenic Geosmithia species and other fungi. A molecular technique for direct analysis of infected walnut tissue will simplify and expedite diagnoses. A recombinase polymerase amplification assay for $G$. morbida was developed in which amplicons were designed for lateral flow strip detection. Based on a consensus sequence for the G. morbida Ef 1-alpha gene, a probe was designed with the 5' end labeled with FAM, the 3' end with a C3 spacer, and an internal abasic nucleotide replacement. Forward and reverse primers were designed to flank the probe, and the primer in the opposite orientation to the probe was labeled at the 5' end with biotin. All combinations of forward and reverse primers and probes were screened against $1 \mu \mathrm{M}$ DNA of G. morbida, Geosmithia fassatiae, and Geosmithia lavendula, and further tested against DNA of Juglans regia and Fusarium solani. The selected primer/probe combination identifies all tested isolates of G. morbida with species-level specificity. Experiments are underway to optimize sensitivity and adapt for field application.

Recombinase Polymerase Amplification Assay for in Field Detection of Tomato Bacterial Spot (Xanthomonas euvesicatoria, X. gardneri, and $X$. perforans)

A. L. STRAYER (1), J. B. Jones (2), M. Paret (3), (1) University of Florida, Department of Plant Pathology, Gainesville, FL, USA; (2) Department of Plant Pathology, University of Florida, Gainesville, FL, USA; (3) North Florida Research and Education Center, University of Florida, Quincy, FL, USA

Bacterial spot, one of the most detrimental diseases of tomato that occurs worldwide, is caused by Xanthomonas euvesicatoria (Xe), X. gardneri (Xg), X. perforans $(\mathrm{Xp})$, and $X$. vesicatoria $(\mathrm{Xv})$. Currently, there is no in-field diagnostic assay available for bacterial spot. In this study, we developed two recombinase polymerase amplification (RPA) assays that can detect $\mathrm{Xe}, \mathrm{Xg}$, and $\mathrm{Xp}$ in crude plant samples in the field using a portable detection instrument. Three RPA exo probes and two primer sets were designed to amplify regions of the $h r p B$ gene of Xe, $\mathrm{Xg}$, and Xp. The Xg RPA assay amplified DNA extracted from 18 pure cultures of Xg and 24 crude plant samples infected with Xg. The Xe RPA assay amplified DNA extracted from 36 pure cultures of Xe and Xp, and 63 crude plant samples infected with either Xe or Xp. The Xp RPA assay only amplified DNA extracted from 18 pure cultures of Xp. Thus, the Xe RPA assay can be used to diagnose bacterial spot caused by either Xe or Xp in the field, which can be differentiated using the Xp RPA assay following isolation in the laboratory. Although an additional RPA exo probe and primer set was designed to detect Xv, it was unable to amplify DNA extracted from pure cultures of Xv. Since Xv is becoming less prevalent worldwide, it could be considered as the least important bacterial spot pathogen. Since the Xe and Xg RPA assays detected Xe, Xg, and Xp in crude plant samples, it holds great potential as an in-field diagnostic tool. 
Direct RT-PCR assay for virus detection and eriophyoid species identification

T. DRUCIAREK (1), M. Lewandowski (2), I. E. Tzanetakis (1), (1) University of Arkansas, Fayetteville, AR, USA; (2) Department of Applied Entomology, Warsaw University of Life Sciences - SGGW, Warsaw, POLAND

Eriophyoid mites (Acari: Eriophyoidea) are important plant pests, causing direct damage and, most importantly vectoring plant viruses. Because of their size (averaging $200 \mu \mathrm{m}$ in length) and concealed lifestyle (living in secreted or confined microhabitats) about $90 \%$ of eriophyoid mite fauna is speculated to be undiscovered. We have developed a novel direct Reverse Transcriptase - Polymerase Chain Reaction (direct RT-PCR) assays for species barcoding and virus detection in individual mites. Bypassed extraction steps prevent loss of nucleic acids during the extraction and lower the risk of crosscontamination. RT step not only allows for the detection of RNA viruses but also provides additional template of the fragments used for species barcoding (Cox 1, 28S rRNA), enhancing the sensitivity and PCR yields when compared to other methods. Results indicate that direct RT-PCR serve as fast and cost effective method for virus detection and species identification using individual mites.

Quantification of Xylella fastidiosa in pecan (Carya Illionoinensis) plant tissues

A. HILTON (1), F. Cao (2), X. Wang (3), L. J. Grauke (3), Y. K. Jo (1), (1) Texas A\&M University, College Station, TX, USA; (2) Nanjing Forestry University, Nanjing Shi, Jiangsu Sheng, CHINA; (3) USDA-ARS Pecan Breeding and Germplasm Repository, Somerville, TX, USA

Pecan Bacterial Leaf Scorch (PBLS) caused by xylem-limited bacterium, Xylella fastidiosa, is a chronic, debilitating disease in species of Carya. PBLS has been recently identified in California, Arizona, New Mexico, and Texas. As new reports of incidence emerge, it is critical to uncover the epidemiology of this pathogen to combat PBLS. The first aim of this study was to determine the distribution of the bacterium within a seedling. A quantitative PCR platform was developed using a novel Xylella-specific TaqMan probe. The bacterial concentration of roots, shoots, and leaves in infected 'Elliott' pecan seedlings were determined. X. fastidiosa was detected in the DNA isolated from roots and shoots, and roots were found to contain the highest titer levels. Our second aim was to determine the relationship between pecan nut quality and concentration of $X$. fastidiosa. We collected 150 nuts from a known PBLS-infected 'Cape Fear' tree and measured nut density on individual nuts. Nut volume was calculated by measurement of buoyancy and used to determine nut density (mass/vol). Three categories were established to represent nut densities: high (.72 g/cc), medium (.62 g/cc) and low $(.52 \mathrm{~g} / \mathrm{cc})$. DNA will be isolated from the cotyledons of each nut, and the concentration of $X$. fastidios $a$ will be determined. Pearson's correlation of coefficient will be used to determine the relationship between $X$. fastidiosa concentration and nut density. Information on the correlation between nut quality and presence of Xylella will be useful when determining seed selection strategies for distribution and cultivation of pecan rootstocks.

Fungi associated with canker and regressive death in Aristotelia chilensis growing in Southern Chile E. X. BRICENO, O. Montenegro, Universidad Austral de Chile, Valdivia, CHILE

Maqui (Aristotelia chilensis) is a Chilean native tree known by producing berries with high antioxidant content. Its cropping area has increased continuously across years, and several local ecotypes have been selected for fruit yield and quality. Commercial orchards located at the Los Rios and Los Lagos Regions, Southern Chile, have shown several disease symptoms on productive wood, such as dry and humid corky like cankers on trunk, branches and twigs. In addition, cutting death and root development delays have been observed in several Maqui nurseries. A number of samples were collected for these symptoms, and several pathogens isolated. Pathogen identification was conducted using morphological evaluations, and later corroborated phylogenetically utilizing ITS4 / ITS5 sequences. Neofusicoccum spp. was isolated from cuttings, Cylindrocarpon sp. and Dicostroma sp. from corky and humid looking cankers, and Diaporthe sp., from cankers associated with regressive death. Pathogenicity tests were carried out on semi-woody branches to fulfill Koch's postulates. All identified pathogens were recovered from individual plants or coexisting in the same Maqui plant.

\section{Development of multiplex viroid rapid detection system for Solanaceae plants and seeds}

C. F. Wu (1), Y. Tseng (1), C. H. Lee (2), Y. K. Chen (1), F. J. JAN PHD (1), (1) Department of Plant Pathology, National Chung Hsing University, Taichung, TAIWAN; (2) Ph.D. Program in Microbial Genomics, National Chung-Hsing University, Taichung, TAIWAN

Solanaceae plants including tomato, potato, eggplant, and tobacco are commercially important crops, widely planting around the world. Viruses and viroids are the obligatory parasitic pathogens which could infect and cause severe yield loss on Solanaceae plants. Viroids are single strand circular RNA which could be transmitted by mechanical operation or seeds. Several viroids which can be transmitted by seeds are important quarantine pathogens in many countries including Taiwan. Six quarantined viroids including Columnea latent viroid (CLVd), Pepper chat fruit viroid (PCFVd), Potato spindle tuber viroid (PSTVd), Tomato apical stunt viroid (TASVd), Tomato chlorotic dwarf viroid (TCDVd), and Tomato planta macho viroid (TPMVd) are the major concerns. The objective of this study was to develop the rapid one step reverse transcription polymerase chain reaction (RT-PCR) and RT- Loopmediated Isothermal Amplification (LAMP) system for detecting these six viroids simultaneously in seeds and infected plants. The infectious DNA clones of the above-mentioned six viroids were successfully constructed and inoculated into plants. The infectious clones after being inoculated into tomato seedlings showed stunting, stem necrosis, and chlorosis symptoms which were very similar to those inoculated with mechanical means. Degenerate primer sets for one-step RT-PCR and specific primer sets used for RT-LAMP were designed and tested. The primers could simultaneously detect six target viroids. This multiplex system conjunction with modified methods for rapid total RNA extraction can be used for detection of viroids from seeds and field-collected samples.

Foliar stage of gumming disease present in sugarcane plantations in Mexico H. V. SILVA-ROJAS (1), A. Rebollar-Alviter (2), A. Valdez-Balero (3), M. L. Osnaya-Gonzalez (4), (1) Colegio de Postgraduados, Edo de Mexico, MEXICO; (2) Univ Autonoma Chapingo, Morelia, Michoacan, MEXICO; (3) Colegio de Postgraduados, Montecillo, NM, MEXICO; (4) Colegio de Postgraduados, Campeche, MEXICO

n 2012, gumming diseases caused by Xanthomonas vasicola pv. vasculorum was identified in sugarcane plantations in Mexico. Only foliar phase was

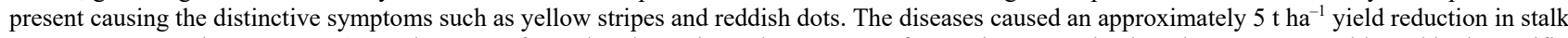
sugar content. During 2013-2014 a study was performed to determinate the presence of gumming systemic phase in sugar cane cultivated in the pacific and central regions of Mexico. For this goal, stalks with symptomatic leaves were collected and bacterial isolates were recovered from nutrient agar medium. Isolates were identified by PCR and sequencing of $16 \mathrm{~S}$ rDNA gene. The diseases was present only at the leaves. No systemic stage of the disease was observed in stalk samples. In Mexico, the disease was detected in the region between the coordinates $18^{\circ} 50^{\prime} 59^{\prime \prime} \mathrm{N}$ and $98^{\circ} 57^{\prime} 46^{\prime \prime} \mathrm{W}$ to $18^{\circ} 05^{\prime} 21^{\prime \prime}$ and $93^{\circ} 21^{\prime} 23 \mathrm{~W}$ with temperature and relative humidity conditions of 23.5 to $25^{\circ} \mathrm{C}$ respectively and $70 \% \mathrm{RH}$. Thus, it is concluded that the systemic phase of the disease does not develop under this environmental conditions. 
Automated primer design for DNA-based detection of the emerging potato pathogen Dickeya dianthicola

S. KARIM (1), R. R. McNally (1), A. Dereeper (2), A. O. Charkowski (1), L. R. Triplett (3), (1) Colorado State University, Fort Collins, CO, USA; (2) IRD, Cirad, Univ Montpellier, IPME, Montpellier, FRANCE; (3) Department of Plant Pathology and Ecology, The Connecticut Agricultural Experiment Station, New Haven, CT, USA

Soft-rotting Enterobacteria (SRE) comprise a subset of bacterial plant pathogens including agronomically important genera Pectobacterium sp. and Dickeya sp. Several Pectobacterium and Dickeya species represent significant pathogens of potato, but identifying these species with PCR is made difficult by their close relatedness and frequent genetic exchange. Numerous SRE species continuously impact potato yields but the recent emergence of Dickeya dianthicola, an invasive pathogen from Europe, now challenges disease management and diagnostic procedures across North America. To address this concern, we developed an automated comparative primer design pipeline, Uniqprimer, and used it to design primers for DNA-based detection of $D$. dianthicola. Uniqprimer performed alignments of six $D$. dianthicola genomes to 74 non-target genomes, designed primers based on regions of divergence, and mapped the primer pairs back to the genomes to confirm specificity. To demonstrate the specificity and sensitivity of our primers, we performed conventional and quantitative PCR assays. All primers were evaluated and confirmed for specificity to D. dianthicola using diverse bacterial isolates and infected potato tissues. Our results demonstrate that specific and sensitive PCR assays designed using Uniqprimer can aid in diagnosis and disease management of the emerging potato pathogen $D$. dianthicola. Uniqprimer is being tested for public release in the Galaxy web platform.

Improvement of LCHV-1 detection by conventional RT-PCR and Real Time PCR protocols N. FIORE (1), C. Fernández (2), A. Zamorano (1), (1) University of Chile, Santiago, CHILE; (2) Universidad de Chile, Facultad de Ciencias Agronómicas, Santiago, CHILE

Sweet cherry is one of the most important fruit crops in economical income in Chile, placing the country in the first place in cherry production in the southern hemisphere. From October 2015 to March 2016, a survey was performed in the main sweet cherry producing regions of Chile, collecting samples from 223 trees. Several viruses were detected by RT-PCR, but not Little cherry virus 1 (LChV-1), using primer pairs indicated in literature. Nineteen samples were randomly selected for small RNA sequencing in Illumina MiSeq platform and sample reads were trimmed using FASTX and assembled using VELVET. Four samples presented contigs that matched with LChV-1 Genbank references, indicating the presence of new variants of the virus in Chilean samples. As a consequence and in order to improve the detection of LCHV-1 by conventional RT-PCR and Real time PCR TaqMan, new specific primer pairs and a molecular probe were designed using the 3' untranslated region of the virus genome. Using the total nucleic acid extracts stored at $-80^{\circ} \mathrm{C}$, the same 223 samples were analyzed again, obtaining $15 \%$ and $30 \%$ of positive samples with conventional RT-PCR and Real time PCR TaqMan, respectively. These new detection tools allowed the improvement of the detection of LChV-1, being Real Time PCR the most sensitive protocol. In addition, this last method was developed for the first time for the detection of LChV-1.

Identification of fungal pathogens associated with cassava root rot in Thailand

S. Duchanee (1,2), R. Sangpueak (1), S. Wongkeaw (1), P. Phansak (3), N. BUENSANTEAI (4), (1) School of Crop Production Technology, Suranaree University of Technology, Nakhonratchasima, THAILAND; (2) National Science and Technology Development Agency, Pathum Thani, THAILAND; (3) Nakhon Phanom University, Nakhon Phanom, THAILAND; (4) School of Crop Production Technology, Suranaree University of Technology, Nakhorn Ratchasima, THAILAND

Cassava root rot (CRR) is the most serious diseases of cassava in Thailand, especially Northeastern region. The aim of this study was to identify the CRR causal agent. The study was carried out by collecting samples with root rot symptoms from various cassava planting areas including 3 districts of Nakorn-Ratchasima provinces, Thailand. The fungal pathogens were isolated from CRR lesions, the typical symptoms were often accompanied by wet, soft or dry rot symptom, and black pycnidia were found on the stalk and propagative stakes. The 139 representative samples were obtained into 5 genera according to the colony and spore morphology using a standard morphological study protocol. Lasiodiplodia spp. was the most frequently found fungus, constituting approximately $54 \%$ of the total, followed by Fusarium spp., Neoscytalidium sp., Phytophthora spp., Sclerotium sp. and other fungal genera which were found at 29, 7, 4, 1 and 5\%, respectively. The pathogenicity test of representative 33 single-spore isolates showed that they could cause stem and root rot symptoms on the inoculated susceptible cassava cv. Rayong 72 under both moist-chamber and green house conditions, the isolate L11HSR2 was the most virulent. By using 3 primers including ITS1/ITS4, Ef1-688F/Ef1-1251R and Bt2a/Bt2b primers to amplify the DNAs from 8 representative isolates, only the primer of the EF1- $\alpha$ region was effective in differentiating the isolates at species level and had an agreeable result with that identified by the standard morphological technique. When these eight isolates was compared against GenBank's database using the Mega BLAST program, and the alignment data using the NJ, UPGMA and ML methods, could be identified as L. theobromae, L. euphorbicola and N. hyalinum. This is, so far, the first report of fungal pathogens complex species associated with CCR disease in Thailand.

Occurrence of Grapevine fanleaf virus in Russia

E. Porotikova (1), U. Dmitrenko (1), A. Kamionskaya (1), S. VINOGRADOVA (1,2), (1) Research Center of Biotechnology, Russian Academy of Sciences, Moscow, RUSSIA; (2) Russian State Agrarian University - Moscow Timiryazev Agricultural Academy, Moscow, RUSSIA

Grapevine fanleaf virus (GFLV) is one of the most severe viruses of grapevine. It occurs worldwide, causes more than $50 \%$ yield loses and decrease sugar content and titratable acidity (Andret-Link et al., 2004). GFLV is transmitted by nematode Xiphinema index. To investigate the GFLV occurrence in Russia the survey of Crimea vineyards has been conducted in 2014-2017. Total of 937 leaf samples with virus symptoms were collected. Short internodes, zigzag growth of shoots, yellow discoloration of leaves were observed. Samples were analysed by RT-PCR with the specific to GFLV primers, followed by sequencing of the PCR products. GFLV was found in $2.3 \%$ of the samples. GFLV was detected in combination with RSPaV $(0.51 \%)$. The combination of GFLV, GVA and GLRaV-1 occurs in $0.13 \%$ of samples and combination of GFLV, RSPaV and GLRaV-3 occurs in $0.13 \%$ of samples. Nowadays we develop immunochromatography assay for rapid GFLV detection in the vineyard.

NextRAD sequencing unravels the genetic diversity of cassava-colonizing Bemisia tabaci

E. N. WOSULA (1), W. Chen (2), Z. Fei (2,3), J. P. Legg (1), (1) International Institute of Tropical Agriculture, Dar Es Salaam, TANZANIA; (2) Boyce Thompson Institute, Ithaca, NY, USA; (3) USDA-ARS Robert W. Holley Center for Agriculture \& Health, Ithaca, NY, USA

Bemisia tabaci is a vector of cassava mosaic begomoviruses and cassava brown streak ipomoviruses which cause significant cassava yield losses in Africa. Cassava-colonizing B. tabaci comprise several cryptic species that cannot be distinguished morphologically and so are separated by sequencing the mitochondrial DNA cytochrome oxidase I (mtCOI). The objectives of this study were to (i) determine the effectiveness of the mtCOI marker for delineating cassava B. tabaci haplogroups and (ii) determine if there is gene flow among these haplogroups. Ninety-five whitefly specimens collected from cassava in eight African countries were genotyped using NextRAD sequencing, and their phylogeny and population genetics were investigated 
using the resultant single nucleotide polymorphism (SNP) markers. SNP marker and mtCOI data obtained from the same insect were compared. Eight genetically distinct groups were identified based on mtCOI, while phylogenetic analysis using SNPs identified six major groups. STRUCTURE analysis identified four ancestral B. tabaci populations that have contributed alleles to the six SNP-based groups. Significant gene flows were detected between several of the six SNP-based groups. This study reveals that $\mathrm{mtCOI}$ is not an effective marker for phylogenetic classification of cassava-colonizing $B$. tabaci, and that more robust SNP-based multilocus markers should be developed. Significant gene flows between populations could lead to the emergence of haplogroups that might alter the dynamics of cassava virus spread and disease severity in Africa. Continuous monitoring of the genetic composition of whitefly populations should be an essential component in efforts to combat cassava viruses in Africa.

Effect of environmental temperature on transmission of mollicutes by Dalbulus maidis leafhopper in maize E. DE OLIVEIRA SABATO (1), E. C. Landau (2), B. D. A. Barros (2), (1) EMBRAPA-CNPMS, Sete Lagoas, BRAZIL; (2) Embrapa, Sete Lagoas, BRAZIL

The objective of this work was to evaluate the effect of winter-spring temperatures on transmission and latent period of maize bushy stunt (MBS) phytoplasma and Spiroplasma kunkelii by D. maidis. In one screen house experiment, where the temperature was daily recorded from the climatic station of the experimental farm of Embrapa, Sete Lagoas, State of Minas Gerais, Brazil, half of a healthy leafhopper population was confined in maize infected with spiroplasma and the other half in maize infected with phytoplasma, for 6 days as acquisition access period (AAP). After that, leafhoppers were feeding with healthy seedlings. Transmission tests were performed with one leafhopper per seedling for each mollicute, with 12 replicates, at 12, 20, 30, 40 days since the first day of AAP. In addition, at 40 days, one leafhopper from AAP-phytoplasma and other from APP-spiroplasma were confined together in 24 maize seedlings. The access period for inoculation (API) was 3 days. Until flowering, only at latent period of 40 days, $41.6 \%$ and $58.3 \%$ of plants respectively submitted to phytoplasma or to both mollicutes presented MBS-phytoplasma symptoms. Temperatures during AAP ranged from $8^{\circ} \mathrm{C}$ to $26^{\circ} \mathrm{C}$ and like this until 40 days. During the API- 40 days test, the temperatures ranged from $8^{\circ} \mathrm{C}$ up to $34^{\circ} \mathrm{C}$, and after that, in general, higher than $8^{\circ} \mathrm{C}$ up to $34^{\circ} \mathrm{C}$. Results of plant symptoms and for PCR tests indicated that MBS-phytoplasma is more tolerant to low temperatures than S. kunkelli.

Root-feeding beetles and Leptographium and Grosmannia blue-stain fungi in loblolly pine stands with differing management practices M. BULAND (1), B. Barnes (1), K. Klepzig (2), K. Gandhi (1), C. Villari (1), (1) D.B. Warnell School of Forestry and Natural Resources, University of Georgia, Athens, GA, USA; (2) Joseph W. Jones Ecological Research Center at Ichauway, Newton, GA, USA

Leptographium and Grosmannia fungal species and their root-feeding beetle vectors (Hylastes, Hylobius and Pachylobius spp) have been associated with areas of loblolly pine mortality in Alabama and Georgia. However, the exact species composition, and in particular the frequency of association of individual fungal species with each vector, have yet to be identified. Objectives of our study are to: 1) determine the phenology of loblolly pine rootfeeding beetles in Georgia, 2) assess the frequency of association of Leptographium and Grosmannia spp associated with these beetles, and 3) analyze whether this association varies in stands with differing management practices and across seasons. Root-feeding beetles were collected in two loblolly pine stands that were either regularly prescribed burned or unburned. Individual specimens were used to isolate and identify associated fungi. Speciesspecific PCR primers were developed for each target fungal species identified and a subset of beetles will be tested to determine the frequency of association of individual fungal species with their vectors. Preliminary results show significant variation across the seasons in the catches of $H$. pales, the most abundant beetle species. Beetles catches in the burned site were higher compared to the unburned site. Fungal species currently recovered include L. procerum, G. alacris, and G. huntii. This study is a first step to facilitate future research into these beetles/fungi complexes.

Carrot motley dwarf disease: A good example for synergistic relationships between viruses and between virus and vector N. YOSHIDA, T. Tamada, HOKUREN Agricultural Research Institute, Naganuma, Hokkaido, JAPAN

Carrot motley dwarf disease is caused by a complex of two viruses: carrot red leaf virus (CtRLV, Polerovirus) and carrot mottle virus (CMoV, Umbravirus). Additionally, the diseased plants contain frequently a virus-like RNA called CtRLV-associated RNA (CtRLVaRNA). Those viruses are transmitted by willow-carrot aphid (Cavariella aegopodii). Umbravirus and CtRLVaRNA do not encode a coat protein, and so use the coat protein of a helper virus CtRLV for aphid transmission. In Japan, we found CtRLV, CMoV and CtRLVaRNA-mixed infection and single CtRLV infection in carrot fields from different districts in Hokkaido. In our aphid inoculation tests using CtRLV isolates containing CMoV or CMoV + CtRLVaRNA, CtRLV with $\mathrm{CMoV}$ induced much severer symptoms in carrot plants than CtRLV alone, in which CtRLV accumulation increased. When co-infected with CtRLV with CMoV + CtRLVaRNA, accumulation of CMoV as well as CtRLV enhanced. The aphids transmitted more efficiently CtRLV with CMoV + CtRLVaRNA than CtRLV alone. In our field and laboratory observations, the aphids appeared to propagate more vigorously on mix-infected carrot plants than on non-infected plants. Together, we found that co-infections of polerovirus and umbravirus (and polerovirus-associated RNA) enhance synergistically their virus accumulation, the transmission efficiency, and the aphid feeding preference.

Candidatus Liberibacter asiaticus Forms ER-Associated Replicative Vacuoles inside Diaphorina citri Gut Cells A. LEVY (1), D. Achor (1), M. Ghanim (2), (1) University of Florida, Lake Alfred, FL, USA; (2) Volcani Center, Bet Dagan, ISRAEL

Citrus greening is caused by the phloem-restricted bacteria Candidatus Liberibacter asiaticus (CLas), and is transmitted by the Asian citrus psyllid (ACP) Diaphorina citri. Current management tools for ACPs rely on the application of chemical insecticides, but at present, these strategies are not efficient enough and also result in insect resistance. Blocking the bacteria propagation and/or movement inside the psyllid can be another alternative to prevent transmission, but very little is currently known about the bacteria-insect interactions. Here we used transmission electron microscopy and confocal microscopy to study the accumulation of CLas inside ACP guts. We show that CLas forms a large replicative vacuole inside the ACP gut cells, and that in the presence of CLas, the psyllid endoplasmic reticulum (ER) undergoes a dramatic re-organization to associate with these intracellular bodies. Immunolocalization confirmed that CLas accumulates inside these vacuoles. Our results suggest that after entry into the gut cells, CLas modifies the host ER to create an isolated and safe environment to support its own propagation inside the psyllid. Understanding the molecular interactions that are involved in this process can open new opportunities for controlling CLas.

Feeding behavior of whiteflies associated to the transmission of Torradoviruses J. Jimenez, M. Arantxa, A. FERERES, CSIC, Madrid, SPAIN

Torradoviruses have been reported as the cause of important yield losses on tomato. Although several studies have been performed, the specific mechanism underlying Torradoviruses transmission by whiteflies remains unknown. The objective of this work was to study the feeding behavior of Bemisia tabaci associated to the acquisition and inoculation process of Tomato torrado virus (ToTV). The feeding behavior of single whiteflies was monitored using Electrical Penetration Graphs (EPG) on tomato test plants during the transmission of ToTV. Insects were removed from test plants when 
the following behavioral events were recorded: extracellular stylet pathway (C), intracellular stylet punctures (potential drops: 'pd'), a single episode of salivation on phloem sieve elements (E1), the interphase between salivation and ingestion (E1/E2), and phloem sap ingestion (E2). For acquisition experiments, non-viruliferous whiteflies were monitored on infected ToTV tomatoes and then transferred to healthy test plants for $48 \mathrm{~h}$. Similarly, to study the viral inoculation process, viruliferous whiteflies were removed from healthy tomato test plants after specific EPG signals were recorded. Then, whiteflies were transferred to a second healthy tomato test plant for $48 \mathrm{~h}$ to assess its initial viruliferous status. ToTV transmission to test plants was assessed by RT-PCR. The results of the feeding behavior activities of $B$. tabaci associated to the transmission of ToTV will be reported.

\section{A bacterial plant pathogen employs the metabolism of its insect vector to fulfill its nutritional and energetic needs} N. KILLINY, Citrus research and education center, IFAS, University of Florida, Lake Alfred, FL, USA

Transmission of phyto-pathogenic bacteria by insects involves circulation and propagation of the bacteria within their vectors. The growth of plantpathogenic bacteria in the hemolymph of their vectors indicate that the hemolymph contains all necessary nutrients. In addition to nutrients, "Candidatus Liberibacter asiaticus" (CLas) uptakes energetic nucleotides, such as ATP, from its vector, Diaphorina citri, using ATP translocase. The goal of this study was to investigate the metabolic changes in $D$. citri upon infection with $C$ Las. CLas caused a dramatic alteration in $D$. citri metabolism, particularly in the induction of the glycolysis and the tricarboxylic acid cycles (TCA). In addition, CLas stimulated $D$. citri to produce more ATP and other energetic nucleotides and inhibited their utilization by the insect, resulting in ATP accumulation. These changes secured the required nutrients and ATP for $C$ Las growth. These metabolic changes resulted in a shorter insect lifespan and altered the feeding behavior. Interestingly, the metabolic alteration was greater in the nymphal stages than in adults. These findings increase our knowledge of insect transmission of the persistent-circulativepropagative type of plant pathogens vectored by insects and provides some insights into the mechanism of colonization of CLas in its vector, $D$. citri.

Partnerships between ambrosia beetles and fungi: Varying levels of promiscuity among vectors of the laurel wilt pathogen, Raffaelea lauricola J. R. Saucedo Carabez (1), R. C. PLOETZ (2), J. Konkol (1), D. Carrillo (1), R. O. Gazis (1), (1) University of Florida, Homestead, FL, USA; (2) Tropical Research \& Education Center, University of Florida, Homestead, FL, USA

Culturable fungi were recovered from mycangia of ambrosia beetles associated with trees of Persea humilis (silk bay, one site) and $P$. americana (avocado, six commercial orchards) affected by laurel wilt, an invasive disease that is caused by a nutritional symbiont, Raffaelea lauricola, of an Asian ambrosia beetle, Xyleborus glabratus. With partial sequences of ribosomal (LSU and SSU) and nuclear ( $\beta$-tubulin) genes, multiple operational taxonomic units (OTUs) of fungi were identified, most of which could be assigned to previously described species. Nine OTUs were isolated from Xyleborus bispinatus, five from Xyleborus volvulus, five from Xyleborus affinis, three from Xyleborinus saxesenii, two from X. glabratus and two from $X y l o s a n d r u s$ crassiusculus. Raffaelea spp. predominated in the Xyleborus species and $R$. lauricola was present in all except $X$. saxesenii and $X$. crassiusculus. Raffaelea arxii, $R$. subalba and $R$. subfusca were present in more than a single species of beetle, and $R$. arxii was the most abundant symbiont in both $X$. volvulus and $X$. affinis. Raffaelea aguacate, which had previously been detected only in laurel wilt-affected avocado trees, was detected for the first time in an ambrosia beetle (X. bispinatus). Yeasts (Ascomycota, Saccharomycotina) were common, and distinct populations of these fungi were found in each species of beetle that was examined. We discuss roles yeasts may play in the life cycles of these important pests.

Relationship between Stemphylium vesicarium and onion thrips (Thrips tabaci) in the development of Stemphylium leaf blight disease A. B. LEACH (1), F. S. Hay (2), R. Harding (1), B. A. Nault (1), (1) Cornell University, Geneva, NY, USA; (2) Plant Pathology \& Plant-Microbe Biology Section, Cornell University, Geneva, NY, USA

Stemphylium leaf blight of onion (caused by Stemphylium vesicarium) and onion thrips (Thrips tabaci) are two common causes of leaf defoliation of onion in New York. Onion thrips have previously shown to interact synergistically with other pathogens which exacerbate plant disease. However, the potential relationship between onion thrips and Stemphylium leaf blight is unknown. In a series of controlled laboratory experiments, the relationship between thrips feeding and movement on the development and severity of Stemphylium leaf blight disease were examined. Onions (cvs. 'Avalon' and 'Ailsa Craig') with varying levels of thrips damage ('no damage', 'low damage', or 'high damage') were inoculated with S. vesicarium. Pathogen colonization and leaf dieback were measured after two weeks. In the transmission assays, thrips were exposed to $S$. vesicarium spores for 30 min., and then transferred to onion leaves where plants were monitored for disease development. Initial results from these trials revealed that thrips feeding increased $S$. vesicarium colonization of onion leaves, and increased leaf dieback. Additionally, onion thrips were capable of transferring S. vesicarium spores to onion plants (albeit at a low frequency $5-15 \%$ of the time). These results suggest that increased thrips control may reduce Stemphylium leaf blight disease, and should be investigated in the field.

Are vectors the bottleneck for Orthotospoviruses' fitness during mixed-infection?

K. ZHAO (1), C. Rosa (2), (1) Penn State University, University Park, PA, USA; (2) The Pennsylvania State University, University Park, PA, USA

In order to be efficiently transmitted from one plant to another, plant viruses induce chemical and physical changes in their plant hosts. In addition, plant viruses can modify the feeding preferences and/or behavior of their insect vectors to enhance transmission. Orthotospoviruses are thrips transmitted viruses that cause high economic losses to agriculture worldwide. Mixed-infections of Orthotospoviruses have been reported in recent years, but little is known about thrips preferences under mixed-infection conditions. Our study focuses on how Western flower thrips' preference influences transmissibility of two Orthotospoviruses, Tomato spotted wilt orthotospovirus and Impatiens necrotic spot orthotospovirus, during mixed-infection and if vector preference is responsible for disease prevalence in different crops. Viral fitness during host-to-host transmission is considered to be an important part of overall viral fitness, and our study will enhance understanding of Orthotospovirus epidemiology.

Genome-wide piRNA profiles of the virus transmitting whitefly, Bemisia tabaci during feeding on TYLCV-infected tomato M. Shamimuzzaman (1), D. K. Hasegawa (1), W. Chen (2), A. Simmons (1), Z. Fei (2,3), K. S. LING (1), (1) USDA-ARS, Charleston, SC, USA; (2) Boyce Thompson Institute, Ithaca, NY, USA; (3) USDA-ARS Robert W. Holley Center for Agriculture \& Health, Ithaca, NY, USA

Piwi-interacting RNAs (piRNAs) are 26-31 nucleotide (nt) non-coding small RNAs (sRNAs) commonly found in animals. The whitefly, Bemisia tabaci MEAM1 is a devastating vector capable of transmitting many plant viruses, including Tomato yellow leaf curl virus (TYLCV), to important crop plants. To investigate potential sRNA mediated regulatory mechanisms in whiteflies that are affected by virus acquisition and transmission, we performed genome-wide profiling of piRNAs in whiteflies feeding on tomato plants with or without infection by TYLCV for 24,48 and $72 \mathrm{~h}$. Results revealed that piRNAs were expressed as clusters throughout the whitefly genome. piRNAs ranging from 564,395 to 1,715,652 were identified in sRNA libraries prepared at three time points and aggregated in between 57 and 96 clusters. Comparative analysis across all time points identified 53 commonly expressed piRNA clusters. We also identified five TYLCV-induced and 24 TYLCV-suppressed piRNA clusters. Approximately $62 \%$ of all identified 
piRNAs are derived from non-coding sequences that include intergenic regions, introns, and UTRs with unknown functions. The remaining $38 \%$ of piRNAs are derived from coding sequences (CDS) and repeat elements. Six protein coding genes were targeted by the TYLCV-induced piRNAs, but their function in anti-viral defense or virus transmission is not obvious. TEs targeted by piRNA clusters include both class I retrotransposons such as Gypsy, Copia, and LINEs and class II DNA transposons such as MITE, hAT, and TcMar. Our enhanced understanding of whitefly piRNA pathway might facilitate the identification of novel targets for RNAi control.

Detection of the zebra chip pathogen Candidatus Liberibacter solanacearum in Canadian psyllids

L. KAWCHUK (1), D. Johnson (2), S. Meers (3), J. Lynn (4), (1) Agriculture \& Agri-food Canada, Lethbridge, AB, CANADA; (2) University of Lethbridge, Lethbridge, AB, CANADA; (3) Alberta Agriculture and Forestry, Brooks, AB, CANADA; (4) Agriculture Canada, Lethbridge, AB, CANADA

Zebra chip (ZC) is a relatively new and economically important disease of potato (Solanum tuberosum L.). It has been documented in commercial potato fields in the United States, Mexico, Central America, and New Zealand. ZC is caused by the unculturable phloem-restricted $\alpha 2$-proteobacterium, Candidatus Liberibacter solanacearum (Lso). Disease symptoms include stunting, wilting, leaf rolling, chlorosis and/or purpling, and sometimes plant death. The most striking symptom of this disease is the appearance of dark stripes in infected tubers that alters flavor and makes the products unmarketable. Five Lso strains (hap A to E) have been described in different crops, with only hap A and B being associated with ZC in potato. Both pathogen strains are vectored and transmitted to solanaceaeous plants by the tomato/potato psyllid, Bactericera cockerelli (Šulc), (Hemiptera: Trizoidae). Over 1,000 plant samples and 7,000 yellow sticky cards were collected across Canada between 2013 and 2017. Low but increasing potato psyllid numbers were observed from AB, SK, and MB (between 2 and over 200 potato psyllids per annum). Sequence analysis of the cytochrome oxidase from individual potato psyllids confirmed the presence of Central and Western haplotypes on the Canadian prairies. A late season Lso positive potato psyllid was detected from four locations in southeastern AB in 2017. Sequence of the Lso 16S rDNA indicates the Canadian pathogen closely resembles hap A from WA, ID, and OR. Best management practices are being developed to prevent the occurrence of ZC in Canada.

Settling and feeding behavior of sharpshooter vectors of Xylella fastidiosa on new plum selections apparently resistant to leaf scald disease H. THOMAZI KLEINA (1), K. Kudlawiec (2), M. A. Dalbó (3), J. Lopes (2), L. L. May De Mio (1), (1) Departamento de Fitotecnia, Universidade Federal do Paraná, Curitiba, PR, BRAZIL; (2) Departamento Entomologia e Acarologia, Universidade de São Paulo, Piracicaba, SP, BRAZIL; (3) Empresa de Pesquisa Agropecuária e Extensão Rural de Santa Catarina, Videira, BRAZIL

Plum leaf scald (PLS) is a serious disease caused by Xylella fastidiosa, which is transmitted by sharpshooters (Hemiptera: Cicadellidae: Cicadellinae). Disease management is based on planting healthy nursery trees, eradication of diseased trees and insecticides. PLS has affected the crop expansion in Brazil, but the development of plum selections that apparently are not infected under field conditions has opened new prospects to disease control based on host plant resistance. Here we investigated the morphological differences of leaves and the settling and feeding behavior of two vectors (Bucephalogonia xanthophis and Sibovia sagata) on three plum selections (SC7, SC13 and Leticia). The settling preference was evaluated by counting the number of insects landed on plants of each selection, at 24, 48 and $54 \mathrm{~h}$ after releasing 40 sharpshooters in a choice-test arena. Sap ingestion rates were quantified by measuring honeydew excretions of sharpshooters confined on branches of each selection for $72 \mathrm{~h}$. SC7 selection was less preferred for landing, whereas SC7 and SC13 reduced sap ingestion of both vector species. Comparative light and scanning microscopy analyses indicated that SC7 and SC13 have larger epidermal cells and thick layers of epicuticular wax, which may affect stylet penetration until the xylem vessels, as well as transmission of $X$. fastidiosa. The results suggest that SC7 and SC13 selections show antixenosis resistance to the vectors.

Survey of Rose Rosette Virus and its eriophyid mite vector in the Deep South

K. SOLO (1), S. Collins (1), R. Ochoa (2), G. Bauchan (3), R. A. Henn (4), J. C. Jacobi (5), J. L. Williams-Woodward (6), F. Hale (7), M. R. Hajimorad (8), J. Wilkerson (1), A. S. Windham (7), M. T. Windham (1), (1) University of Tennessee, Knoxville, TN, USA; (2) USDA-ARS, Systematic Entomology Laboratory, BARC, Beltsville, MD, USA; (3) USDA ARS Soybean Genomics and Improvement Laboratory, Beltsville, MD, USA; (4) Mississippi State Univ Extension Svc, Mississippi State, MS, USA; (5) Alabama Coop Ext Svc, Birmingham, AL, USA; (6) University of Georgia Department of Plant Pathology, Athens, GA, USA; (7) Soil, Plant, and Pest Center, Nashville, TN, USA; (8) University of Tennessee, Dept of Entomology \& Plant Pathology, Knoxville, TN, USA

Rose rosette disease has destroyed thousands of roses in the United States. The disease agent, Rose Rosette Virus (RRV), is vectored by the eriophyid mite, Phyllocoptes fructiphilus. Parts of the southeastern United States have remained free of the disease, except for disease introductions that were eradicated. A survey of Alabama, Georgia, and Mississippi plots $(n=204)$ have revealed the southeastern border of RRV. The presence of RRV in symptomatic samples was confirmed by RT-PCR. Samples were also collected at each plot for detection of eriophyid mites, specifically for $P$. fructiphilus. Through isolation, staining, and light microscopy, mite species were identified. Mites were found to be generally distributed throughout the Deep South, however many of these sites contained eriophyid mites that were not $P$. fructiphilus. The reasons that $P$. fructiphilus are not commonly found on roses in the Deep South, as in other locations in the Mid-South, are unknown. The lack of vector populations in the Deep South may explain the absence of RRV in those areas.

Comparison of mycotoxigenic Fusarium genotypes associated with stink bugs and field corn in the mid-Atlantic U.S. A. COOMBER (1), J. Opoku (2), D. Haak (3), H. L. Mehl (4), (1) Cornell University, Ithaca, NY, USA; (2) Virginia Tech TAREC, Suffolk, VA, USA; (3) Virginia Tech, Blacksburg, VA, USA; (4) Virginia Tech Tidewater AREC, Suffolk, VA, USA

Stink bugs, including the invasive brown marmorated stink bug (BMSB), Halyomorpha halys (Stål), and the native brown stink bug, cause significant damage to crops in the mid-Atlantic region of the U.S. Surveys of corn fields have indicated that BMSB damage is associated with increased incidence of Fusarium spp. that cause grain mold and mycotoxin contamination. We explored the relationship between stink bugs and Fusarium, specifically focusing on the potential that stink bugs are a vector for the pathogen. Paired stink bug and corn samples were collected from corn fields in VA, MD, and DE, and Fusarium spp. were isolated from both. To compare stink bug and corn associated populations, we generated genomic tools in the form of molecular markers by bioinformatically mining publicly available data for BMSB and three mycotoxin-producing Fusarium species of interest, $F$. verticillioides, F. proliferatum, and $F$. graminearum. From these datasets we identified over 1500 potential microsatellite markers. We screened these markers to develop a working set of 39. These were used to genotype Fusarium populations isolated from both stink bugs and corn. Diverse Fusarium genotypes were associated with corn and stink bugs, and in many instances corn and stink bug isolates from the same field were genetically distinct. These results provide information about Fusarium movement across the agricultural landscape and may inform management practices that reduce mycotoxin contamination. 
Acquisition of Erwinia amylovora by Drosophila melanogaster

M. T. BOUCHER (1), G. W. Sundin (2), K. D. Cox (1), G. Loeb (1), (1) Cornell University, Geneva, NY, USA; (2) Michigan State University, East Lansing, MI, USA

Fire blight, Erwinia amylovora, is an economically significant disease of rosaceous plants that causes major loss of apple and pear crops across the globe. Diseased tissues ooze a mixture of plant sap, E. amylovora, and bacterial exopolysaccharide, which collectively serves as the primary source of inoculum for new infections. Insects have long been implicated as passive vectors of $E$. amylovora, but recent research suggests that insects, notably flies, play a more intimate role in the evolutionary history of this disease. The purpose of this project is to evaluate interactions between flies and ooze to understand whether E. amylovora manipulates insects as alternative hosts or vectors. Here, we begin detailing how flies acquire E. amylovora using Drosophila melanogaster as a model. Flies were fed ooze out of a capillary tube for 3,6,12,24, and 48 hours to evaluate the effect of exposure time on acquisition. Flies were homogenized and plated on Crosse-Goodman media to obtain E. amylovora colony forming units. We tested whether mating status, hunger state, fly sex, and/or amount of ooze consumed affected E. amylovora counts in individual flies. We expect exposure time to positively affect the overall rate of positive samples, while other variables will affect CFU. The effect of bacterial phytopathogen exudates on insect behavior and vectoring capability is relatively understudied, and a better understanding of such interactions may lead to better vector control strategies in agricultural systems.

Analysis of microbiomes and metatranscriptome of Norway spruce trees naturally infected by the conifer pathogen Heterobasidion sp. A. Kovalchuk (1), M. Mukrimin (1), Z. Zeng (1), T. Raffaello (1), M. Liu (1), R. Kasanen (1), H. Sun (2), F. ASIEGBU (1), (1) University of Helsinki, Helsinki, FINLAND; (2) Nanjing Forestry University, Nanjing, CHINA

Microbiomes associated to crops and forest trees are critical to forestry, agriculture and global food security. Despite significant progress achieved in our understanding of the composition of microbial communities associated with plants, very little is known about the effect of plant pathogens on their structure particularly the least studied forest trees. We investigated the effect of Heterobasidion root rot disease on microbial communities associated with Norway spruce. The sequenced 16S and ITS2 data were analyzed using the mothur operation pipeline. Canonical analysis of principle coordinate (CAP) and principal coordinates analysis (PCoA) were used to visualize the microbial community structure. To identify in-planta expressed pathogen genes, the processed RNA-seq data were mapped against genome of Spruce and Heterobasidion respectively. Results showed that diseased and asymptomatic trees significantly differed in the structure of the microbiome communities residing in their sapwood. Furthermore, inspite of considerable overlap in shared microbiota, metatranscriptomic analysis revealed pronounced differences in gene expression pattern among the individual symptomatic and asymptomatic trees. The distance-based linear model analysis showed that expression levels of several genes with a role in host defense were significantly correlated with the abundance of in planta expressed pathogen transcripts. Our results further provides a baseline for a functional understanding of the dynamics of the microbiome community of conifer trees.

Strawberry anthosphere microbiome structure and functional study of probiotics

D. R. Kim (1), Y. S. KWAK (2), (1) Gyeongsang National Univ, Jinju-si, KOREA; (2) Gyeongsang National University, Jinju, KOREA

Recent researches were demonstrated that links between microbiome diversity and its' ecosystem functions for example plant productivity. Especially, the plant microbiome has been shown to influence the host plant fitness in viability and association of abundant microbe community with plant healthy. Plants can no longer be considered as standalone entities and a more holistic perception is needed. Respectively, plant fitness is therefore a consequence of the plant per se and its microbiota, which collectively form a holobiont. In this study, anthosphere microbial diversity structure with gray molds disease occurrence during strawberry growth period were investigated. Massive pyrosequencing was performed to analysis the microbial communities shifting during strawberry growth season. The strawberry flower (SF) samples, total read count was 933,640 and total operational taxonomic units (OTU) was 53. Then compared pattern of the gray mold disease occurrence and the microbial community shifting during strawberry growth period. As results, the gray mold disease occurrence rapidly increased from SF $8^{\text {th }}$, which was initial stage being the microbe diversity significantly reduced. In addition, direct comparison between Streptomyces OTU number and the gray mold disease occurrence showed that the negative correlation between the two factors. In conclusion, two hypotheses was suggested that gray mold disease occurrence was trigged when anthosphere microbial diversity simplified and Streptomyces may be the key microorganism to suppress the pathogen.

Fungal diversity of developmental stages in table grapes grown in South Africa

P. Carmichael, N. Siyoum, L. Chidamba, L. KORSTEN, University of Pretoria, Pretoria, SOUTH AFRICA

Physiological changes in plant growth affect the diversity and adaptation of microbiota, including fungi. Hence the study aim was to determine fungal communities that characterise table grapes during berry development. The study was done at two agro-ecologically different table grape commercial farms (site A and B). Samples were collected at full bloom, pea size and mature berry stages, from three positions (inside centre, eastern and western peripheral-ends) per site. Total DNA extraction, Illumina sequencing and analysis of 18 pooled samples for fungal diversity targeting ITS1-2 generated a total of 2, 035, 933 high quality sequences with an average length of $150 \mathrm{bp}$. Overall, the pea size stage ( $829 \mathrm{OTUs}$ ) had the richest microbial community, compared to full bloom (569 OTUs) and mature stages (491 OTUs) at all sites. The phylum Ascomycota (77.0\%) and Basidiomycota $(23.0 \%)$ were the most dominant, while the genera, Alternaria (33.1\%) and Cladosporium (24.2\%) were the overall dominant postharvest decay causing fungi throughout the developmental stages. Fungal population diversity varies with different phenological table grape growth stages and it is further influenced by site of production and the position of the vine within a specific vineyard. The information on fungal diversity and succession in table grapes during preharvest growth stages is critical in the development of a more targeted control strategy.

Pyrosequencing-based microbial community analyses according to kiwi-biome organs

M. J. KIM (1), Y. S. Kwak (2), (1) Department of plant medicine, Gyeongsang National University, Jinju, SOUTH KOREA; (2) Department of plant medicine, Gyeongsang National University, Jinju, Gyeongnam, SOUTH KOREA

Plants have a wide microbial diversity both on and inside of their tissues, which are rhizosphere, phyllosphere and endosphere. These microbes play important roles in healthiness of plant such as supply nutrition and enhance resistance against abiotic stresses or various pathogens. Understanding microbiome structure and diversity in plants are important information to reveal the microbial ecology and function in plant-microbe interaction. NGS techniques can lead to novel insights of phytobiome and reveal a relationship between environmental niches and evolution. This study assessed the bacterial and fungal diversity in kiwifruit rhizosphere, sap and pollen using the Illumina MiSeq platform. The experimental specimens were collected cv. Deliwoong kiwifruit tree (Actinidia deliciosa) in Namhae, South Korea. DNAs were extracted, 16S rRNA and ITS regions were amplified and produced amplicon libraries for MiSeq sequencing. In the rhizosphere, Firmicutes, Proteobacteria and Actinobacteria in bacteria and Ascomycota and Zygomycota in fungi occupied as major population. In the sap, Proteobacteria in bacteria and Ascomycota in fungi mostly occupied. Top 15 OTU fungi in the rhizosphere soils mostly belong to Zygomycota but in the saps mostly belong to Ascomycota. Top 15 OTU bacteria in the rhizosphere were belonged 
mostly phyla Actinobacteria and Firmicutes, genus Streptomyces spp. and Bacillus spp., which are known as PGPR strains and in saps were mostly phyla Proteobacteria and Actinobacteria, genus the others. These results showed that much taxa of PGPR and antagonistic microbes against pathogens can be targets for future research in tissue specific or shard microbes in kiwifruit.

The mycobiome of deep soil profiles in no-till dryland wheat

D. C. Schlatter (1), K. Kahl (2), B. Carlson (3), D. Huggins (1), T. C. PAULITZ (1), (1) USDA ARS, Pullman, WA, USA; (2) Dept. of Soil and Water Systems, University of Idaho, Moscow, ID, USA; (3) Dept. Crop and Soil Science, Washington State University, Pullman, WA, USA

Soil edaphic factors affect the composition of fungal communities, but there is a paucity of information on how communities vary with soil depth and landscape characteristics in no-till cropping systems. The Palouse region of eastern Washington is dominated by dryland wheat systems and is characterized by a highly variable landscape with steep, rolling hills. We used high-throughput sequencing of fungal ITS1 amplicons to characterize fungal communities across soil depth profiles $(0-100 \mathrm{~cm}$ from the soil surface) among distinct landscape positions (north-facing, south-facing, bottomand top-slopes) across a no-till wheat field. Fungal communities were highly stratified with soil depth, where deeper depths harbored distinct fungal taxa and more variable, less diverse communities. Fungal communities from deep soils tended to harbor a greater portion of taxa inferred to be pathotrophic or symbiotrophic in addition to saprotrophic lifestyles. Co-occurrence networks of fungal taxa became smaller, more clustered, and had higher ratios of positive:negative associations as soil depth increased. In contrast, differences between communities from north-facing and south-facing slopes were relatively small. Together, these results suggest that upper soil layers harbor highly diverse saprotrophic communities that compete for abundant resources, whereas only a small number of taxa inhabit deeper soil layers, which rely more on pathotrophic or symbiotrophic strategies.

Does inoculation with Pseudomonas fluorescens LBUM223 impact the rhizosphere and geocaulosphere microbiomes of potato? A. NOVINSCAK, R. Roquigny, D. L. Joly, M. Filion, Université de Moncton, Moncton, NB, CANADA

The phenazine-1-carboxylic acid (PCA)-producing Pseudomonas fluorescens strain LBUM223 shows biocontrol potential against Streptomyces scabies, causing common scab of potato. To better characterize the impact of inoculating a specific biocontrol agent under natural field conditions, the microbiomes of the rhizosphere and the geocaulosphere of potato plants were characterized using Next-Gen sequencing. One initial or bi-weekly applications of LBUM223 were performed up to 11 weeks after planting (in addition to non-inoculated plants). Rhizosphere and geocaulosphere (when potato tubers were produced) soils were sampled every two weeks. Following soil DNA extractions, 16S rRNA gene amplification and sequencing was performed using the Illumina MiSeq technology. The QIIME pipeline was used for data analyses. Results were generated from 45 rhizosphere and 27 geocaulosphere samples, for which 63502 and 44469 different operational taxonomical units were observed. Diversity comparisons between both datasets were performed. To our knowledge, it is the first time that the geocaulosphere microbiome is characterized and compared to the rhizosphere. 11 phyla accounted for 95\% of the diversity, with Actinobacteria, Proteobacteria, Chloroflexi and Acidobacteria being the most important ones. Overall, the results obtained suggest that Pseudomonas fluorescens LBUM223 does not significantly interfere with the autochthonous rhizosphere nor geocaulosphere microbiomes.

Influence of boxwood species and cultivars on the rhizosphere microbiome

N. LEBLANC (1,2), J. A. Crouch (3), (1) USDA-ARS, Beltsville, MD, USA; (2) Oak Ridge Institute for Science and Education, Oak Ridge, TN, USA; (3) USDA ARS, Beltsville, MD, USA

There is growing interest in leveraging the microorganisms associated with boxwood and other ornamental plants to improve plant health. However, it is unknown if different species or cultivars of boxwood influence the composition or diversity of plant associated microorganisms. This work characterized the composition and diversity of microorganisms in the rhizosphere of healthy boxwood in the U.S. National Arboretum Boxwood Collection. Soil samples were collected from the rhizosphere of 80 individual plants, representing 4 species of boxwood and 10 cultivars of the common species Buxus sempervirens. Extracted soil DNA was used to create amplicon libraries targeting archaea, bacteria, and fungi. Libraries were sequenced using Illumina MiSeq v3 chemistry and sequence variants were inferred using the Divisive Amplicon Denoising Algorithm (DADA2). Overall, 15580 sequence variants were identified from bacteria, 7526 from fungi, and 276 from archaea. Based on statistical tests and visualization of these data, there was limited evidence that the composition or diversity of these 3 microbial groups was influenced by the 4 boxwood species. However, within the species $B$. sempervirens, bacterial diversity was significantly different among the 10 sampled cultivars. The outcomes of this work suggest the growth of different $B$. sempervirens cultivars in the landscape may alter the functional activity associated with bacteria, such as plant pathogen suppression.

Foliar endophytic microbiome composition and functional capacities vary with soil nutrient inputs L. L. KINKEL (1), L. K. Otto-Hanson (2), Z. Hansen (3), M. Johnson (4), S. Spawn (5), Z. Song (1), G. May (4), E. Seabloom (4), E. Borer (4), (1) Department of Plant Pathology/University of Minnesota, Saint Paul, MN, USA; (2) Department of Plant Pathology/University of Minnesota, St Paul, MN, USA; (3) Michigan State University, East Lansing, MI, USA; (4) University of Minnesota, Saint Paul, MN, USA; (5) UW-Madison, Madison, WI, USA

Endophytic microbes are of significant interest for their potential to influence plant health and productivity in agricultural and natural habitats. Yet the factors that influence the composition or functional capacities of endophytic microbiomes are not well understood. We studied endophytic microbiomes in the C4 prairie grass Andropogon gerardii within a long-term nutrient amendment experiment. Nitrogen, phosphorous, and potassium (NPK) were applied to plots annually since 2007. In fall 2015, mature, non-senescent leaves were harvested from NPK-amended and non-amended (control) plants. Leaves were surface-disinfested, macerated in sterile water, and dilution-plated onto nutrient media. Bacterial and fungal colonies were randomly selected from plates, and purified and stored at $-80 \mathrm{C}$. For every isolate, $16 \mathrm{~S}$ (bacteria) or ITS2 (fungal) sequence was determined. A random set of 10 isolates from each leaf was further evaluated for nutrient utilization profiles using Biolog plates. We found that NPK dramatically altered fungal, but not bacterial endophytic composition. Fungi, but not bacteria, had significantly smaller niche widths in NPK-treated than control leaves. In the absence of NPK, fungi are significantly better competitors for nutrients than bacteria, but this advantage is lost with NPK. These data suggest that widely-used soil nutrient inputs significantly alter the composition and functional capacities of endophytic microbiomes, with implications for crop productivity.

Effects of global warming on plant diversity-soil carbon relationships and implications for assembly of plant-associated microbiomes S. CASTLE (1), Z. Song (1), A. S. Grandy (2), L. L. Kinkel (1), (1) Department of Plant Pathology/University of Minnesota, Saint Paul, MN, USA; (2) University of New Hampshire, Durham, NH, USA

Soil microbial community responses to climate change, though critical to plant health, remain a significant gap in our ecological understanding. In this study we sampled rhizosphere soils from two plant species (Andropogon gerardii and Lespedeza capitata) within a 9-year factorial plant diversity $\times$ 
warming experiment in central Minnesota, USA. We used amplicon sequencing of bacteria and fungi and molecular analysis of soil carbon to enhance our mechanistic understanding of warming-related shifts in soil carbon and its role(s) in mediating microbial community assembly and species interactions. Warming reduced microbial OTU richness, but reductions were greater in monoculture than polyculture plots. Similarly, soil C chemical diversity changed significantly with warming in monoculture, but not polyculture. However, warming-related shifts in microbial communities were not directly related to soil $\mathrm{C}$ chemical richness or composition. Finally, warming led to consistent shifts in fungal composition, though shifts in microbial taxa differed with plant species richness and identity. These data suggest a potential role of plant diversity and plant host in mediating effects of warming on populations of soil microbes. Such shifts in soil microbiomes represent a potentially important feedback to plant health and productivity under future climate warming.

Exploring Microbiome of Medicinal Plants as Biocontrol Agents

I. Iqrar (1), G. S. ALI (2), (1) Quaid-i-Azam University, Islamabad, PAKISTAN; (2) University of Florida, 32703, FL, USA

Plant microbiome plays an important role in plant health and plant-environment interactions, but the microbiome of medicinal plant is less explored. In this report, endophytic bacteria were isolated from five different medicinal plants: Cannabis sativa, Dodonaea viscosa, Fagonia indica, Caralluma tuberculata and Calendula arvensis. On the basis of initial antimicrobial screening, 12 bacterial species that based on 16S rRNA gene sequencing belonged to nine different genera (Serratia, Enterobacter, Paenibacillus, Streptomyces, Pseudomonas, Bacillus, Pantoea, Pseudarthrobacter and Delftia) were selected for further analyses. Antimicrobial assays revealed that E. cloacae, Paenibaillus $\mathrm{sp}$. and P. taiwanensis have strong antiPhytophthora activity. Volatiles produced by $P$. taiwanensis inhibited growth of $P$. parasitica by more than $80 \%$. Ethyl acetate extracts of $S$. marcescens, E. cloacae, Paenibacillus sp., S. alboniger, P. taiwanensis, E. hormaechei and B. tequilensis and D. lacustris also displayed high potency against $P$. parasitica at different concentrations $(4 \mu \mathrm{g} / \mathrm{mL}-400 \mu \mathrm{g} / \mathrm{mL})$. Bacterial extracts showing with high bioactivity $(>80 \%$ inhibition in vitro $)$ were selected for detached-leaf assay against $P$. parasitica. In detached-leaf assay, application of $1 \%$ ethyl acetate bacterial extract of reduced lesion sizes and lesion frequencies caused by $P$. parasitica by 68 to $81 \%$. Over all $P$. taiwenensis showed positive activities for all the assays. Analysing the potential of bacterial endophytes as biological control agents can potentially lead to the formulation of broad-spectrum biopesticides for agriculturally important crops.

The impact of pesticides on bacterial biodiversity in the turfgrass rhizosphere

E. BUCZKOWSI, M. Millican, P. L. Koch, University of Wisconsin, Madison, WI, USA

Pesticides are routinely applied to turfgrass landscapes to manage weed, insect, and disease pests. Understanding the impact of various pesticides on the turf-associated microbiome may have important implications for developing more sustainable pest management strategies. To evaluate how pesticides influence soil bacterial biodiversity, turfgrass plots at the OJ Noer Turfgrass Research Station in Madison, WI were treated with a single herbicide, insecticide, or fungicide application in June of 2017. The pesticides applied represented either a low or high toxicity product based on its mammalian acute oral LD50 assessment. Soil cores were removed at $0,1,3,7,14$, and 21 days after application and frozen at $-80^{\circ} \mathrm{C}$ until further processing. The entire project was repeated in August 2017. Rhizosphere soil was removed, DNA was extracted, and the V4 16S rRNA region was amplified. Polymerase chain reaction products were pooled and sequenced using the Illumina MiSeq platform and raw sequencing data was processed using Mothur. As of this submission the final sequencing results have not been analyzed, but based on previous research in other systems we hypothesize that the microbial communities will initially undergo a structural change and over time return to a community of similar structure and diversity as prior to the pesticide application.

The structure and function of the global citrus root-associated microbiome J. XU, Y. Zhang, N. Wang, University of Florida, Lake Alfred, FL, USA

Plant root-associated microbiome plays important roles in biogeochemical cycles and promoting plant growth and health. However, the genomic, taxonomic and functional contents of plant microbiome remain largely unknown. Here we established a large-scale gene catalog of the root-associated microbiome of citrus, a perennial plant, via deep metagenome sequencing with samples collected from six continents across distinct biogeographic conditions. We compared the gene catalog of citrus root microbiome with human and ocean microbiome gene catalogs. We also sequenced the amplicon of 16S V4 region and fungal ITS2 region for bulk soil, rhizosphere, and root samples across location. We defined the core citrus root-associated microbes and functions and determined the driving forces of the assembly process of plant root-associated microbiome at both microbial community and single genome (MAG) resolution. Our results broadened the genomic resource and view of plant root-associated microbiome, and shed lights on understanding the genomic and evolutionary bases of microbes for the host associated niche specification.

\section{Is Chemical Warfare More Common Among Seed Microbes Than Other Plant Microbes?}

M. A. MARLIN, G. Newcombe, University of Idaho, Moscow, ID, USA

Because endophytic antagonists have shown the ability to significantly reduce disease severity, we need to know where to look for the most effective ones. The seed microbiome is comprised of specialized members, and individual seeds host few microbes. Two mechanisms appear to be responsible. First, seeds are strongly defended by plants, and second, seed microbes actively exclude one another from individual seeds. Following numerous observations of exclusionary interactions between cultured seed endophytes, we hypothesize that seed microbes commonly inhibit one another through chemical means. We further hypothesize this inhibition will be more common and severe in the seed microbiome of a given plant compared with its vegetative counterpart. In this study, we use Western White Pine (Pinus monticola) to test the hypothesis that antagonistic behavior among seed endophytes is stronger than antagonistic behavior among needle endophytes. Agar plugs of actively growing endophytes were plated in dual culture on PDA to measure antagonistic activity between endophytes. Results will be discussed.

The microbiome of soils suppressive to Spongospora diseases of potato

R. FALLOON (1), R. Frampton (1), P. Wright (2), (1) The New Zealand Institute for Plant \& Food Research Limited, Christchurch, NEW ZEALAND; (2) The New Zealand Institute for Plant \& Food Research Limited, Pukekohe, NEW ZEALAND

The plasmodiophorid Spongospora subterranea causes root galling and tuber powdery scab on potato plants, yet these diseases did not develop in potato crops grown successively over 10 years in a long-term crop rotation field trial, indicating that the soil was naturally suppressive to this pathogen. A subsequent project is determining the characteristics of 12 vegetable-growing soils (including the trial soil), selected to represent different geographic regions, cropping histories and soil types, for their suppression of Spongospora diseases. As part of the study, total DNA was extracted from subsamples of each soil. The microbial communities are being characterized, using generic primers targeting variable regions in the 16S rRNA gene for bacteria or 
the rRNA internal transcribed spacer region for fungi. The amplicons are being sequenced using the Illumina MiSeq system. The sequence data will be classified into operational taxonomic units to generate the microbial community profile for each soil. Diversity and network analyses will be used to determine patterns and associations in the sequence dataset. This project also includes detailed determination of the physical and chemical characteristics of the 12 field soils, and aims to describe soil biotic and abiotic factors associated with suppression of $S$. subterranea. The goal is to identify soil factors that may be manipulated as part of integrated management of the economically important yield- and quality-limiting diseases of potato caused by $S$. subterranea.

Seasonal and potato cultivar effects on common scab causing Streptomyces spp. and bacterial communities C. GOYER (1), K. Nahar (1), B. Zebarth (1), D. Burton (2), S. Whitney (1), (1) Agriculture and Agri-Food Canada, Fredericton, NB, CANADA; (2) Dalhousie University, Truro, NS, CANADA

Pathogenic Streptomyces spp. cause common scab (CS) of potato resulting in significant economic losses. How potato cultivar can influence the abundance of pathogenic Streptomyces spp. is unclear. This study evaluated the effects of potato cultivar on pathogenic Streptomyces spp. abundance and diversity of bacterial communities in three spatial locations: bulk soil (BS), rhizosphere (RS) and geocaulosphere (GS) over the growing season. The experiment was conducted in a naturally common scab infested field located in Fredericton, NB, Canada in 2013 and 2014. Two tolerant (Gold Rush, Hindenburg) and two susceptible cultivars (Green Mountain, Agria) were tested. Soil samples were collected from BS, RS and GS soils. When averaged over time, the abundance of pathogenic Streptomyces spp. measured using quantitative polymerase chain reaction, was significantly greater in GS for susceptible than for tolerant cultivars. The abundance of pathogenic Streptomyces spp. increased in BS, RS and GS for susceptible cultivars at 90 days after planting compared with other dates, whereas no changes over time were observed for tolerant cultivars. Principal coordinate analyses indicated that $\mathrm{pH}$, extractable organic carbon, ammonium and nitrate concentrations explained 68 and $76 \%$ of the variability in BS among cultivars in 2013 and 2014, respectively, and $69 \%$ of the variability in RS among cultivars in 2013. This finding suggests that the cultivars influenced the soil chemical properties in BS and RS. Alpha- diversity of bacterial communities was significantly different among BS, RS and GS, but was not significantly different among potato cultivars. Thus, differences in growth of pathogenic Streptomyces spp. among cultivars may reflect cultivar-specific effects on soil chemical properties rather than effects on soil bacterial communities.

Crop-specific microbiome responses to four-year rotational sequences

M. S. BENITEZ PONCE (1), S. Osborne (2), M. Lehman (2), (1) The Ohio State University, Wooster, OH, USA; (2) USDA Agricultural Research Service, Brookings, SD, USA

Crop rotations beyond two years provide multiple benefits to agroecosystems, which result from interactions between plants, soils and microorganisms. Two consecutive years of sampling in the corn and soybean phase of diverse 4-year rotation sequences and a 2-year corn-soybean rotation was performed at a field site in South Dakota. Rotation sequences studied included small grains, legumes and/or oil-seed crops. Plants were sampled at seedling and flowering to evaluate plant vigor and rhizosphere-associated microbiome through amplicon sequencing of bacterial and fungal ribosomal markers. Both years soybean performed best in rotations containing winter wheat, and performance was not different between the 2-year and the 4-year rotations including spring wheat. Soybean rhizosphere mycobiome mirrored this pattern and was driven by differences in abundance of Glomeromycetes and Sordariomycetes across rotation sequences. Soybean rhizosphere-associated bacteria responded to plant stage with higher abundance observed at flowering for taxa across different phyla. For corn, greatest plant vigor was consistently observed in a rotation including spring wheat, particularly when pea preceded corn. Corn rhizosphere-associated fungi and bacteria preferentially responded to plant stage. Further analysis will focus on interactions between rotation sequence, crop species and crop developmental stage to provide insight on microbiome benefits stemming from diversified cropping systems.

Contrasting microbial diversity in conducive and putative suppressive soils to garlic white rot V. LOURENÇO, JR. (1), A. F. T. A. F. Ferreira (2), P. I. A. Romero (2), E. S. G. Mizubuti (3), (1) Embrapa, Brasília, BRAZIL; (2) Universidade Federal de Viçosa, Viçosa, BRAZIL; (3) Universidade Federal de Vicosa, Vicosa, BRAZIL

White rot, caused by Stromatinia cepivorum, is a devastating disease on garlic in Brazil. As disease-suppressive soils provide a valuable resource for studying beneficial microbial communities, the objective of this study was to analyze the microbiota diversity from fields with no record of white rot in Minas Gerais state, Brazil. Five samples were collected from putative suppressive soils in the municipalities of Gouveia (GO, 06, and 06B) and Campos Altos (SH1 and SH2). Three samples from conducive soils were collected in the municipalities of Capim Branco (02 and 02B) and Rio Paranaíba (CO). A total of 218 and 291 fungal and bacterial OTUs were detected, respectively. The alpha diversity values of the samples ranged as follows: richness estimate (12-94), Shannon diversity (3.4-80.7), and Simpson diversity (2.3-66.5). Members of the phyla Acidobacteria and Proteobacteria were more abundant in all locations. The highest bacterial diversity was estimated in CO, 02, 06, SH1, and SH2 samples. For fungi, specimens of the Ascomycota and Basidiomycota phyla were more frequent. Interestingly, the highest diversity and number of unclassified fungi were found in the putative suppressive soils GO, SH2, and 06, suggesting that suppressiveness to white rot is related to microbial abundance and variability. Additional studies should be conducted to identify functional biological entities that can contribute to white rot suppression.

The secret life of bacteria: The interaction of Enterobacter and the soil pathogen Rhizoctonia solani

P. ZHANG (1), K. Obasa (2), J. C. Huguet-Tapia (1), Z. Peng (3), A. Block (4), F. F. White (1), (1) University of Florida, Gainesville, FL, USA; (2) UNIVERSITY OF FLORIDA, GAINESVILLE, FL, USA; (3) University of Florida, GAINESVILLE, FL, USA; (4) USDA, ARS, SAA, CMAVE, Gainesville, FL, USA

Bacteria and fungi in mycorhizosphere both have a profound impact on the health of crops. Various endohyphal bacteria have been isolated from the soil fungus Rhizoctonia solani AG2-2IIIB, which causes brown patch disease in cool season turfgrass, including the Enterobacter sp. strain named En-cren. Endophytic En-cren was shown to contribute to the virulence of the fungal pathogen and it can be released as a free-living bacterium when hyphae are damaged, rapidly migrating along the outside of hyphae. The objectives to this study was to identify the genetic nature of hyphal motility of the bacteria and whether the bacteria contribute to the virulence of the fungal pathogen through the production of phenylacetic acid (PAA). The bacteria can travel by the aerial hyphae of $R$. solani and presumably expand to new niches in the soil environment. Single mutations in flagella hook protein $f l g E$ and type IV pilus secretin pilQ did not prevent hyphal movement. The tests of double mutants of both motility apparatus are in progress. En-cren produces PAA and indole acetic acid (IAA), of which the former has been proposed as a virulence factor of $R$. solani. Mutation of the gene for indole pyruvate decarboxylase suppressed PAA and IAA production in free-living cultures of the bacterium. The study will provide greater understanding of the interaction of bacteria and fungi of the mycorhizosphere and, more specifically, of the broad range soil pathogen Rhizoctonia solani. 
Microbiome associated with tall fescue under drought stress

G. GROBEN, J. Luo, E. M. Walsh, H. Qu, W. A. Meyer, S. A. Bonos, B. Clarke, N. Zhang, Rutgers University, New Brunswick, NJ, USA

The effects microbiomes have on physiological traits in turfgrasses are poorly understood. Drought tolerance is an economically important trait, which can be influenced by certain microbes. One example is the mycorrhizae association, which has been shown to improve drought tolerance of many plant species. In this study, we evaluated the microbiome associated with tall fescue genotypes grown in a rainout shelter after prolonged periods of drought stress. Twelve plants were selected for analysis, comprised of six sets of siblings, one exhibiting a drought tolerant phenotype and the other a susceptible phenotype. The microbiome associated with the shoot, root, and rhizosphere soil was evaluated for each tall fescue half-sib pair. Microbiome analysis was preformed utilizing an Illumina NGS metabarcoding approach that sequenced the $16 \mathrm{~S}$ and ITS barcoding region to determine the composition of the bacterial and fungal communities, respectively. Comparative analysis of the microbiomes associated with the two phenotypes will be used to determine which microbes are associated with improved drought tolerance and drought susceptibility.

Evaluating the Microbiome of Industrial Hemp

A. R. CALA (1), S. Barnett (2), J. Hansen (2), J. Crawford (2), D. Viands (2), L. Smart (1), D. Buckley (2), C. D. Smart (3), (1) Cornell University, Geneva, NY, USA; (2) Cornell University, Ithaca, NY, USA; (3) Plant Pathology and Plant-Microbe Biology Section, Cornell University, Geneva, NY, USA

Industrial hemp is a new crop for New York State, with increasing grower interest in producing the crop for seed, oil, fiber and other uses. While plantmicrobiome interactions can impact nutrient acquisition, pest and disease susceptibility, and other phenotypes in some plants, the composition and function of the hemp microbiome remains poorly characterized. The purpose of this study was to characterize the industrial hemp microbiome and evaluate environmental variability in microbiome composition. During the summer of 2017, field trials of the hemp cultivar 'Anka' were planted in six locations in Tompkins and Ontario counties, New York. Microbiome composition was assessed in bulk soil, rhizosphere soil, root compartment, phylloplane, and flowers. Furthermore, microbial colonization of male and female flowers was followed over a period of three weeks in the field. Composition of the microbiome was assessed in soil and plant tissues by extracting DNA, amplification of bacterial SSU rRNA genes and fungal ITS regions, and Illumina MiSeq sequencing. We describe variance in microbial community composition with respect to plant compartment and geographic location and assess core microbiome composition of industrial hemp. Furthermore, we describe and contrast temporal development of microbiome composition of male and female flowers.

Metacoder and taxa: $\mathbf{R}$ packages for visualization and manipulation of community taxonomic diversity data Z. FOSTER (1), N. Grunwald (1), T. Sharpton (2), S. Chamberlain (3), (1) USDA ARS, Corvallis, OR, USA; (2) Oregon State University, Corvallis, OR, USA; (3) rOpenSci, Berkeley, CA, USA

Metabarcoding is revolutionizing microbial ecology by circumventing the limits of traditional culture-based techniques, but the massive hierarchical (e.g. taxonomic) datasets produced are difficult to plot and manipulate using current tools. Hierarchical data are more challenging to subset and otherwise manipulate than typical tabular data. We have developed the "taxa" package to provide a standard for the storage and manipulation of any data associated with a taxonomy, modelled after the popular dplyr data-manipulation philosophy. In addition, we have developed "metacoder" for parsing, analyzing, and visualizing hierarchical data associated with metabarcoding research. The reliance on color to depict taxa in stacked bar charts and pie graphs limits the number of taxa displayed to the number of discernible colors. Metacoder implements a novel visualization called heat trees that use the color and size of nodes and edges on a taxonomic tree to quantitatively depict up to 4 statistics distributed over a hierarchy. This allows for rapid exploration of data and information-dense, publication-ready graphics. In addition, metacoder provides tools for reading common file formats and evaluating primers and barcode loci using simulated PCR. The metacoder and taxa packages are already being adopted by the community and have been applied to diverse projects including research on gut microbiota, soil microbiota, wastewater communities, and mycorrhizal associations.

Deciphering the Dynamics of the Citrus Microbiome

N. GINNAN, P. E. Rolshausen, M. C. Roper, University of California, Riverside, Riverside, CA, USA

The plant microbiome is an interactive cross-organismal community that influences host productivity, development, and tolerance to stresses. Shifts in the structure of the citrus microbiome that are related to season and plant development are not fully understood. We hypothesize that host phenology and grove management strategies can regulate citrus microbial community dynamics. To test this hypothesis, we are profiling the microbiome of California citrus trees under organic and conventional management. Furthermore, our sampling times mirror key stages in citrus phenology, including: sprouting initiation(flush), flower initiation, full flowering, fruit setting, fruit development, color breaking, and mature fruit. In parallel, using a reductionist approach, we are utilizing gnotobiotic citrus plants to investigate the interactions of individual citrus microbiota in more detail, particularly, in the context of microbial partitioning to specific plant compartments. Additionally, our grove sampling locations include geographical areas of California that have been exposed to the invasive Asian Citrus psyllid, the vector for the Huanglongbing (HLB) associated bacterium, Candidatus Liberibacter asiaticus (CLas), for varying lengths of time. As HLB incidences increase in these areas, we hypothesize that it will be possible to capture critical shifts in the native citrus microbiome as an invasive pathogen becomes established in a given area.

\footnotetext{
Molecular Characterization of the Pathogen and Symbiotic Fungal Community composition in the rhizosphere of common European potato varieties

K. LOIT (1), L. Soonvald (1), A. Astover (2), L. Tedersoo (3), (1) Estonian University of Life Sciences, Chair of Plant Health, Tartu, ESTONIA; (2) Estonian University of Life Sciences, Chair of Soil Science, Tartu, ESTONIA; (3) University of Tartu, Tartu, ESTONIA

Potato (Solanum tuberosum L.) has one of the heaviest demands for fertilizers and pesticides among all vegetable crops. As Arbuscular Mycorrhizal fungi (AMF) are naturally present in the soil, proper management of AMF may provide as a sustainable alternative to reduce the high inputs of fertilizers and pesticides. The aim of the study was to get new knowledge about pathogen and symbiotic fungal community and their interactions in conventionally treated field soil. In total 315 soil and root samples were collected. AMF and pathogenic fungal community composition were assessed by using Illumina MISeq sequencing of ITS region. The results showed the variable richness of AMF and pathogenic fungi throughout the growing season. It was found that pathogenic fungal community composition was influenced by potato cultivar and plant growth stage. In contrast, AMF community was not influenced either by cultivar nor plant growth stage. Despite applied conventional management regimes, plant roots were highly colonized by pathogenic fungi. In contrast, the colonization rate in plant roots by AMF was still relatively low and insufficient to suppress pathogenic organisms.
} 
Seedling diseases caused by Phytophthora spp., Pythium spp. and Phytopythium spp. pose a significant threat to soybean production. To manage loss due to pests and diseases, seeds are commonly coated with treatments containing fungicides and insecticides. However, not much is known about the ecological significance of chemical seed treatments on oomycete communities. We used amplicon sequencing to investigate the ecological dynamics of oomycete communities in soybean rhizosphere soils. The hypothesis was that seed treatments would act as community filters selecting for specific oomycete populations. Rhizosphere soils were collected, in Michigan, over two years from two locations from soybean seedlings treated with commercially relevant fungicides, insecticides and nematicides and sequenced using Illumina MiSeq. Preliminary analyses indicate that alpha and beta diversity was not highly influenced by seed treatments or soybean varieties; however, composition ( $\beta$ diversity) was highly structured by location, indicating that the effects of seed treatment may be subtle and location specific. For example, in one location, indicator species analysis showed that Pythium hypogynum was significantly more abundant in rhizosphere soils of seedlings treated with a combination of fungicides and Pythium hydnosporum was significantly less abundant in samples treated with fungicides and an insecticide. Investigations into other ecological processes in controlled growth chamber studies are currently being used to study oomycete assembly onto treated soybean seeds/seedlings.

\section{Deciphering the complex interactions between the apple microbiota and a biocontrol agent against post-harvest diseases (Pichia anomala strain} K)

A. R. Sare (1), M. H. Jijakli (2), S. MASSART (3), (1) Gembloux AgroBio Tech, Liege University, Gembloux, BELGIUM; (2) Fac Sciences Agronomique, Gembloux, BELGIUM; (3) University of Liège (ULg) - Gembloux Agro-BioTech, Gembloux, BELGIUM

The yeast Pichia anomala strain K is a Biological Control Agent (BCA) against two postharvest apple pathogens (Penicillium spp. and Botrytis cinerea) Progress has been made during the past two decades to understand the modes of action of the strain K through microbiology, enzymatic, genomic, transcriptomic and proteomic studies. Nevertheless, BCAs commercial application has been hampered by low or non-reliable efficacies in comparison to fungicide treatments (Droby et al., 2016). Massart et al. (2015) identified new alternatives to improve BCA efficacy using microbiota. Once applied on the fruit surface, a BCA will face a complex microbiota where ecological interactions such as parasitism, mutualism and commensalism occur, thus affecting its efficacy. In this study, we characterized at large scale the microbiota residing at the surface of apple and evaluate its potential to influence the efficacy of the strain K. Apple fruit samples of seventeen varieties grown in four disease management practices have been collected and their epiphytic microbiota harvested to create a biobank of apple microbiota. Gene centered high throughput sequencing allowed to decipher the bacterial and fungal populations of the microbiota. Biological assays on apple fruits have been carried out by co-inoculating the apple microbiotas with the strain $\mathrm{K}$ and $B$. cinerea. The results of the preliminary assays revealed that the apple microbiota can either raise, drop or have no effect on the efficacy of the strain K. Study is ongoing to identify traits or strains/species which are benefic to the strain K efficacy.

Effect of native New Jersey Pine Barrens bacteria on germination of switchgrass (Panicum virgatum)

P. ENGEL, E. M. Walsh, S. A. Bonos, N. Zhang, Rutgers University, New Brunswick, NJ, USA

The New Jersey Pine Barrens (NJPB), an ecosystem featuring low soil pH and low nutrient levels, is home to the native perennial switchgrass (Panicum virgatum). To improve viability of switchgrass as a biofuel feedstock, germination and establishment rates of switchgrass seeds must be increased. Native switchgrass seeds in the NJPB are colonized by bacteria whose ecological functions are not yet characterized, although seed effects are proposed ranging from inhibition to promotion of germination. In this study, strains of bacteria that belong to genera Pantoea, Bacillus, Burkholderia, and Paenibacillus isolated from NJPB switchgrass seeds were screened for their effects on switchgrass seeds. Commercially available switchgrass seeds from an upland cultivar (Carthage) and a lowland cultivar (Kanlow) were inoculated with the native bacteria. Germination rates and root quality were assessed in a benchtop assay over a period of four weeks. Investigation of the effects of the native bacteria on switchgrass seeds will inform further study of how the seed microbiome can be manipulated to promote switchgrass establishment and reduce negative impacts of bacterial colonization.

Soil fungal diversity during a soybean-cover crop rotation using community sequencing

M. R. MARROQUIN-GUZMAN (1), C. Proctoc (2), J. McMechan (3), R. Werle (4), A. O. Adesemoye (5), S. E. Everhart (1), (1) University of Nebraska, Lincoln, NE, USA; (2) University of Nebraska-Lincoln, Lincoln, NE, USA; (3) University pf Nebraska-Lincoln, Ithaca, NE, USA; (4) University of Nebraska-Lincoln, North Platte, NE, USA; (5) University of Nebraska Lincoln, North Platte, NE, USA

Cover crops represent an important rotational benefit to field crop production systems. In the Midwest, over-wintering cover crops such as oats (Avena sativa L.) and rye (Secale cereale L.) are known to improve disease management, soil properties, and provide an economic benefit from forage production. However, how cover crops impact soil microbial communities and whether longer periods of cover crop provide greater benefit is understudied. In soil ecosystems, fungi play pivotal roles in nutrient cycling, carbon turnover, and soil formation. Moreover, some fungi are known to decompose plant residues, stimulate plant growth and cause plant diseases. Here, our aim is to characterize soil fungal community diversity in a soybeanoat/rye system in three experimental trials in Nebraska. A fallow control treatment was utilized at each location and cover crops consisted of a 50:50 mixture of oats and cereal rye. The soil was sampled at planting and at harvest. Bulk DNA was purified and the ITS region sequenced using the Illumina Mi-Seq platform. A community sequencing approach will be used to analyze and compare fungal diversity within and across locations. Results will contribute to understanding how cover crops impact fungal communities in Nebraskan soils and identify fungi that may be potential indicators of disease incidence and yields.

Influence of temperature on the isolation of water molds using a soil bating technique K. NAVARRO (1), S. Wijeratne (1), A. E. Dorrance (2), (1) The Ohio State University, Wooster, OH, USA; (2) The Ohio State University, Dept. of Plant Pathology, Wooster, OH, USA

Water molds (Pythium and Phytophthora spp.) can cause pre- and post-emergence damping-off of seed and seedlings in soybean. Very wet environmental conditions favor disease development however, varying temperature profiles have been reported. Thus, our objective was to investigate the effects of temperatures on the isolation of water molds using a soil bating technique and compare a culture dependent isolation method (CDIM) to an amplicon-based metagenomics approach (ABMA). Soils collected from soybean fields in northern Ohio were placed in $17.8 \mathrm{~cm}$ pots, flooded for $24 \mathrm{~h}$ and incubated for two weeks at $15^{\circ} \mathrm{C}$ or $25^{\circ} \mathrm{C}$. A susceptible cultivar was then introduced and symptomatic seedlings collected after $4 \mathrm{~d}$. For the CDIM, seedling roots were washed and plated on PIBNIC selective media and corresponding rhizospheric soil collected for the ABMA. The ITS 1 region was then amplified. Sanger sequencing was used to identify isolates collected using the CDIM, while for the ABMA, Illumina Mi-seq was performed. From our CDIM, Pythium sylvaticum and P. ultimum var. ultimum were the most frequently recovered species at both temperatures. However, P. hypogynum, 
P. heterothallicium, $P$. inflatum and $P$. middletoni were only recovered, at $25^{\circ} \mathrm{C}$ and, $P$. torulosum was only recovered at $15^{\circ} \mathrm{C}$. Furthermore, when using ABMA, we were able to identify a greater amount of water mold species including 20 Pythium spp. and 2 Phytophthora spp. demonstrating a greater depth in detection when multiple species with similar morphology are present in the field. Temperatures also seemed to influence species diversity and abundance when using a soil bating technique.

Do grafting and rootstock genotype affect the rhizobiome? A study of tomato systems

R. POUDEL (1,2,3), A. Jumpponen (4), L. Meyer (5), M. M. Kennelly (6), C. L. Rivard (5), K. A. Garrett (1,2,3), (1) Plant Pathology Department, University of Florida, Gainesville, FL, USA; (2) Emerging Pathogens Institute, Gainesville, FL, USA; (3) Institute for Sustainable Food Systems, Gainesville, FL, USA; (4) Biology Department, Kansas State University, Manhattan, KS, USA; (5) Kansas State Research \& Extension Center, Olathe, KS, USA; (6) Department of Plant Pathology, Kansas State University, Manhattan, KS, USA

Root and rhizosphere microbiomes are critical for supporting nutrient exchange between plants and the bulk soil environment, and determining plant performance and health. We explore how rootstock genotype affects these microbiomes, with the hypothesis that rootstock genotypes have distinct microbial communities. We grafted scion BHN589 on tomato rootstock genotypes $(\mathrm{N}=4)$, and profiled the associated root and rhizosphere microbiomes by sequencing hypervariable regions of bacterial 16s rRNA and hypervariable fungal Internal Transcribed Spacer (ITS) regions. The majority of observed microbial taxa were shared across the rootstocks, while a small percentage (less than $3 \%, p<0.05$ ) of microbial variation in ordination analysis was associated with genotype. Study site and root-rhizosphere compartment explained the majority of the observed variation in the microbial community across all the rootstock genotypes. A significantly more diverse community was observed in the higher yielding rootstock (Maxifort) compared to the controls. In addition, our analysis of microbial-association networks suggested higher microbial interactions for Maxifort in both the compartments. These results indicate a potential role of the rhizobiome in mediating the effects of grafting in vegetable production. Future approaches to modification of the rhizobiome may enhance disease management in this and related systems, when rootstocks are used to enhance disease resistance.

The avocado root phytobiome: Microbial community structure under abiotic and biotic stress S. CRANDALL (1), T. Miles (2), (1) California State University Monterey Bay, Seaside, CA, USA; (2) Michigan State University, East Lansing, MI, USA

Phytophthora root rot, caused by the plant pathogen Phytophthora cinnamomi (Pc) can adversely affect the health of avocado (Persea americana) trees, especially when soil salinity is high. However, diagnosing the effect of biotic and abiotic stressors requires time consuming and costly laboratory tests. Previous research shows that bacteria and fungi are prevalent in roots as part of a plant's phytobiome; and the composition and structure of microbial communities change under varying environmental conditions. Identifying the microbes present under stressed environments could rapidly and accurately diagnose avocado health at affordable costs. The primary objective of our research was to screen for root microbes that can serve as indicators of plant stress for avocado trees growing in California. A total of 117 avocado root and soil samples were taken from 18 orchards along the central and southern coast region of California. Soil salinity ranged from $0.68 \mathrm{dS} / \mathrm{m}$ to $5.28 \mathrm{dS} / \mathrm{m}$ across all sites. Real-time PCR results indicate that $34 \%$ of the samples tested positive for Pc. Novel next-generation DNA sequencing was used to identify bacteria and fungi species that are associated with plant stress. Results will prove useful to screen for microbial candidates that may be used in future Pc biocontrol experiments.

Study of seed-borne virome in cucurbits

N. Aboughanem-Sabanadzovic (1), S. SABANADZOVIC (2), (1) Institute for Genomics, Biocomputing and Biotechnology, Mississippi State University, Mississippi State, MS, USA; (2) Dept of Biochem, Mol Biology, Entomology and Plant Pathology, Mississippi State University, Mississippi State, MS, USA

Seed transmission represents an efficient way for the virus survival and introduction into a new crop. In this project, we investigated the totality of seedtransmitted viruses ("seed-borne virome") in cultivated and non-cultivated cucurbits (Family Cucurbitaceae) by applying High Throughput Sequencing (HTS, synonym next generation sequencing) of dsRNAs extracted from plant tissue collected from a pool of young seedlings grown under insect-proof conditions in order to prevent contamination by horizontally transmitted viruses. Majority of tested plants contained visible amounts of high-molecular weight dsRNAs, which were used for library construction and custom-based Illumina sequencing on a MySeq platform $(2 \times 250 \mathrm{bp}$ paired-end reads). Assembly and analyzes of HTS data showed widespread presence of known and novel "persistent viruses" in tested cucurbits (viruses belonging to the families Partitiviridae, Amalgaviridae, Endornaviridae and Totiviridae). Additionally, our work revealed presence of several new viruses belonging to families/genera traditionally containing pathogenic viruses. Our study confirmed suitability of HTS as an unbiased and highly-sensitive approach for virus diagnostics and surveillance purposes.

RNA virome of two important phytopathogenic fungi

N. ABOUGHANEM-SABANADZOVIC (1), P. Deng (2), T. Wilkerson (3), M. Tomaso-Peterson (2), T. W. Allen Jr. (3), S. Sabanadzovic (4), (1) Institute for Genomics, Biocomputing and Biotechnology, Mississippi State University, Mississippi State, MS, USA; (2) Mississippi State University, Mississippi State, MS, USA; (3) Mississippi State University, Stoneville, MS, USA; (4) Dept of Biochem, Mol Biology, Entomology and Plant Pathology, Mississippi State University, Mississippi State, MS, USA

Fifty five isolates of Rhizoctonia spp and Macrophomina phaseolina, two important and polyphagous phytopathogenic fungi collected from agronomic crops (i.e. corn, soybean, wheat, peanut) and turfgrasses in Mississippi, were submitted to dsRNA extraction and subsequent custom-based cDNA library construction and Illumina sequencing at the University of Illinois. Results of this investigation showed presence of high molecular weight dsRNA molecules, an indication indication of on-going infections by RNA viruses, in majority of examined isolates of both fungi. Presence of certain viruses was confirmed by transmission electron microscopy observation of purified preparations from dsRNA-containing fungal isolates. Computer-based assembly of 2X250 pair-end reads resulted in numerous contigs varying from several hundred to 17 thousands nucleotides. Analyses revealed presence of numerous previously reported mycoviruses in both hosts. Nevertheless, in this study we also discovered dozens of new viruses belonging to recognized genera and families of mycoviruses with dsRNA and ssRNA genomes (either positive- or negative-sense), or those representing new taxa. Effects of theses viruses is yet to be fully understood; however some of them resulted associated with phenotypic changes in their respective hosts. Finally, significant number of ORF-containing sequences did match currently available data in GenBank, suggestive of the expanding mycovirus diversity. 
S. Shakya (1), N. GRUNWALD (2), J. E. Weiland (2), V. Fieland (1), B. J. Knaus (2), M. H. Jung (3), C. Maia (3), A. Drenth (4), D. I. Guest (5), E. C. Y. Liew (6), C. Crane (7), B. Scanu (8), T. Jung (3), (1) OREGON STATE UNIVERSITY, Corvallis, OR, USA; (2) USDA-ARS, Horticultural Crops Research Unit, Corvallis, OR, USA; (3) Mendel University, Brno, CZECH REPUBLIC; (4) The University of Queensland, Brisbane, AUSTRALIA; (5) University of Sydney, Eveleigh, AUSTRALIA; (6) The Royal Botanic Gardens and Domain Trust, Sydney, AUSTRALIA; (7) Dept Parks and Wildlife, Perth, AUSTRALIA; (8) Univ of Sassari, Sassari Sardinia, ITALY

Phytophthora cinnamomi is a heterothallic, broad host range plant pathogen causing dieback and root rots of more than 3000 plant species. Several independent studies have suggested the existence of clonal lineages, primarily of the A2 mating type, and rarely sexual reproduction. However, a rigorous study of population diversity at a global scale is currently lacking. We analyzed 197 isolates of $P$. cinnamomi sampled from 11 countries. Genotyping by sequencing was performed using two restriction enzymes (PstI and MspI) and sequenced on the Illumina HiSeq 3000 platform. Raw reads were mapped to the $P$. cinnamomi reference genome using bowtie2 and variants were called with the GATK HaplotypeCaller. We tested the hypothesis of clonal reproduction in $P$. cinnamomi populations. A neighbor-joining tree based on 1,027 variants indicated that populations of $P$. cinnamomi from Southeast Asia (Taiwan \& Vietnam) consisting of A1 and A2 mating type isolates were highly diverse whereas most isolates from the remaining countries formed two distinct clonal lineages of the A2 mating type. A1 mating type isolates from Papua New Guinea, South Africa and Australia grouped within the diverse Taiwanese and Vietnamese populations which were inferred to be partially sexual based on the index of association. This suggests that Southeast Asia might be a candidate center of origin for $P$. cinnamomi as speculated previously. These results provide novel insights into the existence of both mating types in Southeast Asia and probably sexual reproduction that could potentially give rise to novel aggressive genotypes or lineages.

Morphological and molecular variability of Alternaria spp. causing leaf blight of cotton in India S. A. ASHTAPUTRE, V. R. Kulkarni, Department of Plant Pathology, Dharwad, INDIA

Variability with respect to morphological characters revealed that out of 25 isolates of Alternaria spp, 18 showed complete resemblance with Alternaria macrospora and remaining seven isolates resembled to Alternaria alternata. The conidial size varied from $42.43 \times 17.69 \mu \mathrm{m}$ in Garag to $76.20 \times 13.20$ $\mu \mathrm{m}$ Tadakod isolates. As per structural figure description of $A$. macrospora by Ellis, 18 isolates showed complete resemblance with Alternaria macrospora and remaining seven isolates resembles to Alternaria alternata morphologically. In molecular variability study, DNA sequences of twenty five isolates were compared using the bioinformatics tool of the National Centre for Bioinformatics (NCBI) blast programme. PCR amplification of Alternaria spp. with conserved primers ITS1 and ITS4 that yielded an approximately $560 \mathrm{bp} \mathrm{rDNA}$ amplicon product. Based on sequence comparison, the sequences of eighteen isolates were confirmed as Alternaria macrospora and seven as Alternaria alternata and accordingly phylogenetic trees were constructed. The present investigation revealed that there is greater morphological and molecular variability among the different isolates of Alternaria sp. It also showed that the genetic variation among the pathogen from different cotton growing areas of India.

The incidence and pathogenicity of Alternaria leaf spot associated with canola (Brassica napus) in southern Australia H. AL-LAMI (1,2,3), M. You (1,3), M. Barbetti (1,3), (1) School of Agriculture and Environment, Faculty of Science, University of Western Australia, Perth, AUSTRALIA; (2) Department of Biology, Faculty of Science, Mustansiriyah University, Baghdad, IRAQ; (3) The University of Western Australia Institute of Agriculture, Faculty of Science, Perth, AUSTRALIA

Alternaria spp., especially Alternaria brassicae, A. brassicicola, A. raphani, A. alternata, A. japonica and A. tenuissima associated with a range of different disease symptoms in Brassicaceae, including various leaf lesions on cauliflower, broccoli, and oilseed brassicas including canola and mustard. Alternaria spp. can cause serious losses, for example, up to $47 \%$ in Indian mustard (Brassica juncea) and $>70 \%$ in some other Brassica spp. However, as yet, resistance breeding programs in rapeseed-mustard against $A$. brassicae have had minimal success. Alternaria spp. associated with leaf lesions on canola and associated weedy Brassicaceae in Australia were unknown, studies were undertaken to determine which Alternaria spp. were associated with diseased leaves of canola (B. napus) and wild radish (Raphanus raphanistrum) plants across two cropping seasons. More than 400 Alternaria spp. isolates were collected and are being identified using PCR-based assay by sequencing of the ITS region of the rDNA operon, the gene fragment plasma membrane ATPase and also the 'Alt a 1' gene. Fifteen species of Alternaria have now been identified, including A. brassicae, A. alternata and A. infectoria, and thirteen of the different Alternaria spp. were found to be pathogenic on B. napus and B. juncea.

\section{Deciphering the genetic variation of Asian soybean rust pathogen Phakopsora pachyrhizi}

Y. GUPTA (1), H. M. Murithi (2), R. K. Shrestha (1), D. Maclean (1), M. Joosten (3), Y. Yamaoka (4), G. L. Hartman (5), S. Brommonschenkel (6,7), P. van Esse (1), (1) The Sainsbury Laboratory, Norwich, UNITED KINGDOM; (2) Intl Inst of Tropical Agriculture (IITA), Dar Es Salaam, TANZANIA; (3) Wageningen University, Wageningen, NETHERLANDS; (4) Univ of Tsukuba, Tsukuba Ibaraki, JAPAN; (5) USDA-ARS at the University of Illinois, Urbana, IL, USA; (6) Universidade Federal de Viçosa/Departamento de Fitopatologia, Viçosa, BRAZIL; (7) Universidade Federal de Viçosa, Viçosa, BRAZIL

Soybean (Glycine max) is an important legume crop and a rich source for plant proteins and vegetable oil. A major constraint for soybean production is soybean rust which causes yield losses of up to $80 \%$. The disease is caused by the obligate biotrophic fungus Phakopsora pachyrhizi. Extensive efforts have been made to find genetic resistance against $P$. pachyrhizi however, the pathogen has rapidly overcome major resistance genes, Rpp 1-6. The degree and distribution of genetic variation in the pathogen population is key for informed deployment of novel resistance genes but is largely unknown for $P$. pachyrhizi. To understand the field variation and population structure of $P$. pachyrhizi, infected field samples of soybean were collected from different locations across Brazil, East Africa and USA. Next-generation sequencing approach was developed to explore the population structure and genetic variation of $P$. pachyrhizi in these very different geographic locations. Sequencing of field samples will allow us to study sequence polymorphisms in $P$. pachyrhizi. We will aim to obtain a detailed understanding of the effector diversity in the field population of $P$. pachyrhizi. This information will be used for the sustainable deployment of genes that confer resistance to soybean rust.

Diversity of Streptomyces spp. causing potato common scab in Eastern Canada A. NOVINSCAK, C. Hudec, M. Filion, Université de Moncton, Moncton, NB, CANADA

Common scab is a potato disease that significantly decreases the market value of potato tubers following the development of necrotic lesions on their surface. Streptomyces scabiei is the main causal agent of common scab; however, other closely related species, including S. acidiscabies and S. turgidiscabies, have also been shown to cause the disease. In this study, we focused on the genetic and phenotypic diversity of Streptomyces spp. able to infect tubers in Prince Edward Island, the main potato-producing province in Canada. Three hundred and twenty-five pathogenic Streptomyces cultures 
were retrieved from the lesions of potatoes harvested from three separate geographical locations using selective culture media and PCR analyses. Genome fingerprinting analyses using repetitive elements polymerase chain reactions (ERIC- and BOX-PCR) revealed 25 distinct genetic groups. The geographical distribution of these groups was analysed and genomes of predominant species have been sequenced using Next-Gen sequencing. A multilocus sequence analysis based on $16 \mathrm{~S}$ rRNA, gyrB, rpoD and $r p o B$ was performed, as well as sequence analyses of pathogenesis-related genes encoding for thaxtomin synthase $(t x t)$, tomatinase (tomA), and necrosis protein (necl). The core and pan-genomes of the Streptomyces spp. were also identified. The virulence of the various strains is currently being analysed through pathogenicity tests (in vitro and on potato tuber slices).

Genetic recombination in Venturia effusa, causal agent of pecan scab

N. D. Charlton (1), C. H. Bock (2), C. A. YOUNG (1), (1) Noble Research Institute, LLC, Ardmore, OK, USA; (2) USDA ARS, Southeastern Fruit and Tree Nut Research Laboratory, Byron, GA, USA

Venturia effusa causes pecan scab, the most prevalent disease of pecan in the southeastern USA. Mating type idiomorphs were recently characterized and the sexual stage was subsequently produced in vitro. To investigate sexual reproduction and recombination of traits in $V$. effusa, select isolates were crossed in pairwise combinations on oatmeal agar. After a 4 mo cold treatment, the pairings were incubated at $24 \mathrm{C}$ with a $12 \mathrm{~h}$ photoperiod for $14 \mathrm{~d}$ to allow maturation of the ascospores. Ascospore progeny from crosses between isolates NFVe23 (cv. Pawnee) $\times$ NFVe24 (native), Albino (cv. Desirable) $\times$ NFVe47 (cv. Choctaw) and Albino (cv. Desirable) $\times$ NFVe78 (cv. Pawnee) were generated. A total of 32, 13 and 30 single ascospore progeny were isolated from each cross, respectively. The parental isolates and ascospore progeny were screened with eight SSR markers and primers for the mating type idiomorphs. Genotyping using SSR and mating type loci, and phenotyping the crosses with the albino parent, revealed recombination in the ascospore progeny. Based on these markers, the proportion of recombinant progeny were 0.97 for NFVe $23 \times$ NFVe24, 1.00 for Albino $\times$ NFVe47 and 0.90 for Albino $\times$ NFVe 78. Identification of the sexual stage and characterization of recombination in $V$. effusa will provide a better understanding of the impact of the sexual cycle in the epidemiology of pecan scab in the field as well as provide insight into approaches to manage this disease.

\section{Genetic Diversity of Tobacco rattle virus isolates from Potato in the USA}

L. MOYO (1,2), A. Hamid (1), I. Mallik (3), N. C. Gudmestad (3), H. R. Pappu (1,2), (1) Department of Plant Pathology, Washington State University, Pullman, WA, USA; (2) Molecular Plant Sciences Graduate Program, Washington State University, Pullman, WA, USA; (3) North Dakota State University, Department of Plant Pathology, Fargo, ND, USA

Tobacco rattle virus (TRV), an important pathogen of potato, tobacco and ornamental bulbs, consists a segmented, positive sense RNA genome (RNA1, $6.79 \mathrm{~kb}$ and RNA2, $3.86 \mathrm{~kb}$ ). It causes corky ringspot disease characterized by concentric rings and necrotic and brown spots in potato tubers. Virus population dynamics shift frequently due to genetic drift, migration, mutation, natural selection and recombination. This could result in increased genetic diversity and emergence of more virulent and resistance-breaking strains. To elucidate the genetic diversity of TRV, whose first report in USA was in 1946 in Florida, the whole genome of 4 RNA1 and 5 RNA2 TRV isolates from potatoes in different states in the USA were cloned and sequenced to assess its population structure, evolutionary and epidemiological dynamics. Phylogenetic analyses based on nucleotide sequences of the coat protein (CP) and nematode transmission (2b) genes, in RNA2, showed geographical clustering of the US isolates with previously known American isolates, while the European isolates formed a distinct cluster. Low genetic diversity among the American isolates was noticed compared to those from Europe. This was further corroborated by the observed high purifying selection pressure acting on the $\mathrm{CP}$ and $2 \mathrm{~b}$ genes, genetic differentiation and infrequent gene flow between American and European isolates. Based on these sequence analyses, TRV did not seem to have significantly diverged in the USA. Phylogenetic groupings based on RdRp, 1a and $1 \mathrm{~b}$ genes in RNA1 were consistent with geographical clustering of the isolates. This study provided new insights into the genetic diversity of TRV in the USA.

Novel Magnaporthales fungi pathogenic to switchgrass and turfgrasses J. LUO, P. Vines, A. Grimshaw, L. Hoffman, E. M. Walsh, S. A. Bonos, B. Clarke, J. Murphy, W. A. Meyer, N. Zhang, Rutgers University, New Brunswick, NJ, USA

About $50 \%$ of species in the fungal order Magnaporthales are pathogens of monocotyledon plants, including the rice blast fungus Pyricularia oryzae (syn. Magnaporthe oryzae), the take-all pathogen of cereals Gaeumannomyces graminis (syn. Gaeumannomyces graminis var. tritici), and the summer patch pathogen of turfgrass Magnaporthiopsis poae (syn. Magnaporthe poae). From our recent survey of fungi associated with grass roots, we discovered several new lineages in Magnaporthales. Based on multi-gene phylogenetic analysis and morphological and biological characteristics, three new genera (Magnaporthiopsis, Pseudophialophora and Falciphora), ten new species, and five new combinations have been proposed and published. Plant-fungal interaction experiments showed that Pseudophialophora angusta, P. eragrostis, P. magnispora, P. schzachyrii, P. tarda, and $P$. whartonensis that were isolated from grass roots in the New Jersey Pine Barrens had negative effects on the growth of switchgrass (Panicum virgatum). Magnaporthiopsis meyeri-festucae isolated from turfgrasses in New Jersey exhibiting summer patch-like symptoms was demonstrated to be pathogenic to hard fescue (Festuca brevipila) and Kentucky bluegrass (Poa pratensis). Furthermore, our phylogenomic analysis on the Magnaporthales fungi has provided insight into the evolution of pathogenicity and supported our taxonomic treatment of these taxa. This work will serve as the basis for future studies on biogeography, host range, biodiversity, evolution, and the impact of these plant pathogens on grass communities.

\section{The open road: A case study of reproducible research in plant pathology}

Z. Kamvar, S. E. EVERHART, University of Nebraska, Lincoln, NE, USA

Open and reproducible research is quickly becoming an important topic in plant pathology where research output can directly influence management decisions that affect global food supply. Moreover, data sharing among plant pathologists has the potential to reduce cost and increase collaboration overall. While there is a need for plant pathologists to become familiar with concepts and tools of open and reproducible research, few examples specific to our field exist. We recently assessed the population genetic structure of the white mold pathogen, Sclerotinia sclerotiorum from research plots and commercial fields across the United States. Due to the vast amount of work that went into collecting all the data and measurements, we deliberately designed our data analysis such that it was i) auditable ii) reproducible and iii) open and accessible by anyone. We present here this case study of reproducible research in plant pathology as a proof-of-concept standard of open research for data sets of any size, attainable by anyone. In a nontechnical manner, we provide a clear overview of the concepts and mechanisms used to produce a full-fledged publication starting from raw data in the $\mathrm{R}$ programming language. By providing recommendations for open research practices in plant pathology, we hope to foster a zeitgeist that will enable scientists in our discipline to reproduce their research with confidence, creating more efficient sharing and evaluation of data and materials. 
Southwestern dwarf mistletoe (SWDM), Arceuthobium vaginatum subsp. cryptopodum, is the most damaging pathogen on ponderosa pine, Pinus ponderosa, in the Southwest. Although the parasite is native to the region, fire suppression has led to overstocking that may have facilitated increased spread through stands. Incidence of SWDM was previously rated along roadsides in New Mexico National Forests in 1960 and 1987 . Our objective is to map current distribution and incidence of SWDM in the State and identify changes since these previous surveys. Over 150 miles of roadways throughout three Ranger Districts in two New Mexico National Forests were traversed at $15 \mathrm{mph}$ with an observer noting relative incidence of SWDM along 0.1 mile road increments to a width of 66' into the forest (representing 0.8 acres per increment). Incidence was rated from $0-3$, with 0 representing no mistletoe observed, 1 representing less than 33\% incidence, 2 representing 33-66\% incidence, and 3 representing greater than $66 \%$ incidence. Data were georeferenced using GPS, and maps were developed in ArcGIS. Summary statistics were developed and compared to previous surveys to identify changes in SWDM. Infection was not observed across $67 \%$ of the assessed area. Light infection was present in $18.6 \%$ of the area, moderate infection was present in $9.2 \%$ of the area, and heavy infection was present in $5.2 \%$ of the area. Overall, SWDM has spread into previously uninfected areas and increased in incidence.

Investigation of Genotypes and Fungicide Resistance Profiles of Botrytis Populations on Single Strawberry Flowers M. HU (1), G. Schnabel (2), (1) University of Maryland, College park, MD, USA; (2) Clemson University, Clemson, SC, USA

Genotypic variation often can be found in fungal pathogens from different hosts, locations, and different parts of the same plant. However, information about pathogen diversity in single flowers has not been studied in depth. A total of 192 single-spore Botrytis spp. isolates obtained from 19 strawberry flowers were fingerprinted using microsatellite markers. Multiple haplotypes were found in 12 flowers, but seven flowers exhibited a single haplotype. Two different Botrytis species, B. cinerea and B. fragariae, were found co-existing in three flowers. Sensitivity was determined to the fungicides cyprodinil, fludioxonil, fenhexamid, iprodione, thiophanate-methyl, boscalid, fluopyram, penthiopyrad, and isofetamid, which represent six different chemical classes. Multifungicide resistance was commonly observed in both species but resistance to boscalid and penthiopyrad was detected only in $B$. cinerea. Isolates from the same flower, regardless of haplotypes, tended to have identical resistance profiles. Each haplotype was largely associated with a single resistance profile but a single resistance profile was often represented by multiple haplotypes. This study is the first to investigate genotypic and phenotypic diversity in Botrytis spp. isolated from the same tissue. The findings improve our understanding of epidemiology for Botrytis and may be relevant to genotyping and phenotyping studies in other fungal pathogen systems.

Population structure and genetic diversity of Melampsora spp. collected from Salix purpurea in the Northeast United States C. R. CROWELL (1), C. Carlson (2), D. Wilkersen (2), L. Smart (3), C. D. Smart (1), (1) Plant Pathology and Plant-Microbe Biology Section, Cornell University, Geneva, NY, USA; (2) Horticulture Section, Cornell University, Geneva, NY, USA; (3) Cornell University, Geneva, NY, USA

Melampsora spp. willow rust is the most threatening disease of Salix spp. for short rotation coppice bioenergy feedstock production wherever shrub willow is grown. Investigations into this group of pathogens in the Northeast United States are ongoing to elucidate genotypic diversity and aid in successful disease resistance breeding. Previous work has identified two species, M. americana and M. paradoxa as the primary contributors to disease on S. purpurea in the Northeast. Single pustule isolates $(\mathrm{n}=184)$ were collected from wild and cultivated S. purpurea in NY, WV, VT, MI, and PA in 2015, and are being genotyped via genotyping-by-sequencing. Whole genome PacBio sequencing of a single M. americana isolate was performed in collaboration with the Joint Genome Institute's Community Science Program to aid in variant discovery. Preliminary data from 95 samples suggest differentiation of isolates by location of collection, with two sites, Morgantown WV and Portland NY, showing highly diverged individuals. These data, in combination with previously performed detached leaf virulence assays across select host genotypes, will improve breeding efforts for targeted populations in the Northeast United States.

White mold/dry bean: Population structure and phenotypic variation of Sclerotinia sclerotiorum from dry bean in the USA Z. Kamvar (1), B. S. Amaradasa (1,2), R. Jhala (1), S. B. McCoy (1), J. R. Steadman (1), S. E. EVERHART (1), (1) University of Nebraska, Lincoln, NE, USA; (2) University of Florida, Gainesville, FL, USA

The ascomycete pathogen and causal agent of white mold on dry bean, Sclerotinia sclerotiorum, is a necrotrophic pathogen on over 400 known host plants. Currently, there are no known cultivars of dry bean with complete resistance to white mold. For over 20 years, bean breeders have used white mold screening nurseries (WMSN) with natural populations of S. sclerotiorum to screen new cultivars for resistance. It is thus important to know if the genetic diversity in populations of S. sclerotiorum within these nurseries a) reflect the genetic diversity of the populations in the surrounding region and b) are stable over time. Furthermore, previous studies have investigated the correlation between mycelial compatibility groups (MCG) and multilocus haplotypes (MLH), but none have formally tested these patterns. We genotyped 366 isolates of S. sclerotiorum from producer fields and WMSN surveyed over 10 years in 2003-2012 representing 11 states in the United States of America, Australia, France, and Mexico at 11 microsatellite loci resulting in 165 MLHs. Populations were loosely structured over space and time based on analysis of molecular variance and discriminant analysis of principal components, but not by cultivar, aggressiveness, or field source. Our study suggests that breeders should continue to test dry bean lines in several WMSN across the USA to account for both the phenotypic and genotypic variation that exists across regions.

Intra- and inter-field diversity of Xanthomonas translucens isolates associated with natural occurrence of bacterial leaf streak in wheat and barley R. D. CURLAND, C. A. Ishimaru, University of Minnesota, St. Paul, MN, USA

Bacterial leaf streak (BLS) has been a disease of increasing concern over the past decade. Recent studies have shown that both $X$. translucens pv. undulosa (wheat and barley) and X. translucens pv. translucens (barley) are common in the Northern Great Plains. Phylogenetic analyses of multilocus sequence alignments (MLSA) of isolates from this region substantiated the close relatedness of both pathovars. Multilocus sequence typing (MLST) further determined the existence of multiple sequence types of each pathovar at the national and regional scale. Here we evaluated the intra- and interfield scale genetic diversity of bacteria causing BLS. Twenty-five leaf samples from two wheat and two barley fields, showing natural infections of BLS, were collected during the growing season of 2015. Bacteria from individual leaves were isolated on Wilbrink's agar. Colonies characteristic of $X$. translucens were purified and stored. Pathogenicity and relative virulence of isolates were assayed in wheat and barley seedlings. Phylogenies and sequence types were ascertained from analyses of the concatenated sequences of four housekeeping genes ( $r p o D, d n a K$, fyuA and $g y r B)$. Estimates of genetic diversity within and between fields were measured. Knowledge of the range of virulence and sequence types of $X$. translucens. pv. undulosa and $X$. translucens pv. translucens within prominent areas of wheat and barley production can inform breeding strategies for BLS disease resistance. 
Discula destructiva, an exotic pathogen of Cornus spp. in North America: Evidence of independent introductions

D. HADZIABDIC (1), K. Mantooth (2), S. L. Boggess (1), M. T. Windham (1), S. Miller (3), G. Cai (4), N. Zhang (5), M. Staton (6), B. H. Ownley (6), R. N. Trigiano (1), (1) University of Tennessee, Knoxville, TN, USA; (2) Clemson University, Coastal Research and Education Center, Charleston, SC, USA; (3) APHIS PPQ, Linden, NJ, USA; (4) USDA-ARS / Purdue University, West Lafayette, IN, USA; (5) Rutgers University, New Brunswick, NJ, USA; (6) University of Tennessee, Department of Entomology and Plant Pathology, Knoxville, TN, USA

Native to North America, Cornus florida (flowering dogwood) and C. nuttallii (Pacific dogwood) are valued for their ornamental characteristics and as an important food source for many animals due to the high fat content of berries. In the late 1970s, trees in the eastern and western United States were declining which resulted in massive die-offs of entire populations. The fungal pathogen, Discula destructiva was identified as the causal agent of dogwood anthracnose. For the next two decades, this fungus spread throughout most of the native ranges of C. florida and C. nuttallii resulting in high mortality rates. We evaluated genetic diversity and population structure of $93 \mathrm{D}$. destructiva isolates using 47 microsatellite loci. Our results indicated low genetic diversity and the presence of four genetic clusters that corresponded to two major geographic areas, the eastern U.S. and the Pacific Northwest, and to the two time periods when the isolates were collected (pre- and post-1993). Linkage disequilibrium was present in five out of six subpopulations, suggesting that the fungus only reproduced asexually. Evidence of population bottlenecks was found across four identified genetic clusters, and was probably the result of the limited number of founding individuals on both coasts. Our results support the hypothesis that $D$. destructiva is an exotic pathogen with independent introductions on the east and west coasts of the U.S.

Variation of mutations in $A V R$ genes in the field isolates of the tomato wilt fungus, Fusarium oxysporum $\mathrm{f}$. sp. lycopersici T. ARIE (1), S. Hanyuda (2), R. Masuko (2), Y. Ayukawa (2), K. Komatsu (2), (1) Tokyo Univ of Agric \& Tech (TUAT), Fuchu, Tokyo, JAPAN; (2) Tokyo Univ Agric \& Tech (TUAT), Fuchu, Tokyo, JAPAN

Race-cultivar interactions between the tomato wilt fungus, Fusarium oxysporum f. sp. lycopersici (Fol) and tomato (Solanum lycopersicum) are determined by the interactions between the avirulence genes (AVR1, AVR2 and $A V R 3)$ carried by $F o l$ and the corresponding resistance genes $(I, I 2$ and I3) carried by tomato. The avirulence genes often exist on small conditionally dispensable (CD) chromosomes, which are not necessary for growth but are responsible for pathogenicity. Three races have been reported in $\mathrm{Fol}$ and these races can be determined by the combination of carrying avirulence genes; race 1 (AVRI AVR2 AVR3), race 2 (avrl AVR2 AVR3) and race 3 (avrl avr2 AVR3). A collection of isolates of races 2 and 3 of Fol was made

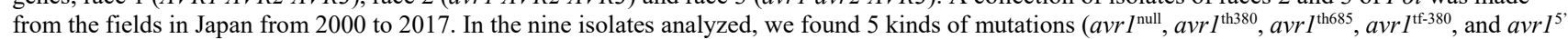
$\left.{ }^{\mathrm{ND}}\right)$ in $A V R 1$, and 4 kinds $\left(a v r 2^{\text {null }}, a v r 2^{\mathrm{G} 121 \mathrm{~A}}, a v r 2^{\mathrm{T} 122 \mathrm{~A}}\right.$, and $\left.a v r 2^{\mathrm{C} 146 \mathrm{~T}}\right)$ in $A V R 2$. None of the nine isolates had the identical combination of mutations in $A V R 1$ and $A V R 2$, even though some of the isolates are phylogenetically very close. These suggest that race differentiation of $F o l$ in the field is more diversified than expected. Because this observation possibly relates to the fact that the avirulence genes exist on CD chromosomes, we will discuss the karyotypes of the field isolates, too.

Genetic Diversity Studies of Potato mop-top virus from Potato in the United States

Y. ZHAI (1), A. Hamid (1), A. Tabassum (1), I. Mallik (2), N. C. Gudmestad (2), H. R. Pappu (1), (1) Department of Plant Pathology, Washington State University, Pullman, WA, USA; (2) North Dakota State University, Department of Plant Pathology, Fargo, ND, USA

Potato mop-top virus (PMTV; Genus: Pomovirus; Family: Virgaviridae) is a soil-borne RNA virus that is becoming increasingly important in potato production in the US in recent years. This virus is transmitted by Spongospora subterranea. To understand the genetic diversity of the virus, the complete genome of six isolates of PMTV collected from different states in the USA were sequenced, analyzed, and compared with previously characterized isolate from the Washington State. The seven USA isolates showed high nucleotide similarities with one another in all seven genes. However, the nucleotide similarity of CPRT gene is least compared to the rest. The phylogenetic trees of the PMTV genes have some extent of similarity but are not dramatically consistent, with the RdRp gene (RNA 1) has the strongest divergence potential. Genetic diversity test showed that PMTV S strain to be more diverse than PMTV M strain and PMTV recombinant. Statistically significant and insignificant negative values of Tajima's D in PMTV $\mathrm{S}$ strain and $\mathrm{M}$ strain, respectively, suggest the dominance of purifying selection and population expansion is operating in those strains. The marginal likelihood mean substitution rate was $5.28 \times 10^{-5}$ subs/site/year, and $95 \%$ highest posterior density (HPD) substitution rate is between $5.29 \times 10^{-5}-5.27$ $\times 10^{-5}$. Our work provides the genomic basis for PMTV diagnosis, prevention, and disease epidemiology in the United States.

Population diversity of Fusarium on the main cereals in China

J. FENG, Institute of Plant Protection, Beijing, CHINA

Wheat, maize and rice are the most important cereals in China. They are all threatened by toxigenic Fusarium, which caused yield loss and mycotoxin contamination. Therefore the knowledge of diversity of Fusarium population on different crops and regions is important for management of the disease and mycotoxin contamination. In recent years, we did a large-scale survey of Fusarium population diversity on wheat, maize and rice in the main grainproducing areas. Samples including head blight, and crown rot of wheat, ear rot and stalk rot of maize and perithecia from rice and maize stubble were collected. More than 2000 Fusarium isolates were isolated and the species and chemotype were identified. F. graminearum and F. asiaticum are the main causal agent of wheat head blight and $F$. pseudograminearum is responsible for wheat crown rot. The maize population showed much higher diversity than wheat at species level. F. graminearum with $15 \mathrm{ADON}$ chemotype can circulate between maize and wheat in Northern China. F. boothii is only found on maize and virulence tests confirmed its different fitness to the hosts. In Southern China, $F$. asiaticum is predominant on both wheat and rice stubble, which revealed rice stubble is the main media for their overwintering. Significant more NIV producers are observed on rice than wheat in the same area, indicating the rice preference of NIV population. In agreement with this, predominant NIV chemotype were also identified in Hunan and Fujian Province, where rice is the main crop and wheat is absent.

Phenotypic and Genotypic diversity in Phytophthora infestans Isolates in Korea M. AKTARUZZAMAN, B. S. Kim, Gangneung-Wonju National University, Gangneung, SOUTH KOREA

The oomycete, Phytophthora infestans (Mont.) de Bary causes late blight disease in potato (Solanum tuberosum L.) worldwide including South Korea. In the present study, we analyzed phenotypic characteristics such as mating type, metalaxyl resistance, fitness composites and physiological race of $P$. infestans isolates in Korea. The DNA-based markers called SSRs were also used for analyzing the genetic diversity among the isolates. For phenotypic characterization, a total of 301 isolates were purified from different locations of Korea in 2013-2016. All the isolates were identified as A1 mating type. Frequencies of isolates resistant $(4.7 \%, 4.9 \%, 9.7 \%$ and $8.5 \%)$, moderately resistant $(75.3 \%, 80.4 \%, 77.3 \%$ and $75.8 \%)$ and sensitive $(20.0 \%, 14.7 \%$, $16.6 \%$ and $15.7 \%$ ) to matalaxyl were found in 2013 to 2016, respectively. Fitness components and composite fitness index of metalaxyl resistant isolates 
were higher compared to metalaxyl sensitive isolates. Total number of physiological race of $P$. infestans was detected 17, 25, 22 and 23 in 2013 to 2016 , respectively. Among them, the races R0.1.2.4.5.6.7.11, R0.1.2.3.4.5.6.7.11, R0.1.2.4.5.6.7.10.11 and R0.1.2.3.4.5.6.7.10.11 were dominant and showed virulence to potato, except R8 and R9. For genetic diversity, we analyzed 172 P. infestans isolates from 2009-2016. In our study, 45 multi-locus genotypes (MLGs), which were classified into four genotypes KR-1, KR-2, RU-1 and US-11. KR-1 and KR-2, where KR-2 was a new genotype.

Phylogenetic analysis of South American Berberis species and their related rust fungi

C. W. BARNES (1), S. Hambleton (2), M. E. Ordonez (3), T. G. Fetch Jr. (4), M. Chaves (5), J. Martinelli (6), G. da Silva (6), S. Bordignon (7), P. Campos (8), R. Gandullo (9), R. Madariaga (10), G. Azzimonti (11), S. German (12), (1) Instituto Nacional de Investigaciones Agropecuarias, Quito, Pichincha, ECUADOR; (2) Agriculture \& Agri-Food Canada, Ottawa, ON, CANADA; (3) Universidad Catolica del Ecuador, Quito, ECUADOR; (4) Agriculture and Agri-Food Canada, Brandon, MB, CANADA; (5) Centre for Temperate Climate Agricultural Research, Pelotas, BRAZIL; (6)

Universidade Federal Rio Grande du Sul, Porto Alegre, BRAZIL; (7) Universidade La Salle, Canoas, BRAZIL; (8) Instituto Nacional de Tecnologia Agropecuaria, Buenos Aires, ARGENTINA; (9) Facultad Ciencias Agrarias de la Universidad del Comahue, Neuquén, ARGENTINA; (10) Instituto de Investigaciones Agropecuarias, Chillan, CHILE; (11) Instituto Nacional de Investigación Agropecuaria, Colonia, URUGUAY; (12) National Institute for Agricultural Research (INIA), Colonia, URUGUAY

New races of both Puccinia graminis and P. striiformis are frequently reported throughout the world. Sexual recombination on a susceptible Berberis species host may have produced some of these new reported races, or could generate future new races. The two centers of diversity of simple-leaved Berberis were separated in the Cretaceous period into South American and Asian populations. In South America there are two distinct subgroups: the Aequinoctiales and the Euaustrales. In 2016 and 2017, we sampled Berberis and Berberis infected with rust fungi in Ecuador belonging to the Aequinoctiales, and in Argentina, Brazil, Chile, and Uruguay belonging to the Euaustrales. DNA sequences from both the host and rust fungus were used for phylogenetic analysis. Within the Euaustrales Berberis, we collected B. laurina, B. microphylla, B. darwinii, B. linearifolia, B. serrato-dentata, and an unknown Berberis species. Within the Aequinoctiales Berberis, we collected B. hallii, B. lutea, B. pichinchensis, B. grandiflora and two unknown Berberis species. Analyses of the rust ITS sequences suggested that at least 8 distinct species were collected. None were identified as either $P$. graminis or P. striiformis based on comparisons with available reference DNA data while only one corresponded to a named species, Edythea quitensis (Ecuador). Most rust fungi had one Berberis host. More sampling is needed to rule out South American Berberis as a host for P. graminis or P. striiformis.

Population structure, virulence and resistance to mefenoxam of Phytophthora capsici in Michigan Y. GUO (1), C. Krasnow (1), M. K. Hausbeck (2), (1) Michigan State University, East Lansing, MI, USA; (2) Department of Plant Soil and Microbial Sciences, Michigan State University, East Lansing, MI, USA

The oomycete plant pathogen Phytophthora capsici is highly dynamic and destructive with a broad host range. In Michigan, it causes economic losses to many important vegetable crops. The objectives of this study were to investigate the population structure and characterize the virulence and mefenoxam sensitivity for nine $P$. capsici populations collected from six counties between 2003 and 2016 in Michigan. Simple sequence repeats (SSR) markers from intergenic regions and effectors/surrounding regions were developed to study the population structure and the potential linkage with virulence parameters. Analysis of molecular variance revealed moderate to high degree of genetic difference among the populations. Significant differentiation $\left(\mathrm{F}_{\mathrm{ST}}\right)$ was identified in over $60 \%$ of the pairwise comparisons among populations, indicating the high genetic diversity of $P$. capsici in Michigan. Bayesian clustering showed some population structure when isolates were grouped by host. Significant difference was detected in mefenoxam resistance among populations. In vitro inoculation indicated significant difference in virulence-related parameters among the populations. General linear model (GLM) and mixed linear model (MLM) based association analyses identified some markers significantly associated with at least one of the virulence parameters. These results expand the understanding of the genetic base of this important pathogen.

Genetic structure of Phoma betae populations on Beta vulgaris in New York and Washington States, USA

L. KOENICK (1), N. L. Knight (1), N. Vaghefi (1), L. du Toit (2), S. J. Pethybridge (1), (1) Cornell University, Plant Pathology \& Plant-Microbe Biology Section, Geneva, NY, USA; (2) Washington State University, Mount Vernon NWREC, Mount Vernon, WA, USA

Phoma betae is an economically important seedborne pathogen of table beet worldwide that is responsible for damping off, foliar disease, and root decay. The majority of seed used in New York is sourced from the Pacific Northwest region of the USA. During the growing season, $P$. betae spreads through polycyclic epidemics resulting from the dispersal of conidia by water splash. $P$. betae may then survive on colonized plant debris in the soil for up to 2 years. $P$. betae is capable of sexual reproduction, producing pseudothecia that release ascospores that can be dispersed long distances via wind. However, the teleomorph has not been observed in New York or Washington. Understanding the population biology of $P$. betae can provide insights into pathogen etiology. No information on population genetics of $P$. betae is available currently. In this study, 10 microsatellite markers were used to genotype $P$. betae isolates from table beet fields in New York $(\mathrm{n}=70)$ and table beet seed crops in Washington $(\mathrm{n}=105)$. Isolates were divided into five populations based on collection location in each state. The genetic diversity, differentiation, and linkage equilibrium of each population were assessed. This information can inform management strategies of $P$. betae in the future.

Genotypic diversity of globally derived isolates of Fusarium oxysporum f. sp. fragariae

P. HENRY (1), C. Borrero (2), M. Aviles Guerrero (3), N. Capote (4), M. H. Nam PhD (5), Y. Hirayama (6), S. Yamazaki (7), J. H. J. Leveau (8), T. R. Gordon (9), (1) University of California, Davis, CA, USA; (2) Fundación de Investigación de la Universidad de Sevilla, Sevilla, SPAIN; (3) Univ de Sevilla, Sevilla, SPAIN; (4) IFAPA Las Torees Tomejil, Seville, SPAIN; (5) Nonsan Strawberry Exp Station, Nonsan, SOUTH KOREA; (6) Nara Prefectural Experiment Station, Nara, JAPAN; (7) Tochigi Prefectural Agricultural Experiment Station, Tochigi, JAPAN; (8) University of CaliforniaDavis, Davis, CA, USA; (9) Department of Plant Pathology, University of California - Davis, Davis, CA, USA

Fusarium wilt of strawberry (caused by Fusarium oxysporum f. sp. fragariae) was first reported in Australia in 1962 and soon after observed in Japan (1969) and South Korea (1974). New reports of this disease surged globally in the early to mid-2000's, coinciding with decreased availability of the fumigant methyl bromide. Studies of genetic diversity in national populations have been conducted in Australia, South Korea, and California, but the methods for assessing diversity are not consistent between publications. Therefore, little is known about genetic diversity in the global population, or the extent to which this pathogen may have been trafficked between countries. Corresponding authors from 15 publications reporting $F$. o. fragariae in 7 countries were contacted for the isolates used in their study. From this query, DNA or cultures were obtained from 27 Australian, 22 Japanese, 18 Korean, 8 Spanish, and 11 Californian isolates ( 86 total). The translation elongation factor 1a (EF-1a; 700bp) and nuclear ribosomal intergenic spacer (IGS; $\sim 2.2 \mathrm{kbp}$ ) loci were sequenced from all isolates and used to construct multi-locus phylogenetic trees. Each isolate was additionally PCR-tested for presence of a locus that is diagnostic of some $F$. o. fragariae strains. Thirty-nine isolates representing diverse haplotypes from each country were tested for pathogenicity on cultivars known to be susceptible and resistant to $F$. o. fragariae in California. Several clades with high bootstrap support included 
isolates from multiple countries, which suggests international movement may have occurred more than once. The challenges to, and opportunities for, preventing future dispersal are discussed.

Identification of Resistance to Wet Bubble Disease and Genetic Diversity in Wild and Cultivated Strains of Agaricus bisporus X. WANG, Y. Li, Y. FU, Jilin Agricultural University, CHANGCHUN, CHINA

Outbreaks of wet bubble disease (WBD) caused by Mycogone perniciosa are increasing across the world and seriously affecting the yield of Agaricus bisporus. However, highly WBD-resistant strains are rare. Here, we tested 28 A. bisporus strains for WBD resistance by inoculating M. perniciosa spore suspension on casing soil, and assessed genetic diversity of these strains using 17 new simple sequence repeat (SSR) markers developed in this study. We found that 10 wild strains originating from the Tibetan Plateau in China were highly WBD-resistant strains, and 13 cultivated strains from six countries were highly susceptible strains. A total of 88 alleles were detected in these 28 strains, and the observed number of alleles per locus ranged from 2 to 8 . Cluster and genetic structure analysis results revealed the wild resources from China have a relatively high level of genetic diversity and occur at low level of gene flow and introgression with cultivated strains. Whol-genome resequencing got the same result and show that wild strains from the Tibetan Plateau and cultivated strains from Europe and America, maybe originated from two different ancestors, evolved parallelly in different ways because of geographic isolation, but there existed genetic exchanges. Moreover, the wild strains from China potentially have the consensus ancestral genotypes different from the cultivated strains and evolved independently. Therefore, the highly WBD-resistant wild strains from China and newly developed SSR markers could be used as novel sources for WBD-resistant breeding and quantitative trait locus (QTL) mapping of WBD-resistant gene of A. bisporus.

MLVA-based diversity analysis of Xanthomonas axonopodis pv. manihotis (Xam) populations in Mali

M. KANTE (1), L. Blondin (2), C. M. Flores M (3), L. Y. Rache Cardenal (4), I. Wonni (5), K. Dagno (6), S. Restrepo (4), A. J. Bernal (7), R. Koebnik (3), V. M. Verdier (8), O. Koita (9), C. Verniere (10), B. Szurek (3), (1) Université de Segou, IRD, LBMA, Segou, MALI; (2) CIRAD-UR Bioagresseurs, Montpellier, FRANCE; (3) IRD, Cirad, Université de Montpellier, IPME, Montpellier, FRANCE; (4) Universidad de los Andes, Bogota, COLOMBIA; (5) Institut de l'Environnement et de la Recherche Agricole (INERA), Bobo Dioulasso, BURKINA FASO; (6) Institut d'Economie Rurale (IER), Prog. Sorgho/ Lab de Phytopathologie, CRRA de Sotuba, Bamako, MALI; (7) Universidad de los Andes, Laboratorio de Interacciones Moleculares de Microorganismos Agr, Bogota, COLOMBIA; (8) IRD, Cirad, Univ Montpellier, IPME, Montpellier, FRANCE; (9) University of Sciences, Techniques and Technologies of Bamako (USTTB), LBMA, Bamako, MALI; (10) CIRAD, UMR BGPI, Montpellier, FRANCE

Cassava Bacterial Blight (CBB) caused by the bacterium Xanthomonas axonopodis pv. manihotis (Xam) occurs on several continents including West Africa where it has recently been reported in Burkina Faso and Ivory Coast, sometimes imposing severe yield losses. The genetic diversity of CBB in Mali, a country bordering both Burkina Faso and Ivory Coast, was investigated in 2015 in two cassava production regions (Bamako and Ségou). Forty strains were isolated from cassava leaves showing angular leaf spots symptoms typical of CBB. Colonies were identified as Xam after specific PCR diagnostic tests and pathogenicity assays. The Koch postulate was further validated. Subsequently, a multilocus variable number of tandem-repeat (VNTR) analysis (MLVA) scheme based on 14 microsatellites discriminated 18 haplotypes out of the 40 isolates. The two populations of Ségou and Bamako were genetically differentiated $(\mathrm{RST}=0.262, \mathrm{P}<0.001$ ). A greater diversity was observed in the population of the Bamako region compared to that of Ségou with an allelic richness of 2.96 and 2.07, respectively. A unique haplotype was shared by strains from Bamako and Ségou but strains from both regions are associated in different clonal complexes supporting epidemiological link between those two regions. New surveys carried out in 2016 in five production areas have made it possible to isolate 29 populations (corresponding to over $300 \mathrm{Xam}$ strains), whose Analysis of the genetic structure is in progress. The data collected will help to rationalize the use of casava variety grown in Mali.

Virulence characterization of Puccinia striiformis f. sp. tritici collections from China, Italy, Mexico, and Ecuador M. Wang PhD (1), A. Wan (2), M. Li (3), M. Maccaferri (4), P. Figueroa (5), C. W. Barnes (6), D. Campana (7), X. CHEN (8), (1) Washington Sate University, Pullman, WA, USA; (2) Washington State University, Pullman, WA, USA; (3) Institute of Agricultural Environment and Resources, Kunming, Yunnan, CHINA; (4) University of Bologna, Viale Fanin, ITALY; (5) Mexican National Institute for Agricultural Research, Obregon, MEXICO; (6) Instituto Nacional de Investigaciones Agropecuarias, Quito, Pichincha, ECUADOR; (7) INIAP Estación Experimental Santa Catalina, Quito, ECUADOR; (8) USDA ARS, Pullman, WA, USA

Puccinia striiformis f. sp. tritici (Pst) causing stripe rust is one of the most important pathogens on wheat worldwide. The fungus evolves into different virulent races to overcome resistance. In 2014-2017, 443 isolates were recovered from collections of China (141), Italy (133), Mexico (123), and Ecuador (46). These isolates were tested with 18 wheat $Y r$ single-gene lines that are used to differentiate Pst races in the U.S. A total of 132 Pst races were detected, including 105 new races. The number of unique races in China, Mexico, Italy, and Ecuador were 54, 25, 24, and 18, respectively. None of the races were shared by more than two countries, but 4 races were shared by China and Italy, 3 by China and Mexico, and 4 by Ecuador and Mexico. The top three Mexican races, PSTv-37 (40.7\%), PSTv-52 (17.1\%), and PSTv-198 (9.8), were similar to those identified in the US in 2016. Overall, virulences to $Y r 9, Y r 43, Y r E x p 2, Y r 6, Y r 7$, and $Y r 44$ were most frequent (81.0-90.3\%); those to $\operatorname{YrTr} 1, \operatorname{Yr} 8, \operatorname{Yr} 76, \operatorname{Yr} 27, \operatorname{Yr} 17, \mathrm{YrSP}$, and $Y r 1$ were moderate (17.8-59.6\%); and those to $\mathrm{Yr} 10, \mathrm{Yr} 32$, and $\mathrm{Yr} 24$ were low (0.5-1.1\%). None of the races were virulent on $\mathrm{Yr} 5$ and $\mathrm{Yr} 15$. Significant variations in frequency were detected for virulences to $\operatorname{Yr} 1$ (10.6\% in Mexico to $85.8 \%$ in China); $\operatorname{Yr} 17$ (11.3\% in China to $71.4 \%$ in Italy); $\operatorname{Yr} 27$ (3.0\% in Italy to $84.6 \%$ in Mexico); and $\operatorname{Yr} 76$ (6.5\% in Ecuador to $83.0 \%$ in China). The information is useful for understanding epidemiology and controlling stripe rust.

Population dynamics of the late blight pathogen in Canada for 2017

R. D. PETERS (1), K. I. Al-Mughrabi (2), F. Daayf (3), A. MacPhail (1), L. Kawchuk (4), (1) Agriculture and Agri-Food Canada, Charlottetown, PE, CANADA; (2) New Brunswick, Agriculture, Aquaculture \& Fisheries, Wicklow, NB, CANADA; (3) University of Manitoba, Winnipeg, MB, CANADA; (4) Agriculture \& Agri-food Canada, Lethbridge, AB, CANADA

Late blight was once again an important disease issue for both potato and tomato crops in Canada in 2017. Plant tissue samples infected with the late blight pathogen were received from several potato and tomato production regions in Canada. Using established protocols, Phytophthora infestans was isolated from these samples and assessed to determine genotype, mating type and metalaxyl-m sensitivity. Pathogen isolates collected from British Columbia potatoes were identified as the US-11 genotype, which is an A1 mating type and highly resistant to metalaxyl-m. Samples received from all other production regions, including Alberta, Manitoba, Ontario and New Brunswick yielded isolates of the US-23 genotype, which is an A1 mating type and sensitive to metalaxyl-m. Although isolates of US-23 were often sensitive to metalaxyl-m early in the season, increased resistance to this chemical pesticide was documented as the season progressed. Greenhouse/storage trials revealed that US-23 was less aggressive on potato foliage than US-8 or US-24, but equally aggressive as the other genotypes on potato tubers. The US-23 genotype has become the predominant genotype in most growing regions across Canada in recent years. However, every year there are fluctuations in the genotypes found, which underscores the importance of 
continuous population monitoring. The epidemiology of late blight has completely changed in Canada with the distribution and spread of new pathogenic strains.

Molecular characterization of Potato virus Y -NTN strain from India

A. Hamid (1), Y. ZHAI (1), S. V. Ramesh (2), H. R. Pappu (1), (1) Department of Plant Pathology, Washington State University, Pullman, WA, USA; (2) ICAR, Kasaragod, Kerala, INDIA

Potato virus $\mathrm{Y}(\mathrm{PVY})$ is a major threat to potato cultivation worldwide. PVY is a dynamic virus that exists as several distinct strains and cause varying degrees of pathogenicity and wide range of symptoms in potato. PVY strain characterization is essential for breeding programs. The complete genome of a potato isolate of PVY (JK12) isolated from the potato producing areas of Jammu and Kashmir, India was characterized. Nucleotide sequence comparisons and phylogenetic analysis with known PVY strains revealed that the isolate was an NTN strain of PVY. At the whole genome sequence level, the JK12 isolate shared the highest identity (99.42\%) with a PVY strain from Germany followed by the ones from the United Kingdom (99.34\%) and Japan (99.33\%). Recombination detection analysis identified two recombination break points and the isolate (JK12) appeared to have resulted from the recombination event between an PVY-N strain from Belgium as the major parent and a PVY-O strain from China as the minor parent. Mutation and recombination could be the basis for the emergence and subsequent establishment of NTN in this region. Furthermore, global evolutionary lineage analysis of all the known PVY strains revealed relatively low nucleotide diversity among the NTN strains. This is the first report of molecular characterization of an NTN strain of PVY from India.

\section{Genetic diversity of Puccinia striifomris f. sp. tritici from China and Sweden}

B. LIU (1), H. Zhang (1), A. Berlin (2), T. Liu (1), L. Gao (1), W. Chen (1), J. Yuen (3), (1) Institute of Plant Protection Chinese Academy of Agricultural Sciences, Beijing, CHINA; (2) Swedish Univ of Agricultural Sciences, Uppsala, SWEDEN; (3) Swedish University of Agricultural Sciences, Uppsala, SWEDEN

Wheat stripe rust, caused by obligate biotrophic fungus Puccinia striiformis f. sp. tritici (Pst), is considered one of the most destructive diseases of wheat worldwide. Since Berberis spp. grow in both China and Sweden, the importance of the alternate host for the genetic diversity of Pst in the two countries were compared. Single-uredinium isolates of Pst from Gansu, Sichuan, and Yunnan provinces of China and Klagstorp, Uppåkra, and Öland regions of Sweden sampled in 2015 were genotyped with 9 simple sequence repeat (SSR) markers. Multilocus genotypes (MLG) were identified and the genotypic diversity for the Chinese population was much higher than the Swedish population. Although not any MLG was common in both countries, a few MLGs from Sweden were closely related to some MLGs from Yunnan. All regional populations except Gansu were in linkage disequilibrium, indicating that only the Pst population in Gansu was likely reproduced sexually through alternate host plants.

How Andean Ralstonia solanacearum potato brown rot strains displace African brown rot strains in the Madagascar highlands A. TRUCHON (1), S. Ravelomanantsoa (2), P. C. Prior (3), C. Allen (1), (1) University of Wisconsin, Madison, WI, USA; (2) National Center for Applied Research on Rural Development - CENRADERU/FOFIFA, Antananarivo, MADAGASCAR; (3) INRA-CIRAD, Saint Pierre, La Réunion, FRANCE

Ralstonia solanacearum (Rs) causes potato brown rot (BR), a serious problem in tropical highlands worldwide. A 2009 survey in Madagascar revealed a widespread BR outbreak. Malagasy potato growers depend on this crop for sustainable food security. Epidemiological and phylogenetic analyses suggested that the recently introduced Andean phylotype II, sequevar 1 (Race 3 biovar 2) Rs strains spread quickly, displacing native African (Phylotype III) Rs strains. Andean strains are highly destructive because they disperse rapidly in potato seed tubers, but there is no published information on transmission of African BR strains. Understanding pathogen transmission is essential for effective disease management. Based on epidemiological data, we hypothesize that African BR strains may not be efficiently tuber transmitted. Using 11 diverse African BR isolates from Madagascar (phylotype III, sequevars 19 and 60) and the well characterized Andean Race 3 biovar 2 strain UW551, we performed comparative bioinformatic and biological analyses to identify differences between Andean and African $R s$ strains that infect potato. We sequenced genomes from one sequevar 19 and one sequevar 60 African strain, and compared their virulence on potato and tomato, as well as their tuber colonization and tuber transmission on potato. Biological differences between Andean and African BR strains may explain the displacement of African Rs strains by Andean strains in the Madagascar highlands.

An international perspective on genetic structure and gene flow in Cercospora beticola populations N. L. KNIGHT (1), N. Vaghefi (1), J. Kikkert (2), M. D. Bolton (3), G. A. Secor (4), L. Hanson (5), S. C. Nelson (6), S. J. Pethybridge (1), (1) Cornell University, Plant Pathology \& Plant-Microbe Biology Section, Geneva, NY, USA; (2) Cornell Cooperative Extension, Canandaigua, NY, USA; (3) USDA-ARS, Red River Valley Agricultural Research Center, Fargo, ND, USA; (4) Department of Plant Pathology, North Dakota State University, Fargo, ND, USA; (5) USDA-ARS, Sugar Beet and Bean Research Unit, Michigan State University, East Lansing, MI, USA; (6) Department of Tropical Plant and Soil Sciences, University of Hawaii, Honolulu, HI, USA

Cercospora leaf spot, caused by the fungus Cercospora beticola Sacc., is an important disease of Beta vulgaris L. (table beet, sugar beet, and Swiss chard) worldwide. Disease impacts include reductions in commodity grading, and quantity and quality of extractable sugars from sugar beet roots. Conidia of $C$. beticola disperse locally by water or wind to initiate and expand disease foci in fields. Mechanisms for long distance pathogen dispersal and epidemic initiation are largely unknown. Studies of populations from Western Europe, Iran, New Zealand, Turkey, and the USA reported high levels of genetic diversity. Moreover, in some populations, equal ratios of two mating types suggests an active, and potentially mobile, teleomorph. In Europe, long distance dispersal of $C$. beticola is implied by evidence of high levels of gene flow between isolates across the region, resulting in a single panmictic population. Furthermore, recurrent clonal lineages shared between the USA and Europe provided evidence for intercontinental genotype flow. The genetic relationships among C. beticola isolates from nine countries (Canada $(n=37)$, Chile $(n=28)$, Denmark $(n=9)$, England $(n=9)$, Germany $(\mathrm{n}=10)$, Italy $(\mathrm{n}=11)$, Sweden $(\mathrm{n}=8)$, Turkey $(\mathrm{n}=7)$, and four states in the USA $(\mathrm{n}=1073))$ were assessed using 12 microsatellite markers. This information will indicate the potential movement of $C$. beticola between regions and has implications for global disease management.

Phylogenetic, morphological, and pathogenic characterization of Alternaria species associated with fruit rot of mandarin in California F. WANG (1), S. Saito (2), T. J. Michailides (3), C. L. Xiao (4), (1) United States Department of Agriculture - Agricultural Research Service, Parlier, CA, USA; (2) USDA ARS, Parlier, CA, USA; (3) University of California - Davis, Parlier, CA, USA; (4) USDA-ARS, Parlier, CA, USA

Alternaria rot caused by Alternaria species is one of the major postharvest diseases of mandarin fruit in California. The aims of this study were to identify Alternaria species using phylogenetic analyses and morphological characteristics and test their pathogenicity to mandarin fruit. In 2015 and 
2016, decayed mandarin fruit with Alternaria rot symptoms were collected from different packinghouses in California and isolations of the putative fungi were made. In total, 177 Alternaria isolates were obtained from decayed fruit which were classified into three species, A. alternata, A. arborescens and A. tenuissima. The identification was based on DNA sequences of the second largest subunit of RNA polymerase II (RPB2), plasma membrane ATPase and Calmodulin gene regions in combination with morphological characters. Of the 177 isolates, 88 isolates (49.7\%) were identified as $A$. alternata, 53 isolates $(29.9 \%)$ were A. arborescens and 36 isolates $(20.3 \%)$ were A. tenuissima. Mycelial growth rate and sporulation varied among the selected isolates of the three species. Pathogenicity tests showed that all three Alternaria species were pathogenic on mandarin fruit at both $5^{\circ} \mathrm{C}$ and $20^{\circ} \mathrm{C}$. Our results indicate that three Alternaria species, A. alternata, A. arborescens, and A. tenuissima, were responsible for Alternaria rot of mandarin fruit in California with $A$. arborescens causing fruit rot on mandarin being reported for the first time.

Investigating the distribution and diversity of Leptosphaeria maculans in northern Idaho

J. PICKARD, J. B. Davis, J. Brown, K. L. Schroeder, University of Idaho, Moscow, ID, USA

Leptosphaeria maculans, the causal agent of blackleg in canola (Brassica napus L.), is a serious threat to oilseed production. Developing plant resistance to blackleg has been a major objective of many canola breeding programs. Previously overlooked in northern Idaho until 2011, blackleg has found a foothold among Brassica crops in this region. Blackleg poses a risk to the northern Idaho canola industry since virtually no selection has been previously carried out to identify disease resistance among locally adapted varieties. Although observed disease symptoms have been minor to date, with little progression into the upper canopy and very few stem cankers, the frequency of infection is widespread. In a survey of 46 canola fields in northern Idaho, 38 were found to display symptoms of blackleg. Isolates of L. maculans (130) and L. biglobosa (10) were recovered from these infected fields. Analysis of $L$. maculans isolates revealed an equal distribution of mating types, suggesting widespread distribution of ascospores and sexual recombination. Utilizing known plant resistance differentials and PCR, the diversity and distribution of avirulence genes was determined. Characterization of local blackleg races has allowed screening of the University of Idaho canola germplasm to find sources of genetic resistance that can be introgressed into high yielding canola lines with adaptation to northern Idaho environments.

Race structure and genetic diversity of the Pyrenophora tritici-repentis population in North Dakota Z. LIU (1), G. Ameen (2), J. Guo (1), (1) North Dakota State University, Fargo, ND, USA; (2) Department of Plant Pathology, North Dakota State University, Fargo, ND, USA

The fungus Pyrenophora tritici-repentis (Ptr) is the causal agent of tan spot, which is a major foliar disease of wheat (Triticum ssp.) worldwide. The fungal pathogen is known to produce three necrotrophic effectors (NEs), and eight races have been identified among globally collected isolates based on the production of different combinations of NEs. In this study, a total of 350 Ptr isolates collected from North Dakota (ND) in the last several years were characterized for race structure and genetic diversity. By using the ToxA and ToxB gene specific primers and greenhouse inoculations, race 1 isolates were found to be predominant $(93 \%)$ with a small percentage of race $2(5 \%)$ and race $4(2 \%)$ isolates. Our results are similar to the previous finding with a population collected in ND two decades ago. A subset of race 1 isolates from different years was analyzed with five microsatellite markers that are dispersed on different chromosomes of the fungal genome. A high level of genetic diversity was detected with each microsatellite marker amplifying an average of 8.4 alleles. This work indicates that the race structure of the Ptr population in ND is relatively stable over the years, but small variability in virulence could exist within race 1 due to the genetic diversity.

Increased aggressiveness of Fusarium pseudograminearum isolates causing crown rot disease on wheat in Western Australia M. KHUDHAIR (1), F. Obanor (2), L. F. Thatcher (3), D. Gardiner (4), K. Kazan (5), E. Aitken (6), (1) The university of Queensland/ CSIRO Agriculture and Food, Brisbane, AUSTRALIA; (2) The Grains Research and Development Corporation (GRDC), Canberra, AUSTRALIA; (3) CSIRO, Perth, AUSTRALIA; (4) CSIRO Agriculture and Food, Brisbane, AUSTRALIA; (5) CSIRO Agriculture and Food, St Lucia, Brisbane, AUSTRALIA; (6) School of Agriculture and Food Scineces, St Lucia, AUSTRALIA

Fusarium crown rot (FCR) caused by the fungal pathogen Fusarium pseudograminearum $(F p)$ is a chronic and serious disease of wheat and barley in many countries, including Australia, as it causes high yield losses and low grain quality. The incidence and severity of FCR in most wheat growing regions of Australia, and particularly in Western Australia (WA), have been increasing in the last decade. To investigate if $F p$ isolates are becoming more aggressive, a total of $103 \mathrm{Fp}$ isolates collected from two intensively sampled wheat fields in WA (Tammin and Karlgarin) in 2008 and 2015 were genetically analyzed. The aggressiveness of the isolates was also tested on seedlings of the susceptible wheat cultivar Mace using a paper-towel bioassay. Overall, the results revealed significant differences between the two sampling years within the two surveyed paddocks with the aggressiveness of isolates sampled in 2015 being significantly $(P<0.0001)$ higher than that of the 2008 isolates. This indicates a shift towards more aggressive isolates occurring within pathogen populations in WA. However, there was no significant $(P<0.5)$ difference in the aggressiveness of isolates from between the two paddocks sampled at either year. Increased aggressiveness of $F p$ isolates collected from the same locations in different years correlates with the recent increases of FCR incidence and severity in WA and this may have implications in managing this important wheat and barley disease.

Assessment of plant pathogenic fungal and oomycete communities in the soil of a long-term fertilization experiment L. SOONVALD (1), K. Loit (1), A. Astover (2), L. Tedersoo (3), (1) Estonian University of Life Sciences, Chair of Plant Health, Tartu, ESTONIA; (2) Estonian University of Life Sciences, Chair of Soil Science, Tartu, ESTONIA; (3) University of Tartu, Tartu, ESTONIA

Many diseases caused by plant pathogens are actually a complex assembly of different species. Therefore, it is important to study them not only on the single species level but also analyse their community structure. The objective of this study was to assess, how mineral and organic fertilization treatment and their application rates affect the pathogenic fungal and oomycete communities in soil. Hence, in 2014 soil samples were collected three times during the growing season from potato field grown in an experimental field for long-term fertilization experiment. Pathogen community composition was assessed by using PacBio SMRT sequencing of full ITS region. Previous studies, which have mainly focused on single pathogenic species, have suggested that organic fertilization treatment decreases the number of pathogenic organisms in the soil. In our study, pathogen richness decreased only substantially with time, while both treatment and fertilizer application rate didn't affect pathogen species richness. However, community composition analysis showed that treatment has the largest source of variation on pathogen communities in soil. Our results thus indicated that different fertilization treatments harbour different pathogen communities. Disease suppression could, therefore, act not only on single species-level but also on a communitylevel. 
When natives become invasive: Population genetic signatures following range expansion in members of thousand cankers disease complex R. O. Gazis (1), S. E. Everhart (2), A. Graves (3), Z. N. Kamvar (2), R. N. Trigiano (4), S. J. Seybold (5), D. HADZIABDIC (4), (1) University of Florida, Homestead, FL, USA; (2) University of Nebraska, Lincoln, NE, USA; (3) Forest Service Forest Health, New Mexico Zone, Albuquerque, NM, USA; (4) University of Tennessee, Knoxville, TN, USA; (5) USDA Forest Service, Pacific Southwest Research Station, Davis, CA, USA

Thousand Cankers Disease (TCD) of trees in the Juglandaceae is a consequence of interactions among a fungal pathogen, Geosmithia morbida, an insect vector, Pityophthorus juglandis, and susceptible plant hosts. In the past two decades, TCD has spread from the western to eastern USA, and has invaded the natural range of four North American species of Juglans. It has also spread to Italy where it affects native English walnut (J. regia). Due to the significant potential spread of the pathogen and/or vector, critical information is needed on the population structure of pathogen and vector, pathways for potential dispersal, and host-parasite co-adaptation. In this study, we elucidate diversity patterns and potential pathways of range expansion from the hypothesized center of origin (Arizona and New Mexico) and a secondary center of invasion (California). A total of 807 G. morbida and 1767 P. juglandis individuals from subpopulations in the western USA were genotyped using 16 and 15 species-specific microsatellite loci, respectively. Our preliminary results indicate generally high genetic diversity, presence of population structure, and evidence of gene flow among subpopulations. High levels of genetic diversity across all groups may be explained by human-mediated movement of infested plant material from multiple sources and on multiple occasions. Our findings support an earlier hypothesis that the pathogen and vector have long been associated in the southwestern USA.

Genetic diversity in Verticillium dahliae population from olive in Lebanon

W. HABIB (1), F. Baroudy (1), K. D. Puri (2), E. Gerges (1), C. Saab (1), K. V. Subbarao (3), F. Nigro (4), (1) Lebanese Agricultural Research Institute, Beirut, LEBANON; (2) University of California, Davis, Salinas, CA, USA; (3) University of California at Davis, c/o U.S. Agricultural Research Station, Salinas, CA, USA; (4) University of Bari, Bari, ITALY

The species Verticillium dahliae Kleb. is a soilborne ascomycete that causes vascular wilts on many plant species. In Lebanon, a high incidence of Verticillium wilt has been recently revealed on olive; however, little is known about the genetic diversity and pathogen population. A representative collection of $V$. dahliae isolates $(n=81)$ from olive was assessed for several phenotypic characters and was genotyped using polymorphic microsatellite loci. Isolates were grouped into three clusters by the UPGMA analyses: cluster I included 28 VCG4B isolates and one VCG2B isolate, all belonging to race 2 and the non-defoliating (ND) pathotype; cluster II included 7 VCG1A isolates belonging to defoliating (D) pathotype and race 2; and cluster III encompassed 43 isolates from VCG2A and two from VCG2B, all with the ND pathotype. Cluster II isolates were the most aggressive and caused defoliation on cotton plants, whereas cluster III isolates induced significantly milder symptoms than cluster I isolates. Furthermore, D isolates were distinct in culture (PDA) and grew faster than ND isolates, with a mean colony diameter of $63.2 \pm 1.7 \mathrm{~mm}$ compared to $39.6 \pm 0.6 \mathrm{~mm}$ after 21 days. The D pathotype was recorded in six orchards in Bekaa and South governates, and MAT1-2 idiomorph was detected in 79 isolates. This phenotypic and genetic characterization of $V$. dahliae population is crucial for the effective management of Verticillium wilt and olive breeding in Lebanon.

\section{Genetic diversity and virulence of Fusarium oxysporum f. sp. vasinfectum (FOV) races causing Fusarium wilt of cotton in the southeastern United States \\ H. HALPERN (1), M. T. Brewer (1), R. C. Kemerait (2), (1) University of Georgia, Athens, GA, USA; (2) University of Georgia, Tifton, GA, USA}

Fusarium oxysporum f. sp. vasinfectum (FOV), the fungus that causes Fusarium wilt of cotton (Gossypium spp.), has recently caused devastating outbreaks in the southeastern United States. FOV is genetically and pathogenically diverse, with six nominal races and eleven distinct genotypes based on sequences at the partial translation elongation factor $(E F-1 a)$ locus and intergenic spacer region (IGS). Some genotypes of FOV are more virulent on cotton than others, and some require plant pathogenic nematodes for virulence. Our research objectives are to: 1) understand how populations of FOV are evolving in the southeastern United States by determining how different genotypes of FOV are related, and 2) understand the relationship between FOV genotype and disease phenotype. We used genotyping-by-sequencing (GBS) to identify SNP variants across 160 genetically, geographically, and temporally diverse FOV isolates. Unique SNP genotypes were used to construct a maximum likelihood tree and to perform K-means hierarchical clustering. We also conducted greenhouse assays to test for differences in virulence among genotypes. Forty-five genetically diverse isolates were inoculated onto three cotton cultivars with differing susceptibilities to FOV. Our results show that there is high genetic diversity in FOV, and some clustering based on previously described races. Additionally, some genotypes of FOV are associated with distinct disease phenotypes on cotton.

Epidemiology and pathogen diversity of Xanthomonas campestris pv. campestris in New York

H. W. LANGE (1), M. Tancos (2), C. D. Smart (3), (1) SIPS, Cornell University, Geneva, NY, USA; (2) USDA ARS FDWSRU, Frederick, MD, USA;

(3) Plant Pathology and Plant-Microbe Biology Section, Cornell University, Geneva, NY, USA

Black rot caused by the bacterium Xanthomonas campestris pv. campestris (Xcc) is often a serious disease of crucifers in New York, impacting yield and storage quality. We have followed the pathogen population in New York to determine its diversity, if it overwinters in weeds or crop residue, which control methods are most efficacious, and how currently grown cultivars differ in susceptibility over diverse seasonal weather patterns. Over 300 isolates have been collected, from 2004-2017, and tested for pathogenicity on cabbage as well as examined for diversity via multi-locus sequence analysis. Studies to identify the frequency of Xcc overwintering in NY and starting epidemics the following year have also been performed. Our findings show that new strains of Xcc enter NY each year, and while weeds can be hosts of Xcc, they are not a major source of inoculum. Copper-based products and plant activators are effective in slowing disease spread in the greenhouse and field. Additionally, resistant cultivars that were tested held up over cool/wet or hot/dry seasons with warmer seasons being more conducive to infection. Increased knowledge of the epidemiology, population diversity, and products that control this bacterium have improved management strategies for growers and identified future areas for research.

\footnotetext{
Relationships among Brazilian and worldwide isolates of Fusarium oxysporum $\mathrm{f}$. sp. lactucae race 1 inferred from IGS-rDNA region and $E F$-1a gene

C. CABRAL (1), M. E. D. N. Fonseca (2), K. R. Brunelli (3), M. Rossato (4), H. Costa (5), L. S. Boiteux (6), A. Reis (6), (1) Embrapa Hortaliças, Brasília-DF, BRAZIL; (2) Embrapa Hortaliças, Brasília, BRAZIL; (3) Sakata, Bragança Paulista-SP, BRAZIL; (4) UPIS, Brasília-DF, BRAZIL; (5) Incaper, Vitória-ES, BRAZIL; (6) Embrapa Hortaliças, Brasilia, BRAZIL
}

Fusarium wilt (Fusarium oxysporum f. sp. lactucae - FOLac), is one of the main lettuce diseases in subtropical regions. Although nationwide surveys indicated the exclusive presence of FOLac race 1 in Brazil, no detailed studies are available providing molecular evidences if these isolates were introduced into the country via contaminated seeds or if they are endemic populations. The translation elongation factor $1 \alpha(E F-1 \alpha)$ gene and rDNA intergenic spacer (IGS-rDNA) region are the most comprehensive databases for comparative analyses of Fusarium isolates. The objective of this work was to assess the genetic relationships of 23 Brazilian FOLac race 1 isolates with isolates of worldwide origin. A consistent single-cluster pattern was observed for FOLac race 1 isolates from Brazil, California-USA, Arizona-USA, Japan, and Italy, as well as the novel race 4 isolates from the 
Netherlands. Our analysis (based upon six single nucleotide polymorphisms identified only in the IGS-rDNA sequence) allowed the identification of intra-race 1 variation with the discrimination of four haplotytes. The low diversity levels and the presence of only a single haplotype across Brazil are strong indications that Brazilian FOLac race 1 isolates are result of recent introduction event(s).

Genetic variation and differentiation in global populations of the wheat leaf rust fungus, Puccinia triticina

J. A. KOLMER (1), M. E. Ordonez (2), S. German (3), K. Xiao (4), A. Fox (4), M. Acevedo (5), (1) USDA ARS, St Paul, MN, USA; (2) Universidad Catolica del Ecuador, Quito, ECUADOR; (3) National Institute for Agricultural Research (INIA), Colonia, URUGUAY; (4) USDA-ARS, St Paul, MN, USA; (5) Cornell University, Ithaca, NY, USA

The leaf rust pathogen, Puccinia triticina is common across all major wheat growing regions worldwide. Collections of $P$. triticina were obtained from common and durum wheat in North America, South America, Europe, South Africa, the Middle East, East Africa, Russia, Central Asia, China, Pakistan and New Zealand in order to determine the genetic diversity within each region and genetic relationship between regions. A total of 831 single uredinial isolates were characterized for virulence to isogenic lines of Thatcher wheat and for molecular genotype at 23 SSR loci. The isolates in East Africa and Europe were the most diverse for the average number of effective alleles per locus, while the populations in Russia and North America were the least diverse. The isolates in Europe and South America had the highest number of multilocus genotypes of 81 and 75, respectively, and were the most diverse for Shannon's genotypic diversity. All populations had significantly higher levels of $H_{o}$ compared to $H_{e}$ at individual SSR loci, and had highly significant values of $I_{\mathrm{a}}$ and $\mathrm{r}_{\mathrm{d}}$ which indicated clonal reproduction. Europe had the highest number of distinct SSR genotype groups with eight, and Russia had only two SSR groups. The populations in North America and South America; Russia and Central Asia; the Middle East and East Africa; were closely related for SSR genotype based on Nei's genetic distance. Based on $k$ means clustering and DAPC of SSR genotypes, isolates virulent to durum wheat were placed into a single separate genetic group, and isolates virulent to common wheat were placed into five groups. Twenty-seven SSR genotypes that were found in different continental regions, also had identical or similar virulence, which indicated historical and current migration of $P$. triticina worldwide.

Population structure of Phytophthora plurivora on Rhododendron in Oregon nurseries

N. CARLESON (1), V. Fieland (2), J. Tabima (1), J. E. Weiland (3), C. Scagel (4), N. J. Grunwald (4), (1) Oregon State University, Corvallis, OR, USA; (2) OREGON STATE UNIVERSITY, Corvallis, OR, USA; (3) USDA-ARS, Horticultural Crops Research Unit, Corvallis, OR, USA; (4) USDA ARS, Corvallis, OR, USA

Phytophthora plurivora is a recently described plant pathogen, separated from the Phytophthora citricola species complex in 2009. Recent studies of Pacific Northwest nurseries have frequently encountered the pathogen, and it has been shown to be among the most dominant species in Phytophthora communities, however, not much is known about its population genetics. We characterized the population structure of $P$. plurivora $(\mathrm{n}=70)$ in a survey of four Oregon nurseries across three different counties with focus on Rhododendron hosts. Samples were identified to the species level using a PCRRFLP assay of the internal transcribed spacer (ITS) region. We used genotyping-by-sequencing (GBS) by digesting with a PstI-MspI enzyme combination and sequencing on an Illumina HiSeq 3000. Variants were called de novo using the Stacks pipeline, resulting in 547 high-quality variants and 69 samples after filtering for low read depth. Based on $F_{\mathrm{st}}$ and discriminant analysis of principal components (DAPC), populations were moderately differentiated among nurseries. P. plurivora was previously assumed to primarily reproduce clonally, and the observed low genotypic diversity within nurseries corroborated this hypothesis. These findings provide an initial perspective on P. plurivora population structure in Pacific Northwest nurseries.

Single-pustule inoculation to examine diversity of the Brazilian orange rust pathogen from various origins and uredia L. PORTO (1), R. S. Arias (2), A. Seiiti Urashima (3), (1) Federal University of Sao Carlos, Araras, BRAZIL; (2) U.S. Department of Agriculture, Tilton, GA, USA; (3) Federal University of São Carlos, Araras, BRAZIL

The orange rust of sugarcane (Puccinia kuehnii) has caused elimination of important cultivars in Brazil since the main strategy of control is through resistant genotypes. The effectiveness of this measure is dependent on the knowledge of pathogen diversity. Nevertheless, the few studies in this area employed fungi collected from multiple lesions. In this process, variants present at lower frequency may not be detected. Therefore, the present study aimed to employ single uredium to examine diversity of $P$. kuehnii from different cultivars as well as among uredia from the same leaf. A total of 285 single uredium from three susceptible cultivars were inoculated on four cultivars and lesion area measured by Assess software, 21 days after inoculation. Genetic variants among uredinium DNA of 24 pustules from the same leaf were genotyped with four SSR markers. Phenotypic data, based on diseased area, identified different variants ranging from 3.12 to $6.45 \%$ in these inoculum sources demonstrating that diverse pathotypes of $P$. kuehnii were affecting each of the susceptible cultivars. Genotypic variation were observed among uredia of the same leaf when using SSRs. Therefore, single-pustule inoculation proved to be effective in detecting variants of the pathogen, even present at very low frequency. Grant: FAPESP (2017/14351-0)

Population structure of Tomato chlorotic spot virus, an emerging tospovirus of tomato and other vegetable plants in the United States S. Zhang, B. POUDEL, University of Florida, Homestead, FL, USA

Tomato chlorotic spot virus (TCSV) is an emerging tospovirus and has established in south Florida. TCSV was first detected in tomato and bell pepper in south Florida in 2012. It has caused significant losses to tomato and bell pepper growers in the region since 2014. Subsequently, TCSV was confirmed in tomato in Ohio in 2013 and New York in 2017. In Homestead, FL, TCSV has been detected in common bean and some weed hosts in vegetable fields in 2017. Under field conditions, TCSV is efficiently transmitted by flower thrips. Virus infection results in severe stunting and eventually death of the plants when plants are infected at an early stage. Whereas, the pants infected by the virus at later stage develop leaf distortion, chlorotic spots, and fruits also develop chlorotic spots making them unsuitable for marketing. Survey of tomato fields conducted in Miami-Dade County, Florida from 2016 to 2017 indicated that TCSV is the most dominant tospovirus in south Florida. Initial and preliminary analysis of sequence data suggested sequence diversity between tomato and pepper isolates of TCSV. So as to understand the population structure and evolutionary aspect of TCSV, more than thirty isolates of TCSV different fields of tomato in Homestead, FL were collected and utilized for genetic diversity. Approximately $30 \%$ of the virus genome was sequenced, analyzed for studying genetic diversity, and protein coding areas were examined for positive/negative selection. Further bioinformatics analysis is being performed to determine if there is any recombination occurring in TCSV population.

Phytophthora species introduced to Southern California restoration plantings on the Angeles National Forest threaten rare species habitat S. J. FRANKEL (1), T. J. Swiecki (2), E. A. Bernhardt (2), C. L. Blomquist (3), S. N. Rooney-Latham (3), K. VinZant (4), (1) USDA Forest Service, Pacific Southwest Research Station, Albany, CA, USA; (2) Phytosphere Research, Vacaville, CA, USA; (3) California Dept of Food \& Agric, Sacramento, CA, USA; (4) USDA Forest Service, Coronado National Forest, Tucson, AZ, USA 
Over the past several years, more than 40 Phytophthora species have been recovered from native plant nursery stock in northern California habitat restoration plantings. These unintentional introductions of $P$. tentaculata, $P$. cactorum, and other species, which were extensive in several cases, potentially undermine the very purpose of the restoration projects because they degrade rather than enhance habitat. In 2016 - 2017, the Angeles National Forest in Southern California conducted an assessment to determine if Phytophthora species were present in restoration areas planted to mitigate habitat loss along utility corridors. Baiting with green pears was used to detect Phytophthora from sampled roots of symptomatic (dead, declining, stunted, or off-color) nursery stock planted at fifteen restoration sites. All locations had plants associated with Phytophthora. Numerous Phytophthora species, including two likely novel or hybrid species, were identified via DNA sequencing. At four source native plant nurseries, groups of container plants grown for restoration were tested by baiting irrigation leachate with green pears. Four Phytophthora species were recovered, three of which were also detected in the field sites. Some of the Phytophthora recoveries were from nursery plants without obvious shoot symptoms. Restoration areas are conservation investments, and these results confirm that infested nursery stock has the potential not only to interfere with restoration success, but to introduce plant pathogens into the wildlands of southern California. Preventive measures are needed to sustain plant health, including production of healthy nursery stock using strict phytosanitary measures, or direct seeding and other changes to restoration practices.

\section{AmpSeq based SNP marker development for population analysis of geographically diverse isolates of hop powdery mildew (Podosphaera macularis)}

W. WELDON (1), L. E. Cadle-Davidson (2), D. H. Gent (3), S. Wolfenbarger (4), B. J. Knaus (5), N. J. Grunwald (6), D. M. Gadoury (1), (1) Cornell University, Geneva, NY, USA; (2) USDA Grape Genetics Research Unit, Geneva, NY, USA; (3) US Department of Agriculture, Agricultural Research Service, Corvallis, OR, USA; (4) Oregon State University, Department of Botany and Plant Pathology, Corvallis, OR, USA; (5) USDA-ARS, Horticultural Crops Research Unit, Corvallis, OR, USA; (6) USDA ARS, Corvallis, OR, USA

The geographic distribution of powdery mildew of hop (Podosphaera macularis) spans all hop growing regions of the northern hemisphere. Notable differences in genetic diversity, mating type, and virulence have been observed across the continental US and Europe. Analysis of structure within populations, or across spatial distributions, typically requires genetic markers such as single nucleotide polymorphisms (SNPs) to characterize individual isolates. The P. macularis 'HPM-663 reference' transcriptome was recently sequenced and assembled de novo. RNA-seq libraries from 87 geographically diverse $P$. macularis isolates were mapped against this reference to generate an initial set of SNPs. Only 196 unique transcripts SNPs shared by at least two samples. Amplicon Sequencing (AmpSeq) primers were designed with the specifications: 1) the amplicon containing each variant should be 240 to 280 base pairs (bp) in length, ideally $260 \mathrm{bp} ; 2$ ) the SNP site should be at least $30 \mathrm{bp}$ from the end of the amplicon; 3) the amplicons may span multiple variants; and 4) the AmpSeq primers should bind sites with minimal homology to all other primer binding sites. Under these conditions, 166 transcripts (84.6\%) were suitable for designing AmpSeq primers to genotype P. macularis populations. This concept of designing markers spanning divergent regions of transcripts provides a promising marker generation approach transferable across diverse species.

\section{Botryosphaeriaceae diversity on Acacia koa and A. heterophylla in Reunion and Hawaiian Islands} F. JAMI (1), S. Marincowitz (1), P. W. Crous (2), B. Slippers (1), J. J. Le Roux (3), D. M. Richardson (3), M. J. Wingfield (1), (1) Forestry and Agricultural Biotechnology Institute (FABI), University of Pretoria, Pretoria, SOUTH AFRICA; (2) Westerdijk Fungal Biodiversity Institute, Utrecht, NETHERLANDS; (3) Department of Botany and Zoology, Centre for Invasion Biology, Stellenbosch University, Stellenbosch, SOUTH AFRICA

Acacia koa and A. heterophylla are commonly occurring native trees on the islands of Hawaii and Reunion, respectively. A recent study suggested that these trees are the same species, separated geographically for more than 1.4 million years. An intriguing question is whether their microbiota is the same, although, they are growing in two different geographical locations. In this study we compared fungi in the Botryosphaeriaceae from these trees. Isolates were identified based on comparisons of sequence data for the rDNA-ITS, LSU, TEF1- $\alpha$ and $\beta$-tubulin loci. In total, ten Botryosphaeriaceae species were identified of which five species were specific to samples from Hawaii and four species to Reunion, with one species common to both islands. The common species, Neofusicoccum parvum, is known to have a wide global distribution and the overall results suggest that $A$. koa and $A$. heterophylla have unique fungal biota in the areas where they occur naturally. Although the trees are genetically very similar (A. heterophylla renders $A$. koa paraphyletic), the results of this preliminary study suggest that they have established unique and independent microbiota.

\section{Diversity of Botryosphaeriaceae on Proteaceae in South Africa, Australia and Portugal}

F. JAMI (1), D. Migliorini (2,3), T. I. Burgess (4), B. Slippers (1), P. W. Crous (5), M. J. Wingfield (1), (1) Forestry and Agricultural Biotechnology Institute (FABI), University of Pretoria, Pretoria, SOUTH AFRICA; (2) Forestry and Agricultural Biotechnology Institute, University of Pretoria, Pretoria, SOUTH AFRICA; (3) National Research Council, Institute for Sustainable Plant Protection, Sesto Fiorentino, Florence, ITALY; (4) Centre of Excellence for Climate Change, Woodland and Forest Health, Murdoch University, Perth, AUSTRALIA; (5) Westerdijk Fungal Biodiversity Institute, Utrecht, NETHERLANDS

The Botryosphaeriaceae is an important and diverse family of latent fungal pathogens of woody plants. Because some species appear to have a worldwide distribution, they are increasingly being used as model organisms to understand patterns in the global movement of latent pathogens. The aim of this study was to consider the diversity of Botryosphaeriaceae on 14 native Proteaceae species across South Africa and Australia, as well as on South African plants grown in Portugal. Ten species of the Botryosphaeriaceae were identified from 200 isolates using sequence data of the ITS rDNA, TEF-1 $\alpha$ and $\beta$-tubulin loci. Surprisingly, Neofusicoccum parvum was found only from Portugal materials, despite its global distribution and common occurrence in Australia and South Africa on the other hosts. Neofusicoccum protearum and N. australe were the only shared species in Australia, South Africa and Portugal, while Diplodia allocellula was found in Australia and South Africa. The other six species were obtained from a single country and include $D$. mutila, D. alatafructa and N. macroclavatum in Australia, and D. pseudoseriata, N. viticlavatum and N. cryptoaustrale in South Africa. The results of this study show that the Botryosphaeriaceae diversity on the Proteaceae is geographically distinct, but there is also evidence for specialist species, such as $N$. protearum, that have been transported globally with plant material.

\section{Diversity of "Candidatus Liberibacter asiaticus" strains in California}

Z. Dai (1), F. Wu (2), Z. Zheng (2), R. K. Yokomi (1), L. Kumagai (3), W. Cai (4), J. Rascoe (4), M. Polek (5), X. Deng (2), J. CHEN (1), (1) USDA, ARS, SJVASC, Parlier, CA, USA; (2) South China Agricultural University, Guangzhou, CHINA; (3) California Dept of Food \& Agriculture, Sacramento, CA, USA; (4) USDA-APHIS-PPQ-S\&T-CPHST, Beltsville, MD, USA; (5) USDA ARS, Riverside, CA, USA

Citrus Huanglongbing (HLB) is a highly destructive disease and associated with a non-culturable bacterium, "Candidatus Liberibacter asiaticus" (CLas). Characterization of CLas strains is critical for HLB management. HLB was found in Florida in 2005 and now is endemic there. In California, CLas was first detected in Los Angeles County in 2012. Since then, the bacterium has been detected in multiple urban locations in southern California. Little is known about the diversity and biology of these $C$ Las strains. In this study, nine CLas strains from six southern California cities were analyzed targeting chromosomal loci and prophage regions based on next generation sequencing (Illumina MiSeq and HiSeq). No variation was detected in 16S rRNA and 
$n r d \mathrm{~B}$ gene sequences. All California CLas strains were of Asiatic origin, rather than Florida, based on ter $\mathrm{L}$ gene grouping. Single Type 1 prophages were found in CLas strains from Anaheim, Cerritos, San Gabriel, and Riverside; Single Type 2 prophage was found in a Hacienda Heights strain; Type 1 and 3 prophages were found in a Cerritos strain, and Type 1 and 2 prophages were found in a La Habra strain. All prophages harbored Miniature InvertedRepeat Transposable Elements (MITEs) with Type B MITEs associated to Type 1 and Type 3 prophages and Type A MITEs associated with Type 2 prophages. This information provides data useful to track the origin and pathway of $C$ Las strains and is critical for the management of the disease.

Deconstructing Australian Fusarium oxysporum species complex using genealogical concordance phylogenetic species recognition S. ACHARI (1,2), B. A. Summerell (3), J. Edwards (1,2), J. Kaur (1), T. Sawbridge (1,2), R. Mann (1), Q. Dinh (1), (1) AgriBio, Bundoora, AUSTRALIA; (2) LaTrobe University, Bundoora, AUSTRALIA; (3) Royal Botanic Gardens and Domain Trust, Sydney, AUSTRALIA

Fusarium oxysporum is an important plant and human pathogen which is readily isolated from agroecosystem and the natural ecosystem soils. Studies have indicated that it is a species complex with many phylogenetic lineages comprised of pathogenic and putatively non-virulent strains. Earlier phylogenetic analysis of Australian isolates using Genealogical Concordance Phylogenetic Species Recognition had identified seven independent evolutionary lineages which delineated into three major clades with two phylogenetic "species". This study was carried out using the barcoding regions of eight nuclear and mitochondrial genes. A recent study using complete protein coding nuclear genes and complete mitochondrial genome has identified three clades in the complex representing three phylogenetic species. The current study intends to revisit the phylogeny of the Australian $F$. oxysporum species complex using complete nuclear gene sequences and complete mitochondrial genome. It will also analyse the position of the Australian isolates in the already identified clades and phylogenetic species. Strains from both the agroecosystem and natural ecosystem will be used for the phylogenetic analysis. Knowledge of the phylogenetic lineages from this study will be important in understanding the limits of genetic exchange, the evolutionary potential and most importantly the population diversity of the Australian F. oxysporum species complex. Such information is important for disease management strategies.

Current diversity of Phytophthora infestans infecting cultivated potato in the Peruvian Andes

A. ASTETE FARFAN (1), W. Perez (2), S. Gamboa (3), H. Lindqvist-Kreuze (4), (1) Universidad Nacional San Antonio Abad del Cusco, Cusco, PERU; (2) International Potato Center, Lima, PERU; (3) International Potato Center, Lima 12, PERU; (4) International Potato Center (CIP), Lima, PERU

Late blight caused by $P$. infestans is the most devastating disease of potato worldwide. To understand the current $P$. infestans population structure in the Peruvian Andes, 701 single-lesion samples were collected from cultivated potato in different agroecological zones at 2016-2017. Genetic diversity was analyzed using the 12-plex SSR markers kit and mtADN. Phenotypic diversity was characterized for mating type, metalaxyl resistance and virulence. SSR analysis showed that population consists of EC-1 and PE-3 clonal lineages variants, that have the mtADN IIa and Ia, respectively. All isolates were A1 mating type. $94 \%$ of the EC-1 variant isolates were resistant to metalaxil, whereas the sensitive proportion (47\%) in the PE-3 variants was bigger than the resistant (35\%). Furthermore, virulence assays detected 41 complex physiological races from 142 selected isolates, the most frequent physiological race was 1,3,4,7,10,11 in both EC-1 and PE-3 variants. Phylogenetic analysis evidenced that isolates share identical SSR genotypes and physiological races in distant regions, that suggests that migration could have occurred. Additionally, no detection of A2 mating type indicate that population reproduction must be asexual. EC-1 clonal lineage continues to dominate across the Peruvian Andes, since $91 \%$ of the isolates were variants of this clonal lineage. The US-1 clonal lineage reported at 2001, appears to be displaced as it was no longer detected infecting cultivated potato.

Genetic and genomic studies of the Korean oak wilt fungus (Raffaelea quercus-mongolicae) provide information of its biology and ecology M. S. KIM (1), H. W. Park (1), J. Jeon (2), Y. H. Lee (2), J. R. Ibarra Caballero (3), J. E. Stewart (3), N. B. Klopfenstein (4), (1) Dept. of Forestry, Environment and Systems, Kookmin University, Seoul, SOUTH KOREA; (2) Seoul National University, Seoul, SOUTH KOREA; (3) Department of Bioagricultural Sciences and Pest Management, Colorado State University, Fort Collins, CO, USA; (4) Rocky Mountain Research Station, USDA Forest Service, Moscow, ID, USA

Raffaelea quercus-mongolicae is associated with oak wilt disease in South Korea, and this ambrosia beetle-vectored fungus has thus far only been found in South Korea. Mongolian oak (Quercus mongolica) is the primary host of R. quercus-mongolicae, and it is widely distributed over central South Korea where it is ecologically important in native forests. The objectives of this study on R. quercus-mongolicae were to assess genetic diversity and population structure using Restriction-site-Associated-DNA sequencing (RAD-seq), sequence the whole genome, and analyze the transcriptome under in vitro conditions. Results of RAD-seq indicated low genetic diversity and no apparent population structure among South Korean populations of $R$. quercusmongolicae, which supports the hypothesis that this fungus was introduced to South Korea. The genomic sequence of $R$. quercus-mongolicae (KACC44405; 27-Mb), along with other Raffaelea spp., will provide valuable resources for comparative genomic analyses and identifying genes that contribute to potential pathogenic relationships between the fungus and host, and potential symbiotic relationships between the fungus and insect vector. Transcriptomic analyses showed that many gene products were predicted to have diverse functions, such as ATP production for growth, recovery under stressed conditions, fluidity of cell membrane, maintenance of cell membrane, and regulation of fungal virulence.

Diversity of Pectobacterium spp. Isolated from Potato (Solanum tuberosum L.) on South Korea

S. JEE (1), G. R. Bak (2), J. G. Choi (2), M. Kwon (2), (1) Rural development administration, Pyeongchang, KOREA; (2) Rural Development Administration, Pyeongchang, KOREA

During 15 years, Pectobacterium species has been changed in classification and new species were suggested. Pectobacterium carotovorum subsp. carotovorum $(\mathrm{Pcc})$ had been studied as main soft rot pathogen in South Korea. We tried to know diversity of soft rot pathogens which are distributed in South Korea with new criteria. Including former collection, 235 strain were collected from potato cultivation area during 2 years, 2016-2017. Checked pathogenicity on potato slice, all collection were cultivated on crystal violet pectate (CVP) medium in three temperature. This collection were classified by $16 \mathrm{~s}$ rDNA sequence and rec A sequence. Isolated from potato, strains showed stronger pectinase activity and better tolerance in high temperature than others which were isolated from Kimchi cabbage and radish. Dickeya genus and Pectobacterium species except Pectobacterium carotovorum (Pc) subspecies were not identified in this collection. Pc subspecies were main pathogen of potato soft rot in South Korea. Pcc constitutes $47 \%$ of the collection, and Pectobacterium carotovorum subsp. brasiliense (Pcb) were $27 \%$. We suggest that Clade I is able to be new Pc subspecies.

Identification and race characterization of melon powdery mildew pathogen in Bukidnon, Philippines M. I. PINOTE, East-West Seed Company, Manolo Fortich, PHILIPPINES 
Powdery mildew is one of the most important diseases affecting melon worldwide. The disease is caused by two genera of obligate fungi: Podosphaera, which is common in subtropical and tropical areas in greenhouse crops and Golovinomyces, which occurs more frequently in temperate and cooler areas under field conditions. Both species are highly variable, as indicated by the existence of two races of Golovinomyces and 31 races of Podosphaeria In the Philippines, only Erysiphe cichoracearum syn Golovinomyces cichoracearum has been reported as the causal pathogen of melon powdery mildew. To establish the correct identity of the melon powdery mildew, identification and race characterization study using 19 melon cultivars was conducted. Based on the presence of the fibrosin bodies, shape, dimensions and length to width ratio of the conidia, melon powdery mildew was identified as Oidium sp., the anamorph of Podosphaera. Race characterization study revealed the presence of race N1 in greenhouse cultivated melon. Furthermore, no race shift of melon powdery mildew has been observed from 2013 to 2014 in East-West Seed Company research farm in Bukidnon, Philippines. The information generated from this study will be useful in breeding for powdery mildew resistance in melon.

The study on the pathogenic fungal diversity on chili pepper and its potential influencial factors

Y. DIAO (1), C. Lei (2), (1) State Key Laboratory of Mycology, Institute of Microbiology, Chinese Academy of Sciences, Chaoyang Beijing, CHINA; (2) State Key Laboratory of Mycology, Institute of Microbiology, Chinese Academy of Sciences, Beijing, CHINA

As an important vegetable crop, Chili (Capsicum spp.) is planted in the world and its production has always been affected by fungal diseases. Although lots of fungicides have been applied in controlling the diseases, they can still bring $20-50 \%$ losses to chili production every year. The lack of understanding of the pathogens affects the choice of suitable fungicides and diseases control. Therefore to achieve better control of fungal diseases in chili peppers, it's essential to reveal the pathogenic fungal diversity. In this study, more than 2000 isolates were collected from diseased chili fruits in 19 countries and further identified by sequencing and morphology observation. The result showed that the main pathogen genera were Alternaria, Colletotrichum and Fusarium. The correlation between fungal diversity and influence factors (geography, chili varieties and capsaicin) were analyzed. The result revealed that chili samples with higher capsaicin have lower fungal diversity, while samples from closer countries harbored more similar fungal communities. Compared to chili varieties, geographic distance and capsaicin played more important role in fungal diversity on chili pepper fruits. Furthermore, we also analyzed the fungal diversity on chili pepper in China between 2015 and 2016. Our result showed that the main diseases were black spot disease, anthracnose disease and rot disease, which are similar to the fungus in other countries in this study, and the main pathogens were Alternaria alternata, Colletotrichum scovillei and C. truncatum. Fungal diversity between southern and northern China showed significant differentiation, and southern regions owned higher fungal diversity than northern regions.

\section{Diversity of Venturia inaequalis in Latvia}

O. SOKOLOVA, I. Morocko-Bicevska, Institute of Horticulture, Dobele, LATVIA

The apple scab, caused by an ascomycetous fungus Venturia inaequalis (Cooke) G. Wint., is one of the most important apple diseases worldwide. Due to the $V$. inaequalis life cycle, in each spring plants are infected by the newly released ascospores representing new genotypes and therefore ensuring the high potential for genetic diversity and adaptation ability of the pathogen. $200 \mathrm{~V}$. inaequalis isolates from 27 apple genotypes from Latvia, and other countries were selected and genotyped using simple sequence repeat (SSR) markers to study genetic diversity of $V$. inaequalis in Latvia. Thirty-one published primer pairs of SSR were screened, and 14 primer pairs that were amplifying one fragment and showing differences of fragment lengths were used for genotyping. The length of amplified fragments was detected with an automated genetic analyzer and obtained data were combined into one single binary data matrix. The data were analysed by cluster analysis (UPGMA) and Principal Component Analysis (PCA) with computer program "Past" in respect to the host genotype, orchard and geographic region. Two main separate groups were identified among Latvian origin isolates according to the region. Some of the Latvian $V$. inaequalis isolates showed genetic similarity to isolates virulent on scab-resistant cultivars from other European countries indicating their potential for further virulence development

Genotypic diversity and reproductive biology of Thekopsora areolata, the causal agent of cherry spruce rust in Norway spruce seed orchards H. Capador, B. Samils, Å. OLSON, Swedish University of Agricultural Sciences, Uppsala, SWEDEN

Swedish forestry is sustained by yearly planting of about 200 million Norway spruce seedlings, produced in seed orchards planted with superior trees. The seed orchards are intensively managed to produce large quantities of high quality seeds. However, today there are a deficit of plants originating from Swedish seed orchards mainly due to irregular flowering as well as to pest and pathogen infections. The fungal pathogen Thekopsora areolata, causal agent of cherry spruce rust, heavily affects Norway spruce seed production. Using newly developed microsatellite markers and a hierarchical sampling strategy, population structure and reproductive mode of T. areolata were investigated. From one location in Norway, two locations in Finland and five locations in Sweden, one aecium per infected cone was analysed. In addition, multiple aecia per scale and cone were sampled at two locations in Sweden The results show an over-all high genetic diversity in $T$. areolata at all hierarchical levels with no genetic structure, indicating high gene flow and random mating. At the cone/scale level non-random mating was observed, for which several distinct hypotheses will be presented. Future research directions and management implications will be discussed.

Genetic diversity analyses in Ralstonia syzygii species

I. SAFNI (1), M. Fegan (2), L. Sly (2), U. Kappler (2), (1) University of Sumatera Utara, Medan, INDONESIA; (2) University of Queensland, St Lucia Brisbane, AUSTRALIA

Recently, the taxonomy of the Ralstonia solanacearum species complex has been revised into three genomic species, including Ralstonia solanacearum, Ralstonia pseudosolanacearum and Ralstonia syzgii on the basis of a polyphasic taxonomy approach with an emphasis on DNA-DNA hybridization and whole genome sequences. Based on some differences of phenotypic and chemotaxonomic analyses, the species of $R$. syzgii is further subdivided into three subspecies, including Ralstonia syzygii subsp. syzygii, R. syzygii subsp. indonesiensis, and R. syzygii subsp. celebesensis. This study utilized genomic fingerprinting Rep-PCR approach (BOX-and ERIC PCR) and Multilocus Sequence Typing (MLST) to analyze the genetic diversity of 27 strains of $R$. syzygii and 4 representative strains of $R$. syzygii subsp. celebesensis respectively. Strains of $R$. syzygii were found highly diverse based on Rep-PCR results. All R. syzygii subsp. celebesensis strains tested with the exception of one strain (UQRS 633), which had distinct band profiles were confirmed as a homogenous species within the $R$. solanacearum species complex by the consistent similarity of their band patterns in rep-PCR experiments. In contrast, using four housekeeping genes ( $a d k, g a p A, g d h A$ and $p p s A)$ and one megaplasmid gene ( $h r p B)$ in MLST analysis, four selected strains of $R$. syzygii subsp. celebesensis showed homogeneity.

Molecular characterization of Colletotrichum spp. isolates associated with southern highbush blueberry in north and central Florida M. VELEZ-CLIMENT, P. Harmon, Department of Plant Pathology, University of Florida, Gainesville, FL, USA 
Blueberry anthracnose is commonly known to be caused by Colletotrichum gloeosporioides and C. acutatum. Each of these has been shown to be a species complex. Defining the cryptic species within a complex is of particular importance because species may differ significantly in virulence, disease severity, host range and geographic distribution. Accordingly, the development of alternative disease management practices may be needed for each species. In this study, isolates of Colletotrichum spp. from symptomatic southern highbush blueberry fruit, stems and leaves were collected from 20132016 in north and central Florida. Colony and conidial characteristics grouped all isolates within the C. gloeosporioides species complex. Phylogenetic analyses of the internal transcriber spacer (ITS), glyceraldehyde-3-phosphate dehydrogenase (GAPDH), chitin synthase (CHS-1), manganese-superoxide dismutase (SOD2), and cytochrome b (CYTB) gene sequences confirmed the morphology-based taxonomic placement and further identified isolates as C. siamense. Isolates were also evaluated for sensitivity to QoI fungicides $\left(10 \mu \mathrm{g} \mathrm{mL}^{-1}\right.$ of azoxystrobin) and a fragment of the CYTB gene was sequenced. Fungicide resistant isolates were found in all plant parts tested and had a G143A mutation in CYTB. The presence of the QoI fungicide resistance mutation in the population indicates that azoxystrobin may no longer be a viable management option for blueberry anthracnose in north and central Florida.

Regional distribution of soilborne diseases in cereal crops in Australia G. VADAKATTU (1), M. Hicks (1), A. McKay (2), D. Gobbett (1), J. Ozman (1), R. Lawes (3), (1) CSIRO, Glen Osmond, AUSTRALIA; (2) SARDI, Glen Osmond, AUSTRALIA; (3) CSIRO, Floreat Park, AUSTRALIA

Soilborne root diseases are major constraints for Australian cereal production, with $>\$ 400$ million annual costs. Cereals cropped continuously are at high risk from soilborne diseases. A multi-disciplinary national project was initiated in 2014 to understand the extent of yield gap in rainfed cereal crops, and identify factors driving yield limitations across Eastern, Southern and Western grain production regions. During 2015 to 2017 crop seasons, wheat and barley root samples were collected at GS31 stage from paired fields (50 plants from five GPS locations on a 100M transect from up to 120 farms p.a.). Roots were scored for the presence and severity of diseases and overall seminal and crown root health (0-5 scale). Eight soilborne root diseases were recorded, and only a few fields $(<20 \%)$ had plants without disease symptoms. Multiple diseases in a single field and agro-ecological region based distribution of specific diseases were commonly observed. Significant levels of soilborne pathogens (PredictaB ${ }^{\circledR}$ DNA test) were found in the pre-crop soil samples. Root rot diseases caused by Rhizoctonia solani AG8, Fusarium pseudograminearum, Pythium spp. and root lesion nematode were the most common. Take-all and Bipolaris root rot were also observed in multiple regions. Brown root rot (Phoma scleroitedes) less commonly recorded. These observations coupled with water-limited yield estimations indicate that root diseases may cause significant portions of the yield gaps in wheat and barley crops. In-crop root assessments are compared with pre-crop pathogen DNA inoculum data to identify links with soil and environmental factors affecting disease impacts.

Fine scale population genetic structure and within tree distribution of mating types of Venturia effusa, cause of pecan scab in the USA C. H. Bock (1), C. A. Young (2), K. L. STEVENSON (3), N. D. Charlton (2), (1) USDA ARS, Southeastern Fruit and Tree Nut Research Laboratory, Byron, GA, USA; (2) Noble Research Institute, LLC, Ardmore, OK, USA; (3) University of Georgia, Tifton, GA, USA

Scab (caused by Venturia effusa) is the major disease of pecan in the southeastern USA. There is no information available on the fine scale population genetic diversity. Four cv. Wichita trees (populations) were sampled hierarchically. Within each tree canopy, 4 approximately evenly spaced terminals (subpopulations) were selected and up to 6 leaflets (sub-subpopulations) were sampled from different compound leaves on each terminal. All lesions on each leaflet were sampled (from 1 to 8 lesions). The isolates were screened against 30 microsatellite markers and mating type was determined. Of 335 isolates there were 165 MLGs (clonal fraction 49.3\%). Nei's unbiased measure of gene diversity was moderate to high ( 0.489 ), and genotypes were evenly distributed. AMOVA demonstrated $92.1 \%$ of variance was explained among isolates within leaflet populations. The vast majority of clones existed on individual leaflets on a terminal ( 72 isolates) compared to other spatial scales ( $\leq 4$ isolates at other strata). Using clone corrected data, the MAT1-1-1 and MAT1-2-1 idiomorphs were at equilibrium (92:73). Both mating types were frequently observed on the same leaflet. The results provide novel information on the characteristics of populations of $V$. effusa at fine spatial scales, and provide insights into pathogen dispersal within and between trees. The proximity of both mating types on single leaflets is further evidence of the opportunity for sexual reproduction in the field.

Genotypic and phenotypic characterization of Stagnospora nodorum blotch of wheat in Virginia N. KAUR (1), H. L. Mehl (1), D. Haak (2), (1) Virginia Tech Tidewater AREC, Suffolk, VA, USA; (2) Virginia Tech, Blacksburg, VA, USA

Parastagnospora nodorum ((Berk.) Quaedvlieg, Verkley \& Crous) (teleomorph Phaeosphaeria nodorum) is a necrotrophic pathogen which causes Stagnospora nodorum blotch (SNB) in wheat. The fungus infects both leaves and glumes, causing significant yield loss in wheat. $P$. nodorum populations have not been well characterized in Virginia, so the objectives of this study were to 1) develop molecular markers to genotype $P$. nodorum isolates, 2) assess genetic diversity within and among pathogen populations in Virginia wheat fields, and 3) evaluate variation in fungicide sensitivity within $P$. nodorum populations. Simple sequence repeat (SSR) markers were designed by mining publically available genomic data for $P$. nodorum. Isolates were collected from 5 different wheat fields in Virginia in 2017 and genotyped with 10 SSR markers. Diversity within and among fields were compared using molecular haplotypes assembled from the molecular markers. In vitro assays were used to screen isolates for sensitivity to strobilurin, triazole, and SDHI fungicides. Management of SNB in wheat can be improved through the use of molecular tools to gain a better understanding of genotypic diversity and fungicide sensitivity within $P$. nodorum populations.

Investigation of Erysiphe necator Population Structure using Amplicon Sequencing (AmpSeq) without Clonal Isolation B. KISSELSTEIN (1,2), L. E. Cadle-Davidson (2), D. M. Gadoury (1), (1) Cornell University, Geneva, NY, USA; (2) USDA Grape Genetics Research Unit, Geneva, NY, USA

Studies of aerially-dispersed fungal pathogens are often limited by: (i) subculturing samples of uncertain purity to achieve clonal lines, and (ii) conducting follow-up bioassays to quantify fungicide resistance or other traits. Amplicon Sequencing (AmpSeq) has the potential to circumvent subculturing by detecting clonality, for targeted bioassay-based validation of known genotypes. In a proof-of-principle study of the haploid fungus Erysiphe necator, from 2015 to 2017 we used 1-cm pieces of tape to collect over 800 samples of conidia from single colonies on grapevine foliage from 5 vineyards. AmpSeq was used to compare heterozygosity (an indicator of paralogy or mixed infection) and allele frequencies for over 200 molecular markers. For the first two years, the median heterozygosity rate was 3\%, but the CYP51 gene (with known paralogs) had sites with $6 \%$ and $28 \%$ heterozygosity, and two other genes had heterozygosity above $70 \%$. Based on markers with low heterozygosity, at least $5.9 \%$ of samples represented mixed infections, suggesting that subculturing to achieve clonality may be unnecessary for some applications. The DMI fungicide resistance allele EnCYP51 A495T was consistently observed in approximately half of samples across all commercial vineyard sites for both years. This approach of genotyping many loci, including genes under selection, enables numerous analyses without clonal isolation. 
Applying population genomics to understand the genomic basis of speciation, pathogenicity and host specialization in Ceratocystis fimbriata sensu lato

T. DUONG (1), A. Hammerbacher (1), M. J. Wingfield (1), S. Chen (1,2), R. Chang (1), F. Liu (1), B. D. Wingfield (1), (1) Forestry and Agricultural Biotechnology Institute (FABI), University of Pretoria, Pretoria, SOUTH AFRICA; (2) China Eucalypt Research Centre, Chinese Academy of Forestry, GuangDong, CHINA

Ceratocystis fimbriata sensu stricto was first descibed in 1890 as the causal agent of sweet potato black rot in the USA. More recently, several cryptic species related to C. fimbrirata s.s. have been described as major pathogens of various tree crops including acacia, eucalypts, mango and pomegranate in Asia, Africa and South America. Studies using phylogenetic analyses, population genetic markers and mating have shown that these species are closely related and in some cases inter-fertile. Consequently, their species boundaries are a source of disagreement and collectively, we treat them as members of C. fimbriata sensu lato. Several studies have revealed that some of these species are host specific. However, nothing is known regarding the genetic basis of their host specificity or pathogenicity. We sequenced the genomes of a relatively large number of $C$. fimbriata s.l. isolates collected from five different hosts on which these fungi are pathogenic. Population genomic analyses revealed that isolates were clustered based on hosts from which they were isolated. Additionally, genomes of these fungi displayed the "two-speed" genome signatures, similar to those known in other plant pathogenic fungi and oomycetes. We are currently identifying and characterizing genes residing in genomic regions with accelerated evolution. The results will provide insights into the genetics of host specialization and speciation in these important species of Ceratocystis s.l.

Population biology and comparative genomics of Claviceps purpurea and other defensive mutualists in the Hypocreales S. Wyka, K. D. BRODERS, Colorado State University, Fort Collins, CO, USA

The genus Claviceps includes many species that are ovarian parasites of Poaceae, causing the disease ergot resulting in the production of sclerotia in place of seeds. The genus recently experienced advances in the delimitation of cryptic species and separate lineages with respect to geographic distribution and host preference. It was also recently discovered that $C$. purpurea produces both cytokinins and auxins during infection. Current outbreaks of ergot on barley in relatively geographically isolated areas has spurred efforts to analyze the population structure, study the evolution of phytohormone production in agriculturally important $C$. purepurea, and examine effects of infection on the host. A phylogenetic analysis of five housekeeping gene loci demonstrated that C. purpruea isolates from the U.S. were distinct from European isolates, but no subpopulations in relation to host preference were observed. A comparative genomic analysis of over 115 species within the Hypocreales revealed orthologs of $C$. purpurea cytokinin biosynthesis genes and putative auxin genes in members of the closely related tribe Balansiae, which contains beneficial fungal endophytes. Together these results indicate a distinct population of $C$. purpurea infecting barley and other grasses in the central plains, and the evolution of phytohormones may play an important role in the ecology and evolution of Claviceps and other species in the Hypocreales as defensive mutualists with a broad host range.

Characterization of cucurbit yellow vine disease strains of Serratia marcescens using whole genome sequencing Z. Matteen, E. L. LITTLE, University of Georgia, Department of Plant Pathology, Athens, GA, USA

Cucurbit yellow vine disease (CYVD), caused by the bacterium Serratia marcescens and transmitted by squash bugs (Anasa tritis), results in wilting and death of cucurbits. The disease was first observed in 1988 and is now endemic in the eastern U.S. Up to $100 \%$ losses have occurred in Georgia. Mechanisms of pathogenicity are unknown. Previous work determined CYVD strains of S. marcescens are genetically distinct. To characterize strain differences, whole genome sequencing was performed on the DNA from diverse $S$. marcescens strains and the aligned sequences compared with $S$. marcescens sequences in the NCBI database. CYVD strain sequences were most similar to each other (>99.97\%) and to two endophytic strains R01-A and 90-166 (98.2\% and 97.7\%, respectively). The remaining non-CYVD strains were more genetically distant. CYVD strains have a reduced genome (15-17\% reduction) compared with non-CYVD strain R01-A. Several predicted genes in the CYVD sequence deletions encode proteins involved in substrate metabolism, explaining the reduced metabolic capability of CYVD strains. Possible pathogenicity proteins were identified in the CYVD unique sequences such as polysaccharide synthesis and toxin production. CYVD strains contain a Type VI Secretion System (T6SS) of unknown function, but not virulence-type T2SS, T3SS, or T4SS common in pathogenic bacteria. Results of this study help explain how this new plant pathogen evolved from a non-pathogenic endophyte.

Phylogenetic characterization of Colletotrichum gloeosporioides isolates from Florida strawberry and non-cultivated hosts M. OLIVEIRA, M. Marin, N. Y. Wang, N. Peres, University of Florida, Wimauma, FL, USA

Colletotrichum gloeosporioides is the causal agent of Colletotrichum crown rot (CCR), a major disease affecting strawberries in the southern United States. Isolates from other non-cultivated hosts around strawberry fields have shown to serve as a source of inoculum for CCR. Recent multi-gene studies defined $C$. gloeosporioides as a complex species comprised of 22 species and one subspecies. The objective of our study was to phylogenetically characterize $C$. gloeosporioides isolates from strawberry and other non-cultivated plants around Florida strawberry fields. Five genomic regions: internal transcribed spacer (ITS), actin (ACT), calmodulin (CAL), chitin synthase (CHS-1), and glyceraldehyde-3-phosphate dehydrogenase (GAPDH) were sequenced from 53 strawberry isolates and seven isolates from non-cultivated hosts (balsam apple, Brazilian pusley, smilax, magnolia, and wax myrtle). Phylogenetic analysis using a maximum likelihood method based on the Kimura 2 model and a discrete gamma distribution revealed that most of the isolates from Florida (77\%) were closely related to C. siamense, whereas two of the strawberry isolates were closely related to C. theobromicola (syn. C. fragariae). The biological importance of these different Colletotrichum species in the C. gloeosporioides species complex as well as fungicide resistance profiles are currently being investigated to determine whether different management strategies are needed in strawberry production fields in Florida.

Genetic, morphological and pathogenic characterization of Chilean isolates of Chondrostereum purpureum

J. CHILIAN (1), D. Grinbergs (2), A. France (1), (1) INIA Quilamapu, Chillan, CHILE; (2) Instituto de Investigaciones Agropecuarias, Chillán, CHILE

Chondrostereum purpureum is a Basidiomycete that cause silver leaf disease. It severely affects yield and quality in several fruit species, such as apple (up to $60 \%$ ) and blueberry $(40 \%)$. The objective was to characterize $C$. purpureum isolates from different hosts and geographic localities, based on their genetic, morphologic and pathogenic features. A survey was conducted through the Chilean fruit production area, from 2014 to 2017 . Thirty nine isolates were obtained from symptomatic woods of 10 different hosts. Isolates were grown in PDA media. Then, their morphology were described and growth measured. DNA was extracted from colonies growing edge and PCR conducted. A sequence-characterized amplified regions (SCAR) was used to group C. purpureum isolates. Apple cuttings cv. Gala Brookfield were inoculate using mycelial plugs and the internal necrosis was measured after $21 \mathrm{~d}$ incubation. Cultural characteristics were similar between isolates from different hosts and geographic origins, however, there were variations on mycelial growth rate ( 8 to $35 \mathrm{~mm}$ in diameter). All isolates were pathogenic showing different virulences, such as Cato isolate (60\% internal necrosis) compared 
to the Osorno's one (30\%). Banding patterns allowed the identification of seven groups. Cluster analysis of this molecular patterns did not match groups based on virulence, morphology, geographic location or host, indicating that $C$. purpureum is highly diverse in Chile.

Characterization of tan spot fungus populations from wheat in Oklahoma

S. SUAREZ, C. D. Garzon PhD, R. M. Hunger, S. M. Marek PhD, Oklahoma State University, Stillwater, OK, USA

Wheat ranks third among economically important crops in the United States. Reduced tillage or no-till practices in Oklahoma have contributed to a higher incidence of fungal leaf spot diseases, particularly tan spot caused by Pyrenophora tritici-repentis (PTR). The occurrence and genetic diversity of PTR in Oklahoma was determined. The identity of 311 isolates collected from 13 winter wheat fields across Oklahoma in 2016 and 2017 was confirmed using PTR-specific PCR primers. The genetic structure of PTR subpopulations from ten Oklahoma counties ( $\mathrm{n}=100)$ in 2016 was assessed using eight simple sequence repeat (SSR) markers. AMOVA showed low but significant genetic differentiation among 2016 subpopulations. Four geographically distant fields were sampled again in 2017. Population structure analysis of the 2016 and 2017 subpopulations indicated significant differentiation among subpopulations as defined by location and year. Our results suggest local distribution and survival of PTR inoculum on site over time, occasional extensive long-distance movement of PTR inoculum, and frequent recombination among PTR populations. Presence of the cultivar-specific toxin genes was determined by PCR. Ninety-five percent of the PTR isolates were positive for toxA and none were positive for tox $B$. Thus, ToxA is the predominant toxin produced by PTR populations in Oklahoma. Further studies will phenotype PTR isolates for toxin production using differential lines of wheat.

Assessment of pathotype variability in Phytophthora sojae populations across the North Central region of the United States

L. WEBER (1), A. E. Dorrance (1), C. A. Bradley PhD (2), E. Byamukama (3), M. Chilvers (4), A. McCoy (4), L. J. Giesler (5), J. E. Kurle (6), D. K. Malvick (7), S. X. Mideros Mora (8), A. E. Robertson (9), K. Wise (2), (1) The Ohio State University, Dept. of Plant Pathology, Wooster, OH, USA; (2) University of Kentucky, Princeton, KY, USA; (3) Department of Agronomy, Horticulture, and Plant Science, South Dakota State University, Brookings, SD, USA; (4) Michigan State University, East Lansing, MI, USA; (5) Univ of Nebraska-Lincoln, Lincoln, NE, USA; (6) University of Minnesota Plant Pathology, Minneapolis, MN, USA; (7) University of Minnesota, St. Paul, MN, USA; (8) University of Illinois at Urbana-Champaign, Urbana, IL, USA; (9) Iowa State University, Department of Plant Pathology, Ames, IA, USA

Phytophthora root and stem rot of soybean, caused by the oomycete Phytophthora sojae, is a devastating disease that accounts for losses of approximately 32 million bushels of soybean across the North Central region of the United States annually. This pathogen is most effectively managed by Rps-gene mediated resistance and consequently, assessment of current $P$. sojae populations is of great importance. The aim of this study was to characterize the pathotype diversity of $P$. sojae populations across the North Central region of the US. Soil was collected from multiple locations in each state with a history of Phytophthora root and stem rot and baited with the moderately susceptible cultivar 'Sloan'. Recovered isolates were pathotyped using 14 soybean differentials to assess their virulence against commonly deployed Rps genes. From over 2600 soil samples collected across 292 locations, recovery efforts have led to the collection of over $800 \mathrm{P}$. sojae isolates, with more than $350 \mathrm{P}$. sojae isolates recovered from Ohio and Kentucky soils alone. From Ohio soil samples, $P$. sojae was recovered from 128 of the 190 soil samples baited, for a recovery rate of $67 \%$. The characterization of these $P$. sojae populations provides greater awareness of the virulence that exists against Rps genes, and which can be effectively deployed to confer resistance against $P$. sojae populations in the North Central soybean producing region of the US.

Relation and occurrence of Fusarium virguliforme, Macrophomina phaseolina, and Heterodera glycines in Tennessee

E. Zuchelli (1), R. Guyer (2), A. Mengistu (3), H. M. Kelly (2), A. MCLAUGHLIN (2), (1) Universidade de Passo Fundo, Rio Grande Do Sul, BRAZIL; (2) University of Tennessee, Jackson, TN, USA; (3) USDA ARS, Jackson, TN, USA

Soil borne pathogens such as Fusarium virguliforme (FG), Macrophomina phaseolina (MP), and Heterodera glycines (HG) can reduce soybean (Glycine $\max$ ) yield by damaging the roots and vascular system and causing plant death. Due to the importance of these pathogens, we aim to evaluate their occurrence across Tennessee. In the fall of 2017, 24 soil samples and 14 root samples were collected from 4 different counties. Soil probes were used to collect $15 \mathrm{~cm}$. deep soil cores targeting the root zone of the previous crop. Soil was elutriated and used to evaluate HG eggs/100 cc soil. Colony forming units (CFU) were evaluated to quantify FG and MP using $0.05 \mathrm{~g}$ and $0.005 \mathrm{~g}$ of root samples, respectively; and $1 \mathrm{~g}$ of soil. Soil was dried and ground, sterilized in $10 \%$ bleach for 3 minutes, rinsed, and distributed into petri plates with selective media for each pathogen. FG was also evaluated by extracting DNA from $0.1 \mathrm{~g}$ of soil and root samples and qPCR was conducted using species specific primers/probes. Presence of all 3 pathogens were observed in 7 samples which included all 4 counties. Correlations and analysis of variance among DNA and CFU of FG; soil CFU and root CFU of FG and MP; and other factors influencing disease development such as no-till practices, cultivar resistance, soil texture, will be conducted and presented.

Characterization of Xanthomonas isolates causing black spot on tomato in South Africa

S. VOU (1), J. van der Waals (1), T. Goszczynska (2), T. Coutinho (1), (1) University of Pretoria, Pretoria, SOUTH AFRICA; (2) Agricultural Research Council, Pretoria, SOUTH AFRICA

Black spot of tomato, caused by seed and seedling-borne black spot Xanthomonads (BSX), is prevalent in South Africa and can cause substantial yield losses of the crop. Multilocus sequence analysis (MLSA) of two housekeeping genes (dnaK and $\operatorname{gyr} B$ ) was used to resolve the taxonomic classification of 30 Xanthomonas strains previously identified as belonging to this genus. Pathogenicity tests on a susceptible tomato cultivar, Red Khaki, and matured green tomato fruits were also undertaken and susceptibility of strains to both $\mathrm{CuSO}_{4}$ and streptomycin was tested in vitro. Plants and fruit were monitored at weekly and on daily intervals, respectively. Disease symptoms were scored and a percentage infection derived for the seedlings and tomato lesion diameters measured with a ruler. Of the 30 isolates, seven species of Xanthomonas were identified, viz: $X$. euvesicatoria, $X$. gardneri, $X$. perforans, $X$. vesicatoria, $X$. campestris pv. campestris, $X$. arboricola pv. populi and X. arboricola pv. juglandis. Five species, $X$. euvesicatoria, $X$. gardneri, $X$. perforans, $X$. vesicatoria and $X$. campestris pv. campestris, caused black spots on both leaves and fruits of the susceptible tomato cultivar. This study thus showed that all four BSX species previously reported to be responsible for black spot disease of tomato elsewhere are present in South Africa. Also, this is the first report of $X$. campestris pv. campestris causing black spots on tomato leaves. All four of the isolates used in pathogenicity tests were resistant to both $\mathrm{CuSO}_{4}$ and streptomycin, therefore other management options will need to be explored.

Characterization of Phytophthora infestans populations from soils of the Ecuadorian Andes

M. S. BENITEZ PONCE (1), J. L. Andrade-Piedra (2), C. D. Garzon PhD (3), P. Gutierrez (4), A. Koch (5), A. Mera (5), L. Pena Zuniga (3), P. Romero (5), M. Vinueza (6), (1) The Ohio State University, Wooster, OH, USA; (2) International Potato Center (CIP), Lima, PERU; (3) Oklahoma State University, Stillwater, OK, USA; (4) Universidad de las Fuerzas Armadas, ESPE, Sangolgui, ECUADOR; (5) Universidad de las Fuerzas Armadas, ESPE, Sangolqui, ECUADOR; (6) International Potato Center, Quito, ECUADOR 
Potato late blight is a major concern for potato production in all regions of the world. In Ecuador, the incidence of foliage infection is high and can severely compromise potato production. Tuber blight, however, is low, which has been attributed to suppressive characteristics of soils where potatoes are produced. Inoculum for tuber infection often comes from infected foliage residue and infected seed tubers. In this work, we characterized populations of Phytophthora infestans in soils as a way to understand incidence and infectivity of soilborne inoculum for tuber infection. Sixty-one isolates were obtained directly from soils from potato-growing fields of two provinces in Ecuador representing the major potato growing regions in the Ecuadorian Andes. Sampling design considered field topography, host developmental stage and soil depth. Greater number of isolates were recovered at a shallower depth and at flowering. All isolates collected were infective in both leaf and tuber assays. Mitochondrial DNA analysis indicated the presence of haplotypes Ia, Ib and IIb and IIa, and suggested the occurrence of clonal lineages EC-3, US-1, EC-1 and US-6, and mating type A1. Furthermore, microsatellite analyses revealed significant population differentiation between provinces. There was no correlation between haplotype, genetic variation and infectivity. These results confirm inoculum pathogenicity, further confirming the suppressive nature of Ecuadorian soils to tuber blight.

Variation of the avirulence gene AvirPib among a worldwide collection of isolates of Magnaporthe oryzae C. FENG (1), Q. Pan PhD (2), B. Dhillon (1), B. Liu (1), J. C. Correll (1), (1) University of Arkansas, Fayetteville, AR, USA; (2) South China Agric University, Guangzhou, Guangdong, CHINA

Blast disease, caused by the fungus Magnaporthe oryzae (Mo), is one of the most destructive diseases of rice. Deploying resistant cultivars, based on the understanding of race population structure, is the most economical and effective way to manage this disease. The Pib gene, which confers high resistance to a wide range of races of $\mathrm{Mo}$, has been used in global rice breeding programs for over 30 years. The objectives of this study were to investigate the $A v r P i b$-based race structure in Africa, Asia, and North and South American populations of $M o$, and then dissect molecular mechanisms underlying variation in $A v r P i b$. The phenotype of all isolates from the four populations was tested on the Pib-monogenic line IRBLb-B. The genotype of each isolate was characterized by triple cycles of PCR assays targeted the whole gene, coding, and upstream regions of $A v r P i b$. High resistance frequencies among isolates in some populations indicated that $P i b$ is still effective in such populations. Various combinations of phenotype/genotype were identified, indicating that genetic diversity of $A v r P i b$ was different in some populations. A total of 15 isolates, selected from each population, were subjected to resequencing of $A v r P i b$. Functionality of novel AvrPib variants, which were not previously detected, were validated by a mutagenesis assay. The results can provide insights on the effectiveness of deploying rice cultivars containing the $P i b$ resistance gene.

Population biology of Fusarium oxysporum associated to banana in Ecuador F. MAGDAMA (1), M. D. M. Jimenez-Gasco (2), (1) Escuela Superior Politécnica del Litoral, Guayaquil, ECUADOR; (2) The Pennsylvania State University, University Park, PA, USA

Ecuador is a leader in banana exports, however, epidemiological studies of Fusarium oxysporum f. sp. cubense (Foc) are still lacking. Assessing the evolution and diversity of pathogenic and nonpathogenic populations of $F$. oxysporum associated to banana within an ecological context would reveal new information for the management of Foc tropical race 4 (FocTR4) threatening global banana production. A collection of 460 isolates was used to perform comparative phylogenetic analyses based on TEF, IGS and RPB1 gene regions. Further analyses included pathogenicity test, VCGs, validation of $F o c$ TR4 detection methods and presence of putative effector genes. Our results suggest that $F o c$ populations from Ecuador are clonal and associated with VCG-0120, indicating a single introduction. Foc is present as endophyte in the roots of Cavendish in areas previously used for the production of 'Gros Michel'. Three out of the four markers currently available in the literature were not reliable for FocTR4 detection and generated false positive PCR reactions. Effector SIX genes, putatively linked to pathogenicity, were found in Ecuador Foc populations but also in nonpathogenic F. oxysporum, suggesting a broader role of these genes in plant-microbe interactions. We propose the consideration of $F$. oxysporum endophytes as key constituents in the evolution of the species and highlight the importance of understanding its true ecological niche for better disease control and risk management.

Diversity of begomoviruses causing disease in peppers (Capsicum spp.) in Asia

L. KENYON, S. L. Shih, L. M. Lee, Y. L. Chan, World Vegetable Center, Shanhua, Tainan, TAIWAN

Chili leaf curl- or Pepper yellow leaf curl-type diseases caused by whitefly-transmitted Geminiviruses (Begomoviruses) have emerged over the last 2030 years as major constraints to pepper (Capsicum spp.) production in many areas of Asia. The diversity of the Begomovirus species and strains associated with the diseases may affect how the diseases can be managed, especially in regard to breeding and selecting for resistance. Using Begomovirus DNA-A component sequences from GenBank and sequences from leaf curl diseased pepper samples collected more recently from different locations across the region, the identity, genetic diversity and phylogenetic relationships of the different causal begomoviruses were assessed. The results indicate that the diseases are usually associated with different virus species or strains in different areas, and the increasing diversity of strains and species in an area is through a combination of movement of known species or strains into the area, and/or the emergence of novel, previously undescribed, species or strain, and/or recombination between different strains and/or species. The possible drivers for the rapid emergence of these different species and strains will be discussed.

The presence of secreted in xylem genes in Fusarium oxysporum f. sp. zingiberi from Australian ginger showing symptoms of Fusarium yellows A. Matthews (1), E. Czislowski (2), D. P. Le (3), J. Wadduwage (4), S. Achari (5), E. AITKEN (2), S. Hamill (6), (1) University of Queensland, Brisbane, AUSTRALIA; (2) School of Agriculture and Food Sciences, The University of Queensland, Brisbane, AUSTRALIA; (3) Department of Agriculture, Narrabri, AUSTRALIA; (4) University of Kelaniya, Kelaniya, SRI LANKA; (5) AgriBio, Bundoora, AUSTRALIA; (6) DEEDI, Nambour, AUSTRALIA

Fusarium oxysporum f. sp. zingiberi (Foz) is the causative agent of Fusarium yellows in ginger. The pathogen causes rotting of rhizomes in field grown ginger plants. This disrupts the vascular system of the plant causing foliage yellowing and premature death. Foz is one of the most significant diseases of ginger. An understanding of the diversity of $F$. oxysporum strains associated with field grown ginger could provide valuable information to manage Fusarium yellows through practices such as preventative measures and early diagnostics. In this study, we sought to establish the genetic diversity of $F o z$ and the presence of secreted in xylem $(S I X)$ pathogenicity genes in these isolates. Thirty four $F$. oxysporum like isolates were recovered from ginger rhizomes of plants showing typical Fusarium yellows symptoms. A phylogeny of these isolates was produced using sequence information from the translation elongation factor region. The isolates of $F$. oxysporum were also screened for the presence of SIX7, SIX9, SIX10 and SIX12. Phylogenetic analysis showed that 23 isolates formed a clade which was distinct from the other isolates of $F$. oxysporum. Interestingly, all of the isolates within this clade were shown to carry the four $S I X$ genes whilst the other isolates of $F$. oxysporum outside of this clade did not carry any of the SIX genes. As there are few options for controlling Foz in ginger crops these results will be valuable for crop disease management and cross-pathogenicity research. 
Genetic characterization of Rathayibacter spp. present in the United States of America (USA)

B. SCHROEDER (1), A. Sechler (2), M. Tancos (3), E. E. Rogers (4), D. Luster (3), W. Schneider (4), T. D. Murray (5), (1) University of Idaho, Moscow, ID, USA; (2) USDA-ARS Foreign Disease/Weed Science Research Unit, Ft. Detrick, MD, USA; (3) USDA ARS FDWSRU, Frederick, MD, USA; (4) USDA ARS FDWSRU, Fort Detrick, MD, USA; (5) Washington State University, Pullman, WA, USA

Rathayibacter toxicus is a Gram-positive plant pathogen that infects Lolium rigidum and other grass species in Australia; it is also a USDA APHIS-listed Select Agent in the USA because it produces corynetoxins in planta that are fatal to grazing animals. None of the other six validly described Rathayibacter species are known to produce toxin, but anecdotal reports of animal deaths raise concerns that a toxigenic Rathayibacter sp. already may be present in the USA. Of the seven species, $R$. rathayi and $R$. agropyri are present in the US. Knowing which other species of Rathayibacter are present is important for developing detection and discrimination methods to help prevent introduction and establishment of $R$. toxicus in the USA. The goal of this study was to characterize 114 Rathayibacter strains isolated from various grass species collected in the Pacific Northwest US since 2013. To do so, the 16S rRNA gene and four housekeeping genes $(r p o B, \operatorname{gyr} B, \operatorname{rec} A$, and $p p k)$ were amplified and sequenced from all strains and seven reference isolates. Phylogenetic analysis of the sequences suggests that there are several undescribed Rathayibacter species present in the USA and there is significant within species diversity. One clade of strains originating in the USA are closely related to the toxigenic strain Rathayibacter sp. EV isolated from Ehrharta villosa var. villosa in South Africa, suggesting that strains present in the USA may be capable of toxin production as well.

Population genomics reveals high mutation rate and divergence among populations of blueberry pathogen Exobasidium maculosum A. ABRAHAMS, M. T. Brewer, University of Georgia, Athens, GA, USA

Exobasidium maculosum is an emerging pathogen of blueberry (Vaccinium spp.) with increasing prevalence in the southeastern United States. The fungus causes Exobasidium leaf and fruit spot and has the potential to cause significant economic loss. Our goal is to identify the genetic basis of high levels of diversity seen among populations of $E$. maculosum. The mutation rate of E. maculosum was calculated using a fluctuation assay that measures the number of spontaneously arising mutants on selective media. Additionally, 15 isolates of $E$. maculosum equally representing the three distinct previously identified populations of Georgia and North Carolina in the southeastern United States and a population from northeastern North America represented by isolates from Maine and Nova Scotia, Canada, were sequenced and investigated for genes involved in rapid divergence and DNA repair pathways. Our results suggest that populations of E. maculosum are divergent, especially near the RAD21 gene, which is involved in DNA repair of double strand breaks. Additionally, results of the fluctuation assay and genomic analyses suggest that the elevated diversity in the pathogen results from a high mutation rate. Pathogens with an increased mutation rate have the ability to adapt quickly giving them a selective advantage in host invasion and manipulation. Gaining insight into the evolution of E. maculosum is beneficial for disease management and understanding disease emergence.

Identification and characterization of microRNA-like RNAs in Fusarium oxysporum f. sp. cubense J. PENG, Chinese Acadey of Tropical Science, hAI, CHINA

Recent discoveries of microRNA-like RNAs (milRNAs) in plant pathogenic fungi have been characterized and are suggested to play a role in pathogenic development and virulence. Fusarium wilt of banana caused by Fusarium oxysporum f. sp. cubense (Foc) is one of the most severe diseases in world. We proposed that Foc could encode milRNAs and therefore the different developmental stages, mycelia and conidia between Foc 1 and Foc4 were deep sequenced for milRNAs identification, of which a total of 133 conserved miRNAs, 88 milRNAs and 133 novel milRNA candidates were identified. The partial milRNAs/milRNAs* were testified by northern blotting. Besides, transient expression milRNA precursor by ago-infiltration in $N$. benthamiana leaves was proved to be an alternative approach for validating milRNAs processing in vivo. Furthermore, the most abundant milR-1, was predicted only target gene in host rather than fungus itself. To elucidate whether milR-1 could silence target mRNA, transient co-expression of milR-1 precursor and GFP fused target mRNA in $N$. benthamiana plants was performed and results confirmed that milR-1 could inhibit the translation of target mRNA. Our finding first proved that Foc could produce milRNAs and suggested possible roles of milRNAs in the Foc-host interaction via trans-kingdom gene silencing.

Application of a new approach for study of virulence variation in cucurbit powdery mildew populations A. LEBEDA (1), E. Kristkova (2), B. Sedlakova (2), J. D. McCreight (3), E. Kosman (4), (1) Palacky Univ in Olomouc, Olomouc-Holice, CZECH REPUBLIC; (2) Palacky University in Olomouc, Department of Botany, Olomouc, CZECH REPUBLIC; (3) United States Department of Agriculture, Agricultural Research Station, Salinas, CA, USA; (4) Tel Aviv University, Institute for Cereal Crops Improvement, Tel Aviv, ISRAEL

Cucurbit powdery mildew (CPM) is caused by two obligate ectoparasites, Golovinomyces orontii s.l. (Go) and Podosphaera xanthii (Px), that are highly variable in virulence. Various systems of CPM race determination and denomination were used (Lebeda et al. 2011). We developed new tools to enhance research of CPM virulence variation (Lebeda et al. 2016). Diversity models were applied to analyses of virulence (disease reaction patterns) variation of 115 isolates of two CPM species, Go and Px, collected in the Czech Republic from 2010 through 2012. Diversity within and distances between Go and Px populations and each other in spatio-temporal context and with regard of original host plant species were analyzed, based on virulence patterns of individual isolates (races) on a set of 21 melon (Cucumis melo L.) race differential genotypes (Lebeda et al. 2016). Significant differentiation among the Go and Px pathogen populations was revealed, and the results clearly demonstrate and confirm that the set of differential C. melo genotypes is well composed because of high differentiation capacity. Differentiation of pathogens among years was significant for both species. No significant difference between Go isolates from different host plant species was established due to high variability among Go isolates, but there was significant host-specific differentiation among Px isolates. The results revealed high virulence variation in isolates of Go and Px, and their spatio-temporal fluctuations. Approach applied in this study provides a complex view of virulence structures of CPM populations, and when completed by race determination and denomination on melon, it may serve as a base to understand virulence variation of these CPM species from a spatio-temporal viewpoint, as well as application of this system in breeding, seed production and cucurbit growing.

How many types of fungal \& oomycete phytopathogens are there? Catastrophy for the bio/hemi/necrotroph divisions J. Hane (1), J. Paxman (1), D. Jones (1), A. Testa (1), R. P. OLIVER (2), P. J. G. M. De Wit (3), (1) Curtin, Perth, AUSTRALIA; (2) Curtin University, Perth, AUSTRALIA; (3) Wageningen University, Rhenen, NETHERLANDS

The classification model that dominates plant pathology is that diseases and pathogens can be divided into three 'trophic' classes, biotroph, hemibiotroph and necrotroph. This pathogen classification leaves much to be desired. Many pathogens are placed by different authors in two or even all three of these classes. Apart from the undisputed fact that all obligate pathogens are all biotrophic, no phenotypic feature can be described as diagnostic. Other pathogens, such as the wilts and those causing root diseases do not obviously comply with the biotroph/hemi/necrotroph trichotomy. There are now 137 fungal and oomycete pathogen genome sequences. With this information resource, we set out to determine whether an objective analysis of these genomic resources could provide a more robust classification system that had predictive power. We propose a novel bioinformatic technique for the 
assessment of fungal trophic phenotypes across multiple species via analysis solely of their gene content. We used the functional annotations of carbohydrate active enzymes (CAZyme). Our analysis led us to conclude that the existing tripartite trophic classification system for filamentous plant pathogens is unsustainable. Instead our analysis identified eight pathogen trophic descriptors and a saprobe trophic class. This study highlights some longstanding anomalies and permits the objective prediction of properties of a species based solely on its CAZyme gene content. We have used the data included in this study to develop and train a predictive tool for CAZyme-Assisted Training And Sorting of TROPHY - CATASTROPHY.

Whole Genome Sequencing and Secretome analysis of Tilletia indica inciting Karnal bunt of wheat Provides Pathogenesis-related genes M. S. GURJAR PHD, R. Aggarwal, ICAR-Indian Agricultural Research Institute, New Delhi, INDIA

Tilletia indica is an internationally quarantined plant pathogen inciting Karnal bunt of wheat. The disease was first reported from India in 1931. The pathogen is genetically highly diverse and unique life style which starts to infect the wheat plant at specific spike development stage. Therefore, Tilletia indica genome assembly size of $33.7 \mathrm{MB}$ was generated with GC content of $55.0 \%$ using HiSeq 2500 and PacBio RSII platforms. A total number of 10,113 genes were predicted with average gene size of 1,945 bp out of which functionally annotated genes were 7,262 . A total number of 3,216 protein coding genes were assigned in different categories including biological process' (1,148 genes), cellular ( 833 genes) and molecular function (1,235 genes). Out of a total number of 1,877 transposable elements, gypsy had the highest count. 5772 simple sequence repeat identified in the genome assembly. The most abundant simple sequence repeat type was trinucleotide having $42 \%$ of all SSRs. In comparative genome analysis suggested 3,751 proteins of Tilletia indica had orthologs in five fungi whereas 126 proteins were unique. In comparative genome analysis suggested 3,751 proteins of Tilletia indica had orthologs in five fungi whereas 126 proteins were unique. Secretome analysis revealed the presence of 1,014 secretory proteins in the genome. Some putative pathogenicity-related genes were identified in genome. The full genome of $T$. indica will provide deeper understanding and provide window to understand the pathogenesis mechanism, fungal life cycle, survival of teliospores and novel strategies for management of Karnal bunt disease of wheat.

RNA Pulling: A novel approach for whole genome sequencing of monopartite ssRNA virus, a case study S. SHARMA (1), A. Sharma (1,2), (1) Punjab Agricultural University, ludhiana, AE, INDIA; (2) Punjab Agricultural University Ludhiana, India, ludhiana, AE, INDIA

Whole genome sequencing is an inevitable step for characterization of any virus. Many a techniques for full genome characterization of viruses have been discovered over the decades from "first-generation" technology to deep sequencing, next gen sequencing, which are the latest additions. But the constraints as of money, resources still exist for many a labs to practice. For the full genome sequencing, traditional approach of designing the primers after aligning the available sequences of full genome of Pepper mottle virus from NCBI was adopted. Total RNA was isolated and cDNA was prepared with olido DT primers as well as reverse primers corresponding to 3' poly A tail, coat protein, NIb, and HC pro regions, respectively. None of the above prepared cDNA were able to yield desired amplicons when used individually. A novel idea of RNA pulling approach by using cocktail of reverse primers corresponding to P1,HCPro-P3,CI,NIa-NIb and CP genes was applied. A single hit PCR was found to be promising approach for whole genome characterization of pepper mottle virus.

Transcriptome profiling reveals the EanI/R quorum sensing regulon in Pantoea ananatis $\mathrm{LMG}_{2665}^{\mathrm{T}}$

S. SIBANDA (1), S. Kwenda (2), C. Tanui (3), D. Shyntum (3), T. Coutinho (1), L. Moleleki (3), (1) University of Pretoria, Pretoria, SOUTH AFRICA; (2) University of Witswatersrand, Johannesburg, SOUTH AFRICA; (3) Department of Microbiology and Plant Pathology, University of Pretoria, Pretoria, SOUTH AFRICA

The plant pathogen, Pantoea ananatis LMG $2665^{\mathrm{T}}$ synthesizes and utilizes acyl homoserine lactones for signaling. The complete set of genes regulated by the EanI/R system in $P$. ananatis LMG $2665^{\mathrm{T}}$ is still not fully known. In this study, RNA-Seq was used to identify the EanI/R quorum sensing (QS) regulon in this bacterium. Two approaches were used in this regard. Firstly, pairwise comparisons of the wild type strain in the absence of $\mathrm{AHLs}_{\mathrm{O}} \mathrm{OD}_{600}$ $=0.2)$ and in the presence of AHLs $\left(\mathrm{OD}_{600}=0.5\right)$ were made. Additionally, pairwise comparisons of LMG $2665^{\mathrm{T}}$ and its $\mathrm{QS}$ mutant at $\mathrm{OD} 600=0.5$ were made. In total, 608 genes were differentially expressed between $P$. ananatis LMG $2665^{\mathrm{T}}$ in the presence of AHLs vs the same strain in the absence of AHLs, and 701 genes were differentially expressed between the wild type strain vs the QS mutant at OD $600=0.5$. A total of 196 genes were commonly differentially expressed between the two approaches. The RNA-Seq data was validated by RT-qPCR. Genes regulated by the EanI/R QS are involved in redox sensing, metabolism, flagella formation and flagella dependent motility, cell adhesion and biofilm formation, regulators, transport, chemotaxis and methyl accepting proteins, membrane proteins, cell wall synthesis, stress response and a large number of hypothetical proteins under the tested conditions. Functional characterization of the QS regulated genes in LMG $2665^{\mathrm{T}}$ could help in the formulation of control strategies for this plant pathogen.

Temporal dynamics of the soil metabolome and microbiome in response to anaerobic soil disinfestation S. S. Hewavitharana, R. Leisso, L. Honaas, D. Rudell, M. MAZZOLA, USDA-ARS, Wenatchee, WA, USA

Anaerobic soil disinfestation (ASD) is a soil-borne disease management practice effective for controlling a broad spectrum of pathogens in a diversity of cropping systems. Chemical and biological modes of action of disease control have been proposed. Temporal dynamics of metabolome and microbiome changes in response to ASD using rice bran were examined. ASD treated soil underwent aerobic, fermentative, and anaerobic phases over a 15-day period. Separate weighted gene correlation analysis (WGCNA) of metabolites and microbial operational taxonomic units (OTUs) revealed that changes in both datasets could be divided into "modules" indicative of similar changes in levels. Co-abundance correlation metabolite networks consisted of production, consumption, and intermediate modules over time. Parallel to the metabolic network, microbial co-abundance networks were composed of modules of OTUs that either proliferated, declined or showed intermediate trends. Specific microbial OTU modules showed significant correlations with particular metabolic modules indicating impact of these microbial modules on production or consumption of those metabolic modules. Adjacent time points had relatively similar microbial communities whereas distant time points were highly dissimilar. Functional attributes of the network of microbial community and community metabolome such as scale free topology may help the soil system to recover from the perturbation delivered by ASD once it is aerated.

The pathogenic mechanism analysis of sugarcane ratoon stunting disease base on histology and transcriptomics Y. GUO, W. Wang, X. Qiu, Fujian Institute of Subtropical Botany, Xiamen, CHINA

Sugarcane ratoon stunting disease (RSD) is worldwide. In this research, we inoculated Lxx to Badila to explore the infecting process, obtained differential gene expression profile of sugarcane interacting with Lxx, and identified genes which were expressed differentially and confirmed involving 
in key metabolic pathways and signal transduction by q-PCR. Detailed results were as follow. 1. Results of in-situ PCR of stem and leaf from infected Badila showed Lxx maily distributed in xylem and phloem in stem, xylem in main vains and bundle sheath cells near upper epidermis, vascular bundle sheath cell wall in mesophyll tissue and the mesophyll cells. Pathological section observed in microscope displayed that cell wall of "wreath" mesophyll cells outside leaf bundle sheath was thin and shrinked irregularly, and sugarcane stem cells of vascular bundles in the xylem and phloem cells were hashed obviously. Lxx quantitative analysis results in infected sugarcane stem showed that the extent of damage in phloem and xylem of vascular tissue and Lxx bacteria planting density were positively correlated; 2 . The differential transcriptome analysis among various stages in infected sugarcanes showed that the differential expressed genes mainly involved in plant hormone signal transduction, phenylalanine metabolism, phenylpropanoids biosynthesis, carbon metabolism, photosynthesis, plants and pathogen interactions. SA activated PR gene expression and induced sugarcane to produce system acquired resistance. Phenylalanine metabolic pathway was also activated responsing to Lxx stress, and defense system was started. The key genes involved in photosynthetic organism carbon fixation were down-regulated, maybe Lxx destroyed photosynthesis is one of the reason for leading to stunting disase in sugarcane.

Genome sequencing and transcriptome analysis of the hop downy mildew pathogen Pseudoperonospora humuli reveal species-specific genes for diagnostics

A. RAHMAN (1), E. Góngora-Castillo (2), M. Bowman (3), K. L. Childs (3), G. David (4), L. M. Quesada (5), (1) NCSU, Raleigh, NC, USA; (2) Centro de Investigación Científica de Yucatán | CICY, Mérida, MEXICO; (3) Michigan State University, East Lansing, MI, USA; (4) USDA ARS, Corvallis, OR, USA; (5) North Carolina State University, Raleigh, NC, USA

Pseudoperonospora humuli is an obligate oomycete pathogen of hop (Humulus lupulus) that causes downy mildew, an important disease in most production regions in the world. The pathogen can cause a systemic infection in hop, overwinter in the rhizomes, and infect propagation material. Nonetheless, no species-specific diagnostic assays or genome sequence are available for $P$. humuli. The genome of the isolate $P$. humuli OR502AA was sequenced using Illumina technology and assembled with ABySS. The assembly had a minimum scaffold length of $500 \mathrm{bp}$ and a N50 of $19.2 \mathrm{Kbp}$. A total number of 18,656 genes were identified using MAKER-standard gene predictions. Additionally, transcriptome assemblies were generated using RNA-seq and Trinity for seven additional $P$. humuli isolates. Bioinformatics analysis of next-generation sequencing (NGS) reads of $P$. humuli and $P$. cubensis (a closely related sister species) identified 242 candidate species-specific $P$. humuli genes that could be used as diagnostic molecular markers. These candidate genes were validated using PCR against a diverse collection of isolates from P. humuli, P. cubensis, and other oomycetes. Overall four diagnostic markers were found to be uniquely present only in $P$. humuli. These candidate markers identified through comparative genomics can be used for pathogen diagnostics or adapted for biosurveillance of airborne sporangia, another important source of inoculum in hop downy mildew epidemics.

Validation of a CANARY ${ }^{\circledR}$ multiplex testing platform for rapid identification of Ralstonia solanacearum

A. A. AHMAD (1,2), D. N. Kalkofen (3), R. Rana (3), M. Carter (4), D. Luster (5), J. J. Lehett (3), A. R. Flannery (3), Q. Huang (2), (1) Department of Plant Pathology, Faculty of Agriculture, Minia University, EL-Minia, EGYPT; (2) Floral and Nursery Plants Research Unit, U. S. National Arboretum, USDA/ARS, Beltsville, MD, USA; (3) PathSensors, Inc., Baltimore, MD, USA; (4) USDA ARS FDWSRU, Fort Detrick, MD, USA; (5) USDA ARS FDWSRU, Frederick, MD, USA

Ralstonia solanacearum (Rs) race 3 biovar $2(\mathrm{r} 3 \mathrm{~b} 2)$ is a bacterial plant pathogen listed by USDA-APHIS as a Select Agent. Accurate and rapid early detection, therefore, is needed for the efficient screening of imported plants to support exclusion of r3b2. PathSensors, Inc. in collaboration with USDAAPHIS and USDA-ARS has developed a high-throughput Rs diagnostic assay based on CANARY ${ }^{\circledR}$ (Cellular Analysis and Notification of Antigen Risks and Yields), an immune-based biosensor technology. Previous assay verification demonstrated that the assay had high specificity and sensitivity for $R s$ including $\mathrm{r} 3 \mathrm{~b} 2$ in inoculated zonal geraniums with very low false positive/negative rates and an analytical detection limit of $100 \mathrm{CFU}$ for both $\mathrm{r} 3 \mathrm{~b} 2$ and non-r3b2 strains. In this study, a blinded third-party validation trial was conducted to simulate a real-world application of the assay. Water control and $R s$ r1b3 drench-inoculated zonal geranium stem samples were tested by the CANARY ${ }^{\circledR}$ assay and compared to plating followed by $R s$-specific colony PCR (P-PCR). The CANARY ${ }^{\circledR}$ assay correctly identified $R s$-inoculated samples, including early asymptomatic samples missed by P-PCR. This result suggests that the CANARY ${ }^{\circledR}$ assay has greater sensitivity which, combined with its speed and ease of use, makes it feasible for early detection screening of imported plants for the presence of $R s$ before further identification of $\mathrm{r} 3 \mathrm{~b} 2$ can be made.

Proteomic Profile of Aspergillus flavus responses to oxidative stress

J. C. Fountain (1), J. Koh (2), M. Pandey (3), W. Zhuang (4), Z. Y. Chen (5), R. C. Kemerait (1), S. Chen (2), R. Varshney (3), B. GUO (6), (1) University of Georgia, Tifton, GA, USA; (2) University of Florida, Gainesville, FL, USA; (3) International Crop Research Institute for the Semi-Arid Tropics, Hyderabad, INDIA; (4) Fujian Agriculture and Forestry University, Fuzhou, CHINA; (5) Louisiana State University Agricultural Center, Baton Rouge, LA, USA; (6) USDA ARS CPMRU, Tifton, GA, USA

Aflatoxin production by A. flavus and related species of fungi is regulated in concert with other secondary metabolites, developmental processes, and stress-responsive enzymes in response to environmental stress. Specifically, oxidative stress has been shown to be a pre-requisite and stimulator of aflatoxin production. This is of particular interest given the observation that drought stress results in compromised host resistance to aflatoxin contamination, and that drought stress results in the accumulation of ROS in host plant tissues. The objectives of this study were to use proteomics to provide insights into the pathogen responses to $\mathrm{H}_{2} \mathrm{O}_{2}$-derived oxidative stress, and to identify potential biomarkers for fungal infection and targets for host resistance breeding. Three isolates, AF13, NRRL3357, and K54A with high, moderate, and no aflatoxin production, and cultured in medium supplemented with varying levels of $\mathrm{H}_{2} \mathrm{O}_{2}$, were examined using an iTRAQ approach. Overall, 1,173 proteins were identified of which 238 were found to be differentially expressed (DEPs). Observed DEPs encompassed metabolic pathways including antioxidants, carbohydrates, pathogenicity, and secondary metabolism. Increased lytic enzyme, secondary metabolite, and developmental pathway expression in AF13 was correlated with increased tolerance to oxidative stress, likely assisting in host plant infection and microbial competition. Elevated expression of energy and cellular component production in NRRL3357 and K54A implies a greater focus on oxidative damage remediation. These trends provide insights into important mechanisms relevant to host plant interactions under drought stress allowing for more targeted efforts in host resistance research.

Genome-wide identification of candidate secretory effector proteins of Colletotrichum tanaceti isolated from Australian pyrethrum R. LELWALA (1), P. Korhonen (1), N. Young (1), J. B. Scott (2), P. Ades (1), R. Gasser (1), P. W. J. Taylor (1), (1) The University of Melbourne, Parkville, AUSTRALIA; (2) University of Tasmania, Burnie, AUSTRALIA

Colletotrichum tanaceti is a foliar fungal pathogen causing anthracnose in pyrethrum; the source plant of the natural insecticide compounds, pyrethrins. The molecular determinants of pathogenesis of $C$. tanaceti to pyrethrum are unknown. Fungal effectors are small secreted proteins driving virulence by manipulating the host response. The objective of this study was to examine the genome of $C$. tanaceti to detect Candidate Secreted Effector Proteins (CSEPs). We report the first draft genome assembly of $C$. tanaceti using Illumina MiSeq platform and annotation supported by RNASeq of saprobic and 
biotrophic stages. The genome comprises 5,245 contigs, an estimated size of 58Mb with $96 \%$ BUSCO completeness. A deduced proteome of 12,051 proteins were used for downstream prediction of the secretome. In-silico analysis pipeline was used to predict the signal peptides, followed by the exclusion of membrane proteins and endoplasmic reticulum targets, to define a secretome of 1,185 proteins. These proteins were used to predict 224 CSEPs, of which 189 lacked Pfam domains. The candidates were further screened for known conserved motifs of effectors and similarity to known effectors. A total of 33 CSEPs with no orthologs in 19 related species and no Pfam domains, were presumed to be $C$. tanaceti-specific. These effector candidates can be utilized for investigating their biological function in pathogenesis and for breeding of pyrethrum for resistance.

Comparative genomic analysis of Fusarium oxysporum f. sp. vasinfectum isolates and their small secreted proteins S. SEO, J. Coleman, Auburn University, Auburn, AL, USA

Fusarium oxysporum f. sp. vasinfectum (Fov) causing vascular wilt disease is one of the most devastating pathogens of cotton (Gossypium spp). To elucidate the molecular mechanisms responsible for host-specificity for pathogenicity on cotton, five Fov isolates were sequenced using PacBio SMRT sequencing and the genomes compared with $10 \mathrm{~F}$. oxysporum genomes that are non-virulent on cotton. Genome analysis revealed that the highly virulent genotype race 4 had the largest genome size and coding genes and repetitive elements, compared to the other four Fov isolates. The genome of $F$. oxysporum comprises both core and accessory regions, and all the genomes of the Fov isolates were able to be divided into these two main architectures based on the genome of $F$. oxysporum f. sp. lycopersici (Fol) as a reference. Comparative analysis among both cotton and non-cotton isolates of $F$. oxysporum focused on small secreted proteins (SSPs), which may function as virulence factors termed fungal effectors. The results showed that all isolates had similar proportions of SSPs based on the whole genome following a universal effector prediction pipeline. A total of 165 proteins were common to all the different formae speciales of F. oxysporum. Importantly, 56 SSPs are unique to the most virulent genotype and 17 SSPs existed only in the cotton Fov isolates. In summary, each Fov isolate genome reflects the diversity of pathogenesis-related genomic features, sharing or differing from each other, and may yield insights into the development of disease management strategies for cotton wilt.

Metabolome and transcriptome analyses to study plant-virus interaction: The case of study Onion yellow dwarf virus - 'rossa di tropea' onion A. TIBERINI (1), F. Mercati (2), F. Araniti (1), A. Ciampa (3), G. Micali (1), A. Taglienti (4), G. Leo (1), M. R. Abenavoli (1), M. T. Dell'abate (3), F. Sunseri (1), L. Tomassoli (4), G. R. Albanese (1), (1) Università degli studi Mediterranea, Reggio Calabria, ITALY; (2) CNR- Istituto di Bioscienze e BioRisorse (IBBR) - U.O.S., Palermo, ITALY; (3) CREA - Centro di Ricerca Agricoltura e Ambiente, Roma, ITALY; (4) CREA - Centro di Ricerca Difesa e Certificazione, Roma, ITALY

The -omics sciences are becoming a fundamental tool to better understand and investigate host-pathogen interactions, highlighting the complexity of plant responses. In this study, in the frame of the SI.ORTO project (SIR-MIUR grant - SIORTO-RBSI149LD5), aimed to evaluate the effects of Onion yellow dwarf virus (OYDV, genus Potyvirus) on the accumulation of nutraceutical compounds in 'Rossa di Tropea' onion (IGP trademark), transcriptomic and metabolomic profiles were obtained using RNAseq and gas chromatography-mass spectrometry (GC-MS)/high resolution angle magic spinning NMR (HR-NMR) respectively. Differentially expressed genes (DEGs) and metabolites (DEMs), in bulb onion samples, healthy versus OYDV-infected, showed, independently, an overall modulation of several metabolites connected to sugar and amino-acid metabolism, and the TCA cycle. Further, magnetic resonance micro-imaging (MRI) on whole bulbs, and determination of volatile organic compounds (VOCs) by GC-MS, highlighted a structural alteration and modulation of compounds related to flavor and organoleptic properties respectively in OYDV-infected bulbs. In agreement with metabolomic and transciptomic profiling, preliminary results showed an actual OYDV modulation of important pathways. This research represents a first and multi-disciplinary study on metabolic modulation in a plant-virus pathosystem ('Rossa di Tropea' onion-OYDV).

Genomic analysis of Xanthomonas arboricola: Pathogenicity and development of a real-time PCR protocol for bacterial spot disease of Prunus spp.

J. M. Garita-Cambronero (1), A. Palacio Bielsa (2), J. CUBERO (3), (1) Instituto Tecnológico Agrario de Castilla y León (ITACyL), Valladolid, SPAIN; (2) Centro Investigacion Y Tecnología Agroalimentaria Aragón, Zaragoza, SPAIN; (3) INIA, Madrid, SPAIN

Xanthomonas arboricola pv. pruni (Xap) causes bacterial spot of stone fruits. The bacteria produce lesions that reduce the marketability of fruit and the yield and vigor of the trees. Xap is within the interesting Xanthomonas genus, which has been intensively studied due its strain specialization and its host range complexity. Comparative genomics of Xanthomonas arboricola revealed the evolutionary history of the pathogenic bacteria of this species, as well as a characterization of factors involved in virulence. Phenotypic assays on Xanthomonas, isolated from Prunus, have revealed the coexistence of nonpathogenic strains phylogenetically different to those pathogenic ones. Taking advantage of the variation in genomic features, a real-time PCR protocol, based on the xopE3 gene, has been developed to differentiate Prunus-pathogenic and non-pathogenic strains of X. arboricola and to refine the diagnosis of this quarantine pathogen. The use of this protocol in conjunction with a previous real-time PCR test based on the gen $f$ ts $X$, showed a high specificity to differentiate pathovar pruni from the other groups of $X$. arboricola. The new real time protocol is a valuable molecular tool for the diagnosis of the bacterial spot of stone fruits and almond and the detection of its causal agent. This work was supported financially by the Instituto Nacional de Investigación y Tecnología Agraria y Alimentaria (INIA) project RTA2014-00018.

Mining the Penicillium expansum proteome to unlock fungal virulence mechanisms during postharvest apple fruit decay W. M. JURICK II PHD (1), H. Beard (2), W. Garrett (3), V. L. Gaskins (1), B. Cooper (2), (1) USDA-ARS Food Quality Laboratory, Beltsville, MD, USA; (2) USDA-ARS Soybean Genomics \& Improvement Laboratory, Beltsville, MD, USA; (3) USDA-ARS Animal Biosciences \& Biotechnology Laboratory, Beltsville, MD, USA

The blue mold fungus, Penicillium expansum, is one of the most globally important postharvest pathogens. The fungus reduces fruit quality, produces harmful mycotoxins, and contributes to food waste. It is a successful saprophyte, and is an aggressive necrotrophic wound pathogen that utilizes complex biochemical processes to overcome the host. To better understand fundamental mechanisms of $P$. expansum virulence, we compared the proteomes of an aggressive, wild-type strain (R19) and a lesser virulent mutant with a T-DNA insertion in the Heat Shock Protein 60 gene (HSP60). Proteins were extracted from mycelia, and peptides were labeled with isobaric tandem mass tags and analyzed by mass spectrometry. More than 3,500 proteins were quantified and mapped to known biochemical pathways in KEGG. In addition, proteins released into the culture medium were separately purified and analyzed. These included at least 100 secreted proteins (i.e. CAZymes) such as aspartic proteases, glucanases, invertases, and pectate lyases. It is likely that these enzymes contribute to decay by digesting host proteins and complex carbohydrates in apple. This study is the most detailed proteomic evaluation of this pathogen to date, and the results will provide a deeper understanding of how this pathogen causes disease. Our end goal is to translate these findings into new controls by interfering with fungal pathways, effectors and processes vital for blue mold decay development during storage. 
Metabolomics approach to elucidate the mechanisms underlying biological control of Fusarium root rot by PGPR L. P. Parikh, A. O. ADESEMOYE, University of Nebraska Lincoln, North Platte, NE, USA

Plant growth promoting rhizobacteria (PGPR) have been used to control plant pathogens and enhance growth. The mechanisms used by many PGPR strains are unclear. A better understanding of the mechanisms could help improve efficacy. Fusarium root rot is a major soilborne pathogen of economic importance in row crops. F. graminearum and F. oxysporum are the two most important species causing Fusarium root rot in Nebraska. Chemical control is the most widely used method in controlling Fusarium root rot pathogens. Biological control, if understood, could be an important component of an effective integrated pest management system. Burkholderia ambifara C628 and Bacillus simplex R180, are PGPR strains with effective biological control against Fusarium root rot. The objectives of this research were to (1) extract, purify, and characterize the secondary metabolites secreted by Burkholderia ambifara C628 and Bacillus simplex R180 and (2) determine the role of the identified secondary metabolites in the biological control activities. These two PGPR strains were screened for lipopeptide antibiotic genes fengycin, bacillomycin, surfactin, iturin, and zwittermicin using PCR primers specific to the genes. Strain C628 showed the presence of only iturin A gene while R180 showed the presence of surfactin, bacillomycin D, and iturin A genes. These metabolites were extracted by acid precipitation from liquid cultures. The cell-free metabolite and crude extract showed antifungal bioactivity against pathogenic strains $F$. graminearum and $F$. oxysporum. The extract is being characterized by chemical analysis through high performance liquid chromatography-mass spectrometry.

The infectious process of Colletotrichum lupini, a major threat for lupin crops G. DUBRULle, F. Pensec, A. Picot, R. Baroncelli, S. Madec, A. Pawtowski, G. Le Floch, Université de Brest, EA 3882, LUBEM, IBSAM, ESIAB, Plouzané, FRANCE

Colletotrichum lupini is a species within the Colletotrichum acutatum species complex (CAsc) causing anthracnose, a serious disease affecting lupin crops worldwide. In addition to its economic impact, $C$. lupini is a useful model to study host speciation process since it is known to infect only lupins. To date, this pathogenic fungus has been poorly described. In this context, our work aims at getting better insight into the genome, the physiology and the infectious process of the fungus. First, based on the recent annotation of two $C$. lupini reference genomes, we identified specific regions of $C$. lupini that are absent in the genome of other species from the CAsc. In order to better understand the infectious process of the fungi, transcriptomic and proteomic analyses were performed on $C$. lupini infected lupin seeds during transition from the biotrophic to the necrotrophic stage. The results allowed us to identify specific pathogenicity gene markers of both stages. The expression of these markers has been further evaluated using a set of $C$. lupini strains. These results together with the description of the physiology of $C$. lupini during the first steps of the infection will significantly increase knowledge in host speciation process and ultimately contribute (i) to the early detection of the fungus in the field and (ii) to the development of new resistant lupin varieties.

Molecular evolutionary genomics and population structure of Iris yellow spot orthotospovirus (Family: Tospoviridae; Genus: Orthotospovirus) A. TABASSUM (1), S. V. Ramesh (2), Y. Zhai (1), R. Iftikhar (3), H. R. Pappu (1), (1) Department of Plant Pathology, Washington State University, Pullman, WA, USA; (2) ICAR, Kasaragod, Kerala, INDIA; (3) Government GC Women's University, Faisalabad, PAKISTAN

Iris yellow spot orthotospovirus (IYSV) is an important virus of Allium sps. Using the global repository of IYSV nucleoprotein (N) gene sequences available in GenBank (a total of 142), temporal dynamics and geographic diversity of the IYSV isolates were investigated to gain better insights into the evolutionary forces influencing genetic population structure of IYSV. Based on In Silico Restriction Fragment Length Polymorphism (RFLP), N gene sequences showed a 79:55 distribution of known IYSV isolates into two major genotypes, IYSV Netherlands (IYSV $\mathrm{NL}_{\mathrm{NL}} ; 55.63 \%$ ) and IYSV Brazil (IYSV ${ }_{\mathrm{BR}} ; 38.73 \%$ ). A little over five percent of the isolates fell in neither group [IYSV other (IYSV $\mathrm{other}_{3} ; 5.63 \%$ )]. Phylogenetic tree and phylogenetic networks largely corresponded to the RFLP pattern. Nucleotide diversity comparisons indicated IYSV $V_{\mathrm{BR}}$ to be least differentiated from IYSV $\mathrm{other}$ compared to the IYSV $V_{\mathrm{NL}}$ type. Two recombinants were detected among all the $\mathrm{N}$ gene sequences that were analysed through RDP4 and RAT programs. The marginal likelihood mean substitution rate was $5.09 \times 10^{-5}$ subs/site/year and $95 \%$ highest posterior density (HPD) substitution rate between $5.11 \times$ $10^{-5}-5.07 \times 10^{-5}$. The substitution rate identified for IYSV $\mathrm{N}$ gene is similar to other plant RNA viruses. Evolution studies of IYSV will help to understand the disease epidemiology and design appropriate disease control measures.

Proteomics analysis based on ITRAQ LC-MS/MS reveals novel roles of hshB in Xanthomonas oryzae pv. oryzicola Y. ZHAO, Z. Song, F. Liu, Institute of Plant Protection, Jiangsu Academy of Agricultural Sciences, Nanjing, CHINA

Xanthomonas oryzae pv. oryzicola $(X o c)$ is the causal agent of the bacterial leaf streak (BLS) which is one of the most serious bacterial diseases in rice (Oryza sativa). Previous research showed that the transcription of the gene $h s h B$ was positively regulated by a diffusible signal factor (DSF)-dependent quorum sensing system, and it was required for the virulence, extracellular protease activity, extracellular polysaccharide production, and resistance to oxidative stress in Xoc. To better understand functions of $h s h$, we conducted a comparative proteome analysis between Xoc wild type strain Rs105 and $h s h B$ mutant by using iTRAQ LC-MS/MS. The results clearly revealed that the expression of 621 proteins had significant changes in $h s h B$ mutant compared to the wild type strain. Among them, the expression of 150 proteins was significantly up-regulated, and 471 down-regulated. Based on our further analysis and experimental results, it showed that $h s h B$ could affect protein expression levels of 32 transcription factors, and 5 of them were demonstrated to be involved in the virulence, cell motility, extracellular polysaccharide production, and resistance to oxidative stress of Xoc. Moreover, the direct interaction of $\mathrm{HshB}$ with fle $Q$ promoter was demonstrated by gel shift assay. To our knowledge, our results revealed the regulation mechanism of $h s h B$ to transcription factors for the first time, and expanded understanding of $h s h B$ in $X$ oc.

Genome architecture and virulence gene dynamics in oat crown rust populations

M. Miller (1), S. Rottschaefer (2,3), A. Persoons (4), C. Hirsch (2), D. Saunders (4), E. H. Stukenbrock (5,6), S. Kianian (2,7), M. FIGUEROA (1), (1) Department of Plant Pathology, University of Minnesota, St. Paul, MN, USA; (2) University of Minnesota, St. Paul, MN, USA; (3) University of Minnesota, Minneapolis, MN, USA; (4) John Innes Centre, Norwich, UNITED KINGDOM; (5) Christian-Albrechts University of Kiel, Environmental Genomics, Kiel, GERMANY; (6) Max Planck Institute for Evolutionary Biology, Plön, GERMANY; (7) USDA-ARS, Cereal Disease Laboratory, St. Paul, MN, USA

Crown rust caused by Puccinia coronata f. sp. avenae $(P c a)$ is the most destructive foliar pathogen of cultivated oat, Avena sativa. Populations of $P c a$ display very high genetic variability as demonstrated by the numerous virulence profiles that can be captured during national and international rust surveys. Such levels of polymorphisms likely confer evolutionary advantages to the pathogen and drive adaptation to plant resistance genes deployed in varieties. During the last two decades, $P c a$ populations in the US have undergone drastic virulence shifts yet the underlying genetic and molecular mechanisms responsible for these changes are not understood. Capitalizing on the recent release of two genome references for $P c a$, a comparative population genomics study of two Pca populations collected in 1990 and 2015, respectively, was launched. Our goal is to determine the population 
structure and dynamics of $P c a$, as well as the evolutionary processes that drive virulence changes over time, particularly in response to selective pressures and occurrence of sexual reproduction. Overall, this work contributes to the understanding of the biology of $P c a$ and enables the development of molecular markers associated with rust virulence.

Pheromone expression in the unisexual fungus, Huntiella moniliformis

A. WILSON (1), M. van der Nest (1), M. Wilken (2), M. J. Wingfield (2), B. D. Wingfield (2), (1) FABI, University of Pretoria, Pretoria, SOUTH AFRICA; (2) Forestry and Agricultural Biotechnology Institute (FABI), University of Pretoria, Pretoria, SOUTH AFRICA

Unisexuality is a reproductive strategy which enables fungi that are normally self-sterile, to reproduce in the absence of a compatible mating partner. The process is well documented in two human pathogens, but has also been described in the wood-infesting Huntiella moniliformis. This fungus is a member of the Ceratocystidaceae, a family that includes known pathogens of agronomic crops and trees. In this study, a comparative transcriptomics approach was used to elucidate the molecular mechanisms responsible for unisexuality in $H$. moniliformis. By sequencing RNA from vegetative and sexuallycompetent isolates of $H$. omanensis, a self-sterile relative of $H$. moniliformis, we identified genes important for sex. This was compared to similar data obtained from $H$. moniliformis to detect significant differences between the two reproductive pathways. Notably, the pheromones in $H$. moniliformis were expressed in a mating-type-independent manner. This contrasted with pheromone expression in $H$. omanensis, which exhibited a strict mating-typedependence. H. moniliformis also expressed both pheromone receptors, suggesting that it can recognize and respond to both pheromones. The results indicated that this unusual pheromone expression system may play a role in the unisexual capabilities of $H$. moniliformis. Given the strong link between unisexuality and pathogenicity in the human pathogens, further investigation into this mating strategy in $\mathrm{H}$. moniliformis would be worthwhile.

The transcriptome of roots of sweet orange tree with symptoms of citrus blight

S. Fu (1), J. Shao (2), A. Roy (3), R. H. Brlansky (4), J. S. HARTUNG (5), (1) Southwest University, Chongqing, CHINA; (2) USDA ARS Molecular Plant Pathology Lab, Beltsville, MD, USA; (3) USDA-APHIS-PPQ-S\&T-CPHST, Beltsville, MD, USA; (4) University of Florida, Lake Alfred, FL, USA; (5) USDA ARS MPPL, Beltsville, MD, USA

Citrus blight is a very important progressive decline disease of commercial citrus in Florida. Diagnosis is made by demonstrating physical blockage of xylem cells that prevents the movement of water through affected portions of the trunk. The etiology of the disease has not been established, although the disease can be transmitted by root grafts, suggesting a viral etiology. We used a direct water uptake assay in citrus trunks to identify symptomatic trees from 4 commercial groves in Florida. Scaffold roots were taken from trees that failed to take up water and total RNA was extracted from phloemenriched root tissues. Seven RNA extracts were used as templates for 100 bp paired-end RNASeq with the Illumina system. The overall pattern of transcription in the seven libraries was very similar and about 200 transcripts $(6 \%)$ were up or down regulated in all seven libraries. These transcripts were limited to pathways that included modifications to cell walls, minor carbohydrate metabolism, lipid metabolism, secondary metabolism (notably terpenes) and mitochondrial electron transport. An analysis of gene ontology showed large alterations to metabolic and cellular processes, biological regulation and cellular component organization. Trehalose metabolism was altered, consistent with a defense response to dehydration or other cellular stress. Identification of specific metabolic pathways altered by citrus blight disease may lead to improved management of the disease.

Whole genome comparisons of the host specific species Ceratocystis fimbriata sensu stricto and $C$. manginecans A. FOURIE (1), R. de Jonge (2), M. van der Nest (1), M. J. Wingfield (1), B. D. Wingfield (1), I. Barnes (1), (1) Forestry and Agricultural Biotechnology Institute (FABI), University of Pretoria, Pretoria, SOUTH AFRICA; (2) Plant-Microbe Interactions, Department of Biology, Faculty of Science, Utrecht University, Utrecht, NETHERLANDS

The fungus Ceratocystis manginecans is a devastating pathogen of trees in fruit orchards and forestry plantations world-wide. It causes canker and wilt disease on a broad range of species, including mango, pomegranate and wattle, but has never been isolated from tuber crops. In contrast, the closely related species $C$. fimbriata sensu stricto infects only sweet potato where it causes black rot. In this study, the genome sequences of three $C$. manginecans isolates and one $C$. fimbriata isolate were determined and compared, to identify genetic differences associated with host specificity. Results showed that $C$. manginecans has a marginally larger genome size $(32 \mathrm{Mb}$ vs $30 \mathrm{Mb})$, which could be attributed to a higher repetitive element content $(18 \%$ vs $13 \%)$, but the species had similar gene numbers $(\sim 7200)$. Only $1 \%$ of the genes was unique to a species, of which many were hypothetical proteins or putative secreted effectors. Equivalent catalogues of CAZYs, peptidases, lipases and secondary metabolite biosynthetic gene clusters were detected in both species. Genome alignments identified exclusive genomic regions containing genes that were only present in $C$. manginecans. Future functional characterization should reveal the roles of the identified unique genes in the biology of these fungi and their ability to thrive in their niches.

The composition and expression of Carbohydrate-Active Enzymes in Rhizoctonia cerealis transcriptome W. LI, X. Ren, H. Sun, N. Wang, H. Chen PhD, Jiangsu Academy of Agricultural Sciences, Nanjing, CHINA

The soilborne fungus Rhizoctonia cerealis, which belongs to the binucleate Rhizoctonia anastomosis group AG-DI, is the pathogen of wheat sharp eyespot in the temperate regions of the world with significant impacts on grain yield and quality. Until now, several genome sequences of $R$. solani isolates which belong to the multinucleate Rhizoctonia, have been published. However, it has very rarely been reported in binucleate Rhizoctonia spp isolates and has never been described in $R$. cerealis. In this study, we sequenced the transcriptome and secretome of $R$. cerealis strain R0301 using Illumina Genome Analyzer sequencing technology, and analyzed the composition and expression of Carbohydrate-Active Enzymes in this fungus. The results indicated that among 21,021 unigene entries of $R$. cerealis transcriptome, $465(2.2 \%)$ could match with one CAZyme family. These putative CAZymes include 44 families of carbohydrate esterases (CE), 255 families of glycoside hydrolases (GH), 146 families of glycosyltransferases (GT), and 19 families of polysaccharide lyases (PL). The species and quantities of CAZymes in $R$. cerealis were similar with the CAZymes in $R$. solani AG1-IA and AG8 genome, which all of them are the monocotyledon pathogen. We further compared the CAZyme expression patterns in $R$. cerealis under three different conditions, the results show that many CAZyme genes were significantly up-regulated during the infection of wheat, which indicated that some of the CAZymes would play important roles in the infection process. This study will serve as a resource for future studies to understand the infection mechanism of $R$. cerealis to wheat.

Genome-based identification of genes involved in pathogen interactions with Brassica crops H. U. Stotz (1), N. Larkan (2), P. Haddadi (3), H. Borhan (4), B. Fitt (1), C. KARANDENI-DEWAGE (1), (1) University of Hertfordshire, Hatfield, UNITED KINGDOM; (2) Armatus Genetics Inc., Saskatoon, CANADA; (3) Agriculture and Agri-Food Canada, Saskatoon, CANADA; (4) Agriculture and Agri-Food Canada, Saskatoon, SK, CANADA 
A wealth of genomic information, deposited in public databases, is available to identify candidate genes that contribute to interactions between crops and their pathogens. Of interest to us are pathogens that pose global threats to production of Brassica oilseed and vegetable crops, including Brassica napus, Brassica juncea, Brassica rapa and Brassica oleracea. We have used published and in-house genome sequences to determine the distribution of genes encoding leucine-rich repeat (LRR) receptors in the genome of B. napus. Specific attention was given to a resistance $(R)$ gene cluster on chromosome A7 that operates against the phoma stem canker pathogen Leptosphaeria maculans. Tests for positive selection were used to decrease the number of candidate LRR genes, which can be used as cloning and breeding targets. In addition, the genomic intervals of known major and quantitative trait loci for resistance against $L$. maculans, Sclerotinia sclerotiorum and the clubroot pathogen Plasmodiophora brassicae were used to determine the specific contribution of genes encoding nucleotide-binding, secreted or transmembrane LRR receptors. Furthermore, work is proceeding to better understand molecular components of compatibility of B. napus to L. maculans and its sister species Leptosphaeria biglobosa. This research is of benefit to our academic and industry colleagues working on plant breeding.

Does transmission mode shape PVY quasispecies? Insights from Illumina deep sequencing W. L. DA SILVA (1), S. Gray (2), (1) Cornell University, Ithaca, NY, USA; (2) Section of Plant Pathology and Plant-Microbe Biology, Cornell University, Ithaca, NY, USA

Surveys indicate that recombinant strains of Potato virus $Y(\mathrm{PVY})$ have emerged in recent years to predominate in the U.S. potato crop, and that the genetic diversity abounds among and within PVY strains. To investigate how transmission modes (insect and mechanical) are contributing to the evolution and diversification of PVY, we designed an experiment to characterize the virus quasispecies through multiple aphid or mechanical passages of three PVY strains $\left(\mathrm{O}, \mathrm{N}\right.$, and NWi). On average, more single nucleotide variations (SNVs) were detected in $\mathrm{PVY}^{\mathrm{N}}$ populations $(250)$ than in PVY ${ }^{\mathrm{O}}(107)$ or PVY ${ }^{N W i}(150)$. Unique SNVs were detected for each transmission mode for each virus strain and more SNVs were detected in aphid transmitted than mechanical inoculated populations. Many of the SNVs were located in cistrons that encode proteins involved in aphid transmission (HC-Pro and CP) or cell-to-cell movement (P3, CI, and CP). Our data suggest that each transmission mode exerts unique selection pressures on the virus population and allows different mutations to accumulate and become fixed. To date, aphid transmission induces more unique changes in the PVY quasispecies than mechanical inoculation. The next step is to link genotypic changes to phenotypes.

Characterizing the epidemiological link between transplant and field outbreaks of bacterial spot on tomato with whole genome sequencing P. ABRAHAMIAN (1,2), S. Timilsina (2), G. V. Minsavage Jr. (2), J. B. Jones (2), E. M. Goss (2,3), G. E. Vallad (1,2), (1) Gulf Coast Research and Education Center, University of Florida, Wimauma, FL, USA; (2) Department of Plant Pathology, University of Florida, Gainesville, FL, USA; (3) Emerging Pathogens Institute, Emerging Pathogens Institute, FL, USA

Bacterial leaf spot (BLS), caused by Xanthomonas perforans $(X p)$, is a major tomato disease. During transplant production, environmental conditions, grower practices and lack of efficient disease control measures contribute to severe BLS outbreaks. In Florida, growers primarily rely on transplant seedlings for field production. Therefore, we hypothesized that disease outbreaks in the field are caused by using contaminated transplant seedlings. Our objective was to monitor and characterize $X p$ outbreaks associated with tomato seedlings in transplant houses and in production fields. We surveyed $X p$ strains from two commercial transplant growers (A and B) and tracked the same seedlings of different cultivars into corresponding fields. A total of 67 strains were sequenced on the Illumina MiSeq2000 platform. The pairwise average nucleotide identity between strains was higher than $99 \%$. Transplant and field strains clustered together by grower, irrespective of cultivar or time. Single nucleotide polymorphism (SNP)-based analysis showed that $90 \%$ and $80 \%$ of field strains showed high genetic similarity to at least one or more transplant strains from grower A and B, respectively. Our SNP-based analysis demonstrated that contaminated seedlings are a major source of introducing $X p$ strains into fields. As a result, BLS management during tomato transplant production is critical to reduce $X p$ levels prior to field planting.

\section{Approaches to effectively use pathogenomics for wheat crop protection against rusts}

G. BAKKEREN (1), H. B. Khalil (2), V. Panwar (3), X. Wang (4), R. Linning (5), D. L. Joly (6), D. Cram (7), M. Jordan (8), B. McCallum (9), B. Saville (10), (1) Agric \& Agri Food Canada, Summerland, BC, CANADA; (2) Department of Genetics, Faculty of Agriculture, Ain Shams university, Cairo, EGYPT; (3) National Research Council of Canada, Morden, MB, CANADA; (4) Agriculture \& Agri-Food Canada, Morden Research \& development centre, Morden, CANADA; (5) Agriculture \& Agri-Food Canada, Summerland Research \& development Centre, Summerland, BC, CANADA; (6) Université de Moncton, Moncton, NB, CANADA; (7) National Research Council of Canada, Plant Biotechnology Institute, Saskatoon, CANADA; (8) Agriculture \& Agri-Food Canada, Morden Research \& Development Centre, Morden, CANADA; (9) Agric \& Agri Food Canada, Morden, MB, CANADA; (10) Trent University, Peterborough, ON, CANADA

To improve our understanding of the globally important wheat rust pathogen, Puccinia triticina (Pt), we have sequenced 95 genomes of Canadian isolates and a variety of transcriptomes of interactions between specific races and Near-Isogenic Lines carrying specific leaf rust $(L r)$ resistance genes. Comparative analyses of high-resolution RNAseq datasets led to the identification of 700 effectors predicted to be secreted and expressed during host infection and allowed us to discriminate between specific resistance responses triggered upon recognition by $\operatorname{Lr} 3$ versus $L r 2 a$ with the latter revealing induction of a significant number of chloroplast-related genes. We were also able to visualize global gene expression reprogramming of a suite of $126 P t$ effectors and wheat genes, and, using CytoScape, revealed gene co-expression networks possibly involved in disease or defense pathways. Transcription of some of the identified wheat genes is likely affected by some effectors having a nuclear localization. Fluorescent chimers of five $P t$ effectors, whose transcript levels were highly associated with wheat transcripts across various interactions, were found to be targeted to diverse plant organelles including the chloroplast in a heterologous Nicotiana benthamiana system. A novel functional effector secretion assay was developed in a cereal-infecting Ustilago species to potentially assist $L r$ gene breeding, and essential fungal pathogenicity genes were targeted in a HIGS approach resulting in increased rust resistance in wheat. Examples will be presented to illustrate that generating genomic resources can drive research innovations for crop protection.

Investigating the Role of Trehalose Metabolism During Ralstonia solanacearum Bacterial Wilt Disease A. MACINTYRE (1), C. Allen (2), (1) University of Wisconsin-Madison, Madison, WI, USA; (2) University of Wisconsin, Madison, WI, USA

Ralstonia solanacearum $(R s)$ is a xylem-dwelling pathogen that causes bacterial wilt disease. Unknown metabolic strategies enable Rs to exploit its harsh, nutrient poor niche. Comparative metabolomic analysis revealed that xylem trehalose levels increased 16-fold upon $R s$ infection. This disaccharide plays many roles in plants and bacteria, including stress tolerance and signaling. Rs and host tomato plants both have multiple pathways to synthesize and degrade trehalose. During $R s$ infection, a tomato trehalose-6-phosphate (T6P) synthase gene is upregulated, indicating that disease alters host trehalose metabolism. On the pathogen side, transcriptional analyses showed that the $R s$ trehalase gene, tre $A$, is highly upregulated during bacterial wilt disease. Rs $\triangle$ treA cannot grow on trehalose as a sole carbon source, and cannot colonize tomato plants as well as wild-type. Together, these observations suggest that trehalose metabolism in tomato and Rs contributes to bacterial virulence and fitness during disease. We are testing this hypothesis using a suite of $R s$ altered trehalose metabolism mutants, including strains lacking one or more of the pathogen's three trehalose synthetic 
pathways, and a type 3 effector with T6P activity. We will determine the effect of these mutations on $R s$ stress tolerance, xylem sugar levels, competitive fitness in planta, and virulence. This is the first study of trehalose biology in the context of a natural host for this destructive pathogen.

Diversity of the Microbacteriaceae, with focus on the plant pathogenic genera Clavibacter and Leifsonia, based on environmental 16S data T. GALHARDO EGREJA RIBEIRO SILVA (1), J. R. Herr (2), (1) Department of Plant Pathology, University of Nebraska, Lincoln, NE, USA; (2) The Center for Plant Science Innovation, University of Nebraska, Lincoln, NE, USA

The family Microbacteriaceae, which contains numerous plant pathogens, are gram-positive bacteria with predominantly high GC content. The most notable genera in this family are pathogens of economically important monocots. For example, Clavibacter is the causal agent of Goss's bacterial wilt and leaf blight in corn and Leifsonia is the causal agent of ratoon stunting disease (RSD) in sugarcane. Taxonomic and phylogenetic diversity of this bacterial family has lagged behind those of other pathogens despite the prevalence of the Microbacteriaceae in many environments (e.g. plant tissue, thermal hot springs, and ocean water). In order to characterize this important family, we mined approximately 1.4 billion $16 \mathrm{~S}$ sequences that had been deposited into NCBI's SRA raw data repository. These were then clustered into 53,696,442 sample-specific OTUs (operational taxonomic units) using the QIIME pipeline. Of those initial OTUs, 180,806 OTUs showed 95\% synteny to OTUs designated as members of the Microbacteriaceae from cultural studies and deposited in the SILVAngs database. A consensus tree was generated from 1,568 MCMC Bayesian phylogenies and consisted of four major sub-familiar lineages which do not conform to previously described subfamilies. We hypothesize that pathogenicity is dependent on genomic diversity and/or the presence of integrated mobile elements.

Dynamics of chromosomal and plasmid-borne copper resistance systems in Xanthomonas perforans populations R. BHANDARI (1), G. V. Minsavage Jr. (2), E. Newberry (3), J. Klein (2), E. M. Goss (2), G. E. Vallad (4), J. B. Jones (2), N. Potnis (3), (1) Auburn University, Auburn, AL, USA; (2) Department of Plant Pathology, University of Florida, Gainesville, FL, USA; (3) Department of Entomology and Plant Pathology, Auburn University, Auburn, AL, USA; (4) Gulf Coast Research and Education Center, University of Florida, Wimauma, FL, USA

Bacterial spot of tomato and pepper is a major problem around the world. Repeated use of copper bactericides has rendered pathogen populations resistant to copper. Bacterial spot xanthomonads have evolved several mechanisms to efflux copper from bacterial cells, including the cop (copper resistance) systems. The Cop system in bacterial spot xanthomonads collected in the 1980s was plasmid-borne, with the exception of a unique chromosomal copper resistance operon characterized in strain XVP26, isolated in Taiwan. Movement of a large copper resistance plasmid (200-300kb) has been described as a mechanism for the spread of copper resistance among pathogenic xanthomonads. We recently identified several copper-tolerant X. perforans (Xp) strains collected in Alabama and Florida between 1990-2016 that do not contain these large plasmids. Analysis of complete genomes of representative strains obtained by PacBio SMRT sequencing revealed the presence of a copper resistance operon encoding CopL, CopA, CopB and $\mathrm{CopF}$ on a genomic island on the chromosome. Further screening of draft genomes of additional $X p$ strains indicated that chromosomal copper resistance system is spread in certain lineages of $X p$ populations in the U.S. Our findings indicate that continuous use of copper bactericides in the field has imposed a constant selective pressure on $X p$ populations leading to a dramatic shift in the balance of chromosomal copper resistance and plasmidencoded copper resistance.

Detection and Characterization of pXFSL21, a Novel Single-Copy Plasmid from Xylella fastidiosa Strain Stag's Leap C. Van Horn (1), F. Wu (2), Z. Zheng (2), J. CHEN (3), (1) USDA-ARS-PWA, Parlier, CA, USA; (2) South China Agricultural University, Guangzhou, CHINA; (3) USDA, ARS, SJVASC, Parlier, CA, USA

Xylella fastidiosa Strain Stag's Leap, originally isolated from Napa Valley, California, is highly virulent in causing Pierce's Disease (PD) of grapevine. Plasmids are extrachromosomal genetic elements associated with bacterial environmental adaptation such as virulence development. In this study, the plasmid status of Strain Stag's Leap was studied. DNA samples extracted from pure culture in PW medium (in vitro) and PD grapevine in greenhouse (in planta) were subject to next generation sequencing (NGS, MiSeq/HiSeq formats). Through de novo assembling and primer walking techniques, a circular plasmid, pXFSL21, of 21,665 bp was identified from MiSeq data (in vitro DNA) and confirmed by HiSeq data (in planta DNA) using referenced assembling. NGS read mapping and PCR experiments indicated that pXFSL21 was single-copied per genome both in vitro and in planta. The plasmid had 27 genes/open reading frames (ORFs) encoding proteins of DNA replication (DR), conjugal transfer (CT), two toxin/antidote (TA) systems, and a multidrug resistance (MDR) efflux pump. BLAST search against current GenBank database (version 222.0) showed that pXFSL21 was unique for its TA and MDR genes among the 46 deposited X. fastidiosa plasmids. However, among the whole genome sequences of 41 X. fastidiosa strains, MDR homologs were found in 29 strains and TA system genes were found in four strains. Phylogenetic analyses suggested genes of DR, CT, TA, and MDR were likely of different origins.

Comparative Ralstonia solanacearum dynamics and metabolomic profiling of advanced potato clones with different levels of bacterial wilt resistance

V. Ferreira (1), N. Denis (1), A. Lopez (2), V. Croce (1), M. Perez (1), F. Vilaró (3), G. A. Galvan (4), M. J. Pianzzola (1), M. Valls (5), G. Moyna (2), M. I. SIRI (1), (1) Bioscience Department, School of Chemistry, Universidad de la Republica, Montevideo, URUGUAY; (2) Departamento de Quimica del Litoral, CENUR Litoral Norte, Universidad de la Republica, Paysandu, URUGUAY; (3) National Institute of Agriculture Research (INIA, Las Brujas), Canelones, URUGUAY; (4) Faculty of Agronomy, CRS, Universidad de la Republica, Montevideo, URUGUAY; (5) Center for Research in Agricultural Genomics, Universitat de Barcelona, Barcelona, SPAIN

Bacterial wilt caused by Ralstonia solanacearum is responsible for substantial losses in cultivated potato crops worldwide. Breeding for bacterial wilt resistance is a major challenge for disease control, especially in regions where the pathogen is endemic. A potato breeding program is conducted in Uruguay based on the introgression of resistance from the wild native species Solanum commersonii. In this work we report the characterization of advanced potato clones with different levels of bacterial wilt resistance. First, we performed the comparison of pathogen colonization and dissemination patterns after plant inoculation with $R$. solanacearum fluorescent and luminescent reporter strains. Resistant clones showed colonization only in roots and the stem base suggesting that resistance in potato is achieved through restriction of bacterial invasion and multiplication in stems. In addition, we performed a metabolomic profiling of roots, stems, leaves and xylem sap based on NMR analysis. Plant samples were collected from non-inoculated plants and from plants soil-inoculated with $R$. solanacearum at 7 dpi. ${ }^{1} \mathrm{H}-\mathrm{NMR}$ spectra of plant metabolites were acquired and subjected to multivariate analyses, allowing differentiation among infected and non-infected plants. The relationship between the metabolite profiles and resistance was also explored. This study paves the way for understanding potato resistance to $R$. solanacearum and for the identification of relevant metabolites involved in this plant-pathogen interaction. 
Genome sequence of the common bean rust pathogen suggests coevolution with its common bean host

O. P. Hurtado-Gonzales (1), J. Diaz Valderrama (2), M. C. Aime (2), M. PASTOR-CORRALES (1), (1) Soybean Genomics \& Improvement Lab, BARC-West, ARS-USDA, Beltsville, MD, USA; (2) Purdue University, West Lafayette, IN, USA

Achievement of durable resistance to Uromyces appendiculatus, the causal agent of the common bean rust disease, is difficult due to the extensive virulence of this pathogen. Yet this diversity segregates into two distinct groups that correspond to the Mesoamerican and Andean gene pools of common bean, suggesting that $U$. appendiculatus has coevolved with its host. To find additional evidence for coevolution, we are using our sequenced data of the genomes of an Andean race (5-0) and a Mesoamerican race (31-1) of U. appendiculatus. De novo assemblies resulted in genomes of $587.6 \mathrm{Mb}$ and 546.7 $\mathrm{Mb}$ for races 5-0 and 31-1, respectively. These assemblies contained a high number of contigs, suggesting a complex and highly repetitive genome that is larger $(>500 \mathrm{Mb})$ than most of the reported genomes of fungal plant pathogens. Over 110,000 simple sequenced repeats (SSRs) were also identified in each genome assembly. Sixteen SSRs markers were designed and tested on a world collection of 46 Mesoamerican and Andean races of $U$. appendiculatus maintained at Beltsville. A neighbor-joining analysis separated these races into two major groups that also corresponded to the Andean and Mesoamerican gene pools of common bean. These results suggest coevolution between $U$. appendiculatus and its common bean host and are crucial to the development of strategies that could result in durable host resistance to the hyper virulent bean rust pathogen.

Potential role of small noncoding RNAs in regulating hypovirulence in Rhizoctonia solani anastomosis group 3 E. R. CHAMPACO (1,2), A. Kitazumi (3), R. P. Larkin (2), S. M. Tavantzis (1), B. G. de los Reyes (3), (1) University of Maine, Orono, ME, USA; (2) USDA-ARS, New England Plant, Soil, \& Water Lab, Orono, ME, USA; (3) Texas Tech University, Lubbock, TX, USA

Double-stranded RNA (dsRNA) elements are frequently associated with fungi. In Rhizoctonia solani anastomosis group-3 (AG3), the $3.6 \mathrm{~kb}$ dsRNA element M2 has been associated with the hypovirulence of Rhs1A1 strain, enabling its use as a biological control agent. Previous studies that examined the role of M2 in downregulating virulence led to a number of hypotheses, but the precise molecular mechanisms are yet to be elucidated. We began to address the hypothesis that M2 might be a possible source of noncoding regulatory RNAs involved in downregulating the expression of virulenceassociated genes encoded in the nuclear genome either by post-transcriptional or transcriptional gene silencing mechanisms. We conducted a parallel analysis of RNA-Seq data of the virulent strain (Rhs1 AP) and hypovirulent strains (Rhs1A1 and Bs69) using mycelial RNAs from cultures grown on isopore membranes overlaid on potato dextrose agar. Over two dozen noncoding regulatory RNAs (miRNA, siRNA) that could potentially arise from M2 were identified. Possible targets of these regulatory RNAs in the nuclear genome were also identified for reverse co-expression analysis.

Understanding the role of root exudation for pathogen germination and attraction, and their application for disease control C. R. WILSON (1), M. A. O. Balendres (2), J. Amponsah (3), D. Nichols (4), R. S. Tegg (1), (1) University of Tasmania, New Town, AUSTRALIA; (2) University of the Philippines, Laguna, PHILIPPINES; (3) University if Tasmania, New Town, AUSTRALIA; (4) University of Tasmania, Sandy Bay, AUSTRALIA

Plant roots release a substantial quantity of primary metabolites (root exudates) into the rhizosphere, the majority of which are low molecular weight organic compounds. These exudates play important roles in interactions between the plant, and other soil biota including soil-borne plant pathogens. Exudate compounds may benefit pathogens, promoting their germination, growth, survival, pathogenesis and reproduction, or may be detrimental inhibiting growth or disrupting motility. With a combined in vitro bioassay, light microscopy and targeted metabolomics approach using HILIC UPLCMS analysis, we demonstrate the importance of potato root exudates for stimulating germination of resting spores of the pathogen Spongospora subterranea f. sp. subterranea. Furthermore, we show these compounds provide a chemotactic signal to attract released zoospores to the host roots enabling efficient infection. We also demonstrate application of this knowledge for novel management of soil inoculum through a 'germinate to exterminate' approach, and propose novel disease management approaches through disruption of pathogen chemical signalling.

Detection of copy number variation for chromosomal sliding windows using high throughput sequencing data in the $R$ environment B. J. KNAUS (1), N. J. Grunwald (2), (1) USDA-ARS, Horticultural Crops Research Unit, Corvallis, OR, USA; (2) USDA ARS, Corvallis, OR, USA

Inference of copy number variation presents a technical challenge because variant callers typically require the copy number of a genome or genomic region to be known a priori. Here we present a method to infer copy number that uses variant call format (VCF) data as input and is implemented in the $\mathrm{R}$ package $\mathrm{vcfR}$. This method is based on the relative frequency of each allele (both genic and non-genic) sequenced at heterozygous positions throughout a genome. These heterozygous positions are summarized by using arbitrarily sized windows of heterozygous positions, binning the allele frequencies, and selecting the bin with the greatest abundance of positions. This provides a non-parametric summary of the frequency that alleles were sequenced at in each window. The method is applicable to organisms that have reference genomes that consist of full chromosomes or sub-chromosomal contigs. It differs from other software designed to detect copy number variation in that it does not rely on an assumption of base ploidy, but instead infers it. We validated these approaches with the model system of Saccharomyces cerevisiae and applied it to the oomycete Phytophthora infestans, both known to vary in ploidy. This functionality has been incorporated into the current release of the R package vcfR to provide modular and flexible methods to investigate copy number variation in genomic projects.

Diversified transcriptional modulation of alternative splicing repertoire during rice-Magnaporthe oryzae interactions J. JEON (1), G. Choi (1), H. Kim (2), G. W. Lee (3), S. Y. Park (4), S. Kim (1), K. T. Kim (1), Y. H. Lee (1), (1) Seoul National University, Seoul, SOUTH KOREA; (2) National Cancer Center, Goyang, SOUTH KOREA; (3) KOREA DNA BANK, Seongnam, SOUTH KOREA; (4) Sunchon National University, Suncheon, KOREA

In post-genome era, one gene to one protein paradigm has shifted to one gene to multiple proteins or functions in whole genome. Among transcript modulating mechanisms, alternative splicing (AS) have potential roles in modulating genomic systems. Although $80.4 \%$ of the genome contains introns, only less than $8 \%$ of genome was revealed as AS containing genes in Magnaporthe oryzae. Similar to transcriptome alterations, AS genes would be reprogrammed during rice-M. oryzae interaction. To unveil ASome networks, we compared genome-wide transcriptional profiles of AS isoforms of fungal pathogen and host plant. We collected infected rice sheaths under microscope to enrich fungal samples as a sequential manner. These samples included vegetative condition and five different infection stages covering pre-penetration, biotrophic, and nectrotrophic stages. In this study, at least one AS isoform of 4,765 genes were found from total conditions, including 3,042 genes from in planta conditions. Two-thirds of isoform patterns showed intron retention, and an increase of this pattern led to the upsizing of ASome infection stages. Among total stages, the largest amount of stage-specific AS isoforms were found in biotrophic stage. We also identified AS isoforms assigned differential function compared with stages by $a b$ initio proteome. This profiling of ASome provides expanded AS repertoire of M. oryzae and shows genome-wide transcriptomic complexity during rice-M. oryzae interactions. 
The wheat pathogen Zymoseptoria tritici senses and responds to different wavelengths of light

C. MCCORISON (1), S. B. Goodwin (2), (1) Purdue University, West Lafayette, IN, USA; (2) USDA ARS, West Lafayette, IN, USA

The ascomycete fungus Zymoseptoria tritici (synonym: Mycosphaerella graminicola) is a major pathogen of wheat that causes the economically important foliar disease Septoria tritici blotch. Despite its importance as a pathogen, nothing is known about the reaction of this fungus to light. To test for light responses, cultures of $Z$. tritici were grown in vitro under white, blue or red light or darkness, and their transcriptomes were compared between each other. There were huge differences in gene expression with over 3,400 genes upregulated in one or more of the light conditions compared to dark, and from 1,909 to 2,573 genes specifically upregulated in the dark compared to the individual light treatments. Differences between light treatments were lower, ranging from only 79 differentially expressed genes in the red versus blue comparison to 585 between white light and red. Analysis of the Gene Ontology (GO) terms showed that those genes related to metabolism were enriched in all three light treatments, while those related to growth and communication were more prevalent in the dark. Interestingly, genes for LysM effectors that have been shown previously to be involved in pathogenicity also were upregulated in one or more of the light treatments, suggesting a possible role of light for infection. This analysis shows that $Z$. tritici can sense and respond to light with a huge effect on transcript abundance.

Dissection of non-host resistance to European pear scab fungus, Venturia pirina using fluorescence phenotyping and transcriptomics S. Johnson (1,2), D. Jones (3), A. Thrimawithana (3), V. Bus (4), H. Ishii (5), J. Bowen (3), C. Deng (3), C. Mesarich (6), K. M. PLUMMER (1,2), (1) Plant Biosecurity CRC, Bruce, ACT, AUSTRALIA; (2) La Trobe University, Bundoora, AUSTRALIA; (3) The New Zealand Institute for Plant \& Food Research Limited, Auckland, NEW ZEALAND; (4) Plant and Food Research, NZ, havelock North, NEW ZEALAND; (5) Kibi International University, School of Agricultural Regional Vitalization, Hyogo 656-0484, JAPAN; (6) Massey University, Palmerston North, NEW ZEALAND

Pear scab, a major fungal disease of pears, is caused by two distinct species of host-specific Venturia: V. nashicola infects Asian pears (Pyrus $\mathrm{x}$ bretschneideri, P. ussuriensis and P. pyrifolia var. culta) and is restricted to Asia; while V. pirina infects European pear (P. communis). The incompatible interactions between European pear and V. nashicola, and Asian pears and $V$. pirina, are likely governed by non-host resistance. The durable nature of non-host resistance is desirable for pome fruit breeding; however, the multi-layered molecular mechanisms underlying this resistance remain poorly understood. Eurasian pears (hybrids of Asian pears crossed with European pears), with varying degrees of resistance to both pear scab fungi, provide a unique opportunity to dissect non-host resistance. Fluorescence microscopy was used to characterise compatible and incompatible phenotypes of $V$. pirina on European, Asian, Eurasian pears and the distant non-host, apple (Malus x domestica). Comparative transcriptomics of interactions, from 3 and 10 days post-inoculation (including mock inoculations), revealed the dynamic molecular mechanisms of multi-layered, non-host resistance. These data will be valuable for the development of genetic markers for scab resistance in pome fruit. The role of effector candidates in coevolution of pear scab fungi with Pyrus spp. is also under investigation using comparative secretomics. Effector candidates may be useful in identifying durable resistance genes for molecular plant breeding, as well as biosecurity diagnostic targets for specific and sensitive detection of the Asian pear scab pathogen, V. nashicola.

Expanded effector families in fruit scab fungi: Venturia inaequalis, $V$. pirina and $V$. nashicola K. M. PLUMMER (1,2), J. Wheeler (2), S. Johnson (1,2), D. Jones (3), D. Jones (4), A. Thrimawithana (3), C. Deng (3), J. Shiller (5), C. Mesarich (6), H. Ishii (7), A. Taranto (8), V. Bus (9), J. Bowen (3), (1) Plant Biosecurity CRC, Bruce, ACT, AUSTRALIA; (2) La Trobe University, Bundoora, AUSTRALIA; (3) The New Zealand Institute for Plant \& Food Research Limited, Auckland, NEW ZEALAND; (4) Curtin, Perth, AUSTRALIA; (5) IRHS, The University of Angers, Beaucouzé, FRANCE; (6) Massey University, Palmerston North, NEW ZEALAND; (7) Kibi International University, Minami-awaji, JAPAN; (8) Australian National University, Canberra, AUSTRALIA; (9) Plant \& Food Research, Havelock North, NEW ZEALAND

Venturia inaequalis, $V$. pirina and $V$. nashicola, are host-specific fungi, causing scab disease on apples, European pears and Asian pears, respectively. Frequent fungicide applications are required for scab control, and fungicide resistance and residues are an on-going issue. Disease-resistant germplasm exists, however it is critical to aim for selection of durable disease resistance, as breeding is slow with these woody, outcrossing hosts. The genetics of host-cultivar resistance to the apple scab fungus follows the gene-for-gene model: pathogen effectors are secreted into the host environment during infection and a subset of these may be recognised as avirulence (Avr) determinants by cognate plant resistance (R) proteins to induce a resistance response. Few $R$ genes, and no Avr genes have been cloned in scab pathosystems. Furthermore, the molecular basis of non-host resistance is unknown. Comparison of predicted secretomes of Venturia spp. revealed genus and species-specific effector candidates that may determine host range. Many orthologues of known fungal effectors were also identified; including Ecp6, AvrLm6 and Ave1. AvrLm6 is represented by an expanded family of over 25 genes in individual Venturia genomes (Leptosphaeria maculans isolates have 2). Ave1 orthologues are represented by over 12 members in each Venturia genome. These, and other effector candidates, are currently being functionally characterized.

Genome comparisons reveal factors responsible for host specificity in the Fusarium fujikuroi species complex

L. DE VOS, C. Dewing, M. van der Nest, M. J. Wingfield, B. D. Wingfield, E. Steenkamp, Forestry and Agricultural Biotechnology Institute (FABI), University of Pretoria, Pretoria, SOUTH AFRICA

The Fusarium fujikuroi species complex comprises numerous socioeconomically important fungi that display high levels of host-specificity. For example, F. circinatum, F. pininemorale and $F$. fracticaudum occur mainly on Pinus species, while their close relatives, $F$. subglutinans and $F$. temperatum infect Zea mays. We investigated the molecular basis for the ability of these fungi to associate with their respective hosts using a comparative genomics approach. For this purpose, genes common and unique to species from the two plant hosts were identified and then compared in terms of the pathways and processes in which they are likely involved. The results revealed substantial interspecies differences regarding the repertoires of genes implicated in carbohydrate and secondary metabolism, as well as those coding for putative effectors involved in host-pathogen interactions. However, we also detected a number of unique commonalities within in each of the two groups of fungi, particularly with regards to the array of putative cell wall degradation enzymes for which they encode. These findings may provide insights into the molecular mechanisms employed by Fusarium species in the F. fujikoroi species complex to infect, inhabit and cause disease on their respective plant hosts.

Variation in gene content of a dispensable chromosome in members of the Fusarium fujikuroi species complex

L. DE VOS (1), R. H. Proctor (2), M. van der Nest (1), E. Steenkamp (1), B. D. Wingfield (1), (1) Forestry and Agricultural Biotechnology Institute (FABI), University of Pretoria, Pretoria, SOUTH AFRICA; (2) Mycotoxin Prevention and Applied Microbiology Research Unit, USDA, Peoria, IL, USA

Members of the Fusarium fujikuroi species complex (FFSC) include important plant pathogens and mycotoxin-producing fungi that have a haploid chromosome number of 12 . The smallest chromosome is dispensable, and evidence indicates that it varies among species and even strains of individual 
species. Whole-genome assemblies of Fusarium species representing the ten well-known mating populations were compared to identify scaffolds corresponding to chromosome 12. Shared and unique genes amongst the different species were determined using a reciprocal BLASTp approach. Chromosome 12 from different species exhibited significant length polymorphism compared to other chromosomes, and displayed remarkably low levels of sequence homology and synteny. Similarity in gene content and organization appeared to be limited to closely related species in each of the three main clades of FFSC. Phylogenetic analyses of the genes on chromosome 12 homologs indicated that most had diverse and non-orthologous origins. Speciesspecific unique genes were dispersed throughout the chromosome, with no clustering. The low sequence and genic homology, as well as the diverse evolutionary trajectories of genes present on chromosome 12 highlights the plasticity of this chromosome. Our future work will investigate the roles that these molecules play in the biology of this important group of fungi.

Evolution of carbohydrate and protein metabolism gene families in Colletotrichum spp.

M. THON (1), S. A. Sukno (1), R. Baroncelli (2), (1) University of Salamanca, Instituto Hispano-Luso de Investigaciones Agrarias, CIALE, Villamayor, SPAIN; (2) University of Western Brittany, Brest, FRANCE

Colletotrichum spp. exhibit a broad diversity of host range, host specificity and reproductive behaviors, making the genus an excellent model for studies of the evolution of these traits. We leveraged the growing number of genome sequences available for Colletotrichum spp. to perform a comparative analysis of gene content to find associations with host range and host specificity. Hierarchical clustering of gene families and phylogenetic analyses revealed lineage specific losses of secreted carbohydrate-active enzymes (CAZymes) and protease encoding genes in species that have narrow host range as well as expansions of these families in the acutatum and gloeosporioides species complexes. Members of these species complexes are broad host range pathogens, suggesting that the higher number in CAZy and protease diversity may be associated with the ability to infect multiple host species. This result suggests that the repertoires of CAZymes and peptidases are the product of recent, lineage specific expansions of these families independently in each species complex. Phylogenetic analyses of the CAZyme and peptidase families revealed that, in contrast to our expectations, gene loss is an important force driving the evolution of gene family size. These results are consistent with the idea that different lifestyles, host and host tissues present different types of carbohydrate substrates to the pathogen this is reflected by each species' CAZyme and peptidase repertoire.

\section{Analysis of Fusarium graminearum pangenome}

A. K. MACHADO (1), R. King (1), M. Urban (1), E. M. Del Ponte (2), D. J. Tessmann (3), K. Kanyuka (1), K. Hammond-Kosack (1), (1) Rothamsted Research, Harpenden, UNITED KINGDOM; (2) Universidade Federal de Vicosa, Vicosa, BRAZIL; (3) Universidade Estadual de Maringa, Maringa, BRAZIL

Next-Generation Sequencing (NGS) technologies allow the capture of the entire genomic sequence present within a species, known as the species pangenome. Here we show the analysis of the pangenome of Fusarium graminearum $(\mathrm{Fg})$, a major fungal pathogen of wheat. We sequenced 15 telomere-to-telomere genomes of $F g$ strains from Brazil and added five publicly available $F g$ genomes into the analysis. In total, 14,798 genes were identified to comprise the pangenome, of which $1111(\sim 7 \%)$ represent accessory genes exclusive to a subset of isolates. The latest version of the genome for $F g$ reference PH-1 strain contains 14,160 genes. Therefore, over 600 genes are predicted to be new genes calls, where more than half lack predicted annotation. Ten new secondary metabolites clusters were identified. These are present in at least one $\mathrm{Fg}$ strain. Six of these clusters are exclusive to one or more Brazilian strains. Single-nucleotide polymorphism (SNP) analyses have been carried out to compare SNP density and differences between core and variable genes with respect to synonymous/nonsynonymous/ nonsense SNPs. This new genomic resource can be used to retrieve biologically relevant information and thereby identify and assess the contribution of the accessory genome to adaptive evolution and phenotypic variation among different $F g$ strains.

Analysis of Chenopodium-virus interactions using Chenopodium quinoa reference genome N. SOLTANI, M. Staton, M. Huff, K. Gwinn, University of Tennessee, Department of Entomology and Plant Pathology, Knoxville, TN, USA

Local lesion hosts prevent systemic spread of viruses, but little is known about the molecular basis of the response. Publicly available RNA-Seq transcriptomic data, previously used for analyses of Chenopodium amaranticolor in response to Cucumber mosaic virus and Tobacco mosaic virus, were re-examined against a recent annotated reference genome of $C$. quinoa. Star was used to map reads, and HTSeq and DESeq were used to count, normalize and analyze differentially expressed genes (DEGs). Sequences of DEGs with adjusted $P$ values $<0.1$ were submitted to Blast2GO to annotate functional classification and assign KEGG and GO terms. Use of a reference genome from a related species was effective for read mapping, with $94 \%$ of reads mapping to the $C$. quinoa genome; $32 \%$ mapped to multiple loci and were not considered further. With this new analysis, 789 DEGs were obtained, which contrasts to 47,346 DEGs in the original paper. Discrepancy may be due to increased resolution provided by mapping to full gene models of $C$. quinoa genome instead of a de novo assembly, or use of stringent statistics that penalize absence of biological replicates. Our re-analysis with the new pipeline revealed DEGs with similar GO terms as the original article, but some regulatory pathways differed. Application of a reference genome from related species of Chenopodium facilitated re-analysis of transcriptome-based viral response and allowed identification of molecular pathways in virushost interaction.

\section{Characterization of a Sudden Death Syndrome (SDS) Core Effector Using Comparative Genomics between SDS-causing and non-SDS-causing Fusarium species}

H. X. CHANG (1), H. Sang (2), L. L. Domier (3), M. Chilvers (2), (1) Department of Plant Soil and Microbial Sciences, Michigan State University, East Lansing, MI, USA; (2) Michigan State University, East Lansing, MI, USA; (3) USDAARS, Urbana, IL, USA

In the United States, sudden death syndrome (SDS) is a destructive soybean disease caused by the soil-borne fungus Fusarium virguliforme. Other species including $F$. azukicola, F. brasiliense, and F. tucumaniae from South America are found to cause SDS foliar symptoms. These fungi produce phytotoxins, which are translocated from infected roots to soybean leaves, and consequently induce interveinal chlorosis and necrosis. With the hypothesis that SDS-causing Fusarium have acquired specialized phytotoxic effectors, this study aims to identify the SDS core effector(s) (SCE) that elicits the distinct SDS foliar symptoms. A comparative genomics approach between nine strains of SDS-causing species and nine strains of non-SDScausing Fusarium species was performed. These 18 strains have 1,100 to 2,300 putative effectors, but after filtering out orthologous genes, only one putative effector was found for the nine strains of SDS-causing Fusarium species. This SCE of F. virguliforme (FvSCE) is a 228 bp gene without available annotation. The FvSCE was cloned into an overexpression system mediated by Soybean mosaic virus, and the recombined vector was bombarded into soybean leaves to visualize its phytotoxicity. A CRISPR-Cas9-directed split-marker approach was used to create the FvSCE knockout mutant. This study will illuminate the function of FvSCE linked to SDS foliar symptoms and provide novel insights into the evolution of core effectors in plant pathogenic fungi. 
Population genomic insights into the evolution of pathogenicity and host range expansion of Xanthomonas perforans to pepper E. NEWBERRY (1), S. Timilsina (2), G. V. Minsavage Jr. (2), J. B. Jones (2), N. Potnis (1), (1) Department of Entomology and Plant Pathology, Auburn University, Auburn, AL, USA; (2) Department of Plant Pathology, University of Florida, Gainesville, FL, USA

Xanthomonas perforans is among four species within the genus Xanthomonas responsible for bacterial leaf spot of tomato and pepper. While the host range of other bacterial leaf spot xanthomonads such as X. euvesicatoria, X. vesicatoria, and X. gardneri encompass a variety of solanaceous plant species, $X$. perforans is generally considered to be restricted to tomato. Recently, a novel $X$. perforans lineage isolated from diseased peppers grown in Florida was described, thus suggesting a recent host range expansion of this pathogen. The objective of the present study was to assess the genetic diversity of $X$. perforans strains that are pathogenic on pepper, and to identify genes that are significantly correlated with this pathogenicity phenotype. We screened a collection of strains that were isolated from diseased tomato and pepper plants grown in Alabama, South Carolina, and Indiana for pathogenicity, and conducted whole genome sequencing. A core genome phylogeny revealed the presence of three distinct lineages within $X$. perforans that were capable of infecting pepper. Genome-wide association identified several genes related to carbohydrate and amino acid metabolism to be significantly correlated with pathogenicity on this host. These findings provide novel gene candidates potentially modulating the host range of $X$. perforans, and are in contrast to previous studies that have emphasized the contribution of effector genes towards host-specificity.

Comparative genomics reveals the role of transposable elements in the evolution of pathogenicity in fungal pathogens of conifers T. DUONG (1), M. J. Wingfield (1), V. Le (2), W. De Beer (3), B. D. Wingfield (1), (1) Forestry and Agricultural Biotechnology Institute (FABI), University of Pretoria, Pretoria, SOUTH AFRICA; (2) Department of Clinical Biochemistry, Aalborg University Hospital, Aalborg, DENMARK; (3) Forestry and Agricultural Biotechnology Institute, University of Pretoria, Pretoria, SOUTH AFRICA

Leptographium wageneri is the causal agent of black stain root disease of conifers. Unlike the majority of other Leptographium spp., this species is a primary pathogen. Three varieties of $L$. wageneri can be distinguished based on optimal growth temperature in culture, the tree hosts that they infect and isozyme profiles. Nothing is known regarding the genetic determinants of pathogenicity in $L$. wagneri. To considser this question, we sequenced, annotated and compared the genomes of the three varieties of $L$. wageneri and the closely related non-pathogenic, $L$. douglasii. Available genomes of related species, including that of the bark-beetle associated and mildly pathogenic blue-stain fungus Grosmannia clavigera were also included in the analyses. The results showed that all three varieties of $L$. wageneri had larger genome sizes than the non-pathogenic species. This was as a consequence of the invasion and expansion of transposable elements (TEs). Interestingly, TE invasion has also occurred in G. clavigera, but to a lesser extent than has occurred in L. wageneri. Although gene catalogues were similar between the pathogenic and non-pathogenic species, genes encoding for small secreted proteins were highly diverse in the pathogens. These genes were found to reside in the TE-rich regions of the genomes. Overall, this study highlights the roles of TEs and TE-assisted evolution in the evolution of pathogenicity in the three varieties of $L$. wageneri and G. clavigera.

Genome-wide analysis of NBS-LRR genes in Indian mustard (Brassica juncea) and prediction of candidate disease resistance genes F. INTURRISI (1), P. E. Bayer (1), H. Yang (2), C. K. K. Chan (1), D. Edwards (1), J. Batley (1), (1) University of Western Australia, Perth, AUSTRALIA; (2) University of Queensland, Brisbane, AUSTRALIA

Brassica juncea (Indian mustard) is a member of the economically important Brassicaceae family. A number of important diseases affect B. juncea, including blackleg and white rust. For crop improvement it's important to identify resistance (R) genes for disease resistance breeding. In this study, a comprehensive genome-wide analysis of nucleotide binding site leucine-rich repeats (NBS-LRR) genes was performed in $B$. juncea along with prediction of candidate $\mathrm{R}$ genes from published resistance loci. The phylogenetic relationship between the $B$. juncea NBS-LRR genes was also studied. The 289 NBS-LRR genes identified in B. juncea were predominately TIR type and $45 \%$ were clustered. The physical position of published disease loci in $B$. juncea was identified through BLAST of markers on the reference genome. Four resistance loci were found for white rust resistance and seven loci were identified for blackleg resistance, with 28 NBS-LRR, 14 receptor-like proteins and 115 receptor-like kinases genes were located within and close by the regions. Eleven $B$. juncea public accessions were re-sequenced to determine genome-wide SNPs associated with the candidate R genes. Genomewide analysis of $B$. juncea NBS-LRR genes provides a valuable resource to identify candidates for novel and functional R genes, which is a key step to enhance resistance against pathogens.

Deciphering floral infection of blueberry pathogen Monilinia vaccinii-corymbosi using comparative genomics and transcriptomics K. BANSAL (1), Y. Zhang (2), A. Yow (3), M. A. Cubeta (3), H. Ashrafi (3), J. A. Rollins (2), (1) University of Florida, Gainesville, FL, USA; (2) Department of Plant Pathology, University of Florida, Gainesville, FL, USA; (3) North Carolina State University, Raleigh, NC, USA

M. vaccinii-corymbosi (Mvc) is a hemi-biotrophic fungus in the Sclerotiniaceae family that causes mummy berry disease on blueberries. Fruit infection is highly specialized as fungal conidia mimic pollen by germinating on stigma and growing in a directed manner towards the ovary. Fungal hyphae eventually colonize the developing berry first within the locules biotrophically, and then the mesocarp necrotrophically, to form mummies. Conidia of Mvc infect and mummify a limited number of Vaccinium species. Members of Sclerotiniaceae family however have varied host ranges, modes of infection and tissue specificities. For example, Sclerotinia sclerotiorum and Botrytis cinerea are necrotrophs with broad host ranges while species of Monilinia and Ciborinia may be necrotrophs or hemibiotrophs with restricted host ranges and tissue specificities. We hypothesize that Mvc secretes proteins to mimic pollen tracking of the stylar canal, to evade the blueberry defense machinery and to establish disease. Comparative genomics of Mvc with other flower-infecting Sclerotiniaceae fungi revealed different families of secreted proteins that may be involved in pollen mimicry and host specificity. Transcriptomic analysis of infected blueberry tissues showed that different families of secreted proteins were expressed differentially during stylar infection and fruit mummification. Ongoing research includes functional analyses to understand the role of identified genes in pathogenicity of Mvc.

The genomes of Ophiosphaerella spp. reveal new insights into the bermudagrass spring dead spot pathosystem N. GRAF GRACHET (1), M. Couger (2), C. A. Young (3), S. Hartson (1), C. D. Garzon PhD (1), S. M. Marek PhD (1), N. R. Walker (1), (1) Oklahoma State University, Stillwater, OK, USA; (2) Oklahoma State Univesity, Stillwater, OK, USA; (3) Noble Research Institute, LLC, Ardmore, OK, USA

Spring dead spot, caused by Ophiosphaerella herpotricha, O. korrae and $O$. narmari, is a destructive soilborne disease of turf type bermudagrass in the southern US. Ophiosphaerella appears to switch from a disease-causing necrotroph in susceptible cultivars to an asymptomatic endophyte in resistant cultivars. Ophiosphaerella spp. are described as necrotrophs; however, the mechanisms of pathogenesis are not well understood. The objective of this study was to annotate the protein coding genes Ophiosphaerella spp. to provide clues into pathogenesis. The genomes of 11 isolates and the transcriptomes of 6 isolates were sequenced. Proteins of one isolate of $O$. herpotrich $a$ were validated by mass spectrometry (MS). The functions of protein coding genes were predicted using many functional annotation tools/databases. Genome sizes ranged from $47 \mathrm{Mb}$ to $70 \mathrm{Mb}$. The number of protein coding genes varied from twelve to fourteen thousand. All isolates had similar abundances of CAZymes (average $=861$ ) with an expansion of 
plant cell wall degrading enzymes AA families 3,4 , and 11 , and CE family 1 . Protein search against PHI-base revealed $\sim 40$ homologs of avirulence genes. In total, 2,884 O. herpotricha protein-genes were validated by MS, and seven were confirmed homologs of avirulence genes. These results indicate Ophiosphaerella utilizes multiple strategies during pathogenesis.

Complete genome sequence of Xanthomonas phaseoli pv. phaseoli G66 reveals a particular repertoire of Type 3 effectors including a novel TAL effector

A. Munoz Bodnar (1), A. Castaneda (2), M. Jain (3), L. CAI (4), D. W. Gabriel (3), (1) University of Florida, Gainesville, FL, USA; (2) ICA, Bogota, COLOMBIA; (3) Institute of Food and Agricultural Sciences, University of Florida, Gainesville, FL, USA; (4) University of Florida, gainesville, FL, USA

Xanthomonas phaseoli pv. phaseoli (Xpp) and Xanthomonas citri pv. fuscans (Xcf) are the causal agents of common bacterial blight (CBB). Strains of both species cause systemic and nearly identical disease symptoms on bean. We determined the complete genome sequence of Xpp U.S. strain G66 through PACBIO sequencing. This genome carries a 5,031,159 bp chromosome and three plasmids of 6,364 bp, 50,429 bp and 37,769 bp. G66 shows a 99.9\% Average Nucleotide Identity (ANI) to previously sequenced Xpp strain CFBP412 (Ruh et al, 2017), suggesting a common ancestor. The entire effector repertoire of G66 was compared against Xcf, revealing 18 effectors that are common to both Xcf and Xpp, seven effectors that are unique to Xcf, and four unique to Xpp: XopAP, XopAU, XopAV and HpaA. Among the common Xcf and Xpp effectors were transcription activator-like (TAL) effectors of the pthA/avrBs3 gene family and found in all CBB strains examined. These TAL effectors are required for both cotton blight and citrus canker disease caused by different subspecies or pathovars of $X$. citri. Such TAL effectors are found in many different xanthomonads, and are often found in all strains causing a particular disease phenotype. G66 carries two potential pthA/avrBs 3 homologs on two different plasmids but only one appears intact. This homolog carries 19 direct repeats and is currently being functionally analyzed.

Whole genome sequence analysis of Xanthomonas perforans shows widespread recombination events S. TIMILSINA (1), J. Pereira (1), G. V. Minsavage Jr. (1), P. Abrahamian (2), F. Iruegas-Bocardo (1), J. C. Huguet-Tapia (1), E. Newberry (3), N. Potnis (3), G. E. Vallad (2), E. M. Goss (1), J. B. Jones (1), (1) Department of Plant Pathology, University of Florida, Gainesville, FL, USA; (2) Gulf Coast Research and Education Center, University of Florida, Wimauma, FL, USA; (3) Department of Entomology and Plant Pathology, Auburn University, Auburn, AL, USA

Bacterial spot is an important disease of tomato in the United Stated caused by multiple Xanthomonas species. In Florida, this disease is caused by $X$. perforans $(X p)$. Over the last 15 years, significant changes have occurred in $X p$ strains isolated from Florida, including shifts in bacterial races, loss of bacteriocins, and changes in effector profiles. Preliminary phylogenetic and genomic studies have identified recombination events between $X$. euvesicatoria $(X e)$ and $X p$. Different phylogenetic groups within $X p$ appear to have differing patterns of recombination. Our objective was to analyze the extent and role of genetic exchange from $X e$ to $X p$ strains. We used whole genome sequences of $X p$ strains collected from the United States between 1991 and 2016. A core gene multilocus sequence analysis identified genes present in all $X p$ strains. Comparing core genes against reference strains $X e$ 85-10 and $X p$ 91-118 identified allelic variation specific to $X e$ and $X p$, respectively. Results show that genetic exchange is distributed throughout $X p$ genomes and includes housekeeping and effector genes. Histidine kinase and TonB receptors were among the genes observed to have recombined in multiple genetic groups. These observations define the extent of genetic exchange driving recent $X p$ evolution.

Genomic insights into the mechanisms of pathogenesis in Raffaelea lauricola, causal agent of laurel wilt disease Y. ZHANG (1), D. Vanderpool (2), J. A. Smith (3), R. C. Ploetz (4), J. A. Rollins (1), (1) Department of Plant Pathology, University of Florida, Gainesville, FL, USA; (2) Division of Biological Sciences, University of Montana, Missoula, MT, USA; (3) School of Forest Resources and Conservation, University of Florida, Gainesville, FL, USA; (4) Tropical Research \& Education Center, University of Florida, Homestead, FL, USA

Laurel wilt, caused by Raffaelea lauricola, is a lethal vascular disease of North American members of the Lauraceae plant family. $R$. lauricola and its primary ambrosia beetle vector Xyleborus glabratus are invasive introductions from Asia. However, there is no report of laurel wilt killing native Lauraceae trees in Asia. To gain insight into the lethality of $R$. lauricola in North America, we generated and compared high quality genomes of $R$. lauricola and the closely related, nonpathogenic $R$. aguacate, each of which are symbionts of ambrosia beetles associated with avocado. The $R$. lauricola genome uniquely encodes small-secreted proteins (SSPs) that are associated with virulence in other pathogens and is enriched in secondary metabolite biosynthetic clusters. The two species also exhibit significant differences in CAZymes that are associated with plant polysaccharide degradation, such as CBM50 (LysM) and CH18. Notably, fungal-secreted LysMs have been reported as a novel class of conserved effectors. We have identified potential adaptations of R. lauricola, which may have enabled it to colonize and cause disease on host plants; pathogen-specific SSPs, secondary metabolites, and LysM effectors are candidate factors for this adaption. Functional analysis of these candidate virulence factors is in progress to gain insight into the mechanisms of pathogenesis in R. lauricola.

Understanding pathogenic success by identifying Ralstonia solanacearum's in planta nutrient sources C. HAMILTON (1), A. MacIntyre (2), C. Allen (3), (1) University of Wisconsin-Madison, madison, WI, USA; (2) University of Wisconsin-Madison, Madison, WI, USA; (3) University of Wisconsin, Madison, WI, USA

The soilborne pathogen Ralstonia solanacearum (Rs) causes bacterial wilt by colonizing plant xylem, causing plants to wilt and die. Rs reduces crop yields at every level of production from small- to large-scale farming operations. Although the xylem environment has been historically characterized as nutrient poor, xylem sap must contain food sources because $R s$ populations in wilting plants exceed $10^{9} \mathrm{CFU} / \mathrm{g}$ stem in tomato. Our group's previous genomic, in planta transcriptomic, and xylem sap metabolomic genomic data sets offer clues about the pathogen's carbon source utilization during disease. This work suggests that sucrose, trehalose, galactose, glucose, gluconate, and myo-inositol could fuel Rs growth in planta. However, we do not know which specific xylem sap carbon sources the pathogen uses. To identify these carbon sources, we measured virulence and competitive fitness of a suite of bacterial mutants lacking catabolic and transport genes for sucrose (scrA), trehalose (treA), galactose (Rsc2755), glucose ( $g l k)$, gluconate ( $g n t K)$, and myo-inositol $(\mathrm{iol} G)$. Additionally, it was previously found that $R s$ grows better in sap from infected plants, which suggested that the $R s$ manipulates host xylem carbon source content. To test the hypothesis that wilt-resistant plants withhold carbon sources from $R s$, we compared the metabolomic profiles of xylem sap from susceptible and resistant tomato plants.

The differential role of plasmids in Clavibacter virulence on tomato

S. THAPA (1), R. L. Gilbertson (2), G. L. Coaker (1), (1) University of California, Davis, CA, USA; (2) Department of Plant Pathology, University of California, Davis, CA, USA 
Clavibacter michiganensis subsp. michiganensis $(\mathrm{Cmm})$ is a Gram positive bacterium that proliferates in the xylem vessels of tomato, causing bacterial wilt and canker. The reference strain NCPPB382 has one chromosome (3.2 Mb) and two conjugative plasmids pCM1 (27.5 kb) and pCM2 (72 kb). Whole genome sequencing of $\mathrm{Cmm}$ strains collected in North America and Europe was performed with Illumina and PacBio platforms. Genomes were de novo assembled with the SPAdes and Hierarchical Genome Assembly Process 3. The sequenced strains have undergone significant alterations in plasmid number, size and gene composition. Most of the sequenced strains possess pCM1 and pCM2-like plasmids with sizes ranging from 27-100 kb. Curing of either plasmid resulted in a significant decrease in virulence. The $\beta-1,4$-endocellulase CelA in pCM1 and serine protease Pat- 1 in pCM2 are highly conserved in most $\mathrm{Cmm}$ strains and were previously demonstrated to be important for virulence. Interestingly, we have identified virulent $\mathrm{Cmm}$ strains lacking either pCM1 (NZ2541) or pCM2 (CASJ001). The absence of pCM1 or pCM2 in particular strains indicates that these plasmids, which are required for virulence in some $\mathrm{Cmm}$ strains, are not universally required for pathogenicity. Rearrangement, gene gain and/or loss in these conjugative plasmids have shaped $\mathrm{Cmm}$ diversity and pathogenicity.

A Global Outlook on the Evolution of Type Three Effectors in Xanthomonads causing Bacterial Spot on Tomato and Pepper

M. JIBRIN (1,2), N. Potnis (3), S. Timilsina (4), G. V. Minsavage Jr. (4), E. Osdaghi (5), S. Vou (6), T. A. Coutinho (7), O. P. Pruvost (8), R. Roach (9), M. I. Siri (10), M. J. Pianzzola (10), A. M. Quezado Duval (11), D. S. Egel (12), T. C. Creswell (13), G. E. Ruhl (13), L. Maynard (14), G. E. Vallad (15), P. D. Roberts (16), E. M. Goss (4), J. B. Jones (2), (1) Ahmadu Bello University, Zaria, NIGERIA; (2) University of Florida, Gainesville, FL, USA; (3) Department of Entomology and Plant Pathology, Auburn University, Auburn, AL, USA; (4) Department of Plant Pathology, University of Florida, Gainesville, FL, USA; (5) Bajgah Shiraz University Shiraz, Shiraz, IRAN; (6) University of Pretoria, Pretoria, SOUTH AFRICA; (7) Centre for Microbial Ecology and Genomics, University of Pretoria, Pretoria, SOUTH AFRICA; (8) CIRAD, St Pierre Reunion Is, FRANCE; (9) La Trobe University, Bundoora, AUSTRALIA; (10) Bioscience Department, School of Chemistry, Universidad de la Republica, Montevideo, URUGUAY; (11) EMBRAPA, Brasilia, BRAZIL; (12) Purdue University, Vincennes, IN, USA; (13) Purdue University, West Lafayette, IN, USA; (14) University of Purdue, West Lafayette, IN, USA; (15) Gulf Coast Research and Education Center, University of Florida, Wimauma, FL, USA; (16) University of Florida, Immokalee, FL, USA

Xanthomonas euvesicatoria (Xe), X. vesicatoria $(X v), X$. perforans $(X p)$ and $X$. gardneri $(X g)$ are generally regarded as the bacteria species causing bacterial spot of tomato and pepper. $X e$ and $X p$ are closely related and may be regarded as a single species. Resistance breeding efforts have often targeted type three effector proteins found in these species. While unique effector profiles have been associated with each species, there is a need to understand the distribution and evolution of effectors on a global scale due to apparent international movement of these pathogens. In this study, we sequenced more than one hundred genomes, representing populations from more than fifteen countries covering Africa, North America, South America, Europe, Asia and Australia. We also included previously sequenced strains representing additional populations from the NCBI database. We utilized comparative genomics and phylogenetic approaches to evaluate the evolution of these effectors across and within species and geographies. Our findings suggest diverse dynamics of effectors, not only between or within species but across geographies. For example, the TAL effector, AvrHah1, originally found in $X g$, is present in $X p$ strains from Nigeria, Mauritius, South Africa and Italy but absent in strains from Florida, USA. This comprehensive global study looking at populations of bacterial spot xanthomonads from different countries in six continents has several implications for disease management.

Deciphering how beet necrotic yellow vein virus overcomes rhizomania resistance genes in sugarbeet through metabolome analysis W. M. Wintermantel (1), C. Broeckling (2), K. L. Richardson (3), K. M. WEBB (4), (1) USDA-ARS, Salinas, CA, USA; (2) Colorado State University, Fort Collins, CO, USA; (3) USDA ARS, Salinas, CA, USA; (4) USDA-ARS, Soil Management and Sugar Beet Research Unit, Fort Collins, CO, USA

Rhizomania, caused by Beet necrotic yellow vein virus (BNYVV), is one of the most important diseases of sugarbeet and is distributed in most growing areas of the world. BNYVV can be controlled through single resistance genes. However, BNYVV strains with the ability to overcome the widely used $R z 1$ gene have been emerging in many production areas. To more fully understand how sugarbeet responds to BNYVV we have utilized a proteomic and metabolomic approach to identify the biological pathways associated with Rhizomania resistance. Earlier proteomic studies demonstrated that a relatively small number of proteins were associated with BNYVV infection and resistance in sugarbeet. We utilized a metabolomics approach to further characterize the functional pathways associated with BNYVV in near isogenic lines containing either the $R z 1$ or $R z 2$ resistance gene. Plants were grown for three weeks in growth chambers in either healthy soil, soil with traditional BNYVV, or soil containing Rzl resistance breaking BNYVV (RBBNYVV). Metabolites were extracted from roots and evaluated by GC-MS and UPLC-MS. There were significant differences among treatments, with primary metabolomic differences associated with strain of BNYVV as compared to healthy (non-infected) sugarbeet. These studies build on the knowledge generated through previous proteomic research, and may lead to identification of targets for genetic modification to break the virus-host interaction.

Unearthing Planet's Plant Virus Modulome: Exploring Plant Virus Proteome Modularity for Taxonomic Classification and Biological Predictions R. TAHZIMA (1,2), A. Haegeman (1), Y. Brostaux (2), K. De Jonghe (1), S. Massart (2), (1) Flanders Research Institute for Agriculture, Fisheries and Food (ILVO), Merelbeke, BELGIUM; (2) University of Liège (ULg) - Gembloux Agro-BioTech, Gembloux, BELGIUM

Current plant virus systematics are often designed for specific well-defined plant viral taxa and may appear cumbersome for unassigned or newly discovered plant virus entities. Therefore, plant virology could directly benefit from comparative analysis for characterizing newly sequenced plant virus isolates in order to aid their taxonomic placement and even to make predictions on their biology. We indeed hypothesized that the information content of the virus genome, or more specifically the virus proteome, is directly linked to its taxonomic classification, but also to its biology (e.g. vector, host plant). To explore these hypotheses, we characterized all available plant viral genome sequences from RefSeq by looking for protein families/domains in their respective open reading frames using existing Hidden Markov Model profiles. Our resulting dataset includes 1319 different plant viruses belonging to 26 families and 120 genera. For all these accessions we evaluated the presence/absence and abundance of different features, for example Protein Families (PFAMs) and Superfamilies (SFs). Through appropriate data mining from this unique dataset, we found features with a high correlation with the current taxonomical classification of the virus, or with the biology of the virus (e.g. vector, host plant). Hence, we can identify several features of interest for improved taxonomical classification and we are possibly able to make predictions about the virus biology. This approach could particularly be useful to explore the biology of all the new viruses recently identified by NGS and to guide further research while providing information for preliminary risk analysis after virus discovery.

Copy number variation appears increased in clonal lineages over sexual lineages of Phytophthora infestans

B. J. KNAUS (1), J. Tabima (2), S. Shakya (3), H. S. Judelson (4), N. J. Grunwald (5), (1) USDA-ARS, Horticultural Crops Research Unit, Corvallis, OR, USA; (2) Oregon State University, Corvallis, OR, USA; (3) OREGON STATE UNIVERSITY, Corvallis, OR, USA; (4) University of California, Riverside, CA, USA; (5) USDA ARS, Corvallis, OR, USA 
The Irish Potato Famine pathogen, Phytophthora infestans (Mont.) de Bary, continues to emerge causing epidemics globally. However, mechanisms that produce clonal lineages that are highly virulent remain uncharacterized. We used high throughput sequencing, from previously published sources as well as our own sequencing, to infer copy number variation, based on $200 \mathrm{kbp}$ sliding windows of heterozygous sites, for $P$. infestans genomes from a global sample of isolates. Instead of observing individuals that were predominantly diploid or triploid we observed individuals that represented a full spectrum of intermediate states from diploid to triploid. Many of the clonal lineages that have led to epidemics exhibit copy number increases, including US-1, US-8, US-11, US-23, and US-24. However, the lineage that is thought to have caused the Great Famine appeared diploid. Isolates collected from Mexico, the center of genetic diversity for $P$. infestans and a region where it is characterized as exhibiting a sexual mode of reproduction, were also predominantly diploid. These results indicate that no simple assumption about the role of copy number variation in $P$. infestans biology can be made and highlights the importance of inference of copy number prior to genetic analysis. Our results present a new perspective of $P$. infestans epidemics as frequently being accompanied by changes in copy number that may contribute to the pathogenicity of the lineage.

\section{Deciphering the mechanism of $E$. coli resistance to a membrane-targeting antimicrobial peptide through genomic and transcriptomic approaches}

R. Rabara, L. Huynh, J. VELÁSQUEZ GUZMÁN, H. Nguyen, S. Basu, S. Zhang, G. Gupta, New Mexico Consortium, Los Alamos, NM, USA

Antimicrobial peptides (AMPs) are essential components of host innate immunity, representing the first line of defense in bacterial clearance. However, bacteria can develop resistance to AMPs. Using Escherichia coli strain BL21-Gold (DE3) as a model, we investigated the mechanism of bacterial resistance to AMPs. The strain was allowed to evolve resistance against an amphipathic 11 residue helical peptide (or P11). The minimal inhibitory concentration (MIC) of the resistant strain is 13-fold higher than that of the wildtype (or susceptible) strain. Genome sequencing of the resistant $E$. coli derivative revealed insertions and deletions in several genes. Through comparative genome analysis, we detected transposase insertions in genes involved in outer membrane (asmA) and lipopolysaccharide (waaP) biosynthesis. The asmA gene encodes assembly protein asmA, which is involved outer membrane assembly; whereas waaP encodes lipopolysaccharide core heptose (I) kinase, required for the heptose phosphorylation in lipopolysaccharide (LPS) core. We also detected a transposase insertion in the Dihydrouridine synthase C (dusC) gene, which encodes tRNAdihydrouridine synthase. Knocking out these three genes resulted in 3-fold increase of the MIC compared to the control. Several mutations in genes that encode proteins are involved in interactions with phospholipids and membrane permeabilization of P11. Overall, our data suggest the collective action of genic and intergenic mutations contribute to resistance. Based on these observations, we designed a next-generation, 26 residue AMP (P26) that overcomes the bacterial resistance mechanism. Transcriptome profiling analysis is underway and should also be highly informative.

Exploring the genome of Metschnikowia fructicola, a biocontrol yeast effective against postharvest diseases E. Piombo (1), N. Sela (2), M. Wisniewski (3), M. Hoffmann (4), M. L. GULLINO (5), M. Allard (4), E. Levin (6), D. Spadaro (7), S. Droby (8), (1) University of Torino - DISAFA, Grugliasco, ITALY; (2) ARO - The Volcani Center - Dept. Plant Pathology, Rishon Le-Zion, ISRAEL; (3) USDA ARS, Kearneysville, WV, USA; (4) Food and Drug Administration (FDA), Division of Microbiology, Office of Regulatory Science, College Park, MD, USA; (5) Agroinnova - University of Torino, Grugliasco, Torino, ITALY; (6) ARO - The Volcani Center - Dept. Postharvest Science, Rishon Le-Zion, ISRAEL; (7) DISAFA and AGROINNOVA, University of Torino, Torino, ITALY; (8) Agricultural Research Organization, The Volcani Center, Rishon Lezion, ISRAEL

The yeast Metschnikowia fructicola has been reported as an efficient biocontrol agent of postharvest diseases of fruit. The mechanisms of action by which $M$. fructicola inhibits postharvest pathogensinclude iron-binding compounds, induction of defence signalling genes, such as PRP and MAPK cascade genes, production of fungal cell wall degrading enzymes and relatively high amounts of superoxide anions. M. fructicola also exhibits chitinase activity and the chitinase gene, $M f C h i$, was highly induced in response to fungal pathogen cell walls. Several studies have examined differential gene expression during the interaction of the yeast, $M$. fructicola, with a host fruit or a postharvest pathogen. In the current work, we report the assembly of the whole genome sequence of two strains of M. fructicola using PacBio and Illumina shotgun sequencing technologies. Using the PacBio, a high-quality draft genome consisting of 93 scaffolds, with an estimated genome size of approximately $26 \mathrm{Mb}$, was obtained. Comparative analysis of M. fructicola proteins with three available closely-related genomes revealed a shared core of homologous proteins. Comparing the genomes of the two M. fructicola strains using a SNP calling approach resulted in the identification of 564,302 SNPs/indels with a total of 2,004 predicted high impact mutations. Based on the assembled genome, sequences were annotated with gene description and gene ontology and clustered in functional groups. Analysis of CAZyme family genes revealed 1,145 putative genes. Transcriptomic analysis of CAZymes in M. fructicola during its interaction with either grapefruit peel tissue or Penicillium digitatum revealed a high level of CAZyme gene expression when the yeast was placed in wounded fruit tissue. The significance of the findings in biocontrol capabilities of M. fructicola will be discussed.

Investigating effector diversity as a source of cultivar-specific pathogenicity across global isolates of the lettuce bacterial leaf spot pathogen E. ROSENTHAL, A. Sebastian, C. T. Bull, The Pennsylvania State University, University Park, PA, USA

Bacterial Leaf Spot of lettuce has a 100-year history in the US, and in the 1990's it reemerged as a significant threat that breeding programs struggled to counter. Multilocus sequence analysis of global Xanthomonas campestris pv. vitians $(X c v)$ isolates using four housekeeping genes and evaluation of plant-pathogen interactions has revealed six groups with cultivar-specific resistance. We hypothesize that differences in effector repertoires cause this variation in cultivar-specificity across the genotypic groups. To test this, the genomes of 21 representative isolates of $X c v$ and relevant types and pathotypes were sequenced, assembled, and annotated. Barcoded libraries were created for each isolate using $2 \mu \mathrm{g}$ of DNA extract and the Illumina TruSeq DNA PCR-Free kit. An equimolar pool of the libraries for each isolate was sequenced using Illumina MiSeq, generating $250 \mathrm{nt}$, paired-end reads. Yield was $185 \mathrm{Mb}$ per sample; with the $X c v$ genome size of $5 \mathrm{MB}$, that computed to $37 \mathrm{x}$ coverage. The sequence data was aligned to a reference genome for assembly and annotation. Bioinformatics tools are being employed to identify sequences belonging to any of the more than 30 known effectors of Xanthomonas plant pathogens. Once these effector repertoires are determined, we will evaluate correspondence between effector repertoire composition and host reaction. This knowledge can be applied in development and deployment of lettuce with resistance across all genotypes of Xcv.

Evolution of necrotrophic phytopathogenic bacteria in the Enterobacteriaceae

R. R. MCNALLY (1), N. T. Perna (2), A. O. Charkowski (1), (1) Colorado State University, Fort Collins, CO, USA; (2) University of Wisconsin, Madison, WI, USA

The emergence of plant pathogenesis by bacteria is the product of convergent evolution. Within the Enterobacteriaceae alone, two groups of bacteria have independently evolved the ability to cause plant disease. Soft rot Enterobacteria (SRE) represent one of these groups and include three genera: Pectobacterium, Dickeya, and Brennaria. While much research has focused on biotrophic bacterial plant pathogens, SRE pathogenicity is characterized by necrotrophy and the production of cell wall-degrading enzymes. Here we report a comparative genomic analysis of the Enterobacteriaceae to elucidate the evolution of necrotrophy in SRE. In total, 307 Enterobacteriaceae genomes were analyzed including 20 genomes from Pectobacterium, 
Dickeya and Brennaria. Predicted proteins were grouped according to orthology and phylogenetically evaluated. Genes gained or lost in SRE bacteria were determined via thresholded RAxML analysis $(\geq 0.75)$ and represent putative evolutionary adaptions for plant necrotrophy. In total, 195 genes were acquired by SRE bacteria while 253 were lost. $7 \%$ of acquired genes represent predicted virulence factors while $53 \%$ are involved in metabolism, signal transduction or transport. Our results indicate that the evolution of necrotrophy in the Enterobacteriaceae required extensive acquisition of methylaccepting chemotaxis proteins and ATP-binding cassette transporters in addition to a small number of cell-wall degrading enzymes.

A novel dsRNA virus stimulates sporulation of Phytophthora infestans and may have contributed to late blight epidemics G. CAI (1), K. L. Myers (2), W. E. Fry (2), B. I. Hillman (3), (1) USDA-ARS / Purdue University, West Lafayette, IN, USA; (2) Cornell University, Ithaca, NY, USA; (3) Rutgers University, New Brunswick, NJ, USA

Phytophthora infestans is the causal agent of potato and tomato late blight. To identify genetic elements contributing to this disease, several dsRNA viruses have been characterized in this organism. One of the viruses, Phytophthora infestans RNA virus 2 (PiRV-2), is 11,170bp in length and without a polyA tail. It contains a large open reading frame (ORF) with short 5'- and 3'- untranslated regions. The ORF is predicted to encode a polyprotein of 3710 aa (calculated molecular weight $410.94 \mathrm{kDa}$ ). This virus lacks significant similarity to any known viruses, even in the RNA-dependent RNA polymerase region. Phylogenetic analysis showed it should be classified into a new virus family. Comparing isogenic strains with or without the virus demonstrated that the virus stimulated sporangia production multiple folds. This virus was faithfully transmitted through asexual reproduction and was found in most strains in the US-8 and US-22 lineages. It may have contributed to the success of these two lineages in recent late blight epidemics. This is the first report that a potential hypervirulent virus may contribute to late blight epidemics and presents a novel target for late blight control.

Molecular characterization of viruses in country beans (Lablab purpureus) in Bangladesh

M. RAHMAN (1), B. Bagewadi (2), A. C. Fayad (3), A. V. Karasev (4), R. Naidu (5), (1) Washington State University, Prosser, WA, USA; (2) University of Agricultural Sciences, Dharwad, INDIA; (3) Virginia Polytechnic Institute and State Univ., Blacksburg, VA, USA; (4) University of Idaho, Moscow, ID, USA; (5) Washington State University - IAREC, Prosser, WA, USA

Country bean (Lablab purpureus L., family Fabaceae) is a widely cultivated legume vegetable for human consumption as a source of protein, carbohydrate, and other essential nutrients in South Asian countries. Viral diseases are one of the major constraints limiting the production of country beans in Bangladesh. During a survey of farmers' fields in Gazipur and Comilla regions, leaves showing bright yellow mosaic symptoms were analyzed to profile viruses present in symptomatic plants. Total RNA from symptomatic leaves were subjected to Next-Generation Sequencing (NGS) using Illumina HiSeq 2000 platform. The quality filtered 125-basepair paired-end reads were assembled de novo into contigs using the CLC Genomics workbench 8.0 software and annotated against the viral Ref Seq database in GenBank by BLASTN program to identify viral sequences present in symptomatic samples. The results indicated the presence of sequences highly similar to Bean common mosaic necrosis virus (BCMNV) in symptomatic samples collected from two distinct geographic regions in Bangladesh. In pair wise comparisons, the coat protein (CP) sequences showed $100 \%$ identity between the two BCMNV isolates and showed $93-94 \%$ identity at the amino acid level with CP sequences of BCMNV isolates reported from the US and East Timor. Phylogenetic analysis of CP sequences showed that BCMNV isolates from Bangladesh formed a separate clade distinct from virus isolates characterized from the US and East Timor.

Host-specific lineages of Bean common mosaic virus in Bangladesh, Cambodia and Nepal S. DAS (1), B. Bagewadi (2), L. Shah (3), K. H. Seng (4), A. C. Fayad (5), A. V. Karasev (6), R. Naidu (7), (1) Washington State University, Prosser, WA, USA; (2) University of Agricultural Sciences, Karnataka, INDIA; (3) International Development Enterprises iDE-Nepal, Kathmandu, NEPAL; (4) International Development Enterprises iDE- Cambodia, Phnom Penh, CAMBODIA; (5) Virginia Polytechnic Institute and State Univ., Blacksburg, VA, USA; (6) University of Idaho, Moscow, ID, USA; (7) Washington State University - IAREC, Prosser, WA, USA

Bean common mosaic virus (BCMV; genus Potyvirus, family Potyviridae) infects legume crops worldwide. This study was aimed at characterizing BCMV isolates collected from yardlong beans (Vigna unguiculate) from Bangladesh, Cambodia, and Nepal, and red kidney beans (=common bean, Phaseolus vulgaris) from Nepal. Yardlong bean leaves showing mild mosaic, vein banding, and deformation symptoms and kidney bean leaves exhibiting puckering, mottling and deformation symptoms were analyzed for the presence of viruses. Total RNA from symptomatic samples were subjected to Next-Generation Sequencing using Illumina HiSeq 2000 platform. The quality filtered reads were assembled de novo into contigs using the CLC Genomics workbench 8.0 software and annotated against the viral Ref Seq database in GenBank by BLASTN program to identify viruses present in symptomatic samples. The results indicated the presence of sequences highly similar to BCMV. The coat protein (CP) sequence of BCMV isolates from yardlong beans originating from the three countries were highly identical, but distinct from those obtained from kidney beans. Phylogenetic analysis of the CP sequences differentiated BCMV isolates from Bangladesh, Cambodia, and Nepal into two distinct clades by original host, with Nepalese common bean isolates clustering with those from different countries and legume hosts and yardlong bean isolates forming a distinct clade aligning with cowpea isolates from China.

Description of a novel mild strain of Citrus tristeza virus in California that reacts with monoclonal antibody MCA13

R. K. YOKOMI (1), V. S. Selvaraj PhD (2), Y. Maheshwari (2), M. Chiumenti (3), M. Saponari (3), A. Giampetruzzi (4), S. Hajeri (5), (1) USDA, ARS, SJVASC, Parlier, CA, USA; (2) USDA ARS SJVASC, Parlier, CA, USA; (3) CNR - Institute for Sustainable Plant Protection, Bari, ITALY; (4) Department of Soil Plant and Food Science, University of Bari, Bari, ITALY; (5) Citrus Pest Detection Program (CCTEA), Tulare, CA, USA

Quick decline caused by Citrus tristeza virus (CTV) destroyed citrus on sour orange rootstock in southern California during the 1930's -40's. However, use of resistant/tolerant CTV rootstocks, certified pathogen-free budwood, and quarantines have limited further economic damage from CTV. Multilocus marker profiles of California CTV isolates revealed presence of multiple genotypes but bioindexing showed two general phenotypes: mild; and various degrees of seedling yellows/stem pitting. To examine the genetic diversity involved, full-length genomes of three California CTV isolates were determined by siRNA sequencing. Phylogenetic analyses differentiated these isolates into the VT genotype (CA-VT-AT39) and a new genotype called S1 (CA-S1-L and CA-S1-L65). The three isolates all reacted with MCA13, a monoclonal antibody used by the Central California Tristeza Eradication Agency (CCTEA) to identify potentially harmful CTV isolates. Bioindexing showed that CA-VT-AT39 was severe but CA-S1-L and CA-S1-L65 were mild. Screening the in planta CTV accessions collected from 1968 to 2011 by the CCTEA for S1 strains resulted in identification of 42 other S1 suggesting it has been present since before 1968 in California. BLAST and phylogenetic analysis of the S1 $p 25$ gene region with other extant CTV sequences from NCBI suggested putative S1-like isolates may occur elsewhere in the Mediterranean and Asian regions. This information is useful for the management of CTV. 
Small RNA-Seq to characterise viruses responsible of Lettuce big-vein disease

A. Bernal-Vicente (1), L. Donaire (2), C. Gómez-Aix (1), M. A. Sanchez Pina (2), M. Juarez Gomez (3), M. A. ARANDA (2), (1) Abiopep S.L., Murcia, SPAIN; (2) CEBAS-CSIC, Murcia, SPAIN; (3) Universidad Miguel Hernandez, Alicante, SPAIN

Lettuce big-vein disease (LBVD) is an emerging disease causing losses ranging from 30 to $70 \%$ in lettuce production worldwide. Several studies have associated this disease with Mirafiori lettuce big-vein virus (MiLBVV; genus Ophiovirus), alone or in mixed infection with Lettuce big-vein associated virus (LBVaV; genus Varicosavirus). We used Illumina small RNA sequencing (sRNA-seq) to detect viruses present in symptomatic lettuce plants from commercial fields in Southern Spain. Data analysis showed the presence of MiLBVV and LBVaV in samples from different plant tissues. Additionally, Ranunculus white mottle virus (RWMV; genus Ophiovirus) was also identified in these samples. Sequences of the coat protein genes were used to infer MiLBVV and LBVaV nucleotide diversities within the analysed samples and to differentiate potential strains. Comparison of the sequences determined in this work with those available in databases allowed the design of universal primers to detect and quantify the accumulation of both MiLBVV and LBVaV. Subsequent analysis of multiple samples from symptomatic lettuce plants of several plots located in Southern Spain showed high rates of MiLBVV and LBVaV mixed infections, with $58 \%$ of the positive samples infected with both viruses, but also significant proportions of single infections, which were more frequent for $\mathrm{LBVaV}(21 \%)$. Moreover, the presence of one or both viruses was detected in 6 out of 10 commercial seed lots obtained from different providers, suggesting the possibility of their seed transmission.

A Cilevirus detected in Hibiscus rosa-sinensis and Citrus sinensis is a strain of Citrus leprosis virus $\mathbf{C 2}$ causing citrus leprosis disease in Colombia A. ROY (1), A. L. Stone (2), G. Leon Martinez (3), J. S. Hartung (4), B. Adducci (1), G. Wei (1), V. A. Mavrodieva (5), M. K. Nakhla (1), W. Schneider (2), R. H. Brlansky (6), (1) USDA-APHIS-PPQ-S\&T-CPHST, Beltsville, MD, USA; (2) USDA ARS FDWSRU, Fort Detrick, MD, USA; (3) CORPOICA, Villavicencio, COLOMBIA; (4) USDA ARS MPPL, Beltsville, MD, USA; (5) USDA APHIS PPQ S\&T CPHST, Beltsville, MD, USA; (6) University of Florida, Lake Alfred, FL, USA

Citrus leprosis virus C2 (CiLV-C2), a quarantine citrus pathogen in the United States, is present in Colombia. A strain of CiLV-C2 infecting Hibiscus rosa-sinensis (CiLV-C2H) was found in Hawaii and Florida. Recently, a mixed infection of CiLV-C2 and CiLV-C2H in sweet orange with leprosis symptoms has been recorded in Colombia. It is not clear if CiLV-C2H alone can produce symptoms in Citrus spp. or if there are synergistic effects between CiLV-C2 and CiLV-C2H. Next Generation Sequencing using the Illumina platform was performed to determine the genomic sequences of CiLV-C2 and CiLV-C2H in Hibiscus and Citrus. The structure of the CiLV-C2 and CiLV-C2H genomes are identical and shared $92 \%$ amino acid identity. RNA1 of CiLV-C2 and -CiLV-C2H contains 2 ORFs whereas RNA2 contains 5 ORFs. The putative coat protein gene sequence of CiLV-C2HFlorida RNA1 shared 91\% nucleotide and 92-93\% amino acid identity with CiLV-C2H-Hawaii and C2H-Colombia. In addition, movement protein sequences encoded by RNA2 of CiLV-C2H-Florida and CiLV-C2H-Colombia are similar and share $91 \%$ nucleotide and $94 \%$ amino acid identity with CiLV-C2H-Hawaii. The ORFs of CiLV-C2H-Florida are more closely related to the CiLV-C2H-Colombia than to the CiLV-C2H-Hawaii. CiLV-C2 and CiLV-C2H genome specific primers were developed to detect both viruses either in single or dual infection. A real-time RT-PCR assay is needed to detect and quantify both the viruses in mixed infection.

Distribution and incidence of sugarcane mosaic in Louisiana and evaluation of recovery from infection J. RICE, J. W. Hoy, Louisiana State University, Baton Rouge, LA, USA

In Louisiana, sugarcane mosaic is currently caused by Sorghum mosaic virus (SrMV). Development of resistant cultivars has resulted in low mosaic incidence. Recently, resistance selection has relied on distinguishing susceptibility/resistance based on natural infections. With decreased infection pressure, susceptibility may escape detection, and mosaic was detected in advanced selections and a new cultivar HoCP $09-804$. To prevent mosaic from re-emerging as an important problem, multiple lines of research were undertaken. Field surveys were conducted in 2016 and 2017 in breeding program yield trials and cultivar increase plots to determine disease incidence and distribution. Mosaic was detected in two of three areas, and incidence in HoCP 09-804 fields ranged from $0 \%$ to $10 \%$. Runs analysis detected aggregation of infected plants in surveyed fields suggesting the source of infection was infected seed cane. Increases in incidence during 2017 ranged from -62 to $+97 \%$, and incidence ranged from 0.2 to $9.4 \%$. "Recovery" evaluated as the production of asymptomatic plants from buds on symptomatic stalks was compared for two cultivars by planting single symptomatic and asymptomatic stalks. L 10-147 had a 19\% frequency of asymptomatic plants growing from symptomatic stalks compared to $2 \%$ for HoCP $09-804$. When apparently recovered plants were assayed by reverse transcription polymerase chain reaction, $67 \%$ of HoCP $09-804$ and $95 \%$ of L 10-147 samples tested negative for SrMV.

Vigna unguiculata as a model system for studying Soybean vein necrosis virus

C. ZAMBRANA-ECHEVARRIA, C. L. Groves, T. L. German, D. Smith, University of Wisconsin-Madison, Madison, WI, USA

Glycine max (soybean) is a major crop grown for animal feed, vegetable oil, and protein for human consumption. Soybean vein necrosis virus (SVNV) is a member of the Tospoviridae and one of two viruses in the family reported to infect $G$. max. SVNV is transmitted by thrips in a persistent propagative manner and is notoriously difficult to manipulate in G. max. In order to further our understanding of the molecular biology of SVNV, we propose to use Vigna unguiculata as a model plant system. Inoculation with SVNV-infected thrips indicated $V$. unguiculata is highly susceptible to SVNV. Plants showed necrotic lesions on leaves, resulting in severe infection and eventual defoliation. SVNV was detected in symptomatic leaves with RT-PCR and by immunodetection of $\mathrm{N}$ protein. Virions were partially purified by ultracentrifugation. Typical tospovirus particles ranging in $80-120 \mathrm{~nm}$ in diameter were observed using transmission electron microscopy and confirmed by RT-PCR. Compared to G. max, SVNV is much easier to manipulate and study in $V$. unguiculata. While, $V$. unguiculata has already been shown to be a host for SVNV, we have demonstrated its usefulness as a model plant system to study SVNV by optimizing detection methods, studying epidemiology, and improving our understanding of virus-host-plant-vector interactions.

Characterization of a Bean common mosaic virus isolate from lima bean (Phaseolus lunatus)

X. FENG (1), G. Orellana (2), J. Green (3), M. J. Melzer (4), A. V. Karasev (5), (1) University of Idaho EPPN Department, MOSCOW, ID, USA; (2) University of Idaho - EPPN, Moscow, ID, USA; (3) University of Hawaii, Honolulu, HI, USA; (4) Department of Plant \& Environmental Protection Sciences, University of Hawaii at Manoa, Honolulu, HI, USA; (5) University of Idaho, Moscow, ID, USA

Lima bean (Phaseolus lunatus L.) is a popular cultivated legume vegetable grown for dry bean or canned bean production in the United States. Viruses affecting lima bean have not yet been well studied. In 2016, two symptomatic $P$. lunatus plants exhibiting mosaic, vein banding, and growth retardation were collected in a public garden in Honolulu, HI. A potyvirus-specific ELISA (Agdia, Elkhart, IN) was initially used to identify potyvirus infection in the two samples. Sequencing of RT-PCR products generated using the universal potyvirus primer set NIb2F and NIb3R and virus-specific ELISA confirmed both samples as positive for Bean common mosaic virus (BCMV). The samples were subjected to biological characterization on a panel of 11 
differential cultivars of common bean (Phaseolus vulgaris), and to molecular characterization through whole genome sequencing. Both samples were found to contain nearly identical BCMV sequences, named BCMV-A1, which were $93 \%$ identical to some BCMV isolates from China. BCMV-A1 induced an unusually severe systemic necrosis in cultivar 'Dubbele Witte' which could result in plant death in 10-15 dpi, and was able to partially overcome resistance genes $b c-1$ and $b c-2$ in common bean. Phylogenetic analysis of the BCMV-A1 sequence, and distinct biological reactions in common bean differentials suggested that isolate A1 represents a novel strain of BCMV.

Complete nucleotide sequence of a DNA virus isolated from Vitis vinifera in India: A symptomless host of Grapevine red blotch virus A. MARWAL, R. Kumar, R. K. Verma, M. Mishra, R. K. Gaur, Mody University of Science and Technology, Sikar, Rajasthan, INDIA

Symptomless grape plants (Vitis vinifera) were found to be anchoring a Grapevine red blotch virus (GRBV) in Punjab province of India. Full length DNA sequence component of the virus was cloned and determined (KU522121; 3,204 bp), showing similarity with monopartite mastrevirus, begomovirus and other Grapevine red-blotch associated virus reported from US and Canada. A series of experiments were performed to identify any possible mixed infections by other members of Geminiviridae family, which were tends to be negative. Assessing infectivity of the cloned virus and symptoms it induces in plants, full-length construct were produced and agroinfiltrated to 15 plants each from Chenopodium album, Lycopersicon esculentum and Vitis vinifera and five plants from each species were taken as negative (not inoculated with the construct). Following agroinfiltration, plants were incubated in a growth chamber at $25^{\circ} \mathrm{C}$ and symptoms were allowed to develop for 2-4 weeks with regular monitoring. None of the experimented plants developed any observable disease symptoms. When no symptoms appeared at the end of four weeks, plants were assayed (old as well as young leaves under investigation) for virus occurrence by PCR diagnostics, with the help of primers designed for KU522121. All the sample plants were positive for geminivirus infection despite the lack of symptoms. This species of Grapevine red-blotch virus might not be well adapted to Indian Vitis vinifera, suggesting it as a reason for symptomless temperament. To our knowledge this is the first report of a symptomless host Vitis vinifera harbouring a Grapevine geminivirus from Indian vineyards.

Comparison the effects of Chrysanthemum stunt viroid, Hop stunt viroid and Citrus exocortis viroid on tomatoes using Agro-inoculation Y. H. CHENG, Taiwan Agricultural Research Institute, Taichung, TAIWAN

Viroids are known as the smallest self-replicating RNAs that infect plants. Symptoms caused by viroids vary depending on the species and varieties of the hosts. Three tomato-infecting viroid, Chrysanthemum stunt viroid (CSVd), Hop stunt viroid (HSVd) and Citrus exocortis viroid (CEVd) have been reported in chrysanthemum or citrus but not on tomato in Taiwan. Aiming in assessing the impact of the above three viroids on popular tomato varieties in Taiwan, the partial tandem repeat clones CS, HS and CE, corresponding to the CSVd-t (LC090813, tomato isolate), HSVd-c (X00524, cucumber isolate) and CEVd-t-10 (EF494692, tomato isolate) were synthesized, cloned into Ti plasmid (pCAMBIA1302) and transformed to Agrobacterium tumefaciens. Activity assay of the three clones were performed by agro-inoculated to tomato with $10^{7}$ cells per inoculation. Four tomato varieties including 2 big-fruit tomatoes (Farmer 301 and Hui-Jiao) and 2 cherry tomatoes (Kim-Ying and Jade girl) were used as host analysis in this study. The inoculated plants were examined and confirmed as infected by viroid using the reverse transcription-PCR method forty days after inoculation. More than $90 \%$ of the tested plants were detected with the corresponding viroid. All plants of four tomato varieties infected with the clone CS and HS were symptomless. Contrary to the previous description, plants of the 4 tomato varieties infected with the clone CE had significant symptoms such as leaf deformation and growth retardation. When the infected tomato plant was used as inoculum, the host range test was confirmed by mechanical inoculation to give the same results. Both CSVd and HSVd are seed-transmission with $2.3 \%$ and $1.2 \%$, respectively. When the plants infected with CEVd were unable to produce fruits and seeds, and the four varieties were the same.

Study of synergistic interaction between two potexviruses, Cactus virus $X$ and Pitaya virus $X$

Y. M. WU (1), C. Y. Huang (2), Y. C. Chang (3), (1) Department of Plant Pathology and Microbiology, National Taiwan University, New Taipei City, TAIWAN; (2) Department of Plant Pathology and Microbiology, National Taiwan University, Taipei City, TAIWAN; (3) Department of Plant Pathology and Microbiology, National Taiwan University, Taipei, TAIWAN

Cactus virus $X(\mathrm{CVX})$ and Pitaya virus $X(\mathrm{PiVX})$, which belong to the genus Potexvirus, often co-infect pitaya plants in Taiwan. To study the interaction of CVX and PiVX, the infectious clones of two viruses were constructed. Based on our northern blot analyses of protoplast and plant inoculation experiments, CVX and PiVX are beneficial to each other's RNA accumulation. These results indicated that synergistic interaction may exist between CVX and PiVX. This is the first report of synergistic effect occurring within different species of the genus Potexvirus. Synergistic interactions are mostly described between unrelated viruses and have been proven due to suppression of RNA silencing in the co-infected plant. Therefore, we want to study whether the synergism between potexviruses is related to RNA silencing. By transiently co-expressing GFP in Nicotiana benthamiana line 16c plant, five viral proteins of CVX and PiVX were separately tested for RNA silencing suppression ability. According to our results, triple gene block protein 1 (TGBp1) and coat protein of CVX and PiVX revealed different degrees of silencing suppression ability. It is suggested that both proteins may be involved in the synergistic interaction. However, the influence of each viral gene on the other virus need further in vivo studies. These experimental results will provide information of how the synergistic interaction works between CVX and PiVX in planta.

\section{Development of novel virus eradication methods for pitaya}

C. CHEN (1), S. H. Lee (2), Y. C. Chang (3), (1) Department of Plant Pathology and Microbiology, National Taiwan University, Tainan, TAIWAN; (2) Department of Plant Pathology and Microbiology, National Taiwan University, New Taipei City, TAIWAN; (3) Department of Plant Pathology and Microbiology, National Taiwan University, Taipei, TAIWAN

Pitaya (Hylocereus spp.) in the family Cactaceae is an important fruit crop in Taiwan. Three potexviruses, Cactus virus X, Pitaya virus X, and Zygocactus virus $X$, have been reported to infect pitaya, and our field surveys showed that almost all pitaya plants in Taiwan are infected by these viruses. In order to obtain virus-free seedlings, we tried to develop new virus eradication methods for pitaya by means of cryotherapy and $24.5 \mathrm{GHz}$ high-frequency microwaves. To achieve our goal, virus-infected pitaya stems were first cultured on MS medium containing plant growth regulators to produce aseptic new shoots which were used for virus elimination experiments. After several tests, vitrification cryotherapy for pitaya was successfully developed, and there were about $60 \sim 83 \%$ survival rate and $40 \sim 70 \%$ regrowth rate of the treated pitaya shoot tips. This result indicates that cryotherapy can be applied to a tropical pitaya plant. In addition, exposure to high-frequency microwaves for 1.5 seconds resulted in a temperature increase to $80 \sim 90^{\circ} \mathrm{C}$ in aseptic pitaya shoots and $30 \sim 40 \%$ of the treated shoot tips remained viable. Because plant tissues irradiated by high-frequency microwaves can reach above thermal inactivation point of viruses within seconds, we expect it may become a novel plant virus eradication method. The successful rates of virus elimination by cryotherapy and high-frequency microwaves will be investigated by multiplex RT-PCR. 
Virus diseases of vegetables in Mali, West Africa

W. B. LEGESSE (1), Y. L. Chan (2), V. Afari-Sefa (3), L. Kenyon (2), (1) World Vegetable Center, Bamako, MALI; (2) World Vegetable Center, Shanhua, Tainan, TAIWAN; (3) World Vegetable Center, Cotonou, BENIN

Vegetables are important sources of diversified nutrients and income in Mali. However, diseases caused by geminiviruses and more specifically species under the genera begomovirus are among the major production constraints in Mali. Tomato leaf curl Mali virus (ToLCMLV), tomato yellow leaf curl virus (ToYLCrV), Okra leaf curl diseases (OLCD) and other members of Geminiviruses are major problems of tomato, hot pepper, okra and African Eggplant. Recent virus disease survey and diagnosis study using PCR amplification of Begomovirus universal primers and ELISA analysis identified several viruses in Mali. In Okra, 11 out of 12 samples tested, were positive to Geminiviruses while in hot peppers, 16 out of 29 samples tested were positive using PCR amplification. Moreover, ELISA test detected 3 PMV, 1 PMMV, 3 WSMOV and 5 PoTV viruses from 11 African eggplant samples tested. Field trials of seven okra varieties were also conducted to observe the tolerance of these varieties to natural virus infection. Well-known varieties such as Batoumambe and Konni were found to be more susceptible than other less popular varieties. In general, prevalence of virus diseases was much higher during the dry and cold seasons than in the rainy season. Consequently, high losses of tomato and hot pepper crops due to viruses are common features. The higher risk of virus diseases in vegetables and specifically on tomato and hot pepper calls for an urgent need to develop virus resistant varieties.

Outbreak of Groundnut ringspot virus, genus Orthotospovirus, in peanut fields in Argentina S. DE BREUIL (1,2), N. Esteban Bejerman (1,2), C. Nome (1), F. Giolitti (1), S. L. Lenardon (3), (1) Instituto de Patología Vegetal (IPAVE), CIAP INTA, Córdoba, ARGENTINA; (2) CONICET, CABA, ARGENTINA; (3) Centro de Investigaciones Agropecuarias (CIAP), INTA, Córdoba, ARGENTINA

Argentina is one of world's leading exporters of peanuts (Arachis hypogaea L.), with the major production area located in the center of the country. During the 2015/2016 growing season, severe outbreaks of a tospovirus-like disease occurred in commercial peanut fields. The aim of this work was to determine the prevalence and geographical distribution of the disease and perform molecular studies of the etiological agent. Diseased peanut plants were observed in 30 of 80 fields surveyed, located in the north-central region of the peanut-growing area. In DAS-ELISA, all samples collected from symptomatic plants reacted strongly with antisera to Groundnut ringspot virus/Tomato chlorotic spot virus (GRSV/TCSV) (Agdia) and Tomato spotted wilt virus (TSWV) (Bioreba). Total RNA was extracted from ELISA-positive tissues and RT-PCR was performed with primers for conserved regions of the GRSV, TCSV and TSWV nucleoprotein $(N)$ genes. PCR amplicons were digested with the HincII and Bst NI enzymes and the restriction patterns agreed with those corresponding to GRSV. This result was confirmed by RT-PCR, using specific primers for GRSV $N$ gene, followed by sequencing of the $\sim 800$-bp amplified fragments, which shared $\geq 97 \%$ nucleotide sequence identity with the Argentine isolate GRSV-AR (KT972594). Our study highlights the importance of understanding the factors that lead to epidemics caused by GRSV and current research is focused on thrips vector dynamics.

The impact of Triticum mosaic and Wheat streak mosaic viruses' co-infection on spring wheat performance D. YABWALO (1), M. A. Langham (1), R. L. Geppert (1), E. Byamukama (2), (1) South Dakota State University, Brookings, SD, USA; (2) Department of Agronomy, Horticulture, and Plant Science, South Dakota State University, Brookings, SD, USA

Triticum mosaic virus (TriMV), a fairly new wheat (Triticum aestivum L.) pathogen, largely co-infects winter wheat with Wheat streak mosaic virus (WSMV), a well-known pathogen that can cause severe yield losses in winter wheat. The combined impact of TriMV and WSMV on spring wheat is not well known. A field study was initiated to investigate the differential performance of diverse spring wheat genotypes against WSMV, TriMV and a mix of WSMV-TriMV, and to determine the effect of WSMV-TriMV co-infection on yield, shoot development and chlorosis in spring wheat. Seven hard red spring wheat cultivars and three elite breeding lines were inoculated with WSMV, TriMV and a 1:1 mix of WSMV and TriMV at Feeke's stage 4. Virus severity (Vscore) was rated on a 1-5 scale where 1=no disease and $5=$ severe chlorosis. Most of the inoculated genotypes developed moderate to severe symptoms but 'Select' consistently responded better than the rest to all viral inocula with predicted cumulative probabilities of 0 and 0.23 at Vscore $=5$ and Vscore $\leq 2.5$, respectively. The rest of the cultivars were above 0.75 probability at Vscore $\leq 2.5$. A negative Pearson's correlation was observed between yield and Vscore $(r=-0.78, p \leq .0001)$. Overall yield reduction by WSMV-TriMV was $75 \%$ compared to $35 \%$ and $39 \%$ reduction due to WSMW and TriMV, respectively. Independently, the two viruses were not significantly different from each other for yield and Vscore. These results suggest that spring wheat performance can severely suffer from WSMV-TriMV co-infection. Therefore, breeding efforts should be devoted to developing resistance to both viruses.

Latent and incubation periods of Cowpea aphid-borne mosaic virus in passionflower

D. MARQUES DE ALMEIDA SPADOTTI, G. Favara, J. A. M. Rezende, University of São Paulo - ESALQ, Piracicaba, BRAZIL

In Brazil, Cowpea aphid-borne mosaic virus (CABMV, genus Potyvirus) is widespread in yellow passion fruit (Passiflora edulis f. flavicarpa) causing woodiness disease. Currently, there are no effective and long-lasting forms of disease control. The objective of this work was to determine the latent and incubation periods of the disease to subsidize the roguing of infected plants as an alternative for disease management. 140 and 120 passion fruit plants were used on each assay, respectively. CABMV isolates from the states of São Paulo, Bahia and Rio de Janeiro were used, and the virus was sap transmitted to health plants. Latent period was evaluated throughout virus recovery from new developed leaf, 3 to 7 days after test-plants inoculation, whereas incubation was evaluated through symptoms and PTA-ELISA. The latent period ranged from 3 to 7 days (average 6 days), and the incubation 4 to 15 days (average 8 days). The same assay was performed with the São Paulo isolate of CABMV and transmission with the aphid Myzus persicae and the results were similar (average latent and incubation periods 8 and 9 days, respectively). Despite the variability of isolates, greenhouse environmental conditions, and inoculation methods, the latent and incubation periods of CABMV are very close, evidencing that the roguing of symptomatic plants can be consistently used to decrease source of inoculum of the virus, consequently diminishing secondary spread.

Identification of a novel endornavirus in Hydrocotyle spp.

C. ESCALANTE GUARDADO (1), R. I. Alcalá-Briseño (2), E. Rodrigues De Souto (3), R. A. Valverde (4), (1) Louisiana State University Agricultural Center, Baton Rouge, LA, USA; (2) Department of Plant Pathology, University of Florida, Gainesville, FL, USA; (3) Univ Estadual de Maringa, Maringa, BRAZIL; (4) Dept of Plant Pathology and Crop Physiology, Louisiana State University Agricultural Center, Baton Rouge, LA, USA

Endornaviruses are RNA viruses reported to infect plants, fungi, and oomycetes. Plant endornaviruses have a genome that ranges from 13.5-17.6 kb, are transmitted vertically, and do not cause symptoms. Endornaviruses have been found infecting many economically important crops but only in a few noncultivated plants. Hydrocotyle species include perennial weeds commonly found in wetlands, home lawns, and turf-covered areas in the United States. Using electrophoretic analyses of dsRNA as an initial detection tool, we conducted a survey for endornaviruses in five symptomless Hydrocotyle species 
collected in south Louisiana. Two species, H. umbellata and H. prolifera, contained endornavirus-like dsRNAs. The dsRNA from both species was purified and sequenced. The sequence of both dsRNAs was similar and contained a single open reading frame, which coded for a polyprotein of 4,773 aa. BLASTp analysis showed that the polyprotein had the highest identity $(50 \%)$ with the polyprotein of Lagenaria siceraria endornavirus. Conserved protein domains included helicase, capsular polysaccharide synthase, glycosyl transferase, and RNA-dependent RNA polymerase. Three other Hydrocotyle species, $H$. bonariensis, $H$. verticillata and $H$. ranunculoides, were endornavirus-negative. These results confirm that in south Louisiana, $H$. umbellata and $H$. prolifera are infected with a novel endornavirus.

PVS ${ }^{\mathrm{P}}$ : A new potato virus $\mathrm{S}$ lineage infecting Solanum phureja in Colombia

D. Vallejo, P. Gutiérrez, M. MARIN, Universidad Nacional de Colombia, Medellin, COLOMBIA

Solanum phureja is an exotic potato crop produced in Andean countries that has a very good export potential due to its organoleptic and nutritional properties. Unfortunately, this crop is seriously affected by a variety of fungi and several uncharacterized viral diseases. In order to have a better understanding of the viruses infecting S. phureja in Colombia, a High-throughput sequencing study using the Illumina HiSeq-2000, was performed in plants showing symptoms of viral infection in Antioquia. Bioinformatic analysis revealed infection by a potato virus S (PVS) strain with low nucleotide sequence identity at the whole genome level (81-82\%) with respect to the Andean $\left(\mathrm{PVS}^{\mathrm{A}}\right)$ and Ordinary $\left(\mathrm{PVS}^{\mathrm{O}}\right)$ lineages. Assembly of this novel PVS isolate, resulted in a genome sequence of $8483 \mathrm{nt}$ (excluding 3' poly-A tail) containing six ORFs encoding proteins RdRp, 25K, 12K, CP, 7K and 11K. Sanger sequencing and phylogenetic analyses revealed that the PVS strain infecting $S$. phureja forms a distinct clade differentiated from the PVS ${ }^{\mathrm{O}}$, PVS and PVS recombinants. We propose the name PVS ${ }^{\mathrm{P}}$ for this new lineage in reference to the host $S$. phureja. Finally, primers for real-time RT-PCR detection of $\mathrm{PVS}^{\mathrm{P}}$ were designed using the complete genome as reference. Primers were validated in 15 tuber samples and 15 leaf samples revealing infection levels of $86.6 \%$ and $60 \%$, respectively. These results highlight the importance of updating current S. phureja seed-certification programs in Colombia.

Early viral infection on sweet potatoes under field conditions

A. FURTADO SILVEIRA MELLO (1), R. Souza (1), M. Silva (1), G. Olegario (2), (1) Embrapa Vegetables, Brasilia, BRAZIL; (2) Embrapa Vegetables, Canoinhas, BRAZIL

Sweet potato is a major crop in many countries and its cultivation usually requires low inputs to produce reasonable yields. The great majority of Brazilian growers use vine cuttings from previous crops to establish areas. This practice is very common on the northeast region of the country where the weather enables the production all year long. The lack of fallow with no crop rotation and use of propagative material harvested in open field increases the spread and accumulation of pathogens in the field, leading to a gradual reduction on yield and root quality. On the South region of Brazil the winter season is very severe not enabling sweet potato cultivation during this period which potentially decreases the spread of pathogens. In Brazil, very little is known about the prevalence and impact of different viruses on sweet potato. The goal of this work is to assess if a single crop cultivation would be sufficient to enable the detection of viruses by ELISA and grafting on the indicator Ipomoea setosa. Virus free plants were planted in Canoinhas, SC during the summer 2016-17 under field conditions and after five months, stems from sweet potato plants were harvested and grafted on the indicator plant. Thirty days after grafting plants presented symptoms and at least one clone was positive by DOT-ELISA. This finding indicates the potential impact of virus transmission on sweet potatoes even on regions with less conducive environment for viruses and its vectors.

Zucchini yellow mosaic virus disease of cucurbits in a tropical environment: Aphid vectors, alternate hosts, and epidemic drivers

R. Clarke (1), M. Kehoe (2), S. Broughton (2), C. G. Webster (2), B. Coutts (2), P. Goldsmith (3), M. Warmington (4), R. A. C. JONES (2,5), (1) Raitech Ltd, Kununurra, AUSTRALIA; (2) DPIRD, South Perth, AUSTRALIA; (3) ORDCO, Kununurra, AUSTRALIA; (4) DPIRD, Kununurra, AUSTRALIA; (5) Institute of Agriculture, University of Western Australia, Crawley, AUSTRALIA

Irrigated crops of pumpkin, melon and watermelon are grown in tropical northwest Australia where agriculture is $<60$ years old. They suffer severe epidemics of the Southeast Asian Zucchini yellow mosaic virus (ZYMV) strain which is absent elsewhere in Australia. Eleven surveys were undertaken in 2015-2017 to establish which aphid species occur, when aphids spread ZYMV during the growing season and where ZYMV and aphids survive outside the growing season. Growing season data collection blocks of watermelon gathered information on aphid numbers and ZYMV incidence. Allyear-round aphid trapping used sticky traps. Samples were collected for virus testing (ELISA, RT-PCR) and aphid identification (light microscopy, PCR). Six aphid species were present, three of which transmitted ZYMV, Aphis gossypii, A. craccivora, and A. nerii. Outside the growing season, ZYMV was rarely detected and only in volunteer cucurbits, wild melon vines and home garden cucurbit plantings. The aphid vector reservoir was much larger: A. gossypii survived on home garden cucurbit crops, volunteer cucurbits, wild afghan melon, wild melon vines, milkweed (Euphorbia hirta), and native rosella (lbemoschus ficulneus); A. craccivora on legume crop plants in home gardens, Sesbania rootstocks in sandalwood plantations and legume weeds; and Aphis nerii on calotrope (Calotropis procera). Underlying environmental factors driving the annual ZYMV epidemics in this remote region will be described.

Overview of occurrence and incidence of plant virus diseases in crop fields in Korea during 2012-2016

M. KIM (1), J. E. Kim (1), W. R. Go (1), J. Kim (1), H. R. Kwak (1), J. K. Seo (2), J. S. Kim (3), H. S. Choi (1), (1) National Institute of Agricultural Science, Wanju, SOUTH KOREA; (2) Seoul National University, Pyeongchang, SOUTH KOREA; (3) Andong National University, Andong-si, SOUTH KOREA

Plant viruses infect various horticultural and agricultural crops, in which they induce severe symptoms causing decrease of desired quality or yield. In Korea, more than one hundred of plant viruses have been reported since the first identification of Rice strip virus in 1966. During 2012-2016, we examined occurrence and incidence of plant virus diseases in major crops in Korea by investigating the total number of 5,256 samples showing viruslike. Virus diagnosis was performed by electron microscopy, RT-PCR, large scale oligonucleotide (LSON) chip analysis, next generation sequencing (NGS), and biological tests. The numbers of virus species detected from the investigated samples were 19 species in 2012,34 species in 2013,39 species in 2014, 33 species in 2015, and 27 species in 2016. The numbers of virus species detected from the investigated samples were total 64 species for 5 years. The newly recorded viruses were Turnip yellow mosaic virus (TYMV) in 2012, Tomato chlorosis virus (ToCV), Plantago asiatica mosaic virus (PIAMV), Butterbur mosaic virus (ButMV), Sweet potato virus C (SPVC), Sweet potato virus 2 (SPV2), Sweet potato symptomless virus 1 (SPSMV-1) and Sweet potato chlorotic fleck virus (SPCFV) in 2013, and Chrysanthemum stem necrosis virus (CSNV), Euphorbia leaf curl virus (EuLCV), East Asia Passiflora virus (EAPV), Cucurbit aphid-borne yellows virus (CABYV) in 2014, and Citrus leaf blotch virus (CLBV) in 2015. In total, 64 virus species were detected from crop fields in Korea during last five years. Top 10 viruses that were most frequently detected were Cucumber mosaic virus (CMV), CABYV, Tomato yellow leaf curl virus (TYLCV), Watermelon mosaic virus (WMV), Broad bean wilt virus 2 (BBWV2), Tomato spotted wilted virus (TSWV), TYMV, ToCV, Beet western yellows virus (BWYV), and Lily mosaic virus (LMoV). 
Deep sequencing of total RNAs in papaya for genome characterization of Papaya ringspot virus Bangladesh strain

I. Hamim (1,2), M. Al Rwahnih PhD (3), W. B. Borth (1), M. J. Melzer (1), D. Gonsalves (4), J. Y. Suzuki (4), M. Wall (4), J. S. HU (1), (1) Department of Plant \& Environmental Protection Sciences, University of Hawaii at Manoa, Honolulu, HI, USA; (2) Department of Plant Pathology, Bangladesh Agricultural University, Mymensingh, BANGLADESH; (3) Foundation Plant Services Facility, University of California, Davis, CA, USA; (4) USDA ARS, Daniel K. Inouye U.S. Pacific Basin Agricultural Research Center, Hilo, HI, USA

Papaya (Carica papaya L.) is an economically important horticultural crop in Bangladesh. During a survey conducted on papaya viruses in different papaya growing regions of Bangladesh in December 2016 to January 2017, nearly 100\% of papaya plants in some locations were observed to have typical symptoms of Papaya ring spot virus (PRSV) infection. Using a PRSV-specific single-tube nested PCR assay, the presence of PRSV was confirmed in several symptomatic plants. PRSV positive samples were then subjected to deep sequencing analyses on an Illumina ${ }^{\circledR}$ NextSeq 500 platform. A nearly complete genome (10282bp) of PRSV-Bangladesh strain (PRSV-BD) was generated. To validate the results of the deep sequencing analysis, 8 pairs of PRSV-specific primers were designed and overlapping DNA fragments were amplified by RT-PCR from an isolate (G52158) collected from Gazipur district. The amplified DNA fragments were cloned and sequenced by Sanger sequencing method and the resulting sequences were found to be $100 \%$ identical at the nucleotide level to the deep sequencing-derived sequence of PRSV-BD. Further analysis of genome using BLASTn showed that PRSV-BD was the most closely related to PRSV-Aus-53C (GenBank accession no. KX655866), a PRSV-W strain reported from cucurbits in Australia.

Molecular analysis indicates that papaya in Bangladesh is a host of multiple begomoviruses I. HAMIM (1,2), W. B. Borth (1), M. J. Melzer (1), D. Gonsalves (3), J. Y. Suzuki (3), M. Wall (3), J. S. Hu (1), (1) Department of Plant \& Environmental Protection Sciences, University of Hawaii at Manoa, Honolulu, HI, USA; (2) Department of Plant Pathology, Bangladesh Agricultural University, Mymensingh, BANGLADESH; (3) USDA ARS, Daniel K. Inouye U.S. Pacific Basin Agricultural Research Center, Hilo, HI, USA

Papaya (Carica papaya) is one of the important fruit crops in Bangladesh. Papaya plants showing severe leaf curl symptoms distinct from those caused by Papaya ringspot virus were observed in several papaya growing districts in Bangladesh during December 2016. These symptoms and the presence of whitefly infestations on these plants suggested the possibility of begomovirus infection. Forty-five symptomatic leaf samples were collected from seven papaya-growing districts in Bangladesh. Degenerate primers (MkBegAF4/MkBegAR) targeting the coat protein and the partial AC2 protein genes of the DNA-A component of begomoviruses were used in PCR assays for detection of begomoviruses in these samples. A $1.3 \mathrm{~kb}$ fragment from DNA-A component of begomoviruses was consistently amplified from 43 out of 45 symptomatic samples. Sanger sequencing and BLASTn analyses of these partial DNA-A component sequences revealed that these virus isolates could be classified into three distinct groups (groups 1, 2, 3). The 29 isolates in group 1 were positive for Tomato leaf curl Bangladesh virus (ToLCBV), while 10 isolates in group 2 and 4 isolates in group 3 were positive for Tomato leaf curl Joydebpur virus (ToLCJoV) and Tomato leaf curl New Delhi virus (ToLCNDV), respectively. The complete DNA-A genome sequences of isolates G34106, P11, and P412 were determined and characterized. G34106, P11, and P412 were most closely related to GenBank accession nos. KM383761 (ToLCBV), KM383750 (ToLCJoV), and KM383742 (ToLCNDV), respectively. All of these begomoviruses had been previously identified as infecting tomato in Bangladesh. Our findings suggest the existence of multiple begomoviruses infections in papaya in Bangladesh, which may pose a serious threat to the papaya industry there.

Rose viruses: Understanding the current status and protecting the future of the UK rose sector I. VÁZQUEZ IGLESIAS (1), N. Boonham (2), A. Fox (2), G. Clover (3), R. Robinson (3), (1) Newcastle University/ Fera Science Ltd., York, UNITED KINGDOM; (2) Fera Science Ltd, York, UNITED KINGDOM; (3) Royal Horticultural Society, London, UNITED KINGDOM

Roses are one of the most important ornamental flowering shrubs grown worldwide and the national flower of England. They contribute $£ 9$ billion to the UK economy, however, $£ 40$ million are lost due to viral infections. Despite this, rose viruses haven’t been studied in detail since the 1980's in the UK and, given the plant health risk posed by -Rose rosette virus- RRV, it is necessary to investigate the current phytosanitary status. RRV (Family: Bunyaviridae, Genus: Emaravirus), described for the first time in the 1940s, is transmitted by the eriophyid mite, Phyllocoptes fructiphilus and has been reported in the US and India. The aim is to future proof rose cultivation in the UK. By better understanding the current situation of rose viruses, we can identify the gaps in our knowledge and future plant health priorities, improving response to new and emerging diseases, such as RRV. Recent advances in molecular diagnostics could help to clarify rose viruses taxonomy and to study their aetiology more effectively. To ascertain the current phytosanitary status of UK rose production we are using a combination of target and non-target diagnostic technologies. ELISA and TaqMan ${ }^{\circledR}$ RT-PCR will be used as an initial screening for known viruses. In addition, using Next Generation Sequencing (NGS) both symptomatic, virus-negative samples and a sub-set of asymptomatic samples will be tested to try to identify any known and unknown viruses. Preliminary screening results will be presented.

Mapping synergistic interaction determinants between Panicum mosaic virus and Satellite panicum mosaic virus C. R. R.V, N. Palmer, G. Sarath, S. Edme, R. Mitchell, S. TATINENI, USDA-ARS, Lincoln, NE, USA

Panicum mosaic virus (PMV; genus Panicovirus; family Tombusviridae) causes mild mosaic symptoms on switchgrass (Panicum virgatum) and other millets. Satellite panicum mosaic virus (SPMV) depends on PMV for its replication and movement. PMV and SPMV synergistically interact in coinfected millets resulting in severe mosaic, yellowing, and stunting of plants. In this study, infectious cDNA clones of a Nebraska isolate of PMV and a Kansas isolate of SPMV (GenBank accession \# M17182) were developed. Symptom phenotypes of PMV-NE alone and PMV-NE+SPMV-K were compared with those of PMV-TX alone and PMV-TX+SPMV-TX on proso millet (Panicum miliaceum). Both PMV-TX and NE isolates elicited mild mosaic symptoms. Co-infection of proso millet by PMV-NE+SPMV-K elicited severe mosaic, yellowing, and stunting compared to moderate symptoms by PMV-TX+SPMV-TX, suggesting that either PMV-NE or SPMV-K contributed to the observed robust synergistic interaction in proso millet. Additionally, co-infection of proso millet by PMV-TX or PMV-NE with SPMV-K but not with SPMV-TX elicited severe symptoms, indicating that SMPV-K was the main contributor to efficient synergistic interaction with PMV isolates. Comparison of the genome sequences of SPMV-K and SPMVTX revealed 11 nucleotide differences with four non-synonymous and three synonymous changes in the coat protein ORF. These genomic differences between SPMV isolates provide the bases for the differential synergistic interaction with PMV.

Prevalence of maize-infecting potyviruses and maize chlorotic mottle virus in the United Republic of Tanzania during 2016-2017 growing seasons

D. P. MASSAWE (1), L. R. Stewart (2), (1) The Ohio State University, Wooster, OH, USA; (2) USDA ARS, Wooster, OH, USA

Viruses in the genus Potyvirus (potyviruses) cause Maize lethal necrosis (MLN) when co-infected with maize chlorotic mottle virus (MCMV). MLN causes up to $100 \%$ yield loss, threatening food security in East Africa where maize is a staple crop. To assess virus prevalence, surveys were conducted 
in 2016 and 2017 in 11 regions located in five agro-ecological zones: Lake (Mara and Mwanza), Northern Highlands (Kilimanjaro, Arusha and Manyara), Coastal (Tanga, Pwani and Morogoro), Central (Dodoma) and Southern Highlands (Iringa and Songwe). For each of 78 fields, 10 samples were collected on a transect and analyzed by ELISA using generic potyvirus and MCMV antisera. Of 780 samples, 338 (43.3\%) tested positive for potyviruses and $176(22.6 \%)$ for MCMV. Prevalence of potyviruses was $43 \%(n=70), 47.1 \%(n=170), 46.9 \%(n=390), 37.5 \%(n=40)$, and $27.3 \%$ $(\mathrm{n}=110)$ in Lake, Northern Highlands, Coastal, Central, and Southern Highland zones, respectively (listed north to south). In the same samples, MCMV showed a strong north to south gradient as expected for emergence from the north, with $40 \%, 35.9 \%, 20.5 \%, 10.0 \%$, and $2.7 \%$ detection in the same zones. Other viruses were also identified, including a polerovirus previously described in China, detected at 37/78 location across zones by RT-PCR. Our study reveals high prevalence of potyviruses across Tanzania's maize growing zones, whereas MCMV shows a gradient that may rapidly shift with virus emergence or be responsive to management.

Distribution and titer of Little cherry virus 2 (LChV2) in Prunus avium in time and space A. WRIGHT, S. J. Harper, Washington State University, Prosser, WA, USA

Little cherry virus 2 (LChV2) is one of the causative agents of little cherry disease, which produces misshapen, unpalatable fruit in infected cherry (Prunus avium) trees, resulting in yield losses for growers. Since its emergence in 2010, this disease has been spreading rapidly in cherry orchards across the Pacific Northwest. A major issue hindering control of this disease is early detection and diagnosis, as the virus is not evenly distributed within a host and titer fluctuates seasonally. In this study, we aim to follow the titer and distribution of LChV2 in different tissues of a single plant across two years. To do so, RNA is being extracted from multiple tissues throughout the year of a 10 -year-old $P$. avium cv. Lambert tree infected with the LC-5 isolate of LChV2. In late summer, titer was greatest in the midrib, being 1.5 to 5.6 times greater than the petiole, 510 times greater than the wood scrapings, and 10,000 times greater than the buds. In mid-fall the results were more variable. Titer in the midrib was 0.9 to 3.1 times greater than the petiole, 0.9 to 4.8 times greater than the wood scrapings, 4.7 to 99 times greater than the roots, and 18 to 38 times greater than the buds. Data collection will continue throughout the year to assess how titer and distribution vary seasonally. Knowing the distribution and titer of LChV2 will allow for better sampling of $P$. avium for LChV2 detection and reduce the occurrence of false negatives.

Bipartite networks of hosts and viromes: Diversity of viruses of papaya orchards, associated weeds and potential vectors in Southern Mexico R. I. ALCALÁ-BRISEÑO (1), K. Casarrubias-Castillo (2), D. López-Ley (3), L. Silva-Rosales (4), K. A. Garrett (5), (1) Department of Plant Pathology, University of Florida, Gainesville, FL, USA; (2) CINVESTAV-IPN, Irapuato, MEXICO; (3) NA, Chiapas, MEXICO; (4) Cinvestav-IPN, Irapuato, Gto, MEXICO; (5) Plant Pathology Department, University of Florida, Gainesville, FL, USA

Intraspecific virus interactions affect disease severity, and interspecific interactions (mixed infections) can result in either synergism or antagonism. At least 15 plant viruses are reported to infect papaya, suggesting the potential for multiple interactions and differences in symptomatology. This work is the first to characterize viral diversity using metagenomics in two regions of Chiapas, one of the largest papaya production regions in Mexico. Plants with three types of papaya symptoms were analyzed: visually asymptomatic, PRSV-typical symptoms, and other viral symptoms. Weeds and insects in areas surrounding papaya orchards were also analyzed. The ecological interactions in the virome were analyzed in a bipartite network framework with two types of nodes, location/host and virus, where links represent an association. There were differences by location and host combination, revealing a structure between a modular and a nested community. This ecological network represents the dynamics of the primary host (papaya), the secondary host (weeds) and potential vectors (insects). We identified known and emergent viruses associated with papaya plants and weeds, and potential associations with vectors. Management of viruses associated with the papaya industry can be improved by sanitizing tools and implementing strategies to control populations of beetles and leafhoppers, the vectors of novel viral species reported in this study.

Differential spread of Potato virus Y (PVY) strains in the field: Implications for the rise of recombinant PVY strains in New Brunswick, Canada T. MacKenzie (1), J. Lavoie (2), X. NIE (3), M. Singh PhD (4), (1) Agricultural Certification Services Inc., Fredericton, NB, CANADA; (2) New Brunswick Department of Agriculture, Aquaculture and Fisheries, Wicklow, NB, CANADA; (3) Agriculture and Agri-Food Canada, Fredericton Research and Development Centre, Fredericton, NB, CANADA; (4) Agricultural Certification Services, Fredericton, NB, CANADA

Potato virus $Y$ (PVY) is a major cause of yield and quality loss in potato crops worldwide. Multiple strains and substrains, including the common strain $\left(\mathrm{PVY}^{\mathrm{O}}\right)$, recombinant $\mathrm{N}: \mathrm{O}$ strain $\left(\mathrm{PVY}^{\mathrm{N}: \mathrm{O}}\right)$ and recombinant potato tuber necrosis $\left(\mathrm{PVY}^{\mathrm{NTN}}\right)$ strain have been recognized. In a survey carried out in 2009 in New Brunswick (NB), Canada, $\mathrm{PVY}^{\mathrm{O}}$ was the predominant strain followed by PVY ${ }^{\mathrm{N}: \mathrm{O}}$ and $\mathrm{PVY}^{\mathrm{NTN}}$ were widespread in potato seed lots. Recently, populations of PVY strains have shifted dramatically toward recombinant strains. A continuous survey of PVY strains in commercial fields of NB from 2009 to 2016 and five field trials tracking PVY spread in the province were conducted to study the current status of PVY strains and their relative rates of spread. PVYO dropped from $82 \%$ of infections in 2010 to $14 \%$ in 2016 , replaced mostly by PVY $\mathrm{PYN}^{\mathrm{NTN}}(64 \%)$ and PVY ${ }^{\mathrm{N}: \mathrm{O}}(22 \%)$. In field trials with potato cultivars Russet Burbank and Goldrush, $\mathrm{PVY}^{\mathrm{NTN}}$ spread most effectively compared to $\mathrm{PVY}^{\mathrm{N}: \mathrm{O}}$ and $\mathrm{PVY} \mathrm{O}^{\mathrm{O}}$. Strain-specific PVY spread varied with the potato cultivar, possibly due to selective PVY hypertensive resistance in Goldrush, mostly expressed at the plant-to-plant transmission level with little difference in transduction to tubers in infected plants.

Initial molecular characterization of a novel emaravirus from Callicarpa (beautyberry) identified by high-throughput sequencing R. L. JORDAN (1), J. Hammond (2), J. F. Murphy (3), (1) USDA-ARS, USNA, Floral \& Nursery Plants Research, Beltsville, MD, USA; (2) USDAARS, USNA, Floral and Nursery Plant Research Laboratory, Beltsville, MD, USA; (3) Auburn University, Auburn, AL, USA

The genus Callicarpa (beautyberry) contains approximately 140 species of perennial deciduous shrubs and small trees. The common name refers to its most well-known ornamental feature - prolific white, lilac, lavender, or purple-colored fruit that covers the shrub in late summer through autumn. Mosaic symptoms were observed on a Callicarpa americana plant growing in Auburn, AL. Total RNA was purified from leaves of this plant, and a custom cDNA library was prepared for paired-end Illumina MiSeq next-generation sequencing (NGS). Raw sequence reads (3,804,206 35-250bp reads) were trimmed of adaptor linkers and de novo assembled into 197,416 contiguous sequences using Geneious Pro R9. Contig sequences were subjected to BLASTX analysis against known viral sequences in GenBank (NCBI) databases and 15 contigs were identified that had significant putative protein homologies with members of the genus Emaravirus. Complete or near-complete sequences were obtained for genomic RNAs 1-4. The in silico translated proteins have $29-62 \%$ amino acid identities with emaravirus orthologs RdRp (RNA 1), glycoprotein precursor (RNA 2), NP (RNA 3), and MP (RNA 4). Results of ongoing NGS and RT-PCR analysis (using Callicarpa emaravirus-specific primers) with other Callicarpa samples will also be presented. Previously, the only virus reported to infect beautyberry was Cucumber mosaic virus. To our knowledge, this is the first report of an emaravirus infecting Callicarpa. 
Application of tissue culture to produce virus-free plants from imported potato germplasm P. Bandla, J. McCallister, C. Kepner, T. Schulden, J. Foster, R. D. FRENCH-MONAR, USDA-APHIS, Plant Germplasm Quarantine Program, Beltsville, MD, USA

Certain plant genera are restricted from entering the United States because they are known to carry plant pathogens that are not readily detected at inspection stations, such as viruses and phytoplasmas. The USDA APHIS Plant Germplasm Quarantine Program (PGQP) imports apples, sugarcane, rice, stone fruit, potatoes, sweetpotatoes, and other crops belonging to these so-called "prohibited" genera. The Potato Quarantine Program uses a variety of diagnostic tools, including molecular and serological tests, indicator plants, and electron microscopy to detect pathogens that could threaten and compromise U.S. agriculture. Accessions that are found to be infected cannot be released and must undergo treatment in tissue culture. In vitro therapy procedures have allowed for the successful elimination of many pathogens, including viruses that are otherwise difficult to eradicate and would have made the germplasm unavailable to stakeholders. Between October 2016 and November 2017, the Potato Quarantine Program tested 117 accessions, including 27 that were previously found to be infected and had received therapy in tissue culture. Using a combination of heat therapy, chemotherapy, and meristem tip culture, pathogens were successfully eliminated from 26 accessions (96\%). This included nine out of the ten accessions infected with Potato Virus $S$ (PVS), all ten accessions infected with potyviruses, and all seven accessions infected with a combination of potyviruses, PVS, and/or Potato Virus $M$ (PVM). The high rate of virus eradication achieved by using multiple in vitro therapy techniques, in combination, has allowed the program to release accessions that would otherwise have been unavailable for release.

Understanding pathogen and environmental drivers of white leaf spot (Pseudocercosporella capsellae) epidemics and their impacts on canola T. MURTZA (1,2,3), M. You (1,2), M. Barbetti (1,2), (1) School of Agriculture and Environment Faculty of Science The University of Western Australia, Perth, AUSTRALIA; (2) The University of Western Australia Institute of Agriculture, Faculty of Science, Perth, AUSTRALIA; (3) Department of plant pathology, Faculty of Agriculture, University of Agriculture, Faisalabad, PAKISTAN

White leaf spot (Pseudocercosporella capsellae) is an important but underestimated disease worldwide on both oilseed and horticultural Brassicas. Canola (Brassica napus) most important oilseed Brassica crop worldwide and in Australia this white leaf spot results in losses up to $30 \%$, especially in highly susceptible varieties. Australia is facing variable and increasing temperatures, elevated drought stress and increasingly unpredictable rainfall that together have been associated with shifts in virulence and increased diversity within the $P$. capsellae population. Australia-wide populations of $P$. capsellae are being studied in relation to their pathogenicity and virulence across different canola genotypes using a cotyledon based screening assay and also assessed for their genetic differences. Increased variability in climate has also been associated with increasing incidence and severity of white leaf spot, likely due to more favourable conditions for development of severe epidemics. The variable Mediterranean climate in south west Western Australia makes it an ideal location to study and model current and future climate effects, and to project future risks, of white leaf spot epidemics on canola. Studies are being undertaken to define the key environmental drivers of white leaf spot epidemics. Significant phenotypic and genetic variation has been identified within the $P$. capsellae population and this is helping to explain the increasing importance and adverse impact of white leaf spot over recent decades.

Identification of temperature-sensitive resistance to Puccinia striiformis $\mathbf{f}$. sp. tritici in Chinese and international differential hosts H. Zeng, J. FENG, R. Lin, W. Fengtao, S. Xu, Institute of Plant Protection, Chinese Academy of Agricultural Sciences, Beijing, CHINA

Temperature affects wheat resistance to Puccinia striiformis f. sp. tritici (Pst). Thirty-one entries of Chinese, international and other tester wheat cultivars were studied in seedling stage at two different day/night temperature regimes $\left(24 / 18^{\circ} \mathrm{C}\right.$ and $\left.14 / 10^{\circ} \mathrm{C}\right)$ to identify the temperature-sensitive resistance of the entries. Four entries, Lutescens 128, Funo, Lee and Carstens V, were confirmed no temperature-sensitive resistance genes. Six wheat cultivars, Early piemium, Fengchan 3, Fulhard, Heines VII, Mentana and Virgilio, have shown temperature-sensitive resistance. Comparison with standard cultivars of 0-3 temperature-sensitive genes, Virgilio resistance to Pst race 10E162 was probably controlled by two temperature-sensitive genes, and Mentana and Fulhard each possessed one temperature-sensitive gene. Virgilio, Fulhard and Mentana as the temperature-sensitive gene resources are useful in breeding for resistance to stripe rust. As the differential hosts of wheat stripe rust, it is necessary to strictly control the temperature without exceeding $18^{\circ} \mathrm{C}$, since infection type may differ due to the different temperature.

Temperature dependent RNA metabolism in Xylella fastidiosa during cold stress and grapevine infection L. BURBANK, USDA-ARS, Parlier, CA, USA

Reoccurrence of Pierce's disease of grapes, caused by Xylella fastidiosa, is known to be influenced by environmental factors, particularly cold temperatures during overwintering. Grapevines in colder regions are often cured of $X$. fastidiosa infection over the winter season, depending on cultivar, time of inoculation, and disease severity. The dynamics of $X$. fastidiosa low temperature adaption and survival during persistent infections in planta is still poorly understood. RNA metabolism is an essential part of bacterial response to low temperature, including inducible expression of RNA binding proteins, helicases, and exoribonucleases. Characterization of two $X$. fastidiosa RNA-binding family cold shock protein (CSP) homologs revealed that neither was cold-inducible at the transcriptional or post-transcriptional level, suggesting a diminished cold adaptation response in this pathogen. However, expression of $X$. fastidiosa cold response RNA helicase, $\operatorname{srm} B$, is reduced in a CSP mutant ( $\triangle \operatorname{csp} 1)$ compared with wild type $X$. fastidiosa, indicating a potential regulatory role of Csp1 in RNA metabolism during temperature response. Understanding the cold-adaptation process of $X$. fastidiosa is important as it relates to the ability of this pathogen to survive through the winter in infected plants depending on geographic location and climatic conditions.

Disease variation of Sphaceloma manihoticola isolates affecting cassava in Barbados A. T. ALLEYNE, The University of the West Indies, Cave Hill Campus, Bridgetown, BARBADOS

Sphaceloma manihoticola, (Bitancourt \& Jenkins) causal agent of Superelongation disease (SED) of cassava Manihot esculenta (Crantz) is an important pathogen in the Caribbean, given its potential to negatively impact the cassava industry. It produces increased quantities of gibberellin $\mathrm{GA}_{4}$ in late stages of infection by over-expression of Smp 450 genes in-planta. This study sought to characterize SED in varying rainfall zones in Barbados. From 2015 to 2017, field experiments were conducted in three rainfall zones: low; intermediate and high; to quantify incidence and SED severity in Barbados. Additionally, fungal isolates were analysed by PCR using primers (SPM-4) designed from the Smp 450 gene cluster. A disease rating scale and digital assessment of disease severity were used to measure SED leaf symptoms, while cassava tuber size and mass were also measured, after production. Results showed high disease incidence (100\%) in each zone with significant differences in disease severity between rainfall zones. No statistically significant differences were seen in tuber mass produced while tuber sizes were significantly different. Fungal isolates also displayed varying morphotypes between zones. A flat, smooth, mucoid orange color was common among isolates in low rainfall areas, while variation in fungal 
morphology with deeply fissured colonies was seen in isolates from the higher rainfall zone. Moreover, five PCR fragments ranging from $300-150 \mathrm{bp}$ were present in these latter isolates amplified with SPM4 PCR primers, but only four fragments (225-150 bp) were present in isolates from the low rainfall zone. These findings (varying patterns of disease severity, morphology and molecular characteristics) provide insights on S. manihoticola isolates and disease severity variation with rainfall and the management of SED in the cassava industry.

Field response of near-isogenic brown midrib sorghum lines to Fusarium thapsinum and effects of controlled water deficit on stalk rot disease D. L. FUNNELL-HARRIS (1), P. O’Neill (2), S. Sattler (2), (1) Wheat, Sorghum, and Forage Research Unit, USDA-ARS, Lincoln, NE, USA; (2) USDA-ARS, Lincoln, NE, USA

To increase digestibility of sorghum [Sorghum bicolor (L.) Moench] biomass for ruminant livestock and lignocellulosic biofuels production, nearisogenic brown midrib (bmr) lines, bmr6, bmr12 and bmr6 bmr12 double mutant in backgrounds RTx430 and Wheatland were developed. Compared with wild-type, $b m r$ lines have reduced lignin, altered lignin composition and altered phenolic metabolite levels due to mutations in monolignol pathway genes whose products synthesize lignin subunits. In previous greenhouse assays, $b m r$ lines were not more susceptible, or were more resistant, to Fusarium stalk rot caused by Fusarium thapsinum, compared to wild-type. This destructive disease can result in lodging under high temperature or drought conditions. To determine if greenhouse results applied to field environments, $b m r$ and near-isogenic wild-type plants were inoculated with $F$. thapsinum at irrigated and dryland sites. Mean lesion lengths of most $b m r$ lines were similar to wild-type; bmr6 bmr12 plants had smaller lesions than corresponding wild-type under irrigation $(\mathrm{P}=0.03)$. To assess $F$. thapsinum virulence under drought condition, wild-type plants were inoculated in the greenhouse with adequate or deficient water regimes. Mean lesion lengths were greater on water-stressed plants than those on plants adequately watered $(\mathrm{P}=0.01)$. Development of this screen provides a way to identify sorghum germplasm with increased resistance or tolerance to Fusarium stalk rot under drought conditions.

Managing Phytophthora root rot on flood stressed woody ornamental plants M. Brown, F. BAYSAL-GUREL, J. B. Oliver, K. Addesso, Tennessee State University, McMinnville, TN, USA

Phytophthora cinnamomi is an oomycete pathogen that attacks woody ornamentals; especially plants exposed to elevated soil moisture levels. During flooding events, Phytophthora root rot often causes damage in nurseries throughout the Southern and Eastern United States, sometimes leading to complete nursery crop loss. In this study, we evaluated the efficacy of fungicides and biofungicides for preventive and curative control of Phytophthora root rot on flooded dogwood seedlings. Fungicides/biofungicides were applied as preventive or curative drench treatments $7 \mathrm{~d}$ pre-flooding or $1 \mathrm{~d}$ postflooding, respectively, to artificially inoculated dogwood, Cornus florida, seedlings. The plants were flooded by maintaining standing water for 1 , 3, or 7 $\mathrm{d}$, with five replications per treatment. After the experiment, plant growth data (fresh weight, root weight, plant height, plant width) were recorded, and roots were assessed for disease severity using a scale of $0-100 \%$ roots affected. Preventive treatments that reduced disease severity compared to a nontreated control included Subdue MAXX at 1, 3 and $7 \mathrm{~d}$ flooding and Orkestra Intrinsic at 1 and $3 \mathrm{~d}$. Compared to the non-treated control, curative applications of Empress Intrinsic and Orkestra Intrinsic reduced disease severity at 1 and $3 \mathrm{~d}$ flooding. Fungicides/biofungicides can be incorporated into an integrated strategy to manage Phytophthora root rot on flood stressed nursery trees.

\section{Silicon fertigation maintains optimum growth and ion homeostasis of maize (Zea mays L.) under combined stresses of cadmium and fungus} (Fusarium spp.)

M. T. JAVED (1), M. Z. Haider (1), M. S. Akram (1), M. Shahid (2), N. Habib (1), N. Iqbal (1), (1) Department of Botany, Government College University, Faisalabad, PAKISTAN; (2) Department of Biotechnology and Bioinformatics, Government College University, Faisalabad, PAKISTAN

Cadmium $(\mathrm{Cd})$ and fungal pathogens in soil-plant systems interfere with plant growth and nutrient acquisition. Plant resistance to abiotic and biotic stresses is a complex phenomenon and involves rebalancing of nutrient homeostasis. Therefore, the current work was aimed at investigating the effect of silicon ( $\mathrm{Si}$ ) on maize growth under $\mathrm{Cd}$ and Fusarium spp. application. A pot experiment was conducted to estimate the combined effect of $\mathrm{CdCl}_{2}$ stress $\left(0,5\right.$ and $\left.10 \mathrm{mg} \mathrm{kg}^{-1}\right)$ and Fusarium spp. on growth and physio-biochemistry of maize with and without $1 \mathrm{mM}$ potassium silicate $\left(\mathrm{K}_{2} \mathrm{SiO}_{3}\right)$. The cytosolic concentrations of cadmium, $\left[\mathrm{Cd}^{2+}\right]_{\mathrm{cyt}}$, and chloride, $\left[\mathrm{Cl}^{-}\right]_{\mathrm{cyt}}$ were monitored from leaf protoplasts by using Leadmium Green (AM) and anion-specific MQAE dye. Fusarium spp. inoculation along with Cd stress significantly reduced the plant biomass and the quantity of photosynthetic pigments due to enhanced $\mathrm{H}_{2} \mathrm{O}_{2}, \mathrm{MDA}$, proline levels and impaired nutrient homeostasis. Subsequently, the maize grown with Si prompted a significant increase in plant biomasses together with decreased reactive oxygen species and enhanced activity of antioxidant enzymes under the combined stresses. In parallel, the $\mathrm{Si}$ application conferred the plants to accumulate the nutrients ( $\mathrm{P}, \mathrm{K}, \mathrm{Ca}, \mathrm{Mg}, \mathrm{Fe}$ and $\mathrm{Mn}$ ) when compared with plants without Si under combined stresses. It can be concluded that $\mathrm{Si}$ inhibited the uptake and acropetal $\mathrm{Cd}$ translocation in maize which in turn improved the growth under Cd and Fusarium spp. stress. Silicon application also maintained the lower level of cytoplasmic $\mathrm{Cd}$ and $\mathrm{Cl}$ in maize leaf protoplasts. The outcomes are important to grow maize plants (likely by preventing the stock rot) in areas affected with Fusarium spp. and Cd contamination.

Copper phytoextraction mediated by Medicago sativa L. (alfalfa) plants plus soil acidification, biodegradable chelant and oomycete combination D. TRUJILLO, E. Salgado, N. Riquelme, A. Cáceres, X. Besoain, Pontificia Universidad Católica de Valparaíso, Quillota, CHILE

High levels of soil copper concentrations cause toxicity on plants determining an abiotic disease. Phytoextraction arises as a possible strategy for cleaning contaminated soils with copper. However, the efficiency of the process is low due to the low biomass generation of plants growing in contaminated soils, the low availability of copper in the soil solution and the limited copper translocation from plant roots to the canopy. The purpose of this work was to search for a methodology to improve phytoextraction efficiency of alfalfa plants, with soil acidification plus a biodegradable chelator and the action of an oomycete. A pot experiment was carried out in a greenhouse for 135 days and harvested twice with a completely random design. Each experimental unit was one alfalfa plant growing in a $3 \mathrm{~L}$ pot with soil containing $650 \mathrm{ppm}$ copper. Oomycete and phosphoric acid $\left(\mathrm{H}_{3} \mathrm{PO}_{4}\right)$ plus the chelator methylglycliciacetic acid (MGDA) for corresponding treatments were added individually and in combination, in addition to a control. Each treatment had 8 replicates. The combination of $\mathrm{H}_{3} \mathrm{PO}_{4}$ plus MGDA and oomycete increased 63.1 and 64.6 times copper concentration at alfalfa canopy in the first and second harvest respectively with respect to control. Therefore, the combined treatment is a feasible technology for soil copper phytoextraction.

Avr-Rps gene expression in an incompatible soybean-Phytophthora sojae interaction: The influence of silicon A. RASOOLIZADEH, H. Sonah, F. Belzile, R. Deshmukh, R. R. Bélanger, Université Laval, Quebec, QC, CANADA

Silicon $(\mathrm{Si})$ is reported to offer protection to plants, particularly against biotrophic and hemibiotrophic pathogens; however, the mechanisms underlying this phenomenon are unknown. In the soybean-Phytophthora sojae interaction, Si has been shown to confer incompatibility between the plant and 
pathogen. Similarly, the expression of $A v r$ genes in P. sojae triggers the expression of corresponding soybean Rps (Resistance to $P$. sojae) genes and leads to resistant phenotypes. The objective of this study was to exploit the incompatible $P$. sojae-soybean pathosystem to investigate how Si fertilization affects the expression of Rps-Avr genes as a means to test the hypothesis that the deposition of $\mathrm{Si}$ in the extracellular matrix (apoplast) interferes with effector-receptor expression. Based on phenotypic responses, both Si-treated and -deprived plants showed resistant phenotypes when they were inoculated with $P$. sojae carrying an $A v r$ gene associated with the corresponding Rps gene in soybean plants. However, based on the expression of $A v r-$ $R p s$ genes, it appears that a differential expression under Si treatment, especially at 4 dpi, would indicate that different mechanisms are at work to explain the incompatible interactions. These results could contribute to explain the elusive prophylactic properties of Si against plant pathogens.

Influence of soil pH and liming on Fusarium crown rot of wheat

K. L. SCHROEDER, A. Leggett, University of Idaho, Moscow, ID, USA

Fusarium crown rot is a common disease of wheat worldwide. In the dryland wheat producing areas of northern Idaho and eastern Washington, Fusarium crown rot is caused by the soilborne pathogens Fusarium culmorum and F. pseudograminearum. Previous research suggests a possible correlation between decreasing soil $\mathrm{pH}$ and decreasing incidence of Fusarium crown rot. With the interest in soil liming to remediate low soil $\mathrm{pH}$ in the region, other potential outcomes of liming are being explored. A series of greenhouse and field studies was conducted to determine the influence of soil $\mathrm{pH}$ and liming on Fusarium crown rot. In inoculated greenhouse studies, a soil with a $\mathrm{pH}$ of 4 was adjusted to 5,6 or 7 with calcium carbonate. The highest severity of Fusarium crown rot was observed at a $\mathrm{pH}$ of 6 with the lowest levels of disease at 4 and 7 . In field trials, sensitive spring wheat varieties were seeded into a soil with a pH of 4.2 or limed with 11.2 metric tons calcium carbonate/ha (NuCal fluid lime) to achieve a pH of 6 in the top $15 \mathrm{~cm}$ of soil. In inoculated plots, there was a trend toward increased severity in the limed plots. While there was a positive yield response for the limed plots, Fusarium crown rot did not influence yield. These data suggest that as fields are limed, the severity of Fusarium crown rot may increase, although the impact is likely to be minimal.

Deficit irrigation and grapevine red blotch disease management

A. KC, A. Rasmussen, A. Levin, Southern Oregon Research and Extension Center, Oregon State University, Central Point, OR, USA

Grapevine Red Blotch Disease (GRBD) is a newly identified disease of grapevines caused by grapevine red blotch virus (GRBV). The economic loss associated with the disease is reduction in fruit quality and delayed ripening. The imposition of moderate water deficits is a common viticultural practice to advance ripening and improve fruit quality. However, the stress experienced by vines under deficit irrigation can potentially amplify the negative effects of GRBD. Research was conducted to understand interaction between GRBV and deficit irrigation on disease development and fruit quality. A split-plot field experiment with two irrigation treatments, a grower control (GC) and a deficit irrigation (DI); and two vine statuses, healthy and infected, were arranged in a randomized complete block design. Deficit irrigation was imposed by irrigating $66 \%$ of the grower standard practice and inline water meters were used to quantify applied water amounts. Vine health status was determined by PCR-based assays as infected or non-infected with GRBV. Disease severity was recorded every week after the first symptom appearance on infected vines. No significant interaction between water and vine health status to disease severity was observed. However, the stem water potential of infected vines was significantly higher compared to healthy vines in both dry and wet treatments. In addition, overall fruit quality of the infected vines was better in the GC irrigation treatment than the DI treatment. The results suggest that keeping vines well-watered may mitigate some of the negative effects of GRBV infection.

Defining factors associated with rapid apple decline in the Southeastern United States

S. M. VILLANI, J. Calvin, R. Kreis, S. Schoof, J. F. Walgenbach, North Carolina State University, Mills River, NC, USA

Rapid Apple Decline (RAD) is an emerging and poorly understood phenomenon that results in the sudden death of apparently healthy, young dwarfing apple trees in high density (HD) planting systems. Erratic environmental conditions, including water stress, are believed to be largely responsible for RAD in Western North Carolina. In 2017 a survey was initiated to evaluate the extent of RAD in NC. Nearly $80 \%$ of young, HD orchards in Western NC had trees that were declining or had recently died from RAD. Within several of these orchards, tree loss exceeded $30 \%$. In addition, biotic agents associated with RAD in NC were investigated. Ambrosia beetles were identified in $100 \%$ of declining trees with Xylosandrus crassiusculus, X. germanus, and Xyleborinus saxeseni accounting for the majority of species observed. The association between fungi and RAD was also determined. From 28 orchard locations, 582 fungal isolates were recovered from the tissue of 3 regions of the tree: graft union, beetle galleries, and scion. Botryosphaeria dothidea, Phomopsis spp., and Fusarium spp., were most frequently recovered, with the majority of B. dothidea and Phomopsis spp. isolated from the graft union. Ambrosia beetle fungal symbionts, Ambrosiella grosmanniae and $F$. solani represented $13.4 \%$ and $16.9 \%$ of fungal isolates recovered from beetle galleries, respectively. Further elucidation of the association of recovered fungal isolates under varying degrees of water stress is ongoing.

Effect of soil-applied protoporphyrinogen oxidase inhibitor herbicides on root rot severity of soilborne pathogens in soybean [Glycine max (L.) merr.]

N. ARNESON (1), L. J. Giesler (2), R. Werle (3), S. E. Everhart (4), (1) University of Nebraska-Lincoln, Lincoln, NE, USA; (2) Univ of NebraskaLincoln, Lincoln, NE, USA; (3) University of Nebraska-Lincoln, North Platte, NE, USA; (4) University of Nebraska, Lincoln, NE, USA

Injury to soybean seedlings can allow infection by pathogens such as Fusarium spp., Rhizoctonia solani, and Pythium spp. Soil-applied protoporphyrinogen oxidase inhibitor herbicides (PPOs) can result in injury under certain environmental conditions during crop emergence. Use of PPOs in soybean production is common in integrated weed management, thus there is a need to understand the relationship between PPO injury and seedling disease. In 2017, a study was replicated at five Nebraska field sites, using a randomized complete block design with $2 \times 3 \times 2$ factorial of two cultivars (sensitive and tolerant to sulfentrazone), three herbicides (glyphosate [GLY], sulfentrazone + GLY, and flumioxazin + GLY), and two seed treatments (ST; with and without fungicide). The objectives were to determine the effects of soil-applied PPOs on root rot and of fungicide ST on root rot when PPOs are used. Seedlings were collected and root rot disease severity measured. At Lincoln, flumioxazin resulted in $6 \%$ increase in root rot severity in the tolerant cultivar compared to GLY $(\mathrm{P}<0.01)$ and sulfentrazone had a $4 \%$ decrease in the sensitive cultivar compared to GLY $(\mathrm{P}<0.01)$. However, at Mead, sulfentrazone with no ST had $10 \%$ less root rot severity $(P<0.05)$. Effects on soybean root rot in Nebraska varied by location with PPO use, yet yield impact was not clear. As PPOs continue to be used, more studies under variable disease pressure and environments are needed to understand this interaction.

Spatial Correlations of Southern Rust and Soil Phosphorus in Corn

J. BAILEY (1), T. N. Spurlock (2), N. Seiter (3), (1) University of Arkansas-Fayetteville, MONTICELLO, AR, USA; (2) University of Arkansas,

Monticello, AR, USA; (3) University of Illinois, Urbana, IL, USA 
Southern rust (SR), caused by the fungus Puccinia polysora, is an economically important corn disease in Arkansas. Due to rapid reproduction by the fungus, its initial identification causes concern for growers. Additionally, scouting and proper fungicide timing can be difficult because farmers typically do not pay a corn scout. Therefore, it would be beneficial to determine a predictive scouting regime for SR. In 2017, four fields were found to have SR at various growth stages. Fields were spatially marked with a GPS unit at 100, 100, 80 and 73 points near Grady, Pickens, Plumerville, and Long Lake, respectively. The fields were rated for SR at detection and then every two weeks after until crop maturity. Ratings were assigned for each GPS point (within 3 meters of a single row) below the ear leaf, at the ear leaf, and above the ear leaf. Spatial distributions and relationships were determined using Moran's I and spatial regression models. After harvest, soil samples were taken at each GPS point and processed at the University of Arkansas Soil Testing Laboratory using the Melich-3 method. Spatial analyses indicated SR spread in an aggregated pattern $(\mathrm{P}=0.05)$. Phosphorus levels $(\mathrm{P})$ within fields varied and were also aggregated $(\mathrm{P}=0.05)$. Positive spatial correlations existed across all fields between $\mathrm{SR}$ and $\mathrm{P}(\mathrm{P}=0.10)$ indicating that differences in SR severity could be controlled by plant growth and P could be a component of a SR predictive model.

\section{A preliminary assessment of potential distributions for Armillaria solidipes and Pseudotsuga menziesii under changing climate within the western USA}

J. W. Hanna (1), M. V. Warwell (1), M. S. Kim (2), J. E. Stewart (3), N. B. KLOPFENSTEIN (1), (1) Rocky Mountain Research Station, USDA Forest Service, Moscow, ID, USA; (2) Dept. of Forestry, Environment and Systems, Kookmin University, Seoul, SOUTH KOREA; (3) Department of Bioagricultural Sciences and Pest Management, Colorado State University, Fort Collins, CO, USA

Armillaria root disease (caused by Armillaria solidipes) causes major mortality and growth loss of Douglas-fir (Pseudotsuga menziesii) in the western USA. How will a changing climate influence Armillaria root disease of Douglas-fir in the western USA? To examine this question, climate-based, species-distribution models using Maximum Entropy were used to predict suitable climate space (potential distribution) in the western USA for $A$. solidipes and its Douglas-fir host, using bioclimatic variables for contemporary climate and a projected future climate. The projected future climate was based on a representative concentration pathway 8.5 , which represents a "business-as-usual" continued rise in greenhouse gas scenario and the global circulation model HadGEM2-ES. With these climate scenarios, results predict that suitable climate for $A$. solidipes and Douglas-fir are highly correlated, with the predicted suitable climate space moving dramatically northward and to higher elevations. These models indicate that this Armillaria root disease will continue to spread to new climatically suitable locations where both pathogen and host co-occur. We further hypothesize that Douglas-fir will have a higher likelihood of susceptibility to A. solidipes as it becomes maladapted due to changing climate. This interaction could accelerate Douglas-fir mortality on sites where it is maladapted, which could lead to a buildup of woody fuels that contribute to higher fire severity.

Temperature adaptation and fungicide sensitivity in Macrophomina phaseolina, the causal agent of charcoal rot on soybean and dry bean V. ORTIZ LONDONO (1), H. Sang (1), H. X. Chang (1), Z. Noel (1), K. Wise (2), M. Chilvers (1), (1) Michigan State University, East Lansing, MI, USA; (2) University of Kentucky, Princeton, KY, USA

Charcoal rot caused by Macrophomina phaseolina can significantly reduce yield and seed quality in soybean and dry bean, especially under hot and drought conditions. Consequently, production is affected mainly in tropical and subtropical regions. However, the incidence of charcoal rot in soybean has recently been increasing in the northern US, in which pathogen temperature adaptation may be involved. To address this, genomes of $96 \mathrm{M}$. phaseolina isolates, collected from soybean and dry bean grown in the northern and southern US, Puerto Rico and Colombia, were sequenced to $23 \mathrm{X}$ coverage using a 150 base-pair paired-end strategy on the Illumina HiSeq 4000 platform. Mycelial dry weight at $15^{\circ} \mathrm{C}$ relative to $35^{\circ} \mathrm{C}$ was used to evaluate the influence of temperature on fungal growth. Some isolates from the northern US grew faster at $15^{\circ} \mathrm{C}$ than isolates from the southern US and Colombia. To assess fungicide sensitivity, relative mycelial growth of $M$. phaseolina isolates challenged against boscalid (SDHI), iprodione (dicarboximide) and prothioconazole (DMI) was used to determine $\mathrm{EC}_{50}$ values. Most isolates were sensitive to boscalid, iprodione and prothioconazole, with $\mathrm{EC}_{50}$ distributions means of $1.55,0.92$ and 0.24 , and ranges of $0.15-28.31,0.63-1.49$ and $0.16-1.17 \mu \mathrm{g} \mathrm{ml} \mathrm{l}^{-1}$, respectively. Combined phenotypic and genomic approaches and population genomics are being used to identify genomic regions and candidate genes involved in temperature adaptation and fungicide sensitivity.

Seasonal variations in rose mosaic disease severity and risks associated with using non-symptomatic plants from contaminated crops S. WRIGHT, B. Babu, L. Ritchie, B. Riddle, G. Knox, M. Paret, North Florida Research and Education Center, University of Florida, Quincy, FL, USA

Rose Mosaic Disease (RMD), caused primarily by Prunus necrotic ringspot virus (PNRSV) and Apple mosaic virus (ApMV), has been an ongoing issue for rose growers in the U.S. Field trials were conducted in North Florida to determine the correlation between the initial RMD severity (\%) compared to the final disease severity (AUDPC) in fall 2014, spring 2015, and fall 2017 seasons, and to understand the risk factors in using non-symptomatic roses from contaminated crops. The severity ratings were taken on 121 Pink Double Knockout roses in fall 2014 and in spring 2015; and on 43 infected rose varieties in fall 2017. There was a very strong positive correlation between initial disease severity and final AUDPC in fall $2014(\mathrm{r}=0.953$; Pearson's correlation $\mathrm{P}=0.01)$, and a positive correlation in spring $2015(\mathrm{r}=0.560 ; \mathrm{P}=0.01)$. A significant number of the non-symptomatic plants became symptomatic during the period of the experiment; $33 \%, 90 \%$ and $34 \%$ in fall 2014 , spring 2015 , and fall 2017 , respectively. The general trend was for disease severity to increase as the temperature increased, and to decrease as the temperature decreased. However there were a few exceptions to this general trend. These results indicated that it might not be safe for a nursery to invest in non-symptomatic roses if rose mosaic disease was confirmed in a small subset of the same crop.

Sustained water stress increases black walnut susceptibility to the pathogen Geosmithia morbida R. A. SITZ (1,2), D. Spitzer (3), J. R. Ibarra Caballero (4), T. Ocheltree (3), J. E. Stewart (4), (1) Department of Bioagricultural Sciences and Pest Management, Colorado State Univeristy, Fort Collins, CO, USA; (2) United States Department of Agriculture Forest Service, Moscow, ID, USA; (3) Department of Forest and Rangeland Stewartship, Colorado State University, Fort Collins, CO, USA; (4) Department of Bioagricultural Sciences and Pest Management, Colorado State University, Fort Collins, CO, USA

Geosmithia morbida is the fungal causal agent of thousand cankers disease (TCD) of walnut, and black walnut (Juglans nigra) is particularly susceptible. This disease has spread throughout the western states, as well as several eastern states, though disease may be more severe in the west due to the increased drought stress from the arid climates. However, neither black walnut response to drought, nor tree susceptibility to TCD under drought conditions is well understood. In this study, we drought stressed black walnuts by maintaining them at $60 \%$ pot capacity, and compared their drought and infection responses to well-watered controls which were maintained at $100 \%$ pot capacity. Physiological measurements including mid-day water potential, stomatal conductance, and photosynthesis were conducted. To quantify canker sizes over time (12 hour, 24 hour, 2 day, 4 day, 8 day, and 16 day measurements), trees were inoculated with G. morbida isolate 1217 using a $5 \mathrm{~mm}$ core punch and the bark was removed to calculate the lesion area. Overall, cankers were larger on trees in the drought stressed treatment, and increased over time $(P>0.05)$. Black walnut trees displayed isohydric 
behavior, as drought stressed trees sustained similar mid-day water potentials when compared to the well-watered treatment $(P>0.05)$. However, photosynthesis and stomatal conductance decreased in drought stressed trees $(P>0.05$ for both measurements). Taken together, these results suggests that $J$. nigra compensates for drought stress by using an isohydric behavior that conserves water through limiting stomatal conductance. This strategy may compromise its ability to defend against the canker pathogen G. morbida.

Do reduced irrigation practices alter opportunistic pathogen dynamics in nursery systems?

J. M. DEL CASTILlO MUNERA (1), J. Lea-Cox (2), B. Belayneh (2), A. Ristvey (3), A. Poret-Peterson (4), C. L. Swett (1), (1) University of California, Davis, CA, USA; (2) University of Maryland, College Park, MD, USA; (3) University of Maryland, Queenstown, MD, USA; (4) USDAARS, Davis, CA, USA

Deficit irrigation allows nurseries to adapt to water shortages and improve water use efficiency, but may enhance opportunistic root and crown pathogens. To better understand and manage effects of deficit irrigation on opportunistic pathogen abundance and diversity in a commercial setting, we looked at both the effect of a single pathogen (Pythium aphanidermatum) and shifts in fungal and oomycete community assemblages (using Illumina MiSeq analysis of ITS1-2 and ITS 6-7 respectively) in poinsettia (Euphorbia pulcherrima). When poinsettias were inoculated with $P$. aphanidermatum under moderate deficit irrigation $(25 \% \mathrm{VWC})$, disease incidence was double that of plants under mild deficit irrigation $(35 \% \mathrm{VWC})$ or saturated irrigation $(45 \%$ VWC) treatments, but the effect was not significant $(P=0.146)$. There was a negative effect of pathogen treatment on shoot growth under the lowest VWC treatment $(\mathrm{P}<0.05)$, but not in the well-watered or mildly stressed plants. In preliminary community analyses, 157 fungal and 8 oomycete genera were present in poinsettia roots, and fungal communities differed between commercial greenhouse and controlled studies. Single organism studies indicate a potential risk for irrigation deficit to enhance losses from opportunistic pathogens; expanded community analyses will more broadly elucidate risks on pathogen recruitment. These studies can be used to develop water-pathogen co-management strategies, to enable water use adaptation.

Light induced resistance to bacterial gall disease caused by Pseudomonas syringae pv. cerasicola in cherry tree (Cerasus $\times$ yedoensis) M. ISHIHARA (1), M. Okuda (2), S. Nishii (3), T. Ikeda (3), (1) Forestry and Forest Products Research Institute, Hokkaido Research Center, Sapporo, JAPAN; (2) Kyoto Perfectural University, Kyoto, JAPAN; (3) Kyoto Prefectural University, Kyoto, JAPAN

A bacterial gall disease of cherry trees caused by Pseudomonas syringae pv. cerasicola leads to a twig blight and decline. Cerasus $\times$ yedoensis is resistant to this pathogen, but is often infected under dark light conditions; therefore, the resistance to bacterial gall disease in $C$. $\times$ yedoensis is thought to be light induced. Inoculation experiments to $C$. $\times$ yedoensis were conducted under various light conditions of light intensity, light quality, and irradiation times using with fluorescent tubes in growth chambers. High light intensity, long irradiation time, and blue light irradiation caused to reduce gall size. We assessed histological and anatomical features of diseased regions to evaluate the effect light intensity and quality on disease development. The inoculated branches treated with medium or weak white light intensity developed bigger galls than those treated with high intensity white light or medium intensity blue light. In the weaker white light conditions, cell death spread across the branch and the large galls developed due to the increased number of callus cells. In the high light treatments, a small region of the gall cortex died and its size remained small, indicating that the pathogen was confined by the hypersensitive reaction of the plant. It was concluded that a light induces the resistance to a bacterial gall in $C$. $\times$ yedoensis. Further, we showed that the ultraviolet irradiation reduced a gall size on $C . \times$ yedoensis twig. It is suggested that the ultraviolet may contribute to the development of resistance of $C . \times$ yedoensis under field conditions.

\section{Liberibacter crescens, a presumed bacterial plant pathogen, forms biofilm in vitro}

E. NARANJO (1), E. Pérez-López (1), M. V. Merfa (1), M. Jain (2), M. J. Davis (3), D. W. Gabriel (2), L. De La Fuente (1), (1) Department of Entomology and Plant Pathology, Auburn University, Auburn, AL, USA; (2) Institute of Food and Agricultural Sciences, University of Florida, Gainesville, FL, USA; (3) Citrus Research and Education Center, University of Florida, Lake Alfred, FL, USA

The Liberibacter genus includes heretofore unculturable plant pathogens that cause considerable economic losses worldwide. The only culturabed species of the genus, Liberibacter crescens (Lcr), has been used as a surrogate for pathogenic $\mathrm{Ca}$. Liberibacter species. The replacement of fetal bovine serum (FBS) by methyl- $\beta$-cyclodextrin (MBC) in BM7 medium (modified medium: mBM7) resulted in a significant increase in Lcr cell attachment. $\mathrm{MBC}$ and FBS concentration gradients showed $0.75 \mathrm{~g} / 1$ was the optimum MBC concentration for Ler viability and biofilm formation, and demonstrated that FBS prevented Lcr biofilm formation. Assays comparing Lcr cell attachment force in microfluidic chambers (MC) for both culture media confirmed that FBS presence in BM7 prevents Lcr cell-surface attachment. Cell adhesion assays showed that Lcr cells aggregated significantly less in BM7 compared with mBM7. Calcofluor white staining of two-week old GFP-Lcr cultures on each media exhibited qualitative differences in cell aggregation and revealed the presence of exopolysaccharides in Lcr biofilm extracellular matrix. Time-lapse microscopy assays in MC with mBM7 showed Lcr cells are non-motile, and form aggregates by remaining joined after cell division. Staining of these aggregates with the LIVE/DEAD ${ }^{\circledR}$ BactLight Bacterial viability kit in MC revealed a high proportion of live cells in Lcr biofilms. This study demonstrates the potential of Lcr to form biofilms under specific growth environments.

Characterization of Ralstonia solanaceraum phage Rs-USA-M1 isolated from a tomato field in Florida, USA H. S. Addy (1,2), A. A. AHMAD (1,3), Q. Huang (1), (1) Floral and Nursery Plants Research Unit, U. S. National Arboretum, USDA/ARS, Beltsville, MD, USA; (2) Department of Plant Protection, Faculty of Agriculture, University of Jember, Jember, INDONESIA; (3) Department of Plant Pathology, Faculty of Agriculture, Minia University, EL-Minia, EGYPT

Ralstonia solanacearum is one of the most destructive plant pathogenic bacteria in the world. In order to control this bacterium, efforts have been made to explore the possibility of using bacteriophages as an alternative control strategy. In this study, we report the isolation of a $R$. solanacearum-infecting phage, designated as phage Rs-USA-M1, from a tomato field in Florida, USA. Electron microscopy revealed that phage Rs-USA-M1 is a member of the family Myoviridae with a long "neck". The phage infected 13 out of 31 tested $R$. solanacearum strains. It had a burst time of about 180 minutes releasing approximately 56 plaque-forming units per cell. The phage has a genome size of 39,309 nucleotides (nt) containing 58 open reading frames. It also contains a 45-nt tRNA-like sequence as a probable attachment attP site, which is well-aligned within a 76-nt threonine tRNA sequence from $R$ solanacearum strain 23-10BR, GMI1000, K60, or Po82. Phylogenetic analysis based on the amino acid sequence of the large terminase, portal, or head protein revealed that phage Rs-USA-M1 belongs to the subfamily Peduovirinae and the Genus P2virus, and is most closely related to Ralstonia phage RSA1. However, the phage Rs-USA-M1 differs from phage RSA1 in amino acid sequence of the integrase, suggesting that the former is a variant of the latter. The potential of using phage Rs-USA-M1 as a biocontrol agent for $R$. solanacearum is currently being studied and will be discussed. 
A New (Type 3) Prophage of “Candidatus Liberibacter asiaticus” in China

Z. ZHENG (1), M. Bao (2), F. Wu (1), C. Van Horn (3), J. Chen (4), X. Deng (1), (1) South China Agricultural University, Guangzhou, CHINA; (2) South Ch, Guangzhou, CHINA; (3) USDA-ARS-PWA, Parlier, CA, USA; (4) USDA, ARS, SJVASC, Parlier, CA, USA

Prophages are important genetic entities of "Candidatus Liberibacter asiaticus" (CLas), a non-culturable $\alpha$-proteobacterium associated with citrus Huanglongbing (HLB). Two CLas prophages have been described, SC1 (NC 019549.1, Type 1) and SC2 (NC 019550.1, Type 2). To explore the prophage repertoire, 523 CLas samples were collected from southern China and surveyed for Type 1 and Type 2 prophages by specific PCR. Eighteen samples were found lacking both prophages. One sample, JXGC, was sequenced using Illumina HiSeq and generated $>100$ million short sequence reads (150 bp per read). Read mapping showed a sequence coverage of $46 \%$ to SC1 and 50\% to SC2. BLAST search using SC1 and SC2 as queries identified three contigs from the JXGC de novo assembly that form a circular Type 3 prophage, P-JXGC-3 (31,449 bp, KY661963). P-JXGC-3 had 36 open reading frames, ten of which were not found in Type 1 or Type 2 prophages, including four genes that encoded a restriction-modification (R-M) system ( $h s d R, h s d S, h s d M 1$ and $h s d M 2$ ). Typed by prophage specific PCR, the CLas strains in southern China contained all combinations of the three prophage types with the exception of a Type 2-Type 3 combination. Based on gene annotation, P-JXGC-3 is not capable of reproduction via lytic cycle. The R-M system was speculated to play a role against Type 1 prophage/phage invasion. The new prophage information will facilitate current research in HLB epidemiology and host-pathogen interactions.

Occurrence of bacterial pathogens, including non-toxigenic strains of Pseudomonas syringae pv. phaseolicola, in bean seed crops in Washington State

M. L. DERIE (1), L. Tymon (2), L. du Toit (3), (1) Washington State University Mount Vernon NWREC, Mount Vernon, WA, USA; (2) Washington State University-Mount Vernon NWREC, Mount Vernon, WA, USA; (3) Washington State University, Mount Vernon NWREC, Mount Vernon, WA, USA

Bean processing and seed crops are grown on 12,000 and 2,700 ha annually, respectively, in the Columbia Basin of Washington. A bean seed quarantine and phytosanitary inspection program was instituted in the region in 1968 to ensure production of pathogen-free seed. In 2009, five bean seed lots grown in the Columbia Basin were destroyed after Xanthomonas axonopodis pv. phaseoli (common bacterial blight) or Pseudomonas syringae pv. phaseolicola (Psp) (halo blight) were detected. A bean seed crop in each of 2012 and 2014 was destroyed after detection of $P s p$. Five bean seed crops were destroyed in 2016 to 2017 after detection of quarantine bacterial pathogens during inspections. In 2016 and 2017, bacteria were isolated from severely symptomatic crops. The 2016 isolates tested positive for presence of the phaseolotoxin genes, but lesions on inoculated bean plants did not develop the chlorotic halo linked to phaseolotoxin production, i.e., the highly virulent isolates were non-toxigenic (Tox ${ }^{-}$). The 2017 isolates also were Tox ${ }^{-}$. Phylogenetic analyses of the gapA, gltA, gyrB, and rpoD genes grouped the 2016 isolates with $P s p$ and the 2017 isolates with P. syringae pv. syringae (bacterial brown spot). Thus, three quarantine bacterial pathogens have been confirmed on bean seed crops in Washington, including highly virulent Tox ${ }^{-}$isolates of $P s p$ causing atypical halo blight.

Evaluation of PGPR strains in multiple crop hosts and predictability of growth promotion efficacy by PGPR traits R. Akinrinlola (1), A. O. ADESEMOYE (2), G. Y. Yuen (1), (1) University of Nebraska-Lincoln, Lincoln, NE, USA; (2) University of Nebraska Lincoln, North Platte, NE, USA

Plant growth-promoting rhizobacteria (PGPR) can increase plant growth and yield by facilitating nutrient availability, stimulating plant growth via hormone production, and inhibiting plant pathogens. The objectives of this study were to (a) assess plant growth promotion properties of twelve sporeforming bacteria on sweetcorn, wheat, and soybean and (b) determine which physiological traits are predictive of growth-promotion efficacy. The bacterial strains were isolated from wheat (Tritium aestivum) rhizospheres and identified via 16S rDNA sequence analysis as species of Bacillus, Paenibacillus and Lysinibacillus. Greenhouse pot experiments with the three test crops grown in a raw field soil-sand potting mix were conducted to assess each strain's growth-promotion potential. The strains were screened in vitro for traits associated with plant growth promotion, including antagonism against bacteria and fungi, mineral nutrient conversion, and growth hormone production. Seed treatment with Bacillus megaterium R181, B. safensis R173, B. simplex R180, and Paenibacillus graminis R200 increased the growth of all test crops compared to water-treated controls. The four strains increased shoot mass by $93-126 \%$ compared to the controls and increased root mass by $127-197 \%$. Seven other strains increased growth only on sweetcorn. Results of in vitro physiological traits showed that no strain was strongly positive for antagonism against Gram-negative bacteria, chitinase activity, or nitrogen-fixation. Strains R173, R180, and R181 differed as to the number and type of traits each expressed, while R200 was negative for all traits tested. Therefore, no single trait was found to be predictive of growth-promotion efficacy of the bacterial strains.

Limited movement of Candidatus Liberibacter asiaticus in split-root citrus provides a model system for local and systemic effects of Huanglongbing

J. D. VELOSO DOS SANTOS (1), M. M. Murata (2), K. Gerberich (3), D. B. Bright (4), E. G. Johnson (2), (1) UNESP Jaboticabal, Jaboticabal, BRAZIL; (2) University of Florida, Lake Alfred, FL, USA; (3) University of Florida Citrus Research and Education Center, Lake Alfred, FL, USA; (4) University of Florida Citrus Research \& Education Center, Lake Alfred, FL, USA

The phloem-limited bacterium Candidatus Liberibacter asiaticus (Las) causes Huanglongbing (HLB), the most devastating citrus disease worldwide. Transmitted by the Asian citrus psyllid, Las quickly moves into the root system and causes root dieback while simultaneously stimulating root growth. In the canopy, Las causes phloem-plugging, blotchy mottle, branch dieback, leaf drop, small and misshapen fruit, and premature fruit drop. To develop treatments for HLB, it is important to understand the movement of Las between leaf and root tissues and local and systemic effects of Las on the tree. In previous split-root experiments, lateral movement of Las occurred rapidly when inoculated above the split. Developing a split-root system with Las only present in one half of the root system would assist movement and pathogenesis studies. By using split-root rhizotrons and graft inoculating below the trunk split as the fall root flush begins, the Las infection remained isolated in the inoculated half of the root system for at least 2 months. Additionally, throughout the fall root flush and into dormancy, Las was undetectable in the canopy. This provides a model system for comparison of root growth, dieback, and transcriptional responses to local infection and systemic effects caused by distant infection with healthy controls. This differential infection of split roots also enable study of conditions that allow upward movement of Las by canopy manipulation.

Bacteriocins play a key role in Pseudomonas syringae competition in the plant environment H. EHAU-TAUMAUNU (1), K. Hockett (1,2), (1) Dept. of Plant Pathology and Environmental Microbiology, The Pennsylvania State University, University Park, PA, USA; (2) The Huck Institutes of Life Sciences, The Pennsylvannia State University, University Park, PA, USA 
Bacteria can employ narrow-spectrum toxins called bacteriocins to inhibit the growth of competing strains closely related to the producer. Bacteriocinbased biocontrol has been proposed as an alternative to broad-spectrum chemical biocides. However, the fitness benefit provided by bacteriocins to producing strains is poorly understood in the plant environment. Here, we investigated the competition between Pseudomonas syringae pv. syringae $(P s y)$ and $P$. syringae pv. phaseolicola (Pph) which are both able to infect and cause disease on Phaseolus vulgaris (common bean). Importantly, Psy encodes a bacteriocin that inhibits $P p h$. A series of in vivo experiments tested the direct competition of $P$ sy, or derived mutants with reduced or abolished virulence and/or bacteriocin production, against $P p h$. The leaf epiphytic and endophytic populations of $P s y$ and $P p h$ were measured at days 0,4 , and 6 after co-inoculation. Additionally, the levels of bacteriocins present were monitored. Co-inoculations with $P s y$ and $P p h(1: 1)$ indicate the bacteriocin producing $P s y$ had a statistically significant increase in population size compared to $P p h$ at days $4(P=0.0002)$ and $6(P=0.0028)$. However, the $P s y$ bacteriocin deficient mutant population remained similar to the $P p h$ population growth. These results demonstrate a fitness benefit for the bacteriocin producing $P s y$ strain against $P p h$ in the plant environment, and begins to aid our understanding of how to effectively employ bacteriocins.

Survey and characterization of Ralstonia solanacearum in solanaceous crops in Tanzania

H. KANYAGHA (1), F. Rotondo (2), A. L. Testen (1), S. A. Miller (3), (1) The Ohio State University, Wooster, OH, USA; (2) The Ohio State University, Wooster, OK, USA; (3) Department of Plant Pathology, The Ohio State University, Wooster, OH, USA

Tomato is one of the most important horticultural crops in Tanzania in terms of productivity and economic importance. However, cases of bacterial wilt, caused by Ralstonia solanacearum, are increasing at an alarming pace, especially in prime tomato producing regions. During a survey conducted from May to July 2017, 128 fields were visited in the Iringa and Mbeya (southern highland), Morogoro and Tanga (coastal and medium altitude), and Arusha (northern highland) regions of Tanzania. Bacterial wilt was detected in the majority (55\%) of fields in solanaceous crop plants, with incidences ranging from $2 \%$ to $80 \%$. Tanga region had the most infected fields and Iringa had the fewest. One hundred twenty-one isolates of $R$. solanacearum were recovered from infected plant tissue and soils (tomato: 59 isolates, potato: 49, eggplant: 5, pepper: 2, soil: 6) and assessed for numerous characteristics All isolates were confirmed as $R$. solanacearum using PCR with species-specific primers 759/760. The isolates were classified into biovar using their ability to utilize cellobiose, lactose, maltose, dulcitol, mannitol, and sorbitol and into phylotype using multiplex PCR with phylotype-specific primers. All 121 isolates belonged to biovar 3 and were confirmed as phylotype III.

\section{Characterization of bacteriophages against Erwinia amylovora and Erwinia pyrifoliae causing fire blight and black shoot blight in apple and pear}

C. S. Oh (1), J. Park (2), Y. R. Song (2), D. H. PARK (3), (1) Department of Horticultural Biotechnology, Kyung Hee University, Yongin, KOREA; (2) Kyung Hee University, Yongin, KOREA; (3) Kangwon National University, Chuncheon, KOREA

The fire blight and black shoot blight in apple and pear have been reported in Korea since 2015 and 1995, respectively. As an environmentally friendly control tool for bacterial diseases, bacteriophages that are the viruses killing only target bacteria very specifically have been considered as phage therapy. In this study, we isolated and characterized 21 bacteriophages against Erwinia amylovora and Erwinia pyrifoliae from soils collected at apple and pear orchards in Korea. All isolated bacteriophages were effective to both E. amylovora and E. pyrifoliae, but not to other closely related bacteria such as Pectobacterium carotovorum, Dickeya zeae and Pantoea agglomerans. Isolated bacteriophages were categorized into three groups by the restriction enzyme digestion patterns of their genomic DNAs. Based on morphology and genomic DNA sequences of these three bacteriophages, two belonged to Myoviridae and one belonged to Podoviridae. Lytic activities of all three bacteriophages were stable up to $40-50^{\circ} \mathrm{C}$, at $\mathrm{pH}$ ranges 3-11, and under 365 $\mathrm{nm}$ UV light, indicating that bacteriophages effective to both $E$. amylovora and E. pyrifoliae are stable under various environmental conditions of apple and pear orchards in Korea. These results suggest that isolated bacteriophages might be used for phage therapy against both E. amylovora and $E$. pyrifoliae together in the field.

Survey of pectolytic bacteria causing blackleg and soft rot in Pennsylvania potatoes A. MAINELLO, C. Ramage, B. K. Gugino, C. T. Bull, The Pennsylvania State University, University Park, PA, USA

Recent outbreaks of potato blackleg and soft rot caused by Pectobacterium spp. and more recently Dickeya spp. across the U.S. mid-Atlantic region have caused yield loss due to poor emergence as well as stem and tuber rot. In order to strategize management and to understand variables influencing pathogen diversity, we must first define the pathogens causing blackleg and soft rot in Pennsylvania (PA). No surveys have been conducted to determine the prevalence of these species associated with disease outbreaks in PA potato fields. Therefore, symptomatic potato plants were collected from PA fields in 2016 and 2017. Suspect pathogens were isolated based on their ability to grow and cause pitting on SL-CVP media. Isolates were tentatively identified based on phylogenetic relationships using neighbor-joining analyses of $16 \mathrm{~S}$ rRNA gene sequences from all isolates as well as Pectobacterium and Dickeya spp. type strains. Various pathogenic taxa, including the dominant taxa Dickeya dianthicola and Pectobacterium carotovorum subsp. carotovorum, have been isolated from symptomatic plants in PA. Understanding how the diverse pectolytic bacteria associated with symptomatic blackleg potatoes in PA may be influenced by factors such as cultivar, location, season and/or soil type is the first step in developing effective management strategies.

Conservation of tunicamycin biosynthetic gene clusters across Rathayibacter species M. TANCOS (1), A. Sechler (1), W. Schneider (2), E. E. Rogers (2), (1) USDA ARS FDWSRU, Frederick, MD, USA; (2) USDA ARS FDWSRU, Fort Detrick, MD, USA

Tunicamycins are a novel class of toxigenic nucleoside antibiotics with broad biological activity, inhibiting both bacterial cell wall biosynthesis and eukaryotic $N$-linked glycosylation. Tunicamycins are produced by several soil-associated Actinomycetes, including the grass-associated select agent Rathayibacter toxicus. Currently endemic to Australia, R. toxicus causes bacterial toxicosis in livestock when high concentrations of tunicamycin are consumed from infected pastures. $R$. toxicus is currently the only known phytobacterium that produces tunicamycin, but similar livestock toxicoses have been reported outside Australia. Genomic analyses of available grass-associated Rathayibacter spp. identified two novel tunicamycin gene clusters (TGCs) in R. iranicus and an undescribed South African Rathayibacter sp. termed 'EV'. The uncharacterized TGCs are similarly sized to the TGC of $R$. toxicus, but differ in gene orientation, order, and count. R. toxicus possesses a putative TGC that spans 14 genes, of which 12 genes (tunA-tunL) appear essential for toxin production. In contrast, the $R$. iranicus TGC has 15 genes, but lacks the conserved gene tunG. Rathayibacter sp. EV possesses 14 genes, including all 12 essential genes, but differs greatly in gene order and orientation from $R$. toxicus. Preliminary bioassays indicate toxin production for both $R$. iranicus and $R$. sp. EV, but chemical analyses are ongoing. Conservation of the TGC within species was also investigated. 
A survey of soft rot pectobacteriaceae along the anthropogenic gradient of the Durance river

M. A. BARNY (1), O. Berge (2), E. Rochelle Newall (3), F. Van Gijsegem (4), (1) INRA, Paris, FRANCE; (2) INRA, Montfavet, FRANCE; (3) IRD, Paris, FRANCE; (4) iEES Paris, Paris, FRANCE

Background: Soft rot Pectobacteriaceae (SRP: Pectobacterium spp. and Dickeya spp.) infect a large number of plant species worldwide, including economically important plants. While the diversity of SRP observed on plants is fairly well described, the presence and diversity of SRP outside the plant context is not known in detail. Objectives: To anticipate disease emergence from environmental reservoirs and to propose regulatory guidelines and good practices for crop health management it is important to gain knowledge of SRP ecology outside of agronomic contexts. Methods: SRP sampling was performed all along the Durance river catchment in winter, spring, summer and autumn 2016 and 2017. This river catchment is interesting because it links alpine streams above the Serre-Ponçon lake to the Mediterranean agricultural basin of Avignon along an anthropogenic gradient. SRP sampling was coupled to detailed analysis of water physicochemical characteristics. Diversity of isolated SRP was further characterized through sequencing of one house-keeping gene. Conclusion: SRP distribution along the river stream was uneven, subjected to seasonal variation, land use and water physicochemical characteristics. This study further revealed a large previously unrecognized SRP diversity and the different ecological behaviours of Pectobacterium spp. and Dickeya spp. The potential virulence on various crops of the isolated SRP is currently under investigation.

Characterization of two new Chromobacterium species isolated from cranberry galls in Massachusetts

K. O’HARA-HANLEY, A. Harrison, S. Gadagkar, S. Soby, Midwestern University, Glendale, AZ, USA

Despite their importance in maintaining the cranberry bog ecosystems of southeastern Massachusetts, the microbiota of wetland bogs have been largely uncharacterized. These bogs are the source of a number of newly-described bacterial species, including Chromobacterium spp. The genus Chromobacterium has been associated with septicemia in mammals, and control of insect pests, but it's role in maintaining wetlands soils microbial populations is unknown. The genus has quickly expanded in recent years, giving rise to the possibility of more undiscovered species. As a first step in understanding the role of Chromobacterium in wetlands soils, we report here two previously uncharacterized bacterial isolates from wild cranberry bogs placed within the genus Chromobacterium (b-proteobacteria) based on 16S rRNA sequence comparison, fatty acid methyl ester composition, and metabolic profile. However, each isolate was determined to be a new species based on the complete genomic sequences and phenotypic characterization. Both isolates produce the antioxidant pigment pyomelanin, and contain multiple genes that confer antibiotic resistance against aminoglycosides, blactams, as well as antibiotic exclusion by low outer membrane permeability and ABC-type efflux pumps. Taken together these results suggest that these new bacterial species are well-adapted to soils with robust microbial populations.

Opportunistic plant pathogenic bacteria: Unravelling meaning and significance

T. COUTINHO (1), C. T. Bull (2), J. Stavrinides (3), (1) University of Pretoria, Pretoria, SOUTH AFRICA; (2) The Pennsylvania State University, University Park, PA, USA; (3) University of Regina, Saskatchewan, CANADA

In medicine, the concept of opportunistic pathogens has been well established and defined. Opportunistic pathogens are those bacteria and fungi that usually do not invade healthy hosts, but may invade individuals having underlying conditions that result in a weakened or immunocompromised state. The concept of opportunistic pathogens was later adopted by the field of plant bacteriology pathology in the early 1970's to refer to bacteria that were considered largely soil-dwelling, but could infect the plant when conditions were favourable. Since then, the term "opportunistic" has been used to refer to those bacteria that are commonly associated with asymptomatic plants, but which periodically cause disease, or those bacteria that are pathogenic only when their plant host is compromised and thus predisposed to infection. The absence of any strict definition has resulted in almost all of the members of the "Big Ten" most important phytopathogenic bacteria being described as opportunistic. In this presentation, we argue that they are those bacteria not typically regarded as plant pathogens, but which periodically cause significant losses to various agricultural crops. We also argue that opportunists exhibit distinct characteristics and meet three specific criteria that separate them from the de facto phytopathogenic bacteria that have evolved to exploit plant hosts.

'Candidatus Liberibacter asiaticus' bacteriophage search and the role of the OmpA protein in Liberibacter species M. SENA VELEZ (1), M. Jain (2), D. W. Gabriel (2), K. M. Jones (1), (1) Florida State University, Tallahassee, FL, USA; (2) Institute of Food and Agricultural Sciences, University of Florida, Gainesville, FL, USA

'Candidatus Liberibacter asiaticus' (CLas), a phloem limited bacterium, causes citrus Huanglongbing (HLB), a psyllid-transmitted disease, which has put millions of citrus trees out of production in citrus growing areas, and for which no effective control method has been described. The lack of an axenic medium for culturing these bacteria is a major hurdle in the study and development of control strategies for HLB. Bacteriophages are highly infective pathogens of bacteria; they can be used as biocontrol agents as well as transduction vectors. Our main objective is to isolate $C$ Las-infecting bacteriophages for the study, modification and control of CLas. Liberibacter crescens $(L c r)$ is the only cultivable Liberibacter species. It has been widely used as a model for the study of CLas. In order to search for CLas-infecting bacteriophages, the CLas OmpA protein was expressed in Lcr. OmpA is known to be a phage receptor in bacteria closely-related to Liberibacter spp. Extracts from CLas-free psyllids produced plaque-like zones of lysis on lawns of $L c r$ expressing CLas OmpA, but not on wild type $L c r$. Our short term goals are focused on optimizing the yield and reproducibility of phage infection. In addition, the effect of the CLas OmpA protein on Lcr growth, stress sensitivity, and biofilm formation is being evaluated. Isolating bacteriophages able to infect $C$ Las opens a new way to modify and study $C$. Liberibacter species, and possibly, more effective control methods.

Characterization of two new Pseudomonas species isolated from cranberry galls in Massachusetts T. CASTANEDA, A. Harrison, S. Gadagkar, S. Soby, Midwestern University, Glendale, AZ, USA

The American cranberry (Vaccinium macrocarpon Ait.) is an important commercial crop in the US. However, little is known about its ecology, especially with regard to its microbiome or opportunistic pathogens. Cranberry stem galls have previously been associated with harvest damage, and the presence of several phytohormone-producing genera of bacteria, including Pantoea, Enterobacter, and Pseudomonas. Bacteria were isolated from cranberry upright (stem) galls in a Massachusetts bog. Of the isolates collected from galls, we placed two within the genus Pseudomonas by means of comparing 16S rRNA gene sequences, fatty acid methyl ester analysis, metabolic activity, and other phenotypic characteristics. Based on whole genome sequencing, one isolate appears to cluster within the Pseudomonas syringae sensu lato group. The other may represent the first isolate from a previously unknown clade within the genus. 
Bacteriocin sensitivity in Pseudomonas syringae depends on growth stage and nutritional status

P. KANDEL (1), K. Hockett (2), (1) Department of Plant Pathology and Environmental Microbiology, Penn State University, University Park, PA, USA; (2) The Huck Institutes of Life Sciences, The Pennsylvannia State University, University Park, PA, USA

Pseudomonas syringae is a plant pathogenic bacterium that causes worldwide diseases and substantial losses in crops of economic importance. Strains of $P$. syringae can produce a variety of bacteriocins, narrow-spectrum proteinaceous toxins that kill strains closely related to the producer. Bacteriocins could be used as eco-friendly antibiotic alternatives that target specific bacterial pathogens. However, as with antibiotics, we anticipate repeated bacteriocin exposure would select for genetic resistance. In selecting for resistant mutants, we observed that a fraction of cells survived bacteriocin treatment in a nonheritable manner consistent with physiological tolerance. Therefore, our objective was to determine conditions that promote bacteriocin tolerance and to investigate the underlying mechanism of this trait. Purified bacteriocin particles were applied to target cells growing under various growth stages (log vs stationary), nutrients (rich vs poor), and temperatures. Cells in low nutrients and stationary phase were found to have increased tolerance. Characterization of the tolerant cells is underway. These results will provide valuable insights in developing bacteriocins as biocontrol agents and suggest optimum conditions for their application to achieve disease management.

Evaluation of small molecules of biofilm-inhibiting compounds for control of bacterial spot of tomato S. Zhang (1), Q. LIU (2), (1) University of Florida, Homestead, FL, USA; (2) UF-TREC, HOMESTEAD, FL, USA

Bacterial spot, primarily caused by Xanthomonas perforans, is a challenge to tomato production of Florida. Infection of tomato seedlings may result in total crop loss, and infection of enlarging fruit can make them unmarketable. However, effective strategies for growers to control this disease are very limited. Exploring alternative approaches for effectively managing this important disease for the tomato industry is imperative. Small molecules of biofilm formation-inhibiting compounds have been extensively studied for improved control of bacterial pathogens in medical research, but little has been done with regards to plant pathogens. In our greenhouse study, three small molecules, i.e. N-acetyl-L-cysteine (NAC), 3-indoleacetonitrile (IAN) and D-leucine, were found effective in significantly $(P<0.05)$ reducing bacterial spot of tomato. NAC at $10 \mathrm{mg} / \mathrm{mL}$, IAN at $1 \mathrm{mg} / \mathrm{mL}$ and D-leucine at 10 $\mathrm{mM}$ significantly reduced disease severity by $55 \%, 91 \%$ and $27 \%$, respectively, compared to the non-treated control. When IAN was applied 3 and 1 day before, the same day, 1 and 3 days after pathogen inoculation, disease severity was reduced by $-6.7 \%, 34 \%, 91 \%, 90 \%$, and $38 \%$, respectively. These results indicate that small molecules of biofilm formation-inhibiting compounds are effective against bacterial spot of tomato and could be incorporated into integrated pest management programs for control of this economically important disease in tomato production.

LC2 and LC1 act as key regulators of bacteriophage SC1 in Liberibacter crescens

A. MUNOZ BODNAR (1), L. A. Fleites (2), M. Jain (3), D. W. Gabriel (3), (1) University of Florida, Gainesville, FL, USA; (2) Boyce Thompson Institute, Gainesville, FL, USA; (3) Institute of Food and Agricultural Sciences, University of Florida, Gainesville, FL, USA

Most Liberibacter asiaticus (Las) genomes sequenced to date harbor bacteriophages with similarity to Florida Las strain UF506 SC1 and SC2. SC1 is maintained as a stable lysogen in psyllids but its lytic phase is activated in periwinkle and citrus. A C2-like (LC2) repressor is encoded on the SC1 bacteriophage that may be involved in the activation of the SC1 lytic cycle in planta. Different Las strains also chromosomally encode either a full length or a truncated version of a C1-like (LC1) repressor: one full and one truncated. The regulatory control mechanisms of the lytic cycle in planta and its demonstrated repression in psyllids is unknown. We hypothesize that both LC1 and LC2 could play important roles in repression of the lytic SC1 genes. Electrophoretic mobility shift assays (EMSAs) revealed that both LC1 versions as well as LC2 binds to the C2 promoter. To functionally test this hypothesis, the $\mathrm{C} 2$ promoter region was fused in both directions with a GFP reporter and these constructs were transformed into Liberibacter crescens (Lcr), along with expression plasmids expressing both LC1 and LC2. Interestingly, LC2 appeared to repress GFP expression, whereas both versions of $\mathrm{LC} 1$ seems to activate the $\mathrm{C} 2$ promoter. Both regulators are molecular targets for Las chemical control.

Enhanced virulence of Xanthomonas citri subsp. citri after coinfection with Apple stem grooving virus in citrus trees A. Iwai (1), M. Kashihara (2), Y. Okano (3), M. Yahata (1), H. HIRATA (1), (1) Graduate School of Integrated Science and Technology, Shizuoka University, Shizuoka, JAPAN; (2) Graduate School of Science and Technology, Shizuoka University, Shizuoka, JAPAN; (3) Graduate School of Agricultural and Life Sciences, The University of Tokyo, Tokyo, JAPAN

Plants may be exposed to or infected with multiple pathogens simultaneously, but there is little information on coinfections by different pathogens. The influence of coinfection with a plant virus and plant pathogenic bacterium was studied by challenge inoculation with Xanthomonas citri pv. citri (Xcc), causal agent of citrus canker, on citrus trees (Citrus natsudaidai) infected with Apple stem grooving virus. After pin-prick inoculation with Xcc, a watersoaked lesion, the first visible symptom of citrus canker, was observed earlier than on leaves without ASGV infection. Similarly, a population of Xcc and the size of citrus canker lesions on ASGV-infected leaves were larger than those on ASGV-uninfected leaves. Enhanced virulence of Xcc with ASGV coinfection was confirmed by coinoculation of healthy citrus with ASGV and Xcc. When a citrus leaf was preinoculated with ASGV using an Agrobacterium infection system then challenge-infiltrated or pinpricked with Xcc, Xcc symptoms were relatively more severe than those in the zone preinoculated with Agrobacterium harboring empty vector as a control. Expression of genes related to canker formation was highly upregulated and that of genes related to plant defense responses was repressed after coinoculation with ASGV and Xcc, suggesting that the defense response against Xcc was suppressed by ASGV infection; thus, controlling latent viral infections is important to control secondary challenge by Xcc.

The biological control of Fusarium wilt of sweet potato using non-pathogenic Fusarium oxysporum and Trichoderma species R. SUTHERLAND (1), T. Mathonsi (1), R. Gouws-Meyer (2), L. Sunette (1), M. Kena (3), D. Oelofse (1), S. Venter (1), (1) Agricultural Research Council, Pretoria, SOUTH AFRICA; (2) Tshwane University of Technology, Pretoria, SOUTH AFRICA; (3) University of Limpopo, SOVENGA, SOUTH AFRICA

Sweet potato is the $7^{\text {th }}$ most important crop globally, and the $2^{\text {nd }}$ most important root crop in South Africa. It is produced by small scale farmers, emerging farmers and some commercial farmers in South Africa. Over the years, sweet potato production has declined as a result of Fusarium wilt of sweet potato. Fusarium wilt of sweet potato is caused by Fusarium oxysporum f. sp. batatas (Fob), a fungal soil-borne pathogen. The disease causes symptoms such as wilting, necrosis, internal stem discoloration and leaf yellowing. Control measures are limited and ineffective against Fusarium wilt of sweet potatoes, which has led to the search for an inexpensive and effective control measure. This has intensified the need to focus research on biological control. Roots and soil samples were collected from healthy sweet potato plants in field trials and Fusarium and Trichoderma spp endophytes were isolated. An in vitro dual assay was conducted to screen the antagonistic ability of Fusarium and Trichoderma isolates against Fob. Trichoderma isolates showed antagonistic ability through competition and parasitizing of Fob, while Fusarium isolates only showed competitive ability, with an inhibitory percentage up to $50 \%$ for Trichoderma and 30\% for Fusarium spp. Inoculation in vivo showed that Trichoderma and Fusarium suppress the disease under greenhouse conditions. According to these observations, both species can be combined and used as bio-control agents as they provide different 
antagonist modes of action. Potentially, as a result of the study, farmers may be able to manage the disease, optimize their production, and consequently contribute to food security and income generation.

Allelochemicals- A Significant Molecules for Control of Soil Borne Plant Pathogens from Rhizobacteria

D. U. PANDYA, Samarpan Science and Commerce College, Gandhinagar, INDIA

Rhizobacteria produced secondary compounds i.e. allelochemicals (like siderophores, antibiotics, volatile metabolites, enzymes, etc.) acts as signals against fungal pathogens. Present study described in vitro and in vivo biocontrol potential of selected strains against charcoal rot diseases of Groundnut (Arachis hypogaea (L.). The Bacillus strain MBCU2 (Bacillus sonorensis- KC741546) and MBCU4 (Bacillus subtilis- KC907389) showed maximum production of volatile metabolites, HCN production, lytic enzymes such as chitinase, $\beta 1,3$ glucanase and cellulase production as compared to other strains. The scanning electron microscopy (SEM) analysis revealed loss of structural integrity of mycelia of Macrophomina phaseolina due to allelochemicals produced by biocontrol strains. Bacillus strain MBCU2 and MBCU4 recorded maximum groundnut growth enhancement with least disease incidence at $80^{\text {th }}$ DAS (Day after sowing) over uninoculated control i.e. 21 and $28 \%$ respectively, under green house experiments. Also these biocontrol strains showed presence of active lipopeptides such as iturin, surfactin and fengycine, confirmed by LC-MS as well as MALDI-TOFF analysis. This study direct linked with integrated disease management strategies for crop protection.

Evaluation of soybean nodule microbiome for biocontrol applications

S. Tokgoz (1), D. Lakshman (2), B. Yertayeva (1), M. H. Ghozlan (3), G. Biliarski (1), A. MITRA (1), (1) University of Nebraska, Lincoln, NE, USA; (2) USDA-ARS, beltsville, MD, USA; (3) Alex Univ, Damnhoor, EGYPT

Legume nodules are known to harbor a host of bacteria besides the Rhizobia. In an effort to identify beneficial bacteria for plant disease control we are studying soybean nodules as the source of these bacteria as our metagenomics analysis of a single soybean nodule identified a large number of diverse bacterial species. Surface sterilized single soybean nodule was used to isolate nodule associated bacterial species. A total of 569 colonies were tested individually against two bacterial and one fungal pathogens on solid media plates for inhibition of pathogen growth. From the initial screening, 56 colonies were selected based on significant growth inhibition of three selected plant pathogens. These colonies were further tested on three other bacterial and two fungal pathogens on solid plates. Fifteen colonies from these were also tested in tomato seedlings against two bacterial pathogens. Both soil and stem inoculation methods were used to infect tomato plants. Bacterial metabolites were extracted from 15 colonies with ethanol and ethylacetate and used to treat tomato seeds. Treated seeds was tested for resistance against two bacterial pathogens. Results show that many soybean nodule associated bacteria strongly inhibit growth of plant pathogens in both in vitro and in vivo assays. Furthermore, the treated plants grew better than untreated control plants. All 56 colonies are being identified by 16s RNA sequencing and MALDI-TOF assays. Efforts are being made to establish a cocktail of these bacteria that can be used for broad-spectrum disease management.

Inhibition of Fungal Growth by Bacterial Volatiles

G. EBADZADSAHRAI (1), A. Harrison (1), H. Bean (2), S. Soby (1), (1) Midwestern University, Glendale, AZ, USA; (2) Arizona State University School of Life Sciences, Tempe, AZ, USA

Bacterial volatile organic compounds (VOCs) are natural chemicals that have been implicated in interkingdom signaling and have potential applications as biological alternatives or supplements to synthetic chemicals for controlling fungi in sustainable agriculture. Sixty-eight bacterial isolates from wild and cultivated cranberry bogs were evaluated for VOC activity against Phytophthora spp., Phomopsis sp., Coleophoma sp., and Trichoderma sp. Volatile emissions from several Chromobacterium spp. inhibited all of the ascomycetes and oomycetes tested. In this study, we compared the volatile metabolomics of wild-type C. vaccinii with spontaneous Quorum-Insensitive (QI) mutants that have lost acyl homoserine lactone receptor function. Wild type strains were significantly more inhibitory against the tested fungi and oomycetes than the QI mutants. VOCs produced by $C$. vaccinii strains and one of the fungi were analyzed by 2D gas chromatography time-of-flight mass spectrometry (GCxGC-TOFMS). The metabolomes of wild type and QI $C$. vaccinii were compared, and candidate inhibitory VOCs from several chemical classes are currently being identified from wild type Chromobacterium.

\section{Effect of temperature and biological control agents on mycelial growth and sclerotia development of Sclerotinia sclerotiorum and Sclerotium rolfsii \\ Z. MERSHA, Virginia State University, Petersburg, VA, USA}

Sclerotinia sclerotiorum $(S s)$ and Sclerotium rolfsii $(S r)$ are soilborne pathogens affecting many vegetables in Missouri. Ss causes a number of diseases including white mold, timber rot and lettuce drop. If unmanaged and when conditions are favorable, these diseases could result in a significant yield loss. $\mathrm{Sr}$ causes southern blight on tomatoes and incidence of the disease has increased recently. Options to control these pathogens are limited due to wide host range, lack of resistant varieties, and sclerotia which can survive long time in the soil. Knowledge of the conducive environment for these pathogens is crucial to growers from the disease management perspective. In 2014 and 2015, in vitro experiments were carried out using a potato dextrose agar (PDA) medium. A single sclerotium was aseptically placed at the center and incubated at different temperatures $\left(4,17,20,25,30,35\right.$ and $\left.40^{\circ} \mathrm{C}\right)$. Radial growth of mycelial colony was measured at 2-day intervals and the time of sclerotia formation was recorded. Mycelial growth was faster and days to sclerotium formation of Ss were shorter at 17,20 and $25^{\circ} \mathrm{C}$ compared to temperatures $>30^{\circ} \mathrm{C}$, which halted development. Coniothyrium minitans and Trichoderma $s p p$. significantly reduced the number of sclerotia produced by $S s$. At $4^{\circ} \mathrm{C}, S s$ grew significantly slow but none of the $S r$ grows at this temperature. For $\mathrm{Sr}$, mycelial growth was faster and days to sclerotium formation were shorter at 25 and $30^{\circ} \mathrm{C}$ than 17 and $20^{\circ} \mathrm{C} . \mathrm{Sr}$ mycelia grew at $35^{\circ} \mathrm{C}$ but no sclerotia were formed.

Biological control activity of rice rhizosphere bacteria and their interactive effects with silica treatment against sheath blight of rice J. LEONARD, Louisiana State University, Baton Rouge, LA, USA

Sheath blight of rice caused by the soil-borne fungus Rhizoctonia solani is one of the devastating diseases of rice worldwide. Bacteria isolated from diverse parts of the rice plant have the ability to inhibit the development of sheath blight. Silica has been found to potentiate defense enzyme activity in rice, thus led to the reduction of the progression of sheath blight lesions. This study aims to develop new biocontrol agents and cultural practices with silica fertilizer for disease management of sheath blight. Bacteria were isolated from rice rhizosphere and pure-cultured on Luria Broth Agar amended with $40 \mu \mathrm{g} / \mathrm{ml}$ of cycloheximide, and screened for their antifungal activity against $R$. solani on Potato Dextrose Agar plates. Among the 534 bacterial isolates tested, forty-nine isolates suppressed the development of $R$. solani in culture media. In the anti-fungal bioassay, the highest significant inhibition zone values range from 1.03 to $1.29 \mathrm{~cm}$. These bacteria are being identified through $16 \mathrm{~s}$ rDNA sequencing, and further tested for their in vivo activities 
in greenhouse and field assays. The additive or synergistic activity of selected bacteria in combination with different amounts of silicate slag, a silica fertilizer obtained as a by-product of the steel and iron industry, will be presented.

Induced Systemic Resistance and Stem Rot Management in Peanut Using Microbial Consortia

N. Haveri (1), P. Boyapati (2), H. K. SUDINI (3), (1) University of Horticultural Sciences, Bagalkot, INDIA; (2) Professor Jayashankar Telangana State Agricultural University, Hyderabad, INDIA; (3) ICRISAT, Hyderabad, INDIA

Peanut, a pre-eminent grain legume, is affected by several biotic stresses of which stem rot disease (Sclerotium rolfsii) is an economically significant one. Sustainable management of stem rot is a viable option keeping in view of human and environmental health issues due to indiscriminate use of fungicides. In our present study, a mixture of fungal (Trichoderma sp. T1) and bacterial (Bacillus sp. B1) antagonists were used to devise sustainable approach against stem rot. Talc based formulations of T1 and B1 strains were screened individually as well as conjunctively under greenhouse and field conditions in two different locations in 2016 on a stem rot susceptible cultivar (TMV 2). Altogether, there were nine treatments including a standard chemical check (azoxystrobin). Results indicate combined applications of T1 and B1 strains (with or without chitin amendment) significantly reduced stem rot (20\% plant mortality) compared to control (100\% mortality). Evidence of induced systemic resistance (ISR) was also studied for seedlings inoculated with bioagents through primed expression of defense enzymes such as phenylalanine ammonia-lyase, peroxidase, polyphenol oxidase, catalase, and total phenols. The challenged seedlings were also screened for presence of pathogenesis related (PR) proteins such as $\beta$-1,3-glucanase and chitinase for evidence of systemic acquired resistance (SAR). Overall our results revealed the effectiveness of these bioagents in sustainably managing stem rot disease besides inducing systemic resistance under the conditions evaluated.

Biocontrol of Pierce's disease of grapevine and citrus greening with a benign strain of Xylella fastidiosa D. L. HOPKINS (1), Y. Benyamini (2), (1) University of Florida, Apopka, FL, USA; (2) Luxembourg Industries Pumol Ltd, Tel Aviv, ISRAEL

Vascular diseases caused by xylem-limited Xylella fastidiosa subspp. and phloem-limited Candidatus Liberibacter spp. result in large economic losses in many agricultural plants. $X$. fastidiosa is rapidly spreading into new hosts and areas. Except for plant resistance, there is no effective control for these diseases. In a trial established in the UC Riverside vineyard in 2011, a pin-pricking technique was used to inject a benign strain of X. fastidiosa (EB92-1) into the xylem vessels of Pinot Noir and Cabernet Sauvignon grapevines in the greenhouse prior to transplanting into the vineyard. Under heavy disease pressure, EB92-1 has provided control of Pierce's disease (PD) for 6 years in Cabernet Sauvignon and Pinot Noir. Compared to only 10\% of the treated vines, 40 percent of the untreated vines had severe PD symptoms. None of the treated vines have died; whereas, $10 \%$ of the untreated have died. Trials were established in Florida in 2014 to evaluate EB92-1 for prevention of citrus greening symptoms. A power drill and syringe were used to inject the biocontrol strain into the tree. After 3 years, $53 \%$ of the untreated, mature trees were dead or dying from citrus greening, compared to only $10 \%$ of the treated. In young trees, there were moderate symptoms in $18 \%$ of the untreated and in $5 \%$ of the treated. Biological control of diseases caused by $X$. fastidiosa, and possibly Candidatus L. subspp., with a benign strain of X. fastidiosa, EB92-1, may be feasible.

Inhibition of Colletotrichum coccodes and Verticillium dahliae by the Biocontrol Agent Penicillium oxalicum in Potato D. FARBER (1), L. D. Porter (2), D. A. Johnson (1), (1) Washington State University, Pullman, WA, USA; (2) USDA-ARS, Prosser, WA, USA

Verticillium dahliae $(V d)$ and Colletotrichum coccodes $(C c)$ are soil-borne pathogens with potential interactive effects, which cause two economically important diseases of potato, Verticillium wilt and black dot, respectively. Both reduce yield and tuber quality. Soil-borne pathogens are difficult to manage by cultural practices, leading to over-use of fungicides. Alternatively, Penicillium oxalicum (Pox) is a biocontrol agent used in integrated pest management (IPM), which has inhibited $V d$ populations on tomato. To investigate inhibition of $V d$ and $C c$ populations on potato, Pox at $3.95 \mathrm{~g}$ of infested corn kernels per liter of soil was co-inoculated with either $3.95 \mathrm{~g} \mathrm{Cc}$ - or $V d$-infested sand per liter of potting mix. Positive controls containing $C c$ or $V d$ without $P o x$ were also included. Potatoes co-inoculated with $C c$ and $P o x$ produced significantly fewer microsclerotia than with $C c$ alone, with means of $1110.4 / \mathrm{g}$ and $3109.2 / \mathrm{g}$ ground potato stem tissue, respectively $(p=0.018)$. However, no significant differences were found in $V d$ microsclerotia counts, tuber weight or disease severity within stem cross-sections between plants co-inoculated with Pox and $V d$ or $C c$ when compared to the positive controls. Results suggest that while Pox may reduce $C c$ and $V d$ populations under greenhouse conditions, field trials, as well as examining the efficacy of Pox at higher concentrations, are needed to validate the IPM use of Pox as an effective biocontrol agent to manage these diseases.

Azospirillum brasilense Sp7 x Gibberella zeae interactions in early growth stages of high carotenoid corn S. B. LADE, University of Lleida, Lleida, SPAIN

High-carotenoid (HC) corn has clear benefits for human health. It is rich in pro-vitamin A and other nutritionally important carotenoids and represents a cost-effective intervention particularly in developing countries. In addition to safety and efficacy studies, $\mathrm{HC}$ must be tested to determine fitness to interact with a diverse array of environmental stresses pests, and pathogens. Gibberella zeae (anamorph Fusarium graminearum) (GZ) has devastating effects on corn when infection takes place early in the plant's lifecycle. Priming seeds with plant growth-promoting rhizobacteria (PGPR) can promote seedling survival and reduce posterior detrimental effects of this pathogen. The aims of this study were to compare HC with its near isogenic white corn inbred line (M37W), and to test how the PGPR Azospirillum brasilense Sp7 (Sp7) interacts with HC to offset symptoms caused by GZ. HC seedlings were co-inoculated with Sp7 and GZ and grown under controlled conditions. Four weeks after planting, the seedlings were tested for disease severity, moisture accumulation, ferric reducing antioxidant power (FRAP), total phenolic content (TPC), and hormone assemblage of the plant immune system. Co-inoculation decreased disease severity in HC, and increased both FRAP and TPC. Results showed that co-inoculation has a synergistic effect with $\mathrm{HC}$, promoting seedling survival and a non-antagonistic relationship between significantly increased $(\mathrm{p}<0.05)$ levels of jasmonic and salicylic acids.

Biocontrol potential of bacteriophage $\mathrm{K} \Phi 1$ in control of pepper bacterial spot

K. GAŠIĆ (1), N. Kuzmanovic (2), A. Prokić (3), M. Ivanović (3), N. Zlatković (3), A. Obradovic (3), (1) Institute for Plant Protection and Environment, Belgrade, SERBIA AND MONTENEGRO; (2) Julius Kühn-Institut, Federal Research Centre for Cultivated Plants (JKI), Braunschweig, GERMANY; (3) University of Belgrade, Faculty of Agriculture, Belgrade, SERBIA AND MONTENEGRO

Xanthomonas euvesicatoria causes bacterial spot and pepper yield losses in Serbia. In order to increase efficacy and sustainability of the disease control strategies, alternative approaches were studied. A strain of bacteriophage $\mathrm{K} \Phi 1$ was isolated from the rhizosphere of diseased pepper plants. The phage showed lytic activity to all $X$. euvesicatoria strains tested and did not lyse other Xanthomonas neither less related species. The strain KФ1 is resistant to chloroform, stable in different media and buffers, sustain $\mathrm{pH} 3-9$, and can be stored at $4^{\circ} \mathrm{C}$ at least two years without decreasing of titer. Copper compounds reduced the phage vitality proportionally to the used bactericide concentration. UV light was detrimental to the phage, but skim milk plus sucrose formulation extended its survival. Whole genome sequence analysis showed that KФ1 phage genome does not carry toxin genes, virulence 
genes, or genes related to lysogeny, indicating its suitability for a phage therapy. Results of the three repeated greenhouse experiments showed that foliar application of KФ1 phage $\left(10^{8} \mathrm{PFU} / \mathrm{ml}\right)$ on artificially inoculated pepper plants significantly reduced the symptom severity compared to the untreated control.

Effect of biological agents (strains-PUCV-VBL) on summer bunch rot of table grapes cv. Thompson seedless in Chile F. CÁDIZ, E. Salgado, X. Besoain, Pontificia Universidad Católica de Valparaíso, Quillota, CHILE

Summer bunch rot (SBR) caused by fungal complex including Botrytis cinerea, Penicillium expansum, Aspergillus niger and Rhizopus stolonifer, is an important disease of tables grapes. We have developed a product with two biological agents (BA), Gluconobacter cerinus and Hanseniaspora hosmophila for SBR control. Successful results were obtained testing BA as biofungicide on SBR in Red Globe (RG) cv. both in vivo and field trials. BA has not been tested in Thompson Seedless (TS) cv. and the literature indicates that the genus Gluconobacter would be one of the causative agents of sour rot in TS. The objective of this study was to evaluate the BA on control of SBR in TS. For this purpose, TS grapes $(n=5)$ were inoculated using the BA mixture in two concentrations. After $24 \mathrm{~h}$, the grapes were inoculated using conidia of SBR causative agents. Non-inoculated and inoculated grapes were left as negative and positive controls. In a second trial in the same way, but using the BA mixture in a new formulation, bunch of grapes cv. TS ( $\mathrm{n}=3$ ) were sprayed. The results of the first trial indicate that the mixture of BA in low and high concentrations could inhibit the development of SBR, but producing a browning of the berries. However, in the second trial, when a new formulation of the BA mixture was used in the group, summer cluster rot was controlled, and browning was not observed in the berries. Sour rot was not observed in either of the two trials.

Effect of 6 Exogenous Soybean Isoflavones on Heterodera glycines

Y. WANG (1), C. Guo (2), X. Zhu (2), Y. Duan (1), L. Chen (2), (1) Shenyang Agricultural University, Shenyang, CHINA; (2) Shenyang Agriculture University, ShenYang, CHINA

Soybean cyst nematode ( $\mathrm{SCN}$, Heterodera glycines) is one of the most destructive pathgons of soybean in many countries. Approximately 3.2 billions are considered lost to SCN each year. Isoflavones are a kind of secondary metabolites formed in the process of legume growth. They are structural component of plants that can be induced when plants are infected by microorganisms. They are also called plant antitoxins. In this article, 6 exogenous soybean isoflavones were selected to identify the inhibition of hatching and mortality of J2. In addition, field efficiency trials of seeds coated with isoflavones were determined. The results showed that all 6 isoflavones could inhibit H. glycines, and genistein had the highest mortality of $45.46 \%$ on $\mathrm{J} 2$ and inhibition rate of $43.48 \%$ on hatching at concentration of $100 \mu \mathrm{g} / \mathrm{mL}$ after $24 \mathrm{~h}$ treatment, also had inhibition rate of $49.47 \%$ at concentration of $50 \mu \mathrm{g}$ $/ \mathrm{mL}$ in field trials. Genistin and daidzein significantly killed J2 and inhibited hatching, but their control efficacies were weaker than those of others in field trials. Glycitin and glycitein had weaker toxicity on J2 and higher inhibition on hatching than genistin and daidzein. Furthermore, daidzin showed the weakest inhibition of $\mathrm{J} 2$ and hatching than other 5 isoflavones in vitro, but its control efficacy in the field showed no significant differences compared with genistein. The research confirmed that soybean isoflavones had positively suppressive ability on $\mathrm{H}$. glycines, especially genistein, showed better comprehensive efficacy. The results suggested that isoflavones played an important role in resistance mechanism of soybean against nematode infection, and also provided clues in control SCN by soybean isoflavones.

Gingerwilt complex is a major threat to Ginger cultivation and food security in north East India

P. RAJA (1), R. Selvakumar (2), A. K. Singh (1), A. K. Pandey (1), (1) College of Horticulture and Forestry, Pasighat, INDIA; (2) Sugarcame breeding institute, Coimbatore, Tamil Nadu, India, coimbatore, INDIA

Cultivation of Ginger in the virgin soil is the age old practice in Arunachal Pradesh, India at the altitude of 100 MSL to 600 MSL. Yield loss upto 90\% was noticed due to rhizome rot in the field where ginger is cultivated continuously. The study was conducted on disease incidence in relation with altitude, rainfall and continuous cultivation. Fusarium oxysporum f. sp. zingiberi was found up to 250 and 600 MSL respectively. Disease incidence was highly correlated with rainfall ( $486 \mathrm{~mm}$ to $1625 \mathrm{~mm}$ ) during planting season (May -June). Disease incidence of 5 to 10,15 to 30 and 60 to 80 per cent was noticed in $1^{\text {st }}, 2^{\text {nd }}$ and $3^{\text {rd }}$ year respectively in the same field and inoculum potential was raised from $3 \times 10^{2}$ to $5 \times 10^{5} \mathrm{cfu} / \mathrm{g}$. Biocontrol agent $(\mathrm{BCA})$ Trichoderma isolates were collected and evaluated for their antagonistic potential in addition to mycelial growth, conidial production and mycelial weight in vitro and in vivo. Further suitable carrier material, shelf life, competitive saprophytic ability reveals that rice bran $\left(15 \times 10^{9} \mathrm{CFU}\right)$ and paddy straw $\left(11 \times 10^{9} \mathrm{CFU}\right)$ supported maximum population. The Trichoderma population was stable at $20^{\circ} \mathrm{C}$ storage temperature $\left(12 \times 10^{9} \mathrm{CFU}\right)$ followed by $25^{\circ} \mathrm{C}\left(10 \times 10^{9} \mathrm{CFU}\right)$. Saprophytic ability of native Trichoderma viride was very high in virgin soil $\left(14.66 \times 10^{9} \mathrm{CFU}\right)$ than sterilized one. Soil application of BCA mixed with Farm Yard Manure (FYM) (20g/kg of FYM) 7 days before sowing has increased the yield upto 56.7\% (31.6t/ha) followed by seed rhizome treatment with BCA @ 2g/l water for 30 minutes plus soil application of BCA@5\% (29.93 t/ha). Different methods of storage of the seed ginger rhizome study revealed that Trichoderma viride $(0.5 \%)$ treated rhizomes stored in the pits $(3 \times 1 \times 3 \mathrm{~m})$ layered with FYM, Trichoderma and sand was the best method.

Impact of Valinomycin, a novel antibiotic produced by Streptomyces sp. S8 in Large patch C. W. JEON (1), Y. S. Kwak (2), (1) Gyeongsang National University, Jinju, SOUTH KOREA; (2) Department of plant medicine, Gyeongsang National University, Jinju, Gyeongnam, SOUTH KOREA

Large patch disease, caused by Rhizoctonia solani AG2-2, is a fungal disease commonly found on Zoysiagrass, it poses a significant threat to golf courses and turfgrass field. In order to rectify such issues, Streptomyces sp. S8 was selected based pyrosequencing and microbial-ecology studies. S8 is known to possess exceptional antagonistic actions against the large patch pathogen found at grass plantations. This study aimed to demonstrate the feasibility of using the strain as a biological agent through a series of root colonization assays and addition to elucidate the microorganisms' antifungal mechanism. The S8 strain root colonization ability was found to be outstanding with over $6 \log \mathrm{cfu} / \mathrm{g}$ of rhizosphere soil by the fourth week of plantation. Subsequent to treatment the $\mathrm{S} 8$ strain suppressed densities of the pathogen, the $R$. solani AG2-2 population density was 0.7 cfu/toothpick on the first week, which rose marginally to 1.2 cfu/toothpick on the fourth week and it was also excellent in terms of controlling the large patch disease. As the result, genome of the S8 strain was sequenced and existence of valinomycin biosynthesis gene was confirmed. The valinomycin biosynthesis gene was composed of the $v l m 1$ and $v / m 2$ genes, which were known to produce substances that have outstanding antifungal activity in previous studies. In order to verify the finding, a deletion mutant was created by CRISPR/Cas9 system in the valinomycin biosynthesis gene, $v \operatorname{lm} 2$. The deletion strain showed significantly reduced antifungal activity against the large patch pathogen. Therefore, it is anticipated that eco-friendly microorganism preparations derived from the $\mathrm{S} 8$ strain can be utilized to control the large patch disease in turfgrass.

Endophytic establishment of Beauveria bassiana in maize, and its effects on plant growth and reproduction of grain aphids (Sitobion avenae) Z. MAHMOOD, T. Steenberg, K. Mahmood, M. Kristensen, Aarhus University, Slagelse, DENMARK 
Mycoinsecticides based on the entomopathogenic fungus Beauveria bassiana can be used in integrated pest management. In addition, it has the potential of establishing a symbiotic relationship with plants and grow as an endophyte, without compromising plant growth, to provide life lasting resistance against insect pests. One-week-old maize plants $(\mathrm{N}=12)$ were sprayed with a spore suspension, while control plants $(\mathrm{N}=4)$ were treated with a Tween suspension. Two weeks after inoculation, seven clip cages per plant, each containing an adult grain aphid (Sitobion avenae), were attached to different leaves and left for seven days. All treated plants contained endophytic Beauveria bassiana as confirmed by plating surface disinfected leaf parts on PDA containing antibiotics. The fungus had neutral effects on plant growth as revealed by measuring plant heights, root dry weights and shoot fresh weights. Mean numbers of offspring per adult aphid in endophytic plants (4.86 \pm 1.14$)$ were significantly reduced by $39 \%$ to that of control plants $(7.92 \pm 1.32)$, $(\mathrm{p}<0.001)$. The study confirms that Beauveria bassiana can establish as an endophyte in maize and shows that aphid population development is reduced considerably.

Biocontrol of Colletotrichum truncatum in seeds of Phaseolus lunatus using essential oil of Schinus terebinthifolius V. N. CARVALHO, G. S. Pereira, E. J. P. Silva, Federal University of Alagoas, Maceió, BRAZIL

The reduction in germination potential of the seed occurs mainly due to the presence of phytopathogenic fungi. The use of essential oils is a proposal of efficient biological treatment that reduces the incidence of pathogens besides being biomolecules that do not impact the environment. Therefore, the objective of the research was the use of essential oils of Schinus terebinthifolius in seeds of Phaseolus lunatus aiming to propose alternatives of treatment in the reduction of pathogens following methodology of extraction and chemical analysis and later application in vitro and in seeds being counted germination, seeds dead, dry and fresh. On the other hand, the oil presented efficiency, highlighting its fungistatic effect and such oil did not influence the germinative events in fava seeds. However, essential oils may contribute to pre-sowing treatment in reducing pathogens as an alternative to the use of fungicides.

Efficacy of a biological control agent, Rhizobium vitis ARK-1, against grapevine crown gall in the United States A. T. Wong (1), A. Kawaguchi (2), M. NITA (3), (1) Virginia Tech, Winchester, VA, USA; (2) Westen Region Agricultural Research Center, NARO, Fukuyama, JAPAN; (3) Virginia Polytechnic Institute and State University, Winchester, VA, USA

Grapevine crown gall is an economically important disease impacting wine grape production around the world. Infection of grapevines by the bacterial pathogen Rhizobium vitis (=Agrobacterium vitis) leads to gall formation, girdling of the vascular system, and eventual vine death, due to the integration of Ti-plasmid segments into the host DNA, which causes hyperplasia of the host plant tissue. Because of the lack of an effective management method, crown gall is managed by removing the diseased vine and replanting with a healthy vine. However, this method does not completely remove the pathogen, which resides in plant debris in the soil. Recently, a new antagonistic $R$. vitis isolate (ARK-1) was found to prevent $90 \%$ of gall formation in grapevines infected with a pathogenic $R$. vitis isolate native to Japan. To test the efficacy of ARK-1 as a biological control agent against $R$. vitis isolates in the U.S., we conducted co-inoculation experiments with four Virginia native $R$. vitis isolates. Their pathogenicity was confirmed with PCR for the Tiplasmid followed by an inoculation assay. ARK-1 and each pathogenic isolate were co-inoculated in 1:1 ratio to tomato or grapevines grown in the greenhouse. Two experiments revealed significant reductions $(P<0.05)$ in both gall incidence and size. Gall incidence in grape was reduced 77 to $100 \%$ and gall size was reduced 97 to $100 \%$, indicating ARK-1 can be used as a tool for grapevine crown gall management in the US.

Biological control of Agrobacterium rhizogenes in hydroponic tomato using Pseudomonas strains

C. CHAGAS DE FREITAS (1), C. G. Taylor (2), (1) The Ohio State University, Wooster, OH, USA; (2) Department of Plant Pathology, The Ohio State University, Wooster, $\mathrm{OH}$, USA

Agrobacterium rhizogenes is the causative agent of Hairy Root Disease (HRD) on many dicotyledonous plants. This disease is characterized by the overproduction and proliferation of transgenic adventitious roots. These roots alter the plant's source-sink relationship to favor root growth over fruit or shoot production. Moreover, under hydroponic conditions, the over-proliferation of roots can block the flow of water in the hydroponic troughs, impairing downstream water and nutrient absorption. HRD has emerged as a problem in hydroponic cultivation throughout Europe and Canada and is now an emerging disease in the greenhouse vegetable production in the United States. Since chemical control of bacterial plant diseases via antibiotics has limited effectiveness and is highly regulated we seek alternative ways to control the disease. We are studying whether members of the Pseudomonas genus (widely known for their ability to inhibit plant-pathogens) can inhibit HRD. We tested the activity of over 50 different Pseudomonas strains and identified three different strains (1B1, 48G9 and 93G8) that were able to reduce bacterial growth in vitro and in planta. Currently these strains are being tested on hydroponic cultivated tomato to determine if HRD can be inhibited. These results suggest that certain Pseudomonas strains have the ability to inhibit $A$. rhizogenes growth and disease development and can be a potential biocontrol agent for hydroponic growers in the future.

Botrytis cinerea control with Thymus vulgaris and Coriandrum sativum essential oils

A. VALIUSKAITE, N. Rasiukeviciute, A. Morkeliune, E. Dambrauskiene, Lithuanian Research Centre for Agriculture and Forestry Institute of Horticulture, Babtai, Kaunas dist., LITHUANIA

The increasing demand for healthy and safe food and increasing resistance to chemical fungicides demanded new plant protection solutions. Nowadays consumers are concentrated on functional and natural food without pesticides residues. Thymus vulgaris and Coriandrum sativum essential oils were screened against Botrytis cinerea. Grey mould is one the of the main strawberry field and postharvest pathogen. The research was carried out at the LAMMC Institute of Horticulture. The T. vulgaris and C. sativum essential oils were extracted from local material. The inhibition of mycelial growth was evaluated at concentrations $200 \mu \mathrm{l} / 1,400 \mu \mathrm{l} / 1,600 \mu \mathrm{l} / 1,800 \mu \mathrm{l} / 1$ and $1000 \mu \mathrm{l} / \mathrm{l}$. Small purified isolate fragment of about 10-mm diameter was placed in the centre of a sterile Petri dish of PDA with different essential oil concentrations. Plates were incubated $22^{\circ} \mathrm{C}$ in darkness and evaluated after $2,4,8$, 10 days. The antifungal activity of T. vulgaris and C. sativum essential oils was compared with chemical fungicides (Signum and Switch 62,5 WG). The results showed that $T$. vulgaris essential oils inhibited completely of $B$. cinerea growth at concentrations $>400 \mu 1 / 1$. Mycelial growth of $B$. cinerea by $C$. sativum was inhibited only by $1000 \mu 1 / 1$. Therefore results of $T$. vulgaris essential oil study provide the basis for developing an effective biocontrol agent to control strawberry B. cinerea.

What makes phenazine-producing Pseudomonas spp. good rhizosphere colonizers? A. ZBORALSKI, A. Novinscak, G. Léger, M. Filion, Université de Moncton, Moncton, NB, CANADA

Phenazine-producing Pseudomonas spp. play an active part in the suppressive ability of particular soils against phytopathogenic agents such as Gaeumannomyces graminis var. tritici or Fusarium oxysporum. Their inhibitory efficiency is strongly related to their ability to colonize the soil 
surrounding plant roots: the rhizosphere. To better characterize the multiple factors involved in rhizosphere colonization, we assembled a collection of 63 worldwide representative phenazine-producing Pseudomonas strains. The different strains were inoculated in the rhizosphere of Arabidopsis thaliana grown in peat-based potting soil. Rhizosphere colonization was then assessed by quantitative PCR using a newly developed TaqMan probe/primer set targeting the phenazine biosynthesis operon. In parallel, we also investigated the metabolic profiles of the 63 strains using the BIOLOG phenotype microarray technology. In total, 270 out of 758 tested substrates were differentially metabolized by the 63 strains. Substrate utilization profiles correlated with previously obtained phylogenomic data. The strains also exhibited differential rhizosphere colonization capabilities on $A$. thaliana. These results have led to the identification of specific genes potentially involved in rhizosphere colonization abilities. Their implication are presently being assessed through a reverse genetic approach.

Biological control of plant-parasitic nematodes in carrot and wheat by the fungus Clonostachys rosea

M. IQBAL (1), M. Dubey (1), M. Viketoft (2), A. Broberg (3), D. F. Jensen (1), M. Karlsson (1), (1) Dept. Forest Mycology and Plant Pathology, Swedish University of Agricultural Sciences, Uppsala, SWEDEN; (2) Dept. of Ecology, Swedish University of Agricultural Sciences, Uppsala, SWEDEN; (3) Dept. of Molecular Sciences, Swedish University of Agricultural Sciences, Uppsala, SWEDEN

Biological control is a promising approach to reduce plant diseases caused by nematodes. We tested the effect of the fungus Clonostachys rosea strain IK726 inoculation on nematode community composition in a naturally nematode infested soil in a pot experiment, and the effect of $C$. rosea on plant health. The numbers of plant-parasitic nematodes extracted from soil and plant roots decreased by 40 to $73 \%$ when $C$. rosea was applied, while nonparasitic nematodes were not affected. Soil inoculation of $C$. rosea increased fresh shoot weight and shoot length of wheat plants by 20 and $24 \%$, respectively, while only shoot dry weight increased by $48 \%$ in carrots. Light microscopy of in vitro C. rosea - nematode interactions did not reveal evidence of direct parasitism. However, culture filtrates of $C$. rosea growing in potato dextrose broth, malt extract broth and synthetic nutrient broth exhibited toxicity towards nematodes and immobilised 57, 62 and 100\% of the nematodes, respectively, within 48 h. A metabolomics approach using ultra-high performance liquid chromatography-mass spectrometry (UHPLC-MS) indicated that culture filtrates of $C$. rosea contained the compound 10hydroxy-8-decenoic acid, previously reported to have nematicidal activity. This study demonstrates that $C$. rosea can control plant-parasitic nematodes and thereby improve plant growth. The most likely mechanism responsible for the antagonism is antibiosis through production of nematicidal compounds, rather than direct parasitism.

Elucidation of the mechanism of action of essential oils to control postharvest diseases of apples and peaches D. SPADARO (1), M. L. Gullino (2), A. Garibaldi (2), K. Santoro (3), (1) DISAFA and AGROINNOVA, University of Torino, Torino, ITALY; (2) Agroinnova - University of Torino, Grugliasco, Torino, ITALY; (3) Agroinnova and DISAFA - University of Torino, Grugliasco, ITALY

Essential oils are considered a powerful and natural resource to control postharvest pathogens of pome and stone fruit. The efficacy of these natural products has been deeply investigated in vitro but only few of them are applied in vivo. Essential oils can be applied by dipping, spraying, or fumigation the fruit surface. Thyme and savory essential oils were successfully applied through biofumigation at $0.5 \%$ and $0.1 \%$ against brown rots on nectarines and peaches. The application of thyme or savory essential oils favored a reduction of brown rot incidence, caused by Monilinia fructicola, but an increase of gray mold, caused by Botrytis cinerea. Tests in vitro confirmed that $M$. fructicola was more sensitive to essential oil vapors than $B$. cinerea. Essential oil volatile components were characterized in storage cabinets during postharvest. The antifungal components of the essential oils increased during storage, but they were a low fraction of the volatile organic compounds in storage chambers. In addition to direct inhibition of pathogen growth, essential oils can induce resistance in the fruit host. Thyme essential oil can promote the expression of the pathogenesis related gene PR- 8 in apple, which is involved in host defense response. Moreover, essential oils showed a positive role in slowing down senescence processes reducing weight loss and preserving vitamin $\mathrm{C}$ and carotenoid content during storage.

Significant in vitro antagonism of the laurel wilt pathogen by endophytic fungi from avocado does not predict their ability to control the disease J. Perez-Martinez (1), R. C. PLOETZ (2), J. Konkol (1), (1) University of Florida, Homestead, FL, USA; (2) Tropical Research \& Education Center, University of Florida, Homestead, FL, USA

Raffaelea lauricola (Ascomycota, Ophiostomatales) causes laurel wilt, a lethal vascular disease of avocado, Persea americana. We examined biological control of the disease with endophytic fungi from avocado. Xylem (the infection court for R. lauricola) of 112 trees (seven commercial cultivars) was sampled, and 64 operational taxonomic units (OTUs) were identified with partial sequences of ITS rDNA. Thirty-two OTUs were evaluated against $R$. lauricola with in vitro dual culture assays, and nine OTUs that strongly antagonized the pathogen in vitro were tested in planta against laurel wilt. In three greenhouse experiments, grafted plants of 'Simmonds' or 'Russell', cultivars that are susceptible to laurel wilt, were inoculated with endophytes and, after 10-16 days, inoculated with $R$. lauricola. As expected, laurel wilt developed in plants that were not treated with endophytes within 14 days of inoculation with the pathogen (positive controls), but did not develop in mock (water)-inoculated plants (negative controls). However, laurel wilt also developed in endophyte-treated plants to the extent observed in the positive controls $(\mathrm{P}=0.05)$. The pathogen colonized plants rapidly and systemically, but the endophytes generally did not colonize xylem more than $2 \mathrm{~cm}$ above the point at which plants were inoculated. We will discuss the management of laurel wilt and the value of in vitro data when developing biological control measures for it and other vascular wilt diseases.

Biological control of sugarcane red rot pathogen Colletotrichum falcatum by native rhizospheric bacteria P. PATEL, R. Krishnamurthy, C G Bhakta Institute of Biotechnology, Uka Tarsadia University, Bardoli, INDIA

Red rot, caused by Colletotrichum falcatum is a severe disease of sugarcane. To develop a new biocontrol product we collected 226 rhizobacteria from the six sugarcane variety and screened against three pathogenic Colletotrichum falcatum strains, namely cfNAV, cfCHA and cf8436. Accordingly best five antagonistic rhizobacteria were chosen for further in vitro assay by dual culture technique on PDA and in vivo assay by pot trial on highly susceptible sugarcaane variety $\mathrm{CoC} 671$ under green house conditions for 60 days. Sugarcane plants without any microbial treatment were served as control. All five isolates were characterized for biochemical and plant growth promoting activities such as IAA production, Phosphate mobilization, Nitrogen fixation and siderophore production. Molecular identification by $16 \mathrm{~S}$ rRNA gene sequencing reveals five strains as Ochrobactrum intermedium TRD14; Acinetobacter sp PK9; Bacilli $s p$ RSC29; Bacillus $s p$ KR91 and Escherichia $s p$ VRE34. In dual culture assay Acinetobacter $s p$ PK9 gave maximum $71.64 \pm 1.34 \%$ inhibition against cfCHA followed by Escherichia sp VRE34 against cfNAV (60.44 $\pm 2.38 \%)$. In pot trail, sugarcane plants separately inoculated with each pathogen were died within one month while co-inoculation of each antagonist protected the plant for two month and also supported good growth. In case of Escherichia $s p$ VRE34 treatment, plant height and stem diameter were increased from $13.27 \pm 0.67$ inches to $24.03 \pm 1.40$ inches and $6.07 \pm 0.45 \mathrm{~mm}$ to $9.87 \pm 0.93 \mathrm{~mm}$ respectively. All the five isolates have shown antagonistic activity and in both in vitro and in vivo conditions. In conclusion, mentioned isolates with potential to suppress red rot pathogen efficiently and growth promotion in sugarcane can be use for making biofertilizer/biopesticide against red rot disease for sustainable agriculture. 
Examining MAT1-1 strain as biocontrol agent against aflatoxigenic Aspergillus flavus in maize

J. M. S. LUIS (1), I. Carbone (1), G. A. Payne (1), M. A. Weaver (2), T. S. Isakeit (3), R. Heiniger (4), K. L. Bowen (5), P. Ojiambo (1), (1) North

Carolina State University, Raleigh, NC, USA; (2) USDA ARS, Stoneville, MS, USA; (3) Texas A\&M University, College Station, TX, USA; (4) North

Carolina State University, Plymouth, NC, USA; (5) Auburn University, Auburn, AL, USA

Maize production in the Southern United States is frequently threatened by infection with toxigenic strains of Aspergillus flavus that contaminate kernels with aflatoxin. Biocontrol using nonaflatoxigenic $A$. flavus strains can reduce aflatoxin contamination by $\leq 95 \%$. Efficacy can be maximized when the biocontrol strain is genetically similar to the native population of A. flavus in the soil. The commercial biocontrol products in the United States, AF36 and AflaGuard, and most reported biocontrol strains are MAT1-2. In this study, we are examining the efficacy of using a MAT1-1 strain (RMb10) in reducing aflatoxin contamination and its influence in the native population of A. flavus in the soil. AflaGuard and RMb10 were applied in replicated trials in North Carolina, Mississippi and Texas in 2016, and including Alabama in 2017. Soil samples (100 g) were collected from 20 georeferenced points in each field before treatment with biocontrol and at harvest. We recovered a range of 1- 979 colony forming units of $A$. flavus per gram of soil, where colony counts are higher during harvest compared to pre-treatment with biocontrol. A set of 30 isolates per soil sample collection are being processed for double digest RAD sequencing to examine the population structure of $A$. flavus in each trial. Results of the study could be useful in developing additional biocontrol products for managing aflatoxin contamination in maize.

Control of late blight (Phytophthora infestans) on tomatoes using biologicals

E. GACHANGO (1), K. Mccorkle (2), M. Twizeyimana (2), P. E. Hammer PhD (3), A. Thomas (4), S. Ronyak (1), K. Tyson (2), K. Craig (1), B. Espejo (5), R. Dale (1), V. Pham (6), (1) AgBiome, LLC, Durham, NC, USA; (2) AgBiome, Inc., Durham, NC, USA; (3) AgBiome, Research Triangle Park, NC, USA; (4) North Carolina State University, Raleigh, NC, USA; (5) AgBiome, Inc, Research Triangle Park, NC, USA; (6) AgBiome, Durham, NC, USA

Management of tomato late blight, caused by Phytophthora infestans (Mont.) de Bary, has primarily been achieved through application of conventional fungicides. However, due to increase in resistant pathogen populations, agricultural biotechnology industry has aggressively pursued the discovery of biologicals for control of plant pathogens. At AgBiome, we discover microbial-based solutions for agriculture by leveraging a diverse and growing collection of fully sequenced microbes from the plant-soil microbiome. The microbial strains are screened in bioassays against a broad range of plant pathogens. Biological activity against $P$. infestans on tomato, cv. Money Maker, was assessed in the laboratory using detached leaf disk assay. Phytophthora infestans, genotype US-23, isolated from tomatoes in North Carolina was used. The leaf disks were treated by spraying the microbial strains onto the surface, followed by inoculation with $P$. infestans mycelial suspension after $24 \mathrm{~h}$. Several microbial strains showed high activity against $P$. infestans and were tested twice to confirm the activity. The strains that maintained high level of activity were selected and will be tested for control of Phytophthora capsici and P. infestans in the greenhouse, followed by field evaluations in 2018.

\section{Effect of seed treatment with Bradyrhizobium japonicum on soybean sudden death syndrome (Fusarium virguliforme) in irrigated and natural} fields

T. T. Huynh, S. S. NAVI, X. Li, X. B. Yang, Iowa State University, Ames, IA, USA

Our greenhouse studies had showed seed treatment with Bradyrhizobium japonicum isolates can suppress soybean sudden death syndrome (SDS). In natural condition, soil moisture and temperature in mid-July are ones of the factors leading high soybean sudden death syndrome incidence and severity in Iowa. However, the frequency and amount of irrigation can improve potential yield under field condition. Field studies were conducted from 2014 to 2016 to assess the efficacy of seed treatment using B. japonicum (BJ) in both irrigated and non-irrigated fields. Seeds were treated with BJ at $6 \mathrm{~mL} / \mathrm{kg}$ seed and planted in randomized block design. Overhead sprinkler were run from R1 to R6. Results showed higher nodules and higher shoot and root weights per plant at V5 in BJ treated plots compared with untreated plots in both systems. Also, SDS foliar symptoms incidence was reduced from $8 \%$ to $15 \%$ in irrigated and from $3 \%$ to $23 \%$ in non- irrigated fields. An increase of yield was recorded for sprinkler irrigation at 0.06 metric ton/ha on average and either for natural system at 0.024 metric ton/ha. Three year field studies and two greenhouse experiments clearly indicated that BJ seed treatment has plant health benefits and yield advantages over untreated controls. More studies on using different inoculant types and effect comparison with other commercial products have been conducting.

Biological Control for Grapevine Crown Gall by Nonpathogenic Rhizobium vitis Strain ARK-1

A. KAWAGUCHI (1), Y. Noutoshi (2), M. Nita (3), (1) Westen Region Agricultural Research Center, NARO, Fukuyama, JAPAN; (2) Okayama University, Okayama, JAPAN; (3) Virginia Polytechnic Institute and State University, Winchester, VA, USA

Crown gall of grapevine, which is caused by tumorigenic Rhizobium vitis, is the most important bacterial disease of grapevine throughout the world. Screening tests of biological control agent resulted in a discovery of a nonpathogenic $R$. vitis strain ARK-1. By soaking grapevine roots with a cell suspension of strain ARK-1 prior to planting in the field, ARK-1 treatment significantly reduced the number of plants with crown gall symptoms. Several field trials results indicated that ARK-1 was very useful in the fields, not only for grapevine but also for diverse plant species. In experiments where a mixture of ARK-1 and the tumorigenic strain at a 1:1 cell ratio was examined in vitro and in planta, expressions of the virulence genes virD2 and virE2 of tumorigenic strain were significantly lower. The suppression of virulence genes, which can result in a reduction of gall formation and the pathogen population, seems to be a unique mechanism of ARK-1. These results indicate that ARK-1 is a promising new agent to control grapevine crown gall.

Efficacy of Piperaceous plant extracts in controlling chili anthracnose

P. WIRIYAJITSOMBOON (1,2), P. Junthong (1), C. Suwanphakdee (1), T. Leepasert (1), (1) Kasetsart University, Lat Yao, Chatuch, Bangkok, THAILAND; (2) Kasetsart University, Bangkok, THAILAND

The development of biopesticides has been intensively researched since problems of environmental pollution, pathogen resistance, and residual toxicity due to the use of synthetic fungicides in agriculture have developed and been addressed. The objective of this research was to investigate the efficacy of crude extracts from Thai native plants in the genus Piper (Piperaceae) as an alternative to chemical fungicides in controlling chili anthracnose. The antifungal activity of crude extracts against Colletotrichum capsici was investigated in vitro and in vivo. Samples of plant extract from 14 species were screened against C. capsici at the concentration of $100000 \mathrm{ppm}$ on an agar medium using the disc diffusion method (Heatley, 1944). The results showed that four plant extracts including P8 and P12 obtained from P. kongkandanum, and P14 and P15 obtained from P. macropiper inhibited the growth of the fungus. The crude extracts showing positive results were applied on 'Jin da' chili fruits at the concentration levels of 20000, 40000, 60000, 80000, and $100000 \mathrm{ppm}$ prior to inoculation with a fungal mycelial disc. Three sets of controls were applied with sterile distilled water and azoxystrobin and the untreated fruits. Disease severity was assessed eight days post inoculation by measuring the lesion diameters. P12 and P15 crude extracts completely 
inhibited lesion development at 80000 and 40000 ppm, respectively. The activity of Piperaceous plant extracts against $C$. capsici may be useful at controlling anthracnose of chili production without chemical use.

Biological control of soybean diseases and growth promotion of soybean (Glycine max) by beneficial bacteria

R. CALDERON (1,2), J. H. Ham (3), J. C. E. Ontoy (4), (1) College of Agriculture, Benguet State University, Benguet, PHILIPPINES; (2) Department of Plant Pathology \& Crop Physiology, Louisiana State University, Baton Rouge, LA, USA; (3) Louisiana State University, Baton Rouge, LA, USA; (4) Univ of Philippines, San Pablo City, Laguna, PHILIPPINES

The use of beneficial bacteria having antagonistic activity against pathogens or plant growth promoting activity would be a viable strategy to lessen chemical inputs for crop production. The aims of this study were to screen, identify and characterize bacteria from the soybean rhizosphere that promote the growth and health of soybean. From the 1,440 soybean-associated bacteria (SABs) isolated from the soybean rhizosphere, 46 SABs showed significant antifungal activities. The eleven most promising bacteria, mostly identified as Bacillus sp., were further tested in laboratory and greenhouse conditions. Soybean seeds were treated with each SAB and carboxymethylcellulose (CMC) as a sticker before sowing. In pathogenicity tests conducted in plastic pouches with the fungus Rhizoctonia solani, soybean seedlings treated with SAB4, SAB7, SAB8, and SAB10 exhibited significantly less symptomatic area compared to the untreated control ( $5-7 \%$ vs. $50-70 \%$ root tissue lesions of individual seedlings). The second best group of the SABs was SAB1, SAB3, SAB6 and SAB9, which showed $10-25 \%$ symptomatic area. Effects of SAB2 and SAB11 treatments were not significantly different from the untreated control and the CMC-coated seeds. Under greenhouse conditions, the soybean plants treated with SAB9, SAB6, SAB7, and SAB10 were significantly taller and more vigorous than the plants without any treatment or treated with a commercial product. These SABs are potential biological agents for disease management and growth promotion of soybean, which may lead to reduced use of synthetic pesticides and fertilizers resulting in more sustainable soybean production.

A meta-analysis of endophytic colonization efficacy of Beauveria bassiana across the plant kingdom

S. YERUKALA, B. H. Ownley, University of Tennessee, Department of Entomology and Plant Pathology, Knoxville, TN, USA

Endophytic isolates of the insect pathogenic fungus Beauveria bassiana can protect against plant pathogens. However, little is known about the diversity of plant taxa and tissues colonized by B. bassiana, or the extent of colonization. To fill these knowledge gaps, a meta-analysis of published studies was conducted using a random-effects model. We collected 69 results from the Web of Science database, and screened studies that reported $B$. bassiana colonization percentage. A total of 613 studies was extracted. Moderator analysis was used to identify 39 experimental factors that influenced $B$. bassiana plant colonization. In these studies, tissue cultured banana, pine, sorghum, and tomato were the most frequently tested plants; $B$. bassiana was applied mainly on foliage or seed; and colonization efficacy was determined mostly in leaf and root tissues. Across studies, endophytic plant colonization by B. bassiana was $41 \%$ in different plant taxa, with most studies reporting 30 to $70 \%$. Colonization percentage was greatest on stems and leaves. The percentage of total variation across studies that is due to heterogeneity rather than chance was $I^{2}<50 \%$. Thus, our findings provide a quantitative review of endophytic colonization by $B$. bassiana across a range of plant species, which may enhance current understanding of $B$. bassiana as an endophyte, and provide new information for development of sustainable, eco-friendly disease management strategies.

Improvement of fitness and viable cell qPCR monitoring in a biological control strain of Lactobacillus plantarum N. Daranas, B. Esther, J. Francés, J. Cabrefiga, E. Montesinos, A. BONATERRA, University of Girona, Girona, SPAIN

Lactobacillus plantarum PM411 is a biological control agent with broad activity against several quarantine plant pathogenic bacteria. A physiological adaptation strategy was used to increase tolerance of the strain to water stress, consisting of hyperosmotic and acidic conditions during growth. The combined-stress adaptation treatment in PM411 provides a protection not only against water stress, but also a better survival under harsh conditions in the aerial plant surfaces. This beneficial effect is related to a more consistency in bacterial disease suppression and it is expected that the combined methodology could be applied to improve other biocontrol agents and their formulation. To monitor strain PM411, a viability qPCR assay for its detection and quantification in plant materials has been developed. The method uses a nucleic acid-binding dye in combination with the specific qPCR for selectively detecting and enumerating viable bacteria. This method confirmed some limitations of fitness on the plant surface under unfavourable low relative humidity $(\mathrm{RH})$ conditions.

Use of Streptomyces spp. as biocontrol agents of wheat crown rot caused by fusaria

E. M. COLOMBO, A. Kunova, C. Pizzatti, M. Saracchi, M. Pasquali, DeFENS, Università degli Studi di Milano, Milano, ITALY

Crown rot of wheat is caused mainly by Fusarium species and in specific climatic conditions can reduce production up to $50-90 \%$. Biocontrol methods are an interesting approach to satisfy the increasing demand for alternatives to chemical fungicides. Streptomyces spp. act as growth promoters as well as biocontrol agents. Therefore, they are promising candidates for seed treatments, which combine their ability to produce a wide range of biologically active molecules with the potential to establish tight interactions with plants. Forty-seven Streptomyces spp. strains were selected among 1500, isolated from roots or rhizosphere of various crops. Their ability to reduce mycelial growth (up to $80 \%$ ) of five Fusarium graminearum and $F$. culmorum strains $(N=5)$, representing geographical, genotypic and toxigenic diversity, was determined in vitro. Streptomycetes showing inhibition $>20 \%$ were further tested in vitro on wheat seedlings (cv. Bandera) for their ability to reduce disease symptoms. The root-necrosis was reduced up to $50 \%$ after 4 dpi. Moreover, the strains reduced the severity of crown rot up to $80 \%$ after 6 dpi in comparison to the untreated control. The selected strains did not inhibit the growth of the seedlings at the tested condition. Further in vivo studies are foreseen to assess their plant growth promoting ability. Until now, our results confirm that streptomycetes are a promising source for developing organic seed treatments.

Biopreservation of fresh cut fruits of avocado treated with the essential oils from aerial parts of Artemisia afra, incorporated in Gum Arabic O. ADEOGUN, A. Maroyi, A. Afolayan, University of Fort Hare, Alice, SOUTH AFRICA

The bio-preservation activity of $1 \%$ and $3 \%$ concentrations of essential oil from the aerial parts of Artemisia afra distilled through Solvent Free Microwave Extraction method (SFME) and incorporated into an edible coat from Gum Arabic, on the enhancement of shelf life of fresh cut of Avocado fruits were assayed for 28 days at $4 \pm 1{ }^{\circ} \mathrm{C}$. The essential oil was analyzed using GC-MS system. The antioxidant components (total phenolics, ascorbic acid and carotenoid contents), $\mathrm{pH}$, browning potential, total soluble solids, microbial loads, fruits microstructures analysis with the aid of scanning electron microscope, colour determination, antioxidant capacity through DPPH method and visual acceptability were used to determine the potential of coated essential oil from A. afra as an agent of preservation of fresh cut of Avocado fruits. Results showed that the coated essential oils at 1\% and 3\% concentrations significantly $(\mathrm{P}<0.05)$ enhanced the shelf life of the fresh cut fruits of Avocado compared to uncoated fresh cut fruits of Avocado. The 
study showed that the $3 \%$ coated oil concentration showed higher enhancement than $1 \%$ concentration. Based on our findings, the preservation potential of essential oil incorporated in Gum Arabic showed that it could enhance the shelf life of fresh cut of Avocado fruits.

\author{
Efficacy of some plant extracts against Colletotrichum gloeosporioides (Penz.) the cause of anthracnose disease of yam (Dioscorea rotundata \\ Poir.) \\ J. Kwodaga, B. Badii, E. SOWLEY, University for Development Studies, Tamale, GHANA
}

Anthracnose disease caused by Colletotrichum gloeosporioides (Penz.) is a major constraint to yam production in Africa. Due to the harmful effects of synthetic fungicides on the environment and human health, this study tested aqueous and ethanol extracts of Azadiratcha indica (A. Juss), Balanites aegyptica (L. Delile), Jatropha curcas (L.), Khaya senegalensis (Desr. A. Juss) seeds, Icacina oliviformis (Poir. J. Raynal) leaves and Capsicum sp. (L.) fruit, for their effectiveness against $C$. gloeosporioides in vitro and in vivo. The incidence and severity of the disease was determined in Laribako and Pona cultivars of yam, during the 2016 cropping season. Two fungicides Manlax and Rainmancoz served as positive controls. The experiment was laid out in a Randomised Complete Block Design (RCBD) with 15 treatments and 4 replications. The treatments were applied at 2-week intervals from 5 to 15 weeks after planting. For the in vitro study, Manlax and Rainmancoz had $100 \%$ inhibition. Although all the plant extracts significantly (p $\leq 0.05)$ inhibited mycelial growth of $C$. gloeosporioides in vitro, fruit extract of Capsicum sp. at $100 \%$ had the highest inhibition rate (74.8\%) while seed extract of $K$. senegalensis at $75 \%$ concentration had the least (31.5\%). Under field conditions, all the plant extracts reduced the incidence and severity of yam anthracnose leading to increased tuber yield. Fruit extract of Capsicum sp. had the highest potential for the control of yam anthracnose disease.

Aflatoxin biological control beyond research-scale towards commercial-scale adoption

T. FALADE (1), A. Adebowale (1), A. Ortega-Beltran (1), G. Okpachu (1), P. J. Cotty (2), R. Bandyopadhyay (1), (1) International Institute of Tropical Agriculture, Ibadan, NIGERIA; (2) USDA-ARS, University of Arizona, Tucson, AZ, USA

Nigeria requires over 9 million tons of maize annually for human consumption and animal feed. However, market-sold maize samples have reported prevalence of 19 to $100 \%$ aflatoxin contamination with levels as high as $1000 \mathrm{ng} / \mathrm{g}$. This poses food safety and economic risks to Nigeria's population through chronic exposure to aflatoxins, lowered animal productivity and reduced access to premium markets. IITA, in collaboration with USDA-ARS and partners have developed a biocontrol product, Aflasafe ${ }^{\mathrm{TM}}$, that reduces aflatoxin levels by over $80 \%$. To increase adoption and use of Aflasafe, the AgResults project gives premium prizes in the market as a 'pull-mechanism' to reward farmers whose products achieve reduced aflatoxin levels. This is done via agribusiness implementers who train and distribute Aflasafe ${ }^{\mathrm{TM}}$, aggregate produce, ensure testing, and secure orders from premium markets. Within four years of operating this system, the number of implementers has grown from 4 to $24(500 \%)$, area treated increased from 1,500 to $>20,000$ ha (1233\%), number of farmers using Aflasafe ${ }^{\mathrm{TM}}$ increased from 1,000 to $>13,000(1200 \%)$; and aggregation of aflatoxin-safe maize (less than $20 \mathrm{ppb}$ ) increased from 2,000 to $>39,000$ tons $(1850 \%)$. As a result, farmers are producing safer food for themselves and the population, with improved income from premium prices received, demonstrating that the pull-mechanism is effective tool for increasing Aflasafe ${ }^{\mathrm{TM}}$ adoption and use for reducing aflatoxin contamination.

Inhibition of mycelial growth of some fungi associated with diseased mango by Indigenous Bacilli

N. GORASHI, H. Mudawi, Environment, Natural Resources and Desertification Research Institute, Khartoum, SUDAN

Mango (Mangifera indica L.) is a fruit of tropical and subtropical parts of the world, and it is one of the cash crops in Sudan. The production and hence export is affected by different plant pests and diseases, of which fungi are the main threats. Biological control gains importance over chemical one for healthy and safer production. The objective of this study is to evaluate the inhibitory potential of 9 Sudanese Bacilli against 15 fungi isolated from diseased mango parts. Mango diseased parts were brought to the laboratory and the associated fungi were isolated. The bioactivity of indigenous bacilli against these fungi was evaluated in a dual culture method. $4 \mathrm{~mm}$ discs of each fungus was placed in the centre of the petri dish and the bacilli under the test were streaked at the periphery of the Petri dish while a streek without bacteria was done to serve as the control. The diameter of the fungi was measured daily and the inhibition was calculated. Significant differences in the sensitivity of fungi exposed to the nine bacilli and the activity of the bacilli were observed. The inhibition percentage for the normal growing fungi ranged from $75.42 \%$ for Nattrrassia mangifera exposed to B1, to $49.04 \%$ for Rhizoctonia solani exposed to B1. For the slow growing fungi, it ranged from $63.30 \%$ for Alternaria solani affected by B1, to $40.55 \%$ for the same fungus exposed to B2 However, for the very slow growing, it ranged from $40.67 \%$ for Fusarium solani exposed to B1, to $24.13 \%$ for $P$. glomerata exposed to B3. The most promising strains were B1, followed by B4 and B5 and so their minimum inhibition concentrations are under consideration and will be followed by molecular identifications for them. This research is considered at its infancy and will be continued. From these three strains at least one will be formulated and commercialized after evaluation under nursery and field conditions.

Contribution of native plasmids to fitness and fire blight biocontrol efficacy of Pantoea vagans strain C9-1

J. KLEIN (1), K. B. Johnson (2), J. E. Loper (3), V. O. Stockwell (4), (1) Department of Plant Pathology, University of Florida, Gainesville, FL, USA; (2) Department of Botany and Plant Pathology, Oregon State University, Corvallis, OR, USA; (3) College of Agricultural Sciences, Oregon State University, Corvallis, OR, USA; (4) USDA ARS, Horticultural Crops Research Unit, Corvallis, OR, USA

Pantoea vagans strain C9-1 is a registered biocontrol agent for fire blight of pear and apple caused by Erwinia amylovora. C9-1 suppresses growth of $E$. amylovora on stigmas via competition and antibiosis, aided by gene products encoded on its three native megaplasmids. pPag3 is a member of the Large Pantoea Plasmid (LPP-1) plasmid group and was proposed to play a critical role in environmental fitness. We cured C9-1 of two plasmids: pPag2 (166 $\mathrm{kb}), \mathrm{pPag} 3(530 \mathrm{~kb})$, and both pPag2 and pPag3, and evaluated colonization and survival of C9-1 and derivatives from bloom to fruit harvest. In experimental orchards, loss of pPag2 and/or pPag3 did not affect establishment, growth, or survival on apple and pear flowers through petal fall compared to the wild type C9-1. Loss of pPag2 did not affect survival on fruit. However, population sizes of C9-1 lacking pPag3 were lower than C9-1 on apple fruit in two of five trials. We conducted disease efficacy trials with C9-1 and a derivative lacking pPag3. C9-1 cured of pPag3 reduced the incidence of disease to levels similar to the wild-type C9-1. Overall, loss of pPag3 (LPP-1) did not reduce epiphytic fitness on apple and pear flowers or affect C9-1's ability to reduce disease incidence; nonetheless, C9-1 derivatives without pPag3 occasionally exhibited reduced survival on maturing fruit, approximately one month after application to flowers.

Pursuit of Native Fungal Biocontrol Agent Trichoderma for Nepal and Ohio

R. B. KHADKA (1), F. Rotondo (2), S. A. Miller (1), (1) Department of Plant Pathology, The Ohio State University, Wooster, OH, USA; (2) The Ohio State University, Wooster, OK, USA 
Experiments were conducted to assess the biocontrol mechanisms of 39 Trichoderma isolates from diverse microclimatic domains in Nepal and Ohio, USA. Trichoderma species identified by internal transcribed spacer gene sequencing were: T. asperellum $(\mathrm{n}=15)$, T. koningiopsis $(\mathrm{n}=15)$, T. harzianum $(\mathrm{n}=3)$, T. ovalisporum $(\mathrm{n}=2), T$. cerinum $(\mathrm{n}-1), T$. citriviride $(\mathrm{n}-1), T$. hamatum $(\mathrm{n}=1)$ and $T$. ghanense $(\mathrm{n}-1)$. Standard agar plate assays were conducted to evaluate growth inhibition by Trichoderma isolates of Rhizoctonia solani, Phytophthora capsici and Pythium ultimum by competition, mycoparasitism, and antibiosis through production of volatile and non-volatile inhibitors. The efficacy of these isolates in suppressing radish root rot caused by R. solani was tested under greenhouse conditions. Four isolates of T. koningiopsis (T-17, T-28, T-29 and T-3) showed more than $60 \%$ inhibitory activity against the pathogens by volatile metabolites. Two isolates each of T. asperellum (T-18, T-30) and T. koningiopis (T-29, T-25) inhibited pathogen growth through non-volatile metabolites by more than 50\%. Six isolates of T. koningiopsis (T-25, T-1, T-16, T-21, T-22, and T-8) and two isolates of $T$. asperellum (T-27, T-30) outperformed the others in confrontation and in planta experiments. The majority of the effective Trichoderma isolates were recovered from tropical and subtropical climates in Nepal. These isolates merit further evaluation for biocontrol and other industrial applications.

Effect of Nemarioc-AL, Nemafric-BL and Penicillium simplicissimum on avocado root rot and mineral nutrient concentrations N. D. MAMPHISWANA (1), M. Kena (2), P. W. Mashela (1), (1) University of Limpopo, Sovenga, SOUTH AFRICA; (2) University of Limpopo, SOVENGA, SOUTH AFRICA

Worldwide, Phytophthora cinnamomi-induced avocado root rot is a major disease, which causes rotting of feeder roots and general tree decline, with poor nutrient and water uptake, which result in plant death. Following in vitro efficacy trials, the fermented plant extracts [Nemarioc-AL (AL), Nemafric-BL (BL)] and biological agent [Penicillium simplicissimum (PS)] were investigated on avocado root rot suppression in a $2 \times 2 \times 2$ factorial experiment under greenhouse conditions. Treatments were arranged in a randomized complete block design, with nine replicates. At 24 weeks after inoculations, the second order interaction had no significant effects on plant growth variables; whereas the first order interactions had some significant effects. The AL $\times$ BL and BL $\times$ PS interactions were each not sufficiently effective in reducing root rot and foliar symptoms in comparison to individual factors. For instance, AL reduced root rot by $30 \%$, whereas AL $\times$ PS interaction reduced the variable by $4 \%$. Second order interaction for nutrient elements was, except for nitrogen in root tissues, not significant. Relative to the control, AL $\times$ BL $\times$ PS interaction increased nitrogen in root tissues by $89 \%$, which was lower than those of individual factors. First order interactions such as AL $\times$ BL, BL $\times$ PS, AL $\times$ PS were significant for number of nutrient elements in root and/or leaf tissues. However, the combined effect of the products was lower than when applied individually. Results suggested that the three products should be applied separately in the management of avocado root rot.

Essential oils as pepper seeds treatment for Colletotrichum gloeosporioides control D. M. DO NASCIMENTO, P. Leite dos Santos, A. Zanin Kronka, São Paulo State University, Botucatu, BRAZIL

Colletotrichum gloeosporioides is one of the most important pathogens in the pepper crop. This fungus can be transmitted by seeds, which represent a way of this pathogen dissemination. Seeds treatment is recommended for this disease management. Aiming to reduce the use of chemical products in this crop, the action of eight essential oils (rosemary, citronella, clove, copaiba, eucalyptus, mint, basil and tea tree) on C. gloeosporioides was investigated. The in vitro activity of the essential oils on the fungus was evaluated by adding $0.25 \%, 0.50 \%$ and $0.75 \%$ of each essential oil to the potato-dextrose-agar medium (BDA). Then, a fungal mycelial disk was transferred to the center of each plate, incubated at $22^{\circ} \mathrm{C}$ and 12 hours photoperiod. Mycelial growth and sporulation were evaluated. Clove, citronella, eucalyptus, mint and basil essential oils, regardless of the concentration, totally inhibited the pathogen development. After the first experiment, the effect of these five essential oils on the sanitary and physiological quality of pepper seeds infected by $C$. gloeosporioides was investigated. For seeds treatment, the concentration of $0.25 \%$ of each essential oil was adopted. Sanitary quality, germination, seedling length, emergence of seedlings, emergence speed index and dry mass were evaluated. The essential oil of clove was efficient in reducing $C$. gloeosporioides incidence in the seeds, with no damages for their physiological quality.

Root extracts from Medicago truncatula effectively inhibit rice blast (Magnaporthe oryzae) disease K. HAYDON, K. Korth, University of Arkansas, Fayetteville, AR, USA

Plants produce a range of secondary metabolites for defense against insects and pathogens. These include saponins, a structurally diverse group of compounds with varied pesticidal activities that are relatively non-toxic to humans, and therefore prime candidates as natural compounds to control plant disease. Rice blast, caused by the ascomycete Magnaporthe oryzae, is the most serious disease of rice (Oryza sativa), causing up to $30 \%$ of global yield losses every year. To determine the effect of natural plant compounds on rice blast development, saponins were extracted from the roots of hydroponically-grown Medicago truncatula. Either crude or C18 column-purified extracts were sprayed on three-week-old CO39 cultivar rice plants, both several hours before and three days after inoculation with a $10^{5}$ spore $/ \mathrm{ml}$ suspension of $M$. oryzae strain Guy 11 . Representative leaves were imaged seven days post inoculation and lesion number and diseased area were quantified. Spore suspensions were also applied to PDA plates containing root extract-soaked filter paper disks, and resulting zones of inhibition were measured. Both crude and enriched saponin extracts significantly decreased lesion number and area on plants, and produced measureable zones of clearing on in vitro cultures. Saponin-enriched extracts performed as well as the synthetic fungicide epoxiconazole. If produced on a large scale, root extracts can potentially be used as alternative means to manage rice blast disease.

Botanical extracts as an alternative crop protection agent: Towards climate smart crop protection

L. MATSAUNYANE (1), S. Mahlangu (1), L. Ledwaba (1), N. Legodu (2), N. Baloyi (1), R. Maluleka (1), M. Lodwick (1), K. Ntushelo (3), D. Mogotlane (4), D. Oelofse (1), L. Sunette (1), I. Du Plooy (1), S. Venter (1), S. Moephuli (1), (1) Agricultural Research Council, Pretoria, SOUTH AFRICA; (2) Department of Agriculture, Forestry and Fishery, Pretoria, SOUTH AFRICA; (3) University of South Africa, Florida, Gauteng Prov, SOUTH AFRICA; (4) Gauteng Department of Agriculture and Rural Development, Johannesburg, SOUTH AFRICA

Pressures introduced to the agricultural sector by climate change include increase in temperature increase in irrigation demand, and increase in crop diseases. Various methods are currently used to bacterial pathogens and these methods have varying degrees of effectiveness. A study was initiated on weed plants as potential crop protection agents will offer safer, effective, inexpensive and biodegradable solution. In the study, the antibacterial properties of extracts prepared from weed plants were investigated against economically important vegetable bacterial pathogens. Previous studies showed that the most common vegetable diseases found by emerging farmers in the Gauteng Province include cabbage soft rot, tomato bacterial speck and spot, bean leaf spot, and potato blackleg and soft rot. Our study selected weeds that were readily accessible to the Gauteng farmers, and these were Lantana camara L. and Melia azedarach L. Antibacterial studies were done against Pectobacterium carotovorum subsp. carotovorum (Pcc), $P$. carotovorum subsp. brasiliensis (Pcb), Xanthomonas campestris pv. campestris and Burkholderia andropogonis. Results from the minimum inhibitory concentration (MIC) showed that $2.5 \mathrm{mg} / \mathrm{ml}$ Lantana-and Melia-based extracts inhibited the growth of all selected bacterial pathogens by $100 \%$ and $80 \%$, respectively. Evaluation of the mode of bacterial inhibition of the weed extracts, analyzed through microscopy, showed that both extracts caused 
significant morphological changes to all of the selected bacteria, which was visualized as large outpouching of the bacterial cell wall. The results show that both weed extracts are effective antibacterial agents and have the potential to be used as alternative biological crop protection agents.

Attempts to use Coriandrum sativum essential oil to reduce seed pathogens

A. VALIUSKAITE, E. Dambrauskiene, L. Sernaite, R. Karkleliene, N. Rasiukeviciute, Lithuanian Research Centre for Agriculture and Forestry Institute of Horticulture, Babtai, Kaunas dist., LITHUANIA

The increasing resistance to chemical fungicides drawn attention to the natural products antimicrobial activity. Essential oils are biodegradable and ecofriendly botanical products that are accumulated in various plants. The aim of this study was to evaluate antimicrobial effect of coriander essential oil against horticultural crops seed-borne pathogens. The research was carried out at the LAMMC Institute of Horticulture. The Coriandrum sativum essential oil was extracted from local material. The $C$. sativum essential oil efficacy against seed-borne fungi was evaluated by planting naturally contaminated onion and cucumber seeds on Petri plates containing potato dextrose agar (PDA). The PDA was cooled to $45^{\circ} \mathrm{C}$, and essential oil was added: $200 \mu \mathrm{l} / 1,400 \mu \mathrm{l} / 1,600 \mu \mathrm{l} / \mathrm{l}, 800 \mu \mathrm{l} / 1$ and $1000 \mu \mathrm{l} / 1$. The antifungal activity of $C$. sativum essential oil on cucumber and onion revealed that several concentration differently inhibited bacterial and fungal pathogens. Evaluation of the efficiency of essential oil on seed-borne pathogens at a concentration of $1000 \mu \mathrm{l} / 1$ completely inhibited the pathogens. The concentration of $200 \mu \mathrm{l} / 1$ least inhibited bacterial and fungal pathogens. The essential oils as biofungicides would help to control seed-borne pathogens without increasing chemical fungicides resistance.

Mechanism research of transcriptional regulator LeClp in the biosynthesis of WAP-8294A2 in Lysobacter enzymogenes H. XU (1), Y. Zhao (1), G. Qian (2), F. Liu (1), (1) Institute of Plant Protection, Jiangsu Academy of Agricultural Sciences, Nanjing, CHINA; (2) College of Plant Protection, Nanjing Agricultural University, Nanjing, CHINA

Lysobacter enzymogenes OH11, isolated from rhizosphere soil of pepper, is an important bacterial biocontrol agent. OH11 has a bright future in biocontrol application as it could produce WAP-8294A2. WAP-8294A2 is a crucial factor in biocontrol process of L. enzymogenes OH11. On the basis of sequencing genome, an important global regulator LeClp protein was identified from strain OH11 and shown to regulate the WAP-8294A2 biosynthesis via an indirect and uncharacterized mechanism(s). In the present study, we aim to identify the key factor(s) for modulating WAP-8294A2 biosynthesis in the downstream of LeClp by using ChIP-Seq、 bacterial one-hybrid、EMSA、Co-IP、 bacterial two-hybrid as well as other methods and elucidate the mechanism(s) of this factor(s) in the regulation of LeClp. Furthermore, it is another objective to understanding regulatory mechanism(s) of this factor(s) in modulating WAP-8294A2 biosynthesis. This results of the study is an important aid to generate genetically modified L. enzymogens strain with high-yeild WAP-8294A2.

Determining the antifungal activity of Bacillus species against Fusarium graminearum

C. JIMENEZ-QUIROS (1), Ö. Baysal (2), P. Nicholson (3), M. Tor (1), (1) University of Worcester, Worcester, UNITED KINGDOM; (2) Muğla Sitk1 Koçman University, Muğla, TURKEY; (3) John Innes Centre, Norwich, UNITED KINGDOM

The Fusarium head blight (FHB) is caused by Fusarium graminearum $(F g)$ in cereals crops. The pathogen affects the spikelets producing symptoms with white chalky appearance and shrunken grains. Sometimes symptoms are not visible but the grains lodged with high levels of mycotoxins such as Deoxynivalenol. We wanted to determine whether biocontrol agents such as Bacillus could be used to control Fg. For this purpose, in vitro antagonism assays were performed with Bacillus subtilis strains including QST713, FZB24 and EU07 to assess the suppression of Fg. Plugs of Bacillus were placed in medium PDA/NA with a plug of the fungus and incubated for 4 days $\left(28^{\circ} \mathrm{C}\right)$. Fungal growth was assessed by taking pictures and evaluating with a software. Fungal cultures without Bacillus plugs were used as controls. Similarly, assays were also performed with the cell-free culture supernatant of the bacteria that were grown in broth for 24,48 and $72 \mathrm{~h}$. Supernatant was filtered through $0.22 \mu \mathrm{m}$ membranes and filtrates were used for antimicrobial activity. Filtrates $(100 \mu 1)$ were normalised using Bradford assay, spread over the media, and one plug of the fungus was placed over the medium. The $B$. subtilis strain EU07 showed the highest inhibition of fungal growth in both plug and filtrate tests, followed by QST713 and FZB24. Currently we are carrying out comparative genomics of these Bacillus strains as well as transcriptomics on EU07-Fg interactions. Latest results will be presented.

Investigating the effect of a plant extract-based product and chitosan on the incidence of Fusarium head blight of wheat M. DESHAIES (1), P. Ward (1), F. Doohan (2), (1) Auranta, Dublin, IRELAND; (2) University College Dublin, Dublin, IRELAND

The fungus Fusarium graminearum causes Fusarium head blight of wheat and produces mycotoxins in grains, resulting in up to $50 \%$ yield losses. This project aims to investigate the effect of two products on this disease; a plant extract-based product (Auranta Harvest, produced by the company Auranta), and chitosan, a polysaccharide found in fungal cell walls. To determine their impact on the fungal infection, wheat heads were inoculated with $F$. graminearum spores or Tween-20 at mid-anthesis. The heads were treated one day before or after inoculation with water, Auranta Harvest or chitosan. Infection percentages were assessed at days 7, 14 and 21 post-inoculation, and yield analysis was performed. In pre-inoculation treatments, the area under the disease progression curve was on average $18 \%$-days for the treatments with water and Auranta Harvest, and $8 \%$-days for the chitosan. A significant increase in grain production was observed in plants treated with chitosan. In post-inoculation treatments, the area under the disease progression curve was on average 18,10 and 8\%-days for the treatments with water, Auranta Harvest and chitosan respectively. However no significant increase in yield was observed with any of the treatments. Our results show that Auranta Harvest does not stimulate resistance to infection, but that it may have antifungal activity. We also show that chitosan may enhance resistance against Fusarium head blight, and that it could have an antifungal activity.

Endophytic bacteria as biocontrol agents of Moniliophthora roreri, a cacao (Theobroma cacao) pathogen C. P. Rojas (1), L. Rodriguez (2), M. A. Jaramillo (3), P. JIMÉNEZ (3), (1) Universidad de los Andes, Bogota, COLOMBIA; (2) Universidad Militar Nueva Granada, Bogota, COLOMBIA; (3) Universidad Militar Nueva Granada, Cajicá, COLOMBIA

Moniliophthora roreri, the causal agent of frosty pod rot, is a great constraint for cacao (Theobroma cacao) production in Colombia. Since efforts to control this fungus have high environmental costs, alternatives such as the use of microorganisms in biological control schemes are needed. Therefore, this study aimed to assess the potential of cacao endophytic bacteria as biocontrol agents of this fungus. Endophytes were isolated from healthy cacao leaves at three farms in Cundinamarca, Colombia. A leaf was collected at each of three different positions on the trees (apical, medium high, and stem base), and all endophytes from each leaf were recovered. A total of 142 endophytic bacteria were isolated, screened in vitro for antagonism against $M$. roreri, and identified molecularly using the 16S rRNA region. Also, 2058 fungi were isolated, and fungal colonization increased with the leaf age (lower leaves rendered $54 \%$ of the isolated fungi). Contrary to fungi, apical leaves rendered the highest amount of bacterial isolates (44\% of the total). Out of the 142 bacteria tested, 83 showed antagonism activity against $M$. roreri, with colonial growth inhibition greater than $60 \%$. Those bacteria isolated from 
apical leaves produced the largest pathogen growth inhibition. The results of this study indicate that the community of endophytes changed with leaf age, and that most of the bacterial endophytes of cacao have biocontrol potential activity against $M$. roreri. Financed by Universidad Militar Nueva Granada Project: CIAS-2301.

Plant pathogen biocontrol potential of Pseudomonas strains isolated from Chilean wild flora

P. Vega-Celedón (1), M. VALENZUELA (1), A. Vergara (1), X. A. Besoain (2), M. Carvajal (1), M. Seeger (1), (1) Universidad Tecnica Federico Santa Maria, Valparaiso, CHILE; (2) Pontificia Universidad Católica de Valparaíso, Quillota, CHILE

Chilean microbial biodiversity is widely unexplored. The aim of this study was to evaluate the biocontrol potential of Pseudomonas strains, isolated from Chilean non-disturbed ecosystems and from agricultural crops against plant pathogens. Diverse isolates were collected from the rhizosphere of wild flora in different regions of Chile. Eight isolates were identified as Pseudomonas using 16S rRNA gene sequencing. In order to determine their potential as biocontrol agents, antimicrobial assays were carried out against Clavibacter michiganensis subsp. michiganensis, Pseudomonas syringae pv. syringae, Pectobacterium carotovorum, Phytophthora cinnamomi and Botrytis cinerea. Two of the isolates showed high inhibition against all these plant pathogens. In the case of $C$. michiganensis subsp. michiganensis, the antagonistic effect of strains isolated from wild flora, were compared with the effect of five antagonistic Pseudomonas strains isolated from tomato fields. Most of the Pseudomonas isolates collected from wild flora showed higher antagonistic effect against $C$. michiganensis subsp. michiganensis compared with those isolates collected from tomato plants. This study demonstrates the biotechnological potential of these bacteria isolated from non-disturbed ecosystems. In planta assays and additional beneficial properties of the strains will be evaluated.

Induction of apoptosis in the anthracnose fungi by Bacillus subtilis

Y. H. Chen, Y. H. Lin, Y. Yeh, T. P. HUANG, Department of Plant Pathology, National Chung Hsing University, Taichung, TAIWAN

Anthracnose on passion fruits caused by Colletotrichum species is a destructive disease hindering the production. The main objectives are to select and identify native antagonistic microorganisms against anthracnose fungi on passion fruits; to exploit their potential application in disease control and growth promotion; and to investigate the putative control mechanisms. The strain 151B1 isolated from rhizosphere soils in Taichung city, Taiwan was classified as the Bacillus subtilis based on the analysis of 16S rRNA sequences, DNA polymorphism, physiology and biochemistry tests and by Biolog System III. B. subtilis 151B1 showed inhibitory effect on mycelial growth and conidial germination of Colletotrichum sp. N-PF1. Application of $B$. subtilis 151B1 culture broth increased survival rates of passion fruit cuttings with or without inoculation of anthracnose fungi compared to the water and medium controls. It also enhanced the plant height, numbers of leaves, nodes, leaf width, and shoot fresh weights of passion fruit seedlings compared to the controls. Treatment of culture filtrates from strain 151B1 resulted in cell death and chromatin fragmentation, and caused reduction in mitochondrial membrane potential and energy metabolism of Colletotrichum sp. N-PF1, suggesting its function in triggering apoptotic-like cell death. In conclusion, $B$. subtilis 151B1 is a potential biocontrol agent for anthracnose disease of passion fruits. Our findings also suggested that aberrant hyphal morphology and inhibition of spore germination of anthracnose fungi caused by culture filtrates of B. subtilis 151B1 may in part attribute to their ability in triggering program cell death of the fungi.

Biological control of charcoal rot of sorghum by plant growth-promoting actinomycetes S. GOPALAKRISHNAN, V. Srinivas, N. Naresh, G. Alekhya, M. Sreevidya, R. Sharma, ICRISAT, Hyderabad, INDIA

Macrophomina phaseolina is one of the important pathogen of sorghum causing charcoal rot disease in post rainy season, particularly when there is a long spell of drought. Nine strains of Streptomyces spp. (BCA-546, BCA-698, CAI-8, CAI-17, CAI-21, KAI-26, KAI-27, MMA-32 and SAI-13) and one strain of Amycolatopsis sp. (BCA-696), demonstrated previously to have potential for plant growth-promotion on sorghum and chickpea, were evaluated for their antagonistic potential against $M$. phaseolina by dual culture assay, secondary metabolite production assay and in greenhouse and field disease screen. All the ten strains of actinomycetes inhibited M. phaseolina both in dual culture as well as in secondary metabolite production assays, but only five of them (BCA-546, CAI-21, KAI-26, KAI-27 and MMA-32) were notable under greenhouse and field conditions when evaluated against sorghum charcoal rot using tooth pick method of inoculation; these strains significantly reduced the disease. This study indicates that the selected five Streptomyces strains, BCA-546, CAI-21, KAI-26, KAI-27 and MMA-32, have the potential to control charcoal rot disease in sorghum.

\section{Isolate variability in Phytophthora tentaculata aggression and potential for biological control} T. L. WIDMER, USDA ARS FDWSRU, Ft. Detrick, MD, USA

Phytophthora tentaculata $(\mathrm{Pt})$ is a pathogen first described in 1993 from Germany on Origanum vulgare (oregano) and has been reported from the Netherlands, Spain, Germany, and Italy. It has recently been reported in California in 2012 on native plants including Diplacus aurantiacus subsp. aurantiacus (orange sticky monkey flower). Very little is known about this species and its behavior. The purpose of this study was to investigate the differences in aggression of Pt isolates and whether Trichoderma asperellum has the potential as a biological control agent (BCA) against it. Oregano seedlings were transplanted in potting mix amended with millet seed colonized by Pt isolates from Germany (2 isolates), Netherlands (1), Spain (1), and the USA (3) at rates of 0.25 or $0.5 \%(\mathrm{v} / \mathrm{v})$. After 8 weeks, seedling survival among the isolates was significant and varied from $96 \%$ for one of the Germany isolates to $0 \%$ for the Spain isolate, which compared to $96 \%$ for the non-infested controls. The average top dry weight for the controls was 0.33 $\mathrm{g}$ and ranged from $0.45 \mathrm{~g}$ for one of the Germany isolates to $0.02 \mathrm{~g}$ for one of the USA isolates. There was no difference between the two rates. Incorporation of wheat bran colonized by T. asperellum isolate 04-22 into potting mix infested with a Pt isolate from the USA not only improved survival (from 42 to $100 \%$ ) but significantly improved the top dry weight $(1.36 \mathrm{~g})$ compared to non-amended controls $(0.65 \mathrm{~g})$. This study shows that there is a difference in aggression among isolates and that $T$. asperellum has the potential as a BCA. This study will help in understanding and mitigating the threat that $P$. tentaculata has towards the native plant industry in CA and other potential hosts around the world.

Screening A Soybean Cyst Nematode Mycobiome for Potential Natural Antagonists in Search of Biological Control Agents and Biopesticides D. RAJENDRAN (1), K. Bushley (2), S. Chen (3), (1) Plant Pathology, University of Minnesota, St Paul, MN, USA; (2) Plant and Microbial Biology, Universty of Minnesota, St Paul, MN, USA; (3) University of Minnesota, Waseca, MN, USA

Soybean is the second most important crop for the United States, and Soybean Cyst Nematodes (SCN), its most important pathogen, can cause upto 40\% yield loss with no visible above-ground symptoms until later in the season. Effective chemical control agents and fumigants against SCN are detrimental to the environment and animal safety, and hence banned. Crop rotation and genetics are current solutions to manage the disease, while not sustainable. In this light, Biological control agents, especially fungal agents that can sporulate and withstand harsh winters of major soybean states of USA, are attractive and promising options. We have isolated about 6000 fungal samples associated with SCN cysts over 3 years and 6 total crop sequences. These 
isolates were then grouped together as morphotypes based on their appearance on potato dextrose agar, and representatives from each morphotype were identified using their ITS1 barcoding region and BLAST search against NCBI database. The morphotypes were further collapsed into 321 clusters based on $99 \%$ similarity in ITS1 barcoding region using uclust function in qiime. Representatives from each cluster were examined for their ability to withstand freeze-thaw, infect clean SCN cysts and eggs, produce spores, and toxic metabolites that can interfere with SCN egg-hatch and mobility. Many Nectriaceae clusters including Fusarium, Ilyonectria and Cylindrocarpon exhibit high parasitism as well as produce metabolites that inhibit egg hatching upto $90 \%$ with respect to control. A few candidate biocontrol agents were identified for greenhouse trials while chemically prospecting those producing toxic metabolites for safe bionematicides.

\section{Influence of applying microbial agents on the quality of sweet pepper}

Y. R. LIANG (1,2), F. C. Liao (2), T. P. Huang (1), (1) Department of Plant Pathology, National Chung Hsing University, Taichung, TAIWAN; (2) Taiwan Agricultural Chemicals and Toxic Substances Research Institute, Taichung, TAIWAN

Plant growth-promoting rhizobacteria have been indicated as a potential agronomic material for the growth promotion in many crops. Here, experiments were conducted by applying Trichoderma harzianum T23-1, Bacillus amyloliquefaciens Ydj3 and Streptomyces species Cu4 on sweet pepper grown in commercial greenhouses in Taiwan for assessing their potential in growth promotion. These three strains were selected by their enzyme productivity and antagonistic ability against Phytophthora capsici, Botrytis cinerea and Colletotrichum gloeosporioides. Random complete block design was used with three replicates for the treatments with T. harzianum T23-1, B. amyloliquefaciens Ydj3 and Streptomyces species Cu4, and the control. The three strains were applied once per month on sweet pepper in commercial greenhouse and horticultural traits including fruit weight, thickness of fruits, weight of seeds, sweetness ( ${ }^{\circ}$ Brix) and Vitamin C content, were recorded. The highest fruits sweetness was observed by the treatment with strain T23-1 (5.89 \pm 0.08 ${ }^{\circ}$ Brix), followed by the treatment with strain $\mathrm{Cu} 4\left(5.64 \pm 0.13{ }^{\circ} \mathrm{Brix}\right)$. The Vitamin $\mathrm{C}$ content of the sweet pepper was significantly higher by the treatment with strain Ydj3 (799 $\pm 18.39 \mathrm{mg} / \mathrm{g})$, and the treatment with the strain Cu4 $(760.72 \pm 14.67 \mathrm{mg} / \mathrm{g})$ compared to the control $(730.76 \pm 14.38 \mathrm{mg} / \mathrm{g})$. These results suggested that Streptomyces species Cu4 may play a role of quality promotion on sweet pepper and application of the three strains on sweet pepper crops may be potential practice for farmers to derive higher quality of sweet pepper.

A non-pathogenic Fusarium oxysporum W5 effectively controls "Bakanae" disease by competing with the pathogen on rice flowers and seedlings H. SAITO (1), K. Komatsu (2), T. Arie (3), (1) Tokyo Univ Agric \& Tech (TUAT), Fuchu, Tokyo, JAPAN; (2) Tokyo Univ Agric \& Tech (TUAT), Fuchu, Tokyo, JAPAN; (3) Tokyo Univ of Agric \& Tech (TUAT), Fuchu, Tokyo, JAPAN

"Bakanae" (means foolish seedling), caused by Fusarium fujikuroi, is one of the most important diseases of rice (Oryza sativa) in Japan. Though chemical fungicides (e.g. ipconazole) are effective in the control of "Bakanae" at present, the future emergence of fungicide resistant strain of the pathogen is concerned. Therefore, we tried to establish a novel biocontrol method of "Bakanae" using non-pathogenic Fusarium. We obtained 70 isolates of non-pathogenic Fusarium spp. from the rice plants in "Bakanae" outbreak fields in Japan. We selected a non-pathogenic F. oxysporum W5 as a candidate of biocontrol agent for "Bakanae". W5 presented clear "Bakanae" control activity in the rice plants generated from the seeds obtained from the flower treated with W5 bud cell suspension when flowering. Thus, we consider that spraying rice flower with W5 is an effective method of "Bakanae" control. Subsequently, we observed the dynamics of $F$. fujikuroi expressing RFP and W5 expressing eGFP on the rice plant. Compared to the single infection of $F$. fujikuroi, the frequency of $F$. fujikuroi fluorescence was remarkably reduced under the presence of W5. Interestingly, the fluorescence of W5 was not affected much by the presence of $F$. fujikuroi. Additionally, W5 did not inhibit the growth of $F$. fujikuroi on dual culture assay on potato dextrose agar (PDA). These results suggested that W5 competes with $F$. fujikuroi for nutrition and/or space on the rice plant tissues.

\section{Burkholderia gladioli can inhibit Burkholderia glumae in rice seedlings affected with bacterial panicle blight}

C. A. Riera-Ruiz (1), J. Castro-Lara (2), M. I. Jimenez Feijoo (3), J. CEVALLOS-CEVALLOS (3), (1) CIBE-ESPOL, Guayaquil, ECUADOR; (2) Escuela Superior Politécnica del Litoral, Guayaquil, ECUADOR; (3) Escuela Superior Politecnica del Litoral, ESPOL, Guayaquil, ECUADOR

Rice is the most consumed crop worldwide and the basis of the diet in many countries but its production is being threatened by the bacterial panicle blight disease (BPB) caused by either Burkholderia glumae or B. gladioli. However, the interaction of both pathogens in planta is still poorly understood. Isolates of B. glumae and B. gladioli were plated on PDA individually or as a combination of both pathogens. Similarly, rice seedlings were inoculated with each pathogen separately or in a 50\% mixture. Inhibition halos were measured in the Petri dishes whereas progression of disease severity was assessed in inoculated and control seedlings. Pathogens were recovered from infected plants in Petri dishes and each colony-forming unit was identified as B. glumae or B. gladioli by PCR using specific primers. Isolates of B. gladioli inhibited the growth of B. glumae in vitro, with average inhibition halos of $29.6 \mathrm{~mm}$. Both pathogens produced similar BPB disease progression but no significant interaction effect was observed in the plants. The recovery levels of both pathogens was similar in seedlings infected with each bacteria individually. However, more than $90 \%$ of the bacteria recovered from symptomatic seedlings infected with the $50 \%$ mixture of both pathogens were identified as B. gladioli and no B. glumae was observed in infected plants during the first three weeks of the disease. Results show the competition of both pathogens rice plants.

The biocontrol of black rot disease of okro caused by Macrophomina phaseolina in Nigeria D. B. OLUFOLAJI (1), M. E. Udoh (2), (1) Federal Univ of Technology, Akure, NIGERIA; (2) The Federal University of Technology, Akure, Akure, NIGERIA

Macrophomina phaseolina, the fungal pathogen of black rot disease of okra causes great loss in seed germinability and seedling establishment. Trichoderma harzianum an antagonistic biocontrol fungus and mancozeb chemical fungicide were bioassayed for antifungal attributes on the pathogen in Nigeria. T. harzianum and mancozeb were separately inoculated on the agar plates at 24, 48 and 72 hours before and after the introduction of $M$. phaseolina for both Prophylactic and therapeutic treatments. The inhibitory effect of $T$. harzianum and mancozeb were assessed. In vivo studies, involved the soaking of 20 okra seeds in spore suspension of T. harzianum at for 24 and 48 hours separately before and after the introduction of M. phaseolina for prophylactic and therapeutic treatments respectively. It was observed that at 72 hours $T$. harzianum had $92.11 \%$ level of mycelial growth inhibition on $M$. phaseolina, while mancozeb had $94.50 \%$ level of inhibition. In vivo, prophylactic treatment gave 50.00 and $66.65 \%$ for germination and seedlings emergence at 24 and 48 hours respectively which were significantly $(\mathrm{p} \leq 0.05)$ higher than the therapeutics which gave 13.35 and $02.72 \%$ respectively. This is an indication that both the biocontrol agent and the chemical fungicide were able to inhibit the mycelial growth of the pathogen. However the biocontrol agent could be more preferable for disease control since it would be environment friendly and do not exhibit mammalian toxicity.

Diversities of gut endosymbionts in Cowpea beetle on resistant and susceptible cowpea varieties O. Adegbemile, O. ALABI, A. Aduramigba-Modupe, University of Ibadan, Ibadan, NIGERIA 
Insects harbor endosymbionts that supply essential nutrients and detoxify toxic substances in their food hosts. Resistant populations of Cowpea Beetle (CB) (Callosobruchus maculatus F.) are known to develop overtime on resistant cowpea (Vigna unguiculata [L.] Walp.) varieties due to activities of endosymbionts. However, information on role of gut endosymbionts in CB causing development of resistant populations is limited. Therefore diversities of endosymbionts in CB guts was investigated. Five pairs of newly-emerged adults were introduced into 20g of each of IT96-610, IT81D-994 (resistant varieties) and IfeBrown (susceptible variety). Adult emergence and mean developmental period (days) were assessed. Diversities and counts of bacteria and fungi from guts of $F_{2}$ beetles from each of the variety were determined. Sex ratio of 1 male: 3 females was recorded from adults on IT81D-994, while a 1:1 ratio was obtained from the other varieties. Development of beetles was prolonged on IT96-610 (51.0 2.5$)$ and IT81D-994 (51.0 33.0$)$ compared with Ifebrown (39.0 22.0). Bacterial (Bacillus sp., Staphylococcus sp., Micrococcus sp., Streptococcus sp.) and fungal (Aspergillus candidus, Penicillium alfredil, Collentrotrichum gloeosporiodes, Aspergillus terreus) strains were isolated from beetles' guts. Beetles on Ifebrown had the highest bacterial count $\left(12.25 \times 10^{4} \mathrm{CFU} / \mathrm{mL}\right)$ and least count was $6.5 \times 10^{4} \mathrm{CFU} / \mathrm{mL}$ from IT96-610. Highest fungi count, $14.0 \times 10^{3} \mathrm{CFU} / \mathrm{mL}$, was from beetles on IT96-610, while the least $\left(6.0 \times 10^{3} \mathrm{CFU} / \mathrm{mL}\right)$ was from Ifebrown. IT96-610 and IT81D-994 prolonged development of beetles, while IT81D-994 altered the sex ratio. Use of antibiotics to treat cowpea will destroy endosymbionts in beetles' guts and consequently reduce damage of seeds in store.

Elucidation of the mechanisms employed by Bacillus mycoides BM02 in controlling tomato Fusarium wilt J. J. WU (1,2), J. W. Huang (1,2,3), W. L. Deng (1,2,3), (1) Ph.D. Program in Microbial Genomics, National Chung Hsing University and Academia Sinica, Taichung, TAIWAN; (2) Biotechnology Center, National Chung Hsing University, Taichung, TAIWAN; (3) Department of Plant Pathology, National Chung Hsing University, Taichung, TAIWAN

Bacillus mycoides in the $B$. cereus group forms rhizoidal colonies on solid medium, and the strain BM02 isolated from tomato rhizoplane in Taiwan has proven to have promising biocontrol activity. The de novo assembled complete genome of BM02 $(5,782,184 \mathrm{bp})$ is composed of one chromosomal DNA and four plasmids, and subsequent genome mining revealed several gene clusters for synthesizing secondary metabolites might be involved in biocontrol activity. Application of formulated BM02 reduced the disease severity of tomato Fusarium wilt, and activated defense-related genes in SA (PRIa) and ET (LeACO5) signaling pathways in tomato roots that were co-inoculated with BM02 and Fusarium oxysporum f. sp. lycopersici Fol-04. Microscopic observation revealed that BM02 was able to interfere with the infection of GFP-tagged Fol-04 by reducing conidia germination, inducing abnormal hyphae, and inhibiting the formation of appressorium-like structure on tomato roots. The crude extracts of siderophores with iron chelating activity from the culture filtrate of BM02 were found to inhibit conidia germination and stimulate abnormal hyphae formation of Fol-04 in vitro; further analysis, including the chemical structure of the bioactive compound(s), are in progress to determine the biological activities of BM02. Altogether, BM02 has the capacities of activating plant defense responses and producing antifungal metabolites that were collectively involved in controlling tomato Fusarium wilt disease.

Characterization of the effect of HSAF from Lysobacter enzymogenes on Fusarium graminearum

Y. ZHAO, C. Cheng, F. Liu, Institute of Plant Protection, Jiangsu Academy of Agricultural Sciences, Nanjing, CHINA

Fusarium graminearum is a major pathogenic fungus that causes Fusarium head blight (FHB) disease on various cereal crops. Heat-stable antifungal factor (HSAF), which was found in Lysobacter enzymogenes, belongs to the polycyclic tetramate macrolactam family (PTM). HSAF was demonstrated to be a powerful antifungal agent showing activity against a number of plant pathogens, and effective in inhibiting $F$. graminearum. In this study, we investigated the effect of HSAF on the mycelia and conidia of $F$. graminearum $\mathrm{PH}-1$. We determined that the effective concentration of HSAF to inhibit $50 \%$ mycelial growth of PH-1 was $4.1 \mu \mathrm{g} / \mathrm{mL}$ ( $\mathrm{EC}_{50}$ value). The production and germination of conidia were completely inhibited with $6 \mu \mathrm{g} / \mathrm{mL}$ and 1 $\mu \mathrm{g} / \mathrm{mL}$ HSAF, respectively. Furthermore, we observed that different concentration of HSAF treatment led to morphology changes of hypha and conidia, such as the occurrence of wavy hyphae with increased branching, more germinating spots of conidia and swelling conidia. To further study the effect of HSAF on subcellular morphology of PH-1, we conducted TEM observation and found that $5 \mu \mathrm{g} / \mathrm{mL}$ HSAF could result in plasmolysis, invagination of plasmalemma and more lipid bodies. Further exploration on the mechanism of action of HSAF will provide clearer insight into its use as a fungicide to control FHB disease.

\section{Management of brown blight in tea caused by Colletotricum gloeosporioides by crude and purified leaf extract of Xanthium strumarium} D. SAHA, R. Kumar, A. Saha, University of North Bengal, Siliguri, INDIA

Tea (Camellia sinensis) is an economically important plantation crop of India but is susceptible to attack by several fungal pathogens. The study was aimed to develop botanical fungicides which could be a safer alternative to the harmful chemical fungicides. A fungal pathogen was isolated from the leaves of tea plants showing symptoms of brown blight from the tea plantations in Darjeeling. The pathogen was identified as Colletotricum gloeosporioides following PCR amplification and sequencing of the ITS region (Gen Bank accession Number KC493156) and its pathogenicity was confirmed through verification of Koch's Postulates. For controlling the disease, crude leaf extracts of Xanthium strumarium was tested for antifungal activity in vitro against the pathogenic isolate by agar cup assay method. Bioassay guided fractionation of the crude extract led to the isolation of two antifungal compounds, 8-epi-xanthatin (1) and 8-epi-xanthatin-1 $\beta, 5 \beta$-epoxide (2). The structures were elucidated by IR, NMR and ESI-MS spectroscopy data analysis. Determination of MIC of the pure compounds by micro-dilution bioassay method revealed that antifungal activity of $\mathbf{1}$ was higher than $\mathbf{2}$ The water insoluble extracts emulsified in water effectively controlled brown blight of tea in the green-house and its efficacy was comparable to the fungicide bavistin. The results show that $X$. strumarium leaves may be used as a source of botanical fungicidal preparation for controlling brown blight in tea.

Bacillus strains in the control of bacterial wilt in tomato

A. Thomas (1), J. Jarvis (2), D. SARAVANAKUMAR PHD (3), (1) UWI, Port of Spain, TRINIDAD AND TOBAGO; (2) The University of the West Indies, St. Augustine, TRINIDAD AND TOBAGO; (3) University of the West Indies, St. Augustine, Trinidad, TRINIDAD AND TOBAGO

Bacterial wilt caused by $R$. solanacearum considered as a serious challenge in the cultivation of tomato in the Caribbean islands. The overuse of conventional fungicides has lost its efficacy against the bacterial wilt pathogen. Therefore, it is necessary to develop the control strategy for this wilt disease. In the current research, our preliminary screening and characterization of native rhizobacterial strains allowed to evaluate the Bacillus subtilis OG2A and Bacillus amyloliquefaciens CHAG1D, PRIN6, ML5 against $R$. solanacearum in tomato plants under pot culture. The $B$. subtilis OG2A and $B$. amyloliquefaciens CHAG1D, PRIN6, ML5 strains were applied as soil drenching in tomato plants, followed by challenge inoculation of $R$. solanacearum through root injury method. The disease incidence was recorded at 7 days interval after inoculation of pathogen. At 30 and 37 days after inoculation (DAI) of the pathogen, 73.33 and 100 percent wilt incidence was recorded respectively in untreated control plants. At the same time, the plants treated with B. subtilis OG2A $(33.33 \% ; 60.00 \%)$ and B. amyloliquefaciens PRIN6 $(33.33 \% ; 60.00 \%)$ recorded significantly less wilt incidence at 30 and 37 DAI compared to the untreated control. The high wilt incidence was also recorded in chemical treatment as that of untreated control. The promising control of B. subtilis and B. amyloliquefaciens against bacterial wilt in tomato has offered the potential for developing them as bioagents. 
Biological management of collar, stem and root rot disease complex and white grubs using Trichoderma harzianum and Metarhizium anisopliae in groundnut

P. SHARMA (1), P. P. Jambhulkar (2), M. Raja (3), V. K. Agrawal (4), A. S. Baloda (4), G. S. Rathore (3), A. C. Mathur (4), S. Javeria (3), (1) Sri Karan Narendra Agriculture University (SKNAU), Durgapura, Jaipur, INDIA; (2) Agricultural Research Station (MPUAT Udaipur), Banswara, INDIA; (3) Sri Karan Narendra Agriculture University (SKNAU), Jobner, Jaipur, INDIA; (4) Rajasthan Agricultural Research Institute, Durgapura, Jaipur, INDIA

The experimental study was conducted in two agroclimatic conditions of Rajasthan, zone IV B southern humid zone and III A semi-arid eastern zone in humid conditions for ecofriendly management of two major biotic stresses in groundnut crop caused by Aspergillus sp., Macrophomina phaseolina, Sclerotium rolfsii and Rhizoctonia solani in the form of collar, stem and root rot disease complex along with attack by Holotrichea consanguinea (white grubs). Under in vitro studies, the combined application of Trichoderma harzianum $\left(4.3 \times 10^{6} \mathrm{cfu} / \mathrm{ml}\right)$ and Metarhizium anisopliae $\left(2.3 \times 10^{6} \mathrm{conidia} / \mathrm{ml}\right)$ reduced disease incidence in a range of $40-50 \%$ and suppressed the egg hatching and killed $1^{\text {st }}$ and $2^{\text {nd }}$ instar larvae of white grubs from $78 \%$ to $100 \%$. Soil application with $T$. harzianum and $M$. anisopliae enriched farm yard manure $(1: 20)+$ seed treatment with $T$. harzianum + drenching twice with $T$. harzianum was found very effective to reduce the disease complex and two times drenching with $M$. anisopliae significantly reduced $68 \%$ white grub population as compared with control. Another two treatments as fungicidal check of soil application by neem cake and seed treatment with Thiophanate Methyl $450 \mathrm{~g} / \mathrm{L}+$ Pyraclostrobin $50 \mathrm{~g} / \mathrm{L} \mathrm{FS}$ and Carbendazim 12\% + Mancozeb 63\% WP were found more effective than application of BCAs to reduce disease incidence and the Imidacloprid 17.8 SL was used as chemical check against white grub which was found at par with application of $M$. anisopliae but also reduced infestation of white grubs to increase pod yield by $33.6 \%$.

Development of a botanical plant protection product from Larix by-products to protect grapevine from Plasmopara viticola L. TAMM, Research Institute of Organic Agriculture FiBL, Frick, SWITZERLAND

BACKGROUND: Plant extracts might provide sustainable alternatives to copper fungicides, which are still widely used despite their unfavourable ecotoxicological profile. Larch bark extract and its constituents, larixyl acetate and larixol, have been shown to be effective against grapevine downy mildew (Plasmopara viticola) under semi-controlled conditions. The aim of this study was to reduce the gap between innovation and the registration of a marketable product, namely to develop scalable extraction processes and to evaluate and optimise the performance of larch extracts under different conditions. RESULTS: Toxicologically and technically acceptable solvents like ethanol were used to extract the active compounds larixyl acetate and larixol from bark in sufficient amounts and their combined concentration could be increased by up to $39 \%$ by purification steps. The combined concentration of larixyl acetate and larixol from larch turpentine could be increased by up to $66 \%$. The MIC100 against P. viticola in vitro (6-23 $\mu \mathrm{g}$ mL $\left.{ }^{1}\right)$ and the EC50 in planta under semi-controlled conditions $\left(0.2-0.4 \mathrm{mg} \mathrm{mL}^{-1} \mathrm{Q} 4\right)$ were promising compared with other plant extracts. In vineyards, efficacies of larch extracts reached up to $68 \%$ in a stand-alone strategy and $84 \%$ in low-copper strategies. CONCLUSION: Larch extracts represent valid candidates for copper reduction in organic vineyards, and their development into a sustainable plant protection product might be feasible.

Biological control of powdery mildew on cucurbits

K. MCCORKLE, E. Gachango, K. Craig, R. Dale, B. Espejo, P. E. Hammer PhD, V. Pham, S. Ronyak, A. Thomas, M. Twizeyimana, K. Tyson, AgBiome, Inc., Durham, NC, USA

Cucurbit powdery mildew, caused primarily by the ascomycete Podosphaera xanthii, is an important foliar disease of cucurbits worldwide. Symptoms include early chlorotic spots, necrosis, leaf curling, defoliation, and small and sunburned fruit. An increase in fungicide applications to manage this disease has led to a pathogen population shift towards reduced levels of fungicide sensitivity. In response, industry has pursued the research and development of biological controls that have a broad spectrum of antifungal activity with multiple modes of action. AgBiome, an agriculture biotech company, focuses on microbial-derived crop pest solutions by mining the diversity of its vast collection of over 40,000 fully-sequenced microbes. Isolated microbes are screened for activity in bioassays on multiple foliar pathogens, including $P$. xanthii, using high-throughput detached leaf assays. Utilizing our powdery mildew assay, leaf disks ( $35 \mathrm{~mm}$ diameter circle each) were sprayed with the microbial strain, a day after, they were inoculated with a conidial suspension $\left(10^{5}\right.$ conidia/mL and $200 \mu$ l per leaf disk). Disease severity was rated 5 days post inoculation and percent activity relative to the inoculated control was determined. Strains having greater than $70 \%$ activity against $P$. xanthii were confirmed and progressed through the pipeline for greenhouse bioassays and field testing. In 2017, we identified multiple strains with confirmed activity on P. xanthii which will be sent for field screening in 2018. Strains maintaining a high level of activity will be further characterized by additional field evaluations.

Evaluation of Bacillus and Pseudomonas strains for bio-control activities on sheath blight and bacterial panicle blight of rice A. MAHARJAN (1), J. H. Ham (2), M. Kang (3), (1) Louisiana State University Agricultural Center, Baton Rouge, LA, USA; (2) Louisiana State University, Baton Rouge, LA, USA; (3) Kangwon National University, Chuncheon, SOUTH KOREA

Sheath blight (ShB - caused by Rhizoctonia solani) and bacterial panicle blight (BPB - caused by Burkholderia glumae) are economically important rice diseases in Louisiana and other rice-growing regions. Fungicides and oxolinic acid are applied to manage ShB and BPB, respectively, but these chemical methods are not sustainable economically and ecologically. To develop alternative biotic materials for disease management, rice-associated bacteria were screened based on their antagonistic activities against the pathogens by growth-inhibition plate assays, and three strains each of Bacillus spp. and Pseudomonas spp. were selected for evaluation in the field for ShB and BPB, respectively. Rice plants were artificially inoculated with $R$. solani at the tillering stage, while B. glumae was inoculated at $30 \%$ heading. Selected antagonistic bacteria were applied via spraying on the sheath (for ShB) or panicles (for BPB) of plants $24 \mathrm{~h}$ after inoculation. A field trial in 2017 revealed that one Bacillus strain REB 711, significantly suppressed ShB development compared to the untreated control, although it was less effective than the fungicide azoxystrobin. Efficacy of the Pseudomonas strains for suppression of $\mathrm{BPB}$ could not be determined in the field trial due to low disease pressure. Related research activities include characterization of the antimicrobial compounds from the bio-control agents and combined application of bio-control agents.

Understanding the interaction between biocontrol agent Lysobacter enzymogenes and soybean fungal/oomycetes pathogens M. YU (1), J. Nian (2), C. Bradley (2), Y. Zhao (3), (1) UNIVERSITY OF ILLINOIS, Urbana, IL, USA; (2) University of Illinois, Urbana, IL, USA; (3) University of Illinois at Urbana-Champaign, Urbana, IL, USA

Lysobacter enzymogenes strain $\mathrm{C} 3$ (LeC3) is a potential biocontrol agent for plant diseases caused by fungi and oomycetes. Understanding the interaction between $\mathrm{LeC} 3$ and soybean pathogens at molecular level could improve its biocontrol efficacy. In this study, we evaluated the effectiveness of LeC3 in inhibiting hyphae growth or spore germination of soybean fungal/oomycete pathogens, and identified and characterized important LeC3 
genes during this process. Results showed that $\mathrm{LeC} 3$ strongly inhibited hyphae growth or spore germination of Cercospora sojina, Fusarium virguliforme, Macrophomina phaseolina, Phytophthora sojae, Pythium sylvaticum, Rhizoctonia solani, Septoria glycines, and Sclerotinia sclerotiorum, suggesting its broad spectrum activities. Screening a LeC3 mutant library identified 74 mutants that no longer inhibited S. sclerotiorum growth. Characterization of nine selected mutants revealed that these mutants no longer inhibited hyphae growth of $S$. sclerotiorum and significantly suppressed spore germination of $F$. virguliforme. Furthermore, production of four extracellular enzymes and secretion of the heat stable antifungal factor (HSAF), a fungal specific antibiotic, was significantly decreased in all nine mutants. Collectively, our findings demonstrated that LeC 3 could be very effective in controlling soybean fungal/oomycetes diseases and suggested that the nine genes play critical roles in the production and secretion of extracellular enzymes and HSAF.

Augmentation of in-furrow applied insecticides with a superabsorbent polymer to improve management of spotted wilt of peanut J. HAYNES (1), A. K. Culbreath (2), N. Smith (3), D. J. Anco (1), (1) Clemson University, Blackville, SC, USA; (2) University of Georgia, Tifton, GA, USA; (3) Clemson University, Columbia, SC, USA

Spotted wilt of peanut (Arachis hypogaea L.) is a common disease that causes severe economic losses in peanut producing regions around the world. The causal agent is Tomato spotted wilt orthotospovirus (TSWV), which is transmitted by species of thrips (Thysanoptera: Thripidae) with western flower thrips (Frankliniella occidentalis) and tobacco thrips (F. fusca) being of particular importance in the southeastern United States. Field trials were conducted in 2017 to determine if management of spotted wilt and subsequent productivity of peanut could be improved by applying a superabsorbent polymer $(2.24 \mathrm{~kg} / \mathrm{ha}$ ) with standard in-furrow insecticides at the time of planting. To determine this, insecticides (phorate and imidacloprid) were individually applied with or without polymer across varieties susceptible (FloRun 157 or TUFRunner 511), moderately susceptible (Georgia 06G), and resistant (Sullivan and TifNV-High O/L) to TSWV. Untreated controls were included in all trails. The study utilized a randomized complete block design and was conducted in three locations across South Carolina and Georgia. Stunting of plants was significantly reduced (reduction of $8 \%, P<0.05)$ when susceptible varieties were treated with phorate and the polymer. Polymer-associated effects on thrips counts and damage, phytotoxicity and yield were not significant $(P>0.05)$ across locations. While additional experiments are needed, these results suggest that superabsorbent polymer could be used to mitigate the symptoms of spotted wilt on susceptible varieties.

Etiology and management of Septoria leaf spot on stevia A. KOEHLER, D. Shew, North Carolina State University, Raleigh, NC, USA

Stevia (Stevia rebaudiana) is a new perennial crop in the US, produced for leaves that contain numerous glycosides extracted for use as nonnutritive sweeteners. In 2015, leaf lesions caused by a Septoria sp. were observed in multiple locations in North Carolina. Lesions were present throughout the season but spread rapidly and coalesced to cause significant defoliation during favorable environmental conditions in September, prior to harvest. Type culture isolates of Septoria steviae, reported in Japan in 1982, were obtained for comparison to NC isolates. All isolates were sequenced for six loci: actin, $\beta$-tubulin, calmodulin, internal transcribed spacer, RNA polymerase II second largest subunit, and translation elongation factor-1alpha. Isolates from NC were identified as S. steviae. In 2017, efficacy trials were conducted at two locations in NC, testing seven fungicides and one biological control. Products were applied twice late in the growing season and plants were rated for percent of leaf area with lesions. Trials were combined for analysis and there were significant treatment effects $(P<0.001)$. All products tested reduced lesion area compared to the control $(57 \%$ leaf area covered at first harvest). In vitro fungicide trials were conducted to document the initial sensitivity profiles of NC $S$. steviae isolates to a range of fungicide chemistries. Successful management of Septoria leaf spot will be critical to the successful establishment of stevia in the US.

The application of 8-hydroxyquinoline and its copper complex as seed treatment to seeds of corn and cucumber D. YANG, H. Yuan, Institute of Plant Protection, Chinese Academy of Agricultural Science, Beijing, CHINA

8-hydroxyquinoline is a compound with antimicrobial activity. It can provide control against fungi and bacteria. But it can also present phytotoxic activity. In this study, its phytotoxic activity on crop seeds and the pathway of eliminating its phytotoxicity were assessed. After being soaking in the aqueous solutions of 8 -hydroxyquinoline, the germination of corn seeds decreased from $96.7 \%$ to $10.0 \%$ when the concentration of 8 -hydroxyquinoline increased from $50 \mathrm{mg} \mathrm{L}^{-1}$ to $1000 \mathrm{mg} \mathrm{L}^{-1}$. But 8-hydroxyquinoline did not affect the germination of cucumber seeds in the same tested dose range. Our result demonstrated that the Murashiage \& Skoog medium solution can resume the germination of corn seeds if the 8-hydroxyquinoline treated corn seeds were subsequently soaked in the Murashiage \& Skoog medium solution for 8h. Further study showed metallic ions in the Murashiage \& Skoog medium solution contributed to resuming the germination of corn seeds. But if only one kind of metallic ion, e.g. $\mathrm{Fe}^{2+}, \mathrm{Zn}^{2+}, \mathrm{Mg}^{2+}$ and $\mathrm{Cu}^{2+}$, was applied, the germination of corn seeds cannot be assumed to the same level of control. To ensure its antimicrobial activity, the copper complex of 8hydroxyquinoline can be used without phytotoxicity as seed treatment.

Biopriming - as a component of Integrated Disease Management Strategy for the eco-friendly management of Pod Blight Complex of Soybean in India

L. RAO (1), S. Jahagirdar (2), S. D.S. (1), (1) University of Agricultural Sciences, Dharwad, Dharwad, INDIA; (2) Univ of Agricultural Sciences Dharwad, Dharwad, AK, INDIA

The efficacy of bio priming of soybean seeds as a component of integrated management strategy against pod blight complex was tested during two years field trial conducted at Ugarkhurd research station in northern Karnataka in India during 2016-17 and 2017-18. The results indicated the efficacy of seed treatment with Carboxin + Thiram @ $2 \mathrm{~g} / \mathrm{Kg}$ of seed+ foliar spray with Trifloxystrobin+ Tebuconazole $(0.7 \mathrm{~g} / \mathrm{l})$ at 50 and 70 DAS in minimizing pod blight complex. Bio priming of soybean seeds with Pseudomonas fluroscens@8 g/Kg of seed + Coir pith + Jelly+ foliar spray with Neem Seed Kernel Extract @ $0.5 \%$ at 50 and 70 DAS was found promising in minimizing pod blight complex as it exhibited an on par performance with seed treatment with Mancozeb + Carbendazim at $2 \mathrm{~g} / \mathrm{Kg}$ of seed + foliar spray with Carbendazim + Mancozeb $(0.2 \%)$ at 50 and 70 DAS though found significantly inferior to seed treatment with Carboxin + Thiram @ $2 \mathrm{~g} / \mathrm{Kg}$ of seed+ foliar spray with Trifloxystrobin + Tebuconazole (0.7 g/l) at 50 and 70 DAS. However with respect to seed yield, bio priming of seeds with Pseudomonas fluroscens@ 8 g/Kg of seed + Coir pith + Jelly+ foliar spray with Neem Seed Kernel Extract@ $0.5 \%$ at 50 and 70 DAS was found on par with seed treatment with Carboxin + Thiram @ $2 \mathrm{~g} / \mathrm{Kg}$ of seed+ foliar spray with Trifloxystrobin + Tebuconazole $(0.7 \mathrm{~g} / 1)$ at 50 and 70 DAS.

Effect of Seed Treatments on Sudden Death Syndrome and Yield of Soybean Y. R. KANDEL (1), C. A. Bradley PhD (2), M. Chilvers (3), F. M. Mathew (4), A. U. Tenuta (5), D. L. Smith (6), K. A. Wise (2), D. S. Mueller (1), (1) Iowa State University, Ames, IA, USA; (2) University of Kentucky, Princeton, KY, USA; (3) Michigan State University, East Lansing, MI, USA; (4) 
South Dakota State University, Brookings, SD, USA; (5) Ontario Ministry of Agric \& Food, Ridgetown, ON, CANADA; (6) University of Wisconsin, Madison, WI, USA

Seed treatments have recently been registered for management of sudden death syndrome (SDS) caused by Fusarium virguliforme, one of the most devastating diseases of soybean (Glycine max). We evaluated seed treatment fungicides fluopyram (ILeVO ${ }^{\circledR}$ ), thiabendazole (Mertect 340- $\mathrm{F}^{\circledR}$ ), and a biochemical pesticide, saponins of Chenopodium quinoa (Heads Up ${ }^{\circledR}$ ) against their standard base seed treatment in a total of 14 field experiments in Illinois, Indiana, Iowa, Michigan, South Dakota, Wisconsin, and Ontario, Canada during 2015 and 2016. Each of these treatments were evaluated on SDS-resistant and -susceptible cultivars. In 2015, the fluopyram treatment had the least amount of SDS among all fungicides, with nearly $35 \%$ less root rot (RR) and 54\% lower foliar disease index (FDX), while achieving 6.2\% greater yield than the base seed treatment. Resistant cultivars had $30 \%$ less RR and 75\% lower FDX, with $11 \%$ greater yield than susceptible cultivars. In 2016, no seed treatments were significantly different from their base seed treatment, except fluopyram, which had 45\% less RR and 44\% lower FDX. Seed treatment had no effect on yield in 2016. Resistant cultivars resulted in $23 \%$ lower RR and $81 \%$ lower FDX, with $10 \%$ greater yield, compared to susceptible cultivars. Fluopyram resulted in the greatest reduction in disease compared to the other seed treatment products and can be used as a complement to resistant cultivars for managing SDS.

Evaluation of fungicides and application timings for management of sorghum anthracnose in the mid-Atlantic region of the U.S. B. ACHARYA (1), T. N. O'Quinn (2), W. Everman (2), H. L. Mehl (3), (1) Virginia Tech, Suffolk, VA, USA; (2) North Carolina State University, Raleigh, NC, USA; (3) Virginia Tech Tidewater AREC, Suffolk, VA, USA

Demand for locally sourced animal feed has increased grain sorghum production in the mid-Atlantic. Sorghum anthracnose (Colletotrichum sublineola) reduces grain yield up to $50 \%$, but management recommendations have not been developed for the mid-Atlantic where warm, wet conditions favor disease. Using a $6 \times 4$ factorial design, five fungicides (Priaxor, Headline, Quadris, Aproach, Tilt) and four application timings (single applications at boot, flowering, or 14 days after flowering; and applications at all three timings) were compared for anthracnose control and yield response in sorghum over 10 site-years in VA and NC from 2015-2017. Anthracnose severity was rated biweekly, and grain yield was determined at harvest. Effects of fungicide, timing, site-year and their interactions were significant $(\mathrm{P}<0.0001)$. Disease onset and final severity varied among locations. Priaxor and Headline reduced anthracnose, and three applications resulted in significantly lower disease and higher yield compared to single applications. For single applications, flowering timing provided the most consistent reductions in anthracnose compared to the untreated control. Pyraclostrobin is an active ingredient in Priaxor and Headline, and in in-vitro fungicide sensitivity tests, this fungicide was the most effective for inhibiting C. sublineola growth. Results indicate a single well-timed application of a pyraclostrobin-containing fungicide controls anthracnose and protects sorghum grain yield.

Dynamics of fungicide sensitivity in Venturia effusa and fungicide efficacy under field conditions

J. R. STANDISH, T. B. Brenneman, K. L. Stevenson, University of Georgia, Tifton, GA, USA

Venturia effusa, which causes scab, the most damaging disease of pecan, has developed resistance to fungicides that were once very effective. A 2-year study was conducted to explore the relationship between laboratory-based sensitivity to fentin hydroxide (TPTH) and tebuconazole (TEB) in $V$. effusa and the efficacy of these products in managing scab under field conditions. Scab epidemics were monitored on trees each receiving ten applications of TPTH (Super Tin 4L), TEB (Orius 3.6F), azoxystrobin (AZ; Abound), AZ + TEB, TPTH + TEB, or no fungicide (NTC). Sensitivity of $V$. effusa on leaflets from treated trees was assessed in June and September of both years. In 2016, mean relative germination (RGe) on $30 \mu \mathrm{g} / \mathrm{ml} \mathrm{TPTH}$ was $11 \%$ and $41 \%$ (June and Sept., respectively), and in 2017, RGe was 4\% and 1\%. Mean relative growth (RGr) on $1 \mu \mathrm{g} / \mathrm{ml} \mathrm{TEB}$ in 2016 was $46 \%$ and $35 \%$, and $69 \%$ and $56 \%$ in 2017. Leaf and nut scab intensity were significantly lower in both years on trees treated with AZ, AZ + TEB, or TPTH + TEB when compared to NTC and TEB-treated trees. Compared to the NTC, TEB did not significantly reduce leaf scab in 2017 or nut scab in either year, indicating that an RGr value between $46 \%$ and $69 \%$ is likely to result in a control failure on TEB-treated trees. Although better control was expected, TPTH reduced scab with RGe values between $1 \%$ and $41 \%$. These results will be valuable in developing critical fungicide sensitivity thresholds to better predict fungicide performance.

Resistance inductors for potato late blight management in Peru

K. SANABRIA (1), W. Perez (1), J. L. Andrade-Piedra (2), D. Sánchez (3), (1) International Potato Center, Lima, PERU; (2) International Potato Center (CIP), Lima, PERU; (3) HORTUS S.A, Lima, PERU

The effectiveness and efficiency of commercial resistance inductors (RIs) for LB control in the central highlands of Peru were studied. Susceptible plants of cv. Yungay were sprayed with PKplus ${ }^{\circledR}$, Manvert Biolet ${ }^{\circledR}$, Miconic $^{\circledR}$ and Manvert Silikon ${ }^{\circledR}$, and then they were inoculated with a complex P. infestans isolate under laboratory and greenhouse conditions. PKplus ${ }^{\circledR}$ and Manvert Biolet ${ }^{\mathbb{B}}$ were the most effective in disease control. Both products were selected for field trials and were applied on four potato cultivars with different levels of susceptibility in three locations during the rainy season. Treatments were PKplus $^{\circledR}$, Manvert Biolet ${ }^{\mathbb{\circledR}}$; and propineb (alone or alternated); a Decision Support System (DSS, using systemic and/or contact fungicides); and a plot without fungicides. Relative area under the disease progress curve (RAUDPC), yield, environmental impact (EI) and marginal rate of return (MRR) were registered in all experiments. The most effective (low RAUDPC, high yield and low EI) and efficient (MRR $>50 \%$ ) treatments were PKplus ${ }^{\circledR}$ and propineb alone, PKplus ${ }^{\circledR}$ alternated with propineb and the DSS in susceptible cultivars; PKplus ${ }^{\circledR}$ and Manvert Biolet ${ }^{\circledR}$ alternated with propineb, and propineb alone in a moderately resistant cultivar; and propineb alone in a resistant cultivar. These results showed that it's possible to control potato LB in a profitable and environmentally-friendly manner, even in susceptible cultivars during the rainy season.

A novel alternative to copper bactericide: Magnesium based nanomaterials for management of tomato bacterial spot

Y. Y. LIAO (1), A. L. Strayer (1), Z. Huang (2), S. Santra (2), J. C. White (3), A. Mukherjee (3), R. De La Torre-Roche (3), L. Ritchie (4), J. Colee (5), G. E. Vallad (6), J. H. Freeman (4), J. B. Jones (7), M. Paret (4), (1) University of Florida, Department of Plant Pathology, Gainesville, FL, USA; (2) NanoScience Technology Center, Department of Chemistry, University of Central Florida, Orlando, FL, USA; (3) Connecticut Agric Exp Station, New Haven, CT, USA; (4) North Florida Research and Education Center, University of Florida, Quincy, FL, USA; (5) Department of Statistics, University of Florida, Gainesville, FL, USA; (6) Gulf Coast Research and Education Center, University of Florida, Wimauma, FL, USA; (7) Department of Plant Pathology, University of Florida, Gainesville, FL, USA

Bacterial spot incited by four Xanthomonas spp., can cause major yield losses of tomato. In Florida, there has been no effective chemical control strategy utilizing bactericides as presence of copper-tolerant strains has rendered $\mathrm{Cu}$-based bactericides ineffective. In addition, EPA has recently posted guidelines suggesting limiting the amount of copper bactericides that can be applied on several vegetable crops. Therefore, finding an effective alternative to copper is very critical. In this study, we demonstrate that a non-formulated magnesium oxide nanoparticles (MgO) have high bactericidal activity against $X$. perforans. In greenhouse and field experiments, bacterial spot disease severity was significantly reduced by $\mathrm{MgO}$ applications at 200 $\mu \mathrm{g} / \mathrm{mL}$ compared to the untreated control (UT), whereas the grower standard treatment, copper-mancozeb (K+M), was not significantly different from 
$\mathrm{UT}(\mathrm{p}=0.05)$. In order to determine if nanoparticles accumulated in fruit, inductively coupled plasma mass spectrometry analysis of the fruits from MgO treated plots did not significantly alter levels of $\mathrm{Mg}, \mathrm{Cu}, \mathrm{Ca}, \mathrm{K}, \mathrm{Mn}, \mathrm{P}$ and $\mathrm{S}$ compared to fruit in the UT. In our current work, formulated Mg-based nano-materials significantly reduced disease severity to as low as $100 \mu \mathrm{g} / \mathrm{mL}$ compared with $\mathrm{Cu}+\mathrm{M}$ and $\mathrm{UT}(\mathrm{p}=0.05)$ in two greenhouse studies. This demonstrated for the first time antibacterial potential of $\mathrm{Mg}$ against $X$. perforans and its potential use against bacterial spot of tomato.

Fungicide efficacy for control of foliar and fruit diseases on pomegranate in Florida

K. V. XAVIER (1), G. E. Vallad (2), (1) Gulf Coast Research and Education Center; University of Florida, Wimauma, FL, USA; (2) Gulf Coast Research and Education Center, University of Florida, Wimauma, FL, USA

Pomegranate (Punica granatum L.) has emerged as an important alternative crop in Florida. However, anthracnose fruit rot and leaf spot, caused by Colletotrichum spp., can cause high yield losses. The efficacy of biopesticides and systemic and contact fungicides was tested against leaf spot and fruit rot on pomegranate. Replicated trials were performed in Plant City, FL (var. 'Angel Red') and Parrish, FL (vars. 'Christina' and 'Wonderful'). Treatments included the biopesticides Serenade (Bacillus subtilis) and Tenet WP (Trichoderma spp.), the systemic fungicides Merivon (fluxapyroxad + pyraclostrobin) and Topsin (thiophanate-methyl), and contact fungicides Penncozeb (mancozeb) and Captan, plus a non-treated control. Based on AUDPC, all contact and systemic fungicides statistically reduced leaf spot severity compared to the non-treated control across the two trial sites. However, the relative efficacy of the biopesticide treatments differed at the two sites. In Plant City, all fungicide treatments statistically reduced fruit infection compared to the non-treated control; whereas in Parrish, only the systemic fungicides statistically reduced fruit infection. These results demonstrate that contact and systemic fungicides were more effective than the biopesticides in reducing foliar leaf spot and fruit rot. However, an integrated disease management program including cultural practices must be considered for commercial production of pomegranate in Florida.

Evaluation of copper alternatives and enhancers for managing almond bacterial spot caused by Xanthomonas arboricola pv. pruni in California S. E. HAACK (1), H. C. Förster (1), J. E. Adaskaveg (2), (1) Department of Plant Pathology and Microbiology, University of California, Riverside, CA, USA; (2) Department of Microbiology and Plant Pathology, University of California, Riverside, CA, USA

Bacterial spot of almond, caused by Xanthomonas arboricola pv. pruni, is a new and important disease in California, especially on cv. 'Fritz'. In the spring, gumming mesocarp lesions may cause fruit drop and later penetrate into the endocarp, lowering crop quality and reducing yield. Copper compounds are registered and used prior to late petal fall, but effective rates can cause phytotoxicity on leaves. With additional rainfall, supplemental treatments may be required following leaf emergence and thus, new rotational bactericides or copper activity enhancers are needed. Strains $(\mathrm{n}=72)$ from six counties were all copper-sensitive, with no reduction in growth at $10 \mu \mathrm{g} \mathrm{MCE} / \mathrm{ml}$, reduced growth at $20 \mu \mathrm{g} \mathrm{MCE} / \mathrm{ml}$, and no growth at $25 \mu \mathrm{g}$ $\mathrm{MCE} / \mathrm{ml}$. In-vitro mean minimum inhibitory concentrations (MICs) of mancozeb, mancozeb in the presence of copper (10 $\mu \mathrm{g} \mathrm{MCE} / \mathrm{ml})$, and oxytetracycline were $2.02,0.04$, and $0.07 \mu \mathrm{g} / \mathrm{ml}$, respectively. The experimental salicylaldehyde benzoylhydrazone (SBH) was not inhibitory at 80 $\mu \mathrm{g} / \mathrm{ml}$, but the mean MIC was $0.31 \mu \mathrm{g} / \mathrm{ml}$ in the presence of copper $(10 \mu \mathrm{g} \mathrm{MCE} / \mathrm{ml})$. In field studies, reduced copper rates mixed with mancozeb were highly effective against the disease without causing phytotoxicity. Oxytetracycline efficacy was moderate to low, possibly due to UV degradation. Adjuvants to improve oxytetracycline efficacy and testing of SBH in the field may provide new treatments in managing bacterial spot over an extended growing season.

Chemical treatments inhibiting germination of wheat rust, clover anthracnose, canola blackleg and rice blast spores

M. Barbetti (1,2), P. BARUA (1,2), K. L. Bayliss (3), M. You (1), V. Lanoiselet (4), (1) The University of Western Australia, School of Agriculture and Environment, Perth, AUSTRALIA; (2) Plant Biosecurity Cooperative Research Centre, ACT, AUSTRALIA; (3) Murdoch University, Murdoch, AUSTRALIA; (4) Department of Primary Industries \& Regional Development, Western Australia, Perth, AUSTRALIA

In vitro studies were undertaken to determine the effects of five chemical fungicide/disinfectant treatments [Tilt 250 EC (propiconazole), Amistar 250 SC (azoxystrobin), Sporekill, (didecyl dimethyl ammonium chloride), Farmcleanse (Alkali metal salts of alkylbenzene sulfonic acid and coconut diethanolamide) and Virkon S (potassium peroxymonosulfate)] in preventing spore germination of Puccinia graminis f. sp. tritici, Kabatiella caulivora, Leptosphaeria maculans and Magnaporthe oryzae. Germination was inhibited by all fungicides and disinfectants. Maximum reductions in spore germination were obtained at manufacturer's recommended concentration and concentrations above, while concentrations below were less effective than the recommended concentration. Overall, Amistar and Tilt were the most effective of the chemicals tested, reducing germination of $M$. oryzae $L$. maculans and $P$. graminis f. sp. tritici spores by $>75 \%$. However, the extent to which germination of fungal spores was inhibited was dependent on the pathogen. Sporekill was the least effective, inhibiting spore germination across all the pathogens by $<15 \%$. Additional studies undertaken to define the effectiveness of the same fungicides/disinfectants for the same pathogens inoculated on five common carrier materials, metal, fabric, wood, paper, and rubber, also showed Amistar and Tilt the most effective. Results highlight a necessity for re-evaluating the requirement for decontamination procedures for carrier materials that have perhaps long been mistakenly considered of low biosecurity risk in regards to the movement of exotic fungal plant pathogens and their races.

Characterisation and management of Rhizoctonia associated with sunflower seedlings in South Africa

S. C. LAMPRECHT, T. Phasoana, C. Spies, Y. Tewoldemedhin, Agricultural Research Council-Plant Health and Protection, Stellenbosch, SOUTH AFRICA

Poor establishment is a major yield limiting factor in sunflower production in South Africa. Surveys conducted during 2014 and 2015 showed that many potential fungal soilborne pathogens are associated with seedlings. Rhizoctonia species and anastomosis groups (AGs) were among these potential pathogens. Eighty-four Rhizoctonia isolates were obtained and identified by sequencing the internal transcribed spacer (ITS) regions. Of these, 37 isolates were multinucleate and 47 binucleate. The multinucleate AGs included 2-2LP (4.8\%), 3TB (1.2\%), 4-HGI (9.5\%), 4-HGIII (2.4\%), $R$. zeae (9.5\%), and an unidentified multinucleate AG (SB-3, 16.7\%). The binucleate AGs included A (19.0\%), F (7.1\%), K (7.1\%), Q (3.6\%) and four unidentified binucleate AGs (SB-1, 7.1\%; SB-2, 3.6\%; SB-4, 4.8\% and UNR-1, 3.6\%). Glasshouse trials showed that AGs 2-2LP, 4-HGI, 4-HGIII and SB-3 significantly reduced seedling survival to $53.4,2.8,88.3$ and $7.9 \%$ respectively compared to $96.7 \%$ survival for the control. Seed treatment with a compound with active ingredients fludioxonil, mefenoxam and thiamethoxam significantly improved survival to $87.5,39.7,92.9$ and $88.5 \%$ for AGs 2 2LP, 4-HGI, 4-HGIII and SB-3 respectively, compared to $95.8 \%$ for the control. The binucleate AGs did not cause a significant reduction in survival. Findings indicate that Rhizoctonia spp. may contribute to poor establishment of sunflower, and seed treatment can significantly reduce the impact of these pathogens. 
Effect of post-application irrigation and the usage of soil surfactants on fungicide movement and efficacy W. J. HUTCHENS (1), J. P. Kerns (2), T. Gannon (1), (1) North Carolina State University, Raleigh, NC, USA; (2) Department of Entomology and Plant Pathology, North Carolina State University, Raleigh, NC, USA

Soilborne plant pathogens are challenging to control in turfgrass systems due to the low solubility of many fungicides and their high affinity to bind to organic matter. The most effective approach to deliver fungicides into the root system is through the use of soil surfactants, high application volumes, and post-application irrigation. The effect of post-application irrigation on fungicide movement in soil has not been well characterized. A laboratory experiment was conducted to determine how various post-application irrigation amounts $(0,0.3175,0.635,1.27$, and $2.54 \mathrm{~cm})$ both with and without the use of the soil surfactant polyoxyalkylene polymers $\left(25.47 \mathrm{~L} \mathrm{ha}^{-1}\right)$ affect movement of the fungicide myclobutanil. A growth chamber experiment was also conducted to determine the influence of post-application irrigation amounts on efficacy of propiconazole for managing summer patch disease of 'Penn A-4' creeping bentgrass caused by Magnaporthe poae. In the laboratory experiment, regardless of irrigation treatment, at least $76.5 \%$ of the recovered fungicide remained in the top $5.08 \mathrm{~cm}$ of soil when applied without a soil surfactant. The only treatments to have significant (>5\%) fungicide residues below $5.08 \mathrm{~cm}$ were 1.27 and $2.54 \mathrm{~cm}$ of irrigation. Post-application irrigation of $2.54 \mathrm{~cm}$ coupled with the soil surfactant resulted in the greatest fungicide movement. In the growth chamber experiment, post-application irrigation with $0.635 \mathrm{~cm}$ of water resulted in increased turf quality and root length compared to the other three treatments. These data support the importance of post-application irrigation when applying fungicides in turfgrass systems to control soilborne pathogens.

Agronomic and economic evaluation of fungicide seed treatments for soybean production in the Mid-Atlantic United States A. KNESS (1), N. M. Kleczewski (2), (1) University of Maryland Extension, Forest Hill, MD, USA; (2) University of Illinois, Urbana, IL, USA

Soybean (Glycine max) is a major agricultural commodity in the Mid-Atlantic region. Although seedling pathogens such as Fusarium spp., Pythium spp. and Rhizoctonia solani occur sporadically in the region, some growers routinely utilize fungicide seed treatments. Reasons for this include perceived plant health benefits and insuring disease management. To address whether commonly used seed treatments provide benefits in the absence of disease, field trials were established in 2017 at four locations (Georgetown, DE; Queenstown, MD; Beltsville, MD; and Keedysville, MD) lacking history of seedling diseases. Treatments included metalaxyl + fluxapryoxad + pyraclostrobin; fluopyram; trifloxystrobin; and an untreated control applied at commercial rates to the variety SS4514NR2 by a local seed dealer. Treatments were replicated five times per site and arranged in a spatially balanced, random complete block design. Emergence 14 days post planting and yield were recorded, and data analyzed using a mixed model. Treatment effects were separated using Fisher's LSD. Seed treatments marginally increased emergence $(\mathrm{P}=0.06)$ but did not significantly increase yield and resulted in a 3 $14 \%$ yield decrease when compared to the controls in Georgetown $(\mathrm{P}=0.04)$ and Queenstown $(\mathrm{P}=0.02)$. Economic analysis indicated that seed treatments reduced net income by $\$ 337$ hectare $^{-1}$. This study will be repeated in the future to better understand the impacts of seed treatments in disease-free situations.

Xylella fastidiosa virulence in planta with copper supplementation

Q. GE (1), P. Cobine (1), L. De La Fuente (2), (1) Auburn University, Auburn, AL, USA; (2) Department of Entomology and Plant Pathology, Auburn University, Auburn, AL, USA

Xylella fastidiosa $(\mathrm{Xf})$ is a gram negative, xylem-limited plant pathogenic bacteria that causes disease in many economically important crops worldwide. Copper is a widely-used antimicrobial agent on $\mathrm{Xf}$ hosts. While the effects of copper have been extensively studied for foliar pathogen control, it is unknown what affect it has on a xylem-colonized pathogen. Previous results from our group showed that concentrations of $\mathrm{CuSO}_{4}$ between 5-200 $\mu \mathrm{M}$ increase biofilm formation in vitro, while high concentrations $(>200 \mu \mathrm{M})$ of $\mathrm{CuSO}_{4}$ inhibited biofilm formation. In this study, we focused on in planta experiments to unveil the influence of copper in Xf-caused diseases using tobacco as a model for infection. Xf-infected and non-infected plants were watered with tap water, and water supplemented with $4 \mathrm{mM}$ and $8 \mathrm{mM} \mathrm{CuSO}_{4}$. Symptom progression was observed, and sap and leaf ionome analysis was performed by inductively coupled plasm optical emission spectroscopy. Uptake of $\mathrm{Cu}$ was confirmed by increased concentrations of $\mathrm{Cu}$ in sap of plants treated with $\mathrm{CuSO}_{4}$-amended water. In independent experiments sap copper concentrations for $4 \mathrm{mM}$ supplementation ranged from 10-50 $\mu \mathrm{M}$, while 8 $\mathrm{mM}$ supplementation resulted in 50-100 $\mu \mathrm{M} \mathrm{Cu}$. In vitro this concentration range resulted in enhanced biofilm formation. The symptoms of leaf scorch in the $\mathrm{Cu}$-supplemented plants showed a trend towards more severe at later timepoints. Based on the results, we proposed that the plant copper homeostasis machinery controls the level of copper in xylem preventing it from becoming elevated to a level that that would lead to bacterial inhibition. Further study will focus on how bacteria population and colonization in planta is influenced by copper.

\section{Refinement of peach cover spray programs for sustainable management of brown rot} N. LALANCETTE, L. Blaus, Rutgers University, Bridgeton, NJ, USA

Brown rot is typically controlled with site-specific fungicides applied during the preharvest period. This practice has resulted in development of fungicide resistant Monilinia fructicola, the causal agent of brown rot. However, recent research has shown that the protectant fungicide captan applied during the prior cover spray period can contribute 50 to $69 \%$ control of rot at harvest, thereby reducing the size of the $M$. fructicola population exposed to the site-specific materials. Thus, the objectives of this study were to determine the minimum rate and optimum timing of captan cover sprays that still contribute effectively to brown rot control. Results showed that control with captan was rate dependent, with 51, 69 , and $78 \%$ control observed at 2.24 , 2.80 , and $3.36 \mathrm{~kg} / \mathrm{ha}$, respectively. Treatment timings indicated that the final two cover sprays yielded control equivalent to the full season seven-spray program. Estimation of fungicide levels on fruit, using an in vivo bioassay, showed that residual activity was the primary mechanism for control. As hypothesized, mid-season cover sprays were ineffective. However, $40 \%$ control exerted by the three earliest cover sprays indicated that significant quiescent infections may be occurring on green fruit. These findings show that multi-site protectant fungicides applied during late cover sprays can contribute significantly to brown rot management. Additional data are needed on the significance of quiescent infections.

The effect of plant resistance inducing products on fire blight (Erwinia amylovora) infection and severity on apple B. L. LEHMAN (1), K. A. Peter (2), (1) Penn State Fruit Research and Extension Center, Biglerville, PA, USA; (2) Penn State University, Biglerville, PA, USA

Fire blight is a well-known destructive bacterial disease of pome fruit that can cause significant annual production losses and tree death. Management regimes consist of streptomycin applications at bloom to control blossom blight. In some years, even when blossom blight is controlled, outbreaks of shoot blight can occur and cause major fire blight epidemics, especially in newly planted high density plantings. Experiments were conducted at the Penn State Fruit Research and Extension Center in a greenhouse on one year-old 'Gala' trees grafted onto M.26 rootstocks and a field trial on ten year-old 'Gala' trees grafted onto B.9 rootstocks to test the ability of several products to activate a plant's systemic acquired resistance (SAR) and reduce shoot blight severity. Products tested included Bacillus strains, copper products, plant-based products, and glycerol, as well as Actigard (a registered SAR 
product), which was used as a SAR standard for comparison. While none of the products provided the level of control demonstrated by Actigard, treatments using Cueva, Vacciplant, and Regalia showed a significant reduction in severity over the control in either the greenhouse or field trials. In addition, the application of glycerol and some Bacillus products also resulted in a slight reduction in severity. This study identifies some products that may be incorporated into a shoot blight management regime for young and newly planted orchards during bloom and post-bloom.

\section{Zinc nanoparticles for potential control of Huanglongbing on citrus}

M. M. MURATA (1), S. Santra (2), E. G. Johnson (1), (1) University of Florida, Lake Alfred, FL, USA; (2) NanoScience Technology Center, Department of Chemistry, University of Central Florida, Orlando, FL, USA

Huanglongbing (HLB), caused by the phloem-limited fastidious bacterium Candidatus Liberibacter asiaticus (CLas), has caused tremendous economic losses for citrus industries worldwide. It is considered the most destructive disease in citrus and no cure is available for growers. The development of affordable and sustainable chemicals for HLB management is essential for the future of citriculture. We aim to test the antimicrobial activity of the nanozinc oxide material, Zinkicide ${ }^{\mathrm{TM}}$, against CLas. This product is a plant nutrient-based nanoparticle with bactericidal activity that translocates systemically after spray or soil drench application. Preliminary HLB data from citrus canker field trials showed some efficacy, but efficacy correlated with application rate and interval. Application rate and timing needs optimization. Phytotoxicity was assessed in Sour orange seedlings and wilt symptoms were observed only at high Zinkicide ${ }^{\mathrm{TM}}$ concentration (above 3,000 ppm). Three-year old grafted inoculated CLas-positive Hamlin sweet orange plants were treated with Zinkicide ${ }^{\mathrm{TM}}$ at 1,600 ppm every 7, 14, and 28 days under greenhouse conditions. Antimicrobial activity against CLas was evaluated by transcriptional activity in leaves. Zinkicide ${ }^{\mathrm{TM}}$ spray and drench applied weekly or biweekly significantly reduced Clas activity. These results showed that Zinkicide nanoparticles are a promising tool against HLB and that the current field trial application schedules need to be modified.

\section{Management of bitter rot of apple under optimal conditions for disease development in Georgia orchards} P. M. BRANNEN, University of Georgia, Athens, GA, USA

Bitter rot of apple, caused by members of the Colletotrichum acutatum and C. gloeosporioides species complex, is a major disease of apples in the southeastern U.S., and hot, humid/wet conditions in Georgia are generally conducive to disease development. As warmer conditions prevail, bitter rot has actually increased in importance, and management has become more difficult. New fungicides have been recently registered for management of bitter rot, but recommendations for utilization in spray programs were lacking. Over multiple years, Captan + ProPhyt (potassium phosphite) applications have provided significant management of bitter rot $(\mathrm{n}=3 ; 89.3 \%$ control), and this combination was utilized as the chemical standard for comparison of new fungicides and combinations of fungicides in multiple years of testing. Pristine (pyraclostrobin + boscalid), Merivon (fluxapyroxad + pyraclostrobin) and Luna Sensation (fluopyram + trifloxystrobin) consistently provided equivalent control to that of the standard, but Pristine + ProPhyt was not better than the standard in any trial. Under severe disease conditions, a three-way combination of Captan + ProPhyt combined with Manzate Prostick (mancozeb) provided better control than Captan + ProPhyt alone. Three-way combinations with Captan + ProPhyt combined with Merivon, Inspire Super (difenoconazole + cyprodinil), or Omega 500F (fluazinam) were not as consistent in their benefit over the standard.

Control of Citrus Huanglongbing (HLB) via Trunk Injection of Plant Activators and Antibiotics

J. HU (1), J. Jiang (2), N. Wang (3), (1) School of Plant Sciences, University of Arizona, Tucson, AZ, USA; (2) College of Agronomy Jiangxi Agricultural University, Nanchang, CHINA; (3) University of Florida, Lake Alfred, FL, USA

Citrus Huanglongbing (HLB) or greening is a devastating disease of citrus worldwide and no effective control measure is currently available. In this study, eight plant activators and three antibiotics were evaluated in 3 field trials for their effect to control HLB by trunk injection of young and mature sweet orange trees. Results showed that 4 trunk injections of several activators including salicylic acid, oxalic acid, acibenzolar-S-methyl and potassium phosphate provided significant control of HLB by suppressing Las titer and disease progress. Trunk injection of penicillin, streptomycin and oxytetracycline hydrochloride resulted in excellent control of HLB. In general, antibiotics were more effective in reduction of Las titer and HLB symptom expressions than plant activators. These treatments also resulted in increased yield and better fruit quality. In summary, this study presents information regarding controlling HLB via trunk injection of plant defense activators and antibiotics, which helps citrus growers in decision-making regarding developing an effective HLB management program.

Efficacy of various physiochemical seed treatments for controlling poppy downy mildew in Papaver seed lots T. THANGAVEL (1), J. B. Scott (2), S. J. Jones (3), R. Gugulothu (4), C. R. Wilson (5), (1) University of Tasmania, Newtown, Hobart, AUSTRALIA; (2) Tasmania Institute of Agriculture, Burnie, AUSTRALIA; (3) University of Tasmania, Burnie, AUSTRALIA; (4) University of Tasmania, Hobart, AUSTRALIA; (5) University of Tasmania, New Town, AUSTRALIA

Opium poppy (Papaver somniferum), is a commercially valuable crop, grown for the production of pharmaceutical alkaloids. The Australian poppy industry is the leading producer of legal opiates in the world. Downy mildew, caused by two oomycete species, Peronospora meconopsidis and Peronospora somniferi, is one of the most destructive diseases in commercial poppy growing regions, including Australia. To reduce downy mildew primary inoculum loads in commercial production, a series of physiochemical treatments of poppy seed were trialed. These included hypochlorite and electrolysis water solutions, heating via either steam or hot water, and microwave treatment of seed. Following treatment, seeds were tested for germination and emergence percentages. Downy mildew infestation rates of seed post-treatment were measured via quantitative PCR (qPCR) using species-specific primers. Treated seed was also sown into commercial potting mix to assess downy mildew transmission rates from seed to seedling with infection confirmed via qPCR. Optimal disease control was observed from treatment with electrolysis water (400 ppm for 5 min) which reduced $P$. somniferi infection incidence from 0.82 to $0.07 \%$ in 3 week old seedlings. Seed germination and emergence rates were also significantly improved by this treatment. Thus physiochemical treatments have the potential to reduce the seed transmission of downy mildew in poppy production seeds without detrimental effects on seed viability.

Control of Huanglongbing through penicillin $\mathbf{G}$ trunk injection

X. SUN (1), F. Aldeek (2), D. Jones (1), A. Jeyaprakash (1), G. S. Hodges (1), W. Hammack (2), J. M. Cook (2), (1) Division of Plant Industry, Florida Department of Agriculture and Consumer Services (FDACS), Gainesville, FL, USA; (2) Division of Food Safety, FDACS, Tallahassee, FL, USA

A 2-year field trial was set up to evaluate the effect of penicillin G injection into Huanglongbing (HLB) infected citrus trees on Candidatus Liberibacter asiaticus (Las) titers, tree health, yield, and bacterial community profiles from within foliage and rhizosphere. Penicillin $\mathrm{G}$ injected into citrus trunks was detected through a bioassay and found to be rapidly but unevenly transported into citrus leaves, fruits, and roots within 24 to 48 with its concentrations declining by 72 hours. With the presence of penicillin $\mathrm{G}$ at a lethal concentration ( $>0.1$ PPM) inside citrus tissues for only about seven days, Las titers in 
the leaves of the treated citrus trees were largely reduced. Whole genome sequencing data indicated that foliage-associated bacterial profiles did not change much and penicillin resistant genes were not detected in those bacteria in leaves where penicillin G was detected. Penicillin G was not detected in soils surrounding the roots, indicating that the antibiotic did not exit the roots. Preliminary measurements indicated increases in canopy density and fruit yield from trees treated with penicillin G. At time of harvest, penicillin $\mathrm{G}$ was not detected 132 days post-injection and total residue of its metabolites was less than $7 \mathrm{ppb}$ in Hamlin sweet orange fruit at 132 days and less than $0.5 \mathrm{ppb}$ in Valencia sweet orange fruit at 257-264 days post-injection using a LC/MS testing method. Through this trial, we believe that penicillin G treatment of HLB infected citrus through trunk injection could be utilized as an option for citrus growers in combating HLB in Florida.

Fungicide efficacy trials for the management of maize white spot in Paraná, Brazil A. CUSTODIO (1), L. Moreira (2), M. Nunes (1), N. Suhcoski (1), L. Borsato (1), R. Bianco (1), (1) Instituto Agronômico do Paraná, Londrina, BRAZIL; (2) Cooperativa Agropecuária Mourãoense, Campo Mourão, BRAZIL

In Brazil, maize white spot (MWS) caused by the bacterium Pantoea ananatis was initially described as caused by the fungus Phaeospharia maydis. This is one of the most important diseases for the second crop maize, reducing grain yield up to $60 \%$ in susceptible hybrids. Currently, there is evidence that some foliar fungicides of different modes of action (MoA) can also control this bacterial disease. In the present study, we report the field efficiency of fungicides for MWS control in maize hybrid and their impact on grain yield. Eight field trials were carried out in two distinct locations in Paraná, Brazil, during the growing seasons of 2016 and 2017. Ten commercial foliar fungicides were tested. The fungicide treatments showed differences for MWS severity and grain yield. Under less pressure of MWS, only six foliar fungicides were observed lower disease severity and higher grain yield. On the other hand, fungicidal mixtures containing pyraclostrobin + fluxapyroxad + epoxyconazole and trifloxystrobin + prothioconazole resulted in lower disease severity and higher yield under severe MWS pressure. The specifics MoA fungicides were among the most effective for MWS control and to improve maize yield over most of the trials. Additional experiments must be done in order to understand the obtained results. A working group has been organized to determine the field efficacy of fungicides against maize diseases.

TRIVAPRO $^{\circledR}$ fungicide controlled rust and leafspot diseases and increased yield in corn

T. L. HARP (1), J. F. Hadden (1), E. C. Tedford (1), S. Sajjala (2), (1) Syngenta Crop Protection, Greensboro, NC, USA; (2) Syngenta Crop Protection, Minnetonka, MN, USA

TRIVAPRO ${ }^{\circledR}$ was introduced by Syngenta Crop Protection in 2016 for use on corn, soybeans and cereals. The product contains three active ingredients (azoxystrobin, propiconazole and Solatenol ${ }^{\circledR}$ fungicides) each with a different mode of action that provides strong efficacy and long-lasting performance of rust and leafspot diseases in corn. In twenty-five large scale field trials in 2015 and 2016, TRIVAPRO ${ }^{\circledR}$ provided robust disease control and increased yield in corn, on average, by nearly $25 \mathrm{bu} / \mathrm{A}$ over the untreated plots. When evaluated across an additional twenty-two corn field trials in 2015 and 2016 comparing a single R1 application to Headline $\mathrm{AMP}^{\circledR}$ and Stratego ${ }^{\circledR}{ }^{\circledR}$ YD fungicides, TRIVAPRO ${ }^{\circledR}$ provided superior and longer-lasting disease control and an average of a $10 \mathrm{bu} / \mathrm{A}$ yield advantage over the other fungicide treatments. In all trials, TRIVAPRO ${ }^{\circledR}$ demonstrated an observable long-lasting greening and crop enhancement benefit across numerous corn hybrids and locations across the corn belt.

High levels of resistance to phosphonate fungicides in the hop downy mildew pathogen, Pseudoperonospora humuli D. H. GENT (1), B. Claassen (2), (1) US Department of Agriculture, Agricultural Research Service, Corvallis, OR, USA; (2) Oregon State University, Corvallis, OR, USA

Phosphonate fungicides are an important component of disease management programs for downy mildew on hop (caused by Pseudoperonospora humuli). In two fungicide trials conducted in commercial hop yards in Oregon in 2016, weekly applications of the highest labeled rate of a phosphorous acid fungicide provided no suppression of downy mildew at one location and approximately $50 \%$ disease suppression at the second location. These trials suggested potential resistance to phosphorous acid in the population of $P$. humuli. Isolates of the pathogen collected from a farm reporting disease control failures following use of phosphonate fungicides were assayed for sensitivity to phosphorous acid. The median concentration of phosphorous acid needed to reduce the incidence of infection by $50 \%$ was 1.9 times the maximum labeled rated of the fungicide FUNGI-PHITE. Further, there was a linear relationship between sensitivity to phosphorous acid and fosetyl-Al, indicating cross resistance between these compounds. Follow up field trials found that neither the highest labeled rates of a phosphorous acid product or fosetyl-Al significantly reduced downy mildew as compared to nontreated plots where resistant isolates were present, whereas fungicides with different modes of action reduced disease to varying levels. Further sampling is planned to determine how widespread resistance to phosphonate compounds may be in Oregon.

Mefentrifluconazole - a broad spectrum fungicide for use in turfgrass and ornamentals R. J. KEESE (1), J. S. Barnes (2), J. H. O’Barr (3), (1) BASF, Res Triangle Park, NC, USA; (2) BASF Corp, Res Triangle Park, NC, USA; (3) BASF Corp, Palmyra, PA, USA

A new active ingredient, mefentrifluconazole, is under development in the United States and Canada for the control of turfgrass and ornamental pathogens. Research from internal, university, and private cooperators has indicated that the active ingredient is highly effective in controlling diseases such as dollar spot (Sclerotinia homoeocarpa, brown patch (Rhizoctonia solani), anthracnose (Colletotrichum graminicola), summer patch (Magnaporthe poae) and brown ring patch (Rhizoctonia circinata var. circinata). The proposed classification by FRAC (Fungicide Resistance Action Committee) is Group 3 - demethylation inhibitor. Mefentrifluconazole is being investigated both as a solo product and in combination with pyraclostrobin. Research testing was conducted as RCBD field trials, and data were evaluated using ANOVA and Duncan's New MRT for means comparisons at a significance level of $\mathrm{p}=0.05$. For turfgrass, rates of mefentrifluconazole tested ranged from 250 to $1000 \mathrm{~g}$ ai/ha. In ornamentals, a solo product is in development and has provided control of leaf spots, powdery mildew, rust and scab, and soilborne diseases such as Cylindrocladium and Thielaviopsis. Foliar use rates tested in ornamental research trials ranged from 88 to $234 \mathrm{~g}$ ai/ha. Research results will be presented for turf and ornamentals. Mefentrifluconazole is being reviewed as a Reduced Risk candidate by the US EPA with registration expected in 2019.

Mefentrifluconazole - A new broad-spectrum fungicide for use on row and specialty crops S. L. WALKER, J. S. Barnes, T. R. Bardinelli, BASF Corp, Res Triangle Park, NC, USA

Mefentrifluconazole $\left(\right.$ Revysol $\left.^{\circledR}\right)$ is a new fungicide active ingredient under development in the United States and Canada by BASF Corporation for control of key fungal diseases of pome fruits, stone fruits, tree nuts, grapes, potato, corn, soybeans and other crops. The proposed classification by FRAC (Fungicide Resistance Action Committee) is a Group 3 demethylation inhibitor. Research in internal, university, and private cooperator trials has indicated Revysol is highly effective at controlling important diseases such as apple scab (Venturia inaequalis), blossom blight and brown rot of stone 
fruits and almonds (Monilinia spp.); powdery mildew of grapes (Erysiphe necator), Alternaria diseases of potato and tree nuts (Alternaria spp.), and Cercospora diseases of corn and soybean (Cercospora spp.). Trial data indicate Revysol was effective controlling these diseases and others at a rate range of $73-146 \mathrm{~g}$ ai/HA. Testing of Revysol was conducted as RCBD field trials in multiple locations tested over several years. Data were evaluated using ANOVA and Duncan's New MRT for means comparisons at a significance level of $\mathrm{p}=0.05$. Trial results will be presented. Revysol is being reviewed by the EPA as a Reduced Risk candidate. Registration is expected in 2019.

Potency of difenoconazole against nine postharvest pathogens of pome fruit A. AMIRI, L. K. Pandit, K. Reiser, Washington State University, Wenatchee, WA, USA

Difenoconazole (DFC) was registered as a postharvest fungicide pre-mixed with fludioxonil in 2016. Herein, we report on the efficacy of DFC against Penicillium expansum (Pe), Botrytis cinerea (Bc), Neofabraea perennans (Np), Alternaria alternata (Aa), Mucor piriformis (Mp), Lambertella cornimaris (LCM), Sphaeropsis pyriputrescens (Sp), Phacidiopycnis washingtonensis (Pwa) on apple and Phacidiopycnis pyri (Pp) on pear. Non-wounded Fuji apples and d 'Anjou pears were inoculated with spore suspensions at $5 \times 10^{4}$ spores $/ \mathrm{ml}$ at an experimental orchard prior to harvest for all pathogens except Pe, Mp, and LCM, which were inoculated post-harvest on wounded fruit. Orchard-inoculated fruit were harvested 10 days post-inoculation and treated by dipping for $1 \mathrm{~min}$ in a DFC (Thesis) suspension at $0.3 \mathrm{~g} /$ liter, whereas wounded fruit used for Pe, Mp, and LCM were treated preventively with DFC and inoculated $4 \mathrm{~h}$ post-treatment. Four replicates of 20 -fruit each was used for each pathogen and fruit were stored for 6 months at $1{ }^{\circ} \mathrm{C}$ in a regular atmosphere to determine disease incidence and severity. DFC applied curatively controlled Pwa, Pp, Sp and Aa effectively after 6 months of storage whereas a moderate efficacy was observed for Bc and Np. Preventive DFC application provided a high efficacy against Pe and LCM but not Mp. The high DFC potency against most postharvest pome fruit pathogens observed indicates the fungicide would be an effective tool in future management programs.

Adepidyn ${ }^{\circledR}$ a new fungicide for Fusarium Head Blight and foliar disease control in wheat

N. GLYNN PHD (1), E. C. Tedford (2), T. L. Harp (2), (1) Syngenta, Vero Beach, FL, USA; (2) Syngenta Crop Protection, Greensboro, NC, USA

Fusarium Head Blight (FHB) is a threat to wheat production in many parts of the world. Management of FHB has been primarily achieved through a limited number of De-Methylation Inhibitor (DMI) triazole fungicides. However, robust control under field conditions can be challenging with DMI's because they require a narrow window of application (BBCH 65) and represent only a single mode of action, which can result in high selection pressure on the pathogen and reduced efficacy upon repeated use. Adepidyn ${ }^{\circledR}$ fungicide is a carboximide succinate dehydrogenase inhibitor (SDHI) under development from Syngenta and the only carboximide SDHI to demonstrate high potency against diseases caused by Fusarium. In field trials conducted across North America, Adepidyn ${ }^{\circledR}$ fungicide was effective against FHB when applied from early ear emergence through flowering (BBCH 55 to BBCH 65), offering a wider application window than current DMI fungicides. Furthermore, it provides an alternative mode of action for controlling the disease, providing cereal growers a new tool to manage FHB and enhancing the sustainability of current DMI fungicides. In field trials, the performance of Adepidyn ${ }^{\circledR}$ fungicide matched or exceeded the efficacy observed by DMI fungicides, enhanced the duration of control and helped to reduce mycotoxin levels. Adepidyn ${ }^{\circledR}$ fungicide also has activity towards important leafspot diseases in cereals including Septoria sp., Pyrenophora sp. and powdery mildews. The wide spectrum of activity, extended window of application combined with its high potency, alternate mode of action and long residual control will make Adepidyn ${ }^{\circledR}$ fungicide a useful tool for FHB and foliar disease management in cereals upon registration.

Evaluation of novel Zinc-based antimicrobial formulations to control growth and biofilm formation of Xanthomonas citri and Liberibacter crescens

H. MENDIS (1), S. Santra (2), M. Young (3), P. Rajasekaran (3), E. G. Johnson (4), L. De La Fuente (5), (1) Auburn University, Auburn, AL, USA; (2) NanoScience Technology Center, Department of Chemistry, University of Central Florida, Orlando, FL, USA; (3) University of Central Florida, Orlando, FL, USA; (4) University of Florida, Lake Alfred, FL, USA; (5) Department of Entomology and Plant Pathology, Auburn University, Auburn, AL, USA

Zinkicide ${ }^{\mathrm{TM}}$ and TSOL are Zn-based antimicrobial formulations developed for treating citrus bacterial pathogens 'Candidatus Liberibacter asiaticus' and Xanthomonas citri. This study investigated the effects of Zinkicide ${ }^{\mathrm{TM}}$ and TSOL on growth and biofilm formation of ' $\mathrm{Ca}$. L. asiaticus' surrogates $X$. citri and Liberibacter crescens in batch cultures and under flow conditions using microfluidic chambers (MC). X. citri and L. crescens were grown in SB and BM7 media, respectively, and treated with serial dilutions of Zinkicide ${ }^{\mathrm{TM}}$ and TSOL to test inhibition of growth and biofilm formation. $X$. citri and $L$. crescens were also introduced to MC and inhibitory action of Zinkicide ${ }^{\mathrm{TM}}$ and TSOL on biofilm formation was recorded using time-lapse video imaging microscopy. The minimum inhibitory concentration (MIC) of Zinkicide ${ }^{\mathrm{TM}}$ and TSOL for X. citri was $35 \mathrm{ppm}$ and 40ppm, respectively. MIC of TSOL for L. crescens was $40 \mathrm{ppm}$. Both Zinkicide ${ }^{\mathrm{TM}}$ and TSOL have bactericidal effect on $X$. citri above $60 \mathrm{ppm}$ and $150 \mathrm{ppm}$ respectively. Time-lapse video imaging showed that untreated $X$. citri grown in MC formed biofilm whereas $X$. citri treated with $30 \mathrm{ppm}$ Zinkicide ${ }^{\mathrm{TM}}$ did not form any biofilm. Furthermore, Zinkicide ${ }^{\mathrm{TM}}$ and TSOL inhibited further growth of already formed $X$. citri biofilm at $30 \mathrm{ppm}$ and $60 \mathrm{ppm}$, respectively, in MC. Results confirmed that Zinkicide ${ }^{\mathrm{TM}}$ and TSOL are effective against $X$. citri and $L$. crescens in vitro and inhibit growth and biofilm formation under flow conditions.

\section{Adepidyn ${ }^{\circledR}$, a new fungicide for the control of gummy stem blight in conventional cucurbit production} C. COLLAZO-GONZALEZ, Syngenta Crop Protection, Vero Beach, FL, USA

Adepidyn ${ }^{\circledR}$ is a new fungicide under development from Syngenta under the carboximide succinate dehydrogenase inhibitor (SDHI) mode of action. Adepidyn $^{\circledR}$ fungicide has proven effective in the control of gummy stem blight (GSB) caused by the pathogen Stagonosporopsis cucurbitacearum. GSB is one of the most common and devastating foliar disease of all members in the cucurbitaceae. Stagonosporopsis cucurbitacearum attacks the leaves, stems, and fruit of cucurbits, limiting yields and production of high quality fruit. Once established, the disease is challenging to control under conducive environmental conditions. Conventional farming operations rely on chemical management to attain high yields and quality fruit. In multiple field trials across the South East USA, Adepidyn ${ }^{\circledR}$ fungicide has stood out as an effective control measure of GSB when applied preventively in a foliar spray program. Adepidyn ${ }^{\circledR}$ fungicide has a long residual activity and can offer control to other common diseases of cucurbits like, powdery mildew. Efficacious control of GSB by Adepidyn ${ }^{\circledR}$ fungicide coupled with its extended residual activity, will provide growers with an alternative and reliable mean to control GSB, once the fungicide has received regulatory approvals

Effects of pyriofenone on the infection of wheat plants by Blumeria graminis $f$. sp. tritici

N. Akihiro (1), O. Munekazu (2), S. MITANI (1), (1) Ishihara Sangyo Kaisha, Ltd., Kusatsu, JAPAN; (2) Ishihara Sangyo Kaisha, Ltd., Osaka, JAPAN 
Pyriofenone (Property, Kusabi, Prolivo) is a new fungicide discovered and developed by Ishihara Sangyo Kaisha, Ltd. It is highly active against a broad spectrum of powdery mildew diseases including wheat powdery mildew at $90 \mathrm{~g}$ a.i./ha. The purpose of this study was to understand the biological mode of action of pyriofenone against Blumeria graminis f. sp. tritici on wheat plants. Wheat seedlings in polyethylene pots were sprayed with test solutions before or after inoculation with $B$. graminis. At 2 to 8 days after inoculation, leaf segments were observed with a light microscope. Pyriofenone inhibited all stages of the infection process (including appressorium formation, haustorium formation, mycelial growth, and conidium formation) except for conidial germination; however, it exhibited a greater effect on haustorium formation than on appressorium formation. In addition to its strong preventative effects, pyriofenone exhibited strong curative and inhibitory effects on conidium development. These data indicate that pyriofenone can help suppress powdery mildew epidemics and confer a high level of field control over fungal infestations.

Evaluation of fungicide timing for management of Phoma black stem of sunflowers

M. GILLEY (1), J. Halvorson (1), B. Berghuis (1), S. Fitterer (2), D. Carruth (2), B. Hansen (1), F. M. Mathew (3), S. G. Markell (1), (1) North Dakota State University, Fargo, ND, USA; (2) BASF ND Research Farm, Davenport, ND, USA; (3) South Dakota State University, Brookings, SD, USA

Phoma black stem, caused by Phoma macdonaldii, was identified in $80 \%$ of North American sunflower fields surveyed in 2015. Preliminary data suggested that FRAC 11 fungicides may reduce disease severity, but the economic return and timing of fungicide application was unclear. The objective of this study was to evaluate the timing of pyraclostrobin application on two oil type sunflower hybrids under natural infection. In May 2017, two adjacent fungicide trials were established in North Dakota. Both trials were planted in four-row plots and arranged in a randomized complete block design with four replicates. Fungicide was applied to the middle two rows of plots singly and in combination at V8, R1 and R4. A disease severity index was calculated by multiplying incidence (number of stems infected) and severity (mean number of stem lesions) on ten arbitrarily selected plants in each plot. Infection developed in both trials with $100 \%$ incidence in the non-treated control (NTC) plots. All fungicide timing combinations that included an application at R1, as well as a double application at V8 and R4, significantly $(\mathrm{P} \leq 0.05)$ reduced disease severity for both hybrids. Yield was significantly $(\mathrm{P} \leq 0.05)$ higher than the NTC for all fungicide treatments except V8 and R4 alone in one of the trials; whereas, no significant yield differences were observed for the other trial. Under high disease pressure, fungicide applied prior to flowering may improve yield on some hybrids.

Sensitivity to DMI, SDHI and phenylpyrrole fungicides of Helminthosporium solani causing silver scurf on potato in the US N. ROSENZWEIG (1), G. Olaya (2), K. Frost (3), A. J. Gevens (4), N. O. Nelson (5), G. L. Steere (1), P. S. Wharton (6), P. Samohano (1), (1) Michigan State University, East Lansing, MI, USA; (2) Syngenta Crop Protection, Vero Beach, FL, USA; (3) Oregon State University, Hermiston Agricultural Research \& Extension Center, Hermiston, OR, USA; (4) University of Wisconsin-Madison, Madison, WI, USA; (5) University of Idaho, Twin Falls, ID, USA; (6) University of Idaho, Aberdeen Research and Extension Center, Aberdeen, ID, USA

Silver scurf (SS) caused by Helminthosporium solani is a persistent problem for potato production and is of particular importance in temperate regions. The disease is common in potato seed tubers and can become an issue in storage and negatively impact marketability of ware potatoes that are stored for longer periods of time. Therefore, effective fungicides for management of the disease are essential. The objectives of this monitoring program were to: 1 . collect SS-infected potato tubers from commercial storages across US production regions; and 2. determine the sensitivity of the populations of $H$. solani. to fungicides in three classes, including: DMI fungicides [difenoconazole (DFZ)]; SDHI fungicides [solatenol, (STL), sedaxane (SDX)]; and phenylpyrrole fungicides [fludioxonil (FDL)]. Tubers with symptoms of SS were sampled from potato storages in Idaho, Michigan, Oregon and Wisconsin. Mono-conidial isolates were obtained to determine sensitivity to each fungicide above. A spiral gradient dilution method was used to estimate the fungicide concentration which caused a $50 \%$ inhibition of fungal growth $\left(\mathrm{EC}_{50}\right)$ in vitro for all isolates. A total of $26,33,33$, and 34 isolates were screened against DFZ, STL, SDX, and FDL respectively and the mean $\mathrm{EC}_{50}$ values were 13.9 (min: 0.1, max: 77.6 ), 9.5 (min: 0.10, max: 105.0), 9.7 (min: 0.10, max: 65.9) and 45.8 (min: 0.1, max: 105.0) for DFZ, STL, SDX, and FDL respectively. The sensitivity response to the two SDHI fungicides SDX and STL, revealed cross sensitivity among isolates.

Miravis $^{\circledR}$ : A new fungicide for control of Fusarium wilt in cucurbits

J. RAPICAVOLI (1), K. R. Whitten Buxton (2), J. F. Hadden (3), (1) Syngenta Crop Protection, Rancho Mission Viejo, CA, USA; (2) Syngenta Crop Protection, Vero Beach, FL, USA; (3) Syngenta Crop Protection, Greensboro, NC, USA

Members of the plant family Cucurbitaceae are affected by several special forms of the destructive vascular wilt pathogen Fusarium oxysporum. While the use of resistant cultivars remains one of the most economical methods for control, long-term survival of chlamydospores in the soil and the evolution of new races makes management of Fusarium wilt extremely difficult. Under development by Syngenta Crop Protection, Miravis ${ }^{\circledR}$ is a new fungicide for control of Fusarium wilt in cucurbits. Miravis ${ }^{\circledR}$ contains the active ingredient Adepidyn ${ }^{\circledR}$ fungicide (pydiflumetofen), a novel, potent a.i. in the new Nmethoxy-(phenyl-ethyl) pyrazole carboxamide group of the carboxamide chemical class. Soil applications of Miravis ${ }^{\circledR}$ demonstrated exceptional control of Fusarium wilt compared to chemical standards. Field trials conducted across the Southern United States over the past several years have consistently demonstrated both a reduction in disease incidence and crop enhancement benefits leading to higher yields.

Secondary effects of pyriofenone against several important plant pathogens

O. Munekazu (1), N. Akihiro (2), Y. Nonaka (2), H. Hayashi (2), Y. ABE (2), S. Mitani (2), (1) Ishihara Sangyo Kaisha, Ltd., Osaka, JAPAN; (2) Ishihara Sangyo Kaisha, Ltd., Kusatsu, JAPAN

Pyriofenone (Property, Kusabi, Prolivo) is a new fungicide developed by Ishihara Sangyo Kaisha to control powdery mildew on various crops. This study investigated the secondary effects of pyriofenone on other pathogens. Antifungal activity testing revealed that pyriofenone strongly inhibited the mycelial growth of Rosellinia necatrix and had moderate effects on Botrytis cinerea, Magnaporthe oryzae, Pseudocercosporella herpotrichoides, and Helminthosporium sacchari. Pyriofenone also inhibited conidial formation by $B$. cinerea, conidial germination and appressorium formation by $M$. oryzae, and pycnidium formation by Zymoseptoria tritici in vitro. In planta, pyriofenone exhibited strong preventive and curative activity against white root rot on Japanese pear (Pyrus pyrifolia) and moderate activity against rice blast in pot tests. The control of gray mold with a single foliar spray of pyriofenone was limited, but mixture with other botryticides resulted in better control than with each separate fungicide alone, in both pot and field trials Although pyriofenone is used specifically to control powdery mildew, when mixed with fungicides from other classes it can also control several other important diseases.

The use of prohexadione calcium and a systemic acquired resistance inducer to manage fire blight without antibiotics A. WALLIS (1), M. W. Choi (2), K. D. Cox (2), (1) PPPMB Cornell University, Plattsburgh, NY, USA; (2) Cornell University, Geneva, NY, USA 
Fire blight, a bacterial disease of pome fruit caused by Erwinia amylovora, is one of the most important diseases affecting commercial apple production. It has become increasingly problematic with the widespread adoption of high density orchards and the warming climate. Antibiotics are the most effective management tool, but raise concern due to the potential for antibiotic resistance. Alternatives include systemic acquired resistance (SAR) inducers, which prime host defense responses, and prohexadione calcium $(\mathrm{PhCa})$, a plant growth regulator that inhibits internal invasion of the pathogen. We evaluated the SAR inducer Regalia and various PhCa programs compared to untreated controls and standard antibiotic programs for their effects on blossom and shoot blight, crop load, and shoot growth for two years. Antibiotics provided excellent control ( $<5 \%$ blossom \& shoot blight incidence) compared to control trees. Single applications of the SAR inducer and $\mathrm{PhCa}$ at pink provided good control alone $(<30 \%)$, but control improved when treated with copper prior to inoculation $(<10 \%)$. Tree growth and productivity were not affected by the end of the season. Multiple, low rate $\mathrm{PhCa}$ applications provided excellent shoot blight control, but prolonged programs slightly impeded shoot growth. Future work should further refine PhCa programs and evaluate effects on plant growth in the absence of E. amylovora.

Effective supplementation of silicon by root against stem canker of pitaya (Hylocereus polyrhizus)

S. N. MOHAMED SIDIQUE (1), R. H. Rosli (1), M. H. Mohd (2), X. Jin (3), (1) Universiti Malaysia Terengganu, Kuala Terengganu, MALAYSIA; (2) Universiti Sains Malaysia, Penang, MALAYSIA; (3) National Sun Yat-Sen University, Kaohsiung, TAIWAN

In Malaysia, red-fleshed pitaya fruit (Hylocereus polyrhizus) is a high commercial value plant but prone to disease infections especially stem canker that causing plant breakdown after 3 years of growing. Therefore, our work to determine the effect of silicon nutrient (Si) application on pitaya plant growth and under biotic stress (stem canker disease) caused by Neoscytalidium dimidiatum. Silicon nutrient was applied once a week as root applications at the concentration of $1.5 \% \mathrm{Si}(\mathrm{v} / \mathrm{v}), 2.5 \% \mathrm{Si}(\mathrm{v} / \mathrm{v})$ and $5 \% \mathrm{Si}(\mathrm{v} / \mathrm{v})$, which was using the grower's conventional fertilizer applications and naturally infected. The control plants were untreated without any Si nutrient. There is a potential role of Si in delaying the development of stem canker when we observed a reduction of severity of the stem canker compare to the untreated. Results showed that the disease severity was below $10 \%$ rotted stems of pitaya plants treated with both $1.5 \% \mathrm{Si}(\mathrm{v} / \mathrm{v})$ and $2.5 \% \mathrm{Si}(\mathrm{v} / \mathrm{v})$ whereas $10-50 \%$ rotted stems of pitaya plants treated with $5 \% \mathrm{Si}(\mathrm{v} / \mathrm{v})$. The delay of the epidemic development could give more time to manage disease outbreaks and reduce the amount of fungicides usage. Additionally, the application of Si through the roots produced significantly physiological changes in the plant including increased number of new shoots, longer shoot length and early flowering. Pitaya plants also demonstrate effect of stronger and thicker stems that may retard the germination of pathogen. Silicon uptake in pitaya offer a promising outlook to improve biotic stress tolerance in a sustainable manner.

Fungicide sensitivity of the Indian sub-clonal variants of the Phytophthora infestans 13 A2 lineage S. GUHA ROY (1), T. Dey (2), D. Cooke (3), (1) West Bengal State University, Kolkata, INDIA; (2) West Bengal State University, Barasat Kolkata, INDIA; (3) James Hutton Institute, Dundee, SCOTLAND

Phytophthora infestans is a highly adapted oomycete pathogen of potato and tomato, aggressive genotypes of which often migrate to a new geographical region causing huge crop losses. Large sub-clonal variations within the European 13_A2 (blue 13) lineage were recently identified from a severe late blight epidemic in Eastern India in 2014. The fungicide sensitivity of these GPS-tagged and characterised populations $(\mathrm{n}=59)$ was carried out to determine the baseline sensitivity against the active ingredients of the five commercial fungicides Curzate ${ }^{\circledR} \mathrm{M}-8$ (Cymoxanil 8\% + Mancozeb 64\%), Indofil M- 45 (Mancozeb 75\%), Acrobat ${ }^{\circledR}$ (Dimethomorph 50\%), Amistar ${ }^{\circledR}$ (Azoxystrobin 23\%) and Aliette ${ }^{\circledR}$ (Fostyl- al 80\%). The fungicide sensitivity data was examined in relation to the 24 Multi Locus Genotypes (MLGs) derived from our previous studies and host (potato and tomato). Dose response and $\mathrm{EC}_{50}$ values were calculated for each isolate using an in vitro agar growth assay with 5-6 doses. No insensitivity was observed for any fungicide as the $\mathrm{EC}_{50}$ values were within the range of prescribed field doses for each fungicide. Among these fungicides, Amistar ${ }^{\mathbb{B}}$ has the lowest $\mathrm{EC}_{50} \mathrm{value}<0.1$ $\mathrm{ug} / \mathrm{ml}$ in all isolates. Minor variation in relation to host and MLG was observed and will be related to prior management practices. This baseline sensitivity information for this region will help growers manage their use of fungicides for effective control of this exotic pathogen population.

Use of fungicides to enhance grain yield and reduce disease levels in cultivated wild rice C. Castell-Miller (1), D. A. SAMAC (2), D. K. Malvick (3), (1) Univ of Minnesota, Saint Paul, MN, USA; (2) USDA-ARS, St Paul, MN, USA; (3) University of Minnesota, St. Paul, MN, USA

Attaining high grain yields in cultivated wild rice is frequently hampered by fungal diseases mostly caused by Bipolaris spp. In 2013, 2014, and 2015 grain yield reductions occurred, with losses as high as $33.5 \%$ compared to years of low disease pressure. An integrated disease management system has been implemented, but the limited disease resistance available in cultivars cannot cope with the disease pressure that often occurs and growers must rely on fungicides. The two most used fungicides contain azoxystrobin (targets fungal respiration) alone or in combination with propiconazole (disrupts the ergosterol biosynthesis pathway). The objective of this study was to evaluate the effectiveness of these fungicides to control foliar diseases in wild rice paddies. We conducted on farm experiments in 2016 and 2017 in four Minnesota locations. Fungicides were used in single or combined applications, with an untreated control under natural fungal infection. Percent diseased leaf area was determined in upper leaves in each treatment. Application of fungicides enhanced grain yield and decreased the level of foliar diseases in the upper leaves, although statistically significant differences $(P<0.05)$ among treatments for both variables were not observed in all the years and locations. Disease severity and grain yield were negatively correlated. Responsible use of fungicides can reduce foliar diseases and enhance grain yield in cultivated wild rice.

Evaluation of fungicides for control of Ceratocystis fimbriata and Rhizopus stolonifer on sweetpotato H. COLLINS, L. M. Quesada, North Carolina State University, Raleigh, NC, USA

North Carolina is the number one sweetpotato producer in the United States, over 90,000 acres are harvested every year in the state, and approximately $30 \%$ of the crop is exported. Ceratocystis fimbriata, the causal agent of black rot, and Rhizopus stolonifer, the causal agent for Rhizopus soft rot, are economically important postharvest pathogens of sweetpotato. However, few active ingredients are labeled for disease control in sweetpotato, even fewer products are allowed for use in roots for export, and little is known regarding the efficacy of products. To identify fungicides that reduce disease, sweetpotatoes were wounded and then inoculated with either $R$. stolonifer or $C$. fimbriata. Fungicide treatments were applied to roots as sprays or dips, roots were incubated at $24^{\circ} \mathrm{C}$ for 29 days for black rot and $26^{\circ} \mathrm{C}$ for 21 days for Rhizopus, and rated for disease incidence at 14,21 , and 29 days for black rot, and 7, 14, and 21 days for Rhizopus. Our results revealed that Mertect 340F as a dip or spray (thiabendazole), and sprays of Mentor (propiconazole), Orius 3.6F (tebuconazole), Chairman (fludioxonil + propiconazole), and Stadium (azoxystrobin + fludioxonil + difenoconazole) provided significant reductions in black rot incidence. While Chairman, Orius, and Stadium provided significant reductions in Rhizopus incidence. Our findings are a first step towards expanding the number of registered products for postharvest disease control in sweetpotato. 
Evaluation of nematicide seed treatments for control of root knot nematode (RKN) and soybean cyst nematode (SCN) in soybean in the midAtlantic U.S.

S. AHMED, L. Byrd-Masters, H. L. Mehl, Virginia Tech Tidewater AREC, Suffolk, VA, USA

Plant parasitic nematodes are a major constraint to soybean production, but few effective, economical nematicides are available for their control. The objective of this study was to evaluate nematicide seed treatments for control of root knot nematode (RKN) and soybean cyst nematode (SCN) in soybean production areas in the mid-Atlantic U.S. In 2017, trials were conducted in Virginia (Suffolk, Chesapeake) and North Carolina (Moyock). Avicta, Clariva, Poncho/VOTiVO, and ILeVO seed treatments were compared to aldicarb in-furrow and a no nematicide control. Nematode populations, plant stand, and yield were assessed. Suffolk and Chesapeake had moderate populations of SCN, and Moyock had high RKN. Nematicide treatments did not increase plant stand or yield compared to the control at any of the locations. At Suffolk, aldicarb and Poncho/VOTiVO treatments had 4.6 and 2.8 times less SCN than the control, respectively, but at Chesapeake, treatments did not reduce SCN. At Moyock, RKN and root galling did not vary significantly among treatments, but aldicarb and ILeVO had numerically lower RKN. Results indicate nematicide seed treatments may suppress nematode populations, but overall control and yield protection are minimal when nematode pressure is high. Additional studies are needed to quantify long-term effects of seed treatments on nematode populations and benefits of combining seed treatment and resistant soybean varieties for nematode management.

Comparing protection afforded by different organic alternatives to conventional fungicides for reducing scab on pecan C. H. BOCK (1), D. I. Shapiro-Ilan (2), B. Wilkins (3), D. Wells (4), L. Wells (5), J. H. Brock (5), M. W. Hotchkiss (6), R. F. Mizell (7), (1) USDA ARS, Southeastern Fruit and Tree Nut Research Laboratory, Byron, GA, USA; (2) USDA/ARS, Southeastern Fruit and Tree Nut Research Laboratory, Byron, GA, USA; (3) Auburn Univ, Fairhope, AL, USA; (4) Auburn University, Auburn, AL, USA; (5) University of Georgia, Tifton, GA, USA; (6) USDA ARS, Byron, GA, USA; (7) University of Florida/IFAS, Quincy, FL, USA

Pecan scab (Venturia effusa) is the major yield-limiting disease in the southeastern USA. Although conventional fungicides are available to manage the disease, there is no comparison of organic methods (organically produced nuts attract a higher price). In 2011, 2012, 2014, 2015 and 2016 trees of cv. Desirable were treated with Bordeaux mixture (BM), Compost tea, Sodium Bicarbonate, Serenade, Serenade + Kocide 2000, Sulfur, Nordox 75WG, or Regalia (R). Exact number of sprays depended on the year. Samples of fruit were assessed for severity of scab. Only in 2011, a dry year, did the sulfur treatment have more severe scab compared to any other treatment $(\mathrm{F}=2.9, \mathrm{P}=0.007)$. The control $(\mathrm{C})$, and the other treatments were not significantly different to each other. In all remaining years, the $\mathrm{C}$ had significantly more severe scab compared to some $(2012[\mathrm{~F}=11.6, \mathrm{P}<0.0001], 2014$ [F=14.6, $\mathrm{P}<0.0001]$ and $2015[\mathrm{~F}=14.6, \mathrm{P}<0.0001])$ or all other treatments $(2016[\mathrm{~F}=15.5, \mathrm{P}<0.0001])$. Trees treated with $\mathrm{R}$ had less severe scab compared to the $\mathrm{C}$ $(2012[\mathrm{C}=9.7 \%, \mathrm{R}=2.81 \%], 2014[\mathrm{C}=70.0 \%, \mathrm{R}=24.8 \%], 2015[\mathrm{C}=22.1 \%, \mathrm{R}=4.61 \%]$ and $2016[\mathrm{C}=7.18 \%, \mathrm{R}=3.22 \%])$. BM did significantly reduce scab severity on fruit in three of five years, in 2012 (4.5\%), 2015 (3.33\%) and $2016(2.27 \%)$ compared to the C. Other treatments were less consistent. Although organic fungicide options reduce scab, using resistant cultivars will be more reliable for organic pecan production in the southeastern USA.

Efficacy of Bordeaux mixture to control pecan scab in large-plot experiments

C. H. Bock (1), D. I. Shapiro-Ilan (2), M. W. HOTCHKISS (3), L. Wells (4), R. F. Mizell (5), (1) USDA ARS, Southeastern Fruit and Tree Nut Research Laboratory, Byron, GA, USA; (2) USDA/ARS, Southeastern Fruit and Tree Nut Research Laboratory, Byron, GA, USA; (3) USDA ARS, Byron, GA, USA; (4) University of Georgia, Tifton, GA, USA; (5) University of Florida/IFAS, Quincy, FL, USA

Venturia effusa causes scab, the most important disease on pecan in the southeastern USA. Organic fungicides have not been widely tested for efficacy against scab on susceptible cultivars. A large-plot experiment was used to test the efficacy of the traditionally-used fungicide against scab, Bordeaux mixture (BM), on trees of cv. Stuart at two sites (Colquitt and Byron) in GA. The experiments were conducted in 2011, 2012, 2014,2015 and 2016 (Colquitt only in 2014 and 2015). BM was applied 6 times using an orchard sprayer as follows: $4 \mathrm{lb} \mathrm{CuSO}$ : $1 \mathrm{lb}$ hydrated lime, for the $1^{\text {st }}$ spray; followed by $6 \mathrm{lb} \mathrm{CuSO}_{4}: 2 \mathrm{lb}$ hydrated lime for the $2^{\text {nd }}-6^{\text {th }}$ sprays applied in 100 gallons per acre. Fruit samples were assessed for scab severity twice. At the time of the $1^{\text {st }}$ assessment, only in 2011 did BM reduce scab severity $(\mathrm{F}=56.4, \mathrm{P}<0.0001$; control $=4.14 \%$ fruit area diseased, $\mathrm{BM}=1.08 \%$ area diseased (a $73.9 \%$ reduction). $\mathrm{BM}$ significantly reduced severity of scab at the time of the $2^{\text {nd }}$ assessment at Byron in $2011(\mathrm{~F}=12.3, \mathrm{P}=0.0005)$ and 2014 ( $\mathrm{F}=4.4$, $\mathrm{P}=0.04)$, and at the Colquitt location in $2014(\mathrm{~F}=30.0, \mathrm{P}<0.0001)$ only. The percentage reduction in severity of scab was $43.9 \%, 16.2 \%$ and $24.4 \%$, respectively. In 2015 at the Colquitt location there was an increase (16.3\%) in the severity of scab on fruit of $\mathrm{BM}$-treated trees $(\mathrm{F}=8.8, \mathrm{P}=0.003)$. The limited control of scab by BM indicates resistant cultivars are preferable in organic production systems in scab-prone areas.

Adepidyn fungicide: Baseline sensitivity and cross resistance patterns in Alternaria alternata from almonds G. OLAYA (1), R. Linley (1), K. Edlebeck (1), T. L. Harp (2), R. S. Bounds (2), (1) Syngenta Crop Protection, Vero Beach, FL, USA; (2) Syngenta Crop Protection, Greensboro, NC, USA

Adepidyn ${ }^{\circledR}$ fungicide (pydiflumetofen) is the active ingredient of Miravis ${ }^{\circledR}$, a new fungicide currently under development by Syngenta. Miravis ${ }^{\circledR}$ is a carboxamide fungicide and is the first member of a new chemical group among the succinate dehydrogenase inhibitor fungicides (SDHI, FRAC code 7), the N-methoxy-(phenyl-ethyl)-pyrazole-carboxamides. The baseline sensitivity for Alternaria alternata was established using 40 isolates identified to be wild-type, sensitive to other SDHI fungicides, and with no identified mutations conferring resistance to SDHI fungicides. All monoconidial isolates were obtained from Alternaria blight infected almond leaves from California. The Adepidyn ${ }^{\circledR}$ fungicide baseline sensitivity distributions $\left(\mathrm{EC}_{50}\right.$ values) of the A. alternata isolates ranged from 0.003 to $0.785 \mathrm{mg}$.ai/L, with a mean of $0.056 \mathrm{mg}$.ai/ $/ \mathrm{L}$. In another study, the in vitro sensitivity of 102 isolates was determined for Adepidyn ${ }^{\circledR}$ and Solateno $l^{\circledR}$ fungicides, boscalid and fluopyram. This group of isolates contained wild-type isolates and isolates identified to have mutations conferring resistance to SDHI fungicides. The in vitro sensitivity revealed an incomplete cross resistance pattern between Adepidyn ${ }^{\circledR}$ and Solatenol ${ }^{\circledR}$ fungicides, boscalid, and fluopyram. Adepidyn ${ }^{\circledR}$ fungicide demonstrated the highest intrinsic activity among these fungicides and across all the isolates with or without mutations in the sdh subunits (SdhB-H277Y, SdhB-H277L, SdhC-H134R, SdhC-H135R, SdhD-D123E, SdhD-

H133R/P/T, SdhB-H277Y+SdhC-H134R and H277Y+SdhD-H133R). Boscalid was the least active compound, and the activity of solatenol was similar to fluopyram.

Efficacy of fluopyram seed treatment in management against Fusarium brasiliense, a new dry bean root rot pathogen in Michigan K. OUDMAN, J. L. Jacobs, A. Bryne, M. Chilvers, Michigan State University, East Lansing, MI, USA

Fusarium root rot of dry bean is caused by several species in Michigan. According to a dry bean Fusarium root rot survey conducted in Michigan from 2014 to 2017, the most common species are within the Fusarium solani species complex (FSSC) clade 2, such as F. cuneirostrum and F. phaseoli. However, a new species to North America was also isolated from dry beans in high abundance: F. brasiliense (unpublished data). F. brasiliense is 
endemic to South America and is known to be a causal agent of soybean sudden death syndrome (SDS) despite being isolated from many dry beans in Michigan. F. virguliforme, a SDS pathogen of the same clade, is another prevalent species in Michigan on soybeans. As soybeans and dry beans are often grown in the same locations in Michigan and both $F$. virguliforme and $F$. brasiliense are widespread in these areas, it is necessary to understand the impact of these two species on dry beans. An inoculated field trial was set up in the summer of 2017 to test the aggressiveness of $F$. brasiliense and $F$. virguliforme on two dry bean cultivars and the efficacy of fluopyram (ILeVO; Bayer CropScience) seed treatment. $F$. brasiliense significantly decreased shoot mass and increased root rot ratings. However, fluopyram seed treatment significantly decreased root rot ratings in the inoculated plants, a finding complimented by quantitative PCR assays that revealed significantly decreased pathogen presence in roots treated with the fluopyram seed treatment as compared to the non-treated controls. F. brasiliense is a new concern for dry bean growers, however fluopyram has potential to control this pathogen.

Sensitivity to eight fungicide chemical groups of Colletotrichum fioriniae, the cause of anthracnose of pistachio in California P. D. S. F. LICHTEMBERG (1), R. Puckett (2), G. Gusella (3), H. Doussoulin (1), D. Felts (1), T. J. Michailides (1), T. Carraro (4), (1) University of California - Davis, Parlier, CA, USA; (2) University of California, Davis/Kearney Agricultural Research and Extension Center, Parlier, CA, USA; (3) Università degli Studi di Bari Aldo Moro, Bari, ITALY; (4) Universidade Federal do Paraná, Curitiba, BRAZIL

In 2016 and 2017, pistachios of cv. Red Aleppo showed severe anthracnose symptoms in fruit, leaves, and rachises in a few orchards located in Glenn County, California. In total, 39 isolates identified as Colletotrichum fioriniae were assessed for multiple resistance to different fungicide groups such as the quinone outside inhibitors (QoIs) azoxystrobin and trifloxystrobin, the demethylation fungicide inhibitors (DMIs) metconazole and propiconazole, the succinate dehydrogenase inhibitors (SDHIs) fluopyram and penthiopyrad, and the anilino pyrimidines (APs) cyprodinil and pyrimethanil. Results indicated that DMI fungicides consistently inhibited mycelial growth at dosages such as $0.37 \mu \mathrm{g} / \mathrm{ml}$ (ranging from 0.10 to $0.71 \mu \mathrm{g} / \mathrm{ml}$ ) for metconazole and $0.79 \mu \mathrm{g} / \mathrm{ml}$ (ranging from 0.34 to $1.41 \mu \mathrm{g} / \mathrm{ml}$ ) for propiconazole. The $\mathrm{EC}_{50}$ value for SDHI penthiopyrad was $0.09 \mu \mathrm{g} / \mathrm{ml}$ ( $\mathrm{ranging}$ from 0.04 to 0.28 $\mu \mathrm{g} / \mathrm{ml}$ ) and no inhibitory effect was observed for fluopyram. The AP fungicides cyprodinil and pyrimethanil did not inhibit mycelial growth. The QoI sensitivity values for azoxystrobin ranged from 83.81 to $1,000.88 \mu \mathrm{g} / \mathrm{ml}$ (avg. $647.82 \mu \mathrm{g} / \mathrm{ml}$ ) and for trifloxystrobin from 108.35 to $956.39 \mu \mathrm{g} / \mathrm{ml}$ (avg. $609.13 \mu \mathrm{g} / \mathrm{ml}$ ). Molecular characterization of the cyt $b$ gene revealed that all 37 isolates tested had the mutation G143A. A protective fungicide efficacy test performed after wounding detached pistachio leaves of cv. Red Aleppo revealed that metconazole and penthiopyrad can best control C. fiorioniae lesions. The least control was obtained with fluopyram and trifloxystrobin fungicides. These results will certainly support future studies on resistance and resistance management of pistachio anthracnose in California.

\section{Development of organic pea seed treatments with efficacy against Pythium seed rot} L. D. PORTER, USDA-ARS, Prosser, WA, USA

In 2016, organic peas were grown on 1,347 ha in the state of Washington and on 3,726 ha nationally. A major limitation to organic pea production is seed rot and seedling damping off caused by Pythium spp., particularly Pythium ultimum. Twenty-six organic pea seed treatments were evaluated in two field trials in 2017 to assess the efficacy of these products in improving multiple growth parameters including seedling emergence and yield. Nordox 75 WG (a.i. 83.9\% cuprous oxide), and GEX170008 (a.i. not disclosed), a commercial organic seed treatment developed by Germains Seed Technology, significantly $(P \leq 0.05)$ increased emergence compared to water-treated seed by 23.3 and $57.9 \%$ in field 1 , respectively, and 27.4 and $37.9 \%$ in field 2 , respectively. Nordox and GEX170008 increased yields compared to the water control by 40.6 and $77.9 \%$ in field 1 , respectively, and 23.0 and $5.1 \%$ in field 2, respectively. The Pythium disease pressure was not as severe in field 2 as in field 1 . Despite these increases in yield, only the $77.9 \%$ increase in field 1 was significantly $(P \leq 0.05)$ greater than the water-treated control. GEX170008 is one of the first non-biological organic seed treatment to provide significant protection against Pythium seed rot resulting in more uniform emergence and improved yields in pea. Currently Nordox 75 WG and GEX170008 are not registered for organic use on peas as seed treatments.

Enhancing the constitutive resistance in Capsicum annuum $\mathrm{L}$. fruits against anthracnose development C. Mahendranathan (1), N. ADIKARAM (2), (1) Eastern University, Sri Lanka, Chenkalady, SRI LANKA; (2) National Institute of Fundamental Studies, Kandy, SRI LANKA

This study was undertaken to enhance natural disease resistance of Capsicum annuum L. cvs. HYW and CA-8, against anthracnose and postharvest quality, through some selected pre- and postharvest treatments. C. acutatum was shown to be associated with chili anthracnose for the first time in Sri Lanka. Potassium silicate $\left(\mathrm{Kasil}^{\circledR}\right)$ was applied as a postharvest treatment at concentrations, 0 (control), 100, 200, and $400 \mathrm{mg} / \mathrm{l}$ and found that the concentration at $200 \mathrm{mg} / \mathrm{l}$ was effective. Elicitor treatment, at $200 \mathrm{mg} / \mathrm{l}$, was done once in a week for 4 weeks, it resulted in significantly reduced anthracnose disease when the harvested fruits were challenge-inoculated with C. acutatum. Postharvest application of potassium silicate (Kasil $\left.{ }^{\mathbb{B}}\right)$, at the same concentration reduced anthracnose lesion area by $25-100 \%$, compared to the untreated controls. Postharvest spray treatment of chili at mature, green stage with potassium silicate at $200 \mathrm{mg} / \mathrm{I}$, reduced the severity of anthracnose development by $34-100 \%$. Spore germination assay revealed that potassium silicate has no antifungal effect on conidia of C. acutatum. Potassium silicate (Kasil ${ }^{\circledR}$ ) treated fruits, inoculated with $C$. acutatum after harvest, showed greater accumulation of phytoalexins. Related (PR) Proteins such as $\beta$-1, 3-glucanase are considered to play an important role in plant disease resistance.

\section{Assessing the Curative and Residual Control of Wheat Powdery Mildew with Fungicides}

C. Scanlan (1), N. M. KLECZEWSKI (2), (1) Dept. of Plant and Soil Science, University of Delaware, Newark, DE, USA; (2) University of Illinois, Urbana, IL, USA

Fungicide efficacy trials in wheat are often conducted in the field, and researchers often cannot separate direct curative or residual activity from the impact of a fungicide on inoculum buildup, arrival, and spread. To address this, a set of greenhouse experiments were conducted on the fungicides Prosaro, Caramba, Trivapro, Priaxor, and Stratego YLD using a local isolate of powdery mildew (Blumeria graminis (DC.) Speer f. sp. Tritici). Curative activity was assessed by inoculating 14-day-old pots of Roane wheat 5 days prior to fungicide application. Residual activity was assessed by inoculating plants at 42 days after fungicide application. Disease severity was determined 10 days after inoculation. Each fungicide by inoculation treatment was replicated 6 times per experiment and each experiment was replicated twice. In 5-day curative trials Trivapro reduced disease severity by $99 \%$. Stratego YLD, Prosaro, and Trivapro reduced disease by 71, 63 and 59\% in 42-day residual experiments, respectively. In field trials, Caramba is considered to have superior curative control. Similarly, Stratego YLD often is not considered to provide excellent residual control for fungal diseases, whereas Trivapro is marketed as having superior residual control compared to other fungicides. These results indicate that fungicide performance in the field may be due to other factors that may influence perceived curative and residual product performance. 
Investigating fluopyram as a seed treatment against soybean cyst nematode in the presence of $\boldsymbol{F}$. virguliforme

M. ROTH (1), J. L. Jacobs (1), S. Napieralski (2), A. Byrne (1), F. W. Warner (1), M. Chilvers (1), (1) Michigan State University, East Lansing, MI, USA; (2) University of Wisconsin, Madison, WI, USA

Soybean cyst nematode ( $\mathrm{SCN}$ ) and Fusarium virguliforme are major threats to soybean production throughout the US and require careful management for optimal yields. Management strategies for both pathogens are similar and include using genetically resistant varieties, implementing diverse crop rotations, and using seed treatments containing a nematicide or fungicide for each pathogen, respectively. A commercial seed treatment formulation containing fluopyram, ILeVO, has been marketed against SCN. Fluopyram inhibits the enzyme succinate dehydrogenase in fungi and has shown efficacy against $F$. virguliforme in vitro and in field studies as a seed treatment. Field studies were conducted on 25 varieties across 2014-2016 in a location naturally infested with both $\mathrm{SCN}$ and $F$. virguliforme. Each variety was treated with a base seed treatment or the base treatment plus ILeVO. Soybean variety had the largest impact on SCN reproduction and fecundity. However, the addition of ILeVO showed a reduced number of SCN eggs and juveniles in the soil post-harvest for 19 varieties, and a reduced SCN fecundity in 13 varieties. A follow-up greenhouse study with two varieties in SCN and $F$. virguliforme infested soil also showed that ILeVO significantly reduced the number of SCN females compared to the base treatment. Quantification of SCN DNA using qPCR on greenhouse root samples showed a reduction in SCN DNA in roots containing ILeVO seed treatments compared to treatments without ILeVO.

Picarbutrazox sensitivity of different Pythium species

G. OLAYA (1), D. Ireland (2), F. Brandl (3), P. Pedersen (2), A. E. Dorrance (4), A. E. Robertson (5), (1) Syngenta Crop Protection, Vero Beach, FL, USA; (2) Syngenta Crop Protection, Greensboro, NC, USA; (3) Syngenta Crop Protection, Basel, SWITZERLAND; (4) The Ohio State University, Dept. of Plant Pathology, Wooster, OH, USA; (5) Iowa State University, Department of Plant Pathology, Ames, IA, USA

Picarbutrazox is the active ingredient of a novel seed treatment fungicide developed by Syngenta. Picarbutrazox belongs to the tetrazolyloximes chemical group of fungicides and its mode of action is still unknown (FRAC code U17). It has no known cross resistance to other oomycete compounds. Picarbutrazox targets oomycete plant pathogens and control diseases caused by Pythium, Phytophthora and downy mildews. Under lab, greenhouse and field conditions Picarbutrazox has shown to have broad spectrum of Pythium species control. The sensitivity to Picarbutrazox of 199 isolates belonging to more than 20 species of Pythium was determined. Pythium isolates were recovered from symptomatic plants including corn, soybean and other crops. Isolates were identified to species level using morphological and molecular tools. Another group of Pythium isolates were obtained from collections kept at Universities. Mycelial growth inhibition in vitro was quantified using the following Picarbutrazox concentrations: $0,0.00001,0.0001,0.001,0.01$ and $0.1 \mathrm{mg}$.ai/L. All species of Pythium tested were sensitive to Picarbutrazox. The sensitivity distribution (EC50 values) across all Pythium species tested ranged from 0.000031 to 0.004236 with a mean of $0.000434 \mathrm{mg}$.ai $/ \mathrm{L}$. The Picarbutrazox sensitivity of more than 20 species of Pythium have been documented and the high in vitro intrinsic activity of this fungicide against Pythium species has been demonstrated. In addition, the efficacy of Picarbutrazox as a seed treatment against Pythium diseases of corn, soybeans and other crops has been demonstrated under field conditions.

$\mathbf{N}$-acetylcysteine: A new alternative to control citrus canker

S. PICCHI (1), A. Alves de Souza (2), M. J. Festa Franzini (1), M. Machado (1), M. Takita (1), H. Coletta-Filho (1), (1) Centro de Citricultura Sylvio Moreira" - Agronomic Institute (IAC), Cordeiropolis, BRAZIL; (2) IB - UNICAMP, Campinas, BRAZIL

Citriculture is one of the most important commodities in the international market, and especially in Brazil, since it is the world's biggest orange juice exporter. Among the citrus diseases, citrus canker, caused by the bacterium Xanthomonas citri subsp. citri, has caused significant economic losses worldwide. There are no effective control measures besides cupric compounds applications, which continuous use may promote resistant strains selection, not to mention the limited efficacy and negative impacts on the profits for growers. So, we developed a new product for pulverization on citrus orchards (NACsolution) which is based on the antioxidant, anti-adhesive and antibacterial properties of the molecule N-acetil-cysteine (NAC), a cysteine analogue widely used in human health due to its low toxicity. NAC impairs biological activities of $X$. citri such as EPS production, cellular viability, biofilm formation and motility. Also, it has also impacted the bacterial adhesion on leaf surfaces, an important part of the disease cycle. Field evaluations revealed that an area with initial severity of $40 \%$ decreased this value in $17 \%$, and in areas with initial severity above $50 \%$, this reduction reached $48 \%$. These results were obtained after one year of NACsolution application and we used cupric compounds applications as a control. Therefore, NACsolution seems to be an alternative to block disease progression, being a sustainable alternative to the control of citrus canker.

Seed treatment versus in-furrow fungicide effects on plant stand establishment and control of Rhizoctonia crown and root rot in sugar beet J. R. BRANTNER (1), A. K. Chanda (2), (1) University of Minnesota, Crookston, MN, USA; (2) Department of Plant Pathology, University of Minnesota, Crookston, MN, USA

Rhizoctonia solani is the most common sugar beet root pathogen in Minnesota and North Dakota, causing damping-off and Rhizoctonia crown and root rot (RCRR). In-furrow fungicides have been used but sometimes reduce emergence. New seed treatments are available that are more convenient for growers and safer during emergence. A field trial was conducted in R. solani-infested soil in 2016 and 2017 to compare four seed treatments and three in-furrow fungicides for stand establishment and control of $R$. solani in sugar beet. In 2016, the trial was replanted June 24 due to water damage. This late planting date resulted in warm soil temperatures and high disease pressure during stand establishment. In-furrow fungicides resulted in significantly $(P=0.05)$ higher plant stands at 4 weeks after planting, higher number of harvested roots, and lower severity and incidence of RCRR compared with seed-treatment fungicides. The 2017 trial was planted May 11 under cooler, drier soil conditions less favorable for emergence and disease. In-furrow fungicides resulted in significantly lower stand at 4 weeks after planting compared to seed treatments, but there were no significant differences in the number of harvested roots or RCRR ratings. Under high disease pressure, in-furrow fungicides provide better disease control than seed treatments, but environmental effects on both emergence and disease pressure influence the relative benefit.

Picarbutrazox: A novel compound for the control of seedling damping-off caused by Pythium spp.

F. BRANDL (1), A. Dutton (1), D. Ireland (2), G. Olaya (3), C. Yokota (4), R. Kuwahara (4), (1) Syngenta Crop Protection, Basel, SWITZERLAND; (2) Syngenta Crop Protection, Greensboro, NC, USA; (3) Syngenta Crop Protection, Vero Beach, FL, USA; (4) Nippon Soda, Tokyo, JAPAN

Pythium species are serious and widespread plant pathogens causing damping-off and seedling blight in many crops worldwide. In row crops like corn or soybeans Pythium diseases are limiting factors by reducing the optimized plant stand for high yields. The increase of Pythium diseases is due to shifts towards earlier planting dates and reduced tillage systems. Both agronomic factors favor the incidence of Pythium diseases on seedling establishment as soils become cooler and contains higher moisture compare to more conventional planting schemes. Pythium diseases could be caused by one or many different species of Pythium. Inter and intraspecific variation of Pythium isolates have been documented for pathogenicity and response to fungicides. 
Picarbutrazox is a novel fungicide discovered by Nippon Soda Co Ltd Japan and developed globally by Syngenta for the control of oomycete diseases when applied as seed treatment in corn, soybeans, canola, cereals and other crops. It belongs to the chemical group of tetrazolyloximes with a novel but no elucidated mode of action. Benchmarked against other oomycete compounds no cross resistance has been detected for Picarbutrazox. Field and glasshouse trials from 2016 to 2018 have demonstrated, robust and consistent activity of Picarbutrazox against all pathogenic Pythium species in corn and soybeans at very low use rates. Picarbutrazox is a reliable and high-performing technology that address the huge variability in pathogenicity of the Pythium species. The compound has an excellent seed safety and mixture compatibility with additional fungicides, insecticides and nematicides.

Picarbutrazox will complement the leading seed treatment oomycete control portfolio of Syngenta Seedcare consisting of mefenoxam and azoxystrobin.

Field efficacy of systemic acquired resistance inducers for fire blight management and pathogenesis-related protein gene expression in $\mathrm{Malus}$ domestica

R. KREIS, S. M. Villani, North Carolina State University, Mills River, NC, USA

Fire blight on apple caused by Erwinia amylovora can be a significant problem for apple growers causing blossom and shoot blight. Application restrictions and resistance concerns associated with streptomycin and other antibiotics, necessitate the evaluation of alternative products for fire blight control. Products including Lifegard (Bacillus mycoides isolate J), Actigard (acibenzolar-S-methyl), and MBI-10612 (Marrone Bio Innovations) that induce systemic acquired resistance (SAR) were evaluated for efficacy against fire blight and for pathogenesis-related (PR) protein gene expression. Treatments were applied at $20 \%, 40 \%, 80 \%$, and full bloom on Malus $x$ domestica 'Rome Beauty' in 2017. Treatments with SAR inducers resulted in significantly less blossom blight than the untreated control (UTC) and the Lifegard treatment resulted in significantly less shoot blight than the UTC. PR protein gene expression was evaluated in apple blossoms $24 \mathrm{~h}$ and 7 days after initial applications and evaluated in leaves $24 \mathrm{~h}, 7$ days, and 5 weeks after initial application. The PR-1 gene was found to be 1.23 and 1.17 times greater in leaf and blossom tissue respectively after $24 \mathrm{~h}$ of treatment with Lifegard than the UTC. Gene expression of PR-1 was also 1.58 and 2.09 times higher in the leaves and blossoms 7 days after treatment with Lifegard and 2.58 times greater after 5 weeks. Further work is being done to compare PR gene expression in other treatments with PR-1, PR-2, and PR-8.

Zinc nanoparticles mitigate some fruit symptoms of Huanglongbing on citrus

E. G. JOHNSON (1), M. M. Dewdney (1), M. Danyluk (2), J. H. Graham (1), S. Santra (3), (1) University of Florida, Lake Alfred, FL, USA; (2) Citrus Research \& Education Center, University of Florida, Lake Alfred, FL, USA; (3) NanoScience Technology Center, Department of Chemistry, University of Central Florida, Orlando, FL, USA

Huanglongbing (HLB) is a devastating citrus disease caused by the phloem-limited pathogen Candidatus Liberibacter asiaticus. HLB results in yield loss through small or misshapen fruit, preharvest fruit drop, and unripe, bitter juice. Delivery of bactericidal agents to citrus phloem is the greatest challenge in developing chemical therapies for HLB. Through a collaborative effort, we developed a novel zinc-based nanoparticle bactericide (Zinkicide ${ }^{\mathrm{TM}}$ ) using plant derived compounds. These particles are designed to be small enough $(2-5 \mathrm{~nm})$ to travel systemically through the phloem and to be metabolized by the plant after reaching their target, eliminating the concern of residues in the fruit. The synthesis protocol was designed to be simple and inexpensive for large quantity production. Zinkicide ${ }^{\mathrm{TM}}$ worked effectively as a soil drench to control citrus canker in a proof of concept greenhouse study demonstrating systemic efficacy. Multiple field trials in grapefruit and sweet orange resulted in increased fruit size from Zinkicide application. However, results suggest that application rates need optimization for dilution of active particles from systemic movement. Systemic efficacy in larger trees requires a combination of spray and soil drench. Disparities between canker (21-day applications) and HLB (28-day applications) trials suggest that residual efficacy is shorter than expected and clustering of applications may provide better efficacy than full year coverage.

\author{
A Quantitative Synthesis of the Efficacy and Profitability of Conventional and Biological Fungicides for Botrytis Fruit Rot Management on \\ Strawberry \\ L. CORDOVA (1), F. Dalla Lana (2), P. A. Paul (3), N. Peres (1), (1) University of Florida, Wimauma, FL, USA; (2) Ohio State University, Columbus \\ 43210, OH, USA; (3) The Ohio State University, Wooster, OH, USA
}

Botrytis fruit rot (BFR) is a major disease that affects strawberry production in Florida and worldwide. BFR management relies on frequent fungicide applications. A meta-analysis was conducted on the outcome from nine field trials to evaluate the efficacy and profitability of conventional and biological fungicides compared to a non-treated control (NTC). All trials were conducted in Florida between the 2005/06 and 2016/17 growing seasons. Fungicide treatments were applied weekly and plots were harvested twice a week for yield and BFR incidence quantification. Treatments were grouped into five categories: NTC, multi-site only (thiram), standard (captan alternate with fludioxonil + cyprodinil), Bacillus, and 'others' (miscellaneous biologicals). Following primary analyses, a random effects network meta-analytical model was fitted to estimate the mean yield response of each treatment group and to compare groups in $\mathrm{kg} / \mathrm{ha} / \mathrm{week}$. Thiram and the standard treatment provided an increment in yield of 195.8 and $242.3 \mathrm{~kg} / \mathrm{ha} / \mathrm{week}$, respectively, compared to the NTC. The estimated yield difference between Bacillus and NTC, and 'others' and NTC were not significantly different from zero, suggesting that weekly applications of these products did not provide a yield benefit. The results of this analysis provide growers information that aids their decision-making process regarding BFR management.

Effect of a foliar fungicide applied at flowering on corn standability at harvest A. E. ROBERTSON, Iowa State University, Department of Plant Pathology, Ames, IA, USA

In the U.S. Corn Belt, foliar fungicide applications on corn have increased over the past decade. Some farmers have observed that corn that is treated with a fungicide at flowering is less susceptible to lodging prior to harvest. Field trials were established at six Iowa State University Research and Demonstration farms in 2017. Each field trial was set up as a $2 \times 5$ factorial randomized complete block design. Plots were either sprayed with a fungicide at growth stage R1, or left unsprayed. Plots were harvested on 5 dates, each separated by 4 to 7 days, starting when grain moisture was approximately $23 \%$. Immediately prior to harvest, the push test was done on 100 consecutive plants in each plot and percent lodging was recorded. No fungicide $\times$ harvest date interaction was detected on lodging. Percent lodging increased with later harvest dates $(\mathrm{P}<0.01)$. At 4 of the 6 locations, a fungicide application reduced percent lodging $(\mathrm{P}<0.01)$. These data suggest that when harvest is delayed, farmers should harvest plots with no fungicide prior to fungicide-applied plots due to the greater risk for poor standability and difficulty harvesting in those fields with no fungicides.

Sensitivity of Septoria glycines isolates to quinone outside inhibitor (QoI) fungicides

D. NEVES (1), A. Wang (2), J. D. Weems (1), C. A. Bradley PhD (1), (1) University of Kentucky, Princeton, KY, USA; (2) University of Illinois, Urbana, IL, USA 
Brown spot, caused by Septoria glycines, is a common foliar disease of soybean (Glycine max). Quinone outside inhibitor (QoI) fungicides have been effective in managing brown spot, but the risk of selecting strains with resistance to this class of fungicides is considered high. Septoria glycines isolates from Illinois and Kentucky were tested in vitro using azoxystrobin, pyraclostrobin, and trifloxystrobin amended media to determine the effective concentration in which $50 \%$ conidial germination was inhibited relative to a non-amended control $\left(\mathrm{EC}_{50}\right)$. The mean $\mathrm{EC}_{50}$ values of azoxystrobin, pyraclostrobin, and trifloxystrobin were $0.6583,0.0962$, and $0.1396 \mu \mathrm{g} / \mathrm{ml}$, respectively. Isolates with high $\mathrm{EC}_{50}$ values were identified, and sequencing of the cytochrome $b$ gene from isolates with high $\mathrm{EC}_{50}$ values showed they exhibited a transversion of a guanidine to cytosine at the second position in the codon 143, which is known as the G143 mutation. A single discriminatory dose of azoxystrobin, which was completely inhibitory for the QoIresistant strains but allowed growth of resistant strains, was used to measure the sensitivity of 171 isolates. QoI-resistant isolates made up $29 \%$ of the $S$. glycines tested. Greenhouse experiments showed that QoI fungicides provided a high level of control of brown spot caused by QoI-sensitive isolates, but poor control of brown spot caused by QoI-resistant isolates.

Evaluation of fungicides for management of rust on oil seed and confection sunflower hybrids

B. BERGHUIS (1), M. Gilley (1), J. Halvorson (1), B. Hansen (1), B. Schatz (2), S. Fitterer (3), D. Carruth (3), B. Benson (4), M. Halvorson (2), S. G. Markell (1), (1) North Dakota State University, Fargo, ND, USA; (2) NDSU Carrington Extension Center, Carrington, ND, USA; (3) BASF ND Research Farm, Davenport, ND, USA; (4) Mycogen Seeds, Breckenridge, MN, MN, USA

Two types of sunflower hybrids are produced in the United States; oil seed and confection (non oil). In 2016, approximately 0.64 M Ha were planted, $84 \%$ of which were oilseed and the remainder confection. Sunflower rust, caused by the fungal pathogen Puccinia helianthi, is an economically important disease of sunflowers. Recent research demonstrated that several fungicides were efficacious and an economically viable management tool for sunflower rust. However, the vast majority of the work was done on the confectionery type sunflowers, widely considered to be more susceptible to the disease. Additionally, many "new" fungicides have recently become available to sunflower growers. The objective of this study is to evaluate the efficacy of new fungicides on oil seed and confection sunflower hybrids for rust management. Oilseed and confection sunflower hybrid trials were planted in four row plots in a Randomized Complete Block Design with four replicates in two locations in 2016 and 2017. Trials were artificially inoculated with Puccinia helianthi urediniospores. Twelve different fungicide treatments were applied at growth stage R5. Rust severity was visually evaluated at growth stage R6 and R7. Significant rust severity differences between fungicides and the non-treated control and among fungicides were observed in all confection and oilseed trials. Efficacy of new fungicides tended to be consistent within FRAC groups and will be useful management tools for growers.

Novel Copper-Composites for Management of Bacterial Spot of Pepper Caused by Xanthomonas euvesicatoria Q. FAN (1), S. Kunwar (2), M. Young (3), S. Santra (4), J. B. Jones (5), M. Paret (6), (1) University of florida, Quincy, FL, USA; (2) University of Florida, Quincy, FL, USA; (3) University of Central Florida, Orlando, FL, USA; (4) NanoScience Technology Center, Department of Chemistry, University of Central Florida, Orlando, FL, USA; (5) Department of Plant Pathology, University of Florida, Gainesville, FL, USA; (6) North Florida Research and Education Center, University of Florida, Quincy, FL, USA

Bacterial spot caused by Xanthomonas euvesicatoria is one of the most serious diseases of pepper in Florida and throughout the world. The widespread occurrence of copper tolerant strains of $X$. euvesicatoria have limited the efficacy of copper bactericide application, widely used for managing bacterial spot of pepper. In this study, we investigated the antibacterial effect of three novel copper-composites, Core-shell copper (CS-Cu), Mixed-valence copper $(\mathrm{MV}-\mathrm{Cu})$, and Fixed-quat copper (FQ-Cu) in in vitro and in greenhouse experiments against copper tolerant strains of $X$. euvesicatoria. All concentrations $(100,200,500$ and $1000 \mu \mathrm{g} / \mathrm{ml})$ of CS-Cu and FQ-Cu effectively killed bacteria within $1 \mathrm{~h}$ and MV-Cu killed all the cells at $24 \mathrm{~h}$, in vitro. In the first greenhouse experiment, plants treated with all copper-composites, at all concentrations $(100,200$, and $500 \mu \mathrm{g} / \mathrm{ml})$ had disease severity (AUDPC) statistically equivalent to or better than the growers' standard copper-mancozeb. All three concentrations of FQ-Cu, 200 and 500 ppm of CS$\mathrm{Cu}$ and 100 and $200 \mathrm{ppm}$ of MV-Cu showed significantly less AUDPC compared to Kocide 3000 . Phytotoxicity was observed at 500 ppm of CS-Cu and FQ-Cu. In the second greenhouse experiment, all concentrations of CS-Cu and $200 \mathrm{ppm}$ of MV-Cu significantly reduced AUDPC compared to coppermancozeb. FQ-Cu at all concentrations were statistically equivalent to copper-mancozeb. When compared to Kocide 3000 , all three concentrations of $\mathrm{CS}-\mathrm{Cu}, 200$ and $500 \mathrm{ppm}$ of MV-Cu and $500 \mathrm{ppm}$ of FQ-Cu showed significantly less AUDPC. This study highlights the potential of copper-based nanomaterials for effective management of bacterial spot of pepper.

A case study of misapplication of the fungicide flutriafol to grapevines in Texas

D. N. APPEL (1), S. McBride (2), (1) Department of Plant Pathology and Microbiology, Texas A\&M University, College Station, TX, USA; (2) Department of Plant Pathology and MIcrobiology, Texas A\&M University, College Station, TX, USA

A major limiting factor in winegrape production in Texas is cotton root rot, caused by Phymatotrichopsis omnivora. Chemigation with Topguard Terra ${ }^{\circledR}$ (flutriafol) is used to control cotton root rot under a 24 (c) Special Local Need label. According to the label, the maximum annual rate of application is $15.3 \mathrm{fl}$ oz./ac., either in one spring application or in split spring and post-harvest fall applications. In September 2016, a grower using flutriafol for disease suppression reported unusual symptoms in the grape varieties muscat, mourverdre and chardonnay. The symptoms included severe interveinal chlorosis and necrosis, marginal necrosis, and leaf curl on the older leaves. The new growth that developed subsequent to the misapplication was asymptomatic. The grower reported applying the full rate of flutriafol in July through a subsoil irrigation system that delivered product directly to the root zone of the grapevine, rather than the standard drip irrigation. Plant and soil samples were collected for flutriafol analyses and sent to a commercial testing lab. Levels of flutriafol in the symptomatic foliage were 40-112 ppm. The new growth had levels of $10 \mathrm{ppm}$. Corresponding soil levels of flutriafol ranged from $0.05-0.46 \mathrm{ppm}$. The maximum levels were greater than 5 times those detected in soils treated using recommended methods in previously tested vineyards. This episode was an example of chemical damage due to unintended misapplication and serves as a warning to the dangers of deviating from label recommendations for control of cotton root rot with flutriafol.

Evaluation of fungicide seed treatments for their efficacy in controlling blackleg of canola S. G C Upadhaya (1), V. Chapara (2), M. Rahman (1), L. E. DEL RIO MENDOZA (3), (1) North Dakota State University, Fargo, ND, USA; (2) NDSU, Langdon, ND, USA; (3) North Dakota State Univ, Fargo, ND, USA

Blackleg, caused by Leptosphaeria maculans, causes greatest yield losses when canola plants are infected before reaching the six-leaf growth stage. The objective of this study was to evaluate the efficacy of newly-registered seed treatments to control blackleg. Five treatments were applied to seeds of susceptible cultivar Westar and evaluated in greenhouse at NDSU greenhouse facility, Fargo, ND and in field trials at Langdon and Prosper, ND in 2017 using a RCB design with four replications. In greenhouse, plants were inoculated once with pycnidiospores suspensions at cotyledon stage and three times before plants reached the four-leaf stage in field trials. Field plots also were supplemented with blackleg-infested canola residues. Disease severities were assessed 12 days after inoculation and at harvest time in greenhouse trials. Yield and disease severities were assessed at harvest in field 
trials. All treatments reduced disease severity compared to the non-protected control at seedling stage in greenhouse, but only Obvius (fluxapyroxad + pyraclostrobin + metalaxyl) and Helix (metalaxyl + fludioxonil + sedaxane + difenoconazole + thiamethoxam) showed significant $(P<0.05)$ reductions in severity at harvest. In field trials, Maxim (fludioxonil) reduced disease severity at Prosper $(P<0.05)$ but not at Langdon $(P<0.05)$. None of the treatments improved yields significantly $(P<0.05)$ compared to non-treated control. This study will be repeated in 2018.

Performance of cotton seed treatments under different planting dates and seeding rates

S. YOUNG (1), J. E. Woodward (2), (1) Texas Tech University, Lubbock, TX, USA; (2) Texas A\&M AgriLife Extension Service, Lubbock, TX, USA

Seedling disease of cotton, caused by Rhizoctonia solani, is capable of affecting germination of cottonseed, emergence, survival and development of seedlings. Many management options exist for early season pests. Two field trials were conducted in 2017 to evaluate combinations of early season pest management options. Treatments consisted of two planting dates (early and optimal), two seeding rates ( 2 and 4 seed per foot), and four seed treatments (with increasing levels of early season pest protection), arranged in a split-split-split plot design with four replications. Overall, the application of additional fungicides increased stands over the base treatment by 1.8 to $9.4 \%$ at location 1 and 6.7 to $19.0 \%$ at location 2 . Improvements in yield were achieved with additional inputs in five of the eight scenarios. At location 1, yield improvements of 6.2 to $7.4 \%$ were achieved at the later planting date. An opposite trend was observed at location 2, where yield improvements were greater for the earlier planting date ranging from 4.1 to $15.3 \%$. Differences in trends between planting dates at the two locations were most likely due to weather conditions during seedling emergence. Use of seed treatment fungicides, insecticides and nematicides has increased over the past decade as chemical management options have become more readily available. Additional studies determining the risk of early season pest pressure and use of combinations of such products are warranted.

Effect of fungicide and nematicide seed treatments alone and in combination on cotton stand and yield

J. E. WOODWARD (1), M. L. Cartwright (2), S. Young (3), R. Roper (3), (1) Texas A\&M AgriLife Extension Service, Lubbock, TX, USA; (2) Texas A\&M University, Lubbock, TX, USA; (3) Texas Tech University, Lubbock, TX, USA

Seed cost is the largest expenditure producers have when planting cotton (Gossypium hirsutum L.). While all commercial seed comes with a broadspectrum base fungicide package, many of the premium products sold contain additional fungicide or nematicide/insecticide treatments. Producers often question the need for such products. Field studies were conducted in 2016 and 2017 to compare a non-treated control (TRT 1), a nematicide/insecticide seed treatment (TRT 2), a base fungicide seed treatment (TRT 3), and the combination of the base fungicide seed treatment and the nematicide/insecticide seed treatment (TRT 4) under two seeding rates. Stands for TRT 1 and TRT 2 were similar within a seeding rate and across years. Stand improvements over TRT 1 across the two years and two seeding rates ranged from 20 to $52 \%$ and 26 to $68 \%$ for TRT 3 and TRT 4 , respectively. Similar trends were observed for lint yield when combined across years and seeding rates. Yields for TRT 1 and TRT 2 did not differ. The addition of a fungicide treatment increased yield by $214 \mathrm{~kg}$ ha- 1 over the control, whereas, including a nematicide/insecticide with the fungicide treatment improved yields an additional $93 \mathrm{~kg}$ ha- 1 . This response was in the absence of nematodes or thrips, suggesting a synergism exists between the fungicide and nematicide/insecticide seed treatments evaluated.

White mold incidence, severity and lima bean yield response to fungicide application timing in the Mid-Atlantic Region H. DEMISSIE, PhD Student at the University of Maryland, College Park, MD, USA

Sclerotinia sclerotiorum (Lib.) de Bary causes white mold disease, which results in high yield loss on lima bean. Fungicides with active ingredients boscalid (Endura 70W) or thiophanatemethyl (e.g. Topsin M) are currently used to manage white mold, yet no specific application guidelines exist for lima bean in the Mid-Atlantic region. Efficacy trials were conducted from $2014-2017$ with Endura $\left(0.76 \mathrm{~kg} \mathrm{ha}^{-1}\right)$ fungicide at four locations in Delaware. Treatments included fungicide application timing at $20 \%$ flowering; one week later; two weeks later; three weeks later; at $20 \%$ flowering plus one week later (double treated); and non-sprayed. Disease incidence (DI) was measured as the number of infected plants $\mathrm{m}^{-1}$ section of row. Disease severity (DS) was rated as percentage of infected tillers per plant within each $\mathrm{m}$ section. Lima bean yield was recorded as $\mathrm{kg}$ from $6 \mathrm{~m}^{2}$ subsection of row area. All data were analyzed using Proc GLM in SAS. Both DI $(P<0.0005)$ and DS $(P<0.0002)$ were significantly influenced by fungicide application timings and years. There were significant $(P<0.0001)$ interactions between the application timing and years. Double treated was the best application timing, and significantly reduced the DI and DS compared to the non-sprayed plots and those sprayed three weeks after $20 \%$ flowering. Yield was significantly $(P<0.0001)$ different between locations and years. There was significant $(P<0.015)$ but weak negative correlation $(\mathrm{r}=-0.187)$ between DI and yield.

Fungicide strategies to manage wheat stem rust S. Baraibar (1), S. German (1), L. Pareja (2), S. A. PEREYRA (1), (1) National Institute for Agricultural Research (INIA), Colonia, URUGUAY; (2) Polo Agroalimentario y Agroindustrial, CENUR, Universidad de la Republica, Paysandu, URUGUAY

Stem rust (SR), caused by Puccinia graminis f. sp. tritici, is one of the most destructive diseases of wheat in the Southern Cone of America. The main factor contributing to this has been the increased use of susceptible cultivars. Medium-long term efforts pursue the development of adapted lines resistant to local and Ug99+races (potential risk). However, the optimization of short-term strategies comprising the use of foliar fungicides are needed to manage SR. Field trials were established in 2015 and 2016 in Uruguay to evaluate application moments of metconazole+epoxiconazole (first symptoms, ZGS65 and ZGS71) and nozzle types (CJ03 ${ }^{\circledR}$, TTJ60 ${ }^{\circledR}$ ) in SR susceptible (cv. B601) and intermediate (cv. B501) cultivars. Six commercial fungicides, including combinations of DMIs, QoIs and SDHIs and a non-treated and a complete treatment were evaluated in cv. B601 in both years. Fungicide application at disease onset significantly $(\mathrm{p} \leq 0.05)$ reduced SR severity, AUDPC and increased grain yield, test weight and thousand-kernel weight as compared to the non-treated and applications at ZGS71. Epoxiconazole and metconazole were detected in harvested grain if applied at ZGS71. Yet, concentrations were below the maximum residue levels (CODEX and EU). Significant differences were found among fungicides for SR severity, if applied at first symptoms. These results suggest that fungicides can effectively reduce SR severity and increase grain yield in susceptible cultivars if applied at disease onset.

Effect of paclobutrazol on laurel wilt on redbay (Persea borbonia) and the laurel wilt pathogen, Raffaelea lauricola S. M. ADAMS (1), J. A. Smith (2), (1) The University of Florida \& The Morton Arboretum, Gainesville, FL, USA; (2) School of Forest Resources and Conservation, University of Florida, Gainesville, FL, USA

Paclobutrazol belongs to the triazole group of DMI fungicides. Its fungicidal mode of action is as a sterol biosynthesis inhibitor, although it is more commonly used as a plant growth regulator in horticulture and arboriculture. Laurel wilt is a fatal disease of members of the Lauraceae caused by the fungus Raffaelea lauricola. Since R. lauricola was first identified in the United States in 2003 it has caused widespread death of redbay (Persea borbonia), swamp bay (P. palustris), sassafras (Sassafras albidum), and more recently it has been found in avocado (P. americana). The effects of 
varying concentrations of paclobutrazol from 0-400 parts per million (ppm) have been tested in vitro on $R$. lauricola. Paclobutrazol has a fungistatic effect on R. lauricola at concentrations lower than $400 \mathrm{ppm}$ and significantly slows the growth in concentrations less than $12 \mathrm{ppm}$. However, paclobutrazol does not appear to have fungicidal effects, as regrowth is observed once subcultured back onto unamended malt extract agar. How paclobutrazol changes the redbay host, by possibly changing physiological processes, tissue morphology, and disease response can help us understand resistance and susceptibility in the species better. This will contribute to future plant selection and breeding programs to mitigate the effects of this disease.

Genetic diversity and phylogeny of Fusarium species associated with panama disease of banana in Jordan A. Almomany, N. M. SALEM, M. Tahat, The University of Jordan, Amman, JORDAN

Fusarium species are a cosmopolitan group of fungi which infect banana and cause serious diseases as Fusarium wilt (panama disease) and crown rot disease. Panama disease of banana, caused by Fusarium oxysporum f. sp. cubense (Foc) tropical race 4 (TR4), was reported for the first time in Jordan in 2013. Its continued expansion and wide distribution in Jordan Valley presents undoubtedly significant risks to banana plantations. To address this we conducted comprehensive surveys for banana Fusarium wilt in 60 different banana farms during 2016-2017, in North and South Jordan Valley. Around 759 samples were collected from "Cavendish" banana plantations showing leaves yellowing and pseudostems internal vascular discoloration. The incidence of panama disease ranged from $25-100 \%$ with varied severity. Morphological identification of fungal isolates was done using conidial and hyphal structures. Further molecular identification of 40 selected Fusarium isolates was carried out by amplifying the internal transcribed spacer (ITS) region of the conserved ribosomal DNA (rDNA) and the translation elongation factor $1 \alpha$ (TEF) DNA region, followed by sequencing. Of the 40 Fusarium isolates, 52\% were identified as Foc TR4 that was recovered from most banana farms across Jordan Valley. Five other Fusarium species including $F$. brachygibbosum, F. equiseti, F. musae, F. proliferatum and F. verticillioides were also recovered. All TEF sequences of Fusarium species were compared and analyzed phylogenetically with maximum-likelihood. Our phylogenetic approach clearly indicates that diverse Fusarium species and genetic groups are associated with panama disease in Jordan, and thus raises the necessity of further epidemiological and molecular investigations.

Solarization effects on the soil microbiome at an organic vegetable farm in the Pacific Northwest (USA) N. Redekar, C. Trammell, J. Eberhart, J. L. PARKE, Oregon State University, Corvallis, OR, USA

Soil solarization is effective for managing certain soilborne plant pathogens and weeds without using chemical pesticides. Little is known about nontarget effects on soil microbial communities. We compared the soil microbiomes from solarized and non-solarized treatments at an organic vegetable farm in western Oregon. DNA was extracted from soil samples collected at the beginning and end of the four-week trial, and the 16S (bacteria and archaea) and ITS1 (fungi and oomycete) amplicons were sequenced using the Illumina MiSeq platform. Sequences were quality filtered and queried against Greengenes (for 16S), UNITE (for fungal ITS) and an in-house oomycete ITS1 reference database for identification at $97 \%$ sequence similarity with known species, followed by diversity analysis using QIIME. Relative OTU abundance was adjusted to account for total DNA in each sample. Soil solarization had a large effect on beta-diversity for fungal and bacterial communities. A decline in total OTU abundance was observed in solarized relative to non-solarized soil. For bacteria, the phyla Bacteriodetes, Acidobacteria, Actinobacteria, and Proteobacteria were most impacted. Fungal and oomycete genera most affected included Monographella, Cercophora, Fusarium, Alternaria, Mortierella, Cryptococcus and Pythium. Understanding solarization effects on the soil microbiome is important for predicting impacts on pathogens and beneficial microbes, and optimizing soil health.

Anaerobic soil disinfestation enhances endemic soil populations of Trichoderma

U. SHRESTHA (1), B. H. Ownley (2), D. Butler (1), (1) University of Tennessee, Plant Sciences, Knoxville, TN, USA; (2) University of Tennessee, Department of Entomology and Plant Pathology, Knoxville, TN, USA

Quantification of Trichoderma spp. in soil treated with anaerobic soil disinfestation (ASD) revealed that endemic soil populations of Trichoderma increased after treatment. Treatments included a sandy clay loam soil amended with labile carbon (C rate of $4 \mathrm{mg} \mathrm{C/g}$ soil at a C:N ratio 30:1), Trichoderma harzianum (RootShield) with and without labile $\mathrm{C}$ amendment, and a non-amended control. All treatments were irrigated and tarped with polyethylene at treatment initiation. After three weeks, soil was sampled at a 0 - to 5 -cm depth of each pot and stored at $4^{\circ} \mathrm{C}$ until analysis. Serial dilutions were used to quantify Trichoderma spp. on Trichoderma-selective medium. Soil DNA was extracted and Internal Transcribed Spacer (ITS) region copy number of $T$. harzianum in the soil DNA was quantified using Taqman qPCR. ASD treatments significantly enhanced populations of endemic Trichoderma spp. with the highest CFU (4.6 log CFU+1/g soil) as compared to the non-amended control ( $4.3 \mathrm{log}$ CFU $+1 / \mathrm{g}$ soil), while $T$. harzianum amended soil alone did not significantly increase total populations of Trichoderma spp. Quantification by qPCR indicated the highest ITS region copies of T. harzianum per milligram of soil in RootShield-amended soil; at the same time, endemic T. harzianum in ASD-treated soil was more than two-fold higher than the unamended control. Enhanced soil populations of endemic Trichoderma by ASD treatment may be an important mechanism of plant pathogen suppression by ASD.

Effects of anaerobic soil disinfestation on Sclerotinia sclerotiorum and Sclerotinia minor in Ohio muck soil A. D. SANABRIA (1), A. L. Testen (1), S. A. Miller (2), (1) The Ohio State University, Wooster, OH, USA; (2) Department of Plant Pathology, The Ohio State University, Wooster, OH, USA

Intensive lettuce production on muck soils leads to the buildup of Sclerotinia sclerotiorum and S. minor, causal agents of lettuce drop. The effects of anaerobic soil disinfestation (ASD) on S. sclerotiorum and S. minor were evaluated under growth chamber and field conditions. In growth chamber trials, ASD amendments molasses, wheat bran, and mustard greens at $20.2 \mathrm{t} / \mathrm{ha}$ and a $2 \%$ ethanol solution were tested in experiments designed to determine the effects of volatile compounds produced during ASD on mycelial growth and production of sclerotia. Amended, flooded muck soils were placed in the bottom of petri dishes and covered with another petri dish bottom with PDA medium and an inoculum plug of S. sclerotiorum or S. minor. Petri dishes were sealed with two layers of parafilm. Significant reductions in the number of sclerotia produced and mycelial growth of $S$. sclerotiorum and S. minor were observed for wheat bran, molasses and ethanol compared to the non-amended, flooded control. Field trials were conducted in plots amended with wheat bran (20.2 t/ha), molasses (10.1 t/ha), or wheat bran $(20.2 \mathrm{t} / \mathrm{ha})+$ molasses $(10.1 \mathrm{t} / \mathrm{ha})$, then flooded and covered; controls were not amended, but flooded and either covered or uncovered. Sclerotia of both pathogens were buried in treated soils and recovered after 4 weeks to assess their viability. Significant reductions compared to the controls in viability of sclerotia of both pathogens were observed for all carbon sources.

Efficacy of biochar in the management of anthracnose disease of pepper A. ADURAMIGBA-MODUPE, A. B. Olaitan, S. A. Adejumo, University of Ibadan, Ibadan, NIGERIA 
Anthracnose disease of pepper caused by Colletotrichum capsici is a potentially devastating disease in most regions where pepper is grown. There is an urgent need to promote cost effective and ecologically-friendly method in the disease management; biochar (a soil amendment) has been used to control many soil borne diseases. This research evaluated the efficacy of biochar produced from Maize Cob (MC) and Locust Bean Waste (LBW) at 4000C to suppress anthracnose disease of two pepper varieties (Capsicum annuum and C. frutescens). Biochar at four rates of 0,5, 10 and 15 t/ha were incorporated into $5 \mathrm{~kg}$ sterilized soil per pot, and replicated four times in a completely randomized design. Three weeks old pepper seedlings were transplanted into each pot and inoculated with spore suspension $\left(1 \times 106 \mathrm{~mL}^{-1}\right)$ of C. capsici grown on PDA using sterile distilled water. The data showed that amendment with $15 \mathrm{t}$ /ha LBW biochar enhanced the growth of the two pepper varieties by $30 \%$ in contrast to similar rate with MC. The incidence of anthracnose disease was significantly suppressed (30 to 45\%) with 5t/ha compared to higher biochar rates with MC being superior. Biochar at $15 \mathrm{t} / \mathrm{ha}$ was between 60 and $80 \%$ effective in the management of anthracnose disease of C. annum; while $5 \mathrm{t} / \mathrm{ha}$ effectively reduced disease progression and severity in C. frutescence within the same range. We concluded therefore that, biochar soil amendment was effective in anthracnose disease suppression in pepper.

Carbon amendments alter nutrient use and pathogen-suppressive potential of soil Streptomyces J. P. DUNDORE-ARIAS (1), L. J. Felice (1), R. Dill-Macky (2), L. L. Kinkel (3), (1) Department of Plant Pathology/University of Minnesota, St Paul, MN, USA; (2) University of Minnesota, St. Paul, MN, USA; (3) Department of Plant Pathology/University of Minnesota, Saint Paul, MN, USA

Organic matter inputs are a common practice for increasing soil microbial activity and biomass in agricultural fields, and can play an important role in disease management. The specific selective impacts of carbon amendments on microbial species interactions and metabolic capacities are not well understood. We investigated the effects of soil carbon amendments on nutrient use and antibiotic inhibitory profiles of Streptomyces in agricultural soils Soil mesocosms were established in sterile jars ( $500 \mathrm{~g}$ soil/jar), and amended at intervals over nine months with glucose, fructose, a nutrient mixture or no nutrient. Over 130 Streptomyces isolates were collected from amended and non-amended mesocosm soils, and nutrient utilization profiles on 95 different carbon substrates were determined using Biolog SF-P2 plates. A subset of isolates $(n=40)$ was characterized for their ability to inhibit one another. Carbon amendments were found to expand niche width, growth efficiency, and niche overlap among soil Streptomyces. Carbon inputs also increased the frequency, but reduced the intensity of inhibitory interactions among soil Streptomyces. These results suggest that carbon amendments to soil may intensify competition for preferred resources and enrich antagonistic phenotypes. Thus, the selective impacts of carbon amendments on soil microbiomes may contribute to the establishment of pathogen-suppressive soils in agricultural systems.

Effect of Active Flower, a nutrient spray containing boron, on disease suppression in cucumber (Cucumis sativus L.) and canola (Brassica napus L.) L. NI, Z. K. Punja, Simon Fraser University, Burnaby, BC, CANADA

Active Flower ${ }^{\mathrm{TM}}(\mathrm{AF})$ is a fertilizer that contains N: $\mathrm{P}: \mathrm{K}(8: 4: 12)$ and boron $(\mathrm{B}, 3 \%)$ with potential for alleviating the severity of plant diseases. Greenhouse cucumber plants 'Tasty Green' treated with three weekly applications of AF at 0.3 and $0.5 \mathrm{ml} / 100 \mathrm{ml}$ had significantly (P $\leq 0.05$ ) lower incidence of powdery mildew caused by Podosphaeria xanthii. Canola (Brassica napus L.) 'Westar' treated with four weekly applications of AF at 0.5 $\mathrm{ml} / 100 \mathrm{ml}$ demonstrated reduced development of Sclerotinia sclerotiorum on detached leaves. Nutrient analyses of leaves receiving four applications of $\mathrm{AF}$ at $0.5 \mathrm{ml} / 100 \mathrm{ml}$ showed that $\mathrm{N}$ and $\mathrm{B}$ levels were enhanced by $22 \%$ and $500 \%$ in canola leaves, and $25 \%$ and $700 \%$ on cucumber leaves, respectively, compared to a water control. Neither $\mathrm{N}$ nor B had a significant effect on fungal pathogen growth when tested at rates of 250,500 and 750 $\mathrm{ppm}$ in potato dextrose broth. Application of $\mathrm{AF}$ at $0.5 \mathrm{ml} / 100 \mathrm{ml}$ significantly $(\mathrm{P} \leq 0.05)$ increased phenolic levels by $200 \%$ in canola leaves and $400 \%$ in cucumber leaves compared to the control. Enzymatic activities of peroxidase and polyphenol oxidase were not significantly affected by AF treatment. The mechanism of action of AF in reducing fungal pathogen development may be associated with physiological changes in the plant is response to $\mathrm{B}$, which may include enhanced lignin formation.

Synergism between food additives and heat to reduce postharvest sour rot of oranges

L. PALOU (1), V. Taberner (1), N. Jerby (2), B. de la Fuente (1), (1) IVIA, Postharvest Technology Center, Montcada, Valencia, SPAIN; (2) Higher Agronomic Institute of Chott-Mariem, University of Sousse, Chott Mariem, Sousse, TUNISIA

Postharvest sour rot of citrus fruit, caused by Geotrichum citri-aurantii, is typically controlled with the application of specific fungicides such as propiconazole or guazatine (currently banned in the European Union). However, there is an increasing interest in the implementation of nonpolluting alternative control methods. In this research, 1 min dips in 3\% aqueous solutions of the food additives sodium methyl paraben (SMP), sodium ethyl paraben (SEP), potassium sorbate (PS) and sodium benzoate (SB), all applied at 20 or $50^{\circ} \mathrm{C}$, were evaluated with 'Barnfield' Navel oranges artificially inoculated with G. citri-aurantii about $24 \mathrm{~h}$ before treatment application. All treatments significantly reduced the incidence (percentage of infected fruit) and severity (lesion diameter) of the disease, and a strong synergy between food additives and heat was observed. After 6 days of incubation at $28^{\circ} \mathrm{C}$, while disease incidence on control fruit (dipped in water at $20^{\circ} \mathrm{C}$ ) was $80 \%$, it was $13,30,43$ and $13 \%$ on oranges dipped in SMP, SEP, PS and SB solutions at $20^{\circ} \mathrm{C}$, and $0,5,10$ and $0 \%$ on oranges dipped in solutions at $50^{\circ} \mathrm{C}$, respectively. Nevertheless, heat increased the slight incidence of rind spots caused by some salt treatments. In a further assay, no significant differences in incidence and severity were found between rinsed (5 s spray with tap water at low pressure) and non-rinsed 'Valencia Late' oranges previously dipped in $3 \% \mathrm{SMP}$ or SB solutions at $20^{\circ} \mathrm{C}$.

Grafted processing tomato for the management of southern blight in California

N. SOLARES (1), J. J. Nunez (2), A. I. Putman (1), (1) University of California at Riverside, Riverside, CA, USA; (2) Univ of California Coop Ext, Bakersfield, CA, USA

Southern blight, caused by the soil borne fungus Athelia rolfsii, is a disease of many crops and is particularly difficult to manage in processing tomatoes. The objective of this research is to evaluate grafting for southern blight management in California processing tomatoes. Two cultivars H5608 and H8504 were evaluated as scions grafted to rootstock of Maxifort, a hybrid cultivar resistant to southern blight, or as non-grafted controls. Yield data was collected from a field trial conducted in soil with A. rolfsii that consisted of $165 \mathrm{~m}$ long plots arranged in a randomized complete block (RCB) with 7 replications. A greenhouse study was conducted to evaluate the performance of each treatment under $0,5,10$, and 20 sclerotia per $100 \mathrm{~cm}^{3}$ of soil. Oneplant pots were arranged in a RCB with 8 replications, and disease severity was rated every 2 to 3 weeks. At 5 and 10 sclerotia per $100 \mathrm{~cm}^{3}$ of soil, disease severity in the greenhouse was statistically similar among grafted H5608, grafted H8504, and non-grafted H8504 on the final three rating dates, whereas disease caused by $A$. rolfsii was significantly more severe in non-grafted H5608 compared to the other three treatments. In the field, yield was not significantly influenced by cultivar, but was significantly higher in grafted plots (by $23 \%$ or $36 \%$ ) compared to the non-grafted treatments. Our results suggest grafting susceptible cultivars to the resistant rootstock Maxifort can reduce southern blight and augment yield. 
Impact of heat and soil moisture stress on chickpea plant infection with fungal pathogens

M. SHARMA (1), R. Ghosh (2), (1) ICRISAT, Patancheru, Telangana, INDIA; (2) International Crops Research Institute for the Semi-Arid Tropics (ICRISAT), Hyderabad, INDIA

Chickpea (Cicer arietinum L.) is one of the essential semiarid tropical legume crops grown in the post-rainy season on stored soil moisture (south Asia and spring-sown Mediterranean) or as a Mediterranean winter crop. In both of these instances, the crop is exposed to terminal drought accompanied with high temperatures. Increase in the frequency of climate extremes is influencing the distribution, establishment and epidemiology of chickpea diseases. Changes in the disease spectrum in chickpea for the past one decade were monitored through extensive surveys. Analysis of disease and weather data indicated shift in the occurrence and distribution of chickpea diseases as well as emergence of new diseases. An extension in the geographical distribution of $F$. oxysporum f. sp. ciceris, wilt pathogen of chickpea has been reported under future climatic scenarios. Several minor diseases like dry root rot (Rhizoctonia bataticola) is becoming more intense in tropical humid areas under high temperature and soil moisture stress. Contrary to this, sporadic occurrence of collar rot (Sclerotium rolfsii) has been noticed under high soil moisture levels. Host resistance, changes in biochemical and molecular profiling influenced by soil moisture levels and rise in temperature in relation to these diseases have also been studied. Extensive research is required in this domain to develop adaptation and mitigation strategies for sustained food security. Breeding being an essential part of crop improvement needs to keep pace with these emerging diseases.

Efficacy of anaerobic soil disinfestation on the germination of sclerotia of Sclerotinia sclerotiorum

T. Mahalingam (1), K. P. Somachandra (2), C. Rajapakse (1), R. ATTANAYAKE (1), (1) University of Kelaniya, Dalugama, SRI LANKA; (2) Regional Agricultural Research \& Development Centre, Bandarawela, SRI LANKA

White mold, caused by Sclerotinia sclerotiorum, is often found in cabbage, bean and carrots in Sri Lanka. Although fungicide applications are a common method to manage the disease, fungicides provide unsatisfactory control since the pathogen can produce survival structures. Anaerobic soil dis infestation (ASD) is a method to control various soil borne pathogens, but studies on the effect of ASD on S. sclerotiorum are limited. Therefore, this study was performed to determine the effect of carbon (C) sources in ASD on the germination of sclerotia of S. sclerotiorum. Cabbage and leek cull piles and durian (Durio zibethinus) husks were tested at two rates. Nylon pouches containing 10 sclerotia were buried in a $\mathrm{C}$ source-amended soil (60 and 100 $\mathrm{mg} / \mathrm{g}$ soil) with four replicates, watered to field capacity, covered with black plastic and incubated at $23^{\circ} \mathrm{C}$ for 6 weeks in trial 1 . Trial 2 was conducted at lower $\mathrm{C}$ rates $(30$ and $60 \mathrm{mg} / \mathrm{g}$ soil) of cabbage and leek cull piles for 3 weeks. After incubation, sclerotia were recovered, cultured in a selective medium and percent sclerotial germination was assessed. No sclerotia germinated from the cabbage leek treatment in trial 1 . The highest germination (89\%) was observed for the aerobic control. No significant difference was detected among durian amendments and the anaerobic control. Sclerotial germination ranged $9-28 \%$ for cabbage and $3-27 \%$ for leek amendments in trial 2 . These treatments significantly reduced sclerotia germination compared to the controls $(89-97 \%)(P<0.05)$. This study shows that ASD using cabbage and leek can be effective in reducing sclerotial germination of the soil-borne pathogen S. sclerotiorum.

Microwave radiation reduces survival of Fusarium pseudograminearum in infected durum wheat stubble T. PETRONAITIS (1), C. Forknall (2), S. Simpfendorfer (3), (1) Department of Primary Industries (DPI), Tamworth, AUSTRALIA; (2) Department of Agriculture and Fisheries, Toowoomba, AUSTRALIA; (3) Department of Primary Industries, Tamworth, AUSTRALIA

Fusarium pseudograminearum $(F p)$ is a stubble-borne fungus which is the main causal species of the disease crown rot across the Australian wheat-belt. Microwave radiation may offer a rapid and chemical-free approach to reduce $F p$ inoculum levels in these residues. Crown and node sections ( $15 \mathrm{~mm})$ of durum wheat stubble infected with $F p$ collected post-harvest from an inoculated field experiment were microwaved using a conventional $1100 \mathrm{~W}$ microwave oven for $0,15,30,60$ and $120 \mathrm{~s}$ either embedded in moist soil or without soil. The survival of $F p$ after microwave radiation was assessed by culturing on 1/4 PDA plus novobiocin. Microwave radiation for 15, 30, 60 and $120 \mathrm{~s}$ without soil reduced recovery of $F p$ to an average of $69 \%, 46 \%$, $30 \%$ and $19 \%$ of stubble sections, respectively, compared to the control $(72 \%)$. Efficacy was improved by embedding stubble segments in moist soil, where microwaving for $15,30,60$ and $120 \mathrm{~s}$ reduced recovery of $F p$ to an average of $34 \%, 0 \%, 1 \%$ and $0 \%$ of stubble sections, respectively. Although microwave radiation significantly reduced the survival of $F p$ in durum wheat stubble in the laboratory, the practicality of this method for crown rot management under field conditions is yet to be established.

New technology for controlling strawberry diseases and arthropods

W. J. JANISIEWICZ (1), B. Evans (1), F. Takeda (1), W. M. Jurick II PhD (2), B. Short (1), T. Leskey (1), (1) USDA-ARS AFRS, Kearneysville, WV, USA; (2) USDA-ARS Food Quality Laboratory, Beltsville, MD, USA

Fungicides are the main weapon for controlling diseases of strawberries; however, their use has various problems including development of resistance in pathogens, restrictions on their use especially in protective culture, and a growing public demand for produce free of pesticide residues. Our research has been focused on developing alternative strategies that address those limitations. We developed PhylloLux ${ }^{\mathcal{O}}$ technology which encompasses night-time UV-C irradiation for $60 \mathrm{sec}\left(12.3 \mathrm{~J} / \mathrm{m}^{2}\right)$ twice a week followed by application of antagonists Aureobasidium pullulans and Metschnikowia pulcherrima. We identified that specific dark periods (2-4 h depending on pathogen) immediately following UV-C treatment prevented activation of a light-induced fungal DNA repair mechanism increasing the killing power of the UV-C treatment 6 to 10-fold and making this approach practical because effective irradiation doses were not toxic to plants and the energy cost was substantially reduced. The treatment was effective against gray mold (Botrytis cinerea), powdery mildew (Podosphaera aphanis) and anthracnose (Colletotrichum spp.). The two antagonists filled the microbial void after UV-C treatment, prevented recolonization by unwanted microbes and increased robustness of the control. In recent studies, nightly irradiation alone vastly reduced infestation by two-spotted spider mites (Tetranychus urticae). The PhylloLux ${ }^{\circ}$ technology also may be applicable in vegetable and ornamental production.

Control of stem-end rot disease in mango fruit cultivar 'Karuthacolomban' using non-agrochemical methods C. Karunanayake (1), A. Kulasinghe (1), S. Somaratne (1), N. ADIKARAM (2), (1) Department of Botany, Faculty of Natural Sciences, The Open University of Sri Lanka, Nawala, SRI LANKA; (2) National Institute of Fundamental Studies, Kandy, SRI LANKA

The local mango cultivar 'Karuthacolomban' is highly susceptible to stem end rot (SER) and presently the disease is controlled by synthetic fungicides. The study investigates alternate methods to reduce SER in mango. At the pre-harvest stage, the amount of potassium fertilizer added to trees was altered. Fifteen year old fruit-bearing trees (6 per treatment arranged as a CRBD) were provided with soil fertilizer at the fruit development stage as recommended, but varying the level of potassium. SER development was assessed in harvested fruits from both natural infections and following artificial inoculation. In a separate postharvest dip application, the effect of selected essential oils (EO) in reducing SER was tested. Six EOs were tested at concentrations 500-1000 $\mu 1$ 1-1 by in vitro Poisoned food bio-assay against Lasiodiplodia theobromae causing SER. The essential oils that gave a 
significant reduction $(\mathrm{P}<0.05)$ of radial mycelial growth in three replicate trials were used in in vivo fruit dip-treatments $(3$ min). In field trials, potassium at $3 \times$ recommended dose reduced natural SER development, but reduction was not significant. SER was reduced significantly $(\mathrm{P}<0.05)$ in artificially inoculated fruits. The essential oils Basil, Cardamom (700 $\mu 1$ 1-1) and Citronella (400 $\mu 1$ 1-1) significantly reduced the L. theobromae growth in vitro and Cardamom oil at $700 \mu \mathrm{l}$ 1-1 significantly reduced the severity of SER in vivo. Neither pre nor postharvest treatments significantly affected sensory properties except for external peel appearance which was altered by EO treatment as indicated by a Principle Component analysis (PCA). Potassium is known to increase firmness of plant organs while EOs act directly on the pathogen in reducing SER in mango fruit.

Suppression of wheat powdery mildew (Blumeria graminis f. sp. tritici) by nighttime exposure to UV-B radiation

L. L. GRANKE, B. Rowland, Dow AgroSciences, Indianapolis, IN, USA

Greenhouse testing is critical to the discovery of new fungicides. Test quality for slow-growing pathogens such as Zymoseptoria tritici can be impacted by powdery mildew if an aggressive fungicide treatment program is not followed. In this study, we found that ultraviolet (UV)-B radiation (305-315 nm, 4-6 W/m $\left.\mathrm{m}^{2}\right)$ applied for $8 \mathrm{~h}(16 \mathrm{~h}$ light: $8 \mathrm{~h} \mathrm{UV-B})$ suppressed wheat powdery mildew by limiting conidial germination, colony development, and sporulation in growth chamber studies. Treatment during the entire $8 \mathrm{~h}$ dark cycle worked better than treatment for $6 \mathrm{~h}$. Treatment for $4 \mathrm{~h}$ was not significantly different from no treatment at all. UV-B was more effective as a 0 day protectant than as a 1 day curative treatment. UV-B applied overhead provided greater suppression of powdery mildew on the adaxial surface of the leaf than the abaxial surface. Wheat that was pre-treated with UV-B daily (16 h light: $8 \mathrm{~h} \mathrm{UV-B}$ ) for 1 month prior to inoculation was as susceptible to $Z$. tritici as wheat that received no UV-B pre-treatment. Nighttime exposure to UV-B appears to be a viable non-chemical strategy for suppression of wheat powdery mildew caused by Blumeria graminis f. sp. tritici.

Long-term effects of potato cropping system strategies on soilborne diseases and soil microbial communities R. P. LARKIN, USDA-ARS, New England Plant, Soil, \& Water Lab, Orono, ME, USA

Cropping systems incorporating soil health management practices, such as longer rotations, disease-suppressive crops, reduced tillage, and/or organic amendments can substantially affect soil microbial communities, and potentially reduce soilborne potato diseases and increase productivity, but longterm research trials are needed. In field trials originally established in 2004, different 3-yr potato cropping systems focused on specific management goals of soil conservation (SC), soil improvement (SI), and disease-suppression (DS) were evaluated and compared to a 2-yr standard rotation (SQ) and a non-rotation control (PP) for their effects on soilborne diseases and soil microbial communities over time. After 12-14 years, SI system produced higher potato yield and microbial activity than all other systems. DS maintained significantly lower disease (black scurf and common scab) severity than all other systems, and increased yield and microbial activity relative to SQ and PP. Soil microbial community characteristics and composition, as determined by fatty acid methyl ester (FAME) profiles and genus-level IDs from 16s rDNA (bacterial) and ITS2 (fungal) amplicon sequencing, were distinctly different among the systems, and may be responsible for disease suppression. These results indicate that soil health management practices incorporated into potato cropping systems can alter soil microbial communities and sustainably reduce soilborne diseases and enhance productivity.

ZnO nanoparticles for postharvest strawberry grey mould control

N. RASIUKEVICIUTE (1), A. Valiuskaite (1), N. Uselis (1), Z. Luksiene (2), (1) Lithuanian Research Centre for Agriculture and Forestry Institute of Horticulture, Babtai, Kaunas dist., LITHUANIA; (2) Institute of Applied Research, Vilnius University, Vilnius, LITHUANIA

It is well known that most of the pathogens can develop high resistance to chemical fungicides. Researchers all over the world are looking for new technologies reducing pesticide consumption. New implements are needed to reduce postharvest losses and control food pathogens. This method is based on measures of zinc oxide $(\mathrm{ZnO})$ nanoparticles. Photosensitization is an innovative approach for eliminating fruit pathogens based on simultaneous use of photoactive compound (photosensitizer) and visible light. This study aimed to evaluate the efficacy of new measures for reducing contamination of pathogenic fungi in strawberry. The research was carried out at the LAMMC Institute of Horticulture. The photosensitization (PH) evaluated in LEDbased light source prototype. Experimental treatments included 1) control, 2) $\mathrm{ZnO}+\mathrm{PH}$, and 3) sterile water $+\mathrm{PH}$. Visually healthy strawberry cv. "Darselect" fruits were incubated with photosensitizer $\mathrm{ZnO}$ for $1 \mathrm{~h}$ and illuminated $30 \mathrm{~min}$. with light $(\lambda=400 \mathrm{~nm}$ with an energy density of $20 \mathrm{~mW} / \mathrm{cm} 2)$. Decontaminated fruits assessed after 4 and 8 days of simulated storage at $5-7^{\circ} \mathrm{C}$. The experimental results revealed that 4 and 8 days after the $\mathrm{ZnO}$ treatment, the number of Botrytis infected fruits reduced by $10 \%$ and $13 \%$. In water treatment, the incidence of the grey mould after a 4-day storage was 9\% and 19\% higher compared to the control and $\mathrm{ZnO}$. Data indicated that $\mathrm{ZnO}$ nanoparticles reduced strawberry contamination with Botrytis cinerea. Such $\mathrm{ZnO}$ nanoparticles properties are a promising tool for the development of effective nonchemical fungicides.

Meta-analysis of chlorine usage as a disinfestant in plant production W. E. COPES, USDA ARS, Poplarville, MS, USA

Chlorine has a long history of usage as a disinfestant to inactivate plant pathogens on tools, equipment, production surfaces and harvested produce surfaces in plant production systems. The main active ingredient of many commercial chlorine sanitizers is sodium hypochlorite, which has been used at rates from 1 to $20 \%$ product and exposure times as short as $10 \mathrm{sec}$ to as long as $60 \mathrm{~min}$. Many studies have shown chlorine disinfestants to be highly effective, while others have shown moderate to poor activity against some plant pathogens and when treating some production surfaces. The current study looks at summary means and variances of chlorine dose and treatment duration used to inactivate bacteria, fungi, viroids and viruses in multiple production stages and applications. A meta-analysis was performed on data from manuscripts presenting plant disease control in production of many agricultural and horticultural plant hosts. Conclusions will be presented describing categorical groupings where differing rates were needed to achieve inactivation of plant pathogens.

Alternative control of Macrophomina phaseolina in soybean through the use of ozone

M. BRACALE (1), M. G. Canteri PhD (1), L. Henrique Fantin (1), K. Braga (1), F. André Araujo (2), C. Hideki Sumida (1), (1) Universidade Estadual de Londrina, Londrina, Paraná, BRAZIL; (2) Universidade Estadual de Londrina, LLondrina, BRAZIL

The ozonation is an alternative method for the control of plant diseases. The objective of the study was to test the viability of ozone in the control of the fungus Macrophomina phaseolina. For the assay, Petri dishes with PDA culture medium containing M. phaseolina were used, which were maintained in BOD in the dark at $28^{\circ} \mathrm{C}$. At 4 days of growth, the Petri dishes were opened and exposed to ozone for 25 minutes at total. Every five minutes, one treatment was withdrawn, totalizing 6 treatments with one control. Each treatment had 4 replicates. After exposure to ozone, the Petri dishes were maintained in BOD in the dark at $28^{\circ} \mathrm{C}$ until the mycelial growth of the control reached the edge of the dish. The evaluation consisted in measuring mycelial growth with the help of a pachymeter. The ozonation process was performed by an ozone generator unit coupled to a hermetically sealed chamber. Exposure to ozone occurred through indoor air circulation. The data were submitted to regression analysis and the models adjusted according 
to the coefficient of determination and beta parameters of the regression. The results obtained presented reduction of mycelial growth of the pathogen with the minimum exposure to ozone. The ozonation technique presented viable results for improvement and execution of more studies. The present work requires repetition and possible addition of longer periods of exposure.

Role of volatile fatty acids in suppression of Sclerotium rolfsii during anaerobic soil disinfestation

K. SWILLING (1), U. Shrestha (1), B. H. Ownley (2), D. Butler (1), (1) University of Tennessee, Plant Sciences, Knoxville, TN, USA; (2) University of Tennessee, Department of Entomology and Plant Pathology, Knoxville, TN, USA

Anaerobic soil disinfestation (ASD) is a non-chemical control method used for soilborne plant pathogens in high value specialty crops. During ASD, volatile fatty acids (VFAs) are produced. Two trials were conducted in an anaerobic chamber to determine if two VFAs found in abundance during ASD (acetic and n-butyric acid) suppressed germination of Sclerotium rolfsii. In trial 1, sclerotia were exposed to VFAs at 4,8 or $16 \mathrm{mmol} / \mathrm{kg}$ soil and soil pH $4.5,5.5$, or 6.5 in sandy soil for 4 days, after which germination was assessed and compared to controls exposed to $\mathrm{HCl}$ or water. In trial 2 , sclerotia were exposed to VFAs at 4 or $16 \mathrm{mmol} / \mathrm{kg}$ soil and soil $\mathrm{pH} 4.5$ or 5.5 in sandy or sandy clay loam soil to assess the impact of clay content on VFAs against $S$. rolfsii. Sclerotia germination was $60 \%$ less than controls in sandy soil with $4 \mathrm{mmol}$ acetic acid $/ \mathrm{kg}$ soil and $4.5 \mathrm{soil} \mathrm{pH}$; there was no suppression at 5.5 or 6.5 soil pH. At 8 and $16 \mathrm{mmol}$ acetic acid/kg soil, germination was almost $100 \%$ less than controls at $4.5 \mathrm{soil} \mathrm{pH} ; 20 \%$ to $60 \%$ less at $5.5 \mathrm{soil} \mathrm{pH}$; and not suppressed at $\mathrm{pH}$ 6.5. Increasing clay content significantly reduced activity of VFAs on sclerotia germination at 4, but not at $16 \mathrm{mmol}$ acetic acid/kg soil. In general, acetic and n-butyric acid equally suppressed sclerotia germination. VFAs are likely an important mechanism of $S$. rolfsii suppression during ASD, and that activity is significantly affected by VFA concentration, soil $\mathrm{pH}$, and soil type

The deposition of silicon linked to the reduction in susceptibility to strawberry powdery mildew C. ASIANA, A. Hall, K. Davies, University of Hertfordshire, Hatfield, Herts, UNITED KINGDOM

An important disease of protected strawberries in the UK is strawberry powdery mildew (Podosphaera aphanis), controlled by the frequent use of fungicides. Our work has shown that the weekly use of a silicon nutrient in the fertigation tubes, results in reduced disease susceptibility. The work reported here aims to quantify deposition of silicon applied through the roots in a glasshouse. The glasshouse plants were treated weekly via the roots either with silicon nutrient or deionised water. After 8 weeks the plants were harvested, then sections of the leaves, petioles and roots were cut and stained with a fluorescence dye, Lyso tracker yellow HCK-123. Samples were examined using a confocal microscope at x 400 magnification and a wavelength set at $450 \mathrm{~nm}$. Results showed that the silicon had accumulated in the epidermis and palisade layer of the leaves, and in the vascular bundles of the petiole and roots. Silicon treated plants contained larger amounts of silicon than the untreated and measurements of fluorescence intensity confirmed this. Silicon treated leaves measured $7.923 \mathrm{~W} / \mathrm{m}^{2}$ and untreated $2.209 \mathrm{~W} / \mathrm{m}^{2}$, treated petioles $7.770 \mathrm{~W} / \mathrm{m}^{2}$ and untreated $1.913 \mathrm{~W} / \mathrm{m}^{2}$, treated root $11.594 \mathrm{~W} / \mathrm{m}^{2}$ and untreated $1.266 \mathrm{~W} / \mathrm{m}^{2}$. This showed that the application of the silicon through the roots is taken up and deposited in the plant system. Analysis of epidemics from the field trial showed statistically significant disease reduction in the treated plants, with silicon deposited in the leaves.

Selection of reference genes to develop an RNA-based viability assay in response to thermotherapy N. THAPA, E. G. Johnson, M. M. Dewdney, University of Florida, Lake Alfred, FL, USA

Huanglongbing (HLB), caused by the bacterium Candidatus Liberibacter asiaticus (CLas), is one of the most destructive citrus diseases to threaten citrus industries worldwide. Thermotherapy is being tested in Florida as a potential management practice for HLB. To ensure thermotherapy's effectiveness, a post-treatment viability assessment of CLas is important. Our overall objective is to develop an RNA-based viability assay for CLas to measure lethality of high temperatures. It will be evaluated by comparing the expression of CLas $16 \mathrm{~S} \mathrm{rRNA}$, and other CLas transcripts if necessary, at different timetemperature treatments $\left(55^{\circ} \mathrm{C} / 0 \mathrm{~s} ; 55^{\circ} \mathrm{C} / 60 \mathrm{~s} ; 55^{\circ} \mathrm{C} / 90 \mathrm{~s} ; 55^{\circ} \mathrm{C} / 120 \mathrm{~s} ; 60^{\circ} \mathrm{C} / 30 \mathrm{~s}\right.$; and untreated control). To study gene-expression, we used qRT-PCR, which is a robust, highly sensitive and reproducible method. Citrus gene expression will be used for normalization because citrus tissue remains viable post-treatment. We studied candidate citrus genes for stable expression throughout the treatments to select reference genes for the viability assay. Nine candidate reference genes were evaluated: GAPDH, TUB, ACT2, GAPC2, FBOX, SAND, EF1 $\alpha, U P L 7$, and UBC21. geNorm and BestKeeper software were used to determine the most stable reference genes for the six different treatments, and among the nine genes, GAPC2, EF1 $\alpha$, and GAPDH were the best candidates. We chose $G A P C 2$ and $E F 1 \alpha$, as reference genes for further gene expression studies.

Effect of crop rotation and tillage on Rhizoctonia root and crown rot and Rhizoctonia solani AG 2-2 soil populations D. L. Fonseka (1), M. Wang (2), W. Iverson (2), W. B. Stevens (2), A. K. KALIL (1), (1) North Dakota State University, Williston Research Extension Center, Williston, ND, USA; (2) USDA-ARS, Sidney, MT, USA

Rhizoctonia root and crown rot (RRCR) caused by Rhizoctonia solani AG 2-2 IV and IIIB is a devastating disease of sugar beet (Beta vulgaris subsp. vulgaris) in Montana and North Dakota. Small grain crops such as barley are not susceptible to $R$. solani AG 2-2 and thus barley-beet rotations are common. Tillage is also recommended for control of residue-borne fungal pathogens such as $R$. solani, and intensive tillage has for many years been common practice in sugar beet production. Growers are increasingly choosing to rotate sugar beet with $R$. solani AG 2-2 susceptible crops such as soybean and corn and practice reduced tillage to improve soil health. It is unclear how these practices will impact inoculum levels and RRCR risk. Barley-beet and corn-soy-barley-beet crop rotations managed with either full or reduced tillage were evaluated for RRCR. Soil populations of $R$. solani AG 2-2 were measured under these treatments using quantitative real-time PCR. Soil samples collected prior to planting contained higher levels of $R$. solani AG 2-2 under the barley-beet rotation, possibly due to the shorter rotation between sugar beet crops. The corn-soy-barley-beet rotation increased crown rot severity in comparison to the barley-beet rotation while there was no difference in root rot severity and post-harvest soil populations of $R$. solani $A G$ 2-2. Reduced tillage did not increase RRCR or soil populations of $R$. solani $A G$ 2-2.

Fungal pathogens associated with maize crown and root rot under maize/legume intercropping system in the Limpopo province, South Africa M. KENA, S. Maake, I. Mariga, L. Molatudi, M. Zerizghy, University of Limpopo, SOVENGA, SOUTH AFRICA

Smallholder farmers in Limpopo practice cereal/legume intercropping to mitigate risks of total crop failure due to various factors including diseases. Knowledge on the effect of this cropping practice on the prevalence of fungal pathogens causing maize crown and root rot is generally lacking in Limpopo despite its wide application. The main aim of this study was to evaluate maize/legume intercropping as a means for managing soil-borne diseases. Field experiments were set up under rain-fed and supplementary irrigation conditions as $2 \times 4$ factorial arranged in randomised complete block design with block replicated 2 times (irrigated experiment) and 3 times (rain-fed experiments). Disease assessment was carried out during seedling stage, flowering and harvesting. Fungal species were isolated from symptomatic roots, crown and stems and identified using morphological and DNA sequence analyses. Intercropping maize with groundnut and cowpea significantly reduced disease severity $(<20 \%)$ in both experiments. A significant increase in 
disease severity ( $>90 \%$ ) was recorded in maize/soybean intercropping under rain-fed experiments. More than $80 \%$ of isolates obtained from symptomatic roots and crown belonged to Fusarium spp with F. oxysporum (98.3\%) being the most dominant. Stenocarpella maydis was also isolated from all treatments except in maize/soybean intercropping. Macrophomina phaseolina was most isolated pathogen in this treatment. Rhizoctonia solani was associated with more than $90 \%$ of seedling damping-off and blight in control treatments. There was more than $50 \%$ reduction in seedling damping-off under maize/legume intercropping in both irrigated and rain-fed trials. Results show that groundnut and cowpea when intercropped with maize can be effective in managing soil-borne maize diseases under smallholder farming systems.

Using nanoparticles of metalloids and metallic oxides in plant disease suppression

W. H. ElMER (1), C. Plaza Pérez (2), C. Ma (3), R. De La Torre-Roche (1), F. J. Ferrandino (1), J. A. LaMondia (4), J. C. White (1), (1) Connecticut Agric Exp Station, New Haven, CT, USA; (2) Federal University of Lavras, Lavras MG, BRAZIL; (3) The Connecticut Agric Expt Station, New Haven, CT, USA; (4) The Connecticut Agric Expt Station, Windsor, CT, USA

Micronutrients, such as $\mathrm{B}, \mathrm{Cu}, \mathrm{Mn}, \mathrm{Mo}$, and $\mathrm{Zn}$, can activate enzymes to initiate defense mechanisms against plant diseases. Other elements like Ag, Ce, $\mathrm{Si}$, and Ti can also affect plant health through a variety of mechanisms. When these elements are applied as nanoparticles (1-100 nm) (NP), they perform better than their larger bulked equivalents. Evidence of basipetal transport has allowed the possibility of using foliar applications of NP to affect root nutrition and response to disease. In separate studies, NP were sprayed at $500 \mu \mathrm{g} / \mathrm{ml}$ onto chrysanthemums, grapes, pumpkins, soybeans, and strawberries. Pumpkins sprayed weekly with either $\mathrm{CuO} \mathrm{NP}, \mathrm{ZnO} \mathrm{NP}$, or conventional fungicides had less powdery mildew than their $\mathrm{Cu}$ and $\mathrm{Zn}$ salts or untreated plants $(P=0.021)$, whereas Chardonnay grapes treated with $\mathrm{NP}$ of $\mathrm{CuO}$ had only slightly less powdery mildew than untreated plants $(P=$ 0.080). There was a tendency for B NP to increase strawberry plants weights when inoculated with Verticillium dahliae. Both $\mathrm{CuO} \mathrm{NP}$ and $\mathrm{MnO} \mathrm{NP}$ increased chrysanthemum weights in soils infested with Fusarium oxysporum f. sp. chrysanthemi $(P=0.050)$. The disease ratings of soybeans sprayed with $\mathrm{NP}$ of $\mathrm{Ag}, \mathrm{B}, \mathrm{Ce}, \mathrm{Cu}, \mathrm{Mn}, \mathrm{Mo}, \mathrm{Si}, \mathrm{Ti}$, and $\mathrm{Zn}$ and grown in soil infested with $\mathrm{F}$. virguliforme were 27,8 , and $10 \%$ lower with $\mathrm{Ag}, \mathrm{Cu}$, and $\mathrm{Mn}$, respectively $(P=0.027)$, when compared to controls. Overall, $\mathrm{NP}$ of $\mathrm{CuO}$ and $\mathrm{MnO}$ may offer a novel approach to improve plant health across many crops.

Design, Operation, and Efficacy of an Apparatus Using Ultraviolet Light to Suppress Powdery Mildew of Strawberry in Open Field Production Systems

R. BORBA ONOFRE (1), J. Batistella Gatto (2), M. Marin (3), D. M. Gadoury (4), A. Stensvand (5), M. Rea (6), A. Bierman (6), N. Peres (3), (1) University of Florida - Gulf Coast Research and Education Center, Wimauma, FL, USA; (2) Universidade de Passo Fundo, Passo Fundo, BRAZIL; (3) University of Florida, Wimauma, FL, USA; (4) Cornell University, Geneva, NY, USA; (5) Norwegian University of Life Sciences, Ås, NORWAY; (6) Lighting Research Center, Rensselaer Polytechnic Institute, Troy, NY, USA

Strawberry powdery mildew caused by Podosphaera aphanis can be destructive in both covered and open field production. Fungicides are the principal means used to suppress this disease. Ultraviolet light (UV) has effectively suppressed diverse powdery mildews in several greenhouse and tunnel production trials. Over two growing seasons, we exposed open field plots of mildew-susceptible strawberry plants to UV light $(254 \mathrm{~nm}, \mathrm{FWHM}<5 \mathrm{~nm})$ approximately $1 \mathrm{hr}$ after sunset either once or twice weekly. The apparatus used was tractor-drawn, and consisted of a dense reflectorized hemicylindrical array of multiple UV fluorescent lamps. Total UV energy at the plant canopy surface plant was approximately $84.5 \mathrm{or} 169 \mathrm{~J} \mathrm{~m}^{-2}$ at tractor speeds of $4.5 \mathrm{~km} \mathrm{~h}^{-1}$ and $2.25 \mathrm{~km} \mathrm{~h}^{-1}$, respectively. Comparative fungicide treatments consisted of cyflufenamid alternated with quinoxyfen every two weeks. In the 2016-17 growing season, UV reduced foliar mildew up to 99\% compared to the nontreated control, and provided greater suppression than fungicide treatments. The 2017-18 trials are ongoing, but treatment separations after one month indicate that UV is again providing suppression equal to or better than biweekly fungicide treatments. Deleterious effects of UV exposures have not been noted on plant growth or yield. Effects of UV treatments on Botrytis cinerea and Colletotrichum acutatum will also be assessed in 2017-18.

Novel sources of resistance to septoria nodorum blotch in the Vavilov wheat collection identified by GWAS H. PHAN (1), K. Rybak (1), S. Bertazzoni (1), E. Furuki (1), E. Dinglasan (2), L. Hickey (2), R. P. Oliver (1), K. C. Tan (1), (1) Curtin University, Perth, AUSTRALIA; (2) Queensland Alliance for Agriculture and Food Innovation, Brisbane, AUSTRALIA

The fungus Parastagonospora nodorum is the causal agent of septoria nodorum blotch of wheat. This disease is the outcome of multiple fungal necrotrophic effector-host sensitivity gene interactions that include SnToxA-Tsn1, SnTox1-Snn1and SnTox3-Snn3. Previous work demonstrated that the triple-knockout strain P. nodorum toxa13 maintained the ability to infect most modern bread wheat cultivars as effectively as the wildtype SN15. This suggests that other effectors and sensitivity genes may be relevant. To search for additional sensitivity/resistance genes, as well as putative genes, a diversity panel of 295 historic wheat accessions from the N. I. Vavilov Institute of Plant Genetic Resources in Russia was acquired. This collection comprises both genetically diverse landraces and historic breeding lines registered from 1920 to 1990 . Both P. nodorum SN15 and toxa13 were assayed on the Vavilov panel. SN15 was more virulent than toxa13. The subset of wheat lines insensitive to all three effectors showed significantly lower levels of disease when infected with SN15. However, the subset were no less susceptible to the toxa13 than the rest of the Vavilov collection. GWAS using SN15 assay detected quantitative trait loci (QTL) on chromosomes 1BS (Snn1), 2DS, 5AS, 5BS (Snn3), 3AL, 4AL, 4BS and 7AS. For toxa13 infection, a similar QTL was detected on 5AS, plus two additional QTL on 2DL and 7DL. This study further revealed that plant breeders had inadvertently selected for effector insensitivity from 1940 onwards. This study will help to identify accessions for development of bi-parental mapping populations to characterize resistance-associated alleles for subsequent retrogression into modern bread wheat.

Virulence of Puccinia triticina and detection of leaf rust resistance genes in different Egyptian wheat genotypes A. S. G. ABDELRHIM (1), H. Abd-Allah (1), M. Abou-Zeid (2), C. Cowger (3), (1) Minia University, El Minia, EGYPT; (2) Agriculture Research Center, Giza, Egypt, Giza, EGYPT; (3) USDA-ARS, Department of Entomology \& Plant Pathology, North Carolina State University., Raleigh, NC, USA

Leaf rust caused by Puccinia triticina is one of the most serious diseases of wheat worldwide. It can cause large reductions in wheat yield; for example, in Egypt, losses may reach 50\%. One of the most effective and durable strategies to control leaf rust is breeding for leaf rust resistant cultivars. In Egypt, more up-to-date information is needed about the virulence of $P$. triticina populations, genetic information about growing wheat cultivars, and sources of effective $L r$ genes. The aim of this study was to characterize the virulence of Egyptian $P$. triticina populations and identify effective $L r$ genes in recent Egyptian bread and durum wheat cultivars, as well as old wheat lines. Using single-gene differentials, we identified virulence phenotypes of $50 P$. triticina isolates collected from 10 Egyptian provinces during the 2015-2016 growing season. The highest-frequency virulence phenotypes were TCTTQ (20\%) and TFTTQ (18\%). Also, using gene postulation assay, $\operatorname{Lr} 16, \operatorname{Lr} 19, \operatorname{Lr} 24, \operatorname{Lr} 36$, and $\operatorname{Lr} 51$ were postulated in several old spring bread wheat lines. SSR and SNP markers were used to detect eight $L r$ genes in 64 Egyptian wheat genotypes. Of those genes, $L r 46$ was detected in most old and new 
genotypes, and $\operatorname{Lr} 9$ was found in two old lines (L1360-3838 and V-3) and one recent cultivar (Sakha-94), $\operatorname{Lr} 19$ was detected in 3 old lines and 2 recent cultivars, while $\operatorname{Lr} 21, \operatorname{Lr} 24, \operatorname{Lr} 34$, and $\operatorname{Lr} 67$ were mainly found in old genotypes. In contrast, $\operatorname{Lr} 26$ was detected in 10 genotypes including 2 old lines.

Systematic phenotyping, comparative transcriptomics and functional validation of genes implicated in apple root resistance traits Y. ZHU (1), M. Saltzgiver (1), J. Zhao (2), Z. Zhou (3), J. Shao (4), R. E. Davis (4), M. Mazzola (5), (1) USDA ARS, Wenatchee, WA, USA; (2) Hebei Academy of Forestry Science, Shijiazhuang, CHINA; (3) Institute of Pomology, Chinese Academy of Agricultural Sciences, Beijing, CHINA; (4) USDA ARS Molecular Plant Pathology Lab, Beltsville, MD, USA; (5) USDA-ARS, Wenatchee, WA, USA

Apple replant disease is a major obstacle to establishing an economically viable apple orchard at replant sites. The primary control method is pre-plant soil chemical fumigation, which is expensive and comes with environmental and regulatory concerns. Maximized exploitation of innate resistance requires elucidation of resistance mechanisms in apple roots. Apple root resistance traits were evaluated for more than 60 accessions from 'Ottawa 3' $\mathrm{x}$ 'Robusta 5' progeny in response to infection by Pythium ultimum, Rhizoctonia solani and Pratylenchus penetrans. Plant survival rates to P. ultimum infection ranged from $5 \%$ to $95 \%$ between more susceptible and resistant accessions. The higher survival rates were correlated with deterred necrosis progression along the infected roots. Resistance to $P$. ultimum among genotypes seems to correspond with that of $R$. solani, but no relationship with resistance to $P$. penetrans was observed. Two RNA-seq based transcriptome analyses allowed identification of candidate genes potentially regulating the differential defense activation patterns. These candidate genes include those functioning at pathogen sensing, defense hormone signaling, and synthesis and transport of secondary metabolites. Selected candidate genes are being validated for their association with resistance traits. Definitive roles of these genes in conferring resistance will be examined through in planta expression manipulation by over-expression and/or knock-out by CRISPR.

Two major and five minor QTL confer adult plant resistance to stripe rust in winter wheat cultivar Skiles L. LIU (1), X. Chen (2), (1) Washington State University, Pullman, WA, USA; (2) USDA ARS, Pullman, WA, USA

Stripe rust, caused by Puccinia striiformis f. sp. tritici (Pst), is one of the most destructive diseases of wheat in the world. Wheat cultivar Skiles has been highly resistant to stripe rust since its release in 2008 in the US Pacific Northwest, but the genetics of its resistance was unknown. This study was conducted to determine the genetics of stripe rust resistance in Skiles. Testing Skiles with various Pst races at seedling and adult-plant stages at different temperatures showed that it has only adult plant resistance against predominant races. To map resistance genes, a doubled haploid (DH) population was developed from a cross between Skiles and susceptible wheat AvS. The DH population was phenotyped under natural infection of $P s t$ in the fields of Pullman and Mt Vernon in 2016 and 2017 and at adult-plant stage with predominant race PSTv-37 under controlled conditions, and genotyped with the 90K Illumina iSelect wheat SNP chip. Molecular mapping identified two major QTL on chromosomes 3BS and 4BL. In addition, five minor QTL were mapped on chromosomes 1BL, 5AL, 5BL, 6BS, and 7DL. Testing the DH population with SSR markers specific to the chromosome arms confirmed the major QTL. Genotyping various wheat lines with the flanking markers of the two major QTL validated the usefulness of the markers. The QTL and closely linked markers are useful in developing wheat cultivars with high-level and potentially durable resistance to stripe rust.

High-density Mapping of an Adult-plant Stripe Rust Resistance Gene Yrbai in Wheat Landrace Baidatou Using the Whole Genome DArT-seq and SNP Analysis

Q. LI (1), J. Guo (1), K. Chao (1), J. Yang (1), W. Yue (2), D. Ma (3), B. T. Wang (1), (1) College of Plant Protection, Northwest A\&F University, Yangling, Shaanxi, CHINA; (2) Tianshui Institute of Agricultural Sciences, Tianshui, Gansu, CHINA; (3) College of Agriculture, Yangtze University, Jinzhou, Hubei, CHINA

Stripe rust, caused by the biotrophic fungus Puccinia striiformis f. sp. tritici (Pst), is one of the most widespread and destructive wheat diseases worldwide. Growing resistant cultivars is an effective approach for controlling of this disease. However, because host resistance genes were easily overcome by new virulent $P$ st races. There is a continuous demand for identifying new effective wheat stripe rust resistance genes and develop closely linked markers for marker-assisted selection (MAS). Baidatou, an old Chinese wheat landrace, has been grown several decades in Longnan region, Gansu Province, where stripe rust epidemics are frequent and severe. In our previous study, a single dominant gene YrBai in Baidatou was identified to control the adult-plant resistance (APR) to Chinese prevalent Pst race CYR33. And the gene was located on wheat chromosome 6DS by four polymorphic SSR and two SRAP markers, with the genetic distances of two closely linked markers 3.6 and $5.4 \mathrm{cM}$, respectively. To further confirm the APR gene in Baidatou and construct the high-density map for the resistance gene, adult plants of $F_{1}, F_{2}, F_{3}$ and $F_{5: 6}$ populations were still inoculated with CYR33 at Yangling field, Shaanxi Province during 2014-2015 and 2016-2017 crop seasons, respectively. The results indicated that a single dominant gene confers the APR to Pst race CYR33 in Baidatou. Using DArT and SNP markers, the APR gene YrBai was further located in $0.8 \mathrm{cM}$ region on wheat chromosome 6D. These closely linked markers developed in this study should be useful for MAS for Baidatou in crop improvement and mapbased clone this gene.

Identification and functional validation of white rust resistance gene in Brassica juncea H. ARORA (1), A. K. Pradhan (1), D. Pental (2), (1) University of Delhi, New Delhi, INDIA; (2) Centre for Genetic Manipulation of Crop Plants, New Delhi, INDIA

White rust caused by Albugo candida is a destructive disease in many agronomically important Brassica species including Brassica juncea, the major oilseed crop of the Indian subcontinent. Almost all the released varieties of $B$. juncea in India are highly susceptible to the disease. Infection of leaf and inflorescence leads to massive yield losses of up to $60 \%$ or more in India. In a previous study, the locus conferring complete resistance to $A$. candida race $2 \mathrm{~V}$ in east European gene pool line, Donskaja-IV, was mapped to linkage group A05 of B. juncea. The bacterial artificial chromosome (BAC) library of Donskaja-IV was screened with the tightly linked marker and the identified BAC clone was sequenced and annotated using Brassica BAC annotation pipeline tool. The annotation revealed that the A05 locus of Donskaja-IV contains a putative resistance (R) gene of coiled coil-nucleotide binding siteleucine rich repeat (CC-NB-LRR) class. Transgenic plants of Varuna, a susceptible but widely cultivated Indian variety, were raised expressing the candidate gene $(B j u D)$ with its native promoter by Agrobacterium mediated gene transfer. Infection assays were performed on $\mathrm{T}_{0}$ plants with $A$. candida isolates collected from different geographical areas of India - Bharatpur, Pantnagar, Meerut, Hisar, Bihar, Morena and Alwar. Transgenic plants showed resistance to all the isolates validating the role of gene $B j u D$ in conferring broad spectrum resistance to different $A$. candida isolates. The results obtained in this study will promote development of white rust resistant lines in mustard and reduce the yield losses attributed to $A$. candida.

A quantitative PCR method for determining relative infection rates of maize callus by Fusarium graminearum in screening for fungal resistance genes

E. T. JOHNSON (1), P. Dowd (2), (1) USDA/ARS/NCAUR, Peoria, IL, USA; (2) USDA ARS, Peoria, IL, USA 
Maize grown in the United States is susceptible to contamination by ear mold fungi. Some of these fungi can produce mycotoxins that are harmful to animals and humans. It is important to identify novel ways of reducing maize ear mold contamination. Some genetic studies of maize over the years have identified quantitative trait loci that contribute to resisting mold contamination. The gene(s) at these loci need to be individually tested in the laboratory to confirm their suspected role in fungal resistance. We developed a laboratory method using quantitative PCR to accurately quantify the amount of Fusarium graminearum in maize callus. This PCR method can detect the fungus 12 hours after placement on the callus. We found that callus expressing a known fungal resistance gene contained much less fungus than callus expressing a gene not expected to contribute to fungal resistance. This methodology using callus can test putative fungal resistance genes more quickly than methods utilizing transgenic plants, which are more costly to produce and maintain. This novel method for evaluating maize genes for resistance against $F$. graminearum holds promise for accelerating the development of new lines that are safer for ingestion by livestock and humans.

Durable resistance against wheat leaf spot group pathogens

B. CORSI (1), S. Holdgate (1), I. Mackay (1), E. Consortium (2), J. Cockram (1), (1) National Institute Agriculture Botany, Cambridge, UNITED KINGDOM; (2) EfectaWheat Consortium, Cambridge, UNITED KINGDOM

The wheat leaf spot group (LSG) of necrotrophic pathogens (Parastagonospora nodorum - Pn, Zymoseptoria tritici-Zt and Pyrenophora tritici-repentis - Ptr, tan spot) are considered the most important fungal leaf spot diseases of wheat in Europe. As part of the ERA-CAPs funded project 'EfectaWheat' we are using an 8-parent wheat Multi-parent Advanced Generation Inter-Cross (MAGIC) genetic mapping population, genotyped with an Illumina 90k single nucleotide polymorphism (SNP) array, to investigate the genetics of LSG resistance in wheat. Representative UK $P n$ and $P t r$ isolates were used to inoculate MAGIC field trials 2017; disease severity and additional phenotypic data were scored. The combined datasets are used in genetic analyses to identify QTLs and possible relationships between wheat genetic loci controlling effectors- and pathogen-mediated host resistance/susceptibility. This information will provide useful tools for the development of improved wheat varieties with increased genetic resistance to LSG pathogens, helping to support sustainable wheat production in Europe.

Exploring the resistance of an interspecific almond $\times$ peach population to Monilinia spp.

J. Usall (1), N. Baró-Montel (1), I. Eduardo (2), C. Casals (1), N. Teixidó (1), R. TORRES (1), (1) IRTA, XaRTA-Postharvest, Edifici Fruitcentre, Lleida, Catalonia, SPAIN; (2) IRTA, Centre de Recerca en Agrigenòmica CSIC-IRTA-UAB-UB, Edifici CRAG, Campus UAB, Bellaterra, Catalonia, SPAIN

Monilinia spp. are responsible for brown rot, one of the most significant stone fruit diseases. Despite the efforts invested in manage and control this disease, the pathogen is not under control. Planting resistant cultivars seems to be a promising alternative but currently, most of the commercial cultivars are susceptible to brown rot. Therefore, the identification of resistant material and further analyses to uncover regions associated with brown rot resistance are necessary. The aim of this study was to conduct a Quantitative Trait Loci (QTL) analysis in an interspecific almond $\times$ peach population. Over two seasons, artificial infections with $M$. fructicola were performed in more than 100 individuals of this backcross one $\left(\mathrm{BC}_{1}\right)$ population derived from a cross between the hybrid plant 'MB 1.37' (obtained from a cross between 'Texas' almond and 'Earlygold' peach) and the peach 'Earlygold' of size $\mathrm{N}=185$. Fruit response to brown rot was assessed in wounded and non-wounded individuals and QTL analysis of traits related to fruit quality and to resistance to brown rot (lesion diameter and number of brown rot infected fruit) was carried out. Phenotypic data indicated differences in the response to M. fructicola inoculation and several non-wounded individuals exhibited resistance to brown rot. For non-wounded fruit, positive correlations were observed between resistance traits and comparisons of the same traits between seasons also resulted in significant correlations. The greater diversity among individuals led identification of locus for the presence of QTLs and could serve as a source of resistance in breeding programs.

Genome-wide mapping of genes controlling resistance to bakanae disease in rice

S. Y. CHEN (1), M. H. Lai (2), C. W. Tung (3), C. L. Chung (1), (1) Department of Plant Pathology and Microbiology, National Taiwan University, Taipei City, TAIWAN; (2) Crop Science Division, Taiwan Agricultural Research Institute, Taichung City, TAIWAN; (3) Depart of Agronomy, National Taiwan University, Taipei City, TAIWAN

Fusarium fujikuroi causes rice bakanae disease and leads to complex symptoms, including slenderness, wide leaf angles, and elongation in plant height and internode. Although the disease affects grain yield and quality, little is known about the resistance resources and genetics in rice. The increasing occurrence and severity of the disease has been reported in several countries of Asia. In this study, we used genome-wide association study (GWAS) to identify resistance quantitative trait loci (QTLs) and genes. 231 diverse accessions from rice diversity panel 1 (RDP1) were artificially inoculated and assessed for resistance using two evaluation methods (visual rating and colonization efficiency). We identified 14 candidate QTLs and a total of 206 genes located within these regions. Among the candidate genes, eight defense-related genes [eg. nucleotide binding site-leucine-rich repeat (NBS-LRR) and dirigent (DIR) genes] and two auxin regulation genes are our top interest for further validation. Besides, one QTL was verified by linkage analysis using a population of 132 recombinant inbred lines derived from IR64 x Nipponbare. Combined GWAS and linkage analysis narrowed the QTL to an 8$\mathrm{kb}$ region with three defense-related genes. This study provides useful information on the resistance performance of 231 accessions and candidate QTLs for bakanae resistance. The outcomes would help resistance breeding and uncovering resistance mechanisms in the future.

\section{Bulked segregant analysis with whole-genome resequencing to map QTL involved in Phytophthora crown and root rot resistance in Cucurbita} pepo

G. VOGEL (1), K. LaPlant (1), M. Mazourek (1), M. Gore (1), C. D. Smart (2), (1) Plant Breeding and Genetics Section, Cornell University, Ithaca, NY, USA; (2) Plant Pathology and Plant-Microbe Biology Section, Cornell University, Geneva, NY, USA

Phytophthora crown and root rot, caused by the broad-host range oomycete pathogen Phytophthora capsici, causes severe yield loss in squash and pumpkin (Cucurbita pepo ssp. pepo) in many areas. No source of complete resistance to P. capsici has been identified in C. pepo, but breeding lines with partial disease resistance were developed at Cornell University, using gray zucchini landrace PI 615089 as a source of resistance. These breeding lines display reduced crown and root rot symptoms when inoculated with several $P$. capsici isolates. The goal of this project is to identify quantitative trait loci (QTL) controlling partial resistance in these lines, potentially a complex genetic architecture. In that light, we employed a bulk-segregant analysis using large populations and deep sequencing of DNA from bulks, providing sufficient statistical power to detect small-effect QTL. Over $13,000 \mathrm{~F}_{2}$ individuals from a biparental population segregating for partial root and crown rot resistance were evaluated in a greenhouse disease screen of seedlings. The most susceptible and resistant individuals were identified and bulked separately, with each bulk constituting approximately $15 \%$ of the total population. Whole-genome resequencing of pooled DNA from bulks will be performed to map QTL by identifying linked variants having significant differences in allele frequencies between the bulks. 
Characterizing the adaptation of Phytophthora nicotianae to partial resistance in tobacco

J. JIN, I. Carbone, D. Shew, North Carolina State University, Raleigh, NC, USA

Phytophthora nicotianae causes tobacco black shank worldwide. The ability of $P$. nicotianae to rapidly overcome single-gene resistance was documented following the widespread deployment of the Php gene in tobacco cultivars in the 1990s. Increased levels of aggressiveness in P. nicotianae have been observed following exposure to tobacco varieties with high levels of partial resistance. Our objective in this study was to characterize the adaptation of $P$. nicotianae to partial resistance in tobacco using phenotypic and molecular approaches. We used in vivo inoculum production and rate of lesion expansion to quantify the adaptation by $P$. nicotianae and found adapted isolates not only have higher infection rates and produce more sporangia than non-adapted isolates, but also cause larger lesions on tobacco stems. The adapted isolates also were subjected to double digest restriction-site associated DNA sequencing (ddRADseq) to identify genetic changes during adaptation. No changes in population structure could be attributed to adaptation on multiple tobacco genotypes; however, to our surprise, isolates showed extremely high diversity given $P$. nicotianae was predominately undergoing asexual reproduction during adaptation. Understanding how $P$. nicotianae adapts to partial resistance in tobacco will inform better resistance deployment strategies and may increase the durability of partial resistance.

A new gene for resistance to Triticum isolates of Pyricularia oryzae stacked with $\mathbf{R m g} 8$ in a local landrace of common wheat W. SHIZHEN (1), A. Soichiro (1), T. Vy (1), I. Yoshihiro (2), Y. Tosa (3), (1) Kobe University, Kobe, JAPAN; (2) kyoto university, kyoto, JAPAN; (3) Kobe Univ, Kobe, JAPAN

Pyricularia oryzae, the causal agent of wheat blast, first arose in Brazil in 1985 and recently spread to Asia (Bangladesh in 2016 and India in 2017). Resistance genes against this new pathogen are very rare in common wheat populations. We screened $\sim 520$ local landraces of common wheat collected worldwide with Br48, a Triticum isolate collected in Brazil, and found a highly resistant, unique accession, GR119. Spikes of GR119 were moderately resistant to a hybrid culture 200R54 lacking AVR-Rmg8 (an avirulence gene corresponding to Rmg8), and highly resistant to its transformant carrying AVR-Rmg8. When $\mathrm{F}_{2}$ seedlings derived from a cross between GR119 and Chinese Spring (CS, susceptible control) were inoculated with Br48, resistant and susceptible seedlings segregated in a 15:1 ratio, suggesting that GR119 carries two resistance genes. When the $\mathrm{F}_{2}$ seedlings were inoculated with $\mathrm{Br} 48 \triangle \mathrm{A} 8$ carrying a disrupted $A V R-R m g 8$, however, the segregation fitted a 3:1 ratio. These results suggests that one of the two genes in GR119 was Rmg8. The other, novel gene was designated tentatively as RmgGR119. Rmg8 and RmgGR119 had complementary modes of actions on fungal infection. The strong resistance of GR119 was attributed to the combined effects of Rmg 8 and RmgGR119.

\section{The genomic RNAs of Tomato spotted wilt orthotospovirus are differentially targeted in infected tomato (Solanum lycopersicum) with or without Sw5 gene}

C. OLAYA (1), S. Fletcher (2), N. Mitter (3), H. R. Pappu (1), (1) Department of Plant Pathology, Washington State University, Pullman, WA, USA; (2) School of Chemistry and Molecular Biosciences, The University of Queensland, St. Lucia, QLD, AUSTRALIA; (3) Centre for Plant Science Queensland Alliance for Agriculture and Food Innovation, St. Lucia, QLD, AUSTRALIA

Tospoviruses cause significant losses to a wide range of agronomic and horticultural crops worldwide. The type member, Tomato spotted wilt orthotospovirus (TSWV) causes systemic infection in tomato, whereas its infection is localized in cultivars carrying the $S w 5$ resistance gene. The response to TSWV infection in tomato cultivars with or without $S w 5$ was determined at the small RNA level using the Illumina HiSeq 4000 platform. Predicted reads were aligned to reference sequences using SCRAM2 software package. The TSWV genome is differentially processed among each of the three viral genomic RNAs -Large (L), Medium (M) and Small (S)- in the $S w 5$ - and $S w 5+$ genotypes. In the $S w 5+$ cultivar, the L RNA had the highest number of viral-small-interfering RNAs (vsiRNAs), whereas in the $S w 5$ - cultivar the number was higher in the S RNA. Among the three-viral genomic RNAs, the distribution of hotspots showed a high number of reads per millions reads of vsiRNAs of 21 and 22 nt class at the 5' and 3' ends of the L and the S RNAs, with less coverage in the M RNA. In the Sw5- cultivar, the nature of the 5' nucleotide-end in the siRNAs varied significantly; reads with 5'adenine-end were the most abundant in the viral infected treatment, whereas cytosine and thiamine were more abundant in the mock control. No such differences were seen in case of resistant genotype. Our results allow identification of potential viral genome targets to be used in RNAi-based control strategies.

Leveraging Natural Variation to Identify New Sources of Resistance to Pseudomonas syringae pv. tomato R. ROBERTS (1), S. Mainiero (1), A. F. Powell (1), S. R. Hind (1), S. R. Strickler (1), G. B. Martin (1,2), (1) Boyce Thompson Institute, Ithaca, NY, USA; (2) School of Integrated Plant Science, Cornell University, Ithaca, NY, USA

Pseudomonas syringae pv. tomato (Pst) causes bacterial speck disease of tomato. The only genetic resistance to Pst is conferred by the $R$ gene Pto, which recognizes the Pst effectors AvrPto and AvrPtoB. However, Pst strains have recently emerged lacking these effectors, rendering Pto-mediated resistance ineffective. Our study aims to identify new sources of genetic resistance against $P s t$ that can be introgressed into tomato breeding lines. Using available whole-genome resequencing data, we leveraged natural variation among tomato heirlooms, breeding lines, and a wild species to screen 216 accessions for new sources of resistance. We inoculated the accessions by spraying Pst mutant strains deleted for different effectors and/or flagellin to help elucidate whether observed disease resistance responses involve effector-triggered (ETI) or pattern-triggered (PTI) immune pathways. Interestingly, our screen uncovered new Pst disease phenotypes beyond the typical speck symptoms, and we have found that some of these phenotypes are simply inherited. Using an assay that measures the production of PTI-related reactive oxygen species, we discovered new accessions that have increased, or conversely, no response to the flagellin peptides flg22 or flgII-28, which are recognized by the pattern recognition receptors FLAGELLIN $\underline{\text { SENSING } 2}$

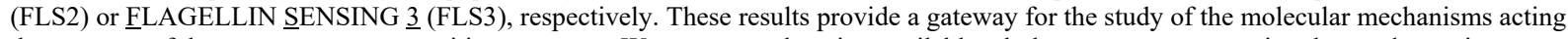
downstream of these two pattern recognition receptors. We are currently using available whole-genome resequencing data and genetic approaches to identify gene candidates that underlie novel resistance or susceptibility to Pst.

Mapping quantitative trait loci (QTLs) controlling adult plant resistance in oat against Puccinia coronata f. sp. avenae

E. S. NAZARENO (1), M. Miller (1), M. L. Carson (2), S. Chao (3), M. Figueroa (1), S. Kianian (2), (1) Department of Plant Pathology, University of Minnesota, St. Paul, MN, USA; (2) USDA-ARS, Cereal Disease Laboratory, St. Paul, MN, USA; (3) USDA-ARS, Northern Crop Science Laboratory, Fargo, ND, USA

Crown rust, caused by the fungus Puccinia coronata f. sp. avenae (Pca), is the most devastating disease in oat worldwide. Losses due to this disease can reach up to $50 \%$, and breeding for resistance is a preferred management method for its cost-effectiveness and sustainability. Adult plant resistance (APR) or partial resistance has been shown to provide durable resistance against $P c a$. In this study, we are mapping APR loci in three recombinant inbred populations generated from three biparental crosses: CI1712 x Otana, PI260616-1 x Otana, and CI8000-4 x Otana. These populations were phenotyped in the Buckthorn nursery in St. Paul, MN for three years (2011, 2012, and 2017). In CI1712 x Otana population, 24 resistant and 24 susceptible lines 
were selected and genotyped using the 6K Oat SNP Chip. Results showed that two QTLs are associated with the resistance phenotype in CI1712: one in Linkage Group 6 (chromosome 14C) with five markers and one in Linkage Group 11 (chromosome 1C) with 13 markers. Kompetitive Allele-Specific PCR (KASP) markers were developed to confirm the presence of these QTLs in the whole population. For PI260616-1 x Otana and CI8000-4 x Otana, a total of 48 resistant lines and 48 susceptible lines were selected for the genotyping. Once the resistance loci from each population are mapped, we aim to pyramid all QTLs into a single line and forward it to breeding programs for the development of new varieties with durable resistance against crown rust.

Multiline breeding and functional analysis of genes for resistance against Magnaporthe oryzae in rice

Y. C. CHEN (1), F. Y. Chang (2), C. L. Chung (1), (1) Department of Plant Pathology and Microbiology, National Taiwan University, Taipei City, TAIWAN; (2) Kaohsiung District Agricultural Research and Extension Station, Pingtung County, TAIWAN

Rice blast can cause serious yield loss in rice production. Due to rapid evolution of Magnaporthe oryzae population, rice resistance to blast is generally easy to be overcome. To effectively control the disease, it is important to keep developing resistant varieties and exploring novel resistant genes. As part of the ongoing effort in our lab, this study continues to introgress resistance genes from 11 IRBLs into Kaohsiung 145 through marker-assisted backcrossing. The progeny were evaluated for blast resistance by artificial inoculation or blast nursery tests, and then selected according to their stable resistance and agronomic performance in the field. Development of Kaohsiung 145 multiline varieties is expected to provide durable resistance to blast fungus. Several candidate blast resistance genes were previously mapped by genome-wide association study (GWAS) using rice diversity panel 1 (RDP1). To validate the gene effects, trait-marker association was tested using available $\mathrm{F}_{2: 3}$ mapping populations. Expression profiles of the candidate genes in susceptible and resistant lines were investigated by qRT-PCR. To further characterize the function of gene(s) of the most interest, we conducted gene cloning and Agrobacterium-mediated transformation to insert the target gene(s) into a susceptible variety. The transformant(s) will be evaluated for resistance against $M$. oryzae. The results will provide useful information for resistance breeding and disease control.

Understanding major gene-mediated resistance in Brassica napus (oilseed rape) against the apoplastic fungal pathogen, Pyrenopeziza brassicae C. KARANDENI-DEWAGE, H. Stotz, B. Fitt, University of Hertfordshire, Hatfield, UNITED KINGDOM

Light leaf spot disease, caused by the fungal pathogen, Pyrenopeziza brassicae, is currently the most damaging foliar disease on winter oilseed rape (Brassica napus) in the UK. Light leaf spot disease management is often reliant on fungicide applications. However, there has been evidence for the development of insensitivity to azole fungicides in UK P. brassicae populations. Deployment of cultivar resistance remains an important aspect of effective management of the disease. Nevertheless, the genetic basis of resistance remains poorly understood and no resistance genes have been cloned. A major gene locus (PBR2) for resistance against $P$. brassicae has been mapped to the bottom end of the $B$. napus chrA01 c. $3.0 \mathrm{cM}$ away from the closest flanking marker. We have identified the physical location of the closest flanking marker on $B$. napus chrA01 using synteny alignment between $B$. napus and $B$. rapa chrA01. The interval between this locus and the telomere corresponds to a c. $1.2 \mathrm{Mbp}$ region in chrA01. Single nucleotide polymorphism (SNP) markers on the corresponding genomic region have been obtained and c. 400 candidate SNPs specific to chrA01 have been identified. Further analysis of this region was done through fine mapping of selected SNPs using KASP marker analysis. As a result, three additional markers linked to the resistance locus were identified, which narrowed down the corresponding chromosomal region to c. $300 \mathrm{Kbp}$. It is expected that this fine mapping will provide tightly linked markers for marker-assisted selection and assist in the isolation, sequencing and functional identification of the resistance gene.

\section{Prediction and screening of candidate resistance genes of Ocimum basilicum in response to the basil downy mildew pathogen Peronospora belbahrii}

G. DeIulio (1,2), K. S. ALLEN (1), R. Pyne (3), R. L. Wick (1,4), J. E. Simon (3), L. J. Ma (1,2), (1) Plant Biology Graduate Program, University of Massachusetts Amherst, Amherst, MA, USA; (2) Department of Biochemistry and Molecular Biology, University of Massachusetts Amherst, Amherst, MA, USA; (3) Department of Plant Biology, Rutgers University, New Brunswick, NJ, USA; (4) Stockbridge School of Agriculture, University of Massachusetts Amherst, Amherst, MA, USA

Basil downy mildew, caused by the biotrophic oomycete Peronospora belbahrii, is a destructive disease of sweet basil (Ocimum basilicum) that results in high yield losses worldwide. Chemical and cultural control options have offered insufficient control for growers, and breeding has been a slow process due to the lack of annotated genetic resources and the necessity for retaining plant morphological and chemical characteristics for marketability. Following phenotypic screening for disease resistance, RNA-seq was used to compare the host-pathogen interactions of a downy mildew resistant sweet basil cultivar 'Mirihani' to a susceptible sweet basil line Rutgers SB22 during the inoculation and infection process. Following a previously-reported de novo-assembly based data analysis pipeline, this data has been used to predict candidate resistant genes uniquely present in the resistant cultivar 'Mirihani'. Both nucleotide-binding leucine rich repeat proteins and receptor-like kinases are represented in the candidate genes. These protein classes are known to act upstream or alongside defense triggering pathways, including plant hormone responses. Analysis of hormone pathways shows that unlike 'Mirihani', SB22 does not accumulate some mRNAs associated with salicylic acid signaling and biosynthesis while both the jasmonic acid and ethylene pathways are consistent between cultivars. The predicted resistance gene sequences were used for primer design and expression profiles observed in the data were validated using qRT-PCR. This research presents the analytical approach for prediction of these candidate resistance genes, as well as the validation of the analysis using gene expression data.

Novel sources of disease resistance in pepper against bacterial spot xanthomonads

N. POTNIS (1), S. Branham (2), J. B. Jones (3), W. P. Wechter (4), (1) Department of Entomology and Plant Pathology, Auburn University, Auburn, AL, USA; (2) USDA, ARS, Charleston, SC, USA; (3) University of Florida, Gainesville, FL, USA; (4) USDA-ARS, Charleston, SC, USA

Bacterial spot Xanthomonas species cause significant disease outbreaks on tomato and pepper in the tropical and subtropical regions throughout the world. Changing pathogen population structure and the geographical distribution have significantly impacted the durability of single/ combinatorial resistance genes currently employed in pepper cultivars in the U.S. These findings reinforce the importance of identifying novel candidates for bacterial spot disease resistance. Our studies involving extensive phenotyping on diverse core germplasm of pepper against Xanthomonas gardneri followed by genotyping-by-sequencing and association analyses yielded highly significant SNPs associated with bacterial spot disease phenotypes. We further screened the pepper genome and identified genes associated with the significant SNPs. Based on annotations, the candidate genes have been found to encode proteins involved in hormone signaling, developmental processes, disease resistance against specific pathogens as well as some involved in basal resistance against multiple viral, fungal and bacterial pathogens. Screening the efficacy of resistant genotypes against different pepper pathogens identified several promising genotypes that impart resistance/tolerance against multiple pathogens of pepper. Potential resistance loci as well as genotypes carrying these loci identified in this study will be employed as a part of resistance gene pyramiding effort against the changing pathogen population structure. 
Reduced stomatal density in wheat and its potential for improving control of foliar pathogens

J. THOMAS (1), J. Sammonds (1), L. Hunt (2), J. Gray (2), (1) National Institute of Agricultural Botany, Cambridge, UNITED KINGDOM; (2) Sheffield University, Sheffield, UNITED KINGDOM

Higher plants evolved in atmospheric conditions where carbon dioxide levels were significantly lower than recent levels. As a result, high stomatal densities were needed for efficient photosynthesis. At higher levels of carbon dioxide, reduced densities may allow efficient photosynthesis without loss of yield. Stomata also act as entry points for some major foliar pathogens, and decreasing stomatal density may thus reduce infection points and the rate of epidemic development. To test this, we investigated the development of two contrasting pathogens in inoculated tests on transformed lines of wheat cv. Fielder with reduced stomatal densities. There was no effect on the development of powdery mildew (Blumeria graminis f. sp. tritici), which enters through epidermal cell junctions (mean $49 \%$ leaf area infected on low density lines and $51 \%$ on the null line). However, in the case of leaf rust (Puccinia triticina), which enters through stomata, mean pustule numbers were significantly lower $(\mathrm{p}<0.05)$ at 7.1 pustules per $\mathrm{cm}$ length of leaf on the line with fewest stomata compared to the null line at 12.8 pustules. Reductions were observed in repeat experiments and additional transformants with higher stomatal frequencies showed higher pustule numbers, though these were still less than the null line. Breeding for reduced stomatal frequency in wheat may offer a means of improving control of some pathogens such as rusts in the field.

Andean landrace G19833 of comon bean is broadly resistant to the common bean rust pathogen

O. P. Hurtado-Gonzales (1), T. Gilio (2), M. PASTOR-CORRALES (1), (1) Soybean Genomics \& Improvement Lab, BARC-West, ARS-USDA, Beltsville, MD, USA; (2) Universidade Estadual de Maringa, Maringa, BRAZIL

The Andean landrace G19833 was sequenced to obtain the reference genome of common bean (Phaseolus vulgaris). Moreover, a large quantity of sequence and genetic resources has been developed for G19833 including BAC libraries, SNP databases, RNAseq from various tissues, and RIL populations, among others. In addition, G19833 have been reported as resistant to the pathogens causing the widespread and devastating anthracnose, angular leaf spot, and Ascochyta blight diseases. However, little is known about the reaction of G19833 to Uromyces appendiculatus, the pathogen that causes the economically important bean rust disease. This pathogen is known for its extensive virulence diversity comprising Mesoamerican and Andean races. We report here the reaction of G19833 to 20 races, 10 Mesoamerican and 10 Andean, of the rust pathogen, representing the broad virulence of Uromyces appendiculatus. G19833 was resistant to all races used in this study that together overcome all known rust resistance genes of common bean. It is also significant that G19833 was resistant to all 10 Mesoamerican races that rendered susceptible all Mesoamerican rust resistance genes of this crop. Thus, these results demonstrate that G19833 is a very valuable source of resistance for the management of the widespread Mesoamerican but also Andean races of $U$. appendiculatus. Results regarding the genetics of the broad spectrum of rust resistance in G19833 will be presented.

Molecular Characterization of Race 1 Bacterial Speck Resistance in a Wild Relative of Tomato

C. MAZO (1,2), M. Vachev (2), S. Mainiero (2), G. B. Martin (1,2), (1) School of Integrated Plant Science, Cornell University, Ithaca, NY, USA; (2) Boyce Thompson Institute, Ithaca, NY, USA

Pseudomonas syringae pv. tomato (Pst) is a persistent pathogen of tomato that causes bacterial speck disease. On tomato, resistance conferred by the $R$ gene Pto is effective against race 0 Pst strains which express the effector proteins AvrPto and/or AvrPtoB; however, race 1 strains of $P s t$, which do not express AvrPto/AvrPtoB but rather a different repertoire of effectors, evade Pto-mediated resistance. In this study, we aimed to characterize a putative $R$ gene discovered in a wild relative of tomato that recognizes AvrRpt2, a bacterial effector widespread in race 1 Pst strains. In Arabidopsis, the $R$ gene RPS2 recognizes AvrRpt2. We found that certain mutations in AvrRpt2 that impair its recognition by RPS2 do not affect its recognition in tomato plants that harbor our putative $R$ gene. This observation suggests there might be a novel AvrRpt2 recognition mechanism in this tomato accession. Additionally, by transiently expressing AvrRpt2 homologs from different bacterial phytopathogens we found that recognition of the effector by the putative $R$ protein correlates with the reported ability of each homolog to cleave Arabidopsis RIN4, which is an indicative of AvrRpt2 cysteine protease activity. Together, these results provide a foundation for investigating molecular mechanisms underlying the interaction between AvrRpt 2 and a new tomato R gene and may lead to the development of Pst race 1 resistant tomato varieties.

Characterization of resistance to Sclerotinia, and its association with plant architecture and composition in lettuce B. E. MAMO PHD (1), N. Adhikari (2), C. Foster (3), L. Rosental (2), R. J. Hayes (2,4), K. V. Subbarao (1), I. Simko (2), (1) University of California at Davis, c/o U.S. Agricultural Research Station, Salinas, CA, USA; (2) USDA, ARS, Crop Improvement and Protection Unit, Salinas, CA, USA; (3) Great Lakes Bioenergy Research Center, Michigan State University, East Lansing, MI, USA; (4) USDA, ARS, Corvallis, OR, USA

Cultivated lettuce (Lactuca sativa L.) is an economically important vegetable crop. Lettuce drop, caused by the fungal pathogens Sclerotinia minor and S. sclerotiorum, causes significant yield loss in all lettuce types. Development of resistant cultivars has been impeded by the lack of resistance in lettuce germplasm. The resistance identified to the disease in lettuce landraces and non-adapted accessions cultivated for seed (oil consumption) is often linked with plant architecture mainly associated with premature bolting. Resistance to crown degradation due to Sclerotinia infection increases with plant age in non-leafy lettuce accessions. Research is underway to understand the relationship between plant architecture and crown degradation, and identify possible mechanisms underlying resistance. Preliminary results indicate that resistance to crown degradation is associated with stem strength and composition. Comprehensive analysis of plant tissue compositions implicated in differentiating susceptible vs. resistant accessions will be presented. For genetic analysis, a recombinant inbred lines (RILs) population derived from a cross between a susceptible cultivar and a resistant accession, and genotyped with molecular markers has been identified. This population is being evaluated for lettuce drop incidence, disease severity and rate of bolting in a field infested with S. minor. The RILs will also be evaluated for stem firmness and composition. QTL analyses of these traits will unravel the genetic mechanism of Sclerotinia resistance, its association with development-related traits and tissue composition in lettuce.

A PCR assay for Verticillium dahliae race 1 resistance in lettuce based on genome sequencing of 60 resistant or susceptible cultivars P. INDERBITZIN (1), S. Reyes-Chin Wo (2), M. Christopoulou (2), R. W. Michelmore (3), K. V. Subbarao (4), I. Simko (5), (1) University of California at Davis, Department of Plant Pathology, Davis, CA, USA; (2) University of California at Davis, Genome Center, Davis, CA, USA; (3) University of California at Davis, Genome Center and Department of Plant Sciences, Davis, CA, USA; (4) University of California at Davis, c/o U.S. Agricultural Research Station, Salinas, CA, USA; (5) USDA, ARS, Crop Improvement and Protection Unit, Salinas, CA, USA

Verticillium dahliae is a soilborne plant pathogen that is a major threat to lettuce production in the Salinas and Pajaro Valleys of central coastal California. In tomato, resistance to $V$. dahliae race 1 is conferred by $V e l$, which encodes a nucleotide binding-leucine rich repeat receptor (NLR) protein. There are two copies of $V e$ in tomato, $V e l$ and $V e 2$, but only $V e l$ provides resistance to $V$. dahliae. In the $V$. dahliae race 1-resistant lettuce cultivar La Brillante, there are three $V e$ homologs, Verticillium resistance $1(V r 1), V r 2$ and $V r 3$, which are $54-57 \%$ similar to $V e 1$ in tomato. Three $V r$ paralogs are 
also present in the $V$. dahliae race 1-susceptible cultivar Salinas, and it is currently unknown which of the $V r$ paralogs confers resistance to $V$. dahliae race 1 in lettuce. To develop a PCR assay to detect $V$. dahliae race 1 resistance in lettuce, we sequenced the genomes of 60 resistant or susceptible lettuce cultivars and extracted the $V r$ gene-like sequences, and found that the La Brillante $V r l$ allele $(L B V r I)$ was present in all resistant cultivars, and absent in all susceptible cultivars. This indicated that $L B V r l$ is associated with resistance of lettuce to $V$. dahliae race 1 . We designed a $L B V r l$-specific PCR assay and validated its accuracy on an additional 90 accessions. This work indicates that $L B V r l$ may be the functional $V r$ allele in lettuce. Gene knock-out assays are underway to prove the function of $L B V r l$ in resistance to $V$. dahliae race 1 .

From plate to paddock: Taking Fusarium crown rot resistance from the lab into the field J. POWELL, Y. Ma, Z. Zheng, C. Liu, CSIRO, St Lucia, AUSTRALIA

Fusarium crown rot, predominantly due to infection by Fusarium pseudograminearum, is a significant constraint on bread wheat, durum and barley production in Australia. Development of varieties with increased resistance leading to yield gain under disease pressure remains the most tractable, longterm solution to this challenge. To this end, four large effect resistance QTL have been identified in bread wheat (on chromosomes 3B, 2D, 5D and 2B) and two in barley (on chromosomes $4 \mathrm{H}$ and $1 \mathrm{H}$ ) respectively and deployed in a breeding program to pyramid and introgress these QTL into elite cultivar backgrounds. Results indicate that combining these QTL effectively decreases the development of disease symptoms and generates lines with yield advantage under disease pressure. Near isogenic lines differing for inclusion of each resistance locus have also been developed to aid the study of the molecular basis of resistance for each of these QTL, facilitate fine-mapping approaches and produce co-segregating markers. Applying RNA-seq approaches to near isogenic lines for the 3B and 4H loci has proven an effective strategy for identifying candidate genes underpinning resistance loci by calling differential gene expression and single nucleotide polymorphisms between resistant and sensitive isolines. Future work will attempt to isolate the underlying causative genes for these QTL and characterise the role these genes play in mediating resistance to fungal diseases.

Genetic control of crown rot of wheat: Applying genetic studies to breeding programs

P. DAVIES (1), V. Paccapelo (2), A. Kelly (2), (1) University of Sydney, Narrabri, AUSTRALIA; (2) Queensland Department of Agriculture and Fisheries, Toowoomba, AUSTRALIA

Crown rot of wheat caused by Fusarium pseudograminearum is a major threat to grain production in Australia and other semi-arid grain growing regions of the world. While resistance to crown rot is available, its control is quantitative in nature, and conveys only partial resistance to this disease. In order to improve the resistance available in elite wheat germplasm, a pre-breeding program was established to pyramid multiple resistance alleles. To aid in this process, a genome wide association study was conducted on results from five resistance trials conducted over three years, using 90K SNP genotypic data. This study identified nine chromosomal regions associated with resistance to crown rot, of which eight corresponded with previously published reports of resistance QTL. This allowed parental selection based on the presence of complementary sets of alleles for resistance, rather than phenotype alone, significantly improving the efficiency of pyramiding. While this research was not aimed at identifying new alleles for crown rot resistance, it is an example of deploying genetic studies within breeding programs to improve the development of enhanced germplasm.

Cell wall polysaccharide architecture and its potential impacts on grapevine susceptibility to Pierce's disease Q. SUN, M. He, University of Wisconsin-Stevens Point, Stevens Point, WI, USA

Pierce's disease (PD) symptom development of grapevine is largely dependent on the systemic spread of the bacterial pathogen $X y$ lella fastidiosa $(X f)$ in a vine's vessel system. A major barrier to this spread is the intervessel pit membrane (PM), a cell wall structure that separates adjacent vessels. It is believed that $X f$ is able to degrade these PMs by attacking various polysaccharides with its cell wall degrading enzymes (CWDEs), enlarging the PMs' porosity and enabling its systemic spread. Therefore, it is important to know the polysaccharide composition and architecture of the PMs. However, the details on these remain unclear. This study focuses on four potential polysaccharide targets of the $X f$ 's CWDEs: fucosylated xyloglucans (F-XyGs), xylans, heavily and weakly methyl-esterified homogalacturonans (HMe-HGs and WMe-HGs). Using immunogold- scanning electron microscopy, we found that these polysaccharides differed in both quantity and spatial distribution in PMs of PD-susceptible Chardonnay vines. Xylans and HMe-HGs were undetectable in the superficial layer of a PM, but gradually increased in quantity toward deeper layers with more xylans than HMe-HGs. F-XyGs occurred in small amount in the superficial layer but were abundant in all the underlayers, while WMe-HGs were abundant in the superficial layer and decreased in quantity toward deeper layers. This information should be valuable for the understanding of $X f$-host vine interactions and the mechanism of grapevine's PD susceptibility.

Comparison of Quantitative Disease Resistance Loci in Soybean Towards Soil Borne Root Pathogens A. E. DORRANCE (1), K. Scott (1), A. K. Stasko (1), C. Gedling (1), S. Karhoff (2), W. Rolling (2), L. McHale (3), (1) The Ohio State University, Dept. of Plant Pathology, Wooster, OH, USA; (2) The Ohio State University, Translational Plant Sciences Program, Columbus, OH, USA; (3) The Ohio State University, Dept. of Horticulture and Crop Sciences, Columbus, OH, USA

Several studies in the past decade have raised awareness of the diversity of species that contribute to soybean stand loss as well as the diversity within species that can impact management. In addition to Phytophthora sojae, more than 40 species of Pythium, Phytophthora sansomeana, and Fusarium graminearum are now recognized as pathogens of soybean. Over the past two decades, numerous sources of resistance have been identified, and the purpose of our studies was to identify the QTL and mechanisms that contribute to resistance. Four recombinant inbred line (RIL) populations from the soybean Nested Association Mapping population were segregating for resistance to P. sojae and one or more Pythium spp. Disease resistance for $P$. sojae was measured in RILs by lesion length with a tray test and root rot or final root weight with a cup assay for Pythium spp. Numerous QTL have been identified; however, the majority are minor and contribute to less than $10 \%$ of the phenotypic variation. In addition, QTL-pathogen specificity within each population was also identified. These results have implications on future breeding efforts to expand the resistance spectrum towards more pathogens. Cultivars bred solely via marker assisted selection will require perfect markers, which are developed from the genes that contribute to a trait of interest.

Forward genetic analysis defines candidate genes for fusiform rust resistance in loblolly pine and avirulence in Cronartium quercuum f. sp. fusiforme

D. ENCE (1), K. M. Smith (2), M. Kirst (1), M. Yandell (3), J. Wegrzyn (4), C. D. Nelson (5), J. Davis (1), (1) University of Florida, IFAS, Gainesville, FL, USA; (2) USDA-Forest Service, Gainesville, FL, USA; (3) Department of Human Genetics, University of Utah, Salt Lake City, UT, USA; (4) University of Connecticut, Storrs, CT, USA; (5) USDA-Forest Service, Southern Research Station, Lexington, KY, USA 
The improved annotated genome of loblolly pine (Pinus taeda L.) may allow fine-mapping of important Mendelian traits such as disease resistance. Cronartium quercuum f. sp. fusiforme (CQF) causes fusiform rust disease on pine, a major disease threat to the timber industry in the southern US. Genetic major gene for gene resistance is the primary approach to control losses. A high priority for breeders and forest managers is to identify candidate resistance genes in pine and avirulence genes in CQF. We conducted bulk segregant analysis of next-generation sequence data from both host and pathogen. In pine, half-sibling progeny from a resistant mother were phenotyped as either resistant or susceptible to CQF. These progeny were sequenced with a custom sequence-capture method targeting a genomic region linked to resistance alleles by prior work. In CQF, analysis of whole-genome sequence of rust grown on resistant or susceptible seedlings identified a $200 \mathrm{kbp}$ region containing several likely effector proteins. In pine, we will test any identified candidate $\mathrm{R}$ genes for interaction with the candidate avirulence genes identified in CQF. By identifying candidates for an interacting avirulence and resistance gene pair in this conifer-rust pathosystem, we will discover markers that will guide breeding and deployment of resistant pine and enable us to identify additional interacting gene pairs that are known to exist but have yet to be molecularly discovered in this pathosystem.

\section{First report of non-2NS resistance to wheat head blast}

G. CRUPPE (1), C. D. Cruz (2), G. L. Peterson (3), L. Calderon Daza (4), M. G. Rivadeneira Caballero (5), P. Singh (6), R. P. Singh (7), H. J. Braun (8), B. Valent (1), (1) Kansas State University, Manhattan, KS, USA; (2) Purdue University, West Lafayette, IN, USA; (3) USDA ARS, Fort Detrick, MD, USA; (4) Wheat Breeding Unit, Wheat and Oilseed Growers Association, Santa Cruz de la Sierra, BOLIVIA; (5) Centro de Investigación Agrícola Tropical, Santa Cruz de la Sierra, BOLIVIA; (6) CIMMYT, El Batan, MEXICO; (7) CIMMYT, Mexico DF, MEXICO; (8) Cimmyt, Mexico, MEXICO

Wheat blast, caused by the fungus Magnaporthe oryzae Triticum (MoT), is a devastating emerging disease affecting S. America and S. Asia. Despite 30yrs of intensive effort, the 2NS translocation from Aegilops ventricosa contains the only source of useful head blast resistance identified to date. Our goa was to identify non-2NS resistance to MoT. At least 1000 elite cultivars, breeding line materials, and wild accessions were tested at 2 biosafety level-3 laboratories in the US and under controlled and field conditions in Bolivia. In controlled conditions, heads were inoculated after full emergence and individually rated for percentage of diseased spikelets. Under field conditions, susceptible spreaders were inoculated at tillering to guarantee sufficient inoculum. Disease incidence and severity were evaluated as the average rating for each meter-row plot. The diagnostic marker Ventriup-LN2 was used to test for the presence of the 2NS translocation. Among the germplasm tested, four non-2NS CIMMYT lines (i.e., CM22, CM36, CM58, and CM61) averaged $6.5,17.5,6.7$, and $0 \%$ of disease severity under controlled conditions. Under field conditions, averages of two locations (2017 growing season) were $3,18.5,0$, and $12 \%$. Meanwhile, susceptible checks averaged $91 \%$ (controlled-environment) and $34 \%$ (field). Additional experiments are being conducted to validate our results. New resistance sources from this study can be incorporated into wheat head blast breeding programs.

Broad-spectrum resistance and susceptibility to bacterial blight and bacterial leaf streak of rice A. M. BOSSA-CASTRO (1), C. Tekete (2,3), C. Raghavan (4), E. Delorean (1), A. Dereeper (3), V. M. Verdier (3), K. Dagno (5), O. Koita (2), G. M. Mosquera (6), H. Leung (4), J. E. Leach (1), (1) Colorado State University, Fort Collins, CO, USA; (2) University of Sciences, Techniques and Technologies of Bamako (USTTB), LBMA, Bamako, MALI; (3) IRD, Cirad, Univ Montpellier, IPME, Montpellier, FRANCE; (4) International Rice Research Institute - IRRI, Los Baños, PHILIPPINES; (5) Institut d'Economie Rurale (IER), Prog. Sorgho/ Lab de Phytopathologie, CRRA de Sotuba, Bamako, MALI; (6) International Center for Tropical Agriculture - CIAT, Palmira, COLOMBIA

Quantitative trait loci (QTL) that confer broad-spectrum resistance (BSR) have been elusive targets of crop breeding programs. Bacterial leaf streak (BLS) and bacterial blight (BB), caused by Xanthomonas oryzae pv. oryzicola (Xoc) and Xanthomonas oryzae pv. oryzae (Xoo), respectively, are responsible for major losses in rice production in Asia and Africa. Controlling these two diseases is particularly important in Sub-Saharan Africa, where no sources of BSR are available in currently deployed varieties. Our goal is to identify novel, broad-spectrum resistance sources to control BLS and BB in rice, using a Multi-parent Advanced Generation Inter-Cross (MAGIC) population, derived from eight elite indica cultivars. MAGIC populations have an increased level of recombination and provide higher precision and resolution to detect QTL. The MAGIC parents and lines were genotyped and phenotyped in both greenhouse and field conditions by screening with diverse strains of Xoc and Xoo. Using genome-wide association and interval mapping analysis, we identified 37 strain-specific QTL, and 14 QTL effective against multiple $X$. oryzae strains. From these, three QTL are pathovarspecific and 11 confer resistance to both pathovars. By detecting phenotypic effects of causal alleles, we have identified resources that will facilitate a better understanding of how the involved genes contribute to resistance or susceptibility. Because the MAGIC founders are elite varieties, the BSR QTL identified can be rapidly incorporated into breeding programs to achieve more durable resistance to BLS and BB.

Genome-wide association mapping of resistance to Fusarium proliferatum in soybean

P. N. OKELLO (1), M. Johnson (1), A. Singh (2), F. M. Mathew (1), (1) South Dakota State University, Brookings, SD, USA; (2) Iowa State University, Ames, IA, USA

Fusarium root rot is an important constraint to soybean (Glycine max L.) production in the United States. In 2014, yield losses due to Fusarium root rot in the United States were 116,000 tonnes (58.4 million dollars). In South Dakota, 10 species of Fusarium were identified causing root rot of soybean, and among a collection of 59 Fusarium isolates representing the 10 species, Fusarium proliferatum isolate FUS026 was determined to be the most aggressive on a susceptible soybean cultivar. In this study, 146 plant introductions from 21 countries and from maturity groups 0 (90) and I (56) in the USDA Soybean Germplasm Collection, were inoculated with isolate FUS026 at planting using the inoculum layer method. At 14 days after inoculation, lesion length caused by F. proliferatum on the soybean roots were measured. One hundred and twenty genotypes in this collection were significantly less susceptible $(P<0.05)$ to isolate FUS026 when compared to the susceptible soybean cultivar, which indicated that these 120 genotypes could be used as potential parental lines to develop soybean varieties with resistance to $F$. proliferatum. A genome-wide association mapping analysis was performed with a total of 34,604 single nucleotide polymorphic (SNP) markers. Thirty five significant markers-trait associations $\left(-\log _{10}(P)>3.0\right)$ were identified across five chromosomes $(1,8,9,10$ and 13). The discovery of these loci through association mapping is a significant step to identify sources of resistance to $F$. proliferatum.

Dissecting interactions of rice major and minor blast resistance genes with yield related components Y. JIA (1), X. Chen (2), X. Wang (3), T. A. Bianco (4), W. Li (5), H. Box (6), (1) USDA ARS, Dale Bumpers National Rice Research Center, Stuttgart, AR, USA; (2) China Agricultural University, Beijing, CHINA; (3) UA/USDA, Stuttgart, AR, USA; (4) USDA ARS, Stuttgart, AR, USA; (5) Jiangsu academy of agricultural sciences, Nanjing, CHINA; (6) USDA ARS, 130, AR, USA

A large recombinant inbred line (RIL) mapping population consisting of 800 individuals was developed to investigate functional interactions of blast $R$ genes, $P i$-ta with $P i$ - $b$, other minor blast resistance QTL, and yield related components with US long grain tropical japonica rice varieties Cybonnet (CYBT) and Saber (SB). CYBT is known to contain Pi-ta (avirulent on blast races, IB1, IB17, and IB49 and virulent on IE1K) and $P i-k m$ (virulent on IB1, IB17, IB49, and IE1k) and SB has Pi-b (avirulent on IB1, IB49, IE1k and partial avirulent on IB49). The entire population was evaluated with Pi-ta and $P i-b$ using gene specific PCR, and with their differential blast isolates/races under greenhouse conditions. A subset of this population (243 
individuals) was used to construct a linkage map with 186 single nucleotide polymorphism (SNP) marker for mapping blast R/QTL, and QTL for 1000 grain weight, number of branches of panicle, panicle length, and weight. A total of seven blast resistance QTL were mapped on chromosomes 2, 3, 8, 9, 10,11 , and 12. Among them, qBR12 (Q1) on chromosome 12 was mapped at the Pi-ta locus accounted for 28.64 to $53.46 \%$ of disease reactions whereas qBR2 (Q2) was located at the $P i-b$ locus accounted 16.85 to $78.37 \%$ of disease reactions. There was a positive additive-by-additive epistatic interaction between $P i$ - $t a$ and $P i$ - $b$ suggesting that $P i$ - $t a$ interacts positively with $P i$ - $b$ in triggering a robust defense response. The remaining five minor resistance QTL have also been identified in other studies with different mapping parents suggesting that these five resistance QTL have coevolved and become domesticated. Relationship of resistance effects of blast $R$ genes/QTL with yield related components will be presented.

Identification of QTLs associated with horizontal resistance in soybean against Phytophthora sojae with an efficient hydroponic system M. DE RONNE (1), C. Labbe (1), A. Lebreton (1), J. Laur (1), C. Dussault-Benoit (1), D. Govare-Monroe (1), F. Belzile (1), L. O'donoughue (2), R. R. Bélanger (1), (1) Université Laval, Quebec, QC, CANADA; (2) CEROM, Saint-Mathieu-de-Beloeil, QC, CANADA

Although the deployment of resistance genes that confer complete immunity against Phytophthora sojae is currently the most attractive means to reduce soybean losses, the constant evolution of new pathotypes can eventually lead to a breakdown in resistance. A complementary approach is to rely on horizontal resistance, or partial resistance, that is not dependent on a gene-for-gene interaction. The objective of this study is to identify QTLs in a soybean population that have been obtained from an early maturing line adapted to Canadian conditions, and a line showing high levels of horizontal resistance both in field observations and greenhouse testing. Altogether, $150 \mathrm{~F} 5$ lines that were advanced by mass multiplication in the field without selection except for natural selection were used for the study. All 150 lines were phenotyped exhaustively for horizontal resistance against $P$. sojae using a hydroponic bioassay allowing direct inoculation of the roots with zoospores. In parallel, all lines were genotyped using a GBS approach and the resulting reads ( $\sim 1 \mathrm{M} /$ line) were used for SNP calling and construction of a genetic map. The association between phenotypic and genotypic data revealed the presence of strong and consistent QTLs for partial resistance of soybean against $P$. sojae. These results should offer new tools to breeders to combine early maturity and $P$. sojae resistance into early maturing germplasm adapted for the expanding soybean production in Canada.

Broad resistance to U.S. powdery mildew isolates in newly developed watermelon germplasm lines

C. KOUSIK (1), M. K. Mandal PhD (2), J. Ikerd (1), S. Adkins (3), W. W. Turechek (4), (1) U.S. Vegetable Laboratory, USDA, ARS, Charleston, SC, USA; (2) ORISE participant, US Vegetable Laboratory, USDA, ARS, Charleston, SC, USA; (3) USDA, ARS, US Horticultural Research Laboratory, Fort Pierce, FL, USA; (4) USDA, ARS, U.S. Horticultural Research Laboratory, Fort Pierce, FL, USA

Powdery mildew (PM) of watermelon caused by Podosphaera xanthii has been occurring more frequently in commercial fields in recent years and is known to result in significant yield reduction. Resistance to PM is available in non-edible pollenizers and in U.S. Plant Introductions (PI), whereas, commercial watermelon cultivars with resistance are lacking. We developed PM resistant red and pink-fleshed watermelon germplasm lines for use in breeding programs after six generations $\left(\mathrm{S}_{6}\right)$ of phenotyping and selection from PI. The lines USVL608-PMR, USVL313-PMR, USVL255-PMR, USVL585-PMR, and USVL278-PMR exhibit high levels of resistance in hypocotyls, cotyledons and true leaves to a local isolate of $P$. xanthii compared to watermelon cultivars Mickey Lee and a susceptible watermelon line USVL677-PMS on which abundant development of conidia was observed. In two field trials in 2017 , resistant lines had $<2 \%$ of leaf area infected compared to $>70 \%$ for USVL677-PMS. Detached true leaves of these lines placed in petri-plates were sprayed with a conidial suspension $\left(10^{5}\right.$ conidia $^{-m l}$ in $0.02 \%$ tween 20$)$ of $P$. xanthii isolates collected from different states (SC, FL, GA, $\mathrm{NY}$ and CA). All PM resistant watermelon germplasm lines were resistant to isolates from different states compared to USVL677-PMS on which severe PM (>70\%) was observed. Watermelon lines with broad resistance to PM will be good sources for incorporating resistance in commercially acceptable cultivars for use across the U.S.

Lectin genes in canola (Brassica napus) confer resistance to Sclerotinia sclerotiorum

L. BUCHWALDT, D. Hegedus, E. Dzananovic, D. Bekkaoui, J. Durkin, F. Fu, J. Nettleton, Agriculture and Agri-Food Canada, Saskatoon, SK, CANADA

Sclerotinia sclerotiorum is an unspecialized fungal pathogen of many broad leaved crops. It causes yield loss in canola worldwide by attacking the stems leading to plant wilt. A gene expression study showed differential upregulation of two lectin genes, concanavalin and curculin, $48 \mathrm{hr}$ after stem inoculation of disease tolerant cultivar Zhongyou (ZY) 821 (China) compared to a susceptible line. Both genes were cloned from ZY821 and DNA sequences were aligned with the reference genomes B. napus (AACC), B. rapa (AA) and B. oleracea (CC) to select gene copies with amino acid variation unique to ZY821. Genes coding curculin on chromosome $\mathrm{C} 6$ and concanavalin on $\mathrm{C} 1$ were selected for separate transformation into susceptible B. napus DH12075 under control of the constitutive CaMV35S gene promoter. Lines with single gene inserts in a homozygous state were identified in $\mathrm{T}_{1}-\mathrm{T}_{3}$ generations. These lines were screened for resistance by inoculating the main stem with a mycelium plug of $S$. sclerotiorum followed by measurement of lesions length 7, 14 and 21 days later. Resistant lines showed $80 \%$ and $50 \%$ reduction of lesions and no plant wilt. Mapping of sclerotinia QTL in a bi-parental DH population derived from ZY821 confirmed QTLs associated with the same lectin alleles on C1 and C6. Both lectin proteins have transmembrane domains. Other domains likely recognize and bind to fungal hyphae and may therefore trigger cell defence. Lectins are known to be abundant in $B$. napus xylem tissue, and may explain why sclerotinia was unable to cause wilt in the transformed plants.

Epigenetic regulation of $\boldsymbol{R h g} 1$, a soybean cyst nematode resistance locus

R. ZAPOTOCNY (1,2), A. F. Bent (1), (1) University of Wisconsin-Madison, Department of Plant Pathology, Madison, WI, USA; (2) University of Wisconsin Madison, Madison, WI, USA

Soybean cyst nematode (SCN; Heterodera glycines) is a major pathogen of soybean. The primary source of resistance against SCN is a locus known as $R h g l$. This $\sim 30 \mathrm{~kb}$ four-gene locus is tandemly repeated up to ten times in resistant varieties. We have previously reported that $R h g l$ transcript abundance and SCN resistance are roughly proportional to Rhgl copy number, and reported differential DNA methylation between resistant and susceptible haplotypes. One hypothesis is that the repetitive nature of the Rhgl locus constrains gene expression due to transcriptional gene silencing. To test this and other hypotheses, we have used $M c r B C$ analysis, treatment with cytosine analogs, and a single-nucleotide-resolution method known as Bisulfite Patch PCR. Our new McrBC analysis revealed shared Rhgl DNA methylation patterns in distinct soybean varieties carrying the same Rhgl haplotype. In a low-sensitivity assay, we did not detect extensive Rhgl demethylation after SCN infection. When roots were treated with the DNA methylation inhibitors zebularine and azacitidine, elevation of Rhgl gene expression was observed in high-copy Rhgl varieties. A trial run of the Bisulfite Patch PCR has revealed more precise data about $R h g l$ and additional experiments are in progress to obtain data on several soybean varieties at multiple timepoints during infection. By determining how this very important locus is regulated during SCN infection, we hope to assist efforts to improve SCN resistance. 


\section{Dissecting black spot resistance in polyploid hybrid roses}

J. D. ZURN (1), D. Zlesak (2), J. M. Bradeen (3), S. C. Hokanson (3), N. Bassil (1), (1) USDA-ARS National Clonal Germplasm Repository, Corvallis, OR, USA; (2) University of Wisconsin River Falls, River Falls, WI, USA; (3) University of Minnesota, St Paul, MN, USA

Devastating foliar diseases, such as black spot caused by Diplocarpon rosae, pose constant threats to the ornamental quality of outdoor grown roses. Black spot is primarily managed though the use of fungicides, however, there is a high demand for resistant roses which require low chemical inputs. To better characterize resistance in four popular polyploid rose cultivars (Brite Eyes ${ }^{\mathrm{TM}}$, High Voltage ${ }^{\mathrm{TM}}$, Lemon Fizz ${ }^{\mathrm{TM}}$, and Morden Blush), phenotyping was conducted with 12 D. rosae races. Additionally, two populations ('Morden Blush' $\times$ Brite Eyes ${ }^{\mathrm{TM}}$ and High Voltage ${ }^{\mathrm{TM}} \times \mathrm{Lemon}$ Fizz $\mathrm{L}^{\mathrm{TM}}$ ) were developed to study the segregation of resistance and to map it with the rose Axiom array. 'Morden Blush' was susceptible to all races while the remaining three cultivars displayed differing disease responses. A 1:1 segregation ratio was observed for the two populations where each individual was either resistant or susceptible to all races tested to date, suggesting resistance is conferred by a single resistance gene in Brite Eyes ${ }^{\mathrm{TM}}$ and Lemon Fizz ${ }^{\mathrm{TM}}$. High Voltage ${ }^{\mathrm{TM}}$ is expected to have a different resistance gene than Brite Eyes ${ }^{\mathrm{TM}}$ and Lemon Fizz ${ }^{\mathrm{TM}}$ based on observed disease responses. Linkage mapping in the 'Morden Blush' $\times$ Brite Eyes ${ }^{\mathrm{TM}}$ population identified a single resistance gene on chromosome 5 and is different from the three previously described genes. Future work will focus on developing tools for marker assisted breeding and incorporating the broad resistances into new cultivars.

Molecular mapping of loci conferring resistance to spot blotch and powdery mildew in barley using the genotyping by sequencing approach Y. LENG (1), M. Zhao (1), J. Fiedler (1), A. Dreiseitl (2), S. Chao (3), X. Li (1), S. Zhong PhD (1), (1) North Dakota State University, Fargo, ND, USA; (2) Agricultural Research Institute Kromeriz Ltd, Kromeriz, CZECH REPUBLIC; (3) USDA-ARS, Northern Crop Science Laboratory, Fargo, ND, USA

Spot blotch caused by Cochliobolus sativus and powdery mildew caused by Blumeria graminis f. sp. hordei are two important diseases in barley. To map genetic loci controlling the resistance to these diseases, a mapping population consisting of 138 recombinant inbred lines from the cross between barley cultivar Bowman and line ND5883 was developed and genotyped using the genotyping by sequencing approach. A total of 2,951 polymorphic SNP markers between Bowman and ND5883 were generated, and 1,821 markers having less than 10\% missing genotypes were used to construct a genetic map. The resulted linkage map consisted of 10 linkage groups covering $865.14 \mathrm{cM}$ in length with a $1.6 \mathrm{cM}$ average distance between markers. This population was then evaluated for infection responses (IRs) at the seedling stage to two pathotype isolates of $C$. sativus and one isolate of $B$. graminis $\mathrm{f}$. sp. hordei. QTL analysis identified three loci $(Q R c s-1 H-P 2, Q R c s-1 H-P 1$ and $Q R c s-7 H-P 1)$ controlling resistance to spot blotch and one locus $(M l a$ 8) conferring resistance to powdery mildew. QRcs- $1 \mathrm{H}-P 2$ located on chromosome $1 \mathrm{H}$ was derived from ND5883 and effective against isolate ND90Pr, while $Q R c s-1 H-P 1$ and $Q R c s-7 H-P 1$, located on chromosome $1 \mathrm{H}$ and $7 \mathrm{H}$, respectively, were derived from Bowman and effective against isolate ND85F The powdery mildew resistance gene Mla 8 is tightly linked to QTL $(Q R c s-1 H-P 2)$ at the short arm of chromosome $1 \mathrm{H}$. Identification and mapping of the QTL and DNA markers associated them will facilitate development of barley cultivars with resistance to spot blotch and powdery mildew.

\section{Genetic analysis of a source of Fusarium wilt resistance in banana from Musa acuminata ssp. malaccensis} E. AITKEN (1), A. Chen (2), J. Dalton-Morgan (2), S. Fraser-Smith (3), J. Batley (4), S. Hamill (5), (1) School of Agriculture and Food Sciences, The University of Queensland, Brisbane, AUSTRALIA; (2) University of Queensland, St Lucia, AUSTRALIA; (3) School of Agriculture and Food Sciences, St. Lucia, AUSTRALIA; (4) University of Western Australia, Perth, AUSTRALIA; (5) DEEDI, Nambour, AUSTRALIA

Tropical Race 4 (TR4) of Fusarium oxysporum f. sp. cubense (Foc) threatens global banana production. This soil-borne fungus enters the roots and colonises the xylem to induce Fusarium wilt. We have identified resistance to Foc, including to TR4, in the wild banana Musa acuminata subsp malaccensis. Pathogenicity tests of F2 progeny showed segregation of a single dominant gene conferring resistance to race 1, sub-tropical race 4 and TR4. Comparison of resistant and susceptible progeny using Genotyping-By-Sequencing analysis revealed SNPs on a genome-wide scale. A region on chromosome 3 showed a strong level of association between SNP zygosity and resistance. This region has now been fine-mapped to reveal a $157 \mathrm{~kb}$ physical sequence containing 28 putative candidate genes, none of which are classic LRR genes. Only 15 genes are predicted to carry non-synonymous SNPs leading to changes in the encoded amino acid sequence. Expression profiles of these candidate genes from inoculated resistant and susceptible plants revealed varying expression levels. SNPs from the gene markers screened across diverse banana genotypes including diploids and cultivated polyploids, identified potential resistance in breeding program germplasm where Malaccensis was a progenitor. Utilisation of a molecular marker for genetic resistance to Foc, including to TR4, will greatly assist banana breeding programs and allow understanding of the mechanisms of resistance at the molecular level.

Characterization of a bacterial leaf streak of rice resistance locus aided by nanopore sequencing A. C. READ (1), S. Carpenter (1), M. Hutin (1), L. R. Triplett (2), A. J. Bogdanove (1), (1) Cornell University, Ithaca, NY, USA; (2) Department of Plant Pathology and Ecology, The Connecticut Agricultural Experiment Station, New Haven, CT, USA

Nanopore sequencing runs generate gigabases of data with average read-lengths limited only by the input DNA library (often well over $20 \mathrm{~kb}$ ). This sequencing technology is suited for resolving challenging sequences including large, repetitive, and rapidly evolving gene families such as the plant nucleotide-binding leucine-rich repeat (NLR) defense genes. We utilized the Oxford Nanopore Technologies USB powered MinION sequencer to generate a draft genome of the American heirloom rice cultivar Carolina Gold Select. The long reads generated by this technology allow assembly of the multiple NLR-encoding $\sim \mathrm{Mb}$ Xol resistance locus. The Xol locus is effective at controlling African strains of the bacterial leaf streak pathogen Xanthomonas oryzae pv. oryzicola (Xoc). Our group recently demonstrated that Xol resistance can be overcome by a truncated TAL effector (truncTALE) from Asian Xoc strains, explaining why Xol is not effective against these strains. Knowledge of the genes underlying the Xol locus empowers our functional studies exploring how resistance is triggered by TAL effectors and suppressed by truncTALEs. We have cloned a candidate NLR from the Xol locus and begun sub-cellular localization and interaction studies. We believe that nanopore sequencing is a powerful tool for rapid determination of the genes in mapped resistance loci and will accelerate the functional analysis of candidate genes.

Discovery and characterization of disease resistance loci using a unique gene copy number variant population H. BASTIAANSE (1), L. Comai (2), A. Groover (1), I. Henry (2), G. Newcombe (3), R. Rousseau (4), (1) USDA Forest Service, Davis, CA, USA; (2) University of California Davis, Davis, CA, USA; (3) University of Idaho, Moscow, ID, USA; (4) Mississippi State University, Mississippi State, MS, USA

Pathogenic fungi that colonize poplar leaves and stems reduce yield and can cause failure of industrial bioenergy plantations. Despite extensive study of poplar pathosystems, the genetic control of poplar resistance to pathogens is still poorly understood, underscoring that new approaches are needed. We developed a unique functional genomics resource based on gene dosage variation in an elite biomass poplar hybrid. We pollinated Populus deltoides with gamma irradiated P. nigra pollen to produce $\sim 800 \mathrm{~F} 1$ seedlings. These contain large-scale deletions and insertions that together tile each chromosome. 
Under natural infection in the field as well as controlled inoculations in the greenhouse, we identified dosage QTLs influencing resistance of poplar to some of its most important fungal diseases: leaf rust and leaf spot (Melampsora sp., Alternaria sp.). Additionally, we investigated the correlation between these disease QTLs and bioenergy/developmental traits QTLs previously identified using the same genotypes. Next, time-course analysis of gene expression during progression of disease symptoms will be performed for selected genotypes, and used to develop predictive models of transcriptional networks underlying disease resistance. A final set of experiments will aim to identify candidate genes for functional analysis by manipulation using CRISPR-Cas9. Such dosage-sensitive candidate genes with significant effects on disease resistance phenotypes could then be exploited in breeding programs through the selection of germplasm with naturally-occurring allelic variation or indels/copy number variation covering resistance loci.

Integrating Host Resistance and Organic Amendments in a Non-chemical Approach to Managing Macrophomina Crown Rot in Strawberries J. WINSLOW (1), M. Mazzola (2), G. J. Holmes (1), K. L. Ivors (1), (1) Strawberry Center, California Polytechnic State University, San Luis Obispo, CA, USA; (2) USDA-ARS, Wenatchee, WA, USA

Macrophomina crown rot, caused by the soilborne fungus Macrophomina phaseolina, is an economically impactful pathogen in strawberry production worldwide. When established, the pathogen can cause extensive plant decline and mortality. In the absence of methyl bromide fumigation, new methods for managing crown rot are needed. In order to examine the combination of strawberry host resistance and organic amendments as an integrated approach for the control of Macrophomina crown rot, a greenhouse experiment was conducted in the fall of 2017. A total of six strawberry cultivars, three resistant (Petaluma, Del Rey, and Fronteras) and three susceptible (Monterey, Albion, and Festival), were grown in inoculated field soil treated with anaerobic soil disinfestation-rice bran, brassica seed meal, and steam, and included a non-amended control. Soil inoculum levels of M. phaseolina were reduced by 99, 70, and 65 percent in ASD-rice bran, brassica seed meal, and non-amended treatments respectively. Preliminary results will be presented.

Silverleaf disease management on blueberry

A. FRANCE (1), D. Grinbergs (2), J. Chilian (1), (1) INIA Quilamapu, Chillan, CHILE; (2) Instituto de Investigaciones Agropecuarias, Chillán, CHILE

Silverleaf disease (Chondrostereum purpureum) was described worldwide in blueberry cv. Brigitta Blue, in 2005, Osorno, Chile. Thereafter, the disease spread into the production area and to new cultivars. In 2014 was detected on rabbiteye, cv. Brithwell. Currently, there are 11 varieties of Vaccinium corymbosum and one of $V$. virgatum affected by the fungus. Silverleaf is difficult to control, because the fungus inhabits deep into the wood. The objective of this work was to evaluate different practices to control silverleaf in blueberry. Treatments were the pruning of symptomatic branches, in vitro test of fungicide and application to the crown, compost Tea applied through drip irrigation, and complete plant pruning at the crown level. Effectiveness was verified through foliar symptoms and ELISA test. The results showed that six out of 14 fungicides were highly active in vitro, but ineffective in plants. Branches pruning partially controlled the disease, because it appeared in the following seasons. Compost Tea was able to delay symptoms expression and reduce the incidence $(\mathrm{P}<0.05)$, but the pathogen was still present. Finally, the complete pruning of the aerial part was the only treatment able to eliminate the pathogen. New sprouts were asymptomatic and pathogen free. Thus, C. purpureum colonize stems and crown heart but not the root system, therefore when the crown heart was exposed to the sun light the pathogen died, making possible the growth of healthy shoots.

Effect of Cultural Practices in Controlling Southern Blight of Potato in the Mid-Atlantic Region

J. GARCIA GONZALEZ, S. L. Rideout, J. R. Pollok, Virginia Tech - Eastern Shore AREC, Painter, VA, USA

Over the past decade, the incidence of southern blight caused by Sclerotium rolfsii Sacc. in potato (Solanum tuberosum L.) has increased on the Eastern Shore of Virginia (ESVA) and surrounding production areas. To date, no potato cultivar is resistant to southern blight, and management has been inconsistent using fumigants and fungicides. This research evaluated the effect of planting date and cultivar selection on reducing potato loss due to southern blight. A factorial design of four planting dates and ten cultivars was evaluated in 2017 and planned for 2018. The field trials were inoculated between 56 and 73 days post-planting with virulent strains of S. rolfsii isolated from ESVA potato fields in 2016. In 2017, earlier planting dates (Mar 7 and 24) produced lower disease incidence (6-60\%), whereas incidence increased (66-100\%) for the later planting dates (Apr 14 and May 10). Total yield and tuber quality (\% marketable) were significantly reduced in the last two planting dates. There were differences in stem and tuber susceptibility between cultivars. 'Accumulator' and 'Atlantic' were identified as cultivars with lower susceptibility in the first two planting dates (10-17\%), and only 'Accumulator' significantly displayed the lowest disease incidence (71-66\%) in the last two planting dates, compared to the other nine cultivars examined (89-100\%). In general, the two early plantings presented a lower incidence of disease, higher yield, and quality; therefore, early planting dates coupled with cultivar choice are recommended as strategies to suppress potato southern blight.

Management of leaf blight and stalk rot diseases in biomass sorghum (Sorghum bicolor) grown in the U.S. Mid-Atlantic C. AGUILAR (1), H. L. Mehl (2), (1) Virginia Tech Tidewater Agricultural Research and Extension Center, Suffolk, VA, USA; (2) Virginia Tech Tidewater AREC, Suffolk, VA, USA

Biomass sorghum is currently being evaluated as an alternative crop and a potential feedstock for cellulosic ethanol production in the mid-Atlantic region. However, as a new crop, research pertaining to optimum agronomic practices is needed. Due to frequent rain events and high relative humidity, disease pressure by the sorghum anthracnose pathogen, Colletotrichum sublineola, is high. Therefore, sixteen high-biomass sorghum hybrids were evaluated at three field sites in Virginia under full-season and double-crop systems. Hybrids were visually screened for anthracnose leaf blight and stalk rot as well as lodging. Hybrid RX6 was the least susceptible to disease and lodging, while lines NS205 and NS204 were the most susceptible. Anthracnose susceptible hybrid NS212 was selected for evaluation of the efficacy of three fungicide formulations [fluxapyroxad + pyraclostrobin (Priaxor); azoxystrobin + propiconazole (QuiltXcel); and propiconazole (Tilt) combined with Priaxor]. Fungicides were applied at three timings, and anthracnose severity in treated plots was compared to a no fungicide control. A significant reduction in anthracnose was observed following a single application of Priaxor early in the growing season $(P<0.0001)$. However, due to chemical costs, fungicide control may not be an economical option for growers, and selection of disease resistant sorghum hybrids for biomass production is currently the best option for anthracnose management.

Effect of chemical, biological fungicides and resistance inducers for the management of blackberry wilt caused by Fusarium oxysporum U. Acosta-Gonzalez (1), J. Hernandez-Castrejon (1), A. REBOLLAR-ALVITER (2), H. V. Silva-Rojas (3), A. Romero-Bautista (1), R. GonzalezVillegas (1), (1) Instituto Tecnologico del Valle de Morelia, Morelia, MEXICO; (2) Univ Autonoma Chapingo, Morelia, Michoacan, MEXICO; (3) Colegio de Postgraduados, Edo de Mexico, MEXICO 
Blackberry wilt caused by Fusarium oxysporum is one of the most important diseases of blackberry in Mexico causing severe losses on established and new plantings. The objective of this study was to evaluate the effect of chemical and biological fungicides and resistance inducers for blackberry wilt management under greenhouse conditions. First, the efficacy of the fungicides prochloraz, thiabendazole, azoxystrobin, thiophanate-methyl,

difenoconazole, triflumizole and potassium phosphite to control six pathogenic $F$. oxysporum isolates was conducted using an in vitro assay. This was followed by a greenhouse trial to test the same fungicides applied pre-inoculation on 5-month old potted plants. In a separate experiment, the effect of commercial biocontrol agents, including a strain of Trichoderma koningiopsis (RCH-169), and resistance inducers (potassium phosphite and acibenzolars-methyl) was quantified. Incidence, severity, fresh weight, dry weight, root length, plant height, and colony forming units (CFU g ${ }^{-1}$ of soil) were obtained. Under in vitro conditions, Prochloraz showed the strongest inhibitory effect $\left(\mathrm{EC}_{50}=0.01 \mu \mathrm{g} \mathrm{mL} \mathrm{m}^{-1}\right)$, followed by triflumizole $\left(\mathrm{EC}_{50}=0.05-0.32 \mu \mathrm{g}\right.$ $\mathrm{mL}^{-1}$ ) and difenoconazole $\left(\mathrm{EC}_{50}=0.09-0.13\right)$. In greenhouse experiments, prochloraz and acibenzolar-S-methyl were most effective in reducing the incidence and severity of blackberry wilt. In the biological control trial, the native strain of $T$. koningiopsis resulted in the lowest incidence (33\%) and severity $(28.43 \%)$, compared with commercial products and the control, including higher fresh weight and lower number of CFU $\mathrm{g}^{-1}$ soil. Together these results indicate some promising treatments for the control of blackberry wilt, which could be integrated into a full season approach to managing the disease in the field.

Understanding the ecology of blueberry rust to improve management in evergreen blueberry production in Australia R. DANIEL (1), K. Scarlett (2), D. Collins (3), (1) NSW Department of Primary Industries, Ourimbah, AUSTRALIA; (2) Forest Research, Farnham, UNITED KINGDOM; (3) NSW Department of Primary Industries, Menangle, AUSTRALIA

More than $80 \%$ of blueberries in Australia are grown in subtropical conditions under an evergreen cropping system. New leaves, susceptible to infection by the fungus Thekopsora minima, the cause of blueberry rust, develop at any time of the year. Severe symptoms may develop and cause defoliation and fruit lesions. Producers currently adhere to strict management guidelines based on calendar sprays of fungicides in the absence of a complete understanding of the disease cycle. Our aim was to identify vulnerable points in the disease cycle that enable targeted, and more effective, application of fungicides. Air sampling, field and greenhouse studies were conducted to determine factors contributing to disease development. In the evergreen system, T. minima survives primarily in infected, symptomatic as well as asymptomatic, leaves retained on the plant. Viability of urediniospores in leaf litter declines to $25 \%$ after 2 weeks. Air sampling has shown that urediniospores are present at any time of the year and are the main source of new infections. Preliminary analyses indicate that spore numbers are greater in humid and wet conditions. The effect of leaf wetness on spore germination and infection needs to be investigated further. We conclude that because spores and new foliage can be present all year round in the evergreen system, fungicide sprays should be targeted when weather conditions are mild and moist.

Microbial diversity and disease suppression in turfgrass landscapes under intensive management M. MILLICAN, E. Buczkowsi, R. Townsend, P. L. Koch, University of Wisconsin, Madison, WI, USA

Maintenance of turfgrass requires routine applications of nitrogen fertilizer, herbicides, insecticides, and fungicides that are beneficial for the turf stand but have broader ecological costs. Understanding the influence of nitrogen and pesticide applications on the turf-associated microbiome is a critical step in understanding how these maintenance regimes impact the surrounding ecosystems and the health and sustainability of the turf phytobiome. High and low acute toxicity herbicides, insecticides, or fungicides were sprayed on tall fescue in Madison, WI and soil samples taken at $0,1,3,7,14$, and 21 days Nitrogen was applied to creeping bentgrass at $0,9.76$, and $29.29 \mathrm{~kg} \mathrm{~N} / \mathrm{ha}$ applied bimonthly and soil cores were removed every four weeks. Bacterial and fungal diversity in both studies was assessed using the 16S rRNA and ITS-2 DNA regions, respectively, and sequenced using Illumina MiSeq. Raw sequencing data was processed using Mothur and statistical analyses were carried out in R. Increasing nitrogen input to $29.29 \mathrm{~kg} / \mathrm{ha}$ results in reduced dollar spot severity nearly to fungicide treated turf. With our study we strive to understand how pesticides, pesticide toxicity, and nitrogen fertilizer impacts microbial diversity and any implications it has on turfgrass disease suppression. These results will deepen our knowledge of how management practices affect the turf phytobiome and help create more sustainable management strategies moving forward.

Ignored fungal pathogen sibling - Leptosphaeria biglobosa

Y. HUANG, A. Javaid, L. H. Gajula, C. Karandeni-Dewage, G. Mitrousia, B. Fitt, University of Hertfordshire, Hatfield, UNITED KINGDOM

In nature, plants are often infected by more than one pathogen and there are many factors affecting their co-existence on their host which lead to changes in their predominance. Phoma stem canker is a damaging disease of oilseed rape (Brassica napus) and Brassica vegetables. This disease is caused by two closely related sibling pathogens, Leptosphaeria maculans and $L$. biglobosa. Since $L$. maculans generally causes stem base canker while $L$. biglobosa causes upper stem lesions, $L$. maculans is considered more damaging than L. biglobosa. Therefore, previous work has mainly focused on $L$. maculans and the importance of $L$. biglobosa in phoma stem canker epidemics has been ignored. However, results of our recent work show that $L$. biglobosa can cause both damaging upper stem lesions and stem base cankers. Furthermore, L. biglobosa is less sensitive to some triazole fungicides than $L$. maculans. The need for effective host resistance to control this disease is greater than ever. However, previous breeding for cultivar resistance has targeted only $L$. maculans; there is no information about cultivar resistance against $L$. biglobosa. Recent studies have shown that cultivars resistant against $L$. maculans are often more susceptible to L. biglobosa. For effective control of phoma stem canker, there is a need to target both L. maculans and $L$. biglobosa. In this talk, the reasons why L. biglobosa has recently increased in importance in phoma stem canker epidemics in the UK will be discussed, using results from field experiments over three seasons and controlled environment experiments.

\section{Integrated management of clubroot-crucial for sustainable oilseed rape production}

A. C. WALLENHAMMAR (1), A. Jonsson (2), (1) Rural Economy and Agricultural Sociery (REAS), Örebro, SWEDEN; (2) Research Institutes of Sweden RISE Agrifood and Bioscience, Skara, SWEDEN

Clubroot, caused by the soil borne pathogen Plasmodiophora brassicae, is a serious threat to oil seed rape (OSR) production and the proportion of clubroot infected fields in Sweden is steadily increasing. Control of clubroot is particularly difficult due to the long persistence of the pathogen in soil. The aim is to develop a concept for integrated control of clubroot in winter OSR crops, supported by DNA-technology, by examining and comparing infestation levels and yield of resistant and susceptible varieties. The objective is to provide an improved decision support and guidelines for growing winter OSR in fields where P. brassicae DNA occurs. Resistant varieties are partially resistant, as they are infected at low levels of pathogen inoculum. Three resistant varieties and a seed mixture of susceptible varieties were established in four field trials in August 2017. P. brassicae was identified in these fields at levels ranging from 5000 to 2.5 million target copies $\mathrm{g}^{-1}$ soil. Soil samples were collected plotwise immediately prior to seeding the trials. Quantification of $P$. brassicae by qPCR analysis was performed and bioassays were carried out in a greenhouse for a six week period to ensure optimal infection. Plants were sampled plot-wise in late autumn and roots assessed for disease symptoms. Preliminary results show a severe infection in susceptible varieties already at 15000 target copies $\mathrm{g}^{-1}$ soil while a low infection occurred at 5000 target copies $\mathrm{g}^{-1}$ soil. 
Marssonina leaf blotch is a fungal disease of apple caused by the ascomycete Diplocarpon mali (anamorph: Marssonina coronaria). The pathogen origins from Asia where it accounts for crop losses of up to 100\%. In Europe it was first detected in Italy in 2001 and is spreading since then in Switzerland and southern Germany (first reports in 2010), mainly in organic apple orchards and in orchards for cider production with a reduced fungicide regime. M. coronaria infects the leaves by using appressoria and shortly afterwards forms haustoria in the host cells. Blackish-violet spots appear on the upper leaf side, sometimes turning into star-like lesions. On the lesions acervuli are produced and eventually the leaves become chlorotic and fall off. First symptoms can be observed in June after a humid period, leaf drop may start two to three weeks later. Severely infected trees can be completely defoliated already in August. The FiBL and KOB Interreg project aims at gaining more knowledge on the biology of the fungus and on control methods in organic farming. For example has the teleomorph Diplocarpon mali not yet been detected in Europe and also the form of overwintering is still unknown. Identification of resistant or less susceptible cultivars, to be directly used in production or as sources of resistance in apple breeding, is a further important pillar in Marssonina control. First results on a population study will be presented.

Evaluation of chemical soil treatment and cultivar on the incidence of powdery scab and Potato mop-top virus in potato A. C. FULLADOLSA (1), Y. Zeng (2), A. O. Charkowski (1), (1) Colorado State University, Fort Collins, CO, USA; (2) Colorado State University, Center, CO, USA

Potato mop-top virus (PMTV) is a necrotic virus of potato and an emerging threat to North American potato production. The powdery scab pathogen Spongospora subterranea f. sp. subterranea (Sss) is the vector of PMTV and has been found in most potato producing regions of the U.S. Sss resting spores, which may harbor PMTV, survive in the soil for many years, even without potato. Neither Sss nor PMTV can be cultured in a laboratory in the absence of a plant host. As a result, there has been relatively little progress in understanding these pathogens and their interactions, and growers have few available management options. We evaluated the effects of two chemical soil treatments on the incidence and severity of Sss and PMTV in six potato cultivars, and on the reduction of Sss soil inoculum. We collected soil samples pre-planting and prior to harvest in each soil treatment block and quantified Sss resting spores using a real-time PCR assay. There was no significant difference of the treatments on the amount of Sss resting spores in the soil, indicating that the efficacy of the soil treatments was low. The variability among samples collected prior to harvest, within each soil treatment, was higher than among those collected pre-planting. This may be due to a differential response of the pathogen to the presence of its host or the cultivar planted in the sampling location. A 50-tuber sample per cultivar-treatment combination was collected at harvest and stored for 90 days at $55^{\circ} \mathrm{F}$. We will determine the incidence and severity of powdery scab on the tubers, and the incidence of PMTV. The information generated through this work will improve our understanding of Sss, its role as a vector of PMTV and will aid the development of improved management practices.

Variability of Colletotrichum truncatum through morphological \& molecular approaches and management of pod blight complex of soybean in India

S. JAHAGIRDAR (1,2), B. G T (1), R. H. Patil (1), (1) University of Agricultural Sciences, Dharwad, Dharwad, INDIA; (2) Univ of Agricultural Sciences Dharwad, Dharwad, AK, INDIA

The association of Colletotrichum truncatum, Alternaria alternata, Macrophomina phaseolina have been observed in causing anthracnose/pod blight of soybean in northern Karnataka. However, Colletotrichum truncatum has been found predominantly associated in causing anthracnose disease. Variability study indicated that, there was much difference in colony colour ranging from cottony white to dark grey, the spore shape was only truncate type and there is no difference with respect to spore size of Colletotrichum truncatum isolates. The results obtained from the cluster analysis revealed that sub cluster groups composed of isolates belonging to same geographical locations with distinct variability. Out of 19 genotypes, the genotypes viz., DSb 12, DSb 20, DSb 23-5 and Kalitur were found highly resistant with a disease grade of one. The genotype JS 335 was highly susceptible to anthracnose with a maximum disease grade of nine. The two year investigation $(2014 \& 2015)$ revealed that the integrated management by seed treatment with Thiram+Carboxin@ $2 \mathrm{~g} / \mathrm{kg}$ of seed followed by spray at 55 and 75 DAS with Trifloxystrobin + Tebuconazole @ $0.1 \%$ was found effective in reducing anthracnose severity and enhancing the yield both at Dharwad and Ugarkhurd. In modular approach study conducted over three years (2013-2015), the chemical module/adapative module have been recommended for the management of major diseases (rust, anthracnose and purple seed stain) and insect pests (defoliaters and Pod borer) in a holistic approach.

Disease control in variety mixtures is not directly linked with yield benefit

A. C. NEWTON (1), D. Guy (1), R. Brooker (2), (1) James Hutton Institute, Dundee, UNITED KINGDOM; (2) James Hutton Institute, Aberdeen, UNITED KINGDOM

The reduction in disease in cereal variety mixtures is normally correlated with the number of component varieties, as is the yield benefit. However, the link between disease and yield is indirect as the effect of disease is only one of many interactions between mixture components that ultimately affect grain and straw yield. Use of fungicide treatments to compare diseased and non-diseased plots can be misleading as varieties interactions are still influenced by microbial challenge. Furthermore, nutrient availability and thereby source-sink interactions affect both disease development and variety interactions. We show that designing mixtures for enhanced yield involves optimising multiple competing and facilitating interactions and that the disease component should be optimised within this complex framework.

\section{An integrated approach to controlling white grubs in forestry}

B. SIVPARSAD, A. Morris, I. Germishuizen, K. Peerbhay, Institute for Commercial Forestry Research, Pietermaritzburg, SOUTH AFRICA

In South Africa (SA), white grubs are the dominant and most economically important soil invertebrate pest during forest establishment. An effective control measure for soil-borne pests is deltamethrin, a chemical banned by the Forest Stewardship Council (FSC) but used by the SA forestry industry under temporary derogation. Therefore, there is an urgent need to find alternative chemical and biological control measures that will comply with FSC regulations in the management of soil pests. This study was initiated to (1) evaluate alternative ecologically sound insecticides and biologicals to reduce the impact of white grub feeding on post-plant performance and (2) monitor the incidence of white grub in selected sites to provide spatial and temporal trends of pest activity. Results of pot trial evaluations indicated that biological products containing azadirachtin, potassium silicate and Beauveria brongniartii, and chemical products containing imidacloprid and clothianidin, protected wattle and eucalypt seedlings from white grub damage. In addition, treatment with imidacloprid and B. brongniartii significantly reduced final mortality of wattle seedlings to $33 \%$ and $50 \%$ respectively. Temporal sampling of selected wattle, pine, and eucalypt sites have indicated that a greater white grub population and diversity is found in wattle sites that are in close proximity to sugarcane fields. Five morphospecies have been tentatively identified based on differential rasta patterns. These include: 
Maladera spp., Pegylis sommeri, Adoretus ictercus, Schizonycha affinis and Schizonycha fimbriate. Results of this study will form a basis for the formulation of an integrated approach for the control of soil-borne pests in forest regeneration.

Evaluating management of stripe rust in North Dakota with foliar applied fungicides and host resistance B. HALLEY, S. M. Meyer, J. Halvorson, C. Schuh, S. Haugen, A. J. Friskop, North Dakota State University, Fargo, ND, USA

Stripe rust (yellow rust; Yr) caused by Puccinia striiformis f. sp. tritici is a cool season disease that can cause yield limitations in North Dakota (ND). Recently, Yr has been detected earlier in the wheat growing season in the Northern Great Plains of the USA. In ND, Yr has been documented as early as Feekes 2 (tillering) in hard red spring wheat. This has increased the risk for economic losses in spring wheat, warranting updated management recommendations. The objective of the research was to determine the effect of fungicide timing in combination with host resistance for Yr management. Five field trials were conducted at three ND locations in 2016-2017. Each trial was a randomized complete block design with a split-plot arrangement. Main plots were three spring wheat varieties varying in Yr susceptibility. Sub-plots were four common fungicide application timing treatments, including a non-treated control, a Feekes 9 (flag leaf) application, a Feekes 10.51 (early-anthesis) application, and sequential application of both Feekes 9 and 10.51. Severity diagrams and area under disease progress curves were generated with data from visual assessments of plants for Yr. Plots were harvested and yield, test weight, and DON were determined. Under high disease pressure, fungicide applications at Feekes 9 and Feekes 10.51 on a susceptible variety resulted in disease control values in excess of $90 \%$. Results will aid in updating management recommendations for ND wheat growers.

Citrus decline a fast spreading disease of Citrus reticulate in South East Asia a quick diagnosis and management technique P. RAJA (1), K. M. Singh (2), P. Nallathambi (3), A. K. Pandey (1), (1) College of Horticulture and Forestry, Pasighat, INDIA; (2) central agricultural university, Imphal, INDIA; (3) ICAR-Indian Agricultural Research Institute, Regional station, Wellington-643231, INDIA

Citrus reticulate is widely cultivated in south east Asian countries. The pest and diseases are major production constraints in this region. Out of diseases, decline leads to death of hundreds of mandarin trees in north eastern states of India. Citrus tristeza virus (CTV) was contributed 30-50, 60-80 per cent decline in 5-10 and 10-15 year old trees respectively. The key symptoms are veinal chlorosis, vein corking, gradual wilting on the tip and branches. For quick detection, the branches are soaked in water with tabolene solution ( $0.5 \%)$ for $48 \mathrm{hrs}$, upon peeling of stem zonal lines, stem pitting are seen. In India, Toxoptera citricida, Toxoptera aurantii is spreading the CTV and active in Dec to March. Upon releasing of viruliferous insects into Citrus limon seedling, similar symptoms were observed. Early detection and replanting with pre-immunized seedling could reduce $90 \%$ incidence followed by $0.01 \%$ dimethoate spray $(60 \%)$. Citrus trunk borer caused $40-60 \%$ decline in $4-10$ year old trees during Sep-Oct months. The grub of the trunk borer bores the tree trunk near ground level horizontally up to the pith and then tunnels vertically and again horizontally for exit during September to November months Presence of chopped saw dust outside the hole is the key symptom to identify this pest. Gradual drying and sudden drooping of premature leaves are common symptoms observed during the study. Experimenting with various management treatments depicted that stem injection with $0.5 \%$ Dichlorvos was the best treatment followed by cent per cent kerosene or petrol then covering with clay. Painting with $10 \%$ Bordeaux mixture with $0.5 \%$ Dichlorvos in the trunk region up to $30-40 \mathrm{~cm}$ height significantly reduced egg laying in the month of June-July. Further works are in progress to develop integrated pest and diseases management modules specific to north eastern states of India.

The biology and etiology of Syzygites megalocarpus, an emerging pathogen of the button mushroom Agaricus bisporus J. DE SOTO, J. A. Pecchia, D. Beyer, Pennsylvania State University, University Park, PA, USA

Syzygites megalocarpus is a fungal (Mucoromycotan) pathogen that infects other fungi, including Agaricus bisporus, the button mushroom. The pathogen recently appeared on a commercial Pennsylvania mushroom farm and has since become a reoccurring problem on many farms. The first objective was to test the effectiveness of two fungicides on S. megalocarpus by measuring mycelial growth rates on plates amended with the fungicides. Neither of the 2 fungicides killed the pathogen or had a great effect on growth rate. The second objective was to establish sporangiospores' thermal death point to determine if current commercial composting parameters would kill the pathogen. Results indicated that S. megaloacarpus sporangiospores have a low thermal death point and did not survive exposure to temperatures above $37^{\circ} \mathrm{C}$ for 30 minutes, indicating that mushroom composting parameters are more than adequate to kill the pathogen. These results imply that infection in cropping systems must be due to secondary contamination of the compost after pasteurization. The third objective was to determine the susceptibility of several, widely used commercial mushroom strains to $S$. megalocarpus infection. Three brown and one white strains were inoculated with both plugs and spore suspensions of $S$. megalocarpus to determine susceptibility levels of different strains as well as to determine if a spore suspension or mycelial plug better initiates disease symptoms. These experiments are still ongoing.

Modifying row cover systems to manage bacterial wilt in organic muskmelon in Iowa, USA

M. L. GLEASON, H. M. Nelson, Iowa State University, Ames, IA, USA

The use of spunbond row covers on muskmelon is limited to early in the season, but organic insecticides used after row cover removal are ineffective and harm beneficial insects. Mesotunnels - nylon mesh row covers - could provide full-season protection with less insecticide use. Four systems were tested on muskmelon in Iowa during 2016 and 2017: spunbond low-tunnels removed at anthesis, mesotunnels removed for two weeks during pollination, fullseason mesotunnels with bumble bees inserted for pollination, and a never-covered control. Insecticides were applied to non-covered plots based on economic thresholds for cucumber beetle. Full-season mesotunnels led to lower bacterial wilt incidence than spunbond low-tunnels and never-covered in 2016, but bacterial wilt was absent in 2017. In both years, full-season mesotunnnels had a higher weight of marketable fruit than never-covered control, but only in 2016 did full-season mesotunnels yield more than spunbond low-tunnels. The number of marketable fruit was greater for the full-season mesotunnels than other treatments in 2016, but yield enhancement was not significant in 2017. No insecticides were applied in the full-season mesotunnels, whereas spunbond low-tunnels required 2 sprays in 2016 and 1 spray in 2017. Mesotunnels may provide a way for organic muskmelon growers to achieve consistently high marketable yield with minimal need for insecticides.

Efficacy of organic treatments for managing tomato foliar and soilborne diseases in WV

R. MAHFUZ, L. Jett, West Virginia University, Morgantown, WV, USA

Early blight, Septoria leaf spot and soilborne wilt diseases of tomato cause significant yield losses on in WV every year. Many small growers in the state do not want to use harsh chemicals. This study determined the efficacy of early blight (EB) resistant varieties, Mountain Fresh Plus F1 (MF+) and Juliet F1 together with the treatments of 'Brandywine' by 1) Kocide-started at pre-bloom on a seven-day schedule; 2) Regalia + Kocide alternated by week starting at pre-bloom; 3) Kocide at first symptom appearance (seven-day interval afterwards); 4) Serenade and 5) Actinovate on a seven-day schedule starting at pre-bloom; and 6) Non-treated control at the WVU certified organic farm in a RCB design with four replications. All treatments except 
Actinovate and Kocide (on symptom appearance) significantly $(\mathrm{P} \leq 0.04)$ reduced EB severity compared to the non-treated control. Another study was conducted with heirloom tomato 'Mortgage Lifter' to investigate the efficacy of bio-fumigants, biological antagonists, and resistant rootstock in managing wilt disease. Thirty-five DAP, grafted (on resistant rootstock "Maxifort") tomato plants showed significantly higher plant vigor compared with non-treated check. All treatments had significantly lower symptomatic leaves than that of non-treated check. Cumulative harvests for six weeks showed that yield from all treatments were significantly $(\mathrm{P}<0.001)$ higher compared with non-treated check. Grafted plants produced $20 \mathrm{lb}$ tomatoes/plant compared with only $11 \mathrm{lbs}$ by non-treated check. Results suggest that organically acceptable methods can reduce both foliar and soilborne diseases if applied on time, and provide significant yield advantage to heirloom tomatoes.

Integrated Fusarium head blight management under irrigation in spring wheat in Idaho J. M. MARSHALL (1), S. Arcibal (2), Y. Dong (3), (1) University of Idaho, Idaho Falls, ID, USA; (2) University of Idaho, Aberdeen, ID, USA; (3) University of Minnesota, St Paul, MN, USA

Fusarium head blight (FHB) is an emerging disease in Idaho due to increased acreage of corn for dairies. A fungicide trial was conducted in southern Idaho for two consecutive years to evaluate the integrated management strategies for reducing FHB and deoxynivalenol (DON) in spring wheat. Four varieties (Klasic, Diva, IDO1202S and IDO851) were planted in a split-plot arrangement with 4 replications, with timing of fungicide application as the main plot and variety as sub-plot. Fungicides were applied at anthesis only (Prosaro) and anthesis +4 days (Prosaro + Caramba, Caramba + Folicur and Proline + Folicur) using a $\mathrm{CO}_{2}$ backpack sprayer at a rate of $20 \mathrm{gal} / \mathrm{A}$. Within 24 to 36 hours of the anthesis fungicide application, a conidial suspension $\left(100,000\right.$ macroconidia/liter) was applied on all plots with a $\mathrm{CO}_{2}$ sprayer with three Teejet 8003 VS nozzles at a ground speed of 1 second per foot at 40 psi. Using the GLIMMIX procedure (SAS 9.4), the variety by fungicide treatment interactions were determined for FHB index, FDK, test weight and yield. Fungicide treatments, especially Prosaro + Caramba, significantly reduced FHB Index, FDK and DON of susceptible (Klasic) and moderately susceptible (IDO1202S and Diva) varieties. The benefit of using the additional post-anthesis fungicide application was only observed in Klasic. Resistance is still the most effective strategy to prevent disease and yield loss as shown by the moderately resistant variety, IDO851. Validation of susceptibility and resistance in the next generation of wheat varieties combined with anthesis fungicide applications will determine economic and strategic benefit of IPM methods.

Effect of cultivar on biocontrol efficacy of Pythium in hydroponic tomato system

L. DEGENRING, A. M. Poleatewich, University of New Hampshire, Durham, NH, USA

Oomycete pathogens cause significant losses in greenhouse production due to their swimming zoospores and wide host range. Many greenhouse producers have utilized commercial biological control agents (BCA), in addition to chemical treatments, to protect plants from these pathogens. Few studies have evaluated the effect of cultivar on BCA colonization and disease suppression efficacy in horticulture crops. This research evaluated the effect of cultivar on biocontrol efficacy in a small scale tomato hydroponic system to identify IPM potential. We predicted that there would be a significant difference in efficacy of BCA between tomato cultivars. Ten cultivars, ranging from those currently used in commercial production to wild relatives, were screened for disease susceptibility using Pythium spp. inoculated at transplant. Four cultivars were selected and evaluated for differential biocontrol efficacy. Biocontrols were applied twice as a drench at transplant to rockwool blocks and at transplant to coco filled pots or rockwool slabs. Plants were challenged 3 days after application with Pythium spp. Root rot, plant height, and dry biomass were evaluated after 3 weeks. This experiment will provide initial data for future research analyzing the causal reasons for varying BCA performances between cultivars and substrates. Results from this research will be shared with growers and biocontrol producers in an effort to improve on-farm performance and increase adoption of biopesticides as plant disease management tools.

Southern blight epidemic: What management options are available to smallholder Common bean farmers in Uganda? P. PAPARU (1,2), C. Mukankusi (3), A. Acur (2), F. Kato (4), A. Kayongo (2), J. Nakibuule (2), S. Nkalubo (2), (1) National Agricultural Research Organization -National Crops Resources Research Institute, P.O. Box 7084, UGANDA; (2) National Agricultural Research Organization -National Crops Resources Research Institute, Kampala, UGANDA; (3) CIAT, Kampala, UGANDA; (4) Centro Internacional De Agricultura Tropical, Kampala, UGANDA

Southern blight caused by Sclerotium rolfsii Sacc. has become a serious disease of common bean in Ugandan. This being a relatively new disease, farmers are not using any management strategies to reduce the impact of the disease on yield. To identify resistant varieties, we evaluated over 500 lines from the Andean Diversity Panel, 132 ALBs (SER 16 x Phaseolus coccineus), lines from Fusarium and Pythium root rot nurseries and Ugandan local germplasm for their reaction to Sclerotium rolfsii. We also validated in farmers' fields root rot management practices such as seed treatment with fungicide (mancozeb and metalaxyl-M), use of NPK 17:17:17 and farm yard manure, and a combination of seed treatment with either of the fertilizers. Untreated seed of the susceptible variety CAL 96 acted as a control. We identified 4 lines with considerable resistance to southern blight, and these are currently being used in our crop improvement programme. Seed treatment and soil fertilization significantly increased seed germination, plant stand at harvest and yield. Seed treatment in combination with NPK 17:17:17 and farm yard manure application increased seed germination by 5-20\%, plant stand at harvest by up to $10 \%$ and yield by $17-33 \%$ over control. As breeding efforts to produce a resistant variety continue, we recommend the use of fungicide seed treatment and soil fertilization to reduce the impact of southern blight and improve yield of common bean in smallholder farms in Uganda.

\section{Managing small patches of field infestation of Plasmodiophora brassicae (clubroot)}

M. R. MCDONALD (1), B. D. Gossen (2), A. Sedaghatkish (1), F. Al-Daoud (1), S. E. Strelkov (3), S. F. Hwang (4), (1) University of Guelph, Guelph, ON, CANADA; (2) Agric \& Agri-Food Canada, Saskatoon, SK, CANADA; (3) Univ of Alberta, Edmonton, AB, CANADA; (4) Alberta Agriculture, Edmonton, AB, CANADA

Clubroot of canola (Brassica napus), caused by Plasmodiophora brassicae, continues to cause crop losses in Canada. Effective methods are needed to reduce the spread of the pathogen within and between fields and to reduce the population of resting spores in soil. A proposed recommendation for treating small areas of infestation is to apply lime, preferably a mixture of quick lime and a standard lime, to increase the soil $\mathrm{pH}$ to 7.4 or above, then seed a perennial cover crop. Other methods to reduce resting spore populations are solarisation with or without fumigation. Resting spores were extracted and treated with propidium monoazide prior to qPCR to quantify the number of viable resting spores in the soil profile. Solarization, achieved by covering soil with totally impermeable film for 16 days, increased mean soil temperatures by about $8{ }^{\circ} \mathrm{C}$ and reduced clubroot severity from $81 \%$ to $35 \%$ on bioassay crop pak choi (Brassica rapa). Adding the fumigant chloropicrin or metam sodium did not further reduce clubroot severity, but yield increased with decreasing clubroot severity. Trials in controlled environment showed that the grass cover crops perennial ryegrass (Lolium perenne) and smooth brome grass (Bromus inermis) reduced the number of viable resting spores in soil after 8 weeks of crop growth. Soil-core samples should be taken to determine when the population of viable resting spores has dropped below an economic threshold. 
Successful Control Cases of Bacterial Canker of Kiwifruit in Korea

G. H. Kim, J. S. Jung, Y. J. KOH, Sunchon National University, Suncheon, KOREA

Bacterial canker disease caused by Pseudomonas syringae pv actinidiae (Psa) is the biggest limiting factor in the cultivation and production of kiwifruit in Korea. Bacterial canker caused by Psa biovar 2 designated Psa2 occurred for the first time on the green-fleshed kiwifruit cultivar Hayward in 1988 and the yellow-fleshed kiwifruit cultivar Hort16A in 2006 in Korea. Psa biovar 3 designated Psa3 began to appear in Korea in 2011. Both biovars are now prevailing in almost all of the kiwifruit growing areas in Korea in recent years. Since bacterial canker has been threatening kiwifruit industry enough to destroy whole kiwifruit vines or orchards in one or a few growing seasons, it is urgent to develop effective control procedures of bacterial canker for kiwifruit growers. They might include various management practices such as disease escaping, cultural practices, blocking of dissemination, early diagnosis, eradication of inoculum sources, chemical control, and trunk injection of antibiotics. On the basis of reduplicative trials on control of bacterial canker caused by Psa2 or Pas3 at naturally infected kiwifruit orchards, we successfully defeated bacterial canker using various combinations of the management practices. Such successful control cases of bacterial canker of kiwifruit can be practically utilized at kiwifruit growers' orchards in order to keep kiwifruit vines healthy in the future.

Integrated management of Rhizoctonia in sugar beet: Resistant varieties, seed treatments and postemergence fungicides A. K. CHANDA (1), J. R. Brantner (2), (1) Department of Plant Pathology, University of Minnesota, Crookston, MN, USA; (2) University of Minnesota, Crookston, MN, USA

Rhizoctonia damping-off and crown and root rot (RCRR) have been the most common root diseases on sugar beet in Minnesota and North Dakota. In 2017, a field trial was established to evaluate an integrated management strategy consisting of a resistant and moderately susceptible variety with seed treatments (penthiopyrad, sedaxane, and fluxapyroxad) in combination with postemergence (PE) azoxystrobin applied at the 4- or 8-leaf stage. Both varieties had similar stands up to 8 weeks after planting (WAP). Seed treatments and untreated control had similar stands at 2 WAP and by 3 WAP all the seed treatments had higher stands compared to the untreated control. At 5 WAP, sedaxane had highest stands, penthiopyrad and fluxapyroxad had intermediate, and untreated control had lowest stands. There were no significant differences between two varieties for stand data and harvest data. Root rot rating, yield, and other harvest parameters were not significantly different for the seed treatments and untreated control. Root rot severity and percent incidence were significantly lower with 4- or 8-leaf PE application compared to no PE. Yield, recoverable sucrose and percent sucrose were not significantly different between azoxystrobin and no azoxystrobin application. Results suggest an integrated approach utilizing a seed treatment to protect seedlings followed by a PE application for mid- to late-season disease pressure.

Agro-economic assessment of the improvements on the management of Fusarium head blight of wheat

R. XIA, D. Hooker, V. Limay-Rios, A. W. Schaafsma, University of Guelph, Ridgetown Campus, Ridgetown, ON, CANADA

Fusarium head blight (FHB), caused by Fusarium graminearum (Schwabe) causes yield loss, quality reduction and mycotoxin contamination (predominately deoxynivalenol (DON)) in wheat. This project is aiming to quantify the economic impacts brought by changes in key agronomic and FHB management practices since a major epidemic in 1996. Field plots, conducted in 2017 consisted of treatments representing agronomic and FHB management practices used in 1996 compared with those used today. Three nitrogen rates $(\mathrm{N}), 5$ winter wheat varieties (one historical and 4 current varieties representing varying levels of susceptibility to FHB) and a fungicide applied at flowering (adepidyn \& non-treated control) were tested. Plots were challenged at $50 \%$ anthesis with spray inoculation followed by mist irrigation. Yield and DON were measured after harvest. Genetic resistance and FHB fungicide individually each contributed approximately $50 \%$ to DON reduction compared with non-treated controls, and combined, they resulted in $75 \%$ reduction. A fungicide application at flowering increased yield by $14-25 \%$ compared to the control. The main effects of genetic resistance and increasing $\mathrm{N}$ did not affect yield. These results will be discussed in the context of historical farm survey data where data on agronomic practices, yield and grain mycotoxin concentration for the epidemic years 1996, 2013 and 2015 were collected. Yield and DON will be modelled quantitatively and converted to economic impacts.

Sustainable control of Strawberry Powdery Mildew

X. Jin (1), A. HALL (2), (1) National Sun Yat-Sen University, Kaohsiung, TAIWAN; (2) University of Hertfordshire, Hatfield, Herts, UNITED KINGDOM

The most serious disease of protected strawberries in the UK is strawberry powdery mildew caused by Podospheara aphanis. This disease can cause $20 \%-80 \%$ yield loss, where $20 \%$ loss is worth $£ 56.8 \mathrm{mp} / \mathrm{a}$. At present the disease is controlled by spraying with fungicides up to 18 sprays a growing season). Most strawberries eaten in the UK are home grown but in addition to financial pressures, growers are under pressure to reduce fungicide sprays and residues. The aim of the work reported here was to devise an integrated control programme, to reduce the inoculum and achieve control with reduced use of fungicides. The programme operates throughout the growing season. Chasmothecia of $P$. aphanis survive on plants and initiate infection in the spring on plants grown for more than one season. P. aphanis also comes onto farms on plants brought in from propagators. Both inoculum sources are reduced by an early season fungicide spray. Regular use of a silicon nutrient once or twice a week in the fertigation tubes throughout the season reduces susceptibility to strawberry powdery mildew. The temperature and humidity necessary for $P$. aphanis germination, growth and spore production have been used to design a web based, real time, rule based prediction system which accumulates the hours of disease conducive conditions. All these measures used in an integrated management programme give good disease control with five fewer fungicide sprays a season.

Site-specific management of spring dead spot of bermudagrass (Cynodon spp.)

J. BOOTH (1), D. S. McCall (2), D. Sullivan (3), J. Friell (4), (1) Virginia Tech, Moseley, VA, USA; (2) Virginia Tech, Blacksburg, VA, USA; (3) TurfScout, Greensboro, NC, USA; (4) The Toro Company, Bloomington, MN, USA

Spring dead spot (SDS) is a reoccurring disease of bermudagrass (Cynodon spp.) in colder regions of adaptation. Ophiosphaerella spp. can infect prior to winter dormancy and predispose the plants to isolated patches of winter damage. Symptoms of dead patches in the turf appear in the spring and can persist for months. Fall fungicide applications have proven most effective for disease suppression. Because symptoms are not present at the time of application, current suppression tactics include multiple fungicide applications across all managed acreage. Despite inconsistent results, turfgrass managers often use affordable fungicides to control costs. Site-specific applications to areas with known SDS outbreaks may result in total fungicide reductions. Precision-guided applications have the potential to reduce costs while maintaining disease suppression. Research objectives include development of methods to map, evaluate, and quantify SDS epidemics as well as the evaluation of site-specific fungicide applications based on historical disease incidence. Aerial imagery was used to create disease maps in spring 2016 and 2017 from five bermudagrass fairways with a history of 
SDS. Treatments were arranged in a completely randomized design across twenty replicated $33 \mathrm{~m}^{2}$ plots to include 1) untreated 2) full-coverage tebuconazole $\left(1.2 \mathrm{~kg}\right.$ ai ha $\left.\left.^{-1}\right), 3\right)$ full-coverage penthiopyrad $\left(1.07 \mathrm{~kg} \mathrm{ai} \mathrm{ha}^{-1}\right)$, and 4) site-specific penthiopyrad $\left(1.07 \mathrm{~kg}\right.$ ai ha ${ }^{-1}$.) Fall treatments were initiated when soil temperatures reached $18^{\circ} \mathrm{C}$. Site-specific treatments reduced fungicide usage by $51 \%$ in 2016 and $65 \%$ in 2017 while providing statistically equivalent suppression when compared to full-coverage plots. This research demonstrates how treated fungicide acreage can be reduced by using site-specific management from SDS distribution maps without compromising efficacy.

Risks associated with use of single drop potato tubers versus cut seed potato pieces for propagation K. M. DUELLLMAN, M. A. Lent, M. C. Bertram, J. C. Randall, University of Idaho, Idaho Falls, ID, USA

Use of single drop potato tubers (small, whole tubers ranging in size from about $50 \mathrm{~g}$ to $70 \mathrm{~g}$ ) for propagation is a common practice outside of North America. However, in the United States, the use of cut seed, where potato tubers of various sizes are mechanically cut into smaller seed pieces (typically targeting a size range of about $55 \mathrm{~g}$ to $70 \mathrm{~g}$ ), is more common. Although the use of single drop seed potatoes may reduce risk of some tuber-borne diseases, the practice can potentially increase the risk of other tuber-borne diseases, such as Rhizoctonia stem canker. Previous work in southeastern Idaho showed cut seed treated with protectant fungicides performed as well as or better than single drop seed for 'Russet Burbank,' and in some years, single drop seed led to increased incidence of Rhizoctonia stem canker. However, this earlier work failed to examine the effect of protectant fungicide seed treatment on the performance of single drop seed. From 2011 to 2017, field trials were conducted in southeastern Idaho to compare performance of cut seed pieces with single drop seed potatoes, either treated with a protectant fungicide or left non-treated. Regardless of seed type used, use of an appropriate protectant fungicide on seed enhanced performance. Based on these results and previous work, the benefits and risks of using cut seed will be discussed.

IPM in soybean - investigations in pairing cultivars, fungicides, and application timings to maximize disease control and yield H. M. KELLY (1), W. J. Jordan (2), (1) University of Tennessee, Jackson, TN, USA; (2) Univ of Tennessee, Jackson, TN, USA

Can a more specific treatment plan be created for managing foliar diseases of soybean that encompasses cultivar, fungicide product, and application number and timing? To investigate this question, field trials arranged in a factorial design were conducted from 2014 through 2017 with 2-4 cultivars of varying disease resistance, multiple fungicides, single, double, and triple fungicide applications, and up to seven different application timings. Disease incidence and severity of frogeye leaf spot, septoria brown spot, and target spot were recorded along with yield. In trials conducted in 2014-2016, cultivars with greater resistance had greater yield, and the number of applications and application timing of fungicides had lower impact on yields compared to susceptible varieties. On average, a single application on resistant cultivars resulted in maximum yield, while susceptible cultivars benefited from additional fungicide applications. Although yield on susceptible cultivars was highest where three applications were made, only single and double fungicide applications increased the return on investment based on estimates of fungicide and application costs and commodity price. In 2017 trials, fungicide product significantly impacted yield, but there was no significant interaction between fungicide product and cultivar. Further results on yield and disease will be discussed along with economics associated with implementing specific treatment plan based on these data.

Fungicide application interval and hybrid tolerance for management of downy mildew (Pseudoperonospora cubensis) in pickling cucumber C. TRUEMAN (1), J. O'Sullivan (2), R. Riddle (2), (1) University of Guelph, Ridgetown, ON, CANADA; (2) University of Guelph, Simcoe, ON, CANADA

Cucurbit downy mildew (CDM) (Pseudoperonospora cubensis) is an important cucumber disease in Ontario, Canada. CDM tolerant hybrids 'Citadel' (CT) and 'Peacemaker' (PM) may allow for longer fungicide application intervals. Early (E) and late (L) seeded trials were completed at Ridgetown (RT) and Simcoe (SI) to evaluate CT, PM, and susceptible 'Vlaspik' (VP) with no fungicide, and seven, 10, and 14-day (d) intervals. At E-RT, CDM severity was low, seven-d VP had $100 \%$ lower area under the disease progress curve (AUDPC) than the untreated, and there was no interval effect on total yield. E-SI had moderate CDM pressure, and AUDPC among hybrids was 78 to $100 \%$ higher with no fungicide than any interval. Total weight was $20 \%$ higher for the 10-d interval than the untreated. High CDM pressure occurred in L-seeded trials. At L-RT AUDPC decreased with decreasing interval, regardless of hybrid tolerance. Results were similar at L-SI except 10 and 14-d intervals were equivalent. Total fruit weight was $80 \%$ higher for all hybrids using a seven or 10-d interval at L-RT, but at L-SI, any fungicide increased yield in VP, while for CT only seven and 10-d intervals increased yield versus no fungicide. There was no interval effect on yield in PM at L-SI and yield was like fungicide treated VP and CT, suggesting higher CDM tolerance. Tolerant hybrids offer potential to reduce fungicide use, but this may depend on CDM pressure. More research is required to develop grower recommendations.

Characterization of MAT1-2-7: A novel MAT gene in the wood-infecting fungus Huntiella omanensis A. WILSON (1), M. van der Nest (1), M. Wilken (2), M. J. Wingfield (1), B. D. Wingfield (2), (1) FABI, University of Pretoria, Pretoria, SOUTH AFRICA; (2) Forestry and Agricultural Biotechnology Institute (FABI), University of Pretoria, Pretoria, SOUTH AFRICA

Sexual reproduction in fungi is controlled by genes at the mating type (MAT) locus. Species of Ceratocystidaceae harbour the MAT1-1-1, MAT1-1-2, MAT1-2-1 and MAT1-2-7 genes at this locus. More specifically, Ceratocystis species, most of which are aggressive plant pathogens, possess all four genes at a single locus, characteristic of self-fertile ascomycetes. In contrast, saprobic Huntiella species possess either the MAT1-1 genes or the MAT1-2 genes, resulting in a self-sterile mating system. A notable exception is the unisexual $\mathrm{H}$. moniliformis, in which single spore isolates can undergo sexual reproduction, despite having only the MAT1-2-1 and truncated MAT1-2-7 genes. In this study, we determined whether the functional MAT1-2-7 plays a role in sexual reproduction by preventing self-fertility as seen in H. moniliformis. A split marker knockout approach was used to replace the MAT1-2-7 gene in H. omanensis, which is a close relative of H. moniliformis, with a Hygromycin resistance cassette. The results showed that while the $\triangle M A T 1-2-7$ strain did not exhibit self-fertility, it does produce structures resembling proto-ascomata. The production of such structures in a self-sterile species hints at a role for MAT1-2-7 in the sexual process. This is the first study to genetically manipulate a species of the Ceratocystidaceae, and the knockout protocol provides a tool to study biologically significant processes in this group of fungi.

Satellite uredinia: An important trait related to Phakopsora aggressiveness

I. PRIMIANO (1), M. Loehrer (2), U. Schaffrath (2), L. Amorim (1), (1) University of Sao Paulo, Piracicaba, BRAZIL; (2) RWTH Aachen University, Aachen, GERMANY

Phakopsora euvitis $(\mathrm{Pe})$ and Phakopsora pachyrhizi (Pp) cause severe rust epidemics on grapevine and soybean, respectively. In contrast to Uromyces appendiculatus $(\mathrm{Ua})$, the bean rust pathogen, both are highly aggressive, causing significant reduction on $\mathrm{CO}_{2}$ assimilation even at low disease severity. A lesion is visible to the naked eye as a discrete area within which uredinia are produced. Comparative studies on lesion expansion were carried out to 
determine a possible correlation with secondary inoculum generation. Single-lesions of $P p$ on soybean, $P e$ on grapevine, and $U a$ on bean were measured every 3 days after the first disease symptoms developed on leaves. Lesion area was evaluated under the stereomicroscope before and after staining with trypan blue, which enabled visualization of mycelial growth inside the leaf tissue. The number of uredinia was counted. All pathosystems showed lesion expansion. Staining showed that mycelial colonization did not extend beyond the lesion border. The number of uredinia of $P p$ and $P e$ within the lesions increased over time (9 to 19 fold), whereas the number of uredinia of $U a$ did not change. Aggressiveness of both Phakopsora species correlated with the high number of satellite uredinia produced within lesions rather than with the total colonized leaf area. Formation of new uredinia within an existing lesion, without the need for a new infection site, seems to be an adaptation strategy of Phakopsora rust.

\section{Evidence for post-mating species barriers in Ceratocystis}

M. WILKEN (1), E. Steenkamp (1), M. J. Wingfield (1), W. De Beer (2), B. D. Wingfield (1), (1) Forestry and Agricultural Biotechnology Institute (FABI), University of Pretoria, Pretoria, SOUTH AFRICA; (2) Forestry and Agricultural Biotechnology Institute, University of Pretoria, Pretoria, SOUTH AFRICA

Species of Ceratocystis (Microascales, Ascomycota) are all homothallic, but can outcross in artificial matings between self-sterile strains. Often, such laboratory crosses produce viable progeny, showing that the species are interfertile under artificial conditions. This sexual compatibility has been used to support the view that closely related species such as $C$. eucalypticola, C. manginecans and C. fimbriata represent host-specific strains of $C$. fimbriata sensu stricto, rather than discrete taxa. In this study, laboratory crosses were made between self-sterile isolates of $C$. fimbriata sensu stricto, $C$. eucalypticola sensu stricto and C. manginecans sensu stricto to consider whether post-zygotic barriers to sexual reproduction might be present. The mating tests produced normal ascomata from which ascospores could be recovered. Single ascospore progeny showed segregation of tested genetic markers, confirming the crosses represented true matings. However, analyses of the progeny showed they were the products of interspecific crosses, with reduced levels of fertility and non-random segregation of unlinked genetic markers. These characteristics are commonly associated with post-mating reproductive barriers found between two distinct taxa. The results provide evidence that sexual compatibility in laboratory tests can be misleading when invoking the biological species concept. Therefore, mating in culture including the production of viable progeny can fail to adequately consider potential post-reproductive barriers.

\section{Mating strategies in the Ceratocystidaceae that includes pathogens of trees and agronomic crop plants} M. WILKEN (1), E. Steenkamp (1), M. J. Wingfield (1), W. De Beer (2), B. D. Wingfield (1), (1) Forestry and Agricultural Biotechnology Institute (FABI), University of Pretoria, Pretoria, SOUTH AFRICA; (2) Forestry and Agricultural Biotechnology Institute, University of Pretoria, Pretoria, SOUTH AFRICA

The Ceratocystidaceae (Microascales) include a biologically diverse group of fungi currently accommodating at least ten distinct genera. Among these, Ceratocystis, Endoconidiophora and Thielaviopsis are well known plant-pathogens, in contrast to Huntiella that are saprobes of little economic importance. Genome sequences are available for more than 25 species spanning seven genera of the Ceratocystidaceae. This resource provides an opportunity to study the mating systems, and more specifically the mating-type genes in these species. In this study the mating-type genes and MATI locus was reconstructed for species in the family. Our results showed that all species capable of unidirectional mating-type switching shared a MAT1 locus structure typical of this reproductive strategy. This included two direct repeats that flank the MAT1-2 genes. Species of Huntiella, Thielaviopsis and Bretziella are known to be heterothallic, but differences in their gene content were evident among the MAT1 loci in these species. Complete and apparently functional MAT1 loci were also characterised from several species that are seemingly asexual, raising the possibility of cryptic sexual cycles in these fungi. The results provide a robust framework to study the evolution of sexual reproduction in not only the Ceratocystidaceae, but within the order Microascales as a whole.

\section{Apiosphaeria guaranitica an important forest pathogen in Central Brazil: Taxonomy and phylogeny} D. Guterres, S. Elias, J. C. DIANESE, UNIVERSIDADE DE BRASÍLIA, BRASÍLIA, BRAZIL

Apiosphaeria guaranitica, the causal agent of brown crust disease of several bignoniaceous hosts, among them Handroanthus and Tabebuia species, have been traditionally recognized as belonging to Phyllachoraceae based on exclusively morphological studies, not supported by any molecular evidence. Thus, for the first time we provide molecular evidence of the link between sexual and asexual forms of the fungus, elucidated the phylogenetic position of $A$. guaranitica based on a multilocus analyses using SSU, LSU, ITS1-5.8S-ITS2, RPB2 and TEF1; and provided the divergence time estimate of this lineage using both fossil-calibration (FC) and secondary calibration (SC) strategies. Our results showed that the natural phylogenetic placement of Apiosphaeria is within Diaporthaceae (Diaporthales), where it represents an ancient lineage of the crown-group of Diaporthaceae, diverging at the late Paleocene at 61.15 (FC) and 60.63 (SC) MYA. This is a pioneer study on the divergence time estimate within Diaporthales based on Spataporthe taylori, a diaporthaceous fossil.

A rapid approach for isolating single fungal spores from rice blast diseased leaves L. FEI, W. Lu, K. Fang, W. Zhao, J. Yang PhD, Y. L. Peng, China Agricultural University, Beijing, CHINA

Single spore isolation is a fundamental approach in plant pathology and microbiology to isolate and identify plant fungal pathogen from diseased field samples. However, routine single spore isolation procedure is time-consuming and has a high risk of contamination by other microorganisms. In this study, we developed a rapid approach for isolating single spore of the fungal pathogen, Magnaporthe oryzae, from rice blast diseased leaves in the field with low potential of contamination. Firstly, fresh diseased leaf with single lesion was directly pasted onto solid water agar plate in the field and was then maintained at four degree. Next, the single fungal spore attached to solid water agar was picked out with needle under stereomicroscope within up to one week, and then the agar plug with a single fungal spore was transferred onto solid oatmeal tomato agar plate for growth and preservation. According to our experience, a skilled technician could isolate over one hundred single spores in one working day with nearly no contamination. This new developed approach is also suitable for isolating single spore from diseased field samples caused by other fungi that produce aerial conidia.

Suppression of Bipolaris spp. by the saprophytic fungus Cladosporium pseudocladosporioides A. ADHIKARI, B. Lane, P. Harmon, E. M. Goss, Department of Plant Pathology, University of Florida, Gainesville, FL, USA

Bipolaris species are important plant pathogens that cause leaf spots, blight, crown rot and melting out diseases of primarily grass hosts. Cladosporium is a fungal genus which has a primarily saprophytic lifestyle, but some species have been reported to have antagonistic effects to biotrophic fungi, including rust pathogens. Recently, we co-isolated Bipolaris and Cladosporium from necrotic lesions on native and invasive understory grasses in Indiana forests. Initial observations suggested that the Cladosporium isolates were parasitic to Bipolaris conidia and appeared to reduce the likelihood of successful 
Bipolaris isolation. Multi-locus sequencing identified Cladiosporium isolates as C. pseudocladosporioides. We examined the antagonistic effect of $C$. pseudocladosporioides on Bipolaris in media assays. Hyphae of C. pseudocladosporioides were able to invade and outcompete Bipolaris colonies. We characterized the interaction between these fungi using light microscopy to look for evidence of pathogenesis. We propose that $C$. pseudocladospoiroides may mediate the interaction between Bipolaris pathogens and grass hosts.

Comparison of five potato-based protocols for conidial production by Calonectria pseudonaviculata S. YANG, P. Kong, C. Hong, Hampton Roads Agricultural Research and Extension Center, Virginia Tech, Virginia Beach, VA, USA

Boxwood blight caused by Calonectria pseudonaviculata $(\mathrm{Cps})$ is an emerging disease of great concern to the ornamental horticulture industries, landscapers, public garden managers, and homeowners in the U.S. and many other countries. The primary objective of this study was to compare five protocols developed in different labs for conidial production, a critical step for successful investigations into Cps biology, disease epidemiology and management. These protocols all utilize potato-based media but they differ in medium type, composition and strength, as well as cultural processes and conditions. The comparative analysis focused on conidial yield and aggressiveness in boxwood infection using two Cps isolates 11D05 and 12A02 recovered from boxwood and sweet box, respectively. The associated time requirement and material cost were also considered. Overall, both isolates were most prolific using the potato dextrose broth (PDB) protocol with $11 \mathrm{D} 05$ producing $3.39 \pm 1.57 \times 10^{7}$ conidia Petri dish $^{-1}$ and $12 \mathrm{~A} 02$ having $2.84 \pm^{2}$ $1.33 \times 10^{7}$ conidia dish $^{-1}$. The resultant conidia were equally, if not more, aggressive in infecting Buxus sempervirens cv. Suffruticosa than those produced with other protocols. The PDB protocol also was more time-efficient and cost-effective than the other protocols evaluated in this study. These results will add quality and productivity to ongoing boxwood blight research, consequently better using the limited resources. Strengths of the four other protocols were also noted and discussed which would be good leads to further improving the PDB protocol.

\section{Ramularia leaf spot of barley in New Zealand}

I. C. HARVEY (1), M. Braithwaite (2), S. Chng (3), R. Warren (3), R. Craigie (4), J. Drummond (4), B. McCloy (5), (1) PLANTwise Services Limited, Lincoln, NEW ZEALAND; (2) Plant Diagnostics Limited, Christchurch, NEW ZEALAND; (3) Plant \& Food Research Ltd, Christchurch, NEW ZEALAND; (4) Foundation for Arable Research, Hornby, NEW ZEALAND; (5) NZ Arable, Templeton, NEW ZEALAND

Ramularia leaf spot of barley caused by the fungus Ramularia collo-cygni (Rcc) was first identified in New Zealand in the early 1980's. Until the last few years, occurrence and damage from Rcc has been sporadic, but the disease has recently become more widespread and an important constraint to barley production. Four aspects of the disease are being actively studied: epidemiology; control with fungicides; development of fungicide resistance in Rcc; and the role and importance of seed-borne inoculum. Appearance of the disease in the field can vary between seasons and fields. Leaf wetness in host growth stages 28-32 relates to disease severity later in affected crops. During the wet 2017 spring, early infections of lower leaves were observed in crops. These may or may not have arisen from seed-borne inoculum, but can lead to rapid disease development resulting in significant losses of green leaf tissue and grain yield reductions. Rcc has been detected in most seed lines tested in Canterbury, and these infections often translated into seedling infections. Isolates of Rcc exhibiting reduced control by SDHI fungicides have been detected in fields, but combinations of some triazoles and protectant fungicides such as chlorothalonil and folpet are still giving effective control. Resistance to strobilurin fungicides has been present in the field for several seasons. Management options using fungicide mixtures and alternations integrated with other management practices can minimise losses from this disease.

Determining the effects of inoculum concentration and wounding on the development of fruit rot of winterberry holly S. LIN (1), F. Peduto Hand PhD (2), (1) The Ohio State University, Columbus, OH, USA; (2) Ohio State Univ, Dept of Plant Pathology, Columbus, OH, USA

Cut stems of winterberry holly carrying brightly colored berries are traditionally used for holiday decorations. In the past few years, a fruit rot caused by a fungal complex including Alternaria alternata, Diaporthe sp., Colletotrichum fioriniae and Epicoccum nigrum, has been associated with significant yield reduction or complete crop loss. Understanding the role of inoculum concentration and wounding on symptoms development may be useful to identify effective management strategies. To this extent, we point inoculated each of the four pathogens separately at four spore concentrations ranging from $10^{3}$ to $10^{6}$ conidia/ml on wounded and unwounded mature winterberry 'Sparkleberry' fruit in a detached fruit assay. Six replicates of ten fruit per treatment were arranged in a randomized complete block design and incubated in a moist chamber. Disease development was evaluated for up to six weeks. All pathogens at all concentrations could infect both wounded and unwounded fruit, but disease on wounded fruit developed faster and resulted in higher incidence. Disease severity increased as spore concentration increased. However, E. nigrum inoculum resulted in low disease severity $(<11 \%)$ at all concentrations. These results indicate that cultural practices that can reduce inoculum concentration in the field and protect fruit from injury may reduce disease severity.

Biological activity of Phaeocryptopus gaeumannii in Christmas trees (Pseudotsuga menziesii var. glauca) in Mexico O. J. Salgado-Feregrino, M. D. J. YÁÑEZ-MORALES, J. Velazquez-Mendoza, M. Jiménez-Casas, I. Alanis-Martínez, Colegio de Postgraduados, Texcoco, MEXICO

In some countries, the ascomycete Phaeocryptopus gaeumannii has reduced the quality and production of the Douglas fir (Pseudotsuga menziesii) ornamental Christmas trees because of severe foliar damage. Little is known about this disease in Mexico, so that appropriate control measures are difficult to develop. This study evaluated various aspects of $P$. gaeumannii biology (via cultured agar isolates, ascospores in pseudothecia and airborne ascospores) and trees quality (incidence, severity and defoliation) over a year. From Summer 2011 to Spring 2012, a 9-year-old ornamental Christmas tree plantation in Valle de Bravo, Mexico State was sampled. Seven needle samples from various branch positions and different needle ages were collected; naturally fallen needles were sampled too. The fungus was identified and sequenced. A pseudothecia opening mechanism was indirectly observed under the scanning electron microscope. Results showed fungal biological activity fluctuated and simultaneously occurred throughout the year. Fungal activity was scarcely shown in fallen needles. A different airborne ascospore peak was detected. The pseudothecia showed an equatorial and polar split, resembling an opening mechanism. In terms of tree quality, the most severe reduction and highest fungal activity were observed mainly in 1year-old needles from the upper section of trees. Defoliation was severe and increased proportionally with increasing needle age. These results were similarly, with a few exceptions, to what is known in Douglas fir (a different variety of Pseudotsuga menziesii) worldwide. This new information on the study area will enable the design of sustainable disease control strategies in Christmas tree plantations.

Pineapple fruit rot caused by Ceratocystis paradoxa and growth studies on two isolates F. OKUNGBOWA (1), D. Esiegbuya (2), (1) University of Benin, Benin City, NIGERIA; (2) Nigerian Institute for Oil Palm Research, Benin City, NIGERIA 
Pineapple (Ananas comosus) is a tropical fruit and good source of minerals and vitamins. Fruit rot of pineapple caused by Ceratocystis paradoxa is a major fungal disease with huge crop losses. Information on effect of the disease on nutritive value is scanty. The aim of this study was to determine the prevalence of pineapple fruit rot caused by C. paradoxa in some markets in Benin City and evaluate the effect of the pathogen on the nutritive value of the fruit and to study pathogen growth under various environmental conditions, with a view to providing information on disease control. Fifty pineapple fruits were examined for symptoms. Small $(5 \mathrm{~mm})$ portions were cultured on Potato Dextrose Agar at $282^{\circ} \mathrm{C}$ for 7 days, using standard mycological procedures. Two isolates ( 1 and 2 ) of $C$. paradoxa were distinguished on the basis of the cultural characteristics and disease severity. Calcium, manganese, potassium, moisture, vitamin $\mathrm{C}$, protein and carbohydrate contents of infected fruits decreased significantly $(\mathrm{p}=0.05)$ while fibre increased. Optimum growth temperature was $30^{\circ} \mathrm{C}$ for both isolates. All light regimes allowed growth with highest growth at $24 \mathrm{hr}$ darkness. Relative humidity of $100 \%$ supported the best growth and $55 \%$, the least. The $\mathrm{pH}$ affected both isolates differently. The physiological responses of the two isolates to environmental factors need further study. The isolates differed in their ability to incite symptoms; the results therefore provide useful information for planning disease control.

Interspecific hybridization involving a rare parental forest pathogen leads to asymmetrical accelerated evolution M. M. GARBELOTTO (1), B. Lockman (2), F. Sillo (3), P. Gonthier (4), (1) UC Berkeley, Berkeley, CA, USA; (2) US FS, Portland, OR, USA; (3) University of Torino / DISAFA, Grugliasco, ITALY; (4) University of Torino / DISAFA, Grugliasco, Torino, ITALY

Hybridization is recognized as a major force in the rapid adaptive evolution of plant pathogens. In a high elevation alpine larch stand in Montana, the dominant pathogenic species Heterobasidion occidentale has hybridized with a rare Eastern North American population of $H$. irregulare. Based on sequence analyses of five loci, we show that: 1)-Hybridization increased allelic diversity, generating alleles absent from either parental populations; 2)Hybrid genotypes are viable and consequential in evolutionary terms; 3 )-Because $H$. irregulare is rare, hybrid progeny has been backcrossing with $H$. occidentale, and thus hybrids carry occidentale mitochondria; 4)-A further consequence of hybrids solely backcrossing with $H$. occidentale has been the isolation and rapid evolution of the irregulare genome, while the same is not true for the occidentale genome; 5)-Based on phylogenetic, Bayesian assignment, and ordination tests, irregulare alleles in hybrids are clearly distinct from all known irregulare alleles from North America and may represent a new species. This discovery demonstrates that larch can be a universal host favoring hybridization, and that hybridization between one abundant and one rare species can result in asymmetrical accelerated evolution of the nuclear genome of the rare parental species leading to speciation.

\section{Colonization dynamics of red raspberry flowers and fruit by Botrytis cinerea}

O. KOZHAR, T. L. Peever, Washington State University, Pullman, WA, USA

Botrytis cinerea, causal agent of gray mold, is the major pathogen of raspberry in the US Pacific Northwest (PNW) and worldwide, and fungicide applications are the main disease management strategy. Fungicides are applied in PNW on a calendar basis rather than based on infection risk because the disease cycle on raspberry is poorly understood. The objective of this study was to determine the dynamics of flower and fruit colonization, including testing the hypothesis that the primary route of infection of raspberry is through flowers. $B$. cinerea was isolated from seven developmental stages of raspberry flowers and fruit in northwestern Washington in 2015-2016. Colonization of flowers by $B$. cinerea was $15 \%$, increased as fruit developed, and peaked on mature fruit (67\%). During flowering, the floral part colonized most frequently was carpel (80\%). As fruit matured, colonization of other floral parts increased. In 2017, $400 \mathrm{~B}$. cinerea isolates were collected from fertilized flowers, as well as green and ripe fruit from 40 raspberry inflorescences in two fields and genotyped using seven microsatellite markers. Number of colonies and genotypic diversity increased as fruit matured. In addition, different multilocus genotypes colonized flowers, green and ripe fruit in the same inflorescence, suggesting multiple infection events, and that flowers are not the major route of infection. These results will help time fungicide applications more effectively to control gray mold.

Emerging diseases of Cannabis sativa $\mathrm{L}$ (marihuana) in Canada

Z. K. PUNJA, Simon Fraser University, Burnaby, BC, CANADA

Cultivation of Cannabis sativa plants in Canada has increased the incidence and severity of previously unreported diseases. Cannabis plants were sampled during 2014-2017 to determine the prevalence of root, foliar and flower-infecting pathogens. Following isolation, pathogenicity studies were conducted to establish which microbes could induce disease symptoms. Pathogens were identified by PCR and sequence analysis of the ITS region. The causal agents of browning and rotting of roots were Pythium dissotocum and P. myriotylum. As well, Fusarium oxysporum and F. solani were isolated from symptomatic roots of plants, which upon reinoculation onto healthy plants, caused stunting, yellowing and wilting. A rot of the flower buds was associated with Botrytis cinerea, which caused botrytis bud rot. In addition, Penicillium olsonii, and to a lesser extent, $P$. copticola, were also recovered from diseased flower buds, and caused penicillium bud rot. A rot of the flower buds was also associated with $F$. solani and $F$. oxysporum, causing fusarium bud rot. Powdery mildew was found to be caused by the pathogen Golovinomyces (Erysiphe) cichoracearum which was detected on vegetatively propagated cuttings of cannabis. Scanning electron microscopic studies provided details on spore germination and sporulation. These pathogens have the potential to reduce product quality wherever cannabis is grown and disease management practices need to be established.

Diagnosis and molecular diversity of $X$. fastidiosa subsp. pauca isolate from olive trees in Brazil

N. SAFADY (1), K. de Campos (2), E. Menoele (3), H. Coletta-Filho (4), (1) Universidade Federal de São Carlos, Araras, BRAZIL; (2) Centro de Citricultura - IAC, Cordeiropolis, BRAZIL; (3) UFSCaR, Araras, BRAZIL; (4) Centro de Citricultura Sylvio Moreira" - Agronomic Institute (IAC), Cordeiropolis, BRAZIL

The bacterium Xylella fastidiosa (XF) is the etiological agent known to infect a hundred of plant species and to cause disease in important crops around the world, including grapevine, citrus, coffee, and stone fruits. Recently in Southeast Brazil, likely in Italy and Argentina, XF was also reported associated with Olive Quick Decline (OQD) disease, causing severe damage in olive trees. So far, few knowledges on XF-olive in Brazil was reported, except that the Sequence Type (ST) 16 of $X$. fastidiosa subsp. pauca (XFp) was the strain found in symptomatic trees. We are investigating the molecular epidemiology of XFp in olive orchards in Southeast Brazil as well as the genetic diversity of bacterium by MLST and SSR markers. Despite of low incidence of XFp in most of sampled orchards, the pathogen was found spread through a broad geographic area and infecting all commercial olive varieties. Data set from MLST markers confirm the ST16 as the main XF genotype in infected trees. However, the faster evolutionary SSR markers shown that isolates genetically distinct were found through the different areas, with polymorphism in 7 off 12 tested loci.

The cacao swollen shoot disease complex in West Africa comprises at least five divergent badnavirus species J. K. BROWN, N. Chingandu, School of Plant Sciences, University of Arizona, Tucson, AZ, USA 
The cacao swollen shoot disease (CSSD) of Theobroma cacao in West Africa is characterized by diverse foliar symptoms, swollen shoots, and tree death. CSSD was previously thought to be caused by a single virus, Cacao swollen shoot virus (CSSV). Current molecular tests confirm positive infection in $<70 \%$ of symptomatic trees, suggesting presence of uncharacterized viruses. Molecular characterization of 61 full-length genome virus sequences from symptomatic trees in Ghana, Togo, Cote d'Ivoire and Nigeria indicated that at least five badnavirus species are associated with CSSD. Analysis of the taxonomic RT-RNase H region confirmed that Cacao swollen shoot CD virus (CSSCDV), Cacao swollen shoot Togo A virus (CSSTAV), and the recently proposed Cacao red vein virus (CRVV) and Cacao red vein-banding virus (CRVBV), are also found in cacao trees exhibiting characteristic CSSD symptoms. All 61 genomes showed similar conserved protein domain architectures, despite the differences in genome arrangement and $70-100 \%$ shared nucleotide identity. CSSV is found in all four countries, except Nigeria, whereas CSSCDV, CSSTAV, and CRVBV appear to be confined to Cote d'Ivoire, Togo, and Nigeria, respectively. In contrast, CRVV has been reported in Ghana and Cote d'Ivoire. Genome sequences and geographical distributions of the CSSD viruses will facilitate development of virus diagnostics, epidemiology studies, and breeding programs for virusresistant cacao varieties.

Two emerging pathogens associated with rapid decline and dieback symptoms in apple detected in Washington, USA S. SZOSTEK, S. J. Harper, Washington State University, Prosser, WA, USA

Material from Honeycrisp apples exhibiting rapid decline and dieback symptoms in Washington state was submitted to the WSU Clean Plant Center Northwest (CPCNW) for diagnosis. Using high-throughput sequencing (HTS), these plants were found to contain Apple stem grooving virus, Apple stem pitting virus, Apple chlorotic leafspot virus, and Apple green crinkle-associated virus, all well established in the United States, as well as Apple hammerhead viroid (AHVd) and Citrus concave gum-associated virus (CCGaV). The presence of AHVd and CCGaV in these samples was verified by RT-PCR and represents the first report of these two pathogens in the U.S., and the first report of CCGaV in apple. A selection of apple material submitted to the CPCNW for diagnostic and virus elimination procedures was then screened by HTS and RT-PCR for AHVd and/or CCGaV. AHVd was detected by both methods in material originating from Washington and Ohio, USA, Japan and Italy. Both methods detected CCGaV in apple plants from Washington and Oregon, USA, and Australia, France, Italy, and Spain. Decline and dieback symptoms were only observed in Honeycrisp infected with $\mathrm{AHVd}$ and $\mathrm{CCGaV}$; the presence of the other viruses did not produce symptoms in neighboring trees suggesting that one or both of these are responsible for the disease observed. Given that AHVd is known to produce trunk swelling and splitting in Gala apple, both pathogens are significant and emerging diseases of apple production worldwide.

Molecular identification of Alternaria species associated with imported industrial hemp seed J. Schoener, R. WILHELM, S. Wang, Nevada Department of Agriculture, Sparks, NV, USA

Industrial hemp (Cannabis sativa L.) seed used for crop cultivation is either imported from foreign countries or produced domestically. In a germination test conducted on imported hemp seeds, a high percentage of fungal growth was observed in the germination tray, suggesting a presence of seed-borne fungal organisms. To isolate and identify those fungal species, fifty hemp seeds (var. Canda) were soaked in $1 \%$ sodium hypochlorite for 15 min with shaking, rinsed in sterilized water, plated on potato dextrose agar amended with streptomycin sulfate (PDA+strep), and incubated at $22^{\circ} \mathrm{C}$ in the dark. While $86 \%$ of seeds germinated, $56 \%$ of seeds had fungal growth, the majority of which had fungal colonies grown from newly germinated tap roots. A total of 29 Alternaria isolates were obtained and maintained on PDA+strep for morphological observations. One type contained 14 isolates with white to gray colonies and another had 15 isolates with gray to black colonies. DNA was extracted from all isolates and PCR was performed using primers ITS1/ITS4 to amplify the ITS region of rDNA. The size of PCR amplicons obtained from 29 isolates ranged from 542 to $600 \mathrm{bp}$. A BLASTn search suggested that these two types of isolates were closely related to Alternaria infectoria and A. tenuissima, respectively. The data suggest that hemp seeds of the Canda variety carried two distinct Alternaria species, but the effects of these two fungal species on the health of seeds or seedlings are not known.

Genetic relatedness and virulence of a novel Ralstonia pseudosolanacearum (Ralstonia solanacearum phylotype I) isolated from Rosa spp. M. BERGSMA-VLAMI, J. van de Bilt, L. Tjou Tam Sin, M. Westenberg, NVWA, Wageningen, NETHERLANDS

A novel R. pseudosolanacearum causing disease in rose has been recently reported. Plants suffered from chlorosis, stunting, wilting and necrosis. Isolates obtained from rose were identified as phylotype I from the 'Ralstonia solanacearum species complex' (RSSC), actually reclassified as $R$. pseudosolanacearum. At the proteomic level, these isolates were accurately identified by MALDI-TOF MS. MLSA positioned these isolates in a distinct clade in phylotype I, exhibiting a clonal structure, when compared with a set of $250 \mathrm{RSSC}$ isolates of worldwide origin. Representative $R$. pseudosolanacearum isolates from rose were assessed for their virulence in two rose cultivars and in solanaceous crops. Significant differences were observed in susceptibility between the two rose cultivars. Additionally, a temperature dependency in susceptibility was observed for both cultivars irrespective of the mode of inoculation. The solanaceous crops showed to be susceptible to the R.pseudosolanacearum isolates from rose. Interestingly, both rose cultivars were able to harbor symptomless infections with other RSSC isolates than those isolated from rose. Our results demonstrated that latent infections in rose do occur even at temperatures as low as $20^{\circ} \mathrm{C}$. This latency poses high risks for the entire floricultural industry as latently infected rose plants might be distributed over various continents, including areas where climatic conditions are optimal for the pathogen.

Infectivity of cloned cacao swollen shoot associated badnaviral genome in Nicotiana benthamiana N. CHINGANDU, J. K. Brown, School of Plant Sciences, University of Arizona, Tucson, AZ, USA

A severe rapid decline symptom phenotype was recently observed in cacao (Theobroma cacao) trees affected with cacao swollen shoot disease (CSSD) in West Africa. At least four badnavirus species, Cacao swollen shoot virus (CSSV), Cacao swollen shoot Togo A virus, Cacao swollen shoot CD virus (CSSCDV) and Cacao red vein virus (CRVV), have been associated with CSSD. To investigate whether the rapid decline phenotype is related to infection by a particular species, the full-length (monomer) genome, and partial dimer of CSSV-G75 isolate from Ghana were cloned and inoculated on 12 Nicotiana benthamiana seedlings using mechanical and agro-infiltration methods, respectively. Symptoms were observed on three of the 12 plants inoculated with the monomer clone, at 20-d post-inoculation (dpi), but not on mock-inoculated, or on the agro-infiltrated plants. Total DNA was isolated from the inoculated plants at 30-dpi and used as template for PCR amplification with CSSV-specific primers, resulting in amplicons of the expected size from monomer-inoculated and partial dimer-inoculated plants. DNA sequences indicated $88-100 \%$ shared nucleotide identity with the cloned CSSV G75 genome. These results show that the cloned CSSV isolate G75 was biologically active and infectious in $N$. benthaminana plants by mechanical transmission and agro-infiltration. Experiments are underway to test infectivity for CSSCDV and CRVV clones using T. cacao seedlings to fulfill Koch's postulates. 
Distribution and impact in Northeastern USA of the emerging disease: Sirococcus shoot blight of eastern hemlock (Tsuga canadensis) I. A. MUNCK (1), W. D. Ostrofsky (2), D. R. Smith (3), G. R. Stanosz (4), (1) USDA Forest Service, Durham, NH, USA; (2) Maine Forest Service, Augusta, ME, USA; (3) University of Wisconsin-Madison, Madison, WI, USA; (4) University of Wisconsin, Madison, WI, USA

In 2009, eastern hemlock was confirmed as a new host of the fungal pathogen Sirococcus tsugae. Because of the potential that hemlock regeneration could be impacted, the objectives of this project were to: 1) delineate the geographic range of Sirococcus shoot blight in the Northeastern USA, 2) verify the association of the pathogenic fungus $S$. tsugae with both shoot blight and needle loss, 3) quantify impact of the disease on eastern hemlock regeneration, and 4) monitor changes in severity during 3 consecutive years. From 2013-2014, 59 long-term monitoring plots throughout the northeastern USA were surveyed. Disease incidence and severity (percent shoot blight and crown defoliation) were assessed for 20 seedlings per plot. Sirococcus shoot blight symptoms were present in most plots (95\%) and on most seedlings $(71 \%)$. For the majority of seedlings, blight affected less than $10 \%$ of shoots, but did range up to $75 \%$. Similarly, needle loss was limited to less than $25 \%$ of the crown for most seedlings. Disease severity was positively correlated with seedling and overstory hemlock density. Using species-specific PCR primers, Sirococcus tsugae was identified from samples collected in New England and New York. In permanent plots, symptom severity increased from 2011 to 2013. Results confirm that Sirococcus shoot blight of eastern hemlock is more widespread in natural forests of northeastern USA than previously known and that impacts can be severe in some locations.

Incidence of head blight complex on wheat and other cereals

S. BANU, M. M. Alam, F. E. Elahi, K. Alam, M. M. Islam, M. M. Islam, Bangladesh Agricultural Research Institute, Joydebpur, BANGLADESH

Infection on wheat head creates panic among the wheat growers, as well as researchers, in Bangladesh. The severity of head infection creates head blight and increases devastating impact on yield and grain quality due to unwanted black-pointed, shrivelled, and infected seeds. Substantial economic loss has occurred in the last couple of years in wheat production of Bangladesh due to the severity of head blight for blast disease, affecting the livelihood of millions of small-scale farmers. To improve the understanding of head infection, comprehensive field survey and studies were conducted in agroecological zones (AEZ) 8, 9, 21, 22, and 28. These AEZs have previous records of severe rice blast incidences. In this study, we have focused on details of pathogens on the head of wheat and other cereals, and revealed that head infection was not only caused by Magnaporthe spp. - the causal organism of blast disease. The other causal organisms were Fusaium spp, Bipoaris spp., and Alternaria spp. Again, infection by Bipolaris spp. was highly variable. The symptoms of head blight caused by these pathogens are easily confusing. The pathogens often co-exist on the same head and in the same field. The results highlight the need for deploying proper assessment of the causal organisms from local fields for effective disease management strategy. Incidences of head blight complex, likely due to climate variability, are challenging for cereal production and a serious threat for regional food security in South Asia.

Phylogeny of pectolytic bacteria associated with recent outbreaks of potato soft rot and blackleg in the United States R. D. Curland (1), R. R. McNally (2), B. T. Webster (1), A. O. Charkowski (2), K. L. L. Perry (3), J. Hao (4), C. T. Bull (5), G. A. Secor (6), N. Rosenzweig (7), S. Johnson (8), R. P. Larkin (9), C. A. ISHIMARU (1), (1) University of Minnesota, St. Paul, MN, USA; (2) Colorado State University, Fort Collins, CO, USA; (3) Cornell University, Plant Pathology \& Plant-Microbe Biology Section, Ithaca, NY, USA; (4) University of Maine, Orono, ME, USA; (5) The Pennsylvania State University, University Park, PA, USA; (6) Department of Plant Pathology, North Dakota State University, Fargo, ND, USA; (7) Michigan State University, East Lansing, MI, USA; (8) University of Maine Cooperative Extension, Presque Isle, ME, USA; (9) USDAARS, New England Plant, Soil, \& Water Lab, Orono, ME, USA

Soft rot diseases of potato are caused by several taxa of bacteria belonging to the newly described family Pectobacteriaceae. Most soft rot bacteria formerly named as Erwinia spp. were reclassified into the genera Pectobacterium and Dickeya. Multiple species of Pectobacterium cause soft rot diseases during field production and storage of potatoes. Recently, Dickeya has been connected with significant losses from blackleg and non-emergence diseases in commercial and seed potato production in the Northeastern U.S. To develop a current depiction of the soft rot pathogens present in the U.S. and to re-evaluate isolate identifications made prior to taxonomic reclassifications, a phylogenetic study of a large collection of pectolytic soft rot bacteria was conducted. The collection included isolates from recent potato soft rot outbreaks around the U.S., historical isolates from academic culture collections, and reference strains from bacterial culture repositories. Phylogenies were constructed using a multilocus sequence alignment (MLSA) of 3 to 5 housekeeping genes. P. carotovorum subsp. carotovorum, P. carotovorum subsp. brasiliensis and P. parmentieri were common within the U.S collection. $D$. chrysanthemi, $D$. dianthicola, $D$. dadantii were also present. The quarantine organism $D$. solani was not detected. By providing new baseline knowledge, this study can aid in monitoring future shifts in soft rot pathogens within the U.S. and inform strategies for disease management.

Araucaria araucana root rot caused by Phytophthora multivora and $P$. citrophthora

E. Gálvez, A. LARACH, N. Riquelme, J. L. Celis, J. Guajardo, X. A. Besoain, Pontificia Universidad Católica de Valparaíso, Quillota, CHILE

Araucaria araucana is an endemic conifer from central and southern Chile, associated with the culture of Mapuche people. It is also important as an ornamental species. During the last year, plants of $A$. araucana have been detected in commercial nurseries, with symptoms of decay, chlorosis, brown necrosis in the leaf apex and dark brown rot in roots. Necrotic tissues obtained from the margin of the root lesions were plated on P5ARP semiselective medium and incubated at $25^{\circ} \mathrm{C}$ for 7 days. From soil samples Phytophthora was recovered employing rhododendrum leaves bates. Phytophthora spp. was isolated from the advance zone of necrotic lesions and replicated to Corn meal agar (CMA), for oomycete purification and its subsequent taxonomic and molecular identification. Two isolates were identified as $P$. multivora and $P$. citrophthora. A pathogenicity test was carried out using these two isolates, inoculating asymptomatic $A$. araucana 2 -year old plants $(\mathrm{n}=3)$, with $100 \mathrm{ml}$ of $1 \times 10^{4}$ propagules $\mathrm{ml}^{-1}$. Three plants were left as negative control adding only sterile distilled water. After 45 days from the inoculation, plants show chlorosis, brunch decay, leaf tip necrosis and severe root rot symptoms. Control plants were symptomless. Phytophthora multivora isolate was more aggressive than $P$. citrophthora isolate. Both species were reisolated from inoculated plants, thus completing Koch's postulates. To our knowledge this is the first reference of these Phytophthora species causing Araucaria araucana root rot in Chile or worldwide.

First detection of Golovinomyces ambrosiae causing powdery mildew on medical marijuana plants in Nevada J. SCHOENER, S. Wang, Nevada Department of Agriculture, Sparks, NV, USA

Marijuana plants (Cannabis sativa L.) are cultivated in registered indoor facilities in Nevada for medical use. Powdery mildew is one of the most common diseases in marijuana cultivation facilities, but it is not known what specific fungal species are associated with cannabis plants. In 2017, a powdery mildew infection was observed on the majority of cannabis plants in a cultivation facility, and all allowable fungicides applied provided only limited control of the disease. To better manage the disease, an accurate identification of the causative agent is necessary in order to select a more targeted fungicide. Infected leaves were placed under a dissecting microscope, and mycelium and spores were blotted with a piece of clear tape and mounted on a slide for morphological examination. The fungus produced ellipsoid conidia without the presence of cleistothecia. To further identify the 
species, $15 \mu 1$ of TE buffer was applied to the leaf surface where a dense mycelial colony was present. Spores were suspended in the TE buffer droplet by repetitive pipetting, and transferred into a microcentrifuge tube. PCR using primers ITS1/ITS4 was performed after heating the spore solution at $100^{\circ} \mathrm{C}$ for 10 minutes. PCR products were purified and sequenced directly. A consensus DNA sequence of $596 \mathrm{bp}$ was obtained and a BLASTn search suggested that this fungus was Golovinomyces ambrosiae. To our knowledge, this is the first report of this species infecting C. sativa.

\section{Emerging viruses in Florida and the Caribbean}

S. ADKINS (1), C. Estevez de Jensen (2), G. Frantz (3), H. C. Mellinger (3), S. Hutton (4), J. E. Funderburk (5), R. Naidu (6), W. W. Turechek (7), (1) USDA, ARS, US Horticultural Research Laboratory, Fort Pierce, FL, USA; (2) University of Puerto Rico, Agro-environmental Sciences, Mayaguez, PR, USA; (3) Glades Crop Care Inc, Jupiter, FL, USA; (4) University of Florida, Wimauma, FL, USA; (5) University of Florida, Quincy, FL, USA; (6) Washington State University - IAREC, Prosser, WA, USA; (7) USDA, ARS, U.S. Horticultural Research Laboratory, Fort Pierce, FL, USA

Multiple thrips-, whitefly- and aphid-transmitted viruses have recently emerged or re-emerged in vegetable and ornamental crops in Florida and the Caribbean. Tomato spotted wilt virus (a thrips-transmitted tospovirus) and Tomato yellow leaf curl virus (a whitefly-transmitted begomovirus) have historically been significant constraints to vegetable and/or ornamental production in this region. With the emergence or re-emergence of additional thrips- and whitefly-transmitted viruses such as Tomato chlorotic spot virus (TCSV), Cucurbit yellow stunting disorder virus (CYSDV), Cucurbit leaf crumple virus $(\mathrm{CuLCrV})$ and Squash vein yellowing virus (SqVYV), pathogen identification and management has become more complex. Aphidtransmitted potyviruses like Zucchini tigré mosaic virus (ZTMV) and non-insect-transmitted tobamoviruses like Tomato mottle mosaic virus (ToMMV) have also begun to appear with greater regularity. Several novel virus species including the ilarvirus Tomato necrotic streak virus (TomNSV) have been detected. New protocols have been developed for rapid and specific identification of these viruses, and to facilitate studies on host and geographic range expansion. Collectively, this information will lead to management strategy improvement. Ongoing tracking of emerging and re-emerging viruses in Florida and the Caribbean will help strengthen agricultural security in the area, which is especially critical in the aftermath of the 2017 hurricanes.

\section{A previously undiscovered Emaravirus associated with witches broom symptoms in blue palo verde (Parkinsonia florida) trees in Arizona} A. S. AVELAR, M. Ilyas, U. Schuch, J. K. Brown, School of Plant Sciences, University of Arizona, Tucson, AZ, USA

Palo verde witches broom disease (PVWBD), of unknown etiology, affects blue palo verde (Parkinsonia florida) trees. Broom symptoms are manifested as dense clusters of shortened, thornless branches with stunted leaves. Recently, the disease has spread widely in Arizona nurseries and landscapes, causing irreversible damage and sometimes requiring tree removal. In this study, the suspected causal agent of PVWBD has been associated with symptomatic trees for the first time. During 2015-2016, leaves, flowers, and seeds were collected from symptomatic and asymptomatic blue palo verde trees. Total RNA was isolated and RNASeq libraries were sequenced using the Illumina Hi-Seq 2500 platform. Four RNA components of 7, 2, 1.4, and 1.5 kilobases were recovered, at 60-65\% similarity (BLASTn) with Wheat mosaic virus (Emaravirus; Fimoviridae), a negative-sense, single-stranded RNA virus with an eriophyid mite vector. Reverse-transcriptase PCR amplification with primers specific for RNAs 1-4 and Sanger sequencing of the cloned amplicons confirmed the presence and absence of four RNA components in symptomatic and asymptomatic samples, respectively. The apparently complete viral genome, and presence of the mite Aculus cercidii (Keifer) infesting palo verde trees, indicates the first association of a previously undescribed emaravirus with witches broom disease of palo verde. The virus name, Palo verde witches broom virus (PVWBV), is proposed.

First report and new hosts of the oak pathogen Diplodia corticola in Wisconsin

D. R. SMITH (1), G. R. Stanosz (2), (1) University of Wisconsin-Madison, Madison, WI, USA; (2) University of Wisconsin, Madison, WI, USA

Diseased oaks (Quercus species) exhibit tip blight, branch and stem cankers, and dieback often attributed to Botryosphaeria species or related anamorphic fungi. Diplodia corticola (teleomorph described as Botryosphaeria corticola) has been identified as part of a disease complex of oaks observed in Europe since the 1980's. Reports of this fungus on the east and west coasts of the United States prompted re-examination of isolates from oaks in Wisconsin. These isolates had been obtained in the late 1990's and early 2000's and previously were identified only as Diplodia species. Phylogenetic analysis of rDNA- ITS sequences (including Diplodia and Botryosphaeria sequences from GenBank) confirmed most Wisconsin isolates from northern red oak (Q. rubra), black oak (Q. velutina), white oak (Q. alba), and bur oak ( $Q$. macrocarpa) as D. corticola. Other isolates from oaks in Wisconsin were $D$. mutila and D. seriata. Wound-inoculation of northern red, white, and bur oak shoots of seedlings in a greenhouse resulted in shoot death and stem lesions, from which the pathogen was reisolated. We conclude that $D$. corticola has been present in the northcentral United States for at least two decades and report two previously unrecognized hosts of this pathogen: white oak and bur oak. The roles of D. corticola and other Diplodia and Botryosphaeria species in the deterioration of oak health in North America merit additional investigation.

Identification of Pectobacterium carotovorum as the causal agent of bacterial canker on Pyrus communis $\mathrm{L}$ in Brazil Y. FRANCO CARDOZA (1), L. Araujo (2), V. Duarte (1), M. G. de Moraes PhD (3), (1) Agronômica - Laboratório de Diagnóstico Fitossanitário e Consultoria, Porto Alegre, BRAZIL; (2) EPAGRI, São Joaquim, SC, BRAZIL; (3) Dept de Fitossanidade Faculdade de Agronomia da UFRGS, Porto Alegre RS, BRAZIL

In June 2016, bacterial canker-like symptoms were observed on Pyrus communis L cv. Yali in one commercial orchard in Monte Castelo, SC. Eighthundred of 1.200 plants were lost by death and, finally, tree eradication. In affected trees, the disease manifested as cankers with exudates on trunks and twigs, and the stems turned brown and slowly died. Bacteria were isolated on King B medium from infected tissues. Biochemical tests including Biolog ${ }^{\mathbb{R}}$ GEN III were conducted and some isolates were selected for whole genome sequencing throught Ion Torrent ${ }^{\mathrm{TM}}$ platform. PCR with Y1/Y2 and PcaF/PcaR primers, specific for P. carotovorum and P. carotovorum subsp. actinidiae respectively were also carried out. A total of 15 bacterial colonies were isolated from infected tissues. All were gram-negative, facultatively anaerobic, rod-shaped and exhibited a strong pectolitic activity in potato and carrot disks. Biolog assay showed that the isolates were homogeneus, and three of them were selected for further tests. The organism with the highest genome and proteome identity (99.8\%) with the isolates was Pectobacterium carotovorum subsp. actinidiae. A product of the expected size was obtained in both especific PCR. Inoculation of pear tree branches with $24 \mathrm{~h}$ old colonies showed that the bacterium was able to colonize the plant tissues. More tests, including kiwifruit inoculation, are in progress to fulfill Koch's postulates. To our knowledge, this is the first report of $P$. carotovorum causing pear bacterial canker-like symptoms in Brazil. Identification and monitoring of this pathogen is very important due to large economic losses that can cause.

First detection of Pythium aphanidermatum crown rot of industrial hemp in Nevada

J. Schoener, R. Wilhelm, M. Yazbek, S. WANG, Nevada Department of Agriculture, Sparks, NV, USA

Industrial hemp (Cannabis sativa L.) is a regulated crop in Nevada. In July 2017, approximately 5-10\% of industrial hemp plants (var. Cherry Wine) observed in a production field exhibited symptoms of crown rot, leaf yellowing, extensive wilt, and eventual death of entire plants. White-colored mold 
on the crown area was frequently observed when a plant was pulled from the ground. To identify the causative agent of the disease, pieces of stem tissue were cut from the margin of the crown rot, surface sterilized, placed onto potato dextrose agar amended with streptomycin sulfate (PDA+strep) and corn meal agar-PARP (CMA-PARP), and incubated at $22^{\circ} \mathrm{C}$ in the dark. Tissue plated on PDA+strep did not yield growth of any pathogens. On CMA-PARP, a fast-growing Pythium was obtained from all pieces of stem tissue plated. The isolate produced oogonia, antheridia, and sporangia on CMA, and morphologically resembled P. aphanidermatum. To further investigate its identity, DNA was extracted from 4 representative isolates followed by PCR using primers ITS1/ITS4 to amplify the ITS region of rDNA. PCR products were purified and sequenced directly. A consensus DNA sequence of 866 bp was obtained and a BLASTn search confirmed that the species was $P$. aphanidermatum. Hemp crown and root rot caused by $P$. aphanidermatum was first reported in a research plot in Indiana in 2017. This is the first detection of $P$. aphanidermatum in a commercially grown industrial hemp crop in Nevada.

New fungal pathogens recently detected on ornamental plants in Italy A. Garibaldi, D. Bertetti, G. Gilardi, M. L. GULLINO, Agroinnova - University of Torino, Grugliasco, Torino, ITALY

In the last five years, several host-fungal pathogen combinations were detected in ornamentals, for the first time in Italy. Infected plants were in farms, nurseries, private and public gardens in Northern Italy. The causal agents of the diseases were identified on the basis of both in vitro as well as in vivo morphological characteristics and molecular analysis. Koch's postulates were fulfilled. Among the soil-borne pathogens, several Fusarium wilt agents were isolated from hosts belonging to Cactaceae, Crassulaceae, Euphorbiaceae, Lamiaceae and Asteraceae, while Verticillium dahliae and V. nonalfalfae were detected on Phlox paniculata and Pelargonium grandiflorum, respectively. Rhizoctonia solani was isolated from Lavandula stoechas, Campanula spp., Lychnis coronaria and Rebutia perplexa. Both Sclerotinia sclerotiorum and Phytophthora cryptogea were detected on species of Asteraceae and Lamiaceae. Among powdery mildews, Golovinomyces magnicellulatus, G. biocellatus and G. cichoracearum were reported on Phlox paniculata, Thymus $\times$ citriodorus "Aureus" and Verbascum nigrum "Album", respectively. Among the causal agents of downy mildews, Peronospora arthurii on Oenothera biennis and Plasmopara obducens on Impatiens walleriana were detected. Coleosporium campanulae on Campanula rapuncoloides, Entyloma gaillardianum on Gallardia aristata and $G . \times$ grandiflora were identified. Several species belonging to different families, in particular Asteraceae and Lamiaceae, are new host for Botrytis cinerea. Leaf necrosis caused by Alternaria spp. were detected on Campanula glomerata, Rudbeckia fulgida and Salvia leucantha. Finally, Pseudonectria buxi was observed on Buxus microphylla.

First report of Tomato chlorotic dwarf viroid and Southern tomato virus infecting greenhouse tomato in Hawaii A. OLMEDO-VELARDE (1), S. Watanabe (1), R. T. Hamasaki (2), M. J. Melzer (3), (1) University of Hawaii at Manoa, Department of Plant and Environmental Protection Sciences, Honolulu, HI, USA; (2) University of Hawaii at Manoa, Department of Plant and Environmental Protection Sciences, Kamuela, HI, USA; (3) Department of Plant \& Environmental Protection Sciences, University of Hawaii at Manoa, Honolulu, HI, USA

In January 2017, greenhouse tomato [Solanum lycopersicum (L.)] plants showing stunting and foliar yellowing, reddening, interveinal chlorosis, and epinasty, were reported from the Island of Hawaii, Hawaii, USA. Initial shotgun cloning and sequencing using double stranded (ds)-RNA extracted from symptomatic plants revealed Southern tomato virus (STV, genus Amalgavirus) was present and a potential causal agent of the disease. Screening of 14 symptomatic and 8 asymptomatic plants by STV-specific reverse transcription (RT)-PCR indicated STV was not clearly associated with the observed symptoms. Symptomatic samples were subsequently subjected to universal Pospiviroid RT-PCR and produced the expected 198 bp amplification product. Cloning and sequencing revealed the product was $99 \%$ identical to Tomato chlorotic dwarf viroid (TCDVd) isolate 'GBVdC_TCDVd04' (GenBank Accession KU714934). A TCDVd-specific RT-PCR assay was developed. This assay, combined with sequencing of amplification products, was used to confirm the identity of the viroid in the symptomatic samples. However, TCDVd was found in asymptomatic tomato samples with this assay, suggesting it may not be the causal agent of the symptoms in tomato. Further investigation is needed to understand the role of STV and TCDVd in the symptomatic and asymptomatic tomatoes, as well as the presence and genetic diversity of this viroid in commercial tomato seeds.

First report and genomic sequence of a new alfalfa marafivirus from France

L. G. NEMCHINOV (1), S. François (2), P. Roumagnac (3), D. S. Mollov (4), D. Filloux (5), (1) USDA-ARS, BARC, Molecular Plant Pathology Laboratory, Beltsville, MD, USA; (2) Laboratory Diversité, Génomes et Interactions Microorganismes Insectes (DGIMI), Montpellier, FRANCE; (3) Laboratory Biologie et Génétique des Interactions Plante-Parasite (BGPI), Montpellier, FRANCE; (4) USDA-ARS, BARC, National Germplasm Recourses Laboratory, Beltsville, MD, USA; (5) Laboratory Biologie et Génétique des Interactions Plante-Parasite (BGPI), Montpellie, FRANCE

Viral infections of alfalfa are widespread in major cultivation areas. A new viral species, provisionally named alfalfa virus $\mathrm{F}$ (AVF), was diagnosed using VANA metagenomics-based approach (PMID:27764211) in alfalfa (Medicago sativa L.) samples collected in Southern France. Nucleotide sequence of the viral genome was determined by de-novo assembly of VANA reads and by 5'/3' RACE systems with viral RNA extracted from enriched viral particles or with total RNA, respectively. The virus shares the greatest degree of overall sequence identity ( $78 \%)$ with Medicago sativa marafivirus 1 (MsMV1) recently deduced from alfalfa transcriptomic data (PMID:28974471). Tentative nucleotide sequence of the AVF coat protein shares $\sim 83 \%$ identity with the corresponding region of MsMV1. Sequence search of a single ORF encoding a polyprotein of 235kDa in Pfam database resulted in identification of five domains, characteristic for the genus Marafivirus, family Tymoviridae: viral methyltransferase, tymovirus endopeptidase, viral (superfamily 1) RNA helicase, RNA dependent RNA polymerase and tymovirus coat protein. AVF genome contains a conserved "marafibox", a 16-nt consensus sequence present in all known marafiviruses. Phylogenetic analysis using MEGA 7 software with complete nucleotide sequences of AVF and other viruses of the family Tymoviridae grouped AVF in the same cluster with MsMV1. Based on the criteria demarcating species in the genus Marafivirus that include overall sequence identity less than $80 \%$ and coat protein identity less than $90 \%$, we propose that AVF represents a distinct viral species in the genus Marafivirus, family Tymoviridae. Geographic distribution of the AVF will be further assessed using serological and molecular methods.

Cryphonectriaceae endophytes in Melastomataceae: Latent pathogens in hiding

G. M. Granados (1), A. R. McTaggart (1), C. A. Rodas (2), J. Roux (1), M. J. WINGFIELD (1), (1) Forestry and Agricultural Biotechnology Institute (FABI), University of Pretoria, Pretoria, SOUTH AFRICA; (2) Forestry Health Protection Programme, Yumbo, COLOMBIA

Species of Cryphonectriaceae can occur as asymptomatic fungal endophytes in Melastomataceae trees and shrubs. These fungi pose threats to biosecurity because they cannot easily be detected by conventional routine quarantine inspections. Host switches in the Cryphonectriaceae have been shown to occur between species of Myrtales including the Myrtaceae and Melastomataceae. The aim of this study was to identify the species of Cryphonectriaceae that occur in the asymptomatic tissues of four native and two introduced species of Melastomataceae that naturally occur alongside Eucalyptus (Myrtaceae) plantations in Colombia. Healthy branch segments were collected in five provinces of Colombia and incubated for two to four weeks to promote sporulation of endophytes. Fruiting bodies of Cryphonectriaceae that emerged were isolated and identified based on DNA sequence data for two gene regions. Three species were identified including Aurapex penicillata, Chrysoporthe cubensis and C. inopina. The results showed that a relatively large 
number of species of Melastomataceae are hosts of the Cryphonectriaceae in Colombia. Some of these such as $C$. cubensis are already emerging as important pathogens of Eucalyptus in that country. Importantly, the risks involved in moving plant tissue with endophytes of the Melastomataceae is high, as they act as reservoirs of aggressive pathogens of Myrtaceae in plantations and natural woody ecosystems.

\section{Grapevine virus $\mathbf{J}$, a novel vitivirus identified in grapevine via high-throughput sequencing}

A. DIAZ LARA (1), D. A. Golino (2), M. Al Rwahnih PhD (3), (1) Department of Plant Pathology, University of California-Davis, Davis, CA, USA; (2) University of California, Davis, CA, USA; (3) Foundation Plant Services Facility, University of California, Davis, CA, USA

To date, nine viruses are formally classified in the genus Vitivirus (family Betaflexiviridae), five of which are reported in grapevine: grapevine viruses A, $\mathrm{B}, \mathrm{D}, \mathrm{E}$ and F. Additionally, three recently discovered viruses, grapevine viruses $\mathrm{H}, \mathrm{G}$ and I are proposed as new members. During the characterization of a white grape selection from Turkmenistan introduced to Foundation Plant Services (UC-Davis), a novel Vitivirus was identified via high-throughput sequencing. Total nucleic acids from the vine were extracted, used for library preparation, sequenced using the Illumina platform and compared against the GenBank virus database. The BLAST analysis detected several sequences (sizes from 4,743 to 7,375 nt) sharing a distant relationship (average identity: $52 \%$ ) with species belonging to the genus Vitivirus. Further characterization resulted in the full genome (7,390 nt) of a putative virus, tentatively named grapevine virus J (GVJ, GenBank MG637048), comprised of five open reading frames. According to the genomic arrangement, sequence homology and phylogenetics, GVJ is a new species in the genus Vitivirus, with the closest characterized virus being GVD. Lastly, a developed RT-PCR assay confirmed the presence of GVJ in the source vine and eight additional vines in a partial survey of the National Clonal Germplasm Repository in Winters, CA. Thus, this work adds a novel species to the list of viruses infecting grapevine and is a basis for future biological studies.

Comparative study of emerging Ralstonia pseudosolanacearum strains

D. J. NORMAN (1), A. M. Bocsanczy (2), P. J. M. Bonants (3), J. van der Wolf (4), M. Bergsma-Vlami (5), (1) Univ of Florida MREC, Apopka, FL, USA; (2) University of Florida MREC, Apopka, FL, USA; (3) Wageningen Plant Research, Wageningen, NETHERLANDS; (4) Wageningen University \& Research, Wageningen, NETHERLANDS; (5) NPPO, Wageningen, NETHERLANDS

Ralstonia pseudosolanacearum (Rps), previously known as $R$. solanacearum phylotype I, one of the causal agents of Bacterial Wilt that was confirmed to be infecting blueberry in multiple counties in Florida in 2016. Unexpectedly, the population structure of strains infecting blueberry is not clonal, instead it includes Rps and $R$. solanacearum (Rs) phylotype IIA strains, which makes detection and analysis of host range more difficult. In the Netherlands, a new Rps strain that infects roses has been isolated and characterized; this strain is virulent and has been found in several countries through Europe. Since 2013, Rps strains have been commonly found on mandevilla production in Florida. We hypothesized that the emerging Rps strains infecting new hosts in Florida are related to the strain infecting roses in Europe and their host range could be determined by differences in their type 3 effector sets. In order to test this hypothesis, we sequenced the genomes of three Rps strains from mandevilla, blueberry and rose (P781, P824, and PD7123 respectively) and we are in the process of comparing their genomes. We are currently evaluating host range of the three strains. Preliminary results show that neither the blueberry (P824) nor the rose (PD7123) Rps strains infect mandevilla plants when wound inoculated and the rose strain is very virulent on southern highbush blueberry.

Emerging pathogens of wasabi (Wasabia japonica) in British Columbia, Canada E. BETZ, Z. K. Punja, Simon Fraser University, Burnaby, BC, CANADA

Wasabi is grown semi-hydroponically in polyethylene greenhouses under humid conditions to simulate the natural riverbed conditions where it is found. Surveys of five greenhouses in the Lower Mainland and on Vancouver Island of British Columbia were conducted during the summers of 2016 and 2017 to identify the major pathogens of this crop. Symptoms observed included wilt, petiole blight, leaf spot, powdery mildew, white rust and root/rhizome rot. Diseased tissues (except for mildew and white rust) were sterilized with $0.4 \%$ bleach and $70 \%$ ethanol and plated onto V8 juice and potato dextrose agars with $100 \mathrm{mg} / \mathrm{L}$ streptomycin. Isolates were first identified by morphology and further confirmed by PCR of the ITS region. In total, 10 fungi/oomycetes were observed on diseased tissues, notably Verticillium isaacii (wilt), Leptosphaeria biglobosa (Phoma leaf spot), Erysiphe cruciferarum (powdery mildew), Albugo candida (white rust), Botrytis cinerea (gray mold), and Fusarium/Pythium (rhizome rot). Verticillium isaacii is previously unreported from wasabi and detached leaf, petiole, and rhizome inoculations were conducted to test pathogenicity. Mycelium was grown for 3 weeks in potato dextrose broth then blended and used to inoculate wounded wasabi tissues. Symptoms of chlorosis, necrosis, and blackening appeared after 7 days. Emerging diseases of wasabi continue to be a threat to commercial production, and management of pathogens is paramount to the success of wasabi farms.

Diplodia seriata and $D$. mutila causal agents of gummy canker in Araucaria araucana trees X. A. BESOAIN, J. Guajardo, A. Larach, N. Riquelme, E. Gálvez, L. Tapia, J. L. Celis, Pontificia Universidad Católica de Valparaíso, Quillota, CHILE

During the last two years a severe decay was observed in Araucaria araucana trees in Araucanía region of Southern Chile and in Valparaíso region of Central Chile. The plants showed arms with dieback symptoms, leaf orange discoloration, canker in the cortex and cream color gummy exudation. In order to determine if these species were responsible for the observed decay two pathogenicity tests were conducted employing 2-year old A. araucana plants $(\mathrm{n}=3)$ for $D$. seriata trial and 10-year old $A$. araucana plants for $D$. mutila. Two isolates of $D$. seriata were recovered from the advance zone of the lesions in southern area (close to Conguillio National park) while two isolates of D. mutila were recovered from the northern area (garden in Quillota city). In both trials plants were inoculated by an injurie and a mycelial plug $(8 \mathrm{~mm})$ of each Diplodia species was introduced in a injurie. Three plants were left as controls for each test, employing only a PDA sterile plug $(8 \mathrm{~mm})$ in injurie. After two months, the inoculated Diplodia plants showed similar symptoms in both trials, arm dieback, orange discoloration and canker in the branches and cream color gummosis. A first report was previously done for D. mutila causing gummy canker in A. araucana, but this is the first report of D. seriata causing gummy canker of Araucaria trees close to the Conguillio National Park. Both species were molecularly detected analyzing ITS, beta tubulin and elongation factor and deposited in GeneBank.

Identification of a new virus from the family Luteoviridae in Miscanthus sinensis D. S. MOLLOV (1), L. Kumar (2), S. Grinstead (1), I. Fuentes-Bueno (1), M. M. Malapi-Wight (2), (1) USDA-ARS, BARC, National Germplasm Recourses Laboratory, Beltsville, MD, USA; (2) USDA-APHIS, Plant Germplasm Quarantine Program, Beltsville, MD, USA

Sugarcane Yellow Leaf Virus (SCYLV) is a widespread and economically important virus infecting sugarcane. Miscanthus, a sugarcane relative, is considered resistant to SCYLV and is used in sugarcane breeding. We detected a new virus in a quarantined Miscanthus sinensis accession using high throughput sequencing (HTS). Leaves exhibiting yellowing symptoms were selected for total RNA extraction and used as templates for ribosomaldepleted RNA library preparation. The resulting libraries were sequenced on an Illumina NextSeq 500 platform. Sequence reads were assembled into 
contigs that revealed a single virus infection. To complete the genome assembly the 5' and 3' ends were cloned, Sanger sequenced, and combined with the HTS data. The complete genome is 5,859 nt and comprises six open reading frames with an organization like SCYLV and phylogenetic relatedness to Luteoviridae. At the whole genome level, the highest nucleotide sequence identity (79\%) was to SCYLV. A BLASTp analysis of the CP amino acid sequences showed highest identity to SCYLV (79-86\%) and wheat leaf yellowing-associated virus (WLYaV) (81\%). The RdRp amino acid sequences had highest identity to SCYLV (79-86\%) and to WLYaV (81\%). These percent identities for both CP and RdRp are below the species demarcation criteria. Identifying and characterizing this new virus is important for developing improved diagnostic methods and will contribute to quarantine and disease management efforts.

\section{Brown Apical Necrosis (BAN) a new problem in English walnut (Juglans regia L.) production in the Biobio Region of Chile} E. A. MOYA-ELIZONDO, J. San Martin, B. Ruiz, Universidad de Concepción, Chillán, CHILE

English (Persian) walnut plantations have had an explosive growth in the Biobío Region in Chile, going from 107 hectares to almost 2,000 in the last 10 years. Walnut blight, caused by Xanthomonas arboricola pv. juglandis, has been the main phytopathological problem affecting these nuts, but from 2013 to date premature fruit dropping during fruit growth in spring, associated with a brown to dark necrosis in the apical area of nuts has been consistently observed. Isolation of different Fusarium and Alternaria species in dropped fruit suggests the presence of the disease complex Brown Apical Necrosis (BAN) in this area. During the 2016/17 season, a follow-up of 16 English walnut trees cv. Chandler and three cv. Serr in a commercial orchard located in Negrete (Biobío Region), showed that between 60 and $100 \%$ of the fruits that fell to the ground between November 28 and January 2 were colonized by Fusarium and Alternaria species. Morphological and molecular analysis (ITS, NS, and $\beta$-tubulin genes) of fungal isolates obtained from walnut fruits indicate the presence of $F$. oxysporum, $F$. culmorum, and A. alternata as part of BAN disease complex in central-southern Chile. A study determined that inoculations with a field isolate of $F$. oxysporum and A. alternata during the flowering period, reproduced BAN symptoms in a $68.8 \%$ and $55.4 \%$, respectively. Actually, we are working in epidemiological studies of BAN disease complex and developing control strategies for its proper management.

Powdery mildew (Sawadaea bicornis) on Rocky Mountain maple (Acer glabrum)

C. JENKINS, C. Nischwitz, Utah State University, Logan, UT, USA

The Rocky Mountain maple (Acer glabrum) is a deciduous tree indigenous to North America. It is found in many western states including Utah, New Mexico, California and Alaska where it provides prevention of slope erosion and wildlife habitat. Trees in Cache Valley in northern Utah began displaying symptoms of powdery mildew infection in the fall of 2017. Thus far, powdery mildew on A. glabrum has only been recorded in Iowa (Uncinula circinata) in 1923 and Idaho (Phyllactinia guttata) in 1973 but the species descriptions did not fit the powdery mildew found in Utah. Based on morphological characteristics (dimensions of chasmothecia and asci, number of appendages) using light microscopy and molecular identification using PCR and DNA sequencing, the powdery mildew was identified as Sawadaea bicornis. Sawadaea bicornis is a European powdery mildew that was first found in the USA on Norway maple (Acer platanoides) and box elder (Acer negundo). To our knowledge this is the first report of Sawadaea bicornis infecting Acer glabrum in North America.

\section{Exotic threats to sugarcane production in Australia}

R. C. MAGAREY, Sugar Research Australia, Tully, AUSTRALIA

Exotic pests and diseases pose a serious threat to Australia's sugarcane industry. As an island continent, strict quarantine has limited the number and type of threats that have entered Australia. Recent research aims for industry pathologists to become familiar with, research and extend information on major threats; these include Sesamia grisescens (noctuid moth borer), Scirpophaga excerptalis (top borer), downy mildew (Peronosclerospora spp), sugarcane streak mosaic virus (SCSMV) and Ramu stunt. These pests and diseases all occur in countries close to northern Australia (Indonesia / Papua New Guinea). Research has investigated pathogen variability; both the Ramu stunt, SCSMV and downy mildew pathogens have shown significant variability with implications for both diagnosis and assessing cultivar resistance. Moth (stalk) borer research has investigated rapid methods for screening for cultivar resistance / tolerance. A shadehouse technique using clusters of plants with a central point of infestation is showing promise. The Australian sugarcane industry is taking all reasonable preventative measures to avoid catastrophic economic outcomes due to unexpected pest or disease incursions.

Tomato chlorotic spot virus, an emerging tospovirus threatening vegetable production in the United States

S. ZHANG (1), K. S. Ling (2), B. Poudel (1), D. Seal (1), M. T. McGrath (3), Q. Wang (4), G. McAvoy (5), (1) University of Florida, Homestead, FL, USA; (2) USDA-ARS, Charleston, SC, USA; (3) Cornell University, Riverhead, NY, USA; (4) Miami-Dade County Extension, Homestead, FL, USA; (5) Hendry County Extension, LaBelle, FL, USA

Tomato chlorotic spot virus (TCSV) is a tospovirus first detected in tomato and bell pepper in south Florida in 2012. Subsequently, TCSV was confirmed in tomato in Ohio and New York. Since 2014, TCSV has caused significant losses to tomato and bell pepper growers in south Florida. TCSV is efficiently transmitted by flower thrips, which are abundant in many regions of the USA. Initial symptoms of TCSV in tomato begin to appear on top leaves as tiny necrotic lesions three weeks after transplanting. Within one week, the symptoms quickly expand to cause bronzing, necrosis, deformation of leaves, and terminal stem and leaf death. In bell pepper, TCSV causes chlorosis on top leaves and necrosis over time. TCSV results in severe stunting and eventually death of the plant if plants are infected at an early stage. Infected plants produce few, if any, fruit with necrotic rings rendering them unmarketable. Field survey from 2016 to 2017 indicated that TCSV has become the dominant tospovirus in south Florida. In 2017, TCSV was also detected in snapbean and purslane in Homestead, FL. Reverse transcription loop-mediated isothermal amplification (RT-LAMP) was developed for specific detection of TCSV from tomato plants. Results of field trials in Homestead, FL indicate that tomato cultivars carrying $s w-5$ gene are resistant to TCSV. Applying insecticides is not highly effective in reducing TCSV incidence, but can minimize secondary spread of TCSV by its thrips vectors.

\section{Contribution of Fusarium spp. to sugarcane yellow canopy syndrome (YCS) in Australia}

S. BASNAYAKE (1), E. Czislowski (2), D. Olsen (3), J. Basnayake (3), E. Aitken (2), A. Geering (1), (1) Queensland Alliance for Agriculture and Food Innovation (QAAFI), St Lucia, AUSTRALIA; (2) School of Agriculture and Food Sciences, The University of Queensland, Brisbane, AUSTRALIA; (3) Sugar Research Australia, Brandon, AUSTRALIA

Yellow canopy syndrome (YCS) of sugarcane, first discovered in North Queensland, Australia, is characterized by prematurely senescing, bright orange leaves in the lower canopy of the plant. Spread of this disorder from northern to southern Queensland and northern New South Wales suggests that this is biotic in nature, although symptoms are likely to be modulated by abiotic stresses. Previous research has shown accumulation of starch in early senescing leaves and also disruption of vascular transport, similar to other well-characterized diseases caused by bacteria and viruses. In order to provide clues as to the aetiology of the disease, a range of antimicrobial agents [streptomycin and oxytetracycline (antibiotics), metalaxyl (systemic fungicide) and 
prochloraz (general fungicide)] were tested on the sugarcane variety Q200 in field trials established in 'YCS hotspots' in Ingham and the Burdekin region in the 2016/17 season. Treatments had no negative effect on crop physiology or growth. Delay in symptom development was observed only in the plants treated with streptomycin. To search for evidence of pathogen infection, vascular regions of plants from the various treatments were examined by light microscopy and a range of fungal and bacterial structures observed mainly in symptomatic leaves. Several Fusarium isolates were obtained from the basal regions of the plant, roots and root rhizosphere. Efforts are being made to identify these isolates to species level and to test for pathogenicity in pot trials.

Current situation of emerging banana viruses in Democratic Republic of Congo

L. MUKWA FAMA TONGO (1), A. Kalonji-Mbuyi (2), G. Romay (3), C. G. Bragard (4), M. L. Iskra-Caruana (5), (1) Plant Clinic InternationalKinshasa, Kinshasa, CONGO, DEM. REP.; (2) Université de Kinshasa, Kinshasa, CONGO, DEM. REP.; (3) Université catholique de Louvain, Louvainla-Neuve, BELGIUM; (4) Universite Catholique de Louvain, Louvain-la-Neuve, BELGIUM; (5) Centre de Coopération Internationale pour l'Agriculture et le Développement, CIRAD, Montpellier, FRANCE

Banana viruses are a major constraint for production and exchange of improvement banana germplasm in the world. Five viruses are regularly listed: Banana bunchy top virus (BBTV), Banana streak virus (BSV), Banana bract mosaic virus (BBrMV), Cucumber mosaic virus (CMV) and Banana mild mosaic virus (BMMV). In Democratic Republic of Congo (DRC), BBTV was reported in 1950 from Yangambi (Kisangani region) and remains the widespread banana virus described in Democratic Republic of Congo. However, in 2013, symptoms of an uncharacterized pathogens were observed in several banana plantations under natural conditions in Bas-Congo province. Consequently, Surveys were conducted in 2014 and 2015 in 50 separate locations of Bas Congo province. Symptomatic and asymptomatic banana and plantain leaf samples were collected within the Province to be screened against the main known viruses. Using Immuno-Capture Polymerisation Chain reaction (IC-PCR) and RT-PCR, the samples were analysed against the four-main Banana streak virus species: Banana streak GF virus (BSGF), Banana streak OL virus (BSOLV), Banana streak IM virus (BSIMV), Banana streak MY virus (BSMYV) and Cucumber mosaic virus (CMV). Results revealed that next to BBTV infection, both BSV and CMV also infect dessert bananas as well as plantains. The fluctuation of agro-ecological conditions could be one of the factors driving emerging viruses in the study area (BasCongo Province, Democratic Republic of Congo). This result suggests the need of the strong strategies for quarantine and resilience for climate change impact for local farms.

Byssochlamys Rot in the Orchard and the Effectiveness of Fungicides against this New Disease M. BIANGO-DANIELS (1), K. Ayer (2), K. D. Cox (2), K. T. Hodge (1), (1) Cornell University, Ithaca, NY, USA; (2) Cornell University, Geneva, NY, USA

Byssochlamys nivea is an important heat-resistant spoilage mold that produces the mycotoxin, patulin. Recent work demonstrates that this species is also a post-harvest pathogen of apples, however the point of infection remains unknown. Latent infection of apples by $B$. nivea in the field may lead to storage and shelf spoilage. To test the hypothesis that $B$. nivea can infect apples in the orchard, a field trial was conducted on 'Gala' apples that were inoculated or mock-inoculated with $B$. nivea. The resulting pre-harvest symptoms were compared to symptoms of Byssochlamys rot under post-harvest conditions. The sensitivity of $B$. nivea to post-harvest fungicides labeled for apples (difenoconazole, fludioxonil, or pyrimethanil) was also determined for $30 \mathrm{~B}$. nivea isolates. We found that $B$. nivea can infect apples through wounds in the orchard, leading to symptoms like those observed post-harvest. For all fungicides tested, percent relative growth (dose of $\mathrm{EC}_{50}$ and $10 \mathrm{x} \mathrm{EC}_{50}$ ) of $B$. nivea isolates from agricultural environments was higher than those from non-agricultural environments. Controlling Byssochlamys rot with fungicides may be complicated by selection for fungicide resistance.

Anthracnose disease ratings on tea (Camellia sinensis) during the growing season in Florida

J. ORROCK, B. Rathinasabapathi, B. Spakes Richter, University of Florida, Gainesville, FL, USA

Tea [Camellia sinensis (L.) O. Kuntze] is being researched as a new specialty crop in the US. In China, anthracnose disease has been reported to cause a $30-50 \%$ yield loss, with $20-40 \%$ incidence. We have made a first report of tea anthracnose in the US, but the impact of this disease on US varieties is unknown. We quantified naturally occurring anthracnose incidence and severity on seven accessions of tea grown in a randomized complete block design in North Central Florida. Severity ratings were scored monthly during the 2017 growing season as a percentage of diseased leaves per plant. Severity ratings for each variety were used to calculate area under the disease progress curve (AUDPC). Accessions differed in incidence and severity of anthracnose. 'Assamica,' 'China Seed' and 'Georgian' had the highest AUDPC for disease severity. Other varieties had significantly lower severities according to Tukey's HSD $(\alpha<0.05)$. The maximum incidence observed was $97.8 \%$ in 'China Seed.' The maximum severity observed was $29.3 \%$ in 'Assamica.' Disease incidence and severity decreased during the initial rapid flushing stages of plant growth, and increased as leaves aged and were exposed to environmental stresses. This work has documented anthracnose disease impacts on US tea varieties and may help shape future directions of tea research, breeding, and recommendations for growers in establishing a novel industry.

Alternaria infectoria species-group member emerges as a wheat pathogen in New York M. FULCHER, G. C. Bergstrom, Cornell University, Ithaca, NY, USA

A recently observed foliar disease of wheat was attributed to isolates from the Alternaria infectoria species-group. This globally distributed species complex contains pathogens and saprobes associated with a variety of host plants, and members of the group exhibiting both of these lifestyles are known to inhabit wheat. We scouted cereal fields in New York for this fungus and recovered it from symptomatic and asymptomatic gramineous hosts, including non-cultivated species. Isolates showed variable pathogenicity on several hosts in greenhouse and laboratory assays. Our wheat pathogenic strains were not identified as a known species based on morphology or a multi-gene phylogeny, and pathogenicity on wheat could not be predicted without plant-based assays. The difficulty of identifying species and distinguishing pathogen from non-pathogen in this group is a challenge for disease monitoring. We are employing comparative genomics to study species delimitation within the entire A. infectoria complex and to search for genetic factors that underlie pathogenicity.

Molecular and biological characterization of Turnip mosaic virus infecting lettuce and chard in Brazil M. R. RIBEIRO-JUNIOR, G. C. Dourado Cruciol, M. F. Moura, B. R. De Marchi, D. N. Nozaki, M. A. Pavan, R. Krause Sakate, São Paulo State University, Botucatu, BRAZIL

Turnip mosaic virus (TuMV) belongs to the Potyvirus genus, and is a major pathogen that infects at least 418 host species. Recently, symptoms of mosaic were observed in lettuce (Lactuca sativa) and in chard (Beta vulgaris subsp. vulgaris) fields, during the spring of 2017 in Sao Paulo State (Brazil). Symptomatic leaves were submitted to ELISA test, using a polyclonal potyvirus antiserum (Agdia, Inc), and all the plants were found positive. 
Total RNA was extracted, followed by RT-PCR reaction using universal primers (W-CIEN/PV1) designed to amplify part of the potyvirus capsid protein (CP) gene, and a fragment of the expected size ( $\sim 850 \mathrm{bp})$ was obtained. This fragment was sequenced for lettuce and chard samples, and both showed nucleotide identity of $96 \%$ with TuMV (GenBank: AB701725.1). Further, the complete sequence of the CP gene ( 1238 bp) was amplified using a specific primers pair (Fwd: TACCTACAAGCAATCTTTG and Rev: GGCAATCGAGATACTATCTC). These amplicons were purified and sequenced, confirming the presence of TuMV in the symptomatic lettuce and chard plants. Bayesian analysis grouped the TuMV isolates from lettuce and chard in the Brassica-Raphanus-[BR] clade, which includes isolates infecting Brassicae and Raphanus species. The TuMV isolate was successfully saptransmitted to Raphanus sativus and Eruca sativa and the infection was confirmed by RT-PCR. To our knowledge, this is the first occurrence of TuMV naturally infecting lettuce and chard plants in Brazil.

Thielaviopsis sp. as the causal agent of Black Rot of Ilex paraguariensis in Argentina

M. RYBAK (1), R. D. French-Monar (2), R. Rybak (1), P. Sansberro (3), E. Garcia (4), (1) Instituto Nacional de Tecnología Agropecuaria, Cerro Azul, ARGENTINA; (2) USDA-APHIS, Plant Germplasm Quarantine Program, Beltsville, MD, USA; (3) Facultad de Ciencias Agrarias (UNNE) Instituto de Botánica del Nordeste (IBONE-CONICET), Corrientes, ARGENTINA; (4) Establecimiento Las Marias, Gobernador Virasoro, ARGENTINA

In 2014, high plant mortality was observed in the main commercial nursery of yerba mate, Ilex paraguariensis (Hill), located northwest of the Corrientes province of Argentina. Symptoms were observed as damaged, necrotic juvenile roots and a conspicuous dieback of twigs of younger plants, which later died by the thousands. In 2017, the same symptoms were observed on adult trees in which, in addition to the branches dieback, mature leaves showed dark black apical necrosis. Microscopically, a very thin, semi-transparent mycelium was observed. The septate hyphae were hyaline at first and difficult to observe. Two types of conidia, endospores and chlamydospores, were observed. Chlamydospores were produced in chains, two to eight spores per chain which separated at maturity and measured 25-60 X 8-11 $\mu \mathrm{m}$. Endoconidia were more abundant than chlamydospores. Endoconidia had rounded ends, varied in size, but were smaller than chlamydospores. Based on morphological characteristics and morphometrics, the fungus was identified as Thielaviopsis sp. To obtain pure cultures, chlamydospores were germinated on PDA. Pathogenicity tests conducted on young healthy plants produced the same symptoms as in the field. The same fungus was reisolated from diseased tissue, thus fulfilling Koch's postulates. Molecular characterization will assist in identifying this pathogen to the species level.

Investigation of new soil born pathogen on soybean (Glycine max) in Tennessee

R. GUYER (1), S. Pate (1), T. G. Garcia (2), V. P. Doyle (2), P. Price (3), H. M. Kelly (1), (1) University of Tennessee, Jackson, TN, USA; (2) Louisiana State University, Baton Rouge, LA, USA; (3) LSU AgCenter, Winnsboro, LA, USA

In August 2017, foliar chlorosis and root necrosis symptoms were observed in a soybean (Glycine max) production field in Hardeman County, Tennessee. Diseased plants (13) were collected from the field, from which 7 unidentified fungal colonies were isolated on potato dextrose agar amended with chloramphenicol and streptomycin sulfate (PDA-CS). Two of the isolates recovered were confirmed using morphological and molecular phylogenetic methods to be closely related to the Xylaria sp. that is known to cause taproot decline. Koch's postulates were performed for both isolates in a greenhouse trial using the soybean variety Asgrow 4632. Inoculum was grown on sterilized soybean stems for one month prior to inoculation. Three treatment groups (non-treated check, sterilized soybean stems, and inoculated soybean stems) were included with four replicates each. Plants were watered every 1-2 days and grown under supplemental lighting with a 16-hr light:8-hr dark cycle with temperatures ranging from $25-35^{\circ} \mathrm{C}$. Foliar symptoms were observed in inoculated plants after 3-4 weeks, and the pathogen was re-isolated from infected roots. The experiment was repeated twice. Taproot decline is an emerging disease, previously reported in Alabama, Mississippi, Louisiana, and Missouri. This is the first report of taproot decline in Tennessee, a disease that has been experimentally shown to be economically significant to soybeans, one of the most important crops produced in Tennessee.

\section{Dissecting a centennial problem for the peony industry}

C. SHAFFER (1), D. Michener (2), N. B. Vlasava (3), I. E. Tzanetakis (1), (1) University of Arkansas, Fayetteville, AR, USA; (2) University of Michigan, Ann Arbor, MI, USA; (3) Central Botanical Gardens NAS, Minsk, BELARUS

Peony, Paeonia lactiflora, Pall. is a perennial ornamental grown around the globe. Lemoine described a disease in the early 1900s with symptoms that include root galls, reduced flowering, and stunted growth with the causal agent remaining elusive. Large scale sequencing was employed to determine whether a virus is associated with the symptoms. About $90 \%$ of 200 accessions from Alaska, Arkansas, Michigan, New York and Oregon tested positive for Cycas necrotic stunt virus (CNSV), a nepovirus that has not been previously reported in the Western Hemisphere but has a significant host range. All isolates sequenced revealed a recombination event close to the 5' terminus of RNA 1 with a Grapevine Anatolian ringspot virus-like virus. The prevalence of the recombinant indicates that it occurred early in the evolutionary history of the virus in peony or it was introduced to peony early in its use as an ornamental. The rest of the virus is $85 \%$ and $90 \%$ identical nucleotide/amino acid respectively to the type isolate of the virus from Japan. The ability of Longidorus and Xiphinema to vector the virus is under investigation as is the host range among ornamentals routinely propagated alongside peony in nursery settings. With the information generated from this project management strategies can be devised to control the virus in the future.

\section{Leaf lesions and fruit warts on pumpkin are caused by Pseudomonas syringae sensu stricto}

L. TYMON (1), K. Bophela (2), S. J. Martins (3), L. Ramos-Sepulveda (4), D. A. Inglis (1), C. T. Bull (5), (1) Washington State University-Mount Vernon NWREC, Mount Vernon, WA, USA; (2) University of Pretoria, Pretoria, SOUTH AFRICA; (3) Pennsylvania State University, University Park, PA, USA; (4) Millersville University, Millersville, PA, USA; (5) The Pennsylvania State University, University Park, PA, USA

In 2015 and 2016, Washington field grown 'Cinnamon Girl' pumpkin leaves and fruit exhibited lesions surrounded by a yellow halo, and wart-like eruptions, respectively. Symptoms were reminiscent of bacterial infections, but atypical of those caused by Pseudomonas syringae pv. lachrymans, the angular leaf spot pathogen in genomospecies 2, phylogroup 1. Pathogenicity of PsAs_2015 isolated from foliage and PsFt1_2015 and PsFt2_2015 from fruit was confirmed on pumpkin seedlings, and the LOPAT profile corresponded to $P$. syringae. A multilocus sequence analysis with $P$. syringae pathotypes, demonstrated that PsAs_2015, PsFt1_2015 and PsFt2-2015 are genetically identical and members of genomospecies 1 phylogroup 2B, thus not related to $P$. syringae pv. lachrymans. Of the genomospecies 1 pathotypes and PsAs_2015, only PsAs_2015, and P. syringae pv. aptata were pathogenic on pumpkin leaves, although $P$. syringae pv. aptata lesions were distinct from those of PsAs_2015. PsAs_2015 did not cause symptoms on beet seedlings whereas $P$. syringae pv. aptata did. 'Baby Boo' pumpkins were either seed, flower, or fruit inoculated with PsAs_2015, or non-inoculated to assess how inoculation timing affects symptoms on fruit. Wart incidence on fruit was significantly correlated with fruit inoculation $(P \leq 0.001)$ as was recovery of PsAs_2015 from symptomatic rinds $(P \leq 0.001)$. Warts on fruit represents a newly observed symptom, likely not the result of systemic infection. 
About Plant Health: Developing new strategies for research communication and public engagement

A. MASINO, T. Llera Duran, A. Garibaldi, M. L. Gullino, Agroinnova - University of Torino, Grugliasco, Torino, ITALY

One of the objectives of the European research programmes is to increase awareness of research and innovation activities, supporting the public recognition of researchers. Of course, communication must combine sciences with entertainment, especially when addressing young audience. In the last years, Agroinnova organized many activities concerning communication and dissemination of research results: each with different and specific format. Among others, teaching in local schools and educational visits (Kids and High Schools to College), summer and winter schools, hands-on experiments and science shows (European Researchers Night), workshops and conferences (Piedmont Research Agriculture Innovation, About Plant Health, Sustainable Development Strategies, Designing the Circular Economy, Open Days), artistic performance (EMPHASIS for the Environment, RESPIRO), etc. Agroinnova focused on the following goals: increasing awareness among the public of the importance of research and the role of plant health in environmental protection; linking the kids experiences to discuss topics and improve key messages; better understanding of the key benefits that research brings to society; lowering the stereotypes about researchers and their profession. Through new formats and technologies, plant pathologists can communicate the results of research and learn more about stakeholders' opinions on how protect the EU agrifood sector from alien and native pests and pathogens.

Plant Pathology on stage. Telling more about science innovation in Horizon 2020 European projects A. MASINO (1), L. Vallarino Gancia (2), I. Ferri (3), B. M. Ferraro (3), A. Bertin (4), M. L. Gullino (1), (1) Agroinnova - University of Torino, Grugliasco, Torino, ITALY; (2) Teatro Stabile di Torino - Teatro Nazionale, Torino, ITALY; (3) Tangram Teatro, Torino, ITALY; (4) Spin-To srl, Torino, ITALY

Dissemination and communication are relevant for European research projects. The overall goal of the Project "Effective Management of Pest and Harmful Alien Species - Integrated Solutions" (EMPHASIS) is to protect European agriculture and forestry systems from native and alien pest's threats. It is a multi-actor approach project focusing research activities on the needs of the stakeholders. With this purpose, EMPHASIS is looking for new ways of engaging with civil society and policy makers. Theatre is considered a place to go in order to get points of reflection and thoughts and show ensures the effective and efficient spread of the scientific results to a wide public. During the European Green Week 2016 Agroinnova brought some renowned experts on the stage of the most impressive theatre of Torino, the Carignano Theatre, to entertain over 400 spectators about the role of plant health on environment protection. In 2017, Agroinnova co-produced RESPIRO, a more show-experience focused on One Health and based on The Diary of Adam and Eve by Mark Twain. The show went on the stage of Carignano Theatre on June 5th (World Environmental Day) and as soon replied, in the frame of an important summer festival of Bardonecchia. Artists performed very well, supported by important national and local newspapers, to sensitize the audience with key messages about the role of plant health for environment and landscape protection.

INRA's flagship program to foster development of solutions for Sustainable Management of Crop Health C. Lannou (1), S. Colleu (2), C. MORRIS (3), (1) INRA, Sophie-Antipolis cedex, FRANCE; (2) INRA, Paris, FRANCE; (3) INRA, Montfavet, FRANCE

Solutions for managing crop health that can be implemented by farmers arise from the interplay of multiple scientific disciplines with complex socioeconomic contexts. To surpass the contributions that disciplinary research provides for crop health, in 2011 France's National Institute for Agricultural Research (INRA) created the flagship program "Sustainable Management of Crop Health" (SMaCH) (http://www.smach.inra.fr/en) as part of 8 programs to address trans-disciplinary societal challenges. SMaCH has leveraged the expertise and motivation of INRA's scientists to target research results that, in particular, will help farmers meet European Union Directive 2009/128/EC that promotes IPM and the ban of about $75 \%$ of currently used active compounds. Since its inception, SMaCH has funded 10's of research projects, networks, workshops, and scholarships (shown in the poster) to 1) encourage, optimize and manage innovations in crop health management, 2) avoid risks to crop health through surveillance and through resilient and robust agrosystems, 3) manage the biodiversity of agrosystems at regional levels, and 4) describe the strategies of stewards and stakeholders of production and marketing networks. At ICPP-2018 SMaCH will promote their research model and strengthen the international dimension of the crop health management strategies they develop. Part of their work will be presented in the concurrent session "Innovative Pest Control Technologies for Smallholder Farmers".

\section{Food Security: The first decade of publication}

R. N. STRANGE (1), P. R. Scott (2), (1) University College London, London, UNITED KINGDOM; (2) ISPP, Oxford, UNITED KINGDOM

The journal, Food Security: the science, sociology and economics of food production and access to food, was inspired by the late Norman Borlaug. He challenged ISPP in 1998 to do something about food security. Realizing that food security articles were spread widely in the literature, we proposed, with the support of a range of distinguished professionals, a new peer-reviewed journal with the broad coverage indicated by its title, including but extending far beyond plant pathology. With Springer as publisher, a quarterly journal of about 300 pages per annum was envisaged, but it rapidly expanded to 6 issues per annum with over 1200 pages for each of the 3 years 2015-2017, and a 2017 impact factor of 2.271. The multidisciplinary journal has four Senior Editors overseeing the broad areas of the physical, biological, nutritional, sociological, economic and political environments, reporting to the Editor in Chief (Richard Strange) and supported by some 40 Associate Editors. We intend that the journal should make its particular contribution to the challenge of why 800 million people are without enough calories and why 2 billion are suffering from hidden hunger (micronutrient and vitamin deficiency) in a still rising population, many of whom are marginalized by global society and live in abject poverty. How should this challenge, in which plant diseases play a significant role, be addressed? Some novel and unconventional approaches may be needed.

Training the next generation: Incorporating student-designed experiments and plant pathology into an undergraduate microbiology classroom A. R. DUNN, New York State Integrated Pest Management Program, Cornell University, Geneva, NY, USA

Designing, conducting, and troubleshooting their own experiments enhances experiential learning and the development of critical thinking skills for undergraduate students, and can make a laboratory course more engaging for both teachers and students. Microbiology students in a 200-level course were given papers describing simple experiments, then asked to identify their own research question and propose a testable hypothesis, refine their question through a literature search, write a protocol, and conduct their experiment. After completing the experiment, students presented their results orally to their classmates and in a written report. Lab activities early in the semester taught basic skills in culturing microorganisms and required students to participate in protocol development. Plant pathogens were included among the available microorganisms because of their real-world relevance and safety (compared to human pathogens), and to expose students to a broader range of career trajectories. Through this process, students asked biological questions, tested hypotheses, and practiced problem-solving. They also analyzed and drew conclusions from their data and developed skills in scientific 
communication. Students enjoyed having ownership of their experiments, and some were very creative. Challenges (and suggestions for overcoming them) of incorporating student-designed experiments into an undergraduate laboratory will also be discussed.

Preparing North Dakota growers for soybean diseases: In-depth training for educators

J. HALVORSON (1), K. Nichols (2), G. L. Tylka (3), D. K. Malvick (4), S. G. Markell (1), (1) North Dakota State University, Fargo, ND, USA; (2) North Dakota Soybean Council, Fargo, ND, USA; (3) Iowa State University, Ames, IA, USA; (4) University of Minnesota, St. Paul, MN, USA

North Dakota soybean acreage has expanded from less than one million acres to over six million acres in the last two decades. Soybean cyst nematode (Heterodera glycines; SCN) was identified in 2003 and by 2009 was found in three counties. Sudden death syndrome (Fusarium virguliforme; SDS), although not yet confirmed in the state, occurs in close proximity in the bordering states of South Dakota and Minnesota. With little to no experience identifying or managing these two important diseases, North Dakota growers were left vulnerable to yield loss. To prepare for the expansion of soybean diseases in North Dakota, the North Dakota Extension service and North Dakota Soybean Council worked with experts from other states to develop in depth field trips/short courses to proactively educate 'critical masses' of agricultural specialists who serve as on-the-ground resources for growers. To monitor learning, pre- and post-tests were administered that asked participants several questions to rate their knowledge level regarding SCN and SDS and to rate the overall experience. In all instances, feedback showed a significant increase in knowledge with overwhelmingly positive comments about all events. As of 2016, SCN has been identified in 19 counties and SDS has not been confirmed. The benefit of having a 'critical mass' of agricultural specialists who can identify and manage these diseases will have a lasting impact on North Dakota's agriculture.

\section{Engaging Undergraduate Honors Students in Plant Pathology}

K. GWINN (1), C. Stripling (2), J. Donaldson (2), C. Stephens (3), (1) University of Tennessee, Department of Entomology and Plant Pathology, Knoxville, TN, USA; (2) University of Tennessee, Agricultural Leadership, Education and Communications, Knoxville, TN, USA; (3) University of Tennessee, Agricultural Leadership Education and Communications, Knoxville, TN, USA

Identification of talented undergraduate students interested in plant pathology as a career is a critical need. Most public institutions in the United States have established honors programs, and honors students are selected based on predicted ability to perform at levels above other students in the classroom and in beyond-the-classroom experiences such as undergraduate research. However, this pool of talented students is often not connected with plant pathology. Programming that fosters academic and social development of academically elite students and provides a gateway to our discipline has been developed at the University of Tennessee. This was accomplished through development of curriculum for a minor in Honors Food, Agricultural, Natural Resources, and Human Sciences. Core classes for the program include freshman seminar, a research ethics, compliance, and methods course, and a 2semester capstone course sequence culminating in an undergraduate thesis/project. In addition, students are required to take three additional honors courses in their major or minor. A minor in Entomology and Plant Pathology (EPP) is offered. Materials to recruit students to honors and EPP minors are being developed. Resources are being created for faculty on how to assess higher-order thinking skills. Faculty in EPP are encouraged to recruit honors undergraduate research assistants, provide opportunities for their thesis research, and develop embedded honors courses in the EPP minor.

\section{Pathogen hunters: Non-scientist engagement in plant disease research}

J. M. HULBERT (1), T. I. Burgess (2), F. Roets (3), M. J. Wingfield (4), (1) Forestry and Agricultural Biotechnology Institute, University of Pretoria, Stellenbosch, SOUTH AFRICA; (2) Centre of Excellence for Climate Change, Woodland and Forest Health, Murdoch University, Perth, AUSTRALIA; (3) Stellenbosch University, Stellenbosch, SOUTH AFRICA; (4) Department of Genetics, Forestry and Agricultural Biotechnology Institute (FABI), Pretoria, SOUTH AFRICA

Cape Citizen Science (http://citsci.co.za) is a plant disease awareness initiative in South Africa. The pilot study was designed to reveal the diversity and distribution of Phytophthora species in the Cape Floral Kingdom. The project has engaged many non-scientists as pathogen hunters and samples have been submitted from several plant producers, botanical gardens, nature reserves, and national parks. Citizen engagement activities have revealed the presence of multiple species of Phytophthora previously undocumented in South Africa and they have facilitated the discovery of at least one novel species. The most recent phase of the project invited citizens to participate in The Cape Town Hypothesis Test, a study designed to promote the early detection of Phytophthora species introductions in urban areas of Cape Town. This pilot study has pioneered the application of several methods of public engagement in plant disease research. Citizens have submitted samples from their home gardens, invited researchers to collect samples on private property, participated in hikes for science and training workshops and they have reported dying plants online. The project has also revealed an abundance of opportunities to involve non-scientists in important microbiological research. Based on our experiences, we recommend establishing similar initiatives to Cape Citizen Science in other parts of the world.

The Cape Town Hypothesis Test: Phytophthora species in urban vs natural areas

J. M. HULBERT (1), T. I. Burgess (2), F. Roets (3), M. J. Wingfield (4), (1) Forestry and Agricultural Biotechnology Institute, University of Pretoria, Stellenbosch, SOUTH AFRICA; (2) Centre of Excellence for Climate Change, Woodland and Forest Health, Murdoch University, Perth, AUSTRALIA; (3) Stellenbosch University, Stellenbosch, SOUTH AFRICA; (4) Department of Genetics, Forestry and Agricultural Biotechnology Institute (FABI), Pretoria, SOUTH AFRICA

The Cape Town Hypothesis Test is the third phase of the pilot study for Cape Citizen Science (http://citsci.co.za/), a project to engage non-scientists as pathogen hunters. This phase was initiated because urban environments provide opportunities for the early detection of Phytophthora invasions. The hypothesis is that the Phytophthora species in the urban areas of Cape Town are different to those in the less disturbed Table Mountain National Park. Citizens were invited to contribute to the hypothesis test by submitting samples from their backyard as well as a few targeted areas in the urban areas. This is the first citizen-driven microbiology based hypothesis test in South Africa. Cape Citizen Science has already demonstrated the efficacy of engaging non-scientists in microbiological research, but the primary aim of this phase of the project was to demonstrate that citizens can contribute to testing hypotheses. In addition, by empowering citizens as biodiversity "protectors", this phase will test the potential to mobilize the public to detect new invasions early.

Laboratory and computer skills for employment-ready students in plant sciences B. SPAKES RICHTER (1), A. M. Poleatewich (2), M. Hayslett (3), K. Stofer (1), (1) University of Florida, Gainesville, FL, USA; (2) University of New Hampshire, Durham, NH, USA; (3) Iowa State University, Ames, IA, USA

Undergraduate introductory plant pathology courses provide the first and often only opportunity most students have to learn critical content and skills in plant disease recognition and management. Many of these students matriculate to entry-level employment in a plant science field, where employers 
expect that their BS degree denotes foundational preparation for job requirements. Yet there has been little communication between employers and course instructors to guide course development, and many of the skills desired by employers are not emphasized in introductory courses or undergraduate curricula. As part of a larger survey to examine gaps between employer expectations and introductory course content, we asked managers of entry-level employees what skills they sought. We report here on specific software and laboratory equipment competencies desired by employers, and in an interactive poster element, provide viewers with opportunity to expand on the initial data set. Our original survey participants indicated that graduates should be adept with Microsoft Office and statistical software. Microscopes ranked first among desired lab equipment competencies, followed in decreasing order by autoclaves, laminar flow/fume hoods, balances, centrifuges, pipettes, thermal cyclers, and pH meters. With knowledge of specific proficiencies desired by our students' future employers, educators in plant pathology can adapt courses to improve employment readiness among our graduates.

The use of direct and indirect methods in seed health testing

G. HIDDINK (1), T. Baldwin (2), L. de Leon Guerra (3), M. Spiekerman (4), C. Hogan (5), (1) Enza Zaden Seed Operations B.V., Enkhuizen, NETHERLANDS; (2) BioGEVES, Beaucouzé cedex, FRANCE; (3) ANOVE, Madrid, SPAIN; (4) Rijk Zwaan Production B.V., De Lier, NETHERLANDS; (5) Sakata Seed America Inc, Salinas, CA, USA

The use of disease-free seeds is an effective measure to prevent the introduction and spread of seedborne pathogens into new production areas. Plant protection authorities and the seed industry pursue the same goal: the free movement of healthy seed lots. Seed health tests should be able to detect the target seedborne pathogen at an appropriate threshold, defined as the amount of infection that could potentially lead to disease outbreaks. The viability and pathogenicity of target organisms are determined by tests such as bioassays, grow outs or pathogen isolation by classical microbiological methods followed by pathogenicity assays, otherwise known as "direct" tests. More recently, "indirect" tests based on PCR and sequencing (NGS) have been developed as pre-screening methods. When pre-screen and direct methods are combined they can improve the speed and effectiveness of seed health tests. Defining the limit of detection and selectivity of a pre-screen test is crucial since its purpose is to detect seed lots that are not infected with the target pathogen(s). Consequently, any positive result with a pre-screen test should be classified as a "suspect" that needs further investigation and verification with a test that confirms the presence of a viable and pathogenic organism. The International Seed Health Initiative for Vegetable Crops considers the development of seed health tests that combine indirect and direct test methods as a necessity to meet Koch's postulates.

\section{Using a Cross-Curricular Approach in a Molecular Diagnostics Course to Reach a Broader Student Audience} M. E. MCKELLAR, J. R. Thompson, Cornell University, Ithaca, NY, USA

A new laboratory course, "Molecular Diagnostics: From Lab to Viñedo," was developed to offer a theoretical and practical introduction to the molecular diagnostic methods employed by researchers in human and veterinary medicine and plant sciences today. Over seven weeks in the fall semester, students practiced molecular diagnostic techniques in an active learning environment. Students then applied their new skills to diagnose grapevine diseases over two weeks during winter break in the Chilean Central Valley. The course utilized a cross-curricular approach to include foreign language and cultural studies. Concurrent enrollment in an appropriate level Spanish language course was required. The fall semester culminated in a cultural festival where students presented lightning talks about Chile, met Chileans from the local community and participated in Chilean cultural activities. Ninety-five percent of the students enrolled in the course majored in biology with a focus on pre-med or pre-vet studies. In addition to providing an international learning experience, the course increased student awareness of careers and research in plant and agricultural sciences by using plant diseases as a model of field study. The multi-disciplinary content of this course could serve as a novel approach for increasing the diversity of non-major students that participate in plant pathology courses.

Know thy enemy: Culturing Candidatus Liberibacter asiaticus is critical to developing new therapies for Huanglongbing O. J. ALABI (1), P. D. Roberts (2), G. Vidalakis (3), (1) Dept. of Plant Pathology \& Microbiology, Texas A\&M University, Weslaco, TX, USA; (2) University of Florida, Immokalee, FL, USA; (3) University of California, Riverside, Riverside, CA, USA

Huanglongbing (HLB) is a destructive disease of citrus. HLB is consistently associated with infection of phloem-inhabiting Candidatus Liberibacter bacterial species, the predominant species being $\mathrm{Ca}$. Liberibacter asiaticus (CLas). However, the inability to obtain CLas in axenic culture has made it impossible to prove Koch's postulate for HLB. In addition, efforts to develop/test therapeutic compounds and evaluation of potential sources of HLB resistance are largely impaired by the inability to culture CLas. To address this key knowledge gap, our project team was funded by a grant from the USDA-NIFA-CDRE entitled "Developing new therapies for Huanglongbing via culturing Ca. Liberibacter asiaticus." The team led by Dr. Dean Gabriel, University of Florida, Gainesville, FL includes 11 co-project directors drawn from seven institutions across the major US citrus producing states of Florida, California and Texas, as well as Colombia, South America. The key objectives of the project are: 1) Concerted, parallel and coordinated efforts towards obtaining viable CLas cultures; 2) Define the influence of physical environment in CLas culturability; 3) Define the role of chemical signaling and co-factors in culturability of CLas; 4) Develop genetic tools that enable delivery of (missing) candidate growth factor genes identified and partially characterized into CLas; and 5) Extending research outcomes to benefit growers and the scientific community.

Plant Pathology in Australia - a brief history

T. Cooke, D. PERSLEY, Department of Agriculture and Fisheries, Brisbane, AUSTRALIA

The history of plant pathology in Australia has been influenced by its geographic isolation, and the establishment of introduced pathogens. Stan Fish (1970) and Neville White (1981) chronicled the early history up until the era of rapid technological advances. They described the pioneering role of plant pathologists whose knowledge underpins current research. European colonisation began with the arrival of the first fleet in 1788. At this time the land was already occupied by Australian Aboriginal people who had lived there for some 50,000 years. They were hunter gatherers and managed the country sustainably so that resources were replenished. Food sustainability became a major issue for European settlers and farms soon developed around their settlements. They grew mainly wheat and raised sheep. The earliest plant pathology investigations began because of wheat rust. William James Farrer (1845-1906) bred rust resistant wheat, and rust research was championed by Daniel Mc Alpine (1849-1932) the "father of plant pathology in Australia". As agriculture increased and diversified, plant disease problems became more prevalent and serious. As well, some pathogens moved from native plants onto introduced hosts. Plant pathologists were soon appointed in State Departments of Agriculture and in the national quarantine service. In the 1920's chairs in plant pathology were established at major universities but the Australasian Plant Pathology Society was not formed until 1969.

Age Demographics and Trends in Graduate Recruitment in Plant Pathology in the US University System D. M. GADOURY, Cornell University, Geneva, NY, USA 
An untold success story of our profession is how challenges posed by future retirements and declining interest in graduate studies were resolved by strategic planning and actions at the societal level. Censuses of the US university system in 2007, 2011, and 2017 enumerated all plant pathology faculty and graduate students. The APS membership database was used to construct the faculty age distribution in 2007 compared to 2017 , and student members were surveyed and interviewed to identify factors that were decisive in selection of plant pathology as a career. Findings of these studies, including the identification of work experience and internships as a principal factor generating commitment to graduate study, were communicated annually to department heads and chairs. Promotion of practical experience to funding agencies and industry resulted in substantial support for internship and work experience programs. Program descriptions were aggregated on APSnet and edited for search engine optimization to raise visibility to online searches. There was a concomitant increase in the number plant pathology graduate students in the US university system from 694 in 2007, to 829 in 2011, and 975 in 2017. Faculty numbers remained unchanged from 2007-17, but the age distribution became markedly bimodal due to new hires. Age demographics, and consistent trends in faculty hiring and retirement indicate a need for graduate programs to operate near this level for the next 10 years.

Critical thinking skills as an integral part of training in plant pathology diagnostic laboratories

S. BEC (1), C. Lapaire Harmon (2), (1) University of Florida, Gainesville, FL, USA; (2) Univ of Florida PDC, Gainesville, FL, USA

The University of Florida Plant Diagnostic Center diagnoses upwards of 3000 diseased plant samples per year. Due to our geographic location and global outreach, we encounter a wide host plant range including temperate, tropical, and subtropical ornamental plants, fruit and vegetable crops, turf, palms and agronomic crops. Diseased plant samples received originate from different ecosystems including, residential properties, golf courses, commercial production greenhouses, orchards, and nurseries. To meet our clientele's needs we rely heavily on undergraduate student workers. Students come to us with vastly different educational backgrounds, knowledge, and skill levels. Most of the undergraduates step into the lab with limited experience and understanding of plant pathology and disease diagnostics. This creates a continuous need for teaching, practice, and use of the acquired skills. With our high sample diversity, we periodically encounter diseases new to the region. Completion of Koch's postulates for a novel disease creates an opportunity for development and sharpening of critical thinking skills, and understanding of botany, microbiology, plant pathology, experimental design, and molecular genetics. Here we present our systematic approach to developing skills crucial for plant disease diagnostics, illustrated by the studies of two bacterial diseases conducted by undergraduate students.

Keys to expanding online education beyond the campus: Ohio State's Master in Plant Health Management

M. M. LEWANDOWSKI (1), A. E. Dorrance (2), L. Canas (3), C. Welty (4), F. Peduto Hand PhD (1), D. Shetlar (5), P. A. Paul (6), A. Londo (7), M. L. Lewis Ivey (8), S. Williams (9), W. Klooster (5), (1) Ohio State Univ, Dept of Plant Pathology, Columbus, OH, USA; (2) The Ohio State University, Dept. of Plant Pathology, Wooster, OH, USA; (3) Ohio State Univ, Ohio Agricultural Research and Development Center, Dept of Entomology, Wooster, OH, USA; (4) Ohio State Univ, Ohio Agricultural Research and Development Center, Dept of Entomology, Columbus, OH, USA; (5) Ohio State Univ, Dept of Entomology, Columbus, OH, USA; (6) The Ohio State University, Wooster, OH, USA; (7) Ohio State Univ Extension, Columbus, OH, USA; (8) Ohio State University, Wooster, OH, USA; (9) Ohio State University, Columbus, OH, USA

Ohio State's Master in Plant Health Management (MPHM) program, administered by the Departments of Plant Pathology and Entomology, is a professional degree that was established to help meet a need for a robust and growing workforce with plant health expertise. The program is part of a trend in graduate education: professional and online degree programs tailored for working professionals. Established in 2012, the program currently has $25+$ mostly non-traditional, part-time and online students. As we evaluate the program's first five years, our ability to adapt to a variety of student interests, provide individualized student advising, expand online course offerings, and relentlessly pursue collaboration opportunities have been keys to the program's growth. Flexibility in the curriculum helps us fit various backgrounds, including students with majors outside of plant health. To provide hands-on experience, we have built collaborations to help students pursue projects in new areas such as precision agriculture, urban farming, and pollinator health. We have also worked with university course designers to use course templates to convert material to online formats. Improvements in video recording and course management systems have helped make online courses more interactive, accessible and attractive to students. Employing students to assist online instructors has been mutually beneficial. We continue to explore opportunities to help pursue career goals.

More "Ideal Plant Clinics" required in the Anthropocene

D. A. HERRON (1), W. De Beer (1), B. Slippers (2), M. J. Wingfield (2), (1) Forestry and Agricultural Biotechnology Institute, University of Pretoria, Pretoria, SOUTH AFRICA; (2) Forestry and Agricultural Biotechnology Institute (FABI), University of Pretoria, Pretoria, SOUTH AFRICA

Globally, plant clinics have played a significant role in limiting crop loss due to pests and pathogens. Even though new plant clinics are being established regularly, there remains a great paucity in capacity to tackle one of the greatest challenges of the Anthropocene: to provide more food and fibre for an escalating global human population on a shrinking land resource base. Plant clinics operate at many different levels but most are equipped to address some of the more immediate issues facing small-scale farmers who wish to know the cause of a problem on their crops. While these clinics are important, there is a need for more advanced clinics that not only answer such questions, but that also play a role in driving/informing research. The "Ideal Plant Clinic" (IPC) should be one that is set up to provide a wide variety of diagnostic services; staffed with well-trained professionals; have suitable infrastructure; draw scientific support from the research community and engage with the stakeholder community through extension. Our experience in establishing a clinic attached to a university, in a research institute, linked to industry, and focused on tree health, provides a model for what an IPC might be. The diagnostic clinic of the Tree Protection Co-operative Programme (TPCP) provides examples of how high-quality research together with pest and disease diagnostics have contributed to improving yields of the South African Forestry Industry over a 20 year period.

Using social media to reach a more diverse audience in Extension programming

A. S. WINDHAM (1), F. Hale (1), D. D. Hensley (2), (1) Soil, Plant, and Pest Center, Nashville, TN, USA; (2) University of Tennessee, Department of Entomology and Plant Pathology, Knoxville, TN, USA

Traditional outreach methods such as group meetings and field days reach a predominately male audience in horticultural commodity groups in Tennessee. A Facebook page was created for the UT Extension Soil, Plant and Pest Center (SPPC). Our goals were to: 1) create timely, illustrated posts of insects and plant diseases to alert growers and gardeners, and aid in identification, 2) inform our audience of educational opportunities, 3) reach an audience new to Extension and our diagnostic lab. Facebook analytics were examined monthly to identify our audience and their participation. In 2017, content from our posts reached over 1.2 million Facebook pages; over 35,000 unique Facebook users interacted or engaged with our page (likes, comments, questions). Users that engaged with our page were evenly distributed across age groups from $25-65+$ yrs. and primarily female (65\%). During the growing season, posts were shared daily by the SPPC staff. The most popular posts were original content accompanied by digital images. Posts were shared by Extension agents, horticultural businesses, master gardeners and gardeners maximized reach of posted content. Challenges of using social media include: time to create posts and interact with users, and documenting impact for reporting. 
Originally born as a response to the threat of Ug99, the Borlaug Global Rust Initiative (BGRI), for 10 years has brought together researchers and institutions around the world to fight the threat of wheat rust. But, global wheat production faces many challenges, including biotic and abiotic stresses beyond rust diseases. It is for this reason that in 2016 the Borlaug Global Rust Initiative (BGRI) started a new phase called Delivering Genetic Gain in Wheat, (DGGW). Within the DGGW, partner institutions will continue to collaborate and build on the successes of the BGRI. Increased efforts will be allocated to the implementation of cutting-edge technologies and methods such as genomic selection and high throughput phenotyping into the CIMMYT wheat breeding program, with the aim to use to shorten breeding cycles, improve genetic resistance, and ultimately develop and deploy varieties of wheat that incorporate climate resiliency and multiple disease resistance for smallholder farmers in politically vulnerable regions. With wheat facing so many challenges, the BGRI aims to engage the broader wheat community beyond the DGGW to foster collaboration among other research projects, including enabling data and knowledge exchange, implementing training sessions targeted to specific groups and creating and maintaining online learning resources. The poster will display progress towards milestones.

Big not always bad. Sustainable agriculture depends on farm

S. SWITEK (1), Z. Sawinska (2), P. Tryjanowski (3), R. Głowicka-Wołoszyn (4), (1) Institute of Zoology, Poznan University of Life Sciences, Poznan, POLAND; (2) Departament of Agronomy, Poznan University of Life Sciences, Poznan, POLAND; (3) Institute of Zoology, Poznan University of Life Sciences, Poznań, POLAND; (4) Department of Finance and Accounting, Poznan University of Life Sciences, Poznan, POLAND

Sustainable production in the European Union is promoted by numerous subsidies for farmers. Besides observing good agricultural practices such as proper pesticide use, farmers must adapt to greening rules since 2014. To receive payment they have to diversify crops, maintain permanent grassland and dedicate $5 \%$ of arable land to ecological focus area (EFA). Farms thus become an important element of environment conservation. The research was conducted in 321 polish farms. $43 \%$ of them had more than 100 ha, around $40 \%$ had between 30 and 100 ha, and $18 \%$ farms had less than 30 ha. Farmers declared their farms most attractive for birds, pollinating insects and game while less attractive for rodents, amphibians, and reptiles. Along with an increase in farm area, the attractiveness rating improved, which was caused by the presence of free-standing trees, field margins, ponds, and ditches. Only the attractiveness assessment for weeds and herbs didn't depend on the farm size. Farmers' survey allows better adjustment of agricultural policy and gives the opportunity to modify and create security activities that are becoming more and more important.

The past, present, and future of plant diagnostic networks in Haiti

J. FAYETTE (1), J. Fulton (1), W. Dantes (1), S. Loubeau (2), K. A. Garrett (1), (1) Plant Pathology Department, University of Florida, Gainesville, FL, USA; (2) AREA project / USAID Feed the Future Programe, Port au Prince, HAITI

Plant diagnostic networks are vital components of plant protection services, providing surveillance for emerging diseases and decision-support for farmers. In Haiti, the national disease reporting by the Ministry of Agriculture has decreased significantly over the last decades. To revitalize the national disease diagnosis reporting, this Ministry has been a key actor in building a plant diagnostic network supported by the AREA project (USAID Feed the Future program). The Bas-Boen plant disease clinic, which is part of a Centre Rural de Developpement Durable, was built by the WINNER project (USAID Feed the Future). It has been functional since 2015 and conducts regular plant disease surveys as well as receiving samples. The objective of our current analyses is to identify how the diagnostic network can best be sustainable after the period of initial support from the USAID program or any international donor, and adaptable to the Haitian context. The major steps for characterizing and implementing this network include: identification of diagnostics expertise across the country, the establishment of a functional plant disease clinic, investment in human resource capacity and lab equipment, the delivery of plant diagnostics workshops, and the creation of a database listing high-priority pests and pathogens of beans, banana, rice, corn, and mangoes. We present how this network supports and expands plant diagnostic services in Haiti.

Importance of seed as an inoculum source for High Plains Virus in sweet corn

C. NISCHWITZ, B. Olson, Utah State University, Logan, UT, USA

High plains virus (HPV) is found in small grains and corn and is commonly transmitted by the wheat curl mite. Seed transmission has been reported but was considered unimportant due to low percentage of infection. In 2016, symptomatic sweet corn was found in fields in Utah with symptoms ranging from chlorotic leaf streaks to stunting and reduced ear set. About four percent of the seedlings showed symptoms within two weeks of emergence in the field. None of the plants died. Yield loss was estimated at 50 percent for the field. Virus testing using ELISA showed that the plants were infected with HPV and confirmed with RT-PCR but no wheat curl mites were found on the plants and the infection pattern in the field was consistent with a seedborne disease. Leftover seed obtained from the grower was tested using ELISA, and 70 percent of the seed tested positive for HPV and was verified with RTPCR. In greenhouse grow out tests of the contaminated seed, three percent of the seedlings showed chlorotic streaks and stunting after three weeks. The results indicate that seed transmission of HPV can be important and result in high yield losses and that seed testing for HPV should be considered. Additional research is necessary to determine if corn variety or virus strain play a role in its significance.

Spatial and temporal heterogeneity in Rhizoctonia solani AG2-2IIIB inoculum density distribution in sugar beet fields M. ZELLNER, M. Nottensteiner, Bavarian State Research Centre for Agriculture, Freising, GERMANY

Rhizoctonia solani AG2-2IIIB, the soilborne fungus that causes Rhizoctonia Crown and Root Rot (RCRR), is responsible for considerable losses in sugar beet yield and quality. The RCRR incidence and severity as well as the location, shape and size of RCRR patches varies between seasons. Investigating the spatial and temporal distribution of the inoculum density (ID) would contribute to clarifying the underlying mechanisms. For a high spatial resolution analysis 49 composite soil samples were taken from a 1 ha grid on a sugar beet field in June 2017. For a large-scale spatial analysis a 15 ha site was probed with three composite samples each derived from an individual 1 ha grid in July 2017. For the temporal analysis soil samples were taken from artificially inoculated sugar beet research plots of a crop rotation field trial in June and September 2013, 2014 and 2015. Viable mycelium was isolated from soil samples by quinoa seed baiting. $R$. solani AG2-2IIIB was quantified by SYBR Green qPCR. The ID was unevenly distributed in the highly resolved 1 ha sampling area with zones of high, medium, low and zero ID. On the large scale two 1 ha sampling areas showed a high ID and one a low ID. The mean ID over all sugar beet research plots decreased threefold until June 2014 followed by a steady increase up to double the initial ID in September 2015. The data show that the ID varies greatly, both spatially and temporally which contributes to the dynamic nature of RCRR. 
Host-to-host transmission rate of Phytophthora ramorum is highest during a relatively short period in mid-winter in California W. SCHWEIGKOFLER, T. Pastalka, S. Sharma, K. G. Suslow, Dominican University of California, San Rafael, CA, USA

The invasive forest pathogen Phytophthora ramorum, causal agent of Sudden Oak Death, spreads via sporangia produced on leaves of woody host plants. We tested the short-distance vertical transmission of Phytophthora ramorum from bay laurel to rhododendrons during the winter $2016 / 17$. Healthy rhododendrons were placed under symptomatic bay laurels and monitored regularly for symptom appearance. Symptomatic rhododendrons were exchanged with healthy ones and exposure time recorded for each transmission event. Phytophthora ramorum was detected from rain water in the canopy of the Bay laurel from November 21 onwards with increasing concentrations. A total of 17 transmission events were detected from December 19, 2016 to February 10, 2017. The average exposure time was 14 to 25 days (N: 15; 88.2\%), but two transmission events (11.8\%) with very short exposure times (four days) were also detected. No transmission was detected after February 10, although favorable climate conditions (including higher than normal rainfall and mild temperatures) persisted for several more weeks, and Phytophthora ramorum propagules were detected regularly from rainwater until April. To our knowledge, this is the first field experiment to study the precise timing of Phytophthora ramorum transmission from a host plant to another. The results will help to better understand the epidemiology of this important plant disease and potentially improve CA forecast models.

Release and dispersal of ascospores of Stagonosporopsis citrulli from colonized watermelon debris

G. RENNBERGER, A. P. Keinath, Coastal Research and Education Center, Clemson University, Charleston, SC, USA

Gummy stem blight (GSB), caused by 3 species of Stagonosporopsis, is a serious disease of cucurbits worldwide. A watermelon field inoculated with $S$. citrulli in Sep 2016 was used as a spore source for the following experiments. Every 2 weeks from Jan to Jul 2017, vine sections from 4 randomly chosen spots in the field were collected, rehydrated, surface disinfested and attached to lids of petri dishes containing agar for 4 days to monitor spore release. Release of ascospores from pseudothecia on vine sections was observed until 19 Jun, almost 10 months after the field was inoculated, and decreased significantly over time in a linear fashion $\left(P<0.0001 ; R^{2}=0.58\right)$. From 25 Oct until 19 Jun seedlings of watermelon (Charleston Grey) and cucumber (Straight 8) were placed at distances of $0,15,31,61,122$ and $244 \mathrm{~m}$ from the center of the field to trap spores on 10 dates. After 48 hours of exposure, trap plants were moved to greenhouse chambers, incubated for 4 days at $>90 \% \mathrm{RH}$ and examined for symptoms of GSB. Symptoms on trap plants placed 0 to $122 \mathrm{~m}$ from the center were observed on both cucurbits. Disease incidence on trap plants decreased significantly with distance and over time $(P<$ 0.0001). Symptoms on trap plants were detected from 25 Oct until 19 Jun. The identity of isolates recovered from spore release plates and trap plants was confirmed as $S$. citrulli using a multiplex PCR. This study demonstrates the importance of ascospores in the dispersal of $S$. citrulli.

Oilseed rape crop debris and potential spread of Leptosphaeria maculans (phoma stem canker) into China

B. FITT (1), A. Qi (1), P. S. Wu (2), C. Rempel (3), R. Lange (4), Z. K. Punja (5), Y. Huang (1), (1) University of Hertfordshire, Hatfield, UNITED KINGDOM; (2) Chinese Academy of Inspection and Quarantine, Beijing, CHINA; (3) Canola Council of Canada, Winnipeg, MB, CANADA; (4) InnoTech Alberta, Vegreville, AB, CANADA; (5) Simon Fraser University, Burnaby, BC, CANADA

Quarantine regulations are designed to prevent establishment of pathogens in new countries. Phoma stem canker, which causes global losses in oilseed rape worth $>£ 1000 \mathrm{~m}$ per annum, is caused by Leptosphaeria maculans and the less damaging L. biglobosa. Chinese surveys provided evidence that only L. biglobosa is currently present in China but that L. maculans is present in crop debris associated with cargoes of oilseed rape seed imported into China. Following our work, which applied models of the spread of $L$. maculans across Canada to assess potential spread across China, in November 2009 the Chinese quarantine agency imposed restrictions on the import of oilseed rape seed into China. Canadian exports to China decreased in 2010 but then returned to previous levels when it was agreed that they could enter China through ports in regions where oilseed rape is not grown. A debate about the amount of crop debris (dockage) permitted in seed cargoes was resolved until 2020, when a memorandum of understanding was signed between China and Canada during a visit by Chinese prime minister Keqiang Li to Canada in 2016. Current modelling work will assess the risks of spread of Leptosphaeria maculans associated with different amounts of debris in cargoes. This work will establish principles that can be applied to help restrict the spread of pathogens to new countries in seed cargoes.

A computer model to simulate the dynamics of mummy berry disease transmission in wild blueberry production H. Qu (1), F. Drummond (2), S. L. ANNIS (2), (1) Chongqing University of Posts and Telecommunications, Chongqing, CHINA; (2) University of Maine, Orono, ME, USA

Monilinia vaccinii-corymbosi (MVC), causing mummy berry, can cause significant crop losses in North American blueberries. Disease levels are affected by interactions among factors, such as inoculum level, variation in blueberry genotypes, pathogen vectors (pollinators), and weather conditions. The goal of this study was to develop a computer simulation model of the infection, spread and effects on the host of MVC in wild blueberry that is dynamic spatially and temporaly, and provides a tool for evaluating changes in abiotic and biotic factors and their impact on disease. Published and field data have been used to estimate the parameters affecting pseudosclerotia germination, ascospore dispersal, primary infection, conidia production, conidia transmission by bees, and secondary fruit infection. The information is included in a GAMA computer simulation model that simulates mummy berry transmission dynamics by computing the effects of plant, fungus and pollinating insects (vectors of conidia), and weather and landscape factors that drive spore dispersal, infection, pollination, and resulting disease loss and yield. The simulated outcomes have been validated with field collected data. The model can be used to evaluate the effects of different scenarios, such as field size, pollinators, and control methods on resulting disease levels. This model can be modified for use in other blueberry cropping systems to determine the effects of inputs on mummy berry severity.

Dispersal of Colletotrichum gloeosporioides by citrus pollen

M. C. de Godoy Gasparoto (1), A. B. Gama (2), S. DE AFONSECA LOURENÇO (2), G. J. Silva Junior (3), L. Amorim (2), (1) UNESP, Registro, BRAZIL; (2) USP, Piracicaba, BRAZIL; (3) FUNDECITRUS, Araraquara, BRAZIL

Colletotrichum acutatum and C. gloeosporioides are the causal agents of Citrus Postbloom Fruit Drop (PFD). Previous experiments showed that citrus pollen can spread C. acutatum. However, no information concerning C. gloeosporioides is available. The hypothesis is that citrus pollen carried by honeybees can spread the fungus from diseased to healthy citrus flowers. The experiment was performed 3 times in controlled conditions. Flowers from potted sweet orange plants were inoculated with a suspension of $1.25 \times 10^{5}$ conidia.ml ${ }^{-1}$ of $C$. gloeosporioides. The plants were kept for 24 hours under moist chamber at $24^{\circ} \mathrm{C}$. Anthers were transferred from inoculated and non-inoculated flowers to healthy flowers. PFD symptoms were evaluated for 3 days. Symptomatic flowers were collected for pathogen isolation on Potato-Dextrose-Agar medium $\left(25^{\circ} \mathrm{C}, 12 \mathrm{~h} / 12 \mathrm{~h}\right.$ for 7 days $)$. DNA was extracted from all isolates and PCR assays confirmed the fungal complex species with the specific primers: CgInt/ITS4. All flowers that received anthers inoculated with C. gloeosporioides showed PFD symptoms. All isolates belonged to the C. gloeosporioides complex. None of the flowers that received non- 
inoculated anthers exhibited PFD symptoms. Images of scanning electron microscopy showed citrus pollen surrounded by conidia of $C$. gloeosporioides on petals 48 hours after the anther transference. This study confirmed that citrus pollen is able to disperse $C$. gloeosporioides.

\section{Dispersal route of Puccinia striiformis f. sp. tritici in China}

X. HU (1), C. Wang (2), B. T. Wang (2), Z. Kang (3), (1) Northwest A\&F University, Yangling, CHINA; (2) Northwest A\&F University, YANGLING, CHINA; (3) Northwest A\&F University, Yangling, Shaanxi, CHINA

Wheat stripe rust, caused by Puccinia striiformis f. sp. tritici (Pst), is an important disease on wheat, seriously threatening wheat production worldwide. China is one of the largest stripe rust epidemic regions in the world. The pathogen sexual reproduction and migration routes between Tibet and the other regions in China are still unknown. In this study, we obtained 961 Pst isolates from 1391 wheat leaf samples from Gansu (277), Shaanxi (253), Sichuan (172), and Tibet (259), composing 13 natural populations, and genotyped them with simple sequence repeat (SSR) markers. The isolates can be divided into two distinct clusters based on DAPC and STRUCTURE analyses. The genetic diversity of Longnan (in Gansu) and Yibin (in Sichuan) populations was the highest and lowest among the 13 populations, respectively. The hypothesis of multilocus linkage disequilibrium was rejected for the populations from Linzhi in the Himalayan, Longnan, Hanzhong, Guangyuan, Mianyang, Liangshan, and Chendu in the South of Qinling Mountains at the level of $P$ $=0.01$, which indicated significant linkage among markers in these populations. Populations in the other regions had extensive gene exchange $\left(N_{m}>4\right)$; little gene exchange was found between Tibet and the other regions $\left(N_{m}<1\right)$. The results suggest that the Tibet epidemic region of Pst is highly differentiated from the other epidemic regions in China.

Epidemiology of spinach downy mildew, including insights on oospore production and global transport on seed S. Kandel (1), B. Mou (1), S. G. Kunjeti (2), K. V. SUBBARAO (3), S. Klosterman (1), (1) USDA-ARS, Crop Improvement and Protection Research, Salinas, CA, USA; (2) Monsanto Company, Bangalore, INDIA; (3) University of California at Davis, c/o U.S. Agricultural Research Station, Salinas, CA, USA

Peronospora effusa is an oomycete pathogen, and the cause of spinach downy mildew. The recent demonstration of viable oospores of $P$. effusa in modern seed lots has raised concerns about the global transport of the pathogen, especially to organic production areas, where control of downy mildew with fungicides is not an option and to areas newly brought under spinach cultivation. The detection of oospores in $>19 \%$ of all seed lots tested, and in the infected leaves from spinach field plots, shows the widespread occurrence of oospores. Moreover, we recently observed the germination of the oospores obtained from spinach leaves, indicating that the oospores may serve as primary inoculum to introduce the pathogen in a growing season. To the best of our knowledge, this is only the second such observation of oospores germination during the last 100 years. Sexual reproduction of $P$. effusa, as in other oomycete systems, rapidly increases genetic diversity of the pathogen, leading to the emergence of new pathotypes. The appearance of new pathotypes makes the task of breeding resistance for this disease difficult. Seed treatment is the most economical option for reducing viable oospore levels in spinach seed lots, as opposed to discarding of infested seed lots or switching them from organic to conventional production. Current research is focused on use of seed treatments to reduce oospore viability, and on elucidation of the potential role of soil-and seed-borne oospores in initiating primary infections on spinach.

Assessment of spore presence for Cercospora beticola as demonstrated by sentinel beets (Beta vulgaris)

L. E. HANSON (1), D. M. Bublitz (2), L. Campbell (3), J. M. McGrath (1), (1) USDA ARS, East Lansing, MI, USA; (2) Michigan State University, East Lansing, MI, USA; (3) USDA Northern Crop Science Lab, Fargo, ND, USA

Cercospora beticola, the causal agent of Cercospora leaf spot (CLS) in Beta vulgaris (sugar, table, and leaf beet), is an important pathogen globally. Disease forecasting models are widely used to aid in CLS management for sugar beet. Most models rely on weather data to predict infection periods but do not include pathogen presence or conditions for spore release. Spore traps have been used to test for pathogen presence but correlate poorly with disease occurrence. This may be due to imprecision in differentiating spores of varying Cercospora species. Further, genetic evidence indicates that a sexual stage may occur, but spore trapping only identified asexual spores. To reduce issues with spore identification, a highly susceptible sugar beet germplasm was used as a live spore trap. Germplasm F1042 was grown in the greenhouse to at least the 10 leaf stage. These sentinel plants were placed in six different field locations for $7 \mathrm{~d}$ and replaced weekly for 6 weeks. Plants were incubated in a humidity chamber for $5 \mathrm{~d}$ to allow spores to infect and monitored for CLS for $21 \mathrm{~d}$. Weather data was taken at the same locations and compared to level of lesion development. Results indicated that infectious spores were present as early as April. A high incidence of infection on sentinel beets was detected $14 \mathrm{~d}$ before disease was observed in local fields. This method can be used to aid in identification of risk periods for spore production to add to forecasting models.

Investigating regional differences in proportions of Leptosphaeria maculans and Leptosphaeria biglobosa in southern England A. JAVAID, L. H. Gajula, B. Fitt, Y. Huang, University of Hertfordshire, Hatfield, UNITED KINGDOM

Phoma stem canker, caused by fungal pathogens Leptosphaeria maculans (Lm) and L. biglobosa (Lb), is responsible for worldwide Brassica napus (oilseed rape) yield losses of over $£ 700 \mathrm{M}$. Previously, it was believed that Lm was more damaging than Lb. Recent work suggests that Lb can cause both upper stem lesions and stem cankers. This work investigated proportions of $\mathrm{Lm}$ and $\mathrm{Lb}$ in Leptosphaeria populations in airborne ascospores and in lesions on leaves/stems of different B. napus cultivars in a field experiment in the UK. Air samplers were operated at four UK sites from September to March in 2015-2016 and 2016-2017. Quantitative PCR analysis of air samples showed differences between sites and seasons in amounts of Lm and Lb DNA. In 2015-2016 there was more Lm DNA than Lb DNA in air samples at three sites, whereas in 2016-2017 there was more Lb DNA than Lm DNA at three sites. The field experiment was done with six cultivars in 2016-2017. The numbers of Lm and Lb lesions on leaves were assessed in autumn/winter and proportions of Lm and Lb DNA in stem cankers were quantified in summer. Cultivars had different proportions of Lm and Lb on leaves and in stem cankers. Cultivars susceptible to both $\mathrm{Lm}$ and Lb had more Lm lesions and greater amounts of Lm DNA in cankers whereas cultivars resistant to Lm had more Lb lesions and greater amounts of Lb DNA in cankers. Therefore, there is a need to breed cultivars with resistance against both $\mathrm{Lm}$ and $\mathrm{Lb}$.

Preservation of genotypic diversity of a fungal pathogen within woody cankers M. DOWLING (1), B. Cox (1), T. Sroka (1), J. Wilson (2), G. Schnabel (1), (1) Clemson University, Clemson, SC, USA; (2) Section of Plant Pathology and Plant-Microbe Biology, Cornell University, Ithaca, NY, USA

Monilinia spp. cause brown rot, blossom blight, and twig cankers on stone fruits worldwide. Cankers are often overlooked as inoculum sources by orchard management strategies, though they may cause infections that remain quiescent until fruit maturity. In this study, we examined blossoms and cankers as diversity storage structures, seeing if multiple genotypes of Monilinia fructicola could infect blossoms and be transmitted to cankers. Fungal 
spores from blossoms and subsequent cankers were collected from 2 orchards in 2015 and 2016. Simple sequence repeat markers were used to genotype 10-20 single spores from four blossom/canker pairs per orchard. Single samples contained up to 5 genotypes, and the average genotype numbers in blossoms and cankers were not significantly different for both years $(p=0.49, p=0.86)$ or farms $(p=0.66, p=0.27)$, showing that a bottleneck effect did not occur during the transition from blossom to canker. The average number of genotypes unique to blossom or canker was also not significantly different in either year $(p=0.12, p=0.87)$ or farm $(p=0.73, p=0.27)$. In conclusion, a single blossom may be infected by 1 or more genotypes of $M$. fructicola, and the canker can store this diversity within a season. This information implicates $M$. fructicola cankers as diversity storehouses. We also found that multiple $B$. cinerea genotypes may infect a blossom, implying that these results may apply to other fungal diseases initiating in reproductive tissue.

Systemic spread of Plasmopara obducens in Impatiens plants with roots exposed to sporangia or oospores N. SHISHKOFF, USDA ARS FDWSRU, Frederick, MD, USA

Since 2011, Impatiens has been threatened by a downy mildew disease caused by $P$. obducens that defoliates and kills the plant. It is not yet clear whether the pathogen spreads primarily through airborne inoculum or whether it can also overwinter in soil. We studied the soil-borne spread of the disease in Impatiens balsamina, a seed-planted annual, and I. walleriana, an annual bedding plant. In two experiments, immersing the root system of six $2-3$ week old seedling of $I$. balsamina in a suspension of sporangia $\left(2 \times 10^{3}\right.$ sporangia/ml for $48 \mathrm{hr}$ and then planting led to a systemic infection of some plants (2-3 plants out of six). In three experiments with $I$. walleriana, adding a sporangial suspension to potting media of eight plants at the 2-leaf stage led to infection of some plants (6-8 plants out of 8). Oospores of $P$. obducens that had been cold-conditioned by exposure to $0 \mathrm{C}$ for one month were added to potting media, and then germinated seed were placed on top, in three experiments for each plant species. Both I. balsamina (in 19-44 plants out of 50) and I. walleriana (8-18 plants out of 38) became systemically infected. Learning to effectively manage this disease is crucial to the health of the bedding plant industry. In particular, a better understanding of the life cycle of the pathogen is necessary. Seed-borne oospores have been reported for $I$. balsamina in India; this species might become systemically infected from seed-borne inoculum, by oospores overwintering in infested litter or by roots coming into contact with vegetative sporangia washed into the soil by rain.

Field infection of virus-free sugarcane by Sugarcane yellow leaf virus in south Florida W. BOUKARI (1), C. Kaye (2), C. Wei (1), M. Hincapie (1), P. C. Rott PhD (1), (1) University of Florida, Belle Glade, FL, USA; (2) US Sugar Corporation, Clewiston, FL, USA

Sugarcane yellow leaf virus (SCYLV), the causal agent of sugarcane yellow leaf, is widespread in Florida. This virus is spread by the aphid vector Melanaphis sacchari or by infected seed cane. We aimed to determine the rate and timing of sugarcane infection under field conditions. Two field trials were set up, one on organic soil and one on mineral soil. Each trial consisted of plots planted with either virus-free or virus-infected seed cane of two commercial cultivars. SCYLV prevalence was determined during three consecutive crop seasons by leaf-blot immunoassay. Virus prevalence varied from $83 \%$ to $100 \%$ in plots planted with infected seed cane regardless of cultivar, location, and crop season. SCYLV was not detected in virus-free plots at first sampling date, but plants in these plots became progressively infected on organic soil in plant cane and first ratoon crops. Sugarcane remained virus-free on mineral soil in plant cane crop and became infected only in first ratoon crop. By the end of the second ratoon crop, the highest virus prevalence was $33 \%$ in sugarcane grown on organic soil whereas only $5 \%$ of plants were infected in sugarcane grown on mineral soil. Differences in sugarcane infection by SCYLV between crop seasons and locations may be related to variation in aphid populations and activity. However, low virus prevalence after three crop seasons suggests that planting virus-free seed cane should limit the impact of SCYLV on sugarcane production in Florida.

\section{Epidemiology of Cytospora leucostoma: A Major Limiting Factor for Colorado Peach Production}

S. MILLER (1), D. Sterle (2), I. Minas (2), J. E. Stewart (3), (1) Colorado State University, Fort Collins, CO, USA; (2) Colorado State University, Grand Junction, CO, USA; (3) Department of Bioagricultural Sciences and Pest Management, Colorado State University, Fort Collins, CO, USA

In western Colorado, Cytospora leucostoma is ubiquitous in peach orchards, and has developed into a major limiting factor of peach production. The annual epidemiology patterns of $C$. leucostoma, specific to the western Colorado climatic conditions are not well known. Epidemiology studies are important for growers to develop well-rounded management strategies targeting seasons of heightened infection periods. Our specific objectives were to: a) monitor spore production and lesion size monthly for one year, b) evaluate mycelial growth when incubated in various temperatures, and c) evaluate conidial germination when exposed to different temperatures. Trends in monthly field spore production, and spore germination assays, indicate a fitness decrease when C. leucostoma is exposed to extreme temperatures and percent relative humidity. Despite this fitness decrease, cankers were shown to sporulate year-round in western Colorado. When colonies were incubated, mycelial growth was quickest and most abundant under $28^{\circ} \mathrm{C}$ and halted at $10^{\circ} \mathrm{C}$ and $37^{\circ} \mathrm{C}$. In monitoring the epidemiology of C. leucostoma, management standards in western Colorado can be adjusted to decrease future infections within existing orchards thereby increasing orchard longevity.

Ceratocystis fimbriata transmission by vegetative propagation in Eucalyptus urograndis

J. K. BURIM CARDOSO, C. De Pieri, A. M. Marcon Ruiz, J. C. Silva, E. L. Furtado, São Paulo State University, Botucatu, BRAZIL

This work aimed to verify the possibility of disseminating Ceratocystis fimbriata by vegetative propagation, and whether the clonal seedlings constitute the inoculum source of this pathogen. For this were chosen parents of the clone SP-519 (Eucalyptus urograndis), susceptible to the pathogen, planted at a location with a history of occurrence of the pathogen, to form of a clonal garden. From these collected buddings, microcuttings were removed and submitted to the process of rooting and acclimation, obtaining 96 seedlings, which were transplanted to a receptacle containing sterilized washed sand, with ferti-irrigation. Three of the 96 plants were observed to die at days 13, 93 and 139, respectively, after transplantation, indicating $3.13 \%$ mortality. Each plant was isolated for morphological and molecular characterization of the pathogen, which demonstrated the presence of $C$. fimbriata. This first observation evidenced that the disease can be transmitted by vegetative propagation. Seedlings are being obtained from these parents. Isolations are being carried out while specific primers are being designed to monitor healthiness by PCR. Seedling producers must be warned of the risk and a healthiness test must be recommended for the formation of parent plants for nursery cultivators.

Potential sources of inoculum and survival of Macrophomina phaseolina in Florida strawberry fields J. BAGGIO (1), N. Peres (2), (1) GCREC - University of Florida, Wimauma, FL, USA; (2) University of Florida, Wimauma, FL, USA

Macrophomina phaseolina, the causal agent of charcoal rot, affects strawberry crowns inducing plant collapse. The fungus survives in the soil through resistant structures (microsclerotia) and is usually controlled by pre-plant fumigation of soil. However, in the 2016-17 Florida strawberry season, roughly $20 \%$ plant mortality still occurred due to charcoal rot where plastic-covered beds were re-used for a second season and crop residue (old strawberry 
crowns/roots) were disposed between beds. Crowns from the previous season were collected from three farms where charcoal rot was reported and $M$. phaseolina was recovered from all samples. After the season, infected crowns were buried in the soil and retrieved every two weeks for three months to quantify survival of $M$. phaseolina. No reduction in the frequency of fungal recovery from crowns was observed during this period. To test the ability of infected crop residue to serve as a source of inoculum for healthy plants, infected crowns were disposed of in the bed alleys or buried next to new plantings of 'Festival', 'Florida Beauty' and 'Winterstar'. After 12 weeks, respective average plant mortality for the three cultivars was 60,43 and $83 \%$ when crowns were placed in the bed alleys, and 53,30 and 63\% when crowns were buried in the beds. Our results suggest that M. phaseolina survives over summer in Florida on old strawberry crowns that do not decompose and may act as source of inoculum for healthy plants.

\section{Control of Zymoseptoria tritici a splash dispersed pathogen by the mean of wheat cultivars mixture; experimental and modelling biophysical approaches}

S. SAINT-JEAN (1), T. Vidal (1), C. Gigot (1), L. Huber (1), C. De Vallavieille-Pope (2), (1) UMR ECOSYS AgroParisTech, INRA, Université ParisSaclay, Thiverval-Grignon, FRANCE; (2) UMR BIOGER AgroParisTech, INRA, Université Paris-Saclay, Thiverval-Grignon, FRANCE

Growing together mixture of cultivars with contrasting level of resistance to disease has been shown as a way of reducing fungal aerial disease severity on crops. In order to improve the design of cultivar mixture for reducing splash dispersed diseases such as septoria tritici blotch, caused by Zymoseptoria tritici, our objective is to understand the role of the canopy architecture of wheat cultivar mixture. Two mechanisms related to the effect of canopy architecture on the epidemiological are studied here. The first one is the impact of architecture of each cultivar on the barrier effect provided by resistant plants among susceptible plants that reduce the dispersion of fungal spore. The second one is the possible modification of the microclimate within the mixture compared to pure stand that could foster some epidemiological processes. We used a mechanistic modelling approach within an explicit description of canopy architecture and splash dispersal processes. Dispersal patterns in cultivar mixtures with either similar (70 cm height) or contrasted straw height $(120 \mathrm{~cm}$ and $70 \mathrm{~cm})$ were compared. Spore interception on susceptible plants was lower when the susceptible cultivar was mixed with a tall resistant cultivar rather than with a resistant cultivar of the same height. This result was confirmed with field experiments with a reduction of AUDPC close to $50 \%$. Field experiments show also that within denser canopy the barrier effect is enhanced but on the other hand the leaf wetness duration increased and could foster the disease. These results suggest that considering cultivar architecture in mixture design is as an additional lever to enhance mixture effects on foliar splash-dispersed diseases.

Crops as hosts of Curtobacterium flaccumfaciens pv. flaccumfaciens in Brazil

D. M. DO NASCIMENTO (1), J. C. Silva (1), J. M. Soman (1), T. A. Fernandes da Silva Junior (2), A. C. Maringoni (1), (1) São Paulo State University, Botucatu, BRAZIL; (2) Universidade do Sagrado Coração, Bauru, BRAZIL

Knowledge of ecological niches of Curtobacterium flaccumfaciens pv. flaccumfaciens (Cff), causal agent of bean bacterial wilt, is essential for the efficient management of the disease. This study evaluated its survival in the phylloplane and rhizosphere of black oat, rattlepod, common bean, sunflower, maize, pearl millet, forage turnip and soybean. The aerial part of the plants was inoculated by spraying bacterial suspension $\left(10^{7} \mathrm{CFU} \cdot \mathrm{mL}^{-1}\right)$ of Feij-2628A strain, resistant to rifampicin and pathogenic to common bean. The soil of the pots was infested with $200 \mathrm{~mL}$ of the same suspension. Cff survival was evaluated weekly, for 42 days, with three plants of each species being collected for each evaluation. After the processing of the soil and phylloplane samples, $100 \mu \mathrm{L}$ of the supernatant were plated in NSARF semi-selective medium, followed by incubation $\left(28^{\circ} \mathrm{C} / 96 \mathrm{~h}\right)$, and qualitative evaluation of Cff colonies. Feij-2628A strain survived for 42 days in the phylloplane of all species evaluated, except in rattlepod (14 days) and maize (28 days). In the rhizosphere, Cff survived for 21 days in soybean, 28 days in black oat, 35 days in pearl millet, maize and forage turnip, and for 42 days in the other species. These results can help producers in the selection of crops for rotation with common bean.

Impact of single-season Potato virus $Y$ epidemics on small mixed-acreage vegetable farms S. RUARK (1), S. Gray (2), (1) Cornell University, Ithaca, NY, USA; (2) Section of Plant Pathology and Plant-Microbe Biology, Cornell University, Ithaca, NY, USA

Potato virus Y (PVY) is a major disease of potato capable of causing severe yield losses. Infected seed potatoes and volunteers are considered the major source of inoculum for the emerging potato crop as well as for other susceptible crops grown nearby. The degree to which PVY moves between solanaceous crops in diverse agricultural settings has not been well-studied. In the Connecticut River Valley in Massachusetts, growers produce a mixture of solanaceous crops including potato, tobacco, tomato, pepper, and eggplant on small adjacent acreages. Between 2015 and 2017 , field surveys were conducted on several small mixed-acreage vegetable farms surrounding Amherst, MA to determine the identity and prevalence of PVY strains present in the region. ELISA and multiplex RT-PCR identified several strains present and moving between potato, tobacco, and tomato fields. We only detected a single strain of PVY in two pepper plants in 2016. No infections were detected in eggplant. PVY management options are limited and minimizing inoculum by planting certified seed is advised, but this may not be cost-effective in all scenarios. A partial budget analysis revealed factors that should be considered prior to implementation of this strategy by growers in this region.

Survival of Xanthomonas fragariae on common materials found in strawberry nurseries H. WANG (1), W. W. Turechek (2), (1) Clemson University, Blackville, SC, USA; (2) USDA, ARS, U.S. Horticultural Research Laboratory, Fort Pierce, FL, USA

Xanthomonas fragariae causes strawberry angular leaf spot, an important disease in strawberry nursery production. To identify potential inoculum sources, the ability of $X$. fragariae to survive was examined on 10 common materials typically associated with strawberry nurseries (cardboard, glass, latex gloves, strawberry leaves, medal, plastic bags, rubber, T-shirt, Tyvek, and wood). Each surface was artificially contaminated with the bacteria and stored at room temperature and $-4^{\circ} \mathrm{C}$, respectively. The bacteria were collected from each surface with PBS-soaked cotton balls at 10 time points from the day of contamination until one year after. During each sampling, the survival rates were examined by testing the bacteria isolated from the PBSsoaked cotton balls by PMA-qPCR, and by rub-inoculating strawberry plants with the cotton balls. The experiment was conducted four times with a different $X$. fragariae isolate per experiment. Results showed that $X$. fragariae survival rates varied by both the material and storage temperature, and the longest survival was observed on cardboard stored at $-4^{\circ} \mathrm{C}$, the conditions which dormant plants are stored in strawberry nurseries. The information provided by this study may contribute to the development of improved sanitation and disease management strategies for strawberry nurseries.

Survival of Xanthomonas campestris pv. campestris in cultivated plants and weeds

J. C. SILVA (1), D. M. do Nascimento (1), T. A. Fernandes da Silva Junior (2), J. M. Soman (1), T. Domingues Tomasini (1), A. C. Maringoni (1), (1) São Paulo State University, Botucatu, BRAZIL; (2) Universidade do Sagrado Coração, Bauru, BRAZIL 
Black rot, caused by Xanthomonas campestris pv. campestris (Xcc), is considered the most important brassica disease worldwide. Once survival niches knowledge of this pathogen is extremely important for the disease efficient management, this work aims to evaluate Xcc survival in phylloplane and rhizosphere of cultivated plants as well as weed endophytic survival. Seven differents botanic families among 10 cultivated plants and 11 weed species were used in the experiments. The $3098 \mathrm{C}$ strain resistant to rifampicin was inoculated by spraying bacterial suspension $\left(10^{7} \mathrm{CFU} . \mathrm{mL}^{-1}\right)$ on aerial part of the plants. The cultivated plants soil was infested with $200 \mathrm{~mL}$ of the same suspension. After soil and phylloplane samples processing and bacterial weeds isolation, $100 \mu \mathrm{L}$ of the supernatant were plated in NSARF semi-selective medium and incubated $\left(28^{\circ} \mathrm{C} / 72 \mathrm{~h}\right)$. Xcc survived for up to 35 days in tomato, wheat, pumpkin, cultivated radish, black oat and cabbage phylloplanes and 21 days in lettuce rhizosphere. The endophytic survival of Xcc was observed in virginia pepperweed, wild radish and bengal dayflower 28 days after inoculation. Results obtained here support the hypothesis that weeds present in areas with black rot history, as well as cultivated plants used in rotation with brassicas, can be an inoculum source of Xcc.

Relationships among measures of wheat blast under field conditions

K. B. MILLS (1), P. A. Paul (2), L. V. Madden (2), J. Salgado (3), (1) Ohio State University, Wooster, OH, USA; (2) The Ohio State University, Wooster, OH, USA; (3) Ohio State Univ, Ohio Agricultural Research and Development Center, Dept of Plant Pathology, Wooster, OH, USA

Wheat blast, caused by Magnaporthe oryzae, affects the leaves and spikes of wheat. However, little is known about the relationship between spike and leaf blast or whether development of the former is influenced by the latter. In 2015, six research plots were planted with a highly susceptible cultivar at each of three sites (OK1, OK2, and CC) in Santa Cruz de la Sierra, Bolivia. Spike blast incidence (INC) and severity (SEV) and leaf blast severity (LEAF) were rated twice a week on a grid of one hundred points in each plot. Ratings began in mid-June, when spikes emerged, and continued until the milky-ripe growth stage in early August. Regression models were fitted to the data to quantify relationships among transformed INC, SEV, and LEAF. There were significantly positive linear relationships $(P<0.05)$ between complementary-log-log transformed SEV $\left(C L L \_S E V\right)$ and square-roottransformed INC (SQRT_INC), between CLL_SEV and CLL_LEAF, and between CLL_INC and CLL_LEAF. An estimated 81 to $89 \%$ of the variation in CLL_SEV was explained by the variation in SQRT_INC; 57 to $71 \%$ of the variation in CLL_SEV was explained by variation in CLL_LEAF; and 48 to $81 \%$ of the variation in CLL INC was explained by CLL LEAF. These results suggest that INC may be a good predictor of SEV, and provide some empirical support for the idea that leaf and spike blast may be associated when the two occur simultaneously in the same field.

\section{Seed transmission of begomoviruses in economic crops}

E. J. KIL (1), H. S. Byun (1), H. S. Choi (2), K. Y. Lee (3), J. K. Kim (4), C. S. Kim (5), S. Lee (1), (1) Sungkyunkwan University, Suwon, KOREA; (2) National Institute of Agricultural Science, Wanju, KOREA; (3) Kyungpook National University, Daegu, KOREA; (4) Chungcheongnam-do Agricultural Research \& Extension Services, Yesan, KOREA; (5) Highland Agricultural Research Institute, Rural Development Administration, Pyeongchang, KOREA

Begomoviruses, single-stranded DNA viruses, cause severe damages to global agricultural production of economic crops. It has long been accepted that begomoviruses could not be transmitted by infected seeds but only through the whitefly Bemisia tabaci, graft and artificial inoculation with infectious clones. However, in these days, begomoviruses such as Tomato yellow leaf curl virus (TYLCV), Sweet potato leaf curl virus and Mung bean yellow mosaic virus have been reported as seed-transmissible viruses in tomato, sweet potato and black gram, respectively. In 2015 and 2016, seeds of TYLCVinfected sweet pepper and white soybean and Tomato yellow leaf curl Thailand virus (TYLCTHV)-infected tomato were collected in Korea and Myanmar. PCR was performed with TYLCV and TYLCTHV specific primer sets (25-100\%), and the amplicons were sequenced. According to the sequence analysis results using a BLAST search, the amplified DNA showed high identity to the previously reported sequences. To analyze virus dissemination, TYLCV and TYLCTHV-specific PCR was also performed with bunches of seedlings germinated from the seeds (five seedlings per pool), and some bunches of seedlings (20-100\%) were confirmed to be TYLCV and TYLCTHV-infected. Virus replication in the seeds and seedlings was verified by the strand-specific amplification method. This is the first report of TYLCV seed transmission in non-tomato plants and the second identification of seed transmission of the TYLCV complex in tomato plants after an initial report of seed transmission of the TYLCV Korea isolate.

Flower blights of macadamia caused by Botrytis cinerea, Pestalotiopsis macadamiae and Neopestalotiopsis macadamiae in Australia O. AKINSANMI, The University of Queensland, Brisbane, AUSTRALIA

Flower blights are significant constraints to macadamia productivity in Australia. Their insidious nature leads to significant yield losses, which are difficult to quantify. Various pathogens belonging to different classes of fungi and oomycetes are associated with flower blights of macadamia. Botrytis cinerea causes Botrytis blight; Pestalotiopsis macadamiae and Neopestalotiopsis macadamiae cause Pestalotiopsis blight; Cladosporium cladosporioides causes Cladosporium blight and Phytophthora capsici and P. palmivora cause Phytophthora blight or wet blight. In Australia, Botrytis blight and Pestalotiopsis blight are the most common flower blights. Epidemiological studies of flower blights including disease incidence and putative sources of pathogen inoculum were examined between and within growing seasons in macadamia cropping systems. Although each pathogen belongs to different groups, has contrasting life cycles and has different optimal weather conditions for infection and disease development, however, similar ecological factors are required for severe damage to macadamia flowers. These findings will contribute to an improved understanding of macadamia cropping systems frameworks. The use of linked simulation models for disease forecasting will support and help to define better management strategies for flower blights of macadamia.

\section{Plant pathogen removal by managed aquifer recharge of fresh tile drainage water for safe reuse as irrigation water in salinized agricultural areas} C. EISFELD (1), J. van der Wolf (2), B. M. van Breukelen (1), J. F. Schijven (3), G. Medema (1), (1) Delft University of Technology, Faculty of Civil Engineering and Geosciences, Delft, NETHERLANDS; (2) Wageningen University \& Research, Wageningen, NETHERLANDS; (3) Utrecht University, Faculty of Geosciences, Utrecht, NETHERLANDS

The project AGRIMAR investigates recycling of fresh tile drainage water (TDW) via managed aquifer recharge (MAR) technology for agricultural purposes to secure water availability. Here, the TDW is collected for storage in brackish aquifers creating a fresh water 'bubble' in the subsurface which gives farmers access to sufficient fresh water to irrigate their crops even in times of drought. The TDW may contain plant pathogens which could be present in the recycled water. To prevent the spread of crop diseases, the removal of selected plant pathogens during aquifer soil passage will be analysed. We focus on three plant pathogenic bacteria of high economic importance, namely Ralstonia solanacearum, Dickeya solani and Pectobacterium carotovorum. As a first experimental approach, we will study the survival of the selected plant pathogens under different representative aquifer conditions. Subsequently, their fate during soil passage is investigated in column experiments and finally in a MAR pilot field site in the Netherlands. The inactivation processes will be described in a mathematical model to compare the model predictions with the experimental results. We will present the outline of the research approach and the first results of batch and column experiments on the fate of these plant pathogens under representative subsurface conditions. 
The pitch canker pathogen Fusarium circinatum: Endophytic on grasses in South Africa

D. A. HERRON (1), E. Steenkamp (2), B. D. Wingfield (2), M. J. Wingfield (2), (1) Forestry and Agricultural Biotechnology Institute, University of Pretoria, Pretoria, SOUTH AFRICA; (2) Forestry and Agricultural Biotechnology Institute (FABI), University of Pretoria, Pretoria, SOUTH AFRICA

Since its first discovery in 1945, the pitch canker fungus, Fusarium circinatum, has been known only as a pathogen of Pinus species and Pseudotsuga menziesii. Recent studies have shown that the fungus is able to infect non-coniferous plants, including eight different grass species and six species of herbaceous plants. In this study, we investigated this association by identifying the Fusarium species inhabiting sixteen species of grass occurring in the understory of pitch canker-affected Pinus patula trees in a South African plantation. Species of Fusarium were identified using their morphological characteristics as well as by phylogenetic inference based on DNA sequences for the beta-tubulin and translation elongation factor-1-alpha gene regions. A number of important Fusarium species were recovered from these grasses, including $F$. circinatum, which was found on five of the sixteen grass species. This study represents the fourth report (two from South Africa, one from Spain and one from the USA) of the pitch canker fungus from grass. The cryptic occurrence of this quarantine pine pathogen in non-coniferous plants highlights a need to consider the role that other plants could play on its biology and distribution and the negative impact it could have on quarantine efforts.

Dispersal of Botrytis cinerea conidia in raspberry fields

O. CARISSE, Agric \& Agri-Food Canada, Saint-Jean-sur-Richelieu, QC, CANADA

Raspberry fruit rot, caused by Botrytis cinerea Pers. ex Pers., is one of the most important fruit diseases of raspberry. Currently, the disease is controlled with applications of fungicides. The improvement of cultural methods for disease management should lessen this dependence on fungicides. However, such improvements would not be possible without knowledge of inoculum sources and dispersal of the pathogen. Dispersal of airborne conidia and incidence of Botrytis fruit rot were monitored at two raspberry plantings during three successive years with meteorological data. The concentrations of conidia were monitored using a 7-day volumetric sampler and rotating-arms samplers. The number of $B$. cinerea conidia in air samples was determined with a real-time qPCR assay. Dispersal of airborne conidia was assessed at $0.5,1.0,1.5,2.0,4$, and $8 \mathrm{~m}$ from a fixed point source of inoculum with rotating-arms samplers placed at $0.45,0.90$, and $1.35 \mathrm{~m}$ from the ground. Each year, the experiment was conducted four times. The coefficient of correlation between the volumetric and rotating-arms samplers placed at 45, 90, and $135 \mathrm{~cm}$ from the ground was significant; and a diurnal pattern of conidial release was observed. During the pre-bloom and bloom period, conidia dispersal gradient $\left(\log \left(\mathrm{conidia} / \mathrm{m}^{3}\right)\right.$ vs distance in $\left.\mathrm{m}\right)$ showed significant flattering at a distance of more than $2 \mathrm{~m}$ from the inoculum source. However, near or at harvest no significant dispersal gradients were observed.

\author{
Management practices of cruciferous crops and edaphic and weather conditions related to clubroot presence in eight geographic regions in \\ Colombia \\ A. Botero Ramirez (1), F. L. PADILLA-HUERTAS (2), C. García (2), (1) University of Alberta Department of Agricultural, Food and Nutritional \\ Science, Alberta, CANADA; (2) Universidad Nacional de Colombia, Bogotá, COLOMBIA
}

Clubroot, caused by Plasmodiophora brassicae, is a major disease of cruciferous crops in Colombia. Nonetheless, there is virtually no information regarding its geographical distribution along the main production areas, neither the environmental factors associated with the disease. On the current study, we surveyed and evaluated the presence of clubroot and its response to management practices and edaphoclimatic factors. In each of 125 horticultural crops fields visited in eight geographic regions, a questionnaire was used to inquire about the prevalence of the disease and crop management methods by the farmer. Soil samples were collected to perform physical and chemical analyses. When cruciferous crops were present, disease intensity was assessed; if not, farmers were asked whether they had ever observed the disease. Clubroot was detected in $53.66 \%$ of the fields. Nariño was the only state where clubroot was not found. Management practices and edaphic and weather conditions were correlated with disease data, negative indices were found between disease and liming $(-0.23)$, annual precipitation $(-0.2)$, and days with rain $(-0.27)$. Positive indices were obtained for inclusion of cruciferous crops in rotation schemes (0.45), phosphorus (0.35), calcium (0.34), magnesium ( 0.25$)$, zinc $(0.24)$, boron $(0.22)$ and sodium $(0.22)$ concentrations in the soil, and cation exchange capacity $(0.35)$. In conclusion, clubroot disease is widely spread along cruciferous production areas in Colombia and its importance is likely to be affected by cropping crucifers, by soil chemistry balances and by precipitation regime.

\title{
Dispersal of Colletotrichum acutatum conidia from citrus and strawberry under controlled conditions
}

A. B. GAMA (1), F. P. Goncalves (2), B. B. Forcelini (3), G. J. Silva Junior (4), L. Amorim (2), N. Peres (5), (1) University of Florida - Gulf Coast Research and Education Center, Wimauma, FL, USA; (2) USP, Piracicaba, BRAZIL; (3) Gulf Coast Research and Education Center, University of Florida, Wimauma, FL, USA; (4) FUNDECITRUS, Araraquara, BRAZIL; (5) University of Florida, Wimauma, FL, USA

Colletotrichum acutatum sensu latu causes postbloom fruit drop of citrus and anthracnose of strawberries. These diseases are usually widespread within infected fields, although primary conidia (PC) are water dispersed to short distances. C. acutatum produces quiescent infections on citrus and strawberry leaves, which eventually produce secondary conidia (SC) that are not in mucilage. Dispersal potential for SC is not known, and we hypothesized that they might be wind dispersed. Strawberry and citrus leaves were inoculated with $5 \times 10^{5}$ and $1 \times 10^{6}$ conidia.ml $^{-1}$, respectively. Secondary conidiation was stimulated in a moist chamber with $1 \%$ sucrose. After $48 \mathrm{~h}$, inoculated leaves were placed in a wind tunnel, with C. acutatum selective media plates placed at 15 to $300 \mathrm{~cm}$ from inoculum source. Winds of $2.5 \mathrm{~m} \cdot \mathrm{s}^{-1}$ were applied for $2 \mathrm{~h}$ over inoculated leaves combined or not with sprays of water or sucrose solution. The experiments were also conducted with symptomatic strawberry fruit, and C. acutatum colonies on Potato Dextrose Agar, sources of PC. C. acutatum colony forming units (CFU) were counted after one week. The exponential model was fitted to data of CFU over distances ( $p=0.05)$. SC were not dispersed when exposed to wind alone. Most of water or sucrose-dispersed SC was limited to short distances, less than $60 \mathrm{~cm}$ from strawberry or citrus leaves, whereas PC water dispersal went further, with abundant conidia found at $3 \mathrm{~m}$ from sources.

The influence of leaf age and cultivar on infection of celery by Colletotrichum fioriniae

S. REYNOLDS (1), M. J. Celetti (2), K. S. Jordan (1), M. R. McDonald (1), (1) University of Guelph, Guelph, ON, CANADA; (2) OMAFRA, Guelph, ON, CANADA

Anthracnose leaf curl, caused by the fungus Colletotrichum fioriniae, is an emerging disease on celery in Ontario, Canada. The objectives of this study were 1) to characterize the early infection process on two celery cultivars, 'TZ 9779' and 'Hadrian', that differ in susceptibility to leaf curl, and 2) to determine the effect of leaf age on disease development. A conidial suspension $\left(1 \times 10^{6}\right.$ spores $\left.\mathrm{mL}^{-1}\right)$ was applied to either the three oldest stalks of 14 week-old plants or immature leaves in the crown. Plants were incubated at $25^{\circ} \mathrm{C}$ in a greenhouse, and assessed over time. The crown developed lesions 48-72 hour post inoculation (hpi), and had significantly more lesions than mature stalks. Leaf curling was delayed on inoculated mature stalks by 10-15 days. Regardless of cultivar or leaf age, leaf curl symptoms developed on non-inoculated leaves of inoculated plants, suggesting that toxins released by 
the pathogen can affect other parts of the plant. Disease development did not differ between cultivars. Conidia germination was lower on the immature leaves (41-43\%) compared to the mature leaves (64-72\%) at 12 hpi, but most conidia have produced melanised appressoria by 96 hpi on either tissue. Quantitative PCR detected the pathogen in asymptomatic mature leaves, with no differences between cultivars. These results demonstrated that immature leaves in the crown are most susceptible to infection, thus chemical control should be directed there.

Using spread models to optimise surveillance for Xylella fastidiosa

A. MASTIN (1), S. White (2), D. Chapman (3), S. R. Parnell (1), (1) University of Salford, Salford, UNITED KINGDOM; (2) Centre for Ecology and Hydrology, Wallingford, UNITED KINGDOM; (3) Centre for Ecology and Hydrology, Penicuik, Midlothian, UNITED KINGDOM

Although surveillance is of central importance to the prevention and control of pests and pathogens, the complex nature of natural biological systems means that it can be challenging to fully account for the ecological and epidemiological characteristics of the pathosystem in question when planning surveillance. Our previous work has demonstrated that failing to account for these issues can lead to suboptimal surveillance performance, both in terms of pathogen detection and cost effectiveness. Using the example of the emerging pathogen Xylella fastidiosa CoDiRO in the Apulia region of southern Italy, we demonstrate how to design a surveillance strategy which incorporates both biological and statistical considerations by linking a spatially explicit model of pathogen spread with a statistical model of a sampling process. Using this model, we apply a simple optimisation routine to identify where best to sample olive tree hosts in the currently "uninfected" region towards the north of Apulia, in order to detect any new incursions at an early stage. By investigating the impact of different spread patterns, we have found that accurately characterising the long distance spread of the pathogen (resulting largely from vector movement) will be key to improving the spatial targeting of early detection surveillance efforts and maximise the probability of early detection of spread beyond the infected region.

Understanding the mechanisms of infection and survival of the maize pathogen Xanthomonas vasicola pv vasculorum M. C. ORTIZ-CASTRO (1), J. M. Jacobs (2), M. C. Plazas (3), J. M. Lang (1), T. A. Jackson-Ziems (4), J. E. Leach (1), A. E. Robertson (5), K. D. Broders (1), (1) Colorado State University, Fort Collins, CO, USA; (2) Univeristy of Wisconsin-Madison, Madison, FRANCE; (3) Universidad Catolica de Cordoba, Cordoba, ARGENTINA; (4) University of Nebraska-Lincoln, Lincoln, NE, USA; (5) Iowa State University, Department of Plant Pathology, Ames, IA, USA

Bacterial leaf streak of corn, caused by Xanthomonas vasicola pv. vasculorum (Xvv), is an emerging disease of maize in North and South America. The disease has been found in Argentina and the United States affecting sweet corn, popcorn, and grain corn. Based on the combined $\$ 52.4$ billion value of the maize industry, early reports of $X v v$ disease severity, and lack of management methods, this emerging pathogen represents a serious economic threat to U.S. maize production. The primary goal of this research is to provide a basic understanding of the mechanism of infection and survival of the maize bacterial leaf streak pathogen. Through genetic transformations of the bacteria with fluorescent proteins, several inoculation strategies, and confocal microscopy, we were able to describe the mode of entry of this pathogen into the corn plant. In addition, we found that there is a significant phenotypic diversity between $X v v$ isolates across different regions. Strains from Colorado and Nebraska, where the disease was first reported, showed the highest and lowest levels of virulence. Finally, through litter studies at multiple locations, we demonstrated that infected residue left on the soil surface harbored significantly greater quantities of $X v v$ than infected residue buried $10 \mathrm{~cm}$ below the surface. These findings provide a more complete understanding of the bacterial leaf streak disease cycle and will aid in the development of management strategies that may limit $X v v$ 's distribution within corn fields and prevent further spread to other corn producing regions.

Huanglongbing spatial pattern in Sao Paulo state, Brazil

K. PAZOLINI (1), J. H. Arruda (1), G. A. Chinelato (1), A. Mastin (2), S. R. Parnell (2), J. Belasque Jr. (1), (1) University of São Paulo, Piracicaba, BRAZIL; (2) University of Salford, Salford, UNITED KINGDOM

Huanglongbing (HLB) is considered the most devastating disease of citrus worldwide, and is known to occur in many citrus growing regions worldwide, including Brazil, the USA and China. Quantifying the spatial nature of spread of the causative bacterium can be a key to understanding the epidemiology of the pathogen, which can then be used to inform and improve control measurements. The aim of this study was to determine the spatial pattern of HLB in plots under strict management in São Paulo state, Brazil. For this, modified Ripley’s K-function, ordinary runs analyses and kernel density estimation were performed on a total of 81 annual "snapshots" of the spatial distribution of infection in 22 commercial citrus plots (each composed of approximately 13,840 trees over 24.9 ha) between 2013 and 2017. These plots were managed with HLB control based on, at least, four inspections per year for detection and rouging of symptomatic trees and regular insecticide sprays. Trees rouging incidence and vector detection on yellow stick cards on the 22 plots ranged from 0.7 to $23.4 \%$ and 0 to 1.9 adults/yellow stick card/assessment, respectively. Results of the modified Ripley's K and ordinary runs analyses found no evidence of aggregation on the 22 plots studied, suggesting that primary spread from surrounding areas predominates. This finding was also supported by kernel density estimation, which found the highest density of suspected infected trees around the plot periphery.

Evaluation and Identification of Oospores on Cucurbit Downy Mildew Infected Field Samples

J. G. JONES (1), K. L. Everts (1), C. Salgado-Salazar (2,3), J. A. Crouch (2), (1) University of Maryland College Park, Lower Eastern Shore Research and Education Center, Salisbury, MD, USA; (2) Mycology and Nematology Genetic Diversity and Biology Laboratory, USDA-ARS, Beltsville, MD, USA; (3) Oak Ridge Institute for Science and Education, Oak Ridge, TN, USA

Pseudoperonospora cubensis, which causes cucurbit downy mildew (CDM), is one of the most devastating pathogens of cucurbit crops worldwide. The pathogen is heterothallic, with two mating types (A1 and A2) both recently found in the United States. Pseudoperonospora cubensis largely reproduces asexually via sporangia capable of long distance spread, but sexual reproduction resulting in oospores that serve as survival structures is possible, if both mating types are present. To date, no data of naturally occurring oospores within the field have been recorded, although the survival structure can be induced under laboratory conditions. However, putative $P$. cubensis oospores were observed and photographed in CDM infected squash leaves from field samples in Maryland and Delaware during 2016-17 growing seasons. Oospore inoculation on detached leaves did not result in any disease symptom development. A custom, pathogen-specific molecular probe, for use in fluorescent in situ hybridization (FISH), is currently being used to verify the oospores are $P$. cubensis and not another oomycete pathogen. If confirmed, cucurbit growers and plant breeders will need to be aware that the virulence of $P$. cubensis could shift again, with the introduction of new genetic variation from sexual recombination.

Effect of flower age and antibiotic treatment on the population dynamics of Erwinia amylovora on apple flower stigmas S. M. SLACK (1), J. Schachterle (1), E. Sweeney (1), E. Pochubay (2), C. A. Outwater (1), G. W. Sundin (1), (1) Michigan State University, East Lansing, MI, USA; (2) Michigan State University, Traverse City, MI, USA 
Fire blight, a disease of pome fruit, is caused by the gram-negative bacterium Erwinia amylovora. If left unchecked, fire blight can decimate an orchard in as little as one season. Pathogen growth on flower stigmas is a critical first step prior to the initiation of blossom blight symptoms. E. amylovora populations can attain sizes of $1 \times 10^{7}$ colony forming units (CFU) per flower if conducive environmental conditions occur. We examined the growth under field conditions of a marked E. amylovora strain on stigmas of flowers previously open for 1, 3, or 5 days. In 1 day open flowers, populations could increase 3-4 logs in 3 days with temperatures as low as 12C. Flowers open 3 days prior to inoculation also supported similar increases in population, however the response was cultivar dependent. Flowers inoculated after being open for 5 days did not reach carrying capacity, and the population crashed by the end of the sampling period. The results indicate that younger flowers are more conducive for E. amylovora population growth. Antibiotics were also applied before or after inoculation on 1 day old flowers and populations were tracked for 4 days. The results indicate the effectiveness of different antibiotic chemistries was dependent on weather and inoculation timing. From these data, we are developing a better understanding of how flower age, antibiotic timing, and weather influence E. amylovora population dynamics and flower infection.

\section{Water mediated virus transmission: Sources, detection and inactivation}

M. RAVNIKAR (1), D. Kutnjak (1), N. Mehle (1), A. Pecman (1), K. Bačnik (1), J. Kosel (2), M. Dular (2), A. Filipić (1), D. Dobnik (1), J. Zel (1), G. Primc (3), M. Mozetic (3), I. Gutierrez Aguirre (1), (1) National Institute of Biology, Ljubljana, SLOVENIA; (2) University of Ljubljana, Faculty of mechanical engineering, Ljubljana, SLOVENIA; (3) Josef Stefan Institute, Ljubljana, SLOVENIA

Environmental waters constitute a potential source of plant viruses. In this study we aimed to confirm if effluents from waste water are also home for plant viruses and if these remain infective, even after their trip through the wastewater plant. In order to detect the low virus concentrations present in water, we used either an efficient concentration system based on CIM monolithic chromatography or an easy-to-use tangential filtration system, Aquafilter. For virus detection after concentration, we used the most sensitive molecular method, quantitative PCR (qPCR). The integrity of the plants viruses was confirmed using transmission electron microscopy (TEM). The infectivity of plant viruses such as Pepper Mild Mottle virus and Tomato Mosaic Virus in concentrated water samples was confirmed by inoculation of test plants. To obtain a wider picture of the viral composition, we also analyzed the metagenome (Virome) of such samples using high throughput sequencing, which allowed us to find new viruses in the aqueous environment from where the samples were collected. Wastewater effluents are used as irrigation waters in many countries, where due to climate conditions, water is scarce. From our findings it is clear that a safe recycling of such waters would require methods that are able to inactivate viruses in water environment in an efficient and cost effective manner. We have tested the ability to inactivate viruses of two physical treatments, hydrodynamic cavitation and plasma. Both methods efficiently inactivated two model viruses (MS2 bacteriophage and Potato virus Y) in water, reaching up to 4 log reductions of the viral infectivity in the case of MS2, and showing up as two promising options to inactivate virus in water samples.

\section{Dispersal patterns of Fusarium circinatum in North Florida loblolly and slash pine forests across two growing seasons}

T. QUESADA (1), J. Hughes (1), K. Smith (2), P. James (1), K. Shin (1), C. Staub (1), M. Marsik (1), J. A. Smith (3), (1) University of Florida, Gainesville, FL, USA; (2) USDA Forest Service, Gainesville, FL, USA; (3) School of Forest Resources and Conservation, University of Florida, Gainesville, FL, USA

Pitch canker is caused by the necrotrophic fungus Fusarium circinatum, which causes episodic outbreaks that can result in significant losses to the timber industry. Previous studies on the phenology and dispersal of this fungus have been previously studies, but knowledge remains uncertain. These mixed results may be due to variation in host species, study location, weather patterns, and other factors. Collecting additional data from a different region and host may help better understand the dispersal of $F$. circinatum in comparison to previous studies. In this study, spore samples were collected from three locations in Northern Florida, USA over two growing seasons, in 2016 and 2017. We used in-house spore traps followed by quantitative PCR using species-specific primers to detect and quantify the abundance of DNA from airborne $F$. circinatum spores in all three locations. While low quantities, under 10 picograms, of $F$. circinatum DNA were detected per sample throughout the entire collection period, results from the first year of sampling suggest a higher peak of 50 to 150 picograms of $F$. circinatum DNA in late May and early June in all three sites, followed by a minor peak of 10 to 50 picograms in early August. By repeating this experiment for a second season we expect to validate these results, and the inclusion of weather data throughout both collection periods will provide information on whether $F$. circinatum release is influenced by particular weather patterns.

Transmission of Magnaporthiopsis maydis from maize seeds to seedlings N. A. Gonzalez, G. P. MUNKVOLD, Iowa State University, Ames, IA, USA

Magnaporthiopsis maydis (syn. Harpophora maydis) is a seedborne fungus that causes late wilt of maize, a disease that is economically important in several parts of the world, but does not occur in the Americas. Potential introduction of M. maydis into the United States is considered a serious threat to U.S. agriculture because of its probable impact on maize production and trade. Although the fungus is known to be seedborne, there is little information regarding the occurrence of seed-to-seedling transmission. In this study, maize seeds were inoculated using a water-restriction method, which resulted in external seed contamination. Approximately 1,000 inoculated seeds were planted in individual pots in a restricted access growth chamber. After 4 weeks, coleoptile and mesocotyl tissues were dissected and surface-disinfested, and individually subjected to PCR analysis. M. maydis was detected by PCR in mesocotyl tissues of $6(0.94 \%)$ of 639 seedlings that emerged from inoculated seeds. All seedlings from noninoculated seeds tested negative. Although disease symptoms could not be observed during the short duration of the experiment, and we did not attempt to re-isolate the fungus from seedlings, these results indicate that $M$. maydis can potentially be seed transmitted, suggesting the continued need for careful phytosanitary measures to prevent its movement with seeds. Further work is needed to clarify the risk of seed transmission from naturally contaminated seeds.

Potential for seed transmission of Xanthomonas vasicola pv. vasculorum on maize collected from fields in the United States S. ARIAS (1), C. C. Block (1), D. A. Mayfield (1), K. D. Broders (2), T. A. Jackson-Ziems (3), G. P. Munkvold (1), (1) Iowa State University, Ames, IA, USA; (2) Colorado State University, Fort Collins, CO, USA; (3) University of Nebraska-Lincoln, Lincoln, NE, USA

Bacterial leaf streak of maize, caused by Xanthomonas vasicola pv. vasculorum (Xvv), was first reported in the U.S. in 2016 and spread quickly to multiple states, leading to speculation that it was introduced on seeds. However, the potential for seed transmission of $X v v$ is unknown. A preliminary study demonstrated that $X v v$ was readily transmitted to seedlings from seeds that had been soaked in concentrated bacterial suspensions, but seed transmission from naturally-contaminated seeds has not been shown. In 2016, we obtained open-pollinated seeds from 37 moderately to heavily-diseased commercial production fields in Colorado, Nebraska, and Iowa, but were able to isolate live Xvv cells from just two of the 37 seed lots. In an initial grow-out study, 4000 seeds from a popcorn field with disease incidence of approximately $90 \%$ infected plants were planted in a greenhouse and grown for four weeks. No disease symptoms were observed in this period, but TaqMan PCR results of 10-seedling pooled samples suggested that $X v v$ may have been present, but asymptomatic, in seedlings. Twenty samples had $\mathrm{Ct}$ values $<31$, and two samples were below $\mathrm{Ct}=24$, in the range of the positive controls. Although the risk of seed transmission seems to be low, it is critical to evaluate the potential for seed-to-plant transmission from naturally 
diseased fields in order to protect trade and prevent movement of the pathogen if seed is proven as a pathway. Additional grow-out plantings are in progress.

'Candidatus Liberibacter asiaticus' cells remain viable in citrus seeds for several months

M. V. MERFA, E. Pérez-López, L. De La Fuente, Department of Entomology and Plant Pathology, Auburn University, Auburn, AL, USA

Citrus huanglongbing (HLB) is currently the most devastating citrus disease worldwide, and in the US it is associated with the heretofore unculturable bacterium 'Candidatus Liberibacter asiaticus' (CLas). Although this pathogen is not seed-transmitted, several reports have shown that CLas can be found in citrus seeds, and our research group has been using seeds collected from symptomatic fruits as initial inoculum for research related to CLas. Thus, the goal of our study was to determine for how long CLas is viable in seeds of fruits stored in the laboratory at different temperatures. As a measurement of CLas viability, transcripts of the rpoB gene were quantified by RT-qPCR at different time points using fruits from trees showing HLB symptoms. The gene coxl from plant was used as internal control. We found that CLas remained viable in seeds for a period of three to six months after infected citrus fruits were sent to our laboratory in Alabama from Florida, Texas and Colombia. On the other hand, rpoB transcripts were not detected in control CLasinfected seeds when ampicillin was added. Factors influencing fruit decay reduced CLas viability. This is the first study using mRNA to assess viability of CLas in plant material, and results showed that this method could be applied to other viability studies of this microorganism.

Automated spore capture and decision support for air borne disease control

N. BOONHAM (1,2), W. Charlton (3), I. Brittain (1), D. McCluskey (4), R. Kaye (4), M. Andreou (5), (1) Fera Science Ltd, York, UNITED KINGDOM; (2) Newcastle University, Newcastle upon Tyne, UNITED KINGDOM; (3) Bayer Crop Science, Cambridge, UNITED KINGDOM; (4) University of Hertfordshire, Hatfield, UNITED KINGDOM; (5) Optisense Ltd, Horsham, UNITED KINGDOM

Precision farming approaches to disease control require measurements of disease spread into the crop, enabling precise actions to be made. Frequently spraying is done prophylactically because fungicides have a more powerful preventative mode of action that curative properties. To enable more precise timing of spraying in wheat crops we developed an automated instrument (SporeSentry), capable of performing isothermal DNA amplification tests based on LAMP chemistry. The instrument is battery powered and facilitates 7-day autonomous sampling of airborne inoculum trapped using a novel cyclone air handling system. DNA is extracted from the airborne inoculum within a microfluidic liquid handling consumable, which then transfers the DNA to reaction tubes, pre-loaded with lyophilised reagents. LAMP tests are completed, and the results are reported using the phone network directly into a disease forecasting system enabling near real-time risk prediction. The instrument can perform up to 8 independent tests on each DNA sample, and LAMP assays are available for a range of diseases and crops. In the 2018 growing season, the instrument will be used in wheat crops alongside conventional spore sampling devices enabling performance comparisons of SporeSentry with established qPCR tests for septoria leaf blotch, brown and yellow rust and enabling exploration of improvements in disease control.

Sporulation and dispersal of the biological control agent Aspergillus flavus AF36 under field conditions in nut crops in California M. T. Garcia-Lopez, J. Moral, R. JAIME, R. Puckett, M. A. Doster, T. J. Michailides, University of California, Davis/Kearney Agricultural Research and Extension Center, Parlier, CA, USA

Aflatoxins, produced by Aspergillus section Flavi, are potent natural carcinogens and widely regulated in foods and feeds. California nut crops are occasionally contaminated with aflatoxins. The biocontrol based on atoxigenic strains of $A$. flavus is a proven method to reduce aflatoxin contamination in crops and is approved for use in nut crops in California. However, a significant portion of treatments may not be fully successful due to poor sporulation or loss of the product. To optimize biocontrol treatments in nut crops, information about the epidemiology of the atoxigenic strain is essential. The objectives of present studies were to describe the behavior of the biocontrol agent and determine optimal conditions for sporulation and dispersal in orchards. The dispersal of the fungus across vertical and lateral dimensions from the source was studied and results indicate that the fungus successfully spreads upwards and laterally, but the rate of dispersal was exponentially inverse to both height and distance. Rapid sporulation of the product is required to avoid product loss and for optimal dispersal. Our results also indicate that the product has optimal sporulation at a soil water content between 13 and 18\%. Product under suboptimal conditions had delayed sporulation and was exposed to arthropod predation (Oniscidea spp. and ants), while on soils with excess water content it decayed. This information is useful to optimize biocontrol treatments in nut crops.

Strawberry runner colonization by Fusarium oxysporum f. sp. fragariae

A. M. PASTRANA LEON (1), D. C. Campbell (2), T. R. Gordon (3), (1) Post Doctoral Scholar, DAVIS, CA, USA; (2) University of California, Davis, DAVIS, CA, USA; (3) Department of Plant Pathology, University of California - Davis, Davis, CA, USA

Strawberry production in California requires coordinated action between high-elevation nurseries and fruit production fields. At high-elevation nurseries, mother plants are established in the spring that produce runners (= stolons), which rise to new plants (= daughter plants). In the autumn, daughter plants are used to establish plantings in commercial fruit production fields in coastal regions of California. Fusarium wilt of strawberry, caused by the soilborne fungal pathogen Fusarium oxysporum f. sp. fragariae (Fof), is a growing threat to the strawberry production industry worldwide. Symptoms of the disease typically include stunting, reduced productivity, necrosis, wilting, crown discoloration, and eventual plant death. One possible explanation for the increasing incidence of Fusarium wilt in fruit-production fields is the introduction of the pathogen from high-elevation nurseries via infected transplants. The present study confirmed that $F$ of moves through stolons of infected mother plants and infects daughter plants without inducing visible symptoms. This provides a potential mechanism for movement of the pathogen from high elevation nurseries to production fields that were not previously affected by Fusarium wilt. Preliminary results suggest there may be a difference in colonization frequency between strawberry cultivars.

Fusarium pseudograminearum anti-apoptosis gene FpBirl is required for conidiation and pathogenesis

L. CHEN (1), X. Geng (2), Y. Ma (2), Y. Hou (2), H. Li (2,3), (1) Department of Plant Pathology, Henan Agricultural University, Zhengzhou, CHINA;

(2) Henan Agricultural University, Zhengzhou, CHINA; (3) Department of Plant Pathology, Henan Agricultural University, Zhengzhou, CHINA

Apoptosis plays crucial roles in physiological processes from development to adaptive responses. Certain aspects of apoptosis have been recently verified in eukaryotic microbes, but not in Fusarium species yet. Here we identified a FpBirl in Fusarium pseudograminearum, a homolog of Saccharomyces cerevisiae BIR1 which is the unique inhibitors of apoptosis protein. Two BIR domains at the N terminal of FpBirl protein were identified. FpBirl was up-regulated during both early and later infection. Compared with the infection on susceptible wheats at early stage which is overexpressed approximately 3-fold, FpBirl was induced over 5-fold in the incompatible interaction. Two FpBirl deletion mutants were obtained by the PEG-mediated protoplast stable transformation, and more apoptosis cells were observed in $\Delta$ fpbirl. $\Delta$ fpbirl mutated mycelia showed similar growth rates to that of WT, but was thinner and less branched under microscopic examination. Conidia of $\Delta f$ pbirl was much less than that of the WT, and only 
$24 \%$ of the mutants conidia was visible or germinated very short germ tubes at $6 \mathrm{~h}$, while approximately $50 \%$ of WT conidia had germinated and produced germ tubes longer than the width of conidia under the same conditions. Furthermore, deletion of FpBirl gene reduced the virulence in the compatible interaction. Upon the wheat coleoptiles infected with mycelia, the lesion sizes of $\Delta f p b i r l$ were reduced to $50 \%$ of WT at $3 \mathrm{dpi}$. Under a pot inoculation experiment, the wheat seedlings inoculated with $\Delta$ fpbirl remained healthy at 14 dpi, while almost all wheat seedlings were infected by the WT. These results suggested that FpBirl is involved in mycelial morphology, conidial production and germination, and virulence of $F$. pseudograminearum.

\section{Downy mildew (Hyaloperonospora parasitica) pathotypes in Australia}

A. MOHAMMED (1,2,3), M. You (1,3), M. Barbetti $(1,3)$, (1) School of Agriculture and Environment Faculty of Science, The University of Western Australia, Perth, AUSTRALIA; (2) Department of Plant Protection, Faculty of Agriculture, University of Kufa, Najaf, IRAQ; (3) The University of Western Australia Institute of Agriculture, Faculty of Science, Perth, AUSTRALIA

Downy mildew disease, caused by the pathogen Hyaloperonospora parasitica, is endemic in Brassica growing regions worldwide. The disease particularly effects younger plants and can reduce crop productivity, even when plants are infected at a later stage of growth. Isolates of $H$. parasitica collected initially in 2006-2008 from Western Australia and subsequently in 2015-2016 across southern Australia were inoculated onto cotyledons of $>25$ diverse Brassica hosts under controlled environment conditions at $18^{\circ} \mathrm{C}$ day and $13^{\circ} \mathrm{C}$ night with a $12 \mathrm{~h}$ photoperiod. At 7 days post-inoculation, disease severity was assessed on a $0-9$ scale, where $0=$ no disease, $9=$ heavy sporulation, cotyledon collapsed. Host responses ranged from no visible symptom or only a hypersensitive response, to systemic spread and abundant pathogen sporulation in highly susceptible genotypes. There were significant effects of isolates, host genotypes, as well as a significant interaction between both. A group of six Brassica genotypes was identified to be ideal for use as host differentials and then utilized to characterize eight different pathotypes of $H$. parasitica using an octal classification based on the virulence of the isolates across each host differential. This is the first classification of $H$. parasitica pathotypes for Australia. This new ability to define pathotypes provides a basis for monitoring changes in $H$. parasitica populations over time. In particular, it will allow early warning of development of new pathotypes able to overcome any current host resistances deployed commercially in the oilseed and vegetable Brassica industries.

Differential pathogenicity and genetic diversity among Fusarium pseudograminearum isolates from Huang-huai wheat growing region of China H. LI (1), H. Zhou (2), X. He (2), H. Yuan (2), X. Zhang (2), (1) Department of Plant Pathology, Henan Agricultural University, Zhengzhou, CHINA; (2) Dept. of Plant Pathology, Henan Agricultural University, Zhengzhou, CHINA

Fusarium pseudograminearum (teleomorph Gibberella coronicola) is the main causal agent of Fusarium crown rot (FCR) of wheat, an important soilborne disease throughout Huang-huai wheat growing region in China. To date, however, the pathogenicity differentiation and genetic diversity of this pathogen have been rarely reported. In this study, pathogenicity test of $34 F$. pseudograminearum isolates in greenhouse and analysis of genetic diversity of a collection of 192 F. pseudograminearum isolates using universal rice primers (URP) PCR method were carried out. The result of the disease index value ranging from 6.67 to 91.11 indicated the obvious pathogenic differentiation in F. pseudograminearum populations, but not correlated with geographic origin. Further clustering analysis of $192 \mathrm{~F}$. pseudograminearum isolates resolved at least 8 sub-clusters with $87.12 \%$ of the polymorphic loci. For the 8 geographical populations, the genetic diversity within each population is significantly higher than among populations, whereas there might be partial gene flows among different populations. UPGMA dendrogram based Nei's genetic distance indicated that eight populations were divided into two main groups including Hebei and three Henan populations, with Shanxi, Shaanxi and two Shandong populations. In general, both pathogenicity and molecular variation of these isolates were not correlated significantly with geographic origin that would make the controlling of this disease more difficult. These results will contribute to monitoring the dynamic of FCR caused by $F$. pseudograminearum and meanwhile providing a guideline for plant breeding efforts and developing other disease management strategies aimed at reducing FCR in Huang-huai wheat growing region of China.

Verticillium dahliae from asymptomatic hosts likely emerged from sympatric potato crops in the Columbia Basin of Washington D. L. WHEELER (1), J. K. S. Dung PhD (2), D. A. Johnson (1), (1) Washington State University, Pullman, WA, USA; (2) Oregon State University, Madras, OR, USA

Verticillium dahliae is a pathogen and an endophyte. The relatedness of isolates from asymptomatic and symptomatic hosts and the contribution of inoculum from asymptomatic hosts is unknown. We tested the hypotheses that: (i) isolates from asymptomatic hosts are genotypically and phenotypically different from isolates from symptomatic hosts; and (ii) there is migration from the former to the latter. $V$. dahliae from symptomatic potato and mint hosts and asymptomatic mustard and grass hosts were genotyped with 10 microsatellite markers. Genotypic diversity and evenness was generally greater among isolates from asymptomatic hosts than symptomatic hosts. Population differentiation, as assessed with discriminant analysis of principal components and analysis of molecular variance, was detected among isolates from asymptomatic hosts and mint $(P \leq 0.01)$ but not potato $(P \geq$ 0.07). Multilocus genotypes from potato were also detected from asymptomatic hosts and unidirectional migration of $V$. dahliae from potato to asymptomatic hosts was more likely than competing scenarios. $V$. dahliae isolates from asymptomatic hosts caused wilt and produced microsclerotia on potato but produced microsclerotia without causing wilt on brown mustard and barley. The origin of $V$. dahliae isolates from asymptomatic hosts in the Columbia Basin of WA is likely potato. The stability, prevalence, and impact of asymptomatic infections needs to be determined to inform disease management strategies.

\section{The Secreted in Xylem Gene Profile of the Spinach Fusarium Wilt Pathogen}

A. BATSON (1), T. Peever (2), L. du Toit (3), (1) Washington State University, Mount Vernon, WA, USA; (2) Washington State University, Pullman, WA, USA; (3) Washington State University, Mount Vernon NWREC, Mount Vernon, WA, USA

The maritime Pacific Northwest (PNW) is the only region of the United States suitable for production of spinach seed, which requires a long day length and cool, dry summers to yield high quality seed. The greatest limitation to spinach seed production in the PNW is Fusarium wilt caused by Fusarium oxysporum f. sp. spinaciae $(F o s)$. There are no known races of Fos and resistance to this pathogen appears quantitative. Unique combinations of 14 effector genes called Secreted in Xylem (SIX) genes, originally identified in F. oxysporum f. sp. lycopersici, have been detected in other formae speciales (ff. spp.). PCR assays were used to target all 14 SIX genes of $>40 F$. oxysporum isolates representing ff. spp. cepae, ciceris, lycopersici, pisi, and spinaciae. Out of 19 Fos isolates tested, 10 had SIX8 and 17 had SIX14. The other ff. spp. had various combinations of 14 SIX genes that have been reported previously. Two and four isolates that were not pathogenic to spinach but were obtained from spinach plants or from soil in which spinach was grown, also tested positive for SIX8 and SIX14, respectively. Phylogenetic analyses of SIX8 and SIX14 DNA sequences indicated that the Fos isolates are divergent from those of other ff. spp. tested. Characterizing the SIX effector profile of Fos will assist with developing molecular diagnostic tools for the pathogen and breeding cultivars with improved resistance to Fusarium wilt. 
Characterization of Fusarium graminearum salicylate hydroxylases and their potential role in wheat pathogenesis G. HAO (1), M. Vaughan (2), T. Naumann (2), S. Mccormick (2), A. Kelly (2), T. J. Ward (2), (1) USDA-ARS, Fort Pierce, FL, USA; (2) USDA/ARS/NCAUR, Peoria, IL, USA

Fusarium graminearum is the major causal agent of Fusarium head blight (FHB), which reduces crop yield and can contaminate grains with various mycotoxins. The phytohormones including salicylic acid (SA) plays an important role in regulating plant resistance against pathogens. Comparative genomic analyses of three North American F. graminearum populations (NA1, NA2 and NA3), identified six putative salicylate hydroxylase (Sah) homologs present in all three populations, and one unique Sah homolog, designated FgSahA, only present in strains belonging to the NA2 population. Wheat $\mathrm{FHB}$ assays with $\mathrm{FgSahA}$ deletion mutants $(\mathrm{Fg} \Delta \mathrm{SahA})$ indicate that $\mathrm{FgShaA}$ plays a modest but significant role in virulence. Gene expression data showed that transcription of SA defense signaling marker genes including PR1, PR1a and PR2 were significantly upregulated in wheat heads inoculated with $\mathrm{Fg} \Delta \mathrm{SahA}$ compared to the wild type strain, although no significant differences in SA concentrations were observed. Attempts to determine if FgSahA is an active salicylate hydroxylase by enzyme assay have been inconclusive. Recombinant FgSahA did not degrade SA using colorimetric plate assay, but this may be due to its inability to fold correctly as indicated by its poor solubility. Expression studies also showed that the FgSahA and another homolog (FgSahB) were highly induced by addition of SA in media. Functional characterization of salicylate hydroxylases in F. graminearum will improve our understanding of how these pathogens manipulate plant defenses and cause FHB. Furthermore, understanding population differences may reveal population specific adaptions and novel control measures.

\section{Managing Pratylenchus brachyurus in agricultural integrated systems using the tropical forage legume 'Estilosantes Campo Grande' in Brazil} C. FERNANDES, J. Verzignassi, C. Queiróz, T. Pereira, Embrapa, Campo Grande, BRAZIL

Losses caused by Pratylenchus brachyurus $(\mathrm{Pb})$ has become increasingly common in soybean, maize and cotton crops in Brazil, due to the use of crops in succession or rotation susceptible to the nematode. Currently, Crotalaria spp. has been the main choice of resistance to Pb. This study aimed to evaluate the hostability of the forage legume 'Estilosantes Campo Grande (ECG)', a multiline composed of improved genotypes of Stylosanthes capitata (Sc) and S. macrocephala (Sm) to Pb. Two trials were carried out at Embrapa Beef Cattle, Campo Grande-MS, Brazil. The first one was sown in pots containing $\mathrm{Sc}$ and $\mathrm{Sm}$. After 30 days from emergence (DAE), 1000 specimens of $\mathrm{Pb}$ were inoculated and 90 days later, the number of $\mathrm{Pb}$ in the root mass and soil was assessed, and the reproduction factor (RF) was calculated. RFs ranged from 0.01 to $0.02 \mathrm{for} \mathrm{Sc}$ and Sm, respectively, compared to the soybean cultivar Vmax RR, with RF=7.22. In another trial, $\mathrm{Sc}, \mathrm{Sm}$ and the controls Crotalatria spectabilis and soybean Vmax RR were sown in a natural infested area, with a history of high $\mathrm{Pb}$ populations. After $90 \mathrm{DAE}$, the density of $\mathrm{Pb}$ in the roots mass of Sc and Sm behaved similarly to $C$. spectabilis, with 5.0, 0.0 and 17.5 specimens of $\mathrm{Pb} / 10 \mathrm{~g}$ of roots, respectively. For soybean Vmax RR, 3229 individuals of $\mathrm{Pb}$ were observed in the same root volume. Thus, ECG can be considered a bad host for this nematode, being an excellent option for the management of Pb in infested areas in Brazil.

\section{Distribution and colonization of human opportunistic pathogen of Fusarium oxysporum in tomato}

\section{J. WANG, W. H. Chung, National Chung Hsing University, Taichung, TAIWAN}

Fusarium oxysporum $(\mathrm{Fo})$ is widely distributed over soils, plant tissues, organic subtracts and water. Fo could cause Fusarium wilt in plants, and also make diseases on human such as keratitis, ringworm of nails and allergy etc. Fo is often found in the patient with low immunity. Thus, it is possible that the patients with low immunity have high risk to be infected by $F o$. The Human Pathogens On Plants (HPOPs) are pay attention due to the risk of $F o$ on agricultural products. Moreover, the human opportunistic pathogen and plant pathogen of $F o$ were closely based on molecular phylogenetic analyses. Consequently, the human opportunistic pathogen of $F o$ might have chance to penetrate into the plant tissues. It is necessary to carry out whether the human opportunistic pathogen of $\mathrm{Fo}$ can penetrate into agricultural products. For detecting the human opportunistic pathogen of $\mathrm{Fo}$, the specific primers (primer5/ST33-R) were developed based on the sequences polymorphism of intergenic spacer region (IGS) of ribosomal DNA from a human opportunistic pathogen of $F o$ isolates. In addition, the MCC2074 isolate was used to study the colonization and distribution in tomato plants and fruits. Results showed that the MCC2074 could colonize in tomato tissues by wound inoculation without symptoms showing. However, the MCC2074 was limited in roots, peg, hypocotyl and the basal stem tissues by using soil infestation method. The colonization of MCC2074 in tomato and cucumber fruits indicated that the MCC2074 could penetrate into fruits tissue and colonize in pericarp wall, locular cavity, and columella.

Effector diversity within the US-23 clonal lineage of Phytophthora infestans

M. SUDERMANN (1), M. Armstrong (2,3), J. Tze-Yin Lim (4), I. Hein (2,3), C. D. Smart (5), (1) Plant Pathology and Plant Microbe Biology Section, Cornell University, Geneva, NY, USA; (2) Cell and Molecular Sciences, The James Hutton Institute, Dundee, UNITED KINGDOM; (3) Division of Plant Sciences at the James Hutton Institute, University of Dundee, Dundee, UNITED KINGDOM; (4) Columbia University, New York, NY, USA; (5) Plant Pathology and Plant-Microbe Biology Section, Cornell University, Geneva, NY, USA

Phytophthora infestans causes late blight in tomatoes and potatoes. The pathogen was responsible for the Irish Potato Famine in the 1840s, and continues to have significant economic impacts world-wide. The goal of this study is to understand population diversity within the US-23 clonal lineage, which is currently the predominant lineage in the USA. Previous work from our lab based on a genome-wide SNP analysis has shown that diversity exists within this lineage. In a continuation of the work, we are now studying effector diversity within the US-23 clonal lineage using a target enrichment technique called PenSeq, which captures over 500 effectors from the $P$. infestans genome. The effector sequences of 12 isolates of $P$. infestans from the US-23 clonal lineage were re-sequenced through PenSeq. Preliminary results showed relatively little effector diversity when comparing presence or absence of known avirulence determinants within each isolate. For example, Avrl doesn't appear to be present in any of the 12 US-23 clonal lineage isolates tested. $A v r 3 a$ and $A v r 3 b$ appear to be present in all of the isolates tested. AVR3a is represented by two different alleles and the isolates showed some differences in alleles. Two of the isolates contained only the EM form, while the remaining isolates were heterozygous for EM and KI. This proves to be interesting because the encoded protein AVR3 $\mathrm{a}^{\mathrm{KI}}$ activates resistance in potatoes but AVR3 $\mathrm{a}^{\mathrm{EM}}$ does not. Detached leaf assays will be conducted to validate the preliminary results. RNA-seq will be used to determine expression levels of each effector in each isolate. A complete analysis of the entire effector repertoire of the 12 US- 23 isolates is in progress.

Virulence testing of South African Venturia inaequalis inoculum using qPCR

T. A. KOOPMAN (1), J. C. Meitz (2), B. Cecilia (1), K. Tobutt (1), C. L. Lennox (2), (1) ARC Infruitec-Nietvoorbij, Stellenbosch, SOUTH AFRICA; (2) Stellenbosch University, Stellenbosch, SOUTH AFRICA

Venturia inaequalis, the causal agent of apple scab, is one of the most important diseases of apple (Malus pumila), locally and internationally. There is eighteen known races of $V$. inaequalis and differences in single spore isolate virulence have been observed. Isolates had co-evolved with the cultivar and can only infect the cultivar it originates from. The objective was to determine virulence of $V$. inaequalis from South Africa using single spore isolates from different cultivars. Samples were collected from several cultivars in four apple growing regions. Lines were generated from single spores and 
conidia produced on cellophane media. Virulence testing of 17 single spore isolates was performed on 'Golden Delicious'/'Royal Gala' ('GD'/'RG'), 'Fuji' and the rootstock 'M793', with visual symptoms evaluated after 14 days. A qPCR technique was used to quantify the amount of fungal DNA in the leaf tissue after 7 and 14 days post- inoculation. Results showed differential infection ability and not all isolates could infect and sporulate on all the cultivars tested. Some single spore isolates caused chlorotic spots without any sporulation and might have presented a delayed sporulation if incubated for 21 days. The fungal DNA concentration was the lowest in inoculations of 'GD'/'RG' and highest in 'M793'. The single spore isolate LOSL 206-1, from 'Cripps Red', was the most virulent isolate on 'RG' and isolate OBL 210-1, from 'Braeburn', the most virulent on 'M793'. Isolates that caused chlorosis also had high amounts of fungal DNA present in leaf tissue. Single spore isolates differed among themselves with respect to their ability to infect and sporulate on different cultivars. The results of the visual scoring and qPCR were comparable and the qPCR method showed clear advantages for the detection and quantification of the pathogen in asymptomatic apple leaves.

Determining the warm-season turfgrass host range of Curvularia malina sp. nov., the ink spot pathogen H. RENFROE, M. Tomaso-Peterson, Mississippi State University, Mississippi State, MS, USA

Curvularia malina is a novel pathogen causing conspicuous chocolate brown to black patches, referred to as ink spot, in bermudagrass and zoysiagrass on golf course putting greens and fairways in the southeastern United States. Black, eye-spot lesions are produced on leaves of affected plants. Three additional warm-season turfgrasses (WST) were included in a study to determine the host range of $C$. malina sp. nov. Surface-disinfested seed of centipedegrass, seashore paspalum, bermudagrass, zoysiagrass, and nodes of St. Augustinegrass were planted in C. malina-infested soilless mix. Thirteen inoculated and five non-inoculated replicates were included for each host. Disease severity, based on percent foliar necrosis, and shoot and root dry weights were determined 8 weeks after emergence. Zoysiagrass displayed the greatest disease severity at $54 \%$ followed by bermudagrass with $13 \%$ severity. Shoot and root dry weights of centipedegrass and seashore paspalum were reduced significantly when compared to their non-inoculated controls. Foliar lesions were observed on leaf sheaths and stolons of St. Augustinegrass; however, no differences in shoot and root dry weights were observed. C. malina was isolated from foliar tissue of all WST except seashore paspalum. These results indicate centipedegrass and St. Augustinegrass may serve as hosts under natural conditions and extends the host range of C. malina, the ink spot pathogen.

Weedy grasses as a potential reservoir of the pathogen causing bacterial leaf streak of wheat K. E. LEDMAN, R. D. Curland, C. A. Ishimaru, R. Dill-Macky, University of Minnesota, St. Paul, MN, USA

Bacterial leaf streak (BLS) of wheat, caused by Xanthomonas translucens pv. undulosa, has been prevalent in Minnesota wheat fields for the past decade. Sources of the pathogen include infested seed and crop debris. Weeds are also considered a potential inoculum source, but little is known about the genetic similarities among isolates of $X$. translucens from these different hosts. The objectives of this study were to isolate $X$. translucens from poaceous weeds in Minnesota, determine pathogenicity of weed isolates on wheat and barley, and to evaluate phylogenetic relationships of isolates from weeds and those from small grains. Bacteria were isolated from 130 samples representing 12 grass species collected in and around wheat fields. Isolates exhibiting characteristic colony morphology on Wilbrink's medium were purified and evaluated further. The majority (68/98) of the isolates were identified as $X$. translucens by $16 \mathrm{~S}$ rDNA sequencing. A subset (47) of these isolates were infiltrated into leaves of wheat and barley seedlings and found to cause disease. Phylogenies of the weed isolates were constructed using sequence alignments of four housekeeping genes $(r p o D, d n a K, f y u A$ and $g y r B)$. Phylogenies from multilocus sequence analysis show that these isolates are closely related to known $X$. translucens pathovars, most commonly $X$. translucens pv. undulosa. Our findings suggest that poaceous weeds serve as a reservoir of inoculum for the bacterial pathogen inciting BLS of wheat.

\section{Determinants of aggressiveness in Fusarium graminearum}

M. SALAZAR, F. Kolb, S. X. Mideros Mora, University of Illinois at Urbana-Champaign, Urbana, IL, USA

Fusarium graminearum, the causal agent of Fusarium head blight (FHB) of wheat, is a devastating pathogen that causes yield and quality losses to its host. Other than deoxynivalenol (DON), little is known about pathogenesis compounds used by F. graminearum to infect wheat. To identify essential fungal pathogenesis genes and determine whether host resistance selects for the isolate aggressiveness, a paired strategy of isolate and transcriptome characterization of naturally infected wheat lines was implemented. A total of 197 F. graminearum infected samples were collected in 2016 from research sites at five Illinois locations using University of Illinois soft red winter wheat improvement program plots. Wheat lines used as a source of samples included an FHB resistant line, a moderately resistant line, a moderately susceptible line, and two highly susceptible lines. RNA extraction of twelve selected samples yielded high-quality RNA. The results of differential fungal gene expression between moderately resistant, moderately susceptible, and highly susceptible interactions will be presented. To determine functional pathogen aggressiveness, pathogen strains isolated from the same samples used for RNAseq were used to inoculate wheat cultivars with varying levels of resistance in field and greenhouse experiments. Statistical analysis is currently underway for both field and greenhouse aggressiveness assays. Preliminary data analysis suggests the existence of two types of isolates. The first group was composed of isolates that had low levels of aggressiveness on both susceptible and resistant wheat lines. The second group was composed of isolates that were significantly more aggressive on a susceptible wheat line but not aggressive on a resistant wheat line.

Uncovering host range for the maize pathogen Harpophora maydis

O. DEGANI (1,2), S. Dor (1,2), S. Graph (1), M. Dafny-Yelin (1), O. Rabinovitz (3), (1) Migal - Galilee Research Institute, Kiryat Shmona, ISRAEL; (2) Tel-Hai College, Tel-Hai, ISRAEL; (3) Extension Service, Ministry of Agriculture and Rural Development, Beit Dagan, ISRAEL

The fungus Harpophora maydis is a soil-borne and seed-borne vascular wilt pathogen that causes severe damage to sensitive maize hybrids throughout Israel, Egypt, India and other countries. It can undergo pathogenic variations and survive as spores, sclerotia or mycelia on plant residues. Maize, Lupinus termis (lupine) and apparently cotton are the only known hosts of H. maydis. Identification of other plant hosts that can assist in the survival of the pathogen is an important step in restricting disease outbreak and spread. Here, a greenhouse pathogenicity trial series accompanied by Real-time PCR tracking confirmed the presence of the fungus' DNA inside the roots of cotton plants grown on infested soil. Moreover, we identified the presence of $H$. maydis in Setaria viridis and in watermelon (Malali cv.). Infected watermelon plants had delayed emergence and development, were shorter, and had reduced root and shoot biomass. $H$. maydis infection damaged the root biomass and phenological development of cotton plants but caused only mild symptoms in S. viridis. In liquid minimal medium, H. maydis growth was enhanced in the presence of maize or cotton root powder. While watermelon root powder had a minor influence on fungal dry weight, it caused a dramatic increase in pathogen laccase production. These findings are an important step towards uncovering the host range and endophytic behavior of $H$. maydis, and encourage expanding this evaluation to other plant species.

Virulence profiling of Phytophthora sojae based on genomic signature of avirulence genes

G. ARSENAULT-LABRECQUE (1), H. Sonah (1), A. Lebreton (2), C. Dussault-Benoit (2), G. Marchand (3), A. G. Xue (4), B. J. Knaus (5), N. J. Grunwald (6), R. R. Bélanger (2), (1) Université Laval, Québec, QC, CANADA; (2) Université Laval, Quebec, QC, CANADA; (3) Agriculture and 
Agri-Food Canada, Harrow, ON, CANADA; (4) Agriculture and Agri-Food Canada, Ottawa, ON, CANADA; (5) USDA-ARS, Horticultural Crops Research Unit, Corvallis, OR, USA; (6) USDA ARS, Corvallis, OR, USA

Phytophthora sojae, causing Phytophthora Root Rot (PRR), has been present in Canada since 1950. The expansion of Canadian soybean over the years has given this pathogen a new niche to establish its devastating presence. The best method to control its spread is through the use of soybean varieties carrying resistance genes (Rps) that provide immunity against $P$. sojae isolates with the corresponding avirulence gene $(A v r)$. Breeders are thus confronted with the need to introgress Rps genes based on the $P$. sojae pathotypes found in the environment; however, this information is constantly incomplete because of the rapid evolution of the pathogen, and confounded by unwieldy phenotyping methods. This project aims to determine the presence and distribution of virulence profiles (pathotypes) of $P$. sojae in Canada, based on the seven most important Rps-Avr relationships. For this purpose, a collection of $31 \mathrm{P}$. sojae isolates, representing the most common pathotypes found in Canadian fields, were targeted for whole-genome sequencing. Different gene mutations directly linked to seven Avr genes from P. sojae, mostly gene suppressions and SNPs, were discovered. These findings corroborate some previous reports, and highlight new findings, while demonstrating specificity linked to the geographical context. Based on these genomic signatures, a multiplex PCR is being developed to help breeders in the selection of specific resistance genes against PRR.

Emerging understanding of the pathogenesis of Rhizoctonia zeae in row crops S. KODATI, A. O. Adesemoye, University of Nebraska Lincoln, North Platte, NE, USA

Rhizoctonia zeae is a multinucleate Rhizoctonia, reported as a major soil borne pathogen in turf but not well reported in row crops. While a literature search showed a brief report of $R$. zeae on corn, there is none for soybean or wheat. In a statewide survey of Rhizoctonia spp. in row crops in Nebraska from 2016-2017, a surprisingly large number of $R$. zeae isolates were recovered. The objective of this study was to determine the taxonomic diversity of the recovered R. zeae isolates and examine their pathogenicity on corn, soybean, and wheat. A total of 71 R. zeae isolates (34 in 2016 and 37 in 2017 ) were recovered from 17 fields in 9 counties of the state in 2016 and 2017. Isolates were identified through morphology and sequencing with internal transcribed spacer (ITS) 4 and 5 primers. Pathogenicity tests were conducted by inoculating all isolates onto soybean through a repeated rolled towel assay and greenhouse assay methods. Visual rating of symptoms on the root and crown was done to determine disease severity. Strains that were virulent on soybean were also screened for pathogenicity on wheat and corn. Among a total of 71 R. zeae isolates collected, $60(84.5 \%)$ were pathogenic on soybean. Among them, eleven strains showed high aggressiveness and virulence on all the three crops at levels that were not significantly different $(\mathrm{p}=$ 0.05 ) from the virulent Rhizoctonia solani. Multiple strategies are being explored to further understand pathogenesis in $R$. zeae.

Pathogenicity and host specialization of Ceratocystis spp. associated with rapid `ōhi'a death (ROD) in Hawai'i L. S. Sugiyama (1), B. Luiz (2), W. Heller (3), L. M. KEITH (1), (1) USDA-ARS, Hilo, HI, USA; (2) University of Hawaii at Hilo, Hilo, HI, USA; (3) University of Hawaii at Manoa, Hilo, HI, USA

Two new species of Ceratocystis are attacking and killing 'ōhi'a (Metrosideros polymorpha), Hawai'i's most abundant and ecologically important native tree. On Hawai`i Island, hundreds of thousands of trees have died from these pathogens, a phenomenon known as Rapid `Ōhi`a Death (ROD). We report here a study to determine the host specialization of these two pathogens. Hosts evaluated included M. polymorpha, Syngonium podophyllum, Platanus $\times$ acerifolia (London plane), Colocasia esculenta (taro), Coffea arabica (coffee) and Ipomoea batatas (sweet potato). Pathogenicity tests were conducted using stem injection or a stem flap wound method with inoculum prepared from 7 day-old cultures. Most M. polymorpha plants inoculated with `ōhi`a isolates wilted 14 to 28 days post-inoculation (DPI) and died 28 to 365 DPI. In contrast, disease was not observed on Syngonium, London plane, taro, coffee or sweet potato plants inoculated with `ōhi'a isolates. Our results indicate that the two Ceratocystis spp. from 'ōhi a specifically cause disease on their respective host. However, it is not yet known if other plant species may serve as asymptomatic carrier hosts or if there is potential for the pathogens to develop new host specificities by hybridizing with other strains. In order to protect Metrosideros forests state-wide and throughout the Pacific, expanded investigation of the host range, genetic diversity and distribution of Ceratocystis species causing ROD is critical.

Characterization of the infection cycle of Phytophthora betacei during disease development on tree tomato (Solanum betaceum) N. GUAYAZAN (1), M. Mideros (1), S. Schornack (2), S. Restrepo (1), (1) Universidad de los Andes, Bogota, COLOMBIA; (2) Sainsbury Laboratory, University of Cambridge, Cambridge, UNITED KINGDOM

Phytophthora betacei is a recently described oomycete plant pathogen closely related to Phytophthora infestans sensu stricto. This plant pathogen infects tree tomato (Solanum betaceum) crops, but is not able to infect potatoes or tomatoes, the main hosts of $P$. infestans. The aim of this study was to characterize the infection cycle of $P$. betacei using microscopy and molecular approaches. To this end, susceptible tree tomato plants were inoculated with a sporangial solution, and the progression of the disease was monitored based on six epidemiological parameters. The results indicated $P$. betacei displays a highly variable disease phenotype. To understand the infection cycle at the cell and molecular level, one aggressive strain was selected and inoculation assays were performed. Samples collected from the inoculated plants 3, 6, 9, 12, 24, 48, 72, and 96 hpi were visualized using Scanning Electron Microscopy (SEM) and light Microscopy (trypan blue staining). Progression of the disease was validated with qPCR by assessing the expression two infection-stage specific markers: Hmpl and Cdc14. Results indicated that P. betacei differs from P. infestans in that the infection cycle of the former is characterized by a longer biotrophic stage, greater lesions and greater sporulation capacity when evaluated on the same host. Varying levels of expression were detected for both genes along the infection cycle, and their profile was consistent with the results observed on the whole plant inoculations. Furthermore, transcriptomic analyses on time course inoculation samples revealed a highly regulated pathogen and host dynamics. De novo assembly of $P$. betacei transcriptome enabled identification of conserved infection-promoting as well as unique novel effector genes. This study provides insights into the interaction between $P$. betacei and S. betaceum.

Two type III effectors are sufficient to transform nonpathogenic or pathogenic bacteria into host-specific gall-forming pathogens G. Nissan (1), L. Chalupowicz (2), G. Sessa (3), I. BARASH (1), S. Manulis-Sasson (4), (1) Tel Aviv University, Tel Aviv, ISRAEL; (2) ARO, The Volcani Center, Rishon LeZion, ISRAEL; (3) Tel Aviv University, Tel-Aviv, ISRAEL; (4) ARO The Volcani Center, Rishon LeZion, ISRAEL

Pantoea agglomerans $(\mathrm{Pa}$ ), a widespread commensal bacterium, has evolved into host-specific gall-forming pathogens on beet (Beta vulgaris) and gypsophila (Gypsophila paniculata) by acquisition of a plasmid-born type III secretion system (T3SS) and effectors (T3Es). The pathovar Pa pv. betae (Pab) elicits galls on beet and gypsophila whereas Pa pv. gypsophilae (Pag) elicits galls on gypsophila and a hypersensitive response (HR) on beet. $\mathrm{HsvB}$ and $\mathrm{HsvG}$ are paralogous host specific T3Es that present in both pathovars and act as transcription factors in beet and gypsophila, respectively. PthG incites virulence in gypsophila and HR on beet, whereas PseB is exclusively present in Pab. The cosmid pHIR11 containing the T3SS of Pseudomonas syringae was mobilized by triparental mating into the nonpathogenic bacteria Pa 3-1 and Pseudomonas fluorescence (Pf). HsvB, HsvG, PthG and PseB were then mobilized either individually or in various combinations into Pa3-1 and $P f$, as well as into the pathogenic bacteria Erwinia amylovora, Dickeya solani and Xanthomonas euvesicatoria, respectively, followed by inoculation into beet and gypsophila. Results obtained indicated 
that the assemblage of HsvB and PseB incited galls on beet, whereas the assemblage of HsvG and PthG caused galls on gypsophila in all the tested nonpathogenic or pathogenic bacteria. Thus, different combinations of two T3Es were sufficient to elicit galls on either beet or gypsophila in a host-specific manner.

Effect of seedborne Alternaria infectoria on susceptibility of wheat seedlings to Fusarium pseudograminearum

S. C. LAMPRECHT, E. Retief, Y. Tewoldemedhin, Agricultural Research Council-Plant Health and Protection, Stellenbosch, SOUTH AFRICA

Seedborne fungi can affect the susceptibility of seedlings to soilborne pathogens. Alternaria infectoria is the dominant seedborne fungus on many commercial wheat cultivars in South Africa. This study investigated the effect of seedborne A. infectoria on the susceptibility of wheat seedlings to Fusarium pseudograminearum, an important soilborne pathogen of wheat in South Africa. Seedborne A. infectoria was eliminated from seed of 12 wheat cultivars using hot water treatment at $45^{\circ} \mathrm{C}$ for $3 \mathrm{hrs}$. Four isolates each of the two fungi were used to prepare spore suspensions of $2.5 \times 10^{5} \mathrm{spores} / \mathrm{ml}$ for each fungus, as well as a spore suspension containing a mixture of the two fungi also containing $2.5 \times 10^{5}$ spores of each fungus $/ \mathrm{ml}$. Hot water and non-hot water treated seed of the 12 wheat cultivars were plated on potato-dextrose agar for 5 days to germinate. The seedlings were then dipped in either the Alternaria, Fusarium or the mixed spore suspensions and planted in a pasteurized $\left(83^{\circ} \mathrm{C}\right.$ for $\left.60 \mathrm{~min}\right)$ sand, perlite and soil $(1: 1: 1 \mathrm{mixture})$ growth medium in a glasshouse $\left(25^{\circ} \mathrm{C}\right.$ day, $15^{\circ} \mathrm{C}$ night temperatures). Control seedlings were dipped in sterile water only. Crown rot severity was evaluated 28 days after planting. Results showed that $A$. infectoria did not affect the susceptibility of wheat seedlings to $F$. pseudograminearum and will therefore not interfere with screening of seedlings for resistance/tolerance to F. pseudograminearum.

Establishment a gene silencing system in Verticillium dahliae and identification of a novel gene required for microsclerotia formation and virulence

D. XIONG, Y. Wang, C. Tian, Beijing Forestry University, Beijing, CHINA

The genome of Verticillium dahliae has been sequenced and annotated, but incorrect gene annotations and unidentified transcribed regions persist. In this study, we established a dsRNA-induced gene silencing system with a plasmid carrying two convergent opposing RNA polymerase II promoters in $V$. dahliae. Subsequently, using RNA-Seq combined with reverse-transcription PCR, we identified a novel transcribed gene, Nag1, located in a cluster of putative secondary metabolic genes whose roles remain unknown. Functional analysis of Nag1 by dsRNA-mediated gene silencing revealed that loss of Nag1 significantly decreased fungal growth and conidial production. In addition, Nag1-silenced mutants exhibited obvious defects in microsclerotia formation and fungal virulence. Consistent with phenotypic observation of the reduction in microsclerotia formation, melanin production and expression of genes involved in melanin biosynthesis were markedly reduced in Nag1-silenced mutants. Overall, our data suggest that Nag1 acts as an important regulator of fungal development, pathogenicity, microsclerotia formation, and secondary metabolism in $V$. dahliae.

Pathogenicity and phylogeny of Fusarium oxysporum causing cucurbit wilting in Taiwan

C. C. CHUNG, C. J. Wang, Y. J. Chen, W. H. Chung, National Chung Hsing University, Taichung, TAIWAN

Cucurbitaceae is one of important vegetable and widely distributed over the word. Several important diseases are common during growth season, such as Fusarium wilt, downy meildew and anthracnoe. Among these diseases, the Fusarium wilt caused by $F$. oxysproum $(F o$ ) is most important disease in cucubit production area. Currently, more than 150 formae speciales have been recoded throughout the world. Although formae speciales show the pathogenic specificity on their host, the cross-pathogenicity has been found in different formae speciales. Previous studies domonstrated that certain formae speciales of $F$. oxysporum causing cucurbit wilt could show cross-pathogencity between different cucubit. In this study, the $F$. oxysproum obtained from cucumber, bitter gourd and loofah were used to examine the cross-pathogenicty between the three cucurbits and carried out their phylogeny. The results indicated that $F$. oxysproum isolates from cucubmer and bitter gourd could infect loofah and $F$. oxysporum isolates from loofah could infect cucumber at $20^{\circ} \mathrm{C}$ based on root dipping method. In addition, some of loofah plants could be infected by $F$. oxysporum isolates from bitter gourd at $28^{\circ} \mathrm{C}$ with root dipping method. For phylogeny analysis, the $F$. oxysporum isolates from three cucurbits did not associate with host specificity based on $I G S$ and $E F-1 \alpha$ sequences. However, the nucleotides of secreted in the xylem $(S I X)$ protein 6 could separe the $F$. oxysporum isolates from three cucurbits.

Genomic basis for host adaptation in Puccinia striiformis

C. XIA (1), X. Chen (1,2), (1) Washington State University, Pullman, WA, USA; (2) USDA ARS, Pullman, WA, USA

Stripe rust fungi, Puccinia striiformis f. sp. tritici (Pst) and P. striiformis f. sp. hordei (Psh), are devastating pathogens of wheat and barley, respectively. However, the genomic basis of their host adaptation is not clear. To understand the evolutionary mechanisms of the different formae speciales, genomic DNA and cDNA of a Pst isolate and a $P s h$ isolate were sequenced using next generation sequencing technologies. The assembled genomes with high continuity enabled us to determine their genome differences and understand the evolutionary history of Pst and Psh. The divergence of Pst and Psh, occurred 8.12 million years ago, has been driven by high nucleotide mutation rates. Extensive losses of gene families in both Pst and $P s h$ have occurred separately after the divergence from their most recent common ancestor, in contrast to very few gene family gains, resulting in a large number of formspecific genes. These form-specific genes have unique genomic features compared to the conserved genes, including 1) significantly shorter in length; 2) significantly less expressed; 3) significantly close to transposable elements; and 4) redundant in pathways. Moreover, 116 and 119 genes were found to be exclusively expressed in $P s t$ and $P s h$, respectively. Our data indicate that the different events of gene family losses, resulting in form-specific genes, have separate $P$ st from $P s h$ and that the form-specific genes are responsible for their adaptation to different cereal crops.

Functional analysis of the MSP18 root-knot nematode virulence gene in rice

M. Grossi de Sa (1), A. S. Petitot (1), M. E. Lisei de Sa (2,3), I. Mezzalira (2), M. A. Beneventi (2), D. X. Amora (2), E. V. S. Albuquerque (4), M. F. Grossi de Sa (2), D. FERNANDEZ (1,2), (1) IRD, CIRAD, Univ Montpellier, IPME, Montpellier cedex 5, FRANCE; (2) Embrapa Cenargen, Brasilia DF, BRAZIL; (3) EPAMIG, Uberaba, MG, BRAZIL; (4) Embrapa Recursos Geneticos e Biotecnologia, Brasilia, BRAZIL

Several root-knot nematodes (RKN) species are responsible for rice (Oryza sativa) production losses in Brazil, Asia or Africa. Successful infection is likely achieved by effector proteins produced in the nematode esophageal gland cells and released into host plant cells. Here we show that the Meloidogyne incognita MSP18 esophageal gland cell protein is conserved in the Meloidogyne javanica and Meloidogyne graminicola species infecting rice. The MSP18 gene was upregulated throughout all nematode parasitic stages in rice. Transient expression assays in onion cells suggest that MSP18 is addressed to the cytoplasm of the host cells. Overexpression of MSP18 in rice enhanced M. javanica and M. graminicola reproduction, indicating that the MSP18 protein facilitates RKN parasitism. Transient expression assays in tobacco showed that MSP18 suppressed the INF1-triggered programmed cell 
death, suggesting that MSP18 can interfere with the plant defense pathways. Data obtained significantly broaden our knowledge of molecular players contributing to nematode pathogenicity, and highlight MSP18 as a novel RKN virulence effector able to modulate host immunity.

Molecular interactions that influence virulence contributions of the IPI-O family of Phytophthora infestans effectors S. ABDULLAH (1,2), Y. Lin (3), Y. Chen (4), D. A. Halterman (5), (1) University of Wisconsin, Madison, WI, USA; (2) UW, Madison, WI, USA; (3) University of Wisconsin, Madison, Madison, WI, USA; (4) University of Wisconsin-Madison, Madison, WI, USA; (5) USDA ARS, Madison, WI, USA

Phytophthora infestans, causal agent of potato late blight, is a destructive pathogen that is a frequently recurring problem worldwide. Several resistance genes exist in potato to counter against this pathogen, but the majority has been overcome after introgression into popular potato varieties. The $R B$ gene, derived from Solanum bulbocastanum, has effector recognition specificity to members of the IPI-O family. Recognition of the IPI-O1 allele by $R B$ elicits a hypersensitive resistance response while IPI-O4 can suppress this response. We have carried out several experiments to determine the virulence contributions of IPI-O1 and IPI-O4 during infection, and to identify host proteins involved in IPI-O recognition/suppression using coimmunoprecipitation and yeast two-hybrid. Our results indicate that both IPI-O1 and IPI-O4 contribute to $P$. infestans virulence, but their impact is influenced by the pathogen genotype. Protein interaction studies have identified both cytosolic- and membrane-localized host proteins that interact with IPI-O and will help to elucidate the function of these effectors in pathogen virulence. Together, we hope that our understanding of the function of the ubiquitous IPI-O effector will assist us in identifying or developing improved host resistance genes in potato.

Identification of genomic regions associated with host specificity and aggressiveness in Ceratocystis species

A. FOURIE, M. J. Wingfield, B. D. Wingfield, M. van der Nest, L. de Vos, I. Barnes, Forestry and Agricultural Biotechnology Institute (FABI), University of Pretoria, Pretoria, SOUTH AFRICA

The genus Ceratocystis sensu stricto includes more than 30 species of fungi that are pathogens of fruit and forest trees as well as agronomic crop plants. The type species, C. fimbriata sensu stricto, is host specific and causes black rot on Ipomoea batatas. Ceratocystis manginecans is genetically closely related to C. fimbriata s.s. and causes cankers and wilt on Acacia spp., mango and pomegranate, amongst others, but is not pathogenic to I. batatas. Despite their increasing economic importance, there is a paucity of knowledge regarding the factors that influence the host specificity and pathogenicity of these Ceratocystis spp. In this study, an interspecific cross was made between a C. fimbriata isolate from I. batatas and a C. manginecans isolate from A. mangium. Seventy $\mathrm{F}_{1}$ progeny isolates were selected to investigate the inheritance of pathogenicity, mycelial growth rate and conidial production. Whole genome-based sequence analysis allowed construction of a linkage map that consisted of 467 SNPs, distributed across eight linkage groups and that spanned $1200 \mathrm{cM}$. We subsequently identified one highly significant QTL associated with growth rate on MEA, one associated with pathogenicity on A. mangium and two QTLs associated with pathogenicity on I. batatas. Candidate genes present in the QTLs are currently being characterized. These will be identified by investigating the presence/absence of genes in a species or nucleotide variations between the species.

\section{Investigating host preference of Acidovorax citrulli, the causal agent of bacterial fruit blotch of cucurbits} M. ZHAO, R. R. Walcott PhD, The University of Georgia, Athens, GA, USA

Acidovorax citrulli causes bacterial fruit blotch of cucurbits (BFB), and can be assigned to two groups: I and II. The association of group I and II $A$. citrulli strains with different cucurbit hosts strongly suggests host preference. We observed significant differences in pathogenicity between representative group I and II $A$. citrulli strains on watermelon and melon fruits, but not on foliar tissues. Thus, we hypothesized that $A$. citrulli host specificity occurs in cucurbit fruit, but not foliar tissues. The objective of this study was to assess differences in cucurbit host preference between group I and II $A$. citrulli strains under field conditions. This is important for understanding the factors that control virulence and to develop effective strategies to manage BFB. We planted a mixed plot with four cucurbit species in Tifton, GA USA and initiated a BFB outbreak with representative group I and II $A$. citrulli strains. We observed that $30 \%, 60 \%, 67 \%$, and $71 \%$ of $A$. citrulli-positive watermelon, melon, pumpkin, and squash leaf samples were infected with the group I strain, respectively. However, $100 \%$ of the symptomatic watermelon fruit samples were infected with the group II strain, while $86 \%$ of symptomatic melon fruits were infected with the group I strain. These data support the hypothesis of $A$. citrulli host specificity in cucurbit fruits but not foliage under natural field conditions. Further studies will confirm and explore the nature of this host specificity.

Thioredoxinand glutaredoxin systemsrequired for oxidative stress resistance, fungicide sensitivityand virulenceof Alternaria alternata H. LI, Zhejiang University, Hangzhou Zhejiang, CHINA

This study determines the function of thioredoxins and glutaredoxinsin the phytopathogenic fungus Alternaria alternatavia analyzing mutants from the targeted deletion of genes encoding thioredoxin peroxidase (Tsal), thioredoxin reductase (Trr 1$)$, glutathione reductase $(G l r l)$ and glutathione synthetases (Gsh1 and Gsh2). Trr1, Glr1, Gsh1, Gsh2 but not Tsal are required for growth and conidiation. Reduced growth and conidiation seen in the Trr1 or Glr1 deletion mutant can be restored by glutathione. Deletion mutants showing growth inhibition by oxidantsare defective for $\mathrm{H}_{2} \mathrm{O}_{2}$ detoxification and induce smaller lesions on citrus leaves. Trrl, Glrl but not Tsal also contribute to $\mathrm{NaCl}$ resistance. Glrl is required for sorbitol resistance and responsible for resistance to mancozeb, boscalid but not chlorothalonil fungicides, a novel phenotype that has not been reported in fungi. Trrl is required for resistance to boscalid and chlorothalonil fungicides while Trrl confers susceptibility to mancozeb. Tsal deletion mutant displays wild-type sensitivity to test fungicides. The expression of Tsal and Trr 1 is regulated by the oxidative stress responsive regulators Yap1, Hog1 and Skn7. The expression of Tsal but not $\operatorname{Tr} r 1$ is also regulated by the NADPH oxidase. The results indicate that the capability to resist oxidative stressis required for virulence of $A$. alternata.

\section{A genetic locus determining pathogenicity of Pantoea ananatis}

Y. TAKIKAWA, Y. Kubota, Shizuoka University, Shizuoka, JAPAN

Pantoea ananatis $(\mathrm{Pa})$ is a gram-negative plant pathogenic bacterium. Pa strains are divided into three groups (Group I, II and III) depending on their pathogenicity on rice, onion and melon and ability to induce hypersensitive response (HR)-like reaction in tobacco (Kido et al. 2010). The whole genome analysis and transposon-tagging analysis on one rice isolate SUPP2219 (Group I) suggested that a genetic region spanning ca. $19 \mathrm{~kb}$ in length existed exclusively in the known genomes of plant pathogenic $P a$ strains but not in saprophytic $P a$. Tn-5 insertion in some genes of this region abolished the virulence of the isolate. This genetic region was tentatively named as PASVIL (Pantoea ananatis specific virulence locus). PCR and Southernhybridization tests revealed that PASVIL existed in all of Group I strains examined but not in Group II and III strains. In complementation tests, one large clone (pL422), spanning nearly entire region of PASVIL (ca. $18 \mathrm{~kb}$ ), not only restored the virulence of the mutants, but also turned non-pathogenic Group III strains virulent. PASVIL contains at least 19 ORFs, coding membrane proteins, proteins for amino-acid transport and metabolism, and transposases, adjacent to tRNA-Phe gene at one border. The GC content of PASVIL was ca. $39 \%$, significantly lower than that of whole genome average $53 \%$. It was deduced that PASVIL might constitute a pathogenicity island, encoding pathogenicity determinant(s) not described before in Pantoea spp. 
Identification and functional characterization of the toxin produced by Colletotrichum fragaricola in strawberry

S. S. YU (1), T. H. Lee (2), M. S. Lee (3), C. L. Chung (1), (1) Department of Plant Pathology and Microbiology, National Taiwan University, Taipei City, TAIWAN; (2) Institute of Fisheries Science, National Taiwan University, Taipei City, TAIWAN; (3) School of Pharmacy Taipei Medical University, Taipei City, TAIWAN

Strawberry production in Taiwan has severely suffered from anthracnose, which is caused by three Colletotrichum species including $C$. karstii, $C$. fructicola, and $C$. fragaricola sp. nov. (a potentially new and dominant species). To investigate the mechanism underlying pathogenesis of $C$. fragaricola, this research focuses on the identification and functional characterization of toxins produced by C. fragaricola. We optimized toxin production by supplementing the medium with strawberry leaf extract and developed a leaf disc bioassay method for toxin evaluation. The culture filtrate of C. fragaricola caused necrosis on leaf discs and induced anthracnose symptoms on detached leaves of strawberry. Assays on the leaf discs of several dicot and monocot crops suggested the lack of host specificity. Pretreatment with heat or proteinase $\mathrm{K}$ did not inhibit the necrosis-inducing activity of the culture filtrate, indicating that the active ingredient is a thermostable and non-protein substance. We then isolated and identified the water-soluble toxic chemical(s) by employing Diaion HP20 open column chromatography, high performance liquid chromatography, mass spectrometry, and nuclear magnetic resonance. To uncover the role of the toxin in pathogenesis, Agrobacterium-mediated transformation and mung bean radicle growth bioassay method were used to screen for toxin-deficient mutants. The results will provide new insights into the mechanisms of $C$. fragaricola infection in strawberry.

\author{
Changes of epidemiological components and histopathology in infection process of UV-B induced mutant strains of Puccinia striiformis f. sp. \\ tritici \\ Y. ZHAO, P. Cheng, H. G. Wang, Department of Plant Pathology, China Agricultural University, Beijing, CHINA
}

Planting resistant cultivars is one of the key measures to control wheat stripe rust caused by Puccinia striiformis f. sp. tritici (Pst), while their resistances are often lost because of the virulence variation of Pst. In this study, the urediospores of a Chinese major physiological race CYR 32 were irradiated under the UV-B intensity of $250 \mu \mathrm{w} / \mathrm{cm}^{2}$ (at which the relative lethal rate of urediospores was $90 \%$ ) to screen virulent mutants on a wheat cultivar Guinong 22 that cannot be infected by CYR32. Radiation times of the UV-B radiation doses $\left(\mathrm{LD}_{50}\right.$ and $\left.\mathrm{LD}_{90}\right)$ for which the relative lethal rates of urediospores were $50 \%$ and $90 \%$ under the intensity of $150 \mu \mathrm{w} / \mathrm{cm}^{2}$ were determined. The effects of UV-B radiation of $\mathrm{LD}_{50}$ and $\mathrm{LD}_{90}$ on the epidemiological components and the changes of histopathology under 1-h UV-B radiation $\left(150 \mu \mathrm{w} / \mathrm{cm}^{2}\right)$ of virulent mutant strains were investigated. Two virulent mutant strains were obtained and named as CYR32-5 and CYR32-61. The radiation times of $\mathrm{LD}_{50}$ for CYR32, CYR32-5 and CYR32-61 were 90, 180 and $120 \mathrm{~min}$, respectively, and that of $\mathrm{LD}_{90}$ were 200, 300 and $230 \mathrm{~min}$, respectively. The results showed that the incubation period was prolonged and that the infection efficiency, lesion expansion rate and area under disease progress curve decreased after UV-B radiation. It was demonstrated that UV-B radiation reduced the germination rate of urediospores, the numbers of hyphal branches, haustorial mother cells and haustoria, and the linear lengths of hyphae.

Weeds like survival niches of Xanthomonas campestris pv. campestris

K. TELES GIROTTO (1), J. C. Silva (1), D. M. do Nascimento (1), T. A. Fernandes da Silva Junior (2), J. M. Soman (1), T. Domingues Tomasini (1), A. C. Maringoni (1), (1) São Paulo State University, Botucatu, BRAZIL; (2) Universidade do Sagrado Coração, Bauru, BRAZIL

Weeds compete with crops for resources and are host pathogens such as the bacterium Xanthomonas campestris pv. campestris (Xcc), causal agent of crucifer black rot. The aim of this study was to identify symptomatic hosts and epiphytic colonization in the weed phylloplane. Weeds were collected in a crucifer production area with a history of black rot in the municipality of Pardinho, São Paulo, Brazil and sent to the laboratory. Isolation of the symptomatic plants and the processing of the phylloplane samples were performed, plated in NSCAA semi-selective medium, incubated $\left(28^{\circ} \mathrm{C} / 72 \mathrm{~h}\right)$ and the characteristics of colonies were observed. Raphanus raphanistrum, Bidens pilosa and Sonchus oleraceus were confirmed as hosts and the presence of the bacterium was verified in the phylloplane of Eleusine indica, Solanum americanum, Ageratum conyzoides, Commelina spp., Galinsoga parviflora and Leonurus sibiricus. The identity of the isolates was confirmed by PCR with the specific primers HrcCF2 and $\mathrm{HrcCR} 2$, and by pathogenicity test. Based on the results, it is recommended the eradication of these weed species in areas with black rot occurrence for inoculum reduction.

Investigation of the role of Nep1-like protein from Leptosphaeria maculans in planta G. MITROUSIA (1), P. Patel (1), H. J. Schoonbeek (2), B. Fitt (1), C. Ridout (2), (1) University of Hertfordshire, Hatfield, UNITED KINGDOM; (2) John Innes Centre, Norwich, UNITED KINGDOM

Proteins of the superfamily of necrosis and ethylene-inducing peptide 1 (Nep1)-like proteins (NLPs) were examined for their role using the Leptosphaeria maculans-Brassica sp. system. These secreted proteins have been studied in various phytopathogenic microorganisms. They are involved in cell death but also in activation of host innate immune responses. At least one NLP has been identified in L. maculans (Lm_NLP1), the causal agent of phoma stem canker on Brassica plants. A previous study showed that Lm_NLP1 is required for the complete virulence of $L$. maculans and induces HRlike cell death following transient expression in tobacco leaves. Here, a construct for recombinant expression of Lm_NLP1 was generated and the protein was expressed and purified. The purified protein was infiltrated into host plant leaves (Brassica napus) and its cytotoxic activity was examined. Additionally, it is observed that a 24-residue peptide, which includes Conserved Regions I and II from Lm_NLP1, is recognised in a variety of Arabidopsis and Brassica lines as a PAMP, and induces a transient ROS-burst in a dose-dependent manner. This will enable us to determine the role of Lm_NLP1 protein as a whole, as well as the conserved peptide region, in triggering host responses.

Infectivity and pathogenicity of two different Plantago asiatica mosaic virus isolates in lilies

K. KOMATSU (1), M. Tanaka (2), M. Verbeek (3), T. Arie (4), (1) Tokyo Univ Agric \& Tech (TUAT), Fuchu, Tokyo, JAPAN; (2) Tokyo University of Agriculture and Technology, Fuchu, JAPAN; (3) Wageningen Plant Research, WAGENINGEN, NETHERLANDS; (4) Tokyo Univ of Agric \& Tech (TUAT), Fuchu, Tokyo, JAPAN

Plantago asiatica mosaic virus (PlAMV) is a member of the genus Potexvirus in the family Alphaflexiviridae and was has been isolated from a variety of host plants. In particular, PlAMV isolates from ornamental lilies (Lilium spp.) cause necrotic symptoms, which significantly reduces their commercial value. However, it has not been clear whether PIAMV isolates from other host plants can cause necrotic symptoms in ornamental lilies, because they may differ in their infectivity and pathogenicity in the hosts. Also, it remains unresolved whether growth conditions affect infectivity and pathogenicity. In this study, we inoculated an edible lily variety (Lilium leichtlinii) and seven varieties of ornamental lilies with two PlAMV isolates, an ornamental lily 
isolate PlAMV-OL and an edible lily isolate PlAMV-Li1. We performed inoculation test at two seasons, in spring and autumn, in two different growth conditions, in a greenhouse and open field. About two months after inoculation, we examined virus infectivity by quantitative reverse-transcription PCR, and virus pathogenicity by observation symptoms exhibited in plants. We found that PIAMV-OL had stronger infectivity and pathogenicity to both edible and ornamental lilies than PlAMV-Li1. Moreover, we observed higher infection rates of PlAMV-OL in open field than in greenhouse, and higher rates of necrotic symptoms in autumn test than in spring test, suggesting that growth environment including ambient temperatures and season affect infectivity and pathogenicity of PIAMV in lilies. Our study would provide important information for estimating the risk of necrotic disease caused by PlAMV, as well as for cultivation management preventing the occurrence of the disease.

Identification of host transcription modulating effectors in the rice blast fungus

S. KIM (1), S. Y. Park (2), C. Kim (1), S. Kwon (3), J. Choi (4), J. Jeon (5), J. Jeon (1), K. T. Kim (1), H. Chung (1), Y. H. Lee (1), (1) Seoul National University, Seoul, SOUTH KOREA; (2) Sunchon National University, Suncheon, KOREA; (3) Heinrich Heine University, Düsseldorf, GERMANY; (4) Samuel Roberts Noble Foundation, Ardmore, OK, USA; (5) Yeungnam University, Gyeongsan, SOUTH KOREA

Plant pathogens secrete effectors to fabricate infection environment during pathogenesis. A type of effectors that modulate host defense responses has been discovered in plant pathogenic bacteria and oomycetes. However, information about the effector is scarce in plant pathogenic fungi. As a first step to identify host transcription modulating effectors in Magnaporthe oryzae, hemibiotrophic pathogen, 12 genes encoding transcription factor with secretion signal were identified. Half of those were biotrophy associated genes and accumulated at apprerssoria or BIC, a structure for effector secretion. Especially, MoNUM1 and MoNUM2 were detected at host nuclei, and required for full virulence of M. oryzae. In order to reveal host target genes, effector binding element (EBE) was investigated by protein binding microarray: 'CAATCTTC' for MoNUM1 and 'GGAGGTGG' for MoNUM2. In addition, genuine targets were identified in light of expression pattern and EBE presence on promoter, which was validated by yeast one hybrid. OsMYB4 was downregulated by MoNUM1, HPL2 and OsWRKY45 were repressed by MoNUM2. MoNUM2 expression in rice confers increased susceptibility for M. oryzae and decreased susceptibility for Cochliobolus miyabeanus, necrotrophic pathogen. Taken together, MoNUM1 and MoNUM2 act as transcriptional repressor in host. Furthermore, MoNUM2 is potential confounding factor for lifestyle-dependent defense of rice.

Genomic regions of wheat yellow mosaic virus involved in the pathotypes against wheat cultivars

T. OHKI (1), T. Sasaya (2,3), T. Maoka (4), (1) Hokkaido Agricultural Research Ctr, NARO, Sapporo, JAPAN; (2) National Agricultural Research Center, Tsukuba, Ibaraki, JAPAN; (3) National Agriculture and Food Research Organization, Tsukuba, JAPAN; (4) Hokkaido Natl Agric Research Center, Sapporo, JAPAN

Wheat yellow mosaic virus (WYMV) belonging to the genus Bymovirus, Potyviridae has two, positive-sense, and single-strand genomic RNAs. RNA1 is ca. $7.7 \mathrm{~kb}$ long, and encodes a polyprotein consisted of coat protein and seven putative nonstructural proteins. RNA2 is ca. $3.7 \mathrm{~kb}$ long, and encodes two proteins that constitute a single polyprotein. WYMV is divided into pathotypes I and II by the reaction of wheat differential cultivars "Fukuhokomugi" and "Shiranekomugi"; pathtype I shows systemic infection with both cultivars but pthotype II doesn't infect "Fukuhokomugi". In this study, we investigated the viral genomic regions involved in the pathotypes using infectious clones of pathotypes I and II of WYMV. When the two wheat cultivars were inoculated with reassort viruses which exchanged either RNA1 or RNA2 from the other pathotypes, the reassort virus with RNA1 from pathotype I infected systemically both cultivars as did pathotype I, indicating the involvement of RNA1 in the pathotypes. Infectious clones of the chimeric and point mutated viruses revealed that the replacement of three amino acids in cylindrical inclusion body protein (CI) of pathotype II with those of pathotype I resulted in the same pathogenicity pattern of pathotype I. Both pathotypes I and II showed different host reaction against two cultivars, but successfully multiplied within protoplasts of the two cultivars. These results suggest that pathotypes of WYMV is associated with amino acid changes in CI and ascribes to the inhibition in cell-to-cell and/or long distance movement of pathotype II within wheat host tissues.

Pathological specialization of Venturia nashicola, the cause of Asian pear scab, and resistance of pear cultivars H. ISHII (1,2), K. Nishimura (3), K. Tanabe (4), (1) Institute for Agro-Environmental Sciences, NARO, Tsukuba, JAPAN; (2) Kibi International University, Minami-awaji, JAPAN; (3) National Institute for Agro-Environmental Sciences, Tsukuba, JAPAN; (4) Tottori University, Tottori, JAPAN

Scab is the most serious disease of Asian pears and the control of this disease largely relies on spray application of fungicides. As a result, the isolates of Venturia nashicola resistant to benzimidazole, as well as sterol demethylation inhibitor (DMI), fungicides have been selected and widely distributed in the pathogen populations. To minimize the use of fungicides, the breeding of scab-resistant pear cultivars (cvs.) is important. Furthermore, survey of the pathological specialization of $V$. nashicola is necessary to assess the durability of scab resistance. The race 1,2, and 3 isolates, each differing their pathogenicity to the Japanese pear cv. 'Kousui' and the Asian pear strain 'Mamenashi 12', have been reported previously in Japan. In this study, new other isolates carrying distinct pathogenicity from the known three races were found from China and Taiwan based on inoculation tests using the monoconidial isolates and potted trees of the differential pear cvs. Jingbaili, Yali and others. They were then designated to the races 4,5 , and 6 of $V$. nashicola. It was also found that the race 2 isolates were widely distributed in China. In the inoculation tests, both the Japanese pear cv. 'Kinchaku' and the cv. 'Xiangli' of Pyrus sinkiangensis (fragrant pear in China) showed no visible symptoms on the leaves inoculated with all of the six races so far tested. This high scab resistance of 'Kinchaku' and 'Xiangli' can be utilized for breeding program as useful genetic resources in the future.

Distribution, variation and function of the $A V R$-Pita gene family among clonal lineages of Magnaporthe oryzae in the United States S. Y. PARK (1), C. H. Khang (2), J. C. Correll (3), Y. H. Lee (4), S. Kang (5), (1) Sunchon National University, Suncheon, KOREA; (2) University of Georgia, Athens, GA, USA; (3) University of Arkansas, Fayetteville, AR, USA; (4) Seoul National University, Seoul, SOUTH KOREA; (5) the Pennsylvania state University, University Park, PA, USA

New pathogen races emerge through various change in avirulence $(A V R)$ genes. AVR-Pital and AVR-Pita2, members of the AVR-Pita gene family, but not $A V R$-Pita3, prevent the rice blast fungus Magnaporthe oryzae from infecting rice cultivars carrying resistance $(R)$ gene $P i$-ta. To understand the mechanism underpinning race variation caused by changes in this gene family, we characterized its members among 23 isolates that represent eight clonal lineages of M. oryzae in the United States. Screening of their genomic DNA using three AVR-Pita genes as probes revealed multiple haplotypes, but little variation was observed within individual clonal lineages. All isolates seem to lack AVR-Pita2 but carried a single copy of $A V R$-Pita3. The copy number of $A V R$-Pital varied from 0 to 3. Novel members of the family may be present too. At least six distinct $A V R$-Pital products are encoded. Three of them confer AVR function, making the strains expressing them avirulent to rice containing Pi-ta. Comparison of the AVR-Pita1 protein sequences, in light of virulence of the strains carrying them, and site-directed mutagenesis showed that tyrosine residue at the position 192 is essential for AVR function. Unlike the previously characterized AVR-Pital genes, which are located very close to the telomere, most AVR-Pital genes in the US isolates might not be telomeric. 
Fusarium virguliforme and corn: Exploring temporal field dynamics within an asymptomatic host

A. M. BAETSEN-YOUNG (1), J. L. Jacobs (1), A. Byrne (1), D. S. Mueller (2), D. Smith (3), K. Wise (4), B. Day (1), M. Chilvers (1), (1) Michigan State University, East Lansing, MI, USA; (2) Iowa State University, Ames, IA, USA; (3) University of Wisconsin-Madison, Madison, WI, USA; (4) University of Kentucky, Princeton, KY, USA

The asymptomatic host range of Fusarium virguliforme $(F v)$ includes corn, a common crop rotation with soybean. We tested the hypothesis that corn may serve as an important asymptomatic host reservoir for $F v$ the causal agent of soybean sudden death syndrome, altering $F v$ population dynamics, and management. The objective of this study was to determine the temporal dynamics of corn root colonization by $F v$. Experiments were conducted in IA, IN, and MI for two years, and in WI for one year. Corn roots were sampled at 1,2, 4, 8, 12, and 16 weeks after planting (WAP) in MI, and 4 or 5, 8 and 16 WAP for IA, IN, and WI. DNA was extracted and analyzed by real-time qPCR for $F v$ quantification. Trials were inoculated with $F v$ and contained four cultural treatments (four replicates/treatment) of 1) tillage with corn residue, 2) tillage without corn residue, 3) no-tillage with corn residue, and 4) no-tillage corn with no residue. In 2016, low (ca. $100 \mathrm{fg} / 10 \mathrm{mg}$ root tissue) $F v$ DNA quantities were detected at 1, 2, and 4 WAP in the MI corn, and dropped to below limits of detection at 8 WAP, followed by a gradual increase at 12 and 16 WAP. In 2017, a similar trend was observed in MI, and in trials in IA and IN; however, higher levels of $F v$ DNA was detected across sampling time points. Overall, trials in MI, IA, and IN revealed consistent low-level detection of $F v$ in corn roots. Cultural tillage practices showed inconsistent treatment effect between trials and locations.

Insights into Candidatus Liberibacter asiaticus Pathogenicity and Biology E. WATSON CARTER, N. Wang, University of Florida, Lake Alfred, FL, USA

Citrus Huanglongbing (HLB) is now the most devastating citrus disease worldwide which is caused by the $\alpha$-proteobacteria Candidatus Liberibacter. The most prevalent species that has been associated with HLB is Candidatus Liberibacter asiaticus (CLas), and the most important species, in terms of pathogenicity, to study. Our understanding of the biology and pathogenicity of CLas has been hampered by the difficulty to cultivate the pathogen and traditional genetic study could not be performed. In this study, we will present our progress in understanding the biology and pathogenicity of CLas. Specifically, we will focus on the secretion systems and putative effector proteins and transporters. CLas is known to have 40 ABC-type transporters, some of which are part of the TISS. In addition to the genes encoding the inner-membrane ABC transporter in the TISS, there are also periplasmicmembrane fusion proteins and an outer-membrane porins; coded by genes: HlyD [COG0845], PrtD [COG4618], TolC [COG1538]). Also found by comparative genomics, are putative type effectors, such as an RTX toxin hemolysin (COG1253) in close proximity to the TISS genes. Here we explore the relative amount of gene and protein expression between the two systems, both effector and secretion system associated genes, to determine their role together in comparison to the virulent pathogen.

Examination of the experimental host range of Plantago asiatica mosaic virus

J. HAMMOND (1), K. K. Rane (2), (1) USDA-ARS, USNA, Floral and Nursery Plant Research Laboratory, Beltsville, MD, USA; (2) University of Maryland, College Park, MD, USA

Plantago asiatica mosaic virus (PlAMV) has been detected in lily (Lilium species and hybrids) in multiple countries following dissemination in the international bulb trade. PlAMV is known to be transmitted between lily plants in the soil, without any known vector, and has been identified in a number of other herbaceous species and the woody shrub Nandina domestica (nandina, heavenly bamboo). The potential for establishment of PlAMV in soil in which PlAMV-infected plants have been grown is of concern, and information regarding the experimental host range is therefore needed. Inoculation of a lily isolate of PlAMV to a wide range of plants from taxonomically diverse plant families, including various crop plants, ornamentals, bioassay plants, and weeds, was therefore carried out; infection was assessed by symptom expression, and by double antibody sandwich ELISA using an antiserum raised against the lily isolate. More than 25 plant species representing at least 15 diverse plant families were demonstrated to be experimental hosts, in addition to known natural hosts in four additional families, and in five families from which experimental hosts were also identified. PIAMV was therefore demonstrated to have a broad host range among diverse plant families. Although several experimental hosts were infected only locally, others were systemically infected, either latently or with obvious symptoms, suggesting the risk of establishment of PlAMV where infected crops are grown.

Phytophthora sansomeana host characterization in Michigan field crops

A. MCCOY, J. L. Jacobs, M. Chilvers, Michigan State University, East Lansing, MI, USA

Phytophthora sansomeana is a pathogen of soybean, corn and Douglas fir, differentiated from the P. megasperma complex in 2009. Since then, the pathogen has been found in eleven states, including Michigan. However, little is known about the prevalence and extent of the $P$. sansomeana host range in relation to crops in Michigan. Michigan produces a wide diversity of field crops including corn, soybean, wheat, dry bean and sugarbeet. In addition, cover crops and forage crops are grown including oats, winter rye and alfalfa. Without knowledge of host specificity, crop rotation for disease management may not be an effective means of control. Therefore, we will be examining the host range, pathogenicity and virulence of $P$. sansomeana on eight crops commonly grown in Michigan (corn, soy, wheat, dry bean, sugar beet, winter rye, oats and alfalfa) using a pathogenicity assay conducted in the growth chamber. In addition, sensitivity to mefenoxam, ethaboxam and oxathiapiprolin will be established using a high throughput assay. The combination of host range identification and fungicide sensitivities will allow us to improve management practices for $P$. sansomeana in Michigan.

Association Mapping of Sclerotinia sclerotiorum mid-stalk rot virulence on two sunflower inbred lines

K. BELAY (1), R. Sharma Poudel (1), S. Solanki (2), C. Misar (3), R. Lakin (1), B. D. Nelson (4), R. S. Brueggeman (5), W. Underwood (3), (1) North Dakota State University, Fargo, ND, USA; (2) Plant Pathology Department, North Dakota State University, Fargo, ND, USA; (3) USDA-ARS, Fargo, ND, USA; (4) Plant Pathology 7660, North Dakota State University, Fargo, ND, USA; (5) Department of Plant Pathology, North Dakota State University, Fargo, ND, USA

Sclerotinia sclerotiorum is one of the most destructive pathogens of sunflower in the United States and worldwide. This fungal pathogen has a remarkably broad host range of over 400 dicot plants. However, little is currently known about the virulence strategies that allow $S$. sclerotiorum to successfully infect a wide range of plant hosts. The goal of our project is to identify $S$. sclerotiorum virulence determinants such as protein, small molecule, and small RNA effectors that contribute to disease development on sunflower. We have four main objectives; 1) evaluation of the virulence of a large and diverse collection of S. sclerotiorum isolates on mid-stalk tissues of sunflower; 2) genotyping-by-sequencing of S. sclerotiorum isolates to facilitate association mapping; 3 ) association mapping to identify candidate genetic factors influencing virulence in $S$. sclerotiorum; 4) evaluation of candidate genes using direct knockout and subsequent evaluation of mutant strains for virulence. We have assembled a collection of 234 diverse $S$. sclerotiorum isolates and completed the phenotyping of these isolates for virulence on stems of USDA sunflower inbred line HA207. Additionally, we are phenotyping those isolates on a second, partially inbred line, HA441. We performed genotyping-by-sequencing for 227 isolates to facilitate association mapping. We are also currently working on direct knockout of five candidate genes, using gene replacement and subsequent evaluation of 
mutant $S$. sclerotiorum strains for virulence on the two inbred lines of sunflower. Our initial results indicate that $S$. sclerotiorum isolates exhibit significant differences in virulence on sunflower, indicating the potential for our method to successfully identify genetic factors underlying these differences.

Variation among putative necrotrophic effector genes in host-specialized populations of Corynespora cassiicola

L. G. SUMABAT (1), R. C. Kemerait (2), M. T. Brewer (1), (1) University of Georgia, Athens, GA, USA; (2) University of Georgia, Tifton, GA, USA

Numerous plant-pathogenic fungi secrete necrotrophic effectors (host-selective toxins) that are important determinants of pathogenicity and virulence in species that have a necrotrophic lifestyle. Corynespora cassiicola is a destructive fungal pathogen causing emerging target spot epidemics in the southeastern U.S. Previous findings revealed C. cassiicola populations from cotton, soybean, and tomato showed host specialization. In addition, other studies have identified the necrotrophic effector cassiicolin from $C$. cassiicola isolates, as a critical toxin for virulence on rubber. It is encoded by 6 Cas gene variants. Our goal is to conduct comparative genomic analyses to identify variation among putative necrotrophic effector genes in $C$. cassiicola causing outbreaks in the southeastern U.S. A total of 12 C. cassiicola genomes, representative of different hosts of origin and geographic regions, were sequenced on an Illumina NextSeq platform. Reads for each genome were assembled de novo and searched for known Cas, Tox, and other homologs for effector-encoding genes. Preliminary results revealed all 6 Cas variants encoding for cassiicolin in the different genomes. Moreover, more than one Cas variant was detected in one genome. Knowledge on the diversity of necrotrophic effector genes is critical in understanding the genetic basis of host specialization and disease emergence of target spot of cotton, soybean, and tomato in the southeastern U.S.

Tolerance of Pinus patula hybrids to novel Fusarium circinatum haplotypes from Guatemala and Nicaragua

I. BARNES (1), M. J. Wingfield (1), K. Mongwaketsi (2), F. Jami (1), J. Garnas (3), (1) Forestry and Agricultural Biotechnology Institute (FABI), University of Pretoria, Pretoria, SOUTH AFRICA; (2) Forestry and Agricultural Biotechnology Institute (FABI), Pretoria, SOUTH AFRICA; (3) Department of Natural Resources and the Environment, University of New Hampshire, Durham, NH, USA

Pitch canker, caused by Fusarium circinatum, is one of the most devastating diseases affecting commercial pine forestry, especially in the Southern Hemisphere. A pathogen survey conducted in Guatemala revealed Pinus hartwegii trees in both planted and natural forests in San Carlos Sija, with typical symptoms of pitch canker. These included flagging of the branches and thick, resinous pitch surrounding cankered areas on the stems. Similar symptoms were also observed on a single individual $P$. oocarpa tree in Nicaragua. DNA-based identification of isolates from diseased trees confirmed the presence of $F$. circinatum. Two haplotypes were identified using microsatellite markers and both mating types were present in Guatemala. In South Africa, $P$. oocarpa has been extensively used as a tolerant hybrid partner with other Pinus spp. to offset the negative impact of pitch canker. Tolerance of these hybrids to the newly discovered haplotypes of $F$. circinatum was tested in an inoculation trial. Seedlings representing five commercial families of the $P$. patula $\times$ P. oocarpa hybrid were inoculated and the pure species $P$. tecunumanii LE (low elevation population) and $P$. patula were included for comparative purposes. Tolerance levels were assessed based on lesion development. Results showed that the $P$. tecunumanii (LE) and the $P$. patula $x$. oocarpa hybrids were significantly more tolerant to infection by $F$. circinatum than $P$. patula. Furthermore, the two former species showed no significant difference in tolerance to the pathogen. From this study it is clear that hybrids with $P$. oocarpa have the potential to negate the negative impact of pitch canker in South Africa, while retaining desirable traits from $P$. patula.

Identification of atypical chitin synthase genes horizontally transferred in plant pathogens

I. R. Gonçalves (1), S. Brouillet (2), M. CHOQUER (1), (1) University Lyon 1, Lyon, FRANCE; (2) University Paris 6, Atelier de bioinformatique, Paris, FRANCE

Chitin, the second most abundant biopolymer on earth after cellulose, is found in probably all fungi, many animals (mainly invertebrates), several protists and a few algae, playing an essential role in the development of many of them. It is produced by type 2 glycosyltransferases, called chitin synthases (CHS). In phytopathogenic fungi, several CHS isoenzymes are found and they are not only thought to be essential for their growth but they are also considered to participate as determinants of their pathogenicity. We performed a genome-wide analysis and detected more than 800 putative CHS in proteomes associated with about 130 genomes. Phylogenetic analyses allowed us to propose a robust and unifying fungal CHS classification that is easily accessible through a dedicated website (http://wwwabi.snv.jussieu.fr/public/CHSdb). Concerning the evolutionary history of CHS, this family has mainly evolved via duplications and losses. However, it is likely that several horizontal gene transfers (HGT) also occurred in eukaryotic microorganisms and, even more surprisingly, in the genomes of bacteria and viruses. Interestingly, many of these horizontally acquired CHS are found in plant pathogens, bacteria or fungi. Whether these atipycal CHS activities can represent new virulence factors implied in plant interactions will be discussed in the poster.

$\boldsymbol{N}$. benthamiana as a surrogate host for studying the pathogenicity of $\boldsymbol{A}$. citrulli, the causal agent of bacterial fruit blotch of cucurbits M. KIREMIT (1), B. Zhao (1), G. E. Welbaum (1), R. R. Walcott PhD (2), S. Burdman (3), Z. Wang (1), N. Eckshtain-Levi (4), J. Miao (1), S. M. Traore (5), A. C. Castro (6), (1) Virginia Tech, Blacksburg, VA, USA; (2) The University of Georgia, Athens, GA, USA; (3) The Hebrew University of Jerusalem, Rehovot, ISRAEL; (4) Boyce Thompson Institute, Cornell university, Ithaca, NY, USA; (5) Auburn University, Auburn, AL, USA; (6) Department of Plant Pathology University of Georgia, Athens, GA, USA

Acidovorax citrulli is the causal agent of bacterial fruit blotch (BFB) of cucurbits. Despite its significant agricultural importance, the mechanisms associated with $A$. citrulli pathogenicity have not been extensively investigated. In this study, we used Nicotiana benthamiana and Nicotiana tabacum as surrogate hosts for studying $A$. citrulli virulence and nonhost resistance triggered by bacterial type III-secreted effectors. Two A. citrulli strains, M6 and AAC00-1, that represent the two major groups among $A$. citrulli populations, induced disease symptoms on $N$. benthamiana, but triggered hypersensitive response (HR) on N. tabacum plants. Transient expression of 19 T3S effectors in $N$. benthamiana and $N$. tabacum leaves revealed that three effectors, Aave1548, Aave2708, and Aave2166, could trigger water-soaking-like cell death in N. benthamiana and HR in N. tabacum. Knockout effector gene Aave 1548 in strains M6 and AAC00-1 reduced A. citrulli virulence on N. benthamiana and melon (Cucumis melo L.). Transient expression Aave1548 and Aave2166 effectors triggered nonhost HR in N. tabacum, and this reaction depends on the functionality of the immune signaling component, NtSGT1.

Genetic variability of the avirulence gene AvrLm4-7 among Leptosphaeria maculans isolates by high resolution melting analysis F. CEVALlOS, L. Pena Zuniga, F. Ochoa Corona, J. P. Damicone, Oklahoma State University, Stillwater, OK, USA

The fungal pathogen, Leptosphaeria maculans, is an ascomycete that causes black leg disease of canola (Brassica napus) in Oklahoma and worldwide. Major-gene resistance involves the interaction between avirulence effectors in the pathogen and their corresponding resistance genes in the plant. AvrLm4-7 is an important avirulence effector that is recognized by two resistance proteins encoded by the genes $R \operatorname{lm} 4$ and $R \operatorname{lm} 7$. A rapid detection, and 
discrimination tool for these effectors is needed for characterization of pathogen races. Previously reported internal primers AvrLm7Up1 and AvrLm7LOW2 were used for PCR and high resolution melting analysis. Melting temperatures profiles were determined for $L$. maculans isolates with different avirulence allele profiles. Some of the melting curves obtained using the internal primers were different from those previously reported. To analyze the entire AvrLm4-7 region, its reference nucleotide sequence was retrieved from NCBI-Genbank and aligned using MEGA 6. Two sets of external primers were designed using the software Primer3 (AvrLm47A and AvrLm47B). The melting curves obtained from the external region using AvrLm47A primers were similar among isolates with a functional AvrLm4-7 gene and some isolates with a non-functional AvrLm4-7 gene. Minor variations of the melting curves were detected in the external region using AvrLm47B primers. Most isolates with a non-functional AvrLm4-7 gene were not amplified by any set of primers. The obtained melting profiles allows the examination of the allelic variability among L. maculans isolates.

Screening soybean and corn root colonization by a Fusarium virguliforme natural population

J. CHEN, A. M. Baetsen-Young, M. Chilvers, B. Day, Michigan State University, East Lansing, MI, USA

In the United States, soybean sudden death syndrome is caused by the ascomycete Fusarium virguliforme ( $F v$ ). Previous screenings of $F v$ isolates have revealed differences in aggressiveness on soybean; however, it remains unknown if isolate aggressiveness persists on alternate hosts. In the current study, we asked if corn, commonly rotated with soybean and an asymptomatic host for $F v$, can be colonized by natural populations of $F v$. In 2016 , isolates were collected from soybean roots in a field naturally infested with $F v$, and nine were evaluated for their ability to infect both soybean and corn in a growth pouch based assay. Soybean and corn seedlings, either individually inoculated with $F v$ macroconidia from nine field isolates or $F v$ isolate Mont-1. After 14 days post inoculation, in-planta pathogen growth was assessed using trypan blue staining of roots. Further, root samples were collected for DNA extraction, and real time qPCR assay assessed $F v$ DNA quantities. Over the two-week interval, symptoms developed on the soybean roots but not on corn roots. However, staining revealed $F v$ mycelia growth upon both the soybean and corn roots. The first biological repeat showed a trend of 100 -fold reduction of corn to soybean $F v$ DNA quantities, but different isolate colonization levels were detected within each plant type. Ongoing experiments will uncover if $F v$ has differing patterns of colonization that is consistent in both corn and soybean roots from a natural population.

Investigating the impacts of continuous artificial culture systems on Phytophthora infestans virulence

E. LARSON (1), D. A. Halterman (2), A. J. Gevens (1), (1) University of Wisconsin-Madison, Madison, WI, USA; (2) USDA ARS, Madison, WI, USA

It is widely accepted, and documented for Phythophthora infestans, that continued sub-culturing on artificial media results in virulence reduction. Typically, passage of the cultured pathogens back through host plant tissue brings about a return in virulence, a response that suggests that changes are not due to genetic mutation. Rather, changes may be the result of a more sophisticated and fluid method of regulation. Elucidating this mechanism could shed light on $P$. infestans' ability to rapidly overcome host resistance. We investigated the virulence of 5 P. infestans isolates (Gu68, US-1, US-8, US-23, and US-24) after sub-culturing on artificial media, and we analyzed changes in effector expression within the pathogen at key stages. Each isolate was sub-cultured and maintained at $12^{\circ} \mathrm{C}$ on Rye-A medium for at least 8 times over 6 months. The oldest, most recent, and median sub-cultures of each isolate were tested by plug inoculation on both susceptible ('Katahdin' potato) and resistant ('Katahdin' potato transformed with RB) hosts in a conducive $100 \%$ humidity environment. Disease severity was determined by measuring lesion size and a significant interaction ( $\mathrm{p}=0.054)$ was found between the host type and age of culture. In preparation for expression analysis, a subset of 6 P. infestans effectors were verified by PCR in each of the isolates. RNA was extracted from infected plants and RT-qPCR analyses were conducted to assess changes in effector expression.

Germination stage effects susceptibility to infection of soybean by Pythium species

R. L. MATTHIESEN, A. E. Robertson, Iowa State University, Department of Plant Pathology, Ames, IA, USA

Damping-off caused by Pythium species is an economically important disease of soybean in the United States. There are few data regarding the susceptibility of soybean germination stages to infection by Pythium species. The goal of this research was to compare the susceptibility of five soybean germination stages to infection by two Pythium species. Soybean germination stages include: (i) water imbibition (0-1 day after planting DAP), (ii) radicle growth (1-3 DAP), (iii) emergence (4 DAP), (iv) epicotyl growth (7 DAP), and (v) unifoliate leaf development (10 DAP). Seeds or root tips were inoculated with an agar slurry of $P$. lutarium or $P$. oopapillum at $0,1,2,3,4,7$, or $10 \mathrm{DAP}$ and incubated at $23^{\circ} \mathrm{C}$. Disease incidence and severity were assessed at 2, 4, 7, and 10 days after inoculation (DAI) by measuring lesion length. For $P$. lutarium and $P$. oopapillum, disease incidence was greatest when inoculation occurred at 1 DAP or 2 DAP, respectively. Root rot severity of seedlings inoculated with either Pythium species at 1 DAP and 2 DAP were more rotted compared to those inoculated at other DAP time points. Root rot severity of seedlings inoculated with $P$. lutarium and $P$. oopapillum was lowest when seedlings were inoculated 7 DAP and 10 DAP. These data improve our understanding of Pythium-soybean interaction and demonstrate that soybean is most susceptible to damping-off caused by $P$. lutarium and $P$. oopapillum within 2 days of planting when temperatures are at $23^{\circ} \mathrm{C}$.

Comparative component analysis of Calonectria pseudonaviculata epidemiology on boxwood, pachysandra and sweet box P. KONG, C. Hong, Hampton Roads Agricultural Research and Extension Center, Virginia Tech, Virginia Beach, VA, USA

Boxwood blight caused by Calonectria pseudonaviculata $(\mathrm{Cps})$ poses an increasing threat to the ornamental nursery and landscape industries as well as public and private gardens. This disease was also recently observed on pachysandra and sweet box associated with boxwood plantings in the United States. This study compared four epidemiological components among the three hosts and pathogen isolates from each hosts. Detached leaves of boxwood, pachysandra, and sweet box were inoculated with three isolates from each host and evaluated on infection rate, lesion size and conidium and microsclerotium production. Significant differences were observed in all four components among the plants but in only one component - conidium production - among the $C p s$ isolates. Specifically, a greater infection rate, larger lesions and higher conidial and microsclerotial yields were observed on boxwood compared to pachysandra and sweet box. Cps isolates from boxwood and sweet box produced more conidia than those from pachysandra on all the test plants. These results indicate that boxwood is a preferred host for $C p s$ while pachysandra and sweet box, with lower infection rates, slower disease progression and/or less conspicuous sporulation, could be perfect carriers of this pathogen. Therefore, it is important to consider pachysandra and sweet box as potential pathogen carriers when developing management programs for boxwood blight.

Pathogenic and physiological variability among Macrophomina phaseolina isolates associated with soybean in Ohio H. D. Lopez-Nicora (1), T. I. Ralston (1), A. E. Dorrance (2), T. L. NIBLACK (1), (1) The Ohio State University, Columbus, OH, USA; (2) The Ohio State University, Dept. of Plant Pathology, Wooster, OH, USA

The fungus that causes charcoal rot, Macrophomina phaseolina, is responsible for significant yield loss in soybean worldwide and can also affect corn. A recent study revealed the widespread distribution of M. phaseolina in both soybean and corn fields in Ohio. The objectives of this study were to evaluate the physiological and pathogenic variability of these M. phaseolina isolates as an initial step to understanding its population structure. A total of $200 \mathrm{M}$. 
phaseolina isolates were obtained from soil collected in soybean and corn fields throughout Ohio and used in physiological and pathogenicity studies. For the physiological assay, pure isolates were grown under different temperatures $\left(15,20,25,30,35\right.$ and $\left.40^{\circ} \mathrm{C}\right)$ on potato dextrose agar (PDA) and area under the growing progress curve (AUGPC) were obtained for each isolate. A soybean cut-stem inoculation technique was used to obtain the area under the disease progress curve (AUDPC) to assess the pathogenicity and aggressiveness of $M$. phaseolina isolates. Overall, larger variation in AUGPC between isolates was observed at lower temperatures than at temperatures reported as optimal $\left(30-35^{\circ} \mathrm{C}\right)$ for $\mathrm{M}$. phaseolina growth. All isolates were pathogenic to soybean; however, isolates displayed significant variation in aggressiveness. A subset of isolates was selected based on phenotypic data and geographic location for further genotypic and population genetic analyses.

Assessment of isolates of Fusarium oxysporum f. sp. vasinfectum as seedling pathogens to cotton using a rolled towel assay J. DIAZ (1), V. Maier (1), R. B. Hutmacher (2), M. L. Ellis (1), (1) California State University, Fresno, Fresno, CA, USA; (2) University of California, Davis, Shafter Research and Extension Center, Five Points, CA, USA

Fusarium oxysporum f. sp. vasinfectum (FOV) race 4 can be an extremely virulent wilt pathogen to susceptible cotton varieties. Within the United States, FOV race 4 currently is geographically limited, first identified in California in 2001 and Texas in 2017. In FOV race 4 infested fields the fungus has also been known to cause damping off and seedling mortality. To better assess disease impacts under California field conditions, more needs to be known on the relative pathogenicity of FOV races as early seedling pathogens. Therefore, the objective of this study was to assess the reaction of cotton to seedling infection with different isolates of FOV using a rolled towel assay. During the summer of 2017, isolates of FOV were isolated from symptomatic cotton seedlings from three FOV race 4-infested commercial fields in California. Sixteen isolates were used to inoculate seed of the FOV race 4-moderately resistant Upland cultivar FM-2334 and susceptible Pima cultivar PHY-830. Seeds were individually inoculated with $100 \mu 1$ of a $1 \times 10^{6}$ conidia/ml suspension. At ten days, seedlings were rated using an ordinal rating scale $(1=$ no disease, $5=$ dead $)$. There was a significant difference for isolate and cultivar $(\mathrm{P}<0.0001)$. Based on the ordinal rating scale, averages for isolates ranged from 2.1-3.4 and 3.7-4.3 for the FM-2334 and PHY-830, respectively. Based on these results, the use of the rolled towel assay should be examined as an early screening method for FOV resistant cotton.

\section{A novel recombinant strain of Beet curly top virus collected from pepper in Mexico}

M. ALA POIKELA (1), K. Green (1), O. V. Nikolaeva (1), L. Robles-Hernandez (2), A. C. Gonzalez Franco (3), A. V. Karasev (1), (1) University of Idaho, Moscow, ID, USA; (2) Univ Autonoma de Chihuahua, Chihuahua, MEXICO; (3) Univ Autonomous of Chihuahua, Chihuahua, MEXICO

Beet curly top virus (BCTV) is a curtovirus transmitted by beet leafhopper, and affecting multiple crops in desert areas of North America. A sample positive for BCTV was collected in South-Central region of Chihuahua, Mexico, from a pepper plant exhibiting stunting, yellowing, and leaf curling. Whole genome of the virus isolate, BCTV-Mx, was sequenced and found to represent a new recombinant between BCTV-Worland and BCTV-CFH parental strains. An infectious clone of the virus was designed and used to study biological properties of BCTV-Mx in multiple hosts. BCTV-Mx was able to replicate in Nicotiana benthamiana, in two out of five tested cultivars of sugar beet, and in six tested cultivars of tomato, inducing moderate symptoms, which included stunting, vein clearing, and leaf curling. Based on the unique combination of biological and sequence characteristics, this novel recombinant, BCTV-Mx, is proposed to represent a distinct strain of the virus.

Australian Verticillium dahliae goes against the group - VCG 2A causes severe disease in Australian cotton

P. DADD-DAIGLE (1,2), M. Labbate (1), P. Roy Chowdhury (1), K. Kirkby (2), T. Chapman (2), (1) The University of Technology Sydney, Ultimo, AUSTRALIA; (2) NSW Department of Primary Industries, NSW, AUSTRALIA

Cotton in Australia is a billion-dollar industry. The industry relies on improved cotton varieties, soil and water management, and weed, insect and disease management for sustainability and growth. One of the many factors that can impact yields is the fungal pathogen Verticillium dahliae, responsible for Verticillium wilt. The soil-bourne ascomycete, $V$. dahliae, has been classified into two pathotypes, defoliating (D) and non-defoliating (ND), and into Vegetative Compatability Groups (VCGs). Globally the D VCG1A has been reported to cause the most significant damage, but in Australia the ND VCG2A has been seen to cause more damage in the field than the D VCG1A. Four cotton varieties were inoculated with several Australian $V$. dahliae isolates encompassing VCG1A and VCG2A by root dipping with a concentration of $\sim 1 \times 10^{6}$ conidia. Plants were monitored over a period of 7 weeks and disease severity scored twice weekly. At the end of the monitoring period both the VCG1A isolates and one VCG2A isolate had killed their host while the remaining VCG2A isolates caused only minor disease. This agrees with the field reports that some Australian VCG2A isolates have the ability to cause severe crop damage, a result that has been previously unreported. The identification of highly virulent ND VCG2A strains allows the use of molecular and genomic tools to further understand the nature of virulence in V. dahliae, targeting features not present in other isolates of the same VCG.

Stalk rot of sweet sorghum caused by genetically diverse Fusarium thapsinum strains

V. S. BUSHULA (1), J. Jebril (2), J. F. Leslie (2), T. Tesso (2), C. R. Little (2), (1) University of Pretoria, Hatfield, SOUTH AFRICA; (2) Kansas State University, Manhattan, KS, USA

Sweet sorghum (Sorghum bicolor) is widely used as an alternative feedstock and source for bio-fuel production. Common to all cultivated sorghum, stalk rot diseases affect yield. Fusarium thapsinum is a fungal pathogen causing sorghum stalk rot. Strains of $F$. thapsinum are genetically diverse and little is understood about their potential for stalk rot on sweet sorghum. The objective of this study was to evaluate stalk rot of sweet sorghum by genetically diverse $F$. thapsinum. Stalk inoculations of 23 sweet sorghum genotypes including lines of variable sugar content and their hybrids were performed using four F. thapsinum strains (F00915, F00916, F01153, and F01163) in a complete randomized block design with three replications. There was a significant genotype by strain interaction for lesion length. Comparisons of lesion lengths showed significant differences between strains, with strains F01153 and F01163 having the highest lesion length of $14.3 \mathrm{~cm}$ each, followed by strain F00915 $(13.6 \mathrm{~cm})$ and F00916 $(12.4 \mathrm{~cm})$. This result suggests that there is variation in stalk rot pathogenicity between the strains. Comparisons of lesion length amongst genotypes ranged from low to high resistance to stalk rot disease caused by $F$. thapsinum strains in this panel of sweet sorghums. Our results suggest that genetic variability within $F$. thapsinum strains plays a role in stalk rot pathogenicity and that breeding efforts in sweet sorghum need to consider genetic diversity in the pathogen.

\section{Antioxidant-Mediated Survival of Primed Finger Millet Plants against Blast Disease}

S. PATIL, B. Satyan Kumudini, Jain University, Bangalore, INDIA

Ragi or finger millet (Eleusine coracana) is an indispensable crop in the arid, semi-arid regions of India and countries in Eastern Africa with proven resilience to climate change. It is now being promoted globally to combat malnutrition and calcium deficiency. Blast, caused by Magnaporthe grisea, causes annual yield losses of $\sim 80 \%$. M. grisea is a virulent pathogen on several graminaceous hosts. Seed-priming with bacteria induce antioxidants that act as signaling molecules and alter cellular patterns of the host that enhance resistance during the early stages of infection. The objectives of this study 
were: (i) to select rhizobacteria that promote growth and induce disease resistance; (ii) to evaluate disease resistance by assessing the temporal accumulation of enzymatic and non-enzymatic antioxidants; (iii) to deduce the metabolic profile in primed versus the control plants. Two fluorescent Pseudomonas isolates JUPC113 (GenBank ID. KX010601) and JUPW121 (GenBank ID. KX010602) were used to prime ragi cv Indaf 9 seeds. JUPC121-primed plants inoculated with M. grisea exhibited blast incidence 92\% lower with increased vigor index (3064.00 \pm 3.06 ) than un-primed plants. Significant increases in antioxidant activities and the activities of tyrosine ammonia lyase and phenylalanine ammonia lyase were observed early in primed plants. There was also increased benzoic acid accumulation, a precursor of salicylic acid pathway along with the accumulation of lignin and callose as mechanical barriers for pathogen entry. Gene expression studies during induction of resistance are in progress to deduce the coordinated role of rhizosphere microbes. Development of different formulations for field testing is ongoing.

Evaluation of physiological effect of fungal culture filtrate (FCF) and mycelial cell wall fraction (MCW) of Alternaria sp. on banana plant Z. M. PATEL, S. S. M. Jampala, Sardar Patel University, Bakrol, INDIA

Banana is the fifth largest agricultural commodity in the world trade. Its production has been affected by a vast array of pathogenic fungi causing high economic loss to the farmers. In spite of substantial advances in plant disease control strategies, global food supply is still threatened by a large number of pathogens and pests. In this study, the efficacy of the fungal components (FCF and MCW) prepared from Alternaria sp. has been used as an elicitor for induced resistance in potted and field grown banana plants against a broad spectrum of bacterial and fungal pathogens. Foliar application of elicitor was given thrice at two months of interval from the day of plantation in the field. Leaf samples were collected continuously up to seven days from the day of treatment. Elicitor treated plants showed increase in activity of defence related enzymes like phenylalanine ammonia lyase, peroxidase, polyphenol oxidase and $\beta-1,3$ glucanase as well as high content of total protein, total phenol and chlorophyll as compared to untreated plants. FCF treatment showed higher induction of defense related enzymes in treated plants than MCW treatment. Thoughtful application of the plants own defence mechanism can be useful for integrated disease management in banana.

Physiological response of naturally regenerated Pinus taeda $\mathrm{L}$. saplings to four levels of stem inoculation with Leptographium terebrantis J. MENSAH (1), M. A. Sword Sayer (2), R. Nadel (1), Z. Fan (1), G. Matusick (1), L. G. Eckhardt (1), (1) School of Forestry and Wildlife Sciences, Auburn University, Auburn, AL, USA; (2) Forest Service, Southern Research Station, Pineville, LA, USA

Leptographium terebrantis is an opportunistic root pathogen commonly associated with loblolly pine (Pinus taeda L.) stands that are undergoing a loss of vigor in the southeastern US. In order to understand the relationship between L. terebrantis inoculum density and host physiology, an artificial inoculation study was conducted in a five-year-old naturally regenerated loblolly pine stand over a 24 week period in a completely randomized design. We found that $L$. terebrantis caused sapwood occlusions that increased in severity as inoculum density increased. The occlusions significantly reduced water transport through the stem but did not interfere with fascicle-level stomatal conductance or induce moisture stress in the saplings. The resilience of stomatal conductance among pathogen-infested saplings is attributed to the growth and hydraulic function of new sapwood that developed after artificial inoculation. Results demonstrate that faster-growing families of loblolly pine may be capable of tolerating the vascular root disease when the formation of new sapwood is supported by sustained crown health.

Expression Analysis of Soybean PAL Induced by Biocontrol Rhizobia Sneb183

Y. WANG (1), J. Qiu (2), X. Zhu (2), L. Chen (3), Y. Duan (1), (1) Shenyang Agricultural University, Shenyang, CHINA; (2) Shenyang Agriculture University, ShenYang, CHINA; (3) Shenyang Agriculture University, Shenyang, CHINA

As the most important enzyme in the metabolism of phenylpropanoids. PAL is involved in the synthesis and accumulation of plant secondary metabolites (phytoalexin, lignin and phenolic compounds), and plays an important role in plant disease resistance and can be used as a biochemical indicator of plant disease resistance. Biological and non-biological can induce plant PAL gene transcription at the transcriptional level. Sinorhizobium fredii Sneb183 was selected with ability to induce soybean resistance to SCN. To understand the mechanism of Sneb183 induce the soybean resistance, RtPCR and ELISA were used to investigated the expression patterns of PAL genes and the content of PAL in soybean root during the early stages different time points after Sneb183 inoculation. The results were as follows: RtPCR analysis results revealed that PAL mRNA levels reached to the highest value 4 hours after inoculation, 3.11 times that of the control, and decreased to 1.61 and 1.18 times from 6 to 8 hours after inoculation. It comes to1.81 times When 24 hours after inoculation. The PAL content in soybean roots has similar trend with the PAL mRNA. It is lower than control at 2 hours inoculated, but increases gradually after that. It has two peaks at 4 hours and 24hours after inoculation and 1.22 times and 1.14 times higher than the control respectively. Our results demonstrates that PAL in soybean roots can be induced by Sneb183, the different stage of PAL expression pattern has diverse role in infection, colonization of Sneb183 and resistance to SCN. At first PAL play adverse roles in Sneb183 infection and colonization, but after infection successfully, infected Sneb183 can rapidly induce the expression and accumulation of PAL in soybean root and resist SCN.

Defense response, water balance and photosynthesis in oak leaves infected with purple mold disease caused by Cystotheca wrightii T. IKEDA (1), M. Okuda (2), H. Taneda (3), T. Fujiwara (4), H. Kato (4), (1) Kyoto Prefectural University, Kyoto, JAPAN; (2) Kyoto Perfectural University, Kyoto, JAPAN; (3) The Unniversity of Tokyo, Tokyo, JAPAN; (4) National Institute of Advanced Industrial Science and Technology (AIST), Tsukuba, JAPAN

Quercus glauca leaves infected with the purple mold disease caused by Cystotheca wrightii do not die, but experience a water deficit. To understand in more detail the effect of $C$. wrightii on leaf function, we studied the histological and anatomical features of infected leaves. We also performed a nondestructive X-ray analysis and assessed the water balance and photosynthesis. X-ray images revealed a higher density in infected parts of the leaf than in non-infected parts. In this denser part of an infected leaf, anatomical and histochemical observations showed the formation of a multi-layered wound periderm and the accumulation of tannin and/or suberin. Lignin was also detected in cell walls of wound periderm. Water relations were impacted as well: The cuticular transpiration of infected leaves increased with a lack of epicuticular wax crystals while leaf lamina hydraulic conductance decreased. Hence, infected leaves transpired more while less water was supplied to the leaf mesophyll. Chlorophyll fluorescence measurements revealed a decline in photosynthetic activity in the infected part of leaves. In summary, leaves infected with purple mold disease showed a defense response, had an unbalanced water status, and exhibited a decline in photosynthetic activity.

Development of inoculation methods to understand interactions of phoma stem canker and light leaf spot causal pathogens during leaf infection J. FORTUNE, E. Garcia-Cano, G. Mitrousia, B. Fitt, Y. Huang, University of Hertfordshire, Hatfield, UNITED KINGDOM

UK oilseed yields have not increased in the last 10 years, in part due to yield losses from diseases, such as phoma stem canker and light leaf spot caused by Leptosphaeria maculans and Pyrenopeziza brassicae, respectively. Some cultivars resistant to L. maculans are susceptible to $P$. brassicae. This 
investigation aims to understand interaction between these pathogens during leaf infection. A robust inoculation method is needed so symptoms can develop reliably after inoculation. Conidial suspensions of $L$. maculans $\left(10^{7}\right.$ spores $\left./ \mathrm{ml}\right)$ or $P$. brassicae $\left(10^{5}\right.$ spores $\left./ \mathrm{ml}\right)$ were prepared with $0.05 \%$ Silwet wetting agent. Two oilseed rape cultivars, each with resistance to one of the pathogens, were inoculated using different methods. Infection rates (\% sites for point or $\%$ of leaves for spray inoculation that showed symptoms) were $10 \%, 67 \%$ or $0 \%$ for L. maculans and $15 \%, 17 \%$ or $56 \%$ for P. brassicae when plants were inoculated with filter paper discs soaked with conidia suspension, drops of $10 \mu \mathrm{l}$ of conidial suspension or sprayed with conidial suspension, respectively. Infection rates were low and there is a need to improve inoculation methods. Different resistance responses were observed for L. maculans and P. brassicae. L. maculans triggered a resistance response at the inoculation site and $P$. brassicae triggered a resistance response at the inoculation site and on the main vein. There is a need to further investigate the resistance response to inoculation with both pathogens.

Transgenic expression of a plant defensin in alfalfa (Medicago sativa) leads to increased resistance to crown rot pathogens

A. E. SATHOFF (1), S. Velivelli (2), D. M. Shah (3), D. A. Samac (4), (1) University of Minnesota, Saint Paul, MN, USA; (2) Donald Danforth Plant Science Center, St. Louis, MO, USA; (3) Donald Danforth Plant Science Center, St Louis, MO, USA; (4) USDA-ARS, St Paul, MN, USA

Plant defensins are small cationic peptides with a conserved signature of cysteines. These peptides inhibit the growth of a broad range of fungi and bacteria. Alfalfa crown rot is a disease complex that reduces alfalfa stand density and causes substantial losses in productivity in all alfalfa-growing areas. Currently, there are no effective methods of disease control. To evaluate plant defensins as a potential control for alfalfa crown rot, the amount of defensin needed to inhibit growth of pathogen strains by $50 \%\left(\mathrm{IC}_{50}\right)$ was calculated. MtDef5, a bi-domain defensin from Medicago truncatula, displayed high activity against both bacterial and fungal crown rot pathogens in vitro. MtDef5 had $\mathrm{IC}_{50}$ values against Pseudomonas syringae pv. syringae and Phoma medicaginis of $0.198 \mu \mathrm{M}$ and $1.50 \mu \mathrm{M}$, respectively. Agrobacterium-mediated transformation was used to create transgenic lines of alfalfa (cultivar Regen SY27x) constitutively expressing MtDef5. Expression of the transgene was confirmed by qRT-PCR and by Western blots using the polyclonal anti-MtDef5 antibody. Disease bioassays demonstrated increased resistance against fungal crown rot pathogens, especially against $P$. medicaginis in transgenic lines expressing MtDef5. These experiments show promise for not only controlling crown rot pathogens, but potentially a wide array of economically important fungal and bacterial pathogens through the transgenic expression of a plant defensin.

The potential effect of karrikinolide (KAR $)$ in inducing resistance against Alternaria solani on tomato A. DAKUIDREKETI, E. Aitken, M. Furlong, J. Kochanek, University of Queensland, St Lucia, AUSTRALIA

Karrikinolide $\left(\mathrm{KAR}_{1}\right)$ is a highly active compound belonging to the karrikin family; a novel plant growth regulator, which is generated by wildfires. It is known to stimulate an array of growth responses in a wide range of plants akin to smoke or aqueous extracts of smoke. Recently, $\mathrm{KAR}_{1}$ has been reported to improve plant performance by inducing rapid and vigorous growth of some horticultural crops at concentrations as low as one-part-perbillion. Although the positive effects of $\mathrm{KAR}_{1}$ has shown to be of potential in the physiological growth and development of these crops, essentially nothing is known of the role of $\mathrm{KAR}_{1}$ in plant-pathogen interactions. Therefore, in this study we sought to determine if $\mathrm{KAR}_{1}$ has the capacity to induce resistance in tomato inoculated with Alternaria solani, a necrotrophic fungus that causes early blight. The results of this study demonstrate for the first time that pretreatment of tomato with $\mathrm{KAR}_{1}$ at $1 \mu \mathrm{gL}^{-1}$ reduced the development of disease caused by Alternaria solani and stimulated defence responses particularly in the genes of the salicylic acid pathway GluA and the PR-la genes.

Involvement of tryptophan-derived metabolites in the post-invasive resistance of Arabidopsis thaliana against multiple fungal pathogens A. KOSAKA (1), M. Pastorczyk (2), M. Kaido (1), K. Mise (1), P. Bednarek (2), Y. Takano (1), (1) Kyoto University, Graduate School of Agriculture, Kyoto, JAPAN; (2) Polish Academy of Science, Poznan, POLAND

A hemibiotrophic pathogen Colletotrichum tropicale (Ctro), formerly called C. gloeosporioides, is not adapted to Arabidopsis thaliana but is able to invade the Arabidopsis pen 2 mutant. However, once Ctro enters pen 2, its further expansion is blocked by post-invasive defense. The pen 2 pad 3 mutant exhibits a reduction in post-invasive resistance against $C$ tro. Here we investigated the post-invasive resistance against $C$ tro in the pen 2 cyp $71 A 12$ cyp71A13 mutant. Ctro was inoculated on tested plant lines, and the inoculated leaves were collected and stained with trypan blue. Microscopic observation showed that the triple mutant exhibited enhanced reduction in the resistance compared with pen 2 pad 3 . We further found the post-invasive resistance reduced in pen 2 cyp 71 A12, similar to pen 2 pad3. Similar phenomenon was observed in the interaction between adapted $C$. higginsianum and the same $A$. thaliana mutants. These findings suggest that the post-invasive resistance against hemibiotrophic Colletotrichum fungi needs (i) the CYP71A12-dependent synthesis of indole-3-carboxylic acid and/or related compounds (I3CAs) and (ii) the CYP71A13 and PAD3-dependent synthesis of camalexin. Notably, the pen 2 cyp $71 A 12$ cyp 71 A13 mutant exhibits reduced resistance against the necrotrophic pathogen Alternaria brassicicola, as compared with pen 2 pad3. Therefore, both I3CAs and camalexin are involved in post-invasive resistance against fungal pathogens with distinct infection strategies.

The molecular mechanisms of resistance in tomato induced by Pseudomonas fluorescens Sneb825 against root-knot nematode H. FAN, Y. You, D. Zhao, H. Zhao, X. Zhu, Y. Wang, Y. Duan, L. Chen, Shenyang Agricultural University, Shenyang, CHINA

The root-knot nematode (RKN) is one of the most seriously damaging plant-parasitic nematode in the world and threatens to the production of vegetable crops, including tomato. Biological control with microbial antagonists has received a great deal of attention as a promising measure for reducing the use of fungicides for controlling different plant diseases. Pseudomonas fluorescens Sneb825 is a beneficial bacterium with high biological activity against RKN and screened from our laboratory's biocontrol strain library with the potential applications for biocontrol of RKN. P. fluorescens Sneb825 effectively inhibited Meloidogyne incognita infection in the split-root experiments and increased tomato plant biomass and yield of tomato in the filed experiments. The changes of lignin and ROS content in tomato roots and the expression level of their related genes were detected. The lignin content, hydrogen peroxide content, and superoxide anion radical production rate in tomato roots of irrigated treatments with the metabolite of $P$. fluorescens Sneb825 were higher than those in controls. After inoculating with second-stage juveniles of $M$. incognita, the expression level of lignin biosynthesis related gene $T P X 1$, hydrogen peroxide biosynthesis related gene $R B O H 1$, and peroxidase gene Ep5C in irrigated treatments with the metabolite of $P$. fluorescens Sneb825 reached the maximum level at 5, 10, and 10 days post inoculation, respectively. It suggested that a large accumulation of lignin and ROS in tomato induced by $P$. fluorescens Sneb825 was a defense strategy to against $M$. incognita. The results of our study are beneficial to reveal the biocontrol mechanisms of $P$. fluorescens Sneb825.

Nanochitosan mediated induced systemic resistance against pearl millet downy mildew through nitric oxide generation C. S. NAYAKA, University of Mysore, Mysore, INDIA 
Pearl millet is a staple food for more than 90 million farmers in arid and semi-arid regions of sub-Saharan Africa, India and South Asia. Downy mildew of pearl millet caused by the biotrophic oomycete Sclerospora graminicola is the most devastating disease which impairs pearl millet production causing huge yield and monetary losses. Chitosan nanoparticles (CNP) were synthesized from low molecular weight chitosan having higher degree of acetylation was evaluated for their efficacy against downy mildew disease of pearl millet caused by Sclerospora graminicola. Seed treatment with CNP induced systemic and durable resistance and showed significant downy mildew protection under greenhouse conditions in comparison to the untreated control. Seed treatment with CNP showed changes in gene expression profiles wherein expression of genes of phenylalanine ammonia lyase, peroxidase, polyphenoloxidase, catalase and superoxide dismutase were highly upregulated. Downy mildew protective effect offered by CNP was found to be modulated by nitric oxide and treatment with CNP along with NO inhibitors cPTIO completely abolished the gene expression of defense enzymes and PR proteins.

Transcriptome and GWAS-based approaches to understand the mechanisms of Fusarium fujikuroi resistance in rice D. SPADARO (1), S. Matic (2), I. Siciliano (2), P. Bagnaresi (3), A. Volante (4), M. Aragona (5), A. Infantino (6), M. L. Gullino (7), G. Valè (4), (1) DISAFA and AGROINNOVA, University of Torino, Torino, ITALY; (2) Agroinnova - University of Torino, Grugliasco, ITALY; (3) CREA - Genomic Research Centre, Fiorenzuola (PC), ITALY; (4) CREA - Rice Research Unit, Vercelli, ITALY; (5) CREA - Plant Pathology Research Centre, Roma, ITALY; (6) CREA - Plant Pathology Research Centre, Rome, ITALY; (7) Agroinnova - University of Torino, Grugliasco, Torino, ITALY

Fusarium fujikuroi, causal agent of Bakanae disease, is the main seedborne pathogen on rice. Profiles of defense-related phytohormones and phytoalexins were investigated. In the resistant genotype Selenio, the pathogen induced high levels of sakuranetin and other phytoalexins. In the susceptible genotype Dorella, the pathogen induced gibberellins and abscisic acid, inhibited jasmonic acid, and Bakanae symptoms were observed. A RNA-seq transcriptome study was performed. The basic rice resistance machinery against $F$. fujikuroi involved PR genes, glucanases and peroxidases. The resistance mechanisms activated in the resistant cultivar included WRKY transcriptional factors, MAPK cascades, and cytochrome P450 genes. When the gibberellin production was controlled, Selenio plants activated the jasmonic acid metabolic pathway. A germplasm collection of japonica rice was screened for F. fujikuroi resistance, allowing the identification of accessions with high-to-moderate levels of resistance to bakanae. A genome-wide association study (GWAS) uncovered two genomic regions highly associated with the observed phenotypic variation for response to bakanae infection. A search for candidate genes with a putative role in bakanae resistance was conducted considering all the annotated genes and $F$. fujikuroi-related DEGs included in the two genomic regions highlighting several gene functions that could be involved in resistance, thus paving the way to functional characterization of the resistance loci.

Identifying susceptibility genes for citrus Huanglongbing in sweet orange

F. NOGALES C. VASCONCELOS, Z. Pang, N. Wang, University of Florida, Lake Alfred, FL, USA

The phloem limited bacteria, Candidatus Liberibacter asiaticus, $\mathrm{Ca}$. L. americanus and $\mathrm{Ca}$. L. africanus, are the putative causal agents of citrus Huanglongbing (HLB), which is devastating citrus industry worldwide. Identifying susceptibility genes is crucial to understand its interaction with citrus host and therefore engineer tolerant and resistant plants. In this study, we will present our current progress in identification of putative susceptibility genes against HLB. To identify putative susceptibility genes, we used yeast-two-hybrid system to screen for potential host target proteins of putative virulence factors of $\mathrm{Ca}$. L. asiaticus. We also employed gene expression essays in order to identify differentially expressed genes in HLB diseased trees, giving us insights of microbe-host interactions at molecular level. The CRISPR technology is being used to mutate putative HLB susceptibility genes to test whether they affect citrus resistance or tolerance to HLB.

Intensification on PAMP-triggered immunity confers disease resistance against bacterial soft rot Z. JING-LIN (1), Y. H. Lin (2), (1) National Pingtung University of Science and Technology, Pingtung, TAIWAN; (2) National Pingtung Univ of Science \& Tech, Pingtung, TAIWAN

Bacterial soft rot disease is a devastating disease affecting a variety of plants worldwide. Possible strategy for controlling this disease consists of introducing the expression of the plant ferredoxin-like protein (pflp) gene in plants and usage of beneficial microorganisms. The PFLP-mediated resistance is associated with the intensification of PAMP-triggered immunity (PTI), have been demonstrated. To gain further insights regarding the enhanced intracellular PTI signaling contributed by PFLP, Arabidopsis mutants in MAPK pathway were used to assay the responses triggered by flg22 $2_{\text {stt. }}$ Firstly, we confirmed that the rapid generation of $\mathrm{H}_{2} \mathrm{O}_{2}$, callose deposition, and hypersensitive response (HR) triggered by flg $22_{\mathrm{Pst}}$ was intensified by PFLP. Then, we demonstrated that the flg22 $2_{\text {sst }}$-induced MAPK pathway was intensified by PFLP based on the expression of the FRK1 gene mapk mutants. In addition to the rapid $\mathrm{H}_{2} \mathrm{O}_{2}$ generation, callose deposition and expression of FRK1 were still intensified by Bacillus amyloliquefaciens PMB05 upon the treatment of flg22 $2_{\mathrm{pst}}$. Morever, B. amyloliquefaciens PMB05 confers resistance against soft rot disease. These suggested that the disease resistance enhanced by $B$. amyloliquefaciens PMB05 was associated with the intensification on PTI through signaling in MAPK pathway. Taken together, we showed the intensification on PAMP-triggered immunity by PFLP or B. amyloliquefaciens strain PMB05 could be as a dominant factor to increase resistance against bacterial soft rot.

Intensification on PAMP triggered immunity by Bacillus strains to control bacterial wilt of tomato

T. H. HO (1), Y. H. Lin (2), (1) Department of Plant Medicine, National Pingtung University of Science and Technology, Pingtung, TAIWAN; (2) National Pingtung Univ of Science \& Tech, Pingtung, TAIWAN

Tomato bacterial wilt caused by Ralstonia solanacearum is a devastating disease in tomato production. Currently, there is still no effective chemical to control this disease. To control this disease, reports showed transgenic plants express extracellalar PFLP protein are resistant against bacterial wilt. The mechanism of this resistance is associated with the intensification of harpin-mediafed HR. Therefore, we sought to screen bacterial strains of Bacillus spp. that can enhance harpin-mediated HR and further evaluate their efficacy on controlling bacterial wilt of tomato. Before screening, the harpin from $R$ solanacearum, PopW, was cloned and expressed in pET system. Results revealed that the HR on tobacco leaves could be induced by PopW. Among 9 rhizosphere Bacillus strains we assayed, B. amyloliquefaciens PMB05 was one strain which could intensify PopW-induced HR. Bacterial suspension of PMB05 applied in the rhizosphere of 2-week old tomato seedlings exhibited strong resistant against bacterial wilt. Finally, we demonstrated that the flg22 $2_{\mathrm{RS}}$-induced ROS generation in tomato roots was intensified by B. amyloliquefaciens strain PMB05. Taken together, we concluded that the PAMP triggered immunity intensified by $B$. amyloliquefaciens strain PMB05 confers resistance of tomato plants against bacterial wilt disease.

Lignin reduction in alfalfa (Medicago sativa) does not affect foliar disease resistance D. A. SAMAC (1), S. Ao (2), M. Dornbusch (1), A. Grev (2), S. Wells (2), K. Martinson (2), C. Sheaffer (2), (1) USDA-ARS, St Paul, MN, USA; (2) University of Minnesota, St. Paul, MN, USA 
Disruptions in the lignin biosynthetic pathway have been shown to reduce disease resistance in a number of crops. Recently, genetically modified alfalfa (Medicago sativa) varieties have been marketed with reduced lignin and improved forage quality traits, including increased digestibility by ruminants at later stages of plant maturity. The objective of this study was to compare foliar disease resistance in three reference alfalfa varieties, 54R02, DKA4322RR, WL355.RR, and the reduced lignin variety, 54HVX41, to evaluate the effect of the reduced lignin trait on foliar disease resistance. Alfalfa plants in research plots at three locations in Minnesota were evaluated for percent defoliation caused by foliar pathogens at four maturity stages; early bud, bud, early flower, and flowering; with natural inoculum. Spring black stem and leaf spot, Leptosphaerulina leaf spot, and common leaf spot were observed from June through September in all locations on all varieties. Summer black stem and leaf spot was most prevalent in August on all varieties at one location. The amount of defoliation increased with maturity stage for all varieties. When harvest was delayed until the flowering stage, moderate to severe (32 to 64\%) leaf loss occurred, depending on location. Alfalfa varieties did not differ in percent defoliation at any maturity stage indicating that the reduced lignin trait did not affect foliar disease resistance.

Network analysis to uncover and quantify host defense signaling-dependent virulence effects of Pseudomonas syringae pv. tomato A. TURO, D. Mackey, Ohio State University, Columbus, OH, USA

Interactions between plants and phytopathogenic bacteria are dynamic, with outcomes that depend on the interactions of virulence effectors and host defense pathways. Among the arsenal of effectors of Pseudomonas syringae pv. tomato (Pto), the phytotoxin coronatine (COR) and the functionally redundant type-III effectors AvrE1 and HopM make key contributions to virulence on host plants. While these effectors perform partially overlapping functions by interacting with distinct host targets, an understanding of how their activities are coordinated through downstream pathways is unclear. Arabidopsis thaliana employs a hormone-dependent signaling network to coordinate host defense, including against Pto. COR, AvrE1 and HopM1 directly and indirectly target the salicylic acid, jasmonate, ethylene, and indole glucosinolate-dependent sectors of this network. We have constructed polymutant sets of both Pto and Arabidopsis for all combinations of these effectors and pathways and will measure quantitatively the interaction of these strains and plants, at both the level of bacterial growth and plant responses. This approach will enable network reconstitution to reveal the virulence contributions of individual and interacting effectors in the context of plants disrupted in different sectors of the hormone network. By determining virulence effector activity in the context of the host defense network, we aim to better understand effector-dependent mechanisms of virulence.

Transcriptome profile of Carrizo citrange roots in response to Phytophthora parasitica infection Z. AFZAL (1), J. C. Huguet-Tapia (2), G. S. Ali (1,3), (1) University of Florida, Apopka, FL, USA; (2) University of Florida, Gainesville, FL, USA; (3) University of Florida, 32703, FL, USA

Phytophthora diseases are the most important soil-borne diseases of citrus in almost all citrus groves of the world. Phytophthora parasitica is among the most widespread Phytophthora species, known to cause root rot, foot rot/gummosis and brown rot of fruits in citrus. Current understanding of citrusPhytophthora interaction at the whole genome level is very limited. In this study, we have analyzed the transcriptome of a commonly used Carrizo citrange citrus rootstock in response to $P$. parasitica infection using the RNA-seq technology. In total, we have identified 8284 differentially expressed transcripts (DETs) among $P$. parasitica-inoculated and mock-treated roots. Of these, 4916 genes were differentially expressed at 24 hours post inoculation and 6718 genes were differentially expressed at 48 hours post inoculation, which corresponds to the biotrophic and necrotrophic phases of $P$. parasitica infection cycles, respectively. Gene ontology analysis of DETs suggested substantial transcriptional reprogramming of diverse cellular processes particularly the biotic stress response pathways in Carrizo citrange roots. Many $R$ genes, Pathogenesis Related (PR) genes, autophagy-related genes and several other genes putatively involved in plant immunity and systemic acquired resistance (SAR) were upregulated in response to $P$. parasitica infection. Analysis reported here lays out a strong foundation for future studies aimed at improving resistance of citrus rootstocks to $P$. parasitica infection.

OsGRDP1 Is a Positive Regulator of Cell Death and Disease Resistance by Activate OsAP25 in Rice W. ZHAO, X. Zhao, T. Qiu, T. Zhang, J. Yang PhD, Y. L. Peng, China Agricultural University, Beijing, CHINA

Lesion mimic mutants are valuable materials for unraveling the signaling pathways and molecular mechanisms governing the PCD execution in plants. Here, we identified a dominant mutant spl-D with spontaneous spots-leaf phenotype from T-DNA inserted T1 rice lines. DAB staining showed that intense brown signals were correlated with lesion formation in $s p l-D$ leaves, indicating $\mathrm{H}_{2} \mathrm{O}_{2}$ accumulation might be responsible for spots emergence spl-D exhibited enhanced resistances to both $M$. oryzae and Xoo. Quantitative PCR analysis showed that several defense-related genes known to be involved in SA and JA signaling pathways were constitutively activated in $s p l-D$. TAIL-PCR analysis showed that the T-DNA insertion site locates 495 bp upstream of $O s G R D P 1$. Expression level of $O s G R D P 1$ in spl-D was markedly higher than that in the wild type. Moreover, transgenic lines in which $O s G R D P 1$ driven by maize ubiquitin promoter exhibited similar phenotypes to $s p l-D$, indicating phenotypes of $s p l-D$ were resulted from overexpression of OsGRDP1. OsGRDP1 interacted with an aspartic proteinase OsAP25 in yeast and in vivo. In spl-D, the proteinase activity of OsAP25 was activated, and the lesion formation could be inhibited by pepstain $\mathrm{A}$, an aspartic proteinase inhibitor. In addition, lesion formation of spl-D was accelerated in dark. Taken together, OsGRDP1 is a positive regulator of cell death and disease resistance, which provides a new clue to elucidate the mechanism of lesion formation.

\section{Induced resistance to late blight depends on potato inducibility and on Phytophthora infestans effectors}

C. THOMAS (1), P. Le Boulch (2), D. Andrivon (1), F. Val (2), (1) INRA UMR 1349 IGEPP (Institute of Genetics, Environment and Plant Protection), Le Rheu Cedex, FRANCE; (2) AGROCAMPUS OUEST UMR 1349 IGEPP (Institute of Genetics, Environment and Plant Protection), Rennes Cedex, FRANCE

Pathogen recognition by plants via pathogen-associated molecular patterns leads to PAMP-triggered immunity but pathogens can modulate it via effectors production. Only few studies connect plant responses with parasite ecology particularly in the interaction between Solanum tuberosum and Phytophthora infestans. We hypothesize that potato induced defense triggered by PAMPs from P. infestans culture filtrate (CCF) could affect disease outcome and effector genes expression. Potato genotypes - Bintje, Désirée, Rosafolia - were sprayed with CCF. Detached leaflets were inoculated 48h later with 1 strain of $P$. infestans. Potato defense genes and $P$. infestans effectors expressions were assessed by qRT-PCR from $48 \mathrm{~h}$ to $4 \mathrm{dpi}$. Simultaneously, disease was evaluated by lesion area measurement and sporangia counting. Treatment with CCF increased the expression of defense genes, reduced lesion and sporangia production in Bintje and Désirée. In Rosafolia, it repressed most defense genes but it only increased sporangia number. In Bintje, after treatment and inoculation, defense genes were still induced until 3dpi but were repressed 4dpi. CCF treatment altered also the expression of pathogen effectors depending on time. Analyses are in process for the other genotypes. Our results highlight that efficiency of potato induced resistance depends on both genotype-dependent response and pathogen ecology via effectors expression during disease spreading. 
Dickeya spp. are necrotrophic bacterial pathogens that cause blackleg disease in potatoes. Blackleg disease has resulted in significant economic losses in the United States since 2015 and continues to devastate the potato industry. Breeding for resistance to blackleg in potato cultivars is difficult due to the limited understanding of genetic mechanisms of host-microbe interactions in this pathosystem. To better understand the plant defense response to Dickeya, quantitative reverse transcription polymerase chain reactions (qRT PCR) were performed with cDNA libraries made from total RNA. These RNAs were extracted from susceptible or tolerant diploid potato stems inoculated with $D$. dadantii 3937 or mock buffer at 0 and 12 -hours post inoculation. qRT-PCR experiments between mock and $D$. dadantii inoculated potatoes revealed changes in expression patterns of genes related to plant defense activation, including pathogen-associated molecular pattern-triggered immunity hallmark genes (Pti5, Gras2, Lrr22), the flagellin receptor gene (FLS2), a salicylic acid regulated gene (PR1), and a jasmonic acid dependent gene (TPI-1). Differential expression of these genes could contribute to the tolerance or susceptibility of the two potato lines to Dickeya spp. Our study of targeted potato defense genes expression patterns provides novel information regarding plant defense mechanisms in the Dickeya-potato pathosystem at an early infection stage.

Controlling Sclerotinia sclerotiorum in Glycine max by targeting oxalic acid production using host-induced gene silencing M. MCCAGHEY, A. Ranjan, J. Kurcezewski, D. Smith, M. Kabbage, University of Wisconsin-Madison, Madison, WI, USA

Sclerotinia sclerotiorum, the causal agent of Sclerotinia stem rot (SSR), is a yield-limiting, fungal pathogen of Glycine max. The pathogenic success of $S$. sclerotiorum requires the secretion of oxalic acid (OA), a key virulence factor for this pathogen. Virus-induced gene silencing (VIGS) using Bean pod mottle virus (BPMV) was used to target OA biogenesis in S. sclerotiorum. A sequence of $366 \mathrm{bp}$, corresponding to the fungal oxaloacetate acetylhydrolase (Ssoah1), was cloned into a BPMV vector in an antisense orientation. BPMV constructs were introduced into G. max using particle bombardment, and viral symptoms paired with RT-PCR were used to confirm viral replication prior to inoculation. Disease progress and target mRNA levels were monitored over a five-day period. Remarkably, plants containing BPMV vectors targeting Ssoah1 showed enhanced resistance to $S$. sclerotiorum compared to empty-vector control plants, in three replicated experiments. These results provide evidence supporting host-induced gene silencing targeting virulence factors as a viable strategy to control SSR. Our study also evaluated exogenous applications of dsRNA and sRNA to limit fungal growth and OA production in vitro and in planta. Genetic resistance to SSR is inadequate in commercial soybean varieties, while fungicidal control can be inconsistent and expensive. Herein, we propose that RNAi strategies will provide new tools for resistance to S. sclerotiorum in G. max.

Transcriptomic analysis for differentially expressed genes in response to the phytoalexin gossypol in Fusarium oxysporum f. sp. vasinfectum J. Coleman, A. POKHREL, Auburn University, Auburn, AL, USA

Fusarium oxysporium f. sp. vasinfectum (FOV) is a soilborne filamentous fungus that causes vascular wilt in cotton. In response to pathogens including FOV, cotton plants produce the phytoalexin gossypol as a defense mechanism. This research aims to explore the molecular mechanisms utilized by FOV to tolerate the antimicrobial action of gossypol during infection and colonization of cotton. To identify these mechanisms candidate genes that could be responsible for gossypol tolerance were identified through RNA sequencing. Four RNA samples were extracted from germlings of a virulent race 4 genotype FOV isolate, grown in minimal medium before and after treatment of $80 \mathrm{ug} / \mathrm{mL}$ of gossypol at 1, 2 and 4 hours. RNA sequence data showed upregulation of $\mathrm{ABC}$ and major facilitator transporters and several classes of dehydrogenases when compared with the non-treated FOV control RNA sample. The last phase of this work involves validation of genes identified through RNA sequencing in gossypol tolerance. This will be done through generation of mutants using CRISPR-Cas9 technology. The research findings will help to understand how a pathogen can develop mechanisms to overcome these plant antimicrobial compounds.

Foliar resistance to bacteria in potato

D. A. HALTERMAN, USDA ARS, Madison, WI, USA

Solanaceous crops including tomato, pepper, and eggplant are susceptible to many foliar bacterial pathogens. However, cultivated potato is immune to most pathogenic Pseudomonas and Xanthomonas species. The purpose of this project is to understand the mechanisms involved in limiting infection of bacteria in the foliage of potato. We have previously found that many popular potato cultivars are immune $P$. syringae pv. tomato DC3000, while many wild species accessions of potato are susceptible or tolerant, suggesting that resistance to foliar pathogens may have been selected during cultivation of potato as a food crop. We have used various DC300 strains defective in pathogenesis ( $\Delta \mathrm{hrcC}, \Delta \mathrm{fliC}$, -coronatine). Populations between wild and cultivated potato have also been developed to map the resistance locus using SNP genotyping. The identification of novel genes involved in bacterial resistance will facilitate the development of new varieties of Solanaceous crops.

Phylloxera galls as Plasmopara viticola infection and sporulation sites on leaves of grapevines partially resistant to downy mildew C. HONG (1), J. O. Obuya (2), P. M. Brannen (1), V. Tsolova (2), H. Scherm (1), (1) University of Georgia, Athens, GA, USA; (2) Florida A\&M University, Tallahassee, FL, USA

Downy mildew (Plasmopara viticola) and phylloxera (Daktulosphaira vitifoliae) are two important pests in many grape production areas worldwide. Both species can produce severe symptoms on leaves, but no interactions between them have been reported previously. In 2017, $P$. viticola was found sporulating on galls of phylloxera-affected leaves of interspecific hybrids 'Blanc du Bois' and 'Lenoir' in vineyards in southern Georgia and northern Florida; no signs of infection were observed on leaves not affected by phylloxera. 'Blanc du Bois' is generally considered resistant to downy mildew once leaves have matured. Leaves harboring $P$. viticola were collected and the pathogen was subcultured on Vitis vinifera 'Chardonnay'. Artificial inoculations with two isolates of $P$. viticola (Pvv731S1 and Pva1068) were conducted on 'Blanc du Bois' leaf disks with or without phylloxera galls to determine the effects of the galls on infection efficiency and sporulation of the pathogen. Each leaf disk was inoculated with $5 \mu 1$ of a sporangium suspension $\left(10^{4}\right.$ sporangia/ml), and disks were incubated at $20^{\circ} \mathrm{C}$ for $16-24 \mathrm{~h}$ in the dark followed by 6 days under diurnal light. Preliminary results revealed that phylloxera galls increased infection efficiency of isolate Pva1068 significantly $(P=0.0069$ based on Chi-Square analysis). This is the first report showing that phylloxera galls can compromise resistance to downy mildew and serve as a reservoir for $P$. viticola on resistant grape cultivars.

WRKYs phosphorylated by MAPK regulate chloroplast-mediated ROS burst in plant immunity H. YOSHIOKA (1), H. Adachi (2), M. Yoshioka (1), (1) Nagoya University, Nagoya, JAPAN; (2) The Sainsbury Laboratory, Norwich, UNITED KINGDOM 
Plants have evolved two types of immune systems; pattern-triggered immunity (PTI) and effector-triggered immunity (ETI) often accompanied by HR cell death. WRKY transcription factors function in plant immunity through phosphorylation by MAPKs. However, downstream signaling of the WRKYs is largely unknown. We investigated genes regulated by MAPK-WRKY phosphorylation pathway by RNA-seq. We found that plant immune WRKYs downregulate the large number of photosynthesis-related genes and inhibit photosynthetic $\mathrm{CO}_{2}$ fixation, resulting in generation of reactive oxygen species (ROS) in chloroplasts responsible for cell death. The MAPK-WRKY network controls the chloroplastic ROS burst to establish robust ETI to a plant pathogen. Furthermore, gain- and loss-of-function analyses indicated that six WRKYs are redundantly involved in ETI-triggered chloroplastic ROS burst in Nicotiana benthamiana. We have reported that the MAPK-WRKY cascades in ETI are involved in transcriptional activation of the gene for plasma membrane plant NADPH oxidase in $N$. benthamiana. We propose that plants developed two ROS-generating systems via immune MAPKWRKY network in light-dependent and independent manners.

A standardised set of differential potato cultivars to identify pathotypes in Synchytrium endobioticum G. VAN LEEUWEN (1), K. K. Heungens (2), J. Przetakiewicz (3), M. Boerma (4), L. Dimitrova (5), K. Flath (6), (1) National Reference Centre NPPONL, Wageningen, NETHERLANDS; (2) ILVO, Merelbeke, BELGIUM; (3) Plt Breeding and Acclimatization Inst National, Mazowieckie, POLAND; (4) HLB, Wijster, NETHERLANDS; (5) Central Lab for Plant Quarantine, Sofia, BULGARIA; (6) Julius Kuehn-Institut, Kleinmachnow, GERMANY

Several pathotypes are known for the quarantine potato pathogen Synchytrium endobioticum whose identification is based on their virulence (or avirulence) on specific potato cultivars, known as differentials. In former research, the cultivar Miriam was proved unsuitable for separating pathotypes 6(O1) and 18(T1). In the Euphresco-project "SENDO" (2012-2015), a group of five European laboratories tested a set of differential cultivars using the same bio-assay method, Glynne-Lemmerzahl. Compared with the standard set described in the EPPO Diagnostic Protocol, two new cultivars were tested: cultivars Talent and Logo. Two different pathotypes were tested, pathotype 6(O1) and 18(T1), and each partner tested ten eye fields per cultivar in three independent replications. The reaction of the sprouts was analysed after 25 days of incubation. Results were scored in two classes: 'wart formation' vs 'no wart formation'. Comparing Talent and Logo, it was explicitly shown that Talent was the most suitable candidate to replace Miriam in the differential set. No wart formation occurred when infected with pathotype 6(O1), while in all labs wart formation did occur in Talent infected by pathotype 18(T1). The results of the SENDO-project support the use of one standardized method and one uniform set of differentials to identify pathotypes in S. endobioticum, thus facilitating clear and unambiguous communication between scientists, risk managers and policy makers.

\section{Pathogenicity properties of some fungal species from Colletotrichum acutatum species complex} J. VILCANE, I. Nakurte, N. Rostoks, University of Latvia, Riga, LATVIA

Molecular tools have been extensively used for identification of fungal species in Colletotrichum acutatum species complex, while the differences in their pathogenicity and virulence in crop plants are not sufficiently studied. The aim of the study was to compare morphological progress of infection by C. godetia, C. nymphae and C. fiorianei on artificially inoculated apple fruits, with the changes in levels of jasmonic (JA) and salicylic acid (SA) as measured by HPLC-MS. Apples cv. 'Honeygold' were wound-inoculated with the $0.5 \mathrm{~mm}^{2}$ pieces of fungal mycelia and stored in sealed plastic boxes at $21^{\circ} \mathrm{C}$ for three weeks, along with the non-wounded and wounded, but non-inoculated controls. Inoculations with Penicillium expansum and Monilinia fructigena were done. Similar size lesions developed on apple surface after inoculation with all the three Colletotrichum species. Differences in the sporulation were observed on lesion surface, and the levels of SA and JA were significantly higher in samples with Colletotrichum sporulation. HPLCMS analysis revealed a relatively higher expression of JA in apples inoculated with Colletotrichum compared to samples inoculated with $M$. fructigena and $P$. expansum, as well as, in both controls. Significantly higher level of JA was detected in a sample inoculated with C. fiorianei (isolated from strawberry), while increase in JA was also observed in a sample inoculated with $C$. fiorianei (from raspberry and blueberry). Significantly higher levels of SA were measured in samples inoculated with C. fiorianei from strawberry, raspberry and blueberry. Overall, the results indicated significant link between levels of JA and SA in apple fruits, and the infection by Colletotrichum fiorianei.

Molecular mechanism of high-temperature resistance to yellow rust in Xiaoyan6

J. WANG, F. Tao, H. Shang, X. Hu, Northwest A\&F University, Yangling, CHINA

Stripe rust, caused by Puccinia striiformis f. sp. tritici (Pst), is a devastating disease of wheat (Triticum aestivum) worldwide. A loss of resistance to stripe rust in the wheat cultivars appeared frequently due to production of new virulent race of Pst constantly, whereas, wheat high-temperature seedling plant (HTSP) resistance to Pst is non-race-specific and durable. We identified 28 high-temperature resistance wheat cultivars to Pst including 13 hightemperature seedling variety from 400 landraces and 92 improved wheat in Northwest China since 1989. Among them, Xiaoyan6 maintains its resistance against stripe rust for nearly 30 years, is a typical durable resistant wheat variety. Xiaoyan6 was selected as a model wheat cultivar to study the HTSP resistance to Pst. The transcriptome of Xiaoyan6 under different temperature treatments were sequenced with Illumina technology, and the molecular mechanism was primarily illuminated by analyzing of different expression genes, KEGG pathway enrichment, and protein interaction. The results indicated that receptor like kinase with the help of heat shock proteins can combined with various effectors by interacting with different types of resistance genes, inducing the HR response of Xiaoyn6. In addition, Pst and temperature were detected by receptor like kinase following by the phosphorylation of kinase, transferring the signal to the WRKY transposon factors through the MAPK cascade, and causing the defense responses. The genes such as TaWRKY70, TaRPM1, TaRPS2 were employed to confirm the mechanism. These results can help people understand the mechanism of HTSP, and will make contributions to improve and utilize the resistance of HTSP.

Host induced gene silencing targeting aflM reduced aflatoxin contamination in transgenic corn

Y. RARUANG (1), O. O. Omolehin (1), Q. Wei (2), Z. Q. Han (3), D. Bhatnagar (2), Z. Y. Chen (1), (1) Louisiana State University Agricultural Center, Baton Rouge, LA, USA; (2) Southern Regional Research Center, USDA-ARS, New Orleans, LA, USA; (3) Guangxi Academy fo Agricultural Sciences, Nanning, CHINA

Reducing Aspergillus flavus infection and subsequent aflatoxin contamination in corn through biocontrol and developing resistant cultivars has so far met with limited success. In the present study, a novel host induced gene silencing (HIGS) strategy was employed to suppress the expression of aflM, an A. flavus gene encoding versicolorin, a key enzyme involved in the aflatoxin biosynthesis. An RNAi vector containing a portion of the aflM gene was constructed and introduced into B104 immature embryos through Agrobacterium transformation. Twenty-three transgenic plants were produced from seven independent transformation events. Six of seven transformation events were confirmed by PCR to have the aflM gene. The $\mathrm{T}_{2}$ generation kernels containing the transgene from two events out of four examined had less aflatoxin ( $p=0.01$ and $p=0.08)$ than those without the transgene. Field-inoculated homozygous $\mathrm{T}_{3}$ transgenic kernels also revealed lower aflatoxin $(\mathrm{p}=0.02)$ than kernels from the null or B104 controls. Similar result was observed when the harvested $\mathrm{T}_{3}$ homozygous transgenic kernels were evaluated under kernel screening assay conditions $(\mathrm{p}=0.008)$. In addition, significantly higher levels of aflM gene-specific small RNAs were detected in the transgenic leaf tissue, indicating that the enhanced aflatoxin resistance in the homozygous transgenic kernels is likely due to suppression of aflM expression through HIGS. 
Enhancing type II-resistance in crops through modification of the cell wall polymer callose T. HANAK (1), C. Voigt (2), J. Stuttmann (3), (1) University of Hamburg, Hamburg, GERMANY; (2) University of Sheffield, Sheffield, UNITED KINGDOM; (3) Martin- Luther- University Halle- Wittenberg, Halle (Saale), GERMANY

Food supply and security are two major challenges of mankind due to an increasing world population and climate change. Therefore, new strategies for enhancing plant defence against pathogens are required. To test new approaches in molecular crop breeding, we aim to modify the biosynthesis of the $(1,3)-\beta$-glucan cell wall polymer callose, known for its importance in plant defence and stress response in Brachypodium distachyon a model plant for crops and wheat (Triticum aestivum). We previously showed that overexpression (OE) of the stress-induced callose synthase PMR4 in Arabidopsis thaliana induced complete penetration resistance to powdery mildew through enhanced callose deposition. Now we overexpressed PMR 4 and its possible homolog BdGSL3 in B. distachyon. Both B. distachyon OE lines revealed a so-called type II resistance to the fungal crop pathogen Fusarium graminearum. The pathogen was only able to infect the directly inoculated floret. Enhanced callose deposition in the spikelets phloem prevented further colonization of the spikelet. In wheat lines overexpressing PMR4, we also observed a stronger type II-resistance to $F$. graminearum associated with enhanced callose deposition. CRISPR/Cas9-mediated disruption of BdGSL3 in B. distachyon indicates its involvement in stress-induced callose depositions. In conclusion, we identified callose biosynthesis as a tool for enhancing resistance to fungal pathogens in crops and the involvement of $B d G S L 3$ in stress response.

Antibacterial and plant defence elicitor peptides for plant disease control

E. MONTESINOS, B. Esther, J. Cabrefiga, L. Montesinos, I. Mora, C. Moragrega, I. Llorente, M. Pla, E. Bardají, M. Planas, L. Feliu, University of Girona, Girona, SPAIN

Synthetic peptides offer great possibilities as novel compounds to the control plant diseases. Libraries of synthetic analogs of natural peptides or de novo designed peptides have been prepared by solid phase chemistry and combinatorial approaches. Leads from these libraries have minimal inhibitory concentrations in the range of common antibiotics, and some have additional activity as plant defence elicitors and cell-penetrating peptides. Several of the most promising peptides had low toxicity profiles with moderate susceptibility to proteases. Antimicrobial peptides of the CECMEL11 library have been effective in greenhouse experiments and field trials, against fireblight (Erwinia amylovora) and bacterial blight of pear (Pseudomonas syringae pv. syringae), bacterial spot and canker of Prunus (Xanthomonas arboricola pv. pruni), and bacterial canker of kiwifruit ( $P$. syringe pv. actinidiae), and also in the control of two diseases caused by phytoplasms, and against brown spot of pear (Stemphylium vesicarium). A new generation of synthetic analogs of endogenous elicitor peptides from Prunus (namely PEPs), lacking antimicrobial properties, were effective in protection against $X$. arboricola pv. pruni infections. The peptide BP178, a derivative from the BP100 CECMEL11 peptide, showed antibacterial and plant defence elicitation activity, and was active against Xylella fastidiosa subspecies. The prospects and limitations of these functional peptides in plant protection, in the scenario of emerging diseases and the current regulatory framework will be discussed.

Explore the function of Papain-like cysteine proteases (PLCPs) in citrus resistance against Huanglongbing (HLB) Y. HUANG, University of Florida, Lake alfred, FL, USA

Huanglongbing (HLB) is one of the most devastating diseases of citrus in the world. It is caused by Candidatus Liberibacter asiaticus (Las) and transmitted by Asian citrus psyllid. Papain-like cysteine proteases (PLCPs) are a family of proteins in the plant apoplast which can be classified into nine subfamilies. They are considered as hubs in plant immunity because previous reports have shown that PLCPs can trigger plant defense against bacteria, fungi and nematodes. In addition, PLCPs are common targets of many pathogen effectors. Specially, our previous study showed that multiple putative Sec-dependent effectors of Las interact with PLCPs based on Yeast-two-hybrid assays. Here we will report our recent progress in functionally characterizing PLCPs. Genome editing via the CRISPR technology is being used to generate mutants of PLCPs. Overexpression of PLCPs in citrus and gene silencing of PLCPs are also being conducted to investigate the roles of PLCPs in HLB disease development.

Analysis of two switchgrass ecotypes indicates genetic diversity of a disease resistance gene class that contains a serine-threonine protein kinase L. D. NISSEN, S. M. Smith, The University of Georgia, Athens, GA, USA

Switchgrass (Panicum virgatum L.) is a perennial warm-season grass indigenous to tall grass prairies in North and Central America. The United States Department of Energy chose switchgrass as a potential bioenergy crop for ethanol production due to its rapid growth, high biomass yield, and ability to be cultivated in poor soil and climate conditions and with low inputs. Upland and lowland are major switchgrass ecotypes that have emerged through natural selection. Switchgrass has not been grown in monoculture, so it is not known which pests and pathogens will affect production. The nucleotide binding site (NBS)-leucine-rich repeat (LRR) class of resistance (R) genes is the largest known class of R-genes that plants use to detect the presence of pathogens and induce defense responses. This project has three major objectives: 1) Identify and sequence resistance gene homologues (RGHs) in the two switchgrass ecotypes, 2) Determine the number of RGH haplotypes in the ecotypes, and 3) Analyze the genetic diversity of RGHs in the ecotypes. In this study, a PCR-based approach was used to characterize the genetic diversity of the NBS-LRR RGHs in 37 switchgrass populations derived from 16 states. PCR-amplified RGHs were cloned and sequenced. Phylogenic analysis of RGHs in the two switchgrass ecotypes indicates 1) high genetic diversity, and 2) the predominant class of R-genes contains an LRR receptor-like serine-threonine protein kinase.

Multiple phytohormonal signaling mediates citrus response to the bacterial pathogen Candidatus Liberibacter asiaticus Y. NEHELA, N. Killiny, Citrus research and education center, IFAS, University of Florida, Lake Alfred, FL, USA

Huanglongbing (HLB) is a destructive disease in citrus worldwide. HLB is caused by Candidatus Liberibacter asiaticus (CLas) and transmitted by Asian citrus psyllid, Diaphorina citri. Upon pathogen infection, citrus plants activate their defense responses which are mainly regulated by phytohormones. Herein, we investigated the effect of CLas-infection on the phytohormonal profile of Valencia sweet orange (Citrus sinensis) leaves using GC-MS running in the selective ion monitoring mode (SIM-mode). Overall, we were able to quantify 13 different phytohormones belonging to six groups. Interestingly, CLas-infection alters the levels of all auxins, salicylates, abscisic acid, and their precursors (tryptophan, phenylalanine, and zeaxanthin, respectively), but did not affect the trans-jasmonic acid, gibberellins and cytokinins groups. In addition, the transcript levels of 52 phytohormones' biosynthetic genes were investigated. The expression levels of genes involved in the biosynthesis of auxins, salicylates, and abscisic acid were upregulated after the CLas-infection, while the expression levels of trans-jasmonic acid-, gibberellins-, and cytokinins-biosynthetic genes remained the same. Both the GC-MS-SIM and gene expression results suggest that, in addition to salicylic acid (SA), both auxins and abscisic acid are implicated in activation of SA-mediated pathway against $C$ Las infection. The crosstalk between these groups is the subject of ongoing research. 
Elucidating the Key Roles of Arabidopsis ETHYLENE RESPONSE 1 and ETHYLENE INSENSITIVE 3 in Mediating Plant Susceptibility to Beet Cyst Nematode

S. PIYA, B. Binder, T. Hewezi, University of Tennessee, Knoxville, TN, USA

Ethylene regulates various physiological and developmental processes including plant defense responses. In response to pathogen attack, plants frequently activate the ethylene biosynthesis pathway, leading to the induction of ethylene-dependent defense signaling. The role of ethylene in establishing the compatible interaction between host plants and cyst nematodes has become more apparent. Previous studies have identified ETHYLENE RESPONSE 1 (ETR1) and ETHYLENE INSENSITIVE 3 (EIN3) as positive regulators of Arabidopsis susceptibility to the beet cyst nematode Heterodera schachtii. However, the mechanisms through which ethylene perception and signaling regulate Arabidopsis response to cyst nematode infection remain largely elusive. The aim of this study was to elucidate the role of various components of ethylene perception and signaling in the Arabidopsis- $H$. schachtii interaction. Our results point to a vital role of the ETR1 receiver domain in ETR1-mediated Arabidopsis susceptibility to $H$. schachtii. We identified specific amino acid residues in the ETR1 receiver domain that contribute to the sub-functionalization of the ETR1 to regulate Arabidopsis susceptibility to $H$. schachtii. Pharmacological assays also revealed a role of cytokinin in ETR1-mediated Arabidopsis susceptibility to $H$. schachtii. Our results also pointed into a potent crosstalk between ethylene and salicylic acid signaling during the interaction. Collectively, our results contribute to a better understating of the function of ethylene and its interplay with other stress-related hormones during nematode parasitism of Arabidopsis.

Barley recognition of AvrPphB suggests a programmable system for pathogen protease recognition analogous to PBS1 decoy in Arabidopsis M. CARTER (1), A. Chapman (2), M. Helm (3), G. Fuerst (2,4), R. W. Innes (3), A. J. Bogdanove (1), R. P. Wise (2,4), (1) Cornell University, Ithaca, NY, USA; (2) Iowa State University, Ames, IA, USA; (3) Indiana University, Bloomington, IN, USA; (4) USDA ARS, Ames, IA, USA

Plant defense pathways crucial for disease resistance often involve host intracellular surveillance for pathogen-secreted effectors by nucleotide-binding leucine-rich repeat proteins (NLRs), some of which guard other, effector-targeted host proteins. The Arabidopsis guard NLR, RPS5, initiates HR when it detects cleavage of the decoy PBS1 kinase by the Pseudomonas effector protease AvrPphB. RPS5 can detect PBS1 cleavage even when the AvrPphBrecognition site in PBS1 is changed to that of another protease, expanding the effector recognition capacity of the NLR. Broad conservation of PBS1 in flowering plants implies that PBS1-guarding may also be conserved across species, revealing the potential to customize each plant surveillance system for key pathogens of that species. Given this rationale, our objective was to determine if barley (Hordeum vulgare ssp. vulgare) contains components of a PBS1-RPS5 surveillance system. We identified barley PBS1 homologs that are cleavable by AvrPphB and observed a range of defense responses to AvrPphB in diverse barley lines. We used lines from three families in a nested association (NAM) population to position a single significant locus on chromosome $3 \mathrm{H}$ associated with the AvrPphB response. This locus contains several NLR-encoding genes, including a candidate RPS5 analog that is expressed only in AvrPphB-responding lines. We are undertaking functional assays of the candidate NLR and PBS1 homologs to verify the key proteins in this response that could be modified in future work. Expanding effector recognition through gene editing PBS1 could yield new genetic resistance for barley and other crops against pathogens that use proteases during infection.

Association of a quantitative trait locus with growth of $F$. circinatum

B. SWALARSK-PARRY, E. Steenkamp, A. Hammerbacher, B. D. Wingfield, L. de Vos, Forestry and Agricultural Biotechnology Institute (FABI), University of Pretoria, Pretoria, SOUTH AFRICA

Fusarium circinatum is an important pathogen of pine globally. Taxonomically, F. circinatum resides in the Fusarium fujikuroi species complex (FFSC), where it has been reported to hybridize with its close relative Fusarium temperatum. The $\mathrm{F}_{1}$ progeny from the hybridization allowed for the construction of a genetic linkage map. The genomes of the two parental species have also been determined. In this study we used these available resources to detect and characterize a quantitative trait locus (QTL) associated with growth on malt extract agar medium supplemented with pine extract. The growth rate of the two parental isolates was significantly different $(P<0.05)$, with the majority of the $\mathrm{F}_{1}$ progeny having significantly higher growth rate than the average growth of the two parents. The detected QTL was localised to a region on the $F$. temperatum genome, which contained two genes encoding a SUR7/RIM9-like membrane protein and a FAD-binding domain protein. This entire region was absent in the genome of the $F$. circinatum parent. Low $\mathrm{G}+\mathrm{C}$ content, repetitive sequences and putative transposable elements were characteristic of this region in $F$. circinatum (FSP34). To further understand the role of the presence or absence of these genes in the FFSC, future work will focus on gene knockouts and complementation studies.

Jasmonic acid has a dominant role in Cucumber mosaic virus induced aphid resistance in Arabidopsis thaliana T. TUNGADI (1), S. Groen (2), A. Murphy (1), J. P. Carr (1), (1) University of Cambridge, Cambridge, UNITED KINGDOM; (2) New York University, New York, NY, USA

The Cucumovirus Cucumber mosaic virus (CMV) has a very broad host range which includes important crop plants and is non-persistently transmitted by the generalist aphid, Myzus persicae. CMV infection of Arabidopsis thaliana reduces M. persicae growth rate and reproduction, and inhibits prolonged aphid feeding. This might promote virus transmission rates. CMV manipulation of host plant innate immunity may determine whether CMV induces susceptibility or resistance to aphids. The exact mechanisms underlying these phenomena are not known. We used Arabidopsis single and combinatorial mutants in salicylic acid (SA), jasmonic acid (JA), ethylene and PAD4-mediated signalling pathways. We explored whether one or more of these immune signalling pathways contribute to CMV-induced resistance to $M$. persicae. Because the CMV $2 \mathrm{~b}$ protein enhanced SA-dependent signalling but blocked JA-dependent signalling in Arabidopsis, we investigated whether JA signalling is required for CMV-induced aphid resistance. CMV-induced aphid resistance is decreased in the coil-16 Arabidopsis mutant that is unable to perceive JA and the resistance is completely abolished in the dde2-2 mutant which is deficient in JA signalling. CMV-induced aphid resistance still occurred in SA-deficient sid 2 mutant and NahG transgenic plants. This indicates that SA is dispensable. CMV-induced aphid resistance occurs in PAD4 single mutant but was neutralised in dde2-2/pad4-1 double mutant plants. Aphid resistance is also neutralised on CMV-infected dde2-2/sid2-2, dde2-2/ein2-1/pad4-1 and in dde2-2/pad4-1/sid2-2 mutant plants. These results show that JA-signalling is needed for CMV-induced aphid resistance in Arabidopsis.

Induction of defense by elicitors from different origins in Potato genotypes against Phytophthora infestans R. LOPES MARTIN (1), P. Le Boulch (2), A. Schwarzenberg (3), E. Nguema-Ona (3), F. Val (2), (1) Agrocampus-Ouest / INRA UMR IGEPP, Rennes, FRANCE; (2) AGROCAMPUS OUEST UMR 1349 IGEPP (Institute of Genetics, Environment and Plant Protection), Rennes Cedex, FRANCE; (3) CMI Roullier, Saint Malo, FRANCE

In potato crop, Phytophthora infestans, the causal agent of late blight, is mainly controlled with pesticides. Biocontrol methods such as those inducing plant defenses could contribute to reduce their use. Several elicitors have been showed to induce potato defense responses in controlled conditions but 
they are not efficient in the field. Our hypothesis is that defense induction depends on both the origin of the applied elicitors and the potato genotype. To assess it two potato genotypes with different levels of resistance toward late blight were treated with PAMPs from $P$. infestans, a green algae extract or BABA. Four-week old leaf tissues were analysed 48 hours after treatment using non-target metabolomics analyses thanks to UPLC-QTOF-MS ${ }^{\mathrm{e}}$. Ours results show that the accumulation of secondary metabolites such as phenylpropanoids, flavonoids and alkaloids are induced after elicitation, some of them (chlorogenic acid and rutin) being potential defense markers. Notably, chaconine, a specific molecule of potato, is more induced with the algae's extract than with PAMPs. These metabolites will be validated and quantified with specific standards. Principal Components Analysis show the significantly difference between the genotypes. Moreover, to complete the investigation of our hypothesis, metabolomic datasets analysis is in progress to identify defense markers. Finally, in order to understand the defense induction pathways specific potato defense genes will be studied.

Chemical defence responses of Australian Acacia trees to infection by Ceratocystis albifundus and C. manginecans A. Hammerbacher, B. SWALARSK-PARRY, B. D. Wingfield, Forestry and Agricultural Biotechnology Institute (FABI), University of Pretoria, Pretoria, SOUTH AFRICA

Australian Acacia mearnsii and A. manginum are considered highly productive timber plantation trees in the southern hemisphere. However, these trees are highly susceptible to infections by Ceratocystis albifundus and C. manginecans respectively, which both cause high losses in wood and pulp production. The aim of this study was to determine how Acacia trees defend themselves against infections by these pathogens and the basis for their susceptibility. Artificial inoculation of young saplings from both tree species was conducted. Analysis of the defence hormones produced in response to fungal colonization revealed that both tree species produce adequate levels of the plant defence hormone jasmonic acid-isoleucine, which is an early signal for initiating defence mechanisms against infection by necrotrophic pathogens. However, tannin levels in both tree species were down-regulated after infection. On the other hand, an increase in flavonoid concentrations in infected saplings was observed. Further research will elucidate if the tradeoff between flavonoids and tannins influences susceptibility of Australian Acacia trees during infection by Ceratocystis species.

The impact of the grapevine trunk disease fungus Lasiodiplodia on the physiological responses of different grapevine cultivars P. REIS (1), G. Pinto (2), A. Cerqueira (2), C. Jesus (2), M. Mota (1), F. Fontaine (3), A. Alves (2), C. Rego (1), (1) Instituto Superior de Agronomia, LEAF, Universtiy of Lisbon, Lisboa, PORTUGAL; (2) University of Aveiro, Aveiro, PORTUGAL; (3) Université de Reims Champagne Ardenne, Reims, FRANCE

There is still little information about the effective risks that Lasiodiplodia spp., particularly Lasiodiplodia theobromae, pose to vineyards or how different cultivars respond to pathogen attack. To better understand the impact of Lasiodiplodia spp. on grapevine physiology, two-year-old potted plants of four Portuguese varieties, Aragonez (=Tempranillo), Touriga Nacional, Touriga Franca and Alvarinho, were inoculated with six L. theobromae, from different geographic origins, and one L. mediterranea isolates, which are responsible for causing diverse symptoms, including bud necrosis and perennial cankers. Plants were kept in a greenhouse under controlled conditions and the effects on plant physiological processes such as water relations, photosynthetic performance and defence mechanisms, were measured after 10 weeks. Leaf water potential, leaf gas exchange and chlorophyll fluorescence were assessed, and leaf samples were collected to assess malondialdehyde (MDA) and proline contents. Results indicate that cultivars had different responses towards pathogens inoculation, with a general tendency towards water potential decrease and increase of proline content, while photosynthetic activity and MDA content varied amongst the tested cultivars. These differences might be related with host-pathogen interaction, suggesting that the cultivar and the inoculum aggressiveness might play specific roles regarding Lasodiplodia spp. in grapevines that have to be considered.

Differential roles of the plant secondary metabolite melatonin in plant-host resistance and pathogen suppression M. K. MANDAL PHD (1), C. Kousik (2), (1) ORISE participant, US Vegetable Laboratory, USDA, ARS, Charleston, SC, USA; (2) U.S. Vegetable Laboratory, USDA, ARS, Charleston, SC, USA

Since the 1950s, research on the animal neurohormone melatonin, has focused on its multi-regulatory effect on patients suffering from insomnia, cancer, and Alzheimer's. Previous studies on melatonin in plants have focused primarily on plant growth and development. However, studies on the physiological function of melatonin in host-pathogen defense mechanism are lacking. This study provides insight on how application of melatonin, an environmental-friendly immune inducer, can boost plant immunity and suppress pathogen growth in a field situation where fungicide resistance and lack of genetic resistance are major problems. We evaluated the effect of spray-applied melatonin and also transformed watermelon plants with the melatonin biosynthetic gene SNAT to determine the role of melatonin in plant defense. Increased melatonin levels in plants were found to boost resistance against the foliar pathogen Podosphaera xanthii (powdery mildew), and the soil-borne oomycete Phythophthora capsici in watermelon and other cucurbits. Transcriptomic data suggests that melatonin alters the expression of genes involved in both PAMP and ETI mediated defenses. Twenty seven upregulated genes were associated with constitutive defense as well as initial priming of the melatonin induced plant resistance response. Our results indicate that developing strategies to increase melatonin levels in specialty crops such as watermelon can lead to resistance against diverse filamentous pathogens.

Effect of a biostimulant on Resistance Gene Expression in Wheat

A. TWAMLEY (1), A. Feechan (2), M. Gaffney (3), (1) School of Agriculture and Food Science, University College Dublin, Belfield, Dublin, IRELAND; (2) University College Dublin, Dublin, IRELAND; (3) Alltech Ltd, Dunboyne, Co. Meath, IRELAND

High wheat yield losses are recorded globally each year due to fungal pathogens. Since many fungal pathogens build up resistance to fungicides, novel and alternative methods of pathogen control need to be developed. An innovative approach to improving productivity in crops challenged by biotic stress, is to stimulate the plant's own defence mechanisms. In a primed state, plants may better respond to biotic and abiotic stresses. The aim of the present project was to determine if a fermentation-based biostimulant can prime plant defences by inducing the expression of endogenous defencerelated genes. Two-week-old wheat seedlings were spray treated with the biostimulant and then inoculated with spores of powdery mildew. Disease assays show a significantly reduced number of pustules/area following treatment with the biostimulant. Tissue samples were also taken at time points pertaining to different developmental stages of the disease. Significantly higher expression of genes associated with the salicylic acid dependent signalling pathway were observed in treated plants compared to untreated plants $(\mathrm{p} \leq 0.05)$. These genes are often associated with the elicitation of plant defence responses to specific biotrophic pathogens, such as powdery mildew. Future work will seek to better elucidate the genes and pathways that are induced by the biostimulant in wheat.

Transcriptional responses of Escherichia coli $0157: \mathrm{H7}$ during plant immunity and plant disease A. LOVELACE, B. Kvitko, University of Georgia, Athens, GA, USA 
Human enteric pathogens such as Escherichia coli $\mathrm{O} 157: \mathrm{H} 7$ are frequently associated with produce-borne illness, even though they are generally unable to replicate efficiently in association with plant tissues. In part this is due to the broad spectrum microbe-restricting plant immune response. The plant pathogenic bacterium Pseudomonas syringae has evolved to overcome plant immunity resulting in conditions that are favorable for bacterial multiplication. Human enteric pathogens are able to exploit the permissive conditions created by $P$. syringae to opportunistically achieve high loads in plant tissues which could represent an important source of produce contamination. Our research aims to characterize the response of human enteric pathogens in the context of plant immunity and disease. The interactions between E. coli O157:H7, Pseudomonas syringae pv. tomato and model hosts Arabidopsis thaliana and Nicotiana benthaminana were characterized in a co-inoculation assay. We observed that $P$. syringae creates a conducive environment for proliferation of $E$. coli in the apoplast with E. coli populations increasing significantly when co-inoculated with $P$. syringae but not when co-inoculated with a type III secretion system mutant. Transcriptome analysis of $E$. coli under disease-mimicking and plant immune conditions through a disease reconstitution assay reveals unique responses to the conditions human pathogens experience during exposure to plant immunity and plant disease.

Evaluation of inactivated fungal extracts as defense inducers against fungal diseases in strawberry

S. MOSCHEN (1,2), V. Hael Conrad (1,2), J. C. Diaz Ricci (2,3), S. M. Salazar (1), (1) Instituto Nacional de Tecnología Agropecuaria (INTA), EEA Famaillá., Famaillá, ARGENTINA; (2) Consejo Nacional de Investigaciones Científicas y Técnicas, CONICET, Buenos Aires, ARGENTINA; (3) INSIBIO - Facultad de Bioquímica, Química y Farmacia, Universidad Nacional de Tucumán, San Miguel De Tucumán, ARGENTINA

Argentina is one of the four main strawberry producers in South America. This crop is susceptible to a large number of diseases and fungal infections are the most important biotic stress. Nowadays, there is a trend towards the use of natural biocontrol agents instead of synthetic fungicides in order to enhance immunity to microbial infections in crops. The aim of this work was to evaluate the protective capacity of inactivated microbial extracts against two of the main fungal pathogens in strawberry (Botrytis cinerea and Colletotrichum acutatum). Fungal extracts of local strains (Colletotrichum acutatum and Acremonium strictum $)$ were obtained and thermally inactivated $\left(121^{\circ} \mathrm{C}, 20 \mathrm{~min}\right)$. The extracts were applied by aspersion on Arabidopsis and strawberry leaves. After 5 days of induction, the plants were infected with a suspension of B. cinerea and C. acutatum. The infection progress was evaluated during 30 dpi showing a significant decrease in plants pre-treated with the microbial extracts. The capacity of inducing a Pattern Triggered Immunity response in Arabidopsis was also evaluated by growth inhibition using Col-0 and FLS2, EFR and CERK1 triple mutant (Fec) seedlings and by qPCR evaluating the expression of defense related genes such as PR1, FRK1, WRKY70 and WRKY 53. This work demonstrated that the inactivated extracts were able to induce protection against fungi, which provides a valuable and natural alternative for the biocontrol in agronomically important crops.

Involvement of hormone pathways in early onset of TSWV resistance

J. WALLS III, C. Rosa, The Pennsylvania State University, University Park, PA, USA

The salicylic (SA) and jasmonic (JA) acid pathways are extensively studied for their implications in plant defenses against pathogens and herbivores. However, there is less work on the role of the abscisic acid (ABA) pathway, the pathway more well-known for its involvement in stresses such as drought, on plant defenses and resistance against plant viruses. In this study we sought to elucidate the role of the ABA pathway in resistance of tomato cv. Better Boy inoculated with Tomato spotted wilt orthotospovirus (TSWV). Transcript levels of genes in the SA, JA, and ABA pathways were measured via qPCR 4 hours post-inoculation (hpi). Plants were also tested for successful infection via ELISA several weeks after inoculation. Plants that were ultimately positive for TSWV showed significant differences in gene regulation in the JA and ABA pathways 4 hpi when compared to plants that were ultimately ELISA negative for TSWV, suggesting a role of these two pathways in early onset of resistance. These results may have widespread implications in virus disease management and pinpoint genes that can serve as targets for genome editing for TSWV-resistant plants.

Investigating the role of secreted proteases in citrus defense response during CLas infection

J. FRANCO (1), T. Liebrand (1), K. Clark (2), L. Zeng (2), V. Ancona (3), W. Ma (2), G. L. Coaker (4), (1) University of California, Davis, Davis, CA, USA; (2) University of California, Riverside, Riverside, CA, USA; (3) Texas A\&M University, Kingsville, Wescalo, TX, USA; (4) University of California, Davis, CA, USA

Huanglongbing (HLB) is a devastating citrus disease causing considerable economic loss. The inability to culture the HLB causal agent, Candidatus Liberibacter asiaticus (CLas), has provided significant challenges in understanding its pathogenesis. To identify defense pathways targeted by $C$ Las, we performed a differential quantitative proteomic study of the phloem from graft-inoculated and mock-inoculated Navels 10 months post-infection. In total, we identified 1401 proteins by mass spectrometry. Multiple induced proteins were secreted proteases, including glycosyl hydrolases, serine proteases, and papain-like cysteine proteases (PLCPs). In other plants, PLCPs are immune induced proteases that can be inhibited by diverse pathogen classes. Additionally, immune related PLCPs were identified by gene expression analysis of Navels treated with salicylic acid. To test whether $C$ Las targets PLCPs, mass spectrometry was used to quantify PLCP activity and abundance in field-grown uninfected and infected Navel samples. Multiple PLCP subfamilies increased in abundance during infection. Interestingly, the SAG12 subfamily increased in abundance, but not activity during infection. These results imply that specific PLCPs are defense activated and inhibited during CLas infection. Data will also be presented on the abundance and activity of specific serine proteases after infection.

Chemical genomics reveals resistant soybean line inhibits Sclerotinia sclerotiorum by targeting its ergosterol biosynthesis pathway A. RANJAN (1), J. Piotrowski (2), M. Kabbage (1), (1) University of Wisconsin-Madison, Madison, WI, USA; (2) Yumanity Therapeutics, Cambridge, MA, USA

In nature, plants employ various strategies to overcome pathogen attacks including production of antimicrobial compounds. We study the interaction between the fungal pathogen, Sclerotinia sclerotiorum, and soybean. While screening soybean lines for resistance to $S$. sclerotiorum, we observed a distinct red discoloration at the site of inoculation in resistant soybean lines, accompanied by a complete arrest of fungal growth. We hypothesized that the resistant lines produce antifungal compounds in response to $S$. sclerotiorum challenge. We recovered the red stem extract (RSE), and assessed its effect on fungal growth. S. sclerotiorum growth was markedly inhibited in the presence of RSE. The mode of action and the antifungal activity of RSE were further tested by performing chemical genomics in a yeast mutant library. Mutants of genes involved in phospholipid and ergosterol biosynthesis were significantly sensitive to RSE. The chemical genomics profile of RSE was compared to other known drug profiles, and was found to have significantly similar activity to ergosterol biosynthesis targeting drugs. This was further substantiated by the significant decrease in ergosterol levels in $S$ sclerotiorum when challenged with RSE. Overall, we show that resistance to $S$. sclerotiorum in soybean involves the upregulation of antifungal activity, targeting ergosterol biosynthesis in the fungus. This work may help uncover novel bioactive antimicrobial compounds with agricultural and pharmaceutical importance. 
Friend or foe: The genetics of an endophytic tree pathogen infection

L. Bezuidenhout, A. Marsberg, A. Postma-Smidt, M. J. Wingfield, B. SLIPPERS, Forestry and Agricultural Biotechnology Institute (FABI), University of Pretoria, Pretoria, SOUTH AFRICA

A poorly understood aspect of plant biology is the role played by fungal endophytes that infect healthy plant tissues without inducing symptoms. The fungus Botryosphaeria dothidea has been recognised both as an endophyte and a pathogen on a wide range of woody plant species. It is not known how $B$. dothidea is able to infect its hosts without triggering a host defence response. In this study, transcriptome data were used to investigate the molecular responses of $E$. grandis and $B$. dothidea during an active infection at 24 hours, 48 hours and 7 days post-infection. A weak host response was observed at 48 hours, characterised by the differential expression of 129 genes, which included the up-regulation and down-regulation of key defence genes such as the jasmonate/ethylene transcription factor, MYB15, and the salicylic acid receptor, NPR5, respectively. Data from differential expression analyses of the $B$. dothidea in planta transcriptome revealed the expression of genes involved in nutrient acquisition, detoxification and cell wall degradation. Transcriptome data suggested that this tree-endophyte interaction is one of tolerance regardless of the weak plant defence response observed. The study provides powerful hypotheses to further explore the importance of fungal tree endophytes.

Drought-acclimated Arabidopsis plants have increased bacterial disease resistance that requires a functional RD21A

Y. Liu (1), K. WANG (1), Q. Cheng (2), X. Zhang (1), B. Zhao (1), (1) Virginia Tech, Blacksburg, VA, USA; (2) Nanjing Forestry University, Nanjing, CHINA

As one major abiotic stress, drought could significantly reduce crop yields. It has recently been revealed that drought stress also has a strong influence on the plant immunity. However, the molecular mechanism of the drought-regulated immunity has not been well studied. We demonstrated that droughtinduced gene RD21A, encodes a cysteine protease, which plays a key role in the drought-induced immunity. The well-watered wild type Arabidopsis (Col-0) plants were more susceptible to a coronatine defective strain DB29 of Pseudomonas syringae pv. tomato DC3000 (Pst-DC3000(DB29)) than that have a temporary drought treatment. However, this drought-induced defense was compromised in the $r d 21 a$ mutant background. Yeast two-hybrid and co-immunoprecipitation assays uncovered that RD21A interacts with ubiquitin E3 ligase SINAT4. Transient expression results indicated that RD21A could be degraded by SINAT4 in vivo. Consistent with the phenotype of null mutant $r d 21 a$, the overexpression of SINAT4 also compromised the drought-induced immunity to Pst-DC3000 (DB29). Furthermore, we also demonstrated that bacterial type III effector AvrRxo1 delivered by Pst-DC3000 (DB29) interacts with both SINAT4 and RD21A in vivo. Protease enzyme assays demonstrated that AvrRxo1 could enhance the E3 enzyme activity of SINAT4, and facilitate the degradation of RD21A in vivo. These results highlight RD21A has a key role in the drought-induced immunity, which can also be targeted by the pathogen virulence effectors.

Effects of engineered nanomaterials on plant innate immune responses

K. EFFERTZ (1), P. Deka (2), E. Magnuson (1), A. Bezbaruah (2), R. S. Brueggeman (1), (1) Department of Plant Pathology, North Dakota State University, Fargo, ND, USA; (2) Department of Civil and Environmental Engineering, North Dakota State University, Fargo, ND, USA

Engineered nanomaterials (ENMs) have potential applications in agriculture including uses as pesticides and fertilizers. Plant-ENM interactions induce defense responses resulting in physiological reprogramming that can alter host-pathogen outcomes. Genetic resistance in barley line CI5791, effective against the necrotrophic fungal leaf pathogen Pyrenophora teres $f$. teres (Ptt), was compromised after root exposure to Zinc Oxide nanoparticles (ZnONPs). RNAseq analyses revealed spatial and temporal suppression of jasmonic acid (JA) responses, effective against necrotrophic pathogens and upregulation of salicylic acid responses effective against biotrophic pathogens, which are antagonistic to the JA responses. These transcriptome analyses may explain the compromised resistance observed. However, to understand the mechanisms by which plants recognize ENMs we developed a model where HvNDR1, a homolog of the Arabidopsis NDR1 gene, is part of a plasma membrane localized receptor complex which interacts with ENMs modulating host cell physiology that alter subsequent pathogen resistance mechanisms. We present data utilizing post-transcriptional gene silencing in barley and Arabidopsis mutants of the putative $N d r l$ complex components showing altered responses after nanoparticle treatment suggesting a role in ENM recognition and responses. Understanding plant-ENM interactions is essential for future development of ENMs for agricultural applications.

Systemic root-to-shoot defense signaling induced by arachidonic acid and extract of the brown seaweed, Ascophyllum nodosum D. Lewis (1), S. M. Robinson (1), H. Little (2), G. L. Coaker (1), R. M. BOSTOCK (1), (1) University of California, Davis, CA, USA; (2) Acadian Seaplants Limited, Dartmouth, NS, CANADA

Eicosapolyenoic fatty acids (EP) are integral components of oomycete pathogens that act as pathogen associated molecular patterns (PAMP) capable of inducing resistance in plants. EPs include arachidonic acid (AA), a robust elicitor of oxylipin pathway genes and plant defense in pepper and tomato roots. Oxylipin pathway enzymes produce antimicrobial divinyl ethers and the defense hormone jasmonic acid. A commercial extract from the seaweed Ascophyllum nodosum (Acadian Seaplants Ltd.; ANE) is a stimulant of plant growth that contains EPs and may also harbor the ability to induce resistance to fungal-like pathogens of Solanaceous crops similarly to AA. To investigate systemic signaling and the overlap in mechanism of action between the compounds, transcriptional analyses of oxylipin and defense marker genes were conducted. The roots of hydroponically grown tomato and pepper were treated with AA or ANE. Tissue was harvested at different times after treatment to assess local (root) and systemic (leaf) induction of target genes associated with induced resistance. Relative transcript abundance of 9- and 13-oxylipin pathway genes and jasmonate- and salicylate-inducible markers was quantified by qRT-PCR. Root treatment with either ANE or AA dramatically induced the same local and systemic transcripts. These data demonstrate that EP treatment not only protects against a root-colonizing oomycete, but also induces a systemic response in aerial tissue.

Subcellular localization of resistance-associated AAPermease Rhg $_{1}$ in response to soybean cyst nematode infection S. HAN, J. Smith, A. F. Bent, University of Wisconsin-Madison, Department of Plant Pathology, Madison, WI, USA

The Rhgl locus mediates economically valuable resistance of soybean to soybean cyst nematode. One of the three genes that contributes to the resistance

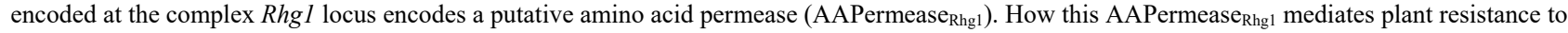
cyst nematodes is largely unknown. Here, we elucidated the subcellular location of AAPermease Rhgl $_{1}$ during SCN infection. Using a custom-raised antibody to track the native protein, we performed transmission electron microscopy immunogold subcellular localization assays in resistant and susceptible roots samples. Samples were examined prior to infection and at time points 3 and 7 days after SCN infection. We also evaluated the cellular location by subcellular fractionation and by immunofluorescence using a wide-field confocal microscope. Upon root invasion, SCN juveniles (J2) move intracellularly, disrupting the cells through which they pass. We observed an increased abundance of small vesicles in penetrated root cells. AAPermease

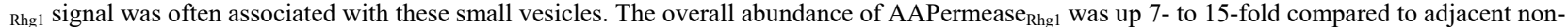


penetrated cells, while in general AAPermease ${ }_{\mathrm{Rhg} 1}$ remained a low-abundance transmembrane protein in non-infected roots and in syncytia. These findings suggest that AAPermease $_{\mathrm{Rhg} 1}$ may contribute to $\mathrm{SCN}$ resistance via a unique localization change, directly surrounding the pathogen as it infects the plant.

The Nec3 gene is a putative negative regulator of pathogen induced programmed cell death in barley G. AMEEN (1), L. Bittara (2), J. Richards (2), S. Solanki (2), T. L. Friesen (3), R. S. Brueggeman (1), (1) Department of Plant Pathology, North Dakota State University, Fargo, ND, USA; (2) Plant Pathology Department, North Dakota State University, Fargo, ND, USA; (3) USDA-ARS, Cereal Crops Research Unit, Northern Crop Science Lab, Fargo, ND, USA

To defend against biotrophic pathogens, plants rely on programmed cell death (PCD) responses that are initiated to isolate and impede colonization. However, necrotrophs often hijack these immunity responses to complete their lifecycle and proliferate. Thus, elucidating mechanisms of PCD suppression or enhancement is important. However, mutagenesis and forward genetics is one of the only options to study these conserved pathways. The nec 3 mutants of barley, predominantly show distinctive large cream to orange necrotic lesions specifically during infection with several necrotrophic pathogens. A g-irradiation induced nec3 mutant in cultivar Bowman background, designated nec3- $\gamma 1$, segregated as a single recessive gene in a nec3- $\gamma 1$ $\mathrm{x}$ cv Quest $\mathrm{F}_{2}$ population when inoculated with $B$. sorokiniana isolate ND85F. The homozygous $\mathrm{F}_{2}$ susceptible progeny, were genotyped with 4 SSR and 25 SNP markers spanning the barley ch. $6 \mathrm{H}$ region where nec 3 was previously mapped at low resolution. The 29 markers were perfectly ordered on the newly released barley genome sequence and nec3 was delimited to an $\sim 0.14 \mathrm{cM}$ region spanning $\sim 16.96 \mathrm{Mb}$ of genomic sequence containing 80 annotated high confidence genes. Exome capture sequencing of three independent nec 3 mutants failed to identify a candidate gene. However, RNAseq analysis of pathogen challenged and control nec3- $\gamma 1$ and wildtype Bowman identified two genes in the delimited region with $>3$ fold down regulation in the nec3- $\gamma 1$ mutant, representing candidate nec3 genes.

Botanical extract of chamomile (Matricaria chamomilla) induces expression of resistance genes in Papaya fruit tree G. CHAVES-BEDOYA PHD, L. Ortiz-Rojas, Z. Galvis-Perez, Universidad Francisco de Paula Santander, Cucuta, COLOMBIA

The papaya fruit tree is affected by different pests and diseases that significantly reduce its production, including viral diseases such as the Papaya ringspot virus (PRSV). PRSV remains a threat to the economic production of papaya worldwide. The use of molecules that promote defense mechanisms in plants turn out to be an alternative in the management of diseases in crops of agricultural importance, such as the papaya fruit tree. Three months old papaya plants were sprayed with 1) botanical extract of chamomile (Matricaria chamomilla) mixed with sulfur, 2) salicylic acid, 3) sulfur solution and 4) water as mock. The aim of the experiment was to test by semi quantitative PCR the ability of the botanical extract to induce the expression of the resistance genes MPK1 and PR1 after mechanically inoculation of PRSV. No statistically significant evidence was found in gene expression. However, we found a higher expression of resistance genes 3 dpi, compared to 7 dpi. Papaya plants sprayed with chamomile-sulfur presented less typical symptoms of the disease caused by PRSV, nevertheless showed moderate symptoms of phytotoxicity.

Baseline sensitivity of Botrytis cinerea isolates to natamycin and its control of gray mold on stored mandarin fruit S. SAITO (1), F. Wang (2), C. L. Xiao (3), (1) USDA ARS, Parlier, CA, USA; (2) United States Department of Agriculture - Agricultural Research Service, Parlier, CA, USA; (3) USDA-ARS, Parlier, CA, USA

Gray mold caused by Botrytis cinerea is an emerging postharvest disease of mandarin fruit in California. Control of postharvest diseases in mandarins relies on postharvest fungicides. However, resistance to multiple mode-of-action fungicides is common in $B$. cinerea populations, leading to failure of decay control. Natamycin is a newly registered biorational fungicide for postharvest use on citrus and other fruits. To establish baseline sensitivity to natamycin, 64 isolates of $B$. cinerea obtained from decayed mandarin with known resistance phenotypes to other fungicides were tested. The effective concentration of natamycin for $50 \%$ reduction in growth relative to control $\left(\mathrm{EC}_{50}\right)$ ranged from 0.324 to $0.567 \mu \mathrm{g} / \mathrm{ml}$ with a mean of $0.444 \mu \mathrm{g} / \mathrm{ml}$ for conidial germination and from 1.021 to $2.007 \mu \mathrm{g} / \mathrm{ml}$ with a mean of $1.578 \mu \mathrm{g} / \mathrm{ml}$ for mycelial growth. The minimum inhibitory concentrations (MIC) were $1.0 \mu \mathrm{g} / \mathrm{ml}$ for germination and $10.0 \mu \mathrm{g} / \mathrm{ml}$ for mycelial growth. No cross resistance between natamycin and other mode-of-action fungicides was detected. Control tests on mandarin fruit inoculated with five different fungicide-resistant phenotypes of $B$. cinerea isolates showed that natamycin significantly reduced incidence and lesion size of gray mold on fruit regardless of fungicide-resistant phenotypes. Our results suggest that natamycin is an effective tool for postharvest gray mold control and management of fungicide resistance in $B$. cinerea.

\section{Pathogenicity, Incidence, and Distribution of Fungi Causing Root Rot in Idaho Sugar Beet Storage Piles C. A. STRAUSBAUGH, USDA ARS NWISRL, Kimberly, ID, USA}

Fungal rots in sugar beet roots held in long-term storage can lead to considerable loss, but the distribution and incidence of fungal rots inside sugar beet piles and pathogenicity for some species is poorly understood. Thus, Idaho sugar beet held in 5 outdoor and 2 indoor piles in 2014 and 2015 were investigated by assessing the root surface area covered by fungal growth and discolored tissue in 9 one-meter square areas per pile using a stratified random sampling design. Pathogenicity was evaluated indoors via plug inoculation in 2015 and 2016. Botrytis cinerea covered 6-22\% of root surface area in indoor piles, while outdoor piles averaged only $0-3 \%$. The 2 outdoor Adelaide piles had less Athelia-like basidiomycete (avg. 0-2\%) than other piles (4-15\%), while no trends were evident for Penicillium-like spp. (0-8\%). Penicillium-like spp. isolated: $60 \%$ P. expansum, $34 \%$. cellarum, $3 \%$. polonicum, and 3\% Talaromyces rugulosus. There were no trends or differences based on sample location in a pile, but the 2 outdoor Adelaide piles had more healthy tissue $(90-96 \%)$ than other piles $(28-80 \%)$. B. cinerea was the most pathogenic $(P>F<0.0001)$ species (avg. $61 \mathrm{~mm}$ of rot) followed by $P$. polonicum and $P$. expansum $(35 \mathrm{~mm}), P$. cellarum $(28 \mathrm{~mm})$, Athlelia-like basidiomycete $(22 \mathrm{~mm})$, and $T$. rugulosus and the non-treated check $(0 \mathrm{~mm})$. Two outdoor piles had negligible fungal growth through proper management, but the management of fungal rot in other piles could be improved.

Integrated management of Penicillium digitatum in citrus fruit using preharvest silicon applications, plus postharvest hot water treatments I. BASDEW (1), M. D. Laing (2), (1) Discipline of Plant Pathology: University of KwaZulu-Natal, Pietermaritzburg, SOUTH AFRICA; (2) University of KwaZulu-Natal, Pietermaritzburg, SOUTH AFRICA

South Africa is the second largest exporter of fresh citrus in the world, after Spain. Postharvest damage by Penicillium digitatum often exceeds $40 \%$ in fruit destined for export markets. Studies were conducted to investigate whether an integrated system combining the effects of preharvest silicon treatments on citrus trees, followed by postharvest hot water treatment (HWT) and a biological control agent, could be used to control green mould of citrus fruit. Fruit were further assessed for the expression of plant protective compounds associated with disease pressure. Navel and Valencia trees were treated with two silicon preparations. Fruit were picked, inoculated with $P$. digitatum spores, and exposed to HWT at $60^{\circ} \mathrm{C}, 62^{\circ} \mathrm{C}$ and $64^{\circ} \mathrm{C}$ for $10 \mathrm{~s}, 15 \mathrm{~s}$ and 20 s, respectively. This was followed by fruit coating with a biological control yeast, B13. Fruit peels were quantitatively assessed for antioxidant, 
flavonoid and phenolic levels. Electron dispersive x-ray spectroscopy showed that silicon is indeed taken up by the tree and transported to fruit. Silicon pretreatments coupled with $\mathrm{HWT}+\mathrm{B} 13$, at $62^{\circ} \mathrm{C}$ and $64^{\circ} \mathrm{C}(15 \mathrm{~s}$ and $20 \mathrm{~s}$ exposure time), provided $98-100 \%$ control of green mould with zero heat damage. Controls (field treatment: water or potassium sulphate) provided $88-93 \%$ protection. Coating fruit with the biological control agent provided long-term disease protection for 6 weeks post-treatment, manifested by zero disease development. A two-fold increase in the levels of antioxidants, flavonoids and phenolics in fruit peels was found, compared to controls. The combined use of silicon and HWT, produced a faster and stronger resistance reaction than either treatment alone.

The ethylene biosynthetic pathway in two major postharvest pathogens Penicillium digitatum and Penicillium expansum: In vitro studies R. TORRES (1), J. Yang (2), L. Vilanova (1), N. Teixidó (1), J. Usall (1), C. Larrigaudière (1), J. Giné-Bordonaba (1), (1) IRTA, XaRTA-Postharvest, Edifici Fruitcentre, Lleida, Catalonia, SPAIN; (2) College of Biosystems Engineering and Food Science, Zhejiang University, Hangzhou, CHINA

In contrast to fungi, higher plants produce ethylene from methionine via the 1-aminocyclopropane-1-carboxylic acid (ACC) pathway. Indeed, two main biosynthetic pathways for ethylene production have been reported in microorganisms including fungi and its utilization does not exclusively depend on the pathogen but rather on the conditions how they are grown. In addition, the role of this hormone in the fungal metabolism of many postharvest pathogens is relatively unknown. Penicillium digitatum and Penicillium expansum are considered widespread fungal pathogens of postharvest rots in citrus and pome fruit, respectively, causing considerable fruit losses. Thus, a deeper understanding of fungal ethylene production could help to design potential disease management strategies in the future. This study aimed at gaining further knowledge on the ethylene production capacity and comparing the ethylene biosynthetic pathway in both $P$. digitatum and $P$. expansum grown under different in vitro conditions as well as trying to understand the possible role of this hormone on their metabolism. Our results showed that $P$. digitatum can produce ethylene over multiple growing conditions whereas P. expansum can produce ethylene exclusively through the 2-keto-4-methylthiobutyric acid (KMBA) pathway and under conditions unlikely to occur in vivo. These results led us to hypothesize that the observed variability in the pathogenicity of $P$. digitatum (a non-host pathogen) and $P$. expansum (a compatible pathogen) in apples may be related to the ability of the fungi to produce ethylene.

Is increased inoculum for Fusarium graminearum an unintended consequence of stay green maize? K. ELI, D. Hooker, A. W. Schaafsma, University of Guelph, Ridgetown Campus, Ridgetown, ON, CANADA

The potential for Fusarium graminearum infection and mycotoxin accumulation in maize and wheat may be increasing with trends in modern maize production. Stay green hybrids are bred to stay physiologically active for a longer period, resulting in increased grain yield. Stay green hybrids stalks may present higher carbohydrate content at harvest, compared to those that senesce earlier and closer to physiological grain maturity. This delay in canopy senescence may accelerate the colonization of Fusarium spp. in fresh stalks after harvest, favoring the development of inoculum and its overwintering on stalks. Coincidently with the introduction of stay green hybrids, it has recently become more common to observe stalks blackened by perithecia soon after maize harvest rather than in the spring, as observed in previous years. The objective of this study is to investigate the relationship between residual carbohydrates and moisture in modern maize hybrids with inoculum production and timing. In this study, 50 commercial hybrids representing a range of stay green characteristics were tested for soluble solids, total solids and moisture content in maize stalks immediately after grain harvest. These stalks will be monitored for perithecia timing of development and their abundance in the spring, and then assessed for ascospore production during winter wheat and maize flowering, comparing inoculum potential of hybrids with differing stay green properties. Results will be presented.

Pantoea agglomerans-Fusarium graminearum interaction for Fusarium head blight management and mycotoxin control Y. CHEN* (1), S. Xu (1), S. Cao (2), Z. Ma (1), (1) Institute of Biotechnology, Zhejiang University, Hangzhou, CHINA; (2) Department of Pharmaceutical Sciences, University of Hawaii, Hilo, HI, USA

Physical and metabolic interactions between bacteria and fungi are widespread in nature and have great environmental, medical and agricultural importance. Yet, the underlying mechanisms are largely unknown. Here, we investigated an antagonistic interaction between a commensal bacterium, Pantoea agglomerans ZJU23, and a disastrous plant pathogenic fungus Fusarium graminearum. ZJU23 was isolated from perithecium of $F$. graminearum and found to secret a broad-spectrum antifungal compound, herbicolin A. We used $\mathrm{Tn} 5$ transposon mutagenesis to create a mutant library, screened approximately 8,000 mutants for loss of herbicolin A production, and recovered 40 mutants. Sequencing of the transposon insertion sites of these mutants revealed multiple independent disruptions of a $50 \mathrm{~kb}$ cluster in the plasmid 2, which was not present in any of the fully sequenced Pantoea genomes. ZJU23 and herbicolin A was significantly inhibited the mycotoxin biosynthesis and virulence of $F$. graminearum. Our current data revealed ZJU23 and herbicolin A has practical implications in controlling important fungal diseases in plant and possibly in animals. The target of herbicolin A towards fungi is under investigating.

Microbial correlates of $F$ usarium biomass and deoxynivalenol content in individual wheat seeds M. G. BAKKER (1), S. Mccormick (2), R. Dill-Macky (3), (1) USDA ARS, Peoria, IL, USA; (2) USDA/ARS/NCAUR, Peoria, IL, USA; (3) University of Minnesota, St. Paul, MN, USA

Fusarium head blight is associated with mycotoxin accumulation in grain, which is a food safety hazard. Aiming to identify novel management approaches for this problem, we used qPCR, gas chromatography, and amplicon sequencing to examine correlations between Fusarium biomass or deoxynivalenol (DON) content and characteristics of the microbial communities inhabiting wheat grain. Microorganisms inhabiting the wheat seed could lessen DON content by reducing Fusarium biomass, or by lowering DON production per unit Fusarium biomass. Samples were collected from a common wheat variety planted across a mist irrigated nursery in St. Paul, Minnesota, in each of two successive years. Individual seeds were analyzed in an attempt to approach the fine spatial scale at which microbial communities are organized. Out of 96 seeds tested in 2016, both Fusarium and DON were present at measurable levels in every seed tested. Out of 192 seeds tested in 2017, DON was detected in only $27 \%$ of seeds. Where measurable, relationships between Fusarium biomass and DON content were strong $\left(\mathrm{r}^{2}=0.78\right.$ in 2016 and 0.72 in 2017; $\left.P<0.001\right)$. Microbiome profiles revealed that for certain taxa, abundance among seeds was correlated with pathogen biomass or with the deviation from the expected relationship between Fusarium biomass and DON content. Seeds collected from the same head harbored microbiomes that were on average more similar to each other than seeds collected from different heads. This indicates some biogeographic patterning to the assembly of the wheat spike microbiome. The microbial communities associated with wheat seeds have the potential to substantially impact the development of Fusarium head blight and the accumulation of mycotoxins in grain. With rigorous technology development, the microbiome of wheat heads may become a target for agricultural management. 
Influence of agronomic factors on fusarium and mycotoxins spectra winter wheat in Poland

Z. SAWINSKA (1), K. Stuper-Szablewska (2), S. Switek (3), R. Andrzejak (4), T. Kosiada (4), R. Głowicka-Wołoszyn (5), (1) Departament of Agronomy, Poznan University of Life Sciences, Poznan, POLAND; (2) Department of Chemistry, Poznan University of Life Sciences, Poznan, POLAND; (3) Institute of Zoology, Poznan University of Life Sciences, Poznan, POLAND; (4) Poznań University of Life Sciences, Department of Phytopathology, Poznan, POLAND; (5) Department of Finance and Accounting, Poznan University of Life Sciences, Poznan, POLAND

Fusarium head blight is one of the most important cereal diseases worldwide. Cereals differ in terms of the main occurring Fusarium species and the infection is influenced by various factors, such as weather and cropping measures. Little is known about Fusarium species in winter wheat in Poland, hence harvest samples from growers were collected in 2016 and 2017, along with information on respective cropping factors. The incidence of different Fusarium species was obtained by using a seed health test and mycotoxins were quantified by GC-MS/MS. With these techniques, the most dominant species, in 2016 is F. poae and in 2017 is F. graminearum, and the most prominent mycotoxin, deoxynivalenol (DON), were identified. Between the two main Poland fungicide protection systems, extensive and intensive, we observed differences with the fusarium lowest incidence and toxin accumulation in fungicide application on flowering (BBCH 65-69) winter wheat. We observed that wheat samples from fields with maize as previous crop had a substantially higher Fusarium incidence and elevated mycotoxins accumulation compared with other previous crops. Further factors increasing Fusarium infection and NIV concentration were high nitrogen fertilisation. Results from the current study can be used to develop optimized cropping systems that reduce the risks of mycotoxin contamination.

Eliciting, antimicrobial and film-forming properties of chitosan applied on fresh fruit and vegetables G. ROMANAZZI (1), S. Bautista-Banos (2), E. Feliziani (1), D. Sivakumar (3), (1) Marche Polytechnic University, Ancona, ITALY; (2) Centro De Desarrollo De Productos Bioticos, Yautepec, Morelos, MEXICO; (3) Tshwane University of Technology, Pretoria, SOUTH AFRICA

Chitosan is a natural biopolymer obtained from crab shells known for its biocompatibility, biodegradability and bioactivity. In human medicine, it is used as stabilizer for active ingredients in tablets, and it is popular in slimming diets. Due to this low toxicity, it is the first compound in the list of basic substances (Reg. EU 2014/563) approved in EU for plant protection, in both organic agriculture and IPM. When applied to plants, chitosan shows a triple activity: i) elicitation of host defenses, ii) antimicrobial activity, and iii) ability to form a film on the treated surface. The eliciting activity of chitosan was studied since the 1990', starting monitoring the activity of enzymes linked to defense mechanisms (chitinase, $\beta-1,3$ glucanase, phenylalanine ammonialyase, etc.) in different fruits (i.e. strawberries and other berries, citrus, table grapes), followed by investigations with qRT-PCR and in the last years with RNA-Seq. The antimicrobial activity of chitosan against a wide range of plant pathogens is confirmed by thousands of in vitro and in vivo studies. Once applied on a plant surface by dipping or spraying, chitosan is able to form an edible coating, whose properties (i.e. thickness, gas and water permeability) depends on the acid in which the biopolymer is dissolved. Several commercial chitosan formulations are available to be used as a biopesticides, and their effectiveness arise from the combination of the three involved mechanisms of action.

Curing apples to control blue mold rot

R. M. VALDEBENITO-SANHUEZA (1), M. Fernandes (2), D. M. Trombert de Oliveira (3), (1) PROTERRA Research Center, Vacaria, BRAZIL; (2) Embrapa Wheat, Passo Fundo, BRAZIL; (3) Universidade Federal de Minas Gerais, Belo Horizonte, BRAZIL

One of the major postharvest problems of apples, in Brazil, is decay caused by fungi including blue mold rot incited by Penicillium expansum that infect fruits through wounds. Careful handling, correct cool chain management adequate cold storage facilities are important to reduce fruit losses. Curing can be achieved by holding fruits at temperatures and humidity conducive to wound healing and detrimental to pathogen attack. In general, the combination of temperature and humidity crop specific. Therefore, the aim of this work was to evaluate wound healing on Fuji apples fruits exposed at different temperatures $\left(10^{\circ} \mathrm{C}\right.$ to $\left.17^{\circ} \mathrm{C}\right)$ and relative humidity $(95$ and $100 \%)$ during different periods. The amount of decay by blue mold during subsequent storage at $17^{\circ} \mathrm{C}$ was also examined. Independent of duration period and temperature the water saturated regime provided better control than $95 \%$ relative humidity. At the water saturated regime the best control was achieved by exposing the apple fruits for 15 hours at temperatures of 15 and $17^{\circ} \mathrm{C}$. At the regime of $15^{\circ} \mathrm{C}$ and $100 \% \mathrm{RH}$, the highest induction of curing and reduced apple infection by Penicillium expansum in wounded Fuji apples was obtained by exposing the fruits for 18 and 24 hours controlling 84.1 and $95.2 \%$, respectively. The cured wounded apple tissues maintained at $15 \mathrm{C} / 100 \%$ RH for 18 hours increased tanin reaction when treated with ferric chloride.

Effect of fresh water algae, Chlorella fusca on improving self-life of organic strawberry in cold storage

C. K. SHIM, M. J. Kim, Y. K. Kim, Y. W. Byeon, J. H. Park, E. J. Han, National Institute of Agricultural Sciences, Wanju-gun, KOREA

The most important cultivation technique of strawberry is to prevent air-borne disease and increase hardness. The aim of this study was to improve organically cultivated strawberry quality and self-life through suppression of plant disease with application of Chlorella fusca. We treated seven days old liquid culture of $C$. fusca $\left(1.5 \times 10^{6} \mathrm{cell} / \mathrm{s} / \mathrm{ml}\right)$ was applied to strawberry in the spray and irrigation method with one week intervals. Untreated control of strawberry fruit and leaves showed the symptoms of powdery mildew and gray mold disease with average $35.3 \%$ and $21.2 \%$ of disease incidence, respectively. Among the application method, the combination of spray and irrigation method was the highest control effect of powdery mildew and gray mold disease with $72.3 \%$ of disease control value. The hardness of strawberry treated with C. fusca was significantly higher than that of untreated by 0.3 $\sim 0.7 \mathrm{~N} / \mathrm{cm}^{2}$. The hardness of strawberries treated C. fusca was $1.4 \mathrm{~N} / \mathrm{cm}^{2}$ higher than that of untreated fruits after 14 days of cold storage. After 14 days of cold storage, the soluble solid content of strawberry was investigated, $\mathrm{C}$. fusca treatment was found to be higher than untreated by average 1.0 brix. Decay fungal growth was significantly lower in the chlorella treatments than in the untreated treatments at 14 days after cold storage. A freshwater alga, Chlorella fusca is a new functional microorganism that needs to be studied for mode of action and effective application techniques to apply other crops in organic farming.

In vitro efficacy of plasma activated water against Colletotrichum alienum

S. Siddique, G. Hardy, K. L. BAYLISS, Murdoch University, Murdoch, AUSTRALIA

Cold plasma (CP) has successfully been used for the decontamination of fresh produce from microorganisms, particularly bacteria that cause food safety issues. CP can be used to produce plasma activated water (PAW) which has been demonstrated to have excellent antimicrobial properties. This study investigated the in vitro efficacy of PAW against Colletotrichum alienum, an important postharvest pathogen of avocado. Three volumes of deionised water, 100, 500 and $1000 \mathrm{ml}$, were treated with CP to generate PAW100, PAW500 and PAW1000, respectively. A conidial suspension of C. alienum isolate WAC-13891 $\left(1 \times 10^{6}\right.$ conidia/ml) was then combined with each PAW in a 1:3 ratio (conidia:PAW) and the percentage of germinating conidia were counted after $12,15,18,24$ and $36 \mathrm{~h}$ of treatment. In addition to treating conidia with freshly prepared PAW, each PAW was also stored for $1,3,7$ or 15 days $\left(25^{\circ} \mathrm{C}\right.$ in the dark) and the germination tests repeated. All three PAW significantly reduced conidia germination compared to the control, even after 15 days of storage. The effect of PAW100 on conidia ultrastructure showed a significant change in the cell wall, plasma membrane and cytoplasm 
compared to untreated conidia as observed by transmission electron microscopy. CP technology is now being investigated as an alternative, chemicalfree treatment for controlling postharvest rots of avocado.

Salmonella Typhimurium reduces the population of several phytopathogens in tomato plants

L. DEBLAIS (1,2), C. M. Vrisman (1), D. Kathayat (2), S. A. Miller (1), G. Rajashekara (2), (1) Department of Plant Pathology, The Ohio State University, Wooster, OH, USA; (2) Food and Animal Health Research Program and Sciences, The Ohio State University, Wooster, OH, USA

Salmonella is an important cause of wide-scale gastroenteritis outbreaks related to the consumption of contaminated fruits and vegetables worldwide. The presence of phytopathogens can influence Salmonella survival in planta; however little is know about the interactions between Salmonella and phytopathogens. Experiments were conducted to assess the interactions of $S$. Typhimurium LT2 (ST) with Xanthomonas gardneri (Xg, the causal agent of bacterial spot of tomato) or with Clavibacter michiganensis subsp. michiganensis ( $\mathrm{Cmm}$, the causal agent of canker of tomato), in three-week-old 'Tiny Tim' tomato seedlings $(\mathrm{n}=64)$. The populations of the plant and foodborne pathogens were monitored weekly for 21 days by counting colonyforming units (CFU). When an $S \mathrm{~T}$ suspension $\left(10^{8} \mathrm{CFU} / \mathrm{ml}\right)$ was co-inoculated with $\mathrm{Xg}(100: 1$ ratio) at the same time using leaf spray inoculation, the $X g$ population was reduced by $0.55-\log$ at 7 days post inoculation (DPI) until 21DPI, where $X g$ was 1.47-log lower in presence of $S T$ compared to the plants infected with $X g$ alone $(\mathrm{P}<0.05)$. Similar results were observed when $S T$ was co-inoculated with $\mathrm{Cmm}(100: 1 \mathrm{ratio})$ at the same time using cotyledon clip inoculation. The $\mathrm{Cmm}$ population was 1.38-log lower in presence of $S$ T at 7DPI until 21DPI (0.54-log reduction) compared to the plants infected with $\mathrm{Cmm}$ alone $(\mathrm{P}<0.05)$. Future experiments will focus on understanding the mechanism(s) causing the reduction of $\mathrm{Xg}$ and $\mathrm{Cmm}$ populations in planta in presence of $S T$.

Is fungicide thermo-nebulization the solution for managing postharvest diseases? A. AMIRI (1), V. Koundal (2), (1) Washington State University, Wenatchee, WA, USA; (2) Washington state University, WENTACHEE, WA, USA

For decades, fungicides have been applied through drenching at harvest or applied in wax on the packing line to protect pome fruit from pathogen infections. Drenching may provide the fungicide right to the fruit surface to protect wounds occurring during harvest and transportation. However, crosscontaminations during the drench and generation of fungicide waste can limit its efficiency. Thermo-nebulization (TNB), i.e. thermo-fogging or thermoaerosolization, is a new concept for applying fungicides postharvest and its efficacy has not been investigated. Trials were conducted at 3 commercial packinghouses in the fall of 2016 and 2017, including artificially-wounded fruit inoculated with Penicillium expansum, Botrytis cinerea, and Neofabraea perennans as well as naturally infected fruit. Fruit were treated with thiabendazole, pyrimethanil, or fludioxonil, applied trough drench or TNB, and stored in controlled atmospheres for 5 to 7 months. Disease incidence and severity were determined and fungicide residue levels on fruit were evaluated. Early results suggest that drench provide a higher efficacy than TNB against the three pathogens inoculated artificially. TNB resulted in higher residue levels than drenching and in room and bin-variability was observed in fungicide residue levels following TNB. Our findings suggest that drenching may be more effective than TNB applications, which on the other hand, can mitigate some food safety and environmental risks in the future.

Mycotoxin analysis of Bt and non-Bt maize from ears inoculated with Fusarium subglutinans and $F$. temperatum and infested with lepidopteran insects

D. A. MAYFIELD (1), F. E. Lanza (2), M. Sulyok (3), R. Krska (3), G. P. Munkvold (1), (1) Iowa State University, Ames, IA, USA; (2) CAPES Foundation, Ministry of Education of Brazil, Brasília, BRAZIL; (3) Department of Agrobiotechnology, University of Natural Resources and Life Sciences, Vienna, AUSTRIA

Prior to 2011, Fusarium temperatum $(\mathrm{Ft})$ was referred to as $F$. subglutinans Group I and the role of $\mathrm{Ft}$ in Fusarium ear rot and mycotoxin contamination in North America has not been established. In 2015 and 2016, field experiments were conducted in Iowa with Fs or Ft silk channel inoculations and with corn earworm (Helicoverpa zea) or European corn borer (Ostrinia nubilalis) infestations on Bt and non-Bt maize. Ear rot severity and insect injury were estimated visually and a multi-mycotoxin analysis was performed on milled grain. In both years, Fusarium ear rot severity and insect injury was lower in the $\mathrm{Bt}$ hybrid, and $\mathrm{Ft}$ inoculation resulted in higher levels of moniliformin compared to $\mathrm{Fs}(P<0.0001)$. In 2016, $H$. zeae infestation increased ear rot severity $(P=0.0452)$ compared to the non-infested control and both insects caused increased moniliformin levels in Ft-inoculated treatments $(P \leq 0.0055)$. In 2015, grain from Fs-inoculated treatments had higher levels of fusaric acid than Ft treatments $(P<0.0001)$. In 2016, Fs-inoculated treatments had the highest levels of fusaproliferin which were increased by insect infestations, whereas Ft-inoculated treatments had the highest levels of beauvericin, also increased by insect infestations $(P<0.0001)$. There were large differences between $\mathrm{Ft}$ strains regarding moniliformin, fusaproliferin, beauvericin, and gibberellic acid levels. The highest levels of fumonisins in 2015 were detected in non-inoculated, non-Bt, insect-infested treatments. However in 2016, insect infested, non-inoculated treatments of both hybrids had the highest fumonisin levels $(P \leq 0.0001)$.

Postharvest fungal decay in onion (Allium cepa L.) storage and the associated risks of Listeria monocytogenes K. H. BRITT (1), T. V. Suslow (2), (1) University of Florida, Gainesville, FL, USA; (2) UC Davis, Davis, CA, USA

In response to an extensive 2012 commercial recall of fresh-cut onions due to Listeria monocytogenes, the presence of field and short-term storage conditions conducive to postharvest decay was investigated as a possible contributing factor to pervasive contamination by $L$. monocytogenes. Following co-inoculation with recognized onion fungal pathogens, Botrytis allii and Aspergillus niger, the survival and growth potential of $L$. monocytogenes in the postharvest storage environment of whole onions, pre-processing was evaluated. L. monocytogenes was shown to survive, but not grow, on the surface of whole onions in optimal storage conditions of $2.5^{\circ} \mathrm{C}$ and $70 \% \mathrm{RH}$, and ambient air atmosphere, as well as in conducive-to-decay storage conditions of $25^{\circ} \mathrm{C}$ and $95 \% \mathrm{RH}$. Net growth was not observed, irrespective of incipient or advanced decay by B. allii or A. niger in those respective environments. $L$. monocytogenes populations inoculated into onion neck-tissue wounds could grow slightly, but not significantly at optimal storage conditions and suboptimal storage conditions of $15^{\circ} \mathrm{C}$ and $75 \% \mathrm{RH}$, regardless of B. allii infections. L. monocytogenes could not grow at conducive-to-disease storage conditions, regardless of onion infection with $A$. niger. B. allii and $A$. niger did not exhibit a positive metabiotic interaction on $L$. monocytogenes survival or net growth when co-inoculated into wounds of whole onions. These results suggest that although raw onions may harbor $L$. monocytogenes, the pervasive environmental detection of the human pathogen and on product was likely the result of other factors associated with the receiving facility.

The Role of Yeasts in the Cranberry Fruit Rot Complex

Z. ZALEWSKI (1), P. S. McManus (2), R. Page (2), (1) University of Wisconsin Madison, Madison, WI, USA; (2) University of Wisconsin-Madison, Madison, WI, USA

Cranberry (Vaccinium macrocarpon) is an economically important fruit crop in North America. Cranberry fruit rot occurs before and after harvest and is believed to be caused by a complex of filamentous fungi. After searching for potential bacterial or yeast pathogens, however, we discovered two 
pathogenic yeast species (Hanseniaspora uvarum and Candida railenensis). In the 2017 season we sampled 10 dry-harvested and 20 water-harvested berry samples from various locations to (i) further explore the potential role of bacteria and yeasts in the fruit rot complex; (ii) determine the prevalence and diversity of pathogenic yeasts; and (iii) assess the effect of harvest method on presence of yeasts and bacteria. In the dry-harvested samples $0-5 \%$ of berries yielded yeasts in the absence of pathogenic, filamentous fungi on PDA, whereas 10-68\% of berries in water-harvested samples yielded yeasts in the absence of filamentous fungi. Isolates are being identified and tested for pathogenicity. Because H. uvarum and C. railenensis were initially discovered in the presence of cycloheximide, we also performed a disc diffusion assay to test their sensitivity to three fungicides used on cranberry (azoxystrobin, chlorothalonil, and prothioconazole). Both yeasts showed high tolerance to the fungicides at doses exceeding field doses by more than 1000 -fold. These data give us important insights into the transmission and control of these new players in cranberry pathology.

Fitness of Aspergillus flavus in soil is affected by temperature and soil microbial community M. DROTT, M. G. Milgroom, Cornell University, Ithaca, NY, USA

Aflatoxin, produced by Aspergillus flavus, commonly contaminates corn and other crops causing acute toxicosis, cancer, immune suppression, and stunted growth in children. Biocontrol strategies have recently been utilized in attempts to decrease aflatoxin contamination by applying large numbers propagules from of non-aflatogenic A. flavus strains to agricultural soils. Despite the increasing use of this method, the relationships between $A$. flavus, the soil community, and aflatoxin have remained unexplored. We hypothesized that aflatoxin production of the fungus mediates its ability to interact with soil microbial communities. To test this hypothesis we compared the fitness of naturally occurring aflatoxigenic and non-aflatoxigenic fungal isolates in autoclaved and natural field soils at suboptimal, optimal and stressful temperatures for $A$. flavus growth $\left(25,37,42^{\circ} \mathrm{C}\right)$. Fitness was measured using qPCR estimates of fungal DNA. In natural soils at $37^{\circ} \mathrm{C}$, the addition of aflatoxins to soil, as well as the ability of the fungus to produce aflatoxin, decreased the fitness of $A$. flavus. At $25^{\circ} \mathrm{C}$ we observed a suppressive effect of the natural soils against both chemotypes of $A$. flavus. We did not observe any differences in fungal fitness in sterile soils. We further investigated the potential for aflatoxin to impact microbial communities using amplicon sequencing at optimal growth temperatures. We observed significant changes in alpha and beta diversity of soil bacterial communities with the addition of aflatoxin and in the presence of aflatoxigenic isolates. We did not, however, observe any specific organisms responsible for the decreased growth we observed in the presence of aflatoxin. Our results provide evidence that aflatoxin production by A. flavus affects the fungus's growth when interacting with soil microbial communities.

Diagnosis and management of postharvest fruit rots of winter squash (Cucurbita maxima) in Oregon's Willamette Valley H. RIVEDAL (1), A. G. Stone (2), K. B. Johnson (1), (1) Department of Botany and Plant Pathology, Oregon State University, Corvallis, OR, USA; (2) Department of Horticulture, Oregon State University, Corvallis, OR, USA

Postharvest fungal rots reduce winter squash profitability in Oregon's Willamette Valley. Commonly, desirable cultivars grown with overhead irrigation show $50-100 \%$ losses by December. Surveys of representative squash fruits in 2016 and 2017 characterized type and severity of postharvest fungal rots. Koch's postulates experiments of novel fungi associated with fruit rots are being conducted. Identified fungi associated with postharvest rots include Fusarium culmorum - a pathogen commonly infecting grasses and some dicotyledonous hosts. We are hypothesizing that this fungus establishes a quiescent infection in fruits via infection of summer flowers. Floral infections may begin when F. culmorum spores in grass debris from previous crop rotations are splashed via overhead irrigation onto squash flowers. If this hypothesis is supported, a proposed management strategy is to grow winter squash under limited irrigation or dryland production. When grown with overhead irrigation, the desirable cultivar 'Sunshine' had up to $100 \%$ storage loss by January, compared to only $5 \%$ storage loss by March when produced under dryland conditions. Additional hypothesized management strategies to reduce irrigation splash include using vetch or brassica mulches that do not host F. culmorum. Field trials comparing dryland, irrigated, and mulched production strategies will be conducted to develop the most effective management strategy for postharvest fruit rots of winter squash.

Fumonisin levels in corn from the Texas High Plains as influenced by harvest date and kernel damage M. L. CARTWRIGHT (1), J. E. Woodward (2), W. Xu (3), J. Bell (4), (1) Texas A\&M University, Lubbock, TX, USA; (2) Texas A\&M AgriLife Extension Service, Lubbock, TX, USA; (3) Texas A\&M AgriLife Research, Lubbock, TX, USA; (4) Texas A\&M AgriLife Extension, Amarillo, TX, USA

In 2017, abnormally high levels of fumonisin were reported in corn in the Texas High Plains. This issue followed periods of above average humidity and below average temperatures that persisted throughout the season. Stalks $(n=100)$ were randomly flagged in a field planted to the hybrid 'DKC62-08'. Ears were collected on 25-Sept and from adjacent plants on 2-Oct and 13-Oct. Sampling dates were prior to, during, and after a period of cool, wet weather. Incidence of Fusarium ear rot was $<5 \%$. After shelling, kernels from five ears were combined, ground, passed through a 20-mesh sieve and assayed with QuickScan Fumonisin Test Kits (Envirologix, Portland, ME). Fumonisin levels varied by date ranging from 1.6 to 31.0 (avg. 9.3 \pm 9.0 ), 0.0 to 7.2 (avg. $4.5 \pm 2.4$ ) and 0.0 to 14.0 (avg. $5.8 \pm 4.8$ ) ppm for the three sampling dates, respectively. Kernels from bulk samples collected on 13-Oct were scored for damage, placed into one of four categories, ground and sieved. Fumonisin levels were determined for three $20 \mathrm{~g}$ sub-samples from each category. Concentrations were lowest for healthy kernels and increased as damage became more severe $\left(\mathrm{R}^{2}=0.945 ; P<0.05\right)$. These results support previous findings regarding the positive relationship between kernel damage and fumonisins; however, additional information on the impact of weather conditions prior to harvest on accumulation of the toxin is needed. Furthermore, the effect of the host plant genetics was not addressed in this study.

The linear mitochondrial genome of the quarantine pest Synchytrium endobioticum; insights in the evolutionary history of an obligate biotroph B. VAN DE VOSSENBERG, Wageningen University and Research, Wageningen, NETHERLANDS

Chytridiomycota (chytrids) inhabit terrestrial and aquatic environments, and represent a basal lineage in true fungi. Most of the described chytridiomycota are free living saprophytes, but several species are notorious pathogens for plants or amphibians. Synchytrium endobioticum is an obligate biotroph chytrid causing potato wart disease. Quarantine measures have been implemented worldwide to control the disease and to prevent its spread. To determine taxonomical relationships, and to gain insights into the evolutionary history of this plant pathogen we assembled and annotated the mitochondrial genome of S. endobioticum and generated mitochondrial genomes for five additional chytrid species. The mitochondrial genome of $S$. endobioticum is a linear 72,865 bp molecule with terminal inverted repeats that encodes 14 mitochondrial genes typically found in fungi. Polymorphisms in $30 \mathrm{~S}$. endobioticum isolates shows clustering in four main mitochondrial lineages, and from our data we conclude that the pest was introduced at least three times in Europe. Strains of pathotype $2(\mathrm{G} 1)$ and $6(\mathrm{O} 1)$ were represented in two mitochondrial lineages, showing that these pathotypes emerged independently. Variations within a strain for polymorphic sites were observed and seem to be consistent in different mitochondrial lineages suggesting that $S$. endobioticum strains are communities of different genotypes with conserved composition. 
Evaluating regional management strategies for avocado laurel wilt

R. CHOUDHURY (1), D. Carrillo (2), L. L. Stelinski (3), E. Evans (2), R. C. Ploetz (4), J. Wasielewski (5), J. H. Crane (2), K. A. Garrett (6), (1) University of Florida, Gainesville, FL, USA; (2) University of Florida, Homestead, FL, USA; (3) University of Florida, Lake Alfred, FL, USA; (4) Tropical Research \& Education Center, University of Florida, Homestead, FL, USA; (5) University of Florida/IFAS Extension, Homestead, FL, USA; (6) Plant Pathology Department, University of Florida, Gainesville, FL, USA

Regional management strategies are critical for the long-term management of diseases that can spread rapidly and persist in the environment, such as laurel wilt. Laurel wilt affects woody plants in the Lauraceae in natural and agricultural ecosystems. The disease is caused by the fungus Raffaelea lauricola and is vectored by several ambrosia beetles. Laurel wilt kills trees shortly after infection, and can spread via rootgrafts. We applied risk assessment to regional management of laurel wilt on avocado, and evaluated the effects of regulatory efforts, natural disasters, and competing ideas for how the disease should be managed. Preliminary analyses suggest regulatory efforts that incentivize removal of diseased trees and discourage abandonment of laurel wilt-affected groves can help to reduce regional disease, and function more effectively than incentivizing or punitive regulations alone. While competing ideas can sometimes lead to development of better management strategies, they slow the adoption of beneficial management tactics, and can lead to increased regional disease. Natural disasters (e.g., Hurricane Irma) may reduce overall disease if affected groves are promptly removed; however, changes in vector behavior and prevalence or reduced management infrastructure that occur afterwards may lead to increased disease. Effective regional management of avocado laurel wilt will require coordination among growers, managers, regulators, and researchers.

The bioSAFE project: Developing tools for the genomic biosurveillance of forest invasive alien pathogens in Canada L. BERNIER (1), P. Tanguay (2), I. Porth (1), W. Hintz (3), C. Landry (4), B. Sinclair (5), G. J. Bilodeau (6), M. Blanchette (7), J. Chapuis (1), P. Hessenauer (4), J. Prunier (1), A. Fijarczyk (4), H. Martin (4), P. Y. De La Bastide (3), N. Feau (8), A. L. Dale (8), K. Hrywkiw (8), R. C. Hamelin (8), (1) Université Laval, Centre d'Étude de la Forêt (CEF), Quebec, QC, CANADA; (2) Natural Resources Canada, Canadian Forest Service, Laurentian Forestry Centre, Quebec, QC, CANADA; (3) University of Victoria Centre for Forest Biology, Victoria, BC, CANADA; (4) Institut de Biologie Intégrative des Systèmes (IBIS), Université Laval, Québec, QC, CANADA; (5) University of Western Ontario, London, CANADA; (6) Canadian Food Inspection Agency, Ottawa, ON, CANADA; (7) McGill University, School of Computer Science, Montreal, QC, CANADA; (8) Department of Forest and Conservation Sciences, University of British Columbia, Vancouver, BC, CANADA

The capacity of forests to provide long-term fibre supply and ecosystem services is threatened by the introduction of Forest Invasive Alien Species (FIAS). The bioSAFE project is developing a biosurveillance pipeline to generate genomic tools that will facilitate biosurveillance to increase preparedness and facilitate early action against FIAS. Dutch Elm Disease [DED] and Sudden Oak Death [SOD] are used as model pathosystems for developing accurate means of detection, identifying pathways of spread, and predicting fitness and outbreak-related epidemiological traits that affect the extent of outbreaks. Ongoing research on DED and SOD includes: 1) comparing whole genomes of the pathogens and their relatives for population genomic studies, 2) identifying genes contributing to parasitic fitness and invasiveness using transcriptomics, 3) genome-wide association studies, 4) quantitative trait locus analysis of progeny from controlled sexual crosses, and 5) developing efficient gene knockdown/knockout protocols for functional validation of candidate genes. Recent results include the assessment of global genomic diversity in pathogen populations, production of knockdown mutants of DED fungi, and development of a target-enrichment panel for Phytophthora spp. The bioSAFE project promises to generate transformative changes to address the challenges of biosurveillance of FIAS by speeding up and improving decision-making to inform FIAS mitigation and management.

Proficiency Testing for Regulatory Plant Pathogen Diagnostics - the United States Model

S. Haymes (1), P. J. Shiel (2), G. Dennis (2), T. S. Robinson (1,3), P. Gautam (1,3), V. A. MAVRODIEVA (1), (1) USDA APHIS PPQ S\&T CPHST, Beltsville, MD, USA; (2) USDA APHIS PPQ S\&T CPHST, Raleigh, NC, USA; (3) North Carolina State University, Raleigh, NC, USA

Regulatory testing of high consequence plant pathogens in the United States (U.S.) is accomplished through a network of university, state, federal and private laboratories. To ensure that molecular diagnostic tests used by these labs are valid for regulatory purposes, the U.S. Department of Agriculture Plant Protection and Quarantine (PPQ) branch established a Proficiency Testing (PT) program administered by the National Plant Protection Laboratory Accreditation Program (NPPLAP). The PT program certifies laboratory analysts to test for the Huanglongbing (citrus greening) pathogens, Plum Pox Virus and/or Phytophthora ramorum using the PT panels designed, produced and validated by the PPQ Beltsville PT group, and distributed to the participants annually. Panel results, submitted on-line, are evaluated by the NPPLAP team. There are over 25 national and two international labs currently participating in one or more PT programs, with over 100 PT certifications issued in the last PT season. The PT program is now established as a useful tool for assuring regulatory bodies and other stakeholders that diagnostic testing is accurate and appropriate for regulatory purposes. Additional benefits of NPPLAP include expanding lab capacity and assistance in the adoption of Quality Management systems for plant diagnostics labs.

National Seed Health Accreditation Pilot Program: Quality management systems approaches to reducing the risk of CGMMV in cucurbit seed T. BRUNS (1), G. P. Munkvold (1), E. V. Podleckis (2), R. L. Dunkle (3), (1) Iowa State University, Ames, IA, USA; (2) USDA, APHIS, Riverdale, MD, USA; (3) American Seed Trade Association, Alexandria, VA, USA

The risk of introducing plant pathogens by seed movement is a critical issue in agriculture. To help fulfill the mission of USDA-APHIS to protect U.S. agriculture, the National Seed Health Accreditation Pilot Program (NSHAPP), has been authorized by USDA-APHIS. NSHAPP is a voluntary program for cucurbit seed importers, administered by the National Seed Health System at Iowa State University Seed Science Center. NSHAPP aims to prevent the introduction of Cucumber green mottle mosaic virus (CGMMV) into the U.S. by organizing the testing of imported seeds. Since 2015, out of over 11,000 lots of imported cucurbit seed, less than $0.4 \%$ of samples have tested positive. Consistent with global discussions on the impact of quality management systems on the risk of pathogen movement with seeds, this program is taking steps to recognize systems approaches to reducing the risk of CGMMV in cucurbit seed. As the systems approach format is refined, the program will be incorporated into a larger framework being developed by USDA-APHIS, called the Regulatory Framework for Seed Health (ReFreSH). A risk assessment has been completed for CGMMV in cucurbit seed production that will aid in the evaluation of quality management system impacts on the risk of CGMMV in seed. This approach may provide an alternative to testing for seeds produced with minimal risk of CGMMV contamination. This program is intended to serve as a model for the broader regulatory framework for seedborne pathogens.

Interceptions of exotic fungi associated with the international movement of medicinal plant material from Asia and the Pacific W. S. K. SUENO, USDA APHIS PPQ, Honolulu Plant Inspection Station, Honolulu, HI, USA 
With the increasing popularity of "alternative" plant-based treatments for health concerns and medical issues comes a concomitant increase in the frequency of these medicinal plants and plant parts being encountered at United States ports of entry. These plants can potentially harbor quarantine significant pests of concern to United States agriculture. Many of these plants are from geographic regions with a paucity of information on fungal diversity, and are on hosts for which the associated fungi may not be well studied or documented. Interceptions of representative medicinal plants and plant parts, primarily from Southeast Asia and the Pacific region, rose steadily during the 2000s, then showed a marked increase starting in the early 2010s. Frequently-recovered fungi included species of Colletotrichum, Pestalotiopsis, Phomopsis, and Phyllosticta. Morphological differences between samples indicated a potential diversity in fungal species infecting the hosts which could, in some cases, be related to host origin.

Impact of accreditation rules on the scope of phytosanitary diagnostic laboratories

P. DE SOUZA TELÓ, V. Duarte, Agronômica - Laboratório de Diagnóstico Fitossanitário e Consultoria, Porto Alegre, BRAZIL

The survey of the botanical species (BS) with import permit by Brazil, between 1997 and 2016, indicated that 214 specific normative instructions compose the country's phytosanitary regulation system. These acts define specific phytosanitary requirements for 2510 combinations among BS, collected plant part (CP), country of origin (CO), and regulated pests (RP), resulting from the combination of 371 botanical species and 35 genera, 68 types of $\mathrm{CP}$ of $76 \mathrm{CO}$ and $697 \mathrm{RP}$. The objective of this work was to evaluate the impact of this scenario on the accreditation of the laboratories of the National Network of Agricultural Laboratories of the Ministry of Agriculture by the Inmetro, an official body of the Brazilian government for accreditation by the ABNT NBR ISO / IEC 17025 - General Requirements for the Competence of Testing and Calibration Laboratories. The conclusion is that the accreditation scope of the laboratories should be constituted by analytical methods versus RP class, disregarding BS and CP.

Next generation sequencing as a tool for pathogen detection in plant introductions grown in quarantine M. M. MALAPI-WIGHT, L. Kumar, R. Turner, J. McCallister, C. Kepner, J. Foster, USDA-APHIS, Plant Germplasm Quarantine Program, Beltsville, MD, USA

The USDA-APHIS Plant Germplasm Quarantine Program (PGQP) is the first line of defense against the entry, establishment, and spread of quarantinesignificant pathogens that could harm U.S. agriculture and environment. Plants maintained at PGQP are tested for multiple pathogens using a combination of molecular methods (i.e. PCR and qPCR), serological tests, and bioassays. However, these tests require previous knowledge of the pathogens infecting each crop. Next generation sequencing (NGS) technologies have provided a powerful alternative for detecting and identifying microorganisms without prior knowledge of their presence in that crop. Our goal at PGQP is to establish NGS as a routine diagnostic tool to detect known and novel pathogens in each imported plant accession. In this study, we used Illumina sequencing-by-synthesis technology to sequence $>70$ quarantined Poaceae clonal accessions that included sugarcanes, turfgrasses, ornamental grasses, and bamboos. Ribosomal-depleted RNA libraries for metagenomics sequencing were generated using the TruSeq Stranded Total RNA with Ribo-Zero Plant Kit and sequenced on an Illumina NextSeq 500 platform. Sequence reads were processed using CLC Genomics Workbench and analyzed using a custom database of 430 viral genomes. A preliminary comparison between conventional laboratory diagnostic tests used by PGQP and NGS technologies suggests that NGS is a powerful tool for diagnostics of pathogens of quarantine importance.

Validating Methods for Eradicating Select Agent and Phylotype I Strains of Ralstonia solanacearum M. HAYES (1), C. Allen (2), (1) UW-Madison, Madison, WI, USA; (2) University of Wisconsin, Madison, WI, USA

Ralstonia solanacearum $(R s)$ is a highly destructive plant pathogen that causes bacterial wilt disease in many diverse crop species, including potato, tomato, and ornamentals. $R s$ can survive for extended periods in soil, water, and plant material, complicating disease prevention and management. Cooltolerant potato- and ornamental-infecting phylotype II (R3bv2) $R s$ strains are quarantine pests in many countries and are highly regulated Select Agents in the U.S., so plant protection officials and researchers need a range of practical, reliable, and validated eradication methods for R3bv2 management and regulatory compliance. Additionally, growers need practical methods to kill $R s$ in contaminated irrigation water, a common source of disease outbreaks. To meet these needs, we measured survival of four R3bv2 strains and two phylotype I strains following treatment with hydrogen peroxide, HuwaSan (chemically stabilized hydrogen peroxide), active chlorine (bleach), heat, ultraviolet radiation, desiccation, and cell lysis steps of two commercial nucleic acid extraction kits. No surviving Rs cells were detected following ten minutes' exposure to 200 ppm hydrogen peroxide, $20 \mathrm{ppm}$ HuwaSan, $50 \mathrm{ppm}$ active chlorine, temperatures above $50^{\circ} \mathrm{C}$, and $30 \mathrm{~s} \mathrm{UV}$ irradiation. All $R s$ strains were susceptible to desiccation on most abiotic and all biotic surfaces tested. Both extraction kits yielded 100\% lysis and no surviving cells. Detailed eradication protocols will be discussed in the context of 1) compliance with Select Agent research regulations and 2) management of a major bacterial wilt outbreak in a greenhouse tomato production facility in West Africa.

Review of quality management systems and accreditation programs to mitigate phytosanitary risk in seed trade S. GARCIA FIGUERA (1), N. McRoberts (2), (1) University of California-Davis, Davis, CA, USA; (2) University of California, Davis, CA, USA

The growth of the seed industry in recent decades, and the complexity of the seed production chain, have resulted in an increasing number of seed consignments being traded globally. Under the current international regulatory framework, countries rely on a series of phytosanitary measures, mainly inspection and certification, to mitigate the risk of introducing regulated pests via seed trade. An initiative called ReFreSH aims to develop a more efficient regulatory framework for seeds that will shift the focus of certification from individual consignments to producers' production systems, which will be certified for the adoption of measures that mitigate pest risk within a systems approach. The idea for ReFreSH is to leverage existing measures used by the seed industry. We reviewed accreditation programs and the mechanisms that the accrediting authorities use to verify compliance. Required measures grouped in common themes such as sanitation and hygiene, use of clean plant material and water management, which suggests consensus about practices that mitigate phytosanitary risk. All of the programs required companies to implement a quality management system and relied on audits to verify compliance. Although seed sampling, seed health testing and treatments emerged as areas that will require more validation and harmonization, the identification of substantial common elements will aid in the development and implementation of ReFreSH.

The National Clean Plant Network: Improving status and availability of clean stock

K. FARRAR (1), M. Al Rwahnih PhD (2), D. Byrne (3), C. A. Clark (4), D. Finch (5), M. Fuchs (6), D. A. Golino (7), R. R. Martin (8), P. Matthews (9), N. Nourse (10), S. Scott (11), S. T. Sim (12), B. Tennis (13), G. Vidalakis (14), (1) Foundation Plant Services, Davis, CA, USA; (2) Foundation Plant Services Facility, University of California, Davis, CA, USA; (3) Texas A\&M University, College Station, TX, USA; (4) Louisiana State University, Baton Rouge, LA, USA; (5) Record Buck Farms, Howey in the Hills, FL, USA; (6) Cornell University, Geneva, NY, USA; (7) University of California, Davis, CA, USA; (8) USDA ARS, Corvallis, OR, USA; (9) Hopsteiner, Yakima, WA, USA; (10) Nate Nourse Consulting, South Deerfield, MA, USA; (11) Clemson University, Clemson, SC, USA; (12) University of California Davis, Davis, CA, USA; (13) Michigan Hop Alliance, Northpost, MI, USA; (14) University of California, Riverside, Riverside, CA, USA 
The National Clean Plant Network (NCPN) is an association of clean plant centers, scientists, educators, state and federal regulators, large and small nurseries, and growers of specialty crops that work to ensure that plant propagation material is clean and available. It was established in 2008 and is funded by the United States Department of Agriculture (USDA). The NCPN clean plant centers and programs produce and distribute asexually propagated plant material free of targeted graft transmissible plant pathogens. Currently there are 30 centers or programs in 24 states and five crop networks that focus on fruit trees, grapes, citrus, hops, berries, roses and sweetpotatoes. The primary focus is on diagnostics, pathogen elimination therapies, establishing and maintaining Foundation collections, and importation and distribution. Activities also include studies of economic benefits of clean stock and education and outreach for stakeholders. The economic benefit of starting a planting with clean stock are large. In grapevines, the benefits of starting with clean grapevine planting stock have been documented in the Northcoast region of California exceeds $\$ 50$ million per year. In fruit trees the economic benefit of one clean plant center in Prosser, WA to nurseries, producers, and consumers based on projected yield loss and quality decline was determined to be approximately \$227 million annually.

Development of assays for the detection and genotyping of regulated plant pathogens using genomic information for identification of molecular markers

G. J. BILODEAU (1), E. Giroux (1), S. C. Briere (1), R. C. Hamelin (2), (1) Canadian Food Inspection Agency, Ottawa, ON, CANADA; (2) Department of Forest and Conservation Sciences, University of British Columbia, Vancouver, BC, CANADA

Engaging in global trade necessitates the implementation of governmental regulations to restrict the movement of organisms on infected plant commodities. These regulations rely heavily on accurate plant disease diagnostics, which can be challenging for fungi/oomycetes that are difficult to isolate and/ differentiate from closely related species. We are developing tools for the identification and genotyping of regulated organisms that will improve these diagnostic efforts. Next-generation sequencing (NGS) can generate sufficient genomic data to help providing answers to biological questions and to identify markers for the development of diagnostic assays. These DNA-based tools are contributing to the genomic toolbox to assess the genetic diversity from the species to the intra-lineage level. The tools we have developed with NGS data included qPCR microsatellite assays, SNPs markers, and AmpliSeq technology to sequence a subset of genes or regions of the genome. These will be presented, using organisms from forestry and agriculture that are included in our regulatory work. Identification assays have been developed using genomic strategies targeting Lachnellula willkommii, European Larch Canker; Phytophthora ramorum, Sudden Oak Death; and Fusarium sporotrichioides in peas, among others. The approach taken to obtain markers by using genomic data and the identification of unique regions will be presented with information regarding assay specificity and sensitivity.

Phytosanitary regulations and ISF's Regulated Pest List Initiative S. THOMAS PHD (1), J. D. Cucuzza (1), R. Ranganathan (2), (1) Monsanto, CREVE COEUR, MO, USA; (2) International Seed Federation, Nyon, SWITZERLAND

The International Plant Protection Convention adopted a commodity standard on the international movement of seeds in 2017. Its purpose is to facilitate trade by identifying phytosanitary measures that can be applied in a multilateral environment, i.e. across multiple countries. Much of the seed trade today is global with seed moving into and out of several countries for parental line increases or commercial hybrid production. Seed trade is negatively impacted as importing countries often set different import requirements for the same crop species resulting in increasingly complex logistics. To better understand when seed is a pest introduction risk (i.e. when seed can be a means for pathogen movement and disease introduction), the International Seed Federation has done an in-depth data review of scientific literature on pests that are being regulated for seed import. The results of this review for regulated pests of 10 vegetable seed species show that a large percentage of import requirements lack a scientific basis. For regulated pests where seed may be a pathway further detailed information has been compiled. An online database of the literature and information is publicly available to growers, industry participants, as well as government regulators.

Virus-tested plant material in Colombia - An appeal for a certification program for important exports

J. CUTLER (1), C. Luechau (1), J. Langer (1), S. von Bargen (1), O. A. Losada (2), F. Casierra-Posada (3), A. Castaneda (4), M. Betancourt Vasquez (5), W. Cuellar (6), E. Arevalo-Peñaranda (7), E. Arvydas Stasiukynas (8), C. Buettner (1), (1) Humboldt-Universität zu Berlin, Phytomedicine Division, Berlin, GERMANY; (2) National University of Colombia, Bogota, COLOMBIA; (3) Pedagogical and Technological University of Colombia - UPTC, Tunja, COLOMBIA; (4) ICA, Bogota, COLOMBIA; (5) CORPOICA, Bogota, Cundinamarca, COLOMBIA; (6) International Center for Tropical Agriculture (CIAT), Cali, Valle del Cauca, COLOMBIA; (7) Instituto Colombiano Agropecuario ICA, Bogota, COLOMBIA; (8) Hacienda Misiones, Cundinamarca, COLOMBIA

The competitiveness of Colombian agriculture in international markets depends on the use of healthy domestic plant material and therefore, virus-free certification can improve quantity and quality of yields and contribute to better trade policy decision-making. The goal of this research is to develop a pilot protocol for routine diagnosis that can be applied in a certification program for virus-tested plant material for several Colombian horticultural products. Three important exports from Colombia have been chosen as model plants for experimentation: ornamental rose (Rosa sp.), cape gooseberry (Physalis peruviana L.), and purple passion fruit (Passiflora edulis Sims). Tests for routine detection of plant viruses affecting these cultivars are being developed based on an inventory of known and novel viruses detected in large and small representative farms in 2016-17. First results of known and novel viruses detected in rose, cape gooseberry and purple passionfruit, respectively by ELISA, RT-PCR and high-throughput sequencing techniques are presented and discussed.

\section{Stone fruit surveys in Texas monitoring for plum pox virus, European stone fruit yellows, phony peach disease, \& light brown apple moth:} 2017-2018

S. C. RHODES, K. Ong, Texas A\&M AgriLife Extension Service, College Station, TX, USA

The Texas Plant Disease Diagnostic Lab continued participation in the National Stone Fruit Survey in 2017, monitoring for pest and pathogens of concern for the stone fruit industry in Texas. The Texas A\&M stone fruit breeding plots and orchards in six different counties in Texas were tested for the presence of plum pox virus (PPV). A total of 462 foliar peach and plum samples were tested for PPV. For virus detection, each sample was tested by ELISA using Agdia ELISA kits following USDA APHIS PPQ and National Plant Diagnostic Network protocols. All 462 samples tested negative for all strains of PPV. Additionally, 834 visual observations were made for European stone fruit yellows (ESFY, causal agent 'Candidatus Phytoplasma prunorum') and phony peach disease (PPD, causal agent Xylella fastidiosa). Disease symptoms were not observed in the surveyed orchards. Jackson traps were also set in each orchard to screen for the light brown apple moth (LBAM, Epiphyas postvittana). A total of 66 traps were collected and LBAM was not observed. These continued survey efforts ensure that stone fruit orchards in Texas remain free of these invasive threats and allow for their early detection if they are introduced. Surveying will continue in 2018, focusing on PPV, ESFY, PPD and LBAM. 
PestLens: A web-based phytosanitary early-warning system

R. NOAR, D. McPhie, S. Emerine, S. Carmack, North Carolina State University, Raleigh, NC, USA

Appropriate and timely safeguarding actions are important to prevent the introduction and minimize the impact of exotic plant pests that can harm United States agriculture and natural resources. PestLens, a cooperative effort between the United States Department of Agriculture's Plant Protection and Quarantine (PPQ) program and the Center for Integrated Pest Management at North Carolina State University, serves as PPQ's phytosanitary earlywarning system that helps PPQ to stay informed about exotic plant pest news so that appropriate safeguarding decisions can be made. The PestLens analysts have expertise in plant pathology, entomology, weed science, and technical communications. Each week, the analysts gather information from a wide range of sources, including hundreds of scientific journals, websites, newsletters, e-mail groups, and automated internet search queries; summarize the new information and provide background knowledge on the pest; and report the information in an e-mail notification that is available to the broader scientific community. The notifications are also available via the PestLens website, which provides a searchable archive of reports for registered users and a secure platform for PPQ action groups to document the safeguarding decisions and actions taken in response to each of the reported phytosanitary events.

Risk assessment for epidemic spread of the quarantined potato pathogen Synchytrium endobioticum in the Republic of Georgia K. F. ANDERSEN (1,2,3), J. L. Andrade-Piedra (4), C. Buddenhagen (2), G. A. Forbes (5), J. Fulton (6), M. Gatto (7), R. Mdivani (8), K. A. Garrett $(1,3,6)$, (1) Institute for Sustainable Food Systems, Gainesville, FL, USA; (2) University of Florida Department of Plant Pathology, Gainesville, FL, USA; (3) Emerging Pathogens Institute, Gainesville, FL, USA; (4) International Potato Center (CIP), Lima, PERU; (5) International Potato Center, Servas, FRANCE; (6) Plant Pathology Department, University of Florida, Gainesville, FL, USA; (7) International Potato Center, Hanoi, VIETNAM; (8) International Potato Center, Tbilisi, GEORGIA

Synchytrium endobioticum (causal agent of potato wart) is a devastating soilborne pathogen. Eradication is difficult and infestation can result in $100 \%$ yield loss, making this a strictly quarantined pathogen worldwide. Emerging epidemics pose a high risk to production in Georgia where potato is an essential staple, grown primarily by smallholder farmers, and yields are among the world's lowest. S. endobioticum was first reported in Georgia in 2014 in a localized outbreak in Adjara. Because pathogen dissemination is primarily via human transport of infested tubers, understanding the local potato seed system is critical. This study was the first to systematically characterize the actors involved in seed and ware potato production and trade in Georgia. To collect this information, an expert elicitation was conducted in 2017 across a broad range of participants from the Georgian potato production sector. We present a model of the current potato seed exchange network for the most important agroecological regions. We integrated network analysis in a risk assessment for S. endobioticum spread in Georgia under 1) no intervention, 2) quarantine, 3) introduction of host plant resistance, and 4) combined quarantine and resistance deployment. Preliminary analyses suggest that under no intervention, risk of spread is high, while rapid and consistent quarantine can be effective. Methods presented here provide a general framework for future seed system risk assessments.

Management of Pest Risks Associated with Plants Imported into the United States for Planting

Y. BALCI, P. Spaine, E. V. Podleckis, N. Somboju, M. Bateman, L. Colon, G. OKeefe, R. Rodriguez-Yanes, I. Singh, D. Hanken, S. Rabindran, USDA, APHIS, Riverdale, MD, USA

Each year millions of plants arrive into the United States for propagation as rooted or unrooted cuttings, bare root plants, plants in growing media, tissue culture plantlets, bulbs, or seeds. Title 7 of the Code of Federal Regulations Part 319.37, addresses requirements and prohibitions for importing plants for planting. The Plants for Planting Manual (https://www.aphis.usda.gov/plant-health/online-manuals) provides the background and procedures for regulating the importation of prohibited plant taxa into the US. When market access is requested for propagation of a prohibited genus, a Pest Risk Analysis (PRA) is conducted by APHIS (Animal and Plant Health Inspection Service). Decisions to allow or deny market access are informed by the findings of the PRA. Relevant information includes the quarantine status of pests of concern identified by the PRA, and whether the importation provides a pathway for the entry and establishment or spread of those pests. Each quarantine pest is assessed for its entry potential, its establishment potential, and the consequences of introduction. Using the findings of the PRA, a risk management strategy is developed, which will address mitigation measures required to prevent entry and establishment or spread of quarantine pests. In certain cases, small quantities of prohibited taxa may be imported for experimental, therapeutic or developmental purposes, under a controlled import permit.

\section{Archival Data and Text Analytics to Track $19^{\text {th }}$ Century Late Blight}

J. RISTAINO, Y. P. Yang, A. C. Saville, R. Guenter, L. Tateosian, NC State University, Raleigh, NC, USA

Phytophthora infestans first caused disease in the US during 1843 around the ports of Philadelphia and New York. Ireland's potato crop was destroyed in 1845. The potato blight caused devastation for many years and led to mass starvation and emigration from the country. There are several theories about the origin of the disease and the source of the 19th century outbreaks. We used historical text documents from contemporary literature of the time to investigate spatial information about disease outbreaks, pathogen origin and spread, and methods of management. The methodologies for automatically extracting information from these voluminous data sources will be discussed. The geographic locations that are proximate in the text to key terms related to potato blight were identified and mapped. Data sources include agricultural documents with extensive discussions of crop yields and failed crop, seed tuber exports and import, weather conditions and along with location names. Natural language processing tools were applied to automate text mining of the data within narrative passages. Specifically, we used text analytics tools from the Natural Language ToolKit (NLTK) and location name extraction and disambiguation from Bericos CLAVIN geoparser. NLTK and CLAVIN were coupled to mine the relationships between locations and reports of potato disease. Interestingly, the maps of US $19^{\text {th }}$ century late blight and modern $21^{\text {st }}$ century disease are strikingly similar. An interactive web mapping tool was developed for users to spatially explore the pertinent data for trends in the emergence of 19th century late blight. New insights from archival data may change our view on the source and spread of this devastating disease.

Pathogenomic analysis of the wheat pathogen Puccinia striiformis f. sp. tritici populations in the United States reveals key effectors G. Wu, B. LYON, K. D. Broders, Colorado State University, Fort Collins, CO, USA

Stripe rust, caused by the fungal pathogen Puccinia striiformis f. sp.tritici (Pst), is one of the most devastating diseases of wheat and is present in all major wheat-growing regions of the world. Stripe rust has traditionally affected cooler regions with higher moisture levels, such as the U.S. Pacific Northwest, but in the last 15 years its geographic footprint has expanded due to incursions of novel Pst races that have unique and broader virulence profiles, are better adapted to warmer environments, and are more aggressive than previously characterized races. Effectors, a group of virulence proteins deployed by the pathogen to manipulate plant cell structures and functions, might contribute to the aforementioned expansion. To better understand the 
diversity, distribution and function of Pst effectors in the pathogen population, we collected 16 field Pst isolates from several regions across the U.S. and one Pst isolate from our greenhouse, and sequenced their transcriptomes. We further assessed the transcriptomes for genome-wide effector diversity and differential expression to identify key effectors that likely contribute to the virulence of Pst. These key effectors could be targets for further molecular characterization as well as be used to devise novel surveillance tools and development of host resistance.

Influence of pulse electromagnetic field and storage period on severity of seed-borne infections of soybean in India

R. HUNJE (1), B. Badiger (2), D. P. Biradar (3), N. K. Biradarpatil (4), (1) Department of Seed Science \& Technology, Dharwad, INDIA; (2) Department of Seed Science \& Technology, College of Agriculture, Hanumanmatti, Dharwad, INDIA; (3) University of Agricultural Sciences, Dharwad, Dharwad, INDIA; (4) College of Agriculture, Vijayapur, Dharwad, INDIA

Pulse electromagnetic field and storage period often influence profile of seed borne infections in Soybean. The study on influence of pulse electromagnetic field and storage period on severity of seed-borne infections was conducted at University of Agricultural Sciences, Dharwad to ascertain the role of different traits and their differential reaction with respect to different varieties. The results identified the seedborne association of Cercopsora kikuchii, Colletotrichum truncatum, Alternaria spp, Phoma spp. and Phompsis spp. There was no significant influence on different dosages of pulse magnetic field on per cent seed infection with maximum (11.67\%) with $100 \mathrm{HZ}$ electromagnetic field and untreated control (12.67\%) which are on par with each other. Among six genotypes maximum per cent seed borne infection (18.33) was recorded in JS 71-05 followed by Co-1 (17.33\%) after 12 months of storage. The correlation of various seed quality parameters with seed infection showed negative correlation in respect of seed germination $(\mathrm{r}=$ $-0.961)$, Field emergence $(\mathrm{r}=-0.834)$, seedling length $(-0.129)$, seedling weight $(\mathrm{r}=-0.864)$, seedling vigour index $(\mathrm{r}=-0.968)$, seed yield $(\mathrm{r}=0.761)$ and seed protein $(\mathrm{r}=-0.814)$. The molecular markers such as Satt 285, Satt 316, Satt 434 and Satt 538 were identified for various traits including seedborne infections. Thus, the study brought a new information on role pulse magnetic field, storage period and molecular markers in seed borne infections of soybean in India.

Molecular markers linked to Verticillium wilt resistance in potato germplasm R. D. S. CHOWDHURY, SDSU, Brookings, SD, USA

Verticillium wilt (VW) of potato (Solanum tuberosum), caused by two different soil-borne fungi Verticillium albo-atrum R \& B or V. dahliae Kleb., is a major limiting factor in potato production throughout North America. Yield losses in potato associated with the disease can reach up to $50 \%$ in severely infested fields. In tomato, resistance to race 1 of Verticillium dahliae is conferred by a dominant Ve gene that has been exploited in breeding programs from more than 50 years. However, previously developed markers within the $V e$ gene in potato are unreliable in predicting resistance. The goal of this project is to identify additional genomic regions that determine VW resistance in potato. An F2 mapping population was developed in our lab by selfing an F1 individual derived from two homozygous diploid parents, S. tuberosum DM1-3 (susceptible to VW) and S. chacoense M6 (resistant to VW). Using a rooted cutting protocol, the population was phenotyped and SNP genotyped. A major QTL in chromosome 1 was identified that explains $31 \%$ of the phenotypic variation. A total of 22 genes are located within the QTL region, and two genes have been selected for further functional validation studies. Using the sequence information of these two genes, are developing markers to distinguish between resistant and susceptible germplasm. The marker information will be a valuable tool for potato breeders interested in selecting for VW resistance.

Variations in transcription profiles induced by herbivore feeding in cassava (Manihot esculenta Crantz) M. RAUWANE, Agricultural resrach Council-Wits University, Onderstepoort, SOUTH AFRICA

Cassava mealybug (Phenacoccus manihoti) was introduced into Africa in the 1970s and can cause yield losses of up to $80 \%$, threatening the food security of over 750 million people. Although biological control of mealybug using the parasitoid wasp: Apoanagyrus (Epidinocarsis) lopezi has been practiced with some success, growing resistant cultivars remains an important means by which to manage the pests. A comparison of differentially expressed genes of two cassava genotypes (AR23.1 and P40/1) in response to mealybug infestation was monitored. The transcriptional responses of the two genotypes differed in time as illustrated by the variations in their expression patterns at 24 and 72 hours of mealybug infestation compared to mock infested. We observed mixed responses depending on the genotypes although there was overall suppression of transcripts and less induction, suggesting suppression of the plant's immune response by the pest. Biological pathway analysis using KEGG revealed significant enrichment of plant hormonal signal transduction for up-regulated DEGs, while plant-pathogen interactions was over-represented in down-regulated DEGs for both genotypes. Stressassociated genes such as 2-oxogluterate (2OG) and the HSP20-like chaperones superfamily proteins were induced in the AR23.1 genotype, and were further validated by real-time RT-qPCR. The study revealed a significantly different response to mealybug infestation in the two genotypes studied, with the resistant genotype (AR23.1) showing a higher proportion of differentially expressed transcripts post infestation. Candidate defence-related genes that were overexpressed in the AR23.1 genotype post infestation will be useful in future functional studies towards the control of mealybugs. 